\title{
Review of particle properties
}

\author{
Particle Data Group
}

R. L. Kelly, C. P. Horne, M. J. Losty, A. Rittenberg, T. Shimada, T. G. Trippe,

C. G. Wohl, and G. P. Yost

Lawrence Berkeley Laboratory, University of California, Berkeley, California 94720, USA

N. Barash-Schmidt

Brandeis University, Waltham, Massachusetts, 02154, USA

C. Bricman, C. Dionisi, M. Mazzucato, and L. Montanet

CERN, European Organization for Nuclear Research, Geneva, Switzerland

R. L. Crawford

Department of Natural Philosophy, University of Glasgow, Glasgow, Scotland

\section{Roos}

Department of High Energy Physics, University of Helsinki, Helsinki 17, Finland

\section{B. Armstrong (Technical Associate)}

Lawrence Berkeley Laboratory, University of California, Berkeley, California 94720, USA

This review of the properties of leptons, mesons, and baryons is an updating of Review of Particle Properties, Particle Data Group [Phys. Lett. 75B (1978)]. Data are evaluated, listed, averaged, and summarized in tables. Numerous tables, figures, and formulae of interest to particle physicists are also included. A data booklet is available.

PACS numbers: $14.20 .-\mathrm{c}, 14.40 .-\mathrm{n}, 14.60 .-\mathrm{z}, 13.90 .+\mathrm{i}$

\section{CONTENTS}

I. Introduction, credits, consultants

II. Selection of data

III. Nomenclature
A. Quantum numbers

B. Particle names

IV. Conventions and parameters for strong interactions

A. Partial-wave amplitudes and resonance parameters

B. Sign conventions for resonance couplings

C. Types of partial-wave analyses

D. Production of resonances

V. Criteria for resonances

VI. Conventions and Parameters for Weak and Electromagnetic Decays

A. Muon-decay parameters

B. $K$-decay parameters

C. $\eta$-decay parameters

D. Baryon decay parameters

VII. Statistical Procedures

A. Unconstrained averaging

B. Constrained fits

Acknowledgments

References (for above sections)
Tables of Particle Properties

$\begin{array}{ll}\text { Stable particles } & \text { S17 }\end{array}$

Addendum $\quad$ S20

Mesons

Baryons

Miscellaneous Tables, Figures and Formulae

Physical and numerical constants (rev.)

Clebsch-Gordan coefficients, spherical harmonics and $d$ functions

$\mathrm{SU}(3)$ isoscalar factors (rev.)

Probability and statistics

Relativistic kinematics (rev.)

Lorentz invariant phase space formulae

Weak interactions of quarks and leptons (new)

Particle detectors, absorbers and ranges (rev.)

Electromagnetic relations (rev.)

Radioactivity and radiation protection

C. M. energy and momentum versus beam momentum (rev.)

Periodic table of the elements (rev.)

Cross section plots (rev.)

Data Card Listings

Illustrative key

Stable particles

Leptons

Mesons

Baryons

Searches

Mesons, $S=0$

$S= \pm 1$

charmed mesons

Baryons, $S=0$

$S=+1$

$S=-1$

S63

$\mathrm{S} 67$

S95

$\mathrm{S} 106$

S117

S162

S1 73

S1 75

$\mathrm{S} 224$

S226

Copyright $(1980$ American Physical Society 
$S=-2$

$S=-3$

charmed baryons

dibaryons

Appendix I. Test of $\Delta I=1 / 2$ rule for $K$ decays

Appendix II. Test of $\Delta I=1 / 2$ rule for hyperon decays

Appendix III. SU(3) classification of resonances

Appendix IV. Growth of information

\section{INTRODUCTION, CREDITS, CONSULTANTS}

This review is an updating through December 1979 of our previous review of particle properties [Particle Data Group (1978)]. As in previous editions we have attempted to make the text as complete and self-contained as possible.

As usual, the results of our compilation are presented in two sections, the Tables of Particle Properties and the Data Card Listings. The Tables summarize the properties of only those particles whose existence is in our judgment experimentally well founded and which have a large probability of standing the test of time. This is a conservative judgment, and surely some genuine resonances are omitted, awaiting confirmation (see section $\mathrm{V}$ below).

The Data Card Listings give up-to-date information, with references, on all reported particles, whether considered well established or not. The Listings also contain mini-reviews on questions of interest.

A history of the Particle Data Group, with a discussion of procedures and problems, has been given by Rosenfeld (1975) and a short survey of the history of some of the constants we compile can be found in Appendix IV.

We have maintained in this review the statistical procedure introduced in 1976 , i.e., we give simultaneously in the Listings the old (labeled "AVG") and new (labeled "STUDENT") average values and errors. Details may be found in Sec. VII.

A pocket-sized Particle Properties Data Booklet, containing the Tables and a reprint of the figures and formulae from the first part of the review, is available on request. For North and South America, Australia, and the Far East, write to Technical Information Department, Lawrence Berkeley Laboratory, Berkeley, CA 94720, USA. For all other areas, write to CERN Scientific Information Service, CH-1211 Geneva 23, Switzerland.

As usual, we wish to emphasize that we compile the experimental results of others. It is inappropriate to give us the credit for their countless hours of effort. We urge that references be given directly to the original data, and we provide complete references in the Data Card Listings for that purpose.

The responsibilities for the various sections can be broken down as follows:

(1) Stable particles: N. Barash-Schmidt, C. P. Horne, M. J. Losty, T. Shimada, and T. G. Trippe.

(2) Meson resonances: C. Dionisi, M. J. Losty, M. Mazzucato, L. Montanet, and M. Roos.

(3) Baryon resonances: C. Bricman. R. L. Crawford, C. P. Horne, R. L. Kelly, M. J. Losty, and C. G. Wohl.

(4) General, including text: All authors.

Consultants: To overcome unavoidable gaps in our coverage, both intellectual and geographical, we have solicited the help of consultants:

- U. Amaldi (CERN),

- W. B. Atwood (SLAC),

- A. Barbaro-Galtieri (Lawrence Berkeley Laboratory),

- V. E. Barnes (Purdue University),

- R. Cahn (LBL),

- M. S. Chanowitz (LBL),

- J. Engler (DESY),

- G. Feldman (SLAC),

- F. Foster (University of Lancester),

- F. Gilman (SLAC),

- G. Goldhaber (Lawrence Berkeley Laboratory),

- R. Hagstrom (Lawrence Berkeley Laboratory),

- F. Mönnig (Karlsruhe),

- R. G. Moorhouse (University of Glasgow),

- O. E. Overseth (University of Michigan),

- S. I. Parker (Lawrence Berkeley Laboratory),

- M. Perl (SLAC),

- R. E. Shriock (SUNY Stony Brook),

- K. Shizaya (Law rence Berkeley Laboratory),

- B. N. Taylor (U. S. National Bureau of Standards). The usefulness of this compilation depends in large part on the interaction between the users and the authors and consultants. We appreciate comments, criticisms, and suggestions for improvements of all stages of data retrieval, processing, evaluation, and presentation.

\section{SELECTION OF DATA}

All particles are considered to fall into one of the three groups:

(1) Stable particles, immune to decay via the strong interaction, including the $\eta$ and the photon and the leptons.

(2) Meson resonances.

(3) Baryon resonances.

The charmed, charmonium, and other new flavor particles have been merged into these groups.

These groups are maintained within the two main parts of the compilation:

(1) Tables of Particle Properties.

(2) Data Card Listings.

The Data Card Listings contain the original information (data, references, etc.), weighted averages, comments, and "mini-reviews". Immediately preceding the Data Card Listings is an illustrative key thereto. We attempt to give complete Data Card Listings up to our closing date (January 1, 1980) for all journals listed in the Illustrative Key. We also include preprints and unpublished conference reports that have come to our attention, but make no claim to completeness.

Roughly $40 \%$ of our encoded results, those set off in parentheses, are not used for averaging. The reasoning is then often given in a footnote below the data. If the reason is not given, it is one of the following:

- The result was presented with no error stated.

- The result comes from a preprint or conference report. It is our experience that such results (and 
particularly the errors) often change before final publication. Accordingly we keep these new results in parentheses until they are published (or explicitly verified to us by the authors).

- It involves some assumptions that we do not wish to incorporate.

- It is of poor quality, e.g. bad signal-to-noise ratio.

- It is inconsistent with other results, e.g. because of different methods employed, rendering averaging meaningless.

- It is not independent of other results, e.g. it is a result from one of several partial-wave analyses all using the same data, again rendering averaging meaningless.

When the data for a particle have received special treatment or present special problems, this is noted in a mini-review in the Data Card Listings.

The Tables of Particle Properties represent the output of weighted averages and some critical judgment. The extent to which "blind" averaging has been tempered with judgment is explained in footnotes to the Tables. In general, however, the footnotes are less complete than is the collection of notes and mini-reviews in the Data Card Listings. The reader is thus encouraged to become familiar with the Data Card Listings and, ultimately, with the original references.

\section{NOMENCLATURE}

\section{A. Quantum numbers}

The symbols $I^{G}\left(J^{P}\right) C_{n}$ represent:

$$
\begin{aligned}
I & =\text { isospin, } \\
G & =G \text { parity } \\
J & =\text { spin (also } s) \\
P & =\text { space parity, } \\
C_{n} & =\text { charge-conjugation parity } \\
& \text { of the isospin multiplet. } \\
& \text { We also use: } \\
B & =\text { baryon number } \\
S & =\text { strangeness, } \\
C & =\text { charm, } \\
l & =\text { orbital angular momentum. }
\end{aligned}
$$$$
C_{n}=\text { charge-conjugation parity for the neutral member }
$$

\section{Mesons}

The charge-conjugation operator $\mathrm{C}$ turns particle into antiparticle and has eigenvalues \pm 1 only for neutral states; so it is useful to define an operator $G$ which has eigenvalues for charged states too. This is usually ${ }^{1}$ defined by

$$
\mathrm{G}=\mathrm{C} \exp \left(i \pi I_{y}\right) \text {. }
$$

A neutral nonstrange, noncharmed state is an eigenstate of $\exp \left(i \pi I_{y}\right)$ with eigenvalue $(-1)^{I}$. Then we can write the eigenvalue equation for the whole multiplet as

$$
G=C_{n}(-1)^{I},
$$

where $C_{n}$ ( $n$ for neutral) is the eigenvalue $C$ would have

\footnotetext{
${ }^{1}$ Most texts define it as in Eq. (1); see e.g. Gasiorowicz (1966); however, sometimes the rotation is taken about $I_{x}$. The difference between the two conventions is mentioned in a footnote in Källen (1964).
}

if applied to the neutral member of the multiplet. Thus, for a $\pi^{\circ}, \mathrm{C}$ has the eigenvalue +1 , and since $I=1, G$ $=-1$. For a charged pion, there are no eigenvalues corresponding to $\mathrm{C}$ and to the isospin rotation, but Eqs. (1) and (2) still give $G=-1$.

Consider a meson as a bound state of fermion-antifermion, e.g. quark-antiquark $\bar{q} q$, with orbital angular momentum $l$, and with the two fermion spins coupling to give a spin $s$. Then one can show that the chargeconjugation eigenvalue [defined as in Eq. (2)] is

$$
C_{n}=(-1)^{l+s} \text {. }
$$

Eqs. (2) and (3) combine to give

$$
G=(-1)^{l+s+I} \text {. }
$$

The parity is

$$
P=-(-1)^{l} \text {. }
$$

Eqs. (3) and (5) combine to give

$$
C_{n} P=-(-1)^{s} \text {, }
$$

so all singlets $\left({ }^{1} S_{0},{ }^{1} P_{1}, \ldots\right)$ have $C_{n} P=-1$, and all triplets $\left({ }^{3} S_{1}, \ldots\right)$ have $C_{n} P=+1$. For proofs of the above, see our 1969 text [Particle Data Group (1969)] and Appendix by $C$. Zemach.

If, instead of $\bar{q} q$, we consider the meson as a state of boson-antiboson (e.g. $A_{2}-\bar{K} K$ ), it turns out that some signs cancel, and Eqs. (3) and (4) (not Eq. (5)!) apply unchanged. Of course, the mesons are often spinless, so $s$ is zero, but the equations are more general. Eqs. (3) and (4) can be considered as selection rules forbidding many decays.

We now use Eqs. (3) and (4) to introduce the concept of "Abnormal- $C_{n}$ " mesons, i.e. mesons that cannot be composed of $\bar{q} q$. For this, it is sufficient to consider the SU(3) subgroup of the full unitary group of flavors, containing the $u, d$, and $s$ quarks in a $\{3\}$ representation.

This triplet of quarks is of course defined to have isospin and hypercharge properties such that $\bar{q} q$ can combine (according to the SU(3) relations $\{3\} \otimes\{3\}$ $=\{8\} \oplus\{1\})$ so as to form only octets and singlets. The non-observation of "exotic" mesons (i.e., mesons in larger SU(3) representations, or mesons requiring at least a $q \bar{q} q \bar{q}$ structure) is of course a direct consequence of the naive quark model. States coupling directly to proton-antiproton channels are sometimes interpreted as "baryonium", requiring $q \bar{q} q \bar{q}$ structure, but this interpretation is model-dependent, and no manifestly exotic mesons have been found. It is slightly less obvious that even some octets are forbidden by the model, namely those with $\left(J^{P}\right) C_{n}=\left(0^{ \pm}\right)_{-},\left(1^{-}\right)_{+}$, $\left(2^{+}\right)-, \ldots$. Such states are not observed, and this is an additional success of the naive quark model classification scheme.

In what follows, do not confuse "Abnormal- $C_{n}$ " with "Normal" or "Abnormal" $J^{P}$, both of which are allowed by the quark model. The series $J^{P}=0^{+}, 1^{-}, 2^{+}, \ldots$ is called Normal because $P=(-1)^{J}$ as for normal spherical harmonics, and $J^{P}=0^{-}, 1^{+}, \ldots$ is called Abnormal.

The top part of Table 1 shows all the low angular momentum states that can be formed from $\bar{q} q$. Note that half of the $J^{P}$ states can be formed by both a triplet and a singlet $\bar{q} q$ state, e.g. ${ }^{3} P_{1},{ }^{1} P_{1}$, or ${ }^{3} D_{2},{ }^{1} D_{2}$. 
TABLE I. Orbital excitations of the $\bar{q} q$ system, and corresponding mesons. For the distinction between Abnormal $J^{P}$ and Abnormal $C_{n}$, see text following Eq. (6) in Section III. Strange and charmed mesons share the same values of $J^{P}$ as the $I=0$ and 1

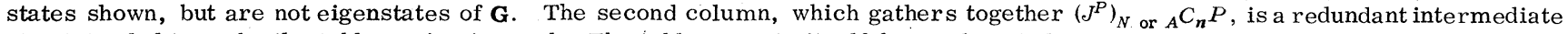
step intended to make the table easier to read. The table repeats itself for each radial excitation.

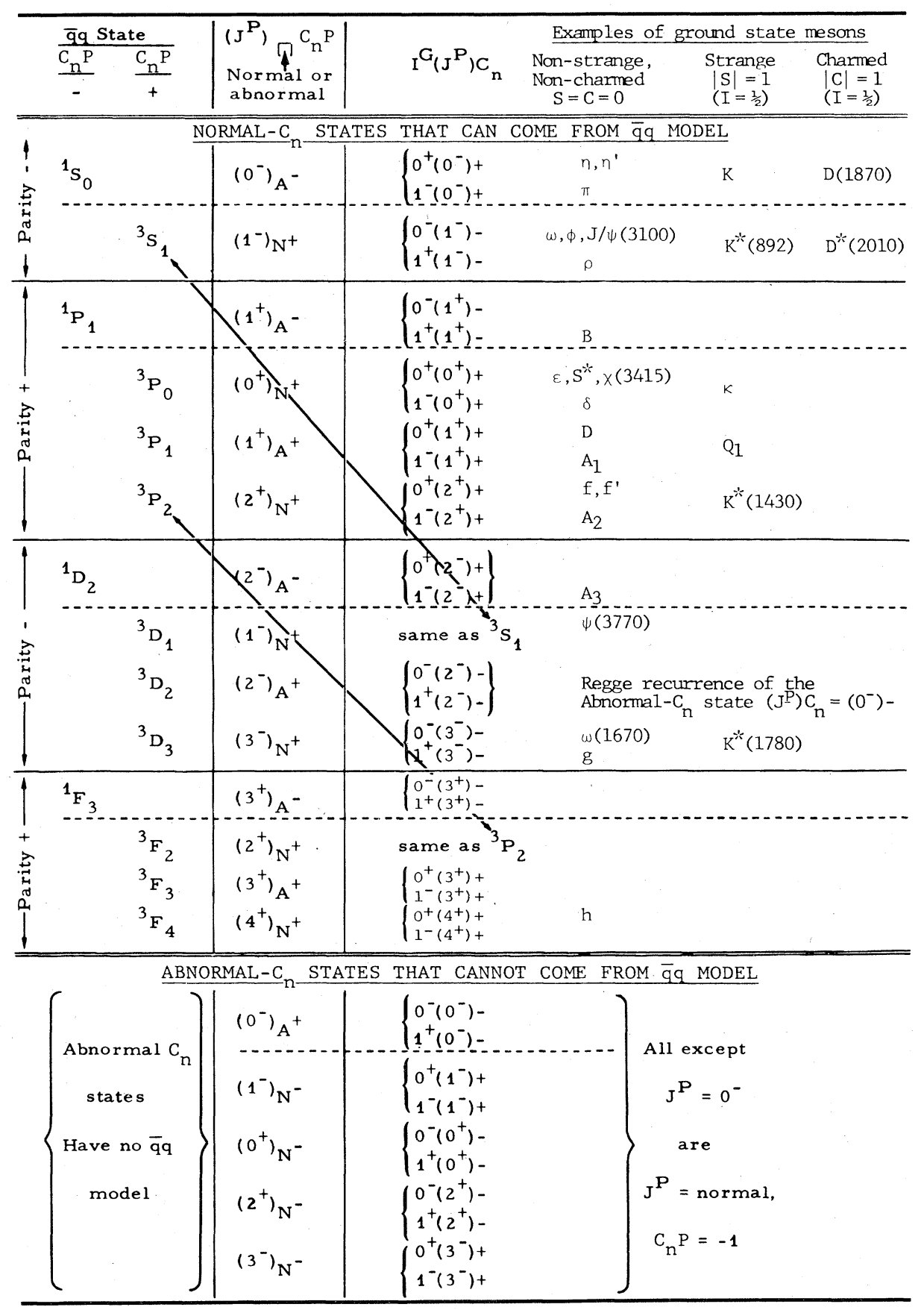


Equation (3) shows that ${ }^{3} P_{1}$ and ${ }^{1} P_{1}$ have opposite $C_{n}$, so the $\bar{q} q$ model allows both. But the states ${ }^{3} P_{0}$ and ${ }^{3} P_{2}$ have no ${ }^{1} P$ counterparts. According to Eq. (6) they have $C_{n} P=+1$, and with the $\bar{q} q$ model there is no way to form a state with a $J^{P}$ of ${ }^{3} P_{0,2}$ (i.e. $J^{P}=$ Normal) and with $C_{n} P=-1$. As mentioned, such octets have not shown up. With the help of Table I one can also see that the special state ${ }^{1} S_{0}, C_{n} P=+1$, cannot be formed, so has Abnormal $C_{n}$.

When, in addition to the $l$-excitation, the re are radial excitations of the $\bar{q} q$ system, Table I repeats itself, and we need a radial quantum number $n$ for each repetition ( $n=1$ for the ground state). Examples of first radial excitations, $n=2$, are $\rho^{\prime}(1600), \psi(3685)$, and $\Upsilon^{\prime}(10060)$. Examples of further possible radial excitations can be found in the $\psi$ and $\Upsilon$ families.

\section{General remarks}

Well-established quantum numbers are underlined in the Tables of Particle Properties (except for stable particles, where most of the quantum numbers are established). We have used what evidence is available (sometimes flimsy) to guess many of the remaining ones, and we have indicated with "?" ones (in the Baryon Table) for which there is almost no evidence.

As is customary, we define antiparticles as the result of operating with CPT on particles, so both share the same spins, masses, and mean lives. Whenever there is a particularly interesting test of CPT invariance we include it in the Stable Particles Table.

\section{B. Particle names}

If a meson has a well-accepted colloquial name, we use it. If not, we name it by a single symbol which specifies its baryon number $B$ ( $=0$ for mesons), its isospin $I$, its strangeness $S$ and charm $C$, and, for a non-strange, non-charmed meson, its $G$ parity.

The name conventions for mesons are given in the first part of Table II.

TABLE II. Particle name conventions.

\begin{tabular}{lllll}
\hline \multicolumn{1}{c}{ Name } & $I$ & $S$ & $C$ & $G$ \\
\hline Mesons & & & & \\
$\eta$ & 0 & 0 & 0 & + \\
$\omega, \phi, \psi, T^{\mathrm{a}}$ & 0 & 0 & 0 & - \\
$\rho$ & 1 & 0 & 0 & + \\
$\pi$ & 1 & 0 & 0 & - \\
$K$ & $1 / 2$ & \pm 1 & 0 & \\
$D$ & $1 / 2$ & 0 & \pm 1 & \\
$F$ & 0 & \pm 1 & \pm 1 & \\
Baryons & & & & \\
$N$ & $1 / 2$ & 0 & 0 & \\
$\Delta$ & $3 / 2$ & 0 & 0 & \\
$Z_{0}, Z_{1}$ & 0,1 & +1 & 0 & \\
$\Lambda$ & 0 & -1 & 0 & \\
$\Sigma$ & 1 & -1 & 0 & \\
$\Xi$ & $1 / 2$ & -2 & 0 & \\
$\Omega$ & 0 & -3 & 0 & \\
$\Lambda_{c}$ & 0 & 0 & 1 & \\
$\Sigma_{c}$ & 1 & 0 & 1 & \\
\hline \hline
\end{tabular}

${ }^{a}$ We use the symbol $\omega$ for those $I^{G}=0^{-}$mesons which are mainly $u \bar{u}$ and $d \bar{d}$ quark states; $\phi$ for those which are mainly $s \bar{s}$ quark states, $\psi$ for mainly $c \bar{c}$ states, and $T$ for mainly $b \bar{b}$ (hypothesized) states.
For some pairs of mesons with supposedly identical quantum numbers, we also use primes; e.g. $\eta, \eta^{\prime} ; f, f^{\prime}$; $\rho, \rho^{\prime}$. Note that primes and subscripts do not carry any further specific meaning.

For baryons no attempt has beøn made to attach a subscript about $J$ and $P$. The name conventions are given in the second part of Table II. For stable baryons of each $I$ and $S$ we use the symbol standing alone; for resonances, the mass is in parentheses [i.e. $N(1688)$, $\Lambda$ (1405), $\Sigma(1765)$, etc.]. The $J^{P}$ assignments are reported in the Baryon Table as $\frac{1}{2}+, \frac{3}{2}-, \frac{5}{2}+$, etc., and also by the symbols $P_{11}, D_{13}, F_{15}$, which refer to the $\pi p$ or $K p$ partial-wave amplitude in which the resonant state occurs (the first subscript refers to the isospin state: $2 \times I$ for $N$ and $\Delta$ and just $I$ for $Z, \Lambda$, and $\Sigma$ ). When two or more baryons have identical quantum numbers we warn the reader by adding primes to the spectroscopic symbol as explained in footnote (a) of the Baryon Table.

\section{CONVENTIONS AND PARAMETERS FOR STRONG INTERACTIONS}

\section{A. Partial-wave amplitudes and resonance parameters}

The vast majority of information concerning baryon resonances comes in the form of partial-wave analyses. In addition data concerning meson resonances ( $\pi \pi$, $K \pi, \pi \pi \pi)$ are, with increasing frequency, being subjected to partial-wave analyses. We thus find it natural to introduce the resonance parameters which we compile in terms of a Breit-Wigner approximation for the partial-wave amplitude.

In general the elastic amplitude for a given angular momentum $l$ may be written as

$$
T_{11}=\frac{\eta \exp (2 i \delta)-1}{2 i},
$$

where $\eta$ is the absorption parameter $(0 \leqslant \eta \leqslant 1)$ and $\delta$ is the phase shift. The subscripts 11 on $T$ denote scattering from channel 1 to channel 1 (e.g. $\pi \pi \rightarrow \pi \pi$ or $\bar{K} K \rightarrow \bar{K} K$ ).

In Fig. 1 we show an Argand plot of the elastic partial wave amplitude $T_{11}$. It illustrates geometrically how the real parameters $\eta$ and $\delta$ are related to the real and imaginary parts of $T_{11}$. Many examples of such Argand plots may be found in the Baryon Data Card Listings.

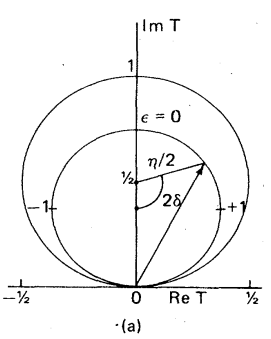

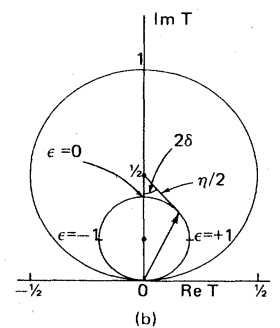

FIG. 1. Argand plots for the elastic partial wave amplitude $T_{11}$. The outer circles are the unitarity bound $(\eta=1)$. The inner circles correspond to the Breit-Wigner approximation of Eq. (2) for (a) $x_{1}=\Gamma_{1} / \Gamma=0.75$ and (b) $x_{1}=0.4$. Note: $\epsilon$ $=/ 2(M-E) / \Gamma$. 
Consider the so-called non-relativistic Breit-Wigner approximation for $T_{11}$ :

$$
T_{11}=\frac{1}{2} \Gamma_{1} /\left(M-E-\frac{1}{2} i \Gamma\right),
$$

where $E$ is the c.m. energy or invariant mass, $\Gamma_{1}$ and $\Gamma$ are the elastic and total widths, and $M$ is the resonance mass. Equation (2) is, of course, not the only possible description of a resonant amplitude; but it suffices to illustrate the properties of partial-wave amplitudes which we associate with resonance behavior in the absence of any background in the same partial wave (see, e.g., the $\pi N D_{15}$ and $F_{15}$ waves in the Baryon Data Card Listings). Usually the widths contain barrier-penetration factors which can vary rapidly with energy. Near threshold, $\Gamma_{1}(E)$ should start up as $q^{2 l+1}$ (also true for the inelastic width $\Gamma_{\beta}$ ). Various $E$ dependences are then used for $\Gamma_{1}$, mostly of the form

$$
\Gamma_{1}(E) \propto \frac{(q R)^{2 l+1}}{\text { const }+\ldots+(q R)^{2 l}} ;
$$

see Jackson (1964), Pišrút and Roos (1968), and Barbaro-Galtieri (1968).

The BW approximation to the amplitude for an inelastic process leading from channel 1 to channel $\beta$ $(\pi \pi \rightarrow \bar{K} K$ or $\bar{K} N \rightarrow \Sigma \pi$, for example) is

$$
\begin{aligned}
T_{1 \beta} & =\frac{1}{2}\left(\Gamma_{1} \Gamma_{\beta}\right)^{1 / 2} /\left(M-E-\frac{1}{2} i \Gamma\right) \\
& =\left(x_{1} x_{\beta}\right)^{1 / 2}\left[\frac{1}{2} \Gamma /\left(M-E-\frac{1}{2} i \Gamma\right)\right],
\end{aligned}
$$

where

$$
\Gamma=\sum_{1}^{N} \Gamma_{\beta}, x_{\beta}=\Gamma_{\beta} / \Gamma,
$$

and $x_{1}$ (called the elasticity) is often written $x_{e}$. (Note that in the Data Card Listings we use the symbol $P_{\beta}$ to denote $x_{\beta}$.) The channel cross section $\sigma_{1 \beta}$ for the reaction $1-\beta$, for spin $0-$ spin $1 / 2$ scattering, is

$$
\sigma_{1 \beta}=4 \pi \lambda^{2}\left(J+\frac{1}{2}\right)\left|T_{1 \beta}\right|^{2},
$$

where $J=l \pm \frac{1}{2}$.

The important features of Eq. (4) which characterize resonant behavior in the Argand diagram ( $\operatorname{Im} T_{1 \beta}$ versus $\operatorname{Re} T_{1 \beta}$ ) are:

energy variation given by circles with diameter $\left(x_{1} x_{\beta}\right)^{1 / 2}$ and maximum amplitude at $E=M$ of

$$
T_{1 \beta}^{\max }=i\left(x_{1} x_{\beta}\right)^{1 / 2}
$$

a maximum in the speed near resonance, given approximately by

$$
\text { "Speed" (res) }=\left|d T_{1 \beta} / d E\right|_{E=M}=\frac{2\left(x_{1} x_{B}\right)^{1 / 2}}{\Gamma(E)},
$$

for slowly varying $\Gamma(E)$. These features may be related to the $\eta, \delta$ representation of $T_{11}$. Thus when $E=M, \delta$ is either $90^{\circ}\left(x_{1}>\frac{1}{2}\right)$ or $0^{\circ}\left(x_{1}<\frac{1}{2}\right)$ and $\eta$ dips to its minimum value.

These simple properties can be used to judge the presence or absence of resonance behavior in an Argand plot, but do not necessarily constitute the criteria we use (see Sec. V). It must also be kept in mind that Eqs. (2) and (4) are only approximations to the "true" amplitude. The simple picture given above can be distorted by various effects: the presence of "background" in the same partial wave as the resonance,

two resonances in the same partial wave overlapping in energy,

the resonant energy $M$ being close to an inelastic channel threshold, in which case a $K$-matrix-like parametrization is more appropriate,

the speed of the resonance being very slow so that the resonance is very broad, and the Breit-Wigner formula a bad approximation.

\section{B. Sign conventions for resonance couplings}

Consider the partial width $\Gamma_{\beta}$ of a resonance decaying into the channel $\beta$. We can always define a coupling constant such that

$$
\Gamma_{\beta} \propto G_{\beta}^{2} \text {. }
$$

In this case the inelastic amplitude in the BreitWigner approximation, Eq. (4), will go as

$$
T_{1 \beta} \propto G_{1} G_{\beta} /\left(M-E-\frac{1}{2} i \Gamma\right),
$$

where $G_{1}$ is the coupling constant for the elastic channel. In the context of exact $S U(3)$ symmetry the relative signs of the product $G_{1} G_{\beta}$ for different resonances are often useful as a consistency check on SU(3) assignment of baryon resonances. See Appendix II for further details.

In the Data Card Listings for baryon resonances, we tabulate measured values for $\left(x_{1} x_{\beta}\right)^{1 / 2} \propto G_{1} G_{\beta}$. When the sign of the amplitude is determined, it is given; absence of an explicit sign indicates that it is undetermined (not that it is positive). For $\Lambda$ and $\Sigma$ resonances, the signs are chosen according to the convention advocated by Levi-Setti (1969) and used in the table of SU(3) Isoscalar Factors presented in this review. Thus the signs multiplying the Breit-Wigner amplitudes for $\bar{K} N \rightarrow \Sigma(1385) \rightarrow \Sigma \pi, \Lambda \pi$ and $\bar{K} N \rightarrow \Lambda(1405)$ $-\Sigma \pi$ are simply the product of the phases of the appropriate isoscalar factors. This convention is shown in Fig. 2, adapted from Levi-Setti (1969).

\section{Types of partial-wave analyses}

Partial-wave analyses (PWA) are classified into three categories in the Data Card Listings: energyindependent partial-wave analyses (IPWA), energydependent partial-wave analyses (DPWA), and modeldependent partial-wave analyses (MPWA), in increasing order of the number of explicit supplementary hypotheses that are used to extract the amplitudes from experimental data.

In an IPWA, data at different energies are analyzed separately. Usually each partial wave included in the fit is allowed to vary freely (subject to unitarity constraints) over some large region, and waves whose angular momenta are above some cutoff value are assumed to be negligible. The sharp cutoff in angular momentum resolves continuum ambiguities in the solution (such as the overall phase ambiguity), but there remains a finite number of indistinguishable "best" solutions (i.e., solutions corresponding to identical physical observables) which have been codified by Barrelet (1972). In addition, there are generally some 
SU(3) RELATIVE SIGN OF RESONANT AMPLITUDES

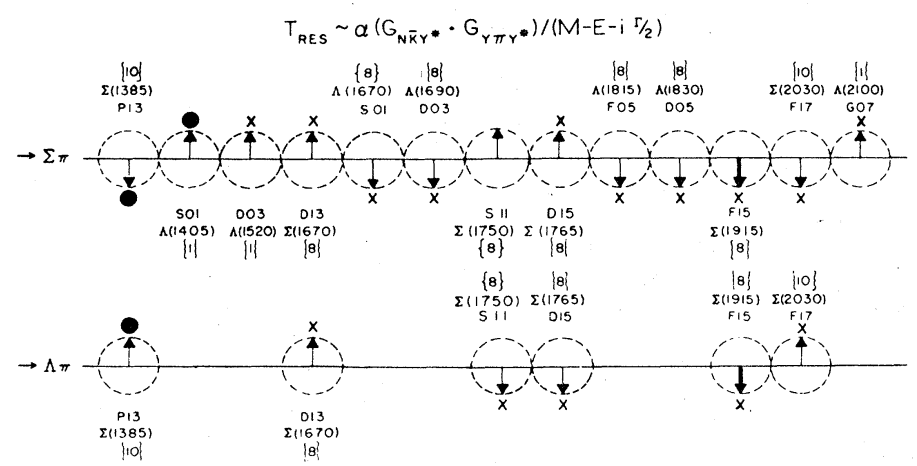

FIG. 2. Plot adapted from Levi-Setti (1969) showing the sign convention adopted here for the $\Sigma \pi$ and $\Lambda \pi$ amplitudes. Once the signs of one $I=0$ and one $I=1$ amplitude are fixed, the others can be measured relative to these two. Arrows here indicate signs predicted by $\mathrm{SU}(3)$; $\times$ marks indicate the observed phases; indicates phase chosen according to sign convention described in text. The $\Sigma(1915)$ predictions have been changed from Levi-Setti's original figure. nearby solutions (and their associated Barrelet ambiguities) which have chi-squared values close to the minimum one.

At the end of the analysis a choice is made among these many solutions, usually on the basis of energy continuity. A popular criterion for making this choice is the shortest path technique in which the total "length" of the preferred solution is chosen to be a minimum. The definition of "length" used here is not universal but is usually closely related to the total geometrical length of the lines representing the various partial-wave amplitudes in Argand plots (see the baryon section of the Data Card Listings for examples of Argand plots). Various other criteria which are also used in some analyses are, e.g., matching with known solutions at low energies, the presence of known resonances in the final results, and limited inelasticity in high partial waves.

In a DPWA, data at different energies are fit simultaneously by using an energy dependent parametrization of the partial-wave amplitudes. The parametrization is usually chosen to include both resonances and nonresonant background of some sort and an attempt is made to keep it as "model independent" as possible. Often the data are grouped into several energy bins which are fit separately rather than trying to fit the whole energy range under consideration simultaneous1y. One of the main advantages of DPWA over IPWA is that sparse data spread over many different energies can be analyzed, e.g., nearly all $S=-1$ analyses are DPWA. In addition, the built-in energy continuity helps to resolve the ambiguities that plague IPWA and eases the problems associated with resonance parameter extraction. The price one pays for these advantages lies in the danger of systematic error in the amplitudes and poor fits to the data if the parametrization is poorly chosen or insufficiently flexible.

An MPWA also uses an energy-dependent parametrization, but one based on explicit model-dependent theoretical assumptions such as Regge exchanges. This technique is usually applied to reactions where the data are incomplete. There is, of course, no sharp distinction between DPWA and MPWA, and a well chosen MPWA parametrization may actually be less biased than a model-independent but poorly chosen DPWA parametrization.

\section{Production of resonances}

Hereby, we mean the observation of statistically significant peaks in invariant mass plots or, loosely, in integrated cross sections. Many meson resonances are of this type. We expect most of these peaks to be associated with Breit-Wigner behavior in appropriate Argand plots; thus the $\rho$ meson peak in $\pi \pi$ mass plots is firmly related to the $I=1, l=1 \pi \pi$ phase shift passing through $90^{\circ}$.

From mass plots we can determine $M, \Gamma$, and the approximate branching ratios

$$
x_{\alpha} / x_{\beta}=\Gamma_{\alpha} / \Gamma_{\beta} \text {. }
$$

In the case of total cross sections, the peak above background gives us, using the optical theorem, the product $\left(J+\frac{1}{2}\right) x_{e}$ :

$$
\sigma^{\text {tot }}(E=M)=4 \pi \lambda^{2}\left(J+\frac{1}{2}\right) x_{e} .
$$

\section{CRITERIA FOR RESONANCES}

An experimentalist who sees indications of a resonance in some energy (or mass) region will of course want to know what has been seen in that region in the past; hence, we strive to have the Data Card Listings serve as an archive for all substantial claims for resonances.

For the Tables of Particle Properties, on the other hand, we wish to be more conservative and to include only those peaks or resonances which we feel have a large chance of survival. An arrow $(\rightarrow)$ at the left of the Tables of Particle Properties indicates that a questionable candidate has been omitted from the Table, but that it can be found in the corresponding part of the Data Card Listings. One's betting odds for survival are of course subjective; therefore no precise criteria can be defined. Very slow speeds ( $\varepsilon$ and $\kappa$ ) make it quite difficult to decide what is a resonance and what is not. For more detailed discussions, see the minireviews in the Listings. In what follows we shall attempt to specify some guidelines.

(a) When energy-independent partial-wave analyses are available (mostly for $N^{*}$ 's), approximate BreitWigner behavior of the amplitude appears to us to be the most satisfactory test for a resonance. We can check that the Argand plot follows roughly a lefthand 
circle, and that the "speed" of the amplitude also shows a maximum near the resonance energy; further, there should be data well above the resonance, showing that the speed again decreases. Indeed proper behavior of the partial-wave amplitude could accredit a resonance even if its elasticity is too small to make a noticeable peak in the cross section.

Of course even if Argand plots are available, it may still be a matter of opinion as to what behavior constitutes a resonance. Such an example is the $Z_{0}(1780)$ state seen in $K N$ total cross-section experiments and in partial-wave analysis. The partial-wave analyses of Giacomelli (1974) and Martin (1975) find preferred solutions which exhibit a resonance-like loop in the $P_{01}$ wave near $1740 \mathrm{MeV}$. However, Giacomelli et al. and Martin point out that, despite the resonantlike appearance of the loop, the evidence for resonant energy dependence is inconclusive. Thus we omit the $Z_{0}(1780)$ from the Baryon Table. A similar quandary has existed for some time concerning the $Z_{1}(1900)$, and it too has been omitted from the Tables.

(b) When there are insufficient data to perform energy-independent analyses, one often resorts to energy-dependent partial-wave analyses (mostly for $Y^{*}$ 's). In this case Breit-Wigner behavior is an input. We therefore require that resonance solutions be found by several different analyses, preferably in different channels $(\bar{K} N \rightarrow \bar{K} N, \pi \Sigma$, etc.), before putting the claim in the table.

(c) Partial-wave analyses of three-body final states $(\pi N \rightarrow \pi \pi N)$ are now available. While these analyses are based on the isobar model $(\pi N \rightarrow \rho N, \pi \Delta$, etc.) and are subject to theoretical objections of varying importance, they provide increasingly reliable information on inelastic decay modes of otherwise established resonances.

(d) Most mesons, $\Xi *$ peaks, and high mass $N^{*}$ and $Y^{*}$ peaks fall into a category for which no partialwave analyses exist. In general we accept such peaks if they are experimentally reliable, of high statistical significance or observed in several different production processes.

Thus, we enter into the Tables of Particle Properties only states for which there is experimentally convincing evidence, and we expect that most of these will be confirmed as resonances.

\section{CONVENTIONS AND PARAMETERS FOR WEAK AND ELECTROMAGNETIC DECAYS}

\section{A. Muon-decay parameters}

The $\mu$-decay parameters describe the momentum spectrum ( $\rho$ and $\eta)$, the asymmetry ( $\xi$ and $\delta)$, and the helicity $(h)$ of the electron in the process $\mu^{ \pm} \rightarrow e^{ \pm}+\nu$ $+\bar{\nu}$. Assuming a local and lepton-conserving interaction, the matrix element may be written as

$$
\sum_{i}\left\langle\bar{e}\left|\Gamma_{i}\right| \mu\right\rangle\left\langle\bar{\nu}\left|\Gamma_{i}\left(C_{i}+C_{i}^{\prime} \gamma_{5}\right)\right| \nu\right\rangle
$$

where the summation is taken over $i=S, V, T, A, P$. Using the definitions and sign conventions of Kinoshita and Sirlin (1957), we have for the momentum parameters

$$
\begin{aligned}
& \rho=\left[3 g_{A}^{2}+3 g_{V}^{2}+6 g_{T}^{2}\right] / D, \\
& \eta=\left[g_{S}^{2}-g_{P}^{2}+2 g_{A}^{2}-2 g_{V}^{2}\right] / D,
\end{aligned}
$$

for the asymmetry parameters:

$$
\begin{aligned}
& \xi=\frac{6 g_{S} g_{P} \cos \phi_{\mathrm{SP}}-8 g_{A} g_{V} \cos \phi_{\mathrm{AV}}+14 g_{T}^{2} \cos \phi_{T T}}{D}, \\
& \delta=\left[-6 g_{A} g_{V} \cos \phi_{\mathrm{AV}}+6 g_{T}^{2} \cos \phi_{T T}\right] / D \xi,
\end{aligned}
$$

and for the parameter describing the helicity of the electron:

$$
h=\frac{2 g_{S} g_{P} \cos \phi_{\mathrm{SP}}-8 g_{A} g_{V} \cos \phi_{\mathrm{AV}}-6 g_{T}^{2} \cos \phi_{T T}}{D} .
$$

Here

$$
\begin{aligned}
& D=g_{S}^{2}+g_{P}^{2}+4 g_{V}^{2}+6 g_{T}^{2}+4 g_{A}^{2}, \\
& g_{i}^{2}=\left|C_{i}\right|^{2}+\left|C_{i}^{\prime}\right|^{2},
\end{aligned}
$$

and

$$
\cos \phi_{i j}=\operatorname{Re}\left(C_{i}^{*} C_{j}^{\prime}+C_{i}^{\prime} C_{j}^{*}\right) .
$$

The quantities $g_{i}$ are defined to be real non-negative numbers, and the $\phi_{i j}$ are phase angles between the $i$ type and $j$-type interactions. Under the assumption of two-component neutrinos $C_{i}^{\prime}=-C_{i}$ and $C_{j}^{\prime}=-C_{j}$, the $S, P$, and $T$ terms vanish, and $\phi_{\mathrm{AV}}$ is the phase angle between $C_{A}$ and $C_{V}$ in the complex plane.

By using the above equations and the experimental determinations of $\rho, \eta, \xi, \delta$, and $h$, limits can be placed on $g_{S} / g_{V}, g_{A} / g_{V}, g_{T} / g_{V}, g_{P} / g_{V}$, and $\phi_{\mathrm{AV}}$. The results, given in the Data Card Listings, assume neither twocomponent neutrinos nor time-reversal invariance. If, however, two-component neutrinos are assumed, then $\sin \phi_{\mathrm{AV}}$ is the amplitude of time-reversal violation. Note that most experiments study only the upper end of the spectrum where $\rho$ and $\eta$ are highly correlated, so they can only report $\rho$ for $\eta \equiv 0$ and $\eta$ for $\rho \equiv \frac{3}{4}$. The values for $\rho$ and $\eta$ we use here were obtained by combining measurements of both upper and lower ends of the spectrum and turn out to be nearly uncorrelated.

Note also that the radiative corrections are unambiguous only when $g_{S}=g_{T}=g_{P}=0$. The same limits on $g_{A} / g_{V}$ and $\phi_{\mathrm{AV}}$ are obtained, however, as when $g_{S}, g_{T}$, and $g_{P}$ are left free.

Current values for the asymmetry parameters as well as $\left|g_{A} / g_{V}\right|$ and $\phi_{A v}$ are given in the Addendum to the Stable Particle Table. In addition, upper limits on $\left|g_{S} / g_{V}\right|,\left|g_{T} / g_{V}\right|$ and $\left|g_{P} / g_{V}\right|$ are given in the $\mu$ section of the Stable Particle Data Card Listings.

\section{B. K-decay parameters}

\section{Dalitz plot for $K \rightarrow 3 \pi$ decays}

The Dalitz plot distribution for the $\tau$ mode $\left(K^{ \pm}-\pi^{ \pm} \pi^{ \pm} \pi^{\mp}\right)$, the $\tau^{\prime}$ mode $\left(K^{ \pm} \rightarrow \pi^{0} \pi^{0} \pi^{ \pm}\right)$, and the $\tau^{0}$ mode $\left(K_{L}^{0} \rightarrow \pi^{+} \pi^{-} \pi^{0}\right)$ of $K$ decay can be parametrized by a series expansion such as that introduced by Weinberg (1960). We use the form

$$
\begin{aligned}
|M|^{2} \propto 1 & +g \frac{s_{3}-s_{0}}{m_{\pi^{+}}^{2}}+h\left(\frac{s_{3}-s_{0}}{m_{\pi^{+}}^{2}}\right)^{2} \\
& +j \frac{s_{2}-s_{1}}{m_{\pi^{+}}^{2}}+k\left(\frac{s_{2}-s_{1}}{m_{\pi^{+}}^{2}}\right)^{2}+\ldots,
\end{aligned}
$$


where $m_{\pi^{+}}^{2}$ has been introduced so as to make the coefficients $g, h, j$, and $k$ dimensionless, and

$$
\begin{aligned}
& s_{i}=\left(P_{K}-P_{i}\right)^{2}=\left(m_{K}-m_{i}\right)^{2}-2 m_{K} T_{i}, \quad i=1,2,3, \\
& s_{0}=\frac{1}{3} \sum_{i} s_{i}=\frac{1}{3}\left(m_{K}^{2}+m_{1}^{2}+m_{2}^{2}+m_{3}^{2}\right) .
\end{aligned}
$$

Here the $P_{i}$ are 4-vectors, $m_{i}$ and $T_{i}$ are the mass and kinetic energy of the $i$ th pion, and the index 3 is used for the odd pion.

The coefficient $g$ is a measure of the slope in the variable $s_{3}$ (or $T_{3}$ ) of the Dalitz plot, while $h$ and $k$ measure the quadratic dependence on $s_{3}$ and $\left(s_{2}-s_{1}\right)$, respectively. The coefficient $j$ is related to the asymmetry of the plot and must be zero if $C P$ invariance holds ( $C$ stands for charge conjugation throughout the discussion in this section). Note also that if $C P$ is good, $g$ must be the same for $\tau^{+}$and $\tau^{-}$, and similarly for $h$ and $k$.

Since different experiments use different forms for $|M|^{2}$, in order to compare the experiments we have converted to $g, h, j$, and $k$ whatever coefficients have been measured. See the mini-review in the $K^{ \pm}$section of the Stable Particle Data Card Listings for details on this point. The results are given in the Addendum to the Stable Particle Table and in the $K^{ \pm}$and $K_{L}^{0}$ sections of the Stable Particle Data Card Listings.

Relations among $\tau^{ \pm}, \tau^{\prime \pm}$, and $\tau^{0}$ are predicted by the $\Delta I=\frac{1}{2}$ rule. See Appendix $I$ for these relations and a discussion of this rule.

\section{Form factors in $K_{\mathcal{L} 3}$ leptonic decays}

Assuming that only the vector current contributes to these decays, we write the matrix element as

$$
M \propto f_{+}(t)\left[\left(P_{K}+P_{\pi}\right)_{\mu} \bar{u}_{l} \gamma_{\mu}\left(1+\gamma_{5}\right) u_{\nu}\right]+f_{-}(t)\left[m_{l} \bar{u}_{l}\left(1+\gamma_{5}\right) u_{\nu}\right],
$$

where $P_{K}$ and $P_{\pi}$ are the four momenta of $K$ and $\pi$ mesons; $m_{1}$ is the lepton mass; $f_{+}$and $f_{-}$are dimensionless form factors which can depend only on $t$ $=\left(P_{K}-P_{\pi}\right)^{2}$, the square of the four-momentum transfer to the leptons. $f_{+}$and $f_{-}$are relatively real if timereversal invariance holds for these decays. $K_{\mu_{3}}$ experiments measure $f_{+}$and $f_{-}$, while $K_{e 3}$ experiments are sensitive only to $f_{+}$because the presence of the lepton mass makes the $f_{\text {- }}$ term negligible.

(a) $K_{\mu 3}$ experiments.

Analyses of $K_{\mu 3}$ data frequently assume a linear dependence of $f_{+}$and $f_{-}$on $t$, i.e.

$$
f_{ \pm}(t)=f_{ \pm}(0)\left[1+\lambda_{ \pm}\left(t / m_{\pi}^{2}\right)\right] \text {. }
$$

Most $K_{\mu_{3}}$ data are adequately described by Eq. (3) for $f_{+}$and a constant $f_{-}$(i.e. $\left.\lambda_{-}=0\right)$. There are two equivalent parametrizations commonly used in these analyses:

(1) $\lambda_{+}, \xi(0)$ parametrization. Analyses of $K_{\mu 3}$ data often introduce the ratio of the two form factors

$$
\xi(t)=f_{-}(t) / f_{+}(t) \text {. }
$$

The $K_{\mu 3}$ decay distribution is then described by the two parameters $\lambda_{+}$and $\xi(0)$ (assuming time reversal invariance and $\lambda_{-}=0$ ). These parameters can be determined by three different methods:

Method $A$. By studying the Dalitz plot or the pion spectrum of $K_{\mu 3}$ decay. The Dalitz plot density is [see, e.g. Chounet et al. (1972)]:

$$
\rho\left[E_{\pi}, E_{\mu}\right) \propto f_{+}^{2}(t)\left[A+B \xi(t)+C \xi(t)^{2}\right],
$$

where

$$
\begin{aligned}
& A=m_{K}\left(2 E_{\mu} E_{\nu}-m_{K} E_{\pi}^{\prime}\right)+m_{\mu}^{2}\left(\frac{1}{4} E_{\pi}^{\prime}-E_{\nu}\right), \\
& B=m_{\mu}^{2}\left(E_{\nu}-\frac{1}{2} E_{\pi}^{\prime}\right), \\
& C=\frac{1}{4} m_{\mu}^{2} E_{\pi}^{\prime}, \\
& E_{\pi}^{\prime}=E_{\pi}^{\max }-E_{\pi}=\frac{m_{K}^{2}+m_{\pi}^{2}-m_{\mu}^{2}}{2 m_{K}}-E_{\pi} .
\end{aligned}
$$

Here $E_{\pi}, E_{\mu}$, and $E_{\nu}$ are respectively the pion, muon, and neutrino energies in the kaon center of mass. The density $\rho$ is fit to the data to determine the values of $\lambda_{+}, \xi(0)$, and their correlation.

Method $B$. By measuring the $K_{\mu_{3}} / K_{e 3}$ branching ratio and comparing it with the theoretical ratio [see, e.g., Fearing et al. (1970)] as given in terms of $\lambda_{+}$and $\xi(0)$, assuming $\mu-e$ universality:

$$
\begin{aligned}
\Gamma\left(K_{\mu 3}^{ \pm}\right) / \Gamma\left(K_{e 3}^{ \pm}\right)= & 0.6457+1.4115 \lambda_{+}+0.1264 \xi(0) \\
& +0.0192 \xi(0)^{2}+0.0080 \lambda_{+} \xi(0), \\
\Gamma\left(K_{\mu 3}^{0}\right) / \Gamma\left(K_{e 3}^{0}\right)= & 0.6452+1.3162 \lambda_{+}+0.1246 \xi(0) \\
& +0.0186 \xi(0)^{2}+0.0064 \lambda_{+} \xi(0) .
\end{aligned}
$$

This cannot determine $\lambda_{+}$and $\xi(0)$ simultaneously but simply fixes a relationship between them.

Method $C$. By measuring the muon polarization in $K_{\mu_{3}}$ decay. In the rest frame of the $K$, the $\mu$ is expected to be polarized in the direction $\mathbf{A}$ with $\mathbf{P}=\mathbf{A}$ / $|\mathbf{A}|$, where $\mathbf{A}$ is given [Cabibbo and Maksymowicz (1964)] by

$$
\begin{aligned}
\mathbf{A}= & a_{1}(\xi) \mathbf{p}_{\mu} \\
& -a_{2}(\xi)\left\{\frac{\mathbf{p}_{\mu}}{m_{\mu}}\left[m_{K}-E_{\pi}+\frac{\mathbf{p}_{\pi} \cdot \mathbf{p}_{\mu}}{\left|\mathbf{p}_{\mu}\right|^{2}}\left(E_{\mu}-m_{\mu}\right)\right]+\mathbf{p}_{\pi}\right\} \\
& +m_{K} \operatorname{Im} \xi(t)\left(\mathbf{p}_{\pi} \times \mathbf{p}_{\mu}\right) .
\end{aligned}
$$

If time-reversal invariance holds, $\xi$ is real, and thus there is no polarization perpendicular to the $K$-decay plane. Polarization experiments measure the weighted average of $\xi(t)$ over the $t$ range of the experiment, where the weighting accounts for the variation with $t$ of the sensitivity to $\xi(t)$.

(2) $\lambda_{+}, \lambda_{0}$ parametrization. Some of the more recent $K_{\mu 3}$ analyses have parametrized in terms of the form factors $f_{+}$and $f_{0}$ which are associated with vector and scalar exchange respectively to the lepton pair. $f_{0}$ is related to $f_{+}$and $f_{-}$by

$$
f_{0}(t)^{\circ}=f_{+}(t)+\left[t /\left(m_{K}^{2}-m_{\pi}^{2}\right)\right] f_{-}(t) .
$$

Here $f_{0}(0)$ must equal $f_{+}(0)$ unless $f_{-}(t)$ diverges at $t=0$. The earlier assumption that $f_{+}$is linear in $t$ and $f_{-}$is constant leads to $f_{0}$ linear in $t$ :

$$
f_{0}(t)=f_{0}(0)\left[1+\lambda_{0}\left(t / m_{\pi}^{2}\right)\right] \text {. }
$$

With the assumption that $f_{0}(0)=f_{+}(0)$, the two parametrizations, $\left(\lambda_{+}, \xi(0)\right)$ and $\left(\lambda_{+}, \lambda_{0}\right)$ are equivalent as 
long as correlation information is retained. $\left(\lambda_{+}, \lambda_{0}\right)$ correlations tend to be less strong than $\left(\lambda_{+}, \xi(0)\right)$ correlations.

The experimental results for $\xi(0)$ and its correlation with $\lambda_{+}$are listed in the $K^{ \pm}$and $K_{L}^{0}$ sections of the Stable Particle Data Card Listings in Sec. XIA, XIB, or XIC depending on whether method $A, B$, or $C$ discussed above was used. The corresponding values of $\lambda_{+}$are listed in subsection $\mathrm{L}+\mathrm{M}$.

Because current experiments tend to use the $\left(\lambda_{+}, \lambda_{0}\right)$ parametrization, we have added a subsection L0 for $\lambda_{0}$ results. Wherever possible we have converted $\xi(0)$ results into $\lambda_{0}$ results and vice versa.

(b) $K_{e 3}$ experiments.

Analysis of $K_{e 3}$ data is simpler than that of $K_{\mu 3}$ because the second term of the matrix element assuming a pure vector current [Eq. (2) above] can be neglected. Here $f_{+}$is usually assumed to be linear in $t$, and the linear coefficient $\lambda_{+}$of Eq. (3) is determined.

If we remove the assumption of a pure vector current, then the matrix element for the decay, in addition to the terms in Eq. (2), would contain

$$
+2 m_{K}\left(f_{S} \bar{u}_{\imath}\left(1+\gamma_{5}\right) u_{\nu}+\left(2 f_{T} / m_{K}\right)\left(P_{K}\right)_{\lambda}\left(P_{\pi}\right)_{\mu} \bar{u}_{l} \sigma_{\lambda \mu}\left(1+\gamma_{5}\right) u_{\nu},\right.
$$

where $f_{S}$ is the scalar form factor, and $f_{T}$ is the tensor form factor. In the case of the $K_{e 3}$ decays where the $f$. term can be neglected, experiments have yielded limits on $\left|f_{S} / f_{+}\right|$and $\left|f_{T} / f_{+}\right|$.

The $K_{e 3}$ results for $\lambda_{+},\left|f_{S} / f_{+}\right|$, and $\left|f_{T} / f_{+}\right|$are listed in the subsections $L+M, F S$, and $F T$, respectively of the $K^{ \pm}$and $K_{L}^{0}$ sections of the Stable Particle Data Card Listings.

See also the Note on $K_{l 3}^{ \pm}$and $K_{l 3}^{0}$ Form Factors in the $K^{ \pm}$section of the Stable Particle Data Card Listings for additional discussion of the $K_{\mu 3}^{0}$ parameters, correlations, and conversion between parametrization and also for a comparison of the experimental results.

\section{3. $\mathrm{CP}$ violation in $K^{0}$ decays}

We list parameters for four different reactions in which $C P$ can be tested [for details, see Okun and Rubbia (1967), Steinberger (1969), and Wolfenstein (1969)].

(a) $K_{S} \rightarrow \pi^{+} \pi^{-} \pi^{0}$.

The quantity measured here is the ratio of amplitudes

$$
A_{S}\left(K_{S} \rightarrow \pi^{+} \pi^{-} \pi^{0}\right) / A_{L}\left(K_{L} \rightarrow \pi^{+} \pi^{-} \pi^{0}\right) \equiv x+i y .
$$

If $C P T$ invariance holds and there is no $I=3$ state present, then $x$ can be neglected and $C P$ violation would be observed as a nonzero $y$. We give the result for Eq. (4) in the $K_{L}^{0}$ section of the Stable Particle Table and under Branching Ratio $R 4$ in the $K_{S}^{0}$ section of the Stable Particle Data Card Listings. Our procedure is to assume that $x=0$, and to list $\left(A_{S} / A_{L}\right)^{2}$ in the form of a branching ratio.

(b) Charge asymmetry in $K_{L} \rightarrow 3 \pi$ decays.

As mentioned above, the presence of a term in $\left(s_{2}\right.$ $-s_{1}$ ) in expression (1) describing the Dalitz plot distribution for $\tau^{ \pm}, \tau^{0}$ decays of $K$ mesons would be an indication of $C P$ violation. Experimenters have used several forms for this $C P$-violation term. As described in the mini-review in the $K^{ \pm}$section of the Stable Particle Data Card Listings, we have converted all results to coefficient $j$ in Eq. (1) above. The latter is listed among the $C P$-violating parameters at the back of the $K_{L}^{0}$ section of the Stable Particle Data Card Listings. Note that only upper limits have been reported for this quantity.

(c) Asymmetry in the $K_{L} \rightarrow \pi^{\mp} l^{ \pm} \nu$ decays.

The quantity measured and compiled here is

$$
\delta=\frac{\Gamma\left(K_{L} \rightarrow \pi^{-} l^{+} \nu\right)-\Gamma\left(K_{L} \rightarrow \pi^{+} l^{-} \nu\right)}{\Gamma\left(K_{L} \rightarrow \pi^{-} l^{+} \nu\right)+\Gamma\left(K_{L} \rightarrow \pi^{+} l^{-} \nu\right)} .
$$

This asymmetry violates $C P$ invariance. If $C P T$ is good, for a pure $K_{L}^{0}$ beam, $\delta$ can be written as

$$
\delta=2\left[\left(1-|x|^{2}\right) /\left(|1-x|^{2}\right)\right] \operatorname{Re} \epsilon,
$$

where $x$ is the $\Delta S=\Delta Q$-violating parameter defined in section B.4, and $\epsilon$ is the parameter of the expansion

$$
\begin{aligned}
& \left|K_{L}\right\rangle=[(1+\epsilon)|K\rangle-(1-\epsilon)|\bar{K}\rangle] /\left[2\left(1+|\epsilon|^{2}\right)\right]^{1 / 2}, \\
& \left|K_{S}\right\rangle=[(1+\epsilon)|K\rangle+(1-\epsilon)|\bar{K}\rangle] /\left[2\left(1+|\epsilon|^{2}\right)\right]^{1 / 2} .
\end{aligned}
$$

We give $\delta$ in the Addendum to the Stable Particle Table. In addition, in the $K_{L}^{0} C P$-violation section of the Stable Particle Data Card Listings, we list $\delta$ separately for $K_{L}^{0} \rightarrow \pi \mu \nu$ and $K_{L}^{0} \rightarrow \pi e \nu$.

(d) $K_{L} \rightarrow 2 \pi$ decay.

The relevant parameters are

$$
\begin{aligned}
& \eta_{+-}=A\left(K_{L} \rightarrow \pi^{+} \pi^{-}\right) / A\left(K_{S} \rightarrow \pi^{+} \pi^{-}\right) \\
&=\left|\eta_{+-}\right| \exp \left(i \phi_{+-}\right), \\
& \eta_{00}=A\left(K_{L} \rightarrow \pi^{0} \pi^{0}\right) / A\left(K_{S} \rightarrow \pi^{0} \pi^{0}\right) \\
&=\left|\eta_{00}\right| \exp \left(i \phi_{00}\right), \\
& \epsilon, \text { defined in Eqs. (5) above, and } \\
& \epsilon^{\prime}=\frac{1}{2} i \sqrt{2} \exp \left[i\left(\delta_{2}-\delta_{0}\right)\right] \operatorname{Im}\left(A_{2} / A_{0}\right) .
\end{aligned}
$$

Here, $A_{i}$ and $\delta_{i}$ are the amplitude and phase of $\pi \pi$ scattering at the $K$ mass, defined by

$$
\begin{aligned}
& \langle I=0|T| K\rangle=\exp \left(i \delta_{0}\right) A_{0}, \\
& \langle I=2|T| K\rangle=\exp \left(i \delta_{2}\right) A_{2} .
\end{aligned}
$$

Wu and Yang (1964) have derived the relationships

$$
\eta_{+-}=\epsilon+\epsilon^{\prime}, \quad \eta_{00}=\epsilon-2 \epsilon^{\prime} \text {. }
$$

We give $\eta_{+-}, \eta_{00}, \phi_{+-}$, and $\phi_{00}$ in the Addendum to the Stable Particle Table. The phases are measured directly, whereas the magnitudes $\eta_{+-}$and $\eta_{00}$ are derived parameters. We use, as far as we can, the directly measured quantities as input and calculate $\eta_{+-}$and $\eta_{00}$ from the values given by our constrained fits. Therefore, if one looks at the Data Card Listings, most of the $|\eta|$ measurements appear in the form of branching ratios, with appropriate comments. We then give the values of $\eta_{+-}$and $\left|\eta_{00}\right|^{2}$ in a separate list at the end of the $C P$-violating parameters section of the $K_{L}^{0}$ section of the Stable Particle Data Card Listings. 


\section{4. $\Delta S=\Delta Q$ rule in $K^{0}$ decays}

The relative amount of $\Delta S \neq \Delta Q$ component present is measured by the parameter $x$, defined as

$$
x=A\left(\bar{K}^{0} \rightarrow \pi^{-} l^{+} \nu\right) / A\left(K^{0} \rightarrow \pi^{-} l^{+} \nu\right) .
$$

We list $\operatorname{Re}\{x\}$ and $\operatorname{Im}\{x\}$ for both $K_{e 3}$ and $K_{\mu 3}$ at the end of the Stable Particle Data Card Listings and give values in the Addendum to the Stable Particle Table.

\section{C. $\eta$-decay parameters}

\section{1. $C$-violation in $\eta$ decays}

As a test of possible $C$-violation in electromagnetic interactions, a number of experiments have looked for possible charge asymmetries in the decays $\eta \rightarrow \pi^{+} \pi^{-} \pi^{0}$ and $\eta \rightarrow \pi^{+} \pi^{-} \gamma$. We list the following parameters:

(a) The left-right asymmetry

$$
A=\left(N^{+}-N^{-}\right) /\left(N^{+}+N^{-}\right),
$$

where $N^{( \pm)}$means the number of events with the $\pi^{( \pm)}$ energy greater than the $\pi^{(F)}$ energy in the $\eta$ rest frame.

(b) The sextant asymmetry

$$
A_{s}=\frac{N_{1}+N_{3}+N_{5}-N_{2}-N_{4}-N_{6}}{N_{1}+N_{2}+N_{3}+N_{4}+N_{5}+N_{6}}
$$

for the decay $\eta \rightarrow \pi^{+} \pi^{-} \pi^{0}$. The numbers refer to the sextants of the Dalitz plot [see, for example, Layter (1972)]. $A_{s}$ is sensitive to an $I=0 C$-violating asymmetry.

(c) The quadrant asymmetry $A_{q}$, defined in a similar way as $A_{s}$, but with each sector of the Dalitz plot now containing $\pi / 2$ rather than $\pi / 3$ radians. $A_{q}$ is sensitive to an $I=2 C$-violating final state.

(d) The $d$-wave contribution to the $C$-violating amplitude in the decay $\eta \rightarrow \pi^{+} \pi^{-} \gamma$. The upper limit for this contribution is measured by the parameter $\beta$, defined by

$$
d N / d|\cos \theta| \propto \sin ^{2} \theta\left(1+\beta \cos ^{2} \theta\right),
$$

where $\theta$ is the angle between the $\pi^{+}$and the $\gamma$ in the dipion center of mass. A term proportional to $\cos ^{2} \theta$ could also be due to $p$ - and $f$-wave interference.

We list $A$ for the decay modes $\eta \rightarrow \pi^{+} \pi^{-} \pi^{0}$ and $\eta$ $\rightarrow \pi^{+} \pi^{-} \gamma, A_{s}$ and $A_{q}$ for the decay $\eta \rightarrow \pi^{+} \pi^{-} \pi^{0}$, and $\beta$ for the decay $\eta \rightarrow \pi^{+} \pi^{-} \gamma$ in the $\eta$ section of the Stable Particle Data Card Listings.

\section{Dalitz plot for $\eta \rightarrow \pi^{+} \pi^{-} \pi^{0}$}

The Dalitz plot for the decay $\eta \rightarrow \pi^{+} \pi^{-} \pi^{0}$ may be fit by the distribution

$$
|M(x, y)|^{2} \propto 1+a y+b y^{2}+c x+d x^{2}+e x y .
$$

Here,

$$
x=\sqrt{3}\left(T_{+}-T_{-}\right) / Q, \quad y=\left(3 T_{0} / Q\right)-1,
$$

$T_{+}, T_{-}, T_{0}$ are the kinetic energies of the $\pi^{+}, \pi^{-}$, and $\pi^{0}$ in the $\eta$ rest system, and $Q=m_{\eta}-m_{\pi^{+}}-m_{\pi^{-}}-m_{\pi 0^{\circ}}$. The coefficient of the term linear in $x$ is sensitive to $C$-violation due to an $I=0$ or $I=2$ final state. We list papers presenting determinations of the parameters $a, b, c$, and $d$ in the $\eta$ section of the Stable Particle
Data Card Listings. However, we do not tabulate values of these parameters because the assumptions made by different authors are not compatible and do not allow comparison of the numerical values.

\section{Dalitz plot for $\eta \rightarrow \pi^{+} \pi^{-} \gamma$}

The Dalitz plot for the decay $\eta \rightarrow \pi^{+} \pi^{-} \gamma$ may be fit to the expression

$$
|M|^{2} \propto 1+2 \alpha z,
$$

where

$$
z=\frac{2}{3} \sum_{i=1}^{3}\left[\frac{3}{m_{\eta}-3 m_{\pi}}\left(E_{i}-\frac{1}{3} m_{\eta}\right)\right]^{2}=\frac{\rho^{2}}{\rho_{\max }^{2}} .
$$

Here $E_{i}$ is the energy of the $i$ th pion in the $\eta$ rest frame, and $\rho$ is the distance to the center of the Dalitz plot. We list the parameter $\alpha$ in the $\eta$ section of the Stable Particle Data Card Listings.

\section{Baryon-decay parameters}

\section{1. $A / V$ ratio for baryon leptonic decays}

Consider the decay

$$
B_{i} \rightarrow B_{f}+l+\nu \text {. }
$$

Assuming $V, A$ theory, neglecting "induced" scalar, "induced" pseudoscalar, and axial weak-magnetism terms, and neglecting the $q^{2}$ dependence of the form factors, the baryon part of the matrix element for these decays may be written [Goldberger and Treiman (1958)] as

$$
\left\langle B_{f}\left|\gamma_{\lambda}\left(g_{V}-g_{A} \gamma_{5}\right)+\left(g_{W} / m_{B_{i}}\right) \sigma^{\lambda \nu} q_{\nu}\right| B_{i}\right\rangle,
$$

where $B_{i}$ and $B_{f}$ represent initial and final baryons, $g_{A}$ and $g_{V}$ the axial and vector coupling constants, $g_{W}$ the weak magnetism coupling constant, and $q_{\nu}$ the sum of the lepton momenta. Here the Pauli representation is used for the $\gamma$ matrices. The ratio $g_{A} / g_{V}$ may be written as

$$
g_{A} / g_{V}=\left|g_{A} / g_{V}\right| \exp (i \phi),
$$

where $\phi$ is $0+n \pi$ if time reversal holds [see Jackson et al. (1957)].

Experiments on the leptonic decays of baryons other than the neutron have generally assumed $\phi$ to be either 0 or $\pi$, and have thus measured the magnitude and sign of $g_{A} / g_{V}$. In studying neutron beta decay, however, experiments have been sensitive enough to measure $\phi$ more precisely, and we include the phase angle in our Listings for this case. It is consistent with time-reversal invariance, and by using the above definition of the matrix element with the Pauli representations, the value of $g_{A} / g_{V}$ in neutron beta decay is negative.

Due to statistical limitation the weak magnetism form factor $g_{W}$ is usually assumed from $C V C$ and $\mathrm{SU}(3)$, so only $g_{A}$ and $g_{V}$ are determined experimentally. This determination is accomplished in a variety of ways.

(a) The lepton-neutrino angular correlation provides a measure of the absolute value of $g_{A} / g_{V}$ [for relevant formulas, see, e.g., Albright (1959)].

(b) The up-down asymmetry of the lepton from polarized baryon decays provides a measure of $g_{A} / g_{V}$ with its sign [for relevant formulas, see, e.g., Albright 
(1959)).

(c) The lepton spectrum, given enough statistics, provides a measure of $g_{A} / g_{V}$ with its sign [for relevant formulas, see, e.g., Bender (1968)].

(d) The polarization of the decay baryon, from polarized or unpolarized initial baryon, also provides $g_{A} / g_{V}$ with its sign [for formulas, see, e.g., Willis and Thompson (1968)].

(e) The presence of a term proportional to

$$
\sigma_{B_{i}} \cdot\left(\mathrm{p}_{e} \times \mathrm{p}_{\nu}\right),
$$

where the initial baryon is polarized or

$$
\sigma_{B_{f}} \cdot\left(\mathrm{p}_{e} \times \mathrm{p}_{\nu}\right),
$$

where the polarization of the decay baryon is observed provides a measure of the deviation of $\phi$ from 0 or $\pi$, and is thus a test of time-reversal invariance [see, e.g., Willis and Thompson (1968)].

We compile the ratio $g_{A} / g_{V}$ with its sign, for those decays for which it has been measured.

All the coupling constants and decay rates for baryon leptonic decays are related by Cabibbo's theory [Cabibbo (1963)], extended to six quarks (and three mixing angles) by Kobayashi and Maskawa (1973). A recent fit to this theory has been done by Shrock and Wang (1978).

\section{Asymmetry parameters in nonleptonic hyperon decays}

The transition matrix for the hyperon decay may be written as

$$
M=s+p(\sigma \cdot \mathrm{q}),
$$

where $s$ and $p$ are the parity-changing and the parityconserving amplitudes, respectively; $\sigma$ is the Pauli spin operator, and $q$ is a unit vector along the direction of the decay baryon in the hyperon rest frame.

The asymmetry parameters are defined by the relations

$$
\begin{aligned}
& \alpha=2 \operatorname{Re}\left(s^{*} p\right) /\left(|s|^{2}+|p|^{2}\right), \\
& \beta=2 \operatorname{Im}\left(s^{*} p\right) /\left(|s|^{2}+|p|^{2}\right), \\
& \gamma=\left(|s|^{2}-|p|^{2}\right) /\left(|s|^{2}+|p|^{2}\right) .
\end{aligned}
$$

With the transition matrix (6), the angular distribution of the decay baryon, in the hyperon rest system, is of the form

$$
I=1+\alpha \mathrm{P}_{Y} \cdot \mathrm{q} \text {, }
$$

where $\mathrm{P}_{Y}=\langle Y|\sigma| Y\rangle$ is the hyperon polarization.

In the notation of Lee and Yang (1957) the polarization $\mathrm{P}_{B}$ of the decay baryon is ${ }^{2}$

$$
\mathbf{P}_{B}=\frac{\left(\alpha+\mathbf{P}_{Y^{\circ}} \mathbf{q}\right) \mathbf{q}+\beta\left(\mathbf{P}_{Y} \times \mathbf{q}\right)+\gamma \mathbf{q} \times\left(\mathbf{P}_{Y} \times \mathbf{q}\right)}{1+\alpha \mathbf{P}_{Y} \cdot \mathbf{q}},
$$

where $P_{B}$ is defined in that rest system of the baryon obtained by a Lorentz transformation along $q$ from the hyperon rest system in which $q$ and $P_{Y}$ are defined.

\footnotetext{
${ }^{2}$ Note that Lee and Yang (1957) contains a misprint. The minus sign in the definition of $\beta$ should be replaced by a 2 . In addition, our unit vector $q$ is the direction of the baryon, whereas their unit vector $\mathbf{p}$ is the direction of the pion.
}

Note that $\alpha$ is the helicity of the decay baryon for unpolarized hyperons.

The three parameters $\alpha, \beta$, and $\gamma$ satisfy the relation

$$
\alpha^{2}+\beta^{2}+\gamma^{2}=1 \text {. }
$$

It is then convenient to describe hyperon nonleptonic decays in terms of the two independent parameters $\alpha$ and the angle $\phi$ defined by

$$
\begin{aligned}
& \beta=\left(1-\alpha^{2}\right)^{1 / 2} \sin \phi, \\
& \gamma=\left(1-\alpha^{2}\right)^{1 / 2} \cos \phi,
\end{aligned}
$$

which has a more nearly gaussian distribution than $\beta$ or $\gamma$. Evidently

$$
\begin{aligned}
& -\frac{1}{2} \pi \leqslant \phi \leqslant \frac{1}{2} \pi \text { for } \gamma>0, \\
& +\frac{1}{2} \pi \leqslant \phi \leqslant \frac{3}{2} \pi \text { for } \gamma<0 .
\end{aligned}
$$

In discussing time-reversal invariance, the quantity of interest is $\Delta$, defined by

$$
\begin{aligned}
& \alpha=2|s||p| \cos \Delta /\left(|s|^{2}+|p|^{2}\right), \\
& \beta=-2|s \| p| \sin \Delta /\left(|s|^{2}+|p|^{2}\right) ;
\end{aligned}
$$

that is, $\Delta$ is the phase angle of $s$ relative to $p$. Evidently

$$
\begin{aligned}
& -\frac{1}{2} \pi \leqslant \Delta \leqslant \frac{1}{2} \pi \text { for } \alpha>0, \\
& +\frac{1}{2} \pi \leqslant \Delta \leqslant \frac{3}{2} \pi \text { for } \alpha<0 .
\end{aligned}
$$

Under the assumption of time-reversal invariance, the angle $\Delta$ must satisfy the relation

$$
\Delta=\delta_{s}-\delta_{p},
$$

modulo $\pi$, where $\delta_{s}$ and $\delta_{p}$ are the pion-baryon scattering phase shifts at the appropriate energy and for the appropriate isospin state. For $\Lambda$ decay, assuming the validity of the $|\Delta I|=\frac{1}{2}$ rule,

$$
\Delta=\delta_{s}-\delta_{p}=(7.0 \pm 1.0) \text { deg. }{ }^{3}
$$

In the Stable Particle Data Card Listings we give $\alpha$ and $\phi$ for each decay since they are the most closely related to the experiments and are essentially uncorrelated. Whenever necessary we have changed the signs of the reported values, so as to agree with our conventions. In the Stable Particle Table we give $\alpha, \phi$, and $\Delta$ with errors; and for convenience we also give the central value of $\gamma$, without an error.

\section{STATISTICAL PROCEDURES}

We divide this discussion on obtaining averages and errors into two sections:

A. the unconstrained case, or "simple averaging", and

B. the constrained case.

In what follows, the term "error" means one standard

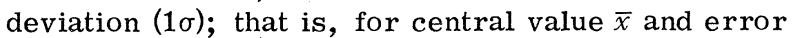
$\delta \bar{x}$, the range $\bar{x} \pm \delta \bar{x}$ constitutes a $68.3 \%$ confidence interval.

\footnotetext{
${ }^{3}$ This value for $\delta_{s}-\delta_{p}$ is derived from the phase-shift analyses by Ayed (1976). The error is our estimation of the uncertainty allowing for possible correlations.
} 


\section{A. Unconstrained averaging}

We first describe the standard procedure we have used for several years to determine averages and errors. We then discuss a second method, which we feel offers a less conservative, but possibly more accurate, estimate of errors.

\section{Standard procedure-Gaussian distribution with scale factor}

We begin by assuming that measurements of a given quantity obey a gaussian distribution, and thus we calculate a weighted average and error

$$
\begin{aligned}
& \bar{x} \pm \delta \bar{x}=\left(\sum_{i} w_{i} x_{i} / \sum_{i} w_{i}\right) \pm\left(\sum_{i} w_{i}\right)^{-1 / 2}, \\
& w_{i}=\left[1 /\left(\delta x_{i}\right)^{2}\right],
\end{aligned}
$$

where $x_{i}$ and $\delta x_{i}$ are the value and error, respectively, reported by the $i$ th experiment, and the sums run over $N$ experiments. We also calculate $\chi^{2}$ and compare it with its expectation value of $N-1$.

If $\chi^{2} /(N-1)$ is less than or equal to 1 , and there are no known problems with the data, we accept the above results.

If $\chi^{2} /(N-1)$ is very large, or if there is prior knowledge of extremely large inconsistencies between experiments, we may choose not to average the data at all. Alternatively, we may quote the calculated average, but then give an educated guess as to the error; such a guess is generally a quite conservative estimate designed to take into account known problems with the data.

Finally, if $\chi^{2} /(N-1)$ is greater than 1 , but not to such a large extent, we still average the data, but then try to make up for this fact in two ways:

(i) We plot an ideogram to guide the reader in deciding which data might be rejected before selected averages are made. An example of such an ideogram is given in Fig. 3 below. Each experiment appearing

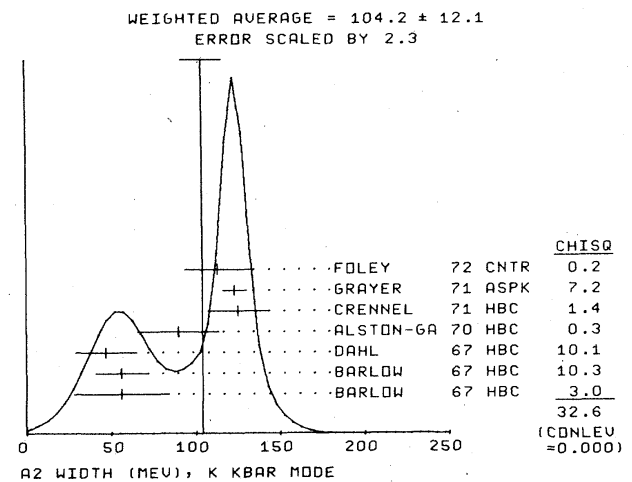

FIG. 3. Ideogram of early measurements of the $A_{2}$ width, as determined from the $K \bar{K}$ mode. The vertical line indicates the position of the weighted average, while the horizontal bar atop the line gives the error in the average after scaling by the SCALE factor. Only those experiments indicated by + error flags were precise enough to be accepted in the calculation of the SCALE factor; the column on the far right gives the $x^{2}$ contribution of each of these experiments. Occasionally, less precise experiments are included in the calculation of the weighted average, but not SCALE; they have $\perp$ error flags. in the plot is represented by a gaussian with central value $x_{i}$, error $\delta x_{i}$, and area proportional to $1 / \delta x_{i}$. The choice of area is a somewhat arbitrary one; it is based on the assumption that an experimenter will work to reduce his (or her) systematic errors until they are slightly smaller (but seldom much smaller) than the statistical errors. Thus, as a bubble chamber physicist gets more events, he (or she) will use them both to reduce the statistical errors and to study the biases. Our confidence that a significant systematic error has not been made in a given experiment, as compared with other contradictory experiments, then tends to go up as $1 / \delta x_{i}$.

But why not assign a weight $1 / \delta x_{i}^{2}$, as is done when computing a weighted average? We feel that this is equivalent to assuming that large systematic errors are as infrequent as large statistical fluctuations, and that this is unrealistic.

We emphasize the difference between least-squares averaging (where the weighting factor is the inverse square of the error) and the ideograms prepared for visual display. The former arithmetic is of course best if one has statistically distributed input, and yields a narrow gaussian distribution centered at the weighted mean. The ideogram (often multipeaked and certainly not gaussian) is based on the opposite hypothesis that some of the input is systematically in error. The idea behind least-squares averaging is that experiments 1 , 2 , 3 , etc., are all valid (so we should multiply their probabilities). Our ideograms are based on the assumption that 1 or 2 or 3 , etc., is valid, "hedged" with 1 / $\delta x_{i}$ betting odds; we then add their probabilities. Both approaches cannot simultaneously be right; we leave it to the reader to choose. A glance at the ideogram will show, however, that the discrepancy is often not severe for reasonably distributed input.

(ii) The second way in which we try to take account of $\chi^{2} /(N-1)$ being greater than 1 is to scale up our quoted error $\delta \bar{x}$ in Eq. (1) by a factor

$$
\text { SCALE }=\left[\chi^{2} /(N-1)\right]^{1 / 2} \text {. }
$$

Our reasoning is as follows. Since we do not know which one or more of the experiments are wrong, we assume that all experimentalists underestimated their errors by the same scale factor (2). If we scale up all input errors by this factor, $\chi^{2}$ returns to $N-1$, and of course the output error scales up by the same factor.

If all the experiments have errors of about the same size, the above (straightforward) procedure for calculating SCALE is carried out. If, however, we are to combine experiments with widely varying errors, we must modify the procedure slightly. This is because it is the more precise experiments that most influence not only the average value $\bar{x}$, but also the error $\delta \bar{x}$. Now, on the average, the low-precision experiments each contribute about unity to both the numerator and the denominator of SCALE, hence the $\chi^{2}$ contribution of the sensitive experiments is diluted, i.e., reduced. Therefore, we evaluate SCALE by using only experiments for which the errors are not much greater than those of the more precise experiments. Explicitly, to calculate SCALE we use only the most sensitive experiments, i.e., those with errors less than $\delta_{0}$, where 
the ceiling $\delta_{0}$ is (arbitrarily) chosen to be

$$
\delta_{0}=3 N^{1 / 2} \delta \bar{x} \text {. }
$$

Here $\delta \bar{x}$ is the unscaled error of the mean of all the experiments. Note that if each experiment had the same error $\delta x_{i}$, then $\delta \bar{x}$ would be $\delta x_{i} / N^{1 / 2}$, so each individual experiment would be well under the ceiling on SCALE.

This scaling approach has the property that if there are two values with comparable errors separated by much more than their stated errors (with or without a number of other experiments of lower accuracy), the error on the mean value $\delta \bar{x}$ is increased so that it is approximately half the interval between the two discrepant values.

We wish to emphasize the fact that our scaling procedures for errors in no way affect central values. In addition, if one wishes to recover the unscaled error $\delta \bar{x}$, one need only divide the given error by the SCALE factor for that error.

\section{A second procedure-Student's distribution}

The second method of averaging data, described in detail in Roos et al. (1975), relies upon an empirical determination of the distribution of the residuals for the ensemble of data appearing in the Review. The residual for the $i$ th measurement of a quantity with average value $\bar{x}$ is defined as

$$
h_{i}=\left(x_{i}-\bar{x}\right) / \delta x_{i} .
$$

Roos et al. select several different subsamples of the data, and show that the residuals for each subsample have approximately the same properties; in particular, their first few even moments are similar. Since the distributions have longer tails than a gaussian, the authors choose to represent them by a distribution function having such a property, namely the Student distribution

$$
S_{n}(h / c)=K\left[1+\frac{(h / c)^{2}}{n}\right]^{-(n+1) / 2} .
$$

Here $K$ is a normalization constant, and $n$ and $c$ are parameters which the authors then fit to the combined sample of data. The resulting empirical distribution is

$$
S_{10}(h / 1.11)=0.351\left[1+\frac{(h / 1.11)^{2}}{10}\right]^{-11 / 2} \text {. }
$$

Note that the shape of $S_{10}$ is somewhere between that of a gaussian $\left(=S_{\infty}\right)$ and that of a Breit-Wigner $\left(=S_{1}\right)$.
The proposed method of averaging the data for a given quantity then consists of finding the value of $\bar{x}$ which maximizes the log-likelihood function

$$
\log \mathcal{L}\left(\left\{x_{i}\right\} \mid \bar{x}\right)=\sum_{i} \log \left[S_{10}\left(\frac{x_{i}-\bar{x}}{1.11 \delta x_{i}}\right)\right]
$$

the sum here is again taken over all $N$ measurements of $x$. The error $\delta \bar{x}$ is determined by finding the variation in $\bar{x}$ needed to decrease the log-likelihood by $1 / 2$ :

$$
\log \mathcal{L}\left(\left\{x_{i}\right\} \mid \bar{x}\right)-\log \mathscr{L}\left(\left\{x_{i}\right\} \mid \bar{x} \pm \delta \bar{x}\right)=\frac{1}{2} .
$$

\section{Comparison of procedures}

Both of the procedures described above adopt a partially empirical approach to the problem that measured values for the quantities tabulated in this Review do not exhibit the gaussian behavior naively expected. (This problem, it should be noted, persists even when careful attempts are made to resolve difficulties and inconsistencies in the data prior to averaging.)

The first approach operates on a quantity-by-quantity basis and adjusts the error in each case so that no scaled $\chi^{2} /(N-1)$ is greater than 1 . This is obviously rather conservative, since even if the data obeyed a gaussian distribution, about half of the quantities would be expected to have $\chi^{2} /(N-1)>1$.

The second approach, on the other hand, assumes that (provided we first eliminate quantities with obvious, known problems) all quantities have the same theoretical distribution function, namely the fairly longtailed $S_{10}(h / 1.11)$. With this supposition, if a particular quantity has a large $\chi^{2}$, it is assumed to be just a happenstance, occasioned by a random fluctuation into the long tails, and no special scaling for this quantity is done. This procedure thus results in generally smaller, or less conservative, error estimates for quantities having $\chi^{2} /(N-1)>1$. (However, it should be noted that, because of the overall scale of $1.11 \mathrm{ap}-$ pearing in the empirical Student's distribution, the errors for quantities with $\chi^{2} /(N-1) \leqslant 1$ are actually increased by about $10 \%$.) Table 3 shows some comparisons of sample results from the two procedures, using data from the 1978 edition of the Review. Shifts in both $\bar{x}$ and $\delta \bar{x}$ can be observed, especially where SCALE $>1$.

\begin{tabular}{|c|c|c|c|c|c|c|c|}
\hline \multirow{2}{*}{$\frac{\text { Particle property }}{\rho^{0} \text { mass }(\mathrm{MeV})}$} & \multicolumn{2}{|c|}{$\begin{array}{l}\text { Pure } \\
\text { gaussian } \\
\bar{x} \pm \delta \bar{x}\end{array}$} & \multicolumn{3}{|c|}{$\begin{array}{c}\text { Standard method: } \\
\text { gaussian + scale factor }\end{array}$} & \multicolumn{2}{|c|}{$\begin{array}{l}\text { Proposed method: } \\
\text { Student's distribution } \\
\bar{x}_{ \pm} \delta \bar{x}\end{array}$} \\
\hline & 770.23 & \pm 0.65 & 770.23 & \pm 0.88 & 1.3 & 770.25 & \pm 0.82 \\
\hline$\eta^{\prime}$ mass $(\mathrm{MeV})$ & 957.57 & \pm 0.25 & 957.57 & \pm 0.25 & 1.0 & 957.57 & \pm 0.28 \\
\hline$\phi$ mass $(\mathrm{MeV})$ & 1019.62 & \pm 0.16 & 1019.62 & \pm 0.24 & 1.5 & 1019.68 & \pm 0.21 \\
\hline$K_{L}^{0}$ mean life $\left(10^{-8} s\right)$ & 5.158 & \pm 0.042 & 5.158 & \pm 0.042 & 1.0 & 5.158 & \pm 0.046 \\
\hline$\nu^{+}$mean life $\left(10^{-10} s\right)$ & 0.8015 & \pm 0.0053 & 0.8015 & $5 \pm 0.0053$ & 1.0 & 0.8015 & $5 \pm 0.0058$ \\
\hline$\chi^{-}$mean life $\left(10^{-10} s\right)$ & 1.483 & \pm 0.011 & 1.483 & \pm 0.015 & 1.4 & 1.481 & \pm 0.012 \\
\hline$K^{ \pm} \rightarrow \pi^{ \pm} \pi^{+} \pi^{-}(\%)$ & 5.521 & \pm 0.075 & 5.521 & \pm 0.098 & 1.3 & 5.533 & \pm 0.089 \\
\hline$\Lambda \rightarrow p \pi^{-}(\%)$ & 63.99 & \pm 0.49 & 63.99 & \pm 0.49 & 1.0 & 63.98 & \pm 0.55 \\
\hline
\end{tabular}

Since the second procedure is a significant departure from the traditional method, we have repeated the previously adopted approach: in the Data Card Listings we give the average-and-error for each quantity cal-

TABLE III. Comparíson of procedures (data from 1978 edition). 
culated both ways; the standard way is labelled at the left with the code "AVG", while the second way is labelled "ST UDENT". In the Tables of Particle Properties, we continue to use the standard procedure-gaussian with SCALE factor. As in the past, a SCALE factor greater than 1 is indicated by the appearance of " $S=\ldots$. next to the value and error.

\section{B. Constrained fits}

Except for trivial cases, all branching ratios and rate measurements are analyzed by the computer program AHR. This program makes a simultaneous least-squares fit to all the data, and outputs the partialdecay fractions $\bar{P}_{i}$, width $\Gamma$, partial widths $\Gamma_{i}$, and their error matrix.

The original version of AHR was written by J. Peter Berge. It is documented separately, and we wish here only to give the simplest nontrivial example that permits us to comment on the error matrix and the scale factor.

Assume that a state has only three partial-decay fractions, $P_{1}, P_{2}$, and $P_{3}\left(\Sigma P_{i}=1\right)$, which have been measured in four different ratios, $R_{1}, \ldots, R_{4}$, where, e.g., $R_{1}=P_{1} / P_{2}, R_{2}=P_{1} / P_{3}$, etc. ${ }^{4}$ Further assume that each ratio has been measured by $N$ experiments (we designate each experiment with a subscript $x$, e.g., $\left.R_{1 x}\right)$. Then AHR finds the best values of $P_{1}, P_{2}$, and $P_{3}$ by minimizing $\chi^{2}$, namely

$$
\chi^{2}=\sum_{r=1}^{4}\left[\sum_{x=1}^{N}\left(\frac{R_{r x}-R_{r}\left(P_{1}, P_{2}, P_{3}\right)}{\delta R_{r x}}\right)^{2}\right] .
$$

In addition to the fitted values $\bar{P}_{i}$, the program calculates an error matrix $\left\langle\delta \bar{P}_{i} \delta \bar{P}_{j}\right\rangle$. We tabulate the diagonal elements $\delta \bar{P}_{i}=\left\langle\delta \bar{P}_{i} \delta \bar{P}_{i}\right\rangle^{1 / 2}$ (except that some errors are scaled according to Eq. (2) as discussed below). In the listings we give the complete error matrix; we also calculate the fitted value of each ratio, for comparison with the input data, and list it below the relevant input, along with a simple unconstrained average of the same input.

Two further comments on the example above.

(1) There was no connection between measurements of the width and the branching ratios. But often we also have information on partial widths $\Gamma_{i}$ as well as total width $\Gamma$. In this case AHR must introduce $\Gamma$ as a parameter into the fit, along with the relations $\Gamma_{i}$ $=\Gamma P_{i}, \Sigma \Gamma_{i}=\Gamma$. When appropriate, we tabulate the $\Gamma_{i}$ along with the $P_{i}$, and give error matrices in the listings.

(2) Note that we do not allow for correlations between input data. We do try to pick those ratios and widths which are as independent and as close to the original data as possible.

In asymmetric errors, we use a continuous function of $\delta(P)^{+}$and $\delta(P)^{-}$in the fitting. When no errors are reported, we merely list the data for inspection.

Hyperon-decay parameters. The program AHR handles any type of input, $\alpha, \phi, \Delta, \beta$, or $\gamma$, according to the definitions of Sec. VI. If for a particular hyperon decay there are data for more than two of the decay

\footnotetext{
${ }^{4}$ We can handle any $R$ of the form $R=\Sigma \alpha_{i} P_{i} / \Sigma \beta_{i} P_{i}^{\prime}$, where $\alpha_{i}$ and $\beta_{i}$ are constants, usually 1 or 0 .
}

parameters, they are analyzed by using the constraint

$$
\alpha^{2}+\beta^{2}+\gamma^{2}=1 \text {. }
$$

Inconsistent constrained data. According to our simple example, which led to Eq. (7), the double sum for $\chi^{2}$ is summed over experiments $x=1$ to $N$, leaving a single sum over ratios

$$
\chi^{2}=\sum_{r} \chi_{r}^{2} .
$$

Even before fitting, some of the $\chi_{r}^{2}$ may be too large. But if we scaled them before fitting, then the scaling would move the central value, contrary to our policy. So we do not scale until after the first fit; then, knowing the fitted $\chi_{r}^{2}$ and its expectation value $\left\langle\chi_{r}^{2}\right\rangle$ we form SCALE factors (just as before), i.e.,

$$
(\mathrm{SCALE})_{r}^{2}=\chi_{r}^{2} /\left\langle\chi_{r}^{2}\right\rangle \text {, }
$$

and if any $(\mathrm{SCALE})_{r}$ is greater than 1 , all $N$ of the measurements of that particular ratio are equally penalized by having their errors inc reased by (SCALE) . $_{r}$. Program AHR then recycles on all the data, those with errors unchanged as well as those with errors increased. We then get new values, $\delta \bar{P}_{i}^{\prime}$ for the errors in the partial decay modes.

Because of the constraint $\left(\sum P_{i}=1\right)$ some SCALE factors may still be greater than 1 even after this second pass. If this is so, the whole procedure (i.e., increasing errors by the new SCALE factors and recycling through AHR) is repeated.

At the end of AHR's final pass we have two measures of the errors for the $\bar{P}_{i}$. One is, of course, the $\delta \bar{P}_{i}^{\prime}$, i.e., the errors in the final fitted values $\bar{P}_{i}^{\prime}$ which include the effects of scaling the input errors. The other measure of the errors is $\left(\bar{P}_{i}-\bar{P}_{i}^{\prime}\right)$, i.e., the shift in the central values of the $i$ th mode between the first (unscaled) fit and the final (scaled) fit. In practice we find that on the average these two measures of the uncertainty are about equal. Rather than selecting just one or the other, our tabulated errors are given by the combination

$$
\left(\delta \bar{P}_{i}\right)_{t a b}=\left[\delta \bar{P}_{i}^{\prime 2}+\left(\bar{P}_{i}-\bar{P}_{i}^{\prime}\right)^{2}\right]^{1 / 2},
$$

where $\bar{P}_{i}$ is the fitted value of the $i$ th partial-decay mode before scaling, $\bar{P}_{i}^{\prime}$ is its value after scaling, and $\delta \bar{P}_{i}^{\prime}$ is the error in $\bar{P}_{i}^{\prime}$. The SCALE factors we finally list in such cases are defined by

$$
(\mathrm{SCALE})_{i}=\left(\delta \bar{P}_{i}\right)_{t a b} / \delta \bar{P}_{i} .
$$

However, in line with our policy of not letting SCALE affect the central values, we give the values of $\bar{P}_{i}$ obtained from the original (unscaled) fits. (The differences between the $\bar{P}_{i}$ calculated with either the scaled or the unscaled errors are, of course, always within the tabulated errors, $\left.\left(\delta \bar{P}_{i}\right)_{t a b}.\right)$

\section{ACKNOWLEDGMENTS}

The Particle Data Group wishes to acknowledge with appreciation the contributions made by Lina BarbaroGaltieri throughout most of the years of the Review of Particle Properties.

We thank all those who have assisted in the many 
phases of preparing this Review. In particular, we acknowledge the usefulness of feedback from the physics community, especially those who have made suggestions or pointed out errors. The comments and suggestions of Dr. Goldschmidt-Clermont were particularly helpful. The European members of the Particle Data Group wish to acknowledge the generous support of CERN, in particular Division EP and Dr. A. Günther and his services.

\section{REFERENCES}

Albright, C.H., 1959, Phys. Rev.115, 750.

Ayed, R., 1976, CEA-N-192, Saclay thesis.

Barbaro-Galtieri, A., 1968, "Baryon resonances," in: $A d-$ vances in Particle Physics, eds. R. L. Cool and R. E. Marshak (Wiley, New York), Vol. 2. See specifically, Table IV and Figs. 10 and 12.

Barrelet, E., 1972, Nuovo Cim. 8A, 331.

Bender, I., V. Linke and H. J. Rothe, 1968, Z. Physik 212, 190.

Cabibbo, N., 1963, Phys. Rev. Lett. 10, 531.

Cabibbo, N., and A. Maksymowicz, 1964, Phys. Lett. 9, 352.

Chounet, L. M., J. M. Gaillard and M. K. Gaillard, 1972,

Phys. Rep. 4C, 199.

Fearing, H. W., E. Fischbach and J. Smith, 1970, Phys. Rev. D 2, 542 .

Gasiorowicz, S., 1966, Elementary Particle Physics (Wiley, New York).

Giacomelli, G., et al., 1974, Nucl. Phys. B71, 138.

Goldberger, M. L., and S. B. Treiman, 1958, Phys. Rev. 11, 354.

Jackson, J.D., S. D. Treiman and H.W. Wyld Jr., 1957,

Phys。Rev. 106, 517.

Jackson, J. D., 1964, Nuovo Cim. 34, 1644.

Källén, G., 1964, Elementary Particle Physics (Addison-Wesley, Reading, MA).
Kinoshita, T., and A. Sirlin, 1957, Phys. Rev. 108, 844.

Kobayashi, M., and T. Maskawa, 1973, Progr. Theor. Phys. 49,652 .

Layter, J. G., J. A. Appel, A. Kotlewski, W. Lee, S. Stein and J. J. Thaler, 1972, Phys. Rev. Lett. 29, 316.

Lee, T. D., and C.N.Yang, 1957, Phys. Rev. 108, 1615.

Levi-Setti, R., June 1969, Rapporteur talk at the Lund Intern. Conf. on Particle Physics (Lund).

Martin, B.R., 1975, Nucl. Phys. B94, 413.

Okun, L. B., and C. Rubbia, 1967, Proc. Heidelberg Conf。 on Elementary Particles, p. 301.

Particle Data Group: N. Barash-Schmidt, A. Barbaro-Galtieri, L. R. Price, A. H. Rosenfeld, P. Söding, C. G. Wohl, M. Roos and G. Conforto, 1969, Rev. Mod. Phys. 41, 109. Particle Data Group: C. Bricman, C. Dionisi, R. J. Hemingway, M. Mazzucato, L. Montanet, N. Barash-Schmidt, R. L. Crawford, M. Roos, A. Barbaro-Galtieri, C. P. Horne, R. L. Kelly, M. J. Losty, A. Rittenberg, T. G. Trippe, G. P. Yost, B. Armstrong, 1978, Phys. Letts. 75B.

Pišút, J., and M. Roos, 1968, Nucl. Phys. B6, 325 .

Roos, M., M. Hietanen and J. Luoma, 1975, Physica Fennica $10,21$.

Roper, L. D., R. M. Wright and B. T. Feld, 1965, Phys. Rev. 138, B190.

Rosenfeld, A. H., 1975, Ann. Rev. Nucl. Sci. 25, 555.

Shrock, R. E., and Ling-Lie Wang, 1978, Phys. Rev. Lett. 41, 692.

Steinberger, J., 1969, CERN Topical Conf. on Weak Interactions, CERN 69-7, p. 291.

Weinberg, S., 1960, Phys. Rev. Lett. 4, 87.

Willis, W., and J. Thompson, 1968, "Leptonic Decays of Elementary Particles," in: Advances in Particle Physics, eds. R. L. Cool and R. E. Marshak (Wiley, New York), Vol. 1, p. 295.

Wolfenstein, L., 1969, in: Theory and Phenomenology in Particle Physics, ed. A. Zichichi (Academic, New York), p. 218. Wu, T. T., and C. N. Yang, 1964, Phys. Rev. Lett. 12, 380. 


\title{
TABLES OF PARTICLE PROPERTIES
}

\author{
April 1980
}

N. Barash-Schmidt, C. Bricman, R. L. Crawford, C. Dionisi, C. P. Horne, R. L. Kelly, M. J. Losty, M. Mazzucato, L. Montanet, A. Rittenberg, M. Roos, T. Shimada, T. G. Trippe, C. G. Wohl, G. P. Yost

(Closing date for data: Jan. 1, 1980)

\section{Stable Particle Table}

For additional parameters, see Addendum to this table.

Quantities in italics have changed by more than one (old) standard deviation since April 1978.

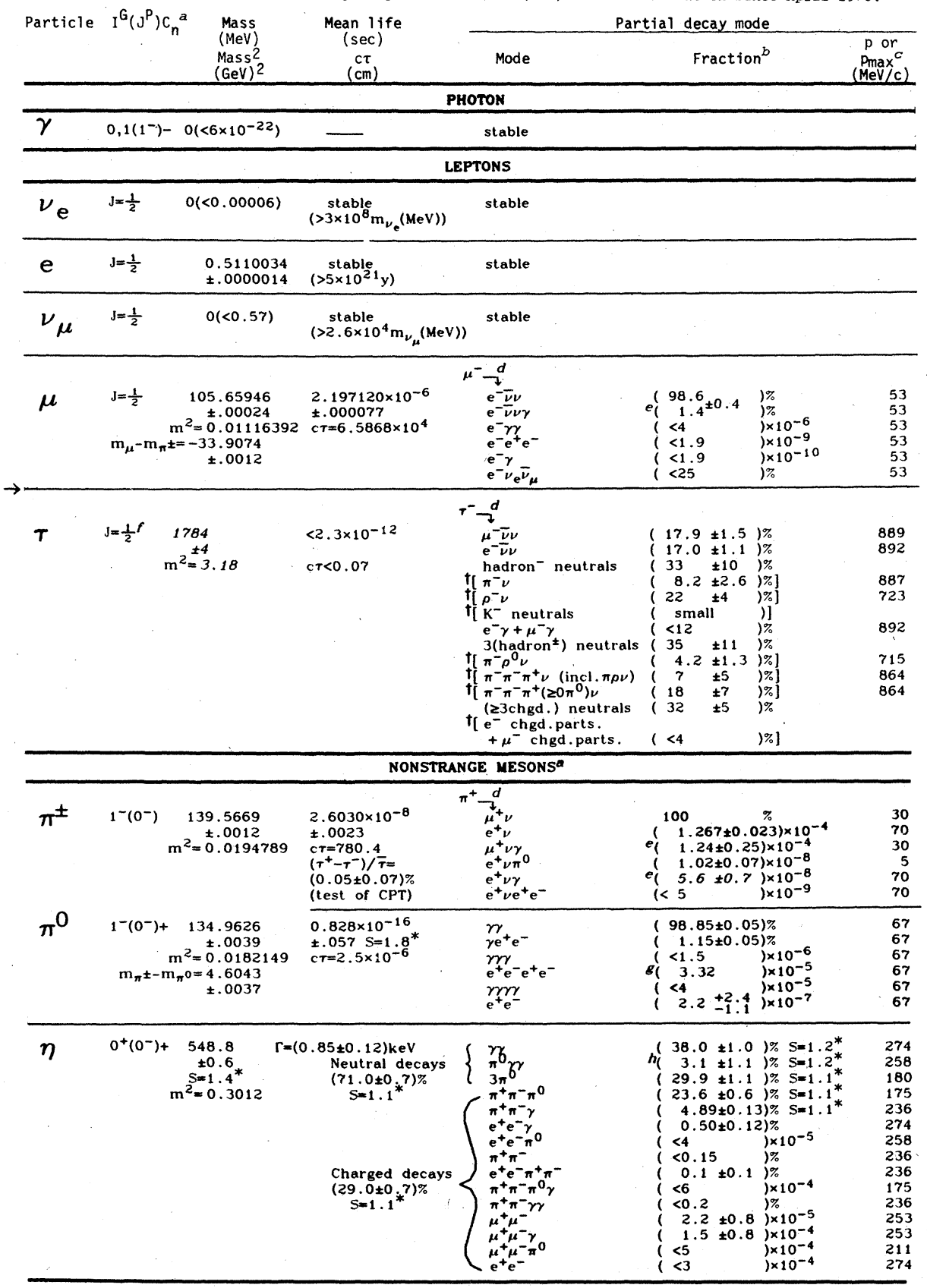


Stable Particle Table (cont'd)

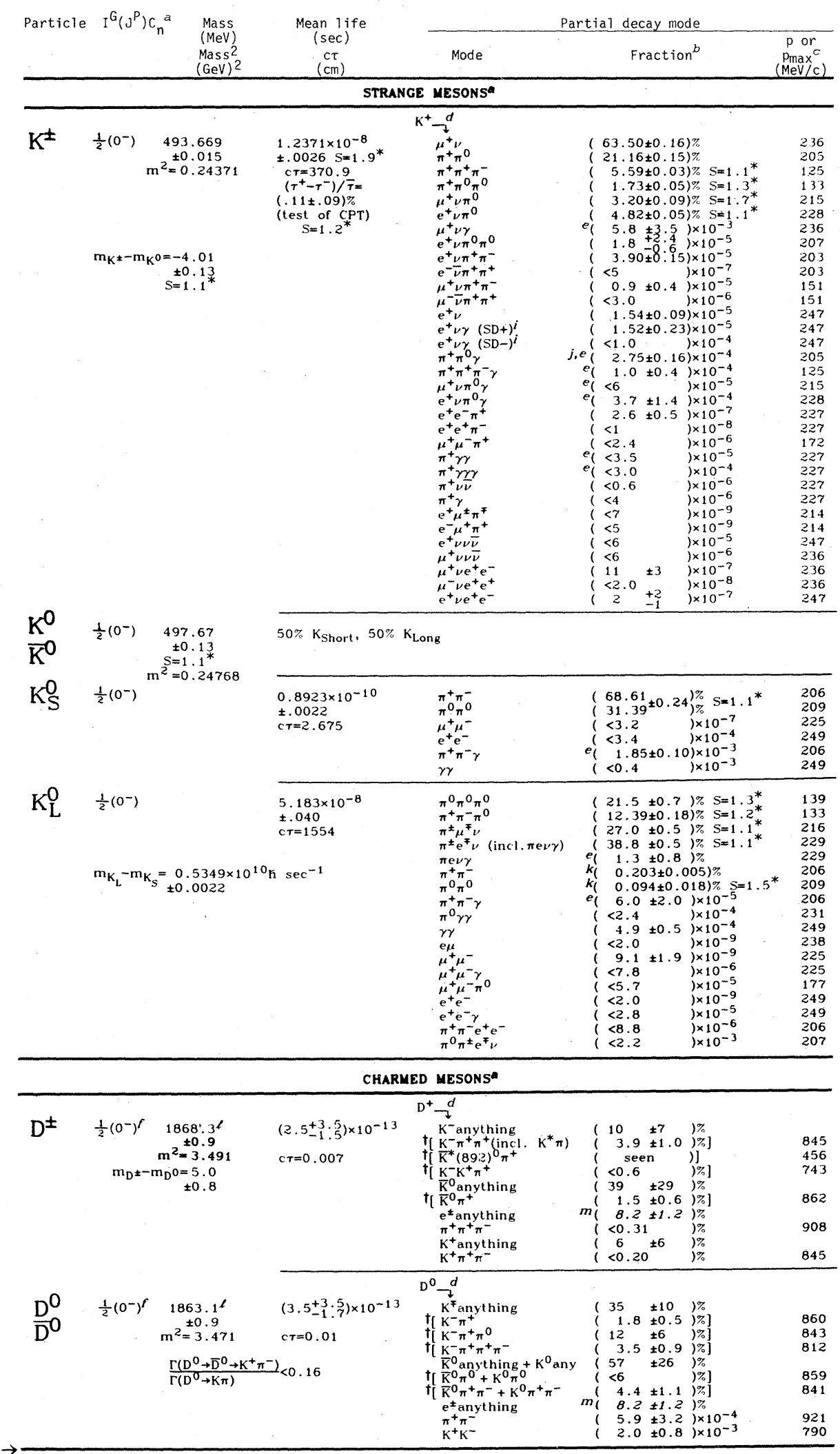


Particle Data Group: Review of particle properties

Stable Particle Table (cont'd)

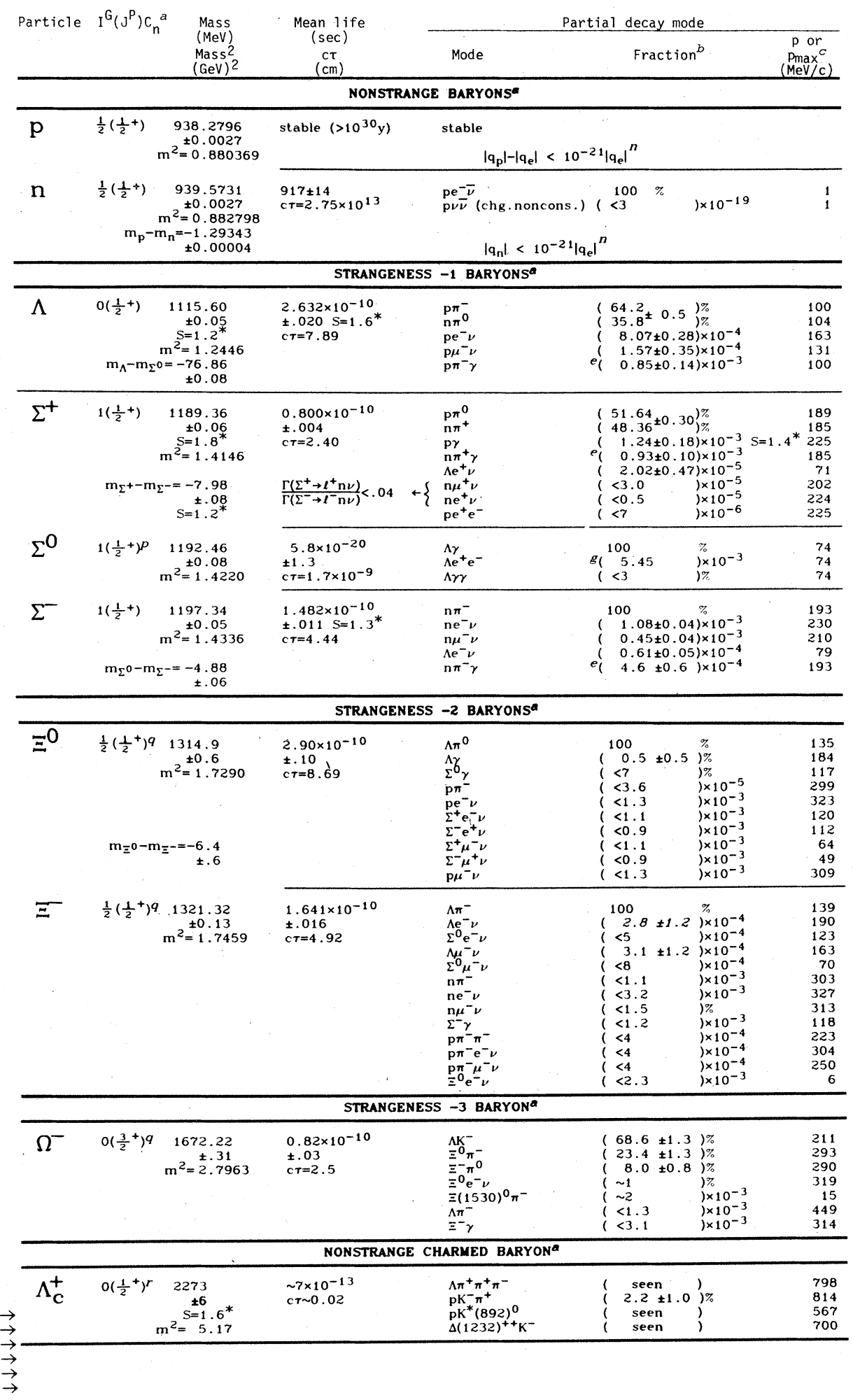


ADDENDUM TO

\section{Stable Particle Table}

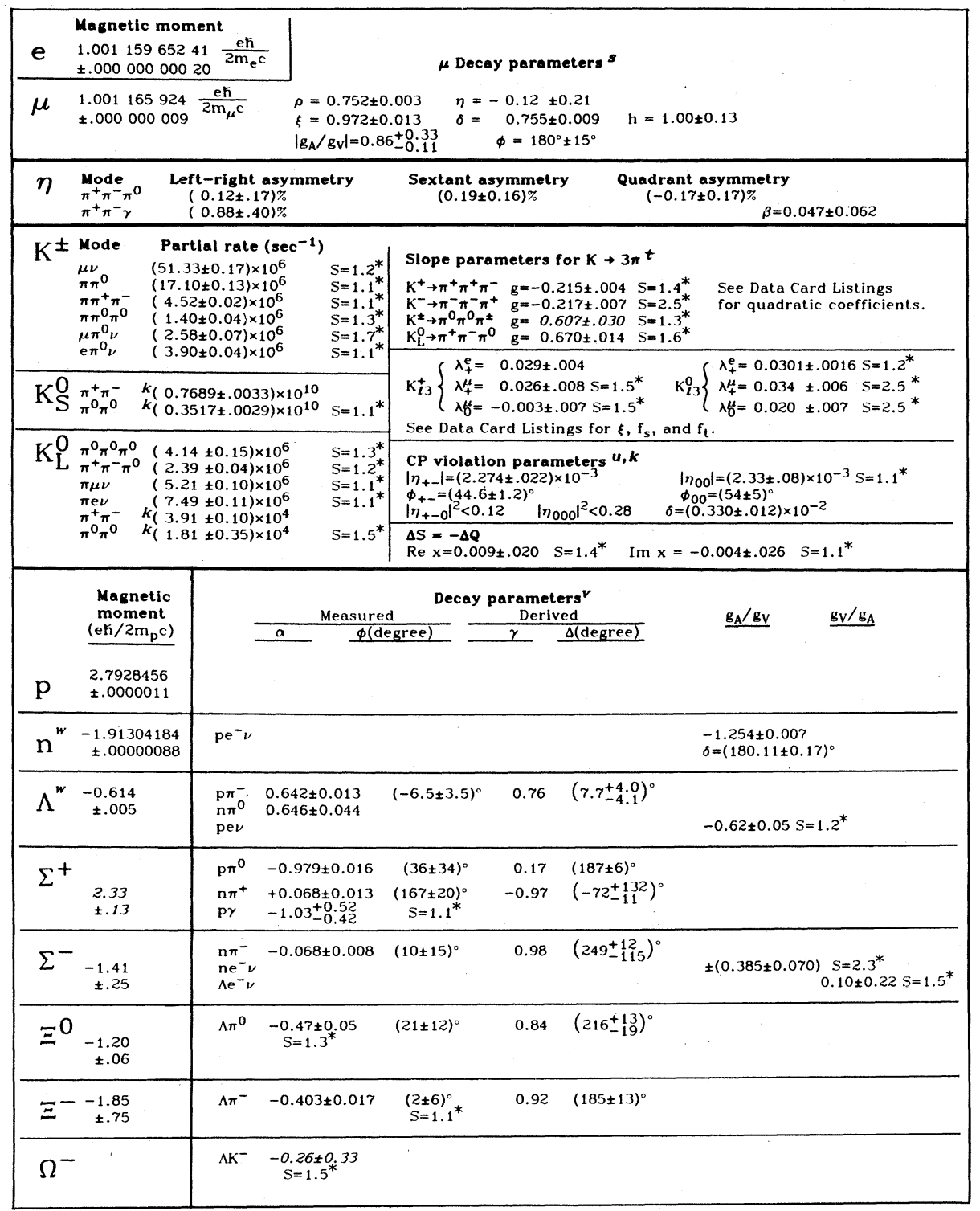




\section{Stable Particle Table (cont'd)}

$\rightarrow$ Indicates an entry in the Stable Particle Data Card Listings not entered in the Stable Particle Table. This is the case for $\nu_{\tau}$, for the charmed-strange meson $\mathrm{F}^{ \pm}$, and for listings of searches for heavy leptons other than $\tau^{ \pm}$, intermediate boson searches, quark searches, magnetic monopole searches, charm seaches, and other particle searches.

* Searches, charm seaches, and other particle searches. have enlarged the error of the mean, $\delta \overline{\mathrm{x}}$; i.e., $\delta \overline{\mathrm{x}} \rightarrow \mathrm{S} \delta \overline{\mathrm{x}}$. This convention is still inadequate, since if $S \gg 1$ the experiments are probably inconsistent, and therefore the real uncertainty is probably even greater than S $\delta \mathrm{x}$. See text, and ideograms in Stable Particle Data Card Listings.

$\dagger$ Square brackets indicate a subreaction of the previous (unbracketed) decay mode.

a. The baryon number $B$, strangeness $S$, and charm $C$ of the hadrons which appear in the tables are as follows:

Mesons $(\mathrm{B}=0)$
$\pi, \eta$
$\mathrm{K}^{+}, \mathrm{K}^{0}$
$\mathrm{~K}^{-}, \overline{\mathrm{K}}^{0}$
$\mathrm{D}^{+}, \mathrm{D}^{0}$
$\mathrm{D}^{-}, \overline{\mathrm{D}}^{0}$

$\begin{array}{rr}\mathrm{S} & \mathrm{C} \\ 0 & 0 \\ +1 & 0 \\ -1 & 0 \\ 0 & +1 \\ 0 & -1\end{array}$

$\begin{array}{crr}\text { Baryons }(B=1) & S & C \\ \text { p,n } & 0 & 0 \\ \Lambda, \Sigma & -1 & 0 \\ \Xi & -2 & 0 \\ n^{-} & -3 & 0 \\ \Lambda^{+} & 0 & +1\end{array}$

b. Quoted upper limits correspond to a $90 \%$ confidence level.

c. In decays with more than two bodies, $p_{\max }$ is the maximum momentum that any particle can have.

d. For simplicity, decay mode charge states are written for the particle shown. For antiparticle modes all particles must be charge conjugated.

. See Stable Particle Data Card Listings for energy limits used in this measurement.

f. Quantum numbers shown are favored but not yet established. See Data Card Listings.

g. Theoretical value; see also Stable Particle Data Card Listings.

b. See note in Stable Particle Data Card Listings.

$i$ Structure-dependent part with positive $(\mathrm{SD}+)$ and negative (SD-) photon helicity.

$j$. The direct emission branching fraction is $(1.56 \pm .35) \times 10^{-5}$.

$k$. The $K_{S}^{0} \rightarrow \pi \pi$ and $K_{\mathrm{L}}^{0} \rightarrow \pi \pi$ rates (and branching fractions) are from independent fits and do not include results of $K_{L}^{0}-K_{S}^{0}$ interference experiments. The $\left|\eta_{+-1}\right|$ and $\left|\eta_{00}\right|$ values given in the addendum are these rates combined with the $\left|\eta_{+-}\right|$and $\left|\eta_{00}\right|$ results from interference experiments.

1. Error does not include $0.13 \%$ uncertainty in the absolute SPEAR energy calibration. Assumes $\mathrm{m}_{\psi}=3095 \mathrm{MeV}$

$m$. This is a weighted average of $D^{ \pm}(44 \%)$ and $D^{0}(56 \%)$ branching fractions

$n$. Limit from neutrality-of-matter experiments. Assumes $\left|q_{n}\right|=\left|q_{p}\right|-\left|q_{e}\right|$.

p. $\mathrm{J}^{\mathrm{P}}$ not measured for $\Sigma^{0}$. Assumed same as $\Sigma^{ \pm}$to allow isotriplet association.

q. $\mathrm{P}$ for $\equiv$ and $\mathrm{J}^{\mathrm{P}}$ for $\mathrm{\Omega}^{-}$not yet measured. Values shown are $\mathrm{SU}(3)$ predictions.

$r . \quad \mathrm{J}^{\mathrm{P}}$ for $\Lambda_{c}^{+}$not yet measured. Values shown are SU(4) predictions.

s. $\left|g_{A} / g_{v}\right|$ defined by $g_{A}^{2}=\left|C_{A}\right|^{2}+\left.\left|C^{\prime} A^{2}, g_{V}^{2}=\right| C_{v}\right|^{2}+\left.\left|C^{\prime}\right|^{2}\right|^{2}$, and $\Sigma\left(e\left|\Gamma_{i}\right| \mu\right\rangle\left\langle\bar{\nu}\left|\Gamma_{i}\left(C_{i}+C_{i}^{\prime} \gamma_{5}\right)\right| \nu\right\rangle$; $\left|g_{A} / g_{v}\right|$ defined by $g_{A}^{2}=\left|C_{A}\right|^{2}+\left|C_{A}^{\prime}\right|^{2}, g_{V}^{2}=\left|C_{V}\right|^{2}+\left|C^{\prime} v\right|^{2}$, and $\sum\left(e\left|\Gamma_{i}\right| \mu\right\rangle\left\langle\nu\left|\Gamma_{i}\left(C_{i}+C_{i} \gamma_{5}\right)\right| \nu\right\rangle_{i}$
$\phi$ defined by $\cos \phi=-\operatorname{Re}\left(C_{A}^{*} C^{\prime}{ }^{\prime}+C^{\prime}{ }_{A} C_{V}^{*}\right) / g_{A} g_{v}$ [for more details, see text Section VI A].

$t$. The definition of the slope parameter of the Dalitz plot is as follows [see also text Section VI B.1]: $|M|^{2}=1+g\left(\frac{s_{3}-s_{0}}{m_{\pi}^{2}+}\right)$

u. The definition for the CP violation parameters is as follows [see also text Section VI B.3]:

$$
\begin{aligned}
& \eta_{+-}=\left|\eta_{+-}\right| \mathrm{e}^{\mathrm{i} \phi_{+-}}=\frac{\mathrm{A}\left(\mathrm{K}_{\mathrm{L}}^{0} \rightarrow \pi^{+} \pi^{-}\right)}{\mathrm{A}\left(\mathrm{K}_{\mathrm{S}}^{0} \rightarrow \pi^{+} \pi^{-}\right)} \quad \eta_{00}=\eta_{00} \mid \mathrm{e}^{\mathrm{i} \phi_{00}}=\frac{\mathrm{A}\left(\mathrm{K}_{\mathrm{L}}^{0} \rightarrow \pi^{0} \pi^{0}\right)}{\mathrm{A}\left(\mathrm{K}_{S}^{0} \rightarrow \pi^{0} \pi^{0}\right)} \\
& \delta=\frac{\Gamma\left(K_{L}^{0} \rightarrow \ell^{+}\right)-\Gamma\left(K_{L}^{0} \rightarrow \ell^{-}\right)}{\Gamma\left(K_{L}^{0} \rightarrow \ell^{+}\right)+\Gamma\left(K_{L}^{0} \rightarrow \ell^{-}\right)}, \quad\left|\eta_{+-0}\right|^{2}=\frac{\Gamma\left(K_{S}^{0} \rightarrow \pi^{+} \pi^{-} \pi^{0}\right)^{C P} \text { viol. }}{\Gamma\left(K_{L}^{0} \rightarrow \pi^{+} \pi^{-} \pi^{0}\right)}, \quad\left|\eta_{000}\right|^{2}=\frac{\Gamma\left(K_{S}^{0} \rightarrow \pi^{0} \pi^{0} \pi^{0}\right)^{C P} \text { viol. }}{\Gamma\left(K_{L}^{0} \rightarrow \pi^{0} \pi^{0} \pi^{0}\right)} \text {. }
\end{aligned}
$$

$v$. The definition of these quantites is as follows [for more details on sign convention, see text Section VI B]:

$$
\begin{array}{l|l|l}
\alpha=\frac{2|s||p| \cos \Delta}{|s|^{2}+|p|^{2}} & \beta=\sqrt{1-a^{2}} \sin \phi & g_{A} / g_{V} \text { defined by }\left\langle B_{f}\left|\gamma_{\lambda}\left(g_{V}-g_{A} \gamma_{5}\right)\right| B_{i}\right\rangle \\
\beta=\frac{-2|s||p| \sin \Delta}{|s|^{2}+|p|^{2}} & \gamma=\sqrt{1-a^{2}} \cos \phi & \delta \text { defined by } g_{A} / g_{V}=\left|g_{A} / g_{V}\right| e^{i \delta}
\end{array}
$$

$w$. For limits on electric dipole moment of $\mathrm{n}$ and $\Lambda$, see Data Card Listings. 


\title{
Meson Table
}

\author{
Aprit 1980
}

In addition to the entries in the Meson Table, the Meson Data Card Listings contain all substantial claims for meson resonances. See Contents of Meson Data Card Listings below.

Quantities in italics are new or have changed by more than one (old) standard deviation since April 1978.

\begin{tabular}{|c|c|c|c|c|c|c|c|c|}
\hline \multirow[b]{2}{*}{ 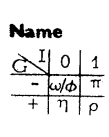 } & \multirow[b]{2}{*}{$\underbrace{\mathbf{I}_{\left(\mathbf{J}^{\mathbf{P}}\right) \mathbf{C}_{\mathbf{n}}}}$} & \multirow[b]{2}{*}{ 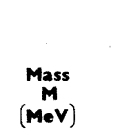 } & \multirow[b]{2}{*}{$\begin{array}{c}\text { Full } \\
\text { Width } \\
\text { r } \\
(\mathrm{MeV})\end{array}$} & \multirow[b]{2}{*}{$\begin{array}{c}\mathbf{M}^{2} \\
\pm \mathbf{r} \mathbf{M}^{(\mathrm{a})} \\
(\mathbf{G e V})^{2}\end{array}$} & \multicolumn{4}{|c|}{ Partial decay mode } \\
\hline & & & & & [ Upper ] & $\begin{array}{l}\text { Fraction }(\%) \\
\text { limits are } 10\end{array}$ & {$\left[\begin{array}{lll}\sigma & (\%)] & \stackrel{p}{p} \\
\mid\end{array}\right.$} & $\begin{array}{l}P_{\text {or }} \\
P_{\max }(b) \\
(\operatorname{MeV} / c)\end{array}$ \\
\hline \multicolumn{9}{|c|}{ NONSTRANGE MESONS } \\
\hline$\pi^{ \pm}$ & $1^{-}\left(0^{-}\right)+$ & $\begin{array}{l}139.57 \\
134.96\end{array}$ & $\begin{array}{l}0.0 \\
7.95 \mathrm{eV} \\
\pm .55 \mathrm{eV}\end{array}$ & $\begin{array}{l}0.019479 \\
0.018215\end{array}$ & See Stable Par & article Table & & \\
\hline$n$ & $0^{+}\left(0^{-}\right)+$ & $\begin{array}{r}548.8 \\
\pm 0.6\end{array}$ & $\begin{array}{l}0.85 \mathrm{keV} \\
\pm .12 \mathrm{keV}\end{array}$ & $\begin{array}{l}0.301 \\
\pm .000\end{array}$ & $\begin{array}{l}\text { Neutral } \\
\text { Charged }\end{array}$ & $\begin{array}{l}71.0 \\
29.0\end{array}$ & $\begin{array}{l}\text { Stable } \\
\text { ticle Table }\end{array}$ & \\
\hline $\begin{array}{l}\rho(770) \\
M \text { and } \Gamma \mathrm{f}\end{array}$ & $1^{+}\left(1^{-}\right)-$ & $\begin{array}{r}776^{\text {ฯ }} \\
\pm 3^{\S}\end{array}$ & $\begin{array}{r}158 \\
\pm 5\end{array}$ & $\begin{array}{l}0.602 \\
\pm .123\end{array}$ & $\begin{array}{l}\pi \pi \\
\pi y \\
e^{+} e^{-} \\
\mu^{+} \mu^{-} \\
n \gamma \\
\text { For upper } 1 \text { imits, see }\end{array}$ & $\begin{array}{l}\approx 100 \\
0.024 \pm .007 \\
0.0043 \pm .0005 \\
0.0067 \pm .0012 \\
\text { seen } \\
\text { footnote }(\mathrm{e})\end{array}$ & $\begin{array}{ll}5 & \text { (d) } \\
2 & \text { (d) }\end{array}$ & $\begin{array}{l}362 \\
375 \\
388 \\
373 \\
194\end{array}$ \\
\hline$\omega(783)$ & $0^{-}\left(1^{-}\right)=$ & $\begin{array}{c}782.4 \\
\pm 0.2 \\
S=1.1^{\star}\end{array}$ & $\begin{array}{r}10.1 \\
\pm .3\end{array}$ & $\begin{array}{l}0.612 \\
\pm .008\end{array}$ & $\begin{array}{l}\pi^{+} \pi^{-} \pi^{0} \\
\pi^{+} \pi^{-} \\
\pi^{0} \gamma^{+} \\
\mathrm{e}^{+} \mathrm{e}^{-} \\
\text {nr } \\
\text { For upper limits, see }\end{array}$ & $\begin{array}{l}89.8 \pm 0.5 \\
1.4 \pm 0.2 \\
8.8 \pm 0.5 \\
.0076 \pm .0017 \\
\text { seen } \\
\text { footnote (f) }\end{array}$ & $S=1.9^{*}$ & $\begin{array}{l}327 \\
365 \\
380 \\
391 \\
199\end{array}$ \\
\hline$n^{\prime}(958)$ & $0^{+}\left(0^{-}\right)+\pi$ & $\begin{array}{r}957.57 \\
\pm 0.25\end{array}$ & $\begin{array}{r}0.28 \\
\pm 0.10\end{array}$ & $\begin{array}{l}0.917 \\
\pm .0003\end{array}$ & $\begin{array}{l}n \pi \pi \\
\rho^{0} \gamma \\
\omega \gamma \\
\gamma \gamma \\
\text { For upper 1imits, see }\end{array}$ & $\begin{array}{r}65.6 \pm 1.6 \\
29.8 \pm 1.6 \\
2.7 \pm 0.5 \\
1.9 \pm 0.2 \\
\text { footnote }(\mathrm{g})\end{array}$ & & $\begin{array}{l}231 \\
164 \\
159 \\
479\end{array}$ \\
\hline$\delta(980)$ & $1^{-}\left(0^{+}\right)+$ & $\begin{array}{l}981^{(\mathrm{h})} \\
\pm 3\end{array}$ & $\begin{array}{l}52^{(h)} \\
\pm 8\end{array}$ & $\begin{array}{l}0.962 \\
\pm .051\end{array}$ & $\mathrm{n}_{\mathrm{K}}^{\mathrm{K}}$ & $\begin{array}{l}\text { seen } \\
\text { seen }\end{array}$ & & 319 \\
\hline $\begin{array}{l}\mathrm{S}^{*}(980) \\
\text { See note }\end{array}$ & $\begin{array}{l}0^{+}\left(0^{+}\right)+ \\
\text {on } \pi \pi \text { and } \mathrm{r}\end{array}$ & 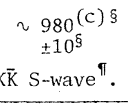 & $\begin{aligned} & 40^{(\mathrm{c}) \S} \\
\pm & 10^{\S}\end{aligned}$ & $\begin{array}{l}0.960 \\
\pm .039\end{array}$ & $\begin{array}{l}\mathrm{K} \overline{\mathrm{K}} \\
\pi \pi\end{array}$ & $\begin{array}{l}\text { seen } \\
\text { seen }\end{array}$ & & 470 \\
\hline$\Phi(1020)$ & $0^{-}\left(1^{-}\right)-$ & $\begin{array}{c}1019.6 \\
\pm 0.1 \\
S=1.3^{*}\end{array}$ & $\begin{array}{l}4.1 \\
\pm .2\end{array}$ & $\begin{array}{l}1.040 \\
\pm .004\end{array}$ & $\begin{array}{l}\mathrm{K}^{+} \mathrm{K}^{-} \\
\mathrm{K}_{\mathrm{L}} \mathrm{K}_{\mathrm{S}} \\
\left.\pi^{+} \pi^{-} \pi^{0} \text { (inc1. } \rho \pi\right) \\
n \gamma \\
\pi^{0} \gamma \\
\mathrm{e}^{+} \mathrm{e}^{-} \\
\mu^{+} \mu^{-} \\
\text {For upper limits, see }\end{array}$ & $\begin{array}{l}48.6 \pm 1.2 \\
35.2 \pm 1.2 \\
14.7 \pm 0.7 \\
1.5 \pm 0.2 \\
0.14 \pm 0.05 \\
.031 \pm .001 \\
.025 \pm .003 \\
\text { footnote (i) }\end{array}$ & $\begin{array}{l}S=1 \cdot 3^{*} \\
S=1.5^{*} \\
S=1.2^{*} \\
S=1.1^{*}\end{array}$ & $\begin{array}{l}127 \\
111 \\
462 \\
362 \\
501 \\
510 \\
499\end{array}$ \\
\hline $\begin{array}{r}\vec{A}_{1}(1100- \\
1300)\end{array}$ & $1^{-}\left(1^{+}\right)+$ & to $\begin{array}{l}1100^{\pi} \\
1300\end{array}$ & $\approx 300^{\pi}$ & $\begin{array}{l}1.44 \\
\pm .36\end{array}$ & $\begin{array}{l}\rho \pi \\
\pi(\pi \pi) \\
\text { S-wave }\end{array}$ & $\begin{array}{l}\text { dominant } \\
\text { seen }\end{array}$ & & $\begin{array}{l}329 \\
558\end{array}$ \\
\hline$B(1235)$ & $1^{+}\left(1^{+}\right)-$ & $\begin{array}{r}1231 \\
\pm 10^{5}\end{array}$ & $\begin{array}{l}129 \\
\pm 10^{\S}\end{array}$ & $\begin{array}{l}1.52 \\
\pm .16\end{array}$ & $\begin{array}{l}\omega \pi \\
{[\mathrm{D} / \mathrm{S} \text { amplitude ratio }=} \\
\text { For upper limits, see }\end{array}$ & $\begin{array}{l}\text { only mode se } \\
=.29 \pm .05] \\
\text { footnote }(j)\end{array}$ & & 348 \\
\hline$f(1270)$ & $0^{+}\left(2^{+}\right)+$ & $\begin{array}{r}1273 \\
\pm 5^{\S}\end{array}$ & $\begin{array}{l}178 \\
\pm 20^{\S}\end{array}$ & $\begin{array}{l}1.62 \\
\pm .23\end{array}$ & $\begin{array}{l}\pi_{1 \pi}+\pi^{+} 2 \pi^{-} \\
K_{\bar{K}} 2 \pi^{-} \\
\pi^{+} \pi^{-} 2 \pi^{0} \\
\text { For upper limits, see }\end{array}$ & $\begin{array}{c}83.1 \pm 1.9 \\
2.9 \pm 0.3 \\
2.8 \pm 0.3 \\
\text { seen } \\
\text { footnote }(\ell) \\
\end{array}$ & $\begin{array}{l}S=1.4^{*} \\
S=1.1^{*} \\
S=1.3^{*}\end{array}$ & $\begin{array}{l}621 \\
558 \\
397 \\
561\end{array}$ \\
\hline$D(1285)$ & $\underline{0^{+}\left(1^{+}\right)+}$ & $\begin{array}{r}1284 \\
\pm 10^{\S}\end{array}$ & $\begin{array}{r}27 \\
\pm 10^{8}\end{array}$ & $\begin{array}{l}1.65 \\
\pm .03\end{array}$ & $\begin{aligned} & K \bar{K} \pi \\
& n \pi \pi \\
&+ {[\delta \pi} \\
& 4 \pi(\text { prob. } \rho \pi \pi) \\
&\end{aligned}$ & $\left.\begin{array}{l}10 \pm 2 \\
49 \pm 6 \\
36 \pm 7 \\
41 \pm 13\end{array}\right]$ & & $\begin{array}{l}303 \\
483 \\
239 \\
564\end{array}$ \\
\hline $\begin{array}{l}\varepsilon(1300) \\
\text { See note }\end{array}$ & $\begin{array}{l}0^{+}\left(0^{+}\right)+ \\
\text {on } \pi \pi \text { and }\end{array}$ & $\begin{array}{l}\sim 1300 \\
\bar{K} \bar{K} \text { S wave }\end{array}$ & $200-400$ & & $\pi \bar{K}$ & $\begin{array}{l}\sim 90 \\
\sim 10\end{array}$ & & $\begin{array}{l}635 \\
423\end{array}$ \\
\hline$A_{2}(1310)$ & $\underline{1}^{-}\left(2^{+}\right)+$ & $\begin{array}{r}1317^{\S} \\
\pm 5^{\xi}\end{array}$ & $\begin{array}{r}102^{\S} \\
\pm 5\end{array}$ & $\begin{array}{l}1.73 \\
\pm .13\end{array}$ & $\begin{array}{l}\rho \pi \\
n \pi \\
\omega \pi \pi \\
\overline{\mathrm{K} K} \\
n^{\prime} \pi \\
\pi \gamma\end{array}$ & $\begin{array}{c}70.0 \pm 2.2 \\
14.6 \pm 1.1 \\
10.6 \pm 2.5 \\
4.8 \pm 0.5 \\
<1 \\
0.45 \pm 0.11\end{array}$ & . & $\begin{array}{l}414 \\
534 \\
360 \\
434 \\
285 \\
651 \\
\end{array}$ \\
\hline
\end{tabular}


Meson Table (cont'd)

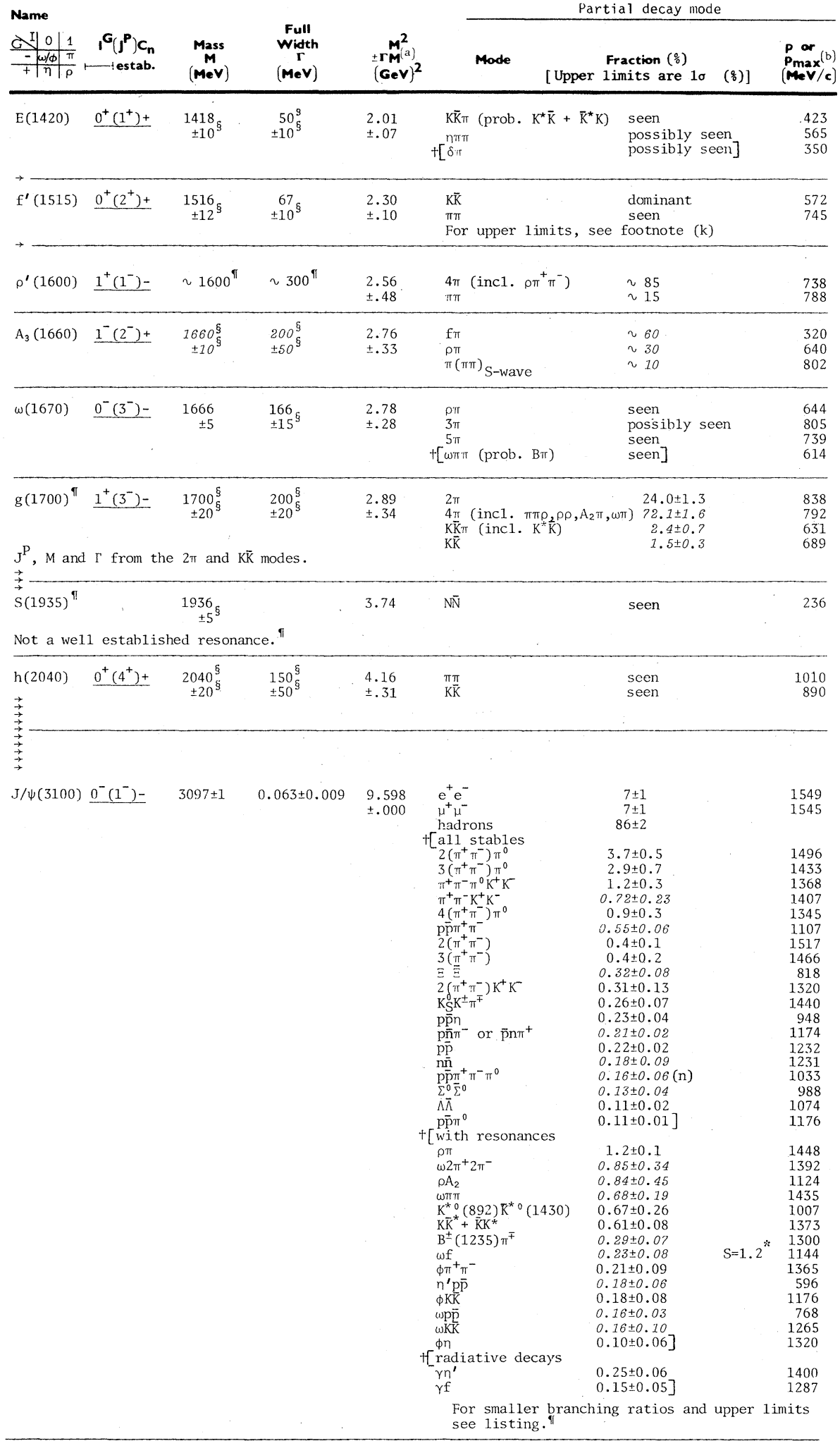


Meson Table (cont'd)

\begin{tabular}{|c|c|c|c|c|c|c|c|c|}
\hline \multirow{2}{*}{ 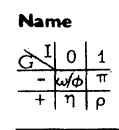 } & \multirow[b]{2}{*}{$\underbrace{\mathbf{I}_{\left(\mathbf{J}^{\mathbf{P}}\right) \mathbf{C}_{\mathbf{n}}}}_{\text {estab. }}$} & \multirow[b]{2}{*}{$\begin{array}{c}\text { Mass } \\
M \\
(\text { MeV })\end{array}$} & \multirow[b]{2}{*}{$\begin{array}{l}\text { Full } \\
\text { Width } \\
\text { r } \\
(\text { MeV })\end{array}$} & \multirow[b]{2}{*}{$\begin{array}{c}\mathbf{M}^{2} \\
(\mathbf{G e V})^{(a)}\end{array}$} & \multicolumn{4}{|c|}{ Partial decay mode } \\
\hline & & & & & [ Upper & $\begin{array}{l}\text { Fraction }(\%) \\
\text { limits are } 10\end{array}$ & $(\xi)]$ & 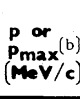 \\
\hline$x(3415)$ & $\underline{0}^{+}\left(0^{+}\right) \pm$ & $3414 \pm 4$ & & 11.655 & 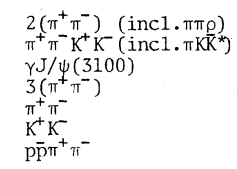 & $\begin{array}{l}4.6 \pm 0.9 \\
3.7 \pm 0.9 \\
2.7 \pm 1.0 \quad(\mathrm{~m}) \\
1.9 \pm 0.7 \\
0.9 \pm 0.2 \\
0.9 \pm 0.2 \\
0.6 \pm 0.2\end{array}$ & & $\begin{array}{r}1678 \\
1580 \\
302 \\
1632 \\
1701 \\
1634 \\
1319\end{array}$ \\
\hline $\begin{array}{l}p_{X} \text { or } \\
(510) \\
J^{P}=1^{+} p\end{array}$ & ceferred. & $3507 \pm 4$ & & 12.299 & $\begin{array}{l}\gamma J / \psi(3100) \\
3\left(\pi^{+} \pi^{-}\right) \\
\left.2\left(\pi^{+} \pi^{-}\right) \text {(incl. } \pi \pi \rho\right) \\
\pi^{+} \pi^{-} K^{+} K^{-}\left(\text {inc1. } \pi K^{*}\right) \\
\pi^{+} \pi^{-} p \bar{p}\end{array}$ & $\begin{array}{c}31.5 \pm 5.2 \\
2.7 \pm 1.1 \\
2.0 \pm 0.6 \\
1.1 \pm 0.4 \\
0.17 \pm 0.11\end{array}$ & $\mathrm{~S}=1.3^{*}$ & $\begin{array}{r}386 \\
1681 \\
1726 \\
1630 \\
1379\end{array}$ \\
\hline $\mathrm{J}^{\mathrm{P}}=2^{+} \mathrm{p}$ & referred. & $3551 \pm 5$ & & 12.610 & 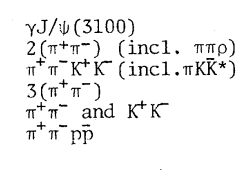 & $\begin{array}{c}15.4 \pm 2.4 \\
2.4 \pm 0.6 \\
2.1 \pm 0.6 \\
1.3 \pm 0.8 \\
0.27 \pm 0.11 \\
0.37 \pm 0.14\end{array}$ & & $\begin{array}{r}425 \\
1748 \\
1654 \\
1704 \\
1407\end{array}$ \\
\hline \multirow[t]{9}{*}{$\psi(3685)$} & $0^{-}\left(1^{-}\right)=$ & $\begin{array}{l}3685 \pm 1 \\
S=1.1 *\end{array}$ & $0.215 \pm 0.040$ & $\begin{array}{r}13.579 \\
\pm .001\end{array}$ & $\begin{array}{c}\mathrm{e}^{+} e^{-} \\
\mu^{+} \mu^{-} \\
\text {hadrons } \\
+\left[\mathrm{J} / \psi \pi^{+} \pi^{-}\right.\end{array}$ & $\begin{array}{c}0.9 \pm 0.1 \\
0.8 \pm 0.2 \\
98.1 \pm 0.3 \\
33 \pm 2]\end{array}$ & & $\begin{array}{r}1842 \\
1839 \\
476\end{array}$ \\
\hline & $\mathrm{m}_{\psi(3685)}$ & $-m_{\psi(3100)}=$ & $\begin{aligned}= & 588.2 \pm 0.9 \\
& S=1.2^{*}\end{aligned}$ & & $+\left[\mathrm{J} / \psi \pi^{0} \pi^{0}\right.$ & $17 \pm 2]$ & & 480 \\
\hline & & & & & $+[\mathrm{J} / \psi n$ & $3.7 \pm 0.4]$ & & 194. \\
\hline & & & & & $\begin{array}{l}+[\pi(\pi) \pi) \pi^{+} \\
+\left[\pi^{+} \pi^{-} K^{+} K^{-}\right.\end{array}$ & $0.16 \pm 0.04]$ & & $\begin{array}{l}1 / 98 \\
1725\end{array}$ \\
\hline & & & & & $+\left[p_{p} \pi^{+} \pi^{-}\right.$ & $0.08 \pm 0.02]$ & & 1490 \\
\hline & & & & & $+\left[2\left(\pi^{+} \pi^{-}\right)\right.$ & $0.05 \pm 0.01]$ & & 1816 \\
\hline & & & & & $+[\gamma \times(3415)$ & $7 \pm 2]$ & & 261 \\
\hline & & & & & $+[\gamma \times \times(3510)$ & $7 \pm 2]$ & & 174 \\
\hline & & & & For smalle & $\begin{array}{l}+[\gamma \times(3550) \\
+\gamma \text { branching ratios an }\end{array}$ & Ind upper 1imits & see List & $\begin{array}{l}132 \\
\text { stings." }\end{array}$ \\
\hline \multirow[t]{2}{*}{$\psi(3770)$} & $\underline{\left(1^{-}\right)-}$ & $\begin{array}{r}3768 \\
\pm 3\end{array}$ & $\begin{array}{l}25 \\
\pm 3\end{array}$ & $\begin{array}{r}14.1998 \\
\pm .094\end{array}$ & $\begin{array}{l}e^{+} e^{-} \\
D \bar{D}\end{array}$ & $\begin{array}{l}.0013 \pm 0.0002 \\
\text { dominant }\end{array}$ & & $\begin{array}{r}1884 \\
243\end{array}$ \\
\hline & $\mathrm{m}_{\psi(3770)}$ & $-m_{\psi(3685)}=$ & $\begin{array}{r}=82.5 \pm 3 . ? \\
S=2.2^{*}\end{array}$ & & & & & \\
\hline$\psi(4030)$ & $\left(1^{-}\right)-$ & $4030^{5} \pm 5^{\S}$ & $52 \pm 10$ & $\begin{array}{l}16.241 \\
\pm 0.210 \\
\end{array}$ & $\begin{array}{l}e^{+} e^{-} \\
\text {hadrons }\end{array}$ & $\begin{array}{l}.0014 \pm 0.0004 \\
\text { dominant }\end{array}$ & & 2015 \\
\hline$\psi(4160)$ & $\left(1^{-}\right)-$ & $4159 \pm 20$ & $78 \pm 20$ & $\begin{array}{l}17.297 \\
\pm 0.324 \\
\end{array}$ & $\begin{array}{l}e^{+} e^{-} \\
\text {hadrons }\end{array}$ & $\begin{array}{l}.0010 \pm 0.0004 \\
\text { dominant }\end{array}$ & & 2079 \\
\hline$\psi(4415)$ & $\left(1^{-}\right)-$ & $4415 \pm 6$ & $43 \pm 20^{5}$ & $\begin{array}{r}19.492 \\
\pm .190\end{array}$ & $\begin{array}{l}\mathrm{e}^{+} \mathrm{e}^{-} \\
\text {hadrons }\end{array}$ & $.0010 \pm 0.0003$ & & 2207 \\
\hline$r(9460)$ & $\left(1^{-}\right)-$ & $9458 \pm 6$ & $\sim 0.060$ & $\begin{array}{l}89.454 \\
\pm 0.0006 \\
\end{array}$ & $\begin{array}{l}\mu_{+}^{+} \mu^{-} \\
\mathrm{e}^{+} \mathrm{e}^{-}\end{array}$ & $\begin{array}{r}2.2 \pm 2.0 \\
2.5 \pm 2.1 \\
\end{array}$ & & $\begin{array}{l}4728 \\
4729 \\
\end{array}$ \\
\hline$T(10020)$ & $\underline{\left(1^{-}\right)-}$ & $10016 \pm 14$ & $<12$ & 100.320 & $\begin{array}{l}\mu^{+} \mu^{-} \\
\mathrm{e}^{+} \mathrm{e}^{-}\end{array}$ & $\begin{array}{l}\text { seen } \\
\text { seen }\end{array}$ & & $\begin{array}{l}5007 \\
5008\end{array}$ \\
\hline & $\mathrm{m}_{\mathrm{T}(10020)}$ & $-m_{\mathrm{T}}(9460)$ & $=559 \pm 7$ & & & & & \\
\hline
\end{tabular}

$\rightarrow \frac{\text { STRANGE MESONS }}{4}$

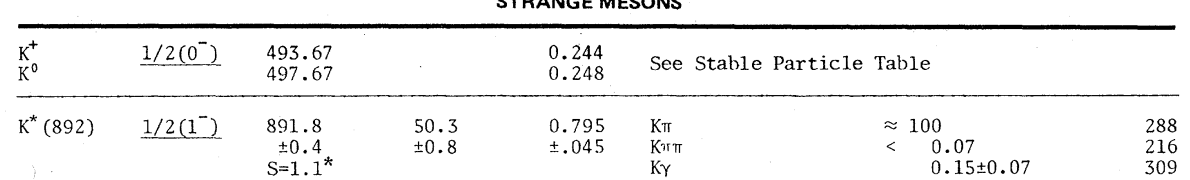

$M$ and $\Gamma$ from charged mode; $m^{0}-m^{ \pm}=6.7 \pm 1.2 \mathrm{MeV}$.

\begin{tabular}{|c|c|c|c|c|c|c|c|}
\hline \multirow[t]{4}{*}{$Q_{1}(1280)$} & $1 / 2\left(1^{+}\right)$ & $\sim 1280$ & ? 120 & 1.64 & $\mathrm{~K} \pi \pi$ & dominant & 501 \\
\hline & & & & & $+\left[K_{\rho}\right.$ & large] & 62 \\
\hline & & & & & $+\left[K^{*} \pi\right.$ & possibly seen] & 307 \\
\hline & & & & & $K a$ & possibly seen & \\
\hline \multirow[t]{3}{*}{$Q_{2}(1400)$} & $1 / 2\left(1^{+}\right)$ & $\sim 1400$ & $\sim 150$ & 1.96 & Кттा & dominant & 576 \\
\hline & & & & \pm .21 & $+\left[K^{*} \pi\right.$ & 2arge] & 399 \\
\hline & & & & & $+[\mathrm{Ko}$ & possibly seen] & 286 \\
\hline
\end{tabular}


Meson Table (cont'd)

\begin{tabular}{|c|c|c|c|c|c|c|c|c|c|}
\hline \multirow{2}{*}{$\begin{array}{l}\text { Name } \\
\begin{array}{rl|l}\text { GI } & 0 & 1 \\
- & \omega / \phi & \pi \\
+ & \eta & \rho\end{array}\end{array}$} & \multirow[b]{2}{*}{$\mathbf{I}^{\mathbf{G}_{\left(J^{P}\right) \mathbf{C}_{n}}}$} & \multirow[b]{2}{*}{$\begin{array}{c}\text { Mass } \\
\text { M } \\
(\mathrm{MeV})\end{array}$} & \multirow[b]{2}{*}{$\begin{array}{c}\text { Full } \\
\text { Width } \\
\Gamma \\
(\mathbf{M e V})\end{array}$} & \multirow[b]{2}{*}{$\begin{array}{c}\mathbf{M}^{2} \\
(\mathbf{G e V})^{2}\end{array}$} & \multicolumn{5}{|c|}{ Partial decay mode } \\
\hline & & & & & Mode & [ Upper & $\begin{array}{l}\text { Fraction }(\%) \\
\text { limits are }\end{array}$ & $\left.1 \sigma \cdot\left(\frac{\%}{0}\right)\right]$ & $\begin{array}{l}\operatorname{por}_{P_{\max }}(\mathrm{b}) \\
(\operatorname{MeV} / \mathrm{c})\end{array}$ \\
\hline$K^{*}(1430)$ & $1 / 2\left(2^{+}\right)$ & $\begin{array}{r}1434^{\S} \\
\pm 5^{\S}\end{array}$ & $\begin{array}{l}100^{\S} \\
\pm 10^{\S}\end{array}$ & $\begin{array}{l}2.06 \\
\pm .14\end{array}$ & $\begin{array}{l}K \pi \\
K^{*} \pi \\
K^{\star} \pi \pi \\
K \rho \\
K \omega \\
K n\end{array}$ & & $\begin{array}{r}49.1 \pm 1.6 \\
27.0 \pm 2.2 \\
11.2 \pm 2.5 \\
6.6 \pm 1.5 \\
3.7 \pm 1.6 \\
2.5 \pm 2.6\end{array}$ & $S=1.1^{*}$ & $\begin{array}{l}623 \\
424 \\
374 \\
327 \\
320 \\
492\end{array}$ \\
\hline$K(1500)$ & $1 / 2\left(0^{+}\right)$ & $\sim 1500$ & $\sim 250$ & $\begin{array}{l}2.25 \\
\pm .36\end{array}$ & К & & seen & & 661 \\
\hline
\end{tabular}

See note on $K \cdot \mathrm{S}$ wave ${ }^{\pi}$.

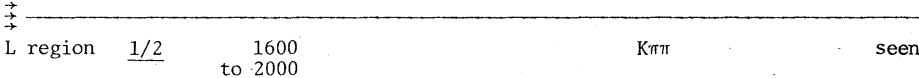

Not a well established resonance .

\begin{tabular}{|c|c|c|c|c|c|c|c|}
\hline \multirow[t]{2}{*}{$K^{*}(1780)^{\pi}$} & $1 / 2\left(3^{-}\right)$ & $\begin{array}{r}1785 \\
\pm 6\end{array}$ & $\begin{array}{l}126 \\
\pm 20^{\S}\end{array}$ & $\begin{array}{l}3.19 \\
\pm .22\end{array}$ & $\begin{array}{c}\mathrm{KN \pi \pi} \\
+[\mathrm{K} \rho\end{array}$ & $\begin{array}{l}\text { large } \\
\text { large] }\end{array}$ & $\begin{array}{l}798 \\
619\end{array}$ \\
\hline & & & & & $\begin{array}{c}+\left[K^{*} \pi\right. \\
K \pi\end{array}$ & $\begin{array}{c}\text { 1arge }] \\
19 \pm 5^{5}\end{array}$ & $\begin{array}{l}660 \\
817\end{array}$ \\
\hline
\end{tabular}

CHARMED, NONSTRANGE MESONS

\begin{tabular}{|c|c|c|c|c|c|c|}
\hline $\begin{array}{l}\vec{D}^{+} \\
\mathrm{D}^{0}\end{array}$ & $1 / 2\left(0^{-}\right)$ & $\begin{array}{l}1868.3 \\
1863.1\end{array}$ & $\begin{array}{l}3.491 \\
3.471\end{array}$ & See Stable Particle & Table & \\
\hline $\mathrm{D}^{*+}(2010)$ & $\begin{array}{l}1 / 2\left(1^{-}\right) \\
m_{D^{*+}}-m_{D^{0}}\end{array}$ & $\begin{array}{l}2008.6<2.0 \\
= \pm 1.0 \\
=145.3 \pm 0.4 \mathrm{MeV}\end{array}$ & 4.034 & $\begin{array}{l}\mathrm{D}^{0} \pi^{+} \\
\mathrm{D}^{+} \pi^{0} \\
\mathrm{D}^{+} \gamma\end{array}$ & $\begin{array}{c}64 \pm 11 \\
28 \pm 9 \\
8 \pm 7\end{array}$ & $\begin{array}{r}40 \\
37 \\
135\end{array}$ \\
\hline$D^{\star 0}(2010)$ & $1 / 2\left(1^{-}\right)$ & $\begin{array}{r}2006.0 \\
\pm 1.5\end{array}$ & 4.024 & $\begin{array}{l}D^{0} \pi^{0} \\
D^{0} \gamma\end{array}$ & $\begin{array}{l}55 \pm 15 \\
45 \pm 15\end{array}$ & $\begin{array}{r}45 \\
138\end{array}$ \\
\hline
\end{tabular}

Contents of Meson Data Card Listings

\begin{tabular}{|c|c|c|c|c|c|c|c|c|}
\hline entry & $I^{G}\left(J^{P}\right) C_{n}$ & $\begin{array}{l}\text { Non-stran } \\
\text { entry }\end{array}$ & $\begin{array}{l}\text { Inge }(S= \\
I^{G}\left(J^{P}\right) C_{n}\end{array}$ & $C=0)$ & entry & $I^{G}\left(J^{P}\right) C_{n}$ & $\begin{array}{c}\text { Strange }(|\mathrm{S}| \\
\text { entry }\end{array}$ & $\begin{array}{l}=1, \mathrm{C}=0) \\
\mathrm{I}\left(\mathrm{J}^{\mathrm{P}}\right)\end{array}$ \\
\hline$\pi$ & $1^{-}\left(0^{-}\right)+$ & $A_{2}(1310)$ & $1^{-}\left(2^{+}\right)+$ & \multicolumn{3}{|c|}{$\rightarrow \mathrm{e}^{+} \mathrm{e}^{-}(1100-3100)$} & K & $1 / 2\left(0^{-}\right)$ \\
\hline$n$ & $0^{+}\left(0^{-}\right)+$ & $E \cdot(1420)$ & $0^{+}\left(1^{+}\right)+$ & $\rightarrow \mathrm{X}$ & $(2830)$ & \multirow{5}{*}{$\begin{array}{l}0^{-}\left(1^{-}\right)- \\
0^{+}\left(0^{+}\right)+\end{array}$} & $\mathrm{K}^{*}(892)^{\prime}$ & $1 / 2\left(1^{-}\right)$ \\
\hline$\rho \quad(770)$ & $1^{+}\left(1^{-}\right)-$ & \multicolumn{2}{|c|}{$\rightarrow X(1410-1440)$} & $\rightarrow U$ & $(2980)$ & & $Q_{1}(1280)$ & $1 / 2\left(1^{+}\right)$ \\
\hline$\omega \quad(783)$ & $0^{-}\left(1^{-}\right)-$ & $f^{\prime}(1515)$ & $0^{+}\left(2^{+}\right)+$ & $J / \psi$ & $(3100)$ & & $\mathrm{Q}_{2}(1400)$ & $1 / 2\left(1^{+}\right)$ \\
\hline$\rightarrow M \quad(940-9$ & 53) & $\rightarrow \mathrm{F}_{1}(1540)$ & 1 (A) & $x$ & (3415) & & $\rightarrow \mathrm{K}^{\prime}(1400)$ & $1 / 2\left(0^{-}\right)$ \\
\hline$n^{\prime}(958)$ & $0^{+}\left(0^{-}\right)+$ & $\rho^{\prime}(1600)$ & $1^{-}\left(2^{-}\right)-$ & $\rightarrow x$ & (3455) & & $K^{*}(1430)$ & $1 / 2\left(2^{+}\right)$ \\
\hline$\delta \quad(980)$ & $1^{-}\left(0^{+}\right)+$ & $A_{3}(1.660)$ & $1^{+}\left(1^{-}\right)-$ & \multicolumn{2}{|c|}{$\mathrm{P}_{\mathrm{C}}$ or $\times(3510)$} & \multirow{3}{*}{$\begin{array}{l}0^{+}(\mathrm{A})+ \\
0^{+}(\mathrm{N})+\end{array}$} & $\kappa(1500)$ & $1 / 2\left(0^{+}\right)$ \\
\hline $\mathrm{S}^{*}(980)$ & $0^{+}\left(0^{+}\right)+$ & $\omega(1670)$ & $0^{-}\left(3^{-}\right)-$ & $x$ & (3550) & & $\rightarrow \mathrm{L}(1580)$ & $1 / 2\left(2^{-}\right)$ \\
\hline$\rightarrow \mathrm{H} \quad(990)$ & & $g(1700)$ & $1^{+}\left(3^{-}\right)-$ & $\rightarrow x$ & $(3590)$ & & $\rightarrow \mathrm{K}^{\star}(1650)$ & $1 / 2\left(1^{-}\right)$ \\
\hline$\phi(1020)$ & $0^{-}\left(1^{-}\right)-$ & $\rightarrow X(1690)$ & & $\psi$ & $(3685)$ & \multirow{6}{*}{$\begin{array}{c}0^{-}\left(1^{-}\right)- \\
\left(1^{-}\right)- \\
\left(1^{-}\right)- \\
\left(1^{-}\right)- \\
\left(1^{-}\right)- \\
\left(1^{-}\right)-\end{array}$} & $\rightarrow \mathrm{K}_{\mathrm{N}}(1700)$ & $1 / 2$ \\
\hline \multicolumn{2}{|c|}{$\rightarrow M(1033-1040)$} & $\rightarrow A_{4}(1900)$ & $1^{-}$ & $\psi$ & $(3770)$ & & $\mathrm{L}$ region & $1 / 2(\mathrm{~A})$ \\
\hline$\rightarrow n_{N}(1080)$ & $0^{+}(\mathrm{N})+$ & $\rightarrow A_{2}(1900)$ & $1^{-}\left(4^{+}\right)+$ & $\psi$ & $(4030)$ & & $K^{*}(1780)$ & $1 / 2\left(3^{-}\right)$ \\
\hline$\Rightarrow M(1150-1$ & \multirow[b]{2}{*}{$1^{-}\left(1^{+}\right)+$} & $S(1935)$ & & $\psi$ & $(4160)$ & & $\rightarrow K^{*}(2200)$ & \\
\hline $\begin{array}{r}A_{1}(1100- \\
1300)\end{array}$ & & h (2040) & $0^{+}\left(4^{+}\right)+$ & $\psi$ & (4415) & & $\rightarrow$ I $(2600)$ & \\
\hline B (1235) & $1^{+}\left(1^{+}\right)-$ & $\rightarrow \mathrm{Tl}(2190)$ & 1 & $\mathrm{~T}$ & $(10020)$ & & \multicolumn{2}{|c|}{ Charmed $(|C|=1)$} \\
\hline$\rightarrow \rho^{\prime}(1250)$ & $1^{+}\left(1^{-}\right)-$ & $\rightarrow X(2200)$ & & $\mathrm{T}$ & $(10400)$ & $\left(1^{-}\right)-$ & $\mathrm{D}(1870)$ & $1 / 2\left(0^{-}\right)$ \\
\hline$f(1270)$ & $0^{+}\left(2^{+}\right)+$ & $\rightarrow$ U0 (2350) & 0 & & & & $D^{*}(2010)$ & $1 / 2\left(1^{-}\right)$ \\
\hline$\rightarrow \eta(1275)$ & $0^{+}\left(0^{-}\right)+$ & $\rightarrow \mathrm{U1}(2400)$ & 1 & & & & $\rightarrow F(2030)$ & \\
\hline D (1285) & $0^{+}\left(1^{+}\right)+$ & $\rightarrow \overrightarrow{\mathrm{N}} \mathrm{N}(1400-36$ & $600)$ & & & & $\rightarrow F^{*}(2140)$ & \\
\hline$\varepsilon(1300)$ & $0^{+}\left(0^{+}\right)+$ & $\rightarrow X(1900-36$ & $600)$ & & & & $\rightarrow$ Exotics & \\
\hline
\end{tabular}




\section{Meson Table (cont'd)}

$\rightarrow \quad$ Indicates an entry in Meson Data Card Listings not entered in the Meson Table. We do not regard these as established resonances. All the entries in the Listings can be found in the Table of Contents of Meson Data Card Listings.

^I See Meson Data Card Listings.

* Quoted error includes scale factor $S=\sqrt{\chi^{2} /(N-1)}$. See footnote to Stable Particle Table.

$+\quad$ Square brackets indicate a subreaction of the previous (unbracketed) decay mode(s).

\$ This is only an educated guess; the error given is larger than the error of the average of the published values. (See Meson Data Card Listings for the latter.)

(a) $\Gamma M$ is approximately the half-width of the resonance when plotted against $M^{2}$.

(b) For decay modes into $\geq 3$ particles, $p_{\max }$ is the maximum momentum that any of the particles in the final state can have. The momenta have been calculated by using the averaged central mass values, without taking into account the widths of the resonances.

(c) From pole position (M - i $/ 2)$.

(d) The $\mathrm{e}^{+} \mathrm{e}^{-}$branching ratio is from $\mathrm{e}^{+} \mathrm{e}^{-} \rightarrow \pi^{+} \pi^{-}$experiments only. The wo interference is then due to wo mixing only, and is expected to be small. See note in Meson Data Card Listings. The $\mu^{+} \mu^{-}$branching ratio is compiled from 3 experiments; each possibly with substantial wo interference. The error reflects this uncertainty; see notes
universality holds, $\Gamma\left(\rho^{0} \rightarrow \mu^{+} \mu^{-}\right)=\Gamma\left(\rho^{0} \rightarrow \mathrm{e}^{+} \mathrm{e}^{-}\right) \times 0.99785$. (e) Empirical limits on fractions for other decay modes of $\rho(770)$ are $\pi^{+} n<0.80^{\circ}, \pi^{+} \pi^{+} \pi^{-} \pi^{-}<0.15 \%$,

(f) Empirical values of fractions for other decay modes of $\omega(783)$ are $\pi^{+} \pi^{-} \gamma<5 \%, \pi^{0} \pi^{0} \gamma<1 \%$, $\eta+\operatorname{neutra1}(\mathrm{s})<1.5 \%, \mu^{+} \mu^{-}<0.02^{\circ}, \pi^{0} \mu^{+} \mu^{-}=(9+5) \times 10^{-}$

(g) Empirical values of fractions for other deciay modes of $n^{\prime}(958): \pi^{+} \pi^{-}<20^{\circ}, \pi^{+} \pi^{-} \pi^{0}<5^{\circ}$, $\pi^{+} \pi^{+} \pi^{-} \pi^{-}<1 \%, \pi^{+} \pi^{+} \pi^{-} \pi^{-} \pi^{0}<1 \%, 6 \pi<1 \%, \pi^{+} \pi^{-} \mathrm{e}^{+} \mathrm{e}^{-}<0.6 \%, \pi^{0} \mathrm{e}^{-} \mathrm{e}^{-}<1.3 \%, \mathrm{ne}^{+} \mathrm{e}^{-}<1.1 \%$, $\pi^{0} \rho^{0}<4 \frac{0}{0}, \mu^{+} \mu^{-} \gamma=(8 \pm 4) \times 10^{-5}$.

(h) The mass and width are from the $n \pi$ mode only. If the $k \bar{K}$ channel is strongly coupled, the width may be larger.

(i) Enpirical limits on fractions for other decay modes of $\phi(1020)$ are $\pi^{+} \pi^{-}<0.03 \%, \pi^{+} \pi^{-} \gamma<0.7^{\circ}$

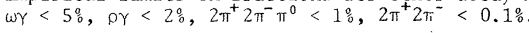

(j) Empirical limits on fractions for other decay modes of $B(1235): \pi \pi<15 \%, K \bar{K}<2 \%, 4 \pi<50 \%$, $\phi \pi<1.5 \%, n \pi<25 \%,(\overline{\mathrm{K} K}) \pi^{ \pm}<8 \%, \mathrm{~K}_{\mathrm{S}} \mathrm{K}_{\mathrm{S}} \pi^{ \pm}<2 \%, \mathrm{~K}_{\mathrm{S}} \mathrm{K}_{\mathrm{L}} \pi^{ \pm}<6 \%$.

(k) Empirical 1 imits on fractions for other decay modes of $\mathrm{f}^{\prime}(1515)$ are $\eta n<50 \%$ $n \pi \pi<30^{\circ}, K_{\bar{K}} \pi+K^{*} \bar{K}<35^{\circ}, 2 \pi^{+} 2 \pi^{-}<320^{\circ}$.

( $\ell$ E) Empirical limits on fractions for other decay modes of $f(1270)$ are $n \pi \pi<1 \%, \mathrm{~K}^{0} \mathrm{~K}^{-} \pi^{+}+$c.c. $<1 \%$, $n n<2 \%$.

(m) Preliminary results from the Crystal Ball experiment give an upper limit of 0.007 , see Meson Data Card Listings.

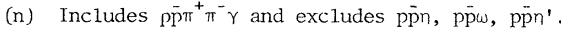

Established Nonets, and octet-singlet mixing angles from Appendix IIB, Eq. (2'). Of the two isosinglets, the "mainly octet" one is written first, followed by a semicolon.

\begin{tabular}{|c|c|c|c|}
\hline$\left(J^{\mathrm{P}}\right) \mathrm{C}_{\mathrm{n}}$ & Nonet members & ${ } 1$ in. & ${ }$ quadr. \\
\hline$\left(0^{-}\right)+$ & $\pi, K, n ; n^{\prime}$ & $-24 \pm 1^{\circ}$ & $-10 \pm 1^{\circ}$ \\
\hline$\left(1^{-}\right)-$ & $\rho, K^{*}, \phi ; \omega$ & $38 \pm 1^{\circ}$ & $40 \pm 1^{\circ}$ \\
\hline$\left(2^{+}\right)+$ & $\mathrm{A}_{2}, K^{*}(1430), \mathrm{f}^{\prime} ; \mathrm{f}$ & $25 \pm 4^{\circ}$ & $26 \pm 4^{\circ}$ \\
\hline
\end{tabular}




\section{Baryon Table}

Apri1 1980

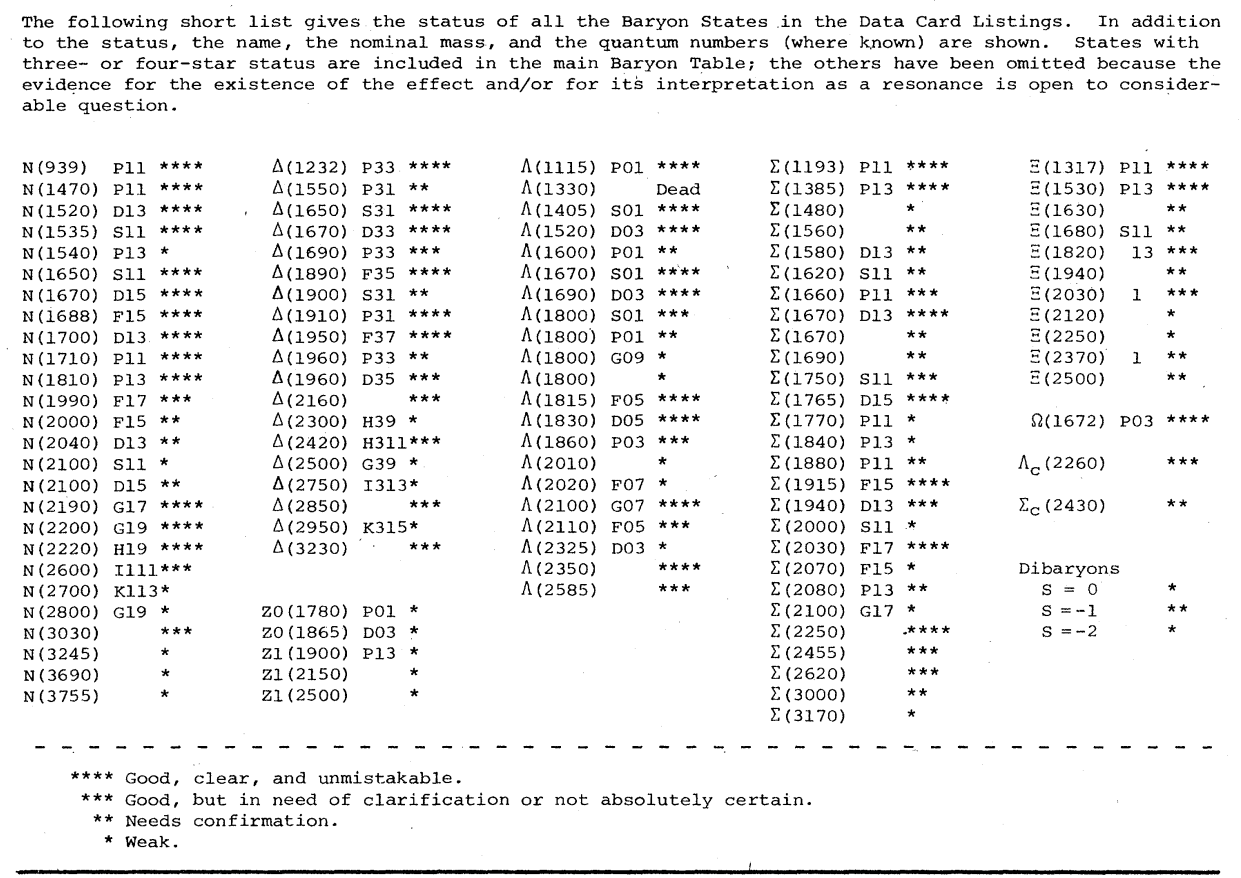

[See notes on $N^{\prime} s$ and $\Delta^{\prime} s, z^{*}{ }^{\prime} s, \Lambda^{\prime} s$ and $\Sigma^{\prime} s, F^{*}{ }^{\prime} s$, and dibaryons at the beginning of those sections in the Baryon Data Card Listings; also see notes on individual resonances in the Baryon Data Card Listings.]

\begin{tabular}{|c|c|c|c|c|c|c|c|c|c|}
\hline \multirow{2}{*}{ Particle $^{\alpha}$} & \multirow{2}{*}{ 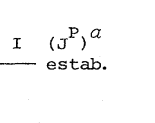 } & \multirow{2}{*}{$\begin{array}{l}\frac{\pi \text { or } \mathrm{K} \mathrm{beam}}{b} \\
\mathrm{P}_{\text {beam }}(\mathrm{GeV} / \mathrm{c}) \\
\sigma=4 \pi \lambda^{2}(\mathrm{mb})\end{array}$} & \multirow[b]{2}{*}{$\begin{array}{l}\text { Mass } \\
M^{c} \\
(\mathrm{MeV})\end{array}$} & \multirow[b]{2}{*}{$\begin{array}{c}\text { Full } \\
\text { Width } \\
\Gamma^{C} \\
(\mathrm{MeV})\end{array}$} & \multirow{2}{*}{$\begin{array}{l}\mathrm{M}^{2} \\
\pm \Gamma \mathrm{M} b \\
\left(\mathrm{GeV}^{2}\right)\end{array}$} & \multicolumn{4}{|c|}{ Partial decay mode $f$} \\
\hline & & & & & & Mode & Fra & $\operatorname{action}^{c}$ & $\begin{array}{l}\mathrm{p} \text { or } d \\
\mathrm{p}_{\max } \\
(\mathrm{MeV} / \mathrm{C})\end{array}$ \\
\hline & & $S=0 \quad I=$ & NUCLEON & RESONAN & ES (N) & & & & \\
\hline $\begin{array}{l}\mathrm{p} \\
\mathrm{n}\end{array}$ & $1 / 2\left(1 / 2^{+}\right)$ & & $\begin{array}{l}938.3 \\
939.6\end{array}$ & & $\begin{array}{l}0.880 \\
0.883\end{array}$ & See & Stable & e Particle & e Table \\
\hline$N(1470)$ & $1 / 2\left(1 / 2^{+}\right) P_{11}^{\prime}$ & $\begin{array}{l}p=0.66 \\
\sigma=27.8\end{array}$ & $\begin{array}{r}1400 \text { to } \\
1480\end{array}$ & $\begin{array}{r}120 \text { to } \\
350 \\
(200)\end{array}$ & $\begin{array}{r}2.16 \\
\pm 0.29\end{array}$ & $\begin{array}{l}\mathrm{N} \pi \\
\mathrm{N} \eta \\
\mathrm{N} \pi \pi \\
{[\mathrm{N} \varepsilon} \\
{[\Delta \pi} \\
{[\mathrm{N} \rho}\end{array}$ & & $\begin{aligned} & 50-65 \\
& \sim 18 \\
& \sim 25 \\
&\sim 7]^{e} \\
&\sim 23]^{e} \\
&\sim 7]^{e}\end{aligned}$ & $\begin{array}{c}420 \\
d \\
368 \\
d \\
177 \\
d\end{array}$ \\
\hline $\mathrm{N}(1520)$ & $1 / 2\left(3 / 2^{-}\right) D_{13}^{\prime}$ & $\begin{array}{l}p=0.74 \\
\sigma=23.5\end{array}$ & $\begin{array}{r}1510 \text { to } \\
1530\end{array}$ & $\begin{array}{r}100 \text { to } \\
140 \\
(125)\end{array}$ & $\begin{array}{r}2.31 \\
\pm 0.19\end{array}$ & $\begin{array}{l}\mathrm{N} \pi \\
\mathrm{N} \pi \pi \\
{[\mathrm{N} \varepsilon} \\
{[\mathrm{N} \rho} \\
{[\Delta \pi} \\
\mathrm{N} n\end{array}$ & & $\begin{array}{l}\sim 55 \\
\sim 45 \\
<5]^{e} \\
\sim 19]^{e} \\
\sim 23]^{e} \\
<1\end{array}$ & $\begin{array}{c}456 \\
410 \\
d \\
d \\
228 \\
d\end{array}$ \\
\hline $\mathrm{N}(1535)$ & 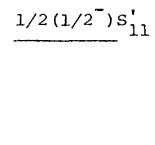 & $\begin{array}{l}p=0.76 \\
\sigma=22.5\end{array}$ & $\begin{array}{r}1520 \text { to } \\
1560\end{array}$ & $\begin{array}{r}100 \text { to } \\
250 \\
(150)\end{array}$ & $\begin{array}{r}2.36 \\
\pm 0.23\end{array}$ & $\begin{array}{l}\mathrm{N} \pi \\
\mathrm{N} n \\
\mathrm{~N} \pi \pi \\
{[\mathrm{N} \rho} \\
{[\mathrm{N} \varepsilon} \\
{[\Delta \pi}\end{array}$ & & $\begin{array}{l}\sim 40 \\
\sim 55 \\
\sim 5 \\
\sim 3]^{e} \\
\sim 2]^{e} \\
\sim 1]^{e}\end{array}$ & $\begin{array}{c}467 \\
182 \\
422 \\
d \\
d \\
243\end{array}$ \\
\hline$N(1650)$ & $1 / 2\left(1 / 2^{-}\right) s_{11}^{\prime \prime}$ & $\begin{array}{l}p=1.05 \\
\sigma=14.3\end{array}$ & $\begin{array}{r}1620 \text { to } \\
1680\end{array}$ & $\begin{array}{r}100 \text { to } \\
200 \\
(150)\end{array}$ & $\begin{array}{r}2.72 \\
\pm 0.25\end{array}$ & $\begin{array}{c}\mathrm{N} \pi \\
\mathrm{N} \pi \pi \\
{[\mathrm{N} \varepsilon} \\
{[\mathrm{N} \rho} \\
{[\Delta \pi} \\
\Lambda \mathrm{K} \\
\Lambda_{\mathrm{K}} \\
\mathrm{N} \\
\mathrm{N} n\end{array}$ & & $\begin{array}{l}\sim 60 \\
\sim 30 \\
<10]^{e} \\
7-21]^{e} \\
4-15]^{e} \\
\sim 10 \\
2-7 \\
\sim 1\end{array}$ & $\begin{array}{c}547 \\
511 \\
d \\
d \\
344 \\
161 \\
d \\
346\end{array}$ \\
\hline
\end{tabular}




\section{Baryon Table (cont'd)}

\begin{tabular}{|c|c|c|c|c|c|c|c|c|}
\hline \multirow[t]{2}{*}{ Particle $^{a}$} & \multirow{2}{*}{$\begin{array}{l}\left.I \quad J^{P}\right)^{a} \\
\text { estab. }\end{array}$} & \multirow{2}{*}{$\begin{array}{l}\frac{\pi \text { or } K \text { beam }}{b} \\
\mathrm{p}_{\text {beam }}(\mathrm{GeV} / \mathrm{c}) \\
\sigma=4 \pi \lambda^{2} \quad(\mathrm{mb})\end{array}$} & \multirow[b]{2}{*}{$\begin{array}{c}\text { Mass } \\
M^{C} \\
(\mathrm{MeV})\end{array}$} & \multirow[b]{2}{*}{$\begin{array}{c}\text { Full } \\
\text { Width } \\
\Gamma^{e} \\
(\mathrm{MeV})\end{array}$} & \multirow{2}{*}{$\begin{array}{l}\mathrm{M}^{2} \\
\pm \Gamma \mathrm{M}^{3} \\
\left(\mathrm{GeV}^{2}\right)\end{array}$} & \multicolumn{3}{|c|}{ Partial decay mode $f$} \\
\hline & & & & & & Mode & $\underset{:}{\operatorname{Fraction}}{ }^{c}$ & $\begin{array}{l}\mathrm{p} \text { or } d \\
\mathrm{p}_{\max } \\
(\mathrm{MeV} / \mathrm{C})\end{array}$ \\
\hline$N(1670)$ & $1 / 2\left(5 / 2^{-}\right) D_{15}^{\prime}$ & $\begin{array}{l}\mathrm{p}=1.00 \\
\sigma=15.6\end{array}$ & $\begin{array}{r}1660 \text { to } \\
1690\end{array}$ & $\begin{array}{r}120 \text { to } \\
180 \\
(155)\end{array}$ & $\begin{array}{r}2.79 \\
\pm 0.26\end{array}$ & $\begin{array}{c}\mathrm{N} \pi \\
\mathrm{N} \pi \pi \\
{[\Delta \pi} \\
{[\mathrm{N} \rho} \\
\Lambda \mathrm{N} \\
\mathrm{N} n \\
\mathrm{~N} n\end{array}$ & $\begin{array}{l}\sim 40 \\
\sim 60 \\
\sim 50]^{e} \\
\sim 5]^{e} \\
<0.3 \\
<0.5\end{array}$ & $\begin{array}{c}560 \\
525 \\
360 \\
d \\
200 \\
368\end{array}$ \\
\hline $\mathrm{N}(1688)$ & $\underline{1 / 2\left(5 / 2^{+}\right) F_{15}^{\prime}}$ & $\begin{array}{l}p=1.03 \\
\sigma=14.9\end{array}$ & $\begin{array}{r}1670 \text { to } \\
1690\end{array}$ & $\begin{array}{r}110 \text { to } \\
140 \\
(130)\end{array}$ & $\begin{array}{r}2.85 \\
\pm 0.22\end{array}$ & $\begin{array}{l}N \pi \\
N \pi \pi \\
{[N \pi} \\
{[N \varepsilon} \\
{[N \rho} \\
{[\Delta \pi} \\
N \eta\end{array}$ & $\begin{array}{l}\sim 60 \\
\sim 40 \\
\sim 22]^{e} \\
\sim 13]^{e} \\
\sim 18]^{e} \\
<0.3\end{array}$ & $\begin{array}{c}572 \\
538 \\
340 \\
d \\
375 \\
388\end{array}$ \\
\hline$N(1700)$ & $1 / 2\left(3 / 2^{-}\right) D_{13}^{\prime \prime}$ & $\begin{array}{l}p=1.05 \\
\sigma=14.3\end{array}$ & $\begin{array}{r}1670 \text { to } \\
1730\end{array}$ & $\begin{array}{l}70 \text { to } \\
120^{g} \\
(120)\end{array}$ & $\begin{array}{r}2.89 \\
\pm 0.20\end{array}$ & 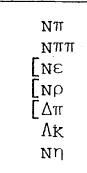 & $\begin{array}{c}\sim 10 \\
\sim 90 \\
<40]^{e} \\
<5]^{e} \\
15-40]^{e} \\
<1 \\
\sim 4\end{array}$ & $\begin{array}{c}580 \\
547 \\
355 \\
d \\
385 \\
250 \\
400\end{array}$ \\
\hline $\begin{array}{c}\mathrm{N}(1710) \\
\ldots\end{array}$ & $1 / 2\left(1 / 2^{+}\right) P_{11}^{\prime \prime}$ & $\begin{array}{l}p=1.20 \\
\sigma=12.2\end{array}$ & $\begin{array}{r}1680 \text { to } \\
1740\end{array}$ & $\begin{array}{c}100 \text { to } h \\
140 \\
(120)\end{array}$ & $\begin{array}{r}2.92 \\
\pm 0.21\end{array}$ & $\begin{array}{l}\mathrm{N \pi} \\
\mathrm{N} \pi \pi \\
{[N \varepsilon} \\
{[N \varepsilon} \\
{[\mathrm{N} \rho} \\
{[\Delta \pi} \\
\Lambda \mathrm{K} \\
\sum \mathrm{K} \\
\mathrm{N} n\end{array}$ & $\begin{array}{c}\sim 20 \\
>50 \\
15-40]^{e} \\
40-65]^{e} \\
10-20]^{e} \\
<5 \\
\sim 10 \\
2-20^{i}\end{array}$ & $\begin{array}{c}587 \\
554 \\
d \\
d \\
393 \\
264 \\
138 \\
410\end{array}$ \\
\hline$N(1810)$ & $\underline{1 / 2\left(3 / 2^{+}\right)} \mathrm{P}_{13}^{\prime \prime}$ & $\begin{array}{l}p=1.26 \\
\sigma=11.5\end{array}$ & $\begin{array}{r}1690 \text { to } \\
1800 .\end{array}$ & $\begin{array}{r}150 \text { to } \\
250 \\
(200)\end{array}$ & $\begin{array}{r}3.28 \\
\pm 0.36\end{array}$ & $\begin{array}{l}\mathrm{N} \pi \\
\mathrm{N} \pi \pi \\
{[\mathrm{N} \varepsilon} \\
{[\mathrm{N} \rho} \\
{[\Delta \pi} \\
\Lambda \mathrm{K} \\
\Sigma \mathrm{K} \\
\mathrm{N} \eta\end{array}$ & $\begin{array}{c}\sim 17 \\
\sim 70 \\
\sim 20]^{e} \\
45-70]^{e} \\
\sim 20]^{e} \\
1-4 \\
\sim 2 \\
<5\end{array}$ & $\begin{array}{l}652 \\
624 \\
468 \\
297 \\
471 \\
386 \\
307 \\
503\end{array}$ \\
\hline $\begin{aligned} & \mathrm{N}(1990) \\
\rightarrow & \end{aligned}$ & $1 / 2\left(7 / 2^{+}\right) F_{17}$ & $\begin{array}{l}p=1.62 \\
\sigma=8.35\end{array}$ & $\begin{array}{r}1950 \text { to } \\
2050\end{array}$ & $\begin{array}{r}100 \text { to } \\
400 \\
(250)\end{array}$ & $\begin{array}{r}3.96 \\
\pm 0.50\end{array}$ & $\begin{array}{l}\mathrm{N} \pi \\
\mathrm{N} \eta \\
\Lambda \mathrm{K} \\
\Sigma \mathrm{K}\end{array}$ & $\begin{array}{l}\sim 5 \\
\sim 3 \\
\text { seen } \\
\text { seen }\end{array}$ & $\begin{array}{l}772 \\
655 \\
562 \\
506\end{array}$ \\
\hline $\mathrm{N}(2190)$ & $1 / 2\left(7 / 2^{-}\right) G_{17}$ & $\begin{array}{l}p=2.07 \\
\sigma=6.21\end{array}$ & $\begin{array}{r}2120 \text { to } \\
2180\end{array}$ & $\begin{array}{l}<400 \\
(250)\end{array}$ & $\begin{array}{r}4.80 \\
\pm 0.55\end{array}$ & $\begin{array}{l}\mathrm{N} \pi \\
\mathrm{N} n \\
\Lambda_{\mathrm{K}}\end{array}$ & $\begin{array}{r}\sim 15 \\
\sim 2 \\
<1\end{array}$ & $\begin{array}{l}888 \\
790 \\
712\end{array}$ \\
\hline$N(2200)$ & $1 / 2\left(9 / 2^{-}\right) G_{19}$ & $\begin{array}{l}p=2.10 \\
\sigma=6.12\end{array}$ & $\begin{array}{r}2130 \text { to } \\
2270\end{array}$ & $\begin{array}{r}200 \text { to } \\
350 \\
(250)\end{array}$ & $\begin{array}{r}4.84 \\
\pm 0.55\end{array}$ & $\begin{array}{l}\mathrm{N} \pi \\
\mathrm{N} n\end{array}$ & $\begin{array}{l}\sim 10 \\
\sim 2\end{array}$ & $\begin{array}{l}894 \\
810\end{array}$ \\
\hline $\mathrm{N}(2220)$ & $1 / 2\left(9 / 2^{+}\right) \mathrm{H} 19$ & $\begin{array}{l}p=2.14 \\
\sigma=5.97\end{array}$ & $\begin{array}{r}2150 \text { to } \\
2300\end{array}$ & $\begin{array}{c}\sim 300 \\
(300)\end{array}$ & $\begin{array}{r}4.93 \\
\pm 0.67\end{array}$ & $\begin{array}{l}\text { NTT } \\
\text { Nn }\end{array}$ & $\begin{array}{r}\sim 20 \\
\sim \quad 1\end{array}$ & $\begin{array}{l}905 \\
811\end{array}$ \\
\hline$N(2600)$ & $1 / 2\left(11 / 2^{-}\right) I_{111}$ & $\begin{array}{l}p=3.26 \\
\sigma=3.67\end{array}$ & $\begin{array}{r}2580 \text { to } \\
2700\end{array}$ & $\begin{array}{l}>300 \\
(400)\end{array}$ & $\begin{array}{r}6.76 \\
\pm 1.04\end{array}$ & $\mathrm{~N} \pi$ & $\sim 5$ & 1014 \\
\hline$\rightarrow$ & $1 / 2(?)$ & $\begin{array}{l}p=4.41 \\
\sigma=2.62\end{array}$ & $\sim 3030$ & $\begin{array}{l}\sim 400 \\
(400)\end{array}$ & $\begin{array}{r}9.18 \\
\pm 1.21\end{array}$ & $\mathrm{~N} \pi$ & $\begin{array}{l}(J+1 / 2) \times \\
<0.1 k\end{array}$ & 1366 \\
\hline
\end{tabular}




\section{Baryon Table (cont'd)}

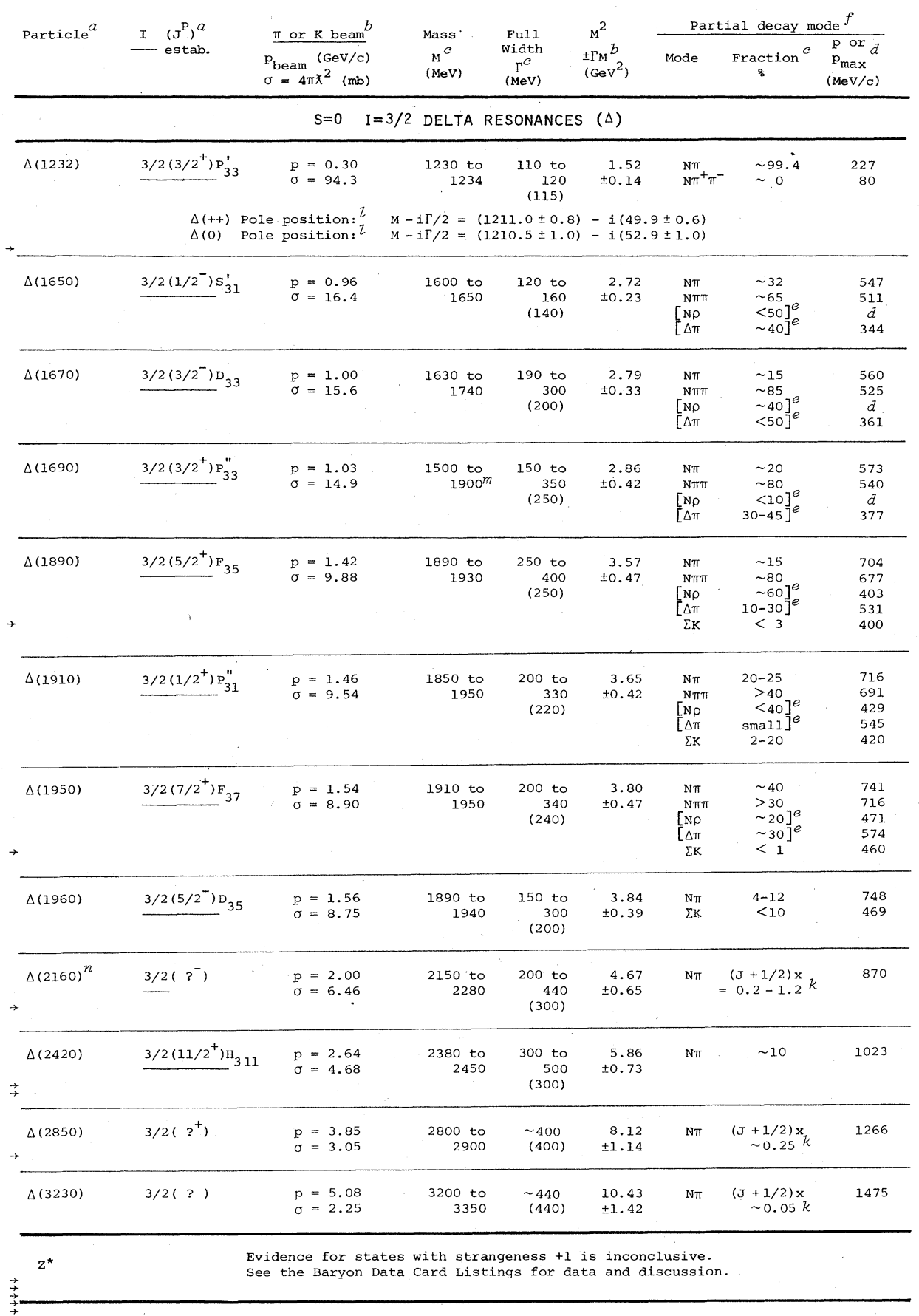




\section{Baryon Table (cont'd)}

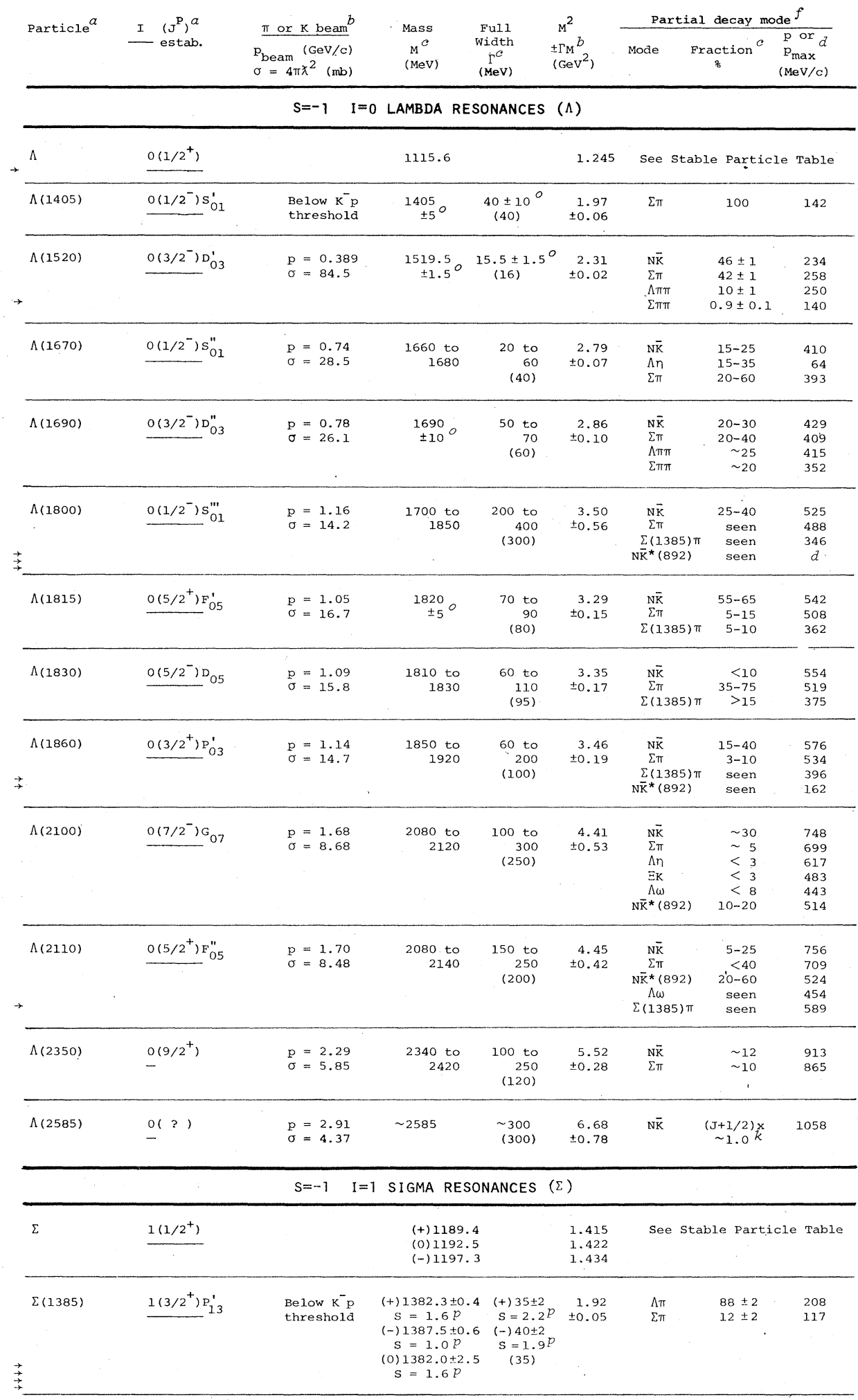




\section{Baryon Table (cont'd)}

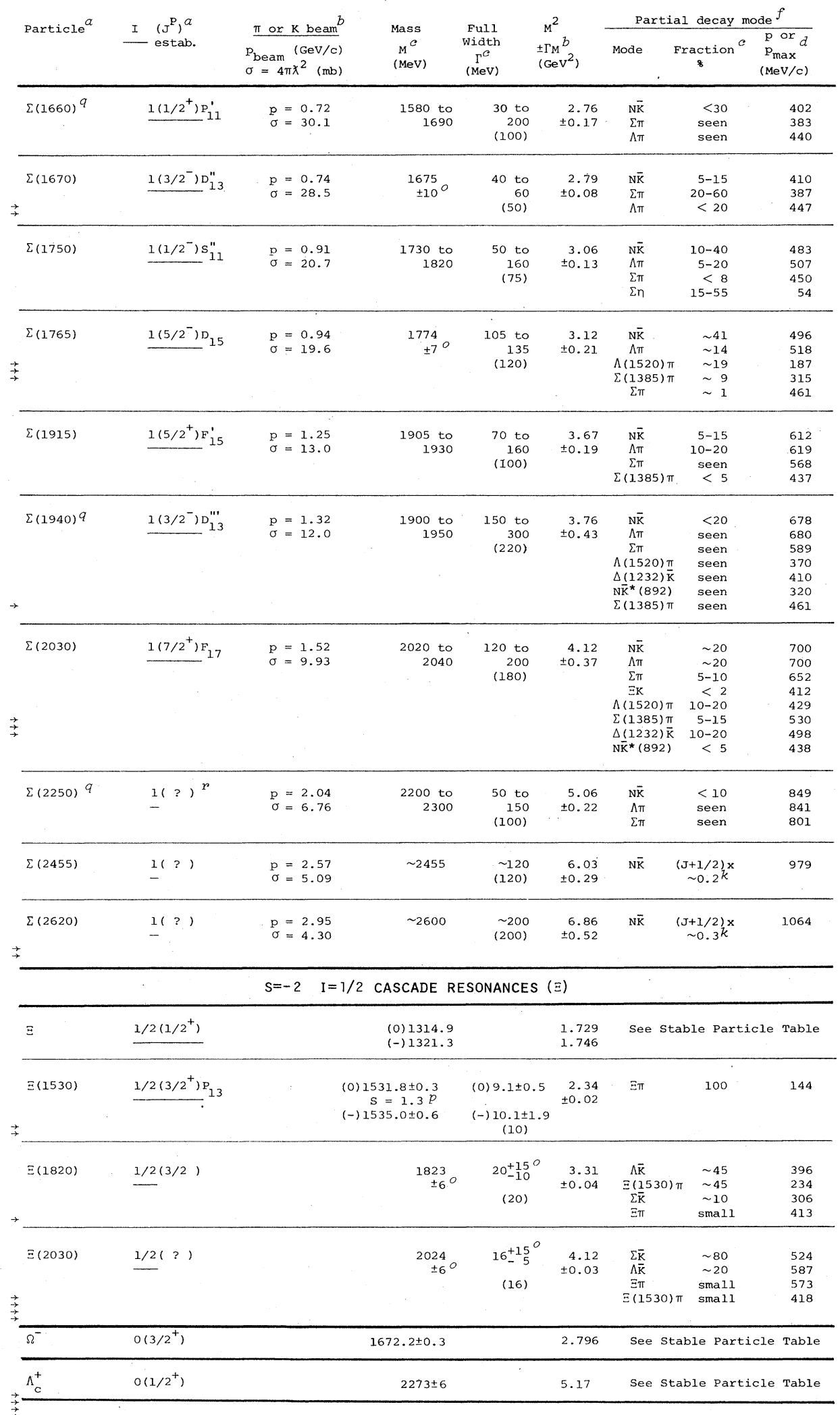




\section{Baryon Table (cont'd)}

For convenience all Baryon States for which information exists in the Baryon Data Card Listings are listed at the beginning of the Baryon Table. States with only a one or two star (*) rating in that list have been omitted from the main Baryon Table; each omitted state is indicated by an arrow in the
left-hand margin of the Table. In the Listings there is an arrow under the name of each state omitted from the Table.

a. The names of the Baryon States in Col. 1 [such as $\mathrm{N}(1470)$ ] contain a nominal mass which is primarily The names of the Baryon states in $\mathrm{Col}$. 1 [such as $\mathrm{N}(1470)]$ contain a nominal mass which is primarily
for purposes of identification. See Col. 4 for actual mass values. The convention for using primes for purposes of identification. See Col. 4 for actual mass values. The convention for using primes
in the spectroscopic notation for the quantum numbers in Col. 2 (such as Pil) is as follows: no prime in the nuantum numbers in Col. 2 (such as $P_{11}$ ) is as follows: no prit there is more than one resonance, the first has been designated with a prime, the second with a double there is more than one resonance, the first has been designate are also given in large print at the beginning of the Data Card Listings for that state. See footnote a. of the Stable Particle Table the strangeness quantum numbers of the baryons; in addition to the names listed there, we also use $N$ and $\Delta$ for $\mathrm{S}=0$ baryons, and $\mathrm{Z}^{*}$ for $\mathrm{S}=+1$ baryons.

b. The numbers in Col. 3 and Col. 6 are calculated using the nominal mass (see $a$. above) for $M$ and the nominal width (see $c$. below) for $\Gamma$.

c. For masses, widths, and branching fractions of most baryons we report here a range instead of an average. Averages are appropriate if each result is based on independent measurements, but inappropriate where the spread in parameters arises because different models or procedures have been applied to a common set of data. The ranges given in the Table are generally chosen to be conservatively large. See the Data Card listings for the individual values obtained in specific analyses. A single value with an approximation sign $(\sim)$ indicates that there is not enough data to give a meaningful interval. A nominal width is included in parentheses in Col. 5; this nominal width is used to calculate the value of $\Gamma M$ given in Col. 6.

d. For two-body decay modes we give the momentum, $p$, of the decay products in the decaying baryon rest frame. For decay modes into $\geqslant 3$ particles we give the maximum momentum, $\mathrm{p}_{\text {max }}$, that any of the particles in the final state can have in this frame. The momenta are calculated using the nominal mass (see $a$. above) of the decaying baryon, and of any isobars in the final state. Some decays which would be energetically forbidden for the nominal masses actually occur because of the finite widths of the decaying baryon and/or isobars in the final state. In these cases, the decay momentum is omitted from Col, 9 and replaced with a reference to this footnote.

e. Square brackets around an isobar decay mode indicate that it is a sub-reaction of the previous unbracketed decay mode.

f. Many of the branching fractions in the Table are extracted from significantly more accurate results on $\sqrt{\mathrm{xx}^{\prime}}$ type couplings obtained in partial-wave analyses. The original $\sqrt{\mathrm{xx}^{\prime}}$ values are given in the Baryon Data Card Listings. For information on radiative decays of $\mathrm{N}^{\prime} \mathrm{s}$ and $\Delta^{\prime} \mathrm{s}$, see the minireview preceding the Baryon Data Card Listings.

g. The range given here does not include the widths of several hundred MeV reported by LONGACRE 75 and LONGACPE 77 .

$h$. The range given here does not include the width of $550 \mathrm{MeV}$ reported by SAXON 80 .

$i$. The range given here does not include the branching ratio of approximately 80 \% reported by FELTESSE 75 .

$k$. This state has been seen only in an energy-dependent fit to total, channel, or fixed angle crosssection data. $J$ is not known; $x$ is $\Gamma_{e l} / \Gamma$.

2. See note on determination of resonance parameters in the Baryon Data Card Listings. Values of mass and width are dependent upon resonance shape used to fit the data. The pole position is much less and width are dependent upon resonance shape used to fit the data. The pole position is much less Data Card Listings) of fits to phase shifts without Coulomb corrections.

$m$. There may be more than one $\mathrm{P}_{33}$ resonance in or near this mass range.

$n$. There is probably more than one $\Delta$ resonance near $2160 \mathrm{MeV}$. The parameter ranges in the Table include the various possibilities. See the Baryon Data Card Listings.

0 . The error given here is only an educated guess; it is larger than the error of the average of the published values (see the Baryon Data Card Listings for the latter).

p. Quoted error includes a S (scale) factor. See second footnote to Stable Particle Table.

q. Because the elastic branching fraction of this resonance is poorly determined, it is not possible to extract inelastic branching fractions from partial-wave couplings. See the Baryon Data Card Listings for the partial-wave couplings.

$r$. Recent partial-wave analyses of the College de France-Saclay group find evidence for a $5 / 2^{-}$and a $9 / 2^{-} \sum$ resonance at this mass. See the Baryon Data Card Listings. 


\section{PHYSICAL AND NUMERICAL CONSTANTS*}

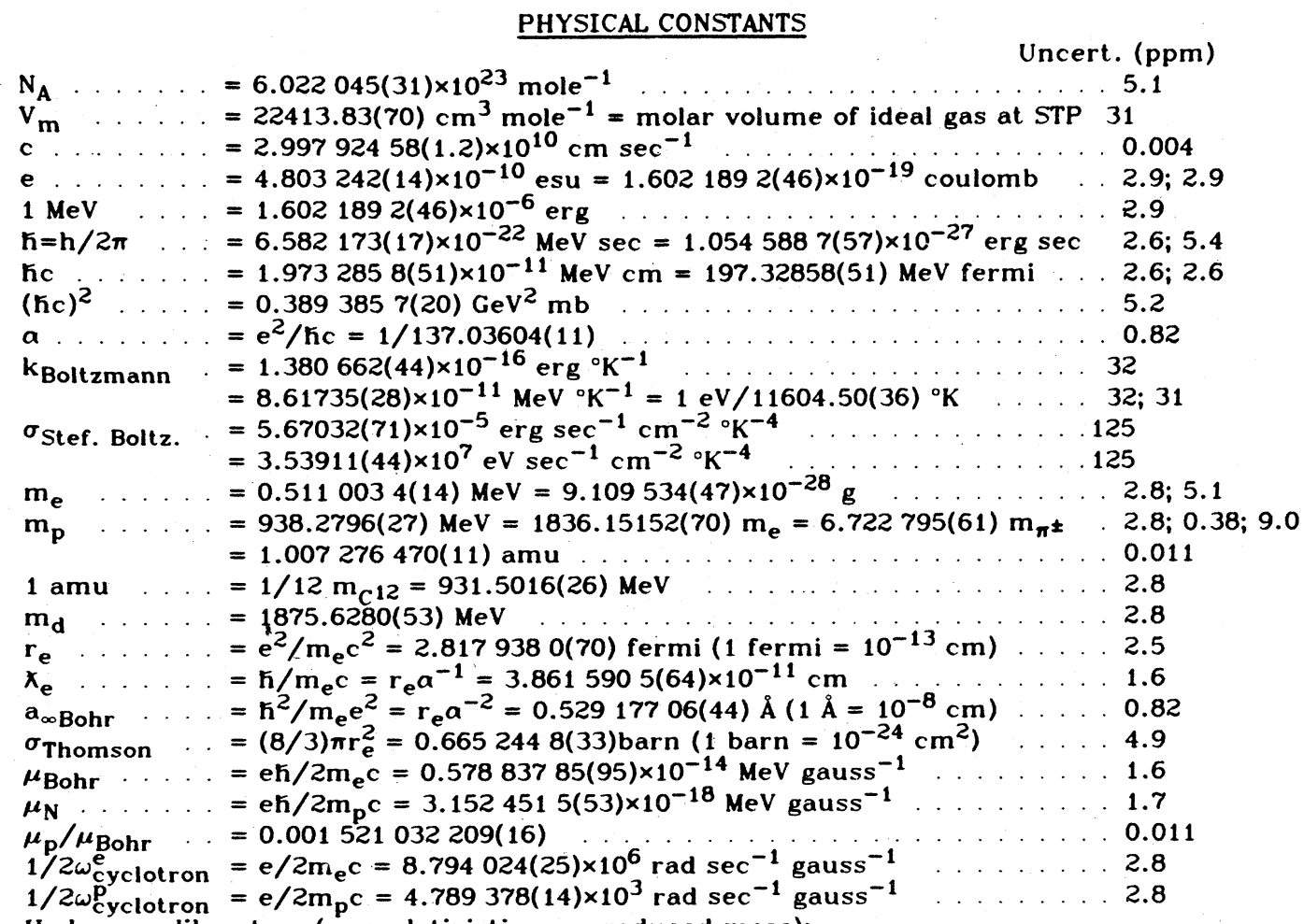

Hydrogen-like atom (nonrelativistic, $\mu=$ reduced mass):

$$
\left.\frac{v}{c}\right)_{r m s}=\frac{z a}{n} ; E_{n}=\frac{\mu}{2} v^{2}=\frac{\mu}{2}\left(\frac{c z a}{n}\right)^{2} ; \quad a_{n}=\frac{n^{2} h}{\mu z c a}
$$

$R_{c o}=m_{e} e^{4} / 2 \hbar^{2}=m_{e} c^{2} a^{2} / 2=13.605804(36) \mathrm{eV}($ Rydberg) . . . . . . . . . 2.6

$$
=\mathrm{m}_{\mathrm{e}} \mathrm{ca} / 2 \mathrm{~h}=109737.3177(83) \mathrm{cm}^{-1} \ldots \ldots . . \ldots 075
$$

$\mathrm{pc}=0.3 \mathrm{H} \rho$ ( $\mathrm{MeV}$, kilogauss, $\mathrm{cm}$ )

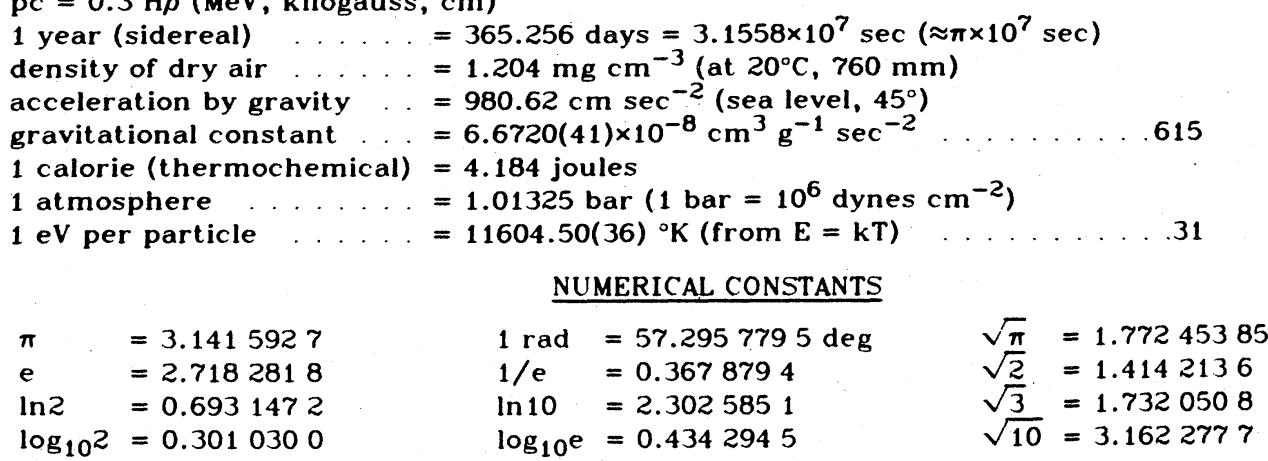

\footnotetext{
*Revised April 1980 by Barry N. Taylor. Originally prepared by Stanley J. Brodsky, based mainly on the "1973 Least-Squares Adjustment of the Fundamental Constants," by E. R. Cohen and B. N. Taylor, J. Phys. Chem. Ref. Data 2,663 (1973). The figures in parentheses correspond to the one-standard-deviation uncertainty in the last digits of the main number. The equivalent uncertainty in parts per million (ppm) is given in the last column. Note that the uncertainties of the output values of a least-squares adjustment are in general correlated, and the general law of error propagation must be used in calculating additional quantities.

The set of constants resulting from the 1973 adjustment of Cohen and Taylor has been recommended for international use by CODATA (Committee on Data for Science and Technology), and is the most up-to-date, generally accepted set currently available. However, since the publication of the 1973 adjustment, a number of new experiments have been completed, yielding improved values for some of the constants: $\mathrm{N}_{\mathrm{A}}=$ $6.0220978(63) \times 10^{23} \mathrm{~mole}^{-1}(1.04 \mathrm{ppm}) ; a^{-1}=137.035963(15)(0.11 \mathrm{ppm})$ [obtained using the Josephson effect]; and $R_{\infty}=109737.31476(32) \mathrm{cm}^{-1}(0.003 \mathrm{ppm})$. But it must be realized that, since the output values of a least-squares adjustment are related in a complex way and a change in the measured value of one constant usually leads to corresponding changes in the adjusted values of others, one must be cautious in carrying out calculations using both the output values from the 1973 adjustment and the results of more recent experiments. A new adjustment is planned for completion by early 1982.
} 


\section{CLEBSCH-GORDAN COEFFICIENTS, SPHERICAL HARMONICS, AND d FUNCTIONS}

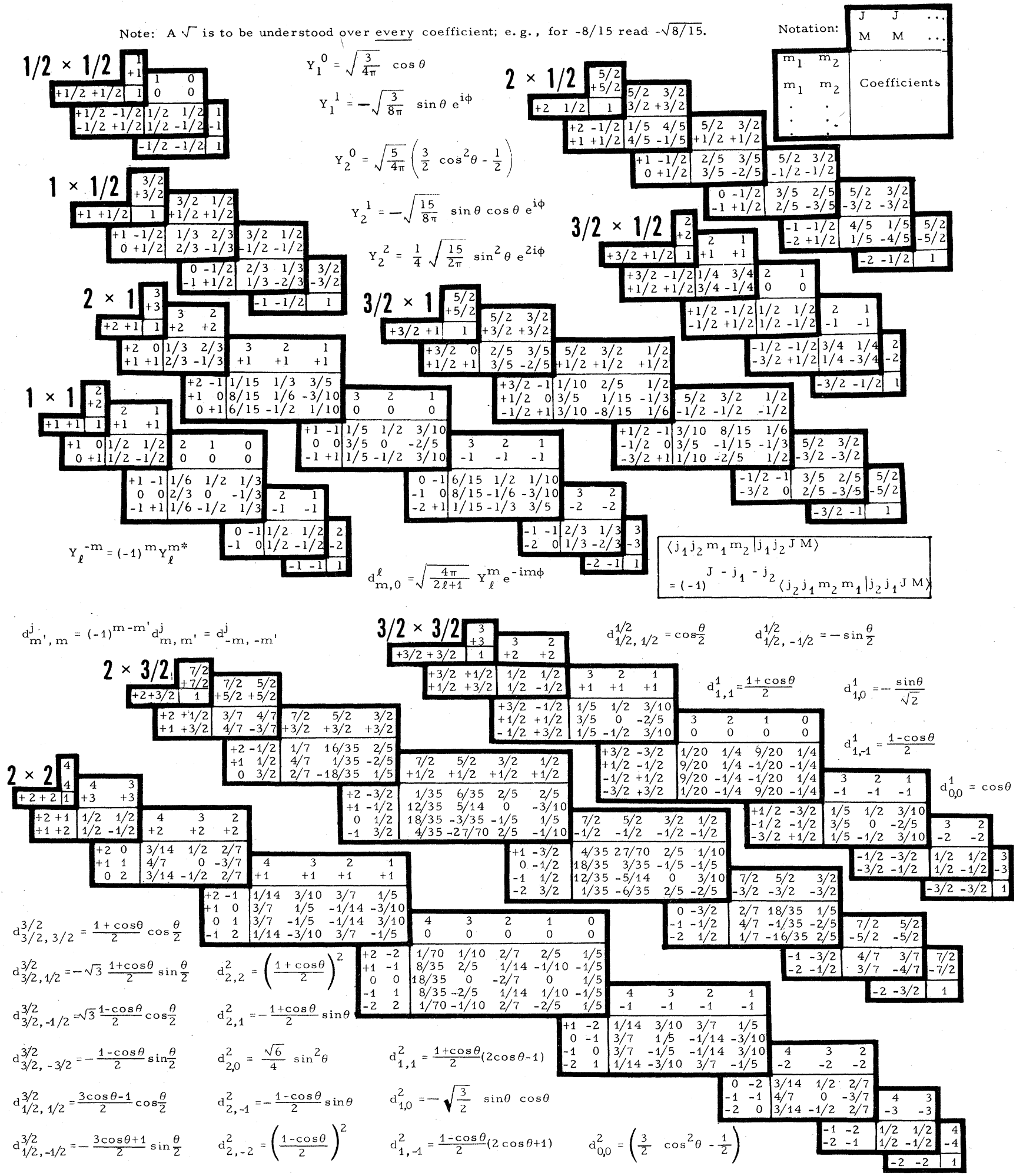

Sign convention is that of Wigner (Group Theory, Academic Press, New York, 1959), also used by Condon and Shortley (The Theory of Atomic Spectra, Cambridge Univ. Press, New York, 1953), Rose (Elementary Theory of Angular Momentum, Wiley, New York, 1957), and Cohen (Tables of the Clebsch-Gordan Coefficients, North American Rockwell Science Center, Thousand Oaks, Calif., 1974). The signs and numbers in the current tables have been calculated by computer programs written independently by Cohen and at LBL. (Table
extended April 1974.) 


\section{SU(3) ISOSCALAR FACTORS}

The most commonly used isoscalar factors, corresponding to the singlet, octet, and decuplet content of $8 \otimes 8$ and $10 \otimes 8$, are displayed at the right. The notation uses particles names to identify the coefficients, so that the pattern of relative couplings can be seen at a glance. We illustrate the use of the coefficient detailed explanation and phase conventions.

A $\sqrt{ }$ is understood over every integer in the matrices; the exponent $\frac{1}{2}$ is a reminder of this. For example, in de Swart's notation the $\Xi \rightarrow \Omega \mathrm{K}$ element of our $10 \rightarrow 10 \otimes 8$ matrix reads

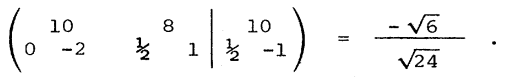

Intra-multiplet relative decay strengths can be read directly from our matrices. Thus, the partial widths for $\Delta^{*} \rightarrow(\mathrm{N} \pi) I=3 / 2$ and $\Omega^{*} \rightarrow(\Xi \bar{K}) I_{=0}$ are in the ratio

$$
\frac{\Gamma\left(\Omega^{*} \rightarrow(E \bar{K})_{I=0}\right)}{\Gamma\left(\Delta^{*} \rightarrow(N \pi)_{I=3 / 2}\right)}=\frac{12}{6} \times(\text { threshold factors }) .
$$

Supplying isospin Clebsch-Gordan coefficients one obtains, e.g.,

$$
\frac{\Gamma\left(\Omega^{*^{-}} \rightarrow \Xi^{0} \mathrm{~K}^{-}\right)}{\Gamma\left(\Delta^{*^{+}} \rightarrow \mathrm{p} \pi^{0}\right)}=\frac{1 / 2}{2 / 3} \times \frac{12}{6} \times \mathrm{tf}=\frac{3}{2} \times \mathrm{tf} .
$$

Partial widths for $8 \rightarrow 8 \otimes 8$ involve a linear superposition of $8_{1}$ (symmetric) and $8_{2}$ (anti-symmetric) couplings. For example,

$$
\Gamma\left(\Xi^{*} \rightarrow \Xi \pi\right) \sim\left(-\sqrt{\frac{9}{20}} g_{1}+\sqrt{\frac{3}{12}} g_{2}\right)^{2} \text {. }
$$

The relation between $g_{1}, g_{2}$ (with de Swart's normalization) and the standard D,F couplings appearing in the interaction Lagrangian,

is

$$
\mathcal{L}=-\sqrt{2} \mathrm{D} \operatorname{Tr}\left([\overline{\mathrm{B}}, \mathrm{B}]_{+} \mathrm{M}\right)+\sqrt{2} \mathrm{~F} \operatorname{Tr}\left([\overline{\mathrm{B}}, \mathrm{B}]_{-} \mathrm{M}\right),
$$

$$
D=\frac{\sqrt{30}}{40} g_{1}, \quad F=\frac{\sqrt{6}}{24} g_{2} \text {. }
$$

Thus,

$$
\Gamma\left(\Xi^{*} \rightarrow \Xi \pi\right) \sim(1-2 \alpha)^{2}
$$

where $\alpha \equiv D /(D+F)$. $\underline{1+8 . \otimes 8}$

$$
(\Lambda)_{1} \rightarrow\left(\begin{array}{lllll}
\mathrm{NK} & \Sigma \pi & \Lambda \eta & \Xi K
\end{array}\right)_{8 \otimes}=\frac{1}{\sqrt{8}}\left(\begin{array}{llll}
2 & 3 & -1 & -2
\end{array}\right)^{\frac{1}{2}}
$$

${ }_{8}+8 \otimes 8$

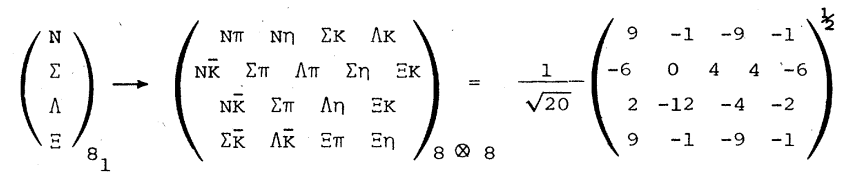

$\mathbb{8}_{2}+8 \otimes 8$

$$
\left(\begin{array}{l}
\mathrm{N} \\
\Sigma \\
\Lambda \\
\Xi
\end{array}\right)_{8_{2}} \rightarrow\left(\begin{array}{ccccc}
\mathrm{N} \pi & \mathrm{N} n & \Sigma \mathrm{K} & \Lambda \mathrm{K} \\
\mathrm{N} \overline{\mathrm{K}} & \Sigma \pi & \Lambda \pi & \Sigma \eta & \Xi \mathrm{K} \\
\mathrm{N} \overline{\mathrm{K}} & \Sigma \pi & \Lambda \eta & \Xi \mathrm{K} \\
\Sigma \overline{\mathrm{K}} & \Lambda \overline{\mathrm{K}} & \Xi \pi & \Xi n
\end{array}\right)_{8 \otimes 8}=\frac{1}{\sqrt{12}}\left(\begin{array}{ccccc}
3 & 3 & 3 & -3 \\
2 & 8 & 0 & 0 & -2 \\
6 & 0 & 0 & 6 \\
3 & 3 & 3 & -3
\end{array}\right)^{\frac{1}{2}}
$$

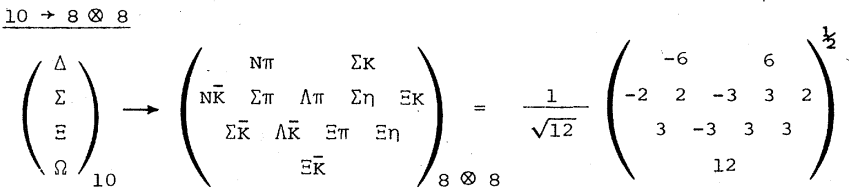

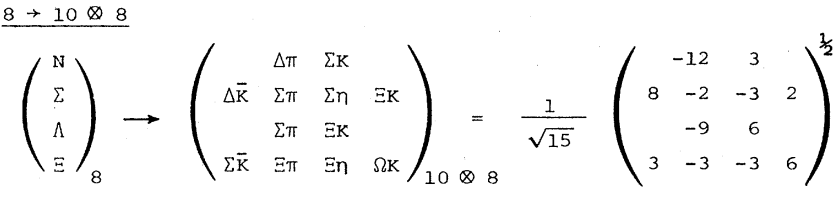

$10 \rightarrow 10 \otimes 8$

$$
\left(\begin{array}{l}
\Delta \\
\Sigma \\
\Xi \\
\Omega
\end{array}\right)_{10} \rightarrow\left(\begin{array}{cccc}
\Delta \pi & \Delta n & \Sigma \mathrm{K} \\
\Delta \overline{\mathrm{K}} & \Sigma \pi & \Sigma \eta & \Xi \mathrm{K} \\
\Sigma \overline{\mathrm{K}} & \Xi \pi & \Xi \eta & \Omega \mathrm{K} \\
& \Xi \overline{\mathrm{K}} & \Omega \mathrm{n} &
\end{array}\right)_{10 \otimes 8}=\frac{1}{\sqrt{24}}\left(\begin{array}{rrrr}
15 & 3 & -6 \\
8 & 8 & 0 & -8 \\
12 & 3 & -3 & -6 \\
12 & -12
\end{array}\right)^{\frac{1}{2}}
$$

SU(n) Multiplicities

The table below gives the multiplicities of the multiplets that occur in $q q, q \bar{q}$, and qqq systems in various $\mathrm{SU}(\mathrm{n})$. Normal mesons are $q \bar{q}$ systems, and normal baryons are qqq systems. Also given are the multiplets that occur in meson-baryon scattering when the meson multiplet is the one to which the pion belongs and the baryon multiplet is the one to which the proton belongs. Complexconjugate representations are indicated by a bar. The two conjugate representations are ind cat (4) by a bar. The two (which contains the SU(3) decuplet) and 20' (which contains the SU(3) contains the $\mathrm{SU}(3)$ decuplet) and
octet). The $\mathrm{C}(\mathrm{N}, \mathrm{M})$ 's are the binomial coefficients $\mathrm{N} ! /[\mathrm{M} !(\mathrm{N}-\mathrm{M}) !]$

\begin{tabular}{l} 
qq \\
\hline $\mathrm{SU}(2): 2 \otimes 2 \rightarrow 3 \oplus 1$ \\
$\mathrm{SU}(3): 3 \otimes 3 \rightarrow 6 \oplus \frac{1}{3}$ \\
$\mathrm{SU}(4): 4 \otimes 4 \rightarrow 10 \oplus 6$ \\
$\mathrm{SU}(n): \mathrm{n} \otimes \mathrm{n} \rightarrow \mathrm{n}(\mathrm{n}+1) / 2 \oplus \mathrm{n}(\mathrm{n}-1) / 2$
\end{tabular}

$$
\mathrm{q} \vec{q} \text { (Mesons) }
$$

SU (2) : $2 \otimes \overline{2} \rightarrow 3 \oplus 1$

$\mathrm{SU}(3): \quad 3 \otimes \overline{3} \rightarrow 8 \oplus$

$\mathrm{SU}(4): \quad 4 \otimes 4 \rightarrow 15 \oplus$

$\mathrm{SU}(\mathrm{n}): \mathrm{n} \otimes \overline{\mathrm{n}} \rightarrow\left(\mathrm{n}^{2}-1\right) \oplus 1$

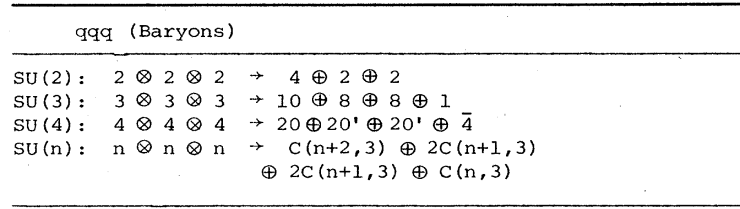

Meson-Baryon Scattering

$\mathrm{SU}(2): \quad 3 \otimes 2 \rightarrow 4 \oplus 2$

$\mathrm{SU}(3): 8 \otimes 8 \rightarrow 27 \oplus 10 \oplus \overline{10} \oplus 8 \oplus 8 \oplus I$

SU (4): $15 \otimes 20^{\prime} \rightarrow 140 \oplus 60 \oplus 36 \oplus 20 \oplus 20^{\prime} \oplus 20^{\prime} \oplus \overline{4}$ 


\section{PROBABILITY AND STATISTICS}

\section{A. PROBABILITY DISTRIBUTIONS AND CONFIDENCE LEVELS}

We give here properties of the three probability distributions most commonly used in high energy physics: Normal (or

Gaussian), Chi-squared, and Poisson. We warn the reader that there is no universal convention for the term "confidence level" as used by physicists; thus, explicit definitions are given for each distribution, and we have attempted to choose definition that correspond to common usage. It is explained below how confidence levels for all three distributions can be extracted from the following figure.

\section{$\chi^{2}$ Confidence Level vs. $\chi^{2}$ for $n_{D}$ Degrees of Freedom}

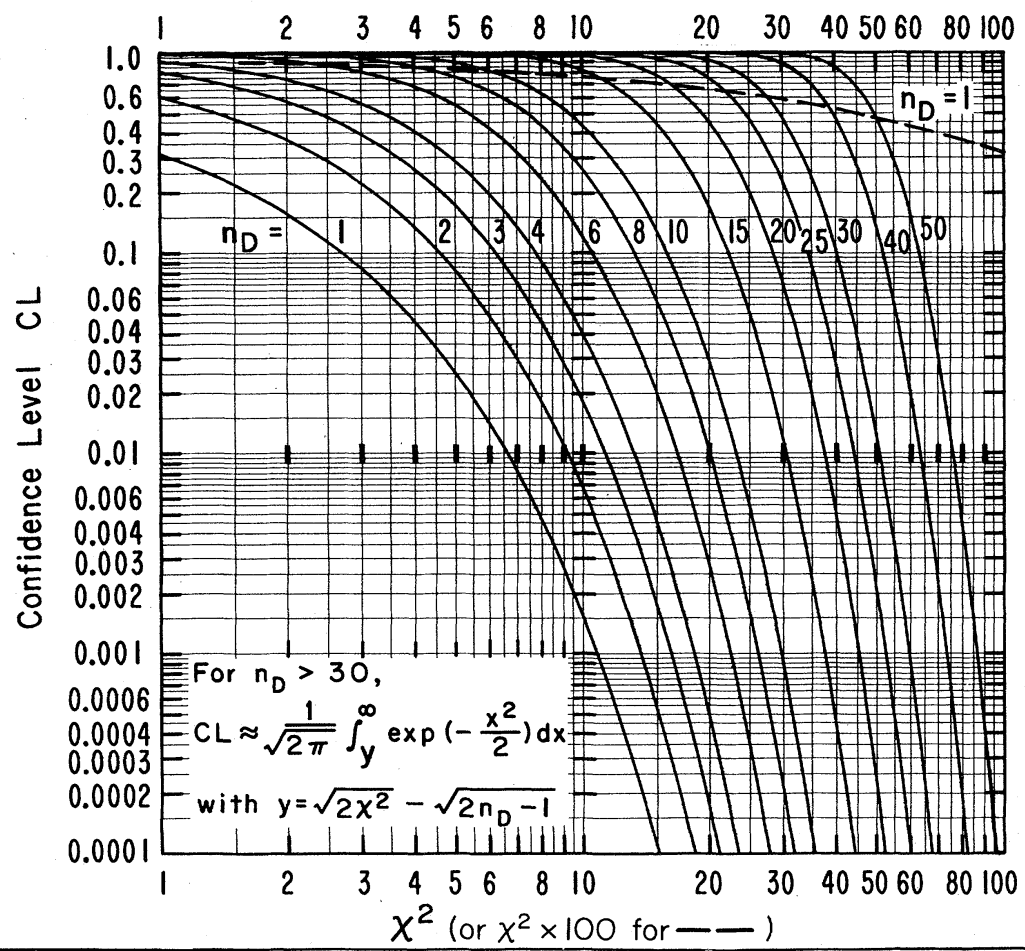

A.1. Normal Distribution

The normal distribution with mean $\bar{x}$ and standard deviation $\sigma$ (variance $\sigma^{2}$ ) is :

$$
P(x) d x=\frac{1}{\sqrt{2 \pi} \sigma} e^{-(x-\bar{x})^{2} / 2 \sigma^{2}} d x \text {. }
$$

The confidence level as sociated with an observed deviation from the mean, $\delta$, is the probability that $|x-\bar{x}|>\delta$, i. e.

$$
C L=2 \int_{\bar{x}+\delta}^{\infty} d x P(x)
$$

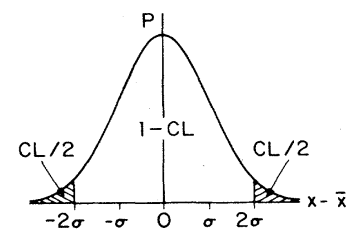

[The small figure in Eq. (2) is drawn with $\delta=2 \sigma$.] C L is given by the ordinate of the $n_{n}=1$ curve in the figure at $x^{2}=(\delta / \sigma)^{2}$. The confidence level for $\delta=1 \sigma$ is $31.7 \% ; 2 \sigma, 4.6 \% ; 3 \sigma, 0.3 \%$. The central confidence interval, $1-\mathrm{CL}$, (which is also sometimes called confidence level) for $\delta=1 \sigma$ is $68.3 \% ; 2 \sigma, 9,5.4 \%$; $3 \sigma, 99.7 \%$. The odds against exceeding $\delta,(1-C L) / C L$, for $\delta=1 \sigma$ are $2.15: 1 ; 2 \sigma, 21: 1 ; 3 \sigma, 370: 1 ; 4 \sigma, 16,000: 1 ; 5 \sigma$, $1,700,000: 1$. Relations between $\sigma$ and other measures of the width: probable error $(C L=0.5$ deviation $)=0.67 \sigma$; mean absolute deviation $=0.80 \sigma ;$ RMS deviation $=\sigma ;$ half width at half maximum $=1.18 \sigma$.

A.2. Chi-squared Distribution

The chi-squared distribution for $\mathrm{n}_{\mathrm{D}}$ degrees of freedom is:

$$
P_{n_{D}}\left(x^{2}\right) d x^{2}=\frac{1}{2^{h} \Gamma(h)}\left(x^{2}\right)^{h-1} e^{-x^{2} / 2} d x^{2} \quad\left(x^{2} \geq 0\right),
$$

where $h$ (for "half") $=\mathrm{n}_{\mathrm{D}} / 2$. The mean and variance are $\mathrm{n}_{\mathrm{D}}$ and $2 n_{1}$, respectively. In evaluating Eq. (3) one may use $\mathrm{D}$ Stirling's approximation: $\Gamma(\mathrm{h})=(\mathrm{h}-1) ! \approx 2.507 \mathrm{e}^{-\mathrm{h}} \mathrm{h}(\mathrm{h}-1 / 2) \times$
$(1+0.0833 / \mathrm{h})$ which is accurate to $\pm 0.1 \%$ for all $\mathrm{h} \geqslant 1 / 2$. The confidence level associated with a given value of $n_{D}$ and an observed value of $x^{2}$ is the probability of chi-squared exceeding the observed value, i. e.,

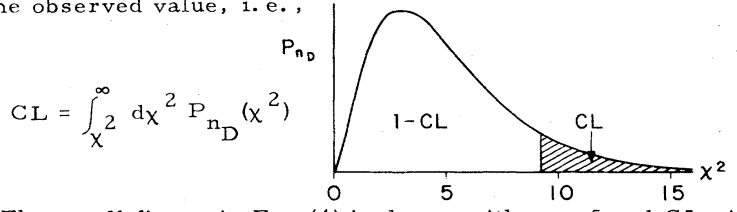

[ The small figure in Eq. (4) is drawn with $\mathrm{n}_{\mathrm{D}}=5$ and $\mathrm{CL}=10 \%$.] $C L$ is plotted as a function of $\chi^{2}$ for several values of $n_{1}$ in the above figure. For large $n_{D}, \chi^{2}$ becomes normally distributed about $\mathrm{n}_{\mathrm{D}}$. Thus,

$$
y_{1}=\left(x^{2}-n_{D}\right) / \sqrt{2 n_{D}}
$$

becomes normally distributed with unit standard deviation. A better approximation, due to Fisher, 1 is that $x$, not $x^{2}$, becomes normally distributed, specifically

$$
y_{2}=\sqrt{2 x^{2}}-\sqrt{2 n D^{-1}}
$$

approaches normality with unit standard deviation. For small $C^{\prime}$ 's in particular, $y_{2}$ is much more accurate than $y_{1}$. Thus,

for $\mathrm{n}_{\mathrm{D}}=50$ and $\mathrm{x}^{2}=80$, the true $\mathrm{CL}=0.45 \%$, but $\mathrm{y}_{1}$ is 3.0 cor responding to a $\mathrm{CL}$ of $0.13 \%$, while $\mathrm{y}_{2}$ is 2.7 corresponding to a CL of $0.35 \%$. 


\section{PROBABILITY AND STATISTICS (Cont'd)}

A.3. Poisson Distribution

The Poisson distribution with mean $\overline{\mathrm{n}}$ is:

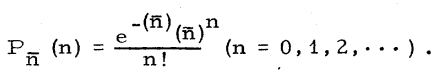

The variance is equal to the mean. Confidence levels for Poisson distributions are usually defined in terms of quantities called

"upper limits" as follows: The confidence level associated with a given upper limit $N$ and an observed value $n_{0}$ of $n$ is the probability that $n>n_{0}$ if $\bar{n}=N$, i. e. ,

$$
\begin{aligned}
\mathrm{CL} & =\sum_{\mathrm{n}=\mathrm{n}_{0}+1}^{\infty} \mathrm{P}_{\mathrm{N}^{(n)}} \\
& =1-\sum_{n=0}^{\mathrm{n}_{0}} \mathrm{P}_{\mathrm{N}}(\mathrm{n})
\end{aligned}
$$

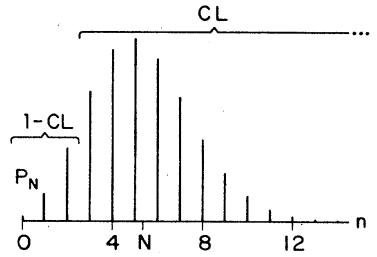

[ The small figure in Eq. (8) is drawn with $\mathrm{n}_{0}=2$ and $\mathrm{CL}=90 \%$.] A useful relation between Poisson and chi-squared confidence levels allows one to look up this quantity on the above figure. Specifically, the quantity $1-C L$ is given by the ordinate of the Specifically, the quantity $1-C L$ is given by the ordinate of the
$\mathrm{n}_{\mathrm{D}}=2\left(\mathrm{n}_{0}+1\right)$ curve at $\chi^{2}=2 \mathrm{~N}$. Thus, $90 \%$ confidence level upper limits for $n_{0}=0,1$, and 2 are given by half the $\chi^{2}$ value corresponding to an ordinate of 0.1 on the $n_{D}=2,4$, and 6 curves, respectively; the values are $\mathrm{N}=2.3,3.9$, and 5.3 .

Tables of confidence levels for all three of the se distributions, the relation between Poisson and chi-squared confidence levels, and numerous other useful tables and relations may be found in and num
Ref. 2.

\section{B. STATISTICS}

We consider here the situation in which one is presented with $\mathrm{N}$ independent data, $y_{n} \pm \sigma_{n}$, and it is desired to make some inference about the "true" value of the quantity represented by single sample point drawn randomly (and independently of the
sing other data) from a distribution having mean $\bar{y}_{n}$ (which we wish to estimate) and variance $\sigma_{\mathrm{n}}^{2}$. (Identification of the true $\sigma_{\mathrm{n}}$ with the $\sigma_{\mathrm{n}}$ datum is an approximation which may become seriously inaccurate when $\sigma_{n}$ is an appreciable fraction of $\mathrm{y}_{n}$.) Some methods of estimation commonly used in high energy physics are given below; see Ref. 3 for numerous applications. Section B.1. deals with the case in which all $\bar{y}_{n}$ are the same, e. g. , several different measurements of the same quantity; Sec. B.2. deals with the case in which $\bar{y}_{n}=\bar{y}\left(x_{n}\right)$, where $x_{n}$ represents some set of independent variables, e.g., cross-section measurements at various values of energy and angle, $x_{n}=\left\{E_{n}, \theta_{n}\right\}$.

B.1. Single Mean and Variance Estimates

(1) If the $y_{n}$ represent a set of values all supposedly drawn from a single distribution with mean $\bar{y}$ and variance $\sigma^{2}$ (i.e., the $\sigma_{n}$ are all the same, but their common value is unknown) then

$$
\begin{aligned}
\bar{y}_{e} & =\frac{1}{N} \sum_{n} y_{n} \text { and } \\
\sigma_{e}^{2} & =\frac{1}{N-1} \sum_{n}\left(y_{n}-\bar{y}_{e}\right)^{2}=\frac{N}{N-1}\left[\left(\bar{y}^{2}\right)_{e}-\bar{y}_{e}^{2}\right]
\end{aligned}
$$

are unbiased estimates of $\bar{y}$ and $\sigma^{2}$. The variance of $\bar{y}_{e}$ is $\sigma^{2} / \mathrm{N}$. If the parent distribution is normal and $\mathrm{N}$ is large, the variance of $\sigma_{\mathrm{e}}^{2}$ is $2 \sigma^{4} / \mathrm{N}$.

(2) If the $\bar{y}_{n}$ all have the common value $\bar{y}$ and the $\sigma_{n}$ are known, then the weighted average

$$
\overline{\mathrm{y}}_{\mathrm{e}}=\frac{1}{\mathrm{w}} \sum_{\mathrm{n}} \mathrm{w}_{\mathrm{n}} \mathrm{y}_{\mathrm{n}} \text {, }
$$

where $w_{n}=1 / \sigma_{n}^{2}$ and $w=\Sigma w_{n}$, is an appropriate unbiased estimate of $\vec{y}$. This ${ }^{n}$ choice of weighting factors in Eq. (11) minimizes the variance of the estimate; the variance is $1 / \mathrm{w}$.

\section{B.2. Linear Least Squares Fit}

A least squares fit of the function $y(x)=\Sigma_{i} a_{i} f_{i}(x)$ to independent data $y_{n} \pm \sigma_{n}$ at points $x_{n}$ (e.g. , a Legendre fit in which the $f_{i}$ are Legendre polynomials and the $a_{i}$ are Legendre coefficients) gives the following estimates of the parameters $a_{i}$ : $a_{e, i}=\sum_{j, n} v_{i j} f_{j}\left(x_{n}\right) y_{n} / \sigma_{n}^{2}$.

Here $\mathrm{V}$ is the covariance matrix of the fitted parameters

$v_{i j}=\overline{\left(a_{e, i}-\bar{a}_{e, i}\right)\left(a_{e, j}-\bar{a} e, j\right)}$,

which is given by

$\left(\mathrm{V}^{-1}\right)_{i j}=\sum_{n} f_{i}\left(x_{n}\right) f_{j}\left(x_{n}\right) / \sigma_{n}^{2}$.

The variance of an interpolated or extrapolated value of $y$ at point $x, y_{e}=\Sigma a_{e, i} f_{i}(x)$, is :

$$
\overline{\left(y_{e}-\bar{y}_{e}\right)^{2}}=\sum_{i j} v_{i j} f_{i}(x) f_{j}(x) \text {. }
$$

For the case of a straight line fit, $y(x)=a+b x$, one obtains the following estimates of $a$ and $b$,

$$
\begin{aligned}
& a_{e}=\left(S_{y} S_{x x}-S_{x} S_{x y}\right) / D, \\
& b_{e}=\left(S_{1} S_{x y}-S_{x} S_{y}\right) / D,
\end{aligned}
$$

where

$$
\begin{aligned}
& S_{1}, S_{x}, S_{y}, S_{x x}, S_{x y}=\Sigma\left(1, x_{n}, y_{n}, x_{n}^{2}, x_{n} y_{n}\right) / \sigma_{n}^{2}, \\
& D=S_{1} S_{x x}-S_{x}^{2} .
\end{aligned}
$$

The covariance matrix of the fitted parameters is:

$$
\left(\begin{array}{cc}
v_{a a} & v_{a b} \\
v_{a b} & v_{b b}
\end{array}\right)=\frac{1}{D}\left(\begin{array}{cc}
s_{x x} & -s_{x} \\
-s_{x} & s_{1}
\end{array}\right)
$$

The variance of an interpolated or extrapolated value of $y$ at point $x$ is:

$$
\overline{\left(y_{e}-\bar{y}_{e}\right)^{2}}=\frac{1}{S_{1}}+\frac{S_{1}}{D}\left(x-\frac{s_{x}}{S_{1}}\right)^{2}
$$

\section{ERROR PROPAGATION}

We consider here the situation in which one wishes to calculate the value and error of a function of some other quantities with errors, e.g. , in a Monte Carlo program. Let $\{y\}$ be a set of random variables with means $\{\bar{y}\}$ and covariance matrix V. Then the mean and variance of a function of these variables are approximately (to second order in $\{y-\bar{y}\}$ ):

$$
\begin{aligned}
& \bar{f} \approx f(\{y\})+\frac{1}{2} \sum_{m n} V_{m n}\left(\frac{\partial^{2} f}{\partial y_{m} \partial y_{n}}\right)_{\{y\}=\{\bar{y}\}}, \\
& \overline{(f-\bar{f})^{2}}=\sum_{m n} V_{m n}\left(\frac{\partial f}{\partial y_{m}}\right)_{\{y\}=\{\bar{y}\}}\left(\frac{\partial f}{\partial y_{n}}\right)_{\{y\}=\{\bar{y}\}} .
\end{aligned}
$$

E. g. , the mean and variance of a function of a single variable with mean $\bar{y}$ and variance $\sigma^{2}$ are:

$$
\begin{aligned}
& \overline{\mathrm{f}} \approx \mathrm{f}(\overline{\mathrm{y}})+\frac{1}{2} \sigma^{2} \mathrm{f}^{\prime \prime}(\overline{\mathrm{y}}), \\
& \overline{(f-\bar{f})^{2}}=\sigma^{2} f^{\prime}(\bar{y})^{2} \text {. }
\end{aligned}
$$

Note that the se equations will usually be applied by substituting some measured quantities, $\{\tilde{y}\}$ say, for the true means, $\{\bar{y}\}$. If, as is often the case, $\tilde{y}_{n}-\bar{y}_{n}$ is of order $\sqrt{V_{n n}}$, then there is no point in keeping the second order terms in Eq. (20) or (22) since point in keeping the second order terms in Eq. $(20)$
the substitution itself introduces first order errors.

1. R. A. Fisher, Statistical Methods for Research Workers

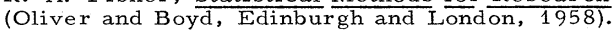

2. M. Abramovitz and I. Stegun, eds., Handbook of Mathematical Functions (National Bureau of Standards, Applied Mathematics Series, Vol. 55, Washington, 1964).

3. W. T. Eadie, D. Drijard, F. E. James, M. Roos, and B..Sadoulet, Statistical Methods in Experimental Physics (North-Holland, Amster $\frac{\text { dam and }}{\text { London, 1971). }}$

Revised and expanded April 1974. 


\section{RELATIVISTIC KINEMATICS}

I. BASICS

(a) Lorentz transformations - - Let $E$ and $\vec{p}$ be the energy and 3-momentum of a particle or system as seen from a certain inertial frame, and let $E^{*}$ and $\vec{p}^{*}$ be the same quantities as seen from a second inertial frame that moves with velocity $\vec{\beta}$ relative to the first. Then starred and unstarred quantities are related by

$$
\left(\begin{array}{l}
E_{p_{k}^{*}}^{*} \\
p^{*}
\end{array}\right)=\left(\begin{array}{cc}
\gamma & -\gamma \beta \\
-\gamma \beta & \gamma
\end{array}\right)\left(\begin{array}{l}
E \\
p_{\|}
\end{array}\right), \quad p_{1}^{*}=p_{1} .
$$

Here $\gamma=\left(1-\beta^{2}\right)^{-1 / 2}$, and subscripts $\|$ and 1 indicate components of $\vec{p}$ or $\vec{p}^{*}$ that are parallel or perpendicular to $\vec{\beta}$ (often $\eta$ is used for $\gamma \beta$ ). The inverse transformation is given by changing $\beta$ to $-\beta$. A particle of mass $m$ at rest in the second frame, so that it is moving at velocity $\vec{\beta}$ relative to the first, has $E^{*}=m$ and $\vec{p}^{*}=0$, so here

$$
E=\gamma \mathrm{m}, \overrightarrow{\mathbf{p}}=\gamma \vec{\beta} \mathbf{m} .
$$

In any frame, the energy, momentum, and mass are related by

$$
E^{2}=p^{2}+m^{2} \text {. }
$$

(b) Four momenta; scalar products -- The 4-momentum vector of a particle or system having energy $E$ and 3 -momentum $\vec{p}$ is

$$
q=(E, \vec{p})=\left(E, p_{x}, p_{y}, p_{z}\right) \text {. }
$$

Conservation of energy and the components of 3-momentum for any process $a+b+\ldots \rightarrow 1+2+\ldots$ may then be written as

$$
q_{a}+q_{b}+\ldots=q_{1}+q_{2}+\ldots .
$$

Although the components of a 4-momentum are different in different frames, the scalar product of any two 4-momenta $q$ and $q^{\prime}$, defined as

$$
\mathbf{q} \cdot \mathbf{q}^{\prime}=E E^{\prime}-\overrightarrow{\mathbf{p}} \cdot \overrightarrow{\mathbf{p}}^{\prime},
$$

is an invariant; i.e., in numerical calculations the same result is obtained in any frame, and in algebraic calculations results obtained in different frames may be equated. For a particle of mass $m$, the scalar product $q \cdot q$ is

$$
\mathrm{q} \cdot \mathrm{q}=\mathrm{q}^{2}=\mathrm{E}^{2}-\overrightarrow{\mathrm{p}}^{2}=\mathrm{m}^{2} \text {. }
$$

The invariant mass $M$ (or total c.m. energy) of an $n$-particle system is given by

$$
\mathrm{M}^{2}=\left(\sum_{i=1}^{\mathrm{n}} \mathrm{q}_{i}\right)^{2}=\left(\sum_{i} \mathrm{E}_{i}\right)^{2}-\left(\sum_{i} \overrightarrow{\mathrm{p}}_{i}\right)^{2},
$$

where $q_{i}=\left(E_{i}, \vec{p}_{j}\right)$ is the 4-momentum of the $i^{\text {th }}$ particle.

(c) Electric and magnetic forces -- In Gaussian cgs units, the force on a particle with charge $q$ moving with velocity $\vec{v}$ in electric and magnetic fields $\vec{E}$ and $\vec{B}$ is

$\vec{F}=q \vec{E}+q \vec{\beta} \times \vec{B}$,

where $\vec{\beta}=\vec{v} / c$. The units are $\vec{F}$ in dynes, $q$ in esu, $\vec{E}$ in statvolts/cm, and $\vec{B}$ in gauss. In mksa units, the force is

$$
\vec{F}=q \vec{E}+q \vec{v} \times \vec{B},
$$

where the units are $\vec{F}$ in newtons ( $1 \mathrm{~N}=10^{5}$ dynes), $q$ in coulombs $\left(1 \mathrm{C} \simeq 3 \times 10^{9}\right.$ esu; each 3 in this section is really $2.9979 \ldots), \vec{E}$ in volts $/ \mathrm{m}(1 \mathrm{~V} \simeq 1 / 300$ stat volt), and $\vec{B}$ in tesla ( $1 \mathrm{~T}$ $=10^{4} \mathrm{G}$ ). The force is zero if $\vec{E}$ and $\vec{B}$ are at right angles, $\vec{\beta}$ (or $\vec{v}$ ) is in the direction $\vec{E} \times \vec{B}$, and $\beta=E / B$ (cgs) or $v=E / B$ (mksa).

In a uniform, static magnetic field, the path of a charged particle is a helix of constant radius $R$ and constant pitch angle $\lambda$, with the axis of the helix being along $\vec{B}$. The momentum is related to the other quantities by

$$
\mathrm{p} \cos \lambda \approx 3 \times 10^{-4} \mathrm{qBR} \text {, }
$$

where the units (very mixed!) are $p$ in $\mathrm{GeV} / \mathrm{c}, \mathrm{q}$ in multiples of the electronic charge $e, B$ in $k G$, and $R$ in $\mathrm{cm}$. The angular velocity about the axis of the helix is

$$
\omega \simeq 3 \times 10^{-4} \mathrm{qB} / \gamma \mathrm{m},
$$

where the units are $\omega$ in $\mathrm{rad} / \mathrm{sec}, q$ in multiples of the electronic charge e, $\mathrm{B}$ in $\mathrm{kG}$, and the energy $\gamma \mathrm{m}$ in $\mathrm{GeV}$.
II. DECAYS

(a) Survival probabilities - - Let a particle have mass $m$ and proper mean life $T_{0}$. In a frame in which its $4-$ momentum is $(E, \vec{p})$, the probability that it survives a time greater than $t$ before decaying is

$$
\text { Prob. }(>t)=\exp \left(-t / \gamma \tau_{0}\right)=\exp \left(-m t / E \tau_{0}\right) .
$$

The probability that it goes a distance greater than $x$ before decaying is

$$
\text { Prob. }(>x)=\exp \left(-x / \gamma \beta c \tau_{0}\right)=\exp \left(-m x / p c \tau_{0}\right) ;
$$

values of $\mathrm{Cr}_{0}$ (in $\mathrm{cm}$ ) are given in the Stable Particle Table. If the particle has charge $\pm e$ and is in a uniform magnetic field $\vec{B}$ [see $I(c)$ ], then the probability that the projection of its helical path on the plane perpendicular to $\vec{B}$ turns through an angle greater than $\theta$ before decaying is

$$
\operatorname{Prob.}(>\theta)=\exp \left(-\mathrm{Cm} \theta / \mathrm{B} \tau_{0}\right) \text {. }
$$

where, if $\mathrm{m}$ is in GeV, $\theta$ in deg, $B$ in $\mathrm{kG}$, and $\tau_{0}$ in sec, then $\mathrm{C}$ is numerically $1.942 \times 10^{-9}$. This last distribution is independent of p or the helical pitch angle $\lambda_{\text {; }}$ its only dependence is geometrical.

(b) Two-body decays - - A particle of mass $m$ decays into two particles, masses $m_{1}$ and $m_{2}$. In the rest frame of $m$, the energies of $m_{1}$ and $m_{2}$ are

$$
\begin{aligned}
& \epsilon_{1}=\left(m^{2}+m_{1}^{2}-m_{2}^{2}\right) / 2 m \\
& \epsilon_{2}=\left(m^{2}+m_{2}^{2}-m_{1}^{2}\right) / 2 m .
\end{aligned}
$$

In this frame, the 3 -momenta of $m_{1}$ and $m_{2}$ are equal and opposite and of magnitude

$$
\begin{aligned}
k & =\left(\epsilon_{1}^{2}-m_{1}^{2}\right)^{1 / 2}=\left(\epsilon_{2}^{2}-m_{2}^{2}\right)^{1 / 2} \\
& =\left\{\left[m^{2}-\left(m_{1}+m_{2}\right)^{2}\right]\left[m^{2}-\left(m_{1}-m_{2}\right)^{2}\right]\right\}^{1 / 2} / 2 m .
\end{aligned}
$$

See also the third paragraph of III(b).

(c) Three-body decays -- A particle of mass $m$ decays into three particles, masses $m_{1}, m_{2}$, and $m_{3}$. The invariant masses $\mathrm{m}_{i j}$ of the 2 -particle systems, where $\mathrm{m}_{i j}^{2}=\left(q_{i}+q_{j}\right)^{2}$, satisfy the relation

$$
m_{12}^{2}+m_{13}^{2}+m_{23}^{2}=m^{2}+m_{1}^{2}+m_{2}^{2}+m_{3}^{2},
$$

so that only two of the three $m_{i j}$ 's are independent. In a rectangular Dalitz plot, $m_{13}^{2}$ (say) is plotted against $m_{12}^{2}$. The kinematic boundaries may be calculated as follows: (i) The lower and upper limits on $m_{12}^{2}$ are $\left(m_{1}+m_{2}\right)^{2}$ and $\left(m-m_{3}\right)^{2}$. (ii) For any $m_{12}^{2}$ between these limits, the lower and upper limits on $m_{13}^{2}$ are given by taking the + and - signs in where $m_{13}^{2}=\left(E_{1}+E_{3}\right)^{2}-\left(p_{1} \pm p_{3}\right)^{2}$

$E_{1}=\left(m_{12}^{2}+m_{1}^{2}-m_{2}^{2}\right) / 2 m_{12}$

$E_{3}=\left(m^{2}-m_{12}^{2}-m_{3}^{2}\right) / 2 m_{12}$

$p_{1}=\left(E_{1}^{2}-m_{1}^{2}\right)^{1 / 2}$

$p_{3}=\left(E_{3}^{2}-m_{3}^{2}\right)^{1 / 2}$.

(These are the energies and momenta of particles 1 and 3 in the rest frame of $\mathrm{m}_{12}$.) The phase-space density is uniform over the areas of both the above and the following form of the Dalitz plot.

In a triangular Dalitz plot, the kinetic energies $T_{1}, T_{2}$, and $T_{3}$ of the final-state particles in the rest frame of $\mathrm{m}$ are plotted as the distances inward from the sides of an equilateral triangle whose altitude is the energy $Q$ released by the decay:

$$
\mathrm{Q}=\mathrm{T}_{1}+\mathrm{T}_{2}+\mathrm{T}_{3}=\mathrm{m}-\mathrm{m}_{1}-\mathrm{m}_{2}-\mathrm{m}_{3} \text {. }
$$

The kinetic energies are related to the 2 -particle invariant masses by

etc.

$$
2 \mathrm{mT}_{1}=\left(\mathrm{m}-\mathrm{m}_{1}\right)^{2}-\mathrm{m}_{23}^{2}=\left(\mathrm{m}_{23}^{\max }\right)^{2}-\mathrm{m}_{23}^{2},
$$




\section{RELATIVISTIC KINEMATICS (Cont'd)}

(d) Four-body decays -- A particle of mass $m$ decays into four particles, masses $m_{1}, m_{2}, m_{3}$, and $m_{4}$. In a triangle (or Goldhaber) plot, the invariant mass of two of the particles is plotted against that of the other $t$ wo, say $m_{34}$ versus $m_{12}$, where $\mathrm{m}_{i j}^{2}=\left(\mathrm{q}_{i}+\mathrm{q}_{j}\right)^{2}$. The kinematic boundaries of this plot are the sides of the triangle whose vertices are at the points $\left(m_{12}, m_{34}\right)$ $=\left(m_{1}+m_{2}, m_{3}+m_{4}\right),\left(m_{1}+m_{2}, m-m_{1}-m_{2}\right)$, and $\left(m-m_{3}-m_{4}\right.$, $\left.m_{3}+m_{4}\right)$. The phase-space density is not uniform over the enclosed area.

III. REACTIONS (MAINLY 2-BODY)

(a) Initial state - - Two particles, masses $m_{1}$ and $m_{2}$, interact. In the lab frame, where particle 2 is at rest, the 4-momenta are $\left(E_{1}, \vec{p}_{1}\right)$ and $\left(m_{2}, 0\right)$. In the c.m. frame, where the 3-momenta are equal and opposite, the 4-momenta are $\left(\epsilon_{1}, \vec{k}\right)$ and

$\left(\epsilon_{2},-\vec{k}\right)$. Then the total c.m. energy $E$ is given by

$$
E^{2}=\left(\epsilon_{1}+\epsilon_{2}\right)^{2}=m_{1}^{2}+m_{2}^{2}+2 E_{1} m_{2} \text {. }
$$

The c.m. energies of particles 1 and 2 are

$$
\begin{aligned}
& \epsilon_{1}=\left(m_{1}^{2}+E_{1} m_{2}\right) / E=\left(E^{2}+m_{1}^{2}-m_{2}^{2}\right) / 2 E \\
& \epsilon_{2}=\left(m_{2}^{2}+E_{1} m_{2}\right) / E=\left(E^{2}+m_{2}^{2}-m_{1}^{2}\right) / 2 E .
\end{aligned}
$$

The c.m. momentum $k$ is

$$
\mathbf{k}=\mathbf{p}_{1} \mathbf{m}_{2} / E \text {. }
$$

See also the expression in $\mathrm{II}(\mathrm{b})$ for $\mathrm{k}$, in which replace $\mathrm{m}$ with $E$.

The velocity of the c.m. relative to the lab is

$$
\beta=p_{1} /\left(E_{1}+m_{2}\right) \text {. }
$$

The parameters for the Lorentz transformation between these frames $[$ see $I(a)]$ are

and

$$
\gamma=\left(\mathrm{E}_{1}+\mathrm{m}_{2}\right) / E
$$

$$
\gamma \beta=\mathrm{p}_{1} / E \text {. }
$$

(b) Two-body final states - - In the reaction $1+2 \rightarrow 3+4$, let the masses be $\mathrm{m}_{i}$ and the final-state c.m. 4-momenta be $\left(\epsilon_{3}, \vec{k}^{\prime}\right)$ and $\left(\epsilon_{4},-\vec{k}^{\prime}\right)$. Then

$$
\begin{aligned}
& \epsilon_{3}=\left(E^{2}+\mathrm{m}_{3}^{2}-\mathrm{m}_{4}^{2}\right) / 2 E \\
& \epsilon_{4}=\left(E^{2}+\mathrm{m}_{4}^{2}-\mathrm{m}_{3}^{2}\right) / 2 E ;
\end{aligned}
$$

and

$$
\begin{aligned}
\mathbf{k}^{\prime} & =\left(\epsilon_{3}^{2}-\mathrm{m}_{3}^{2}\right)^{1 / 2}=\left(\epsilon_{4}^{2}-\mathrm{m}_{4}^{2}\right)^{1 / 2} \\
& =\left\{\left[E^{2}-\left(\mathrm{m}_{3}+\mathrm{m}_{4}\right)^{2}\right]\left[E^{2}-\left(\mathrm{m}_{3}-\mathrm{m}_{4}\right)^{2}\right]\right\}^{1 / 2} / 2 E .
\end{aligned}
$$

Let $\Theta_{3}$ be the lab production angle of particle 3 (the angle between $\vec{p}_{3}$ and $\vec{p}_{1}$ ), and let $\theta_{3}$ be the c.m. production anglc (the angle between $\vec{k}^{\prime}$ and $\vec{k}$ ). These angles are related by

$$
\tan \Theta_{3}=\frac{p_{31}}{p_{3 n}}=\frac{\sin \theta_{3}}{\gamma\left(\cos \theta_{3}+\beta / \beta_{3}\right)} .
$$

where $p_{31}$ and $p_{3}$ are the components of $\vec{p}_{3}$ perpendicular and parallel to $\vec{p}_{1}$, and $\beta_{3}=k^{\prime} / \epsilon_{3}$ is the c.m. velocity of particle 3 . [See III(a) for $\gamma$ and $\beta$.] If $\beta>\beta_{3}$, then particle 3 can only go forward in the lab, the maximum $\Theta_{3}$ being given by

$$
\tan \Theta_{3}^{\max }=\beta_{3}\left(\frac{1-\beta^{2}}{\beta^{2}-\beta_{3}^{2}}\right)^{1 / 2} .
$$

The components of $\vec{p}_{3}$ satisfy

$$
\left(\frac{\mathrm{p}_{34}-\gamma \beta \epsilon_{3}}{\gamma \mathrm{k}^{\prime}}\right)^{2}+\left(\frac{\mathrm{p}_{31}}{\mathrm{k}^{\prime}}\right)^{2}=1 \text {. }
$$

which is the equation of an ellipse with semi-major axis $\gamma \mathbf{k}^{\prime}$ and semi-minor axis $k^{\prime}$. Thus the possible lab momenta of particle 3 are the vectors to the ellipse from the point a distance $\gamma \beta \epsilon_{3}$ back along the major axis from the center of the ellipse.
The results of the preceding paragraph also apply to 2 -body decay. Just set $m_{2}=0$, in which case $E=m_{1}$. [The decayproduct masses are here $m_{3}$ and $m_{4}$, not $m_{1}$ and $m_{2}$ as in II(b).]

The Mandelstam variables $s, t$, and $u$ are the Lorentz scalars defined in terms of the particle 4-momenta $q_{i}$ as

$$
\begin{aligned}
& s=\left(q_{1}+q_{2}\right)^{2}=\left(q_{3}+q_{4}\right)^{2} \\
& t=\left(q_{1}-q_{3}\right)^{2}=\left(q_{2}-q_{4}\right)^{2} \\
& u=\left(q_{1}-q_{4}\right)^{2}=\left(q_{2}-q_{3}\right)^{2} .
\end{aligned}
$$

They satisfy the relation

$$
\mathrm{s}+\mathrm{t}+\mathrm{u}=\mathrm{m}_{1}^{2}+\mathrm{m}_{2}^{2}+\mathrm{m}_{3}^{2}+\mathrm{m}_{4}^{2},
$$

so that only two of the three are independent. Evaluating $s$ in the c.m. frame gives

$$
s=\left(\epsilon_{1}+\epsilon_{2}\right)^{2}=E^{2} \text {. }
$$

and evaluating $t$ and $u$, the 4-momentum-transfer-squared variables, in this frame gives

$$
\begin{aligned}
t & =m_{1}^{2}+m_{3}^{2}-2 \epsilon_{1} \epsilon_{3}+2 k k^{\prime} \cos \theta_{3} \\
& =t_{0}-4 k k^{\prime} \sin ^{2}\left(\theta_{3} / 2\right) \\
\mathbf{u} & =m_{1}^{2}+m_{4}^{2}-2 \epsilon_{1} \epsilon_{4}+2 k k^{\prime} \cos \theta_{4} \\
& =u_{0}-4 k k^{\prime} \sin ^{2}\left(\theta_{4} / 2\right),
\end{aligned}
$$

where $\theta_{4}$ is the c.m. production angle of particle $4\left(\theta_{3}+\theta_{4}=\pi\right)$, and

$$
\begin{aligned}
& t_{0}=t\left(\theta_{3}=0\right)=\left(\epsilon_{1}-\epsilon_{3}\right)^{2}-\left(k-k^{\prime}\right)^{2} \\
& u_{0}=u\left(\theta_{4}=0\right)=\left(\epsilon_{1}-\epsilon_{4}\right)^{2}-\left(k-k^{\prime}\right)^{2} .
\end{aligned}
$$

The differences $\Delta t=t_{0}-t_{\pi}$ and $\Delta u=u_{0}-u_{\pi}$, where $t_{\pi}=t\left(\theta_{3}=\right.$ $\pi)$ and $u_{\pi}=u\left(\theta_{4}=\pi\right)$, are

$$
\Delta t=\Delta \mathbf{u}=4 \mathbf{k k}^{\prime} \text {. }
$$

For elastic scattering, where $m_{1}=m_{3}=m$ and $m_{2}=m_{4}=M$, $t_{0}$ is zero and

And now

$$
t=-2 k^{2}\left(1-\cos \theta_{3}\right)=-4 k^{2} \sin ^{2}\left(\theta_{3} / 2\right) .
$$

$$
u_{0}=\left(m^{2}-M^{2}\right)^{2} / s \text {. }
$$

Evaluating $t$ in the lab frame gives

$$
t=-2 M T_{4} \text {, }
$$

where $T_{4}=E_{4}-M$ is the lab kinetic energy of particle 4. For small-angle elastic scattering,

$$
(-t)^{1 / 2} \approx k \theta_{3} \approx p_{1} \theta_{3} \approx p_{4} \text {. }
$$

where $p_{1}, \theta_{3}$, and $p_{4}$ are lab quantities.

IV. OTHER VARIABLES

(a) Rapidity - For a system of energy $E$ and momentum $\vec{p}$, the rapidity $\mathrm{y}$ is given by

$$
y=\frac{1}{2} \ln \left(\frac{E+p_{M}}{E-p_{M}}\right)=\tanh ^{-1}\left(\frac{p_{M}}{E}\right)=\ln \left(\frac{E+p_{M}}{m_{\perp}}\right) .
$$

where $p_{\|}$is the component of $\vec{p}$ along a particular axis (the "rapidity axis", chosen, for example, parallel to the direction of an incoming beam), and $m_{1}=\left(m^{2}+p_{1}^{2}\right)^{1 / 2}$. Inverting these equations, we find

$$
\begin{aligned}
& \mathbf{E}=\mathrm{m}_{1} \cosh \mathrm{y} \\
& \mathbf{p}_{\mathrm{n}}=\mathrm{m}_{1} \sinh \mathrm{y} .
\end{aligned}
$$

The shape of a rapidity distribution is invariant under a Lorentz transformation between inertial frames with relative motion parallel to the rapidity axis. Such a transformation is given by

$$
y^{*}=y-\ln [\gamma(1+\beta)]=y-\frac{1}{2} \ln \left(\frac{1+\beta}{1-\beta}\right) .
$$

where the sign of $\beta$ is positive in the direction of increasing rapidity and $P_{n}$. 


\section{RELATIVISTIC KINEMATICS (Cont'd)}

(b) Scaling variable, hadron reactions -- In the inclusive reaction $h+2 \rightarrow 3+X$, with $h$ any hadron, Feynman's $x$ for particle 3 is defined as

$$
x=k^{\prime}{ }^{\prime} / k^{\prime}{ }_{\max } \text {. }
$$

where $k^{\prime}$ is the c.m. momentum of particle $3 . k^{\prime}{ }_{\max }$ is obtained [see Sec. III(b)] using the smallest mass $m_{X}$ [called $m_{4}$ in III(b)] consistent with quantum conservation laws. At high energies, $k_{\text {max }}^{\prime} \approx \sqrt{s} / 2$. Rapidity and $x$ are related at large $\sqrt{s}$ by

$$
x \simeq \frac{2 \mathrm{~m}_{1}}{\sqrt{\mathrm{s}}} \sinh \mathrm{y}^{*}
$$

where $y^{*}$ is evaluated in the c.m.

(c) Scaling variables, lepton reactions -- For the inclusive reaction $\ell+2 \rightarrow \ell^{\prime}+\mathrm{X}$, with particles $\ell$ and $\ell^{\prime}$ leptons, we define the 4-vector

so that

$$
q=\left(p_{\ell}-p_{\ell^{\prime}}\right)
$$

$Q^{2} \equiv-q^{2}=2 E_{\ell} E_{l^{\prime}}-2\left|\vec{p}_{\ell} \| \vec{p}_{\ell^{\prime}}\right| \cos \theta-m_{l^{\prime}}^{2}-m_{\ell}^{2} z^{\dagger} 0$

where $\theta$ is the $\ell \rightarrow \ell$ ' scattering angle, and the preceding relation is valid in any frame. Also useful are

and

$$
\begin{aligned}
& \nu=p_{2^{\bullet} q} / m_{2}=\left[E_{\ell}-E_{l^{\prime}}\right]_{L A B}=\left[E_{X}-m_{2}\right]_{L A B} \\
& w=\sqrt{p_{X}^{2}}=\left(-Q^{2}+2 m_{2^{\nu}}+m_{2}^{2}\right)^{1 / 2}=m_{X} .
\end{aligned}
$$

$Q^{2}, \nu$, and $W$ are Lorentz invariants, and the notation "LAB" refers to the reference frame with particle $Z$ at rest. (Note: $\nu$ is sometimes written $\nu=\mathrm{p}_{2} \bullet$ q, leading to the replacement of $\mathrm{m}_{2} \nu$ with $\nu$ throughout.)

Scaling variables in common use include

$x \equiv \omega^{-1}=Q^{2} / 2 m_{2} \nu, 0 s^{\dagger} x s^{\dagger} 1$

and

$$
y=m_{2^{\nu}} / p_{\ell^{\prime}} \mathrm{p}_{2}=\left[\left(E_{\ell}-E_{\ell^{\prime}}\right) / E_{\ell}\right]_{\text {LAB }}, 0 s^{\dagger} \mathrm{y} \leq 1 \text {. }
$$

Both $x$ and $y$ are dimensionless.

Cross sections for inclusive reactions in the energy region where masses are negligible can be written in terms of $E_{\ell}$ and certain pairs of these variables, usually $Q^{2}$ and $\nu, x$ and $y$, or $Q^{2}$ and $x$. If, in any frame, $\left|\vec{p}_{\ell}\right|\left|\vec{p}_{\ell}\right| \propto E_{\ell} E_{l}$ and

$E_{l} E_{l^{\prime}} \sin ^{2}(\theta / 2) \gg m_{l^{\prime}}^{2}$ and $m_{l}^{2}$ (i.e., $m_{\ell}, m_{l^{\prime}}$ small), then

$$
\mathrm{Q}^{2} \approx 4 \mathrm{E}_{\ell} \mathrm{E}_{\ell^{\prime}} \sin ^{2}(\theta / 2)
$$

and

$$
x \approx \frac{2 E_{\ell^{\prime}} E_{\ell^{\prime}} \sin ^{2}(\theta / 2)}{m_{2^{\nu}}} .
$$

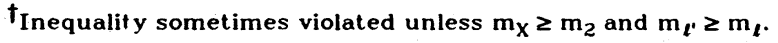

\section{LORENTZ INVARIANT PHASE SPACE FORMULAE}

$$
\begin{aligned}
& \text { For a system of } n \text { particles with overall four-momentum } p \text { and final four momenta } p_{1}, \ldots, p_{n}\left[p_{i}=\left(E_{i}, \vec{p}_{i}\right)\right] \text {, } \\
& \text { Lorentz Invariant Phase Space is given by } \\
& d \operatorname{LIPS}\left(s ; p_{1}, \cdots, p_{n}\right)=(2 \pi)^{4} \delta^{4}\left(p-\sum_{i} p_{i}\right) \frac{1}{(2 \pi)^{3 n}} \prod_{i=1}^{n} \frac{d^{3} \vec{P}_{i}}{2 E_{i}} . \\
& \text { For 2-body: } d \operatorname{LirPS}\left(s, \mathrm{p}_{1}, \mathrm{p}_{2}\right)=\frac{1}{(2 \pi)^{2}} \delta^{4}\left(\mathrm{p}-\mathrm{p}_{1}-\mathrm{p}_{2}\right) \mathrm{d}^{4} \mathrm{p} \frac{\left|\overrightarrow{\mathrm{p}}_{1}^{\mathrm{cm}}\right|}{4 \sqrt{\mathrm{s}}} \mathrm{d} \Omega_{1}^{\mathrm{cm}} \text {. } \\
& \text { For 3-body: } d \operatorname{LIPS}\left(s, p_{1}, p_{2}, p_{3}\right)=\frac{1}{(2 \pi)^{5}} \delta^{4}\left(p-p_{1}-p_{2}-p_{3}\right) d^{4} p_{p} \frac{1}{32 s} d s_{12} d s_{23} d \alpha d \cos \beta d \gamma \text {, } \\
& \text { where } \alpha, \beta \text {, and } \gamma \text { are Euler angles. } \\
& \text { For } a+b \rightarrow n \text { particles or } X \rightarrow n \text { particles, in general }|\mathrm{i}\rangle \rightarrow|\mathrm{f}\rangle \text {, } \\
& \sigma_{i f}=\frac{1}{4 \mathrm{~F}} \int\left|\mathcal{M}_{\text {if }}\right|^{2} \mathrm{~d} \operatorname{LIPS}\left(s ; p_{1}, \cdots, \mathrm{p}_{\mathrm{n}}\right), \\
& \text { or } \quad \Gamma_{i f}=\frac{1}{2 m_{X}} \int\left|\mathcal{H}_{i f}\right|^{2} d \operatorname{LIPS}\left(m_{X}^{2} ; p_{1}, \cdots, p_{n}\right) \text {, } \\
& \frac{\mathrm{d} \sigma}{\mathrm{d} \Omega}=\frac{|\mathcal{M}|^{2}}{(8 \pi)^{2} \mathrm{~s}} \text { or } \frac{\mathrm{d} \sigma}{\mathrm{dt}}=\frac{|\mathcal{M}|^{2}}{64 \pi\left|\overrightarrow{\mathrm{P}}_{\mathrm{a}}^{\mathrm{cm}}\right|^{2} \mathrm{~s}} \text {. }
\end{aligned}
$$

The normalization is such that the optical theorem reads

$$
\left.\operatorname{Im} \mathcal{M}\right|_{t=0}=2\left|\vec{P}_{a}^{\mathrm{cm}}\right| \sqrt{\mathrm{s}} \sigma_{\text {tot }} \text {. }
$$

The choice of Eq. (1) implies a particular normalization of any spinors that may occur in di. The advantage of this normalization is that it greatly simplifies the structure of $\mathcal{H}_{6}$ by putting factors such as $\frac{1}{(2 \pi)^{3}} \frac{1}{2 \mathrm{E}}$ into the phase space where they really belong. In addition, the labels, $i, f$, refer to specific spin (helicity) states, so that the usual "average and sum" rule is implicit. 


\section{WEAK INTERACTIONS OF QUARKS AND LEPTONS}

The "standard" $\mathrm{SU}(2) \otimes \mathrm{U}(1)$ mode $1^{1,2}$ is described here for six quarks and six leptons in left-handed doublets of su(2) weak and right-handed singlets of $\mathrm{SU}(2)_{\text {weak }}\left(\mathrm{T}_{3}=\right.$ third component of weak isospin):

$$
\begin{aligned}
& \begin{array}{c}
T_{3}=+1 / 2 \\
T_{3}=-1 / 2
\end{array}\left(\begin{array}{c}
\nu_{e} \\
e^{-}
\end{array}\right)_{L}\left(\begin{array}{c}
\nu_{\mu} \\
\mu^{-}
\end{array}\right)_{L}\left(\begin{array}{c}
\nu_{\tau} \\
\tau^{-}
\end{array}\right)_{L}\left(\begin{array}{l}
u \\
d^{\prime}
\end{array}\right)_{L}\left(\begin{array}{l}
c \\
s^{\prime}
\end{array}\right)_{L}\left(\begin{array}{l}
t \\
b^{\prime}
\end{array}\right)_{L}, \\
& \mathrm{~T}=\mathrm{T}_{3}=0 \quad \mathrm{e}_{\mathrm{R}}^{-} \quad \mu_{\mathrm{R}}^{-} \quad \tau_{\mathrm{R}}^{-} \quad \mathrm{u}_{\mathrm{R}} \quad \mathrm{d}_{\mathrm{R}} \quad \mathrm{c}_{\mathrm{R}} \quad \mathrm{s}_{\mathrm{R}} \quad \mathrm{t}_{\mathrm{R}} \quad \mathrm{t}_{\mathrm{R}} .
\end{aligned}
$$

Mixing occurs between quarks $d, s, b$ of charge $-1 / 3$ (by convention the charge $2 / 3$ quarks, $u, c, t$, are unmixed) and is expressed by the Kobayashi-Maskawa (KM) mixing matrix ${ }^{2}$

$$
\left(\begin{array}{c}
d^{\prime} \\
s^{\prime} \\
b^{\prime}
\end{array}\right)=\left(\begin{array}{ccc}
c_{1} & s_{1} c_{3} & s_{1} s_{3} \\
-s_{1} c_{2} & c_{1} c_{2} c_{3}+s_{2} s_{3} e^{i \delta} & c_{1} c_{2} s_{3}-s_{2} c_{3} e^{i \delta} \\
-s_{1} s_{2} & c_{1} s_{2} c_{3}-c_{2} s_{3} e^{i \delta} & c_{1} s_{2} s_{3}+c_{2} c_{3} e^{i \delta}
\end{array}\right)\left(\begin{array}{l}
d \\
s \\
b
\end{array}\right),
$$

where $c_{i}=\cos \theta_{i}, s_{i}=\sin \theta_{i}, i=1,2,3$. In the limit $\theta_{2}=\theta_{3}=\delta=0$, this reduces to the usual Cabibbo mixing with $\theta_{1}$ the Cabibbo angle.

$$
\begin{aligned}
& \text { The interaction Lagrangian is } \\
& \mathscr{L}_{\text {int }}=e\left[A^{\alpha} J_{\alpha}^{e m}+\frac{1}{\sin \theta_{W} \cos \theta_{W}} z^{\alpha} J_{\alpha}^{N}+\frac{1}{\sqrt{2} \sin \theta_{W}}\left(w^{+\alpha} J_{\alpha}^{C}+w^{-\alpha} J_{\alpha}^{C+}\right)\right]
\end{aligned}
$$

Here $\theta_{\mathrm{W}}$ is the weak mixing angle in the relations

$$
\begin{aligned}
& \mathrm{W}^{0}=Z \cos \theta_{\mathrm{W}}+A \sin \theta_{\mathrm{W}} \\
& \mathrm{B}=-\mathrm{Z} \sin \theta_{\mathrm{W}}+\mathrm{A} \cos \theta_{\mathrm{W}}
\end{aligned}
$$

which relate the physical fields $A$ (photon) and $Z$ (neutral weak gauge boson) to $\mathrm{W}^{0}\left(\mathrm{SU}(2)\right.$ weak partner of $\mathrm{W}^{+}$and $\left.\mathrm{W}^{-}\right)$and $\mathrm{B}$ (U(1) gauge field). The charged current is written

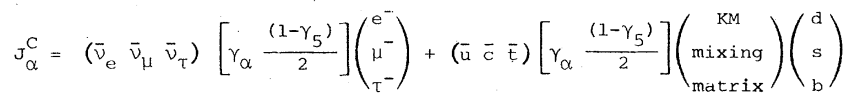

i.e., V-A structure. The neutral current is written

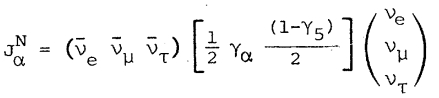

$$
\begin{aligned}
& +(\bar{e} \bar{\mu} \bar{\tau})\left[-\frac{1}{2} \gamma_{\alpha} \frac{\left(1-\gamma_{5}\right)}{2}+\sin ^{2} \theta_{W} \gamma_{\alpha}\right]\left(\begin{array}{l}
e \\
\mu \\
\tau
\end{array}\right) \\
& +\left(\begin{array}{lll}
\bar{u} & \bar{c} & \bar{t}
\end{array}\right)\left[\frac{1}{2} \gamma_{\alpha} \frac{\left(1-\gamma_{5}\right)}{2}-\frac{2}{3} \sin ^{2} \theta_{w} \gamma_{\alpha}\right]\left(\begin{array}{l}
u \\
c \\
t
\end{array}\right) \\
& +(\bar{d} \bar{s} \bar{b})\left[-\frac{1}{2} \gamma_{\alpha} \frac{\left(1-\gamma_{5}\right)}{2}+\frac{1}{3} \sin ^{2} \theta_{w} \gamma_{\alpha}\right]\left(\begin{array}{l}
d \\
s \\
b
\end{array}\right) \text {, }
\end{aligned}
$$

where for fermion $f$ the coupling $\left[\Gamma_{\alpha}^{f}\right]$ has a $V-A$ term depending on $\mathrm{T}_{3}^{\mathrm{f}}$ and a vector term depending on charge $Q_{\mathrm{f}}$ :

$$
\left[\mathrm{r}_{\alpha}^{\mathrm{f}}\right]=\left[\mathrm{T}_{3}^{\mathrm{f}} \gamma_{\alpha} \frac{\left(1-\gamma_{5}\right)}{2}-2_{\mathrm{f}} \sin ^{2} \theta_{\mathrm{W}} \gamma_{\alpha}\right] \text {. }
$$

The effective Lagrangian for exchange of $\mathrm{w}^{ \pm}$and $\mathrm{z}$ between two currents reduces at low $\mathrm{q}^{2}$ to

$$
\mathscr{L}_{\text {weak }}=\frac{G}{\sqrt{2}} 4\left(J^{C} \alpha_{J_{\alpha}} C^{\dagger}+2 \rho J^{N} J_{\alpha}^{N}\right)
$$

with $G / \sqrt{2}=\pi \alpha /\left(2 M_{W}^{2} \sin ^{2} \theta_{W}\right), \quad \alpha=e^{2} /(4 \pi)$, and $\rho=M_{W}^{2} /\left(M_{Z}^{2} \cos ^{2} \theta_{W}\right)$. Assuming the simplest Higgs structure, $\rho=1$, and the $W$ and $z$ masses are related by $M_{Z}=M_{W} / \cos \theta_{W}$. Currently reported values of the weak interaction parameters are

$$
\begin{aligned}
& \left.\begin{array}{l}
\left|\cos \theta_{1}\right|=0.9737 \pm 0.0025 \\
\left|\sin \theta_{1} \cos \theta_{3}\right|=0.219 \pm 0.011
\end{array}\right\} \quad \text { Ref. 3 } \\
& \left.\left|\sin \theta_{1} \sin \theta_{3}\right|=0.06 \pm 0.06\right\} \quad \text { Refs. } 3,4 \text {; } \\
& \left.\theta_{2} \text { and } \delta \text { not determined without }\right\} \quad \text { Ref. } 5 \text {; } \\
& \left.=G_{\mu}=(1.16632 \pm 0.00004) \times 10^{-5} \mathrm{GeV}^{-2}\right\} \text { Refs. } 3.6 \\
& \left.\sin ^{2} \theta_{W}=0.218 \pm 0.025, \quad \rho=0.985 \pm 0.026\right\} \text { Ref. 7. } \\
& \left.\sin ^{2} \theta_{W}=0.228 \pm 0.010, \rho \equiv 1 \text { (fixed) }\right\}
\end{aligned}
$$

The resulting mass estimates for $\mathrm{W}^{ \pm}$and $\mathrm{z}$ are $\mathrm{M}_{\mathrm{W}}=37.3 \mathrm{GeV} / \sin \theta_{\mathrm{W}}$ $=78.1 \pm 1.7 \mathrm{GeV}$, and $\mathrm{M}_{\mathrm{z}}=88.9 \pm 1.4 \mathrm{GeV}$, where the numerical values are obtained using the simplest Higgs structure $(\rho \equiv 1)$.

\section{Lepton-Nucleon Inclusive Scattering}

For reactions $\ell+N \rightarrow \ell^{\prime}+X$, differential cross sections can be written using several choices of independent variables. These are related by

$$
\begin{aligned}
\frac{d^{2} \sigma}{d x d y} & =2 M E_{\ell^{y}}^{2} \frac{d^{2} \sigma}{d \nu d Q^{2}}=2 M E_{\ell^{x}} \frac{d^{2} \sigma}{d x d Q^{2}}=\frac{2 \pi M E_{\ell^{y}}^{2}}{\left|\vec{p}_{\ell}\right|\left|\vec{p}_{\ell^{\prime}}\right|} \frac{d^{2} \sigma}{d \Omega d E_{\ell^{\prime}}} \\
& \cong \frac{2 \pi M E_{\ell^{y}}}{E_{\ell^{\prime}}} \frac{d^{2} \sigma}{d \Omega d E_{\ell^{\prime}}},
\end{aligned}
$$

where $\nu, Q^{2}, x$, and $y$ are defined in the Relativistic Kinematics section IV(c), $E_{\ell}, \vec{p}_{\ell}$ and $E_{\ell^{\prime}}, \vec{p}_{\ell^{\prime}}$ are the incident and outgoing lepton $\mathrm{lab}$ energies and momenta, and $M$ is the target nucleon mass.

Structure Functions $^{8,9}$

For charged current (C.C.) and neutral current (N.C.) reactions, we have

$$
\begin{aligned}
\frac{d^{2} \sigma^{\nu(\nu)}}{d x d y} & =\frac{G^{2} M E_{\ell}}{\pi}\left[\left(1-y-\frac{M}{2 E_{\ell}} x y\right) F_{2}^{\nu(\bar{\nu})}\left(x, \ell^{2}\right)\right. \\
& \left.+\frac{y^{2}}{2} 2 \mathrm{xF}_{1}^{\nu(\bar{\nu})}\left(x, Q^{2}\right) \pm\left(y-\frac{y^{2}}{2}\right) \times F_{3}^{\nu(\bar{\nu})}\left(x, Q^{2}\right)\right],
\end{aligned}
$$

where the upper and lower signs refer to $\nu$ and $\bar{v}$ scattering, respectively, and $F_{3}$ is defined as a positive quantity. The other common structure functions $W_{i}$ are related by $\mathrm{MW}_{1}=\mathrm{F}_{1}, \quad \nu \mathrm{W}_{2}=\mathrm{F}_{2}$, and $U \mathrm{~W}_{3}=-\mathrm{F}_{3}$. For electron and
muon scattering, $\mathrm{F}_{3}=0$, and $\mathrm{G}^{2}$ is replaced by $8 \pi^{2} \alpha^{2} /\left(Q^{2}\right)^{2}$ 


\section{WEAK INTERACTIONS OF QUARKS AND LEPTONS (Cont'd)}

The ratio of the longitudinally to transversely polarized photon absorption cross section is

$R=\frac{\sigma_{L}}{\sigma_{T}}=\frac{1}{2 x F_{1}\left(x, Q^{2}\right)}\left[F_{2}\left(x, Q^{2}\right)-2 x F_{1}\left(x, Q^{2}\right)+\frac{4 M^{2} x^{2}}{Q^{2}} F_{2}\left(x, Q^{2}\right)\right]$.

To compare with the parton-model predictions below, we write for $E_{\ell} \gg M$ :

$$
\begin{aligned}
& \frac{\mathrm{d}^{2} \sigma^{\nu(\bar{\nu})}}{\mathrm{dxdy}}=\frac{\mathrm{G}^{2} \mathrm{ME} \ell}{\pi}\left[\frac{1}{2}\left(2 \mathrm{xF}_{1}^{\nu(\bar{\nu})} \pm \mathrm{xF}_{3}^{\nu(\bar{\nu})}\right)+\frac{1}{2}(1-\mathrm{y})^{2}\left(2 \mathrm{xF}_{1}^{\nu(\bar{\nu})} \mathrm{xF}_{3}^{\nu(\bar{\nu})}\right)\right. \\
&\left.+(1-y)\left(\mathrm{F}_{2}^{\nu(\bar{\nu})}-2 \mathrm{xF}_{1}^{\nu(\bar{\nu})}\right)\right], \\
& \frac{\mathrm{d}^{2} \sigma^{\mathrm{e}} \mu}{\mathrm{dxdy}}=\frac{8 \pi \alpha^{2} \mathrm{ME} \ell}{\left(Q^{2}\right)^{2}}\left[\frac{1+(1-y)^{2}}{2} 2 \mathrm{xF}_{1}^{\mathrm{e}, \mu}+(1-y)\left(\mathrm{F}_{2}^{\mathrm{e}, \mu}-2 \mathrm{xF}_{1}^{\mathrm{e}, \mu}\right)\right] .
\end{aligned}
$$

The Free-Quark-Parton-Model Predictions ${ }^{10}$

For this model in the Bjorken limit $\left(Q^{2}, \nu \rightarrow \infty\right.$ with $x$ fixed), $F_{i}\left(x, Q^{2}\right) \rightarrow F_{i}(x) .8,9$ For spin-1/2 quark partons, we have

$2 \mathrm{FF}_{1}(\mathrm{x})=\mathrm{F}_{2}(\mathrm{x})$, the Callan-Gross relation. Thus, in this

approximation, $R=0$ and there is no ( $1-y)$ term in the cross section.

$$
\text { - (c.c.) } \frac{d^{2} \sigma^{\nu N \rightarrow \mu^{-} x}}{d x d y}=\frac{G^{2} M E_{\ell}}{\pi} 2 x \sum_{q}\left[f_{q}(x)+f_{\bar{q}}(x)(1-y)^{2}\right] \text {. }
$$

For $\bar{v}_{N} \rightarrow \mu^{+} x$, interchange $f_{q}(x)$ and $f_{\bar{q}}(x)$ in the formula. Here $f_{q}(x) d x$ is the number of quarks $q$ in the target nucleon with momentum fraction $x$ to $x+d x$. We include $f_{q}(x)$ and $f_{\bar{q}}(x)$ in the sum only for negative (positive) charged quarks and antiquark in $v(\bar{v})$ reactions.

$$
\text { - (N.C.) } \begin{aligned}
\frac{d^{2} \sigma^{\nu N} \rightarrow v x}{d x d y} & =\frac{G^{2} M E_{\ell}}{\pi} 2 \rho^{2} x \sum_{G}\left\{\left(\varepsilon_{L}^{q}\right)^{2}\left[f_{q}(x)+f_{\bar{q}}(x)(1-y)^{2}\right] .\right. \\
& \left.+\left(\varepsilon_{R}^{q}\right)^{2}\left[f_{q}(x)(1-y)^{2}+f_{\bar{q}}(x)\right]\right\},
\end{aligned}
$$

and the sum runs over all quarks. Here the neutral-current coupling is decomposed according to

$$
\Gamma_{\alpha}^{q}=\varepsilon_{L}^{q} \gamma_{\alpha} \frac{\left(1-\gamma_{5}\right)}{2}+\varepsilon_{R}^{q} \gamma_{\alpha} \frac{\left(1+\gamma_{5}\right)}{2}
$$

with left- and right-handed coupling constants $\varepsilon_{L}^{q}$ and $\varepsilon_{R}^{q}$. In the "standard" SU(2) $\otimes U(1)$ model

$$
\varepsilon_{L}^{q}=T_{3}^{q}-2_{q} \sin ^{2} \theta_{W}, \quad \varepsilon_{R}^{q}=-Q_{q} \sin ^{2} \theta_{W} .
$$

For $\bar{v} N \rightarrow \bar{v} x$, interchange $\varepsilon_{L}^{q}$ and $\varepsilon_{R}^{q}$ in the cross-section formula.

- (E.M.) $\frac{d^{2} \sigma^{e}, \mu}{d x d y}=\frac{8 \pi \alpha^{2} M E_{\ell}}{\left(Q^{2}\right)^{2}} \times \sum_{q} o_{q}^{2}[f(x)+f-(x)] \frac{1+(1-y)^{2}}{2}$.

Comparison with earlier structure function formulas gives:

$$
\text { (c.c.) } \begin{aligned}
F_{2}(x) & =2 x \sum_{q}\left[f_{q}(x)+f_{\bar{q}}(x)\right] ; \\
x_{3}(x) & =2 x \sum_{q}\left[f_{q}(x)-f_{\bar{q}}(x)\right] ;
\end{aligned}
$$

$$
\text { (N.C.) } \begin{aligned}
F_{2}(x) & =2 \rho^{2} x \sum_{q}\left[\left(\varepsilon_{L}^{q}\right)^{2}+\left(\varepsilon_{R}^{q}\right)^{2}\right]\left[f_{q}(x)+f_{\bar{q}}(x)\right], \\
x F_{3}(x) & =2 \rho^{2} x \sum_{q}\left[\left(\varepsilon_{L}^{q}\right)^{2}-\left(\varepsilon_{R}^{q}\right)^{2}\right]\left[f_{q}(x)-f_{\bar{q}}(x)\right] ; \\
F_{i}^{\nu}(x) & =F_{i}^{\bar{\nu}}(x) ; \\
\text { (E.M.) } \quad F_{2}(x) & =x \sum_{q} Q_{q}^{2}\left[f_{q}(x)+f_{\bar{q}}(x)\right] .
\end{aligned}
$$

In the examples below, $u(x), \bar{u}(x), d(x), \bar{d}(x)$, etc., mean $f_{\mathrm{q}}\left(f_{\bar{q}}\right)$ for the individual quark (antiquark) in the proton (for into account.

$$
\begin{aligned}
F_{2}^{\nu p \rightarrow \mu^{-} x} & =2 x[d(x)+s(x)+\bar{u}(x)+\bar{c}(x)], \\
F_{2}^{\bar{\nu}} \rightarrow \mu^{+} x & =2 x[u(x)+c(x)+\bar{d}(x)+\bar{s}(x)], \\
x F_{3}^{\nu p \rightarrow \mu^{-} x} & =2 x[d(x)+s(x)-\bar{u}(x)-\bar{c}(x)], \\
x_{3} \overline{\nu p} \rightarrow \mu^{+} x & =2 x[u(x)+c(x)-\bar{d}(x)-\bar{s}(x)],
\end{aligned}
$$

Hereafter we neglect small contributions of the $s, \bar{s}, c, \bar{c}$ quarks in the sea.

- For charge-symetric nuclei with $q(x)=u(x)+d(x)$, $\bar{q}(x)=\bar{u}(x)+\bar{d}(x)$

$$
\begin{aligned}
& F_{2}^{\nu N \rightarrow \mu^{-} x}=F_{2}^{\overline{v N} \rightarrow \mu^{+} x}=x[q(x)+\bar{q}(x)] \\
& x_{3}^{\nu N \rightarrow \mu^{-} x}=x_{3}^{\bar{v} N \rightarrow \mu^{+} x}=x[q(x)-\bar{q}(x)] . \\
& F_{2}^{e p, \mu p}(x)=x\left[\frac{4}{9}(u(x)+\bar{u}(x))+\frac{1}{9}(d(x)+\bar{d}(x))\right] \\
& F_{2}^{e d}(x) \cong \frac{5}{18} F_{2}^{\nu(\bar{v}) d} \text { C.C. } \\
& \left(\frac{5}{18}: \text { average squared charge of u,d quarks }\right) .
\end{aligned}
$$

1. S. Weinberg, Phys. Rev. Lett. 19, 1264 (1967); A. Salam, in Elementary Particle Theory, edited by N. Svartholm (Almqvist \& Wiksell, Stockholm, 1968), p.367; S. L. Glashow, J. Iliopoulos, and L. Maiani, Phys. Rev. D2, 1285 (1970).

2. M.Kobayashi and K. Maskawa, Prog. Theor. Phys. 49. 652 (1973).

3. R. E. Shrock and L.-L. Wang, Phys. Rev. Lett. 41, 1692 (1978).

4. R. E. Shrock, S. B. Treiman, and L.-L. Wang, Phys. Rev. Lett. 42, 1589 (1979).

5. This determination has been done by, e.g., v. Barger, W. F. Long, and S. Pakvasa, Phys. Rev. Lett. 42, 1585 (1979) W. F. Longr,

6. M. P. Balandin et al., Sov. Phys. JETP 40, 811 (1974).

7. P. Langacker, J. E. Kim, M. Levine, H. H. Williams, and D. P. Sidhu, Univ. of Pennsylvania preprint, Report Coo-3071-243, D. P. Sidhu, Univ. Kef penneylvania preprint, Report, and to be published in proc. Noutrino 1979 Conference, Bergen, Liede

8. J. D. Bjorken, Phys. Rev. 179, 1547 (1969).

9. J. D. Bjorken and E. A. Paschos, Phys. Rev. 185, 1975 (1969).

10. R. P. Feynman, Photon-Hadron Interactions, (W. A. Benjamin, Reading, MA, 1972). 11. E.g., H. Quinn, Proc. Summer Inst. on Particle Physics,
SLAC-215 (1978), 


\section{PARTICLE DETECTORS, ABSORBERS, AND RANGES*}

\section{A. DETECTOR PARAMETERS}

In this section we give various parameters for common detectors. The quoted numbers represent at best an order of magnitude, and are useful only for preliminary design. A more detailed introduction to detectors can be found in "A Consumer's Guide to Particle Detectors," by D. J. Miller, Rutherford Lab Report RL-76-072, July 1976.

A.1 Scintillators: Photon yield $\approx 1 \gamma / 100 \mathrm{eV}$ in plastic

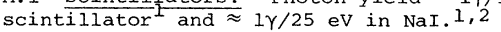

A.2 Cerenkov: ${ }^{3}$ Half-angle $\theta_{C}$ of cone aperture in terms of velocity $\beta$ and index of refraction $n$ :

$$
\theta_{C}=\arccos \left(\frac{1}{\beta n}\right) \sim \sqrt{2\left(1-\frac{1}{\beta n}\right)} \text {. }
$$

Threshold velocity: $\beta_{t}=1 / n ; \quad \gamma_{t}=1 / \sqrt{1-\beta_{t}^{2}}$.

Therefore, $\beta_{t} \gamma_{t}=1 / \sqrt{2 \delta+\delta^{2}}$, where $\delta=n-1$. values of $\delta$ for various commonly used gases are given as a function of pressure and wavelength in Ref. 4; for values at atmospheric pressure, see the Table of Atomic and Nuclear Properties following.

Number of photons $\mathrm{N}$ per $\mathrm{cm}$ :

$$
\begin{aligned}
N & =\frac{\alpha}{c} \int\left(1-\frac{1}{\beta^{2} n^{2}}\right) 2 \pi d \nu=\frac{\alpha}{c} \beta_{t}^{2} \int\left(\frac{1}{\beta_{t}^{2} \gamma_{t}^{2}}-\frac{1}{\beta^{2} \gamma^{2}}\right) 2 \pi d \nu \\
& \approx 500 \sin ^{2} \theta_{c} / c m \text { (visible spectrum). }
\end{aligned}
$$

A. 3 Photon Collection: In addition to the photon yield, one should take into account the light collection efficiency ( $\lesssim 10 \%$ for typical $1-\mathrm{cm}$-thick scintillator), attenuation length $\left(\approx 1\right.$ to $4 \mathrm{~m}$ for typical scintillators $\left.{ }^{5}\right)$, and quantum efficiency of the photomultiplier cathode ( $\leqslant 25 \%)$.

A. 4 Bubble, Streamer, Wire Chambers:

\begin{tabular}{lccc} 
Chamber Type & Accuracy (rms) & $\begin{array}{c}\text { Resolution } \\
\text { Time }\end{array}$ & $\begin{array}{l}\text { Dead } \\
\text { Time }\end{array}$ \\
\hline Bubble & $\pm 75 \mu$ & $\approx 1 \mathrm{~ms}$ & $\approx 1 / 20 \mathrm{~s}^{\alpha}$ \\
Streamer & $\pm 300 \mu$ & $\approx 2 \mu \mathrm{s}$ & $\approx 100 \mathrm{~ms}$ \\
Optical spark & $\pm 200 \mu \mathrm{b}$ & $\approx 2 \mu \mathrm{s}$ & $\approx 10 \mathrm{~ms}$ \\
Magnetostrictive & $\pm 500 \mu$ & $\approx 2 \mu \mathrm{s}$ & $\approx 10 \mathrm{~ms}$ \\
Spark & $\geqslant \pm 300 \mu^{c}, d$ & $\approx 50 \mathrm{~ns}$ & $\approx 200 \mathrm{~ns}$ \\
Proportional & \pm 50 to $300 \mu$ & $\approx 2 \mathrm{~ns}$ & $\approx 100 \mathrm{~ns}$ \\
Drift &
\end{tabular}

${ }_{b}$ Multiple pulsing time.

$60 \mu$ for high pressure.

$c^{300 \mu}$ is for $1 \mathrm{~mm}$ pitch.

Delay line cathode readout can give \pm 150

$e^{\text {parallel to anode wire. }}$

For two chambers.
A.5 Shower Detectors: Typical energy resolutions (FWHM) for incident electron in the $1 \mathrm{GeV}$ range, $\mathrm{E}$ in $\mathrm{GeV}$. For a fixed number of radiation lengths, FWHM in the last three detectors would be expected to be proportional to $\sqrt{t}$ for $t \quad(=$ plate thickness) $\geq 0.2$ radiation lengths. 6

NaI (20 rad. lengths) ${ }^{7} \frac{28}{E^{1 / 4}}$

Lead Glass (14 rad. lengths) $:^{8} \frac{10-12 \%}{\sqrt{E}}$

Lead-Liquid Argon (15.75 rad. lengths) $:^{6} \frac{16 \%}{\sqrt{E}}$

( 42 cells: lead, $2 \mathrm{~mm}$ liquid argon, $\sqrt{\mathrm{E}}$

lead-G10, 2 mm liquid argon)

Lead-Scintillator Sandwich (14 rad. lengths) $)^{9} \frac{22 \%}{\sqrt{E}}$
( 35 cells: $2 \mathrm{~mm}$ lead,

$12.7 \mathrm{~mm}$ scintillator

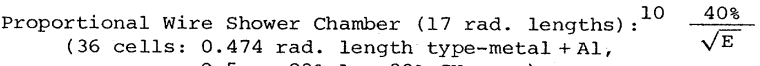
$9.5 \mathrm{~mm} 80 \% \mathrm{Ar}-20 \% \mathrm{CH}_{4}$ gas)

A.6 Proportional Chamber Wire Instability: The limit on the A.6 Proportional Chamber wire Instability: The limit on the when the electrostatic repulsion of adjacent wires exceeds when the electrostatic repulsion of adjacent wires

$$
V \leqslant \frac{\mathrm{sT}^{1 / 2}}{\ell \mathrm{C}}
$$

where $s, \ell$, and $C$ are the wire spacing, length, and capacitance per unit length. An approximation to $C$ for chamber half-gap $t$. and wire diameter d (good for $\mathrm{s} \leqslant \mathrm{t}$ ) gives ${ }^{12}$

$$
\mathrm{V} \lesssim 59 \mathrm{~T}^{1 / 2}\left[\frac{\mathrm{t}}{\mathrm{l}}+\frac{\mathrm{s}}{\pi l} \ln \left(\frac{\mathrm{s}}{\pi \mathrm{d}}\right)\right],
$$

where $\mathrm{V}$ is in $\mathrm{kV}$, and $\mathrm{T}$ is in grams.

A. 7 Proportional and Drift Chamber Potentials: Potential distributions and fields for an array of parallel line charges $q($ coul. $/ \mathrm{m})$ along $z$ and located at $y=0, x=0, \pm a, \pm 2 a, \ldots$, can usually be calculated with good accuracy from (MKSA):

$$
\mathrm{V}(\mathrm{x}, \mathrm{y})=-\frac{\mathrm{q}}{4 \pi \varepsilon_{0}} \ln \left\{4\left[\sin ^{2}\left(\frac{\pi x}{\mathrm{a}}\right)+\sinh ^{2}\left(\frac{\pi y}{\mathrm{a}}\right)\right]\right\} \text {. }
$$

\section{B. COSMIC RAY FLUXES}

The fluxes of particles of different types depend on the latitude, their energy, and the conditions of measurement. Some typical sea-level values ${ }^{13}$ are given below:

Iv flux per unit solid angle about vertical direction crossing unit horizontal area

$\mathrm{J}_{1}$ perpendicular component of total flux crossing unit horizontal area from above

$\mathrm{J}_{2}$ total flux crossing unit horizontal area Total

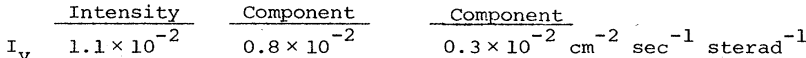

$\mathrm{J}_{1} \quad 1.8 \times 10^{-2} \quad 1.3 \times 10^{-2} \quad 0.5 \times 10^{-2} \mathrm{~cm}^{-2} \mathrm{sec}^{-1}$

$\mathrm{J}_{2} \quad 2.4 \times 10^{-2} \quad 1.7 \times 10^{-2} \quad 0.7 \times 10^{-2} \mathrm{~cm}^{-2} \mathrm{sec}^{-1}$

Very approximately, about $75 \%$ of all particles at sea-level are penetrating, and are muons. The absolute flux of protons ${ }^{\text {at }}-2$ sea-level, in a momentum range $700-1100 \mathrm{MeV} / \mathrm{c}$, is $1.5 \times 10^{-5} \mathrm{~cm}^{-2}$ $\mathrm{sec}^{-1}$ sterad $^{-1}$, or $\sim 0.18$ of all particles. 


\section{PARTICLE DETECTORS, ABSORBERS, AND RANGES (Cont'd)}

\section{PASSAGE OF PARTICLES THROUGH MATTER}

C. 1 Energy Loss Rates for Heavy Charged Projectiles: A heavy projectile (much more massive than an electron) of charge $z_{i n c} e$, incident at speed $B c(\beta \gg 1 / 137)$ through a slowing medium, dissipates energy principally via interactions with the electrons of the medium. The mean rate of such energy loss per trons of the medium. The mean rate of such energy loss per

$$
\begin{aligned}
&\left(\frac{d E}{d x}\right)_{\text {inc }}=\frac{D \rho_{\text {med }} z_{\text {med }}}{A_{\text {med }}}\left(\frac{z_{\text {inc }}}{\beta}\right)^{2} \\
& \times\left[\ln \left(\frac{2 m e^{\gamma^{2} \beta^{2} c^{2}}}{I}\right)-\beta^{2}-\frac{\delta}{2}-\frac{C}{z_{\text {med }}}\right]\{1+\nu\},
\end{aligned}
$$

where $D=4 \pi N_{A} r_{e}^{2} m_{e} c^{2}=0.3070 \mathrm{MeV} \mathrm{cm}{ }^{2} / g$ (see Physical and Numerical Constants Table).

Here $\mathrm{z}_{\mathrm{med}} \cdot \mathrm{and} \mathrm{A}_{\text {med }}$ are the charge and mass numbers of the medium and $\rho_{\text {med }}$ is the mass density of the medium; $I, \delta, C$, and $\nu$ are phenomenological functions. Frequently, the values of $\delta$, $C$, and $\nu$ are negligibly small; the parameter I characterizes the binding of the electrons of the medium. As a rule of thumb, we may as $I \simeq 16\left(Z_{\text {med }}\right) 0.9 \mathrm{eV}$ when $z_{\text {med }}>1$. For realistic media the value of $I$ will vary at the $10 \%$ level from this estimate; for $\mathrm{H}_{2}$, I $=20.0 \mathrm{eV}$. We approximately treat media

$$
\frac{d E}{d x} \approx \sum_{n=1}^{N}\left(\frac{d E}{d x}\right)_{n}
$$

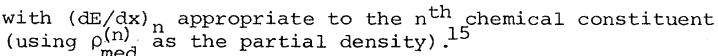

The function $\delta$ represents the density effect upon the energy loss rate; it is non-negligible only for highly relativistic projectiles in dense media. ${ }^{16}$ For ultra-relativistic projectiles, $\delta$ approaches $2 \ell n \gamma+$ constant, where the value of the constant depends upon the density of the medium and its chemical constant depen

The function $C$ represents shell corrections to the energy loss rate. ${ }^{14}$ These effects are non-negligible only for projectiles with speeds not much faster than the speeds of the fastest electrons bound in the medium. The function $\nu$ represents corrections due to higher-order
electrodynamics. 17 These effects become important when $\left|z_{\text {ind }} / \beta\right|$ is comparable to 137. For relativistic unit-charge projectiles, $|\nu|$ is of the order of $1 \%$; positively charged projectiles lose energy more rapidly than do their charge conjugates 17,18

$(\mathrm{dE} / \mathrm{dx})$ inc falls rapidly with $\beta$ until reaching a minimum around $\beta=0.96$ (almost independent of medium), followed by a slow rise. Because of the density effect, the quantity in square brackets approaches $\ln \gamma+$ constant for large $\gamma$.

The value $(\mathrm{dE} / \mathrm{dx})_{\text {inc }} \delta \mathrm{x}$ is the mean total energy loss via interactions with electrons of the medium in a layer of thickness $\delta x$. For any finite $\delta x$, Poisson fluctuations can cause the actual energy loss to deviate from the mean. For thin layers, the distribution is broad and skewed, being peaked below ( $\mathrm{dE} / \mathrm{dx}) \delta \mathrm{x}$, distribution is broad and skewed, being peaked below (dE/dx) $\delta x_{1}$ and having a long tail toward large energy losses. 19 Only for of energy losses become nearly Gaussian. The large fluctuations of the total energy loss rate from the mean are due to a small number of collisions with large energy transfers. The fluctuations are greatly reduced for the so-called restricted energy loss rate, described in section C. 3 .
C.2 Energetic Knock-On Electrons: For a spinless point-charge projectile, the production of high energy (kinetic energy $T$ ) I) electrons is given by (neglecting the spin of the electron):

$$
\begin{aligned}
& \qquad \frac{d N}{d T d x}=\frac{1}{2} D\left(\frac{Z_{\text {med }}}{A_{\text {med }}}\right)\left(\frac{Z_{\text {inc }}}{\beta}\right)^{2} \rho_{\text {med }} \frac{1}{T^{2}}, \\
& \text { for } \quad I \ll T \leqslant T_{\text {max }} ; T_{\max }=\frac{2 m_{e} \beta^{2} \dot{\gamma}^{2} c^{2}}{1+2 \gamma \frac{m_{e}}{M_{\text {inc }}}+\left(\frac{m_{e}}{M_{\text {inc }}}\right)^{2}},
\end{aligned}
$$

where $M_{i n c}$ is the mass of the incident projectile and all other quantities are as in section C.1. This formula does not differ significantly from the precise result, incorporating spin effects, for any projectile (including $\mathrm{e}^{ \pm}$) in the restricted range $I<T<<T$ T $<$; more accurate formulae are available for range $I \ll T<<T$ max; more accurate formulae are available for
various projectiles. 20,21 our formula is inaccurate for $T$ various projectiles. $\simeq \mathrm{T}^{-} \eta$ with $3 \leqslant \eta \leqslant 5.22$

C. 3 Rates of Restricted Energy Loss for Charged Projectiles: The variability of energy loss for heavy projectiles is due primarily to the variability in the production of energetic knock-on electrons. Bremsstrahlung and pair production processes make this variability even greater for electrons than for heavy particles as projectiles (see, e.g., the figure "Fractional Energy Loss for $\mathrm{e}^{+}$and $\mathrm{e}^{-}$in Lead"). If an instrument is capable of isolating these high-energy-loss interactions, then it is appropriate to consider the rate of energy loss excluding them, i.e., a restricted energy loss rate. The mean energy loss rate via all collisions which have energy transfer $\mathrm{T}$ such that $\mathrm{T} \leqslant \mathrm{E}_{\max } \ll \mathrm{T}_{\max }$ is: ${ }^{14}$

$$
\begin{aligned}
\left(\frac{d E}{d x}\right)_{\leqslant E_{\text {max }}} & =\frac{D}{2} \frac{z_{\text {med }} \rho_{\text {med }}}{A_{\text {med }}}\left(\frac{z_{\text {inc }}}{\beta}\right)^{2} \\
& \times\left[\ln \left(\frac{E_{\text {max }} T_{\text {max }}}{I^{2}}\right)-\beta^{2}-\delta-\frac{2 C}{z_{\text {med }}}\right]
\end{aligned}
$$

Notice the overall factor of $1 / 2$.

The density effect causes the restricted energy loss rate to approach a constant, the Fermi plateau value, for the fastest projectiles.

C.4 Multiple Coulomb Scattering through Small Angles: As a charged particle traverses a medium it is deflected via many independent small-angle coulomb scatterings. The bulk of this deflection is due to scattering from the nuclei in the medium. The non-projected (space) and projected (plane) distributions are given approximately ${ }^{23}$ by the Gaussian forms:

$$
\begin{aligned}
& f\left(\theta_{\text {space }}\right) d \Omega \cong \frac{1}{\pi \theta_{0}^{2}} \exp \left(-\frac{\theta_{\text {space }}^{2}}{\theta_{0}^{2}}\right) d \Omega, \\
& g\left(\theta_{\text {plane }}\right) d \theta_{\text {plane }} \cong \frac{1}{\sqrt{\pi} \theta_{0}} \exp \left(-\frac{\theta_{\text {plane }}^{2}}{\theta_{0}^{2}}\right) d \theta_{\text {plane }},
\end{aligned}
$$

where

$$
\theta_{0}=\frac{20 \mathrm{MeV} / \mathrm{C}}{\mathrm{p} \beta} \mathrm{z}_{\text {inc }} \sqrt{\frac{\mathrm{L}}{\mathrm{L}_{\mathrm{R}}}}\left[1+\frac{1}{9} \log _{10}\left(\frac{\mathrm{L}}{\mathrm{L}_{\mathrm{R}}}\right)\right] \text { (radians), }
$$




\section{PARTICLE DETECTORS, ABSORBERS, AND RANGES (Cont'd)}

p, $\beta$, and $z_{\text {inc }}$ are the momentum (in $M e V / c$ ), velocity, and charge number of the incident particle, and $L / L_{R}$ is the thickness, in radiation lengths, of the scattering medium. $L_{R}$ for certain materials is given in the Table of Atomic and Nuclear Properties of Materials. The $1 / \mathrm{e}$ angle, $\theta_{0}$, is a fit to Moliere 24 theory accurate to about $5 \%$ for $10^{-3}<\mathrm{L} / \mathrm{L}_{\mathrm{R}}<10$ except for very light elements or low velocity where the error is about 10 to 208. In this Gaussian approximation, $\theta_{0}$ has the meaning

$$
\theta_{0}=\theta_{\text {space }}^{\text {rms }}=\sqrt{2} \theta_{\text {plane }}^{\text {rms }} .
$$

Beyond angles of about $2 \theta_{0}$, the true distribution function has a long tail which contributes at the level of roughly 1 s of peak height, slowly descending, beyond the point at which the Gaussian would be negligible, to the height expected for single large-angle Rutherford or nuclear scatters.

Other quantities are sometimes used to describe the amount of multiple coulomb scattering: the auxiliary quantities

$\psi_{\text {plane, }} \mathrm{y}_{\mathrm{plane}}$, and $\mathrm{s}$ plane (see the figure) obey:

$$
\begin{aligned}
\psi_{\text {plane }}^{\text {rms }} & =\frac{1}{\sqrt{3}} \theta_{\text {plane }}^{\text {rms }}, \\
\mathrm{y}_{\text {plane }}^{\text {rms }} & =\frac{1}{\sqrt{3}} \perp \theta_{\text {plane }}^{\text {rms }}, \\
\text { and } & s_{\text {plane }}^{\text {rms }}=\frac{1}{4 \sqrt{3}} \mathrm{~L} \theta_{\text {plane }}^{\text {rms }} .
\end{aligned}
$$

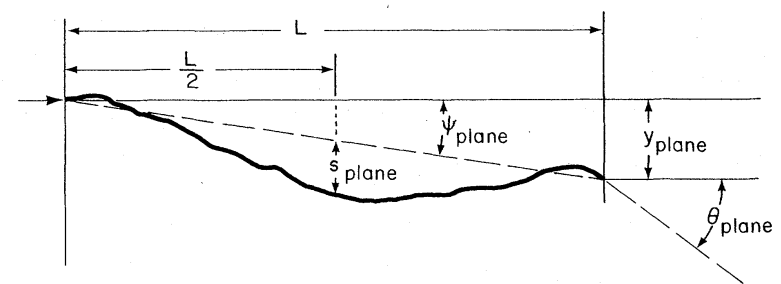

XBL $782-307$

All the quantitative estimates in this section apply only in the limit of small $\theta_{\text {plane }}^{\text {rms }}$ and in the absence of large-angle scatters.

C.5 Electron Range in Lead, Copper, Carbon, and Hydrogen: See figure following.

C.6 Fractional Energy Loss for Electrons and Positrons in Lead: See figure following.

C. 7 Contributions to Photon Cross Section in Lead: See figure following.

C. 8 Photon Mass Attenuation Coefficients, Energy Deposition: See figure following.

D. ATOMIC AND NUCLEAR PROPERTIES OF MATTER

See Table following.
*Prepared April 1974 by Sherwood Parker and Bernard Sadoulet. Revised April 1980 by Sherwood Parker and Ray Hagstrom.

1. Methods of Experimental Physics, L.C.I. Yuan and C.-S. Wu, editors, Academic Press, 1961, Vol. 5A, p.127.

2. R. K. Swank, Ann. Rev. Nuc. Sci. 4, 137 (1954) and G. T. Wright, Proc. Phys. Soc. B68, $92 \overline{9}$ (1955).

3. Methods of Experimental Physics, L.C.L. Yuan and C.-S. Wu, editors, Academic Press, 1961, Vol. 5A, p.163.

4. E. R. Hayes, R. A. Schlutter, and A. Tamosaitis, "Index and Dispersion of Some Cerenkov Counter Gases," ANL-6916 (1964).

5. Nuclear Enterprises Catalogue.

6. D. Hitlin et al., Nucl. Instr. and Meth. 137, 225 (1976). See also W. J. Willis and V. Radeka, Nucl. Instr. and Meth. 120, 221 (1974), for a more detailed discussion.

7. E.B. Hughes et al., IEEE Transactions on Nuclear Science, NS-19, No. 3, 126 (1972)

8. M. Holder et al., phys., Letters 40B, 141 (1972), and J.S. Beale et al., "A Lead-Glass Čerenkov Detector for. Electrons and Photons," CERN Writeup, Intl. Conf. on Instrumentation in H.E.P., Frascati (1973).

9. W. B. Atwood et al., "First Test of a New Shower Detector," SLAC-TN-76-7 (1976). See also J. K. Walker and T. R. Knasel, Rev. Sci. Instr. 37, 913 (1966).

10. R. L. Anderson et al., "Tests of Proportional wire Shower Counter and Hadron Calorimeter Modules," SLAC-PUB-2039 (1977).

11. T. Trippe, CERN NP Internal Report 69-18 (1969).

12. S. Parker and R. Jones, LBL-797 (1972), and A. Morse and B. Feshbach, Methods of Theoretical Physics, McGraw-Hill New York, 1953, p.1236.

13. B. Rossi, Rev. Mod. Phys. 20, 537 (1948).

14. U. Fano, Ann. Rev. Nucl. Sci. 13, 1 (1963).

15. H.A. Bethe and J. Ashkin, Experimental Nuclear Physics, Vo1. 1, E. Segrè, editor, John Wiley, New York, 1959.

16. A. Crispin and G. N. Fowler, Rev. Mod. Phys. 42, 290 (1970).

17. For $z^{3}$ calculations with $z=1$, see J. D. Jackson and R. L. McCarthy, Phys. Rev. B6, 4131 (1972).

18. For an approximate treatment of high-z projectiles, see P. B. Eby and S. H. Morgan, Phys. Rev. A5, 2536 (1972).

19. See, for instance, K. A. Ispirian, A. T. Margarian, and A. M. Zverev, Nucl. Instr. and Meth. 117, 125 (1974).

20. For unit-charge projectiles, see E. A. Uehling, Ann. Rev. Nucl. Sci. 4, 315 (1954).

21. For highly charged projectiles, see J.A. Doggett and L. V. Spencer, Phys. Rev. 103,1597 (1956). A Lorentz transformation is needed to convert these center-of-mass data to knock-on energy spectra.

22. N. F. Mott and H. S.W. Massey, The Theory of Atomic Collisions, Oxford Press, London, 1965.

23. J.D.Jackson, Classical Electrodynamics, John Wiley \& Sons, New York, 1975. V.L. Highland, Nucl. Instr. \& Meth. 129 , 497 (1975); 161, 171 (1979); and earlier references 497 (1975); 161,171 (1979); and earlier references
therein. G. Shen, et al. Phys. Rev. D20, 1584 (1979). Their data, taken at high energies, agrees with Moliere Their data, taken at high energies, agrees with Moliere theory. They find the Highland formula for $\theta_{0}$ to be 11
high for hydrogen and 1 to 58 high for higher-z targets.

24. G.Moliere, z. Naturforsch 2a, 133 (1947) and 3a, 78 (1948). H.A. Bethe, Phys. Rev. 89, 1256 (1953). 


\section{PARTICLE DETECTORS, ABSORBERS, AND RANGES (Cont'd)}

Mean Range and Energy Loss in Lead, Copper, Aluminum, and Carbon

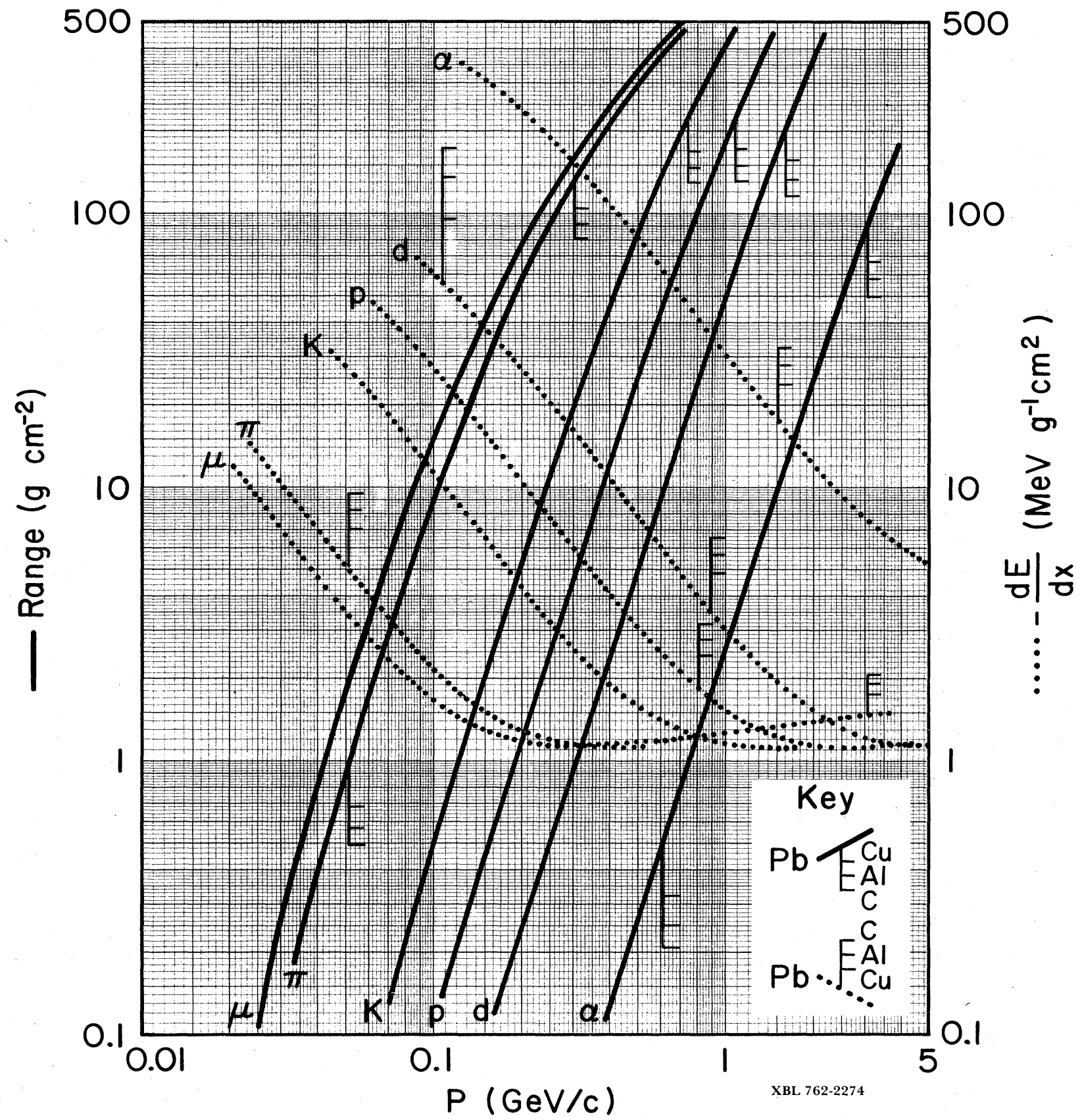

Mean range and energy loss due to ionization for the indicated particles in $\mathrm{Pb}$, with scaling to $\mathrm{Cu}$, $\mathrm{Al}$, and C indicated, using Bethe-Bloch equation (Section C.1 above) with corrections. Calculated using program of Hans Bichsel (UCRL-17538), with density correction added (Hans Bichsel, private communication). See also Joseph F. Janni [Air Force Weapons Laboratory Technical Report No. AFW L-TR-65-150 (1966)]. The average ionization potentials (I) as sumed were: $\mathrm{Pb}(820 \mathrm{eV}), \mathrm{Cu}(320 \mathrm{eV}), \mathrm{A} 1$ (166 eV), and C(77.5 eV). Figure indicates total path length; observed range may be smaller (by 1\% - 2\% in heavy elements) due to multiple scattering, primarily from small energy-loss collisions with nuclei. The functional forms have not been experimentally verified to better than roughly $\pm 1 \%$. For higher energies refer to discussion by Cobb ["A Study of Some Electromagnetic Interactions of High Velocity Particles with Matter," University of Oxford Report HEP/ T/55 (1973)] and by Turner "Penetration of Charged Particles in Matter: A Symposium", National Academy of Sciences, Washington D.C. (1970), p. 48]. Scaling to other beam particles is, to a good approximation, described by the expression on the next page. 
PARTICLE DETECTORS, ABSORBERS, AND RANGES (Cont'd)

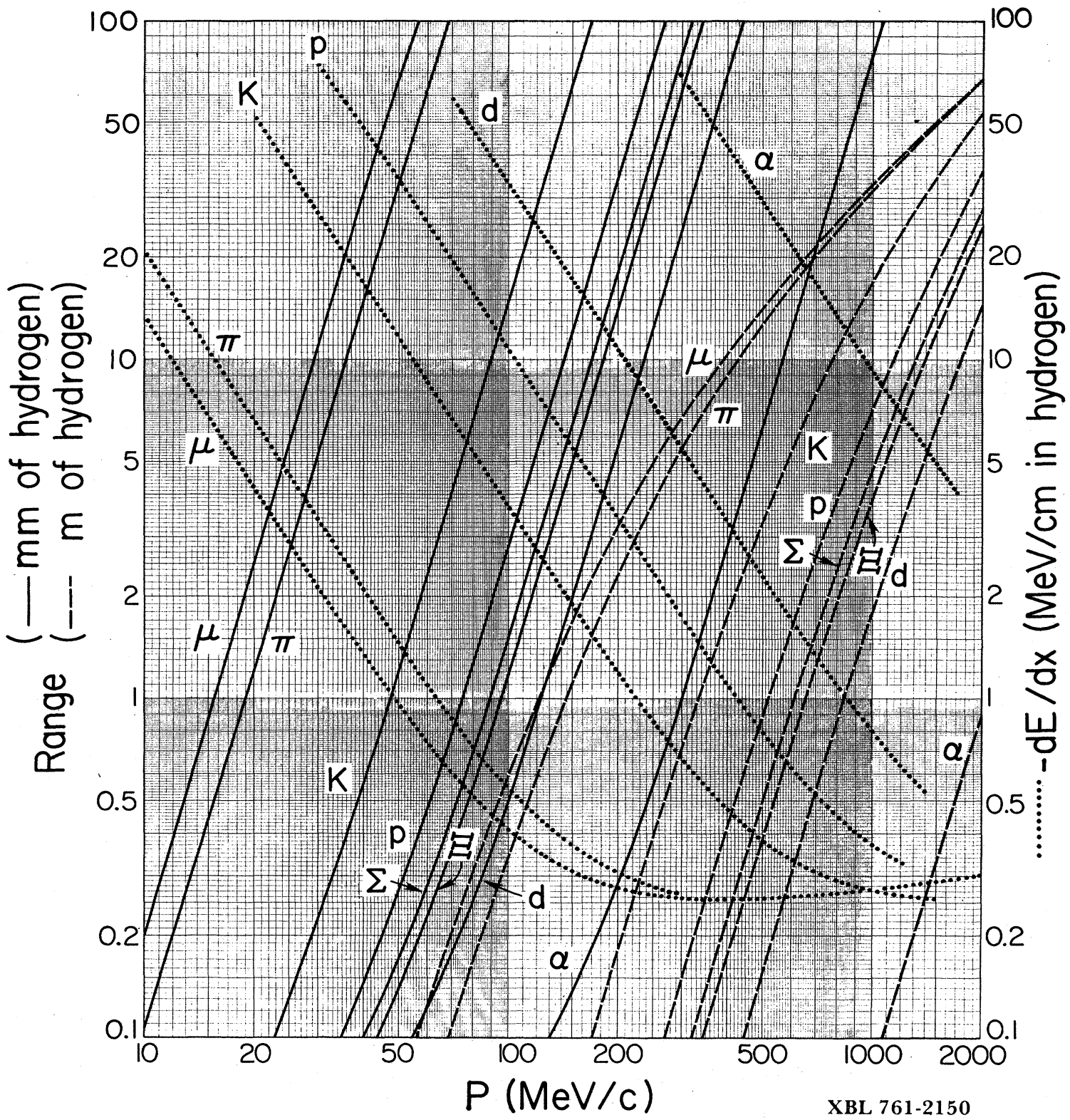

Range and energy loss in liquid hydrogen bubble chamber, based on Bethe-Bloch equation (Section C.1 above), using an average ionization potential for $\mathrm{H}_{2}$ of $\mathrm{I}=20.0 \mathrm{eV}$, which is an approximate average of the experimental result of Garbincius and Hyman [Phys. Rev. A2, 1834 (1970)] and the theoretical result of Ford and Browne [Phys. Rev. A7, 418 (1973)]. Bubble chamber conditions are chosen to be those of Garbincius and Hyman: parahydrogen of density $=0.0625 \mathrm{~g} / \mathrm{cm}^{3}$ (note: range $\propto 1 /$ density), with vapor-pressure 60.8 lb/in (absolute) and temperature $26.2^{\circ} \mathrm{K}$. The functional dependence of the Bethe-Bloch equation is not experimentally verified to better than about $\pm 1 \%$ over large momentum ranges. It should be noted that the number of bubbles per $\mathrm{cm}$ of a track in a bubble chamber is nearly proportional to $1 / \beta^{2}$, not $\mathrm{dE} / \mathrm{dx}$. For the 1 inear portions of the range curves, $R \propto \mathrm{p}^{3.6}$. Scaling law for particles of other mass or charge (except electrons): for a given medium, the range $R_{b}$ of any beam particle with mass $M_{b}$, charge $z_{b}$, and momentum $p_{b}$ is $g i v e n$ in terms of the range $\mathrm{R}_{\mathrm{a}}$ of any other particle with mass $\mathrm{M}_{\mathrm{a}}$, charge $\mathrm{z}_{\mathrm{a}}$, and momentum $\mathrm{p}_{\mathrm{a}}=\mathrm{p}_{\mathrm{b}} \mathrm{M}_{\mathrm{a}} / \mathrm{M}_{\mathrm{b}}$ (i.e., having the same velocity) by the expression:

$$
R_{b}\left(M_{b}, z_{b}, P_{b}\right)=\left[\frac{M_{b} / M_{a}}{z_{b}^{2} / z_{a}^{2}}\right] R_{a}\left(M_{a}, z_{a}, P_{a}=p_{b} M_{a} / M_{b}\right)
$$




\section{PARTICLE DETECTORS, ABSORBERS, AND RANGES (Cont'd)}

\section{Mean Electron Range in Lead, Copper, Carbon, and Liquid Hydrogen}

Mean range of electrons in the continuous-slowing-down approximation, taking into account energy loss by collisions with atomic electrons and by bremsstrahlung; strong fluctuations are to be expected for individual tracks. This range is the total path length; the "practical range" - a common measure of straight-line penetration distance - is shorter because of multiple Coulomb scattering, which becomes increasingly important as the electron slows down. E.g., for a fast electron the rms projected angle due to multiple coulomb scattering reaches 1 radian by the time the electron has slowed to $0.4 \mathrm{MeV}$ in hydrogen, $1.5 \mathrm{MeV}$ in carbon, $9 \mathrm{MeV}$ in copper, and $24 \mathrm{MeV}$ (off scale) in lead. Electron energy deposition and penetration probability vs. range are discussed by L. V. Spencer, "Energy Dissipation by Fast Electrons," NBS Monograph \#1, 1959, and S. M. Seltzer, "Transmission of Electrons through Foils," NBSIR 74, 457 (1974). Electrons which have energy less than $0.2 \mathrm{MeV}$ in $\mathrm{Ar}$

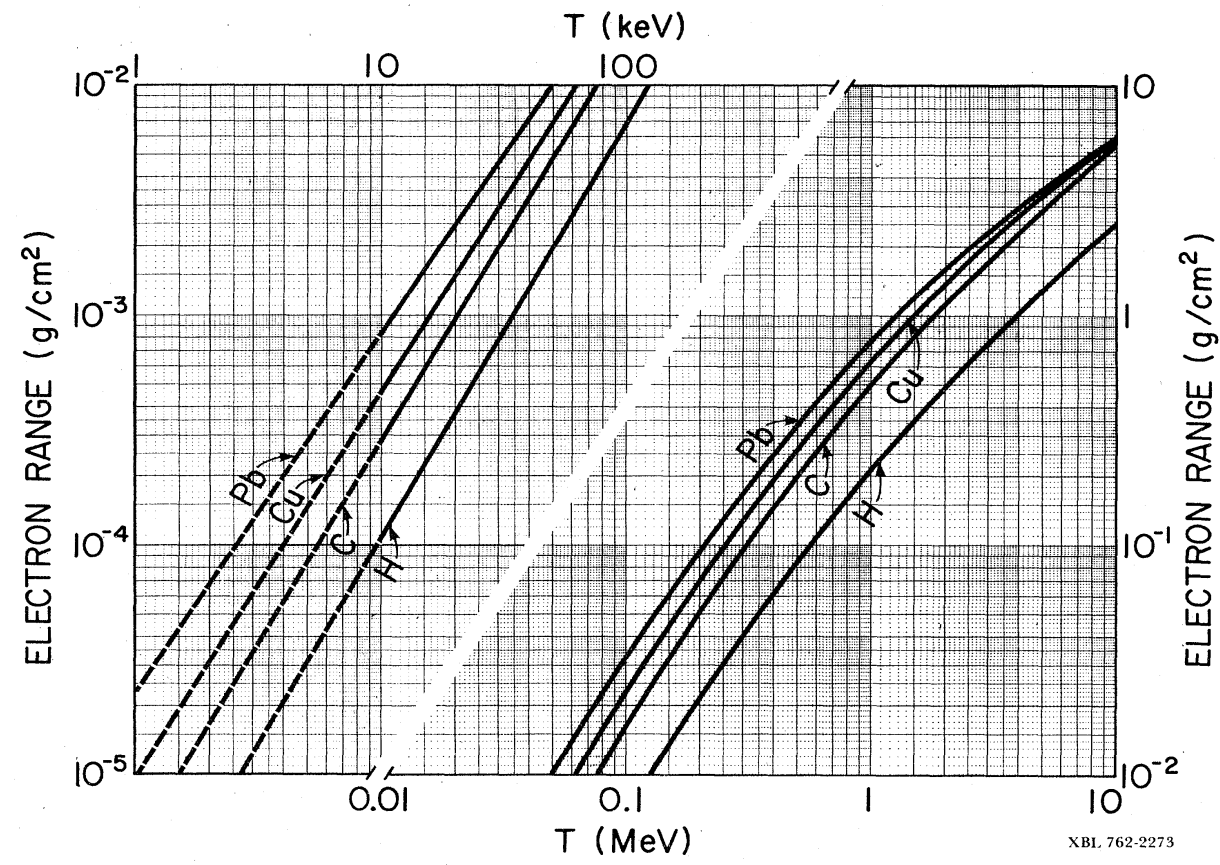

$1.5 \mathrm{MeV}$ in $\mathrm{Cu}, 3.5 \mathrm{MeV}$ in $\mathrm{Sn}$, and $5 \mathrm{MeV}$ in $\mathrm{Pb}$ are likely to deposit $10 \%$ of their energy behind their starting plane. The practical range, $R_{p}$, is defined as that absorber thickness obtained by extrapolating to zero the linearly decreasing part of the curve of penetration probability vs. absorber thickness. Data for Al in the $T$ range of the figure are available, and fit (to $\sim 10 \%) \mathrm{R}_{\mathrm{p}}=\mathrm{AT}[1-\mathrm{B} /(1+\mathrm{CT})] \mathrm{mg} \mathrm{cm}-2$ [a form suggested by $\mathrm{K} .-\mathrm{H}$. Weber, Nucl. Inst. Meth. 25, 261 (1964)], with $\mathrm{A}=0.55 \mathrm{mg} \mathrm{cm}-2 \mathrm{keV}^{-1}, \mathrm{~B}=0.9841$, and $\mathrm{C}=0.0030 \mathrm{keV}^{-1}$. At this penetration depth, 90-95\% of the incident electrons have stopped. Data for other elements are sketchy, but suggest that higher $-z \quad(\leqslant 50)$ elements have $1 \lesssim R_{p} / R_{p}(A l) \lesssim 1.4$ below $\sim 10 \mathrm{keV}$, and $0.6 \lesssim \mathrm{R}_{\mathrm{p}} / \mathrm{R}_{\mathrm{p}}(\mathrm{Al}) \leqslant 1$ above $100 \mathrm{keV}$. The "critical energy" (above which the energy loss due to bremsstrahlung exceeds that due to ionization, and showering becomes important) is $400 \mathrm{MeV}$ for hydrogen, $100 \mathrm{MeV}$ for carbon, $25 \mathrm{MeV}$ for copper, and $10 \mathrm{MeV}$ for lead. The mean positron range may differ from the mean electron range by several percent. See Berger and Seltzer, NASA SP-3012 (1964) and SP-3036, and P. Trower, UCRL-2426, Vol. III, Rev. (1966). 1-10 keV range was obtained by linear extrapolation; in this region the true range may actually lie above the curves.

\section{Fractional Energy Loss for $\mathrm{e}^{+}$and $\mathrm{e}^{-}$in Lead}

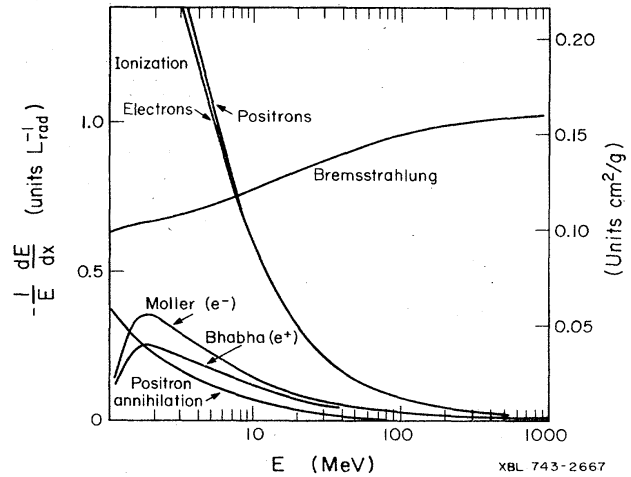

Fractional energy loss per radiation length in lead as a function of electron or positron energy. Electron (positron) scattering is considered as ionization when the energy loss per collision is below $0.255 \mathrm{MeV}$, and as Moller (Bhabha) scattering when it is above.

These figures are adapted from Fig. 3.2 and Fig. 3.3 from Messel and Crawford, Electron-Photon Shower Distri$\frac{\text { bution Function Tables for Lead, Copper and Air Absorbers, Pergamon Press, } 1970 \text {. Messel and Crawford use }}{\mathrm{L}_{\mathrm{r}}(\mathrm{Pb})=5.82 \mathrm{~g} / \mathrm{cm}^{2} \text {, but we have modified the figures to reflect the value given in the Table of Atomic and }}$ Nuclear Properties of Materials (following), namely $\mathrm{L}_{\mathrm{r}}(\mathrm{Pb})=6.4 \mathrm{~g} / \mathrm{cm}^{2}$. The development of electronphoton cascades is approximately independent of absorber when the results are expressed in terms of inverse radiation lengths (i.e., scales on left of plots).
Contributions to Photon Cross Section in Lead

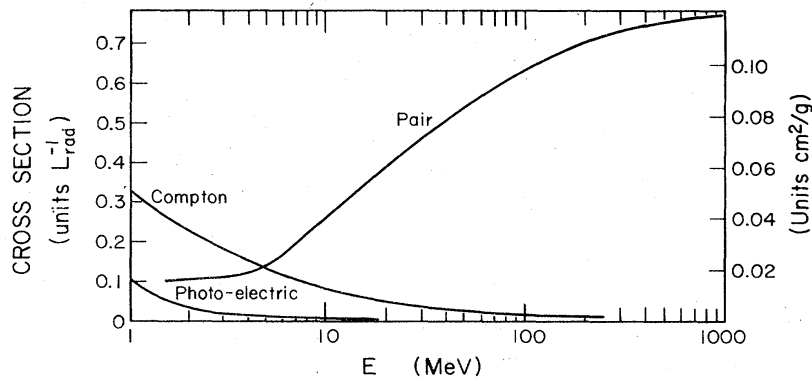

Photon cross section in lead in inverse radiation lengths as a function of photon energy. The intensity of photons can be expressed as $I=I_{0} \exp (-\sigma x)$, where $\sigma$ is read above and $x$ is the path length in radiation lengths. See also figure following. 


\section{PARTICLE DETECTORS, ABSORBERS, AND RANGES (Cont'd)}

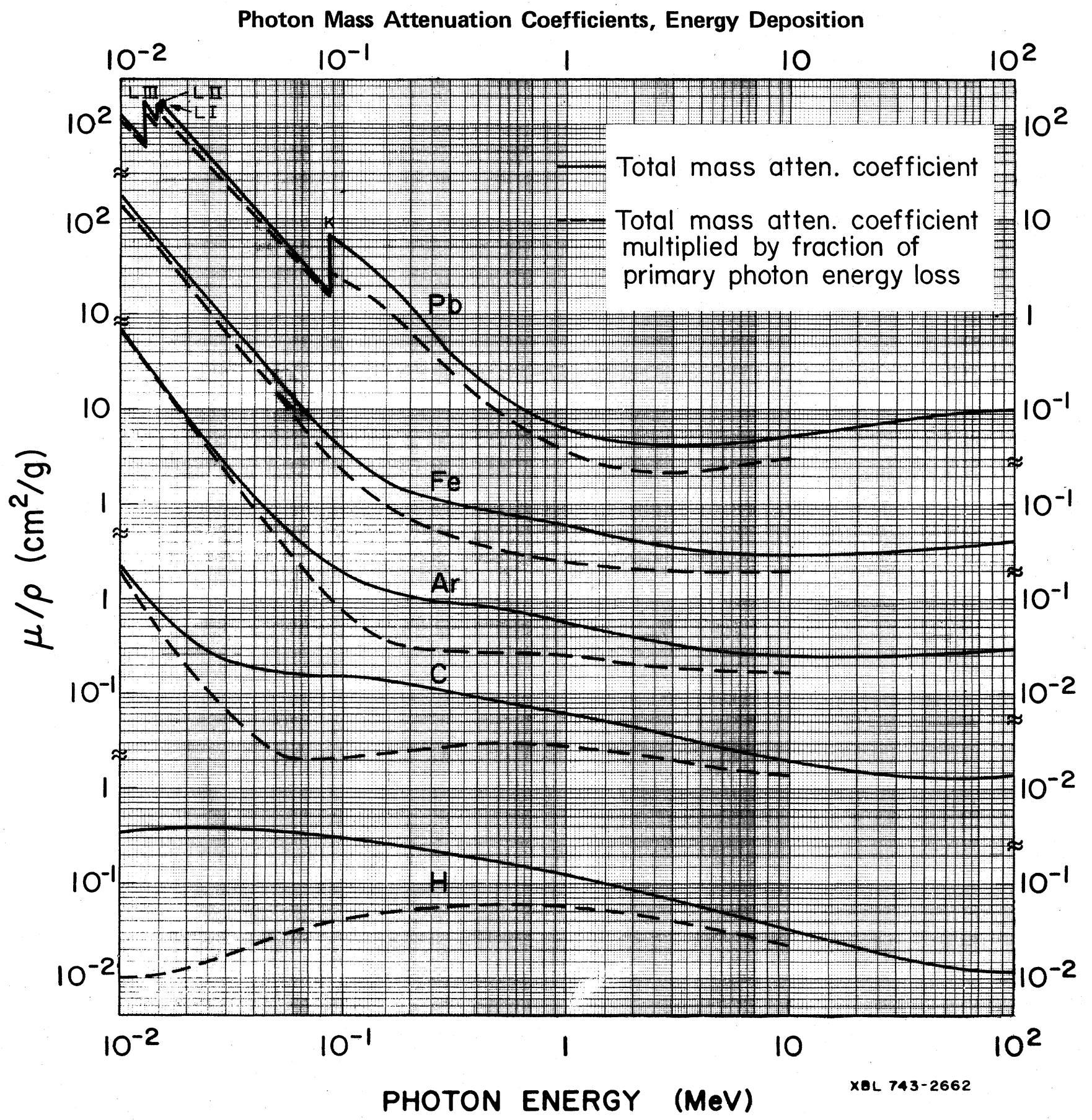

The photon mass attenuation coefficient for various absorbers as a function of photon energy (solid curves). For a homogeneous medium of density $\rho$, the intensity I remaining after traversal of thickness $t$ is given by $I=I_{0} \exp (-\mu t)$. The accuracy is a few percent. Interpolation to other $\mathrm{Z}$ should be done in the cross section $\sigma=(\mu / \rho) \mathrm{M} / \mathrm{N}_{\mathrm{A}} \mathrm{cm}^{2} /$ atom, where $\mathrm{M}$ is the atomic weight of the absorber material and $\mathrm{N}_{\mathrm{A}}$ is Avogadro's number. For a chemical compound or mixture, use $(\mu / \rho)_{\text {eff }} \cong \sum_{i} w_{i}(\mu / \rho)_{i}$, accurate to a few percent, where $w_{i}$ is the proportion by weight of the $i^{\text {th }}$ constituent. $i$ The dashed curve is the mass energyabsorption coefficient, giving $\mu / \rho$ multiplied by the fraction of photon energy deposited in a small volume (assumed large enough to contain the ranges of most secondary. electrons) about the interaction. This fraction is smaller than 1.0 because such processes as Compton scattering and electron bremsstrahlung imply radiation of some of the energy away from the immediate area. From J. H. Hubbell, NSRDS-NBS 29(1969). 


\section{PARTICLE DETECTORS, ABSORBERS, AND RANGES (Cont'd)}

\section{Atomic and Nuclear Properties of Materials*}

\begin{tabular}{|c|c|c|c|c|c|c|c|c|c|c|c|c|}
\hline Material & $\mathrm{Z}$ & A & $\begin{array}{l}\text { Nuclear } \\
\text { cross } \\
\text { section } \sigma^{\mathbf{a}} \\
\text { [barns] }\end{array}$ & \multicolumn{2}{|c|}{$\begin{array}{l}\text { Nuclear collision } \\
\text { length } L_{\text {coll. }}\end{array}$} & $\begin{array}{c}\text { Absorption } \\
\text { length } \lambda^{b} \\
{[\mathrm{~cm}]}\end{array}$ & \multicolumn{2}{|c|}{$d E / d x \min ^{c}$} & \multicolumn{2}{|c|}{$\begin{array}{c}\text { Radiation length } \\
L_{\mathrm{rad}}\end{array}$} & $\begin{array}{c}\text { Density }{ }^{e} \\
{\left[\mathrm{~g} / \mathrm{cm}^{3}\right]} \\
() \text { is for gas } \\
{[\mathrm{g} / \mathrm{l}]}\end{array}$ & $\begin{array}{c}\text { Refractive } \\
\text { index } n ; e \\
\text { () is }(n-1) \times 10^{6} \\
\text { for gas }\end{array}$ \\
\hline $\mathrm{H}_{2}$ & 1 & 1.01 & 0.039 & 43.0 & 607 & 790 & 4.12 & 0.292 & 63.05 & 890 & $\left\{\begin{array}{l}0.0708 \\
(0.090)\end{array}\right.$ & \\
\hline $\mathrm{D}_{2}$ & 1 & 2.01 & 0.074 & 45.1 & 273 & 342 & 2.07 & 0.342 & 126.1 & 764 & 0.163 & 1.128 \\
\hline $\mathrm{He}$ & 2 & 4.00 & 0.134 & 49.6 & 397 & 478 & 1.94 & 0.243 & 94.32 & 755 & $\left\{\begin{array}{l}0.125 \\
(0.178)\end{array}\right.$ & $\left\{\begin{array}{l}1.024 \\
(35)\end{array}\right.$ \\
\hline $\begin{array}{l}\mathrm{Li} \\
\mathrm{Be}\end{array}$ & $\begin{array}{l}3 \\
4 \\
--\end{array}$ & $\begin{array}{r}6.94 \\
9.01 \\
\end{array}$ & $\begin{array}{l}0.215 \\
0.270\end{array}$ & $\begin{array}{l}53.6 \\
55.4 \\
-\end{array}$ & $\begin{array}{r}100.4 \\
30.0 \\
-\end{array}$ & $\begin{array}{r}120.6 \\
36.7 \\
\end{array}$ & $\begin{array}{l}1.65 \\
1.61\end{array}$ & $\begin{array}{l}0.881 \\
2.97\end{array}$ & & 3 & $\begin{array}{l}0.534 \\
1.848 \\
-\end{array}$ & - \\
\hline C & 6 & 12.01 & 0.340 & 58.7 & $\approx 37.8$ & 49.9 & 1.78 & $\approx 2.76$ & 42.70 & $\approx 27.5$ & $\approx 1.55^{f}$ & - \\
\hline $\mathrm{N}_{2}$ & 7 & 14.01 & 0.390 & 59.7 & 73.8 & 99.4 & 1.82 & 1.47 & 37.99 & 47.0 & $\left\{\begin{array}{l}0.808 \\
(1.25)\end{array}\right.$ & $\left\{\begin{array}{l}1.205 \\
(300)\end{array}\right.$ \\
\hline $\mathrm{Ne}$ & 10 & 20.18 & 0.520 & 64.4 & 53.7 & 74.9 & 1.73 & 2.08 & 28.94 & 24.0 & $\left\{\begin{array}{c}1.207 \\
(0.90)\end{array}\right.$ & $\left\{\begin{array}{l}1.092 \\
(67)\end{array}\right.$ \\
\hline Al & 13 & 26.98 & 0.650 & 68.9 & 25.5 & 37.2 & 1.62 & 4.37 & 24.01 & 8.9 & 2.70 & $\therefore-$ \\
\hline Ar & 18 & 39.95 & 0.890 & 74.5 & 53.2 & 80.9 & 1.51 & 2.11 & 19.55 & 14.0 & $\left\{\begin{array}{l}1.40 \\
(1.78)\end{array}\right.$ & $\left\{\begin{array}{l}1.233 \\
(283)\end{array}\right.$ \\
\hline $\begin{array}{l}\mathrm{Fe} \\
\mathrm{Cu} \\
\mathrm{Sn} \\
\mathrm{W} \\
\mathrm{Pb} \\
-\end{array}$ & $\begin{array}{l}26 \\
29 \\
50 \\
74 \\
82 \\
---\end{array}$ & $\begin{array}{r}55.85 \\
63.54 \\
118.69 \\
183.85 \\
207.19 \\
\end{array}$ & $\begin{array}{l}1.160 \\
1.270 \\
2.040 \\
2.810 \\
3.080 \\
\end{array}$ & $\begin{array}{r}79.9 \\
83.1 \\
96.6 \\
108.6 \\
111.7 \\
\end{array}$ & $\begin{array}{r}10.2 \\
9.3 \\
13.2 \\
5.6 \\
9.8 \\
-\end{array}$ & $\begin{array}{l}10.3 \\
18.5 \\
\end{array}$ & $\begin{array}{l}1.48 \\
1.44 \\
1.28 \\
1.17 \\
1.13 \\
-1.0\end{array}$ & $\begin{array}{r}11.6 \\
12.9 \\
9.4 \\
22.6 \\
12.8 \\
\end{array}$ & $\begin{array}{r}13.84 \\
12.86 \\
8.82 \\
6.76 \\
6.37 \\
\end{array}$ & $\begin{array}{l}1.76 \\
1.43 \\
1.21 \\
0.35 \\
0.56\end{array}$ & $\begin{array}{r}7.87 \\
8.96 \\
7.31 \\
19.3 \\
11.35 \\
\end{array}$ & $\begin{array}{l}- \\
- \\
- \\
-\end{array}$ \\
\hline Air & 92 & 238.03 & 3. 380 & $\begin{array}{r}116.9 \\
60.2\end{array}$ & $\begin{array}{l}\approx 6.2 \\
50000^{g}\end{array}$ & $\begin{array}{l}12.0 \\
67500^{g}\end{array}$ & $\begin{array}{l}1.09 \approx \\
1.82\end{array}$ & $\begin{array}{l}=20.7 \\
\quad 0.0022^{g}\end{array}$ & $\begin{array}{r}6.00 \\
36.20\end{array}$ & $\begin{array}{l}\approx 0.32 \\
30050^{\mathrm{g}}\end{array}$ & $\begin{array}{l}\approx 18.95 \\
\left\{\begin{array}{l}0.001205 \\
(1.29)\end{array}\right.\end{array}$ & $5^{g}\left\{\begin{array}{l}1.000273^{g} \\
(293)\end{array}\right.$ \\
\hline $\begin{array}{l}\mathrm{H}_{2} \mathrm{O} \\
\mathrm{H}_{2} \text { (bub } \\
\mathrm{D}_{2} \text { (bub } \\
-----\end{array}$ & bble & $\begin{array}{l}\text { chamber } \\
\text { chamber } 3\end{array}$ & $\begin{array}{l}\left.26^{\circ} \mathrm{K}\right)^{\mathrm{h}} \\
\left.31^{\circ} \mathrm{K}\right)^{\mathrm{h}}\end{array}$ & $\begin{array}{l}58.3 \\
43.0 \\
45.1\end{array}$ & $\begin{aligned} & 58.3 \\
\approx & 683 \\
\approx & 322 \\
- & -----\end{aligned}$ & $\begin{array}{l}78.8 \\
887 \\
403\end{array}$ & $\begin{array}{l}2.03 \\
4.12 \\
2.07 \\
\end{array}$ & $\begin{aligned} & 2.03 \\
& \approx 0.26 \\
& \approx 0.29\end{aligned}$ & $\begin{array}{r}36.08 \\
63.05 \\
126.1\end{array}$ & $\begin{aligned} & 36.1 \\
\approx & 1000 \\
\approx & 900\end{aligned}$ & $\begin{aligned} & 1.00 \\
\approx & 0.063^{\mathrm{h}} \\
\approx & 0.140^{\mathrm{h}}\end{aligned}$ & $\begin{array}{l}1.33 \\
1.112 \\
1.110 \\
------~\end{array}$ \\
\hline $\mathrm{H}-\mathrm{Ne} \mathrm{m}$ & iixtu & re $(50 \mathrm{mo}$ & ole percent) & )$^{i} 62.9$ & 154.5 & 215 & 1.84 & 0.75 & 29.70 & 73.0 & 0.407 & .092 \\
\hline $\begin{array}{l}\text { Propane } \\
\text { Freon } 1\end{array}$ & $\begin{array}{r}\left(C_{3}\right. \\
3 \mathrm{B1}\end{array}$ & $\begin{array}{l}\left.\mathrm{H}_{8}\right)^{\mathrm{j}} \\
\left(\mathrm{CF}_{3} \mathrm{Br}\right)^{\mathrm{j}}\end{array}$ & & 55.0 & $\approx 49.5$ & 73.5 & 2.28 & 0.98 & 45.38 & 111 & $\begin{array}{l}\left\{\begin{array}{l}0.41 \\
(2.0)\end{array}\right. \\
\left\{\begin{array}{c}\approx 1.50^{j} \\
(8.71)\end{array}\right.\end{array}$ & $\begin{array}{l}\left\{\begin{array}{l}1.25 \\
(1005)\end{array}\right. \\
\left\{\begin{array}{l}1.238^{j} \\
(750)\end{array}\right.\end{array}$ \\
\hline $\begin{array}{l}\text { Ilford en } \\
\text { NaI }\end{array}$ & muls & ion & & $\begin{array}{r}88.1 \\
91.9 \\
---1\end{array}$ & $\begin{array}{l}23.1 \\
25.0 \\
-0 .-1\end{array}$ & & $\begin{array}{l}1.44 \\
1.32 \\
-0-0\end{array}$ & $\begin{array}{r}5.49 \\
4.84 \\
------\end{array}$ & $\begin{array}{r}11.02 \\
9.49 \\
----0\end{array}$ & $\begin{array}{r}2.94 \\
2.59 \\
-\end{array}$ & $\begin{array}{l}3.815 \\
3.67\end{array}$ & 1.775 \\
\hline $\begin{array}{l}\text { LiF } \\
\text { Polyethyl } \\
\text { Mylar (C } \\
\text { Polystyre } \\
\text { Lucite, }\end{array}$ & $\begin{array}{l}\text { lene } \\
C_{5} \mathrm{H}_{4} \\
\text { ene, } \\
\text { Plex }\end{array}$ & $\begin{array}{l}\left(\mathrm{CH}_{2}\right) \\
\left.\mathrm{O}_{2}\right) \\
\text { scintillato } \\
\text { iglas }\left(\mathrm{C}_{5} \mathrm{H}_{8}\right.\end{array}$ & $\begin{array}{l}\text { or }(\mathrm{CH})^{k} \\
\left.\mathrm{O}^{\mathrm{O}}\right)\end{array}$ & $\begin{array}{l}61.1 \\
55.7 \\
58.5 \\
57.0 \\
57.7\end{array}$ & $\begin{array}{r}23.1 \\
\approx 59.6 \\
42.1 \\
55.2 \\
\approx 48.9 \\
\end{array}$ & $\begin{array}{l}30.7 \\
78.4 \\
56.1 \\
68.5 \\
65.0\end{array}$ & $\begin{array}{l}1.69 \\
2.09 \\
1.91 \\
1.97 \\
1.97 \\
-\end{array}$ & $\begin{array}{r}4.46 \\
\approx 1.95 \\
2.65 \\
2.03 \\
\approx 2.32 \\
----~\end{array}$ & $\begin{array}{l}39.25 \\
44.78 \\
39.95 \\
43.8 \\
40.55\end{array}$ & $\begin{aligned} & 14.9 \\
& \approx 48 \\
& 28.7 \\
& 42.9 \\
& \approx 34.5 \\
&-\end{aligned}$ & $\begin{aligned} 2.64 \\
0.92-0.95 \\
1.39 \\
1.032 \\
1.16-1.20\end{aligned}$ & $\begin{array}{r}1.394 \\
- \\
1.581 \\
\approx 1.49 \\
\end{array}$ \\
\hline $\begin{array}{l}\text { Spark or } \\
\text { Shielding } \\
\mathrm{CO}_{2} \text { n } \\
\text { Freon } 1\end{array}$ & $\begin{array}{ll}\text { r } & \text { pr } \\
\mathrm{g} & \mathrm{co} \\
2 & (\mathrm{C}\end{array}$ & $\begin{array}{l}\text { oportional } \\
\text { ncrete } \\
\left.{ }^{m} \ell_{2} F_{2}\right)^{n}\end{array}$ & chamber ${ }^{\ell}$ & $\begin{array}{l}65.5^{0} \\
60.4 \\
68.1\end{array}$ & $\begin{array}{c}.030 \% \\
26.2 \\
33800 \\
13800\end{array}$ & $\begin{array}{c}0.022 \% \\
36.8 \\
46000 \\
20200\end{array}$ & $\begin{array}{l}1.70 \\
1.82 \\
1.64\end{array}$ & $\begin{array}{l}0.034 \\
4.25 \\
0.0033 \\
0.0081\end{array}$ & $\begin{array}{l}26.7 \\
36.2 \\
23.7\end{array}$ & $\begin{array}{c}.067 \% \\
10.7 \\
20210 \\
4810\end{array}$ & $\begin{array}{l}0.019 \\
2.5 \\
(1.79)^{n} \\
(4.93)^{n}\end{array}$ & $\begin{array}{c}- \\
(410)^{n} \\
(1080)^{n}\end{array}$ \\
\hline $\begin{array}{l}\text { Freon } 1 \\
\text { Silica Ae }\end{array}$ & $\begin{array}{l}310 \\
\text { eroge }\end{array}$ & $e^{\circ}\left(\tilde{F}_{3}\right)^{n}$ & & $\begin{array}{l}66.0 \\
62.3\end{array}$ & $\begin{array}{r}15000 \\
\approx 311\end{array}$ & $\begin{array}{r}21400 \\
430\end{array}$ & $\begin{array}{l}1.70 \\
1.82\end{array}$ & $\begin{array}{l}0.0072 \\
\approx 0.36\end{array}$ & $\begin{array}{l}27.15 \\
30\end{array}$ & $\begin{array}{l}6380 \\
\approx 150\end{array}$ & $\begin{array}{l}(4.26)^{n} \\
0.1-0.3\end{array}$ & $\begin{array}{c}(720)^{n} \\
1.0+0.25 \rho\end{array}$ \\
\hline
\end{tabular}

*) Table revised April 1980 by J. Engler and F. Mönnig. For details, see CERN NP Internal Report 74-1.

a) $\sigma$ of neutrons $(\approx \sigma$ of protons) at $20 \mathrm{GeV}$ from Landolt-Bornstein, New Series I, Vol. 5 . Energy dependence for all nuclei $\approx 1 / 2$ percent/GeV (from $5-25 \mathrm{GeV}$ ).

b) $\mathrm{L}_{\text {coll }}=\mathrm{A} /(\mathrm{N}, \sigma)$. In the absorption length the elastic scattering is subtracted.

c) For a minimum-ionizing, singly-charged particle in the material. From W.H. Barkas and M.J. Berger, Tables of Energy Losses and Ranges of Heavy Charged Particles, NASA-SP-3013 (1964)

d) From Y.S. Tsai, Rev. Mod. Phys. 46, 815 (1974).

e) Values for solids, or the liquid phase at boiling point, except where noted. Values in parentheses for gaseous phase STP ( $0^{\circ} \mathrm{C}$, 1 atm.), except where noted.

f) Density variable.

g) Gas at $20^{\circ} \mathrm{C}$.

h) Density may vary about $\pm 3 \%$, depending on operating conditions.

i) Values for typical working condition with $\mathrm{H}_{2}$ target: 50 mole percent, $29^{\circ} \mathrm{K}, 7 \mathrm{~atm}$.

j) Values for typical chamber working conditions: Propane $\sim 57^{\circ} \mathrm{C}, 8-10$ atm. Freon $13 \mathrm{~B} 1 \sim 28^{\circ} \mathrm{C}, 8-10$ atm.

k) Typical scintillator; e.g. PILOT $B$ and NE $102 \mathrm{~A}$ have an atomic ratio $\mathrm{H} / \mathrm{C}=1.10$.

l) Values for typical construction: 2 layers $50 \mu \mathrm{m} \mathrm{Cu} / \mathrm{Be}$ wires, $8 \mathrm{~mm}$ gap, $60 \%$ argon, $40 \%$ isobutane or $\mathrm{CO}_{2} ; 2$ layers $50 \mu \mathrm{m} \mathrm{Mylar} / \mathrm{Aclar}$ foils.

m) Standard shielding blocks, typical composition $\mathrm{O}_{2} 52 \%, \mathrm{Si} 32.5 \%$, Ca $6 \%$, Na $1.5 \%$, Fe $2 \%$, Al $4 \%$ plus reinforcing iron bars. Attenuation length $\ell=115 \pm 5 \mathrm{~g} / \mathrm{cm}^{2}$, also valid for earth (typical $\rho=2.15$ ) from CERN-LRL-RHEL Shielding exp. UCRL 17841 (1968).

n) Used in Čerenkov counters, value at $26^{\circ} \mathrm{C}$ and 1 atm. Indices of refraction from E.R. Hayes, R.A. Schluter, and A. Tamosaitis, ANL-6916 (1964).

o) $n\left(\mathrm{SiO}_{2}\right)+2 n\left(\mathrm{H}_{2} \mathrm{O}\right)$ used in Čerenkov counters, $\rho=$ density in $\mathrm{g} / \mathrm{cm}^{3}$. From $\mathrm{M}$. Cantin et al., Nucl. Instr. Meth. 118, 177 (1974). 


\section{ELECTROMAGNETIC RELATIONS}

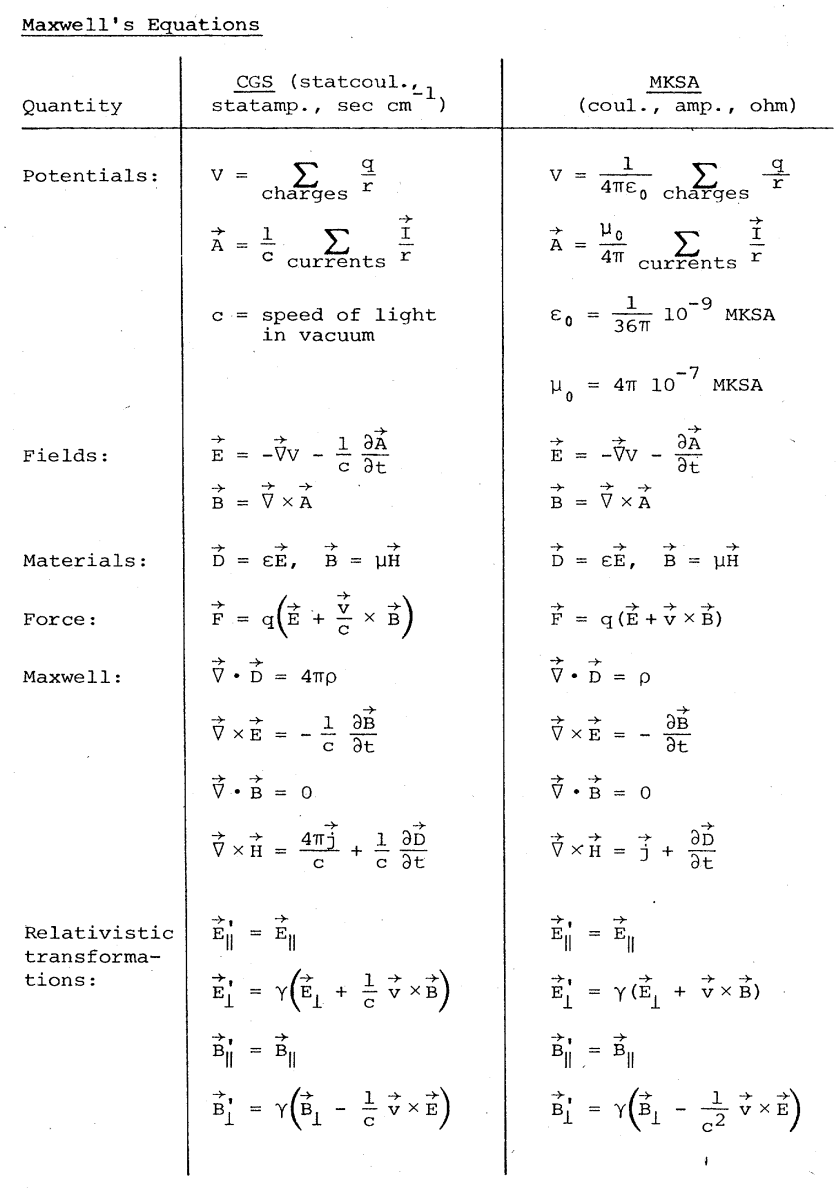

1. Impedance of self-inductance $\mathrm{L}: \mathrm{z}=\mathrm{i} \omega \mathrm{L}$.

2. Impedance of a capacitor of capacitance $C: z=\frac{1}{i \omega C}$.

3. Impedance of a flat conductor of width $w$ at high frequency: $\mathrm{z}=\frac{(1+i) \rho}{w \delta}$

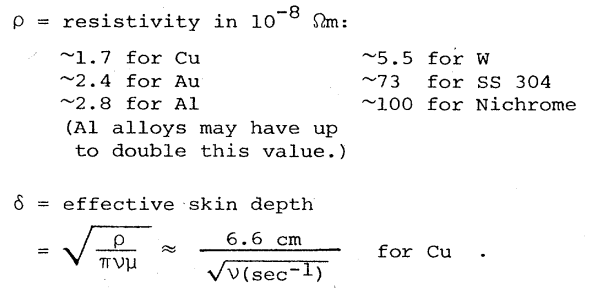

4. Impedance of free space: $z=\sqrt{\mu_{0} / \varepsilon_{0}}=37.6 .7 \Omega$.

Capacitance $C$ and Inductance $L$ per Unit Length (MKSA)

1. For flat plates of width $w$, separated by $d \ll w$ :

$$
\mathrm{C}=\frac{\varepsilon \mathrm{w}}{\mathrm{d}} \quad \mathrm{L}=\mu \frac{\mathrm{d}}{\mathrm{w}} .
$$

2. For coax cable of interior and exterior radii $r_{1}$ and $r_{2}$ :

$$
\begin{aligned}
& C=\frac{2 \pi \varepsilon}{\ln \left(r_{2} / r_{1}\right)} ; \quad L=\frac{\mu}{2 \pi} \ln \left(r_{2} / r_{1}\right) ; \\
& \varepsilon=\text { dielectric constant }\left\{\begin{array}{l}
2 \text { to } 6 \text { for plastics; } \\
4 \text { to } 8 \text { for porcelain, }
\end{array}\right. \\
& \mu=\text { magnetic susceptibility. }
\end{aligned}
$$

Transmission Lines (No LoSS) (MKSA)

Velocity $=1 / \sqrt{L C}=1 / \sqrt{\mu \varepsilon}$.

Impedance $=\sqrt{\mathrm{L} / \mathrm{C}}$.

$\mathrm{L}$ and $\mathrm{C}$ are inductance and capacitance per unit length.

Synchrotron Radiation (CGS)

Energy loss/revolution $=\frac{4 \pi}{3} \frac{e^{2}}{\rho} \beta^{3} \gamma^{4}, \rho=$ orbit radius.

For electrons $(\beta \approx 1), \frac{\Delta \mathrm{E}(\mathrm{MeV})}{r e v .}=0.0885[\mathrm{E}(\mathrm{GeV})]^{4} / \rho$ (meter).

Critical frequency: $\omega_{\mathrm{C}}=3 \gamma^{3} \frac{\mathrm{c}}{\rho}$.

Frequency spectrum (for $\gamma>1$ ):

$$
\begin{aligned}
& I(\omega) \cong 3.3 \frac{\mathrm{e}^{2}}{\mathrm{C}}\left(\frac{\omega p}{\mathrm{c}}\right)^{1 / 3}, \omega<\omega_{C} ; \\
& I(\omega) \cong(1.0,1.6,1.6,0.5,0.08) \frac{\mathrm{e}^{2} \gamma}{\mathrm{C}} \\
& \quad \text { at } \frac{\omega}{\omega_{C}}=0.01,0.1,0.2,1.0,2.0, \text { respectively; } \\
& I(\omega) \cong \sqrt{3 \pi} \frac{\mathrm{e}^{2} \gamma}{\mathrm{C}}\left(\frac{\omega}{\omega_{\mathrm{C}}}\right)^{1 / 2} \mathrm{e}^{-2 \omega / \omega_{\mathrm{C}}}, \omega \gtrsim 2 \omega_{\mathrm{C}} . \\
& \text { The radiation is confined to angles } \lesssim 1 / \gamma \text { relative to } \\
& \text { the instantaneous direction of motion. }
\end{aligned}
$$

See J. D. Jackson, Classical Electrodynamics, 2nd edition
(John Wiley \& Sons, $\frac{\text { New York, 1975) for more formulae and details }}{}$ (Prepared April 1974; revised April 1980.)

\section{RADIOACTIVITY AND RADIATION PROTECTION}

Unit of activity $=$ Curie: $1 \mathrm{Ci}=3.7 \times 10^{10}$ disintegrations $/ \mathrm{sec}$

Unit of exposure dose for $\mathrm{x}$ and $\gamma$ radiation $=$ Roentgen: $1 \mathrm{R}=1 \mathrm{esu} / \mathrm{cm}^{3}=87.8 \mathrm{erg} / \mathrm{g}\left(5.49 \times 10^{7} \mathrm{MeV} / \mathrm{g}\right)$ of air Unit of absorbed dose $=$ rad: $1 \mathrm{rad}=100 \mathrm{erg} / \mathrm{g}\left(6.25 \times 10^{7} \mathrm{MeV} / \mathrm{g}\right)$ in any material Unit of dose equivalent (for protection) $=$ rem: rems (Roentgen equivalents for man) $=\operatorname{rads} \times Q F$, where QF (quality factor) depends upon the type of radiation and other factors. For $\gamma$ rays and $\mathrm{HE}$ protons, $Q F \approx 1$; for thermal neutrons, $Q F \approx 3$; for fast neutrons, $Q F$ ranges up to 10; and for $\alpha$ particles and heavy ions, $Q F$ ranges up to 20 .
Maximum permissible occupational dose for the whole body: $5 \mathrm{rem} /$ year (maximum $3 \mathrm{rem} / \mathrm{calendar}$ quarter)

Fluxes (per $\mathrm{cm}^{2}$ ) to liberate $1 \mathrm{rad}$ in carbon:

$3.5 \times 10^{7}$ minimum ionizing singly charged particles $1.0 \times 10^{9}$ photons of $1 \mathrm{MeV}$ energy (These fluxes are correct to within a factor of 2 for all (These fluxe

materials.)

$\begin{array}{ll}\text { cosmic radiation (charged particles + neutrons) } & \sim 25 \\ \text { cosmic radiation ( } \gamma \text { rays) } & \sim 25\end{array}$

radiation from rocks and air ( $y$ rays)

Cosmic ray background in counters: $\sim 1 / \mathrm{min} / \mathrm{cm}^{2} / \mathrm{ster}$ 


\section{C.M. ENERGY AND MOMENTUM VS. BEAM MOMENTUM}

$$
E_{c m} \mathrm{dE}_{\mathrm{cm}}=\mathrm{m}_{\mathrm{p}} \mathrm{dT}_{\text {beam }}=\mathrm{m}_{\mathrm{p}} \mathrm{v}_{\text {beam }} \mathrm{dP}_{\text {beam }} \approx \mathrm{m}_{\mathrm{p}} \mathrm{dP} \mathrm{P}_{\text {beam }}
$$

\begin{tabular}{|c|c|c|c|c|c|c|c|c|c|c|c|c|c|c|c|c|c|}
\hline \multirow[t]{2}{*}{$\begin{array}{l}\text { PBEAM } \\
(\text { GEV/C) }\end{array}$} & \multicolumn{4}{|c|}{$\begin{array}{c}\text { M. ENERGY----- } \\
\text { (GEV) }\end{array}$} & \multicolumn{4}{|c|}{$\begin{array}{c}\text {-MOMENTUM IN } C . \\
(\text { GEV/C) }\end{array}$} & \multirow[t]{2}{*}{$\mid \begin{array}{c}\text { PBEAM } \\
(\text { GEV/C) }\end{array}$} & $-\cdots-c$ & C. $\underset{\text { MGEV }}{M}$ & $\begin{array}{l}\text { ENERGY- } \\
\text { v) }\end{array}$ & (---- & - MOMEN & TUM & 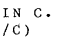 & \\
\hline & $\begin{array}{l}r p, v p \\
\approx e p\end{array}$ & $\pi p$ & $\mathrm{Kp}$ & pp & $\begin{array}{l}\gamma p, v p \\
\approx e p\end{array}$ & $\pi p$ & Kp & $p p$ & & $\begin{array}{l}\gamma_{p}, \nu_{p} \\
\approx e p\end{array}$ & $\pi p$ & $\mathrm{Kp}$ & $\mathrm{pP}$ & $\begin{array}{l}r p, v_{p} \\
\approx \mathrm{ep}\end{array}$ & $p$ & $\mathrm{kp}$ & $p p$ \\
\hline $\begin{array}{l}0.00 \\
0.02\end{array}$ & $\begin{array}{r}.938 \\
.958\end{array}$ & 1.078 & $\begin{array}{l}1.4321 \\
\end{array}$ & 77 & .000 & .000 & .000 & .000 & $\begin{array}{r}1.70 \\
1.72\end{array}$ & $\begin{array}{l}2.0182 \\
2.0027\end{array}$ & 2.0252 & 2.1092 & 5 & .791. & $\begin{array}{r}.788 \\
.793\end{array}$ & 56. & .686 \\
\hline $\begin{array}{l}0.02 \\
0.04\end{array}$ & $\begin{array}{r}958 \\
.977\end{array}$ & $\begin{array}{l}1.079 \\
1.083\end{array}$ & $\begin{array}{l}1.432 \\
1.433\end{array}$ & $\begin{array}{l}1.87 \\
1.87\end{array}$ & $\begin{array}{l}.020 \\
.038\end{array}$ & .011 & $\begin{array}{r}.013 \\
.026\end{array}$ & & $\begin{array}{l}1.72 \\
1.74\end{array}$ & $\begin{array}{l}2.0272 \\
2.0362\end{array}$ & $\begin{array}{l}2.034 \\
2.0432\end{array}$ & $\begin{array}{l}2.1172 \\
2.1262\end{array}$ & 12 & $\begin{array}{r}796 \\
.802\end{array}$ & $\begin{array}{r}793 \\
.799 \\
\end{array}$ & $\begin{array}{l}762 \\
.768\end{array}$ & $\begin{array}{r}.692 \\
.698\end{array}$ \\
\hline 0.06 & .996 & $\begin{array}{l}1.083 \\
1.089\end{array}$ & $\begin{array}{l}1.433 \\
1.434\end{array}$ & $\begin{array}{l}1.87 \\
1.878\end{array}$ & .056 & .053 & $\begin{array}{l}.026 \\
.039\end{array}$ & $\begin{array}{l}.020 \\
.030\end{array}$ & $\begin{array}{l}1.74 \\
1.76\end{array}$ & 2.0452 & 2.0435 & $\begin{array}{l}1342 \\
2.134\end{array}$ & 6 & . & & .774 & .704 \\
\hline 0.08 & 1.015 & 1.096 & 1.4361 & 1 . & .07 .4 & .068 & & .04 & 1.78 & 2.0542 & 622 & 43 & .353 & .813 & .810 & .780 & 10 \\
\hline & & & 439 & & .09 & 085 & & & 1.8 & 2.0642 & 2.0712 & 2.1512 & 2.360 & .818 . & .816 & .785 & .716 \\
\hline & 1.051 & & & & . & & & & & & & & & & & & .721 \\
\hline & $\begin{array}{l}1.069 \\
1.087\end{array}$ & .127 & 1.4451 & 1.882 & .123 & .117 & .09 & .0 & & & & & & & & & \\
\hline $\begin{array}{l}0.16 \\
0.18\end{array}$ & $\begin{array}{l}1.087 \\
1.104\end{array}$ & $\begin{array}{l}1.139 \\
1.152\end{array}$ & $\begin{array}{l}1.4481 \\
1.4531\end{array}$ & $\begin{array}{l}1.883 \\
1.885\end{array}$ & $\begin{array}{l}138 \\
.153\end{array}$ & $\begin{array}{l}.132 \\
.147\end{array}$ & $\begin{array}{r}.104 \\
.116\end{array}$ & $\begin{array}{l}.080 \\
.090\end{array}$ & $\begin{array}{l}1.86 \\
1.88\end{array}$ & $\begin{array}{l}2.0912 \\
2.1002\end{array}$ & $\begin{array}{l}2.098 \\
2.107\end{array}$ & $\begin{array}{l}2.1762 \\
2.184\end{array}$ & $\begin{array}{l}2.381 \\
2.388\end{array}$ & $\begin{array}{l}.835 \\
.840\end{array}$ & $\begin{array}{l}.832 \\
.837\end{array}$ & $\begin{array}{l}.802 \\
.808\end{array}$ & $\begin{array}{r}733 \\
.739\end{array}$ \\
\hline .20 & 121 & .165 & 1.4571 & 87 & .167 & .161 & .129 & 099 & 1.90 & & & & & & & & .744 \\
\hline 0.22 & $\begin{array}{l}1.137 \\
\end{array}$ & 1.178 & & $\begin{array}{l}1.889 \\
1.889\end{array}$ & .182 & .175 & .141 & .109 & 1.92 & 2.1172 & & & 2.402 & .851. & .848 & .819 & .750 \\
\hline $\begin{array}{l}0.24 \\
0.26\end{array}$ & 1.154 & 1.192 & 1.468 & 1.892 & .195 & .189 & .153 & .119 & 1.94 & 2.1262 & 2.1332 & 2.2092 & 2.409 & .856. & .853 & .824 & .756 \\
\hline $\begin{array}{l}0.26 \\
0.28\end{array}$ & $\begin{array}{l}1.170 \\
1.186\end{array}$ & $\begin{array}{l}1.206 \\
1.219\end{array}$ & $\begin{array}{l}1.473 \\
1.480\end{array}$ & $\begin{array}{l}1.894 \\
1.897\end{array}$ & $\begin{array}{r}209 \\
.222\end{array}$ & $\begin{array}{l}.202 \\
.215\end{array}$ & $\begin{array}{l}.166 \\
.178\end{array}$ & $\begin{array}{l}.129 \\
.138\end{array}$ & $\begin{array}{l}1.96 \\
1.98\end{array}$ & $\begin{array}{l}2.1352 \\
2.1442\end{array}$ & $\begin{array}{l}2.142 \\
2.151\end{array}$ & $\begin{array}{ll}2.2177 \\
2.226 & 2\end{array}$ & $\begin{array}{l}2.416 \\
2.423\end{array}$ & $\begin{array}{l}.861 \\
.867\end{array}$ & $\begin{array}{l}.859 \\
.864\end{array}$ & $\begin{array}{l}.829 \\
.835\end{array}$ & $\begin{array}{l}.761 \\
.767\end{array}$ \\
\hline 0.30 & 1.201 & 1.233 & 1.4861 & 1.900 & .234 & .228 & .189 & .148 & 2.0 & 2.1532 & 2.1592 & 2.2342 & 2.430 & $.872^{\circ}$ & .869 & .840 & .772 \\
\hline 0.32 & 1.217 & 1.247 & 1.4931 & 1.903 & .247 & .241 & .201 & .158 & & 2.1962 & 2.2022 & 2.2742 & & .897. & .895 & .866 & .799 \\
\hline 0.34 & 1.232 & 1.261 & 1.5 & 1.9 & .259 & .253 & .2 & & & 2.2 & & & & .922. & .920 & .892 & $\begin{array}{r}.826 \\
8552\end{array}$ \\
\hline $\begin{array}{l}0.36 \\
0.38\end{array}$ & $\begin{array}{l}1.247 \\
1.262\end{array}$ & $\begin{array}{l}1.275 \\
1.288\end{array}$ & $\begin{array}{l}1.507 \\
1.514\end{array}$ & $\begin{array}{l}1.910 \\
1.913\end{array}$ & $\begin{array}{l}271 \\
.282\end{array}$ & $\begin{array}{l}.265 \\
.277\end{array}$ & $\begin{array}{l}.224 \\
.235\end{array}$ & $\begin{array}{l}.177 \\
.186\end{array}$ & $\begin{array}{l}2.3 \\
2.4\end{array}$ & $\begin{array}{l}2.280 \\
2.320 \\
2.32\end{array}$ & $\begin{array}{l}2.286 \\
2.326\end{array}$ & $\begin{array}{l}2.353 \\
2.3922\end{array}$ & $\begin{array}{l}2.534 \\
2.568\end{array}$ & $\begin{array}{r}947 \\
.970\end{array}$ & $\begin{array}{l}.944 \\
.968\end{array}$ & $\begin{array}{l}917 \\
.941\end{array}$ & $\begin{array}{l}.852 \\
.877\end{array}$ \\
\hline 0.40 & 277 & 302 & 1.522 & 1.917 & .294 & 288 & 24 & .196 & 2.5 & 2.36 & .366 & .43 & 602 & .994 & .991 & .96 & 01 \\
\hline 0.42 & 1.292 & 1.315 & 1.5301 & & .305 & .300 & .258 & & 6 & 2.4002 & 2.405 & & & 1.021 & 1.01 & .98 & \\
\hline 0.44 & 1.306 & 1.329 & 1.5 & 1.925 & .316 & .311 & .268 & .214 & & & & & & & & & \\
\hline 0.4 & 1. & 1.342 & 1.5461 & 1.929 & .327 & .322 & .279 & & 8 & & 22 & 22 & & & 6 & 1.03 & .972 \\
\hline 0.48 & 1.335 & 1.356 & $1.554 \mathrm{1}$ & 1.934 & .337 & .332 & .290 & .233 & 2.9 & 2.5142 & 2.5202 & 2.5782 & 2.735 & 1.081 & 1.08 & 1.06 & .995 \\
\hline $\begin{array}{l}0.50 \\
0.52\end{array}$ & $\begin{array}{l}1.349 \\
1.362\end{array}$ & $\begin{array}{l}1.369 \\
1.382\end{array}$ & 1.5631 & 1.938 & .348 & .343 & .300 & .242 & 3.0 & 2.5512 & 2 & 2.6132 & & 01 & 1 & ( & \\
\hline 0.54 & $\begin{array}{l}1.362 \\
1.376\end{array}$ & $\begin{array}{l}1.382 \\
1.395\end{array}$ & & & .358 & .353 & . & & 3. & 2.5882 & & & & & & & \\
\hline 0. & 1.390 & $\begin{array}{l}1.408 \\
\text {. }\end{array}$ & $\begin{array}{l}1.5801 \\
1.5899\end{array}$ & $\begin{array}{l}1.947 \\
1.952\end{array}$ & $\begin{array}{l}.368 \\
.378\end{array}$ & $\begin{array}{l}.3673 \\
.373\end{array}$ & .331 & .269 & 3.3 & 2.6602 & $\begin{array}{l}2.064 \\
2.664\end{array}$ & $\begin{array}{l}.000 \\
2.7181\end{array}$ & 3 & $\begin{array}{l}1.14 \\
1.16\end{array}$ & $\begin{array}{l}1.14 \\
1.16\end{array}$ & $\begin{array}{l}1.12 \\
1.14\end{array}$ & $\begin{array}{l}1.06 \\
1.08\end{array}$ \\
\hline 0.58 & 1.403 & 1.421 & 1.5981 & 1.957 & .388 & .383 & .341 & .278 & 3.4 & 2.6952 & 2.699 & 2.7522 & 2.895 & 1.181 & 1.18 & 1.16 & 1.10 \\
\hline $\begin{array}{l}0.60 \\
0.62\end{array}$ & 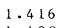 & 4 & 1.60 & 96 & .397 & .393 & .350 & .287 & 3.5 & 2.7292 & 2.7342 & 52 & 2.926 & 1.201 & 1. & 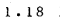 & 1.12 \\
\hline $\begin{array}{l}0.6 \\
0.6\end{array}$ & & & & & .407 & .4 & & & & & & & & & & & \\
\hline $\begin{array}{l}0.6 \\
0.6\end{array}$ & $\begin{array}{l}1.443 \\
1.456\end{array}$ & $\begin{array}{l}1.459 \\
1.472\end{array}$ & $\begin{array}{l}1.6251 \\
1.6344\end{array}$ & $\begin{array}{l}1.973 \\
1.978\end{array}$ & $\begin{array}{r}.416 \\
.425\end{array}$ & $\begin{array}{r}4421 \\
.421\end{array}$ & $\begin{array}{l}3770 \\
.379\end{array}$ & $\begin{array}{l}.304 \\
.313\end{array}$ & $\begin{array}{l}3.7 \\
3.8\end{array}$ & $\begin{array}{l}2.7972 \\
2.8302\end{array}$ & $\begin{array}{l}2.801 \\
2.835\end{array}$ & $\begin{array}{l}2.851 \\
2.884\end{array}$ & $\begin{array}{l}7 \\
8\end{array}$ & $\begin{array}{ll}4 & 1 \\
6 & 1\end{array}$ & 4 & & 1.16 \\
\hline 0.58 & 1.468 & 1.484 & 1.6431 & 1.984 & .434 & .430 & .388 & .322 & $\begin{array}{l}3.8 \\
3.9\end{array}$ & 2.8632 & 2.868 & $\begin{array}{l}.8843 \\
2.9163\end{array}$ & 3.048 & $\begin{array}{l}1.20 \\
1.28\end{array}$ & $\begin{array}{l}1.20 \\
1.28\end{array}$ & $\begin{array}{l}1.24 \\
1.26\end{array}$ & 1.20 \\
\hline 0.70 & 1. & 1.4 & 1.6531 & 1.989 & 3 & .439 & .397. & .3 & 4.0 & 62 & 02 & 2 & 7 & 01 & 1 & 1 & 1.22 \\
\hline $\begin{array}{l}0.72 \\
0.74\end{array}$ & $\begin{array}{l}1.494 \\
1.506\end{array}$ & $\begin{array}{r}1.509 \\
+521\end{array}$ & & 1. & $\begin{array}{r}.452 \\
461\end{array}$ & & & & 4 & $\begin{array}{l}2.9 \\
2.9\end{array}$ & & & & & & & \\
\hline 0.76 & $\begin{array}{l}1.506 \\
1.519\end{array}$ & $\begin{array}{l}1.521 \\
1.533\end{array}$ & $\begin{array}{l}1.6712 \\
1.6812\end{array}$ & $\begin{array}{l}2.00 \\
2.00\end{array}$ & $\begin{array}{r}-461 \\
.470\end{array}$ & $\begin{array}{r}.457 \\
.465\end{array}$ & $\begin{array}{l}415 \\
.424\end{array}$ & $: 34$ & $\begin{array}{l}4 . \\
4 .\end{array}$ & $\begin{array}{l}2.96020 \\
2.992\end{array}$ & $\begin{array}{l}2.964 \\
2.996\end{array}$ & $\begin{array}{l}3.010 \\
3.041\end{array}$ & $\begin{array}{l}6 \\
5 \\
5\end{array}$ & $\begin{array}{l}1.333 \\
1.35\end{array}$ & $\begin{array}{l}1.33 \\
1.35\end{array}$ & 3 & $\begin{array}{l}1.26 \\
1.27\end{array}$ \\
\hline 0.78 & 1.531 & 1.545 & 1.6902 & 2.013 & .478 & .474 & .433 & .364 & 4.4 & 3.023 & 3.027 & 3.0713 & 3.194 & 1.37 & $\begin{array}{l}1.36 \\
1.36\end{array}$ & $\begin{array}{l}1.34 \\
1.34\end{array}$ & 1.29 \\
\hline 0.80 & 1. & 1.5 & 1.6992 & 2.0 & .486 & .482 & & & 4. & & & & & & & & 31 \\
\hline & & & & & & & & & & & & & & & & & \\
\hline $\begin{array}{l}0.84 \\
0.86\end{array}$ & $\begin{array}{l}1.567 \\
1.579\end{array}$ & $\begin{array}{l}1.580 \\
1.592\end{array}$ & $\begin{array}{l}1.718 \\
1.772\end{array}$ & $\begin{array}{l}2.031 \\
2.037\end{array}$ & $\begin{array}{r}503 \\
.511\end{array}$ & $\begin{array}{l}.499 \\
.507\end{array}$ & $\begin{array}{l}.459 \\
.467\end{array}$ & $\begin{array}{r}388 \\
.396\end{array}$ & $\begin{array}{l}4.7 \\
4.8\end{array}$ & $\begin{array}{l}3.115 \\
3.144\end{array}$ & $\begin{array}{l}3.118 \\
3.148\end{array}$ & $\begin{array}{l}3.1613 \\
3.1903\end{array}$ & $\begin{array}{l}99 \\
77\end{array}$ & $\begin{array}{l}1.42 \\
1.433\end{array}$ & $\begin{array}{l}1.41 \\
1.43\end{array}$ & $\begin{array}{l}0 \\
1 \\
1\end{array}$ & $\begin{array}{l}1.34 \\
1.36\end{array}$ \\
\hline 0.88 & 1.591 & 1.604 & 1.737 & 2.043 & .519 & .515 & .475 & .404 & $\begin{array}{l}4.9 \\
4.9\end{array}$ & 3.1743 & $\begin{array}{l}3.148 \\
3.178\end{array}$ & 3.2203 & $\begin{array}{l}3.307 \\
3.335\end{array}$ & $\begin{array}{l}1.43 \\
1.451\end{array}$ & $\begin{array}{l}1.43 \\
1.45\end{array}$ & $\begin{array}{l}1.41 \\
1.43\end{array}$ & $\begin{array}{l}1.36 \\
1.38\end{array}$ \\
\hline 0.90 & 1. & 1.615 & 1.7472 & 2. & .52 & .523 & .484 & .4 & 5.0 & 3. & & & & 61 & 1 & 1 & 1 \\
\hline 0.9 & & 1.6 & & & .5 & & .4 & & & & & & & & & & \\
\hline & 1. & 1.6 & & & .542 & & .500 & & & & & & & & & & \\
\hline $\begin{array}{l}0.96 \\
0.98\end{array}$ & $\begin{array}{l}1.638 \\
1.649\end{array}$ & $\begin{array}{l}1.649 \\
1.661\end{array}$ & $\begin{array}{l}1.775 \\
1.784\end{array}$ & $\begin{array}{l}2.069 \\
2.075\end{array}$ & $\begin{array}{l}.550 \\
.558\end{array}$ & $\begin{array}{l}.546 \\
.554\end{array}$ & $\begin{array}{l}.508 \\
.515\end{array}$ & $\begin{array}{l}.435 \\
.443\end{array}$ & $\begin{array}{l}5.6 \\
5.8\end{array}$ & $\begin{array}{l}3.375 \\
3.430 \\
3\end{array}$ & $\begin{array}{l}3.378 \\
3.433\end{array}$ & $\begin{array}{r}3.417 \\
3.471\end{array}$ & $\begin{array}{l}3.524 \\
3.576\end{array}$ & $\begin{array}{l}1.56 \\
1.59\end{array}$ & $\begin{array}{l}1.56 \\
1.59\end{array}$ & $\begin{array}{l}1.54 \\
1.57\end{array}$ & $\begin{array}{l}1.49 \\
1.52\end{array}$ \\
\hline 1.00 & 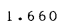 & 2 & 1. & 2. & .565 & .5 & .523 & .4 & 6.0 & 3.4843 & 3. & 3. & 3. & 1.621 & 1.61 & 1.60 & 1. \\
\hline & & & & & & & & & & & & & & & & & \\
\hline 1. & 1. & $\begin{array}{l}1.694 \\
1.705\end{array}$ & 1. & 2. & $\begin{array}{r}.580 \\
587\end{array}$ & $\begin{array}{l}.576 \\
5833\end{array}$ & $\begin{array}{l}.538 \\
5546\end{array}$ & .4 & $\begin{array}{l}6.4 \\
6.6\end{array}$ & 3. & & & & $\begin{array}{ll}7 \\
0 \\
0 & 1\end{array}$ & $\begin{array}{l}1.67 \\
1.70\end{array}$ & $\begin{array}{l}55 \\
58\end{array}$ & $\begin{array}{l}1.61 \\
1.664\end{array}$ \\
\hline $\begin{array}{l}1.00 \\
1.08\end{array}$ & $\begin{array}{l}1.094 \\
1.705\end{array}$ & $\begin{array}{l}1.703 \\
1.716\end{array}$ & $\begin{array}{l}1.831 \\
1.831\end{array}$ & 2.108 & .594 & .591 & .553 & .481 & $\begin{array}{l}6.6 \\
6.8\end{array}$ & $\begin{array}{l}3.642 \\
3.693\end{array}$ & $\begin{array}{l}3.643 \\
3.696\end{array}$ & 3.7313 & 3.827 & $\begin{array}{l}1.70 \\
1.73\end{array}$ & 1.73 & $\begin{array}{l}1.08 \\
1.71\end{array}$ & $\begin{array}{l}1.04 \\
1.67\end{array}$ \\
\hline & & 1.726 & 2 & 2 & .601 & .5 & .5 & .4 & 7.0 & 3. & 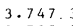 & 13 & 3. & 5 & 1 & 1. & 70 \\
\hline & & & & & & & & & & & & & & & & & \\
\hline $1:$ & $\begin{array}{l}1.7 \\
1.7\end{array}$ & 1.748 & 1.8592 & $2: 1$ & $\begin{array}{r}616 \\
.622\end{array}$ & .612 & .575 & .5 & 7.4 & 3.8 & 6 & & & & 1.81 & 1.79 & 1.7 \\
\hline 1.18 & $\begin{array}{l}1.748 \\
1.759\end{array}$ & $\begin{array}{l}1.739 \\
1.769\end{array}$ & $\begin{array}{l}1.808 \\
1.877\end{array}$ & $\begin{array}{l}2.130 \\
2.142\end{array}$ & .629 & .0626 & .590 & .517 & 7.8 & 3.939 & 3.942 & $\begin{array}{l}3.9264 \\
3.9744\end{array}$ & $\begin{array}{l}4.016 \\
4.062\end{array}$ & $\begin{array}{l}1.83 \\
1.86\end{array}$ & $\begin{array}{l}1.86 \\
1.86\end{array}$ & $\begin{array}{l}1.82 \\
1.84\end{array}$ & $\begin{array}{l}1.80 \\
\text {. }\end{array}$ \\
\hline 1.20 & 0 & 0 & 1. & & .636 & & .5 & & & & & & & & & & \\
\hline & & & & & & & & & & & & & & & & & \\
\hline & $\begin{array}{l}1 . \\
1: 8\end{array}$ & $\begin{array}{l}1.800 \\
1.811\end{array}$ & $\begin{array}{l}1.905 \\
1.914\end{array}$ & $\begin{array}{l}2.163 \\
2.170\end{array}$ & $\begin{array}{r}.650 \\
.656\end{array}$ & $\begin{array}{l}.646 \\
.6533\end{array}$ & $\begin{array}{l}.611 \\
.618\end{array}$ & $\begin{array}{l}.5 \\
.5\end{array}$ & 8 & & & & & & & & \\
\hline 1.28 & $\begin{array}{l}1.812 \\
\end{array}$ & 1.821 & 1.923 & 2.177 & .663 & .660 & $\begin{array}{l}.010 \\
.624\end{array}$ & .552 & $\begin{array}{l}8.6 \\
8.8\end{array}$ & $\begin{array}{l}4.125 \\
4.171\end{array}$ & 3 & $\begin{array}{l}4.158 \\
4.203\end{array}$ & $\begin{array}{l}4.242 \\
4.286\end{array}$ & $\begin{array}{l}1.96 \\
1.98\end{array}$ & $\begin{array}{l}1.95 \\
1.998\end{array}$ & $\begin{array}{l}1.94 \\
1.96\end{array}$ & 1. \\
\hline & & & 1 & & & & & & & & & & & & & & \\
\hline & & & & & & & & & & & & & & & & & \\
\hline 1. & & & 1.9 & 2.1 & & .6 & $\begin{array}{r}.645 \\
6651\end{array}$ & & & & & & & & & & \\
\hline $\begin{array}{l}1.36 \\
1.38\end{array}$ & $\begin{array}{l}1.853 \\
1.863\end{array}$ & $\begin{array}{l}1.862 \\
1.872\end{array}$ & $\begin{array}{l}1.959 \\
1.968\end{array}$ & $\begin{array}{l}2.205 \\
2.212\end{array}$ & $\begin{array}{r}689 \\
.695\end{array}$ & $\begin{array}{l}.685 \\
.692\end{array}$ & $\begin{array}{l}.651 \\
.658\end{array}$ & & & & & & & & & & \\
\hline & & & & & & & & & .8 & 4. & 4.392 & 4.420 & & & & & \\
\hline & & & & & & & & & & & & & & & & $\begin{array}{l}2.10 \\
2.16\end{array}$ & $\begin{array}{l}2.07 \\
2.12\end{array}$ \\
\hline & & & & & & & & & & & & & & & & & \\
\hline & & i. & 2. & $=$ & & & & & & & & & & 8 & 8 & 2.26 & \\
\hline 1.4 & 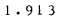 & 1.921 & 2.0132 & 2.247 & .720 & $\because$ & . & & & 4.837 & 4.839 & 4.864 & 4.934 & 2.33 & 2.33 & 2.31 & 2.28 \\
\hline & & & & & & & & & & & 5 & 4. & 5 & 38 & 3 & , & 2 \\
\hline & & & & & & & & & & & & & & & & & \\
\hline & & 1.9 & 2.0 & $\begin{array}{l}2.2 \\
2.2\end{array}$ & $\begin{array}{l}.744 \\
.750\end{array}$ & $\begin{array}{r}.741 \\
747\end{array}$ & $\begin{array}{l}.709 \\
7.15\end{array}$ & $\begin{array}{l}.6 \\
.6\end{array}$ & $\begin{array}{l}13.5 \\
14.0\end{array}$ & $\begin{array}{l}5.120 \\
5.211\end{array}$ & $\begin{array}{l}5.122 \\
5.213\end{array}$ & $\begin{array}{l}5.145 \\
5.236\end{array}$ & & $\begin{array}{l}2.47 \\
2.52\end{array}$ & $\begin{array}{l}2.47 \\
2.52\end{array}$ & $\begin{array}{l}2.46 \\
2.51\end{array}$ & $\begin{array}{l}2.43 \\
2.48\end{array}$ \\
\hline $\begin{array}{l}1.56 \\
1.58\end{array}$ & $\begin{array}{l}1.931 \\
1.961\end{array}$ & $\begin{array}{l}1.959 \\
1.969\end{array}$ & $\begin{array}{l}2.048 \\
2.057\end{array}$ & $\begin{array}{l}2.285 \\
2.282\end{array}$ & .756 & .753 & .721 & $\begin{array}{l}.043 \\
.650\end{array}$ & $\begin{array}{l}14 \\
14\end{array}$ & 5.300 & 5.302 & 5.324 & 5.388 & 2.57 & 2.57 & 2.56 & $\begin{array}{l}2.40 \\
2.53\end{array}$ \\
\hline & & & & & & & & & & & & & & & & & \\
\hline & & & & & .76 & & .7 & & & & & & & & & & \\
\hline & & . & & & .7 & 7 & 7 & & & & & & & 2.70 & $\begin{array}{l}2.70 \\
2.74\end{array}$ & 2.69 & 2.66 \\
\hline $\begin{array}{l}1.66 \\
1.68\end{array}$ & $\begin{array}{l}1.999 \\
2.008\end{array}$ & $\begin{array}{l}2.006 \\
2.016\end{array}$ & $\begin{array}{l}2.091 \\
2.100\end{array}$ & $\begin{array}{l}2.311 \\
2.318\end{array}$ & .785 & $\begin{array}{l}.776 \\
.782\end{array}$ & $\begin{array}{l}.745 \\
.751\end{array}$ & $\begin{array}{l}.674 \\
.680\end{array}$ & $\begin{array}{l}16.5 \\
17.0\end{array}$ & $\begin{array}{l}5.643 \\
5.726\end{array}$ & $\begin{array}{l}5.645 \\
5.727\end{array}$ & $\begin{array}{l}5.666 \\
5.748\end{array}$ & $\begin{array}{l}5.725 \\
5.806\end{array}$ & $\begin{array}{l}2.74 \\
2.79\end{array}$ & $\begin{array}{l}2.74 \\
2.79\end{array}$ & $\begin{array}{l}2.73 \\
2.78\end{array}$ & 2.75 \\
\hline
\end{tabular}

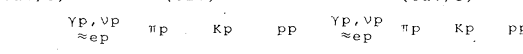
$\begin{array}{lllllllll}17.5 & 5.807 & 5.809 & 5.829 & 5.886 & 2.83 & 2.83 & 2.82 & 2.79 \\ 18.0 & 5.887 & 5.889 & 5.909 & 5.965 & 2.87 & 2.87 & 2.86 & 2.83\end{array}$ $\begin{array}{llllllllll}18.5 & 5.966 & 5.968 & 5.988 & 6.043 & 2.91 & 2.91 & 2.90 & 2.87 \\ 19.0 & 6.044 & 6.046 & 6.066 & 6.120 & 2.95 & 2.95 & 2.94 & 2.91\end{array}$

$\begin{array}{lllllllll}20 & 6.198 & 6.199 & 6.218 & 6.272 & 3.03 & 3.03 & 3.02 & 2.99\end{array}$

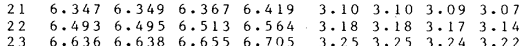

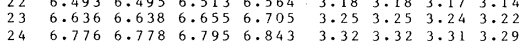
$\begin{array}{lllllllll}25 & 6.913 & 6.915 & 6.932 & 6.979 & 3.39 & 3.39 & 3.38 & 3.36\end{array}$

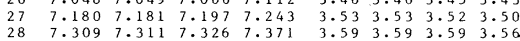

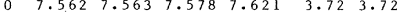
$\begin{array}{lllllllll}32 & 7.806 & 7.807 & 7.822 & 7.864 & 3.85 & 3.785 & 3.84 & 3.86\end{array}$ $\begin{array}{lllllllll}34 & 8.043 & 8.044 & 8.058 & 8.099 & 3.97 & 3.97 & 3.90 & 3.88\end{array}$ $\begin{array}{lllllllllll}35 & 8.158 & 8.160 & 8.174 & 8.214 & 4.03 & 4.02 & 4.02 & 4.00\end{array}$

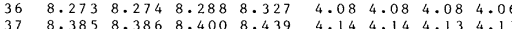

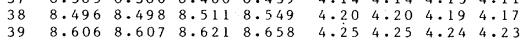

$\begin{array}{lllllllll}40 & 8.715 & 8.716 \quad 8.729 & 8.766 & 4.31 & 4.31 & 4.30 & 4.28\end{array}$

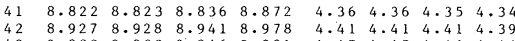

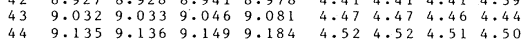

$\begin{array}{lllllllll}45 & 9.237 & 9.238 & 9.251 & 9.286 & 4.57 & 4.57 & 4.56 & 4.55 \\ 46 & 9.338 & 9.339 & 9.352 & 9.386 & 4.62 & 4.62 & 4.62 & 4.60\end{array}$ $\begin{array}{lllllllll}47 & 9.438 & 9.439 & 9.451 & 9.486 & 4.67 & 4.67 & 4.677 & 4.65 \\ 48 & 9.537 & 9.538 & 9.550 & 9.584 & 4.72 & 4.772 & 4.72 & 4.70 \\ 49 & 9.635 & 9.636 & 9.648 & 9.681 & 4.77 & 4.77 & 4.77 & 4.75\end{array}$ $\begin{array}{lllllllll}50 & 9.732 & 9.733 & 9.745 & 9.778 & 4.82 & 4.82 & 4.81 & 4.80\end{array}$

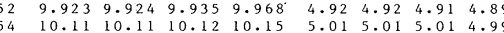

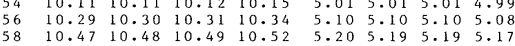
$\begin{array}{lllllllll}60 & 10.65 & 10.65 & 10.66 & 10.69 & 5.28 & 5.28 & 5.28 & 5.26\end{array}$

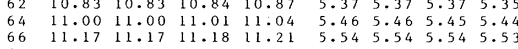
$\begin{array}{lllllllll}70 & 11.50 & 11.50 & 11.51 & 11.54 & 5.71 & 5.71 & 5.71 & 5.69\end{array}$

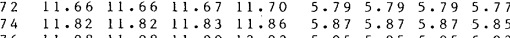
$\begin{array}{lllllllll}76 & 11.98 & 11.98 & 11.99 & 12.82 & 5.95 & 5.95 & 5.95 & 5.93 \\ 78 & 12.13 & 12.14 & 12.14 & 12.17 & 6.03 & 6.03 & 6.03 & 6.01\end{array}$

$\begin{array}{llllllllll}80 & 12.29 & 12.29 & 12.30 & 12.32 & 6.11 & 6.11 & 6.10 & 6.09\end{array}$

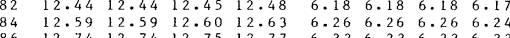
$\begin{array}{lllllllll}84 & 12.59 & 12.59 & 12.60 & 12.63 & 6.26 & 6.26 & 6.26 & 6.24 \\ 86 & 12.74 & 12.74 & 12.75 & 12.77 & 6.33 & 6.33 & 6.33 & 6.32 \\ 88 & 12.88 & 12.89 & 12.89 & 12.92 & 6.41 & 6.41 & 6.40 & 6.39\end{array}$

$\begin{array}{llllllllll}90 & 13.03 & 13.03 & 13.04 & 13.06 & 6.48 & 6.48 & 6.48 & 6.46\end{array}$ $\begin{array}{lllllllll}90 & 13.03 & 13.03 & 13.04 & 13.06 & 6.48 & 6.48 & 6.48 & 6.46 \\ 94 & 13.31 & 13.17 & 13.18 & 13.21 & 6.55 & 6.55 & 6.555 & 6.54 \\ 9 & 13.32 & 13.35 & 6.62 & 6.622 & 6.62 & 6.61\end{array}$

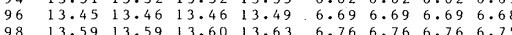

$\begin{array}{lllllllll}00 & 13.73 & 13.73 & 13.74 & 13.76 & 6.83 & 6.83 & 6.83 & 6.82\end{array}$ $\begin{array}{lllllllll}150 & 16.80 & 16.80 & 16.81 & 16.83 & 8.38 & 8.38 & 8.37 & 8.36 \\ 200 & 19.40 & 19.40 & 19.40 & 19.42 & 9.68 & 9.67 & 9.67 & 9.66\end{array}$

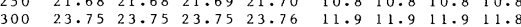
$\begin{array}{lllllllll}350 & 25.65 & 25.65 & 25.65 & 25.66 & 12.8 & 12.8 & 12.8 & 12.8\end{array}$ $\begin{array}{lllllllll}450 & 29.07 & 29.07 & 29.08 & 29.09 & 14.5 & 14.5 & 14.5 & 14.5 \\ 500 & 30.65 & 30.65 & 30.65 & 30.66 & 15.3 & 15.3 & 15.3 & 15.3 \\ 550 & 32.14 & 32.14 & 32.14 & 32.15 & 16.1 & 16.1 & 16.1 & 16.0\end{array}$ $\begin{array}{llllllllll}600 & 33.57 & 33.57 & 33.57 & 33.58 & 16.8 & 16.8 & 16.8 & 16.8\end{array}$ $\begin{array}{llllllllll}650 & 34.94 & 34.94 & 34.94 & 34.95 & 17.5 & 17.5 & 17.5 & 17.4 \\ 700 & 36.26 & 36.26 & 36.26 & 36.27 & 18.1 & 18.1 & 18.1 & 18.1\end{array}$

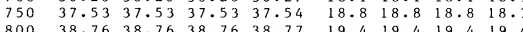

$\begin{array}{lllllllll}850 & 39.95 & 39.95 & 39.95 & 39.96 & 20.0 & 20.0 & 20.0 & 20.0\end{array}$ $\begin{array}{lllllllll}900 & 41.11 & 41.11 & 41.11 & 41.12 & 20.5 & 20.5 & 20.5 & 20.5 \\ 950 & 42.23 & 42.23 & 42.24 & 42.24 & 21.1 & 21.1 & 21.1 & 21.1\end{array}$

$\begin{array}{llllllllll}1000 & 43.33 & 43.33 & 43.33 & 43.34 & 21.7 & 21.7 & 21.7 & 21.6 \\ 2000 & 61.27 & 61.27 & 61.27 & 61.28 & 30.6 & 30.6 & 30.6 & 30.6\end{array}$ $5000 \quad 96.87 \quad 96.87 .96 .87 \quad 96.87 \quad 48.4 \quad 48.4 \% 48.4 \quad 48.4$ $\begin{array}{lllllllll}10000 & 137.0 & 137.0 & 137.0 & 137.0 & 68.5 & 68.5 & 68.5 & 68.5 \\ 20000 & 193.7 & 193.7 & 193.7 & 193.7 & 96.9 & 96.9 & 96.9 & 96.9\end{array}$ $\begin{array}{rrrrrrrrr}50000 & 306.3 & 306.3 & 306.3 & 306.3 & 153 & 153 & 153 & 153 \\ 100000 & 433.2 & 433.2 & 433.2 & 433.2 & 217 & 217 & 217 & 217\end{array}$

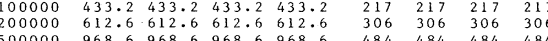
$\begin{array}{rrrrrrrrr}500000 & 968.6 & 968.6 & 968.6 & 968.6 & 484 & 484 & 484 & 484 \\ 1000000 & 1370 & 1370 & 1370 & 1370 & 685 & 685 & 685 & 685\end{array}$ 
Particle Data Group: Review of particle properties

PERIODIC TABLE OF THE ELEMENTS

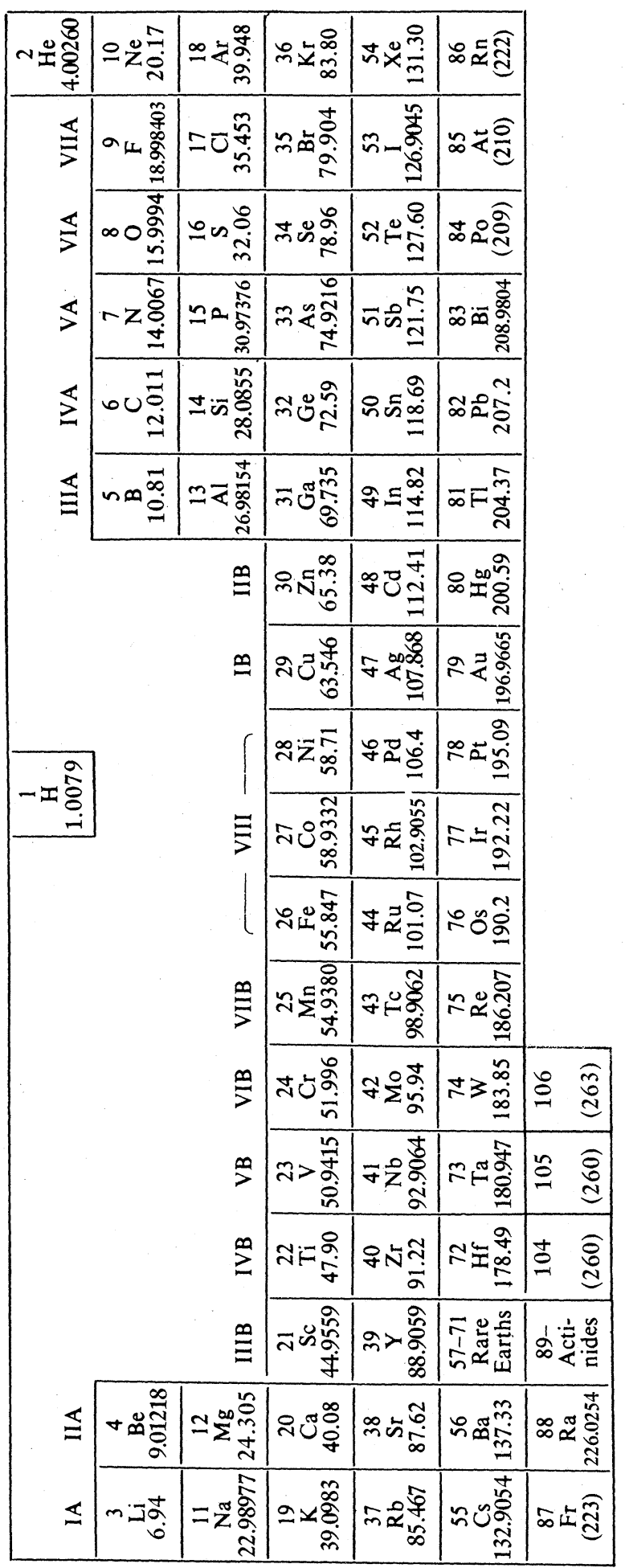

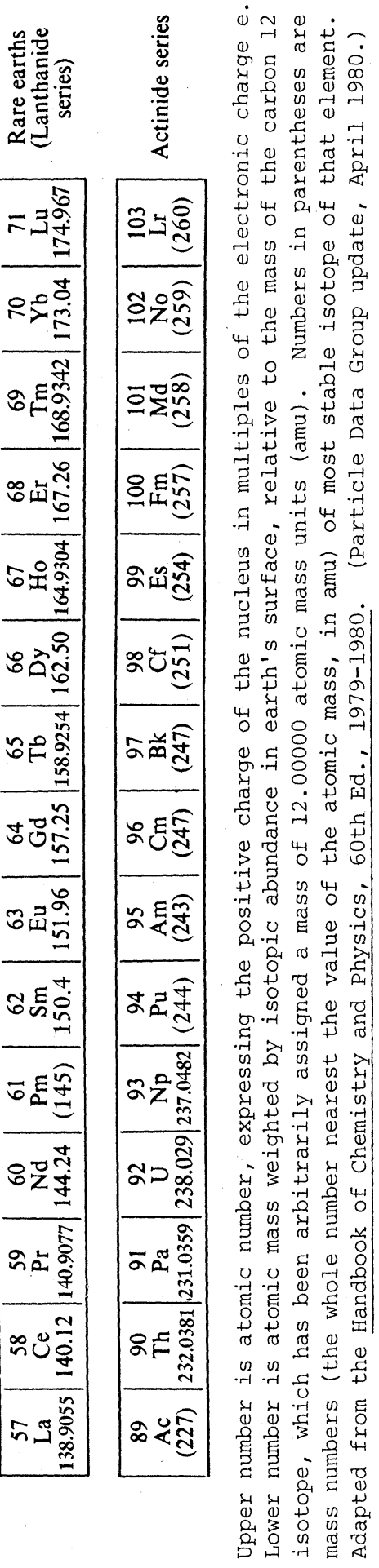




\section{CROSS SECTION PLOTS}

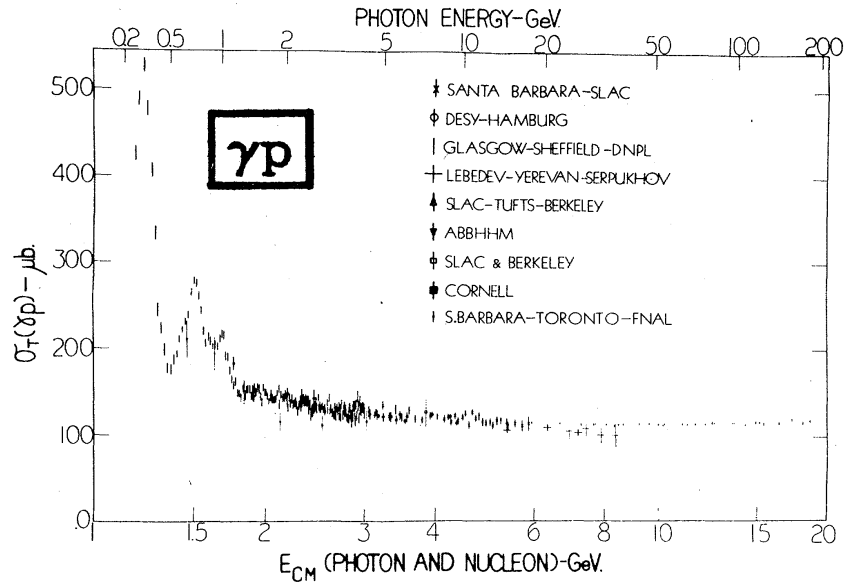

$\gamma p$ total cross section versus photon energy (top scale) and photon-plus-nucleon total center-of-mass energy (lower scale) References: SANTA BARBARA-SLAC: D.O.Caldwell et al., Phys. Rev. D7, 1362 (1973); DESY-HAMBURG: H.Meyer et al., Phys Lett. 33B, 189 (1970); GLASGOW-SHEFFIELD-DNPL: T.A.Armstrong et al., Phys. Rev. D5, 1640 (1972); LEBEDEV-YEREVAN-SERPUKHOV: A.S.Belousov et al., Preprint 19, Moscow (1973), A.S.Belousov et al., Sov. Phys. Doklady 19, 123 (1974), and A.S.Belousov et al., Sov. J. Nucl. Phys. $21(3), 289$ (1975); SLAC-BERKELEYTUFTS: J.Ballam et al., Phys, Rev. D5, 545 (1972); ABBHHM: H.G.Hilpert et al., Phys. Lett. 27B, 474 (1968); SLAC and BERKELEY: J Bajlam et a l. Phys, Rev, Lett. 21,1544 (1968), BERK H J J

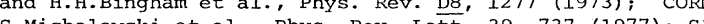

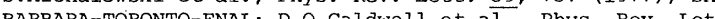
BARBARA-TORONTO-1

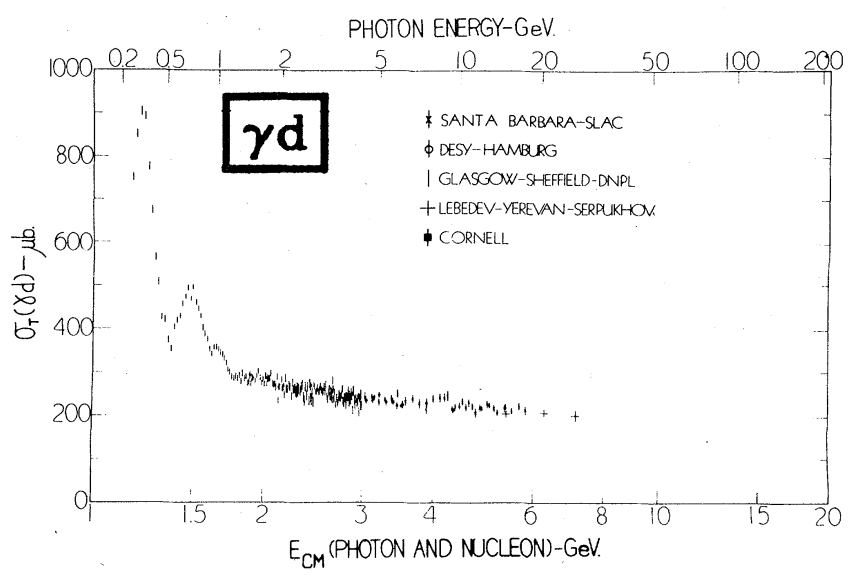

$\gamma$ d total cross section versus photon energy (top scale) and photon-plus-single-nucleon total center-of-mass energy (lower scale). References: SANTA BARBARA-SLAC: D.O.Caldwell et al. Phys. Rev. D7, 1362 (1973); DESY-HAMBURG: H.Meyer et al., Phys. Lett. 33B, 189 (1970); GLASGOW-SHEFFIELD-DNPL:

T.A.Armstrong et al., Nucl. Phys. B41, 445 (1972); LEBEDEVYEREVAN-SERPUKHOV: A.S.Belousov et al., Sov. J. Nucl. Phys. 21.(3), 289 (1975); CORNELL: S.Michalowski et al., Phys. Rev. Lett. 39, 737 (1977). Courtesy Gething M. Lewis, Glasgow.

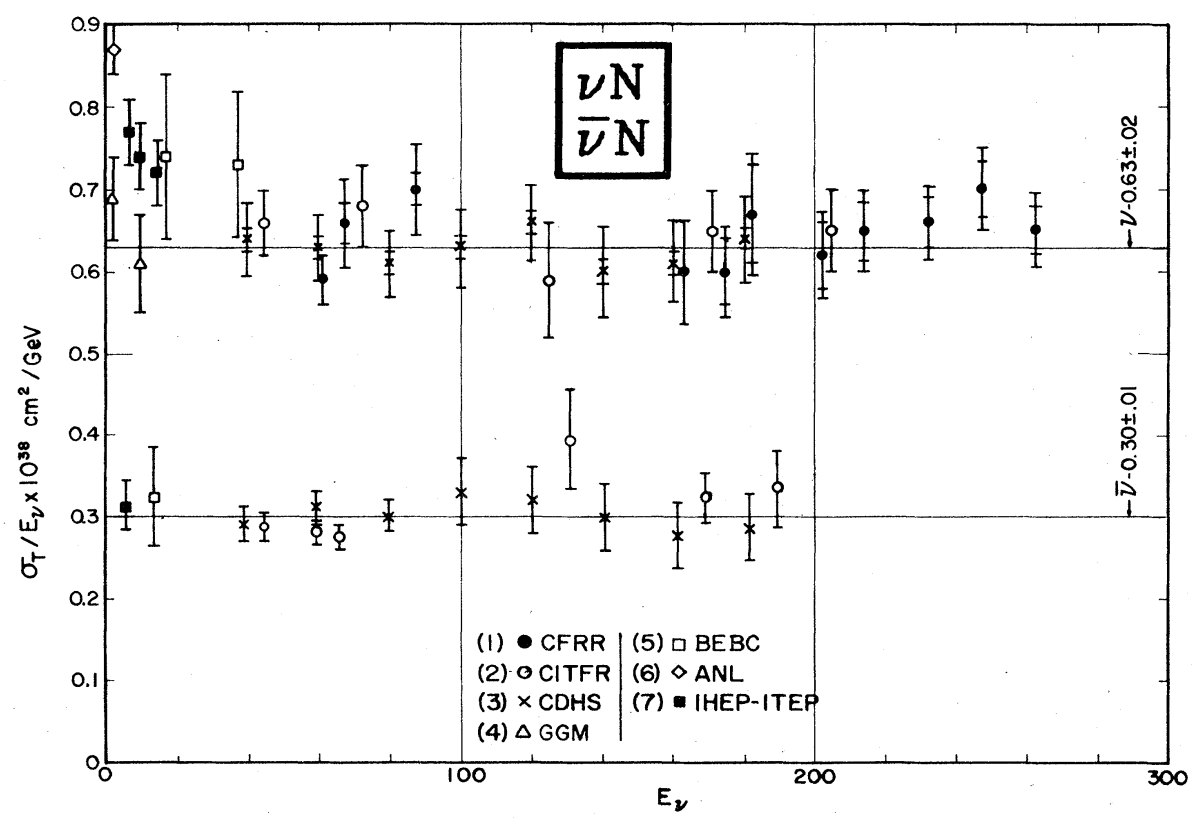

$\sigma_{\mathrm{T}} / \mathrm{E}_{\mathcal{V}}$ for the muon neutrino and antineutrino charged-current total cross section as a function of neutrino energy. The straight lines are averages of all data. (1) B.Barish et al., Cal Tech preprint CALT $68-734$ (1979); (2) B.C.Barish et al., Phys. Rev. Lett. 39, 1595 (1977); (3) J.G.H.de Groot et al., Z. Physik C Particles and Fields I, 143 (1979); (4) S.Ciampolillo et al., Phys. Lett. 84B, 281 (1979); (5) D.C.Colley et al., Z. Physik C - Particles and Fields 2, 187 (1979); (6) S.J.Barish et al., Phys. Rev. D19, 2521 (1979), (7) A.E.Asralyan et al., Phys Lett. 76B, 239 (1978), and A.E.Muklin, Sexpukhov preprint SERP-4-45 (1979) Courtesy D. Theriot, FNAL. 
Particle Data Group: Review of particle properties

\section{CROSS SECTION PLOTS (Cont'd)}

\section{Structure Functions}
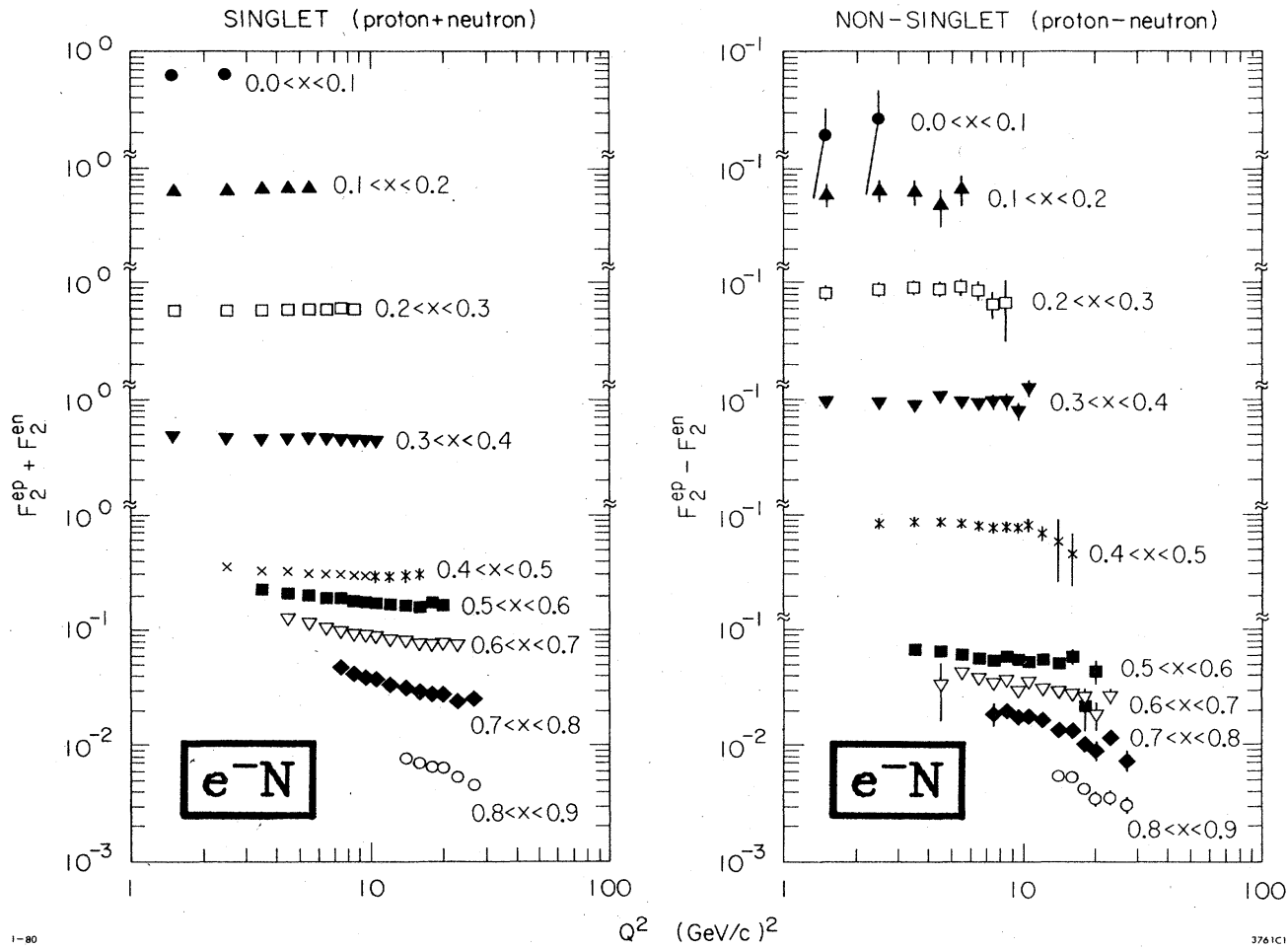

$\mathrm{F}_{2}$ structure functions derived from inelastic electron-nucleon data taken at SLAC $1-4$ with recoil mass $>2$ GeV and four-momentum $\mathrm{F}_{2}$ structure functions derived from inelastic electron-nucleon data taken at SLAC ${ }^{1-4}$ with recoil mass $>2$ GeV and four-momentum trane "Weak Thteractions the data point symols. Corrections for nucleon motion in deuterium have been made. These corrections are small except for $x>0.7$. 2) err was included to account for uncertainties in this correction. References: 1) A. Bodek et al., Phys. Rev. D20, 1471 (1979); 2) W.B.Atwood, SLAC Report No. 185 (1975); 3) M.D.Mestayer, SLAC Report. No. 214 (1978); 4) S.Stein et al., Phys. Rev. D12, 1884 (1975). Courtesy W. B. Atwood, SLAC.
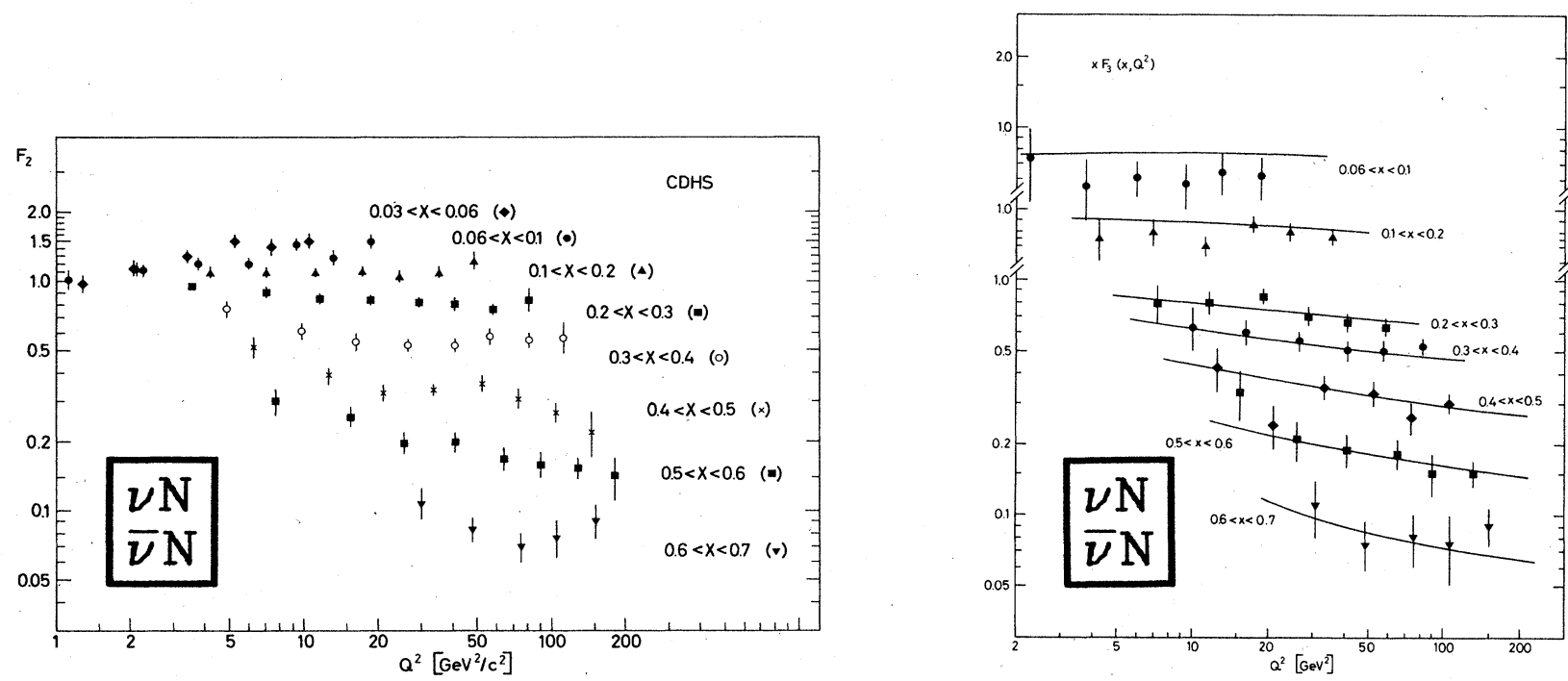

Nucleon structure functions as measured by the CDHS collaboration in high energy (30-200 GeV) charged-current neutrino- and antineutrino-nucleon scattering [J.G.H.de Groot et al., Z. Physik C - Particles and Fields 1, 143 (1979); reproduced by permission]. Definitions, and a discussion of the significance of these structure functions, may be found in the above reference, and also in the "Weak Interactions of Quarks and Leptons" section of the present work. See de Groot. et al., for a discussion of experimental details, including corrections, etc. Curves are based on a QCD parametrization of Buras and Gaemers [Nucl. Phys. B132, 249 (1978)]. 


\section{CROSS SECTION PLOTS (Cont'd)}

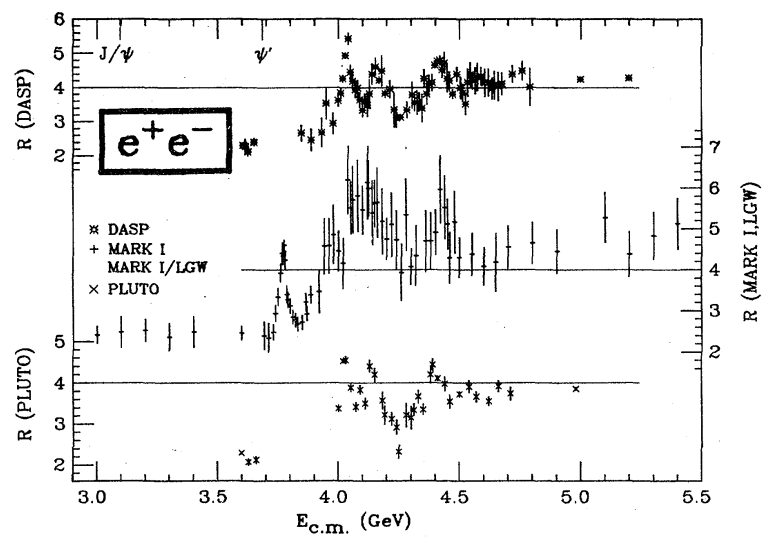

An expanded view of $R$ measurements around charm threshold. See the caption for the figure below for details (we have not combined any data $\mathrm{R}=4$ as an aid to visual comparison of the three sets of data.

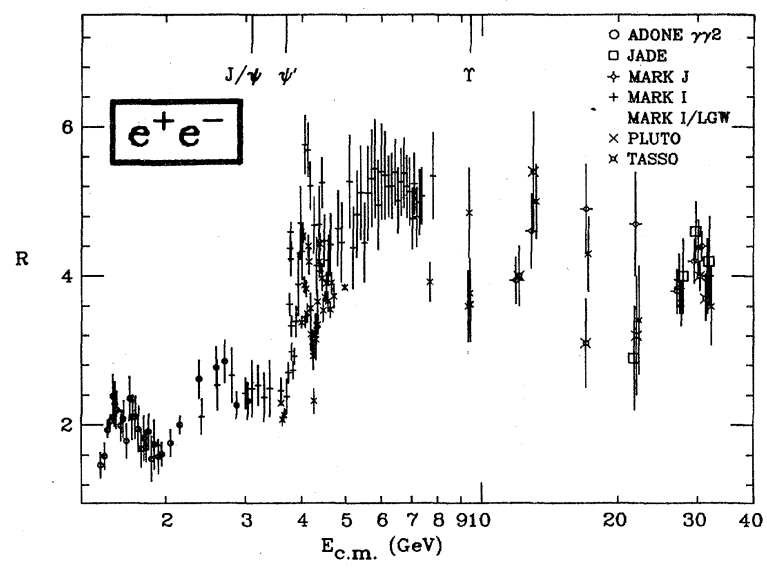

Measurements of $\mathrm{R} \equiv \sigma\left(\mathrm{e}^{+} \mathrm{e}^{-} \rightarrow\right.$ hadrons $) / \sigma\left(\mathrm{e}^{+} \mathrm{e}^{-} \rightarrow \mu^{+} \mu^{-}\right)$, where the annihilation proceeds via one photon. The denominator is a calculated quantity:

$$
\begin{aligned}
\sigma\left(\mathrm{e}^{+} \mathrm{e}^{-}+\ell^{+} \ell^{-}\right) & =(\hbar \mathrm{c})^{2} \frac{\alpha^{2}}{4 \mathrm{E}_{\mathrm{cm}}^{2}} \beta_{\ell} \int_{(4 \pi)} \mathrm{d} \Omega_{\mathrm{cm}}\left(2-\beta_{\ell}^{2} \sin ^{2} \theta_{\mathrm{cm}}\right) \\
& \beta_{\ell \rightarrow 1}=\frac{4 \pi}{3}(\hbar \mathrm{c})^{2} \frac{\alpha^{2}}{\mathrm{E}_{\mathrm{cm}}^{2}}=\frac{86.8}{\left[\mathrm{E}_{\mathrm{cm}}(\mathrm{GeV})\right]^{2}} \mathrm{nb} ; \beta_{\ell}=\frac{\mathrm{P}_{\ell}^{\mathrm{cm}}}{\mathrm{E}_{\ell}^{\mathrm{cm}}} ;
\end{aligned}
$$

for $\mathrm{e}^{+} \mathrm{e}^{-} \rightarrow \mu^{+} \mu^{-}, \beta_{\mu} \cong 1$ for energies shown. Radiative corrections and, where important, corrections for two-photon processes have been made. The J/, $\psi$, and the points in the $\psi^{\prime \prime}$ region are from the MARK I - Lead G1ass Wali (IGW) experiment. For clarity, some of the data points for $\mathrm{E}_{\mathrm{m}}<10 \mathrm{GeV}$ have been combined by us, and some of the points above $10 \mathrm{GeV}$ have been shifted slightly $(<2 \%)$ in $\mathrm{E}_{\mathrm{cm}}$. Systematic errors (not included in the figure) are typiçally between $10 \%$ and 20\%. The horizontal extent of the plot symbols has no special significance. References: ADONE $\gamma \gamma 2$ : C. Bacci et al., PL 86B, 234 (1979); DASP: R. Brandelik et al., PL 76B, Cart submitted to Nucl. Phys. B, H. Newman (private communication); MARK I J.-E.August in et al., PRL 34, 764 (1975), W.Chinowsky, Ann. Rev. Nucl. Sci. 27, 393 (1977); MARK I + Lead Glass Wall: P.A.Rapidis et al." PRL 39, 526 (1977), P.A.Rapidis, Thesis, SLAC-Report-220 (1979); PLUTO: A.Bäcker, Thesis, Gesamthochschule Siegen, DESY F $33-79 / 03$, C.Gerke, Thesis, Hamburg University (1979), Ch. Berger et al., PL 81B 410 (1979), Ch. Berger et al., PL F.C.Porter, Cal Tech.

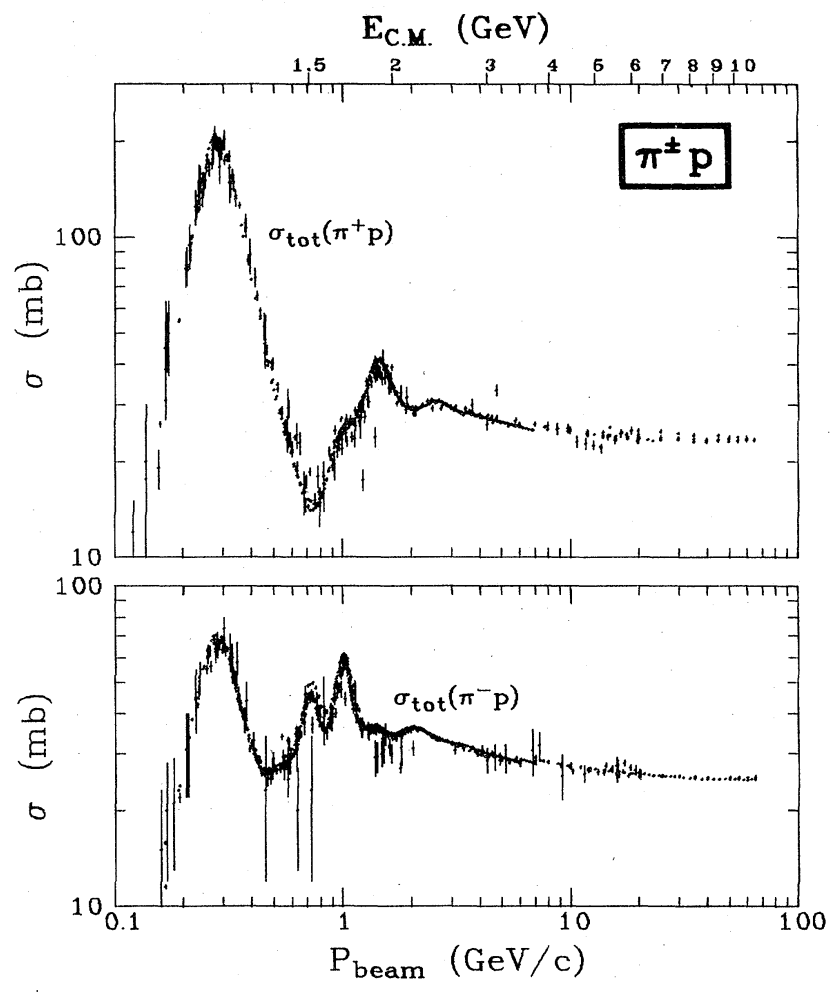

$\pi^{ \pm} p$ total cross-section data from the Particle Data Group compilation " $\pi N$ Two-Body Scattering Data," LBL-63 (1973).

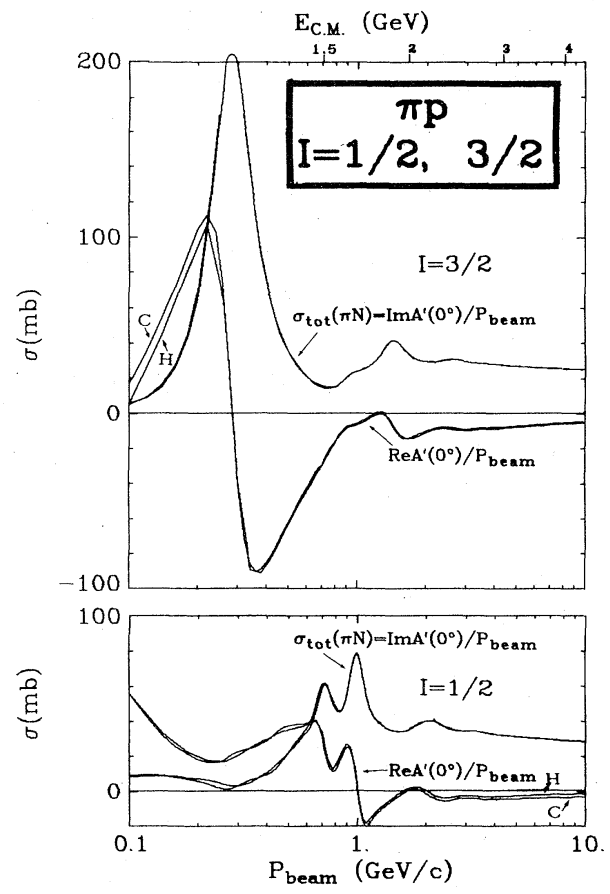
Intexpolations of $\pi \mathrm{N}$ total cross sections for $\mathrm{I}=3 / 2$ and $1 / 2$, and the corre-
sponding real parts of the forward amplitudes as calculated from dispersion relations by A. A. Carter and J. R. Carter (RHEL preprint RL-73-024, 1973; labeled $\mathrm{C}$ above), and by G. Höhlex and H. P. Jakob (private communication, 1972; labeled $\mathrm{H}$ above). The normalization of the curves for each value of $I$ is such that the sum of their squares divided by 19.6 gives do/dt at $0^{\circ}$ in $\mathrm{mb} /(\mathrm{GeV} / \mathrm{c})^{2}$. For visual purposes, these old analyses are fine; for quantita-

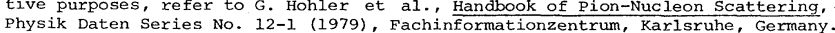


Particle Data Group: Review of particle properties

CROSS SECTION PLOTS (Cont'd)

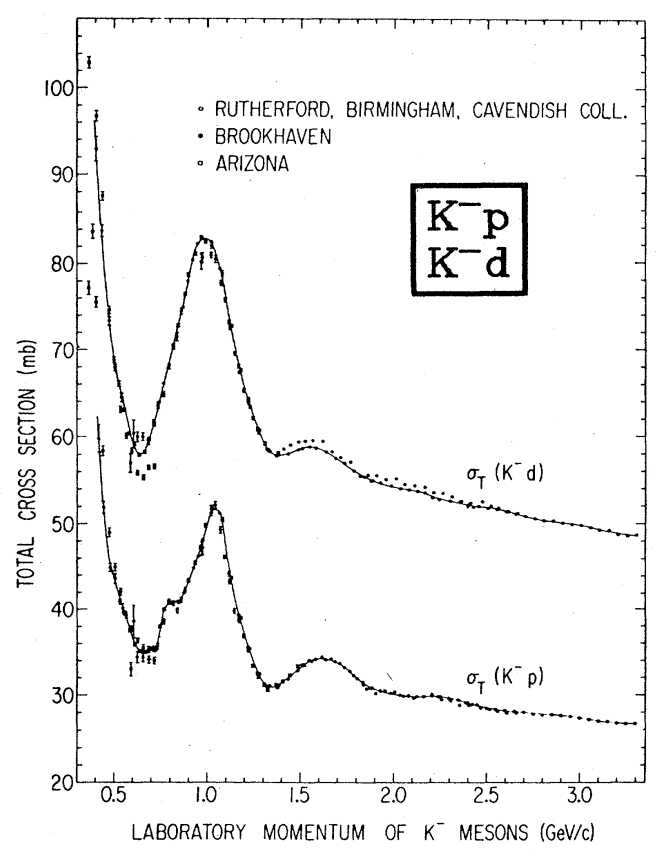

$\mathrm{K}^{-} \mathrm{p}$ and $\mathrm{K}^{-} \mathrm{d}$ total cross-section data compiled by Livet al. Proc. 1973 Purdue Conf. on Baryon Resonances. The solid curve passes through the Brookhaven data.

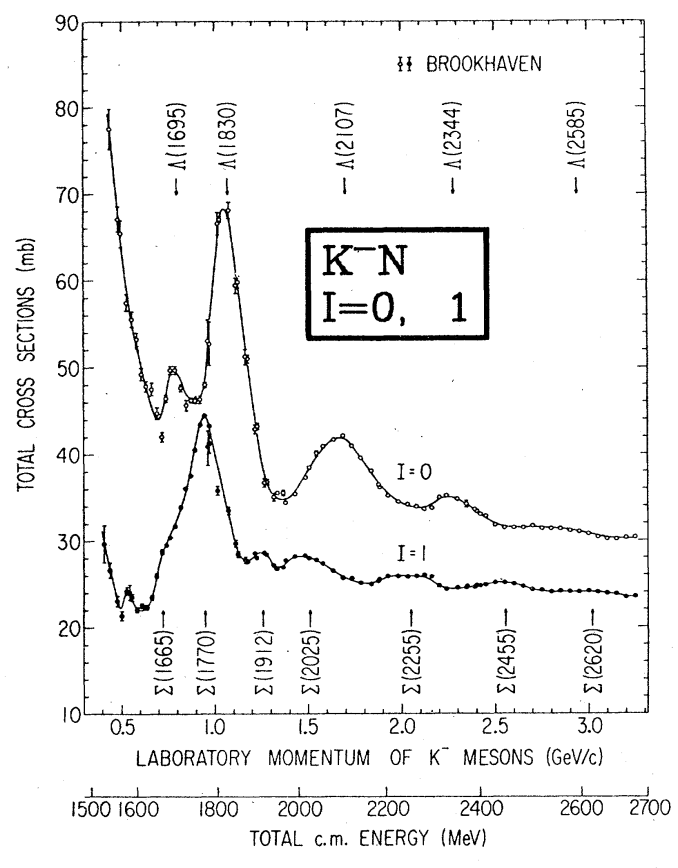

$\mathrm{K}^{-} \mathrm{N}$ total cross sections for $\mathrm{I}=0$ and $\mathrm{I}=1$ below $3.3 \mathrm{GeV} / \mathrm{C}$. Compiled and unfolded by $\mathrm{Li}$ et al., proc. 1973 Purdue Conf. on Baryon Resonances.

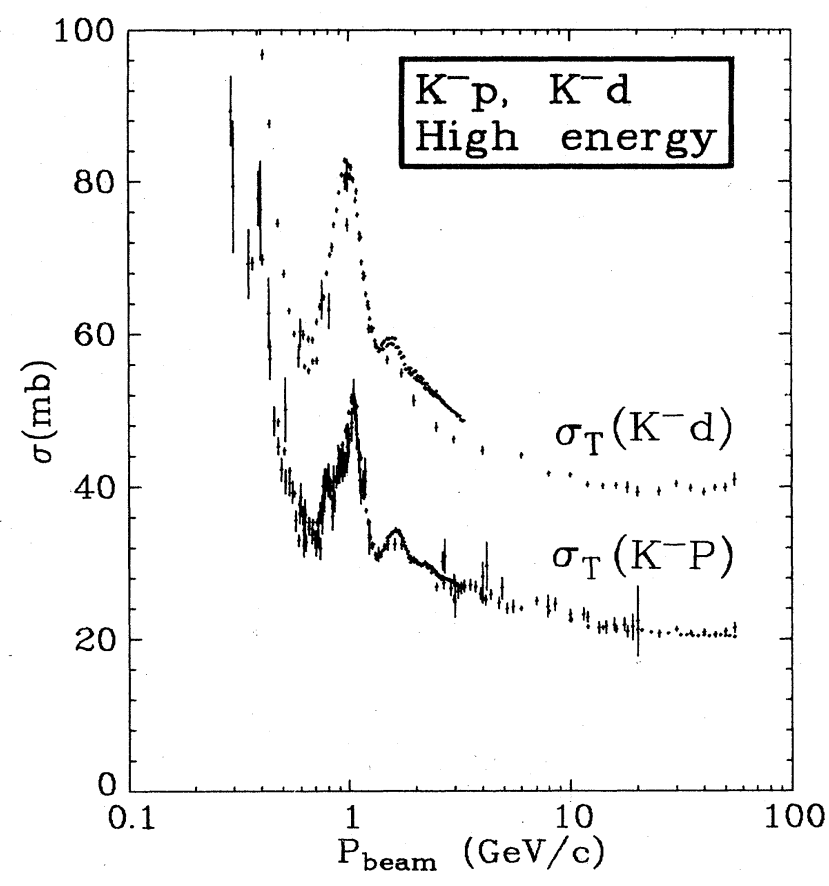

$\mathrm{K}^{-} \mathrm{p}$ and $\mathrm{K}^{-} \mathrm{d}$ total cross-section data. Compilation sources: E. Bracci et al., CERN/HERA $72-2, \mathrm{~K} p$; G. R. Lynch, $\mathrm{K} \mathrm{d}(<3 \mathrm{GeV} / \mathrm{C})$; Particle included.

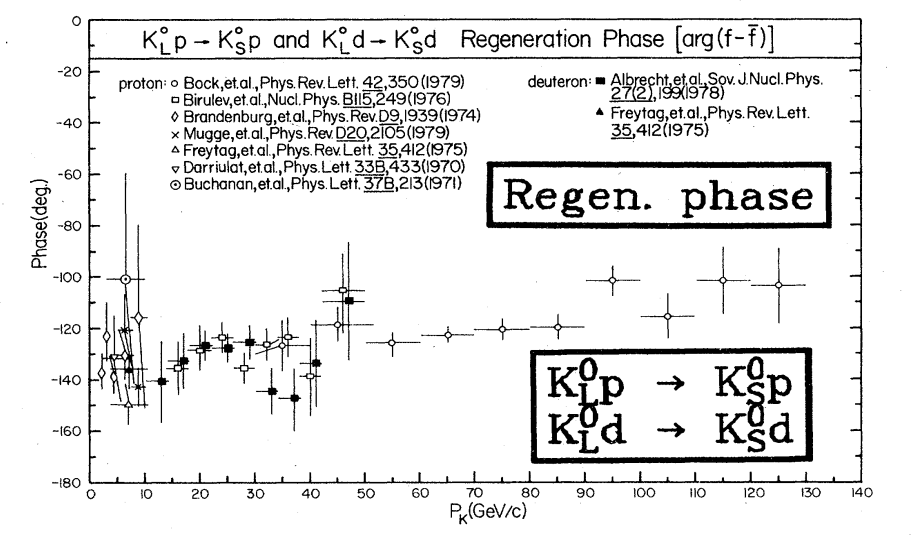

Phases of forward amplitudes for $K_{L}^{0} p \rightarrow K_{S}^{0} p$ (open symbols) and $\mathrm{K}_{\mathrm{L}} \mathrm{d} \rightarrow \mathrm{K}_{\mathrm{S}}^{\mathrm{d}}$ (solid symbols). Courtesy S.Aronson, 


\section{CROSS SECTION PLOTS (Cont'd)}
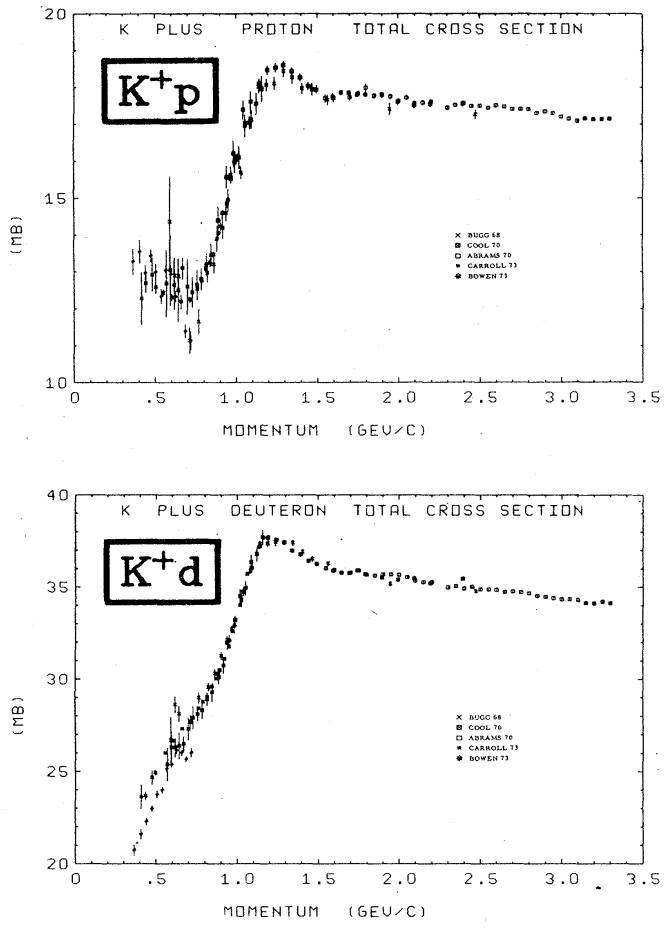

Compilation of $\mathrm{K}^{+} \mathrm{p}$ and $\mathrm{K}^{+} \mathrm{d}$ total cross-section measurements. References can be found in the

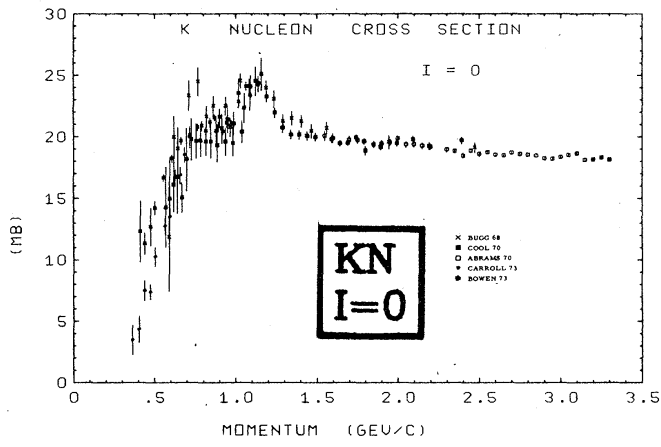

Total cross section for isospin zero KN system. Unfolding of the BUGG 68 and BOWEN 70 and 73
data was done by G. R. Lynch (as in Proc. of 1970 data was done by G. R. Lynch (as in Proc. Of 1970 by the BNI authors. same method of unfolding; the BOWEN 73 unfolded distribution is obtained by a different method [see plot in $\mathrm{z}^{*}$ mini-review in the 1976 edition of this review, Particle Data Group, Rev. Mod. Phys. 48, (1976) ].

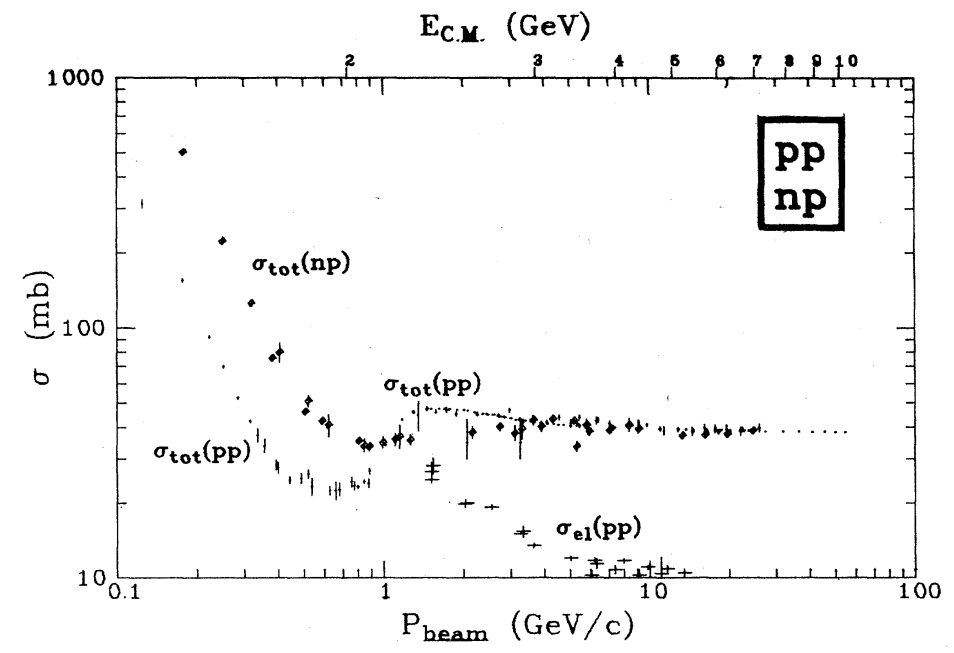

pp and np cross sections from Particle Data Group, "NN and ND some points at higher energies added since original compilation.

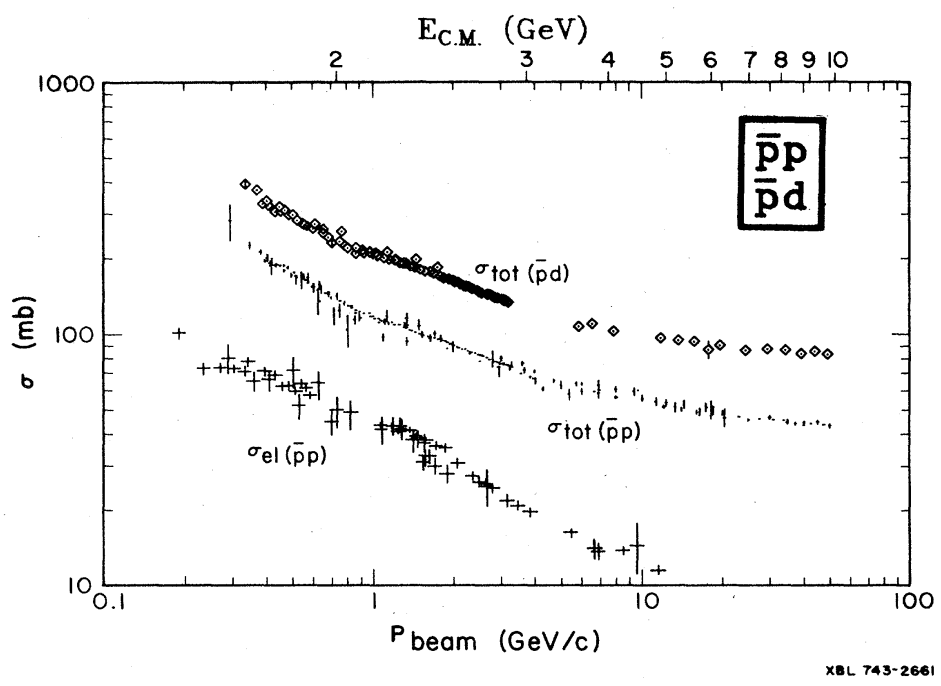

$\overline{\mathrm{p} p}$ and $\overline{\mathrm{p}} \mathrm{d}$ cross sections from Particle Data Group, "A Compilation of $\overline{\mathrm{NN}}$ and $\mathrm{ND}$ Reactions," LBL-58 (1972); some points added since original 
CROSS SECTION PLOTS (Cont'd)

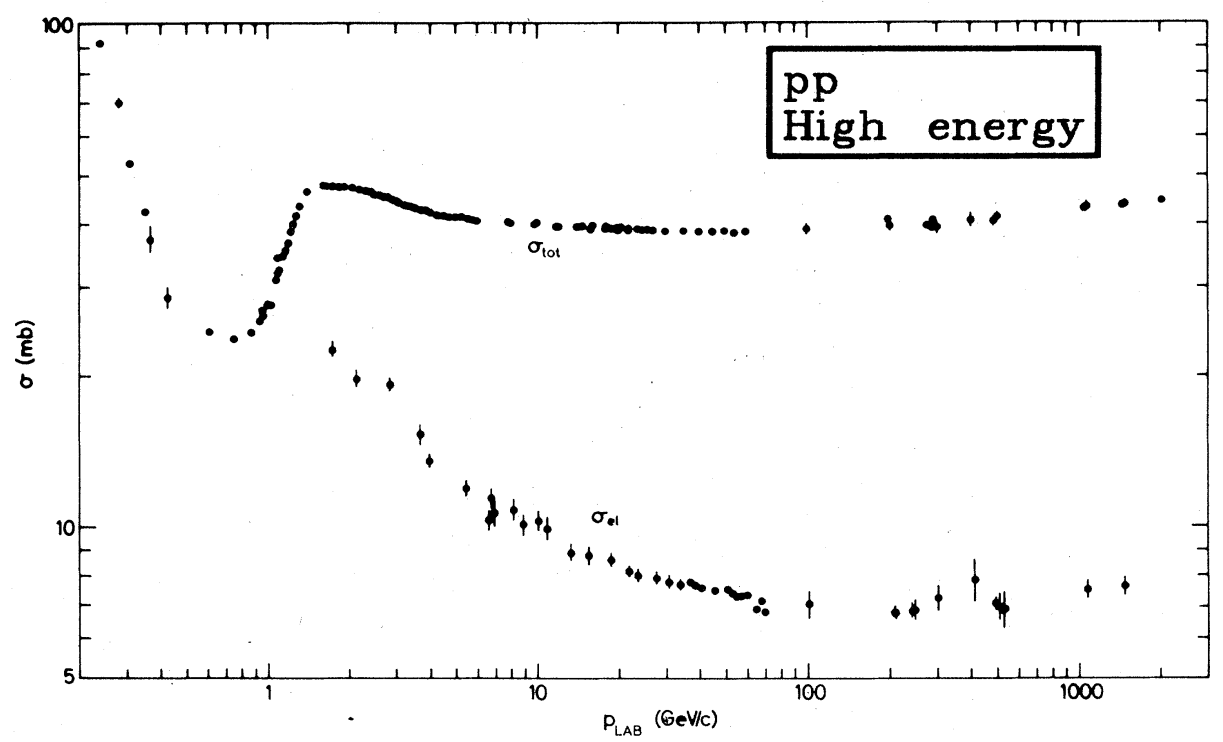

Total and elastic pp cross-section data compiled by U. Amaldi, CERN.

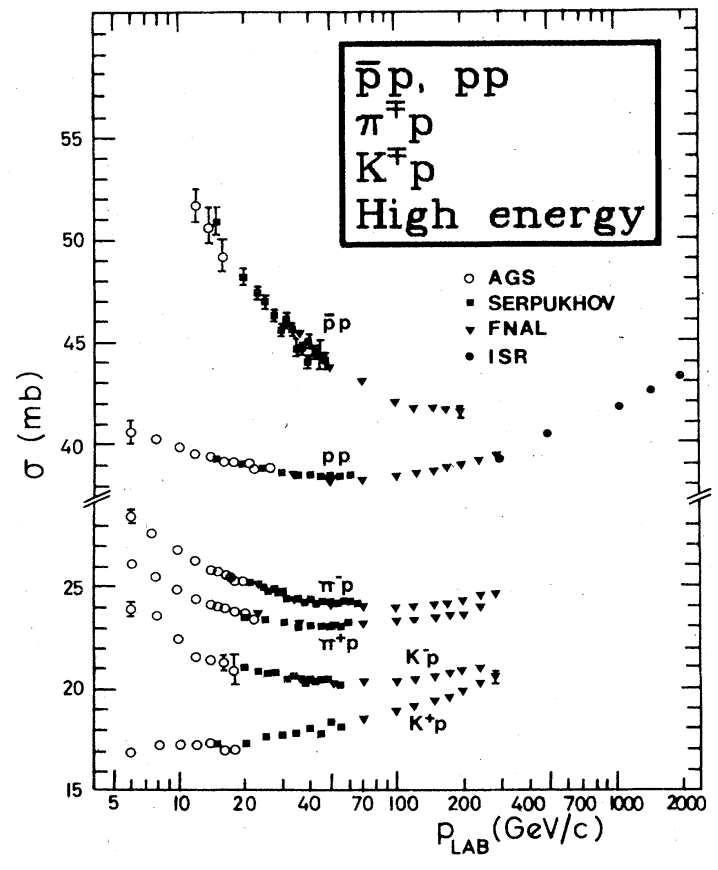

$\mathrm{pp}, \mathrm{pp}, \pi^{-} \mathrm{p}, \pi^{+} \mathrm{p}, \mathrm{K}^{-} \mathrm{p}$, and $\mathrm{K}^{+} \mathrm{p}$ total cross sections versus
$\mathrm{s}(\approx 2 \mathrm{~m} \mathrm{p} \mathrm{p})$, as compiled by U. Amaldi, CERN.

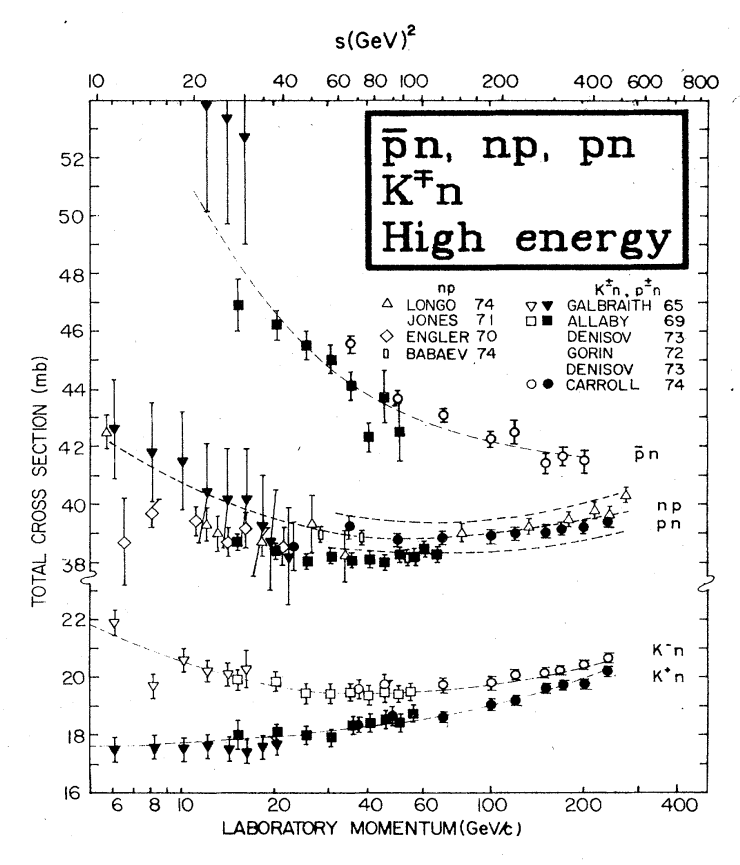

pn, np, pn, $K^{-} n$, and $K^{+} n$ total cross sections versus $s\left(\approx 2 m_{p} p_{1 a b}\right)$, as
compiled by $G$. Giacomelli, CERN. 


\section{DATA CARD LISTINGS}

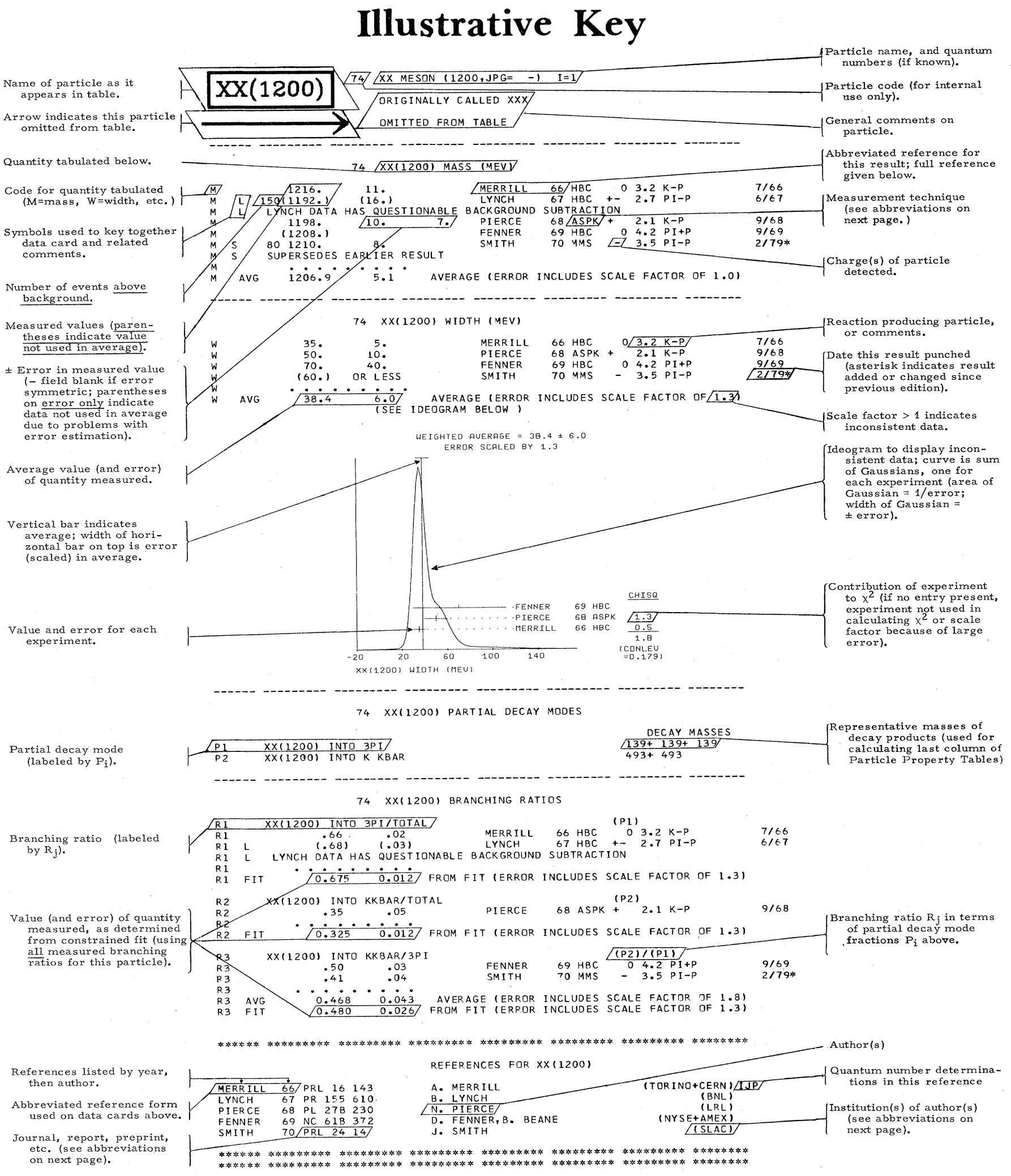

Rev. Mod. Phys., Vol. 52, No. 2, Part II, April 1980 


\section{Illustrative Key (cont'd)}

\section{Abbreviations}

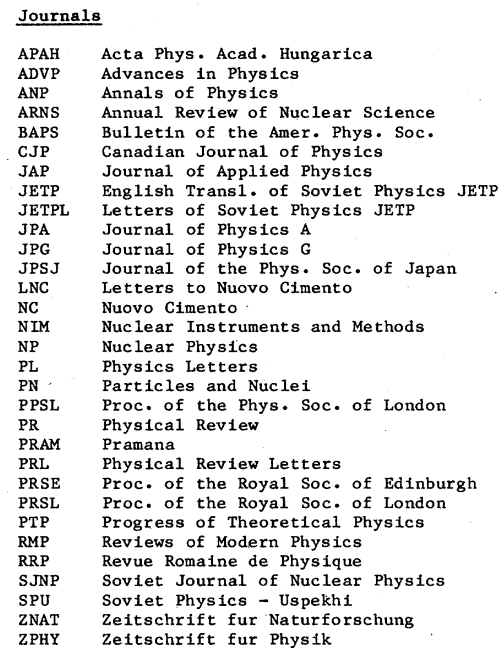

Conferences

Conferences are referred to by the location in which they were held (e.g., DUBNA, BOULDER, LUND, etc.).

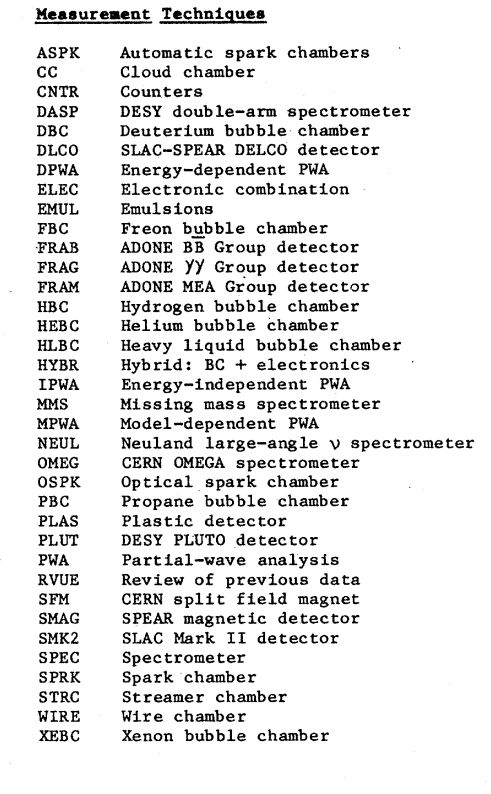

Institutions

\begin{tabular}{|c|c|}
\hline & Technische Univ. Aachen \\
\hline $\begin{array}{l}\text { AARH } \\
\text { ABO }\end{array}$ & $\begin{array}{l}\text { Aarhus Univ. } \\
\text { Abo. Akadem } 1\end{array}$ \\
\hline ADEL & Adelphi Univ. \\
\hline AERE & Atomic Energy Res. Estab. \\
\hline & Alchi Educational Univ. \\
\hline ALBA & State Univ, of New York at Albany \\
\hline ALBE & Alberta Univ., NRC \\
\hline AMST & Univ. of Amsterdam \\
\hline ANKA & Middle East Technical Univ. \\
\hline & Argonne National Lab. \\
\hline ARIZ & Univ. of Arizona \\
\hline ATEN & Nuclear Res. Centre Demokritos \\
\hline ATHU & Univ. of Athens \\
\hline AUCK & Univ. of Auckland \\
\hline BARC & Univ. de Barcelona \\
\hline BARI & Univ. di Bari \\
\hline BART & Bartol Research Foundation \\
\hline BASL & Basel Univ. \\
\hline BEDF & Bedford College \\
\hline BELG & Inst. Interuniv, des Sci. Nuc. \\
\hline BELL & Bell Labs. \\
\hline BERG & Univ, of Bergen \\
\hline BERL & Inst. Hochenergiephys. DAW \\
\hline BERN & Univ. Bern \\
\hline BGNA & Univ. di Bologna \\
\hline BING & State Univ. of New York at Bir \\
\hline B IRM & Birmingham Univ. \\
\hline BNL & Brookhaven National Lab. \\
\hline BOHR & Niels Bohr Inst. \\
\hline BOIS & Boise State Univ. \\
\hline BONN & Univ. Bonn \\
\hline BORD & Univ. de Bordeaux \\
\hline BOST & Boston Univ. \\
\hline BRAN & Brandeis Univ. \\
\hline BRCO & Univ, of British Columbia \\
\hline BRIS & H. H. Wills Phys. Lab., U. of Bristol \\
\hline BROW & Brown Untv. \\
\hline BRUX & Univ, Libre de Bruxelles \\
\hline BUCH & Bucharest State Univ. \\
\hline BUDA & Central Research Inst. of Physics \\
\hline & State Univ, of New York at Buffalo \\
\hline CAEN & Lab. de Phys. Corpusculaire \\
\hline САМВ & Cambridge Univ. \\
\hline CANB & Australian National Univ. \\
\hline CARL & Carleton Univ. \\
\hline CARN & Carnegie-Mellon Univ. \\
\hline CASE & Case Western Reserve Univ. \\
\hline CATH & Catholic Univ, of America \\
\hline CAVE & Cavendish Lab., Cambridge Univ. \\
\hline $\mathrm{CCAC}$ & Community College of Allegheny County \\
\hline CDEF & College de France \\
\hline & Cambridge Elect \\
\hline CENG & CEN, Grenoble \\
\hline
\end{tabular}

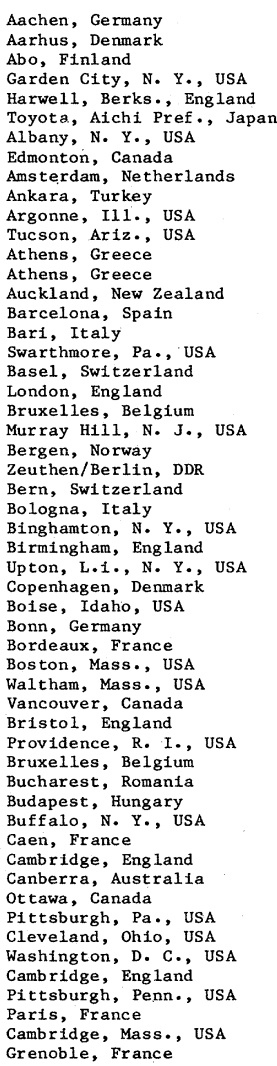

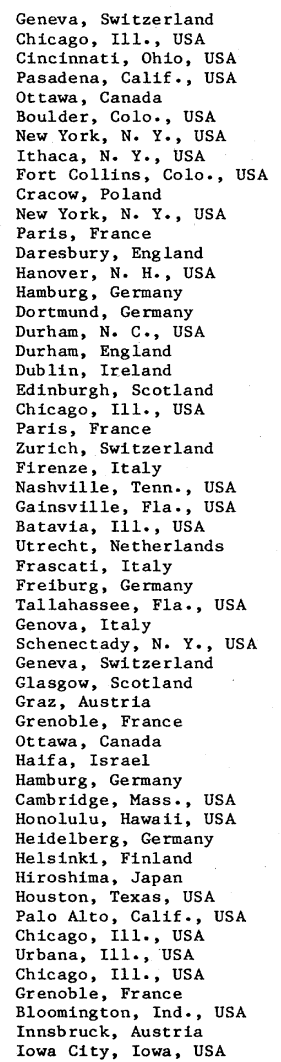




\section{Illustrative Key (cont'd)}

\section{Abbreviations (cont'd)}

\section{Inst1tutions (cont ${ }^{\circ}$ )}

\begin{tabular}{|c|c|c|c|}
\hline $\begin{array}{l}\text { IPN } \\
\text { IPNP }\end{array}$ & $\begin{array}{l}\text { Inst. de Phys. Nucleaire } \\
\text { Inst. de Physique Nucleaire }\end{array}$ & $\begin{array}{l}\text { Orsay, France } \\
\text { Parts, France }\end{array}$ & $\begin{array}{l}\text { OXP } \\
\text { PADO }\end{array}$ \\
\hline & Inst. for Particle Physics of Canada & Montreal, Canada & PATR \\
\hline $\begin{array}{l}\text { IRAD } \\
\text { ISU }\end{array}$ & $\begin{array}{l}\text { Inst. du Radum } \\
\text { Iowa State Undv. }\end{array}$ & $\begin{array}{l}\text { Parts, France } \\
\text { Ames, Iowa, USA }\end{array}$ & PAVI \\
\hline 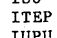 & Inst. for Teor. and Exp. Phys. & $\begin{array}{l}\text { Amoscow, USSR } \\
\text { Mosc }\end{array}$ & PISA \\
\hline$\underset{\text { JUPL }}{\text { IUP }}$ & $\begin{array}{l}\text { Indiana U. - Purdue U. at Indianapolis } \\
\text { Jagelionian Univ. }\end{array}$ & $\begin{array}{l}\text { Indianapolis, Ind., USA } \\
\text { Cracow, Poland }\end{array}$ & $\underset{P \text { PITT }}{\text { PIT }}$ \\
\hline $\begin{array}{l}\text { JAGL } \\
\text { JHU }\end{array}$ & $\begin{array}{l}\text { Jage elionian Undv- } \\
\text { Johns Hopkins Univ. }\end{array}$ & $\begin{array}{l}\text { Cracow, Poland } \\
\text { Baltimore, Md., USA }\end{array}$ & $\begin{array}{l}\text { PPA } \\
\text { PRAG }\end{array}$ \\
\hline & Joint Inst. for Nucl. Research & Dubna, USSR & $\begin{array}{l}\text { PRAG } \\
\text { PRTN }\end{array}$ \\
\hline KANS & $\begin{array}{l}\text { Univ. of Kansas } \\
\text { Und Researcin }\end{array}$ & Lawrence, Kansas, USA & PSLL \\
\hline KARL & Univ. Karlsruhe & Kar1sruhe, Germany & PURD \\
\hline KEK & Nat. Lab for High Energy Phys., Japan & Tsukuba-gun, Japan & REHO \\
\hline KENT & Kent Univ. at Cantebury, Kent & & RHEL \\
\hline KEYN & & Milton Keynes, England & RICE \\
\hline $\begin{array}{l}\text { KIAE } \\
\text { KIEV }\end{array}$ & $\begin{array}{l}\text { Kurchatov Int. of Atomic Bnergy } \\
\text { Physical-Technical Inst. }\end{array}$ & $\begin{array}{l}\text { Moscow, USSR } \\
\text { Kiev, USSR }\end{array}$ & ${ }_{\mathrm{RL}}^{\mathrm{RISO}}$ \\
\hline $\begin{array}{l}\text { KIEV } \\
\text { KINK }\end{array}$ & $\begin{array}{l}\text { Physical-technical inst. } \\
\text { Kinki Univ. }\end{array}$ & $\begin{array}{l}\text { Keve, USRR } \\
\text { Osaka, Japan }\end{array}$ & 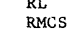 \\
\hline KNTY & Univ. of Kentucky & Lexington, $\mathrm{Ky}$, USA & ROCH \\
\hline KONA & Konan Univ. & Kobe, Japan & ROCK \\
\hline KONS & B. P. Konstantinov Inst, of Nuc1. Phys. & & ROMA \\
\hline LALO & Linear Accelerator Lab, Orsay & Orsay, France & ROSE \\
\hline $\begin{array}{l}\text { LANC } \\
\text { LAPP }\end{array}$ & Lancaster Univ. & Lancaster, England & RUTG \\
\hline & $\begin{array}{l}\text { Lap Univ. } \\
\text { U. C. Los Alamos Scientific Lab. }\end{array}$ & $\begin{array}{l}\text { Annecy, France } \\
\text { Los Alamos, N. M., USA }\end{array}$ & $\begin{array}{l}\text { SACL } \\
\text { SAGA }\end{array}$ \\
\hline LUS & Univ. of Lausanne & Lausanne, Switzeriand & $\begin{array}{l}\text { SAGA } \\
\text { SANI }\end{array}$ \\
\hline & U. C. Lawrence Berkeley Lab. & Berkeley, Calif., USA & SBER \\
\hline LCGT & Lab. di Cosmo-Geofisica del CNR & Torino, Italy & SEAT \\
\hline LEBD & Lebedev Physics Inst. & Moscow, USSR & SEIB \\
\hline LEED & Univ. of Leeds & Leeds, England & SERP \\
\hline $\begin{array}{l}\text { LEHI } \\
\text { LEID }\end{array}$ & $\begin{array}{l}\text { Lehigh UnIv. } \\
\text { Inst. Lorentz. }\end{array}$ & Bethlehem, Pa., USA & SETO \\
\hline LENI & $\begin{array}{l}\text { Inst. Lorentz } \\
\text { Inst. of Nuc1. Phys., USSR }\end{array}$ & $\begin{array}{l}\text { Lelden, Netherrands } \\
\text { Len1ngrad, USR }\end{array}$ & $\begin{array}{l}\text { SFLA } \\
\text { SHEF }\end{array}$ \\
\hline $\begin{array}{l}\text { LENI } \\
\text { LIBH }\end{array}$ & Lab. Interuniv. Belge High Eng. & Bruxelles, Belgium & $\begin{array}{l}\text { SHEF } \\
\text { SHMP }\end{array}$ \\
\hline LINZ & Linz Inst. fur Physik, Kepler Hoch. & Linz, Austria & SIBE \\
\hline LIVP & Liverpool Univ. & Liverpool, England & SIEG \\
\hline LLL & Lawrence Livermore Lab. & L1vermore, Calif., USA & $\sin$ \\
\hline $\begin{array}{l}\text { LOIC } \\
\text { LOM }\end{array}$ & $\begin{array}{l}\text { Imperial col. of Sci, and Tech. } \\
\text { Oueen Mary College }\end{array}$ & London, England & $\begin{array}{l}\text { SLAC } \\
\text { SMAS }\end{array}$ \\
\hline LOUC & $\begin{array}{l}\text { Queen Mary College } \\
\text { University College }\end{array}$ & $\begin{array}{l}\text { London, England } \\
\text { London, England }\end{array}$ & $\begin{array}{l}\text { SMAS } \\
\text { SOFI }\end{array}$ \\
\hline & Westfield College & $\begin{array}{l}\text { London, Eng tand } \\
\text { London, Eng 1and }\end{array}$ & $\begin{array}{l}\text { SOFI } \\
\text { STAN }\end{array}$ \\
\hline NP & Lab. de Phys. Nuc1. et Hautes Energies & Paris, France & $\begin{array}{l}\text { STAN } \\
\text { STEV }\end{array}$ \\
\hline TP & Lab. de Phys. Theor. et Hautes Energies & Paris, France & STLO \\
\hline LRL & U. C. Lawrence Berkeley Lab. & Berkeley, Callf., USA & STOH \\
\hline LSU & Louisiana State Univ. & Baton Rouge, La., USA & STON \\
\hline LUND & $\begin{array}{l}\text { Untv. I Lund } \\
\text { Junta de Rnergia Nuclear }\end{array}$ & Lund, Sweden & $\begin{array}{c}\text { STRB } \\
\text { SUPR }\end{array}$ \\
\hline $\begin{array}{l}\text { MADR } \\
\text { MADU }\end{array}$ & $\begin{array}{l}\text { Junta de Energia Nuclear } \\
\text { Univ, Autonome de Madrid }\end{array}$ & Madrid, Spain & $\begin{array}{l}\text { SURR } \\
\text { SUSS }\end{array}$ \\
\hline $\begin{array}{l}\text { MADU } \\
\text { MANH }\end{array}$ & $\begin{array}{l}\text { Univ. Autonome de Madrid } \\
\text { Manhattan College }\end{array}$ & $\begin{array}{l}\text { Madrid, Spain } \\
\text { New York, N. Y. }\end{array}$ & $\begin{array}{l}\text { SUSS } \\
\text { SYRA }\end{array}$ \\
\hline MANI & Univ. of Manitoba & Winnipeg, Canada & TAMU \\
\hline MANZ & Univ. Mainz & Mainz, Germany & TATA \\
\hline MASA & Univ, of Massachusetts & Amherst, Mass., USA & TELA \\
\hline MASB & Univ, of Massachusetts & Boston, Mass., USA & TEMP \\
\hline $\begin{array}{l}\text { MCGI } \\
\text { MCHS }\end{array}$ & $\begin{array}{l}\text { McGill1 Univ. } \\
\text { Univ, Manchester }\end{array}$ & $\begin{array}{l}\text { Montreal, Canada } \\
\text { Manchester, England }\end{array}$ & TENN \\
\hline $\begin{array}{l}\text { MCHS } \\
\text { MELB }\end{array}$ & $\begin{array}{l}\text { Univ. Manchester } \\
\text { Univ. Of Melbourne }\end{array}$ & $\begin{array}{l}\text { Manchester, England } \\
\text { Parky 11le, Australla }\end{array}$ & TEXA \\
\hline $\begin{array}{l}\text { MELB } \\
\text { MHCO }\end{array}$ & $\begin{array}{l}\text { Uive. of Melbourne } \\
\text { Mount Holyoke College }\end{array}$ & $\begin{array}{l}\text { Parkvile, Australla } \\
\text { South Hadley, Mass., }\end{array}$ & $\begin{array}{l}\text { TMSK } \\
\text { TNTO }\end{array}$ \\
\hline MICH & Univ. of Michigan & Ann Arbor, Mich., USA & тоно \\
\hline MILA & Univ. di Milano & Milano, Italy & TOKY \\
\hline MINN & Univ. of Minnesota & Minneapolis, Minn., USA & TORI \\
\hline MIOH & Miam1 Un 1v. & 1o, USA & TRIU \\
\hline $\begin{array}{l}\text { MIT } \\
\text { MODE }\end{array}$ & $\begin{array}{l}\text { Massachusetts Inst. of Technology } \\
\text { Ist. di Fistca deli Untv. }\end{array}$ & $\begin{array}{l}\text { Cambridge, Mass., USA } \\
\text { Modena, Italy }\end{array}$ & RRST \\
\hline MONP & $\begin{array}{l}\text { Ste. di Fisica dell Univ. } \\
\text { Univ, de Montpel1ier }\end{array}$ & $\begin{array}{l}\text { Modena, I } \\
\text { Montpei1 }\end{array}$ & TWFT \\
\hline MONS & Univ. de $1^{\circ}$ Etat, Mons & Mons, Belgium & UBEL \\
\hline MONT & Univ. de Montreal & Montreal, Canada & UCB \\
\hline MOSU & Moscow State Univ. & Moscow, USSR & UCD \\
\hline MPEI & Moscow Phys. Eng. Inst. & Moscow, USSR & UCI \\
\hline MPIH & Max Planck Inst. fur Phys.-Astrophys. & Heidelberg, Ge rmany & UCLA \\
\hline 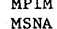 & $\begin{array}{l}\text { Max Planck Int. fur Phys.-Astrophys. } \\
\text { Ist. di Fisica dell Univ. }\end{array}$ & $\begin{array}{l}\text { Munich, Germany } \\
\text { Messina, Itayy }\end{array}$ & UCND \\
\hline MSU & $\begin{array}{l}\text { Ist. di Fisica dell Univ. } \\
\text { Michigan State Univ. }\end{array}$ & $\begin{array}{l}\text { Messina, Italy } \\
\text { East Lans tng, Mich., }\end{array}$ & $\begin{array}{l}\text { UCR } \\
\text { UCSB }\end{array}$ \\
\hline но & $\begin{array}{l}\text { Mt. Holyoke College } \\
\text { Me }\end{array}$ & South Hadley, Mass., USA & ucsc \\
\hline LH & Centre Univ. du Haut-Rhin & Mulhouse, France & UCSD \\
\hline MUNC & Univ. of Munich & , Germany & UMD \\
\hline MURA & Midwestern UnAv. Research Assoc. & Stroughton, Wisc., USA & uncs \\
\hline $\begin{array}{l}\text { NAGO } \\
\text { NAPL }\end{array}$ & $\begin{array}{l}\text { Nagoya Un nv. } \\
\text { Unive dit Napolt }\end{array}$ & $\begin{array}{l}\text { Nagoya, Japan } \\
\text { Napoli }\end{array}$ & $\begin{array}{l}\text { UNH } \\
\text { UPNT }\end{array}$ \\
\hline NASA & $\begin{array}{l}\text { NASA, divaddard Space Flight Center } \\
\text { NASA }\end{array}$ & $\begin{array}{l}\text { Napol1, Itayy., USA } \\
\text { Greenbelt, Md., USA }\end{array}$ & $\begin{array}{l}\text { UPNS } \\
\text { UPPS }\end{array}$ \\
\hline NDAM & Univ. of Notre Dame & Dame, Ind., USA & UTAH \\
\hline $\begin{array}{l}\text { NEAS } \\
\text { NEUC }\end{array}$ & $\begin{array}{l}\text { Northeastern Univ. } \\
\text { Univ, de Neuchatei }\end{array}$ & $\begin{array}{l}\text { Boston, Mass., USA } \\
\text { Neuchate1, Switzer1a }\end{array}$ & $\begin{array}{l}\text { UTRE } \\
\text { VAND }\end{array}$ \\
\hline NEUC & $\begin{array}{l}\text { Univ. de Neuchatel } \\
\text { Nevis Lab. }\end{array}$ & $\begin{array}{l}\text { Neuchate1, Switzerland } \\
\text { Trvington-on-Hudson, } \mathbb{N}\end{array}$ & VAND \\
\hline $\mathrm{JM}$ & $\begin{array}{l}\text { Nevis Lab. } \\
\text { R. K. Univ. Nijmeg }\end{array}$ & $\begin{array}{l}\text { Irvington-on-Hudson, N. } \\
\text { N1fmegen, Netherlands }\end{array}$ & $\begin{array}{l}\text { VICT } \\
\text { VIEN }\end{array}$ \\
\hline NIU & $\begin{array}{l}\text { R. K. Univ. Nijegen } \\
\text { Northern Iilinois Univ. }\end{array}$ & $\begin{array}{l}\text { Netherranc } \\
\text { I11., USA }\end{array}$ & VIRG \\
\hline NORD & Nordisk Inst. for Teor. Atomfys. & gen, Denmark & VPI \\
\hline NOVO & Inst. & No & WARS \\
\hline NRI & $\begin{array}{l}\text { Northern Polytechnic } \\
\text { Naty }\end{array}$ & England & WASH \\
\hline NRL & $\begin{array}{l}\text { Naval Research Laboratory } \\
\text { Northwestern Univ. }\end{array}$ & $\begin{array}{l}\text { Washington, D.c., USA } \\
\text { Evanston, I11., USA }\end{array}$ & $\begin{array}{l}\text { WIEN } \\
\text { WILL }\end{array}$ \\
\hline & Nortnwestern & N. Y., USA & \\
\hline & & io, USA & WOOD \\
\hline & Oregon & $\because$ USA & WUPG \\
\hline & $\begin{array}{l}\text { Oak Ridge Nationa } \\
\text { Univ, de Parts, Fa }\end{array}$ & $\begin{array}{l}\text { dge, Tenn., } \\
\text { France }\end{array}$ & $\begin{array}{l}\text { WUPP } \\
\text { WUSL }\end{array}$ \\
\hline $0 s$ & $\begin{array}{l}\text { Univ. de Parrs, Fac. } \\
\text { Osaka Univ. }\end{array}$ & $\begin{array}{l}\text { Orsay, } \\
\text { Osaka, J }\end{array}$ & WYOM \\
\hline $0 S x$ & Osaka City Univ. & Osaka, Japan & YALE \\
\hline & slo Univ. & Os1o, Norway & YOKO \\
\hline & tawa & ot ta & $2 \mathrm{URI}$ \\
\hline
\end{tabular}

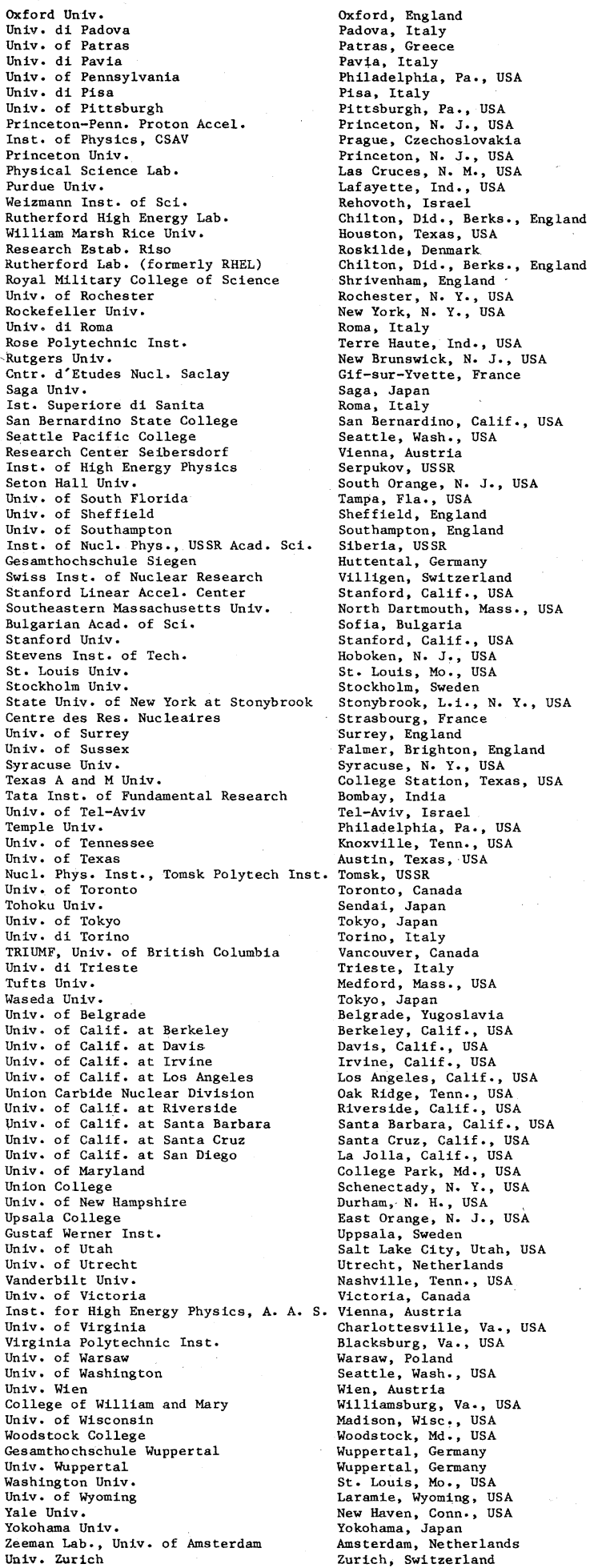




\section{Data Card Listings}

\section{Stable Particles}

\section{For notation, see key at front of Listings.}

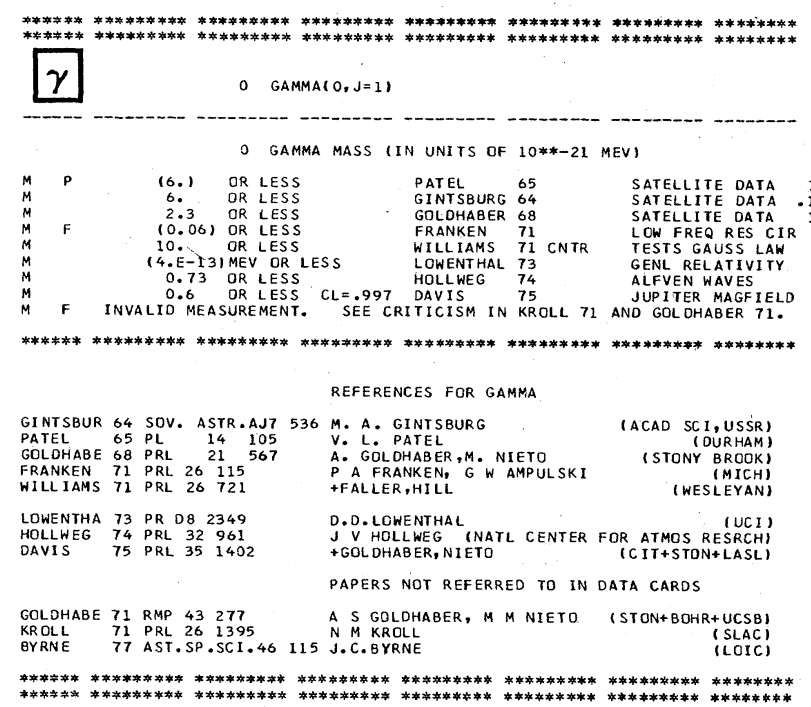

\section{Note on Neutrino Mass Limits}

(by R. Shrock, State Univ. of New York, Stonybrook)

In the conventional case where all neutrinos are assumed to be massless and hence degenerate, it is possible to define the neutrino weak-gaugegroup eigenstates $\nu_{e}, \nu_{\mu}$, and $\nu_{\tau}$ (i.e., the states which couple with unit strength to $e, \mu$, and $\tau$, respectively) to be simultaneously mass eigenstates. However, in the general case of massive (nondegenerate) neutrinos, the gauge-group eigenstates have no wel1-defined masses, but instead are linear combinations of mass eigenstates, which may be labeled $\nu_{1}, \nu_{2}$, and $\nu_{3}$. In the standard $\mathrm{SU}(2) \otimes$ $U(1)$ electroweak theory, ${ }^{1}$ the mixing of the lefthanded components of the mass eigenstates to form gauge-group eigenstates is specified ${ }^{2}$ by a $3 \otimes 3$ unitary matrix $U$ :

$$
\left(\begin{array}{c}
v_{e} \\
v_{\mu} \\
v_{\tau}
\end{array}\right)=u\left(\begin{array}{c}
v_{1} \\
v_{2} \\
v_{3}
\end{array}\right) .
$$

(The right-handed components $v_{i R}$ are singlets.) The lepton mixing matrix $U$ depends on four real parameters, of which three are CP-conserving rotation angles, and the remaining one is a $\mathrm{CP}$ violating phase. It is easy to generalize these remarks to the case of $\mathrm{n}>3$ neutrino species. One should note, however, that there are indications from astrophysics ${ }^{3}$ that $n$ may not be larger than 3 .
Thus, in the general case of $\mathrm{n}$ neutrino species, decays such as ${ }^{3} \mathrm{H} \rightarrow{ }^{3} \mathrm{He}+\mathrm{e}^{-}+\bar{\nu}_{\mathrm{e}}$ and $\pi^{+} \rightarrow \mu^{+}+\nu_{\mu}$, which have been used to set the best upper bounds on the respective neutrino masses, 4,5 really consist of incoherent sums of the separate decay modes ${ }^{3} \mathrm{H} \rightarrow{ }^{3} \mathrm{He}+\mathrm{e}^{-}+v_{i}$ and $\pi^{+} \rightarrow \mu^{+}+v_{j^{\prime}}$ where the $v_{i}, v_{j}$ are mass eigenstates, and $i=1, \ldots, k \leqslant n, j=1, \ldots, k^{\prime} \leqslant n$, with $\nu_{k}$ and $\nu_{k}$, being the heaviest such states allowed by phase space in these two respective decays. The coupling strength for the $i^{\text {th }}$ mode is given for the two decays by the factors $\left|U_{I i}\right|^{2} \equiv\left|u_{e i}\right|^{2}$ and $\left|\mathrm{u}_{2 i}\right|^{2} \equiv\left|\mathrm{u}_{\mu i}\right|^{2}$. There are, in addition, certain kinematic factors depending on $m\left(\nu_{i}\right)$ which enter in determining the branching ratio for the $i^{\text {th }}$ decay mode. Since the off-diagonal elements of the lepton mixing matrix $U$ are constrained to be rather small, the dominant decays are the ones with coupling strength $\left|\mathrm{u}_{i j}\right|^{2}$, where $\mathrm{i}=j$, i.e., ${ }^{3} \mathrm{H} \rightarrow{ }^{3} \mathrm{He}+\mathrm{e}+v_{1}$ and $\pi^{+} \rightarrow \mu^{+}+\nu_{2}$.

It follows that: (1) the old neutrino mass limits quoted in the literature for "m( $\left.\nu_{e}\right)$ ", "m $\left(\nu_{\mu}\right) "$, and $" m\left(\nu_{\tau}\right)$ " are meaningful only, as limits on $m\left(\nu_{i}\right), i=1,2$, and 3 , respectively; (2) a neutrino mass limit cannot be given in isolation - it always contains some implicit dependence on the relevant lepton mixing angles. Fortunately, however, this dependence is relatively unimportant for the dominantly coupled decay modes, i.e., ev $\nu_{1} \mu \nu_{2}$, and $\tau \nu_{3}$, since $\left|U_{i j}\right|^{2}$ is close to unity for $i=j$. Since these decay modes were the ones responsible for the mass limits given previously, the latter can be reinterpreted without significant change or complication as proper limits on $m\left(\nu_{i}\right), i=1,2$, and 3 . This has been done in the Table.

Further neutrino mass limits arising from subdominantly coupled decay modes and other phenomena, such as neutrino oscillations, which involve $U_{i j}$ with $i \neq j$, are dependent upon the unknown lepton mixing angles, and hence we shall not consider them in the present edition.

\section{References}

1. S. Weinberg, Phys. Rev. Lett. 19, 1264 (1967); A. Salam, in Elementary Particle Theory: Relativistic Groups and Analyticity, edited by N. Svartholm (Alquist \& Wiksell, Stockholm, 


\section{Stable Particles}

$\nu, \nu_{e}, e, \nu_{\mu}$

1968), p.367. See also S. Glashow, Nucl. Phys. 22, 579 (1961); S. Glashow, J. Iliopoulos, and L. Maiani, Phys. Rev. D2, 1285 (1970); and, for the $n=3$ case, M. Kobayashi and T. Maskawa, Prog. Theor. Phys. 49, 652 (1973).

2. See, e.g., B. W. Lee and R. E. Shrock, Phys. Rev. D16, 1444 (1977).

3. J. Yang et al., Astrophys. J. 227, 697 (1979); see also footnote 4 in G. Steigman et al., Phys. Rev. Lett. 43, 239 (1979).

4. K. Berkvist, Nucl. Phys. B39, 317 (1972).

5. M. Daum et al., Phys. Rev. D20, 2692 (1979).

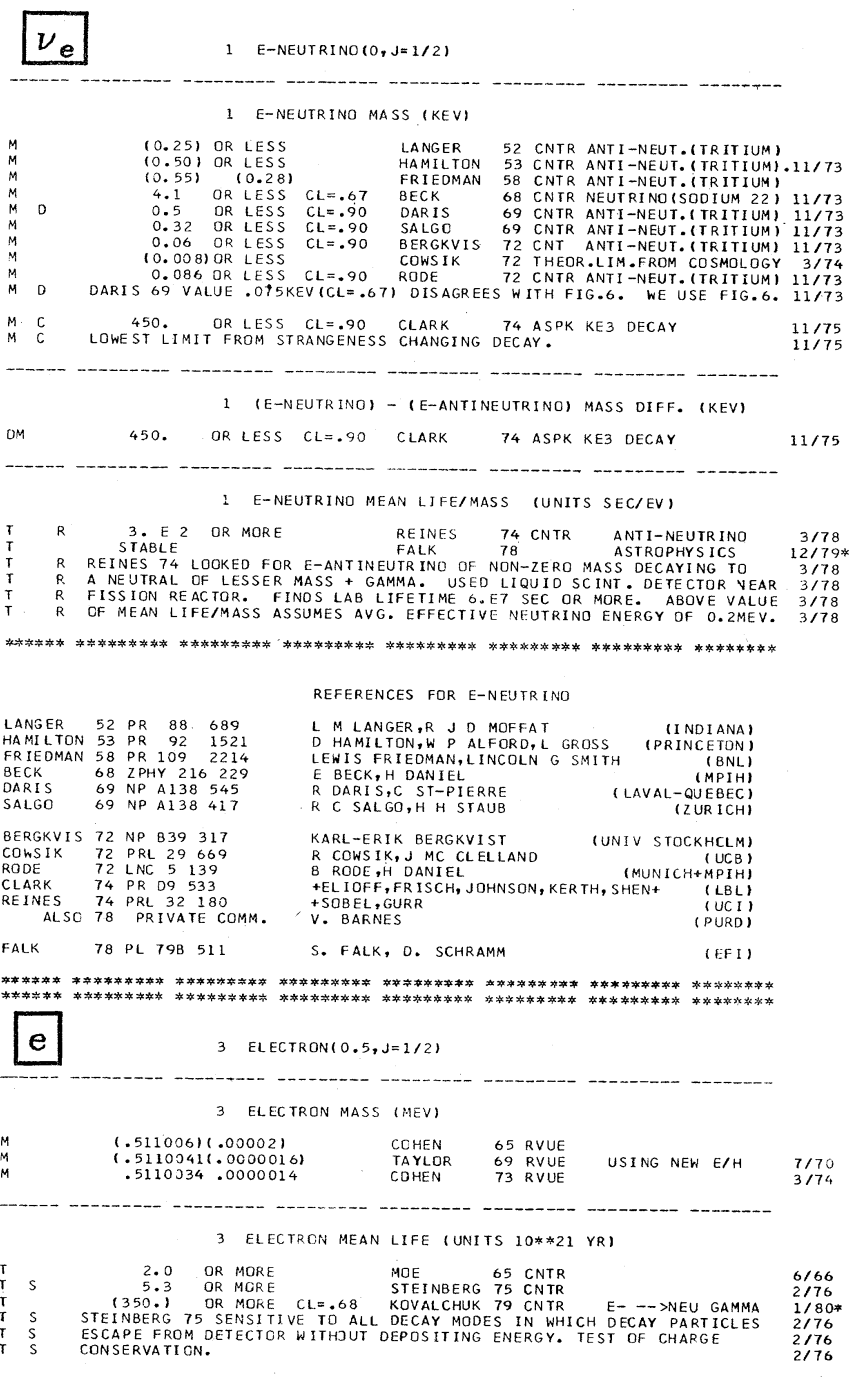

\section{Data Card Listings} For notation, see key at front of Listings.

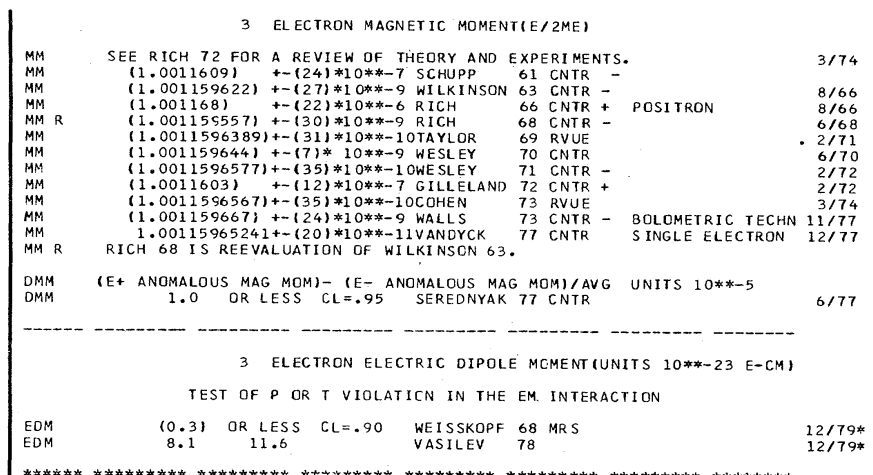

**************************************************************************1

REFERENCES FOR ELECTRON

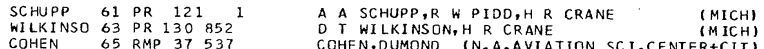

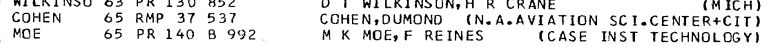

$\begin{array}{lllll}\text { RICH } & 66 & \text { PRL } & 17 & 271 \\ \text { RICH } & 68 & \text { PRL } & 20 & 967\end{array}$

$\begin{array}{lllll}\text { WEISSKCP } & 68 & \text { PRL } & 21 & 1645 \\ \text { TAYLOR } & 69 & \text { RMP } & 41 & 375\end{array}$

$\begin{array}{lllll}\text { WESLEY } & 70 & \text { PRL } & 24 & 1320 \\ \text { WESLEYY } & 71 & \text { PR } & 44 & 1341\end{array}$

$\begin{array}{lllll}\text { WESLEY } & 71 & \text { PR } & \text { A4 } & 1341 \\ \text { GILLELAN } 72 & \text { PR AS } & 38 \\ \text { RICH } & 72 & \text { RMP } & 44 & 250\end{array}$

$\begin{array}{llll}\text { COHEN } & 73 & \text { J.PHYS. CHEM.REF. } \\ \text { WALLS } & 73 & \text { PRL } 31975 \\ \text { STE } & 975 \text {. }\end{array}$

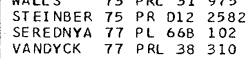

$\begin{array}{lllll}\text { VASILEV } & 78 & \text { JETP } & 47 & 243 \\ \text { KOVALCHU } & 79 & \text { JETPL } & 29 & 145\end{array}$

$A$
$A$ RICH, H H R CRANE

(MICHI
WEISSKOPF, CARRICO, GOULD, LLPWORTH+ (BRANI

(PRIN+UCI+PENN)

$J, C$ WESLEY, A.RICH
$J$
$J$

A RICH,J C WESLEY

KOVALCHUK + (JINR)
*\#A (NUCLEAR RESEARCH INST-USR)

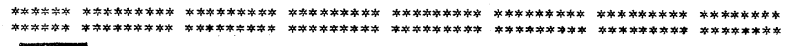




\section{Data Card Listings}

\section{For notation, see key at front of Listings.}

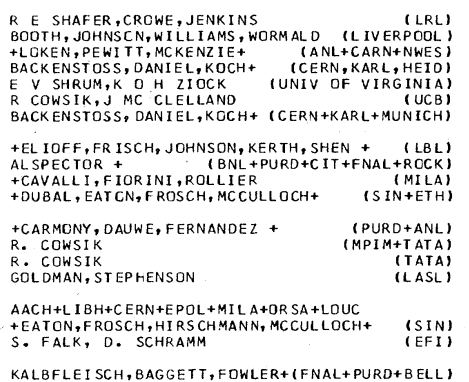

KALBFLEI SCH, BAGGETT, FOWLER+(FNAL+PURD+BELL) $\begin{array}{lllll}\text { DAUA } & 78 & \text { PL } & 748 & 126 \\ \text { FALK } & 78 & \text { PL } 798 & 511\end{array}$

KALBFLEI 79 PRL. 431361 $\mu$

4 MUON(106, $J=1 / 2)$

4 MUON IMASS (MEV)

$M$
$M$
$M$
$M$
$M$
$M$
$M$
$M$
$M$
$M$
$M$
$M$
$M$
$M$
$M$
$M$
-
-

$T$
$T$
$T$
$T$
$T$
$T$
$T$
$T$
$T$
$T$
$T$
$T$
$T$
$T$
$T$
$T$
$T$

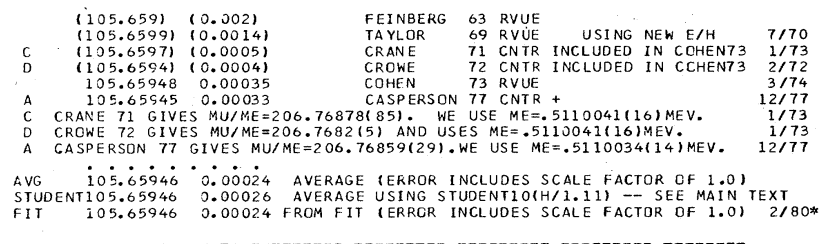

4 MUON MEAN LIFE (UNITS 10**-6)

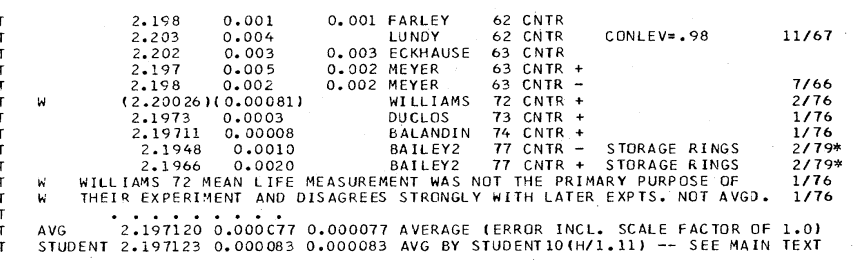

4 MU+/MU- MEAN LIFE RATIO

$\begin{array}{llllllll}\text { DT } & 1.000 & 0.001 & \text { MEYER } & 63 & \text { CNTR } & \text { MEAN LIFE MUT/MU } & 7 / 66 \\ \text { OT } & 1.0008 & 0.0010 & \text { BAILEY } & 79 & \text { CNTR } & \text { STORAGE RING } & 7 / 79 *\end{array}$

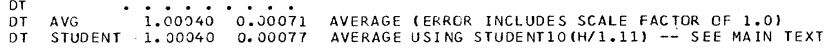

4 MUON ANOMALOUS MAGN. MOMENT (10**-6*E/(2*MU MASS))

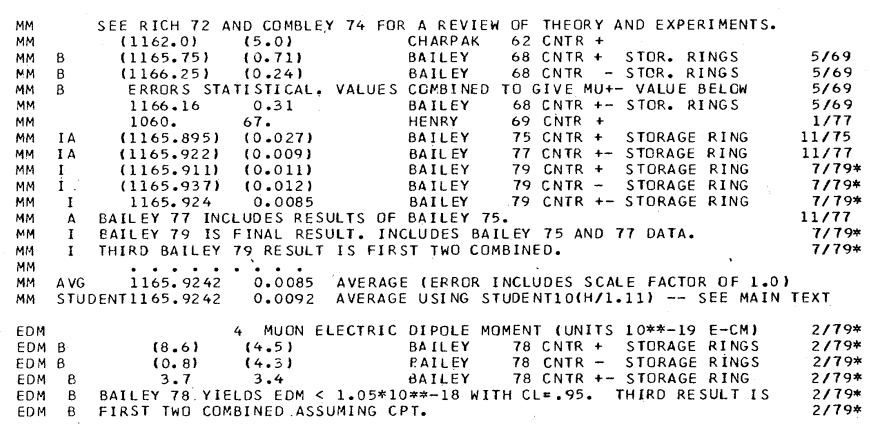

4 MUCN TO PROTON MAGNet IC MOMENT RATIO
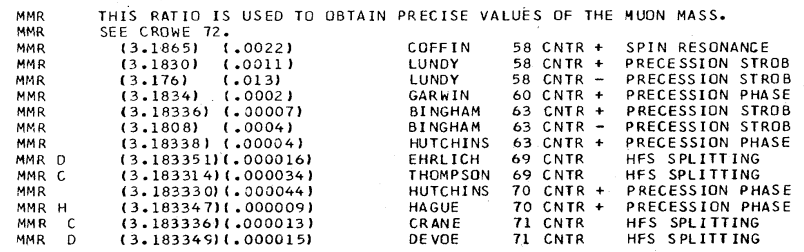

\section{Stable Particles}

$\nu_{\mu}, \mu$

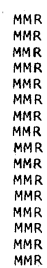

13.18332011.0000131

TE RESUITS T.000008

3.1833402 .0000072

$.1833403: 0000044$

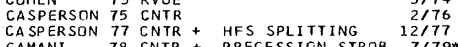

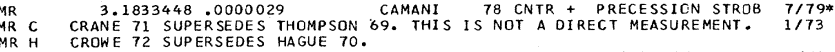

FMR F FAVART 71 ASSUMES A ZERO VALUE FOR THE PROTON POL ARI ZABILITY.

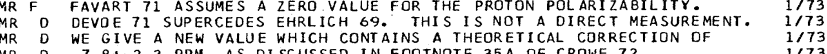

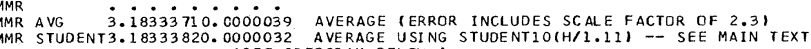
WEIGHTED AUERAGE $=3.1833371 \pm 0.0000039$ ERROR SCALED BY 2.3

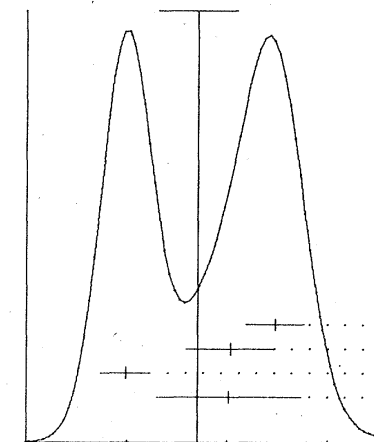

CAMANI 2 B CNTR $\frac{\text { CHISQ }}{7.1}$ CASPERSEN 77 CNTR 0.5 - CASPERSEN 75 CNTR $\quad$ G.2 $\begin{array}{lllllll}3.1833 & 3.1833 & 3.1833 & 3.1833 & 3.1834 & 3.1834 & \text { (CONLEU } \\ =0.001\end{array}$ MUDN TI PRITUN MAGNETIC MUMENT RATIO

4. MUON PARTIAL DECAY MODES

MUON INTO E ANTIIE-NEU) (MU-NEU)

MUON INTO BELECTRONS

MUCN INTO E GAMMA

MUON INTO E (E-NEU) ANTI I MU-NEU)
MUON INTO E ANTI(E-NEU) (MU-NEU) GAMMA

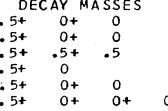

4 MUCN BRANCHING RATIOS

MUON INTO E+2 GAMMA (IN UNITS OF 10**-5) 63 OSPK (P2)/(P1)

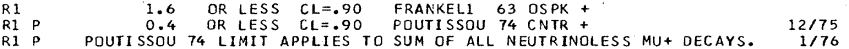
MUON INTO 3 E (IN UNITS OF $10 * *-7) \quad$ (P3)/(P1)

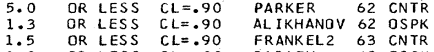

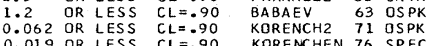

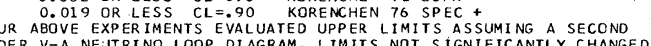

$2 / 72$

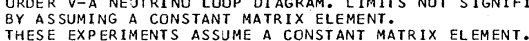

MUON INTO E+GAMMA IIN UNITS OF $10 * *-8)$ (P4)/(P)

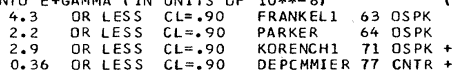

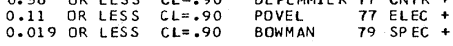

MUON+ INTO (E+ (E-ANT INEU) (MU-NEU)) (P5)/(P1)

FORBI IDDEN BY ADDI TIVE CONSERVATIVATION LAW FOR MUON
MUL TIPL ICATIVE LAW PREDICTS R $4=0.5$

MUON INTO E ANT IIE-NEU) (MU-NEU) GAMMA ASHKIN 27 FVENTS SEEN (P6)/(P))

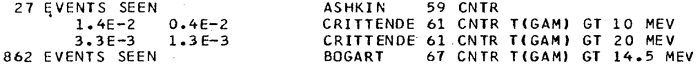

$$
4 \text { MUON DECAY PARAMETERS }
$$

RELATED TEXT SECTION VI A

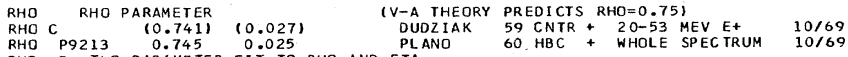
PHO
RHO TWO PARAMETER FI 0.725 TO RHO AND ETAANO PHO C $2276(0.751)(10.034) \quad$ BLOCK 62 HEBC - WHOLE SPECTRUM $10 / 69$

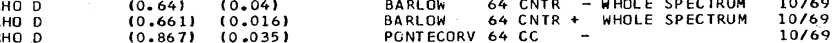
RHO D

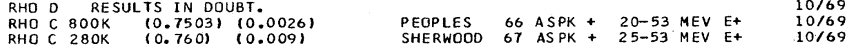

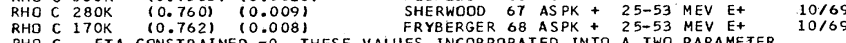

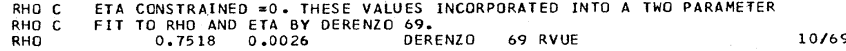

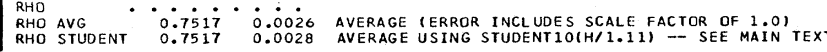




\section{Stable Particles}

$\mu, \nu_{\tau}, \tau^{ \pm}$

\section{Data Card Listings For notation, see key at front of Listings.}

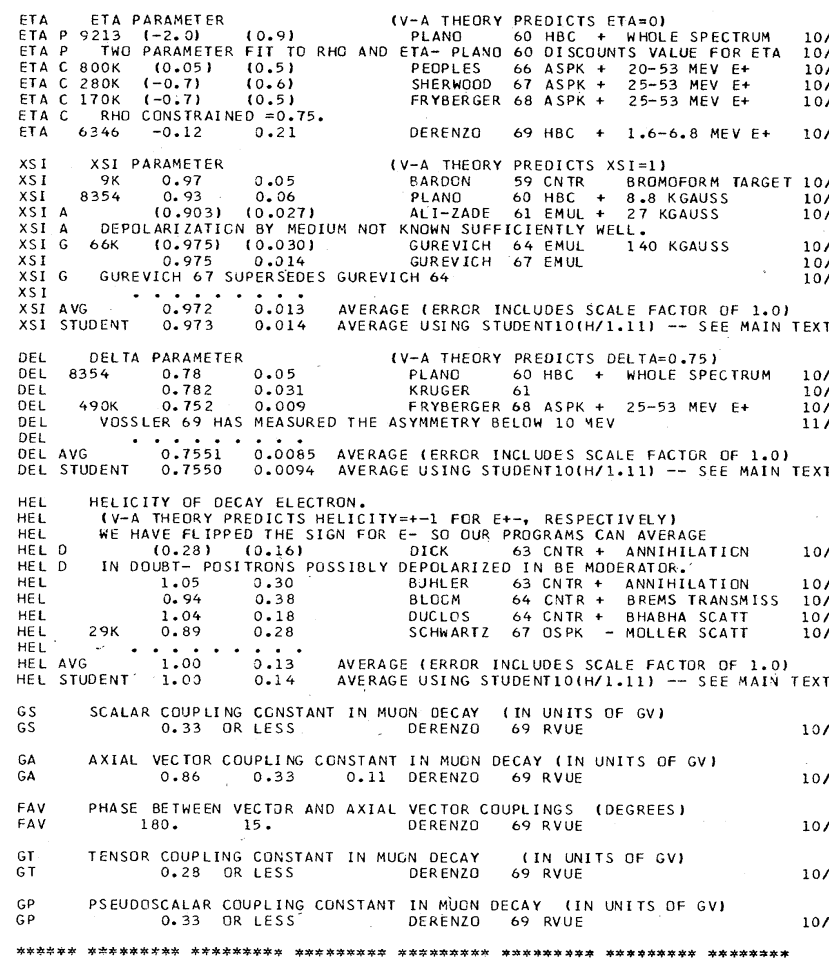




\section{Data Card Listings}

For notation, see key at front of Listings.

Stable Particles

$\tau^{ \pm}, \pi^{ \pm}$

$P_{10}$
$P_{11}$
$P_{12}$
$P_{13}$
$P_{14}$
$P_{15}$
$P_{16}$

TAUt- INTO NEUCTAUI A1t1100 \%+-

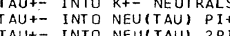

TAUt- INTO NEUUTAUI 2 2PIt- PI-+ IINCL. P9, P1O)

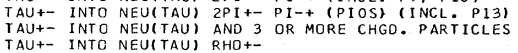

$0+1100$

$0+139$
$0+139+139+139$

o+ 776

\section{FITTED PARTIAL DECAY MODE BRANCHING FRACTIONS}

The matrix below is derived from the error matrix for the fitted partial decay mode branching fractions, $P_{i}$, as follows: The diagonal elements are $P_{i} \pm \delta P_{i}$, where

$\delta P_{i}=\sqrt{\left\langle\delta P_{i} \delta P_{i}\right\rangle}$, while the off-diagonal elements are the normalized correlation coefficients $\left\langle\delta P_{i} \delta P_{j}\right\rangle /\left(\delta P_{i} \cdot \delta P_{j}\right\rangle$. For the definitions of the individual $P_{i}$ ' see the listings above; only those $P_{i}$ appearing in the matrix are assumed in the fit to be nonzero and are thus constrained to add to 1 .

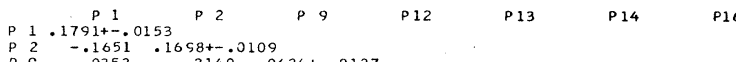

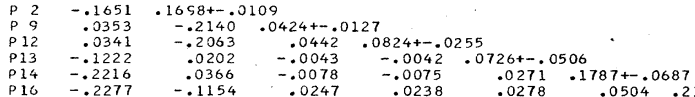

\section{TAU BRANCHING RATIOS}

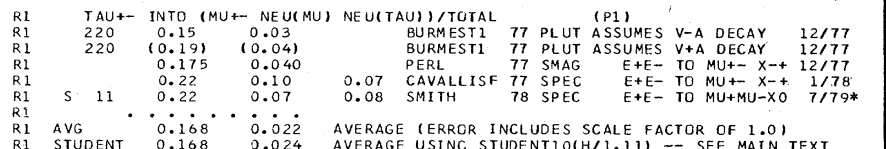

$\begin{array}{llllll}\text { R1 } & \text { AVG } & 0.168 & 0.022 & \text { AVERAGE } & \text { (ERROR INCLUDES SCALE FACTOR OF } 1.01 \\ \text { R1 } & \text { STUDENT } & 0.168 & 0.024 & \text { AVERAGE USINC STUDENIOCH/1.11) S SEE MAIN TEXT } \\ \text { R1 } & \text { FIT } & 0.179 & 0.015 & \text { FREM FIT (ERROR INENUDES SCALE FACTOR OF } 1.01\end{array}$

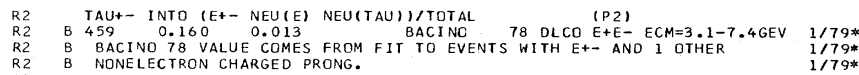

R2 FIT $\cdot 0.10^{\circ} \cdot 0.011$ FROM Fir (ERROR INCL UDES SCALE FACTOR OF 1.01

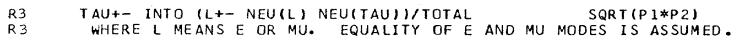

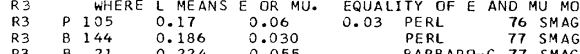

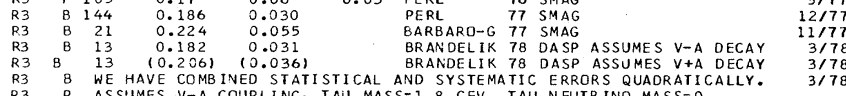

R3
R3 ASSUMES V-A COUPLING, TAU MASS $=1.8$ GEV, TAU NEUTR INO MASS $=0$.
B ASSUMES V-A COUPLING, TAU MASS $=1.9$ GEV, TAU NEUTRINO MASS $=0$.

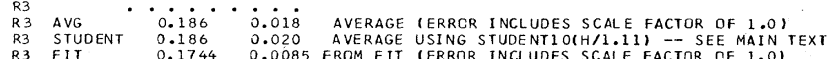

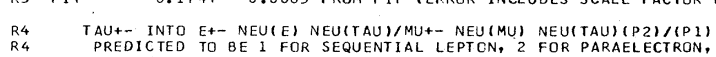

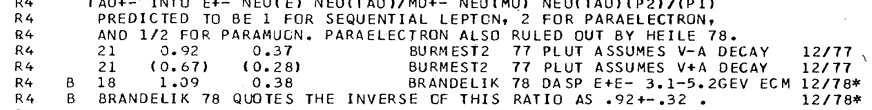

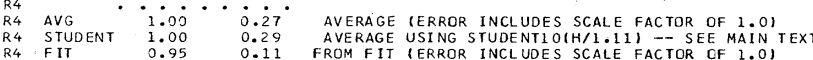

R5 TAU+- INTO (MU+- NEU(MU) NEU(TAU) ) *(E)- NEU(E) NEUITAU) $(P 1) *(P 2)$

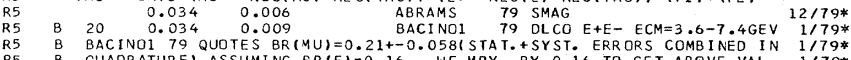

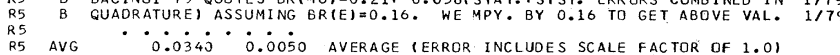

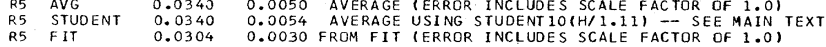

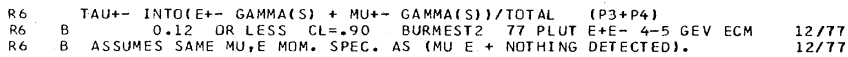

R7 TAU+- INTOLE+- CHARGED PRONG + MU+- CHARGEO PRONG)/TOTAL (P5+PG)

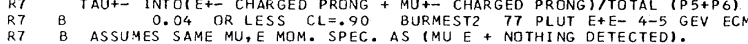

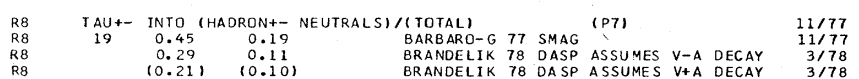

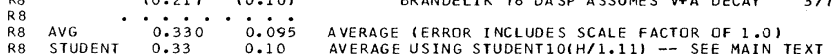

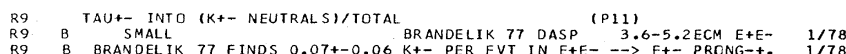

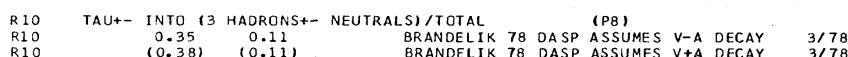

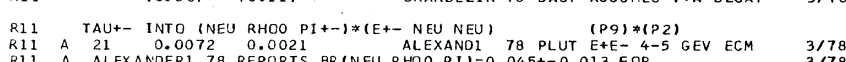

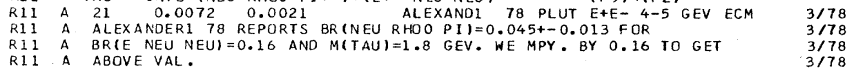

R11 FIT ${ }_{0}$ : $0072 \cdot 0.0021$ FROM FIT (ERRCR INCLUDES SCALE FACTOR OF 1.0 )
R12 TAU+- INTO (NEU A1(11001+-)/TOTAL
R12 A 21 (10.10) $(0.03)$ (P10)

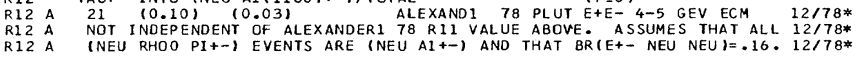

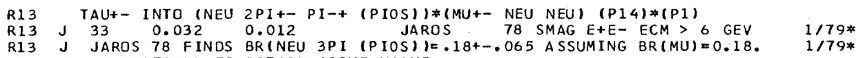

R13 J WE MULTIPLY TO OBTAIN ABOVE VALUE.

- $0.032^{\cdots} \cdot \dot{0} . \dot{0} 12$ from fit (error includes Scale factor of 1.01

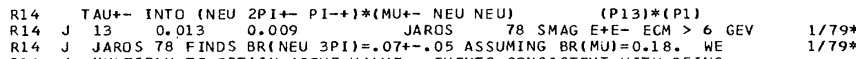

R14 J JAROS 78 FINDS BR(NEU 3PI) $=.07+-.05$ ASSUMING BR(MU) $=0.18$. WE
R14 J MULTIPLY TO OBTAIN ABOVE VALUE. EVENTS CONSISTENT WITH BEING

R14 FIT 0.0130 0.0090 FROM FIT (ERROR INCLUDES SCALE FACTOR OF 1.0 )

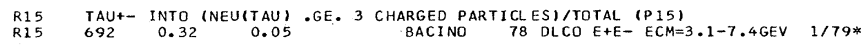

R16 TAU+- INTO (NEU(TAU) PI +-)*(E+- NEU(E) NEU(TAU)) (P12)*(P2)
R16 A 23
0.015

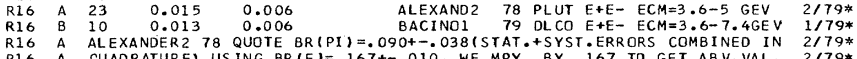

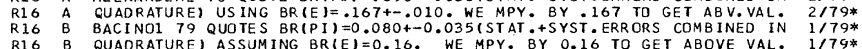

R16 B QUADRATURE) ASSUMING BR $(E)=0.16$. WE MPY. BY 0.16 TO GET ABOVE VAL.

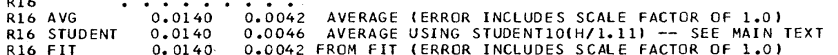

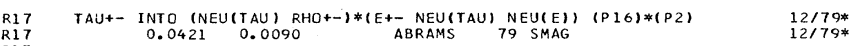

R17 FIT - $0.036 \dot{9} \cdot 0.0069$ FROM Fit (ERROR INCLUDES SCALE Factor of 1.01

$\begin{array}{llll}\text { R18 TAU+- INTO (NEU(TAU) RHO+-)*(MU+- NEU(TAU) NEU(MU)) (P16)*(P1) } & 12 / 79 * \\ \text { R18 } & 0.0329 & 0.0100 & \text { ABRAMS }\end{array}$

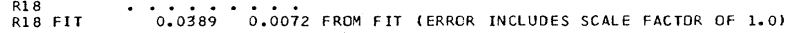

*********************************************************************1

$\begin{array}{lll}\text { PERL } & 75 \text { PRL } 35 & 1489 \\ \text { PERL } & 76 \text { PL } 6384660\end{array}$

REFERENCES FOR TAUU(1800, HEAVY LEPTON

BARBARO- 77 PRL 391058

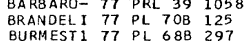

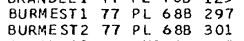

$\begin{array}{llll}\text { CAVALLIS } & 77 & \text { LNC } 20 & 337 \\ \text { PERL } & \text { PL } 70 B & 487\end{array}$

+ ABRAMS, BOYARSKI, BRE IDENBACH
+ FELOMAN, ABR AMS, ALAM, BOYARSKI
+

BARBARO-GALTIERI+ (LBL+NWES+SLAC+HAWA)

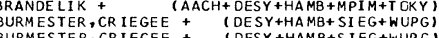

BURMESTER, CRIEGEE + (DESY +HAMB+SIEG +WUPG)

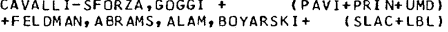

AL EXANDER, CR IEGEE+ (DESY+AACH+SIEG+WUPG)

+FERGUSON, NODULMAN + (UCLA+SLAC+UCI+STON)
+DITYMANN, DUINKER, OLSSON, ONEILL+(DESY+HEID)

ALEXAND 278 PL 78 PL 788 B 78

$\begin{array}{lllll}\text { BACINO } & 78 & \text { PRL } & 41 & 13 \\ \text { BARTEL } & 78 & \text { PL } 778 & 331\end{array}$

$\begin{array}{lllll}\text { BRANDEL I } & 78 & \text { PL } & 738 & 109 \\ \text { HEILE } & 78 & \text { NP } & B 138 & 189\end{array}$

BRANDEL IK + (AACH+DESY+HAMB+ MPIM+TCKY)
+ PERL, ABRAMS, AL AM, BOYARSKI+
(SLAC+LBL) +ABRAMS, ALAM+ (SLAC+LBL+NWES + HAWAI

$\begin{array}{lllll}\text { SAROS } & 78 & \text { PRL } & 40 & 1120 \\ \text { SMITH } & 78 & \text { PR } & 18 & 1\end{array}$

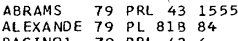

AALAM, BLOCKER, BOYARSKI+ (SLAC+LBL)

ALEXANER + (DESY+AACH+HAMB+SIEG+WUPP)

+FERGUSON, NODULMAN + (UCLA+SLAC+UCI+STON)
+ FERGUSON, NODULMAN +

REVIEWS

$\begin{array}{lll}\text { PERL2 } & 77 \text { HAMBURG SYMP. } & \text { ALSO ISSUED AS SLAC-PUB-2022, M.PERL (SLAC) } \\ \text { FLUGGE } & 77 \text { MESON CONF.BOSTON ALSO ISSUED AS DESY 77-35, G.FLUGGE } \\ \text { (DESY) }\end{array}$

AZIMOV 78 SPU 21225 TFRANKFURT, KHOZE 25 (LENI)

$\begin{array}{lllll}\text { FELDMAN } & 78 & \text { SLAC-PUB-224 } & \text { G.J.FELDMAN (TORYO CONF.1978) } & \text { (SLAC) } \\ \text { PERL } & 78 & \text { SLAC-PUB-2219 } & \text { M. L. PERL (KARLSRUHE SUMMER INST.1978) (SLAC) }\end{array}$

$\begin{array}{lllll}\text { FLUGGE } & 79 & 2 P \text { C1 } 121 & \text { G.FLUGGE } & \text { (DESY) } \\ \text { KIRKBY } 79 & \text { SLAC-PUB-2419 } & \text { J.KIRKBY } & \text { (SLAC) }\end{array}$

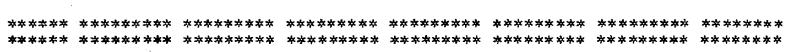

$\pi^{ \pm} \quad 8$ CHARGED PION(140, JPG $=0--1 \quad I=1$

8 CHARGED PION MASS (MEV)

$\begin{array}{llll}139.37 & 0.20 & \text { CROWE } & 54 \text { CNTR } \\ 139.68 & 0.15 & \text { BARKAS } & 56 \text { EMUL + } \\ 139.5771 & 0.0131 & \text { SHAFER } & 57 \\ 1 & \text { CNTR }\end{array}$

$(139.577) \quad(0.013)$

$\begin{array}{ll}139.566 & 0.013 \\ 139.569 & 0.008\end{array}$

$\begin{array}{ll}139.571 & 0.010 \\ 139.5686 & 0.0020\end{array}$

$\begin{array}{lll}139.5667 & 0.0024 \\ 139.5652) & (0.0019)\end{array}$

SHAFER

71 CNTR - MESGNIC ATOMS

BACKNSTO 72 CNTR - MESONIC ATCMS
CNTR - MESONIC ATOMS

BRANDADDO 76 CNTR - MESONIC ATCMS
CARTER 76 CNTR - MESONIC ATOMS

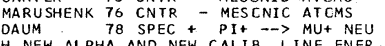

S SHAFER 72 UPDATES SHAFERG 7 WITH NEW ALPHA AND NEW CALIB. LINE ENER.
$B$ BACKENSTOSS 73 CORRECTS BACKENSTOSS 71 WITH NEW VACUUM POL. CALC.

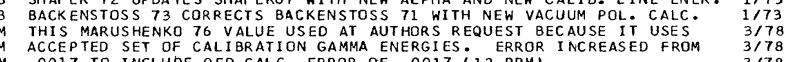

DAUM 78 VALUE DEPENDS ON ASSUMED MU+ MASS M(MU) $=105.65948+\cdots 00035.2 / 778$

$M \quad$ AVG $139.5679^{\circ} 0.0015$ AVERAGE (ERROR INCLUDES SCALE FACTOR OF 1.01

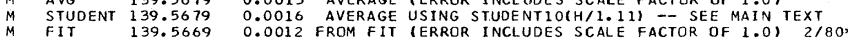




\section{Stable Particles} $\pi^{ \pm}, \pi^{0}$

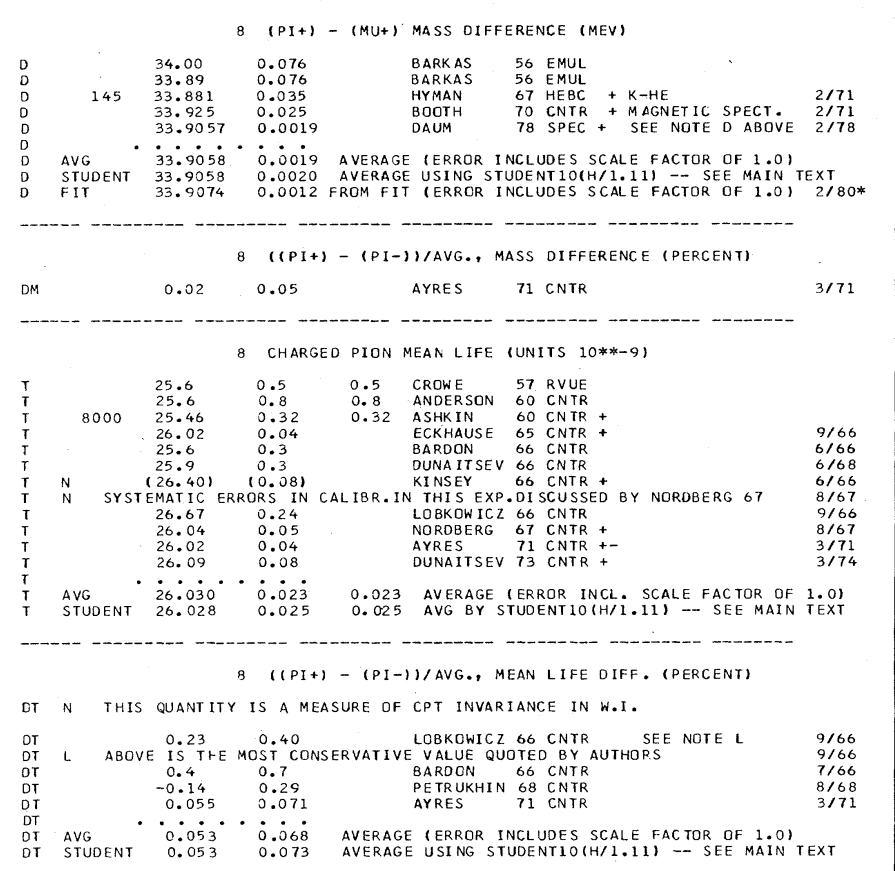

8 CHARGED PION PARTIAL. DECAY MODES

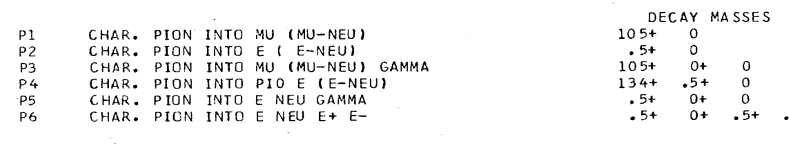

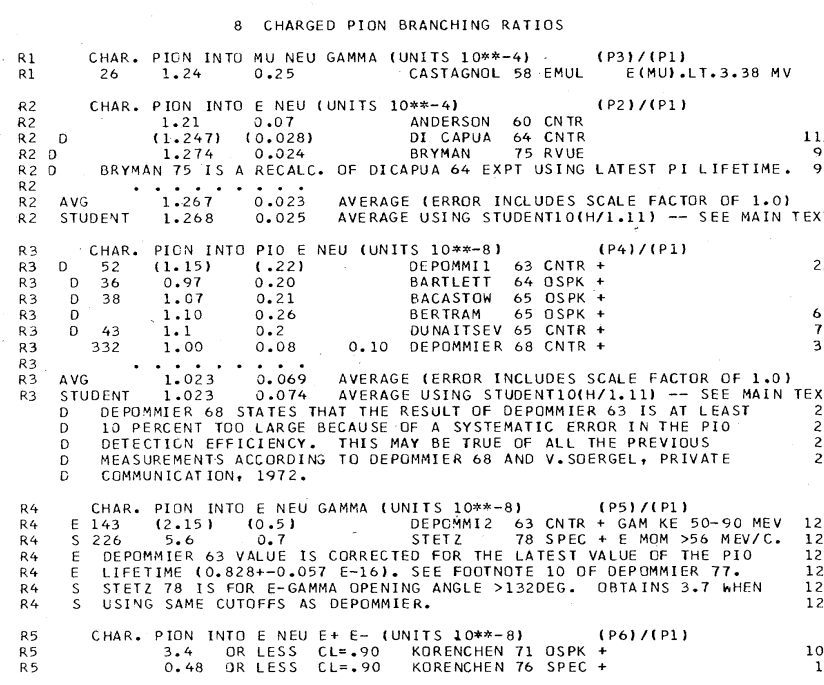

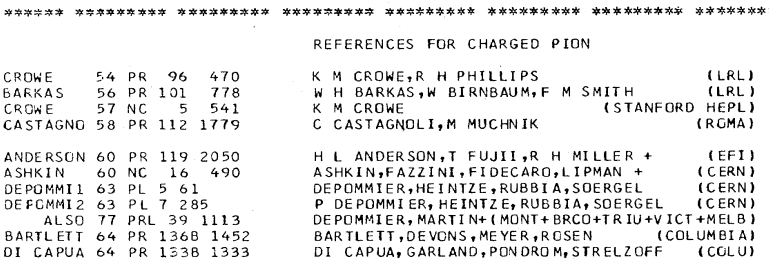

\section{Data Card Listings} For notation, see key at front of Listings.

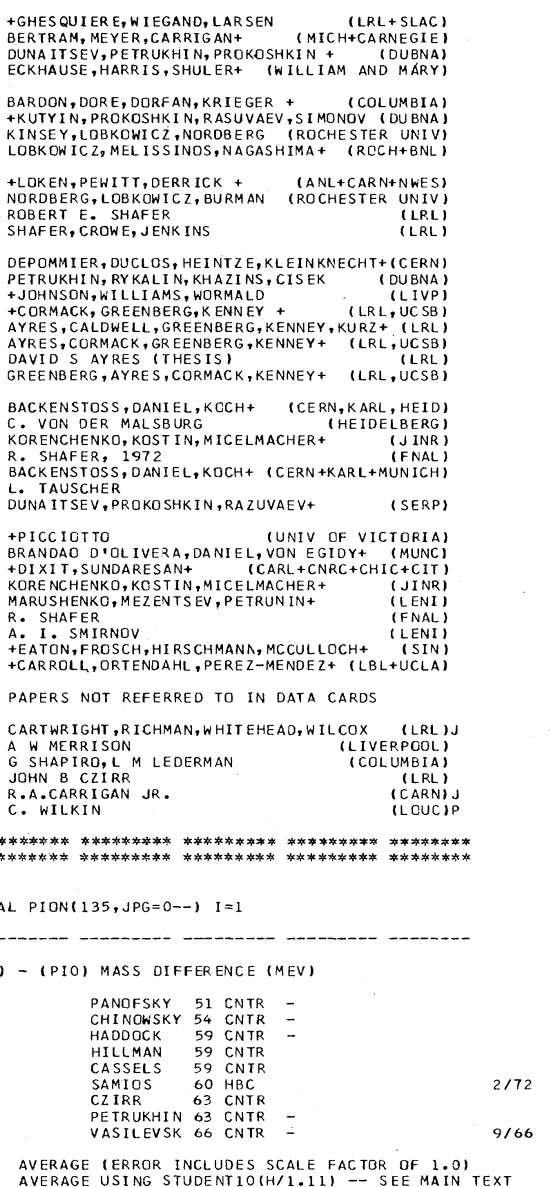

BACASTOW 65 PR 1398407

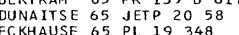

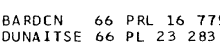

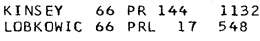

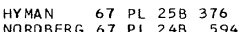
SHAFER 67 PR $163 \quad 145$

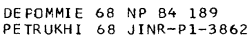
BOOTH
AYRES ALSO 67 PR $157 \quad 1288$

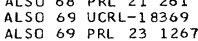
BACKENST 71 PL $36 B \quad 403$

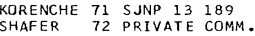
BACKENST 73 PL 438539

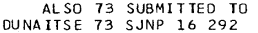
BRYMAN 75 PR 0111337 CARTER 76 PRL 371380 KORENCHE 76 JETP 4435 ALSO 76 PRIVATE COMM
ALSO 78 PRIVATE COMM.

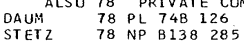

CARTWRIG 53 PR 91677

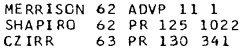

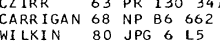

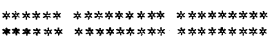

$\pi^{0}$

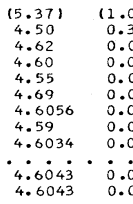
$11 / 75$
975
$9 / 75$ r $2 / 72$ $6 / 66$
$7 / 66$
$3 / 68$ TEXT

2
2722
172
272
172
172

\begin{tabular}{|l|l}
$12 / 77$ \\
$12 / 79 *$ \\
$12 / 77$ \\
$12 / 77$ \\
$12179 *$ \\
$2 / 79 *$ \\
$10 / 71$ \\
$1 / 78$
\end{tabular}

8

$\mid$

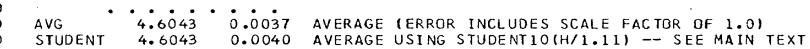

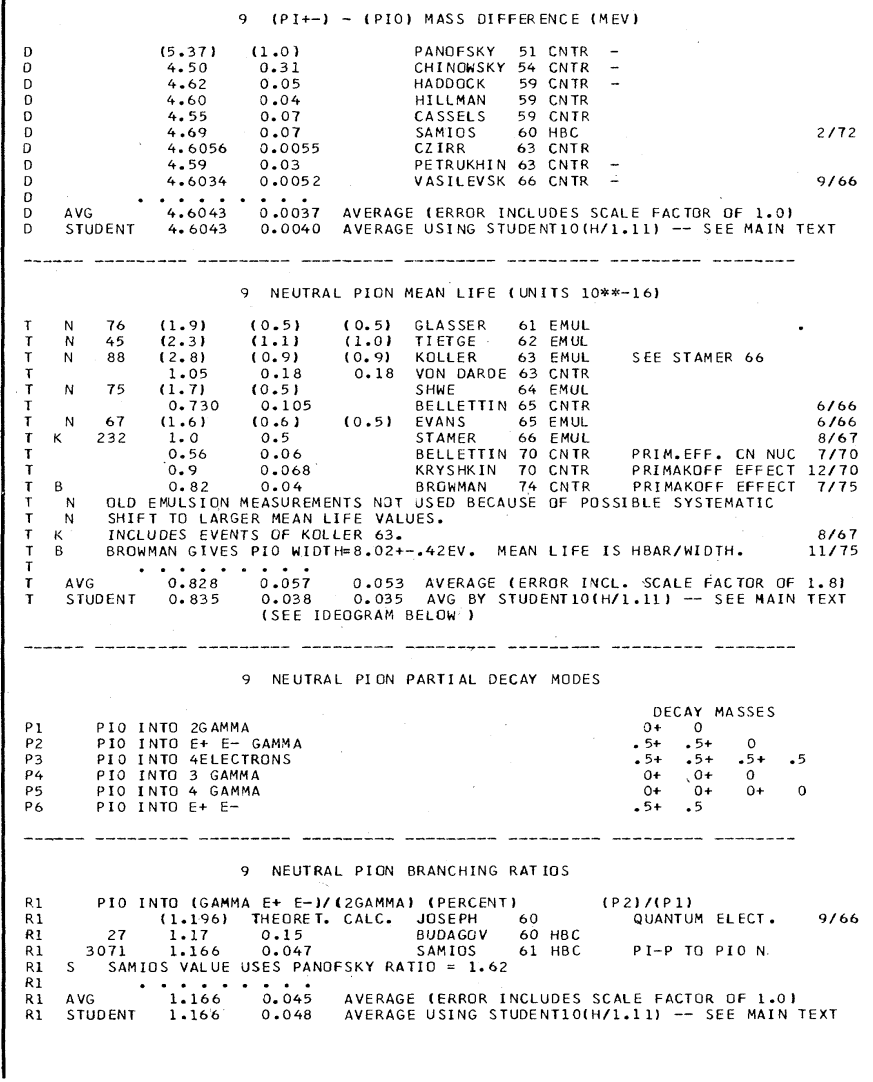




\section{Data Card Listings}

Stable Particles

For notation, see key at front of Listings.

$\pi^{0}, \eta$

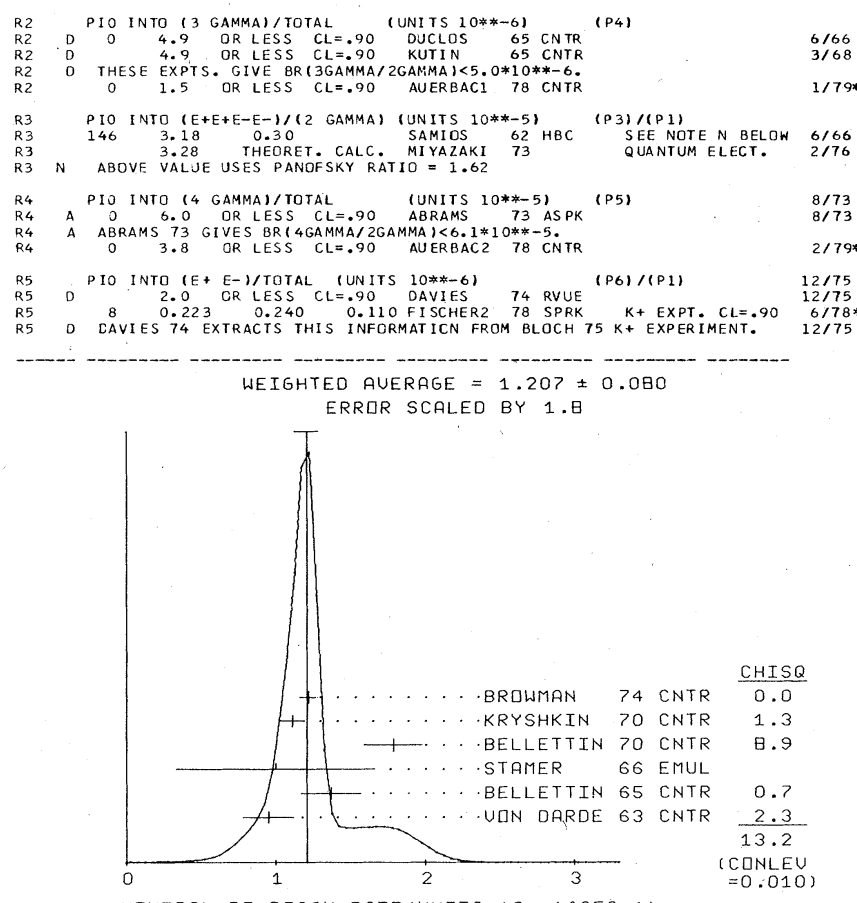

-NEUTRAL PI DECAY RATE(UNITS 10**16SEC-1)

9 NEUTRAL PION ELECTROMAGNETIC FORM FACTOR

THE AMPLITUDE FOR THE PROCESS PIO $\rightarrow-E_{+}$E- GAMMA CONT AINS A FORM FACTOR GAMMA(X**2) AT THE (PIO GAMMA GAMMA) VERTEX

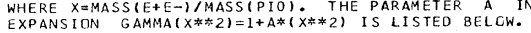

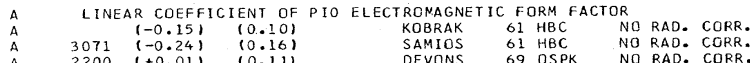

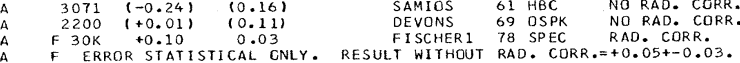

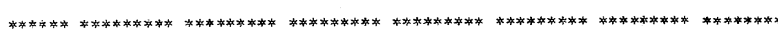

REFERENCES FOR NEUTRAL PION

$\begin{array}{lllll}\text { PANGFSKY } & 51 & \text { PR } & 81 & 565 \\ \text { CHINOWSK } & 54 & \text { PR } & 93 & 586 \\ \text { CA SSELS } & 59 & \text { PPS } & 74 & 92\end{array}$

$\begin{array}{lllll}\text { CASSELS } & 59 & \text { PPS } & 74 & 92 \\ \text { HADOOCK } & 59 & \text { PRL } & 3 & 478 \\ \text { HILMAN } & 59 & \text { NC } & 14 & 887\end{array}$

BUDAGOV 60 JETP 11755

SAMIOS 60 NC 18154

GLASSER $\quad 61 P R \quad 1231014$

$\begin{array}{llllll}\text { KOBRAK } & 61 & N C & 20 & 1115 \\ \text { SAMIOS } & 61 & P R & 121 & 275 \\ \text { SAMIOS } & 62 & P R & 126 & 1244\end{array}$

$\begin{array}{lllll}\text { SAMIOS } & 62 & P R & 126 & 1844 \\ \text { TIEIGE } & 62 & P R & 127 & 1324\end{array}$

$\begin{array}{lrrrrr}\text { CZIKR } & 63 & P R & 130 & 341 \\ \text { KOLLER } & 63 & \text { NC } & 27 & 1405\end{array}$

ALSO 66 STAMER
PETRUKHI 63 SIENA CONF 208
VCN DARD 63 PI

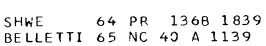

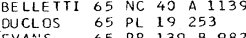

$\begin{array}{ll}\text { EVANS } & 65 \text { PR } 139 \text { B } 982 \\ \text { KUTIN } & 65 \text { JETP LETT } 2243\end{array}$

$\begin{array}{lrrrr}\text { STAMER } & 66 & P R & 151 & 1108 \\ \text { VASILEVS } & 66 & P L & 23 & 281\end{array}$

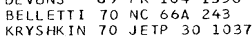

ABRAMS $73 \mathrm{PL} \quad 458 \quad 66$

$\begin{array}{lllll}\text { BROWMAN } & 74 & \text { PRL } & 33 & 1400 \\ \text { CAVIES } & 74 & \text { NC } & 24 A & 324\end{array}$

$\begin{array}{lllll}\text { AUEREAC1 } & 78 & \text { PRL } & 41 & 275 \\ \text { AUERBAC } & 78 & \text { PL } & 788 & 353 \\ \text { FISCHER } & 78 & \text { PL } 733 & 359\end{array}$

W K H PANOFSKY,R L AAMODT, J HADLEY (LRL)

W CHINOWSKY, STEINBERGER
CASSELS, JONES, MURPHY, O.NEILL (COLUMBIA)
CIVERPOOL)

HADDOCK, ABASHIAN, CROWE, CZIRR
HILLMAN, MIDDELKOOP, YAMAGATA, ZAVATTINIICERN)

BUDAGOV, VIKTOR, DZHELEPOV, ERMOL OV + (JINR)

D W JOSEPH
$N$ P SAMIOS
(COLUMBIA)

$R$ G GLASSER, N SEEMAN, B STILLER (NRL)
H.KOBRAK (EFI)

(COLUMBIA+BN)
SAMIOS,PLANO, PRODELL + (COLUMBIA+BNL)

(LRL)
JOHN B CZIRR
(SCLLER, SAYLOR, T HUETTER TENS)

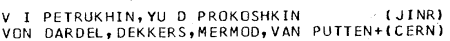

H SHWE, F M SMITH, WH BARKAS (LRL)

DUCLOS, FREYTAG, HEINTZE + (CERN+HEIDELBERG)

OA EVANS
KUTIN, PETRUKHIN, PROKOSHKIN (OXFORO)
(JINR)

STAMER, TAYLOR, KOLL ER, HUETTER+
VASILE VSKY, VI SHNYAKOV, DUNAITSEV + (STEVENS)
(DUENA)

TEEENTHY, NISSIM-SABAT, DI CAPUA+ (COLU + PCMAI

BELLETIINI, BEMPORAD, LUBELSMEY+ (PISA+BCNN)
+STERLIGOV,USOV (TCMSK POLYTECH.INST.)

+CARROLL, KYCIA,LI, MICHAEL, MOCKETT + (BNL)

+DEWIRE, GITTELMAN, HANSON+" (CORN+BING)

+GUY, LIA (BIRMTRHEL+SHMP)

+AUERBACH, HI GHLAND, JOHNSON, +
+AUERBACH, HI GHLAND, JOHNSON, +
(TEMP+LASL)

$\begin{array}{ll}\text { TEXTERMAN, GUISAN, MERMOD, + } & \text { (GEVA+SACL) } \\ \text { +EXTERMANN, GUI SAN, MER MOD, MOREL + (GEVA+SACL) }\end{array}$

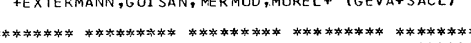

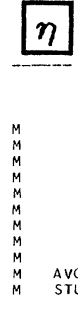

14 ETA(549, JPG $=0-+) \quad I=0$ 14 ETA MASS (MEV)

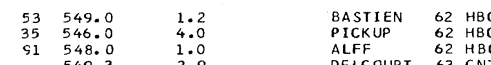

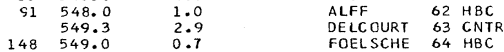

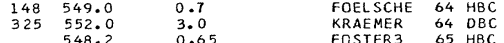

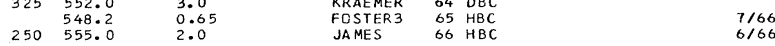

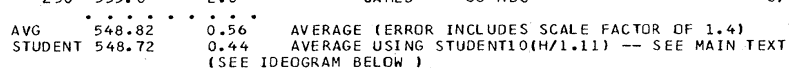

WEIGHTED AUERAGE $=54$ 日. $22 \pm 0.56$

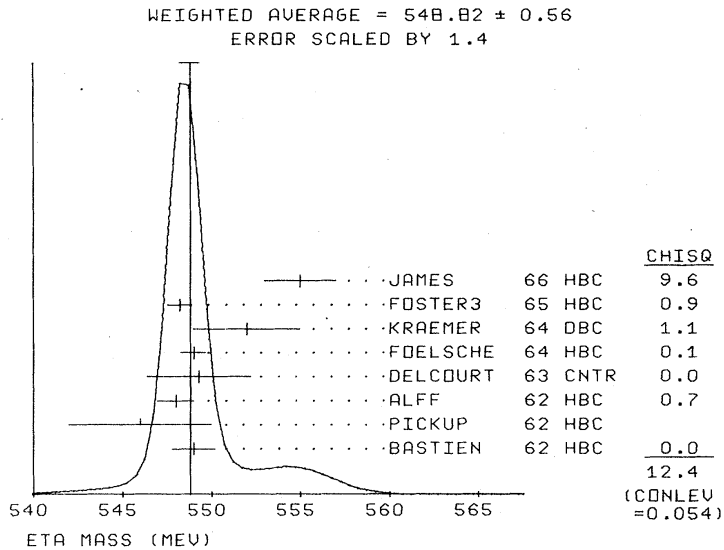

ETA MASS (MEU)

14 ETA WIDTH

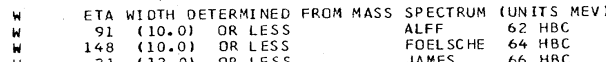

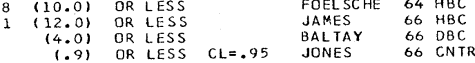

ETA WIOTH OETERMINED FROM DECAY RATE (UNITS KEV)
THIS IS THE PARTIAL DECAY RATE (WI) FOR THE MODE (ETA INTO 2GAMMA

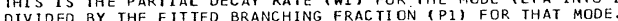

FIT $\bigcup_{0.85}^{\circ} \cdot 0^{\circ}$. $^{\circ}$ fROM FIT

14 ETA PARTIAL DECAY MODES

ETA INTO 3 PIO
ETA INTO PI+ PI- PIO

ETA INTO PI+ PI- GAMMA
ETA INTO E+ E- PIO (VIOLATES C IN E.M.I.)

ETA INTO E+ E- PIO $V$ VIOL

ETA INTO PIO ZGAMMA

ETA INTO E+ E- GAMMA
ETA INTO 2 PIO GAMMA (VIOLATES C)

ETA INTO PI+ PI- PIO GAMMA
ETA INTO PI+ PI- 2GAMMA

ETA INTO MU+ MU-

ETA INTO MU+ MU- GAMMA
ETA INTO MU+ MU- PIO
ETA INTO $I_{+}$PI-

ETA INTO PI+ PI-

DECAY MASSES

$0+130$
$134+134+134$
$139+139+134$

$\begin{array}{lll}134+ & .5+ & .5 \\ 139+139+ & .5+\end{array}$

$\begin{array}{ccc}139+139+ & -5+ \\ 134+ & 0+ & 0\end{array}$

$\frac{5+}{134+}+\frac{.5+}{134+}$

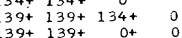

P15

FITTED PARTIAL DECAY MODE BRANCHING FRACTIONS

The matrix below is derived from the error matrix for the fitted partial decay mode ranching fractions, $P_{i}$, as follows: The diagonal elements are $P_{i} \pm \delta P_{i}$, where

$\delta P_{i}=\sqrt{\left\langle\delta P_{i} \delta P_{i}\right\rangle}$, while the off-diagonal elements are the normalized correlation coeffi-

cients $\left\langle\delta P_{i} \delta P_{j}\right\rangle /\left(\delta P_{i} \cdot \delta P_{j}\right)$. For the definitions of the individual $P_{i}$, see the listings

above; only those $P_{i}$ appearing in the matrix are assumed in the fit to be nonzero and

are thus constrained to add to 1 .

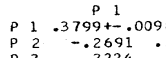

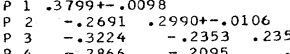

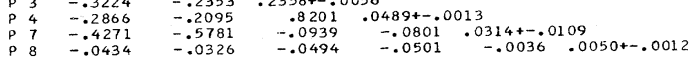




\section{Stable Particles}

$\eta$

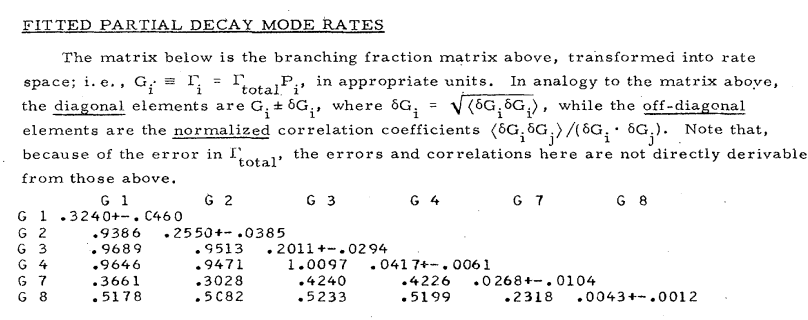

\section{ETA DECAY RATES}

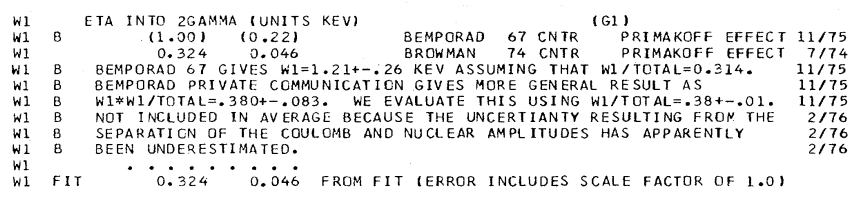

W1 FIT $\cdot 0.324^{\cdots} \cdot 0.00 \dot{6}^{\circ}$ FROM FIT IERROR INCLUDES SCALE FACTOR OF 1.01

14 ETA BRANCHING RATIOS

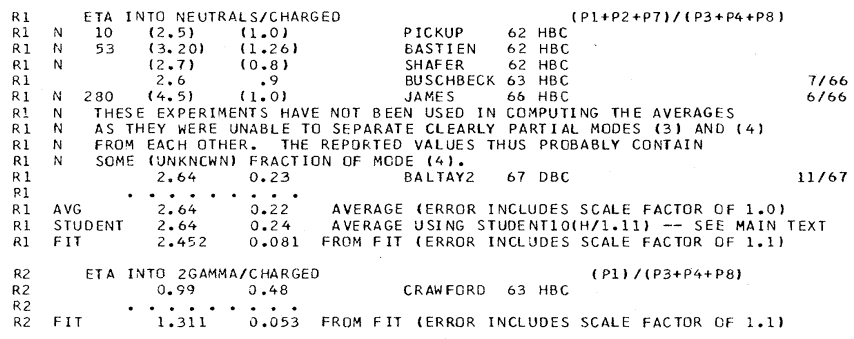

Note on $n \rightarrow \pi^{0} \gamma \gamma$

The discrepancies between various measurements of branching ratios involving $n \rightarrow \pi^{0} \gamma \gamma$ are displayed in the ideogram below, in which all relevant experiments have been converted to a common ratio, $\pi^{0} \gamma r /$ neutrals. Our branching ratio fit does not include DIGIUGNO 66, FELDMAN 67, or the upper limit measurements. See page 43 of "Review of Particle Properties", Physics Letters 39B, No. 1 (1972) for more discussion.

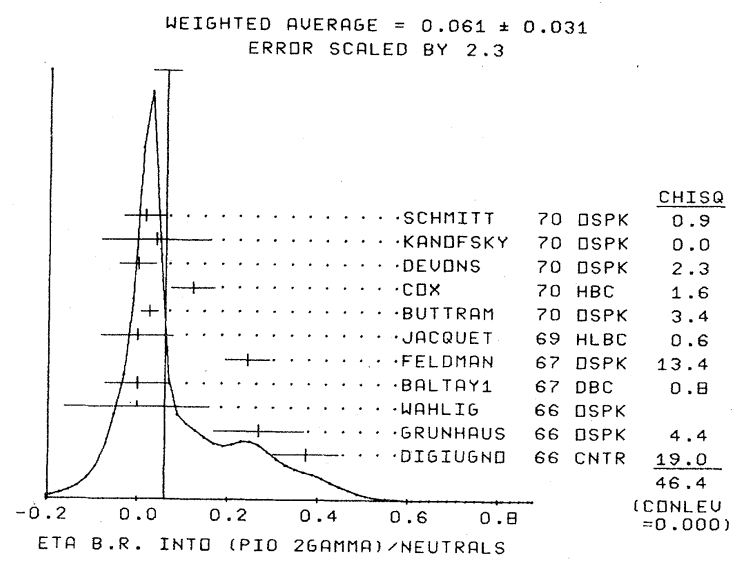

Data Card Listings For notation, see key at front of Listings.
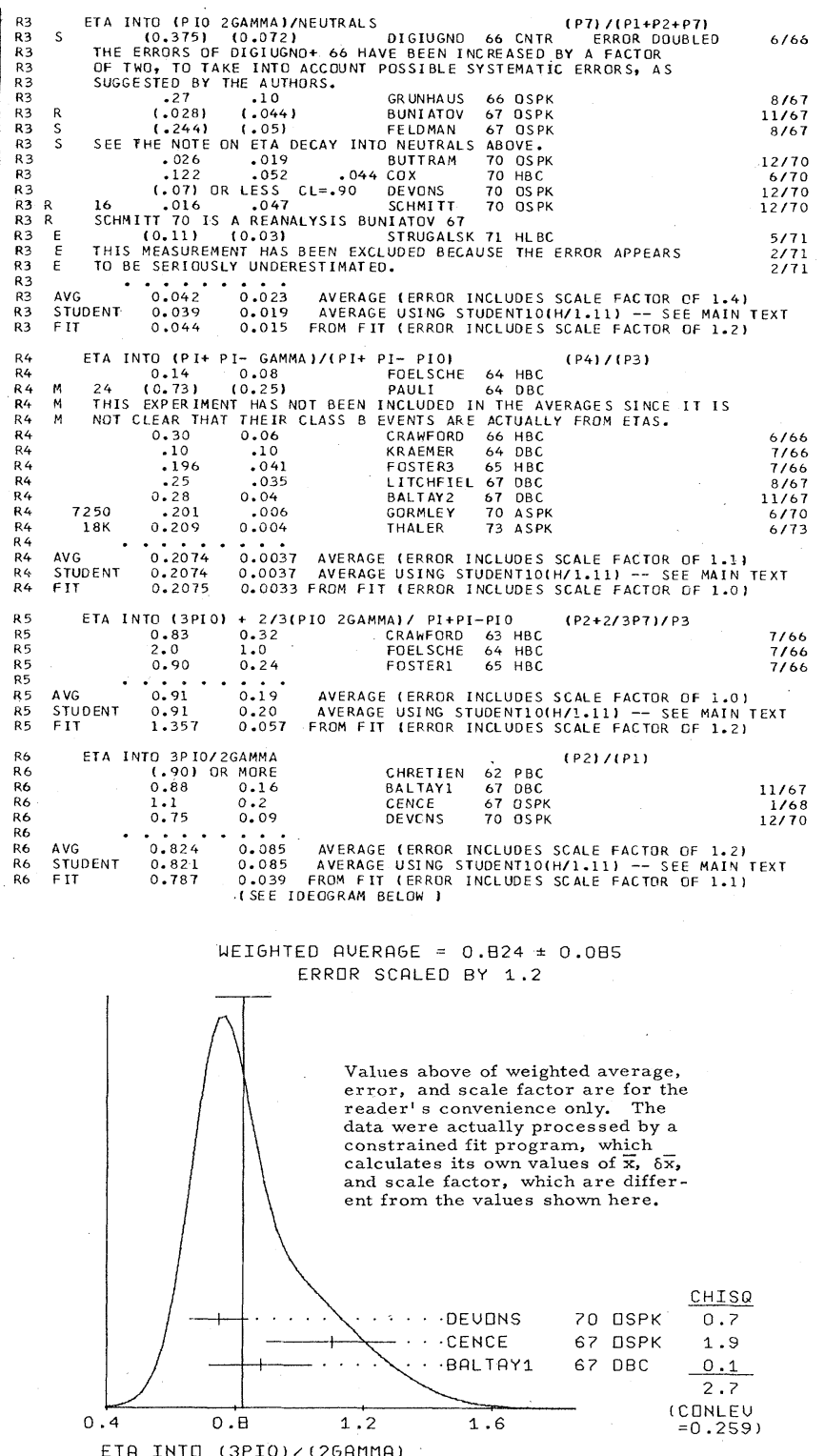

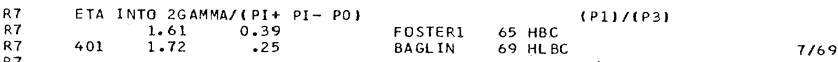

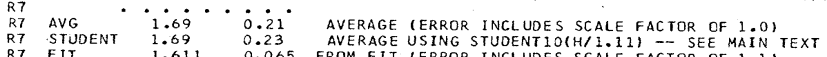

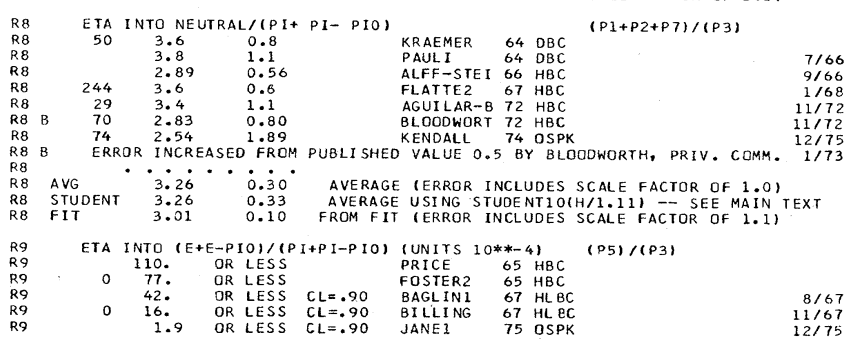




\section{Data Card Listings}

Stable Particles

\section{For notation, see key at front of Listings.}

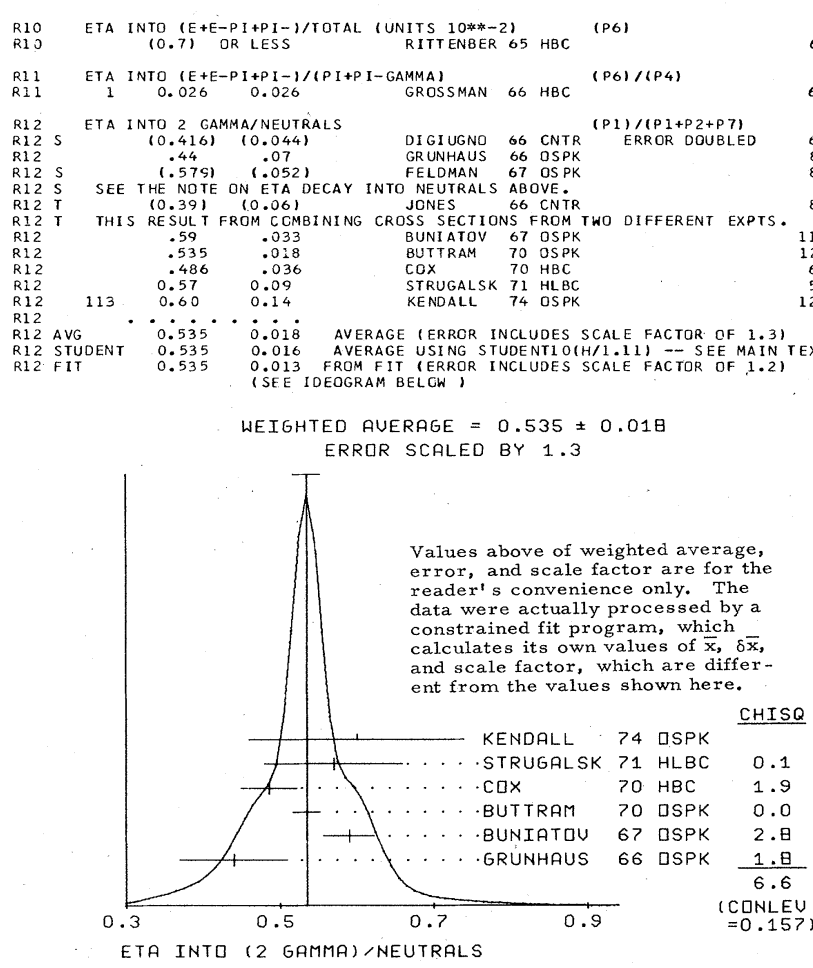

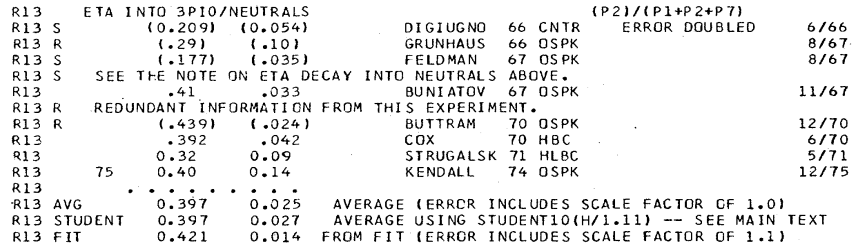

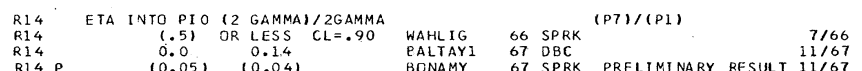
R14
R14 FIT
R14 Fit 0.083 $\cdot 0.030$ FROM FIT (ERROR INCLUDES SCALE FaCtOR OF 1.21 R15 ETA INTO (E+E-PIO)/TOTAL (UNITS $10 * * * 2)$
R15 O
0.7 OR LESS

$\begin{array}{lllll}R 15 & 0.784 \text { OR LESS } & C L=.90 & \text { RAZIN } & 68 \mathrm{HBC} \\ \text { R15 } & 0.084 & \text { OR LES } \\ \text { R15 } & 0.016 \text { OR LESS } & \text { CL }=.90 & \text { MARTYNOV } & 76 \mathrm{HLEC}\end{array}$

R16 ETA INTO 2GAMMA/ $(3 P 10+P 10$ 2GAMMA)
R16
0.80
BACCI

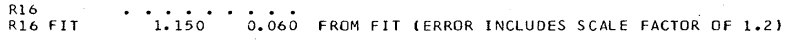

R17 ETA INTO (PI+PI-PIO GAMMA)/(PI+PI-PIO) (UNITS 10**-2)

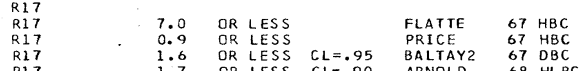

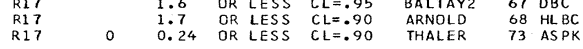

R18 ETA INTO (PI+PI- 2GAMMA)/(PI+PI-PIO)

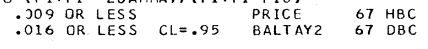

AGLIN2 67 HLBC $\left(P_{2}\right) /\left(P_{3}\right)$

$$
\begin{array}{llllll}
1.3 & .4 & & \text { BAGLIN2 } & 67 & \text { HLBC } \\
1.47 & 0.20 & 0.17 & \text { BULLOCK } & 68 & \text { HLBC } \\
1.50 & .15 & .29 & \text { EAGLIN } & 69 & \text { HLBC }
\end{array}
$$

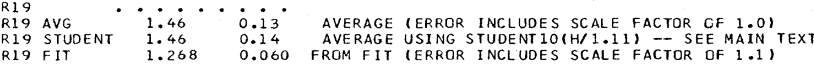

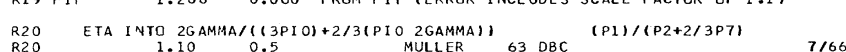

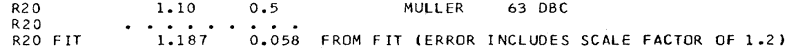

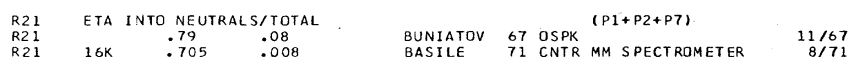
R21
R21 AVG ${ }_{0.7058}^{.705} \cdot 0.0080$ AVERAGE (ERROR INCLUDES SCALE FAC TOR OF 1.0 ) $\begin{array}{llll}\text { R21 STUDENT } & 0.7058 & 0.0086 & \text { AVERAGE USI NG STUDENT1O(H/1.11) - SEE MAIN TEXT } \\ \text { R21 FII } & 0.7103 & 0.0068 & \text { FROM FIT IERRCR INCLUDES SCALE FACTOR OF } 1.1 \text { (1) }\end{array}$

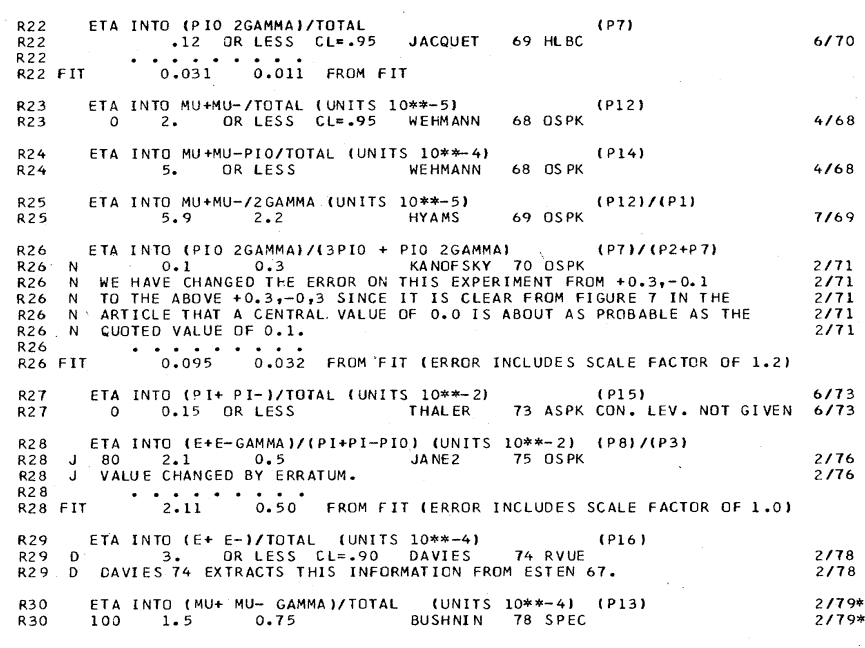

14 ETA C-NONCONSERVING DECAY PARAMETERS

RELATED TEXT SECTION VI C. 1

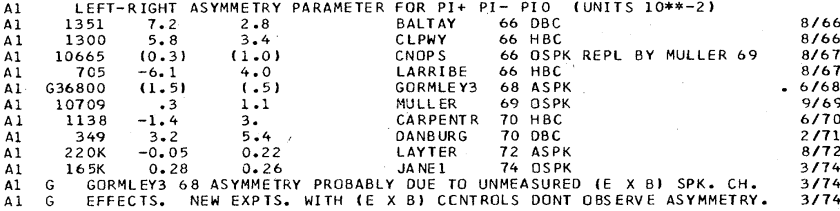

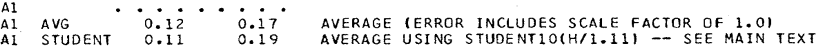

A2 LEFT-RIGHT ASYMMETRY PARAMETER FOR PI+ PI-GAMMA (UNITS 10**-2)

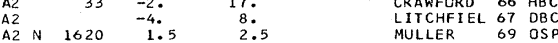

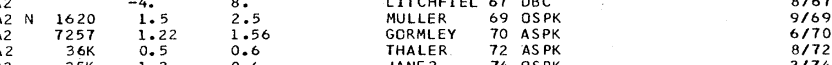

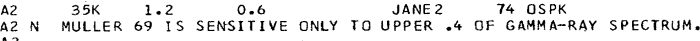

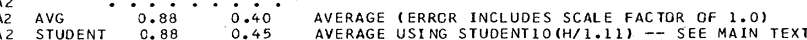

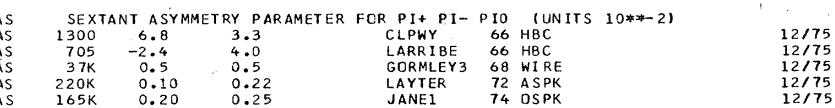

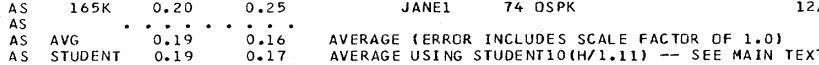

AQ QUADRANT ASYMMETRY PARAMETER FOR PI+ PI- PIO (UNITS 10**-2)

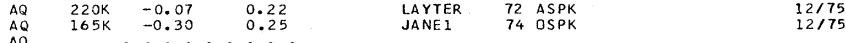

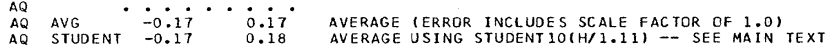
BET BETA FOR ETA TO PI+ PI- GAMMA. SENSITIVE TO D-WAVE CONTRIBUTION. $\begin{array}{lllll}\text { BET } & 7250 & -0.060 & 0.065 & \text { GORMLEY } 70 \text { WI RE } \\ \text { BET L } & 0.12 & 0.06 & \text { THALER } 72 \text { ASPK }\end{array}$ $\begin{array}{ll}B E T & L \\ B E T & L\end{array}$ $\begin{array}{ll} & \\ \text { OEP ENDENGE MAY ALSO COME FRCM P ANO F-WAVE INTERFERENCE. } & 12 / 7\end{array}$ $\begin{array}{llll}\text { BET AVG } & 0.047^{\circ} & 0.062 & \text { AVERAGE (ERROR INCLUDES SCALE FACTOR OF } 1.51 \\ \text { BET STUDENT } & 0.053 & 0.053 & \text { AVERAGE USING STUDENTIOIH/1.11) -- SEE MAIN TEXT } \\ \text { [SEE IDEOGRAM BEL OWI, }\end{array}$ 14 ENERGY DEPENDENCE DF ETA DALITZ PLOT RELATED IEXT SECTION VI C. 2
THE FOLLOWING EXPTS FIT TO ONE

ONE OR MORE DF THE COEFFICIENTS

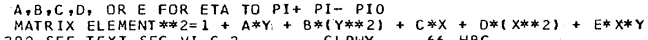
$\begin{array}{lll}1300 & \text { SEE TEXT SEC VI C.2 } & \text { CLPWY } \\ 705 \text { SEE TEXT SEC VI C.2 } & \text { HBC } \\ 7 & \text { LARRIBE } 66 \mathrm{HBC}\end{array}$

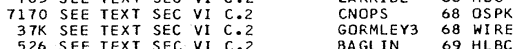

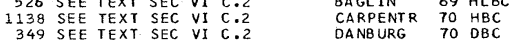

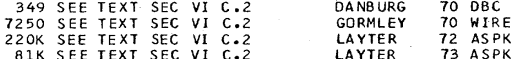




\section{Stable Particles}

$\eta, \mathrm{K}^{ \pm}$

BETA FOR ETA TQ PI+ PI- GAMMA

\section{Data Card Listings For notation, see key at front of Listings.}

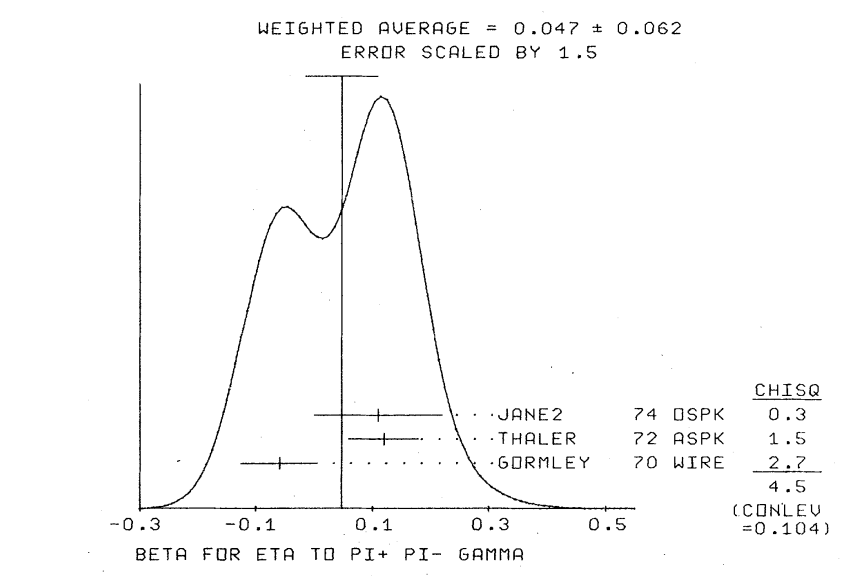

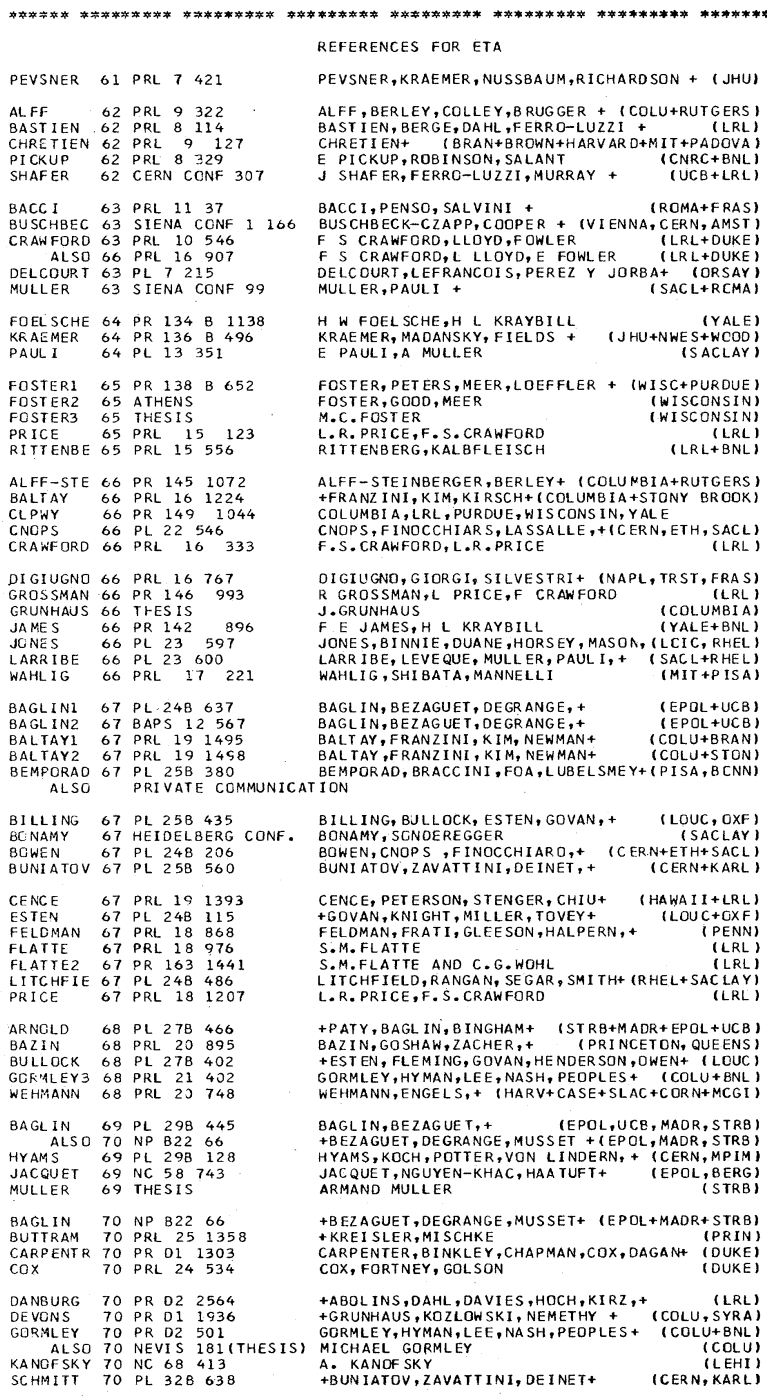

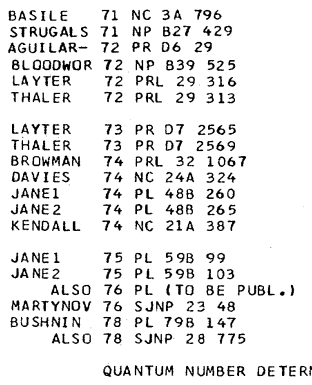

+BOLLINI, DALPIAZ, FRABETTI+ (CERN, BGNA, STRB) +CHUVILO, GEMESY, IVANOVSKAYA+
AGUILAR-BENI TEZ, CHUNG, EISNER, SAMIOS (JINR)
(BNL) BLOOOWORTH, JACKSON, PRENTICE, YOON (TORONTO)
+APPEL, KOTLEWSI, LEE, STEIN, THALR (COLU)
+APPEL, KOTLEWSKI, LAYTER, LEE, STEIN
(CCLU) +APPEL, KOTLEWSKI, LEE, STEIN, THALER (COLU) +DEWIRE, GITTELMAN, HANS ON, LOH + (CORN+BING) +GUY, ZIA I T
+ JONES, LIPMAN, OWEN, PENNEY+ (BHEL +LOWC+SUSS) + JONE S, LI PMAN, OWEN, PENNEY+ (RHEL+LOWC+SUSS)
+ +LANOU, MASSIMO, SHAPIRO + (BROW+BARI+MIT) +GRANNIS, JONES, LIPMAN, OWEN +
+GRANNIS, RONES, RHEL + LCWC)
(GRMAN OWEN + ERRATUM, M.R.JANE, PRIVATE COMMUNICATION. +SALTYKOV, TARA SOV, UZHINSKI I
+DZHELYADIN, GOLOVK IN, GR ITSUCK + (IINR)
(SERP) BUSHNIN, GOLOVKIN, GRITSUK, OZHELYADIN+ (SERP) QUANTUM NUMBER DETERMINATIONS NOT REFERRED TO IN THE DATA CARDS $\begin{array}{llllll}\text { BASTIEN } & 62 & \text { PRL } & 8 & 114 \\ \text { CARMONY } & 62 & \text { PRL } & 8 & 117\end{array}$

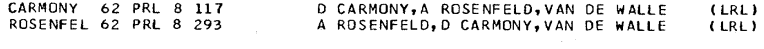

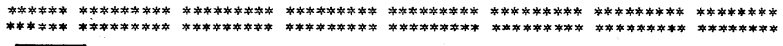
$\mathrm{K}^{ \pm}$ 10 CHARGED $K(494, J P=0-) \quad I=1 / 2$ 10 CHARGED K MASS (MEV)

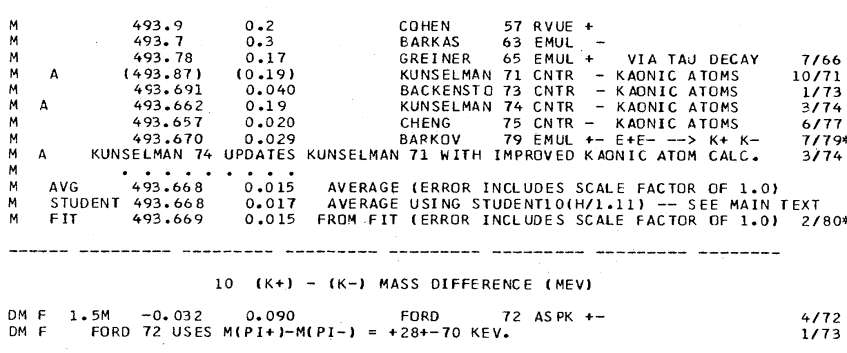

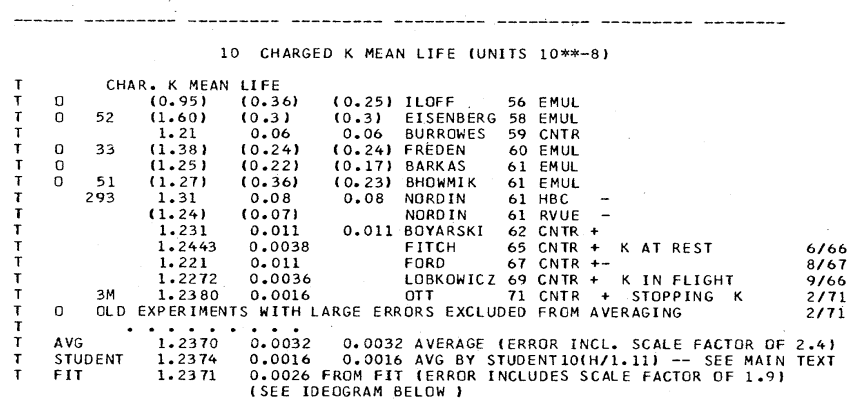

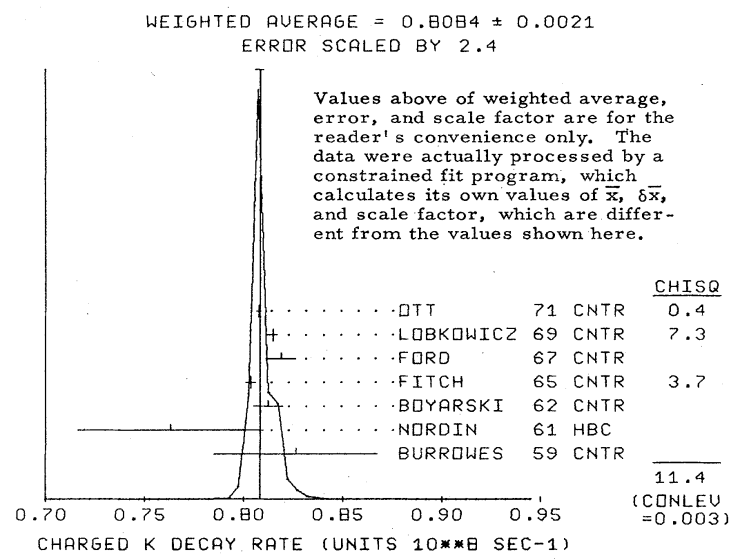




\section{Data Card Listings \\ For notation, see key at front of Listings.}

Stable Particles

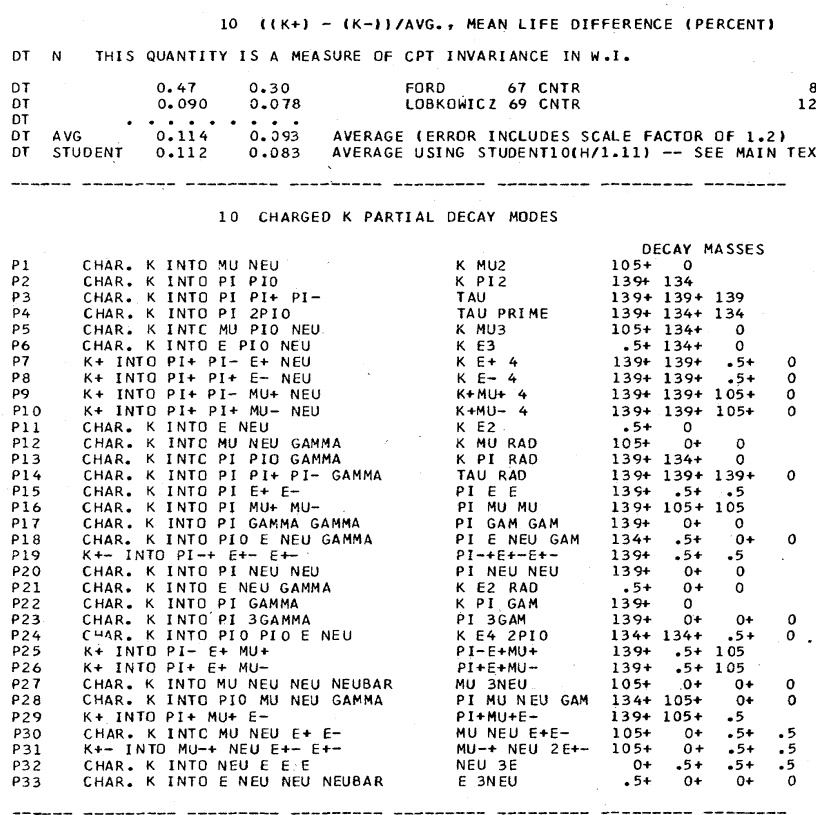

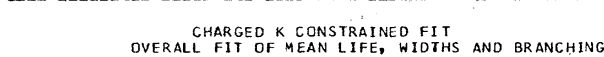

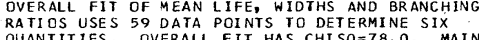

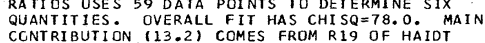 CONT
71 THE SEE NO REASON TO REJECT THIS EXPERIMENT
AT THS THEI}

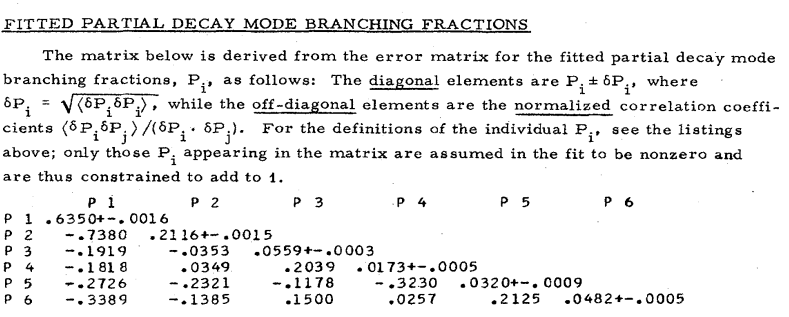

FITTED PARTIAL DECAY MODE RATES

The matrix below is the branching fraction matrix above, transformed into rate space; i.e., $G_{i} \equiv \Gamma_{i}=\Gamma_{\text {total }} P_{i}$, in appropriate units. In analogy to the matrix above, the diagonal elements are $G_{i} \pm \delta G_{i}$, where $\delta G_{i}=\sqrt{\left\langle\delta G_{i} \delta G_{i}\right\rangle}$, while the off-diagonal elements are the normalized correlation coefficients $\left\langle\delta G_{i} \delta G_{j}\right\rangle /\left(\delta G_{i} \cdot \delta G_{j}\right)$. Note that, because of the error in $\Gamma_{\text {total }}$, the errors and correlations here are not directly derivable from those above.

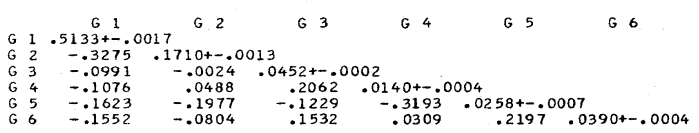

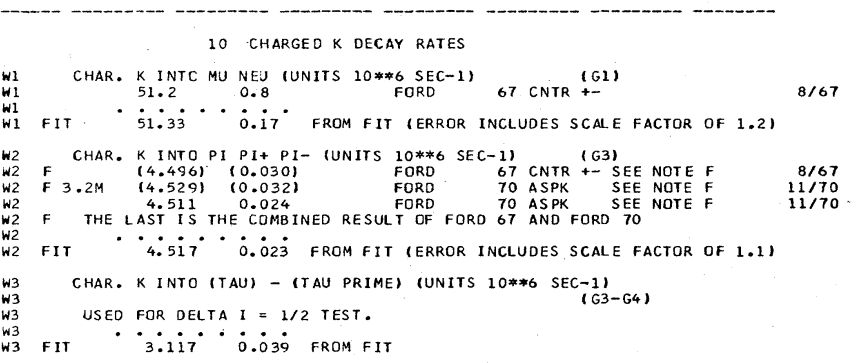

W4 CHAR. K INTO (MU PIO NEU) + (E PIO NEU) (UNITS 10**6 SEC-1) W4 USED FOR DELTA $I=1 / 2$ TEST. P ${ }_{6.484^{\circ}} \cdot \dot{0.089}$ FROM FIT

$$
10(1 K+)-(K-1) / \text { AVG., OECAY RATE DIFFERENCE (PERCENT }
$$

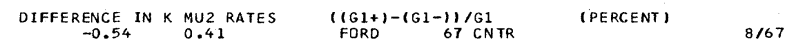

OIFFERENCE IN TAU RATES $((G 3+)-(G 3-)) / G 3$ (PERCENT)

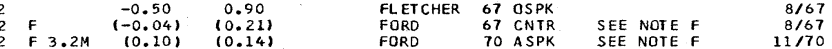

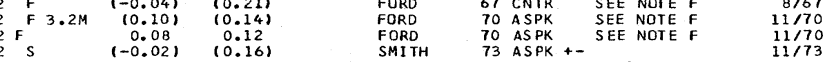
F SECOND FORD 70 VALUE IS FIRST FORD 70 COMB INED WITH FORD 67.
$S$ SMITH 73 VALUE OF D2 IS DERIVED FROM SMITH 73 VALUE OF D3.

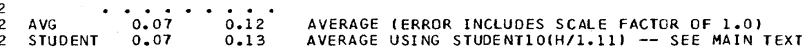
DIFFERENCE IN TAU PRIME RATES ( (G4+)-(G4-1)/AVERAGE (PERCENT)

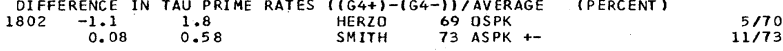

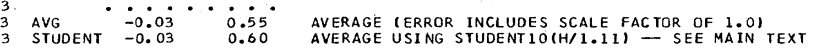
DifFERENCE IN $x$ PI2 RATES
0.8
1.2 DIFFERENCE IN K P P RAD RATES (IG13)-(G13-) / AAVRAGE (PERCENT)

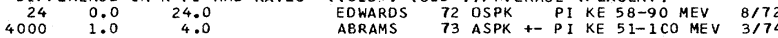

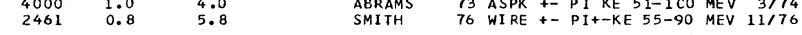

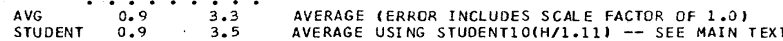

10 CHARGED $K$ BRANCHING RATIOS

O OLD DATA EXCLUDED

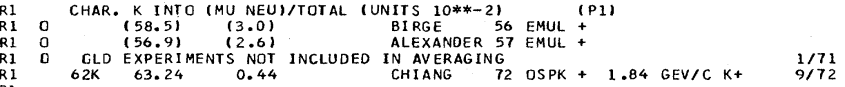

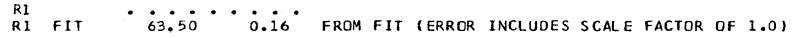

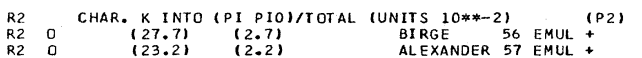

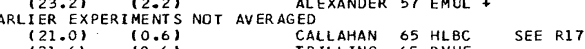

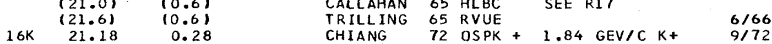

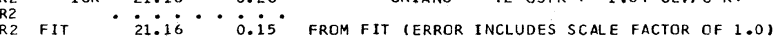

$\begin{array}{llllll}\text { R3 } & & \text { CHAR. K INTO (PI PI+ PI-1/TOTAL (UNITS } & 10 * *-2) & \text { (P3) } \\ R 3 & 0 & (5.6) & (0.4) & \text { BIRGE } & 56 \text { EMUL } \\ \text { R3 } & 0 & (6.8) & (0.4) & \text { ALEXANDER } 57 \text { EMUL }+\end{array}$

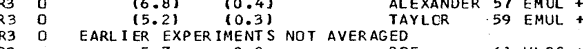

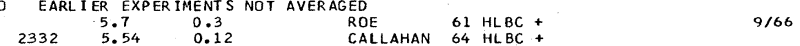

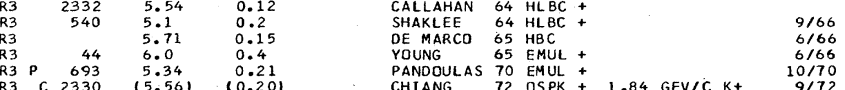

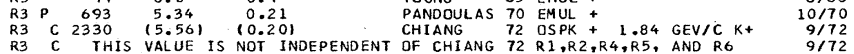

R3 $C$ THIS VALUE IS NOT INDEPENDENT
R3 P TNCLUDES EVENTS OF TAYLOR 59 .

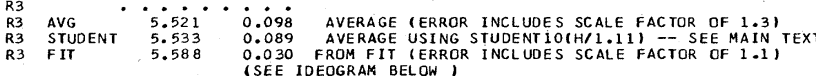

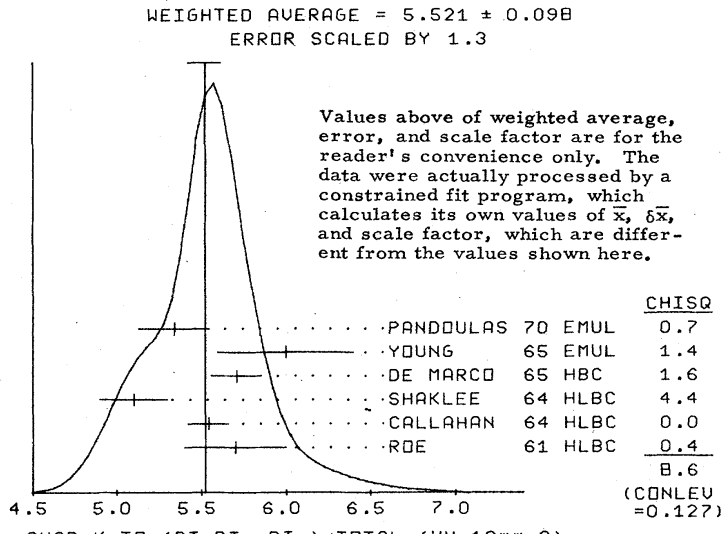

CHAR.K TD (PI PI+ PI-) TTQTAL (UN 10**-2 


\section{Stable Particles} $\mathrm{K}^{ \pm}$

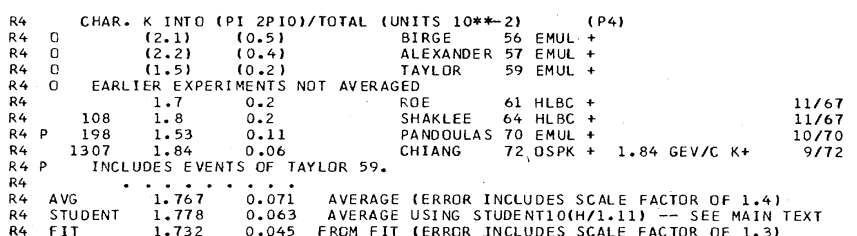

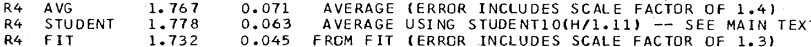
(SEE IDEOGRAM BELOW )
(SE

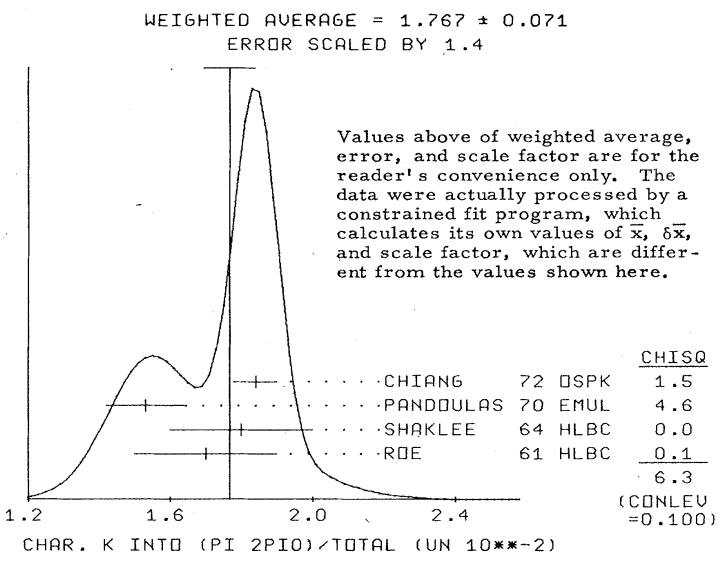

R5 CHAR. K INTC (MU PIO NEU)/TOTAL (UNITS $10 * *-2)$ (P5)

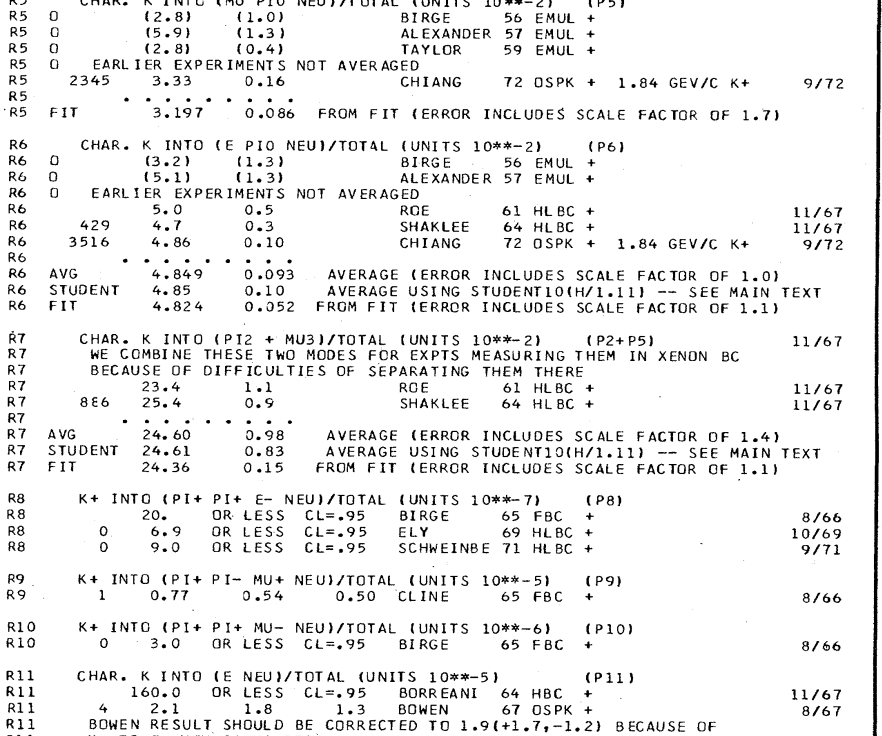

4
R11

R12 CHAR. K INTO (PI GAMMA GAMMA)/TOTAL (UNITS 10**-4) (P17)

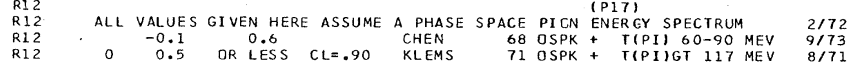

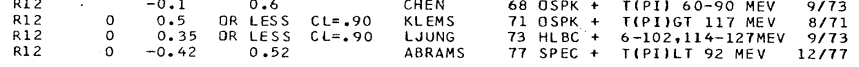

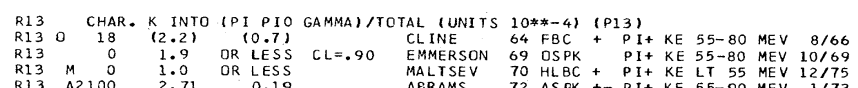

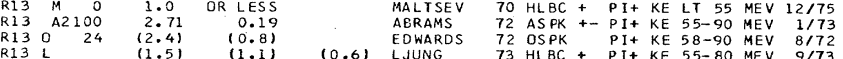

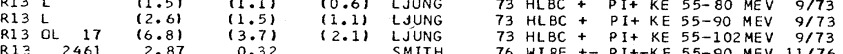
ONLY HIGH STATISTICS EXPER IMENTS ARE AVERAGED.
13 M MALTSEV TO SELECTS LOW PI+ ENERGY TO ENHANCE DIRECT EMISSION CONTR. $3 / 78$
$1 / 76$ R13 A ABRAMS 72 OBSERVES. DIRECT EMISSION BR. RATIO OF $(1.56+-0.35) * 10 * *-5) 1 / 73$

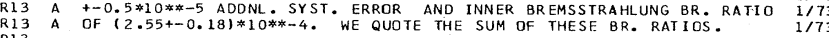

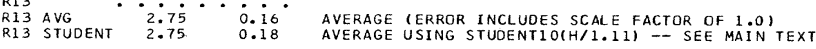

Data Card Listings For notation, see key at front of Listings.

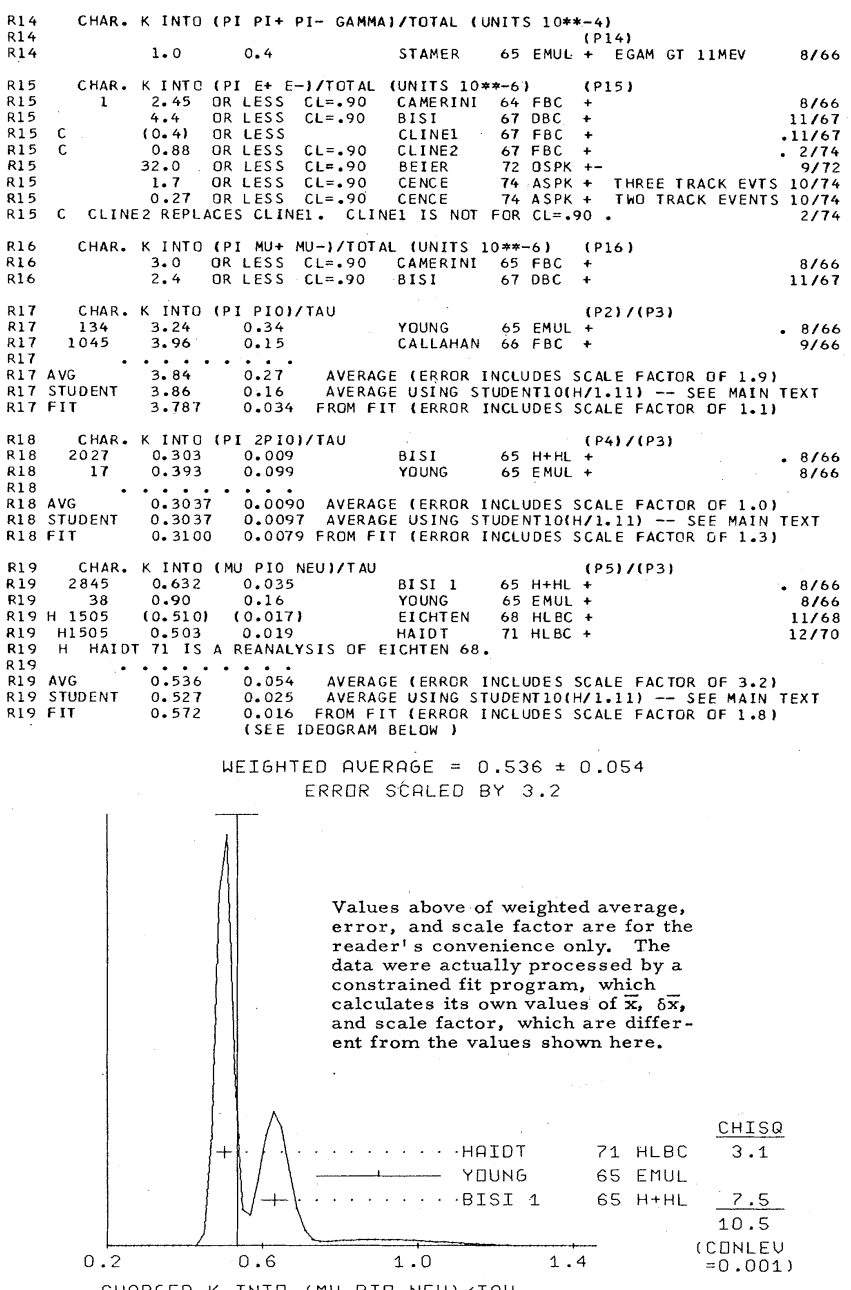

CHARGED K INTO (MU PID NEU), /TAU

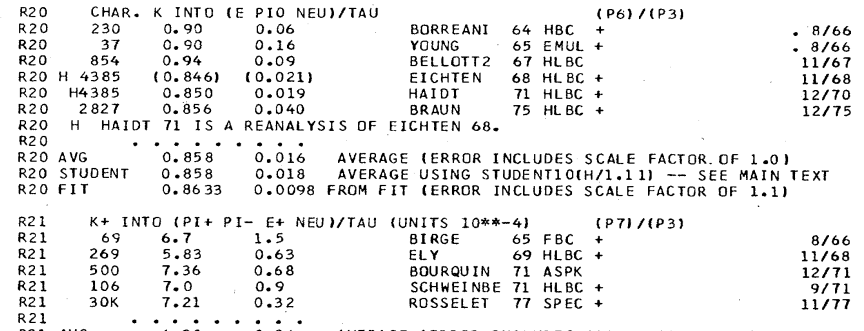

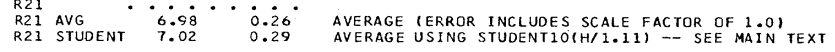

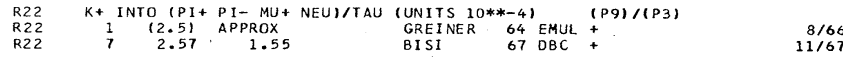

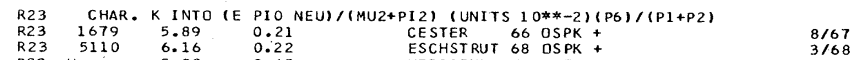

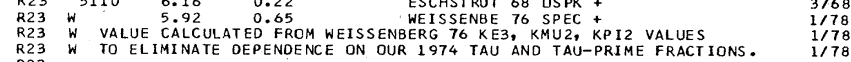

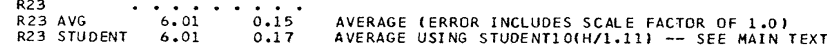

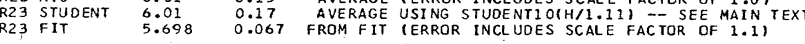




\section{Data Card Listings}

\section{Stable Particles}

\section{For notation, see key at front of Listings.}

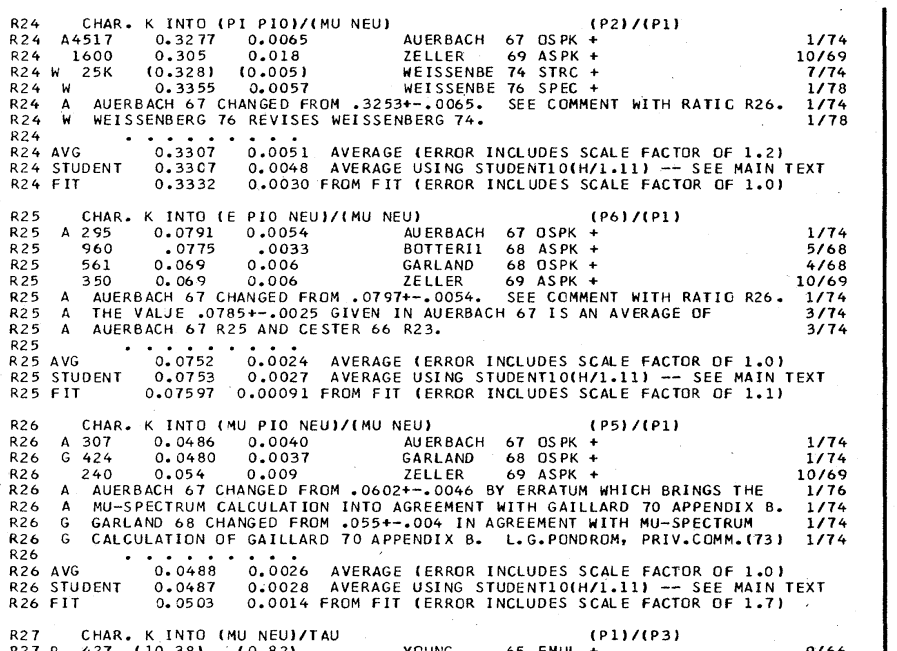

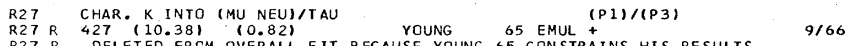

R27 R TO ADO UP TO 1 . ONER ONLY YOUNG MEASURED MU2 DIRECTLY.
$R 27$

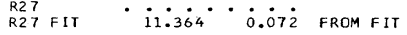

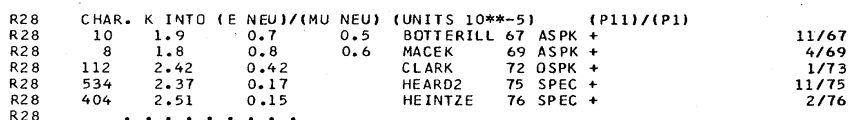

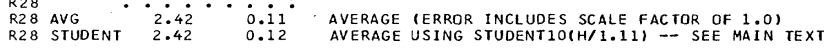

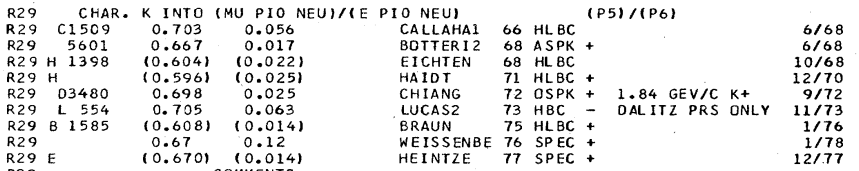

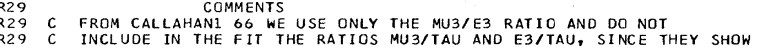

R2 9 C LARGE DISAGREEMENTS WITH THE REST OF THE DATA.

R29 H CNLY INOIVIDUAL RATIOS INCLUDED IN FIT (SEE R 19 AND R20).

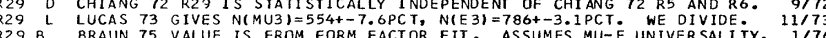

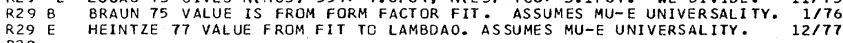

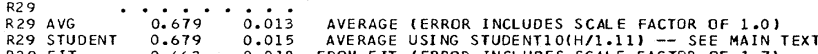

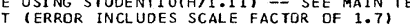

R3O CHAR. K INTO (PIO E NEU GAMMA)/(PIO E NEU) (UNITS 10**-2)

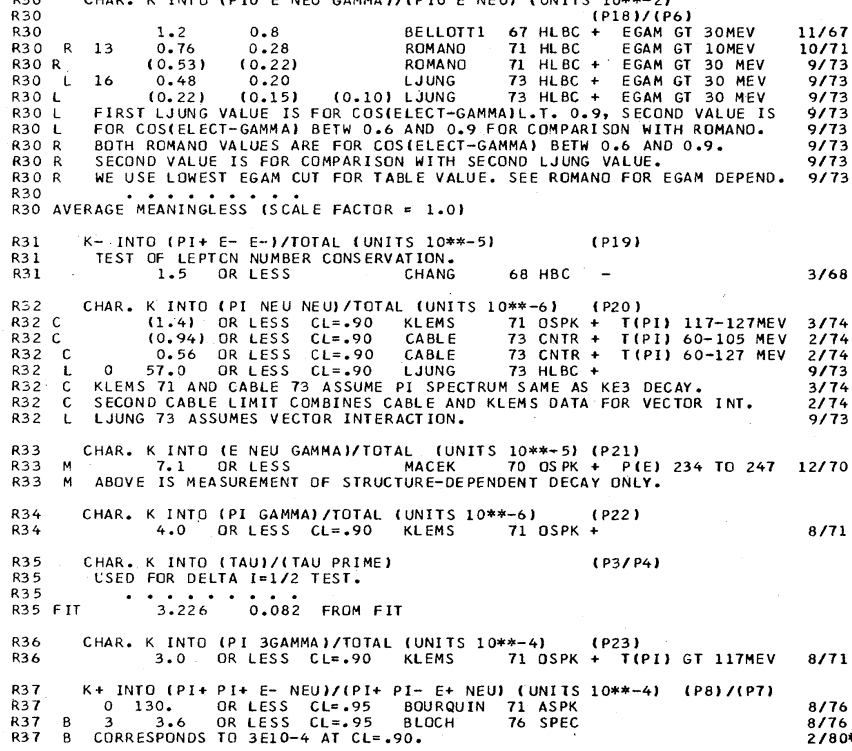

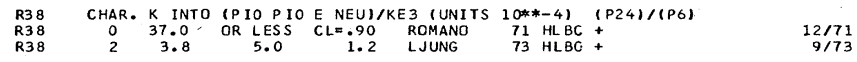

K+ INTO (PI- E+ MU+)/TOTAL (UNITS $10 * *-8) \quad$ (P25)

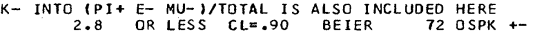

K+ INTO (PI+ E+ MU-)/TOTAL (UNITS $10 * *-8) \quad$ (P26)

$\begin{array}{lllll}R 40 & K-\text { INTO CPI- E- MU+ Y TOTAL IS ALSO INCLUDED HERE } & \\ \text { R40 } & 1.4 & \text { OR LES CL CL.90 BEIER } 72 \text { OSPK +- } & 9 / 72\end{array}$

$9 / 72$

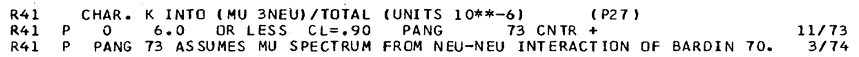

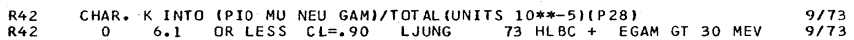

R43 CHAR. K INTO (E PIO NEU)/(PI PIO) (P6)/(P2)

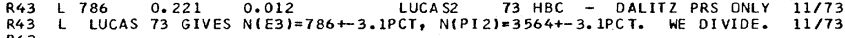

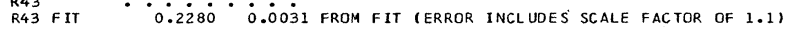

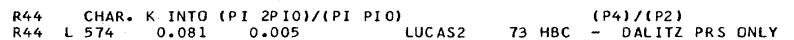

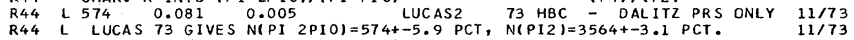

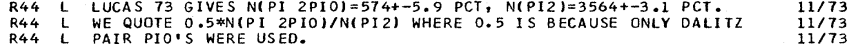

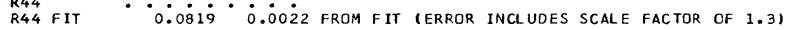

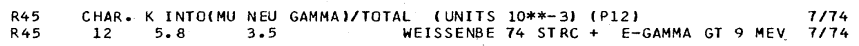

R46 CHAR. K INTO (PI E+ E-1/(PI+ PI-E NEU) (UNITS 10**-3) (P15)/(P7)

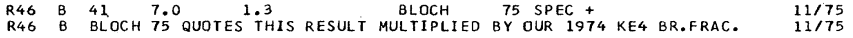

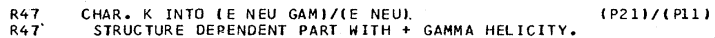

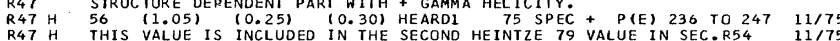

$R 47$
$R$ THIS VALUE IS INCLUDED IN THE SEC

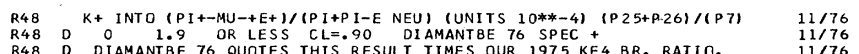

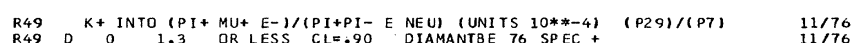

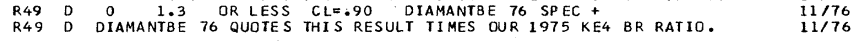

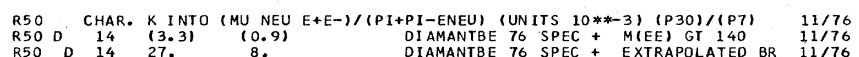

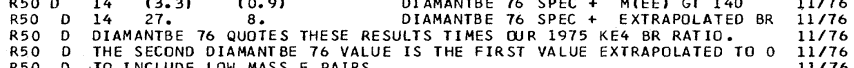

R51 $K+-$ INTO(PI-+E+-E+-)/(PI+PI-E NEU) UNITS(10**-4) (P19)/(P7) $11 / 76$

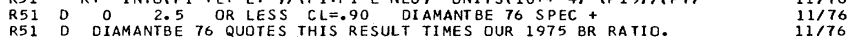

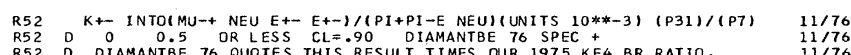

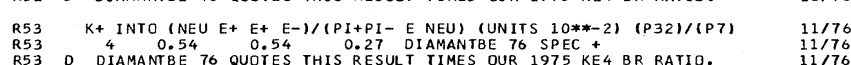

R54 CHAR. K INTO (E NEU GAM)/(MU NEU) (UNITS 10**-5)(P21)/(P1)

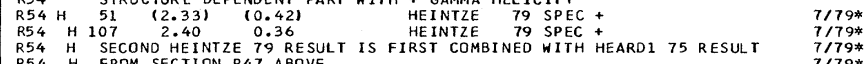

R55 CHAR. K INTO (E NEU GAM)/(MU NEU) (UNITS $10 * *-4)(P 21) /(P 1)$
R55 STRUTURE DEPENDENT PART WITH- GAMMA HELICITY

R55 H STRUCTURE OEPENDENT PART WITH - GAMMA HELICITY

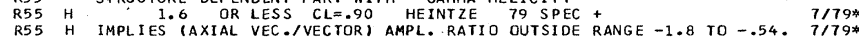

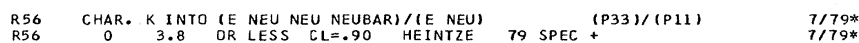

Note on Slope Parameter for $K \rightarrow 3 \pi$ Decays

As was discussed in section VI B.I of the text, for the $3 \pi$ decays of the $K$ mesons we list the slope parameter " $g$ " which is defined, as in that section, by

$$
\begin{aligned}
|M|^{2} \propto 1 & +g \frac{\left(s_{3}-s_{0}\right)}{m_{\pi^{+}}^{2}}+h\left(\frac{s_{3}-s_{0}}{m_{\pi}^{2}}\right)^{2} \\
& +j \frac{\left(s_{2}-s_{1}\right)}{m_{\pi^{+}}^{2}}+k\left(\frac{s_{2}-s_{1}}{m_{\pi^{+}}^{2}}\right)^{2}+\ldots .
\end{aligned}
$$




\section{Stable Particles}

$\mathrm{K}^{ \pm}$

$$
\begin{aligned}
& s_{i}=\left(\underline{p}_{K}-\underline{p}_{i}\right)^{2}=\left(m_{K}-m_{i}\right)^{2}-2 m_{K} T_{i} \\
& s_{0}=\frac{1}{3} \sum s_{i}=\frac{1}{3}\left(m_{K}^{2}+m_{1}^{2}+m_{2}^{2}+m_{3}^{2}\right)
\end{aligned}
$$

$\underline{p}_{K^{\prime}} \underline{p}_{i}$ are the four-vectors for the $K$ and the $i^{\text {th }}$ pion, and the index 3 refers

to the odd pion, i.e., the third pion in the decays listed below.

We refer to the three possible charged decays as $\tau, \tau^{\prime}$, and $\tau^{0}$ :

$$
\begin{array}{ll}
\tau^{ \pm} & K^{ \pm} \rightarrow \pi^{ \pm} \pi^{ \pm} \pi^{\mp} \\
\tau^{ \pm \pm} & K^{ \pm} \rightarrow \pi^{0} \pi^{0} \pi^{ \pm} \\
\tau^{0} & K_{L}^{0} \rightarrow \pi^{+} \pi^{-} \pi^{0}
\end{array}
$$

The measurements of $g$ vary considerably beyond the authors' quoted errors as can be seen in the ideograms associated with the GT+, GT-, and GTP subsections of the $\mathrm{K}^{ \pm}$Data Card Listings and the GTO subsection of the $\mathrm{K}_{\mathrm{L}}^{0}$ Listings. Appendix I discusses tests of the $\Delta I=1 / 2$ rule utilizing these slopes.

There is no indication of a CP-violating asymmetry in $\mathrm{K}_{\mathrm{L}}^{0}$ decay as measured by the coefficient $j$ given in subsection JTO of the $K_{L}^{0}$ Listings. The high-statistics $\tau^{0}$-decay experiment of MESSNER 74 finds significant non-zero quadratic coefficients $\mathrm{h}$ and $\mathrm{k}$. $\mathrm{CHO} 77$, a lower-statistics $\tau^{0}$ experiment, obtains results in agreement with MESSNER 74 but can also obtain good fits with a linear term $(g)$ only. The correlation between the linear and quadratic coefficients changes the CHO $77 \mathrm{~g}_{\tau^{0}}$ from $0.629 \pm 0.017$ (1inear fit) to $0.681 \pm$ 0.024 (quadratic fit). Another experiment, PEACH 77, does not observe this correlation and is in agreement only with the linear fit of CHO 77.

There is some evidence for a non-zero $k$ coefficient from $\tau^{ \pm}$experiments. FORD $72 \quad(1.5 \mathrm{M}$ events) have studied $\mathrm{K}^{ \pm} \rightarrow \pi^{ \pm} \pi^{ \pm} \pi^{\mp}$ and find that the $x^{2} / \mathrm{DF}$ goes from 1.38 to 1.20 for $\mathrm{DF} \approx 150$ when the second order and CP-violation terms are added. However, the authors state that since their coulomb

\section{Data Card Listings For notation, see key at front of Listings.}

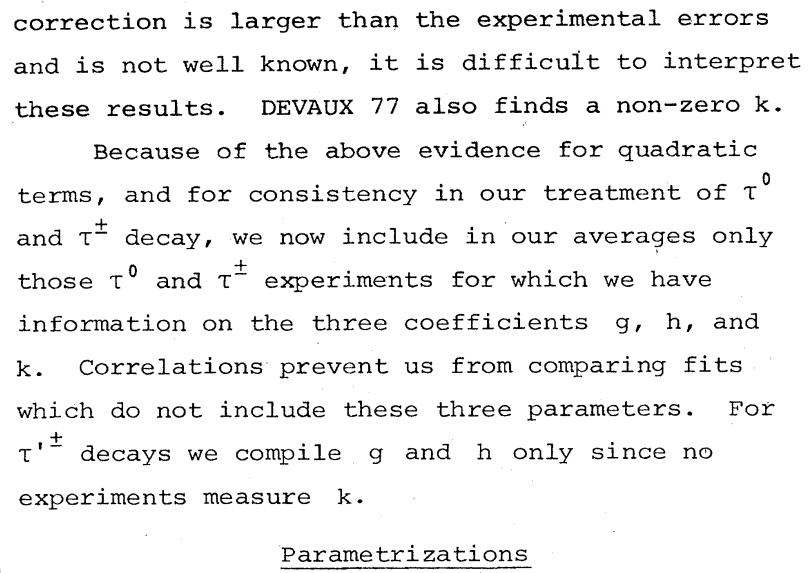

\section{Parametrizations}

In the literature other definitions of slope parameters have appeared. We have converted to the definitions of $g, h, j$ and $k$ in Eq. (1) from whatever experimental quantity has been reported. We give the conversion to the definition (1) for the most widely used parametrizations and tabulate the conversion factors for the reader's convenience.

a) For analysis of charged $\mathrm{K}^{\prime} \mathrm{s}$ and some $\mathrm{K}^{0}$ experiments, the expression often used is:

$$
\begin{aligned}
|M|^{2}= & 1+a_{Y} Y+b_{Y} Y^{2}+a_{Y} X+e_{Y} X^{2} \\
Y & =\frac{3 T_{3}-Q}{Q}, \\
X & =\frac{\sqrt{3}\left(T_{1}-T_{2}\right)}{Q}, \\
Q & =m_{K}-\sum m_{i}
\end{aligned}
$$

The relevant formulae are:

$$
Y=-\frac{3}{2} \frac{s_{3}-s_{0}}{m_{K} Q}+\Delta, \quad X=\frac{\sqrt{3}}{2} \frac{s_{2}-s_{1}}{m_{K} Q}
$$

with

$$
\Delta=\frac{m_{1}-m_{3}}{2}\left(2-\frac{m_{3}+m_{1}}{m_{K}}\right)
$$


Data Card Listings

Stable Particles

For notation, see key at front of Listings.

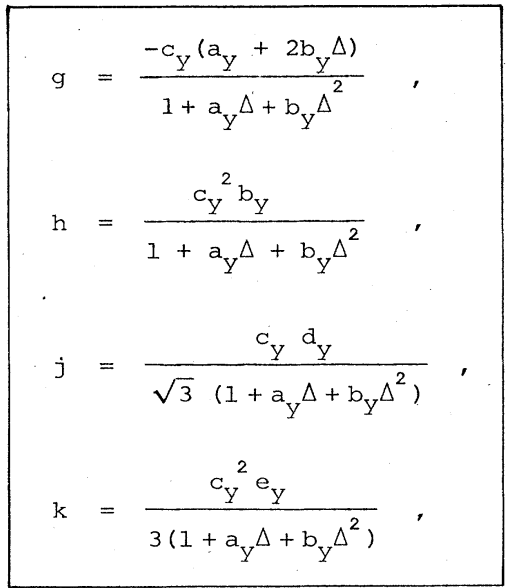

with

$$
c_{y}=\frac{3}{2} \frac{m_{\pi^{+}}^{2}}{m_{K^{2}}}
$$

b) For the analysis of some $\mathrm{K}^{0}$ experiments the expression used is

$$
\begin{aligned}
|M|^{2} & =1+2 a_{t} \frac{m_{K}}{m_{\pi^{+}}^{2}}\left(2 T_{3}-T_{3 \max }\right) \\
& +b_{t}\left(\frac{m_{K}}{m_{\pi^{+}}^{2}}\right)^{2}\left(2 T_{3}-T_{3 \max }\right)^{2},
\end{aligned}
$$

with

$$
\mathrm{T}_{3 \max }=\frac{\left(\mathrm{m}_{\mathrm{K}}-\mathrm{m}_{3}\right)^{2}-\left(\mathrm{m}_{1}+\mathrm{m}_{2}\right)^{2}}{2 \mathrm{~m}_{\mathrm{K}}} .
$$

The relevant transformations are

$$
\mathrm{T}_{3}=-\frac{\mathrm{s}_{3}-\mathrm{s}_{0}}{2 \mathrm{~m}_{\mathrm{K}}}+\frac{2}{3}(1+\Delta)
$$

and

$$
\begin{aligned}
& g=\frac{-2 a_{t}-b_{t} c_{t}}{1+a_{t} c_{t}+\frac{b_{t}{ }^{2} t}{4}} \\
& h=\frac{b_{t}}{1+a_{t} c_{t}+\frac{b_{t}{ }^{2}{ }^{2}}{4}}
\end{aligned},,
$$

with

$$
c_{t}=\frac{2 m_{K}}{m_{\pi^{+}}^{2}}\left[\frac{2}{3} Q(1+\Delta)-T_{3 \max }\right] .
$$

c) Other $\mathrm{K}^{0}$ authors use the same form of matrix element as given in b) above with a linear term only, but define

$$
\mathrm{T}_{\max }=\frac{2}{3} 2 .
$$

The relevant transformation is then

$$
\begin{aligned}
& g=\frac{-2 a_{u}}{I+a_{u^{c} c^{\prime}}}, \\
& c_{u}=\frac{4 m_{K}}{3 m_{\pi^{+}}^{2}} Q \Delta
\end{aligned} .
$$

d) Older $\mathrm{K}^{0}$ analyses were done using

$$
|\mathrm{M}|^{2}=1+\mathrm{a}_{\mathrm{v}} \frac{\mathrm{T}_{3}}{\mathrm{~m}_{\mathrm{K}}} \text {. }
$$

The relevant transformation is then

$$
g=\frac{-c_{v}{ }^{a} v}{1+d_{v} a v}
$$

with

$$
c_{v}=\frac{m_{\pi^{+}}^{2}}{2 m_{k}^{2}}
$$

and

$$
d_{v}=\frac{Q}{3 m_{K}}(1+\Delta)
$$

e) The CP-violating term in $|\mathrm{M}|^{2}$ for $\mathrm{K}_{\mathrm{L}}^{0} \rightarrow$

$\pi^{+} \pi^{-} \pi^{0}$ experiments has been parametrized in several ways. BLANPIED 68 and SCRIBANO 70 use the parametrization given in (b) above with no quadratic term and with an additional $C P$ violating term. BLANPIED 68 parametrizes the CP-violating term as

$$
2 \sigma_{B} \cdot \frac{m_{K}}{m_{\pi^{+}}^{2}}\left(T_{1}-T_{2}\right)
$$

The relevant transformation is then

$$
j=\frac{\sigma_{B}}{I+c_{t} a_{t}}
$$

with $c_{t}$ as defined in (b) above. SCRIBANO 70 parametrizes the $\mathrm{CP}$-violating term as

$$
\frac{2}{\sqrt{3}} \sigma_{\mathrm{S}} \frac{\mathrm{T}_{1}-\mathrm{T}_{2}}{\mathrm{~T}_{12 \max }}
$$

where $\mathrm{T}_{12 \max }$ is the maximum kinetic energy of particle 1 or 2 , the charged $\pi$ 's, given by 


\section{Stable Particles}

$\mathrm{K}^{ \pm}$

$$
T_{12 \max }=\frac{\left(m_{K}-m_{1}\right)^{2}-\left(m_{2}+m_{3}\right)^{2}}{2 m_{K}} .
$$

The resulting transformation is then

$$
j=\frac{m_{\pi^{+}}^{2}}{\sqrt{3} m_{K}{ }^{T_{12 \max }}} \frac{\sigma_{s}}{\left(1+c_{t} a_{t}\right)} .
$$

SMITH 70 gives the asymmetry

$$
\alpha=\frac{\mathrm{N}_{+}-\mathrm{N}_{-}}{\mathrm{N}_{+}+\mathrm{N}_{-}}
$$

where $\mathrm{N}_{+}$is the number of events with $\mathrm{T}_{1}>\mathrm{T}_{2}$ and $\mathrm{N}_{\text {- }}$ is the converse. BLANPIED 68 gives the relation $\sigma_{B}=\alpha / 1.16$ which allows us to use the transformation to $j$ given above for BLANPIED 68 .

For the reader's convenience we give a table of numerical values for $Q, T_{3 \max }, T_{12 \max }, \Delta, C_{y}, C_{t}$, $\mathrm{c}_{\mathrm{u}}, \mathrm{c}_{\mathrm{v}}$, and $\mathrm{d}_{\mathrm{v}}$, obtained using the masses from the current edition.

\begin{tabular}{llcc} 
& $\tau^{ \pm}$ & $\tau^{\prime \pm}$ & $\tau^{0}$ \\
\hline \hline 2 & 74.97 & 84.18 & 83.57 \\
$T_{3 \max }$ & 48.08 & 53.20 & 53.89 \\
$T_{12 \max }$ & 48.08 & 53.99 & 53.12 \\
$\Delta$ & 0.0000 & -0.0790 & 0.0798 \\
${ }^{c_{y}}$ & 0.7895 & 0.7031 & 0.7025 \\
${ }^{c_{t}}$ & 0.0962 & -0.0769 & 0.3204 \\
${ }^{c}$ & 0.0000 & -0.2247 & 0.2272 \\
${ }_{u}$ & 0.0400 & 0.0400 & 0.0393 \\
${ }_{d_{v}}$ & 0.0506 & 0.0523 & 0.0604 \\
\hline \hline
\end{tabular}

\section{References}

See the reference sections of the $K^{ \pm}$and $K_{L}^{0}$ Data Card Listings.

See also the review of T.J. Devlin and J.O. Dickey, Rev. Mod. Phys. 51, 237 (1979), which contains an analysis of $\mathrm{K} \rightarrow 2 \pi$ and $\mathrm{K} \rightarrow 3 \pi$ data in terms of transition amplitudes with appropriate energy dependence.
Data Card Listings For notation, see key at front of Listings.

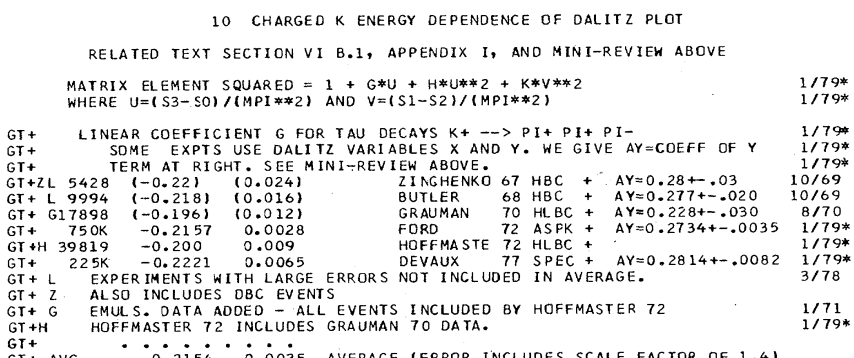

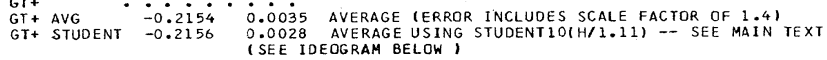

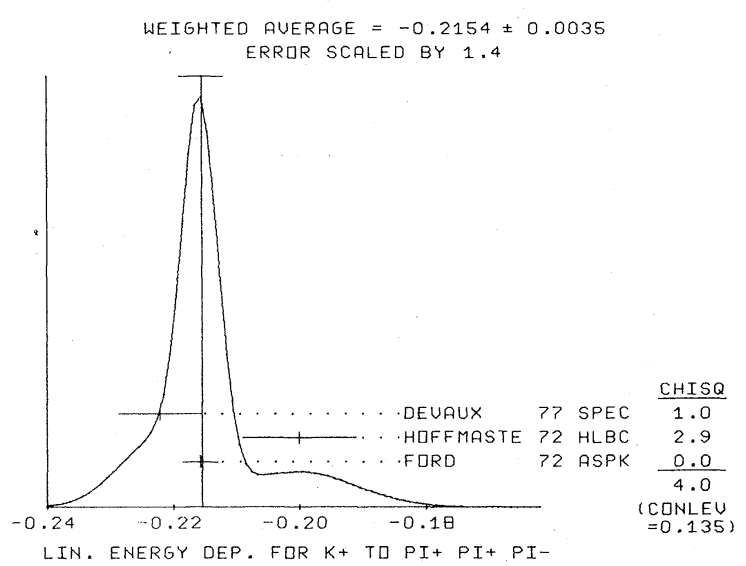

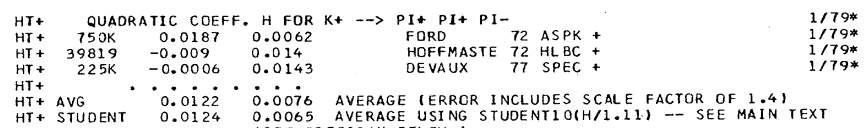
WEIGHTED AUERAGE $=0.0122 \pm 0.0076$

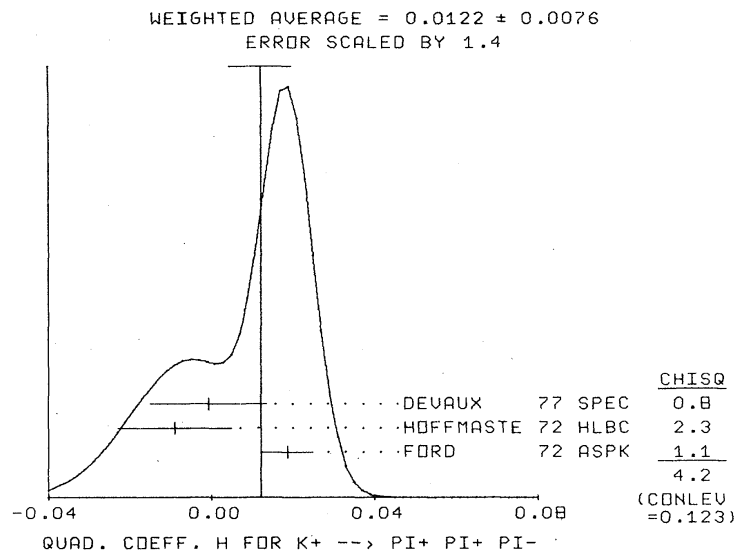

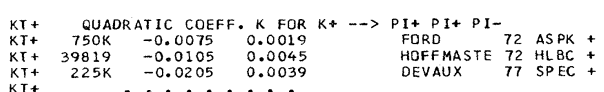

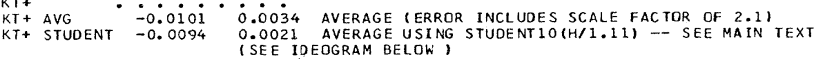




\section{Data Card Listings}

\section{Stable Particles}

\section{For notation, see key at front of Listings.}

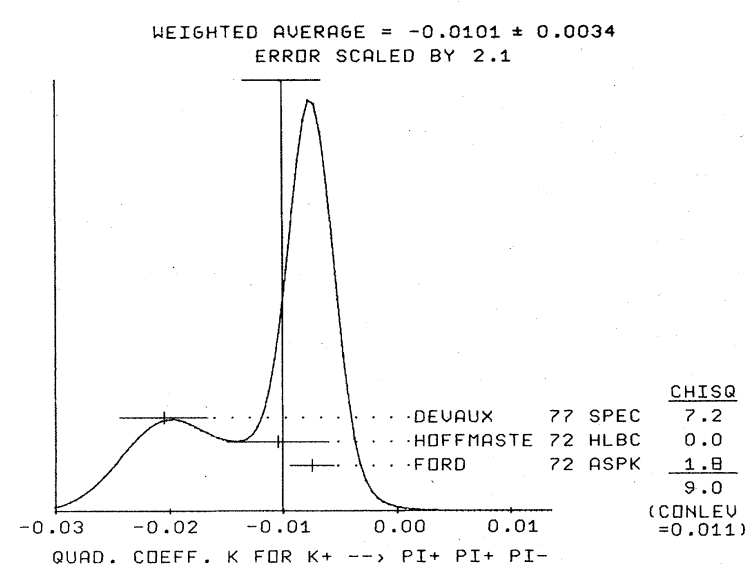

GT- LINEAR COEFFICIENT G FOR TAU DECAYS K- $->$ PI- PI- PI+

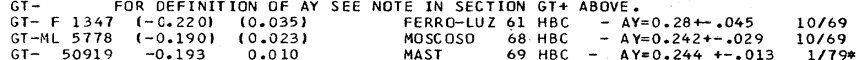

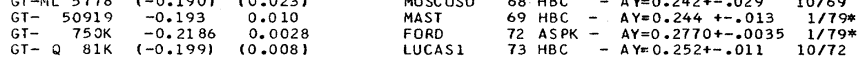

GT- F NO RADIATI VE CORRECTIONS INCLUDED.
GT- $L$ EXPER IMENTS WITH LARGE ERRORS NOT INCLUDED IN AVERAGE.

$3 / 78$

GT-M ALSO INCLUDES CBC EVENTS.
GT- Q QUADRAIC DEPENDENCE IS REQUI RED BY KL EXPTS. FOR COMPARISON WE.
GT- Q AVERAGE DNLY THOSE K+- EXPER IMENTS WHICH QUOTE QUADRATIC FIT VALUES. 1/79*
GT-

\begin{tabular}{l} 
GT- \\
GT- AVG $-0.2167^{\circ} 0.0066$ AVERAGE (ERRRR INCLUDES SCALE FACTOR OF 2.51 \\
GT- STUDENT -0.21730 .0031 AVERAGE USING STUDENT1O(H/1.11) \\
\hline
\end{tabular}

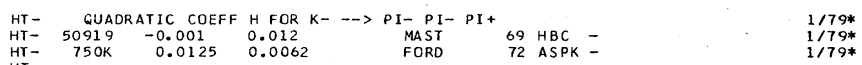

HT- AVG $0.0097^{\circ}$ O.0055 AVERAGE (ERROR INCLUDES SCALE FACTOR OF 1.0 )

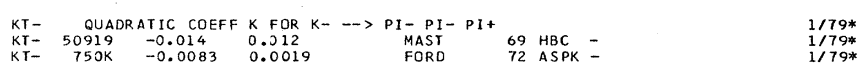

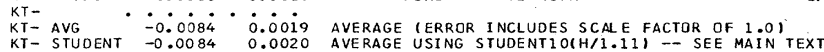

DG $((G T+)-(G T-)) /(1 G T+)+(G T-1)$ IN PERCENT

DG
DG
DG
OG NGON-ZERO VALUE FOR THIS QUANTITY INDICATES CP VIOLATION

GTP
GTP INEAR COEFFICIENT G FOR TAU PRIME DECAYS CHAR,
UNLESS OTHERHISE STATED, ALL EXPTS INCLUDE TERMS QUADRATIC IN

GTP UNLESS OTHERWISE STATED, ALL EXPTS INCLUDE TERMS QUD
GTP (S3-SO)/(MPI**2). SEE MINI-REVIEW ABOVE.

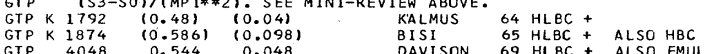

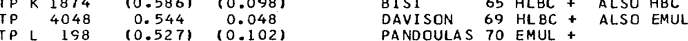

$\begin{array}{lllll}\text { GTP L } 198 & (0.527) & 10.1021 & \text { PANDOULAS 70 EMUL }+ \\ \text { GTP } 1365 & 0.67 & 0.06 & \text { AUBERT } 72 \mathrm{HLBC}+ \\ \text { GTP } & 574 & 10.4841 & 10.0841 & \text { SUCAS2 }\end{array}$

LUCASZ $73 \mathrm{HBC}$ - DALITZ PRS ONLY

$\begin{array}{lccccc}\text { GTP } & 5635 & 0.630 & 0.038 & \text { SHEAFF } & 75 \mathrm{HLBC}+ \\ \text { GTP } & 27 \mathrm{~K} & 0.510 & 0.060 & \text { SMITH } & 75 \mathrm{WIRE}+ \\ \text { GTP L } 4639 & 10.8061 & 10.2201 & \text { BERTRAND } & 76 \text { EMULL } \\ \text { GTP } & & & \end{array}$

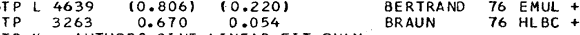

GTP $K$
GTP $L$ AUTHORS GIVE LINEAR FIT ONLY.

GTP
GPP AVG
GTP STUDENT
$0.607^{\circ}$
0.610

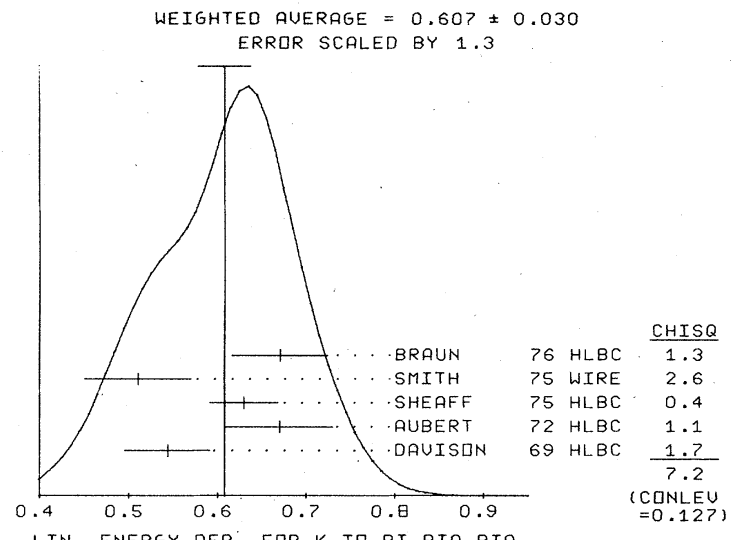

LIN. ENERGY DEP: FQR $K$ TQ PI PIO PIO

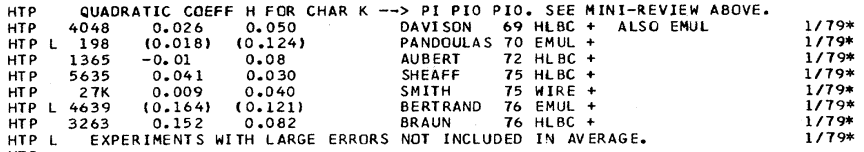

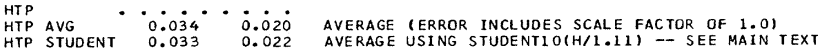

Note on $\mathrm{K}_{\ell, 3}^{ \pm}$and $\mathrm{K}_{\ell, 3}^{0}$ Form Factors

Definitions of the parameters $\lambda_{+}, \xi(0), \lambda_{0}$, $\left|f_{S} / f_{+}\right|$and $\left|f_{T} / f_{+}\right|$and a general discussion of the methods of analysis are given in section VI B.2 of the text.

This note describes the contents of the Data Card Listings for the two $\mathrm{K}_{\mu 3}$ parametrizations, $\left(\lambda_{+}, \xi(0)\right)$ and $\left(\lambda_{+}, \lambda_{0}\right)$, which were discussed in the text. Problems related to our data entries for individual experiments are discussed and a comparison of results is given.

\section{$\underline{K}_{\mu 3}$ Experiments}

The matrix element for $\mathrm{K}_{\mu 3}$ decay, assuming a pure vector current, is given by Eq. (2) in section VI B. 2 of the text. Most experiments appear to be compatible with the assumption that $f_{+}$depends linearly on $t$ and that $f_{\text {_ }}$ is constant. Only DALLY $72\left(\mathrm{~K}_{\mu 3}^{0}\right)$ appears to require $\lambda_{-} \neq 0$ (by about three standard deviations). A single data bin at low $\mathrm{q}^{2}$ seems to be responsible. The effect is not observed in the high-statistics experiment of DONALDSON2 74 (also $\mathrm{K}_{\mu 3}^{0}$ ).

$\lambda_{+}, \xi(0)$ Parametrization: $\lambda_{+}$data from $\mathrm{K}_{\mu 3}$ decay are entered into the $\mathrm{K}^{ \pm}$and $\mathrm{K}_{\mathrm{L}}^{0}$ sections of the Data Card Listings in subsection $\mathrm{L}+\mathrm{M}$. The corresponding $\xi(0)$ values are entered in subsection XIA, XIB, or XIC, depending on whether Method A, B, or $\mathrm{C}$, discussed below and in the text, was used. The data cards contain the values, one-standarddeviation errors $\Delta \lambda_{+}$and $\Delta \xi(0)$, as well as the correlation $\mathrm{d} \xi(0) / d \lambda_{+}$, all indicated on the $\mathrm{e}^{-1 / 2}$

likelihood contour below. The correlations are given on the right side of the $\xi(0)$ data cards.

$\underline{\lambda}_{+}, \lambda_{0}$ Parametrization: This parametrization is used in recent $\mathrm{K}_{\mu 3}$ analyses. To facilitate comparison between experiments, we convert earlier experiments from the $\left(\lambda_{+}, \xi(0)\right)$ parametrization to $\left(\lambda_{+}, \lambda_{0}\right)$ whenever possible (i.e., when $\lambda_{+}$and $\xi(0)$ values, errors, and correlations are given). The 


\section{Stable Particles}

$\mathrm{K}^{ \pm}$

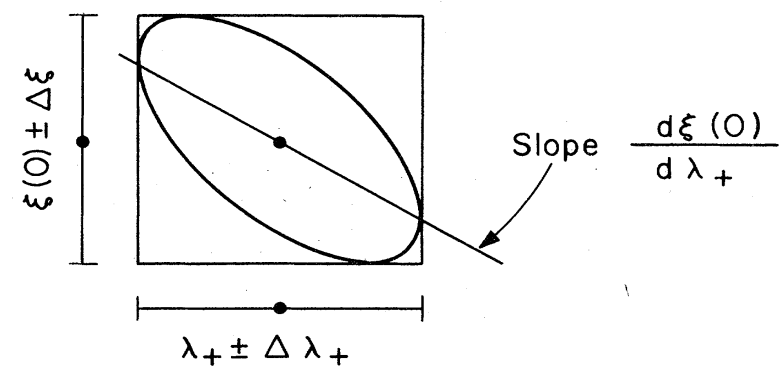

transformation between these parametrizations is:

$$
\begin{aligned}
\lambda_{0} & =\lambda_{+}+\mathrm{a} \xi(0), \\
\Delta \lambda_{0}^{2} & =\left(1+2 \mathrm{a} \frac{\mathrm{d} \xi(0)}{\mathrm{d} \lambda_{+}}\right) \Delta \lambda_{+}^{2}+\mathrm{a}^{2} \Delta \xi^{2}, \\
\frac{\mathrm{d} \lambda_{0}}{\mathrm{~d} \lambda_{+}} & =1+\mathrm{a} \frac{\mathrm{d} \xi(0)}{\mathrm{d} \lambda_{+}},
\end{aligned}
$$

where $a=m_{\pi}^{2} /\left(m_{K}^{2}-m_{\pi}^{2}\right)$. The $\lambda_{0}$ value, the onestandard-deviation error $\Delta \lambda_{0}$, and the correlation $\mathrm{d} \lambda_{0} / \mathrm{d} \lambda_{+}$are given in subsection LO of the data cards.

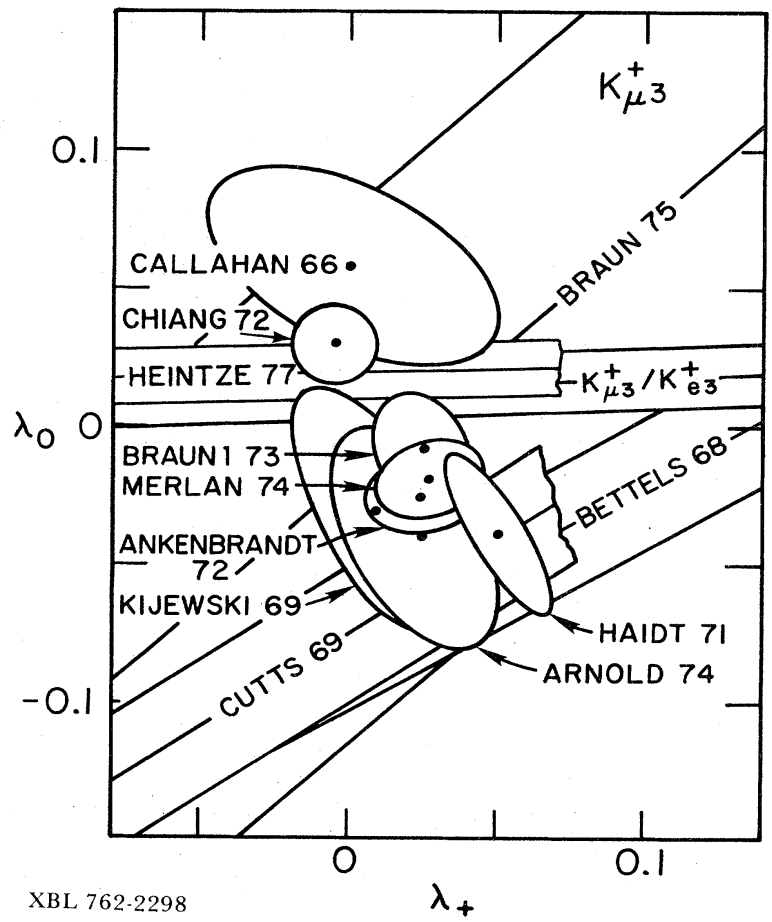

Fig. 1. One-standard-deviation $\left(e^{-1 / 2}\right)$ likelihood contours in the $\left(\lambda_{+}, \lambda_{0}\right)$ plane for $\mathrm{k}_{\mu 3}^{+}$.
Data Card Listings For notation, see key at front of Listings.

We also convert $\left(\lambda_{+}, \lambda_{0}\right)$ results into the $\left(\lambda_{+}\right.$, $\xi(0))$ parametrization whenever possible so that subsection LO is essentially equivalent to the three subsections XIA, XIB, and XIC.

Individual analyses have used a variety of parametrizations. Problems arise when trying to express their results in terms of the parametrizations used here. The discussion of these problems is divided into three sections corresponding to the three methods of analyses discussed in the text.

Method A: Dalitz plot analyses and pion spectrum analyses usually determine $\lambda_{+}$and $\xi(0)$ (or $\lambda_{0}$ ) values, errors, and correlation. Such measurements are entered in the L+M, XIA, and LO subsections. They give rise to the error ellipses shown in Figs. 1 and 2 . These are approximations to likelihood contours.

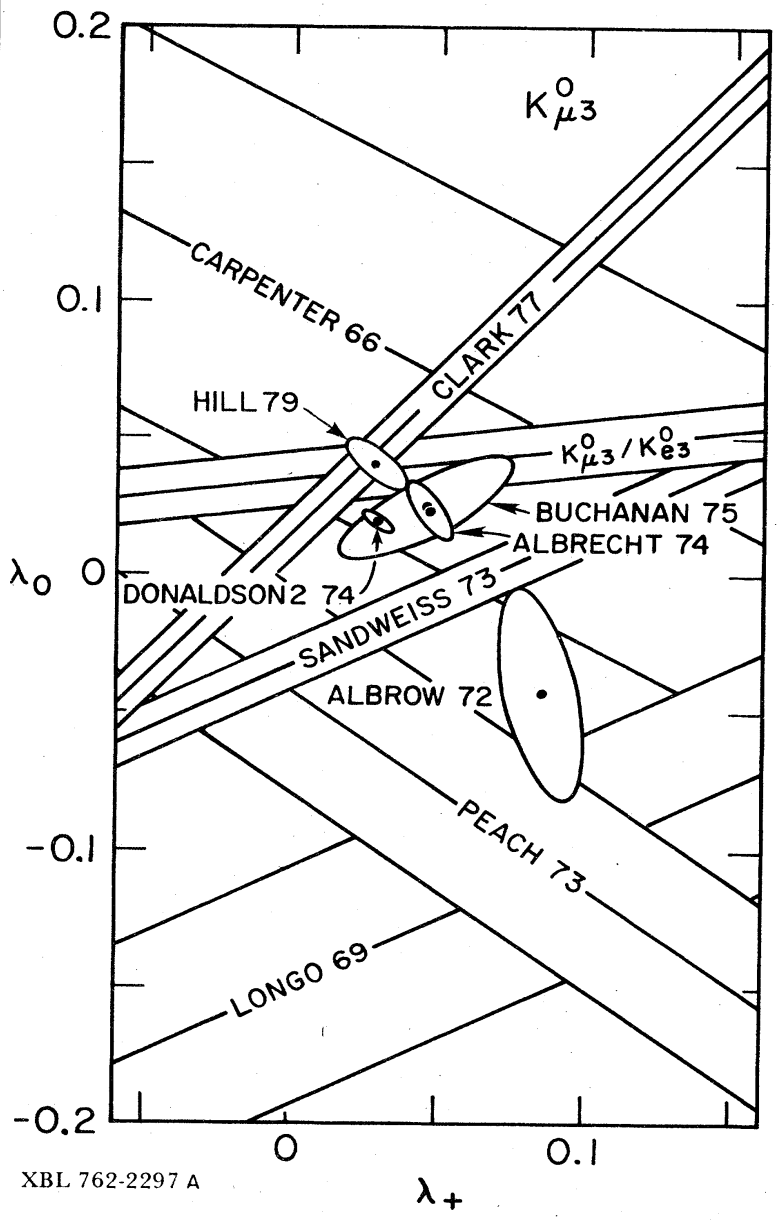

Fig. 2. One-standard-deviation $\left(e^{-1 / 2}\right)$ likelihood contours in the $\left(\lambda_{+}, \lambda_{0}\right)$ plane for $\mathrm{K}_{\mu 3}^{0}$. 


\section{Data Card Listings}

Stable Particles

\section{For notation, see key at front of Listings.}

Some analyses of this type fix $\lambda_{+}$and determine $\xi(0)$, e.g., CARPENTER 66 and PEACH 73 (both $\mathrm{K}_{\mu 3}^{0}$ ). We enter $\xi(0)$ and $d \xi(0) / d \lambda_{+}$in the XIA section and give the fixed $\lambda_{+}$value in the data card footnote. The $\xi(0)$ error is parenthesized because it does not include the uncertainty in the value of $\lambda_{+}{ }^{*}$ These results, transformed to $\lambda_{0}$ measurements, give rise to bands in Fig. 2. These bands are also approximations to the likelihood contours. The actual likelihood bands would not be straight.

In some cases, we alter an error from its published value in order to obtain an error ellipse with a width which matches the error in $\xi(0)$ for fixed $\lambda_{+}$. These adjustments are noted in the $\xi(0)$ data card footnotes, e.g., for CALLAHAN 166 and HAIDT $71 \cdot\left(\mathrm{K}^{+}\right.$subsection XIA), where the published errors and correlation violate the constraint $\left|c_{\lambda \xi}\right|<1$ on the normalized correlation coefficient $\mathrm{C}_{\lambda \xi}$ given by

$$
c_{\lambda \xi}=\frac{\Delta \lambda_{+}}{\Delta \xi} \frac{d \xi(0)}{d \lambda_{+}} .
$$

In some cases, e.g., BRAUN 1 73, the parametrization used is $\lambda_{t^{\prime}} \xi(0), \xi\left(t^{*}\right)$, where $t^{*}$ is the weighted average of $t$ with weighting according to the sensitivity to $\xi$. In this case we do not use $\xi(0)$. It is a badly determined parameter comparable

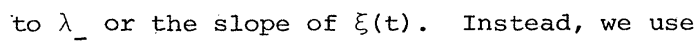

$$
\begin{aligned}
\xi(0) & =\xi\left(t^{*}\right)\left(1+\lambda_{+} t^{*}\right), \\
\frac{d \xi(0)}{d \lambda_{+}} & =\frac{d \xi\left(t^{*}\right)}{d \lambda_{+}}\left(1+\lambda_{+} t^{*}\right)+\xi\left(t^{*}\right) t^{*} .
\end{aligned}
$$

With the BRAUN1 73 values, $\lambda_{+}=0.027, \xi(6.6)=$ $-0.34 \pm 0.20$, and $d \xi(6.6) / d \lambda_{+}=-14$, we obtain

$$
\xi(0)=(-0.40 \pm 0.24)-19\left(\lambda_{+}-0.027\right) ;
$$

or for their fitted $\lambda_{+}=0.025 \pm 0.017$, we get $\xi(0)=-0.36 \pm 0.40$.

Method B: Branching ratio experiments cannot determine $\lambda_{+}$and $\xi(0)$ simultaneously, but simply fix a relationship between them, given in section VI 3.2 of the text. Results are usually quoted as values of $\xi(0)$ at fixed $\lambda_{+}$. We list these results in subsection XIB, but we do not average them because the $\lambda_{+}$values differ. Instead, we compute a combined result by using the relations in the text and our fitted values of $\Gamma\left(\mathrm{K}_{\mu 3}^{ \pm}\right) \%\left(\mathrm{~K}_{\mathrm{e} 3}^{ \pm}\right)$and $\Gamma\left(\mathrm{K}_{\mu 3}^{0}\right) / \Gamma\left(\mathrm{K}_{\mathrm{e} 3}^{0}\right)$, which include the branching ratios from these experiments. The branching ratios from our current edition and the results for $\xi(0)$ and $\lambda_{0}$ evaluated at $\lambda_{+}=0.030$ are

\begin{tabular}{ccc} 
& $\mathrm{K}^{ \pm}$ & $\mathrm{K}_{\mathrm{L}}^{0}$ \\
\hline$\Gamma\left(\mathrm{K}_{\mu 3}\right) / \Gamma\left(\mathrm{K}_{\mathrm{e} 3}\right)$ & $0.663 \pm .018(\mathrm{~S}=1.7)$ & $0.695 \pm .017$ \\
$\xi(0)$ & $-0.20 \pm .15(\mathrm{~S}=1.7)$ & $+0.08 \pm .13$ \\
$\mathrm{~d} \xi(0) / \mathrm{d} \lambda_{+}$ & -11.9 & -10.3 \\
$\lambda_{0}+0.014 \pm .012(\mathrm{~S}=1.7)$ & $+0.037 \pm .011$ \\
$\mathrm{~d} \lambda_{0} / \mathrm{d} \lambda_{+}$ & +0.04 & +0.12 \\
\hline
\end{tabular}

The scale factor $S$ is the amount by which the error has been multiplied in order to compensate for discrepancies in the branching ratios. These $\lambda_{0}$ results give rise to the $\mathrm{K}_{\mu 3} / \mathrm{K}_{\mathrm{e} 3}$ bands in Figs. 1 and 2 .

Method C: Polarization experiments measure $\langle\xi(t)\rangle$, the weighted average of $\xi(t)$ over the $t$ range of the experiment, where the weighting accounts for the variation with $t$ of the sensitivity to $\xi(t)$. Such measurements are entered in subsection XIC.

To reinterpret these results in the $\left(\lambda_{+}, \xi(0)\right)$ parametrization, we recognize that $\lambda_{+}=0$ corresponds to $\xi(t)$ constant (always assuming $\lambda_{-}=0$ ) so that

$$
\left.\xi(0)\right|_{\lambda_{+}=0} \equiv\langle\xi(t)\rangle .
$$

The correlation with $\lambda_{+}$is given by the following relations (valid for small $\lambda_{+}$):

$$
\begin{aligned}
& \xi(0) \approx\langle\xi(t)\rangle\left(1+\lambda+\left\langle\frac{t}{m_{\pi}^{2}}\right\rangle\right), \\
& \frac{d \xi(0)}{d \lambda_{+}} \approx\langle\xi(t)\rangle\left\langle\frac{t}{m_{\pi}^{2}}\right\rangle,
\end{aligned}
$$

where $\left\langle t / \mathrm{m}_{\pi}^{2}\right\rangle$ is the average value of $t$ weighted by the sensitivity to $\xi(t)$. These results, transformed to $\lambda_{0}$ and $\mathrm{d} \lambda_{0} / d \lambda_{+}$values, are entered in subsection $L O$ and give rise to bands in Figs. 1 and 2 . In Figs. 1 and 2, we disregard those polarization measurements for which $d \xi(0) / d \lambda_{+}$is not 


\section{Stable Particles}

$K^{ \pm}$

obtainable. Also we disregard MERLAN 73 because the signs of $\xi(0)$ and $d \xi(0) / d \lambda_{+}$are opposite, whereas the above equation requires them to be the same (since $t>0$ ).

Comparison of $\mathrm{K} \mu 3$ Experiments: Figures $I$ and 2 show the likelihood contours in the $\left(\lambda_{+}, \lambda_{0}\right)$ plane for $\mathrm{K}_{\mu 3}^{+}$and $\mathrm{K}_{\mu 3}^{0}$ respectively.

The $\mathrm{K}_{\mu 3}^{+}$Dalitz plot results (ellipses) shown are fairly consistent and appear to cluster between the $\mathrm{K}_{\mu 3} / \mathrm{K}_{\mathrm{e} 3}$ result and the polarization results of BETTELS 68 and CUTTS 69. The $\mathrm{K}_{\mu 3}^{0}$ results are much less consistent with a small cluster appearing in the neighborhood of the DONALDSON2 74 result.

$\chi^{2}$ fits to the results shown in Fig. 1 and Fig. 2 yield the following values for $\lambda_{+}$and $\lambda_{0}$. The corresponding values of $\xi(0)$ are also given.

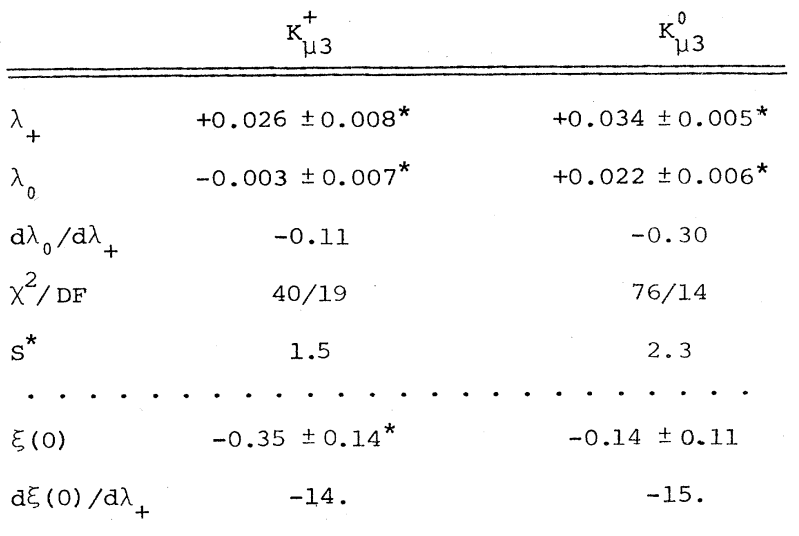

*All errors have been increased by the scale factor $S=\left(X^{2} / D F\right)^{\frac{1}{2}}$ to take into account the discrepancies between measurements.

In view of the large $\chi^{2} / D F$ of these fits, especially $\mathrm{K}_{\mu 3}^{0}$, the fit results should be taken with a grain of salt. The largest contributors to $x^{2}$ in the $\mathrm{K}_{\mu 3}^{+}$case are CHIANG 72 with 8.1 , and the polarization results, BETTELS 68 with 6.8 and CUTTS 69 with 5.5. In the $\mathrm{K}_{\mu 3}^{0}$ case the largest contributors are the polarization results of SANDWEISS 73 with 18, LONGO 69 with 14, and CLARK 77 with 10, and the Dalitz plot results of ALBROW 72 with 11, ALBRECHT 74 with 5.9, and PEACH 73 with 5.5. All other $\chi^{2}$ values were less than 5 . The DONALDSON2 74 result

\section{Data Card Listings For notation, see key at front of Listings.}

$$
\begin{aligned}
& \lambda_{+}=0.030 \pm 0.003 \\
& \lambda_{0}=0.019 \pm 0.004
\end{aligned}
$$

clearly dominates the statistics in the $\mathrm{K}_{\mu 3}^{0}$ case. The $\lambda_{+}$value is consistent with the $k_{e}$ value of $\lambda_{+}$, and with the pole approximation

$$
f_{+}(t)=f_{+}(0) \frac{m_{K^{*}}^{2}}{m_{K^{*}}^{2}-t} \text {. }
$$

Their $f_{0}(t)$ extrapolates linearly to the CallanTreiman point. It is less than two standard deviations from the $\mathrm{K}_{\mu 3} / \mathrm{K}_{\mathrm{e} 3}$ result.

$$
\underline{K}_{\text {e } 3 \text { Experiments }}
$$

The f term of the matrix element [Eq. (2) text Section VI B.2] can be neglected for $\mathrm{K}_{\mathrm{e} 3}$ because it is proportional to the lepton mass. The $f_{+}$term is usually assumed to be linear in $t=q^{2}=\left(P_{K}-P_{\pi}\right)^{2}$, the square of the four-momentum transfer, i.e., the effective mass of the lepton pair. We quote the linear coefficient $\lambda_{+}^{e} \quad(L+E$ on the data cards). There has been some suggestion of departure from linearity [CHIEN $71\left(\mathrm{~K}_{\mathrm{e} 3}^{0}\right)$ and Chounet, Gaillard, and Gaillard ${ }^{1}$ - Review] but no compelling evidence. The $\lambda_{+}$results are fairly consistent and the average values are

$$
\begin{array}{ll}
\mathrm{K}_{\mathrm{e} 3}^{+}: & \lambda_{+}=0.0285 \pm 0.0043 \\
\mathrm{~K}_{\mathrm{e} 3}^{0}: & \lambda_{+}=0.0301 \pm 0.0016 \quad(\mathrm{~S}=1.2)
\end{array}
$$

where the $\mathrm{K}_{\mathrm{e} 3}^{0}$ error has been multiplied by the scale factor 1.2 to compensate for inconsistencies (see ideogram in $\mathrm{K}_{\mathrm{L}}^{0}$ section $\mathrm{L}+\mathrm{E}$ ).

See also the excellent reviews of Gaillard and Chounet, ${ }^{1}$ Chounet, Gaillard, and Gaillard, ${ }^{2}$ and pondrom. 3

\section{References}

1. M. K. Gaillard and L.M. Chounet, "K $\mathrm{K}_{3}$ Form Factors," CERN 70-14 (May 1970), and Phys. Letters 32B, 505 (1970).

2. L. M. Chounet, J.M. Gaillard, and M. K. Gaillard, Physics Reports 4C, 199 (1972).

3. L. G. Pondrom, "Weak Decay Processes," Proc. Particles and Fields 1976, BNL, Oct.6-8, 1976. 


\title{
Data Card Listings
}

Stable Particles

\author{
For notation, see key at front of Listings.
}

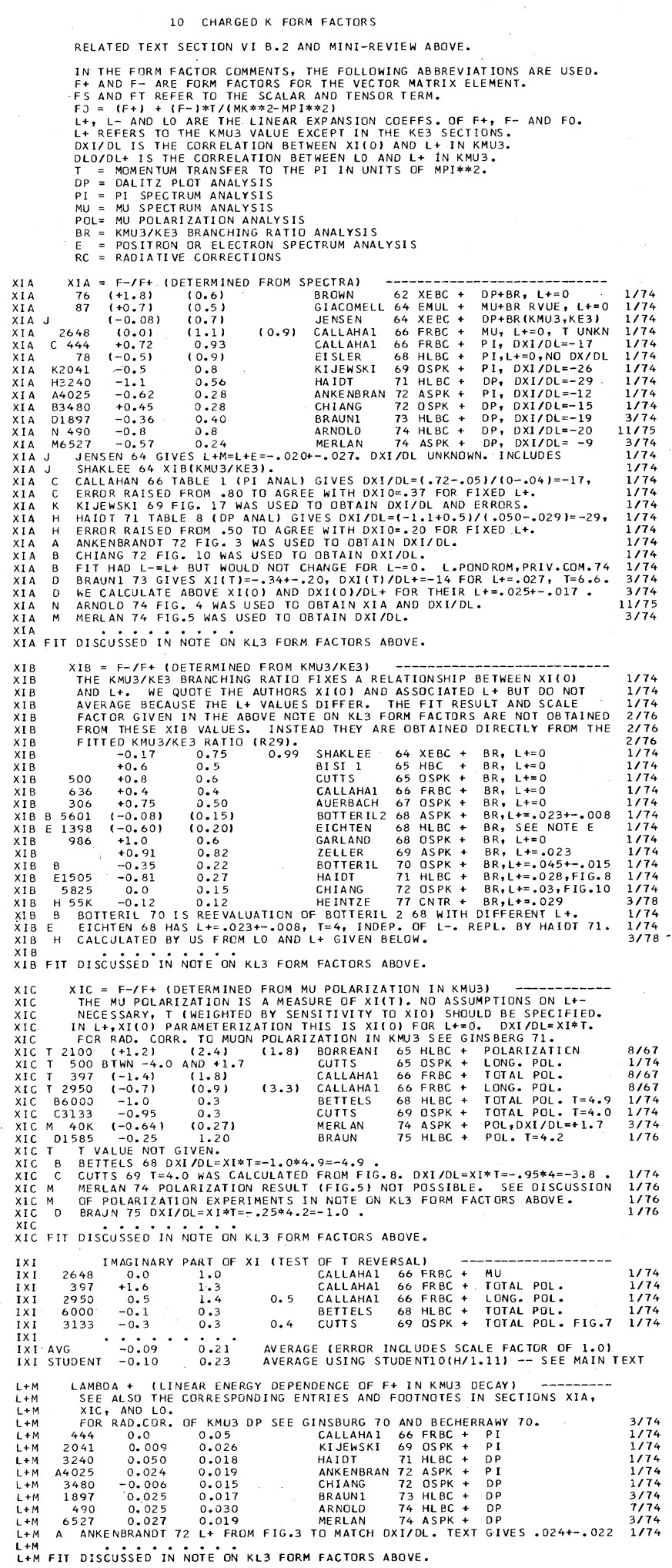

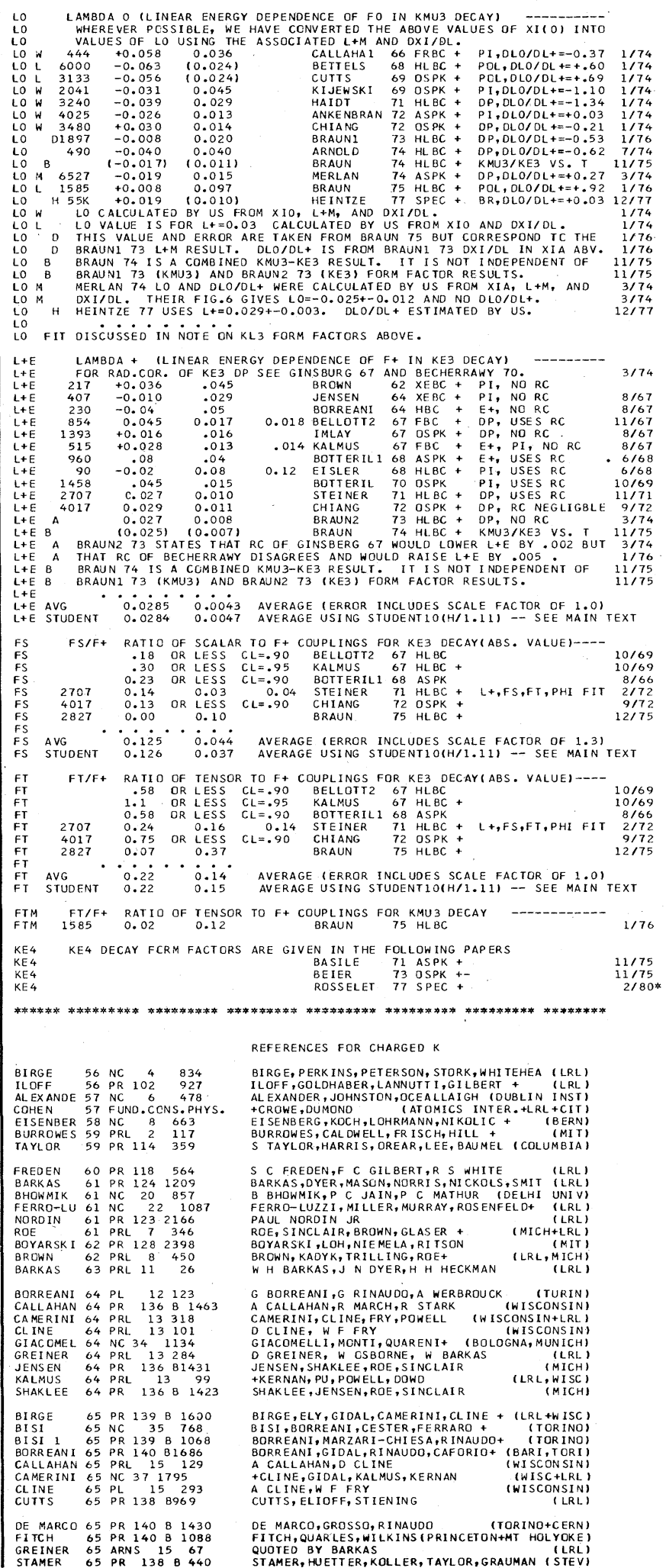




\section{Stable Particles \\ $\mathrm{K}^{ \pm}, \mathrm{K}^{0}$}

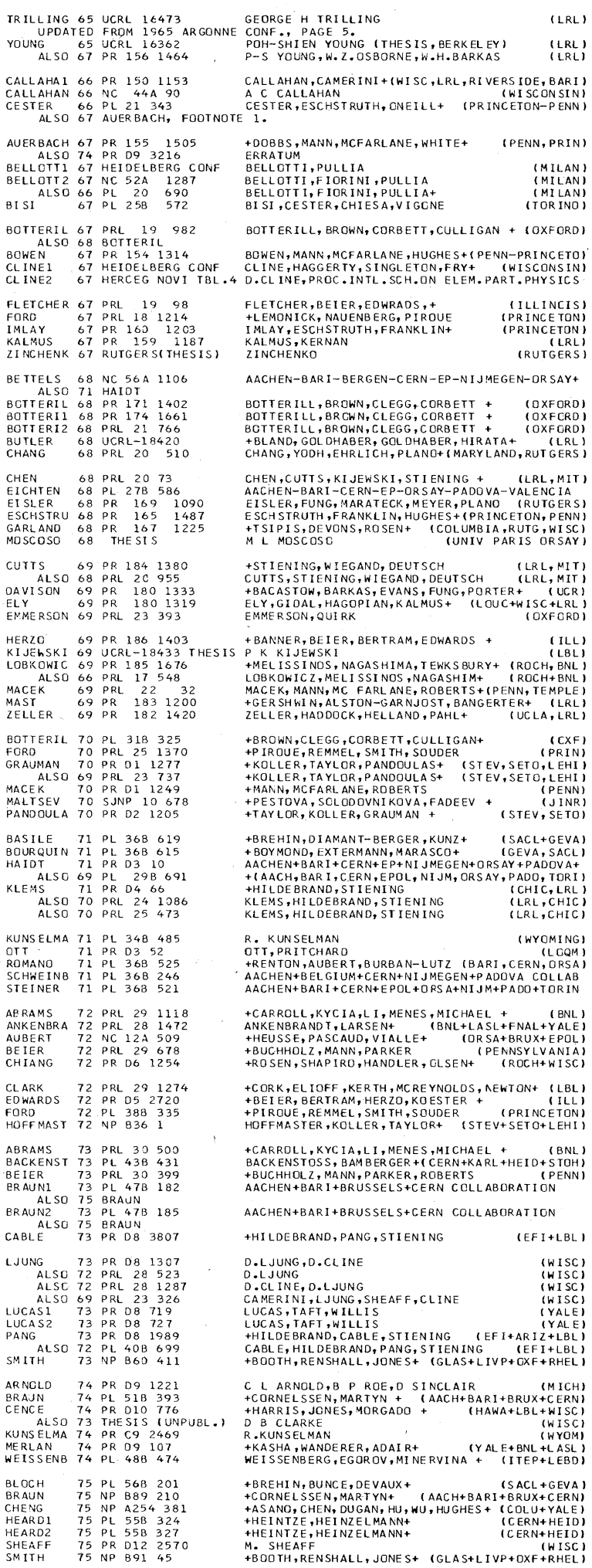

\section{Data Card Listings For notation, see key at front of Listings.}

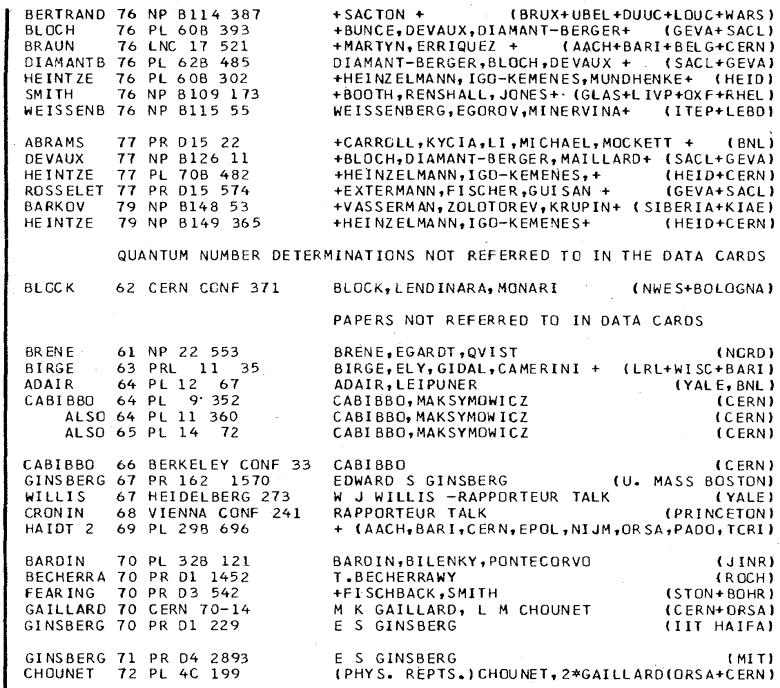

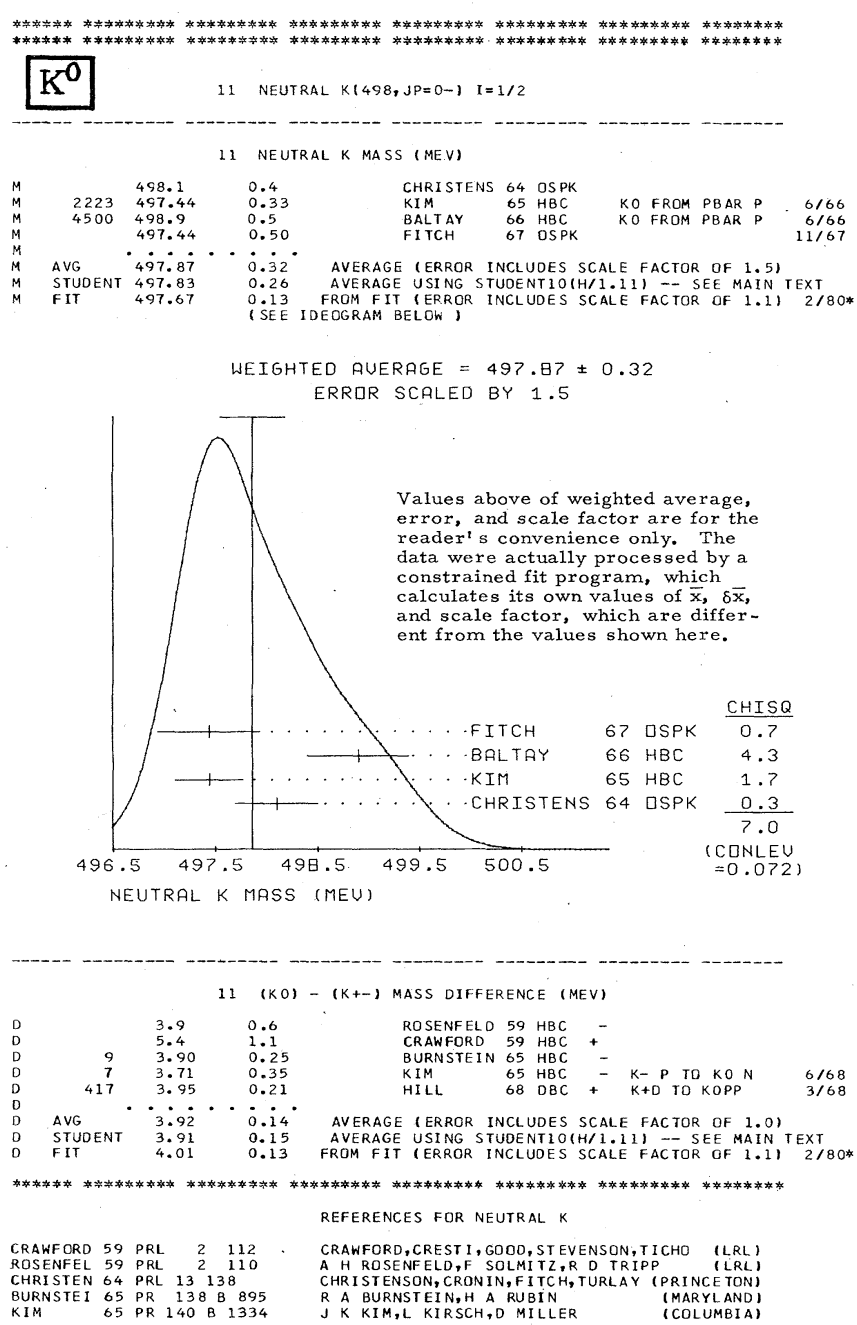




\section{Data Card Listings}

For notation, see key at front of Listings.

\section{Stable Particles}

$\mathrm{K}^{0}, \mathrm{~K}_{\mathrm{S}}^{0}$

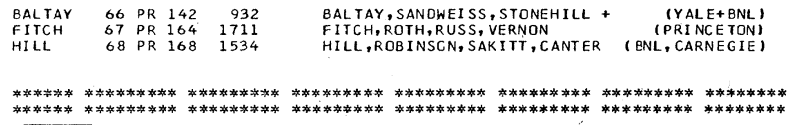

$K_{S}^{0}$

12 SHORT-L IVED NEUTRAL $K(498, J P=0-1 \quad I=1 / 2$

12 KOS MEAN LIFE (UNITS $10 * *-10$ SEC)

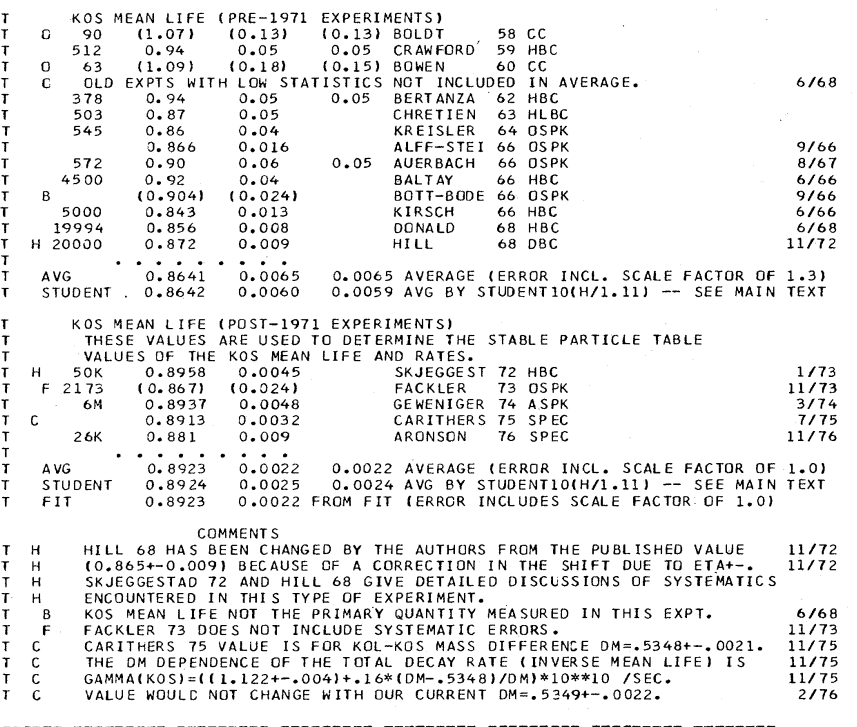

12 kOS partial decay modes

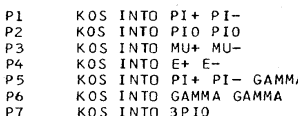

DECAY MASSES
$139+139$

$139+139$
$134+134$
$105+105$
$139+105$

$1.5+$
$139+$
$139+$

$134+134+134$

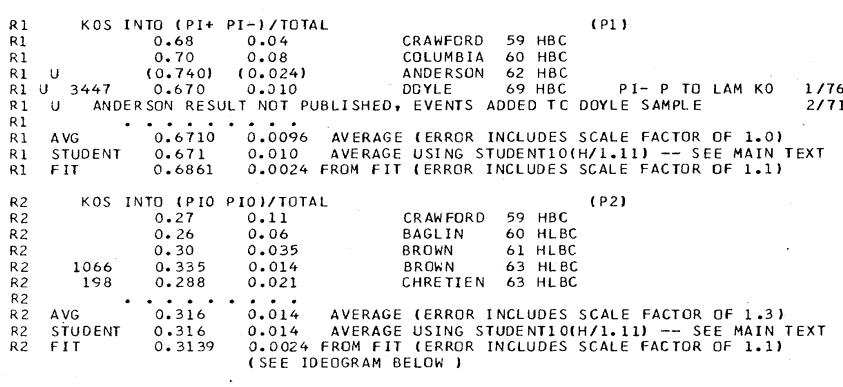

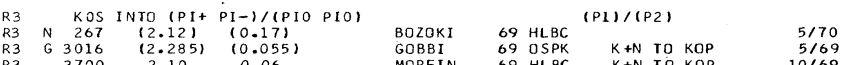

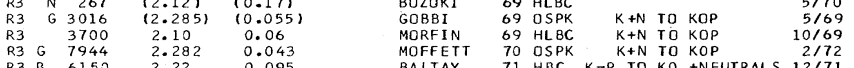

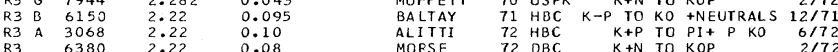

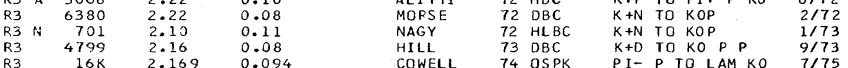

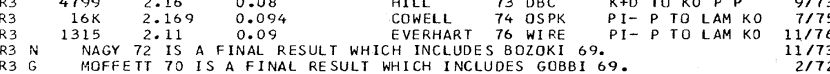

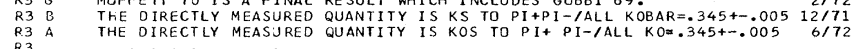

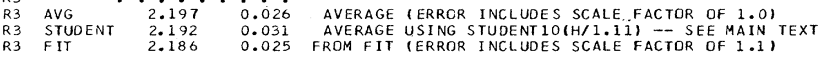

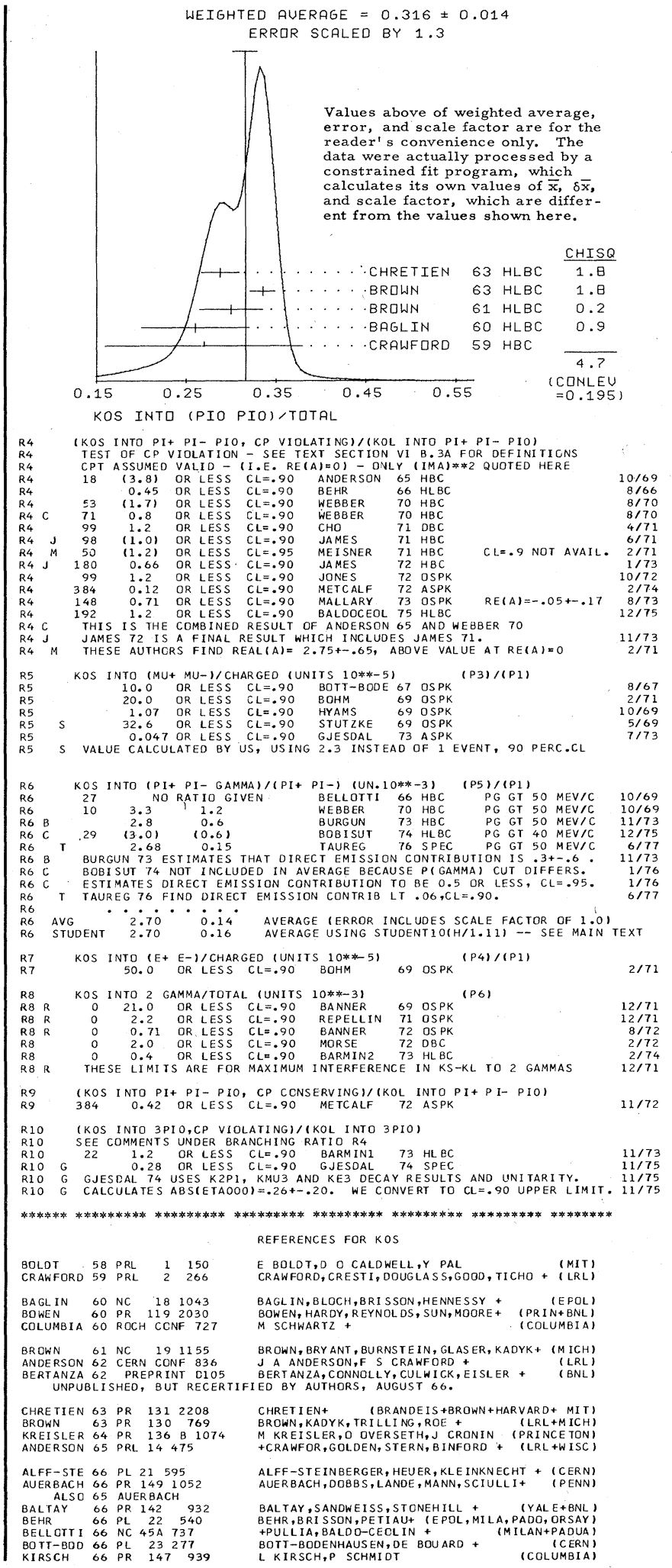




\section{Data Card Listings For notation, see key at front of Listings.}

\section{Stable Particles $\mathrm{K}_{\mathrm{S}}^{0}, \mathrm{~K}_{\mathrm{L}}^{0}$}

\begin{tabular}{|c|c|c|}
\hline $\begin{array}{l}\text { BOTT-BOD } \\
\text { DCNALD }\end{array}$ & $\begin{array}{llll}67 & \text { PL } & 248 & 194 \\
68 & \text { PL } & 278 & 58\end{array}$ & $\begin{array}{l}\text { BOTT-BODENHAUSEN, DE BOUARD, CASSEL+ (CERN) } \\
\text { DONALD, EDWARDS, NI ISAR + (LIVP, CERN, I PNP, CDEF) }\end{array}$ \\
\hline & & \\
\hline & 1882033 & +CRONIN, LIU, PILCHER \\
\hline & & $(A A C H)$ \\
\hline & & +FENYVES, GC \\
\hline & & $\begin{array}{l}\text { J.C. DOYLE } \\
\text { GOBB I, GREEN, HAKEL, ML }\end{array}$ \\
\hline & & $\begin{array}{l}\text { HESSTER) } \\
\text { N(MPIM) }\end{array}$ \\
\hline & & MICHI \\
\hline & 69 & FABA \\
\hline DF & 70 BAPS 15512 & +GOBBI, GREEN, HAKEL, ROSEA \\
\hline $\begin{array}{l}\text { EBGER } \\
\text { ALSO }\end{array}$ & $\begin{array}{l}70 P_{R} D_{1} 1967 \\
69 \text { UCRL } 19226 \text { THESIS }\end{array}$ & $\begin{array}{l}\text { +SOLMITZ, CRAWFORD, ALSTON-GARNJOST } \\
B K \text { WEBBER }\end{array}$ \\
\hline BALTAY & 71 & +BRIDGEWATER \\
\hline & & \\
\hline & 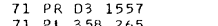 & CANIER,ENGLER, FIS \\
\hline & $71 \%$ & IET, PAUL, PAULI+ \\
\hline REPE & $71 \mathrm{PL}_{3}$ & $\begin{array}{l}\text { +MANN, } \\
\text { +WOLF, CHOLLET, GAILL ARD, JANE+ }\end{array}$ \\
\hline $\begin{array}{l}\text { AL ITTI } \\
\text { BANAER }\end{array}$ & $\begin{array}{ll}72 & \mathrm{PL} \\
72 & 39 \\
72 & \mathrm{PRL}\end{array}$ & $\begin{array}{l}\text { J ALITIT, LE LQUOY, A MULLER } \\
\text { +CRONIN, HOFFMAN, KNAPP, SHOCHET }\end{array}$ \\
\hline & & $=($, PAUL, SAETRE+ (CERN+SACL+OSLO) \\
\hline & 9A 1 & +ABASHIAN, GRAHIAM, MANTSCH, ORR, SMITH+ \\
\hline METCALF & & $\begin{array}{ll}\text { +NEUHOFE, NIERERGALL } & \text { (CERN+IPN+WIEN) } \\
+ \text { NAJEEBERG, BIERMAN, SAGER } & \text { (COLO+PRIN IN UMD ) }\end{array}$ \\
\hline AGY & $72 \mathrm{~N}$ & $\begin{array}{l}\text { +TEL BISZ, VESTITERGOMBI } \\
+\end{array}$ \\
\hline & $69 \mathrm{p}$ & BOZOKI, FENYVES, GOMBOSI, NAC \\
\hline SK J & $72 \mathrm{NP}$ & SK JEGGESTAD, JAMES, MONT ANET + COSLO+CERN+SACL I \\
\hline IN1 & 73 & + BARYLOV, DAVIDENKO, DEMIDO \\
\hline & 73 & \\
\hline & $73 \mathrm{PL} 46 \mathrm{~B}$ & YY, MULLER,PAULI+ \\
\hline$E R$ & 73 PRL 31847 & MPAYRAC \\
\hline & $\begin{array}{llll}73 & P L & 44 \mathrm{~B} & 21 \\
7 & & \end{array}$ & EN, STE I NBERGER+ \\
\hline ALLARY & $\begin{array}{llll}73 & P R & D 8 & 129 \\
73 & P R & D 7 & 195\end{array}$ & $\begin{array}{l}\text { +SAKIT, SAMIOS, BURRIS, ENGLER+ } \\
\text { +BINIE, GALLIVANGOMEZ, PECK. SS }\end{array}$ \\
\hline & & \\
\hline COWE & $74 \mathrm{P}$ & $\begin{array}{l}\text { +HUZIIA, MAITIOL I, PUGLIERIN. } \\
\text { +LEE-FRANZINI, ORCUTT, FRANZINI }\end{array}$ \\
\hline & 74 & DI \\
\hline & 74 & +PRESSER, STEFFEN,STEINBERGER + (CERN+HEID) \\
\hline $\begin{array}{l}\text { ALDOCEO } \\
\text { ARITHER }\end{array}$ & 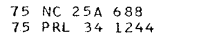 & $\begin{array}{ll}\text { BALDO-CEOL IN, BOBISUT, CAL IMANI }+ & \text { (PADO+WISC) }) \\
\text { CARI THERS, MODIS, NYGREN, PUN + } & \text { (COLU+NYU })\end{array}$ \\
\hline & 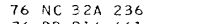 & FI+UCS \\
\hline & $76 \mathrm{PR}$ & , LOWENSTEIN \\
\hline TAUKEG & 76 PL 65892 & YDAK, NA VARRIA \\
\hline & & PAPERS NOT REFERRED TO IN DATA CARDS \\
\hline $\begin{array}{l}\text { BIRGE } \\
\text { MULLER }\end{array}$ & 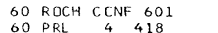 & $\begin{array}{l}\text { GE, P P ELY + } \\
\text { BIRGE, FOWLER, GOOD }\end{array}$ \\
\hline & $61 \mathrm{NC} 221160$ & 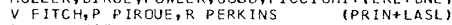 \\
\hline & $\begin{array}{llll}61 & P R & 124 & 1223\end{array}$ & GOOD, MATSEN, MULLER, PICCIONI + \\
\hline & 6 & $\begin{array}{l}(\text { LRL }) \\
(P E N N)\end{array}$ \\
\hline & & GEO \\
\hline
\end{tabular}

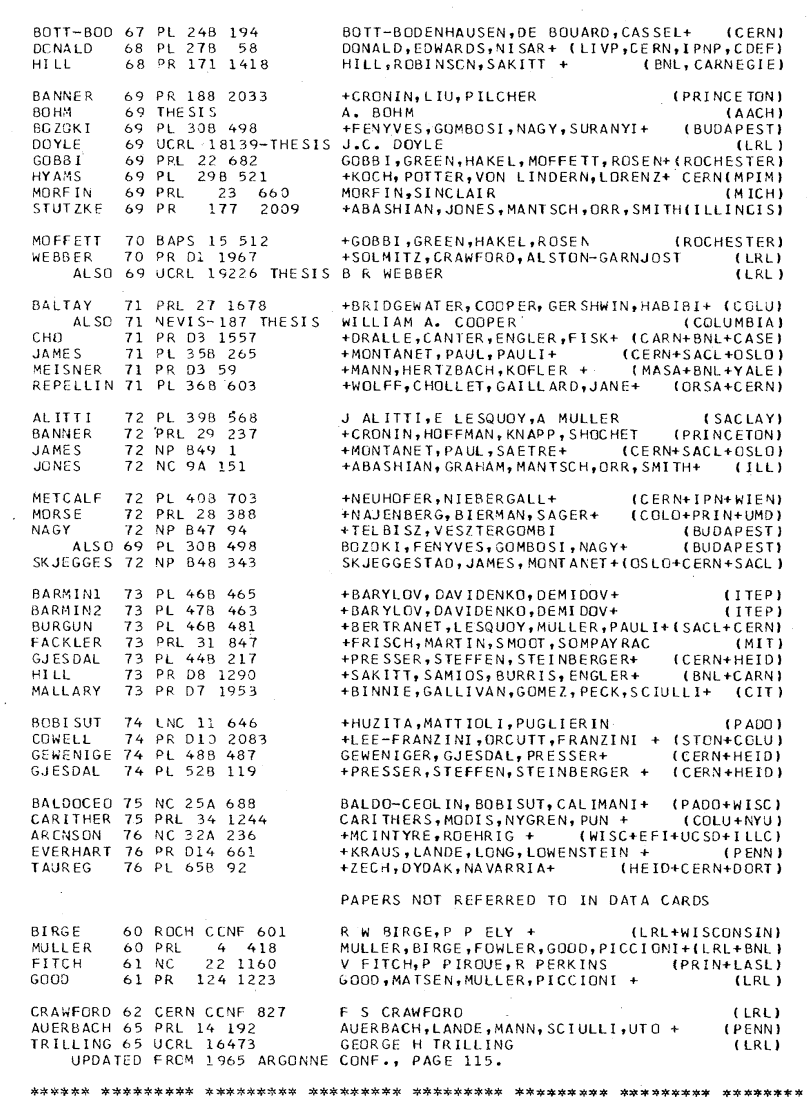

员 13 LONG-LIVEO NEUTRAL K(498, JP=0-1 I=1/2.

13 (KOL) - (KOS) MASS DIFFERENCE
WE GIVE (KOL-KOS MASS DIFFERENCE / HBAR) IN UNITS OF $10 * 10$ SEC-1

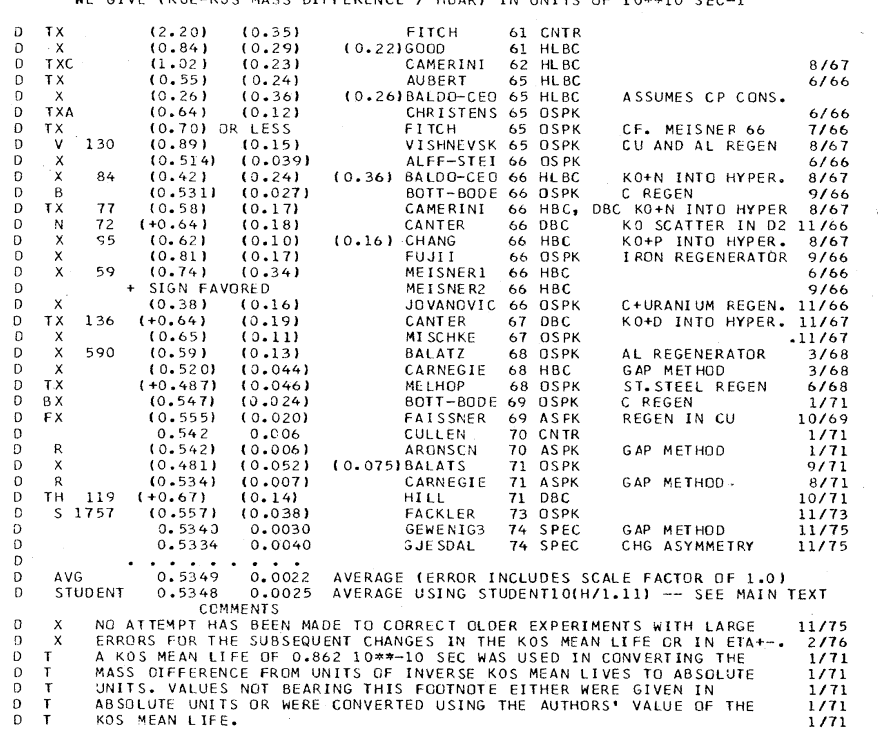

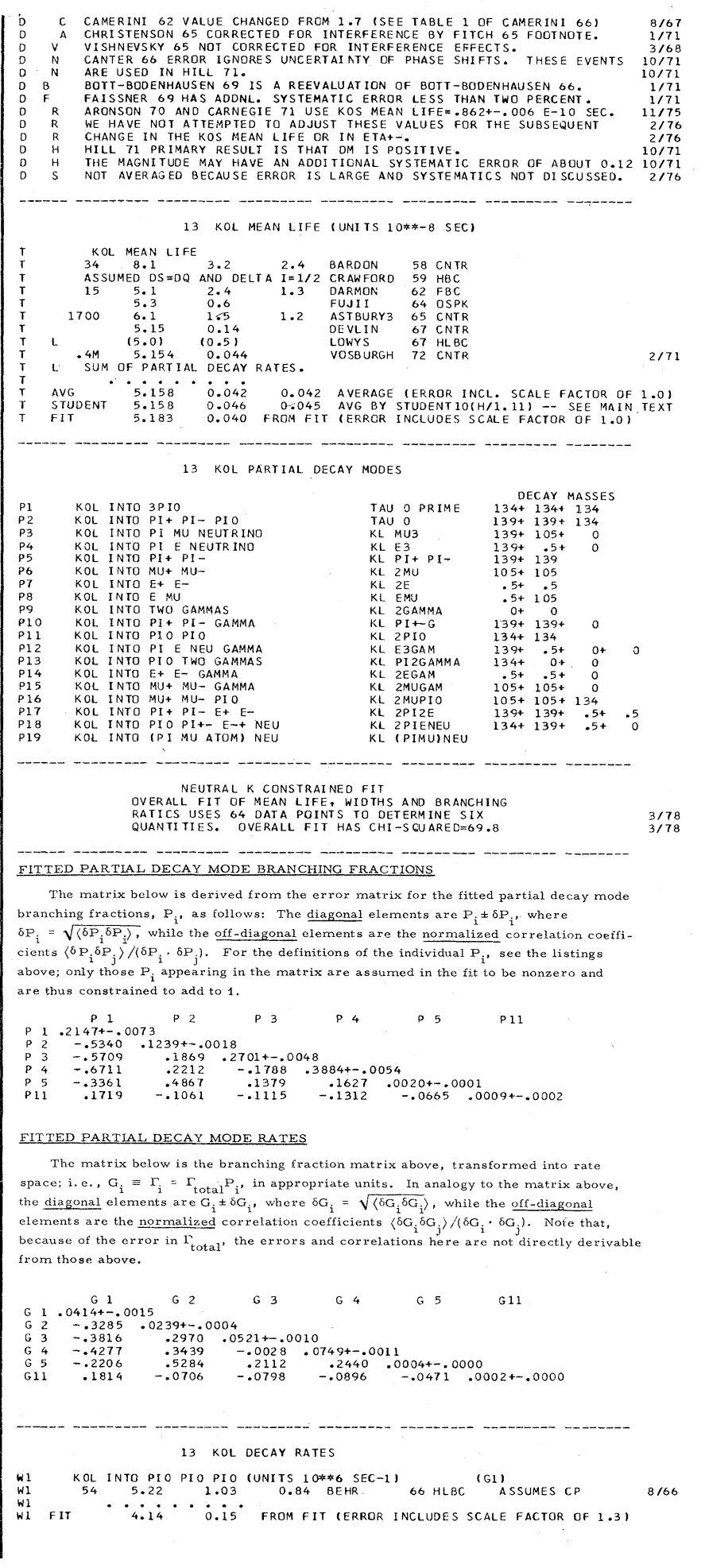




\section{Data Card Listings}

\section{For notation, see key at front of Listings.}

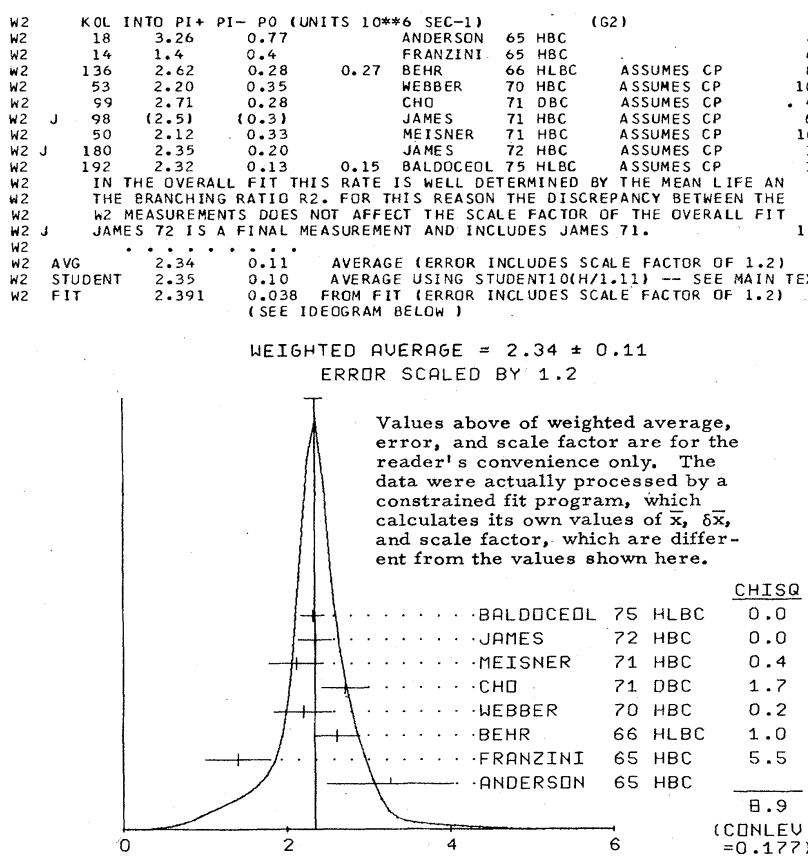

KOL RATE INTO PI+ PI- PIO (10**6 SEC-1)
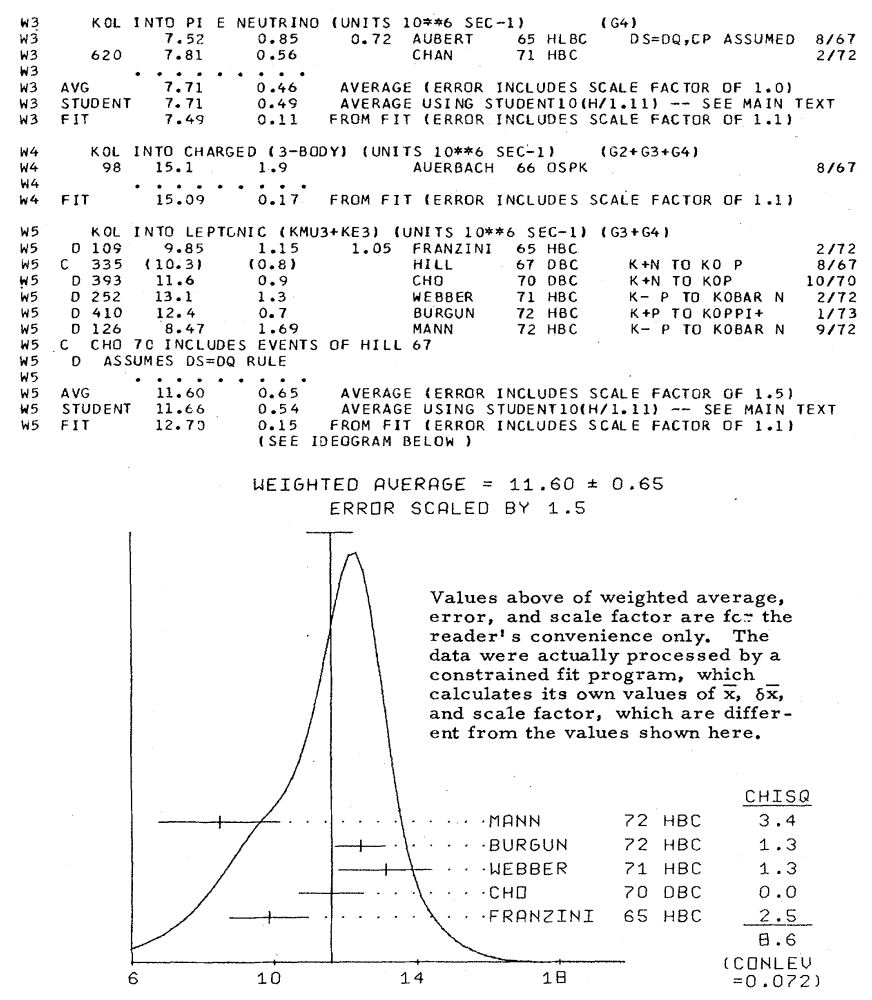

KOL RATE INTD KMU3 + KE3 (10**6 SEC-1)

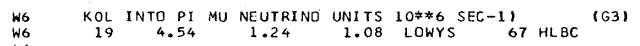

W6 FIT - $5.211 \cdot 0.100$ FROM FIT (ERROR INCLUDES SCALE FACTOR OF 1.11

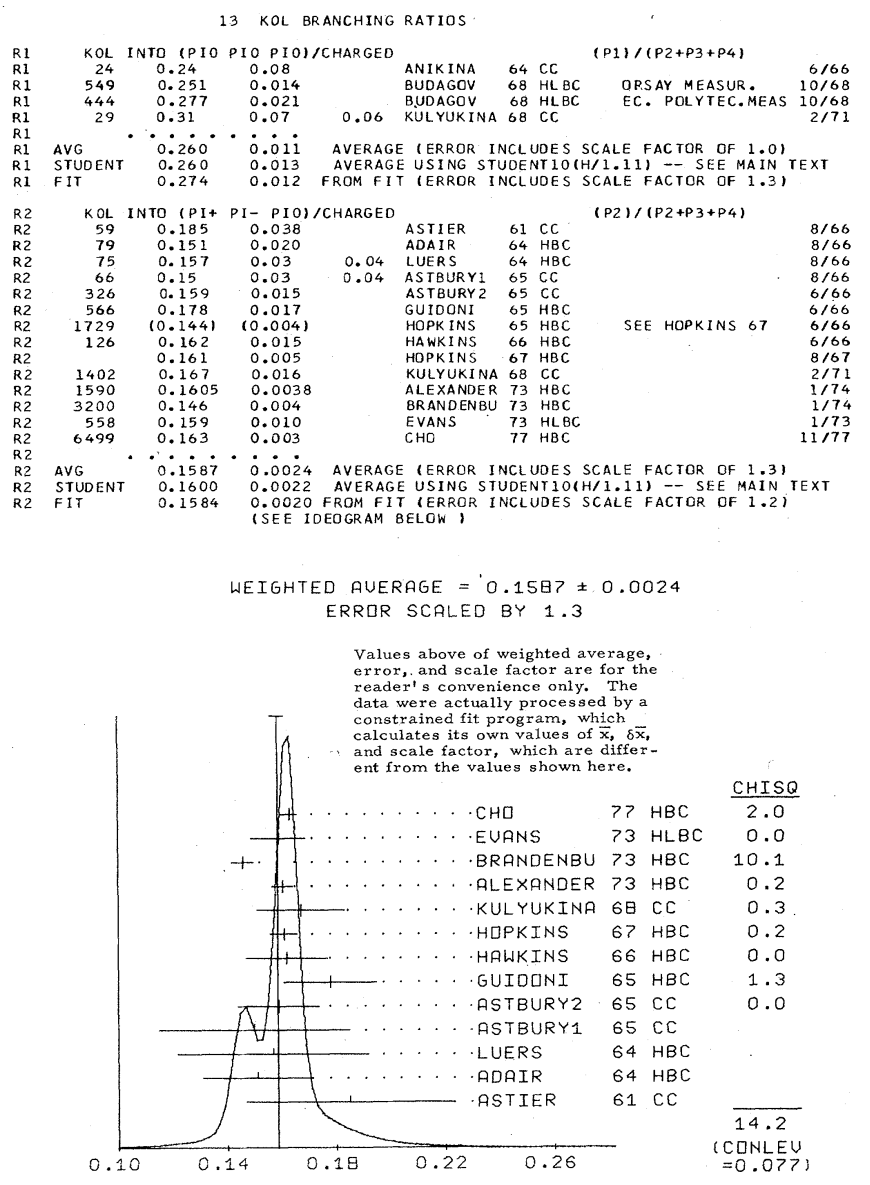

KOL INTO (PI+ PI- PIO) CHARGED

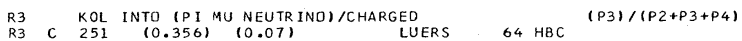

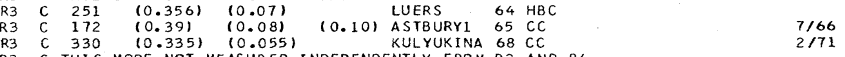

R3 C THIS MODE NOT MEASURED INDEPENDENTLY FROM R2 ANO R4

${ }_{R 3}^{R 3}$ FIT $\because 0.3452 \cdot 0.0051$ FROM FIT

$\begin{array}{lllllllll}\text { R4 } & \text { KOL INTO } 1 \text { PI } & \text { E NEUTRINO } / \text { /CHARGED } & & & & \\ \text { R4 } & 24 & 0.46 & 0.11 & \text { NEAGU } & 61 \mathrm{CC} & (P 4) /(P 2+P 3+P 4) & 2 / 7\end{array}$

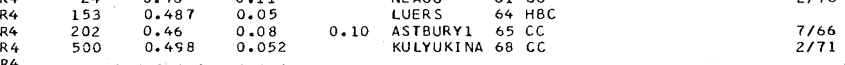

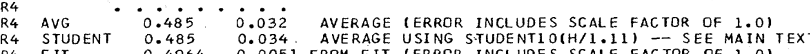

$\begin{array}{lll}R 4 & \text { FIT } 0.4964 & 0.0051 \text { FROM FII }\end{array}$

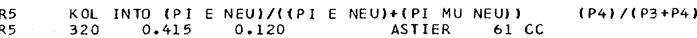

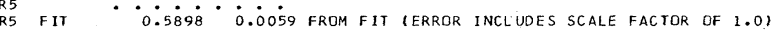

KOL INTO (PI+ PI- PIO)/TOTAL

(P2)

R6 FIT - $0.1239 \cdot 0$. Oois FROM FII

7 KOL INTO (LEPTON PI NEUTRINOI/TOTAL

$(p 3+p 4)$

7 FIT $\because 0.6584^{\circ} \dot{0.0066 \text { FROM FIT }}$

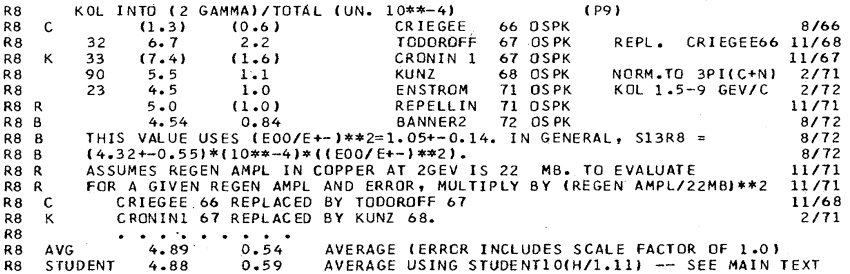




\section{Stable Particles \\ $\mathrm{K}_{\mathrm{L}}^{0}$}

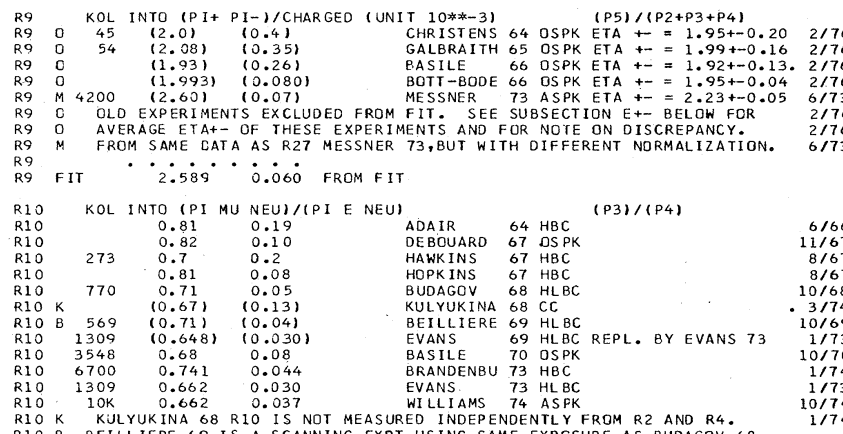

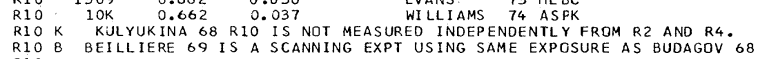

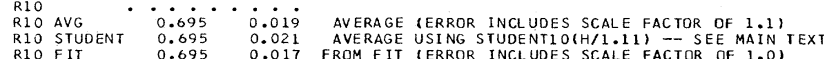

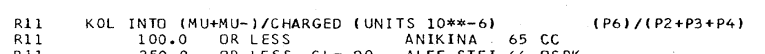

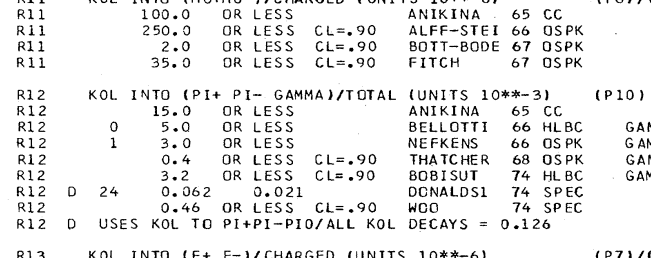

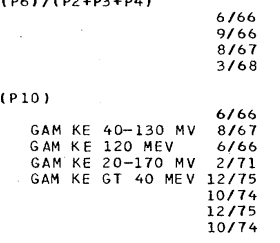

R13 KOL INTO (EE E-1/2CHARGED (UNITS $10 * *-6)$
R13
1000.0

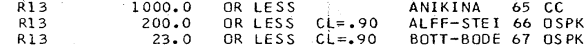

R14 KOL INTO (E MU)/CHARGED (UNITS 10**-4) (P8)/(P2+P3+P4)

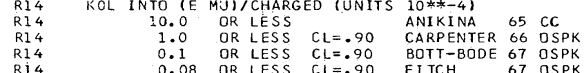

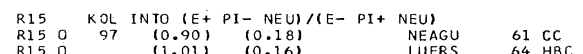

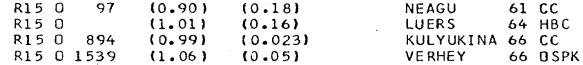

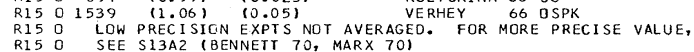

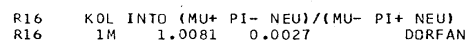

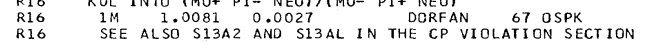

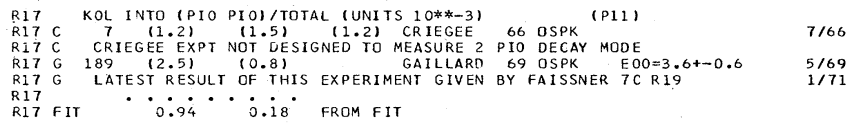

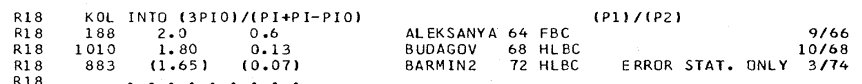

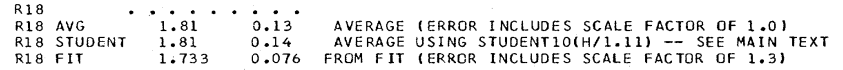

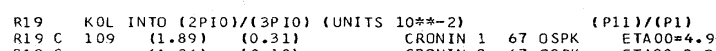

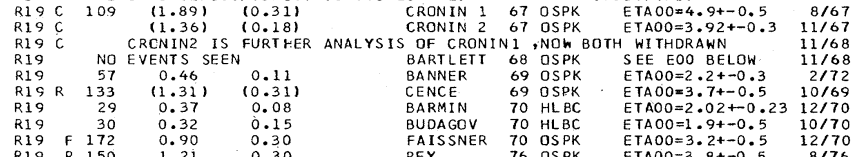

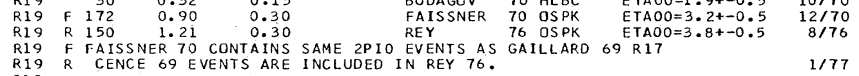

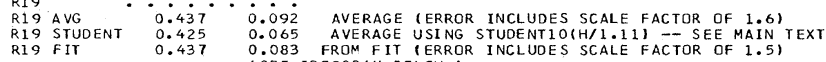

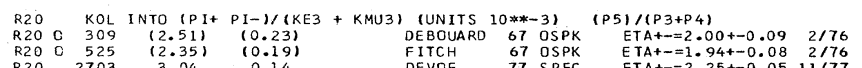

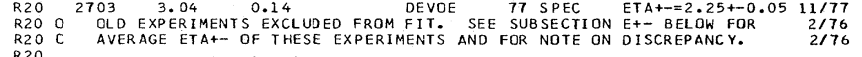

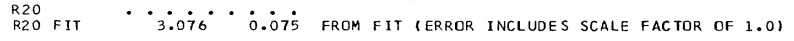

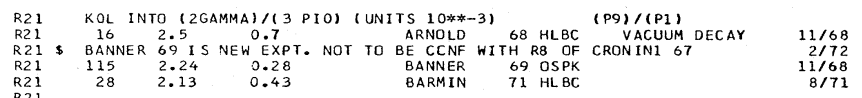

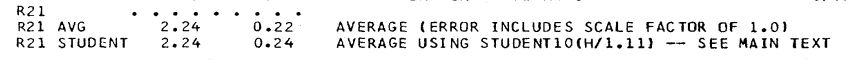
$6 / 66$
$6 / 66$
$8 / 67$

\section{Data Card Listings For notation, see key at front of Listings.}

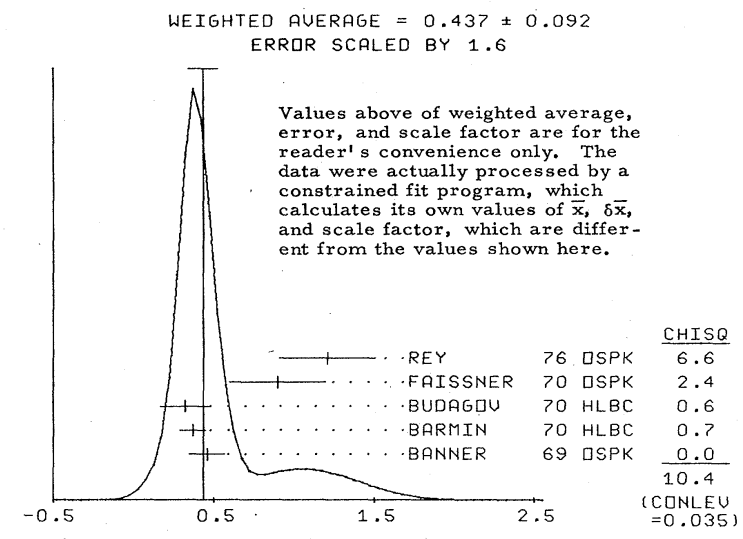

KOL INTD (2PIO)/(3PIO) (10**-2)

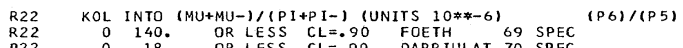

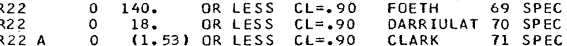

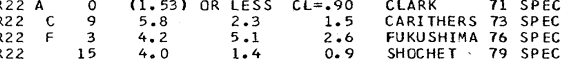

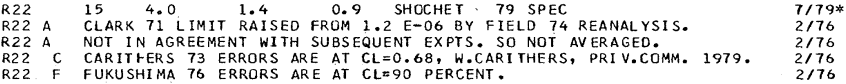

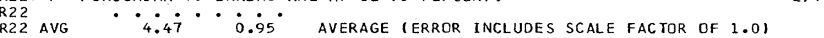
R22 STUDENT 4.5 1.0 AVERAGE USING STUDENTIO(H/1.11) -- SEE MAIN TEX

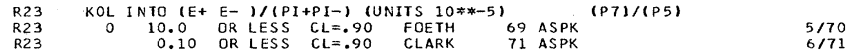

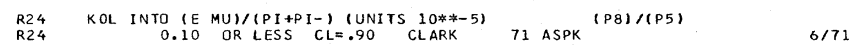

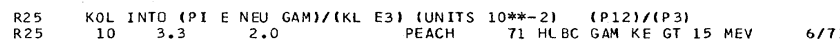

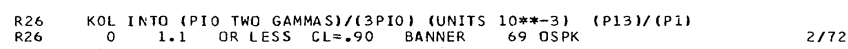

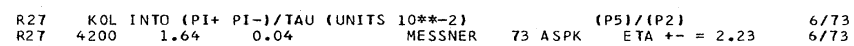

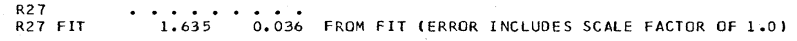

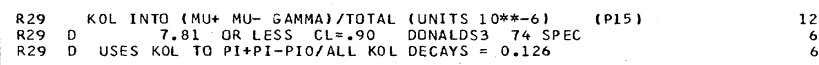

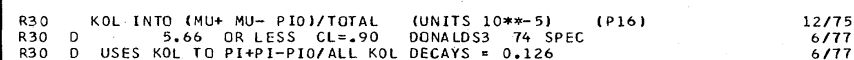
R31 KOL INTO (PI+PI-E+E-) /TOTAL CUNIIS $10 * *-6)$
R31
30.

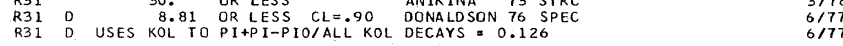

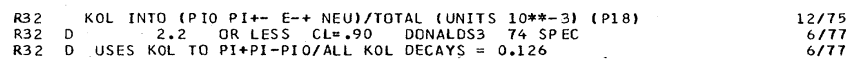

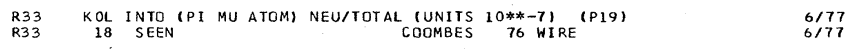
13 KOL ENERGY DEPENDENCE OF DALITZ PLOT

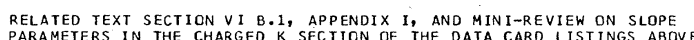

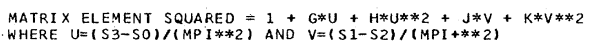

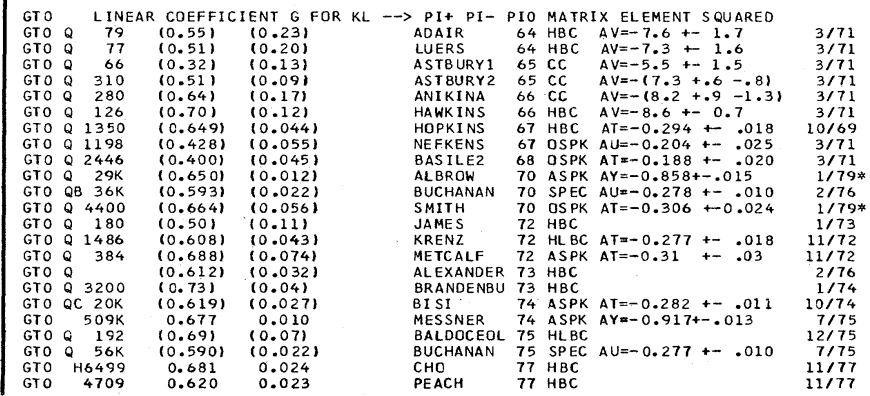




\section{Data Card Listings}

Stable Particles

For notation, see key at front of Listings.

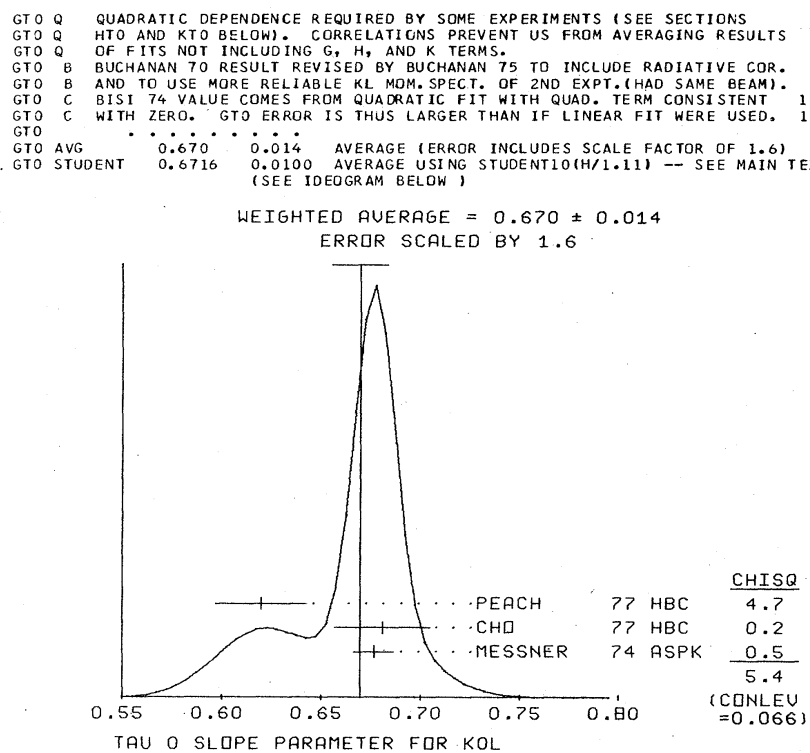

HTO QUADRATIC COEFF. H FOR KL - - P PI+ PI- PIO MATRIX ELEMENT SQUARED

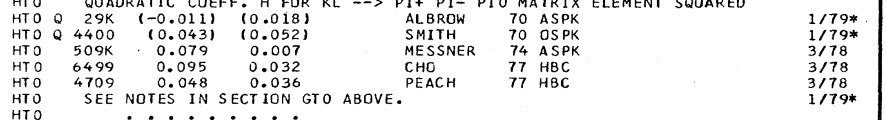

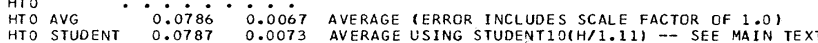

KTO QUUDRATIC COEFF. K FOR KL $\rightarrow$ P PIP PI-P PIO MATR IX ELEMENT SQUARED

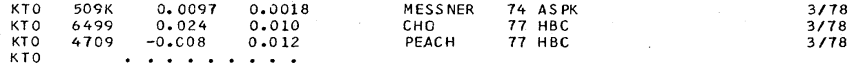

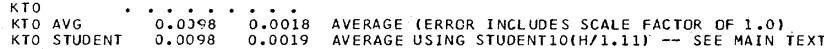

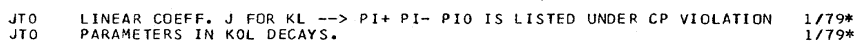

\section{KOL FQRM FACTORS}

RELATED TEXT SECTION VI B. 2 AND MINI-REVIEW ON FORM FACTORS
IN THE CHARGEO K SECTION OF THE DATA CARD LISIINGS ABOVE.

IN THE FORM FACTOR COMMENTS, THE FOLlOWING abBreviations are USEO.

F. ANO F- ARE FORM FACTORS FOR THE VECTOR MARIX ELEMENT.
FS AND FT REFER TO THE SCALAR AND TENSOR TERM.

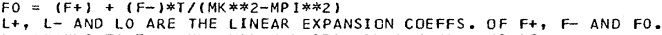

L. REFERS TO THE KMU3 VALUE EXCEPT IN THE KE3 SECTIONS.
OXIJOL IS THE CORRELAT ION BETWEN XICO) AND L IN KMU3.

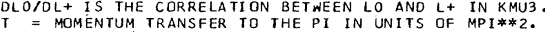

DP $=$ DALIY PLLT ANALYSIS
PI $=$ PI SPECRUMA ANALYSTS

MOU = MU SPECTRUM ANALYSIS

POL $=$ MU POLARIZATION ANALYSIS
$B R=$ KMUI/KE3 BRANCHING RAID ANALYSIS

$B R=$ KMUU/KE3 BRANCHING RATII ANALYSIS
$E$ P POSITRCN OR ELECTRON PPECTRUM ANALYSIS

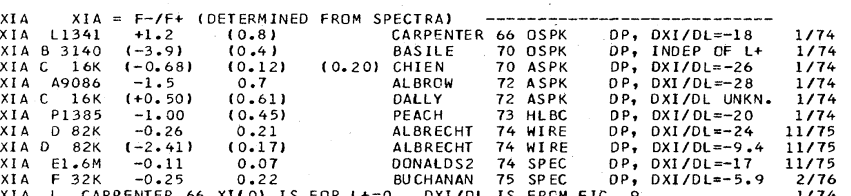

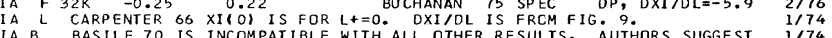

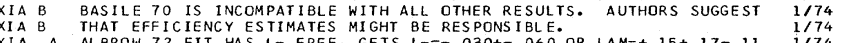

XIA A ALBROW 72 FIT HAS L- FREE, GETS L L =-.030+..060 OR LAM=+.15+.17-.11.

XIA C DALLY T2 IS A REANALYSIS OF CHIEN 70. THE DALLY 72 RESULT IS

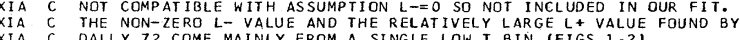

IA C THE (FF+XI) CORRELATION WAS IGNORED.

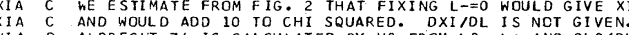

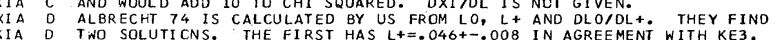

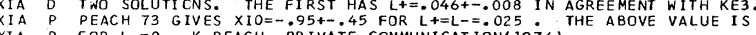

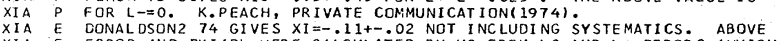

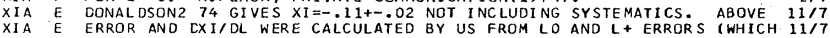

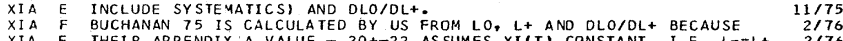

XIA FIT DISCUSSEDO IN NOTE ON KL3 FORM FACTORS IN K+- SECTION OF DATA CARDS.

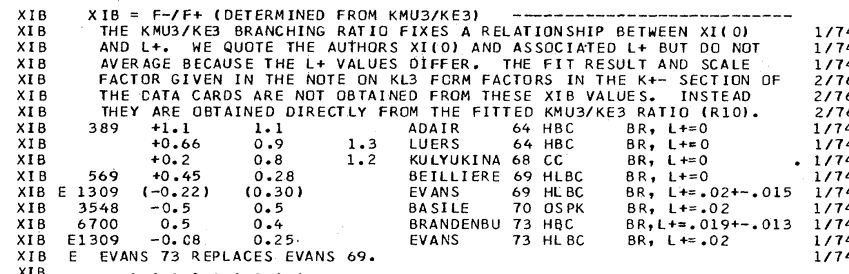

XIB FIT DISCUSSED IN NOTE ON KLZ FORM FACTORS IN Kt- SECTION OF DATA CAROS.

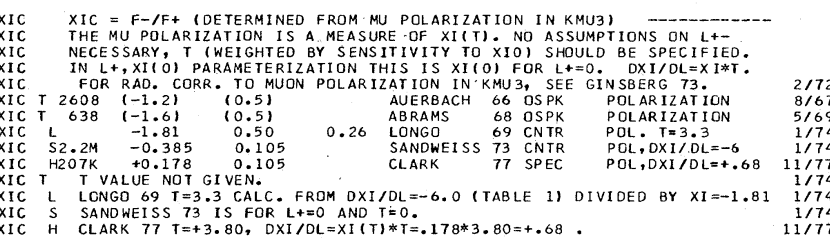

XIC FIT DISCUSSSED IN NOTÉ ON KL3 FORM FACTORS IN K+- SECTION OF DATA CARDS.

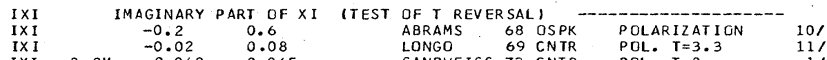

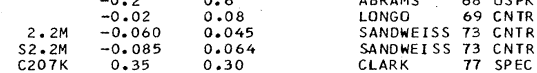

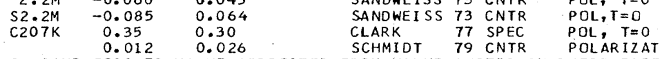

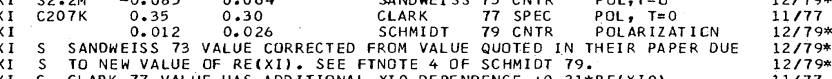

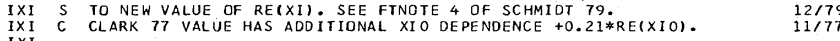

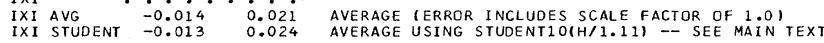

$L+M$
$L+M$
$L+M$

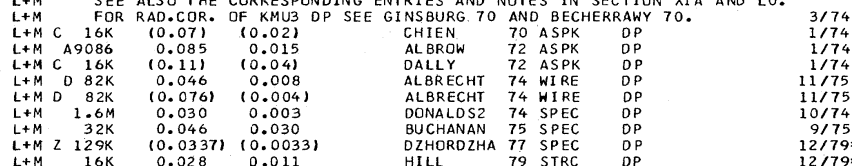

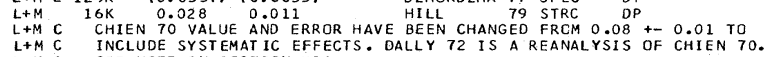

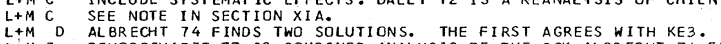

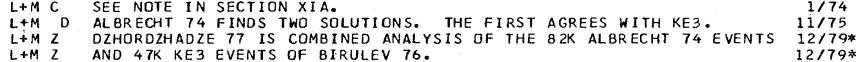

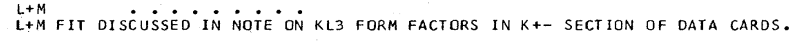

LO LAMBA O (LINEAR ENERGY DEPENDENCE OF FO IN KMUO DECAY
LO WHEREVER POSSIBLE, WE HAVE CONVERTED THE ABOVE VALUES OF XI(O) INTO

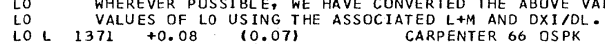

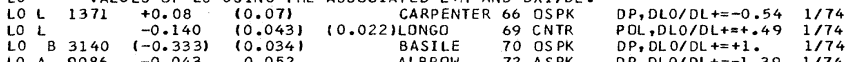

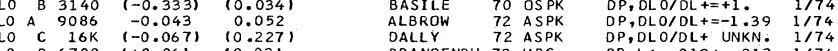

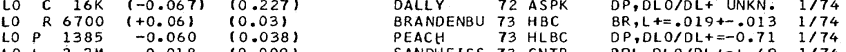

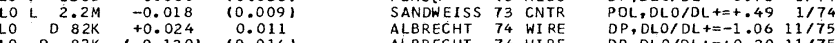

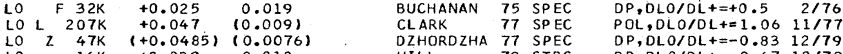

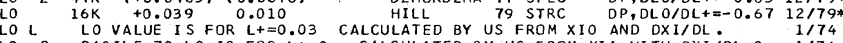

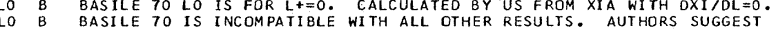

LO B THAT EFFICIENCY ES IMATES MIGHT BE RESPONSIBLE. OXINL. THEY GIVE

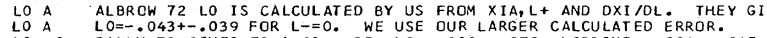

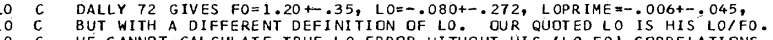

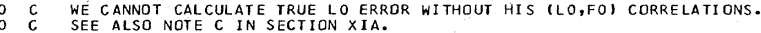

PEACH 73 ASSUMES Lt=0.025. CALCULATED BY US FROM XIO AND DXIO/OLL+.
FIT FOR LO DOES NOT INCUDE YHIS VALUE BUT INSTEAD INCLUDS THE

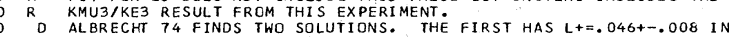

DAGREMENT WITH KE3. DLO/DL+ OBTAINED FROM FIG.2C.

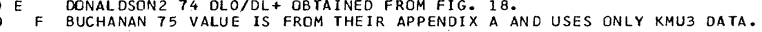

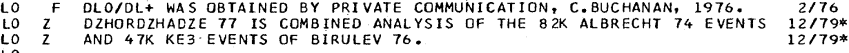

LO FIT DISCUSSE ${ }^{\circ}$ IN NOTE ${ }^{\circ}$ CN KL3 FORM FACTORS IN K+- SECTION OF DATA CAROS. 


\section{Stable Particles \\ $\mathrm{K}_{\mathrm{L}}^{0}$}

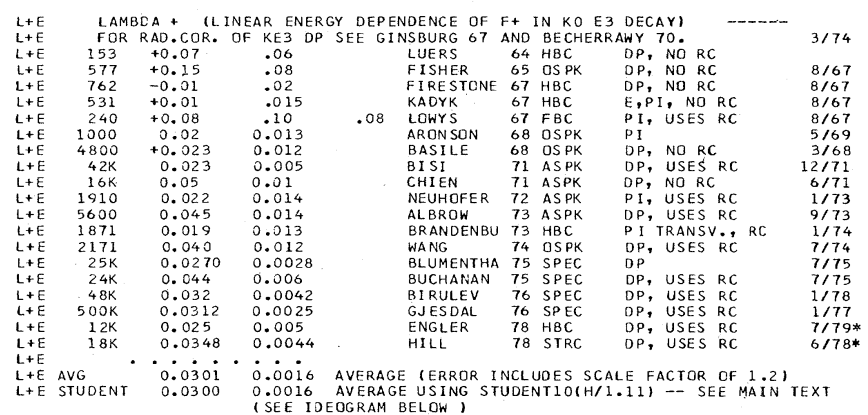

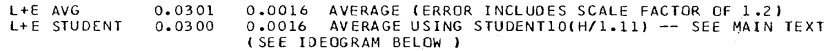
WEIGHTED AUERAGE $=0.0301 \pm 0.0016$

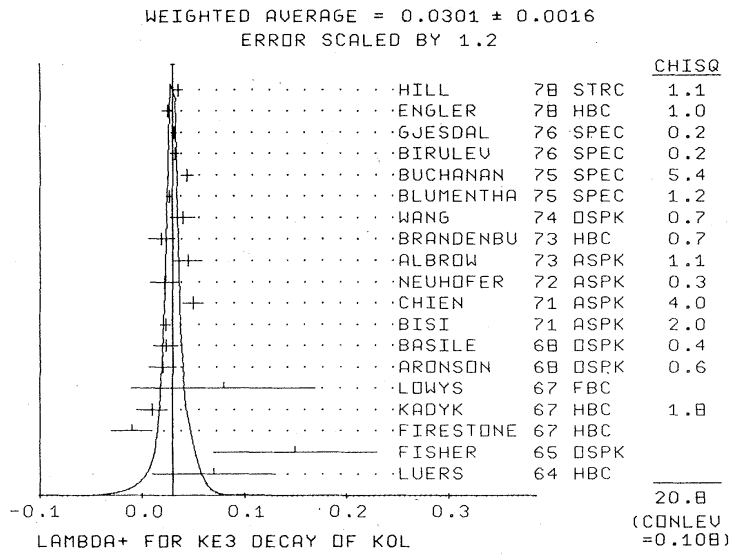

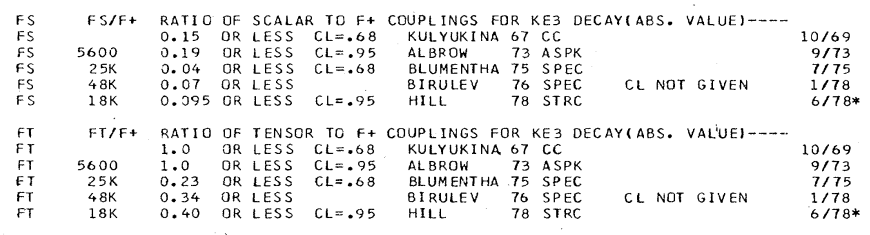

13 CF violation parameters in kOL decays

RELATED TEXT SECTION VI B.3 AND MINI-REVIEW BELOW

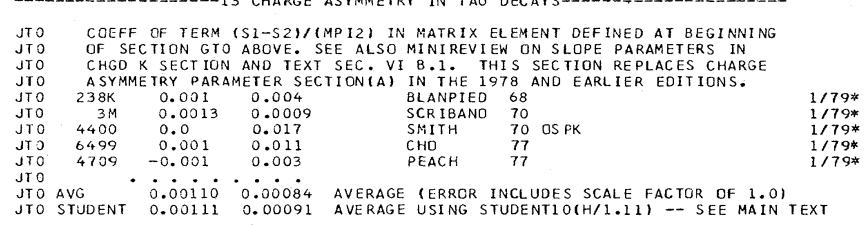

TEXT SECTION VI B.3 C

SUCH a SYMMETRY VIOLATES CP. It is Related to real (EPSiloni.

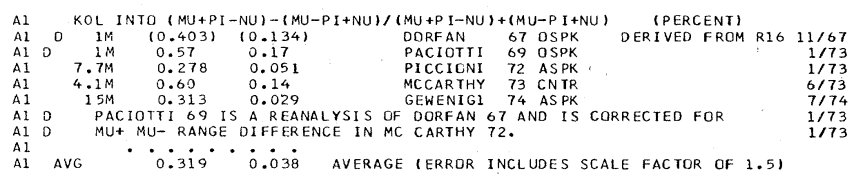

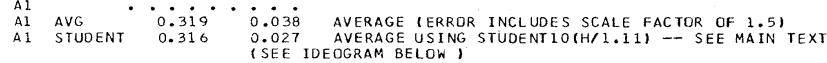

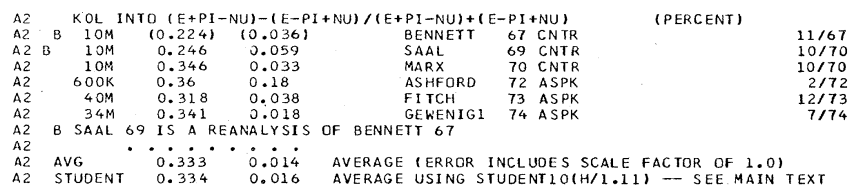

Data Card Listings For notation, see key at front of Listings.

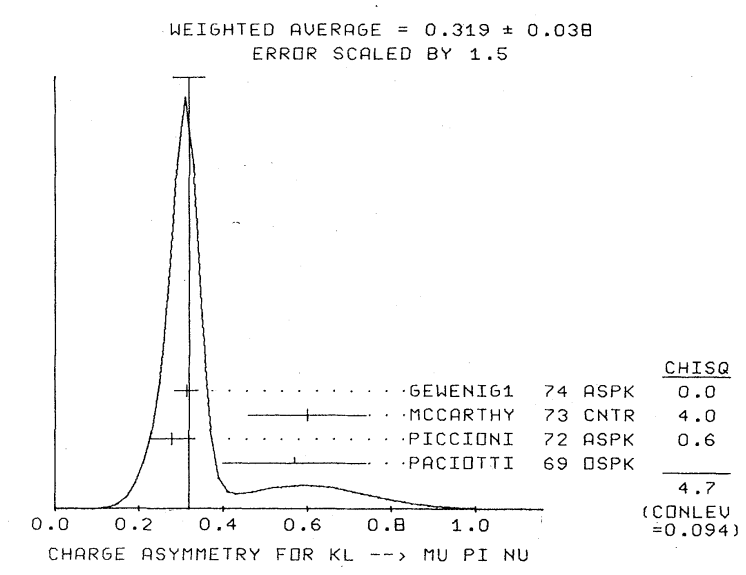

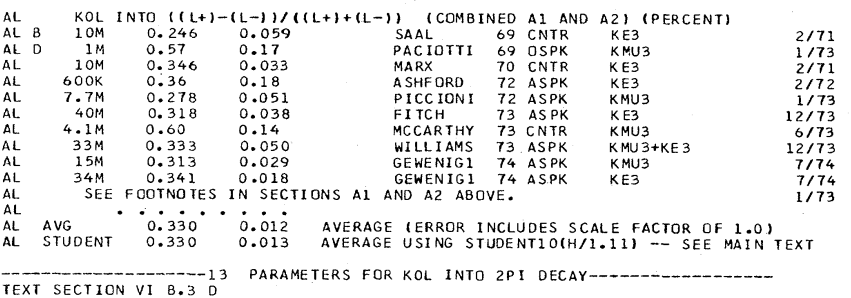

ETAY- $=$ ACKL TO PItPI- IACKS TO PIPPI-)
ETAOO $=$ A(KL TO PIOPIO)/ACKS TO PIOPIO)

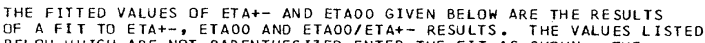

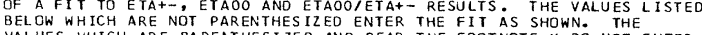
VALUES WHICH ARE PARENHESIZED AND BEAR THE FOOTNOTE X DO NOT ENTER
THE FIT AS SHOHN. THESE EXPRIMENTS GIVE BRANCHING RAT IOS AND ENTER

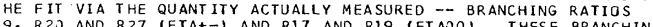
RAT IOS ARE COMBINED WITH CURRENT NORMALIZATIONS AND CURRENT KL AAD KS
MEAN LIVES TO OBTAIN PPI PI RATES. THE EAT- AND ETAOO VALUES OBTAINED

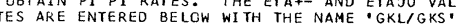

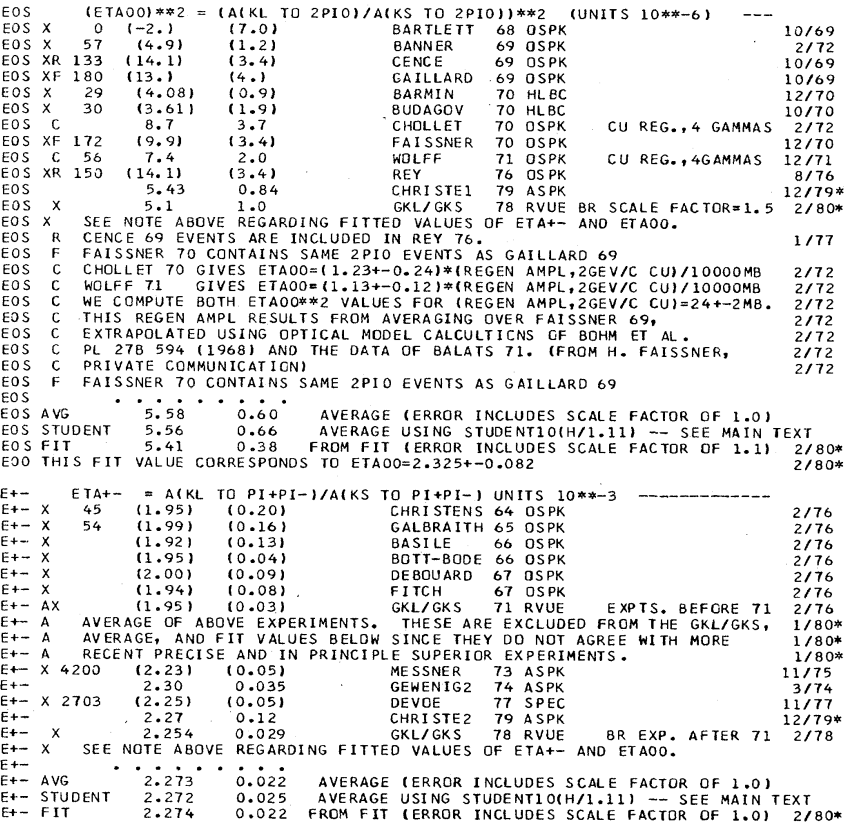




\section{Data Card Listings}

Stable Particles

\section{For notation, see key at front of Listings.}

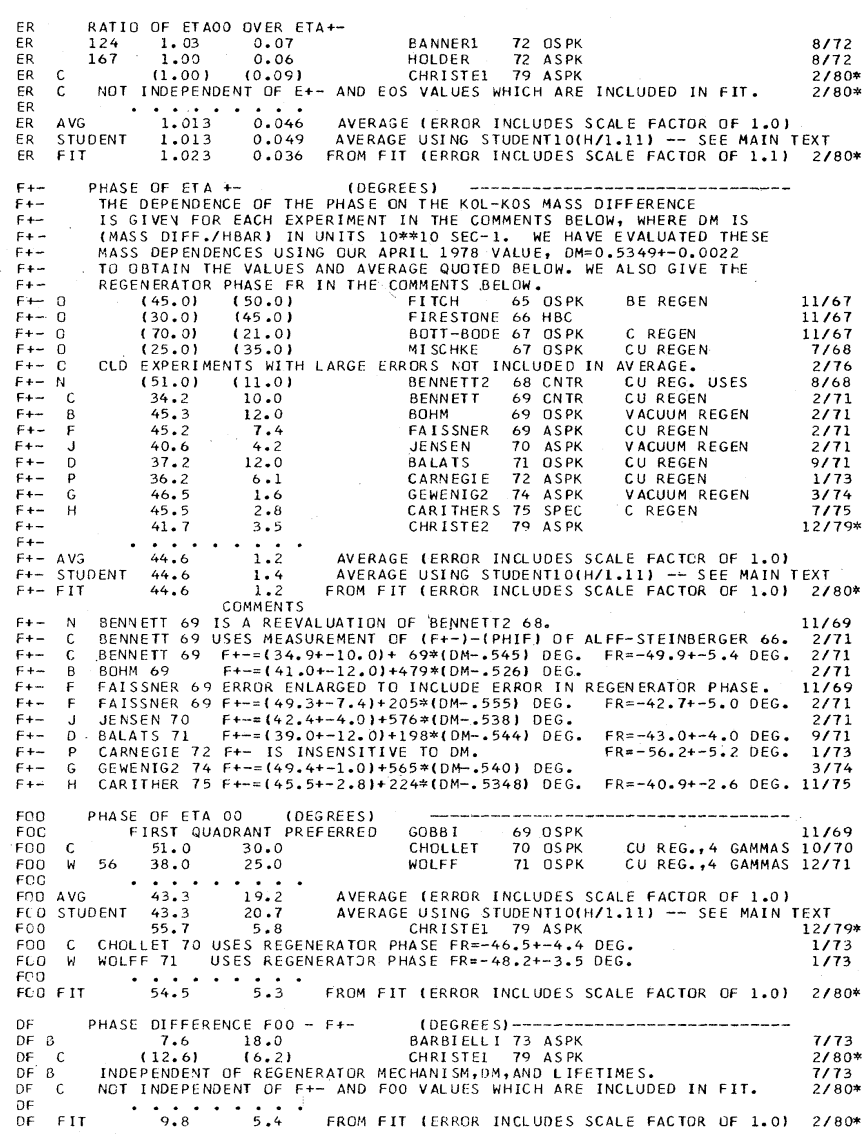

Superweak Model Predictions for $\left|\eta_{00} / \eta_{+-}\right|, \phi_{+-}$and Re $\varepsilon$

In terms of the parameters defined in the text, Sec. VIB(d), the superweak model ${ }^{1}$ predicts that ${ }^{2}$

$$
\left|n_{00} / n_{+-}\right|=1 \text {, }
$$$$
\phi_{+-}=\phi_{00}=\tan ^{-1}\left(\frac{2 \Delta \mathrm{m} \tau}{\hbar}\right) \text {. }
$$

and

$$
\operatorname{Re} \varepsilon=\left|n_{+-}\right|\left[1+\left(\frac{2 \Delta \mathrm{m} \tau}{\hbar}\right)^{2}\right]^{-1 / 2} \text {. }
$$

The latter two expressions and the values of the $\mathrm{K}_{\mathrm{L}}^{0}-\mathrm{K}_{\mathrm{S}}^{0}$ mass difference $\Delta \mathrm{m}=(0.5349 \pm 0.0022) \times 10^{10} \hbar$ $\mathrm{sec}^{-1}$, the $\mathrm{K}_{\mathrm{S}}^{0}$ mean life $\tau_{\mathrm{S}}=(0.8923 \pm 0.0022) \times 10^{-10}$ sec, and the magnitude of the $\mathrm{K}_{\mathrm{L}}^{0} \rightarrow \pi^{+} \pi^{-} / \mathrm{K}_{\mathrm{S}}^{0} \rightarrow \pi^{+} \pi^{-}$ amplitude ratio $\left|n_{+-}\right|=(2.274 \pm 0.022) \times 10^{-3}$, all from the current edition, result in the predictions that

$$
\text { and } \begin{aligned}
\phi_{+-} & =\phi_{00}=(43.67 \pm 0.14)^{\circ} \\
\operatorname{Re} \varepsilon & =(1.645 \pm 0.016) \times 10^{-3} .
\end{aligned}
$$

The above predictions can be compared with the experimental values

$$
\begin{aligned}
& \left|n_{00} / n_{+-}\right|=1.023 \pm 0.036, \\
& \phi_{+-}=(44.6 \pm 1.2)^{\circ}, \\
& \phi_{00}=(54.5 \pm 5.3)^{\circ}, \\
& \operatorname{Re} \varepsilon=(1.621 \pm 0.088) \times 10^{-3},
\end{aligned}
$$

where Ree has been computed using the relation

$$
\operatorname{Re} \varepsilon=\frac{\delta}{2}\left(\frac{|1-x|^{2}}{1-|x|^{2}}\right),
$$

and our current values of the charge asymmetry

parameter for leptonic $K_{L}^{0}$ decay $\delta=(0.330 \pm 0.012) \%$ and the $\Delta S=-\Delta Q$ amplitude (Rex, $\operatorname{Imx})=(0.009 \pm 0.020$, $-0.004 \pm 0.026$ ).

The superweak predictions are in agreement with the data except for the measured value of $\phi_{00}$, which is two standard deviations above the prediction.

This results primarily from the recent CHRISTENSON1 79 measurement $\phi_{00}=(55.7 \pm 5.8)^{\circ}$.

\section{References}

1. L. Wolfenstein, Phys. Lett. 13, 562 (1964).

2. T. D. Lee and L. Wolfenstein, Phys. Rev. 138B, 1490 (1965).

$13 x=(O S=-D Q$ AMPLITUDE) $/(D S=+D Q$ AMPLI I TUDE) RELATED TEXT SECTION VI B. 4

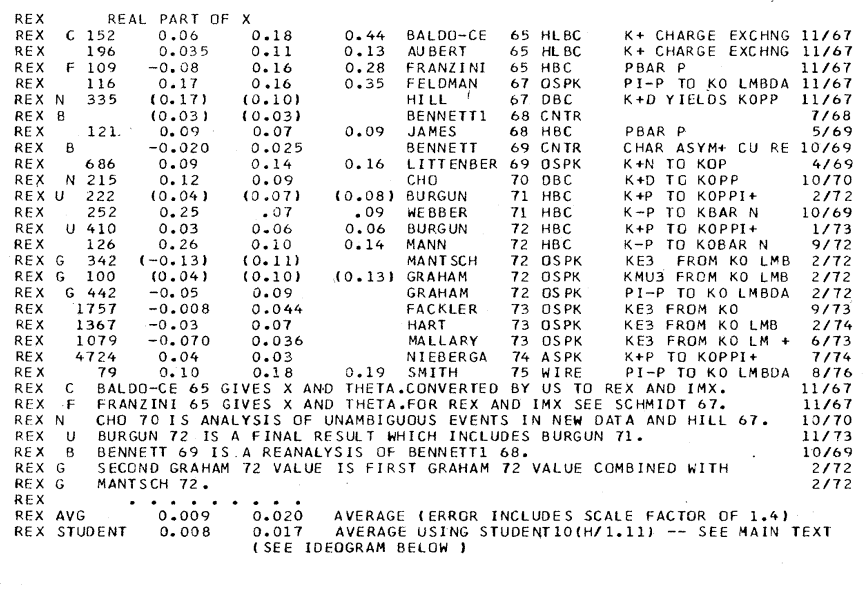




\section{Stable Particles $\mathrm{K}_{\mathrm{L}}^{0}$}

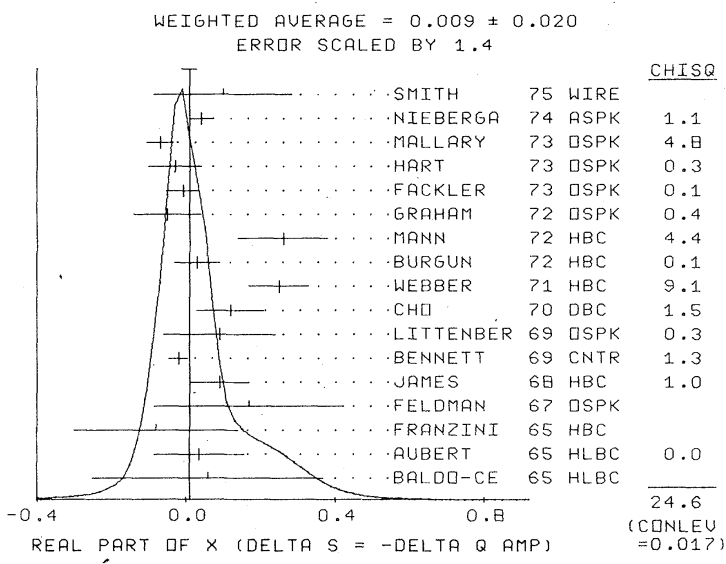

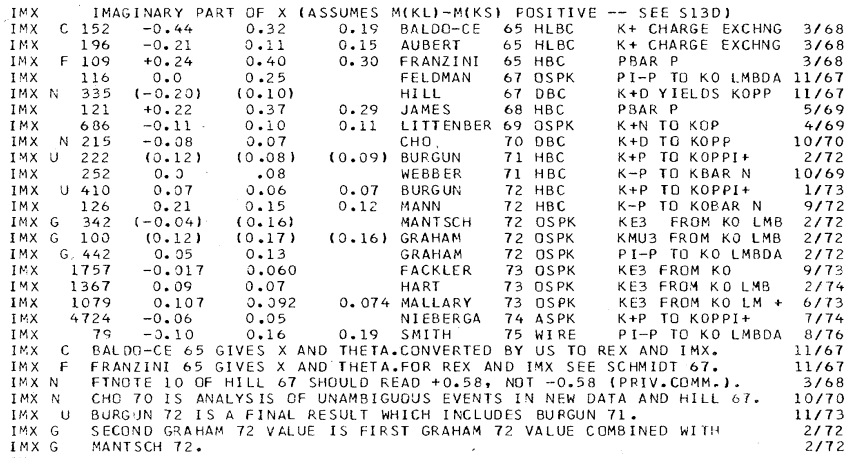

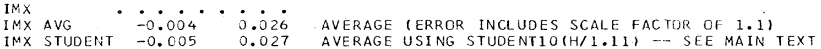

\section{*************************************************************************************}

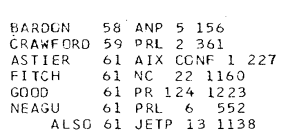

REFERENCES FOR KOL

M BARDON, K LANDF, L LEOERMAN ICOLUMBIA+BNLI)
CRAWFORD, CRESTI, DOUGLASS, GOOD +
(LRL)

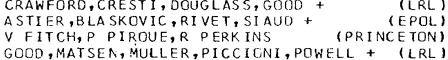

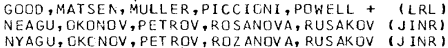

CANERINI 62 PR 128362

CAMERIN, FRY, GADOS, BIRGE, ELY + (WI SC+LRL)
IEPOL)

$\begin{array}{lllll}A D A E R & 64 & \text { PL } 12 & 67 \\ \text { ALEKSANY } & 64 & \text { DUBNA } & 2 & 102\end{array}$

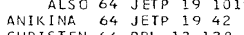

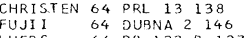

AUERKINA 65 SINR P 2488

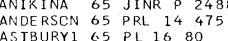

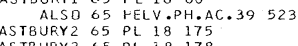

ASTBURY 65 PL 18178

AUBERT 65 PL 1759

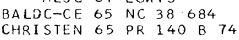

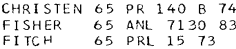

FRANZINI 65 PR 140 P 127

GUIDCNI 65 ARGONNE CONF 49
GGPKINS 65 ARGONNE CONF 67
VISHNEVS 65 PL 18 C 339

ALFF-STE 66 PL 21595

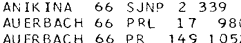

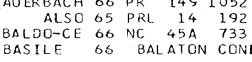

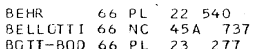

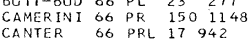

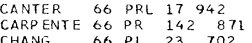

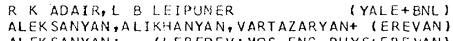

AAEKSANAAN

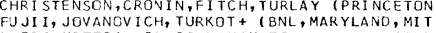

作

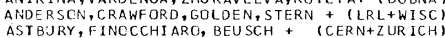

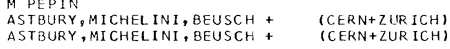
AUBERT, BEHR, CANAVAN, CHOUNET + (EPOL +ORSAY) BALDO-CEOLIN, CAL IMANI, C IAMPOLILLO + (PADO)

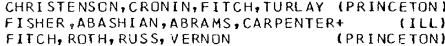
FRANZINI, KIR SCH, PLANO + (COLUMBIA+RUTGERS)

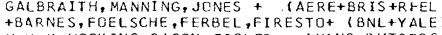
HW K HOPK INS, BACON, EISLER (VAND +R UTGERS)
VI SHNEVSKY, GALANINA, SEMENOV +
(ITEP)

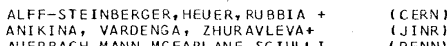

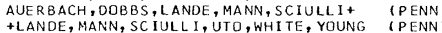

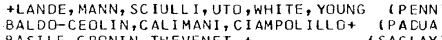
+BRI SSON, BALDO-CEOLIN, AUBERT+ (PADO, EPOL) BOTT-BODENHAUSEN, DE BOUARD, CASSEL+ (CERN)

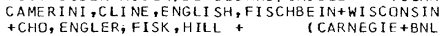
CARPENTER,ABASAN, ABRAMS, FI ISHER (ILLINCIS
CHANG, BASSANO, KIKUCHI, DOOD+ (SYRACUSE, BNL)

\section{Data Card Listings For notation, see key at front of Listings.}

\begin{tabular}{|c|c|}
\hline 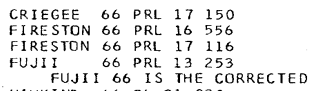 & 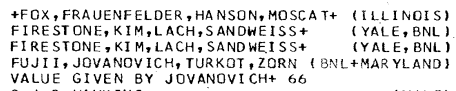 \\
\hline & $\begin{array}{l}\text { CJ E HAK INS } \\
\text { CJ B HAWKINS }\end{array}$ \\
\hline 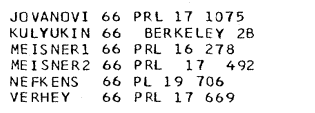 & 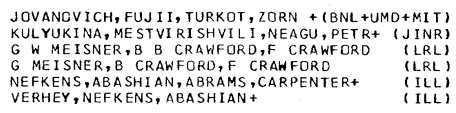 \\
\hline 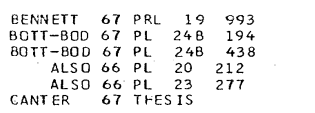 & 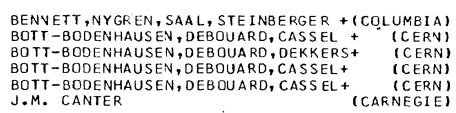 \\
\hline 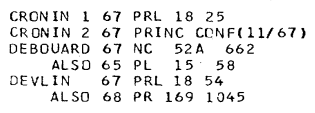 & 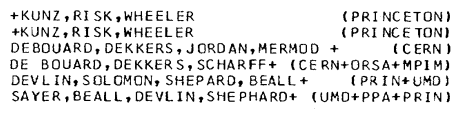 \\
\hline 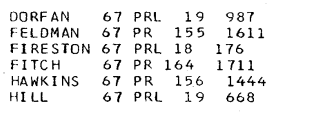 & 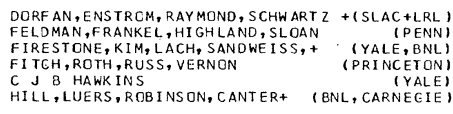 \\
\hline 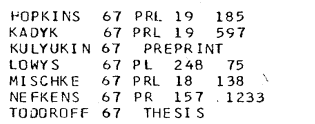 & 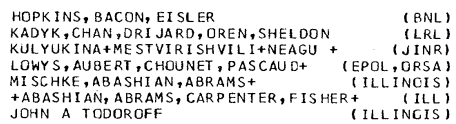 \\
\hline 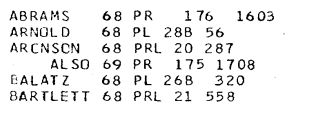 & 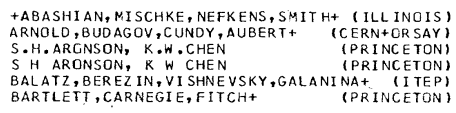 \\
\hline 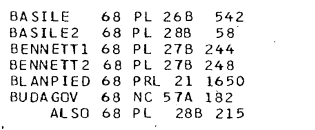 & 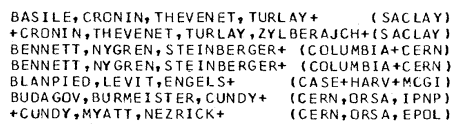 \\
\hline 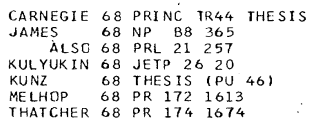 & 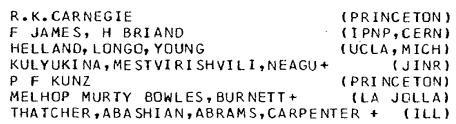 \\
\hline $\begin{array}{l}\text { BANNER } \\
\text { BAPR }\end{array}$ & 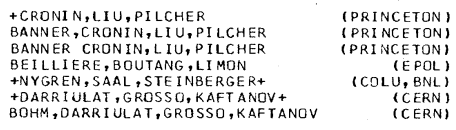 \\
\hline 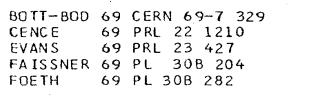 & 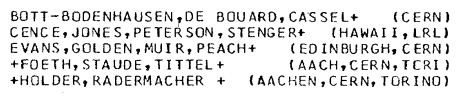 \\
\hline 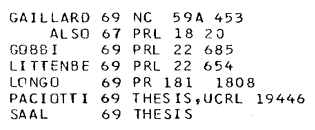 & 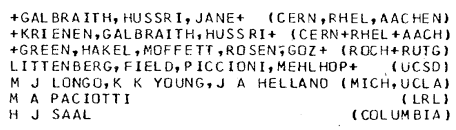 \\
\hline 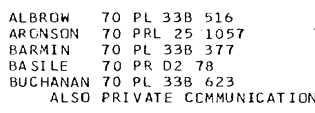 & 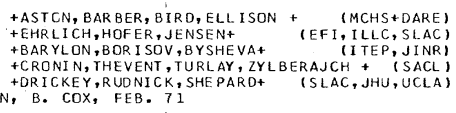 \\
\hline 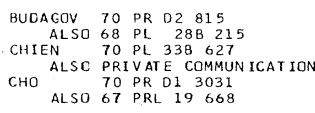 & 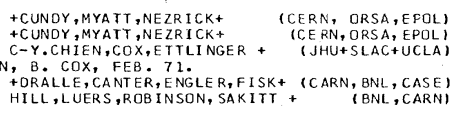 \\
\hline 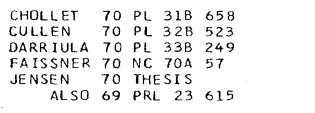 & 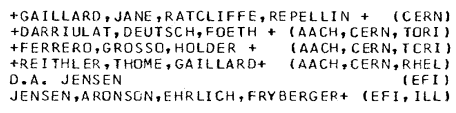 \\
\hline 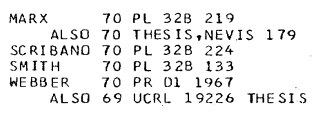 & 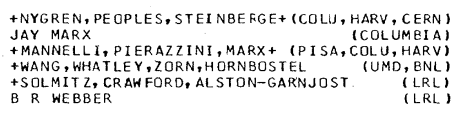 \\
\hline 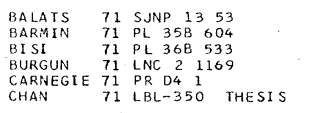 & 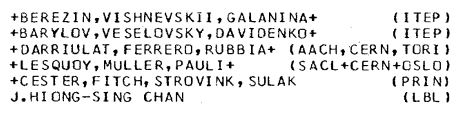 \\
\hline ALSO & \\
\hline
\end{tabular}


Data Card Listings

Stable Particles

For notation, see key at front of Listings.

$\mathrm{K}_{\mathrm{L}}^{0}, \mathrm{D}^{ \pm}$

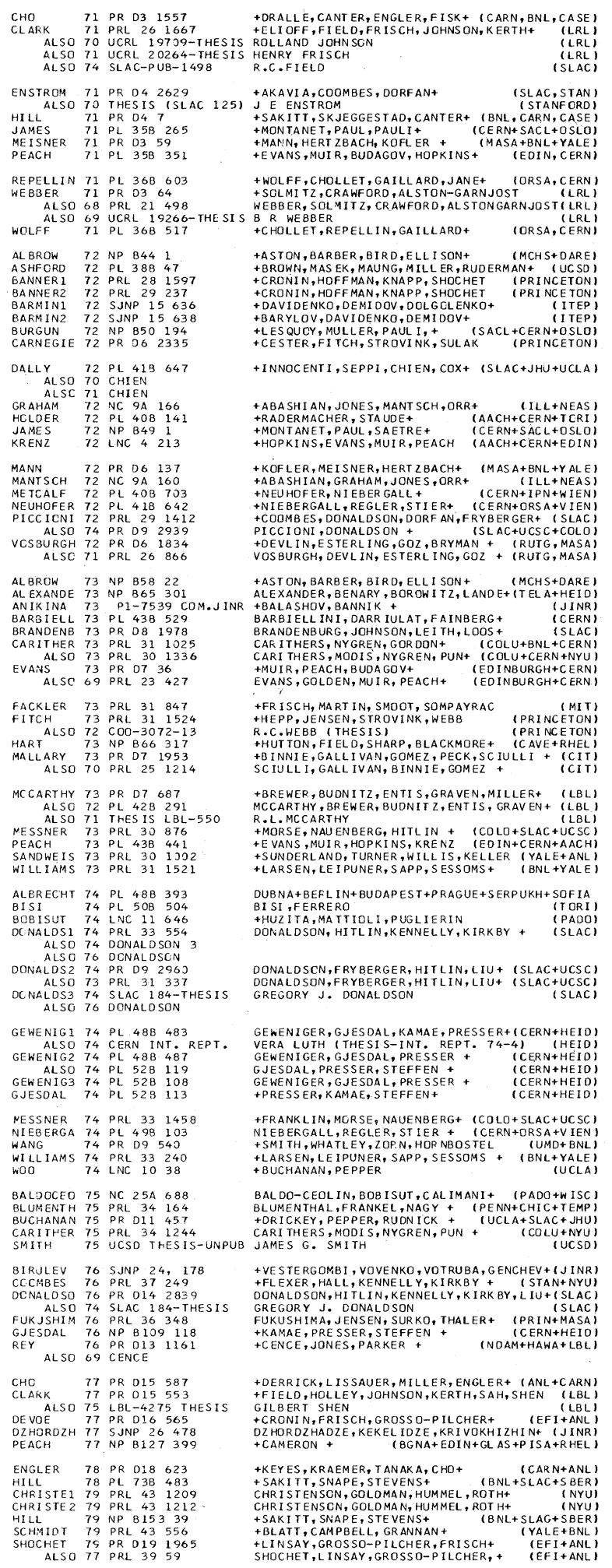

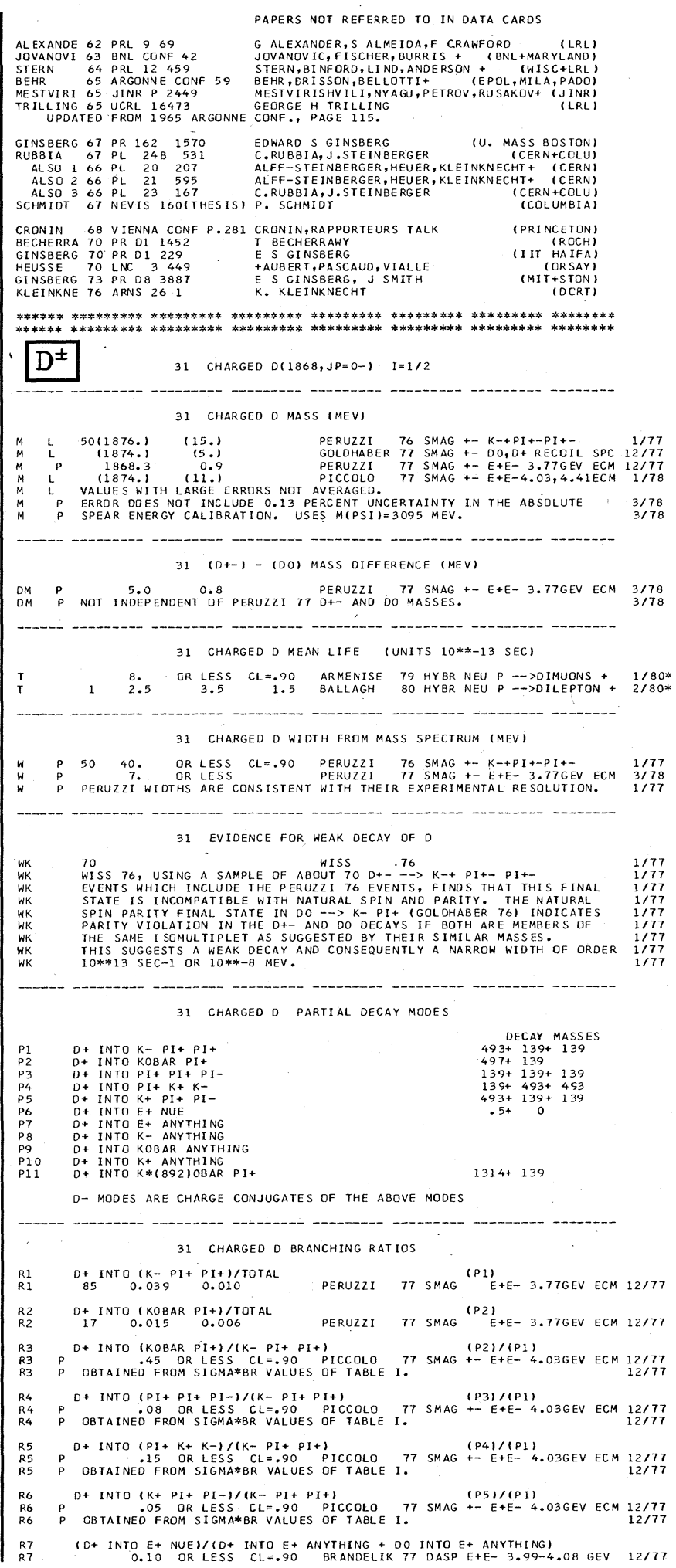




\section{Stable Particles $\mathrm{D}^{ \pm}, \mathrm{D}^{0}, \mathrm{~F}^{ \pm}$}

\section{Data Card Listings For notation, see key at front of Listings.}

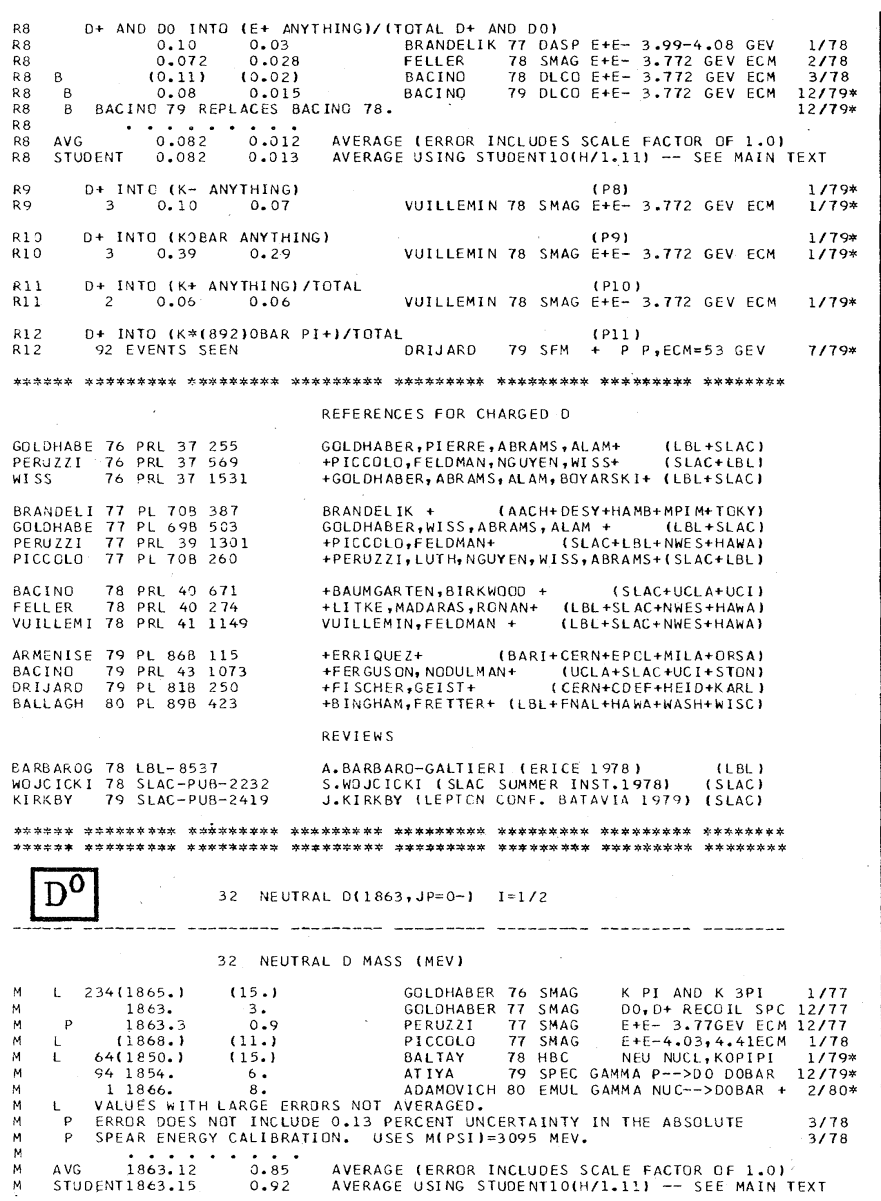

32 neutral 0 mean life (UNits $10 * *-13$ SeC)

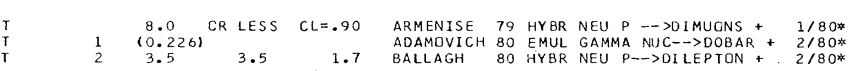

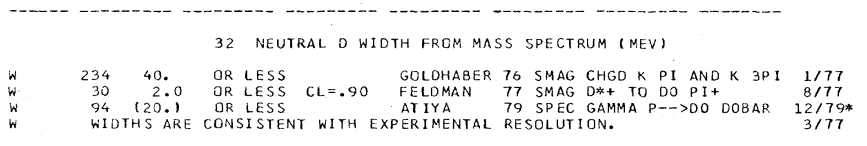

32 Neutral D partial decay modes

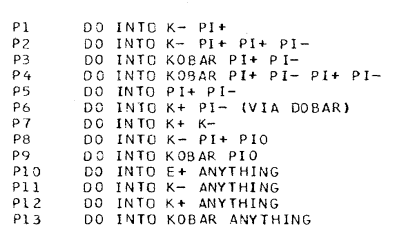

dobar modes are charge conjugates of above modes

FITTED PARTIAL DECAY MODE BRANCHING FRACTIONS

The matrix below is derived from the error matrix for the fitted partial decay mode branching fractions, $P_{i}$, as follows: The diagonal elements are $P_{i} \pm \delta P_{i}$, where $\delta P_{i}=\sqrt{\left\langle\delta P_{i} \delta P_{i}\right\rangle}$, while the off-diagonal elements are the normalized correlation coeificients $\left\langle\delta \mathrm{P}_{i} \delta \mathrm{P}_{j}\right\rangle /\left(\delta \mathrm{P}_{i} \cdot \delta \mathrm{P}_{j}\right)$. For the definitions of the individual $\mathrm{P}_{i}$, see the listing 8 above; only those $\mathrm{P}_{\mathrm{i}}$ appearing in the matrix are assumed in the fit to be nonzero and are thus constrained to add to 1 .

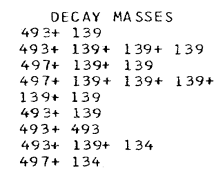

$493+139+134$
$497+134$ 


\section{Data Card Listings}

For notation, see key at front of Listings.

Stable Particles

$\mathrm{F}^{ \pm}, \mathrm{p}, \mathrm{n}$

34 F+-(2030) BRANCHING RATIOS

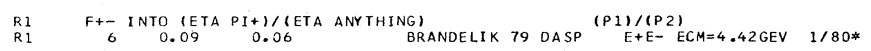
**********************************************************************************1 REFERENCES FOR F+- $\{2030\}$

$\begin{array}{llll}\text { BRANCEL I } 77 \text { PL } 708132 & \text { BRANDELIK }+ & \text { (AACH+ DESY+HAMB +MPI M+ TCKY) } \\ \text { BRANDELI } 79 \mathrm{PL} 80 B & 412 & \text { BRANDELIK+ } & \text { (AACH+ DESY+HAMB +MPIM+TOKY) }\end{array}$

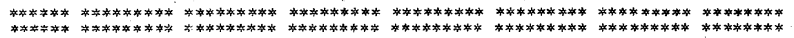

$\mathrm{p}$

16 PROTON (938, J=1/2) $\mathrm{I}=1 / 2$

16 PROTON MASS (MEV)

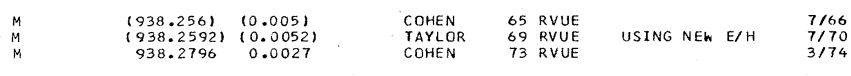

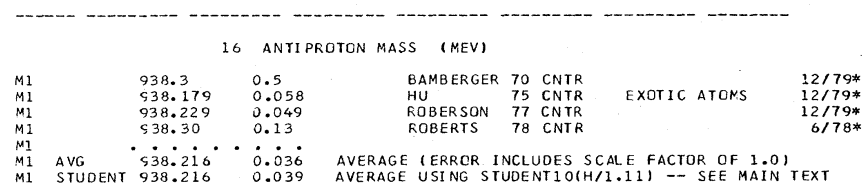

16 PROTON MEAN LIFE (UNITS $10 * 26$ YR)

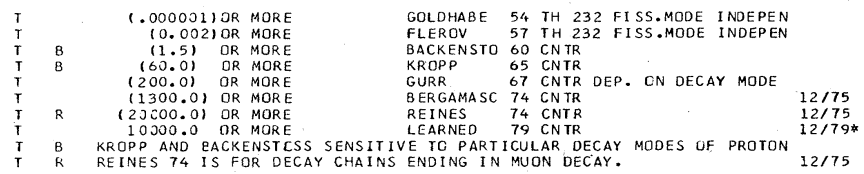

16 ANTIPROTON MEAN LIFE (HOURS)

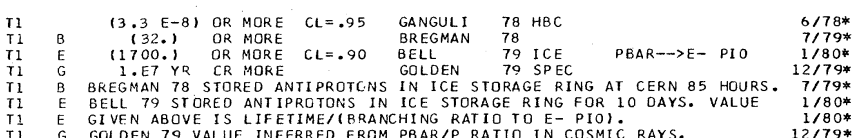

16 PRCTON MAGNET. MOMENT (E/ ZMP)

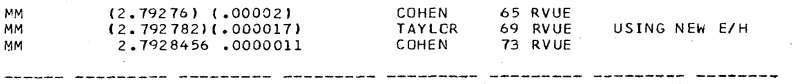

16 ANTIPROTON MAGNETIC MCMENT (E/2MP)

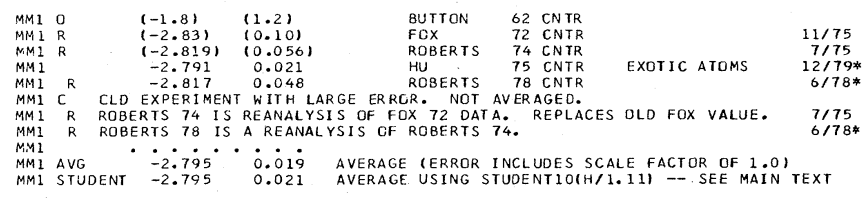

16 PROTON ELECTRIC CI POLE MOMENT (UNITS $10 * *-23$ E CMI

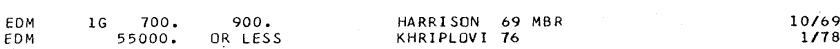

16 PROTON ELECTRON CHARge Difference (UNITS E)

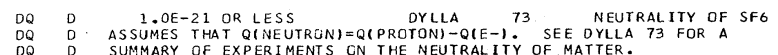

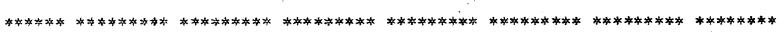

REFERENCES FOR PROTON

GOLOHABE 54 PR 961157 FNGTE2 GOLDHABER, F REINES+ (LOS ALAMOS, BNL)

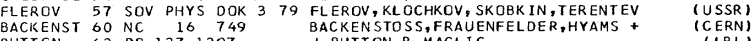

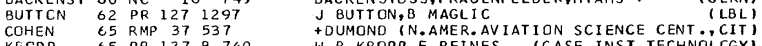

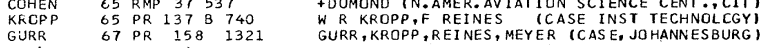

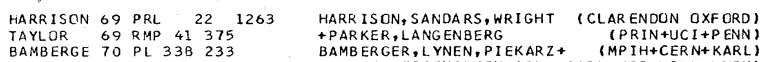

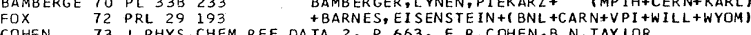

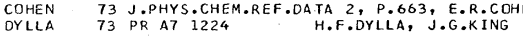

(MIT)

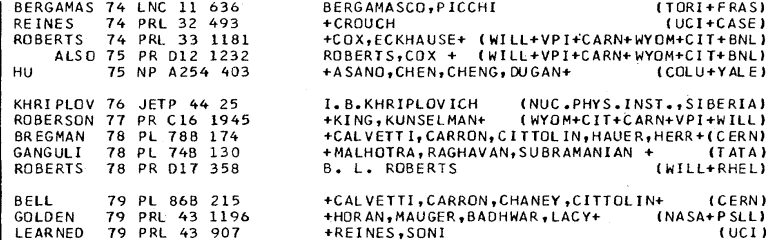

QUANTUM NUMBER DETERMINATIONS NOT REFERREO TO IN THE DATA CARDS

$\begin{array}{llll}\text { KALOGERO } 76.9 R L & 371037 & \text { KALOGEROPCULOS, CHIU, SUDARSHAN } & \text { (SYRA+TEXA)P } \\ \text { FRANKLIN } 77 \text { PR D16 } 910 & \text { JERROLD FRANKLIN } & \text { (HAIF)P }\end{array}$

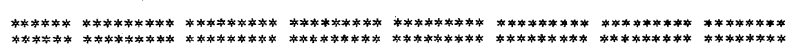

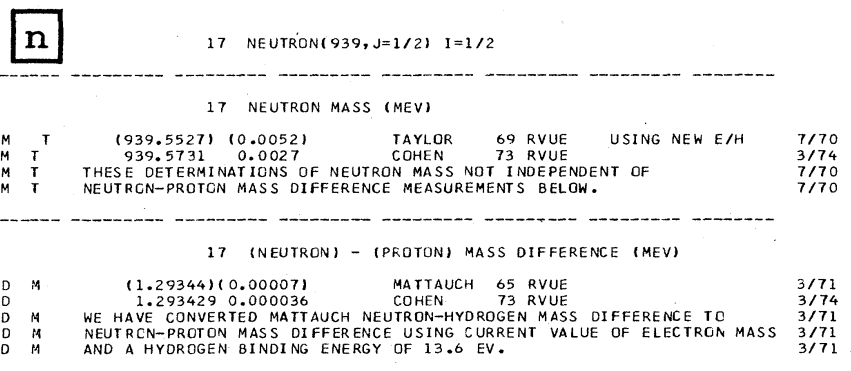

17 NeUtron MEAN LIFE (UNITS 10**3 SEC)

THE MEASUREMENT OF THE NEUTRCN MEAN LIFE BY SOSNOVSKII 59 HAS
BEEN DISCARDED SINCE 1. IT DISAGREES WITH THE BETTER AND MCRE

RECENT RESULT OF CHRISTENSEN $67 .{ }^{2}$. T THE VALUE OF GA/GV DE-
RIVED FRCM THE NEW VALUE OF THE MEAN LIFE AGREES WELL WITH THE

DALUE OBTAINED FROM THE FREE NEUTRON DATA.

$(1.012)(0.021) \quad$ SOSNOVSKI.59 PILE

$\begin{array}{llll}0.918 & 0.014 & \text { CHR ISTENS } 72 \text { PILE } & \\ 0.877 & 0.078 & \text { BONDARENK } 78 \text { PILE } & 6 / 72 \\ 0.917 & 0.014 & 0.014 & \text { AVIRAGE }\end{array}$

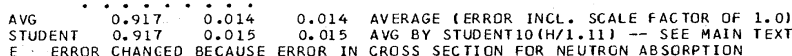

E ERROR CHANGED BECAUSE ERROR
E IN GOLO HAS BEEN REDUCED.

17 NEUtRon MAGNETIC MOMENT (MAGNETONS, 938.2 MEV)

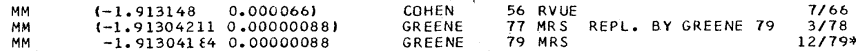

17 NEUTRON ELECTRIC DIPOLE MOMENT (UNITS $10 * *-23$ E CMI

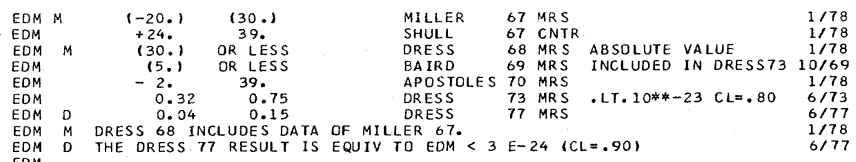

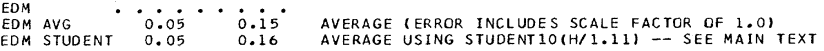

17 NEUTRON CHARGE

SEE SECTION DQ IN THE PROTON DATA CARD LISTINGS ABOVE

17 NEUTRON PARTIAL DECAY MODES

DECAY MASSES

P1 NEUTRON INTO PROTON E- ANTI (NUEI
P2 NEUTRON INTO PROTON NUE ANTI(NUE) $\begin{array}{ccc}938+ & -5+ & 0 \\ 938+ & 0+ & 0\end{array}$

17 NEUTRON BRANCHING RATIOS

R1 NEUTRON INTO (PROTON NUE ANTI (NUE) //PROTON E- ANTI (NUE) (P2)/(P1)

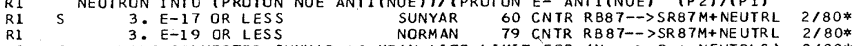

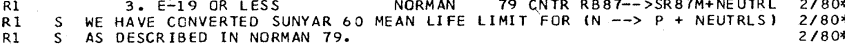




\section{Stable Particles}

n, $\Lambda$

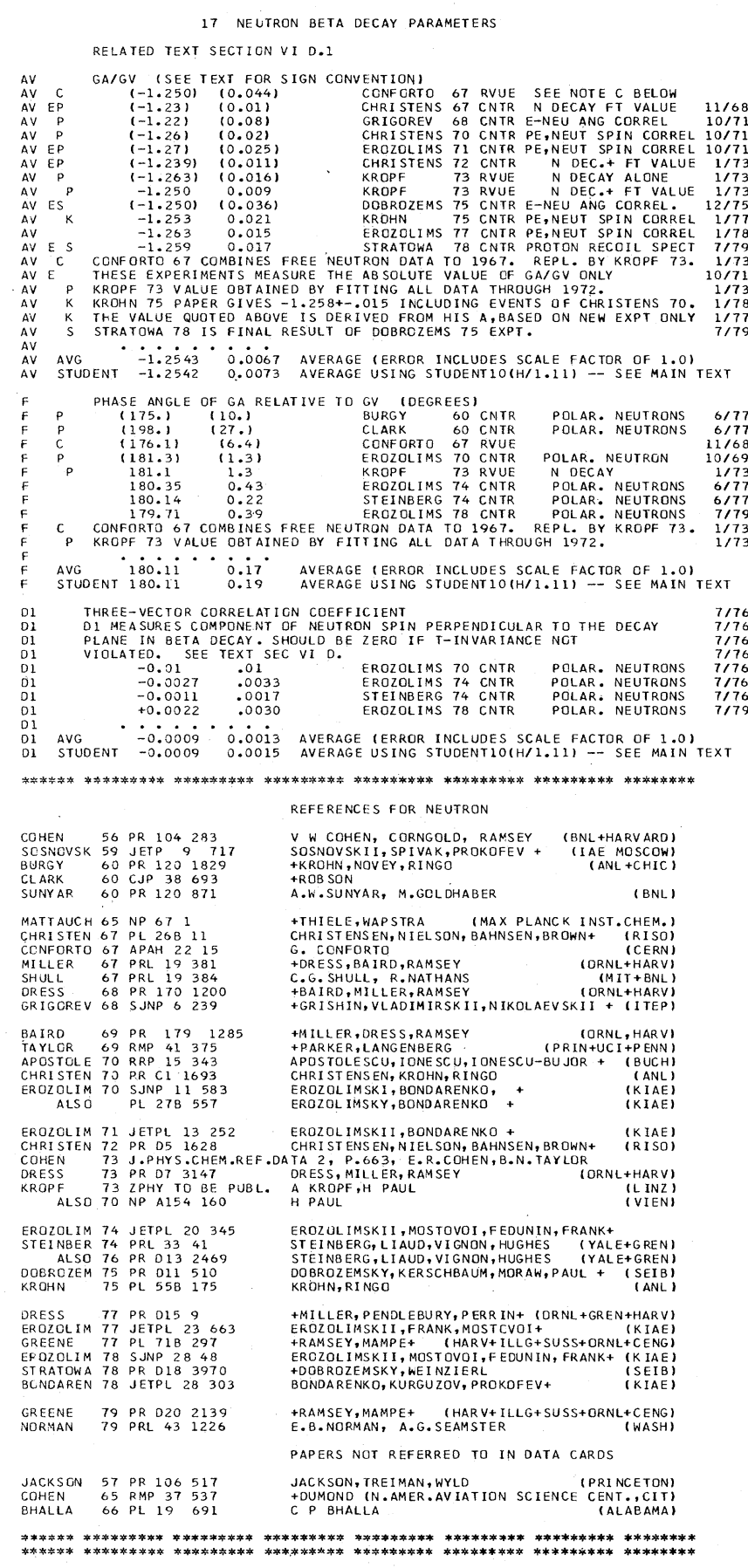

\section{Data Card Listings For notation, see key at front of Listings.}

$\Lambda$ 18 LAMBDA $(1115, J P=1 / 2+1$ I $=0$ 18 LAMBDA MASS (MEV)

SINCE OUR FINAL VALUES FOR THE SIGMA AND LAMBCA MASSES COME FROM
DOING AN OVERALL FIT TO ALL MEASURED MASSES AND MASS DIFFERENCES, WE HAVE USED THE UNCORRELATED MEASUREMENTS FROM SCHM IDT 65 RA THER
THAN THE ONES COMI NG FROM THE OVERALL FIT REPORTED IN THAT PAPER. THAN THE ONES COMING FROM THE OVERAL FIT REPORTED IN THAT PAPER.
SINCE THERE SEEMS TO BE NO CONVINING ARGUMENT AS TO WHY ONE SHOULD

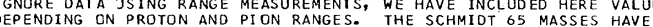
BEEN REEVALUATEO US ING OUR APRIL 1973 PROTON AND CHARGED K AND PI
MASSES. P. SCHMIDT, PRIVATE COMNUNICATION, (1974).

$\begin{array}{lll}1115.44 & 0.12 & \text { BHOWMIK } 63 \text { RVUE + SEE NOTE L BELOW }\end{array}$ ABOVE LAMBOA MASS HAS BEEN RAI SED 35 KEV TO ACCOUNT FOR 46 KEV
INCREASE IN PROTON MASS AND $11 \mathrm{KEV}$ DECREASE IN CHARGED PION MASS.

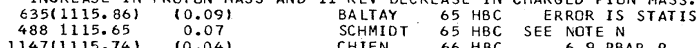

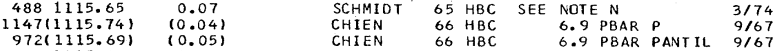
$\begin{array}{ccccc}1115.6 & 0.4 & \text { LONDON } & 66 \mathrm{HBC} & \\ (1116.01 & 10.2) & \text { BADIER } & 67 \mathrm{HBC} & 2.4 \text { PBAR P,LLBAR } \\ 19 \% & \end{array}$

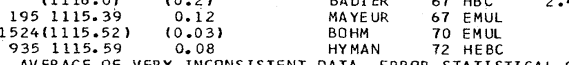
AVERAGE OF VERY INCONSISTENT DATA. ERROR STATISTICAL ONLY. AUTHORS
DETECT SYSTEMATIC EFFECT OF ABOUT . 15 MEV, WHICH THEY ATTRIBUTE $3 / 72$
$3 / 72$
M N BHOWMICK 63 AND MAYEUR 67.
ERROR PURELY STATISTICAL.

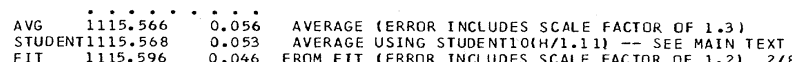
0.053 AVERAGE USING STUDENTIOSH/1.111 - - SEE MAIN TEXT
0.046 FROM FIT (ERROR INCUDES SCALE FACTOR OF 1.2 ) $2 / 80 *$

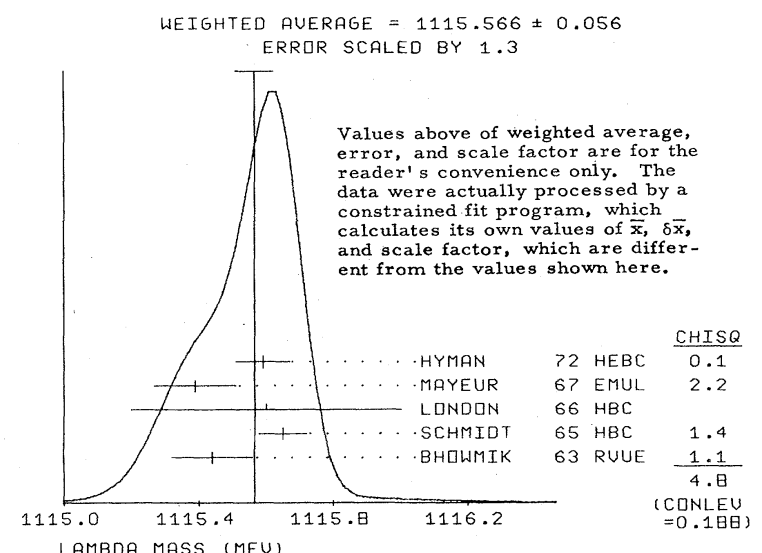

LAMBDA MASS (MEU)

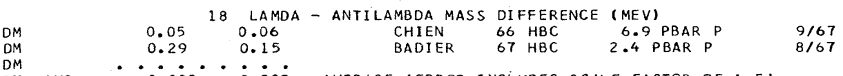

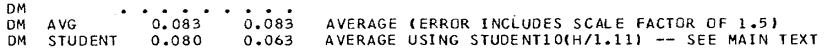

18 LAMBdA MEAN LIFE (UNITS $10 * *-10$ )

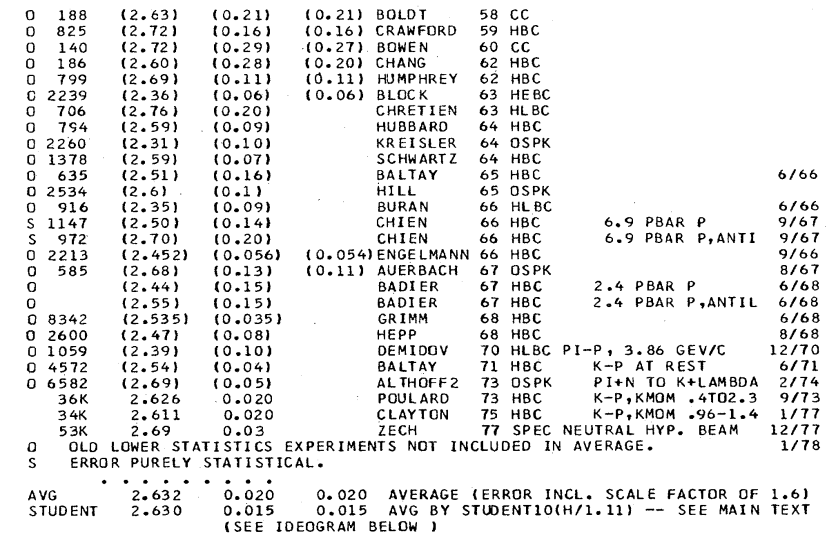




\section{Data Card Listings}

\section{Stable Particles}

\section{For notation, see key at front of Listings.}
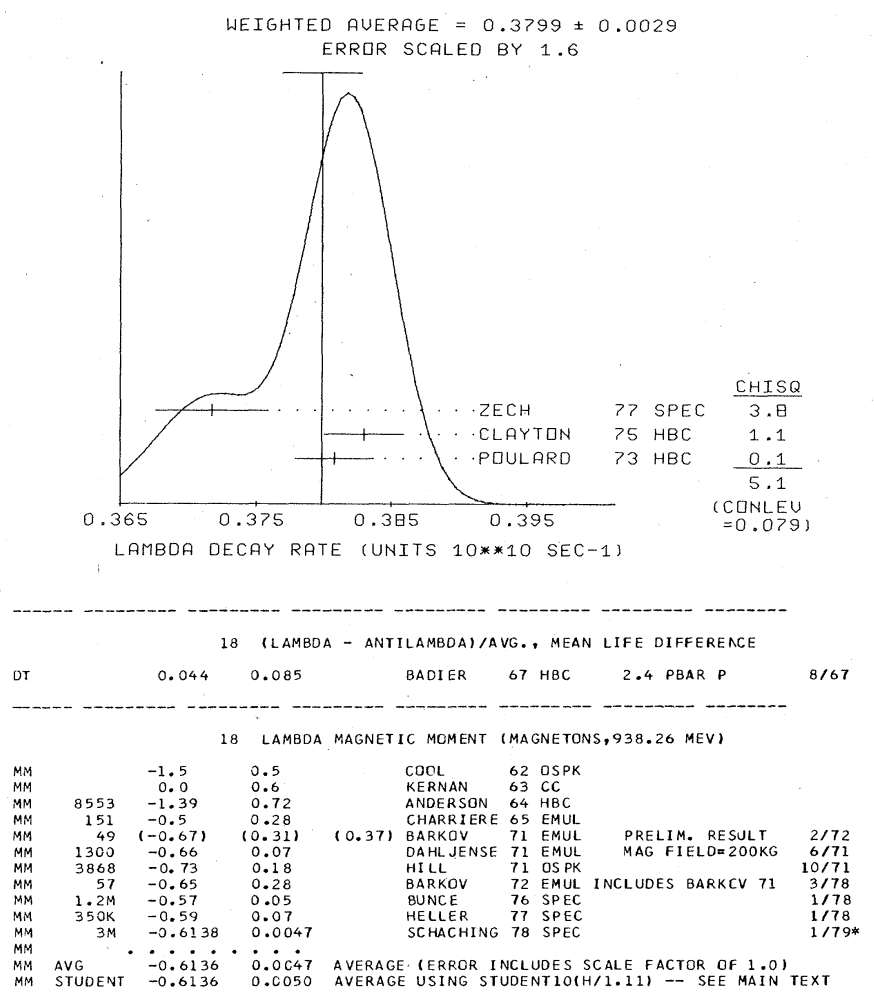

NONZERO VALUE LAMBDA ELECTRIC DIPOLE MCMENT (UNITS $10 * *-14$ E CM)

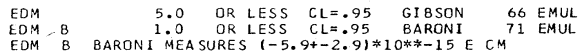

18 LAMBda PARTIAL DECAY MODES

\begin{tabular}{|c|c|c|c|}
\hline & & $\begin{array}{r}\text { DECAY } \\
938+139\end{array}$ & ASSES \\
\hline$p 2$ & LAMBDA INTO NEUTRON PIO & $\begin{array}{l}938+139 \\
939+134\end{array}$ & \\
\hline P3 & LAMBDA INTO PROTON MU- NEUTRINO & $938+105+$ & 0 \\
\hline p4 & LAMBDA INTO PROTON E- NEUTRINO & $938+.5+$ & 0 \\
\hline & LAMBDA INTO PROTON PI- GAMMA & $938+139+$ & 0 \\
\hline
\end{tabular}

18 LAMBDA BRANCHING RATIOS

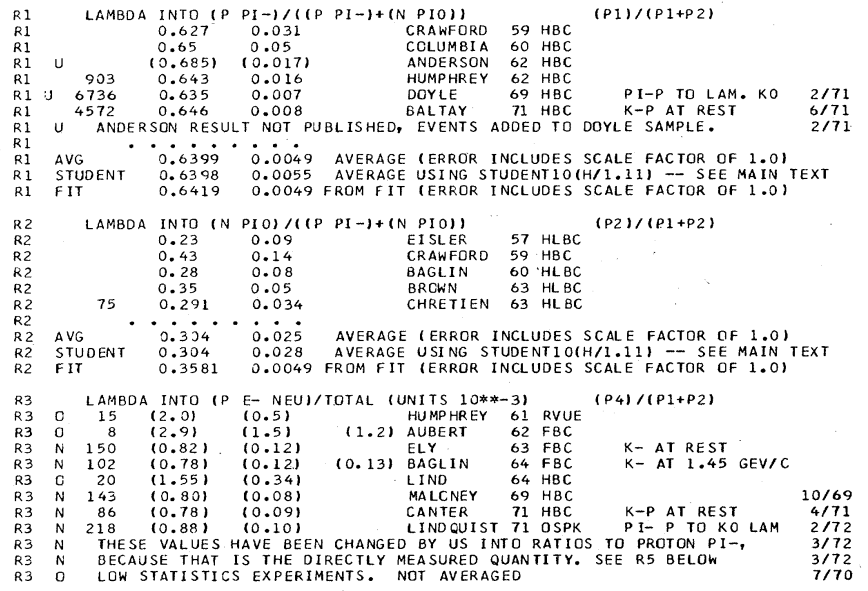

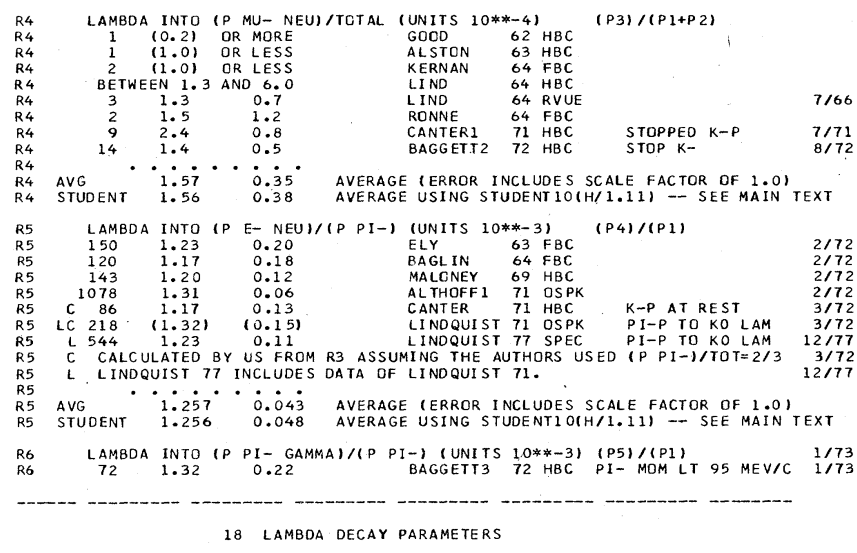

RELATED TEXT SECTION VI D AND APPENOIX III

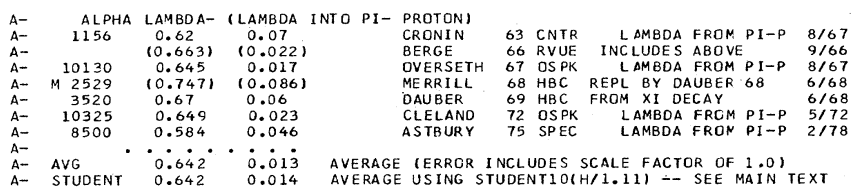

AO ALPHAO /ALPHA- FOR LAMBDA (L. INTO PION/L INTO PI- P)

$\begin{array}{llllllll}A 0 & & 1.10 & 0.27 & \text { CORK } & 60 \text { CNTR } & & \\ \text { AO } & 4760 & 1.000 & 0.068 & \text { DLSEN } & 70 & \text { OSPK PI }+N \text { TO K+ LAMBDA } & 5 / 70\end{array}$

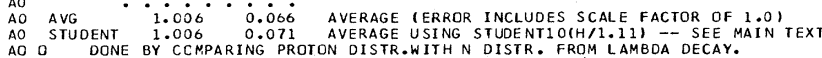

F- PHI ANGLE (SIN(PHI)/COS(PHI) $=$ BETA/GAMMA) (OEGREES)

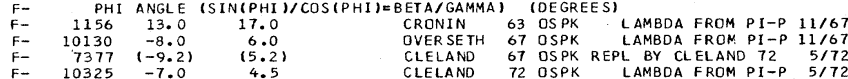

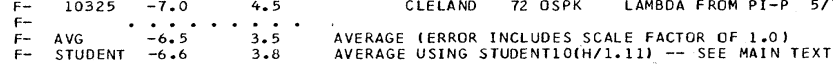

AV GA/GV FOR LAMBDA BETA DECAY (SEE TEXT SEC. VI D.1 FOR SIGN CONV.)

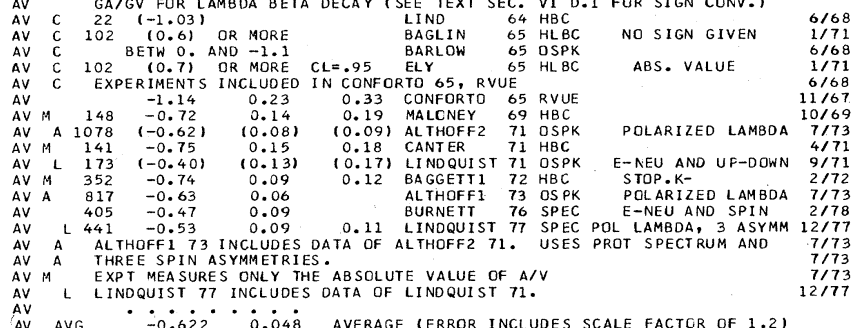

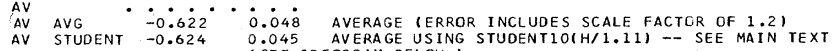

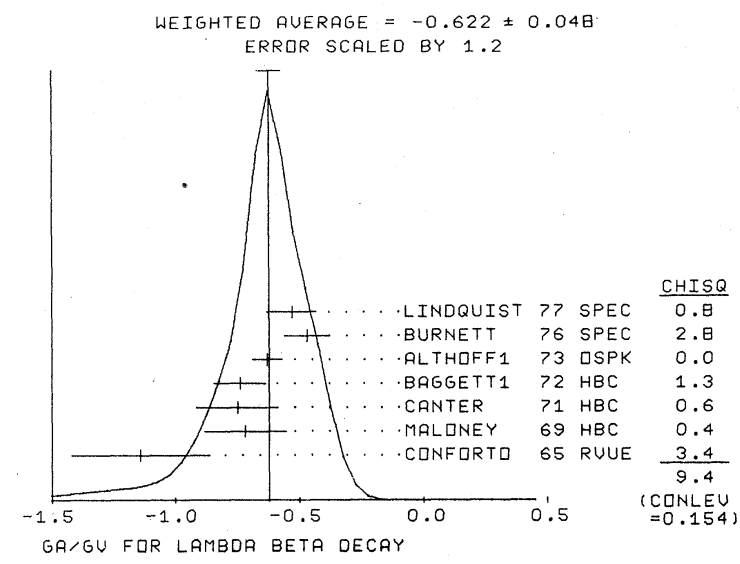




\section{Stable Particles}

\section{$\Lambda, \Sigma^{+}$}

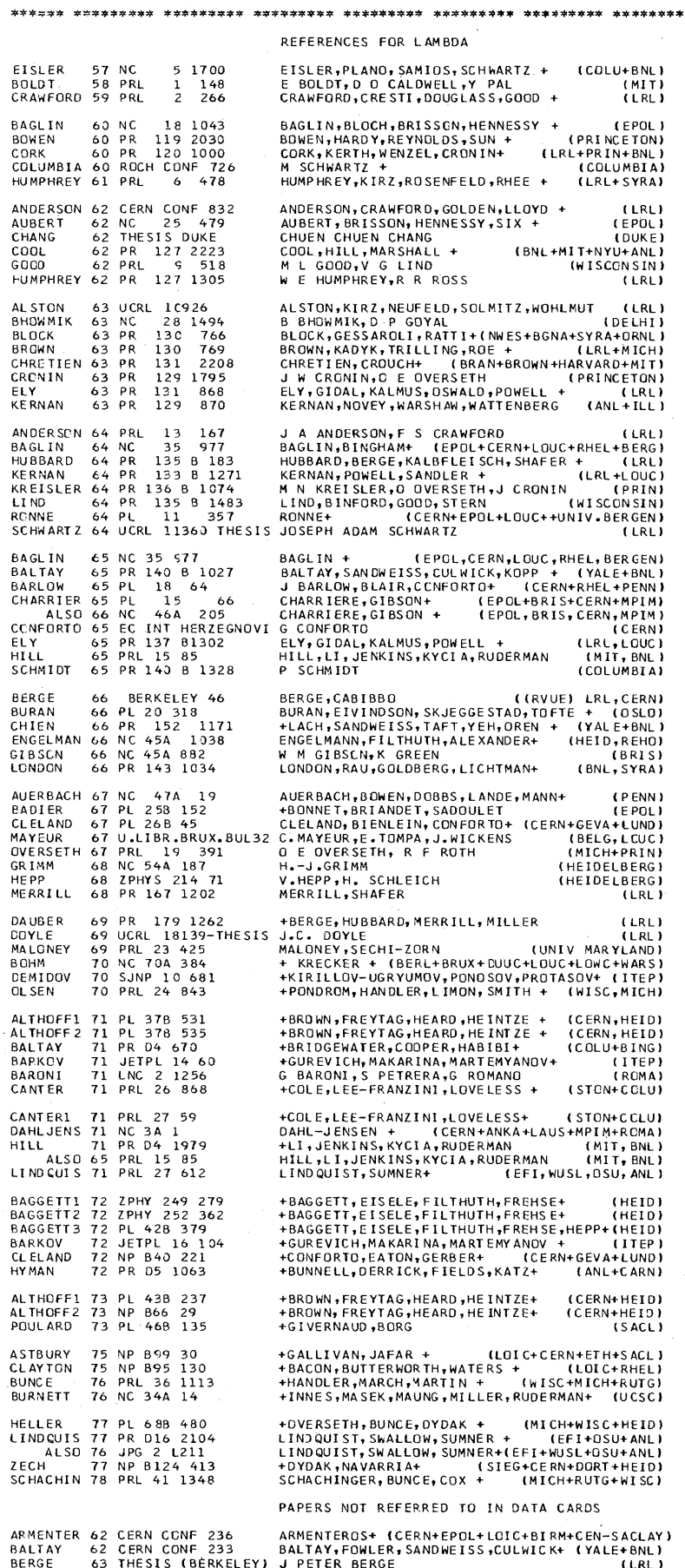

\section{Data Card Listings For notation, see key at front of Listings.}




\section{Data Card Listings}

Stable Particles

\section{For notation, see key at front of Listings.}
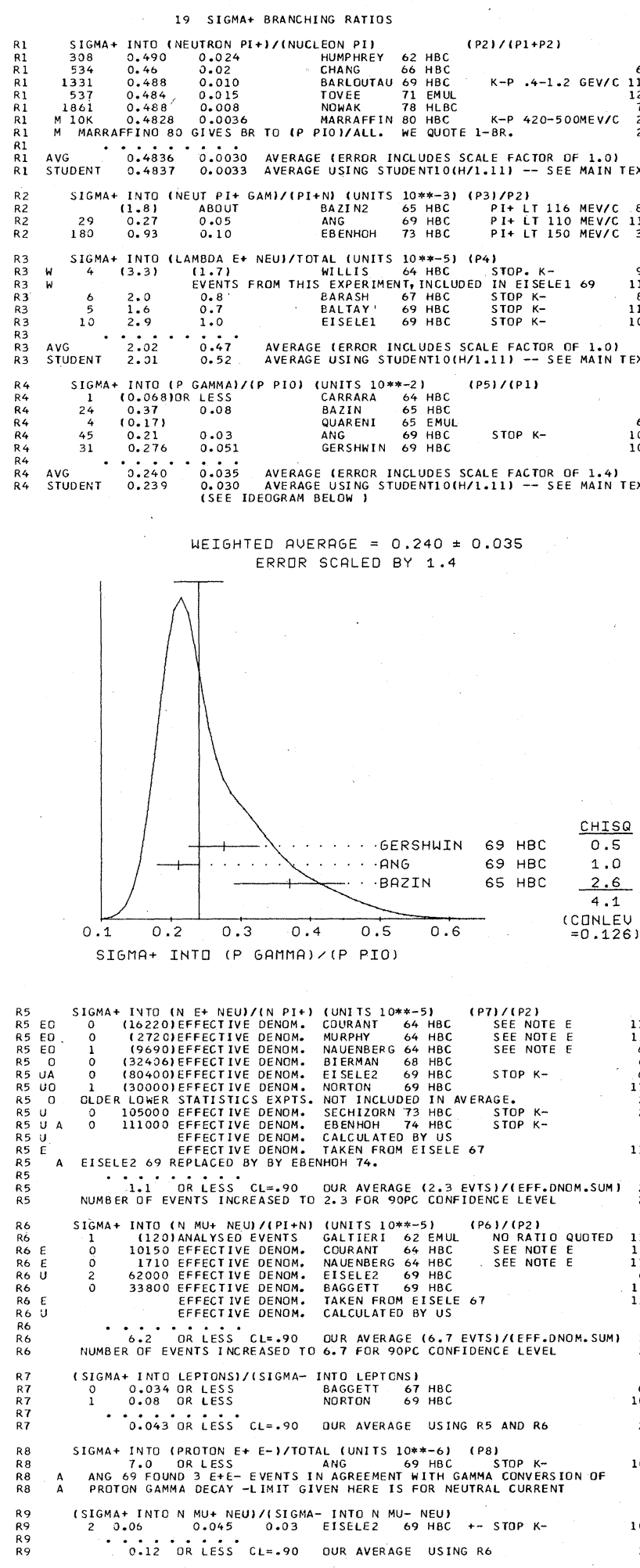

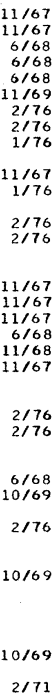

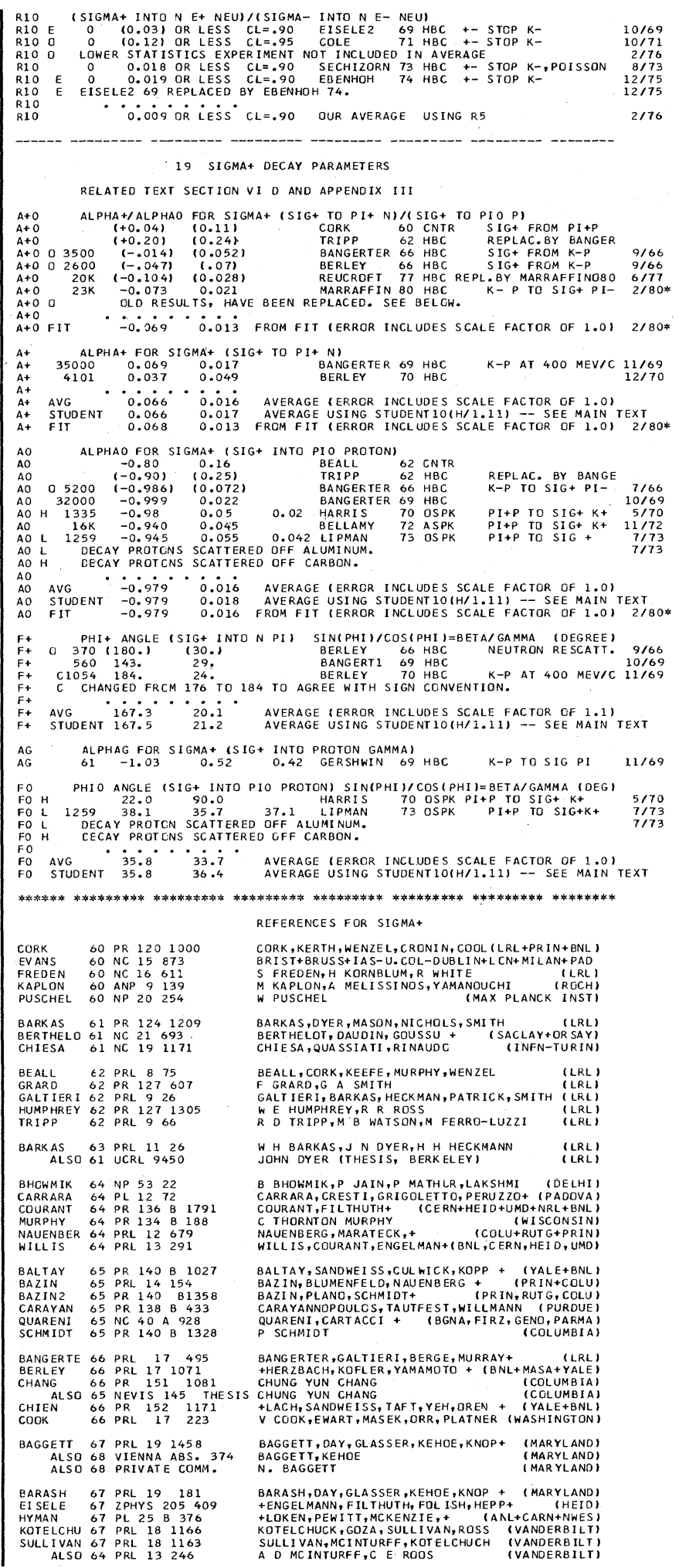


Stable Particles

$\Sigma^{+}, \Sigma^{-}$

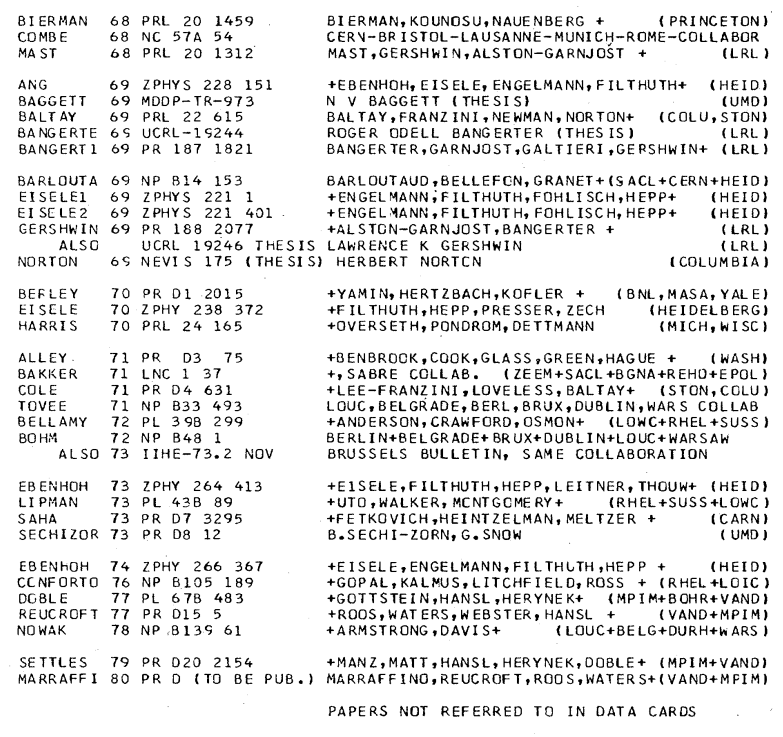

GLASER 58 CERN CCNF 270 GLASER, GOCD, MORRISON (MICH+LRL) QUANTUM NUMBER DETERMINATIONS NOT REFERREO TO IN THE DATA CARDS

$\begin{array}{llllll}\text { TRIPP } & 62 & \text { PRL } 8 & 175 & & \text { R TRIPP,M WATSON,M FERRO-LUZZI } \\ \text { ALFF } & 63 & \text { SIENA CONF } 1205 & \text { (LRL) } \\ \text { ALFF,NAUENBERG, KIRSCH, } & \text { (COLU+RUTG+BNL) }\end{array}$

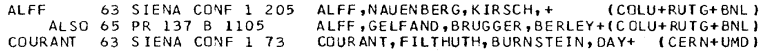

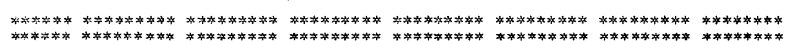

$\Sigma^{-}$

20 SIGMA- $(1198, J P=1 / 2+) \quad I=1$

20 SIGMA- MASS (MEV)

$N$ N See ncte preceding lamboa mass listings

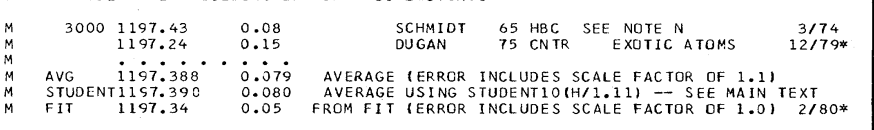

20 (SIGMA-) - (SIGMA+) MASS DIFFERENCE (MEV)

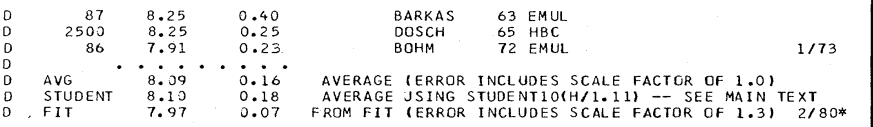

20 (SIGMa-) - (LAMbda) MASS Difference (MEV)

DL N SeE nCte PRECEding lambda mass listings.

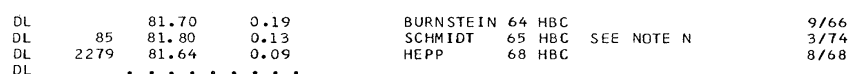

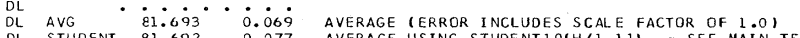

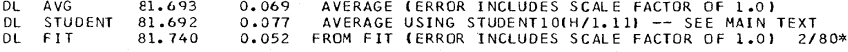

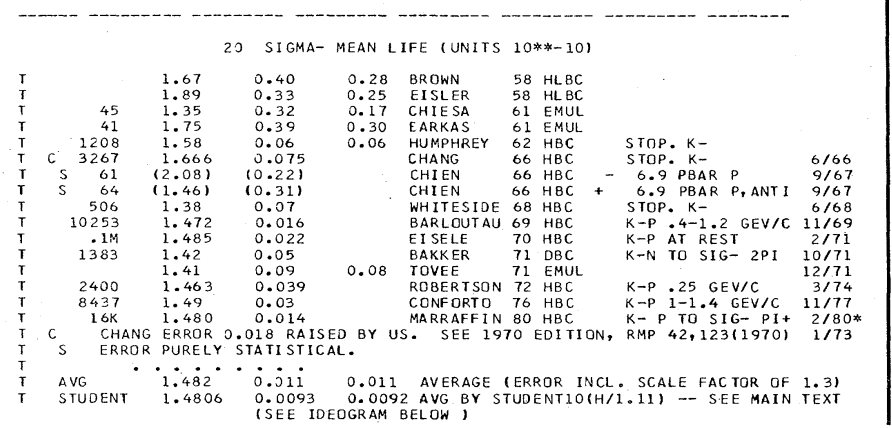

Data Card Listings

For notation, see key at front of Listings.

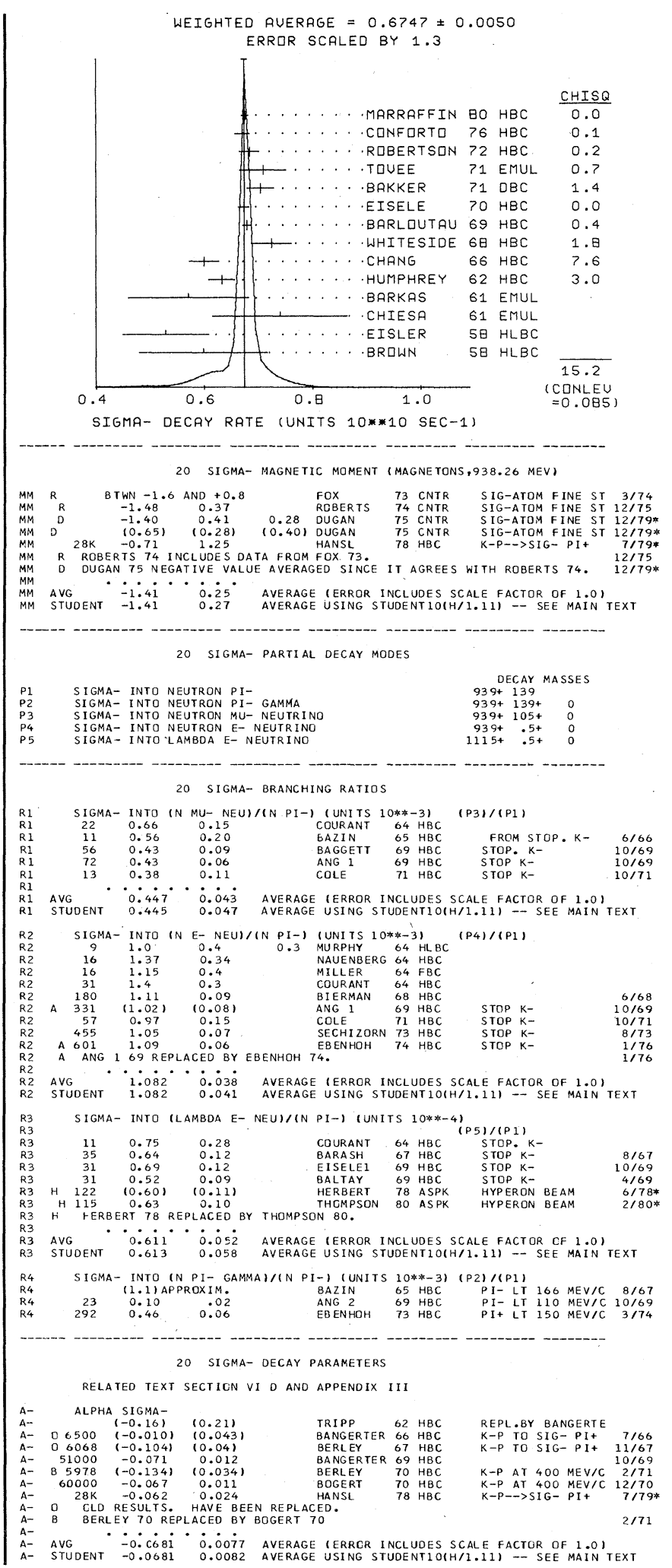




\section{Data Card Listings}

For notation, see key at front of Listings.

Stable Particles

$\Sigma^{-}, \Sigma^{0}$

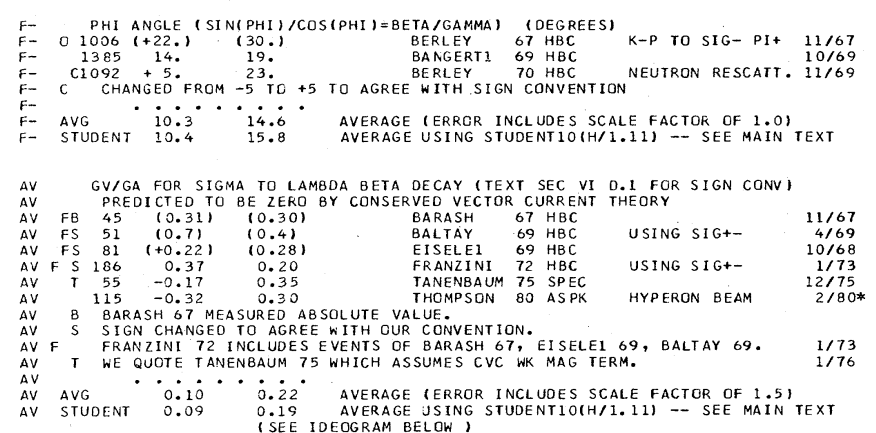

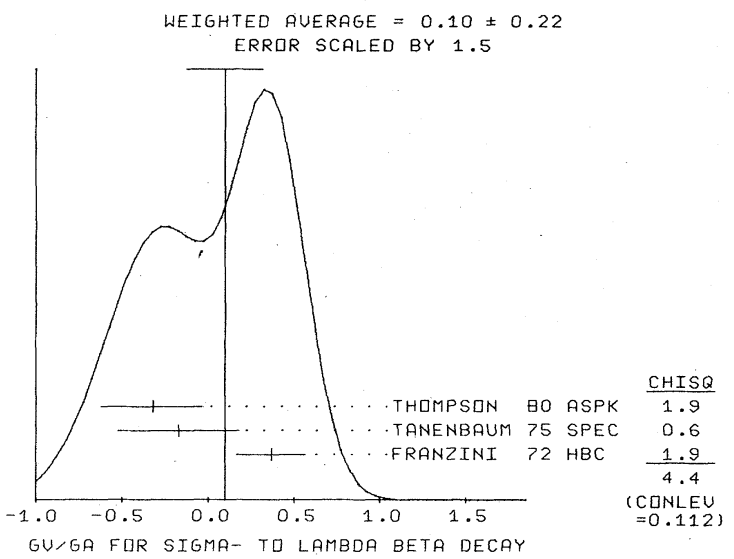

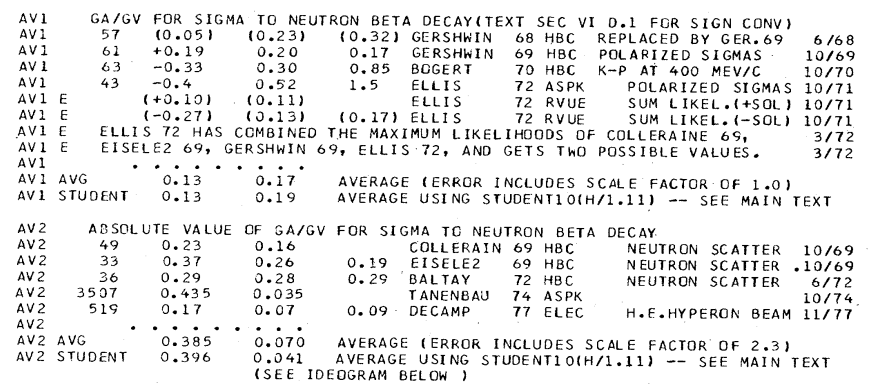

WEIGHTEO AUERAGE $=0.385 \pm 0.070$

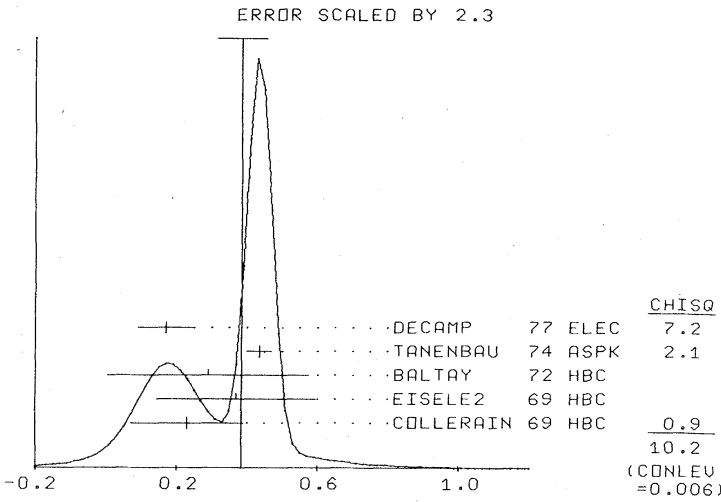

ABS(GA/GU) FUR SIGMA TD N BETA DECAY

\section{$\begin{array}{llll}\text { BRRWN } & 58 & \text { CERN CONF } & 270 \\ \text { EISLER } & 58 & \text { NC } & \text { SER } 10 \\ 10 & 150\end{array}$ \\ $\begin{array}{lllll}\text { BARKAS } & 61 & \text { PR } & 124 & 1209 \\ \text { CHIESA } & 61 & \text { NC } 19 & 19 & 1171\end{array}$

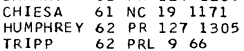

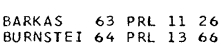

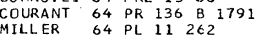

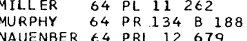

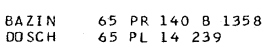

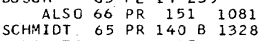

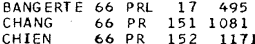 \\ \begin{tabular}{lllll} 
BARASH & 67 & PRL & 19 & 181 \\
BERLEY & 67 & PRL & 19 & 979 \\
\hline & & 19 & 979
\end{tabular}

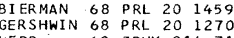 \\ $\begin{array}{lllll}\text { HEPP } & 68 & \text { PPHY } & 214 & 71 \\ \text { WHITESID } & 68 & \text { NC } & 544 & 537\end{array}$

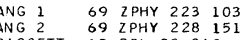 \\ BALTAY 69 PRL $22 \quad 615$

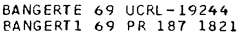 \\ $\begin{array}{llll}\text { BARLOUTA } & \text { OS NP } & 814 & 153 \\ \text { COLLERAI OS PRL } & 23 & 198\end{array}$

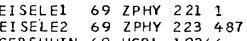 \\ BERLEY 70 PR D1 2015

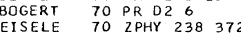 \\ EAKKER 71 LNC 137

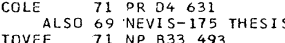 \\ AlTAY 72

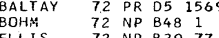

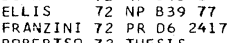 \\ EBENHOH 73 Z $\mathrm{ZHY} 264413$ \\ $\begin{array}{lll}\text { FOX } & \text { FEHZOR } 73 \text { PRL } 311084 \\ \text { SECHIOR } 73 & \text { PR D8 } 12\end{array}$

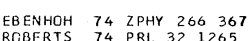

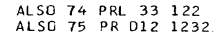 \\ TANENBAU 74 PRL 33175
ALSO 75 TANENBAUM \\ $\begin{array}{llll}\text { DUGAN } & 75 & \text { NP } & A 254 \\ \text { TANENEAU } & 75 & 396 \\ \text { TR } & \text { D12 } 1871\end{array}$

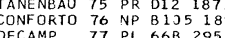

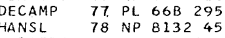 \\ $\begin{array}{lll}\text { HEREERT } & 78 \text { PRL } 40 & 1230 \\ \text { MARRAFFI } & 80 \\ \text { PR D } & \text { TIO BE P }\end{array}$}

MARRAFF I 80 PR D
THEMPSO
TTO PR D D P PJB

$\begin{array}{lllll}\text { BROWN } & 57 & \text { PR } & 108 & 1036 \\ \text { NIETO } & 68 & \text { RMP } & 40 & 140\end{array}$

BROWN, GLASER, GRAVES, PERL,CRONIN + (MICH

BARKAS, DYER, MASON, NICKOLS, SMITH (LRL)
A CHIESA, QUASSIA III, RI RAUDO (TURIN)

W
WE HUPHREY R R ROSS, RINAUDO
R D TRIPP,M WATSON,M FERRO-LUZZI
(LRL)

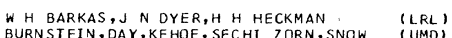

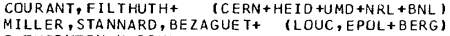
C THORNTON MURPHY
(WI SCONSIN)

BAZIN, PLANO, SCHM IDT + (PR IN+RUTG+COLU) CHUNG YUN CHANG FILTHUT, HEPP, KLUGE. (HEID)

P SCHMIDT

G

C CACH, SANOWEISS, TAFT, YEH, OREN + (YALE+BNL)

BARASH, DAY, GLASSER, KEHOE, KNOP + (MARYAND)
BERLEY, GERTZBACH, KOELER K BIERMAN KOUNOSU, NAUENBERG + (PRINCETON) GERSHWIN, ALSTON-GARNJOST, BANGERT ERT (LRL) H. HEPP, H. SCHLEICH
WHITS IDE,J. GCLLUB
(OBERL IN)

ANG, EISELE, ENGELMANN, FILTHUTH + + (HEID) BAGGEOR, KESELLE, ENGELMANN, FIL THUTH (HEID) BALT AY, FRANL INI, NEWMAN, NORTON+ (COLU, STON) ROGER OOELL BANGERTER (THES IS)
BANGERTER, GARNJOST, GAL TIERI, GERSHWIN+ (LRLL)

BARLOUTAUD, BELLEFON, GRANET+( SACL+CERN+HEID)
COLLERAINE, DAY, GLASSER, KNOP+ (UNIV MARYLAND) TENGELMANN, FILTHUTH, FOHLISCH, HEP P + (HEID) EISELE, ENGELMANN, FIL THUT, FOHL ISCH+ (HEID)
LAWENCE KENNETH GERSHWIN (THESIS) (LRL)

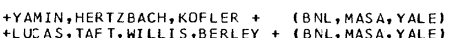
tF IL THUTH, HEPP, PRESSER, ZECH (HEIDELAERG) +, SABRE COLLAB. (ZEEM+SACL+BGNA+REHO+EFOL) TLE-FRANZINI, LOVELESS, BALTAY+ (STON, CLLU)
(COLUMBIA) LOUC, BEL GR ADE, BERL, BRUX, DUBLIN, WARS COLLAB

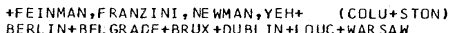
OXFFARE COLUMB IA HEE IOELBERG+MAR YLANO+STONY BROOK
R.M. ROBERTSCN

TE1SELE, FILTHUTH, HEP P LE ITNER, THOUN, (HEDI)

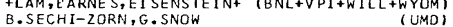

TEISELE, ENGELLANN, FIL LHUTH, HEPP + ${ }^{+}$(HEID) ERRA TUM TO ROBERTS 74 COP I+CARN + WYOM+CI T + BN ROBERTS, COX $*$ (WILL +VP I+CARN WYOM+CIT+BNL)
IANENBAUM, HUNGERBUEHLER + (YALE+FNAL+BNL)

F A SANO, CHEN, CHENG, HU, LI DOFS KY+ (COLUTYALE)

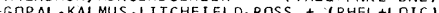
B BADIER, BLAND, CHOLLET, GAILLARD+ (LALO+E POL)

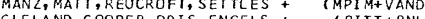
(PITT ENL) PAPERS NOT REFERRED to IN DATA CARDS

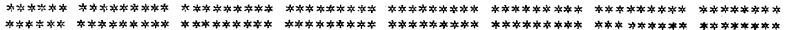

$\Sigma^{0}$

21 S,IGMAO $(1193, J P=1 / 2+1 \quad I=1$

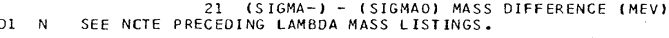

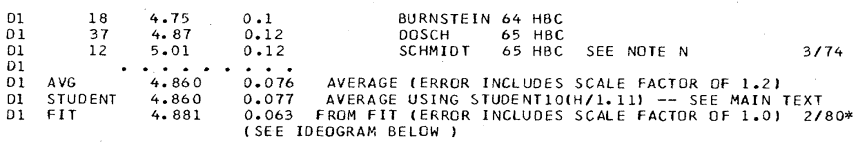

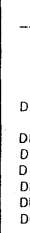

D N See note preceding lambda mass listings.

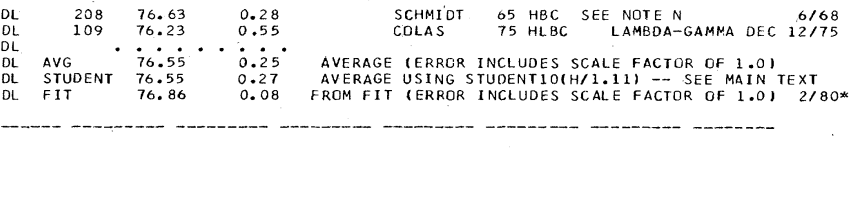




\section{Stable Particles} $\Sigma^{0}, \Xi^{-}$

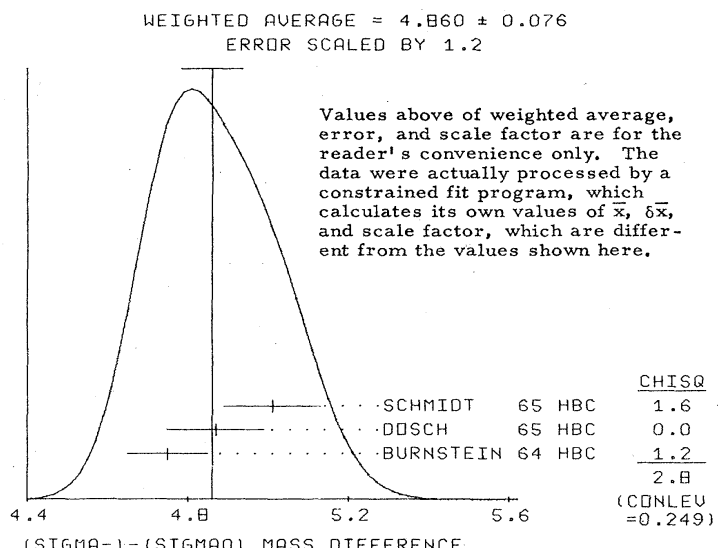

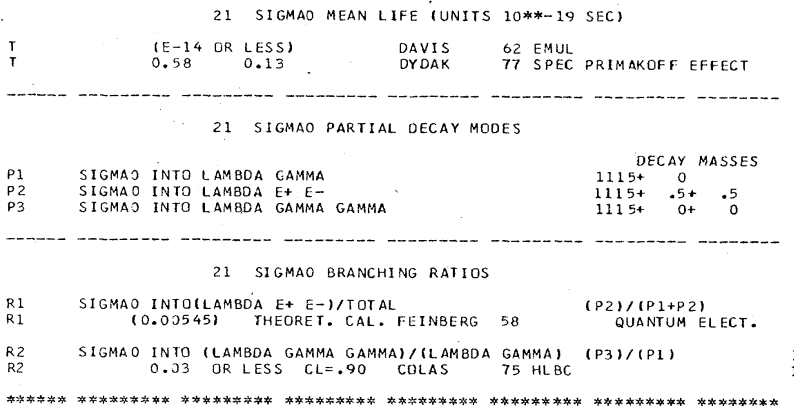

\begin{tabular}{|c|c|c|}
\hline & & REFERENCES FOR SIGMAO \\
\hline $\begin{array}{l}\text { FEINBERG } \\
\text { OALIS } \\
\text { BURNSTEI } \\
\text { DOSCH } \\
\text { SCHMIOT }\end{array}$ & $\begin{array}{l}58 \text { PR } 109.1019 \\
62 \text { PR } 127605 \\
64 \text { PRL } 13605 \\
65 \text { PL } 14235 \\
65 \text { PR } 140 \text { B } 1328\end{array}$ & 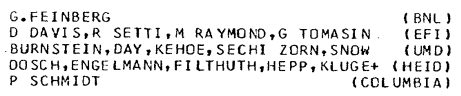 \\
\hline $\begin{array}{l}\text { CCLAS } \\
\text { OYOAK }\end{array}$ & $\begin{array}{l}75 \text { NP } 891253 \\
77 \text { NP B118 }\end{array}$ & $\begin{array}{l}\text { (ORSA) } \\
\text { FARWELL, FERRER, SIX } \\
\text { +NAVARRIA, CVERSETH, STEFFEN+ (CERNAODRT+HEID) } \\
\text { PAPERS NOT REFERRED TO IN DATA CARDS }\end{array}$ \\
\hline COURANT & $\begin{array}{l}63 \text { PRL } 10409 \\
\text { QUANTUM NUMBER }\end{array}$ & $\begin{array}{l}\text { COURANT, FILTHUTH, FRANZINIt (CERN+UMD+NRL) } \\
\text { MINATIONS NOT REFERRED TC IN THE DATA CARDS }\end{array}$ \\
\hline & 3781105 & ALFF;GELFAND, NAUENBE RG+ (COLUM EIA+RUT G+BNL) \\
\hline
\end{tabular}

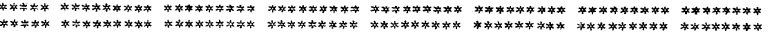

正 $22 \times 1-(1321, \mathrm{~J} P=1 / 2, \quad I=1 / 2$

22 X1- MASS (MEV)

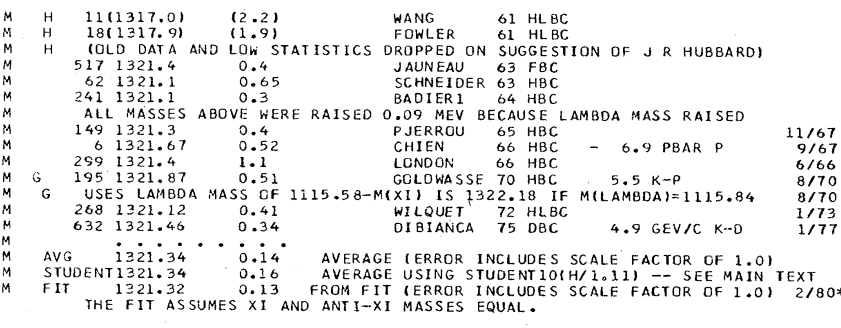
22 ANTI-XI+ MASS (MEV)

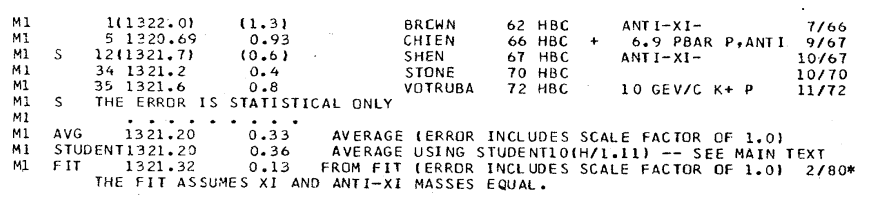

Data Card Listings For notation, see key at front of Listings.

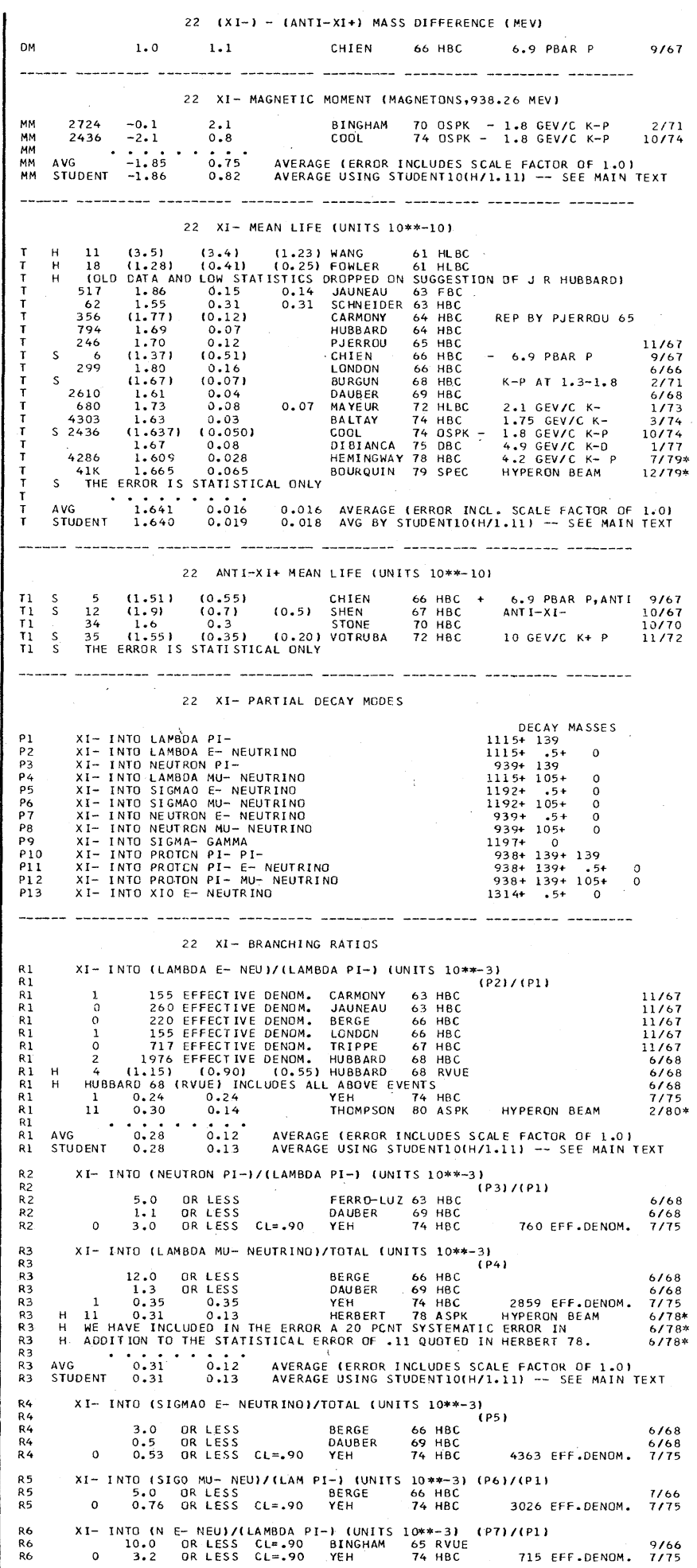




\section{Data Card Listings}

Stable Particles

\section{For notation, see key at front of Listings.}

$\Xi^{-}, \Xi^{0}$

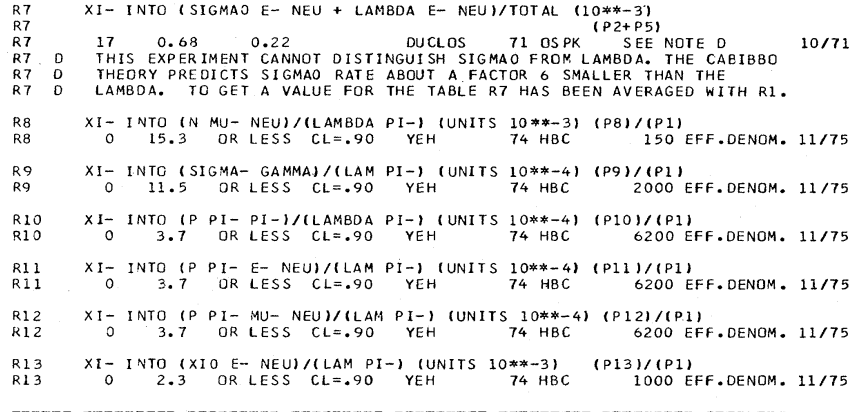

22 XI- DECAY PARAMETERS

RELATED TEXT SECTION VI D ANO APPENOIX III

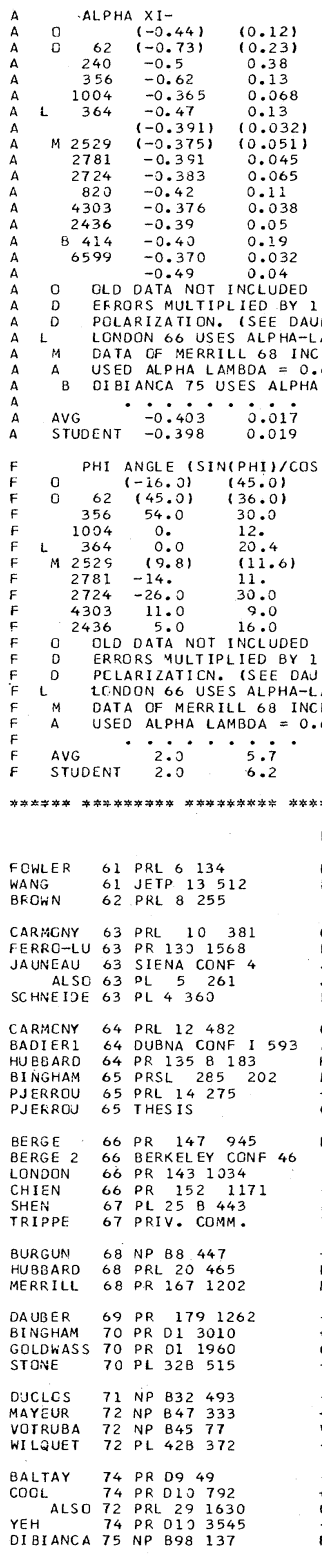

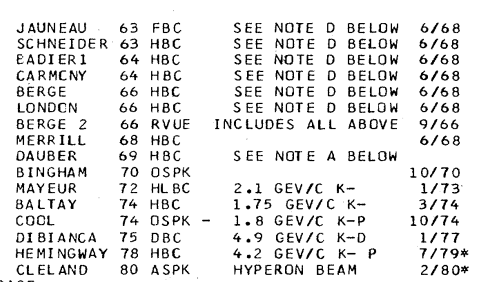
$\begin{array}{lllll}\text { HEMINGWAY } 78 \text { HBC } & 4.2 \text { GEV/C K- P } & 7 / 77 \\ \text { CLEL ANO } 80 \text { ASPK } & \text { HYPERON BEAM } & 2 / 80 *\end{array}$
$6 / 68$

$1 / 77$

VERAGE (ERROR INCLUOES SCALE FACTOR OF 1 .01
AVERAGE USING STUDENTIO(H/1.11) -- SEE MAIN TEXT

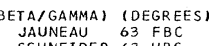

$63 \mathrm{HBC}$

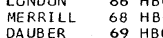

DAUBER

BALTAY

$\begin{array}{ll}68 \mathrm{HBC} & \text { SEE NOTE A BELOW } \\ 69 \mathrm{HBC} & \mathrm{OSC} \\ 70 \mathrm{OSPC} & 1.75 \mathrm{GEV} / \mathrm{C} \mathrm{K} \text { K- } \\ 74 \mathrm{HBC} & 1.30\end{array}$

SEe Note a belon

$1.75 \mathrm{GEV} / \mathrm{C} \mathrm{K}$ K-
$1.8 \mathrm{GEV} / \mathrm{C} K-\mathrm{P}$

TOERADPROXIMATIONS USED FOR XI

DaUber 68.
DaUb.

ERAGE IERROR INCLUDES SCALE FACTOR OF 1 11)
AVERAGE USING STUDENT1O(H/1.11) - SEE MAIN TEXT REFERENCES FOR XI-

FOWLER, BIRGE, EBER HARD, ELY, GOOD, POWELL+( $L R L)$
WANG, T WANG, VI RYASOV, TING, SOLOVEV + (JINR) W WANG, TWANG, VI RYASV, TING, SOLOVEV+ ( (JINR)
BROWN, CULWICK, FOWLER, GAILLOUD + (BNL+YALE)

CARMONY, PJERROU
(UCLA)
FERRO-LUZZI, ALSTON, ROSENFELD, WOJCICKI (LRL

CARRO-LUZZZ, ALSTON, ROSENFELD, WOJCICKI (LRL
JAUNEAU+ IEPOLCERN+LOUC+RHEL +BER GEN JAUNEAU, + (EPOL, CERN, LOUC, RHEL, BERGEN)
(CERN)

CARMONY, PJERROU, SCHLEIN, SLATER, STORK+ ( UCLA) J BADI ER, OEMCULI N, BARL OUT AUD+ (EPOL, SACL, ZEEM)
HUBBARD, BERGE, KALBFLEI SCH, SHAFER + H BINGHAM
SCHLEIN, SLATER, SMI TH, STORK, TICHO (CERN)
(UCLA)

BERGE, EBERHARD, HUBBARD, MERRILL + (LRL) BERGE, CAB IBBO
LONDON, RAU, GOLDBERG, LICHTMAN+(BNL + SYRACUSE) +LACH, SANDWEISS, TAFT, YEH,OREN + (YALE+BNL) B.C.SHEN,A.FIRESTONE, G. GOLOHABER ( UCB+LRL)
(UCLA) +MEYER, PAULI, TALLINI, + (SACL+CDEF+RHEL) MUBBARD, BERGE, DAUBER
MERRILL, SHAFER +BERGE, HUBBARD, MERR ILL, MILLER - (UCSO, WRL)' +COOK, HUMPHREY, SANDER, WILLI AMS + (UCSD, WASH)
(ILLL)
+BERLINSER, SCHULTZ
+ TFREYTAG, HEINTZE, HEINZELMAN, JONES+ (CERN) T +FLIAGI NE, GUY, KNIGHT + (ERUX+CERN +TUFT +LOUC) +BRIDGEWAT ER, COOPER, GER SHWIN+ (COLU+BING) J
+GIACOMELLI, JENKINS, KYCIA,LEONTIC,LI+ (BNL) 作 +GAIGALAS, SMMTH, ZENDLE, EALTAY + (BING+COLU)
F.A.OIBIANCA, R.J.ENDORF
(CARN) $\begin{array}{lll}\text { CARMONY } & 64 & \mathrm{HBC} \\ \text { BERGE } & 66 & \mathrm{HBC}\end{array}$

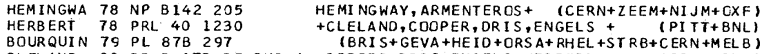
$\begin{array}{llll}\text { CLELAND } & 80 \text { PR } & \text { (TO BE PUB.) +C OOPER, DRIS, ENGELS, HERBERT+ } & \text { (PITT+BNL) } \\ \text { THCMPSON } 80 \text { PR D (TO BE PUB.) +CLELAND, COOPER, DRIS, ENGELS+ } & \text { (PITT+BNL) }\end{array}$

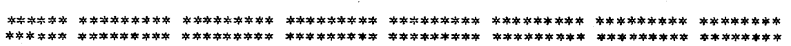
$\pm 0$

23 XI $0(1314, J P=1 / 2) \quad I=1 / 2$

23 XIO MASS (MEV)

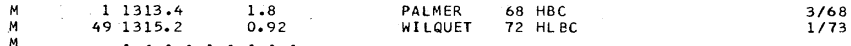

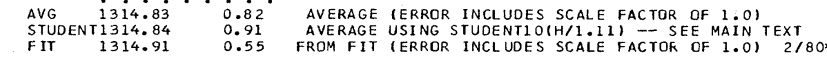
$23\left(X_{1}-\right)-(X I 0)$ MASS DIFFERENCE (MEV)

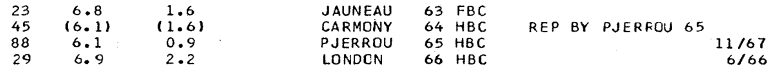

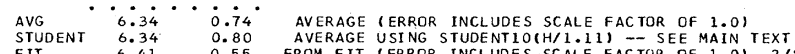
23 XIO MEAN LIFE (UNITS $10 * *-10$ )

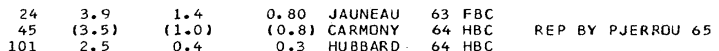

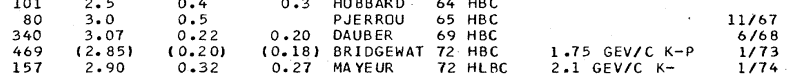

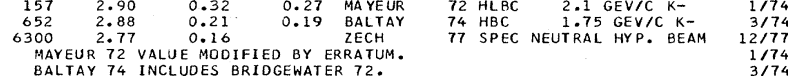

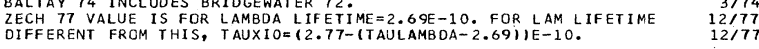

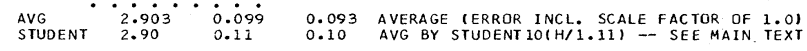

23 XIO MAGNETIC MOMENT IMAGNETONS, 938.26 MEVI

MM $42 \mathrm{~K}-1.20 \quad 0.06 \quad$ BUNCE 79 SPEC $1 / 80 *$

23 XIO PARTIAL DECAY MODES

P1
P2
XIO

P2
P3
PII

P4
P5
PIO INTO SIGMA- E+ NEU
XIO INTO SIGMA+ MU- NEUTRINO

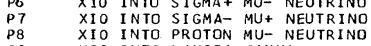

$\begin{array}{lll}\text { P9 } & \text { XIO INTO LAMBDA GAMMA } \\ \text { P1O } & \text { XIO INTO SIGMAO GAMMA }\end{array}$

23 XIO BRANCHING RATIOS

$X I 0$ INTO (PROTON PI-)/(LAMBDA PIO) (UNITS 10**-5) (P2)/(P1)

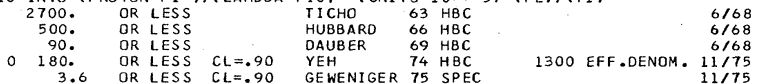
$X I O$ INTO (PROTON E- NEU)/(LAMBDA PIO) (UNITS $10 * *-3)$ (P3)/(P I)

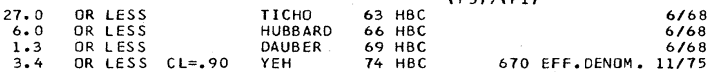
XIO INTO (SIGMA+ E- NEU)/(LAMBDA PIO) (UNITS $10 * *-3)$ (P4)/(PI)

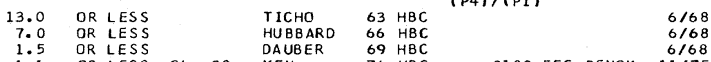

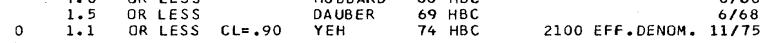
$X$ IO INTO (SIGMA- E+ NEU)/(LAMBDA PIO) (UNITS 10**-3) $($ P5)/(P1)

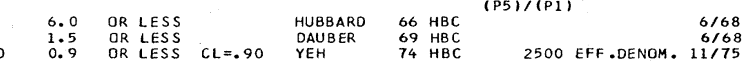

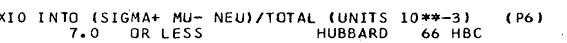

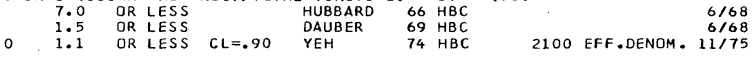
XIO INTO (SIGMA- MU+ NEUI/TOTAL CUNITS $10 * *-3$ ) (P7)

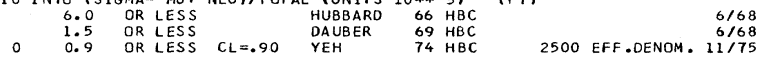

XIO INTO (PROTON MU- NEUI/TOTAL UUNITS 10**-3) (P8)

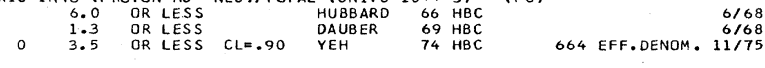




\section{Stable Particles} $\Xi^{0}, \Omega^{-}$
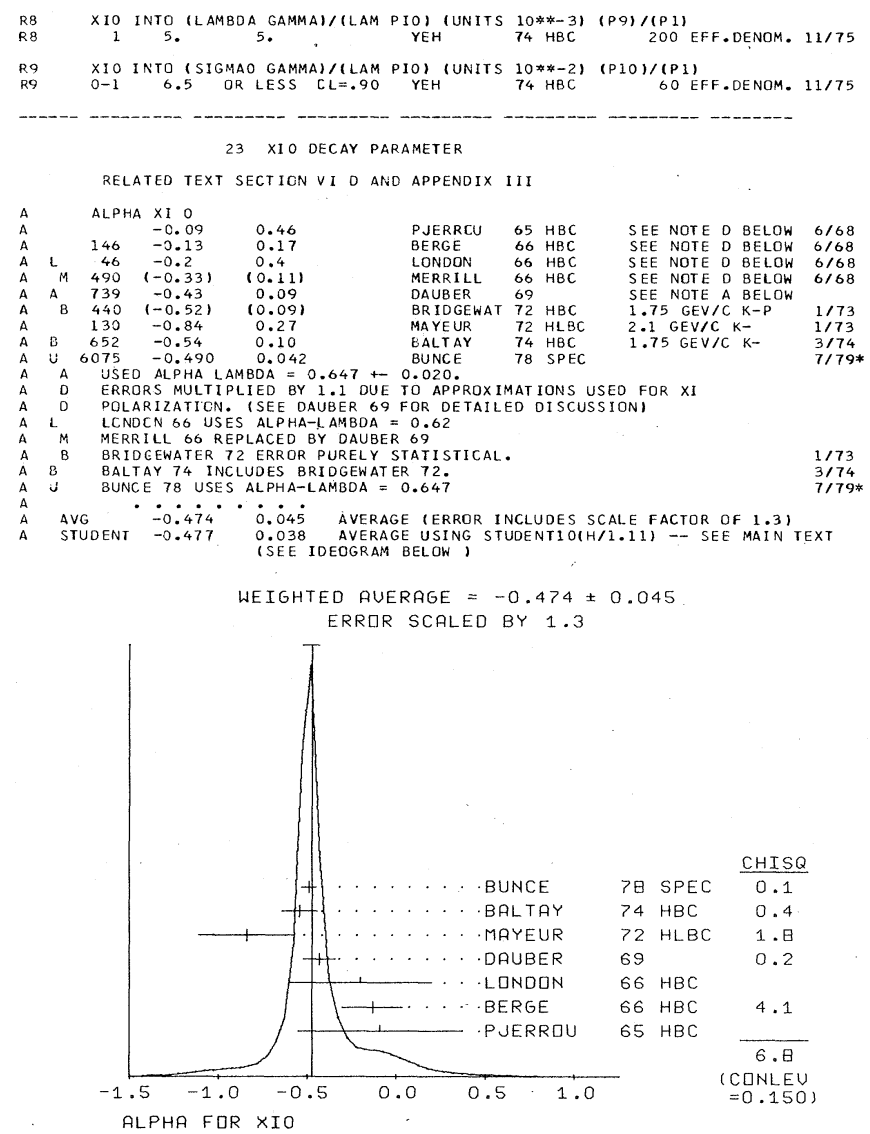

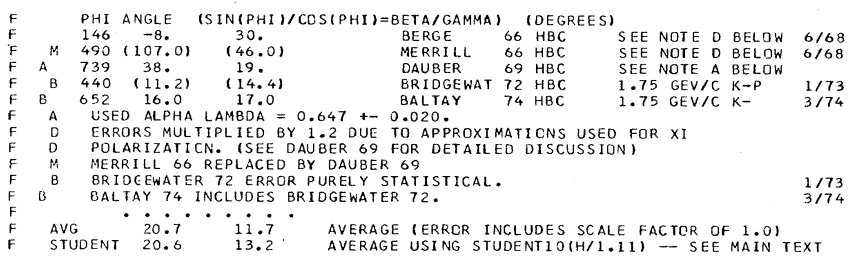

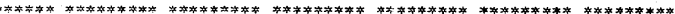

\begin{tabular}{|c|c|c|}
\hline $\begin{array}{l}\text { ALVAREZ } \\
\text { JAUNEAU } \\
\text { ALSO } \\
\text { TICHC }\end{array}$ & 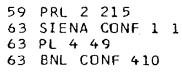 & 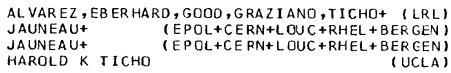 \\
\hline $\begin{array}{l}\text { CARMCNY } \\
\text { HUBEARD } \\
\text { PJERROU } \\
\text { PJERROU }\end{array}$ & 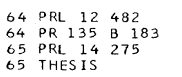 & 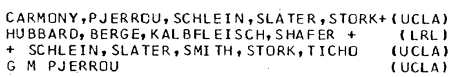 \\
\hline $\begin{array}{l}\text { EERGE } \\
\text { HUBBARD } \\
\text { LENOON } \\
\text { MERRILL } \\
\text { ALSO }\end{array}$ & 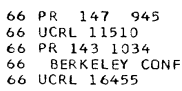 & 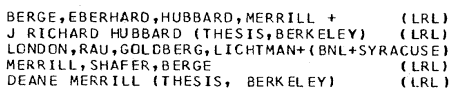 \\
\hline $\begin{array}{l}\text { LMER } \\
\text { UBER }\end{array}$ & $\begin{array}{lccc}68 & P L & 268 & 323 \\
69 & P R & 179 & 1262\end{array}$ & $\begin{array}{l}\text { PALMER, RADOJ ICII, RAU, R ICHARDSO } \\
\text { + BERGE, HUBBARD, MERRILL, MILLER }\end{array}$ \\
\hline
\end{tabular}
BRIOGEWA 72 NEVIS 195 (THESIS) ALBERT BRIDGEWATER P (COLUMBIA)
MAYEUR 72 (BRUX+CERN+TUFT+LOUC)

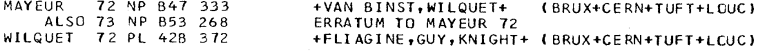

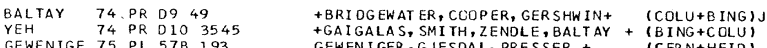

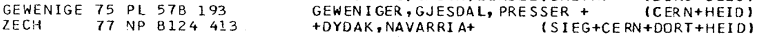

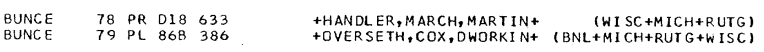

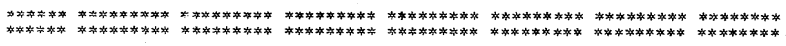

\section{Data Card Listings} For notation, see key at front of Listings.
This year the first results from the CERN hyperon beam experiment are available. BOURQUIN 79 collected a total of some $2400 \Omega^{-}$events and were able to make a very accurate measurement of the mean life. Their value is in agreement with BAUBILLIER 78 and HEMINGWAY 78.

The origin of the discrepancy with DEUTSCHMANN 78 is not known. It could be connected with the fact that the data of DEUTSCHMANN 78 is bubble chamber data at relatively high energy where contamination from $\Xi^{-}$decays might present a problem. In our calculation of the average $\Omega^{-}$mean life below, we do not include the value of DEUTSCHMANN 78. 


\section{Data Card Listings}

Stable Particles

\section{For notation, see key at front of Listings.}

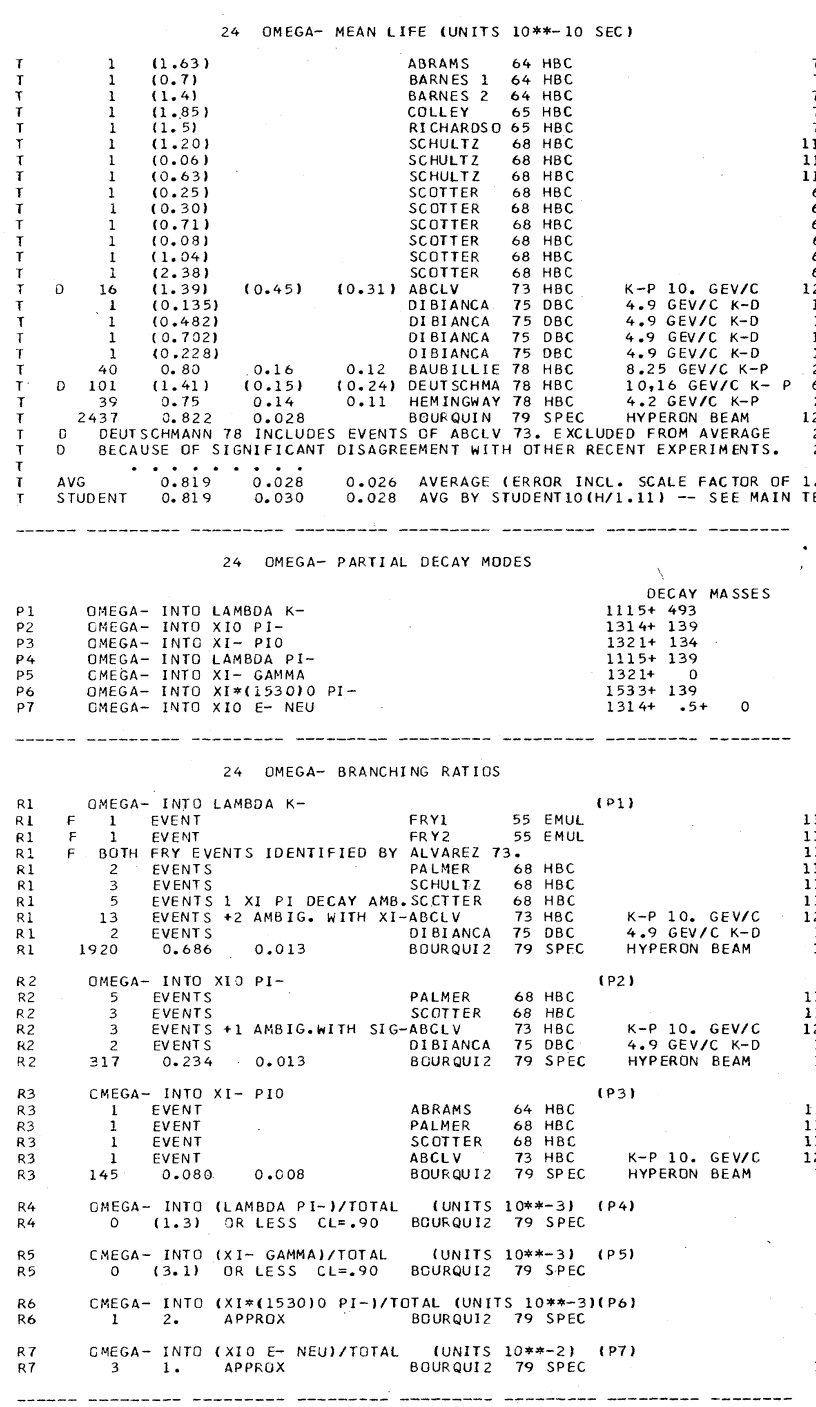

\footnotetext{
24 OMEGA- DECAY PARAMETERS

RELATED SECTION VI D IN TEXT

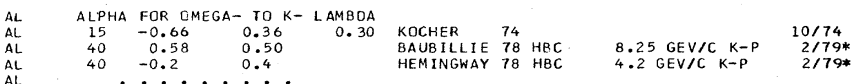

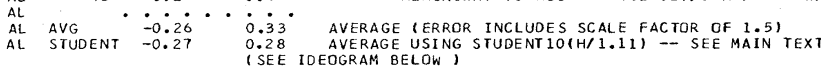

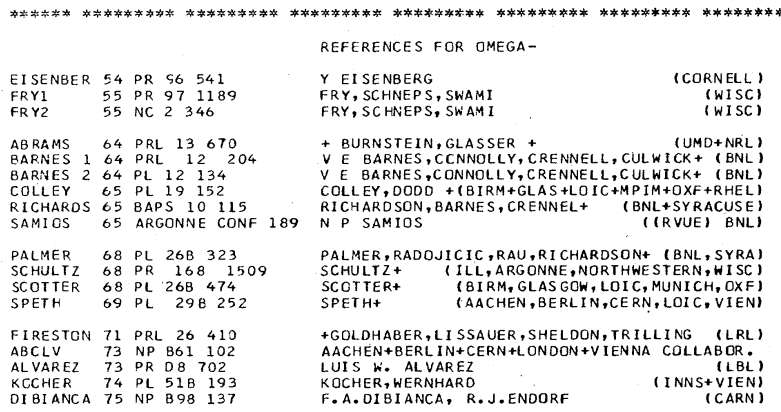

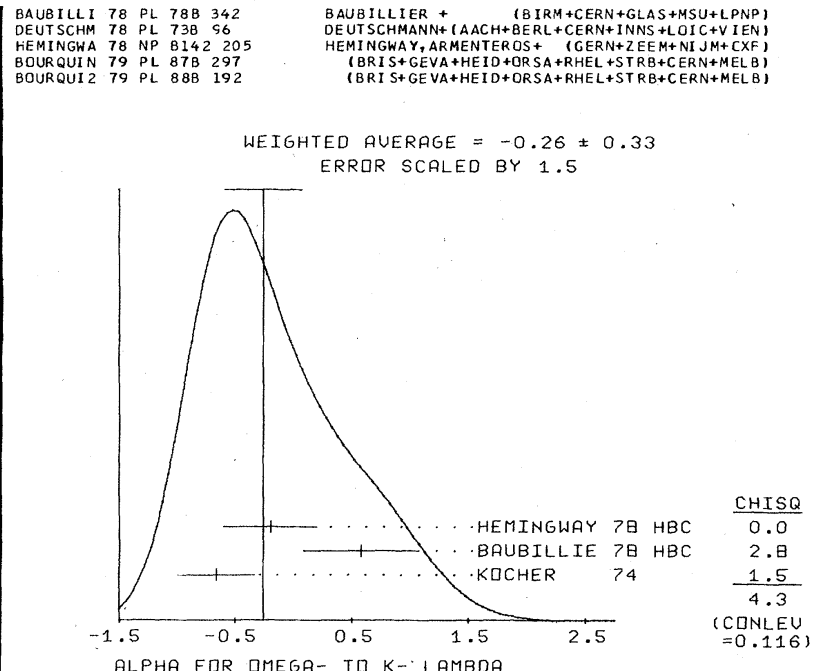

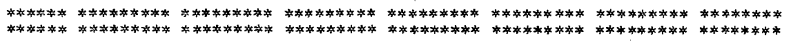

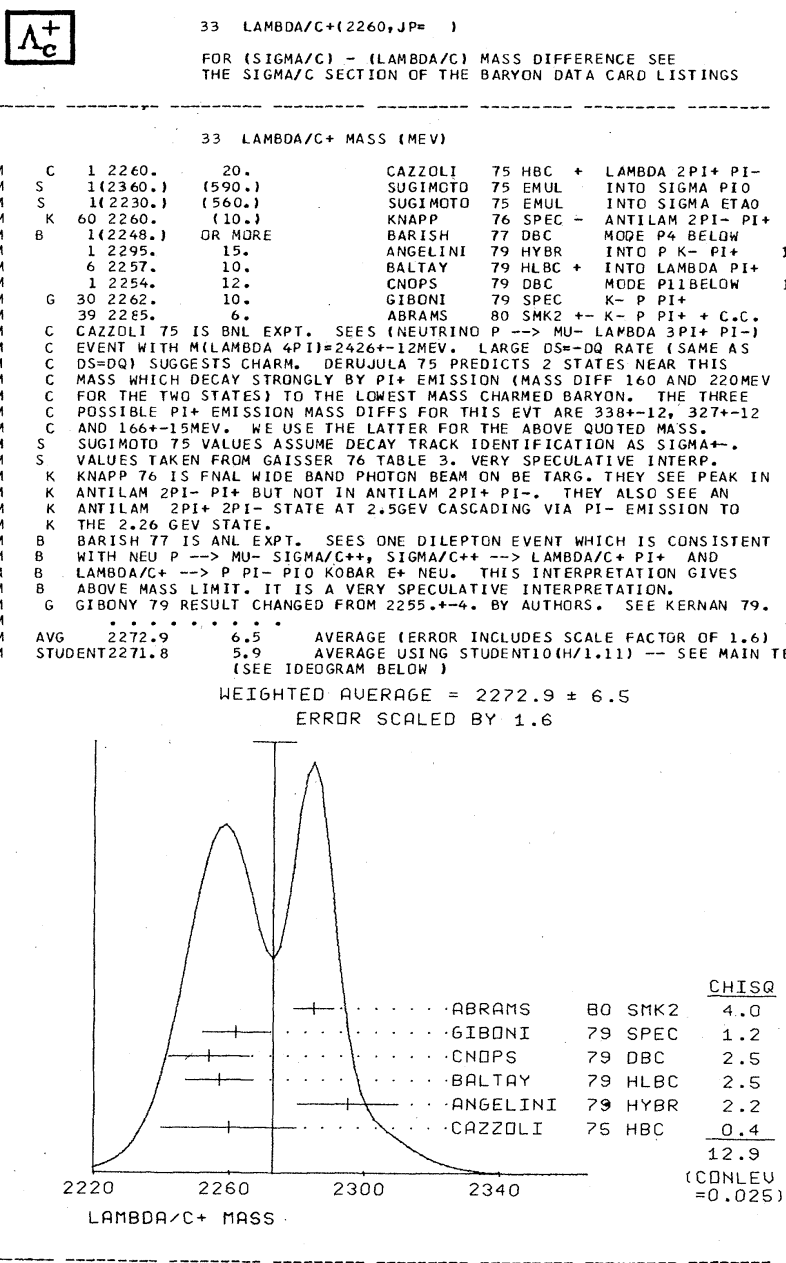




\section{Stable Particles}

$\Lambda_{c}^{+}$, HEAVY LEPTON SEARCHES

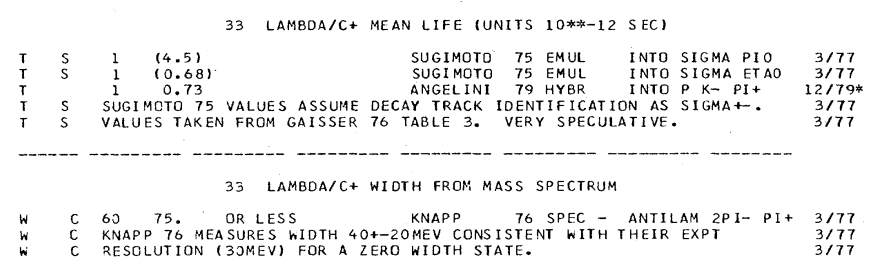

33 LAMBDA/C+ PARTIAL DECAY MODES

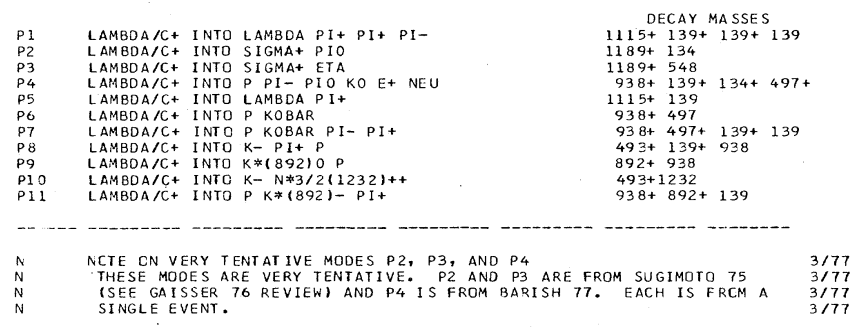

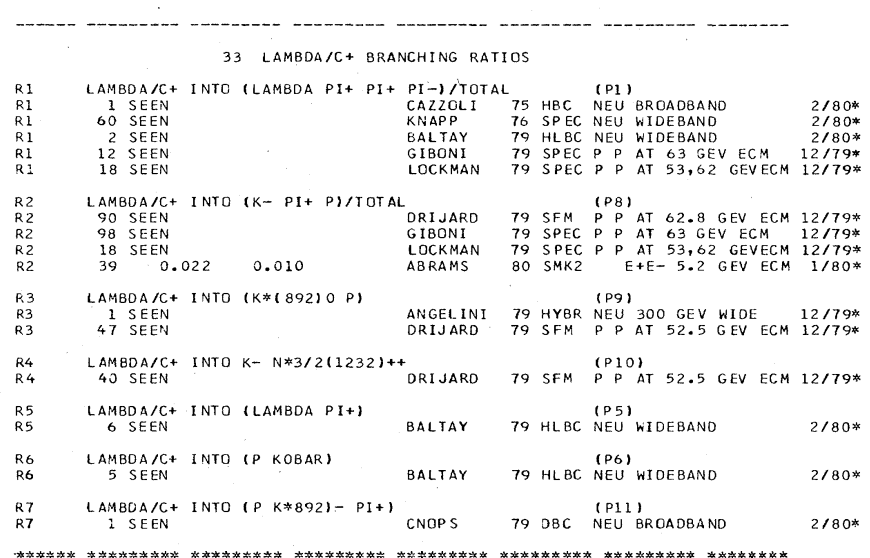

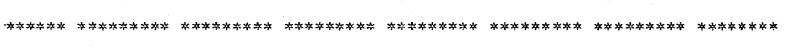

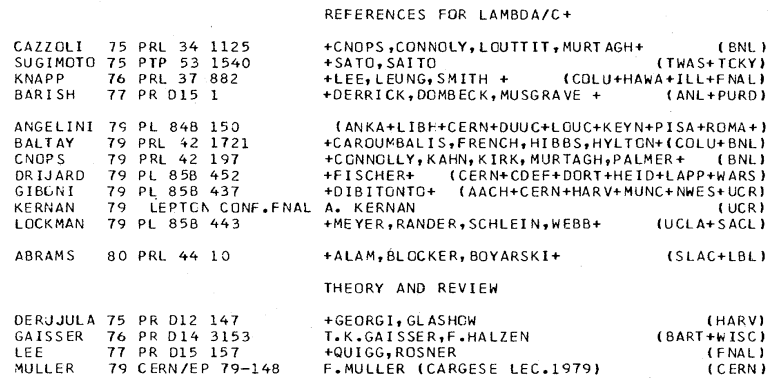

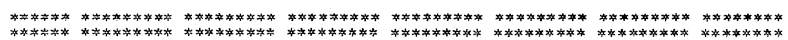

\section{HEAVY LEPTON SEARCHES}

Data on the $\tau^{ \pm}$(1800) heavy lepton are listed in a separate section above, following the $e$ and $\mu$ listings.

The following section contains information on searches for heavy leptons of other types and searches for the $\tau^{ \pm}$in collisions other than $e^{+} e^{-}$.

\section{Data Card Listings For notation, see key at front of Listings.}

Several types of heavy leptons (that is, non-strongly-interacting fermions other than $e$ and $\mu$ ) have been proposed. In the Data Card Listings we distinguish four types. ${ }^{1,2}$ Each has a corresponding antiparticle with opposite charge and lepton number. For convenience we omit writing the antiparticles in the following descriptions. The four types are:

\section{Sequential Leptons $\left(L^{-}, \nu_{L}\right)$. Such a pair} is assumed to have its own separately strictly conserved lepton number $\mathrm{n}_{L}=+1$. This means that the radiative decays

$$
\left.\begin{array}{l}
L^{-} \rightarrow e^{-} \gamma \\
L^{-} \rightarrow \mu^{-} \gamma
\end{array}\right\} \text { are forbidden, }
$$

while the weak decays (assuming $m_{L^{-}}$sufficiently massive)

$$
\left.\begin{array}{l}
L^{-} \rightarrow \nu_{L} e^{-\bar{\nu}} \\
L^{-} \rightarrow \nu_{L} \mu^{-} \bar{\nu}_{\mu} \\
L^{-} \rightarrow \nu_{L} \text { hadrons }
\end{array}\right\} \text { are allowed. }
$$

There could be an increasing mass sequence of such pairs. It is frequently assumed that the neutrinos are massless.

Decay rates are assumed calculable from conventional weak interactions theory. For $\mathrm{L}^{-}$mass between 1 and $3 \mathrm{GeV}$, the branching fraction to each of the two leptonic modes should be roughly $10 \%$ to $20 \%$. For $\mathrm{L}^{-}$mass above $1 \mathrm{GeV}$, the mean life should be $\lesssim 10^{-12} \mathrm{sec}$, too short to be observed in a track chamber. ${ }^{1}$

\section{Paraleptons $\left(\mathrm{E}^{+}, \mathrm{E}^{0}\right)$ and $\left(\mathrm{M}^{+}, \mathrm{M}^{0}\right)$. These pairs} have the same lepton numbers as the opposite-charge ordinary leptons, i.e., $\mathrm{e}^{-}$and $\mu^{-}$, respectively. Radiative decays are again forbidden and decays similar to those allowed for $\mathrm{L}^{-}$are allowed here, e.g.,

$$
\begin{aligned}
M^{+} & \rightarrow \nu_{\mu} e^{+} \nu_{e} \\
\text { or } \quad M^{+} & \rightarrow \nu_{\mu} \mu^{+} \nu_{\mu} .
\end{aligned}
$$

However, the lightest member is not stable as is the case for sequential leptons, so that bizarre decay schemes such as (assuming $\mathrm{m}_{E^{0}}<\mathrm{m}_{E^{+}}$)

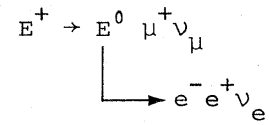




\section{Data Card Listings}

\section{For notation, see key at front of Listings.}

are allowed.

Heavy leptons of this type (and/or a neutral intermediate boson $\mathrm{z}^{0}$ ) are desired in unified gauge theories of weak and electromagnetic interactions to cancel unphysical high energy behavior in such processes as $\mathrm{e}^{+} \mathrm{e}^{-} \rightarrow \mathrm{W}^{+} \mathrm{W}^{-}$. 3

Ortholeptons $\left(\mathrm{F}^{-}\right.$and $\left.\mathrm{N}^{-}\right)$. These have the same lepton numbers as $\mathrm{e}^{-}$and $\mu^{-}$, respectively. They may or may not have associated neutral leptons. Radiative decays are allowed in addition to weak modes similar to those of sequential leptons. The radiative mode can dominate or can be relatively unimportant depending on the model. 4 Decays such as

$$
\mathrm{F}^{-} \rightarrow \mathrm{e}^{-}+\text {hadrons }
$$

are also allowed.

\section{Long-Lived Penetrating Particles. Heavy} leptons could have long mean lives under certain circumstances. For example, if $\mathrm{m}_{\nu_{L}}>\mathrm{m}_{L^{-}}$, then $\mathrm{L}^{-}$, the sequential lepton, is completely stable since its lepton number is conserved.

Experimental Results. The results are summarized in the Data Card Listings below. Mass limits for all types are listed together in subsection $M$. Mass information on the $\tau^{ \pm}$(1800) is no longer included here but has been moved into the new $\tau^{ \pm}(1800)$ section.

The Listings also contain cross-section upper limits reported as results of unsuccessful searches.

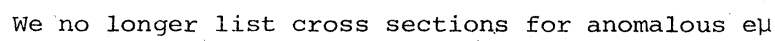
events in $\mathrm{e}^{+} \mathrm{e}^{-}$collisions. These cross sections are consistent with coming from $e^{+} e^{-} \rightarrow \tau^{+} \tau^{-}$where the $\tau^{ \pm}(1800)$ is assumed to be a spin-1/2 Dirac point particle with a mass about $1800 \mathrm{MeV}$.

\section{References}

1. M. L. Perl and P. Rapidis, SLAC-PUB-1496 (October 1974).

2. C. H. Llewellyn Smith, Invited paper presented at the Royal Society Meeting on New Particles and New Quantum Numbers, 11 March 1976, Oxford Ref. 33/76.

3. J.D. Bjorken and C.H. Llewellyn Smith, Phys. Rev. D7, 887 (1973).

4. F. Wilczek and A. Zee, Nucl. Phys. B106, 461 (1976).

\section{Stable Particles HEAVY LEPTON SEARCHES}

PROPERTIES OF THE TAU+-(1800) HEAVY LEPTON AND ITS ASSOCIATED NEUTRINO ARE LISTED SEPARATELY ABOVE FOLLOWING THE E AND MU
LISTINGS. THE FOLLOWING SECTION CONTAINS INFORMATION ON SEARCHES FCR HEAYY LEPTCNS OF OTHER TYPES AND SEARCHES FOR TAU T- IN
COLLISIONS OTHER THAN E+E-. WE LIST MASS LIMITS AND CROSS SECTION UPPER LIMITS REPORTED AS NEGATIVE SEARCH RE SUL TS. WE NO LONGER LIST CROSS SECTIONS FOR THE ESTABLISHED PROCESS E+ E- $\rightarrow$ TAU+ TAU-
AS WAS DONE IN OUR 1977 SUPPLEMENT.

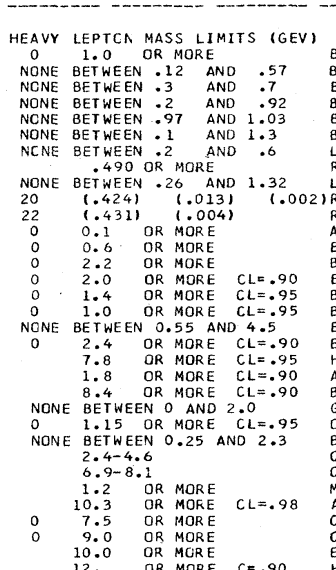

v

COMMENTS
LIMITS APPLY CNLY TO HEAVY LEPON TYPE GIVEN IN COMMENT AT RIGHT ON
CATA CARD CATA CARD. SEE REVIEW ABDVE FOR DESCRIPTION OF TYPES.
IN CCMMENTS BELW: ALL BEAMS ARE MU TYPE NEUTR INO OR ANTINEUTRINO. $L, E, M, F, N$ STAND FOR SE QUENTIAL LEPTCN, PAR
ORTHO-ELECTRCN, ORT HO-MUON RESPECTIVLY.

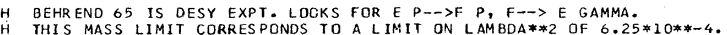
BETOURNE ES IS ORSAY EXPT. LOOKS FOR E P P-PF P. MASS. OF 12
CORRESPONS TO COJPLING CONSTANT LAMBDA*Z GT .OO16, MASS OF .57 TO LAMBDAF*2 GT G.22.
BUONITZ 66 IS CEA EXPT. LOOKS FOR E P-IPF P.

BARNA 68 IS SLAC PHOTOPRODUCT ION EXPT.

BCLEY 68 IS CEA EXPY. LOOKS FOR E P-IPF P. MASS OF . 1 CORRESPONOS LAMBDA*\#2 GT .01

LIBERMAN 69 IS A BNL EXPT MEA SURING MUON BREMSSTRAHLUNG. LICHENSTEIN 70 IS CORNELL EXPT MEASURING E BREMSSTRAHLUNG.

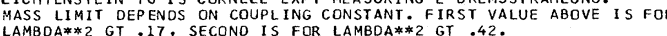
LAMBDA**2 GT. 17 . SECONO IS FOR LAMBDA**2 GT. 42 .
RAMM 70 FINOS PEAK IN MU PI COMB NED MASS PRODUCED BY NEUTRINO INTERACTICNS. HE ALSO CLAIMS EVIDENCE FOR THIS IN KOMUZ DECAYS IN
HBC WHERE PI MU COMBINEO MASS PEAKS IN SAME REGION. CLARK 72 FINOS EVIDENCE FOR COM MU PEAK IN MIGH SNATISTICS KOL ANSORGE 73 LOOKS FOR ELECTRON PAIR PROD AND ELECTRON-LIKE BREMSS. BACCI 73 IS FRASCATI E+E- EXPT. LCOKS FOR F --D E GAMMA. MASS LIMIT DEPENDS ON COUPLING CONSTANT LAMBDA FOR THIS DECAY

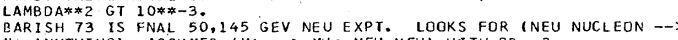
M+ ANYTHING). ASSUMES (M+ $\rightarrow$ MU + NEU NEU) WTTH BRE. ${ }^{3}$. COUPLING TO ORDINARY LEPTONS. SECOND VALUE ALSO ASSUMES COUPLING BUSHNIN 73 IS SERPUKOV 70 GEV P EXPT. MASSES ASSUME MEAN LIFE ABOVE TE-1O AND 3 E-8 RESPECT IVELY. CALCULATTE FROM C

AND 30 GEV MUON PAIR PRODUCIION DATA.
EICHTEN 73 IS CERN $1-10 \mathrm{~T}$ ISE NEU EXPT. LOOKS FOR M+ PRODUCED IN NEU NUCL $\rightarrow$ M HADRCNS ASSUMING 15 PERCENT DECAY TO E+ NEU NEU, WANSON 73 LOOK FOR DEVIATIONS FROM QED IN E+ E- $\rightarrow 2$ GAMMA. THEY
MEASURE THE PRODCT DF THE F MASS * THE COUPLING CONSTANT LAMBOA,

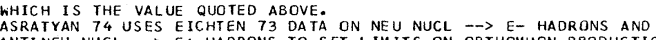
ANTINEU NUCL $\rightarrow-\rightarrow$ E HADRONS TO SET LIMITS ON ORTHOMUON PRODUCTION. BARISH 74 IS FNAL 50,135 GEV NEU EXP T. LOOKS FOR (NEU NUCLEON
M ANYTHING). ASSUMES (M+ - >MU+ NEU NEU) WI TH BR=.3. GITTLESON 74 IS MU P $\rightarrow$ P ORTHOMUON SEARCH. COUPLING CONST ANT LAMBDA**2 IS C.01 FOR MASS UP TO .7 GEV, LIMIT ON LAMBDA**2 RISES TO $<.1$ FOR MASS OF 2.0 GEV.
ORI TO 74 LCOKEO FOR H+H-PAIRS GIVING MU-E PAIRS. MASS LIMIT REFERS COUPLING TO HADRON ASSUMED FROM THEORETICAL MCDELS.

BACCI 77 IS SAME TYPE AS BACCI 73 . LOWER MASS LIMIT CORRESPONDS TO COX 77 ASSUMES TRIMUON EVENTS OF BENVENUTI 277 ARE A NEGATIVE heAVY LEPTCN DEC AYING TO A NEUTRAL HEAVY LEPTON MU - NUBAR.
MEYER T7 LOOKS FOR NARROW NEUTRAL RESONANCE INCE PI IANOIMU PII MEYER 77 LOOKS FOR NARROW NEUTRAL RESONANCE INCE PI) AND(MU PII
CHANNELS PRODCED BY E+E- AT 6.8 GEV (ECM). ASSUMED TO BE DECAY PRODUCT OF THE TAU. SEE SECTION NE BELOW.
ASRATYAN 78 ANALYZES DEPENDENCE OF N.C. C.C. CN ENERGY OF ASSOC.
HADRONS. USES DATA OF HOLDER 77 (PL 728,254$)--N U M U$ INTERACTIONS HADRONS. USES DATA OF HOCDER 77 (PL, 72B, 254)--NUMU INTERACTIONS CNOPS 78 IS FNAL EXPY LOOKING FOR NEUMU NE $-\rightarrow L+(-1$, FOLLOWO BY

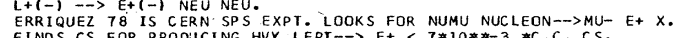
FINDS CS FOR PRODUCING HVY LEPT-
HOLDER 78 IS A CERN NEU EXPT LOOKING FOR NEUMU NUCLEON $\rightarrow$ MU+ AN THING. ASSUMES M+ $\rightarrow$ MU+ 2 NEUMU WITH BR=0.2 .

CCSMOLOGICAL. LIMITS ON MASS OF NEUTRAL HEAVY LEPTONS

NONE 70 EV TO 23 MEV NGNE 30 EV TO 2.5 GEV 50 EV TO 100 KEV
3 EV TO 10 GEV
60 GEV OR LESS

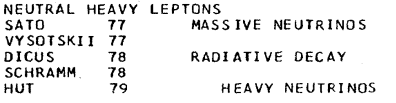

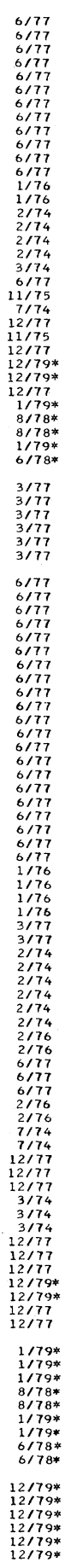




\section{Stable Particles INTERMEDIATE BOSON, QUARK SEARCHES}

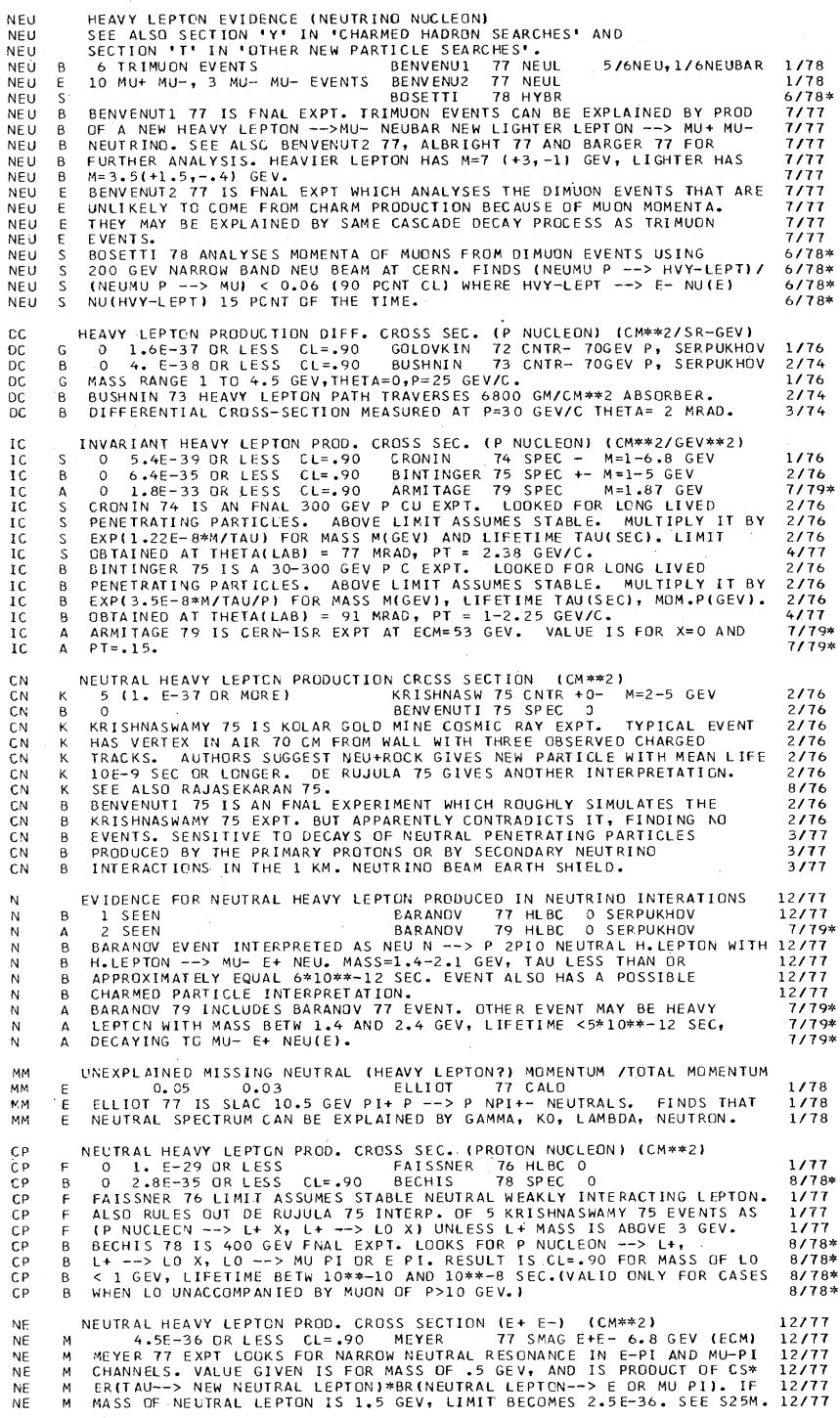

\section{Data Card Listings For notation, see key at front of Listings.}

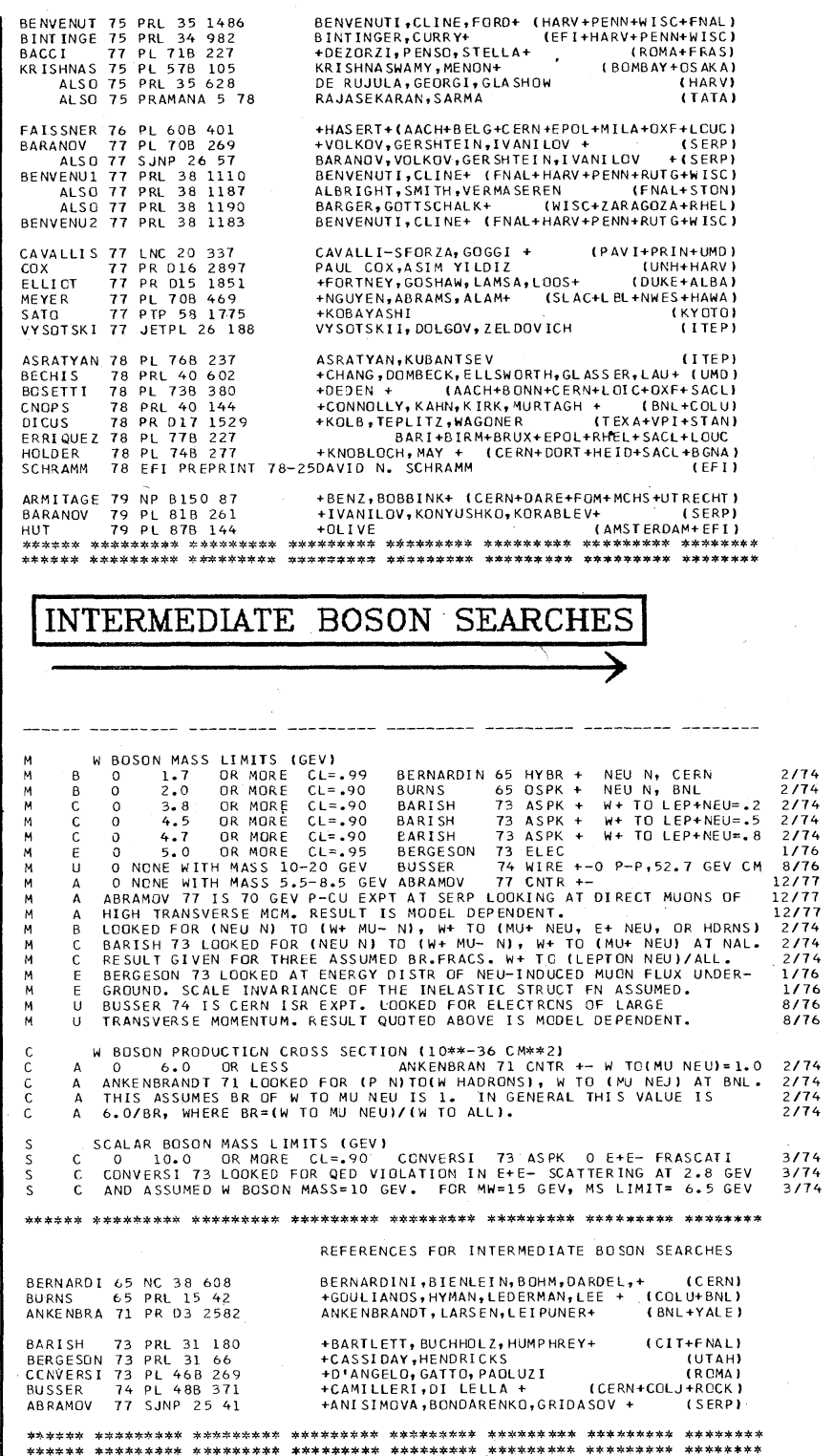

\section{QUARK SEARCHES}

SEARCHES FOR INTEGRALLY CHARGED QUARKS APPEAR ALONG WITH OTHER
SIMILAR SEARCHES IN IOTHER NEW PARTICLE SEARCHES. SECTION BELOW.

Since the last edition, two more instances of charge $\pm 1 / 3$ have been claimed by the stanford group (LARUE 79) using magnetic levitation of heat-treated niobium beads. There has as yet been no independent confirmation of the existence of free quarks.

The best searches for quarks in cosmic rays yield upper limits on the flux of quarks of about 


\section{Data Card Listings}

For notation, see key at front of Listings.
Stable Particles QUARK SEARCHES
$10^{-11} \mathrm{~cm}^{-2}$ ster $^{-1} \mathrm{sec}^{-1}$. Cross-section upper limits established from proton accelerator experiments and calculations based on production models ${ }^{1}$ imply that free quarks, if they exist, have a mass greater than about $5 \mathrm{GeV}$. Mass limits from photon and electron beam searches are slightly lower, but more reliable, depending only on the QED calculations for quark pair production. Limits on free quark concentrations in stable matter vary enormously depending on the source of matter and the technique.

The largely negative result of quark searches does not prove that free quarks do not exist, but indicates that they are hard to find. De Rujula, Giles, and $\mathrm{Jaffe}^{2}$ have considered the question of unconfined quarks in a framework of a renormalizable, spontaneously broken version of $\mathrm{QCD}$, and conclude that: (1) production cross sections are small, (2) interaction cross sections with nucleons are very large, and (3) the physical masses of quarks are probably very large. On this basis, primordial quarks would be expected to be nonintegrally charged, superheavy nucleon complexes.

We group quark searches by experimental technique - proton beams, photon beams, neutrino beams, electron beams, cosmic rays, and stable matter. Proton beam experiments generally measure quark production cross sections (we quote these in section $\mathrm{C}$ ), differential cross-section ratios (section AF), or differential cross sections (sections IC and D). The photon beam experiment measures cross section per equivalent quanta (section DC), and the neutrino experiment measures the ratio of quark events over total events (section NEU). Searches with electron beams may measure differential cross sections (section G) and set limits on the quark mass (section M). Cosmic ray experiments measure quark flux (section F), and searches in stable matter measure quark concentration (section RHO). Most of the accelerator and cosmic ray experiments have searched for fractionally charged particles, but some have searched for massive stable particles which would have low velocity. The latter searches are usually sensitive to a range of charges and may appear in the section below on Other New Particle Searches.

We have relied heavily on the review of
L. W. Jones ${ }^{3}$ for data prior to April 1977.

\section{References}

1. T. K. Gaisser and F. Halzen, Phys. Rev. Dll, 3157 (1975).

2. A. de Rujula, R. C. Giles, and R. L. Jaffe, Phys. Rev. D17, 285 (1978).

3. L. W. Jones, Rev. Mod. Phys. 69, 717 (1977).

QUARK PRODUCTICN CRCSS SECT. FROM PROTON BEAM EXPTS. ICM**2,

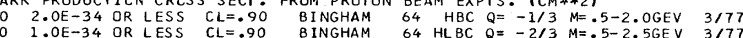

2.0E-35 OR LESS

$1.0 E-34$ OR LESS

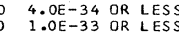

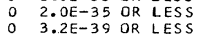

$0.5 E-38$ OR LESS $C L=.90$
0
0

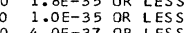

3.0E-39 OR LESS

$3.00-37$ OR LESS $C L=.90$
$1.0 E-36$ OR LESS CLE:90

$3.0 E-34$ OR LESS
$6.0 E-34$ OR LESS
$1.0 E-32$ LES

$\begin{array}{lll}1.0 E-32 & \text { OR LES } & C L=.90 \\ 0 & 1.0 E-32 & \text { OR LES } \\ 0 & \text { LEL } & .90\end{array}$

$\begin{array}{lll}1.0 E-35 & \text { OR LESS } \\ 0 & 1.0 E-35 \text { OR LESS }\end{array}$

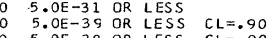

$5.0 E-38$ OR LESS $C L=.90$
$4.00-35$ OR LESS CLE.9.

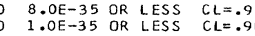

HAGOPIAN 64 CROSS SECT ION INFERRED FR FR

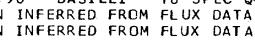

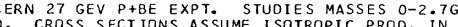

CROSS SECTIONS AT 2 GEV ARE GIVEN HERE. SEE FIG IC PROR MASS DEPEN.

ANTPOV1 69 IS A SERPUKHOV 70 GEV P EXPT. MASS LIMIT FROM NN=NNOC. GIVES RESULTS FOR M=2-5GEV ASSUMING NN-->NNQQ, HADRONIC ORR LEPTONIC QUARKS. WE QUOTE TYPICAL VALUES. P.

ANTSO TSUDIES DIQUARK MASSES

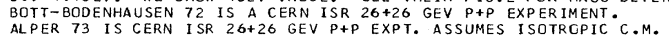
ALPER 73 IS CERN ISR $26+26$ GEV PPP EXPT.
PROOUCTION. SENSIIIVE TO ANY Q 2713 .

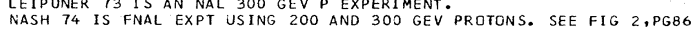
FOR OTHER MASS VALUES AND VARIOUS PRODUCTION MECHANISMS.
FABSAN 75 IS CERN ISR P+P EXPT. INCLUDES RESULTS OF BOTT-BODE 72

ECM $M=52.5$ GEV.

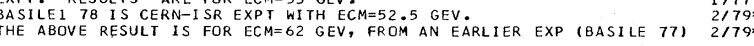

QUARK PRODUCTION FLUX (FLUX QUARKS I FLUX CHARGED PARTICLES)

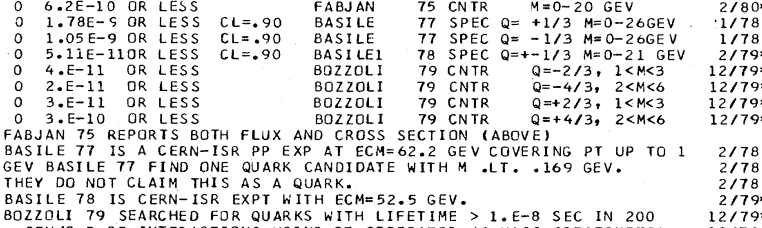

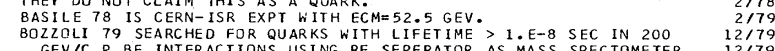

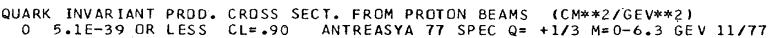

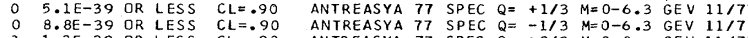

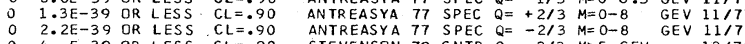

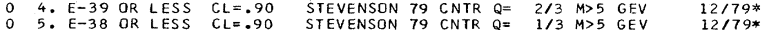
ANTREASYAN 77 LOOKS FOR HIGH TRANSVERSE MOM QUARKS IN 400 GEV P-CU 11177

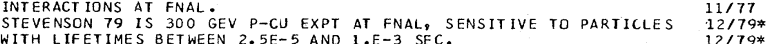

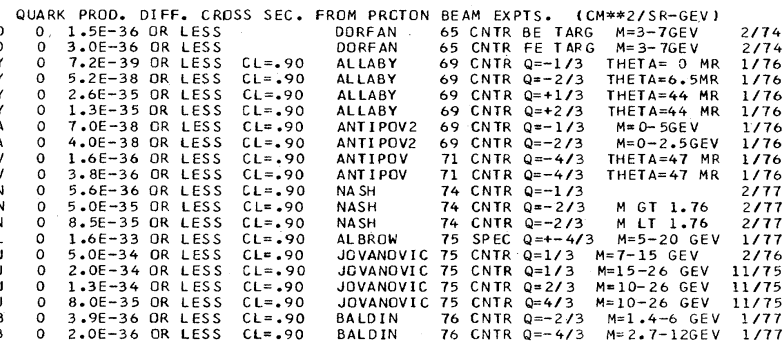




\section{Stable Particles QUARK SEARCHES}

\section{Data Card Listings For notation, see key at front of Listings.}

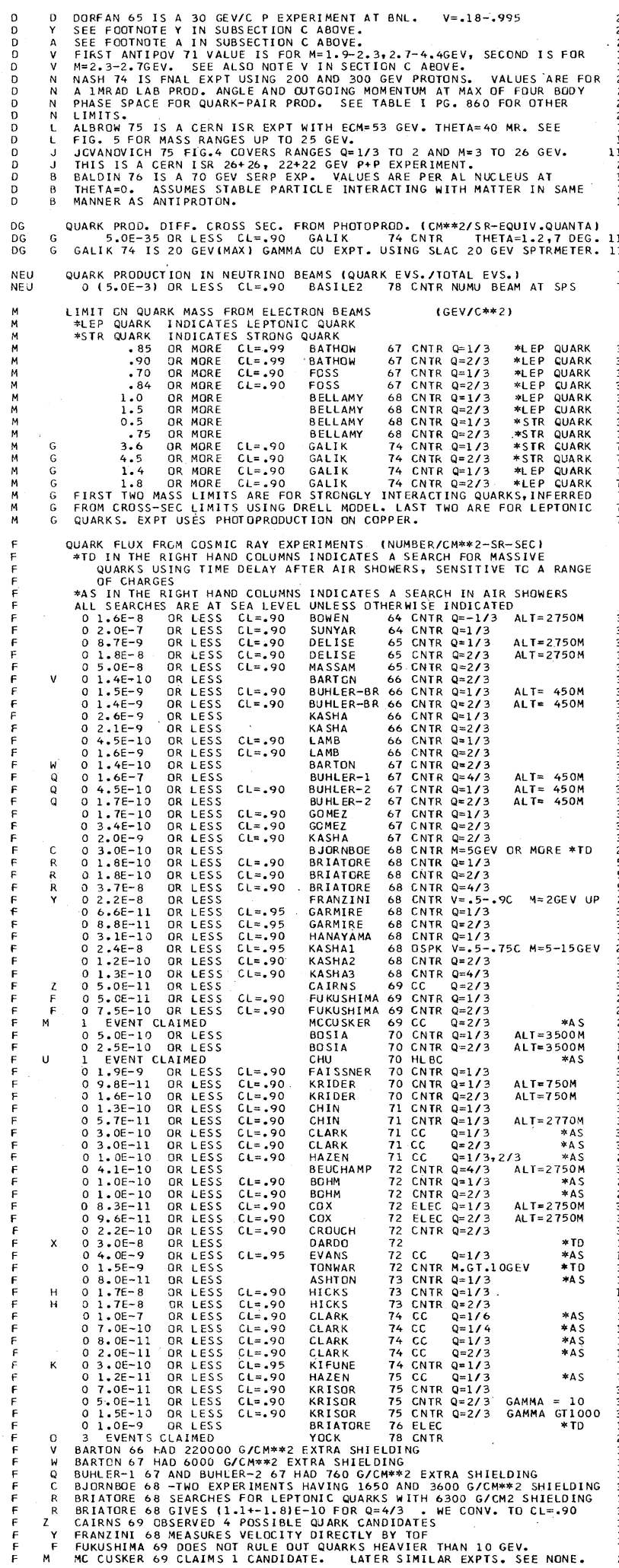

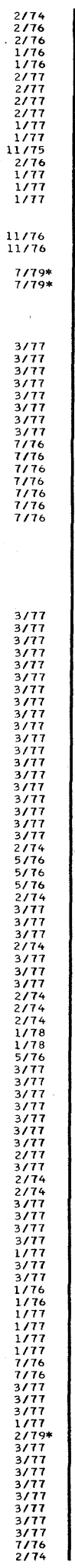

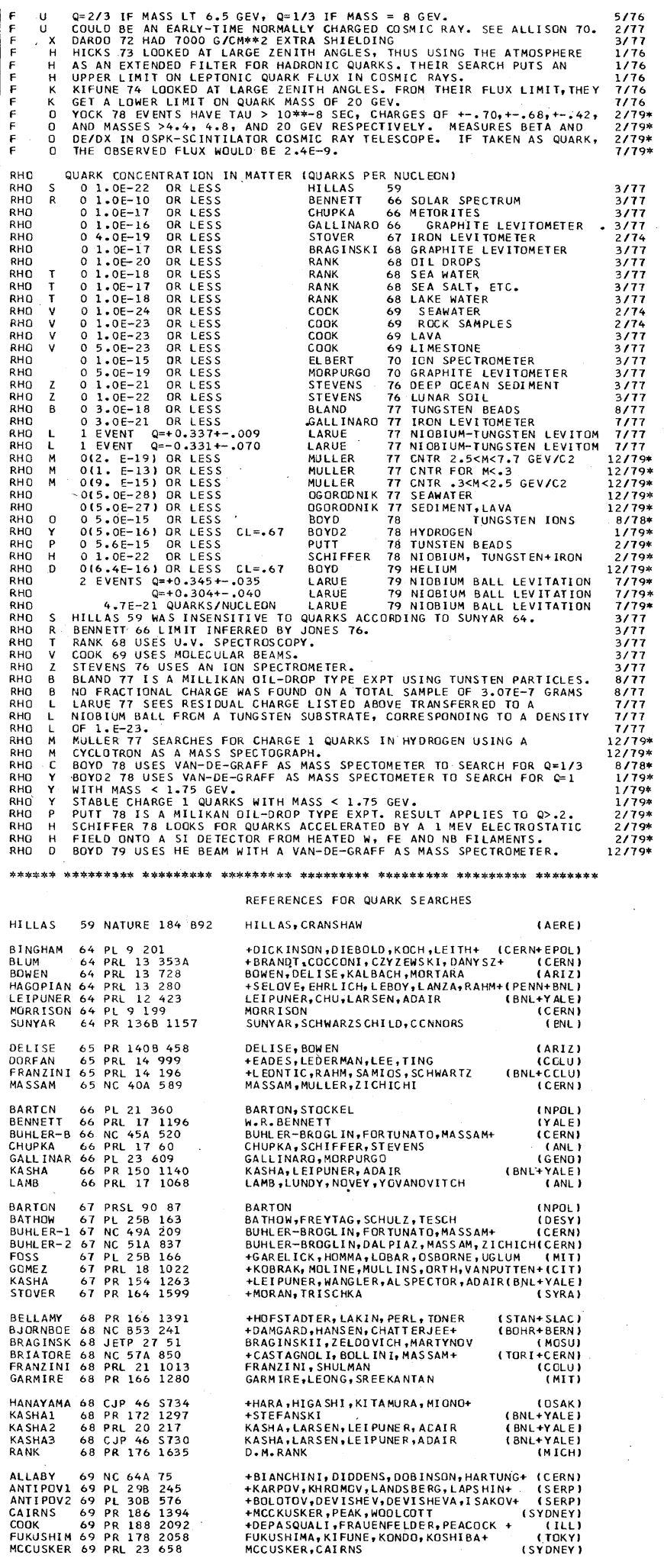


Data Card Listings

For notation, see key at front of Listings.
Stable Particles MAGNETIC MONOPOLE, CHARM SEARCHES
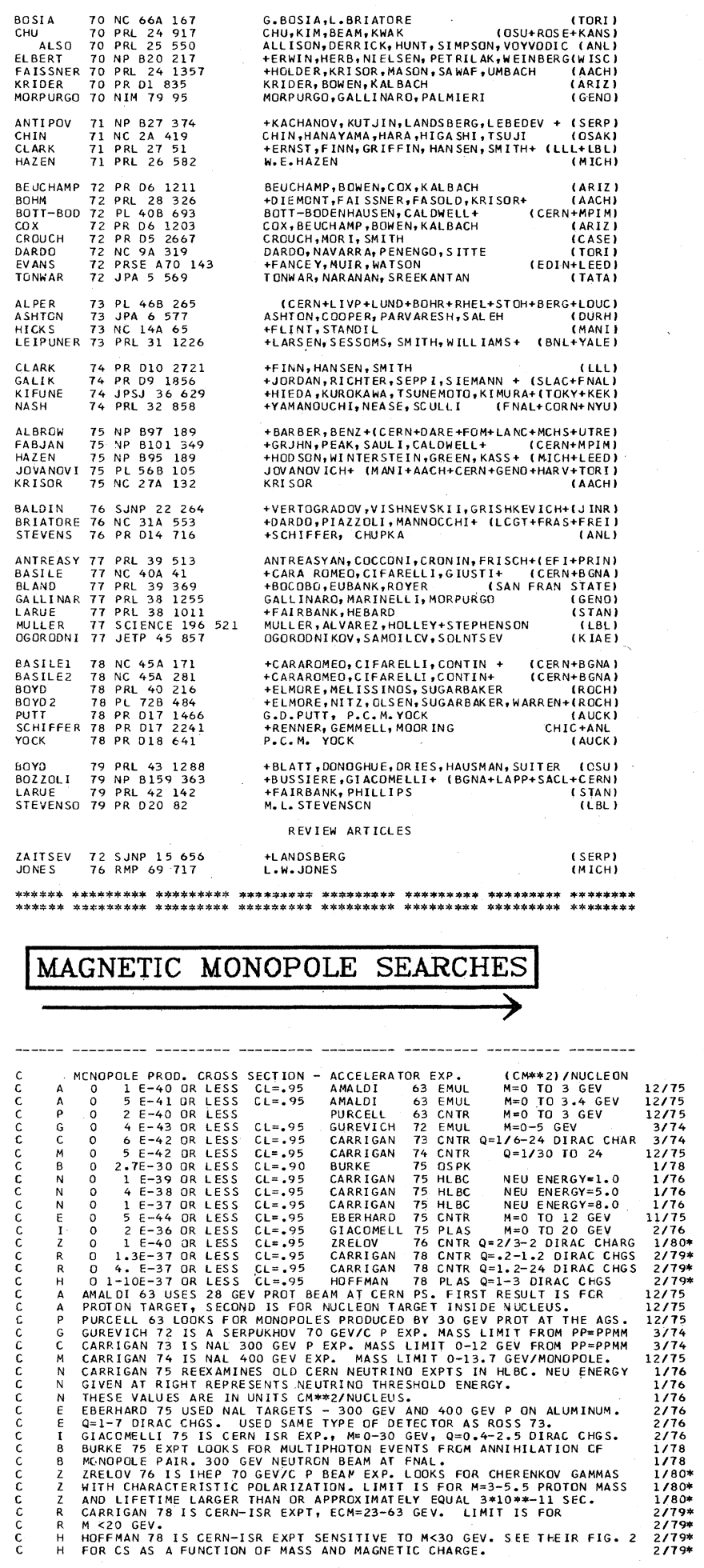

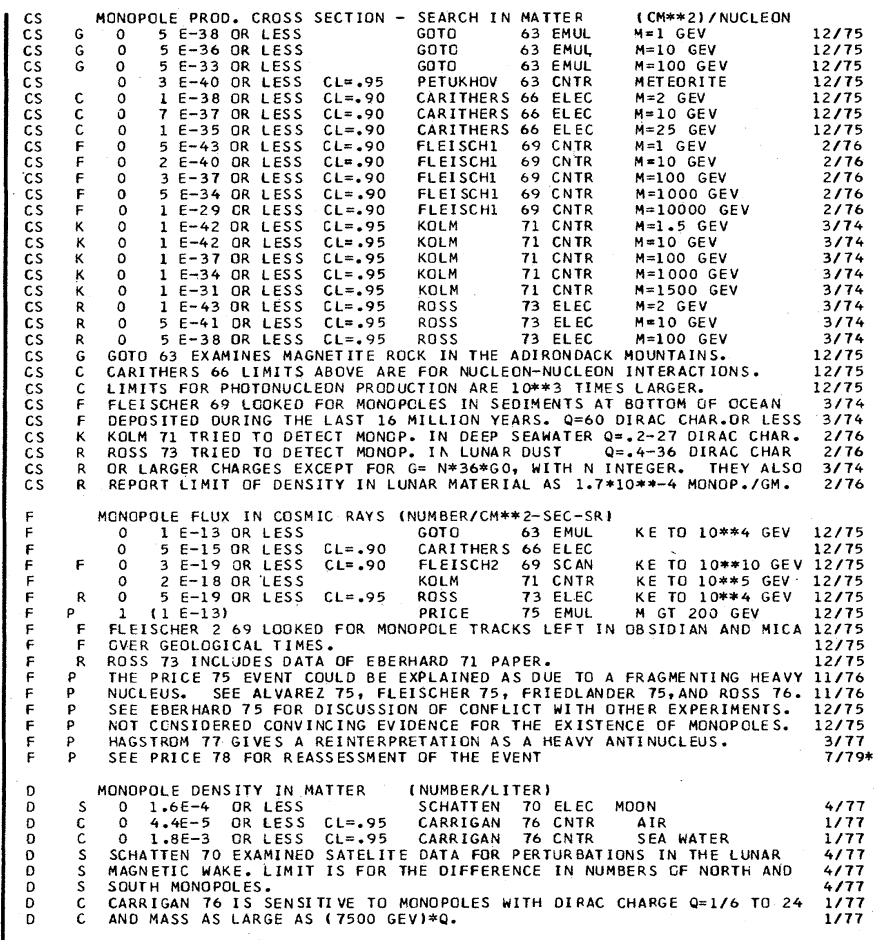

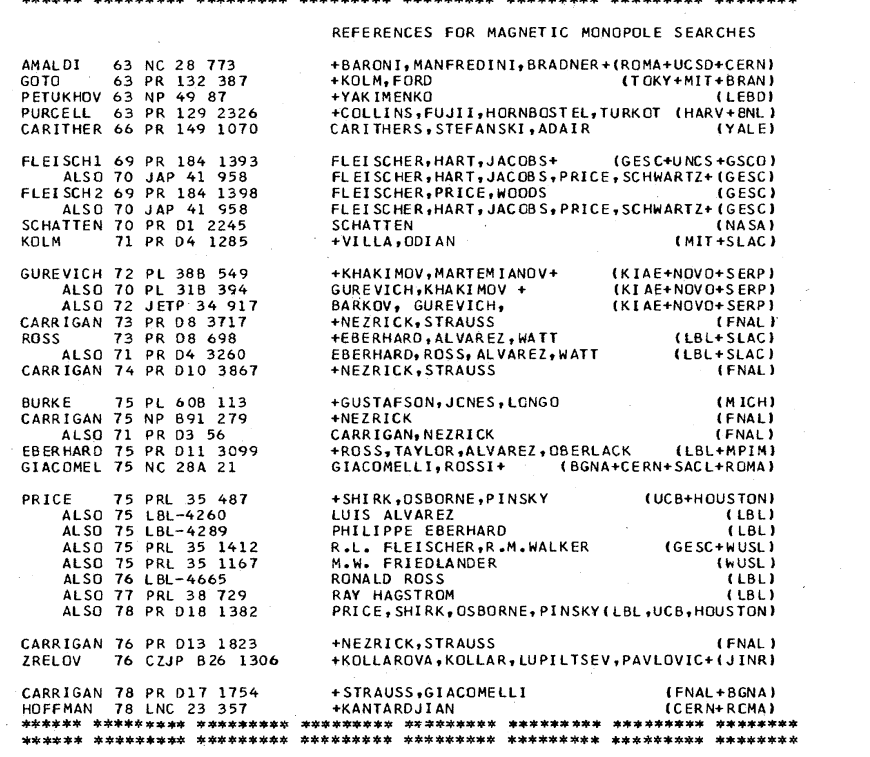

\section{CHARM SEARCHES}

Data on specific charmed states are listed in separate sections in the appropriate places in the Data Card Listings: D, F, and $\Lambda_{C}$ - Stable Particles; $D^{*}, F^{*}$ - Mesons; $\sum_{C}-$ Baryons. 


\section{Stable Particles CHARM SEARCHES}

Evidence for charm not directly relatable to a given state is listed in this section. Neutrinoinduced dilepton events are summarized. Short-lived tracks in emulsions are also dealt with, as are cross-section upper limits for charm searches.

Tri-muon production in neutrino interactions is summarized in the Other New Particle Searches section.

\section{Neutrino-induced Dilepton Events}

Many neutrino experiments have now observed dilepton events. These data are summarized in subsections Y, VO, and VOA. Bubble chamber experiments have observed neutrino-induced $\mu^{-} e^{+}$events associated with strange particle production in the reaction

$$
\nu N \rightarrow \mu^{-} e^{+} K^{0}(o r \Lambda)+\text { anything } .
$$

Dilepton events have no conventional explanation. Production of charmed hadrons, heavy leptons, and intermediate bosons have been proposed as potential explanations. Production of charmed particles (C) in neutrino interactions would be expected to give rise to such events via the mechanism

$$
\begin{aligned}
& \nu_{\mu^{N}} \rightarrow \mu^{-} \mathrm{C}+\text { hadrons } \\
& \longrightarrow \ell^{+} \nu_{\ell}+\text { hadrons, }
\end{aligned}
$$

where the Cabibbo-favored transition would predict a strange particle among the hadrons. Thus the appearance of neutrino-induced dimuon events, $\mu^{-} \mathrm{e}^{+}$ events, and associated strange particles can be understood via the charm mechanism.

\section{Short-Lived Tracks in Emulsions}

The mean life of a weakly decaying charmed meson or baryon of mass $M$ (in GeV) is expected to be in the range ${ }^{1}$

$$
\tau=\left(10^{-11} \text { to } 10^{-13} \mathrm{sec}\right) \times 1 / \mathrm{M}^{5}
$$

with a corresponding mean path length for lab momentum $\mathrm{p}$ (in $\mathrm{GeV} / \mathrm{c}$ ) of

$$
\ell=\frac{\mathrm{pC} \tau}{\mathrm{M}}=(30 \mu \text { to } 3000 \mu) \times \mathrm{p} / \mathrm{M}^{6} \text {. }
$$

Thus even at Fermilab energies, these would be hard to see as tracks in bubble chambers, so emulsion is used. We list data for these experiments in subsections $\mathrm{CC}$ and $\mathrm{EM}$ below.

\section{Data Card Listings For notation, see key at front of Listings.}

\section{Charm Searches}

Experimental evidence for charm production has now been accumulated in various reactions.

Section CP includes several types of evidence for associated charm production in $\mathrm{pN}$ collisions: the prompt $1 \mu$ signal, low mass $\mu^{+} \mu^{-}$pairs with missing energy; opposite-sign Me events; as well as observations of $\mathrm{D}$ and $\Lambda_{\mathrm{C}}$ in the hadronic final

states. Observations of prompt muons in beam dump experiments are listed in section $\mathrm{BD}$.

Sections CG, MU, and D include evidence in photon, muon, and neutrino beam experiments.

Charmed baryon production in $\mathrm{e}^{+} \mathrm{e}^{-}$reactions is listed in section $\mathrm{CE}$; further information can be obtained from the Listings.

\section{References}

1. M.K.Gaillard, B.W.Lee, and J.L.Rosner, Rev. Mod. Phys. 47, 277 (1975).

PROPERTIES OF THE CHARMED D, D** F, F*, LAMBDA/C+, AND SIGMA/C+
STATES ARE LISTED IN SEPARATE SECTIONS. STATES ARE LISTEO IN SEPARATE SECTIONS.
THE FOLLOWING SECTICN CONTAINS INFORMATION ON SEARCHES FOR OTHER CHARMED PARTICLE STATES ANO SEARCHES FOR THE ABOVE STATES IN NEW COLLISION PROCESSES. THESE SEA
LISTED AS CROSS SECTION UPPER LIMITS.

CHARMED BARYON PRODUCTION IN (E+ E-) COLLISIONS (CM**2) 15.6E-35IOR LESS CL $=.90$ PICRGULO 77 SMAG
PICCOLO 77 LOOK AT INCLUSIVE PBAR AND LAMBDA PRDO IN $3.7-7.6$ GEVECM
ETE- AT SLAC. FINDS SHARP RISE IN CS BETW 4.4 AND 5 GEV. EVIDENCE FOR PROD OF CHARMED BARYON IN THAT REGION. FERGUSON 78 FIND INCREASE IN ANTISIGAAA +- PROD BY E+ E- (SLAC) BETWEEN 4 AND 7 GEV (ECM) CONSI STENT WI TH CHARMED BARYON
PRODUCTION. LIMIT IS ON C.S. $*$ BR (AL AMBOAC- $->$ ASIG+- PI-+ PI-1. CHARMED HADRON PRODUCTION CROSS SECTIONS (GAMMA NUCLEON) (CM\#\#2)

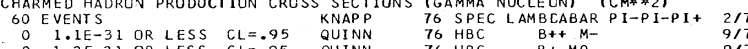

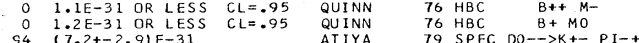
$94(7.2+-2.9) E-31$
1 EVENT NAPP 75 SEES A PEAK AT ME 2.26 ADAMOVICH 90 SPECC DO- DOKARIT IN LAMBDABAR PI+ PI+ PI-. THEY ALSO SEE A LAMBDABAR (4PI) PE PEAK AT

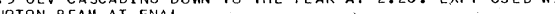
QUINN 76 USED A 9.3 GEV PHOTCN BEAM AT SLAC. SEE TABLES 1 AND 3 FOR
INDIVIOUAL CHANNELS. ABOVE LIMITS ARE FOR ALL CHANNELS WITH ONE OR ATIYA 79 I S FNAL EXPT USING 50-200 GEV PHOTONS. C.S. ASSUMES ERANCHING RATIO OF DO- $>$ K+-P-+ $=018$.
ADAMOVICH 80 SEES THE PRDOUCT ICN AND DECAY OF A DOBAR IN EMULSION
EXPO SED TO SPS GAMMA BEAM IN CCNJUNCTION WITH OMEGA SPECTROMETER.

CHARMED HADRCN EVIDENCE IN MU NUCLEON INTERACTIONS (CM*\#2)

13. E-33) APPROX BUER 79 SEES 449 DIMUONS, 64 TR IMUCNS. CONSI STENT WITH ASSOC IATED $12179 \%$ LISTINGS UNDER S3OMU (CHANG 77 ).

CHARMED HAORON PRODUCTION CROSS SECTION (PI NUCLEON) (CM**2)

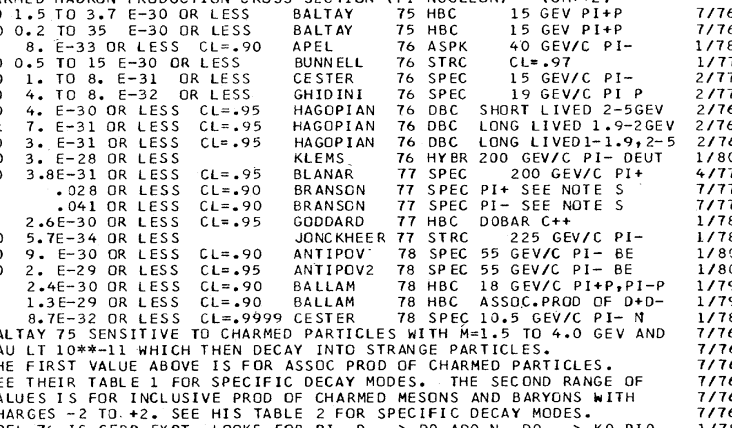




\section{Data Card Listings}

For notation, see key at front of Listings.

\section{Stable Particles CHARM SEARCHES}

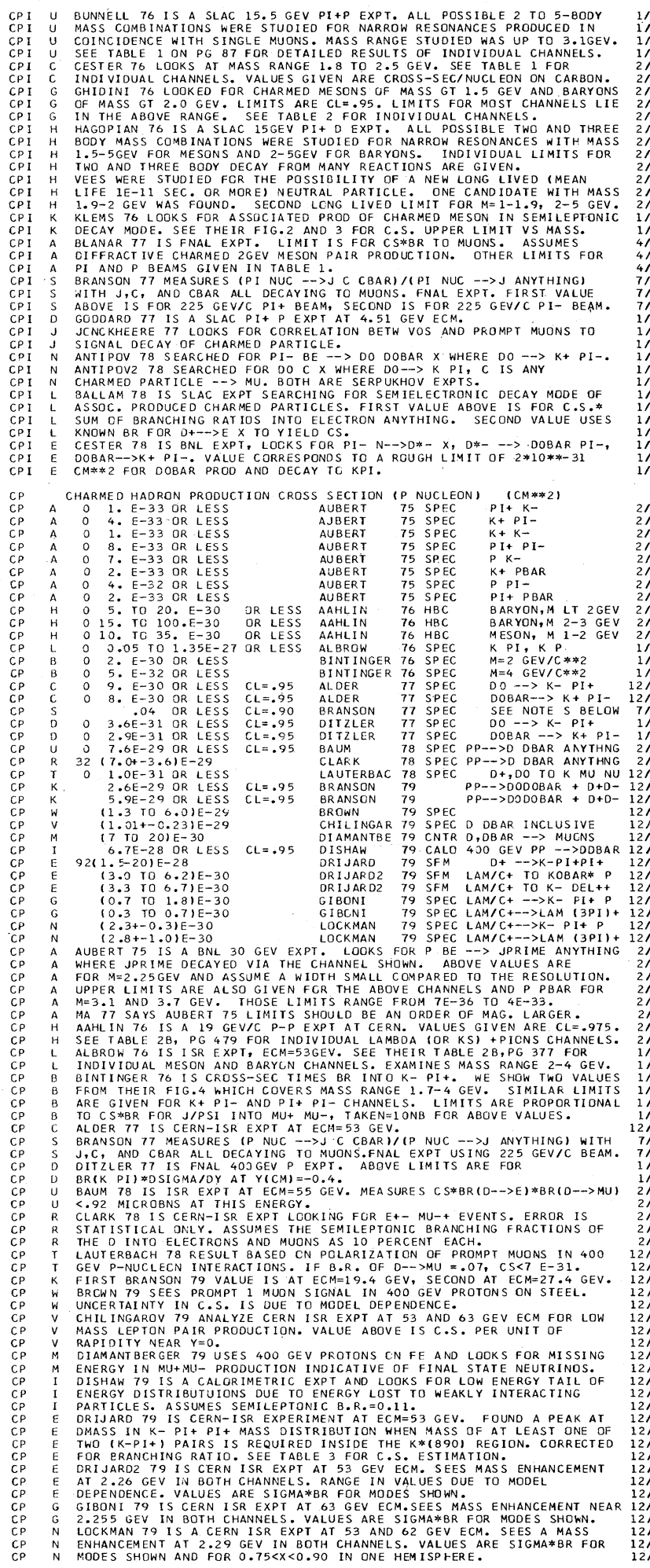

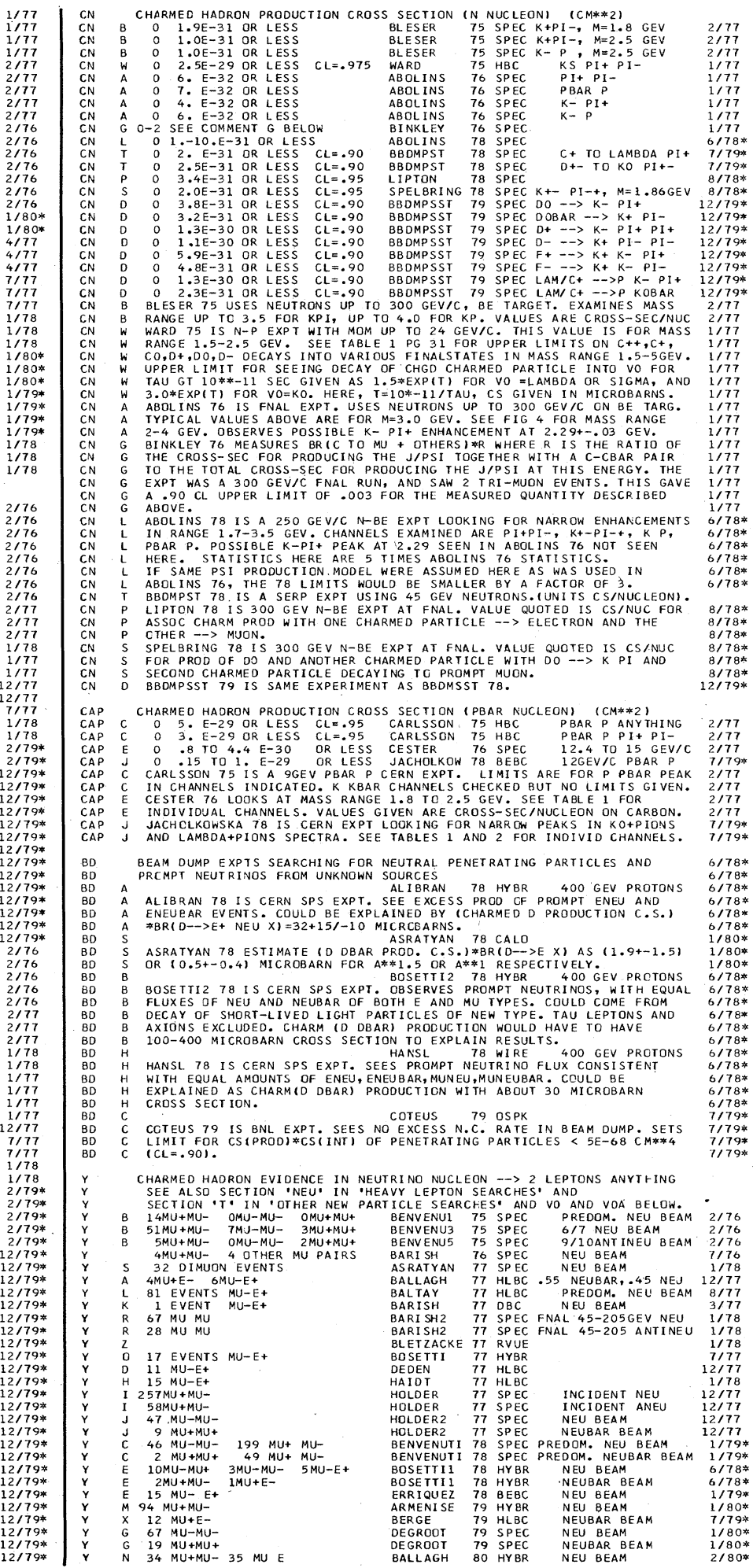




\section{Stable Particles CHARM SEARCHES}

\section{Data Card Listings For notation, see key at front of Listings.}

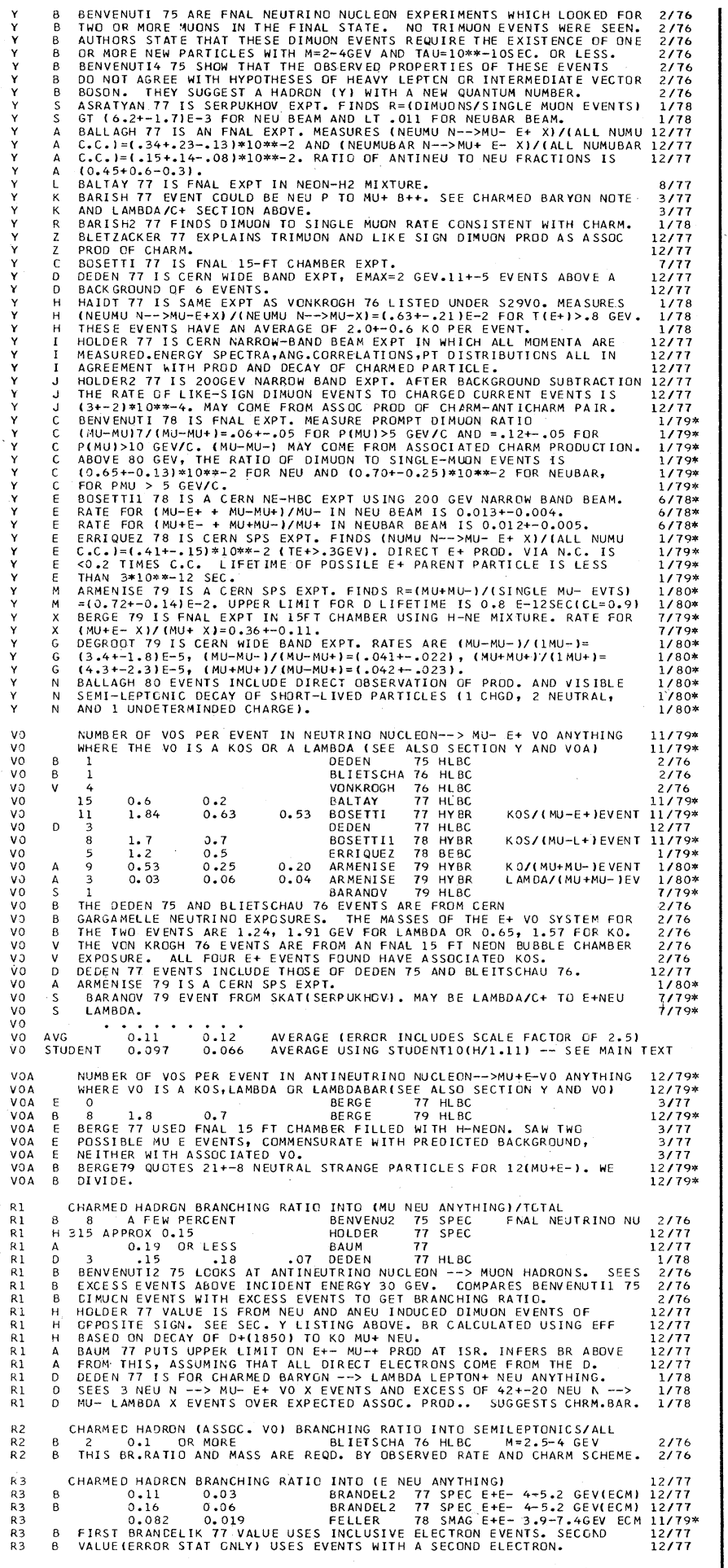

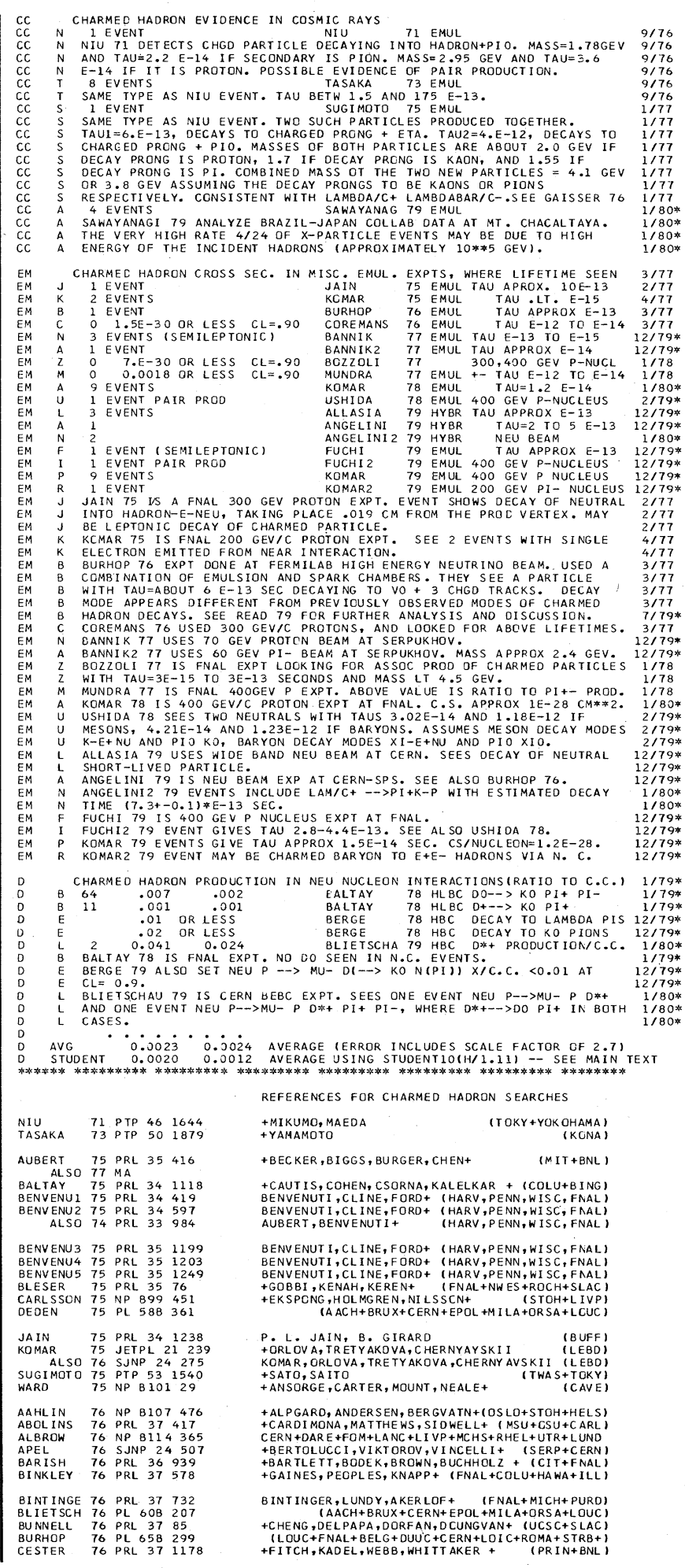




\section{Data Card Listings}

For notation, see key at front of Listings.
Stable Particles OTHER STABLE PARTICLE SEARCHES

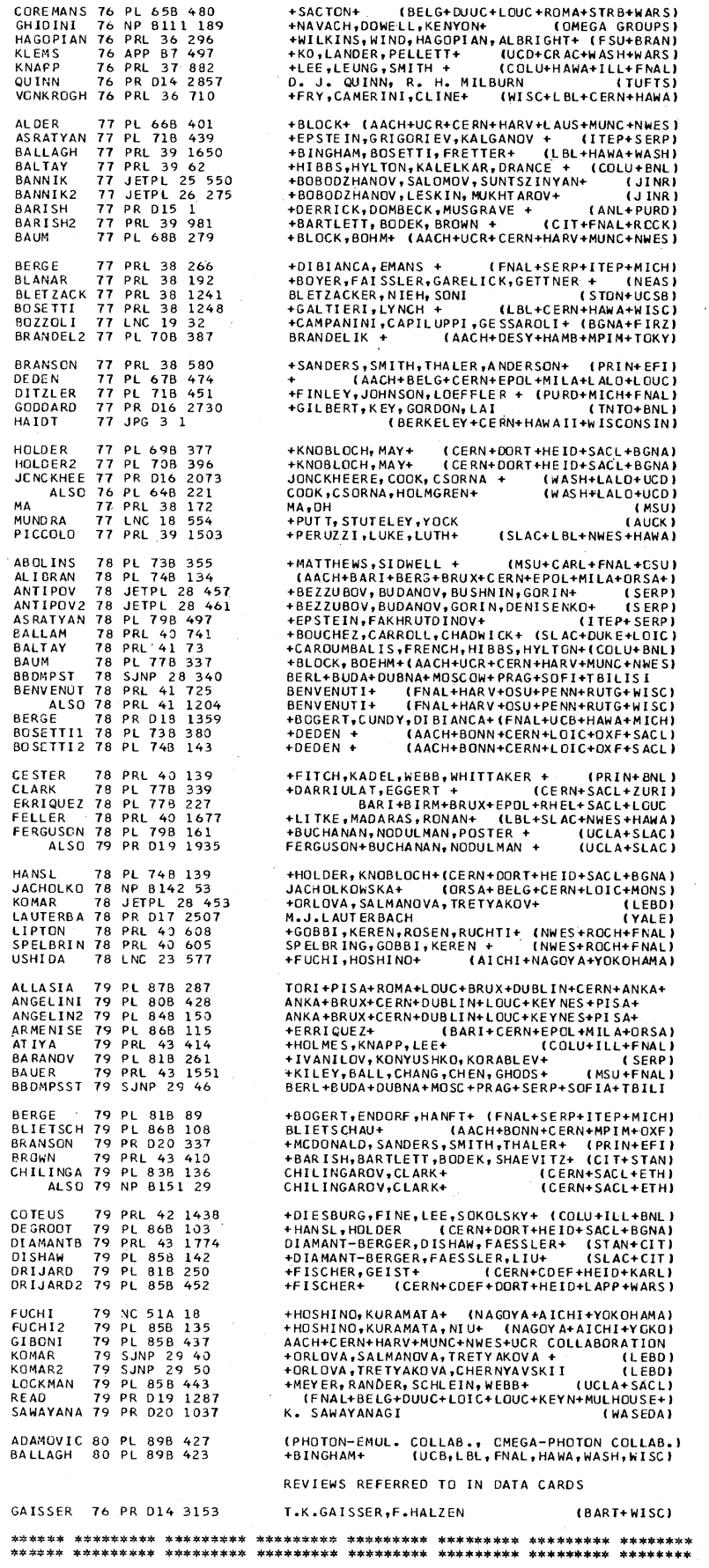

\author{
OTHER STABLE PARTICLE SEARCHES \\ We collect here those searches which do not fit \\ neatly into one of the above search categories. \\ These include axion searches (section $A X$ ), trimuon \\ and four-lepton production in neutrino and anti- \\ neutrino reactions ( $T, F L$ ), and heavy particle \\ searches in accelerator experiments $(\mathrm{CH}, \mathrm{CS}, \mathrm{D}, \mathrm{ICH})$ \\ and in cosmic rays ( $F$ ).
}

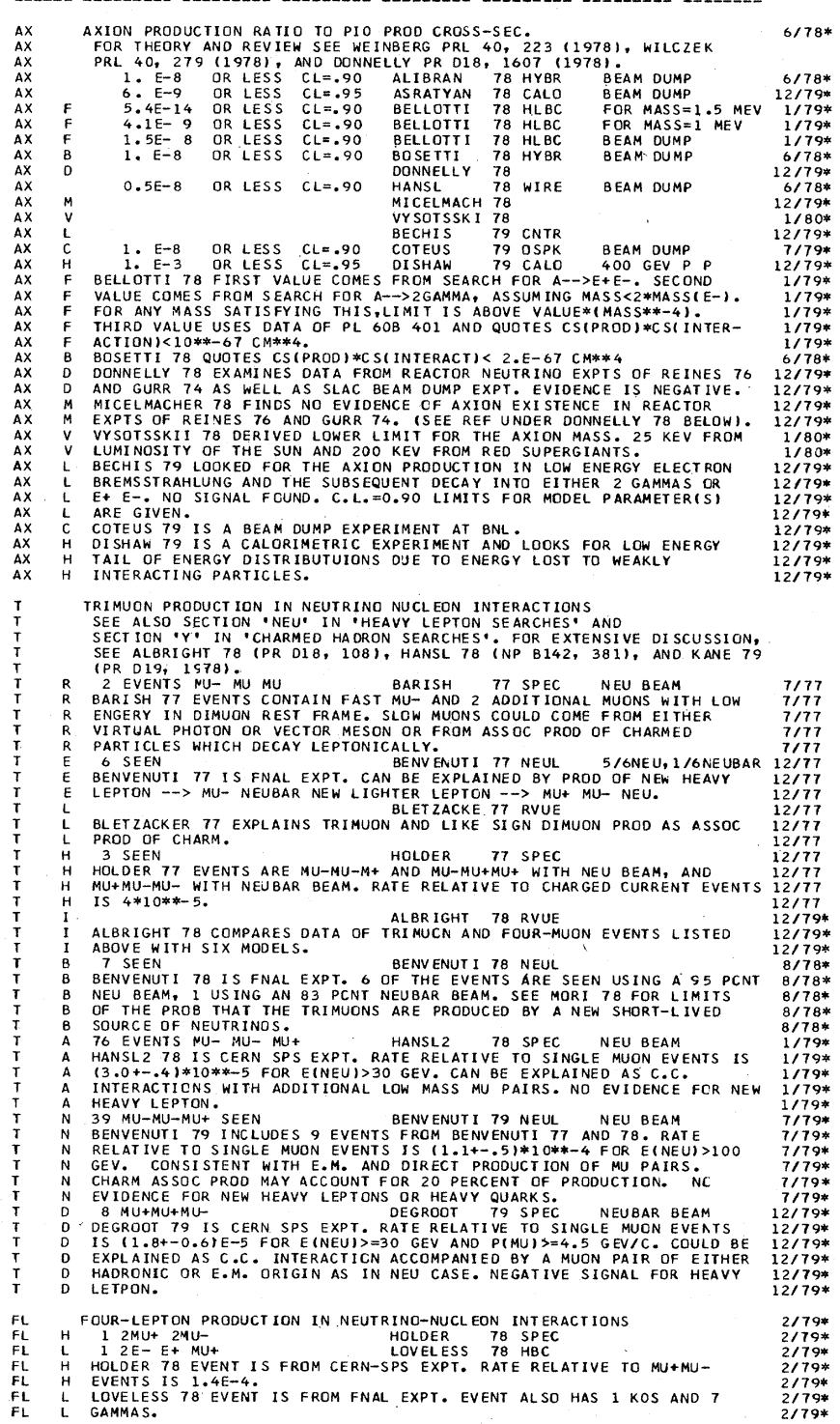




\section{Stable Particles OTHER STABLE PARTICLE SEARCHES}

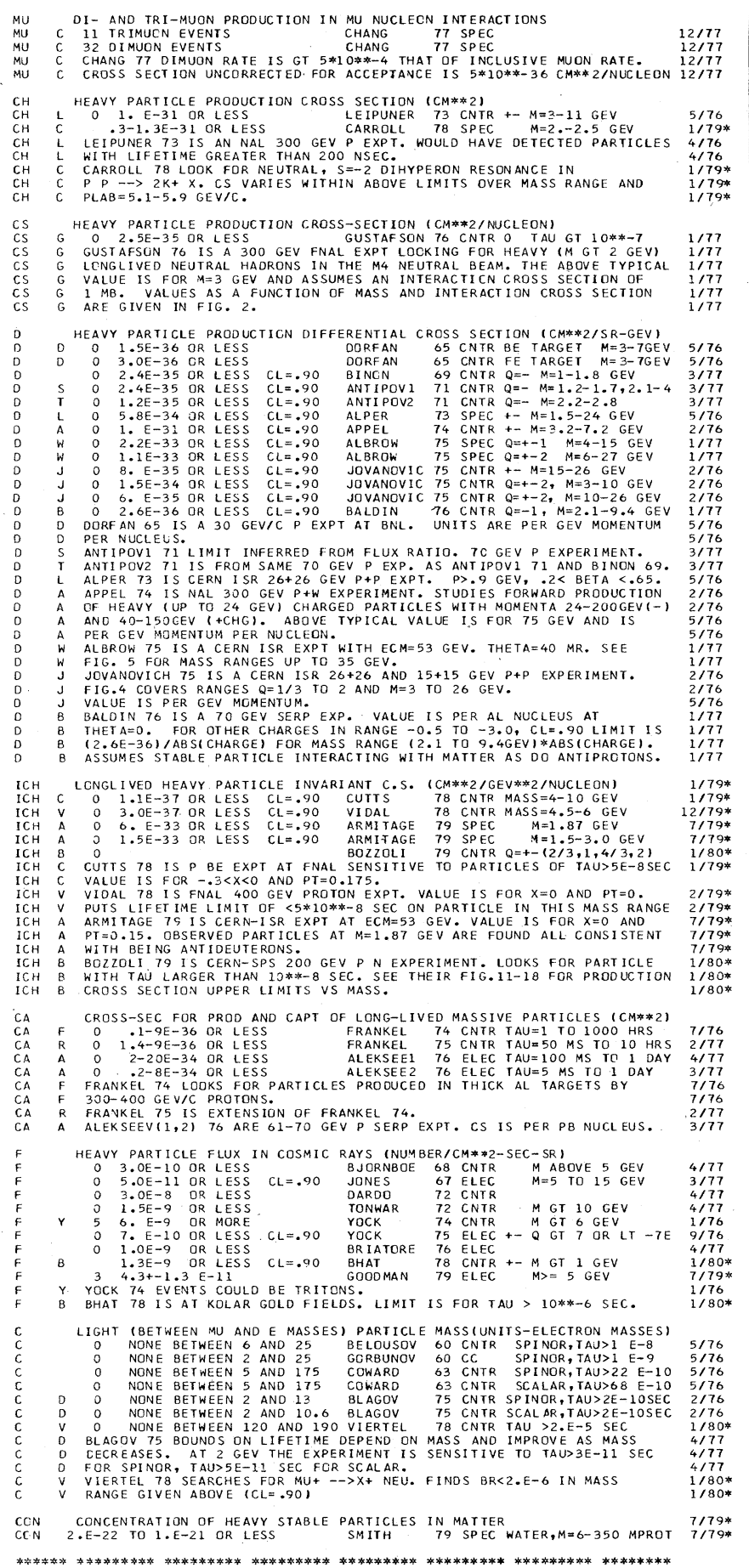

\section{Data Card Listings For notation, see key at front of Listings.}




\section{Data Card Listings}

For notation, see key at front of Listings.

\section{Mesons $\pi^{ \pm}, \pi^{0}, \eta, \rho(770)$}

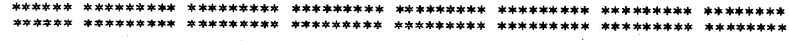
$\mathrm{S}=0, \mathrm{C}=0$ MESON STATES

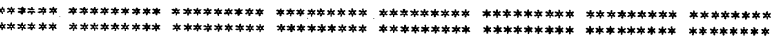

$\pi^{ \pm} \quad 8$ ChARGED PION(140,JPG $\left.=0--\right) \quad I=1$ SEe STABle PARTICLE DATA CARD LISTINGS

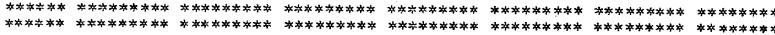

$\pi^{0} \quad 9$ NeUtral. PICN(135, JPG $\left.=0--\right)$ I $=1$

SEE STABLE PARTICLE DATA CARD LISTINGS

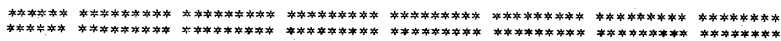

$\eta \quad 14$ ETAL $549, J P G=0-+)$ I=0

See stable particle data card listings

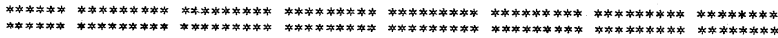

$\rho(770) \quad$ 9 RHO(770,JPG $=1-+1 \mathrm{I}=1$

Note on the $\rho^{0}$ Mass and Width

Because of the broadness of the $\rho$ meson, determinations of the resonance parameters are beset with many difficulties. In physical-region fits, it is well known that the $\rho$ line shape does not correspond to a relativistic Breit-Wigner function with a $\mathrm{P}$-wave width, but requires one further shape parameter (PISUT 68). The same remark applies to the energy dependence of the phase shift $\delta_{l}^{l}$. Different ways of introducing the shape parameter lead to systematic differences in addition to the systematic errors due to different ways of accounting for the background in physicalregion fits, or due to different ways of projecting out the partial waves in phase-shift analyses.

We consider phase-shift analyses more reliable than physical-region fits.

All phase-shift analyses can now be summarized by two pairs of parameters which agree: in an analysis of the BATON 70, HYAMS 73, and PROTOPOPESCU 73 phase shifts, ROOS 75 obtains $M\left(\rho^{0}\right)=(776.3 \pm 0.4)$ $\mathrm{MeV}, \Gamma\left(\rho^{0}\right)=(154.5 \pm 1.0) \mathrm{MeV}$; combining the HYAMS 73 data with more recent data on polarized protons, BECKER2 79 obtains $M\left(\rho^{0}\right)=(776.1 \pm 2.6) \mathrm{MeV}$, $\Gamma\left(\rho^{0}\right)=(161.8+7.6) \mathrm{MeV}$. We base our "educated guess" on these values.

9 RHO MASS (MEVI

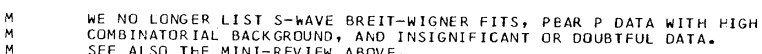

$M$ MIXED CHARGES

$240(752.0)$

$\begin{array}{llllll}\text { ALIITI } & 63 & \text { HBC } & -0 & 1.6 & \text { PI-P } \\ \text { CHADWICK } & 63 & \text { HBC } & \text { t-0 } & 0.0 & \text { PBAR }\end{array}$
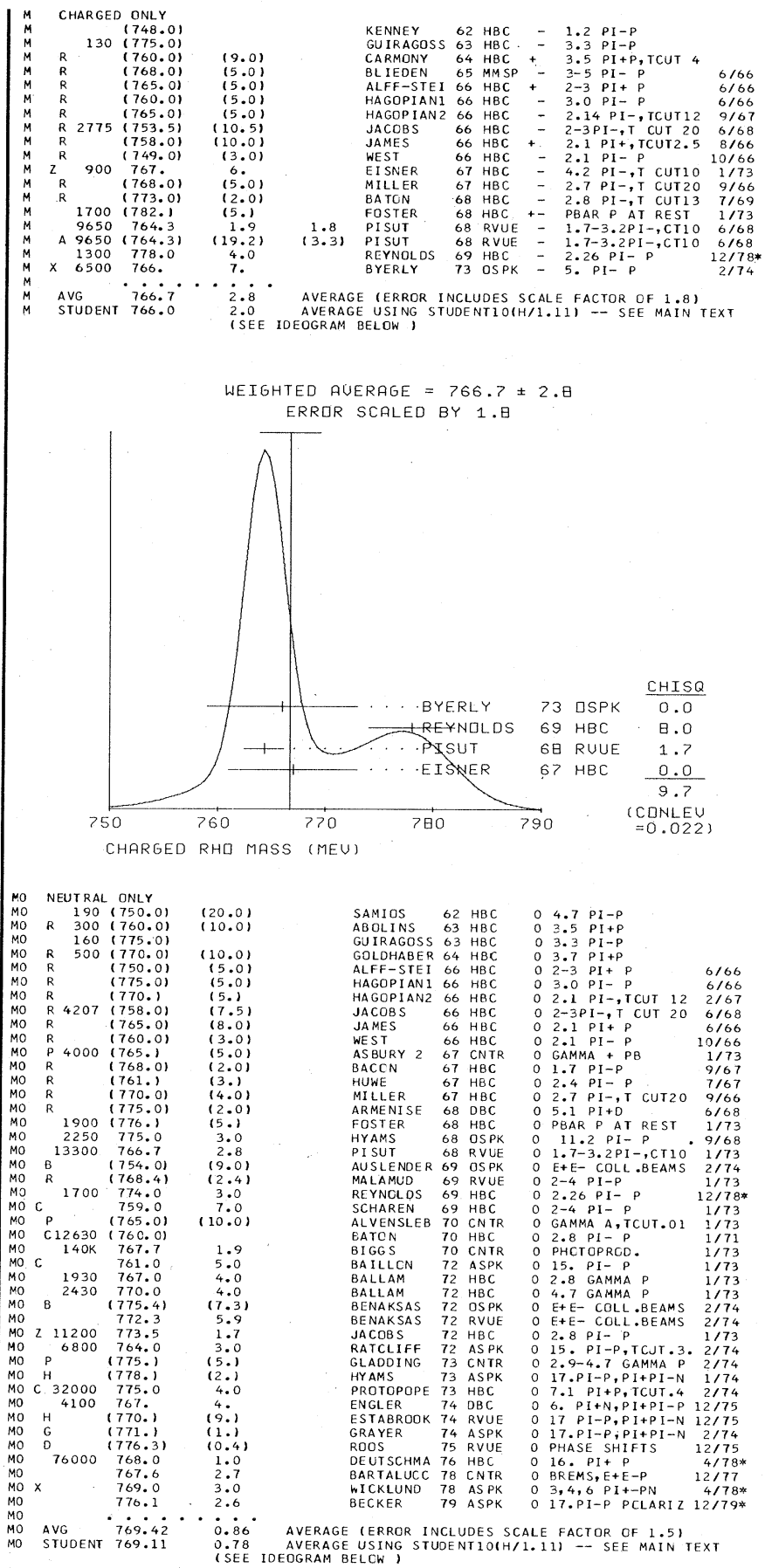

ERRORS ARE 2 STD AND INCLUDE SYSTEMATIC UNCERTAINTIES FROM THEORY

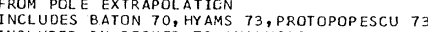
INCLUDED IN BECKER 79 ANALYSIS
FROM PHASE SHIFT ANALYSIS OF GRAYER 74 DATA. FROM PHASE SHIFT ANALYSIS OF GRAYER 74 DAT
FROM PHOTOPROUCTICN, MODEL DEPENDENT.

INCLUDED IN PISUT 68 RVUE

SYSTEMATIC ERRORS ADDED CORRESP. TO SPREAD OF DIFFERENT FIISS.
MASS ERRORS ENLARGED BY US TO WIDTH/SQRT (N), SEE K* TYPED NOTE 


\section{Mesons} $\rho(770)$

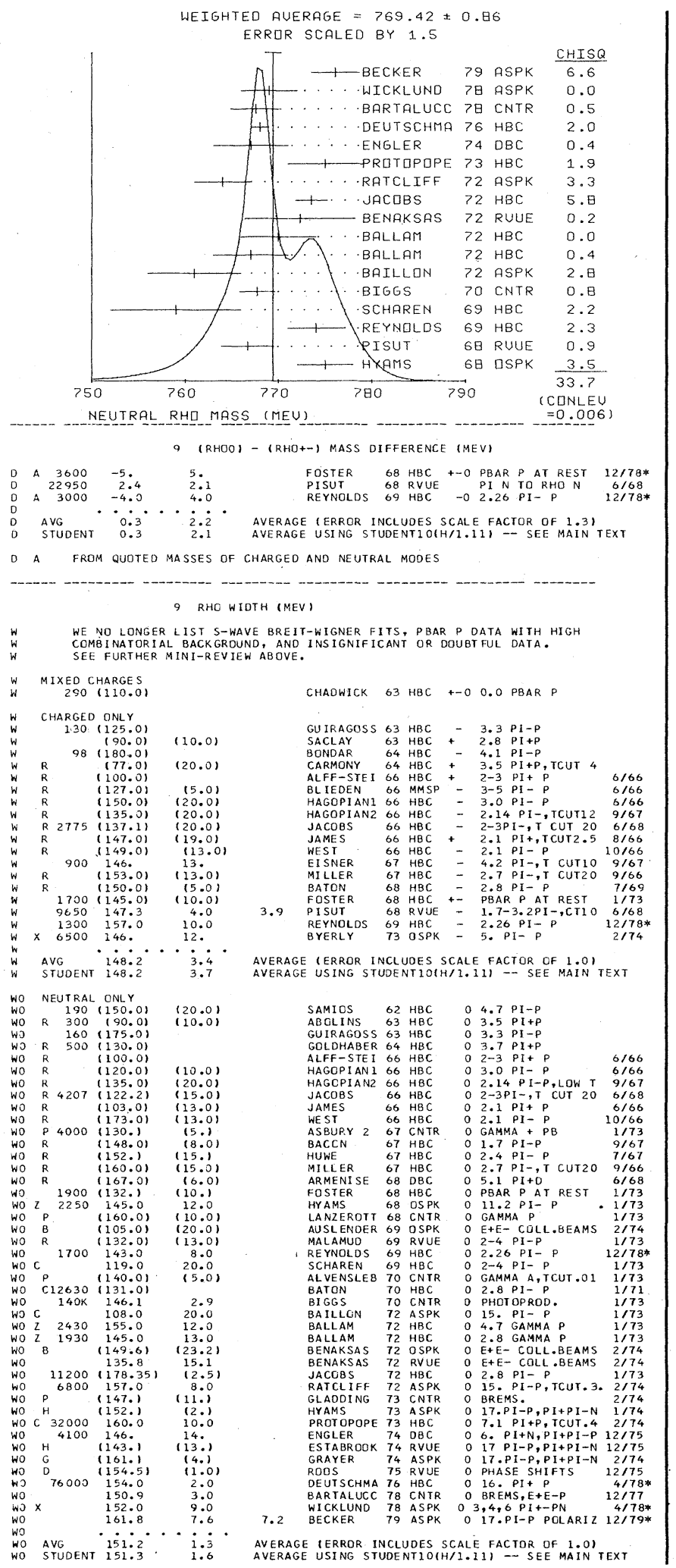

\section{Data Card Listings For notation, see key at front of Listings.}

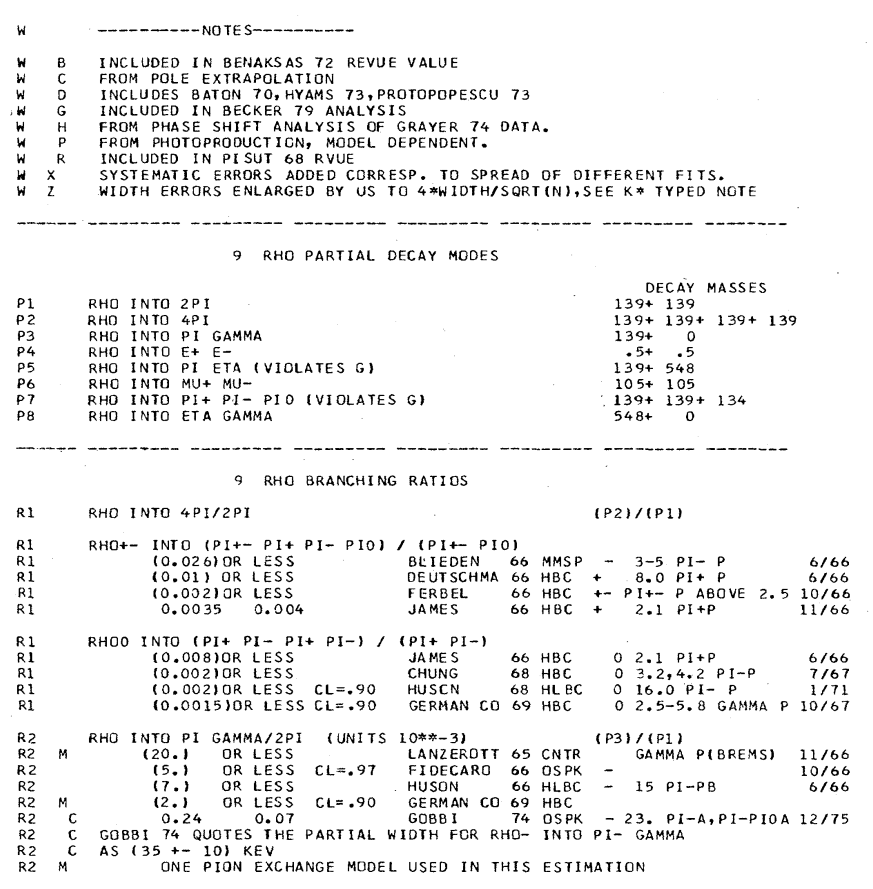

Note on the $e^{+} e^{-}$and $\mu^{+} \mu^{-}$Decays

Extraction of a ratio for $\rho^{0} \rightarrow e^{+} e^{-}$is complicated by interference with $\omega$ decay. In photoproduction, $\gamma_{A} \rightarrow e^{+} e^{-} A$, there is substantial interference between the allowed $\left(\rho^{0}, \omega\right) \rightarrow e^{+} e^{-}$decays. The interference in the colliding-beam reaction $e^{+} e^{-} \rightarrow$ $\pi^{+} \pi^{-}$is due to G-parity-violating mixing of the overlapping $\rho^{0}$ and $\omega$ resonances; it alters the results for the rate $\Gamma\left(\rho^{0} \rightarrow e^{+} e^{-}\right)$only by a small amount. Therefore at present we average only the values from the $\mathrm{e}^{+} \mathrm{e}^{-} \rightarrow \pi^{+} \pi^{-}$experiments.

The same comment applies to the decay $\rho^{0} \rightarrow \mu^{+} \mu^{-}$.

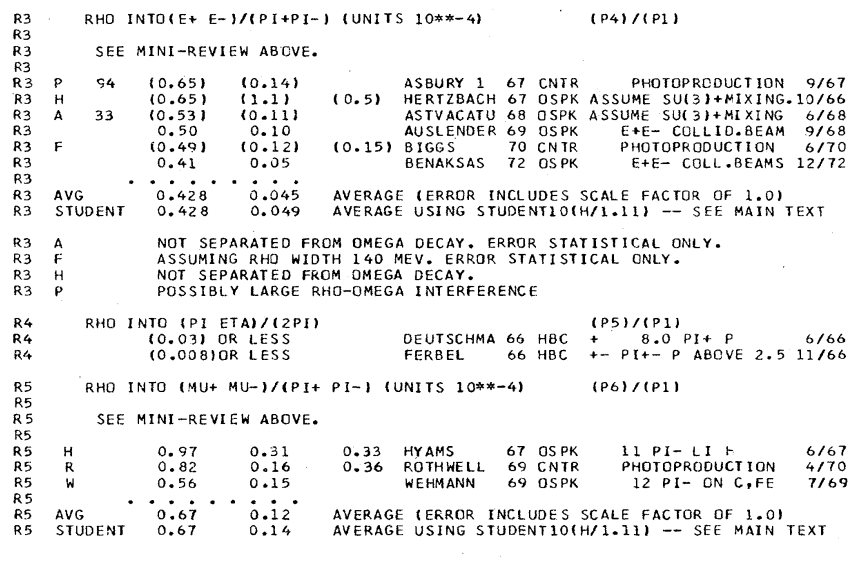




\section{Data Card Listings \\ For notation, see key at front of Listings.}

Mesons

$\rho(770), \omega(783)$

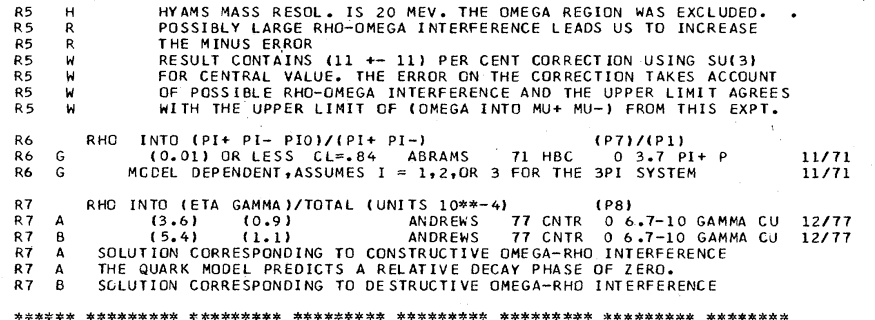

REFERENCES FOR RHO
REF

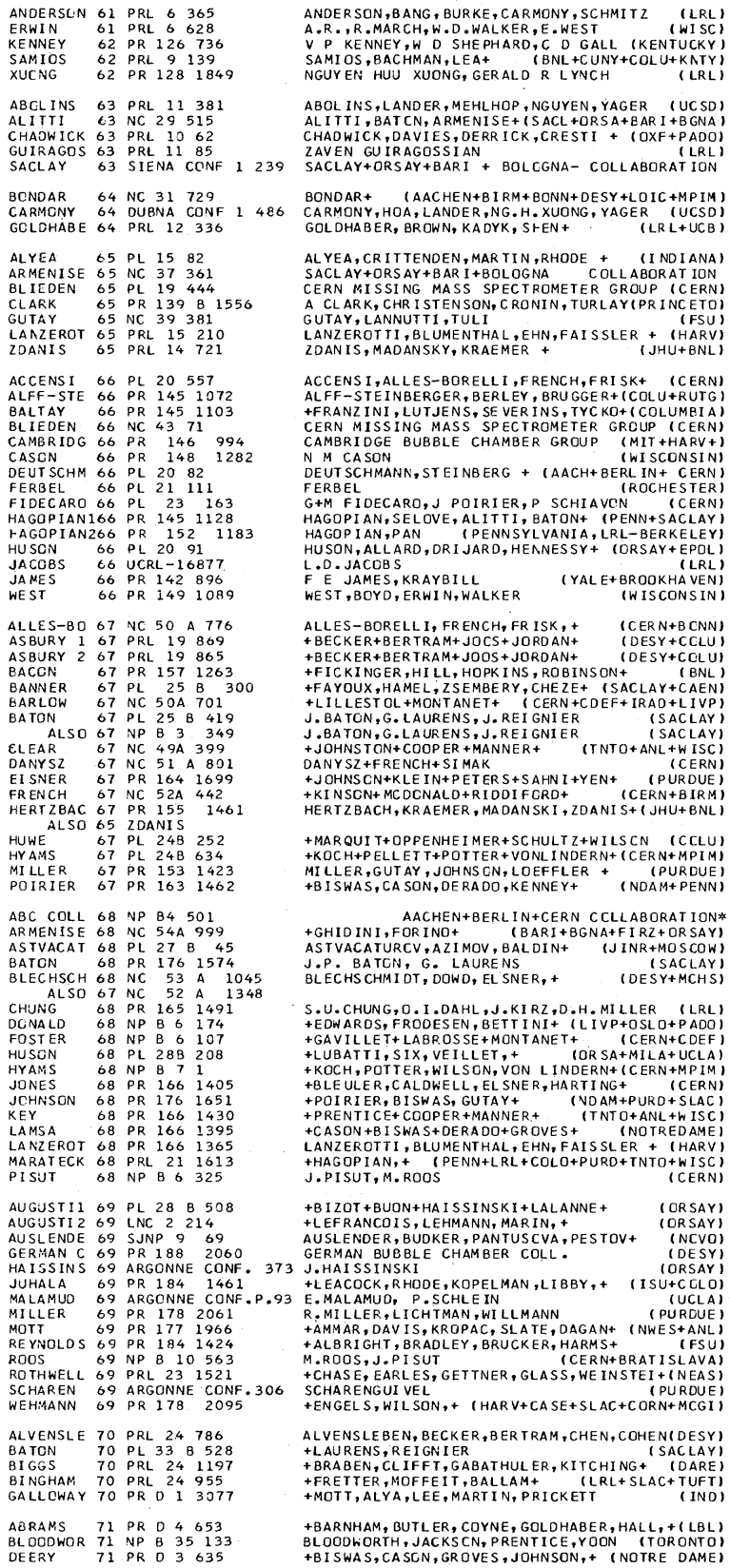

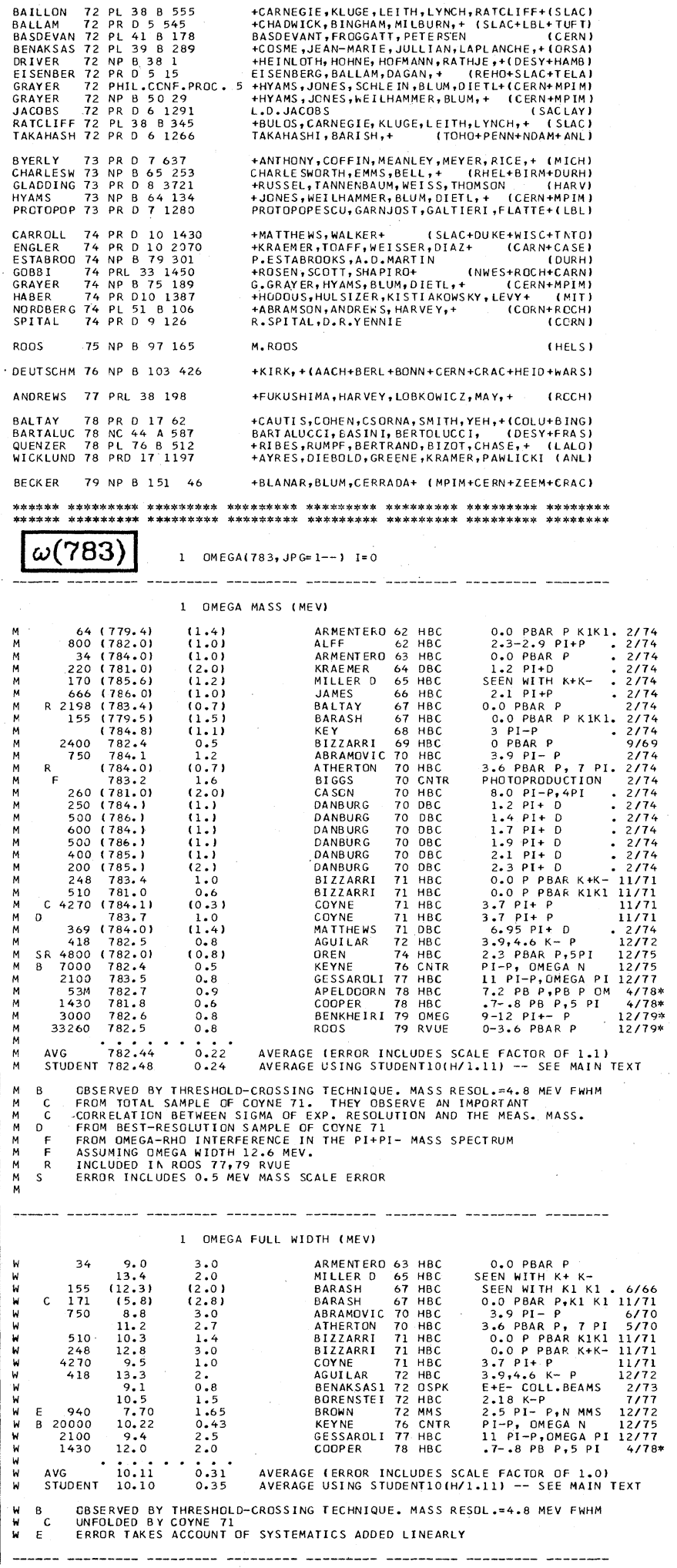




\section{Mesons $\omega(783)$}

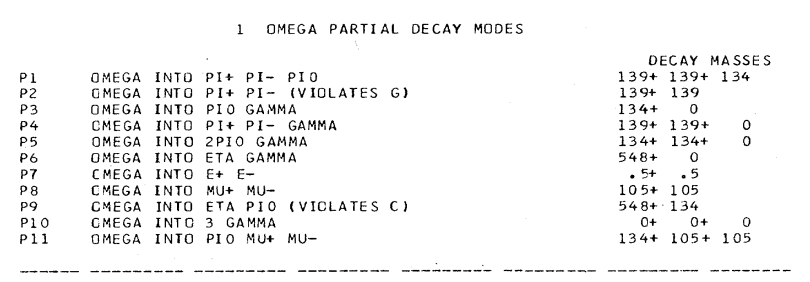

\section{FITTED PARTIAL DECAY MODE BRANCHING FRACTIONS}

The matrix below is derived from the error matrix for the fitted partial decay mode branching fractions, $P_{i}$, as follows: The diagonal elements are $P_{i} \pm \delta P_{i}$, where

$\delta \mathrm{P}_{\mathrm{i}}=\sqrt{\left\langle\delta \mathrm{P}_{\mathrm{i}} \delta \mathrm{P}_{\mathrm{i}}\right\rangle}$, while the off-diagonal elements are the normalized correlation coefficients $\left\langle\delta \mathrm{P}_{i} \delta \mathrm{P}_{j}\right\rangle /\left(\delta \mathrm{P}_{i} \cdot \delta \mathrm{P}_{j}\right)$. For the definitions of the individual $\mathrm{P}_{i}$, see the listings above; only those $P_{i}$ appearing in the matrix are assumed in the fit to be nonzero and are thus constrained to add to 1 :

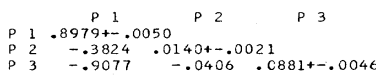

\section{OMEGA BRANCHING RATIOS}

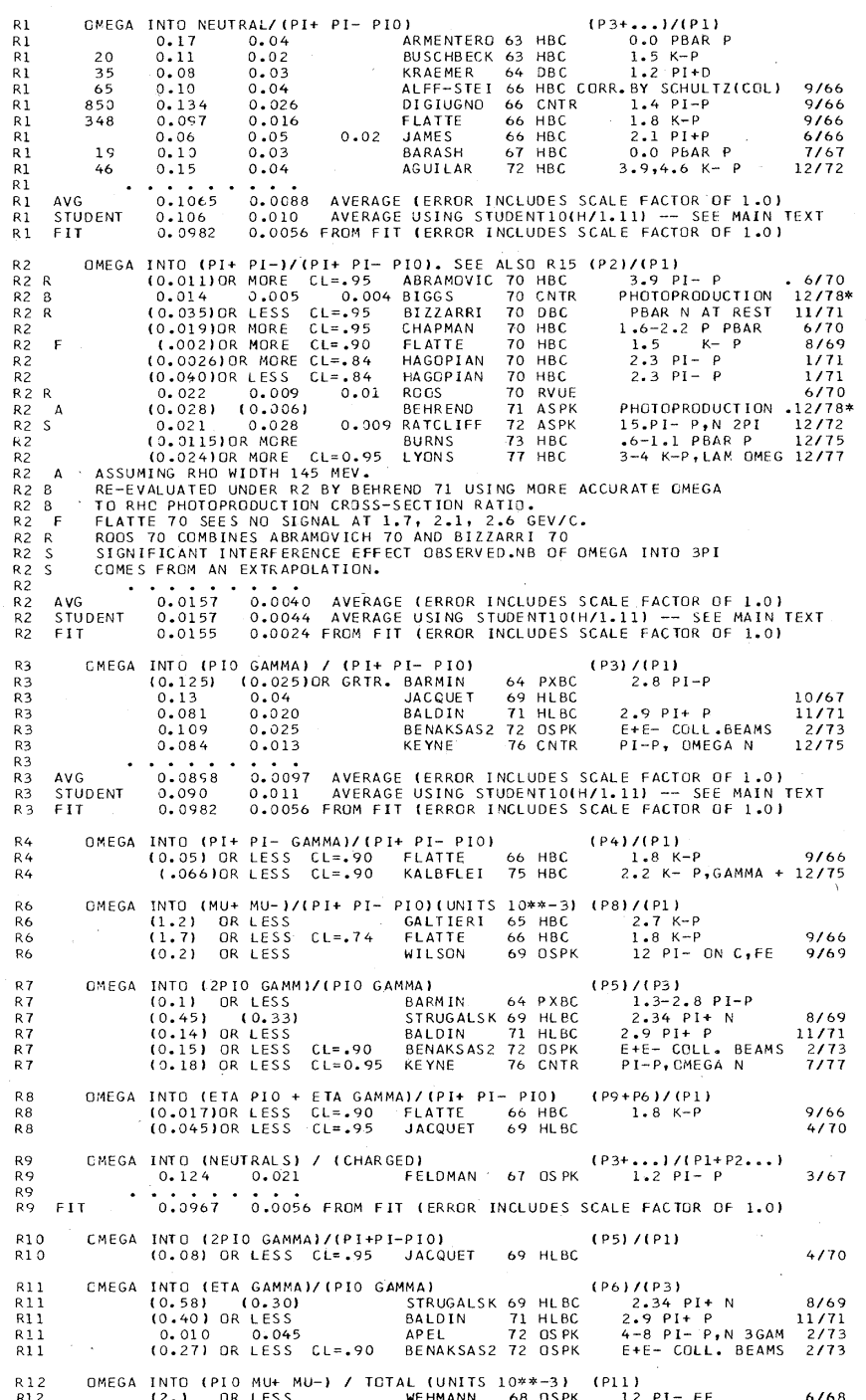

\section{Data Card Listings} For notation, see key at front of Listings.

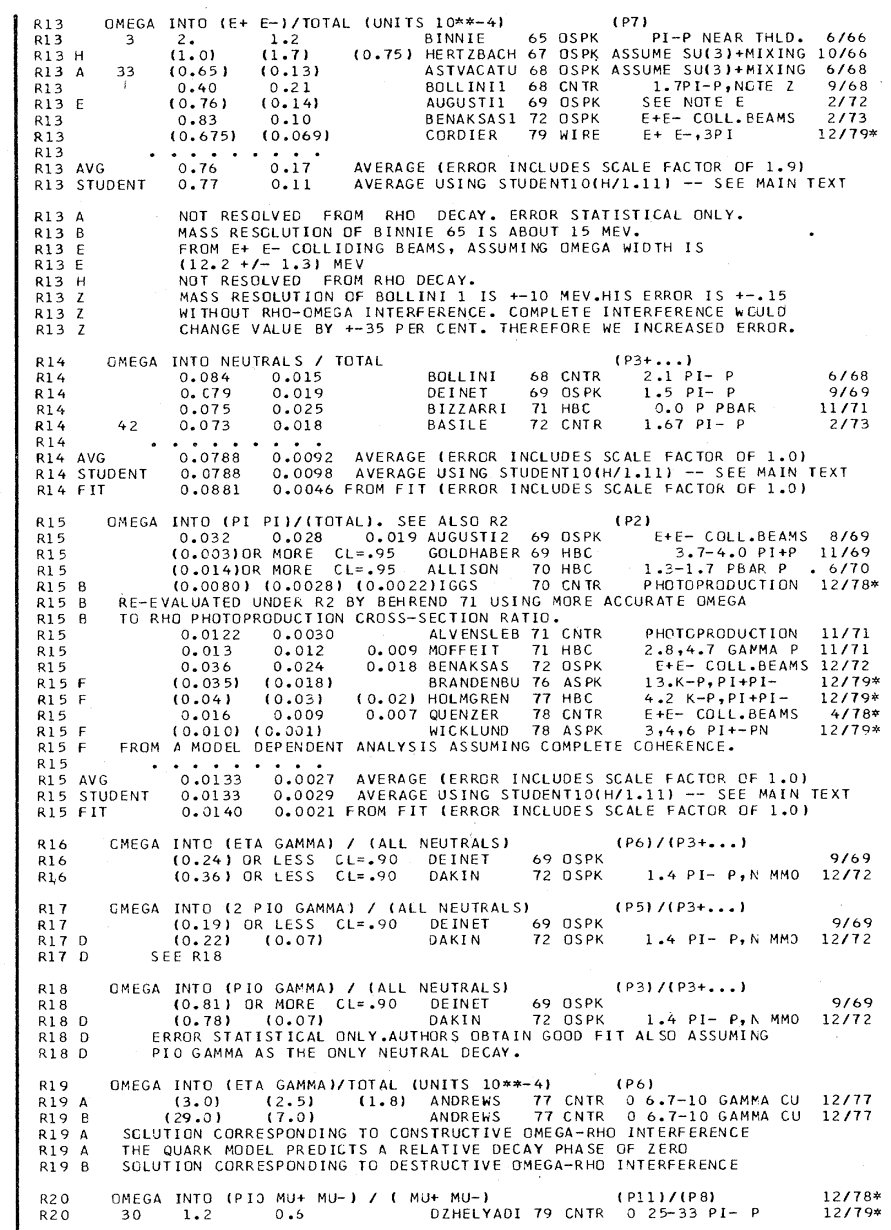

Macich

MAGLIIC
PeESER

ALEF 62 PRL 9325

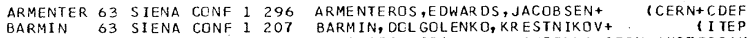

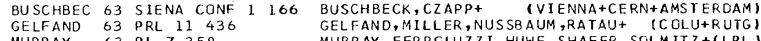

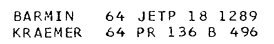

BARMIN, DOL GOLENKO, KRESTNIKOV + (ITEP

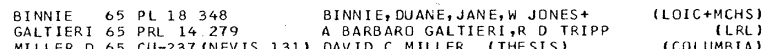

INCLUES DATA OF GELAANO 63 ABODE

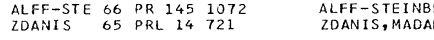

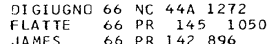

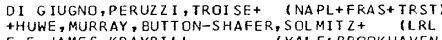

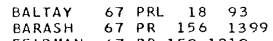

F E JAMES, RRAYGILL TYLL GR BROKKAVEN

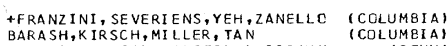

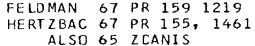

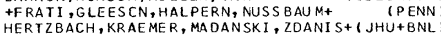

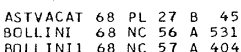

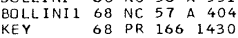

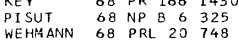

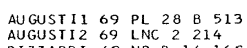

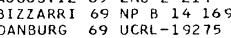




\section{Data Card Listings}

For notation, see key at front of Listings.

Mesons

$\omega(783), \mathrm{M}(940-953), \eta^{\prime}(958)$

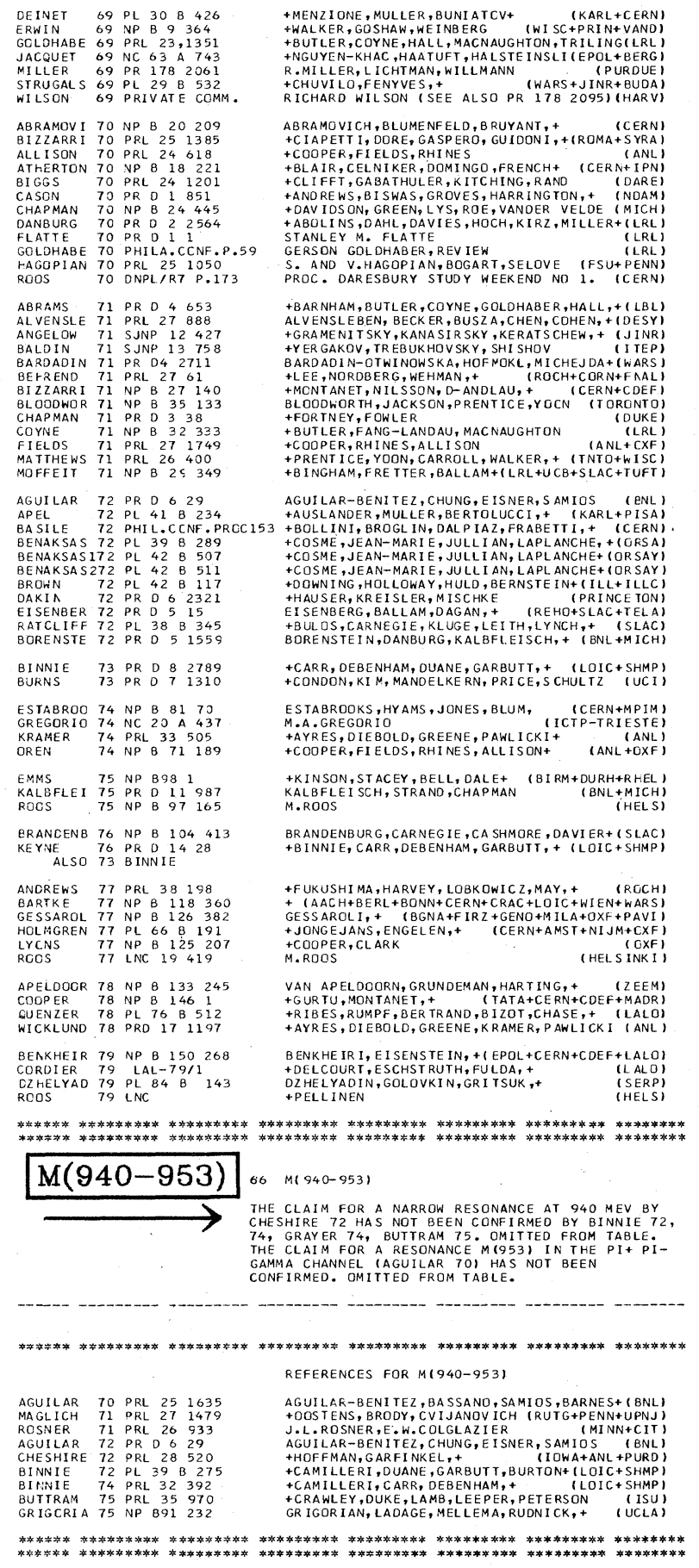

$\eta^{\prime}(958)$ 2 ETA PRIME $1958, \mathrm{JPG}=0-+1$ I $=0$
Note on the $\mathrm{J}^{\mathrm{P}}$ Assignment of $\eta^{\prime}(958)$

From the Dalitz plot analyses of the $n^{\prime} \rightarrow \pi \pi n$ and $n^{\prime} \rightarrow \pi^{+} \pi^{-} \gamma$ decays and from the observation of an $\eta^{\prime} \rightarrow \gamma \gamma$ decay mode, all assignments except $\mathrm{J}^{\mathrm{PC}}=0^{-+}$and $2^{-+}$are excluded. The Dalitz plot analyses favor spin 0 , but cannot rule out spin 2 . The indication of anisotropy in the decay of very forward-produced $\eta^{\prime}$ (KALBFLEISCH 73) has not been confirmed by BALTAY 74, thus again favoring spin 0 , but still not ruling out spin 2 (LEDNICKY 77).

Two recent analyses, however, seem to have finally established the spin 0 assignment of the $\eta^{\prime}$. CERRADA 77 perform a partial-wave analysis of the $n \pi \pi$ system produced in the reaction $K_{p}^{-} \rightarrow n^{\prime} \Lambda$, taking into account the $\eta^{\prime}$ and $\Lambda$ joint decay angular correlations. They conclude that $\mathrm{J}^{\mathrm{P}}$ is unambiguously $0^{-}$. (see also DELAGUILA 77).

ROUSSARIE 77 analyze a large sample of events from the reaction $\pi^{-} p \rightarrow n^{\prime} n$ at beam momenta just above threshold. They verify that the $\eta^{\prime}$ is produced in a relative S-wave state, and thus the Adair condition is satisfied by their total sample of some 1800 events. The decay angular distribution of the $\eta^{\prime}$ is consistent with isotropy, and thus ROUSSARIE 77 conclude that the spin cannot be 2 .

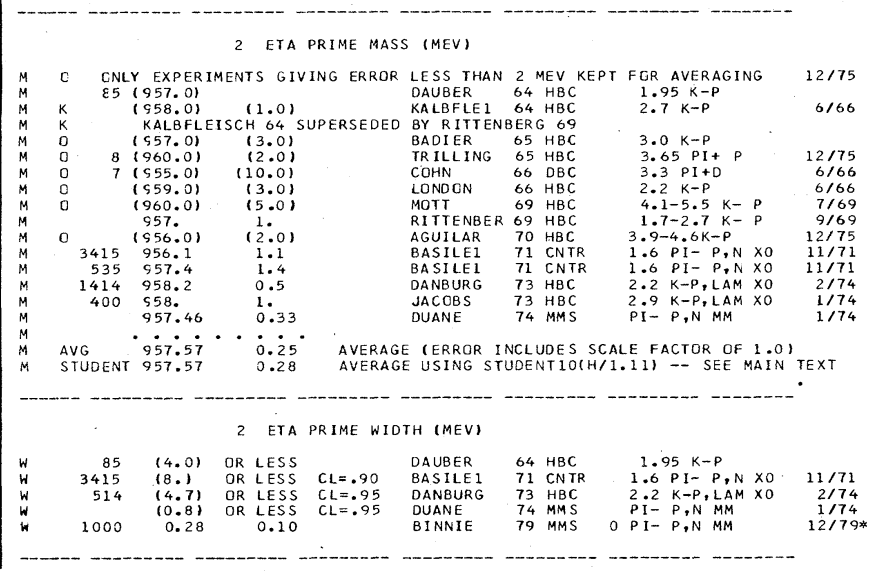




\section{Mesons} $\eta^{\prime}(958)$

\section{Data Card Listings For notation, see key at front of Listings.}

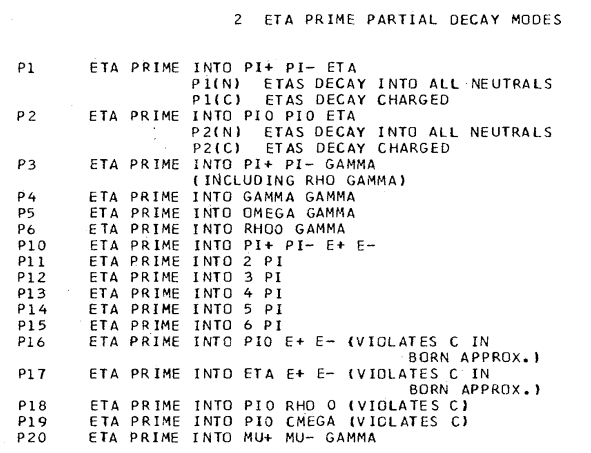

DECAY MASSES
$139+139+548$

$134+134+548$

$139+139+0$

$0+$
$0+78$
$0+7$

$139+176$
$139+139+$
$139+$

$\begin{array}{lll}139+139 & 134 \\ 139+ & 139+134 \\ 139+ & 139+139+139\end{array}$

$134+\quad .5+\quad .5$

$548+\quad .5+\quad .5$

$134+776$
$134+782$

$134+782$
$105+105+0$

\section{FITTED PARTIAL DECAY MODE BRANCHING FRACTIONS \\ The matrix below is derived from the error matrix for the fitted partial decay mode branching fractions, $P_{i}$, as follows: The diagonal elements are $P_{i} \pm \delta P_{i}$, where \\ $\delta P_{i}=\sqrt{\left\langle\delta P_{i} \delta P_{i}\right\rangle}$, while the off-diagonal elements are the normalized correlation coeffi- cients $\left\langle\delta P_{i} \delta P_{j}\right\rangle /\left(\delta P_{i} \cdot \delta P_{j}\right)$. For the definitions of the individual $P_{i}$, see the listings above; only those $P_{i}$ appearing in the matrix are assumed in the fit to be nonzero and are thus constrained to add to 1 .

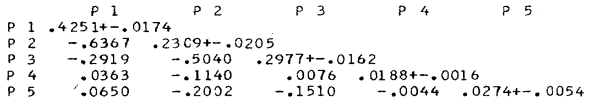

\section{Note on $\eta^{\prime}(958)$ Branching Fractions}

In our calculation of the branching fractions of the $\eta^{\prime}(958)$, we use the decay modes $\eta \pi \pi$ (including $\eta \pi^{0} \pi^{0}$ ), $\rho^{0} \gamma, \omega \gamma$, and $\gamma \gamma$. It is assumed that the rate $n \rightarrow$ neutrals is $71.0 \%$.

In the fit we do not use the constraint

$$
R=\frac{\Gamma\left(n^{\prime} \rightarrow n \pi^{+} \pi^{-}\right)}{\Gamma\left(n^{\prime} \rightarrow n \pi^{0} \pi^{0}\right)}=2
$$

from I-spin conservation. The result of the fit is in agreement with it: $R=1.8 \pm 0.2$.

\section{ETA PRIME PARTIAL WIDTHS (KEV)} W1 ETA PRIME INTU (GAMMA GAMMA) ABRAMS 79 SMAG (G1)
W1 C 23 (5.8) $(2.3)$ HAS BEEN ADOED LINEARLY.

2 ETA PRIME BRANCHING RATIOS

SEE MINI-REVIEW ABOVE.

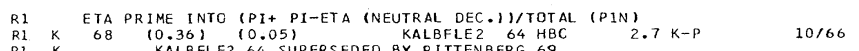

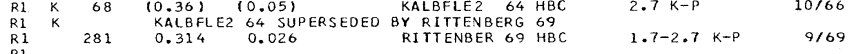

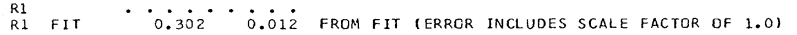

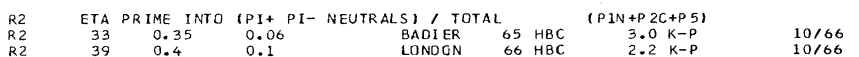

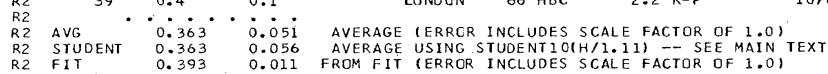

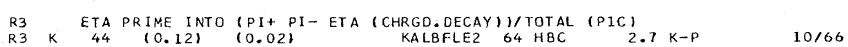

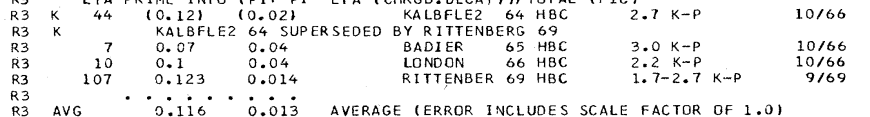

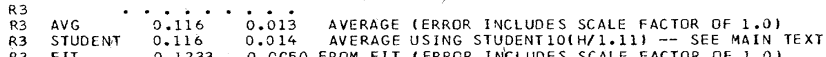




\section{Data Card Listings}

\section{Mesons \\ $\eta^{\prime}(958), \delta(980)$}

For notation, see key at front of Listings.

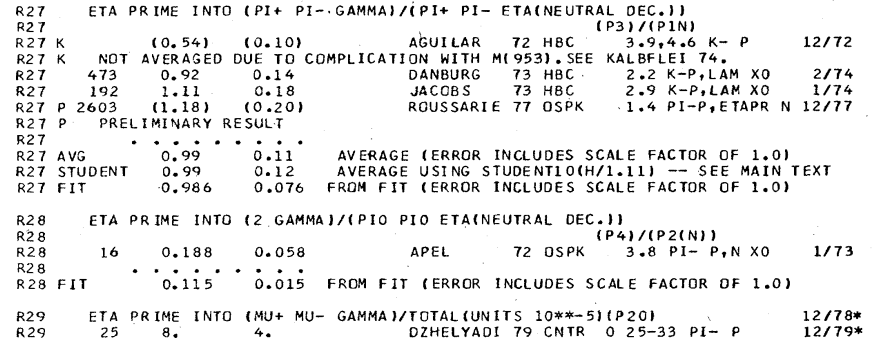

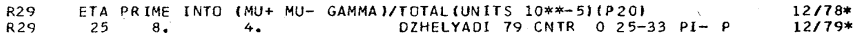

2. ETA PRIME C-NONCCNSERVING DECAY PARAMETER

RELATEO TEXT SECTICN VI C

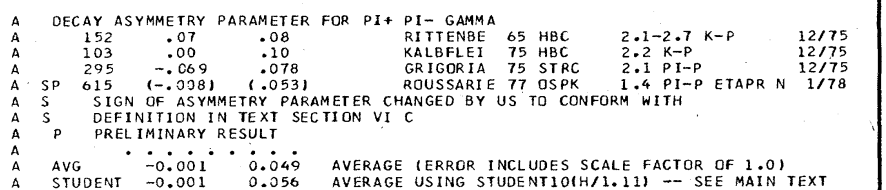

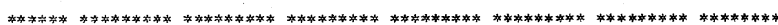

REFERENCES FOR ETA PRIME

$\begin{array}{llllll}\text { DAUBER } & 64 & \text { PRL } 13 & 449 & \\ \text { ALSO } & 64 & \text { DUBNA CONF } 1418\end{array}$

DAUBER, SLATER, SMITH, STORK, TICHO (UCLA)JP

DAUBER, SLA TER, L T SMI TH, STORK, TICHO (UCLA)
+GUNDZ IK, LICHTMAN, CONOLLY, HART, + (SYRA+BNL) +GUNOZIK, LEI TNER, CONNOLLY, HART, + ISYRA+BNL)
KALBFLEISCH, ALVAREZ, BARBARO-GALTIERI, + ILRLIJP

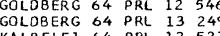

$\begin{array}{lllll}\text { KALBFFLE } & 64 & \text { PRL } & 12 & 52 \\ \text { KALBFLE } 2 & 64 & \text { PRL } & 13 & 349\end{array}$

$\begin{array}{lllll}\text { EADIER } & 65 & P L & 17 & 337 \\ \text { KIENZLE } & 65 & P L & 19 & 438\end{array}$

KIENZLE
RITIENBE 65 P PRL 15556
TRILLING 6.5 PL 19427

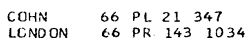

BADI ER, DEMOUL IN, BARL OUTAUD+IEPOL+SACL+AMST KIENZLE, MAGLIC, LEVRAT, LEFEBVRES + (CERN)
RITTENBERG,KALBFLEISCH EFE
(LL+BNL) +BROWN, GOLOHABERS, KAOYK, SCANIO (LRL) COHN, MCCULLOCH, BUGG, CONDO (ORNL+TENN+UCND) LONDON, RAU, SAMI OS, GOLDBERG + (BNL+SYRACUSE) IJP
MARTIN,CRITTENDEN, SCHROEDER
(INDIANA UII BARBARO-GALTIERI, MATI SON, RI TTENBERG+ (LRLII I=0 BARLOUTAUD+ (SACLAY+AMST+BGNA+REHO+EPOL) I
+BUHLER, DALPI AZ, MASS AM+ (CERN+BGNA+STRB)
+AMMAR, MOTT, DAGAN, DERR ICK, FIELOS (NWES+ANL) BARLOUTA 68 PL $26 \quad 8 \quad 674$ $\begin{array}{llllll}\text { BARLOUNA } & 68 & P L & 26 & 8 & 674 \\ \text { BOLINI } & 68 & \text { NC } & 58 & \text { A } & 289 \\ \text { DAVIS } & 68 & P L & 27 & B & 532\end{array}$

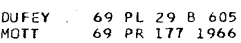
RITTENBE 69 UC $\begin{array}{lllll}\text { AGUILAR } & 70 & \text { PRL } & 25 & 1635 \\ \text { BENSINGE } & 70 & \text { PL } 33 & 33 & 505\end{array}$ BARDADIN 71 PR D 42711

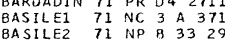

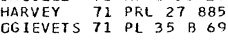

$\begin{array}{llllll}\text { AGUILAR } & 72 & P R & 0 & 6 & 29 \\ \text { APEL } & 72 & P L & 40 & 8 & 680\end{array}$ $\begin{array}{llllll}\text { BLDCDWOR } & 72 & \text { NP } & 8 & 39 & 525 \\ \text { DALPIAZ } & 72 & \text { PL } & 42 & \text { B } & 377\end{array}$ $\begin{array}{llllll}\text { DALPIAZ } & 72 & P L & 42 & \text { B } & 377 \\ \text { RADER } & 72 & \text { PR } & 0 & 6 & 3059\end{array}$ $\begin{array}{llllll}\text { DANBURG } & 73 & P R & D & 8 & 3744 \\ \text { JACBS } & 73 & P R & 0 & 8 & 18\end{array}$ BALTAY 74 PR D9 2999 DUANE 74 PRL 32425

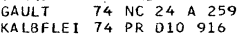
$\begin{array}{lllll}\text { GR IGORIA } & 75 & N P & 891 & 232 \\ \text { KALBFLEI } & 75 & \text { PR } & 011 & 987\end{array}$ $\begin{array}{llllll}\text { CERRADA } & 77 & \text { NP } & B & 126 & 189 \\ \text { DELAGUIL } 77 & \text { PR } & \text { D } 16 & 2833\end{array}$

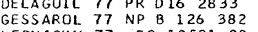
LEDNICKY 77 E 2 - 10E21,22, 23
RCUSARI 77 PREPRINT RCUSSAR I 77 PREPRINT
ALSO 77 BUDAPEST CONF. +GOBBI, POUCHON, CNOPS, + IETH+CERN+SACLIIJ + AMMAR, DAVIS, KR PAC, SLA TE, DAGAN+ (NWES+ANL)
ALAN RITTENBERG (THESIS)
(LRL) I $=0$ aLAN RITTENBERG (THESTS) DWGaNi TNWES chli AGUILAR-BENITEZ, BASSANO, SAM IOS, BARNE S+(BNL)
GENSINGER, ERWIN, THOMPSON, W. D.WALER (WISC) BARDADIN-OTWI NOW SKA, HOF MOKL, MI CHEJ DA+ (WARS) +8OLLINI, DALPIAZ, FRABETTI, + (CERN+BGNA+STRB) +BOLLINI, DALPIAZ, FRABETTI, + +CERN +BGNA+STRB)
+MARQUIT, PETERSON, RHOADES, +
(MINN +MICH) +MARQUIT, PETERSON, RHOADES, +
OGIEVETSKY, TYBOR, ZASLAVSKY
(DUENA) AGUILAR-BENI TEZ, CHUNG, EISNER, SAMIOS (BNL)
+AUSLANDER, MULLER, BERTOLUCCI, + (KARL+PISA) CAMILLERI, DUANE, GARBUT, BURTON+ (LOIC+S HMP BLOOOWORTH, JACKSON, PRENTICE, YOON (TORONTO
+FRABETY I, MASSAM, NAVARRIA, ZICHICHI
(CERN) + KALBFLEISCH, BORENSTEIN, CHAPMAN, + (BNL+MICH) JP
+CHANG, GAUTHER,
(BRAN+UMD+SRA+TUFT) JJP +COHEN, CSORNa, HABIBI, KaLELKAR, + (COLU+BING) JP +BINNI E, CAMILLERI, CARR, CEBENHAM+ (LOIC+SHMP)
(DURH+LOIC+ARIZ) TJONES, SCADRON, THEWS
G.R.KALBFLEISCH
(BNL) GR IGOR IAN, LADAGE, MELLEMA, RUONICK, + (UCLA)
KALBFLEI SCH,STRAND, CHAPMAN
( BNL + M ICH) +WAGNER, BLOCKZIJL, + (CERN+AMST +NIJM+CXF) JP F. DEL AGUILA ANO M.G. DONCEL +M IBARCELCNA) JP R. LEDNICKY
+ERNWEIN,FEL TESSE, BORGEAUD, ROUSSARIE+ ( $(S A C L)$ JP HEMINGWAY REV IEW TALK +ALAM, BLOCKER, BOYARSKI, + (SLAC+LBL) ABRAMS 79 'SLAC-PUB' 2421
ALSO 79 PRL 43477 $\begin{array}{llll}\text { ALSO } & 79 \text { PRL } 43477 \\ \text { APEL } & 99 & \text { PL } 83 \quad 8131\end{array}$ ABRAMS, ALAM, BLOCKER, BOYARSKI,
(SLAC+LBL

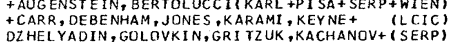

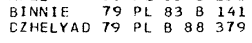

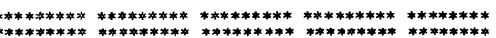

$\delta(980)$ OELLA( 980, JPG $=0+-1 \quad$ I=1

Observations of missing-mass peaks in the 960 $\mathrm{MeV}$ mass region are mostly controversial and are therefore not listed here. Under this entry, we list two types of $I=1$ peaks near the $\mathrm{K} \bar{K}$ threshold:

1. $n \pi$ decays, peaking slightly below the $\mathrm{K} \overline{\mathrm{K}}$ threshold. This defines $I^{G}=1^{-}$and $\mathrm{J}^{\mathrm{P}}=$ normal.

2. $A \mathrm{~K} \overline{\mathrm{K}}$ threshold enhancement with $\mathrm{I}=1$. This association is justified by the remark (ASTIER 67) that the $\mathrm{K} \overline{\mathrm{K}}$ threshold enhancement may be due to a virtual bound state also coupled to the $\eta \pi$ system. In a coupled-channel analysis MORGAN 75 and FLATTE 76 show that the resonance width of the $\delta(980)$ may be much larger than the width observed in the $n \pi$ system. Coupled-channel analyses of more recent $\mathrm{K}^{-} \mathrm{K}^{0}$ and $\mathrm{n} \pi$ data, however, rule out a large width (IRVING 77, MARTIN 78,79).

The low Q-value of the $k \bar{K}$ threshold enhancement and decay distributions of the $\eta \pi$ system favor $\mathrm{J}^{\mathrm{P}}=\mathrm{O}^{+}$. Additional evidence (LIPKIN 69) comes from the absence of a $\rho \pi$ decay mode (GRASSLER 77).

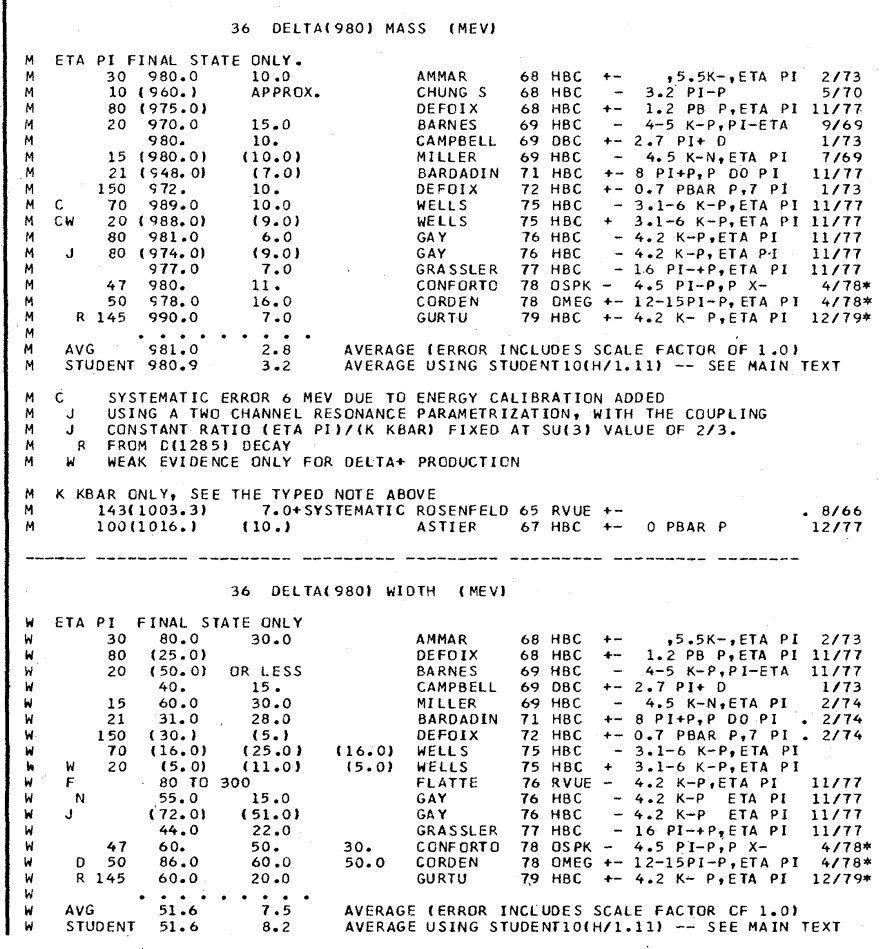




\section{Mesons}

$\delta(980), \mathrm{S}^{*}(980)$

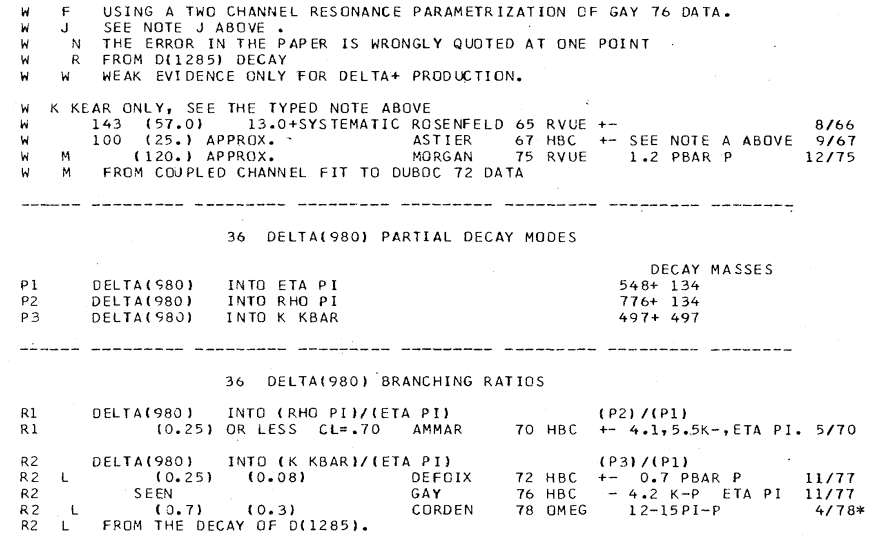

**********************************************************************************

REFERENCES FOR DELTA $(980)$

TURKOT 63 SIENNA CONF 1661 +COLLINS, FUJII, KEMP+ (BNL+PITTSBURGH)

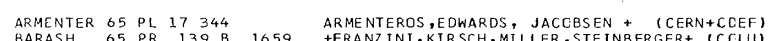

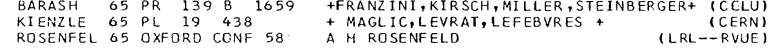

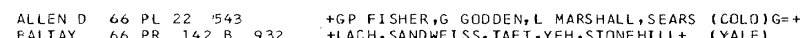

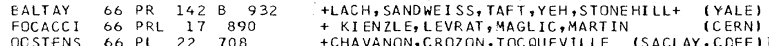

ALLI SCN 67 PL 258619 +CRUZ+ (OXF+MP IM+EIRM+RHEL+GLAS+LEIC)

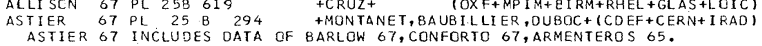

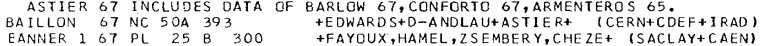

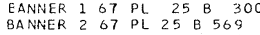

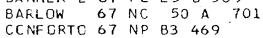

$\begin{array}{lllll}\text { AMMAR } & 68 & \text { PRL } & 21 & 1832 \\ \text { CHUNG } S & 68 & P R & 165 & 1491\end{array}$

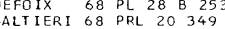

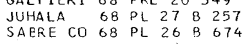

$\begin{array}{lllll}\text { EARNES } & 69 & \text { PRL } & 23 & 610 \\ \text { CANPBELL } & 69 & \text { PRL } & 22 & 1204\end{array}$

$\begin{array}{lllll}\text { JUHALA } & 69 & P R & 184 & 1461 \\ \text { KRUSE } & 69 & P R & 177 & 1951\end{array}$

IPKIN 69 PRL 22212

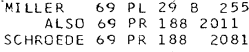

$\begin{array}{llll}\text { ABOLINS } & 70 & \text { PRL } 25469 \\ \text { ANMAR } & 70 \text { PR D } 2430 \\ \text { COOPER } & 70 \text { NP } 823,605 \\ \text { YICU } & 70 \text { THESIS, A } 646\end{array}$

$\begin{array}{lllll}\text { ANDERSCN } 71 & \text { PRL } 26 & 108 \\ \text { BARDADIN } 71 & \text { PR } & 04 & 2711\end{array}$

$\begin{array}{llllll}\text { BINNIE } & 72 & \text { PL } & 39 & \text { B } & 275 \\ \text { CHESHIRE } & \text { PRL } 28 \quad 520 \\ \text { OEFOIX } 72 & \text { NP B } & 44 & 125\end{array}$

$\begin{array}{llllll}\text { CHESHIRE } & 72 & \text { PRL } & 28 & 520 \\ \text { DEFOIX } & 72 & \text { NP } & 42 & 44 & 125 \\ \text { DUBOC } & 72 & \text { NP } & 4 & 46 & 429\end{array}$

$\begin{array}{llllll}\text { DUBGC } & 72 & \text { NP } & 46 & 429 \\ \text { HOLLOWAY } 72 & \text { PHIL. CCNF. PROC }\end{array}$

ATHERTCN 73 PL $43 \quad$ B 249

+CHEZE, HAMEL, MAREL, TEIGER+ (SACLAY+CAEN)
(CDEF+SACL)

$\begin{array}{ll}\text { +MONTANET, O-ANDLAU+ } & \text { (CERN+COEF+IRAD+LIVP) } \\ \text { CONFORTO, MARECHAL+ } & \text { (CERN+CDEF+IPNP+LIVP) }\end{array}$

+DAVIS, KPOPAC, DERRICK,FIELOS,+
+O.DAHL, J. KIRZ, D.H.MILLER +O.DAHL, J. KIRZ, O. H. MILLER (CDEF+IPNP+CERN)
+RIVET, SIAUD, CONFORTO+
BARBARC-GALIIERI, MATISON, RITTENBERG + (LRI) BARBARO-GALT IERI, MAT I SON, RI TYENBERG+ (LRL)
+LEACOCK, RHODE, KOPELMAN, LI IBY + (IOAA+COLO) +LEACOCK, RHODE, KOPELMAN, LI IBY + (IDHA+CCLO)
BARLOUTAUD+
(SACL+AMST+BGNA+REHO+EPOL)

+CHUNG , EISNER, BASSANO, GCLDBERG+ (BNL+SYRA) J.H.CAMPBELL, L ICHTMAN, LOEFFLER, + (PURDUE)
(BARSHON, KWAN WU LAI, +LEACOCK, RHODE, KOPEL MAN, LIBBY, + (ISU+CCLO) KRUSE, LOOS, GOLOWASSER
+MESHKOV
(ILLINOIS)
(REHO+NBS) JP (REHO+NBS)
+MESHKOV
O.H.MILLER, S.L.KRAMER, D.D.CARMONY, +(PURDUE) YEN, AMMANN, CARMCNY, ELSNER, +
SCHROEDER, KERNAN, FISHER, LIBBY, + (ISU +CCLOU) +GRAVEN, MC CARTHY, G.SMITH,L.SMITH+ (LRL +UCD) +KROPAC, DAVI S, DERR ICK, + (KANS+NWES S+ANL+ WI SC)
+HANNER, MUSGRAVE, PCLLARO, VOYVOCIC
TCHIU-PUNG YIOU
(ONL) +DIXIT, (CHIC+ANL +CARL+LASL+CNRC+NAGCYA)
BARDAD IN-DTWINOW SKA, HOF MOKL, MICHE JDA+ (WARS) +CAMI LLERI, OUANE, GARBUTT, BURT ON+ (LOIC+S HMP) + HCFFMAN, GARFINEL,+
+ +NASCIMENTO, BIZZARR1,
(IOWA+ANL+PURD)
(COEF+CERN) + NASCIMENTO, BIZZARRI, +
+GOLOBER, MAKOWSKI, DONALD,+ (COEF+CERN)
(LPNP+LIVP)

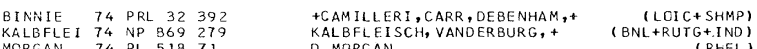

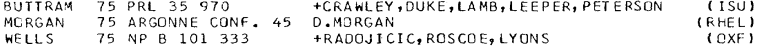
$\begin{array}{lllllll}\text { GAY } & 76 & \text { PL } & 63 & B & 220 \\ \text { FLATTE } & 76 & \text { PL } & 63 & B & 224\end{array}$ $\begin{array}{llllll}\text { GRASSLER } & 77 & \text { NP } & 1 & 121 & 189 \\ \text { IRVING } & 77 & \text { PL } & 70 & 3 & 217\end{array}$

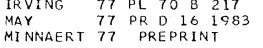

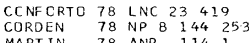
+CHALOUPKA, BLCKZ IJL, HEINEN+(CERN+AMST+NIJM)
(CER. MLATTE
(CER) $+\quad$ (AACH+BERL+BONN+CERN+CRAC+HEID+WARS)

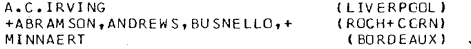
(BOROEAUX) 8+G CONFORTO, KEY+(RHEL + TNTO+CHIC+FNAL+WISC)
+ CORBETT, ALEXANER, (BIRM+RHEL+T ELA+LOWC) ESTABRDO 79 PR D 152678 $\begin{array}{llllll}\text { ESTABRDD } & 79 & \text { PR } & \text { D } & 15 & 2678 \\ \text { GURTU } & 79 & \text { NP } & \text { B } & 151 & 181 \\ \text { MARTIN } & 79 & \text { NP } & \text { B } & 158 & 520\end{array}$ P.ESTABROOKS
+GAVILLET, BLOKZIJL, + (CARL)
+OZMUTLU

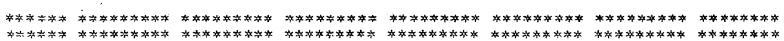

\section{Data Card Listings For notation, see key at front of Listings.}
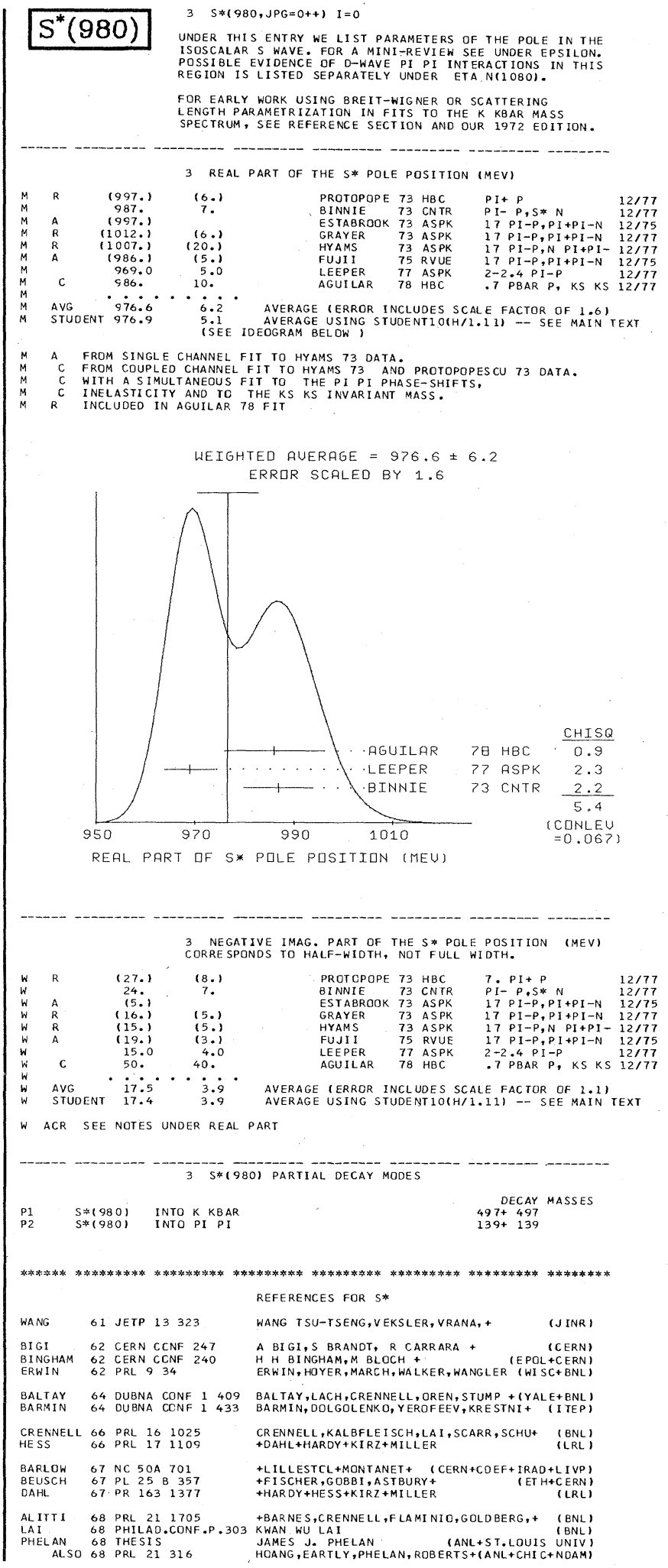


\section{Data Card Listings}

For notation, see key at front of Listings.

\section{Mesons $\mathrm{S}^{*}(980), \mathrm{H}(990), \phi(1020)$}

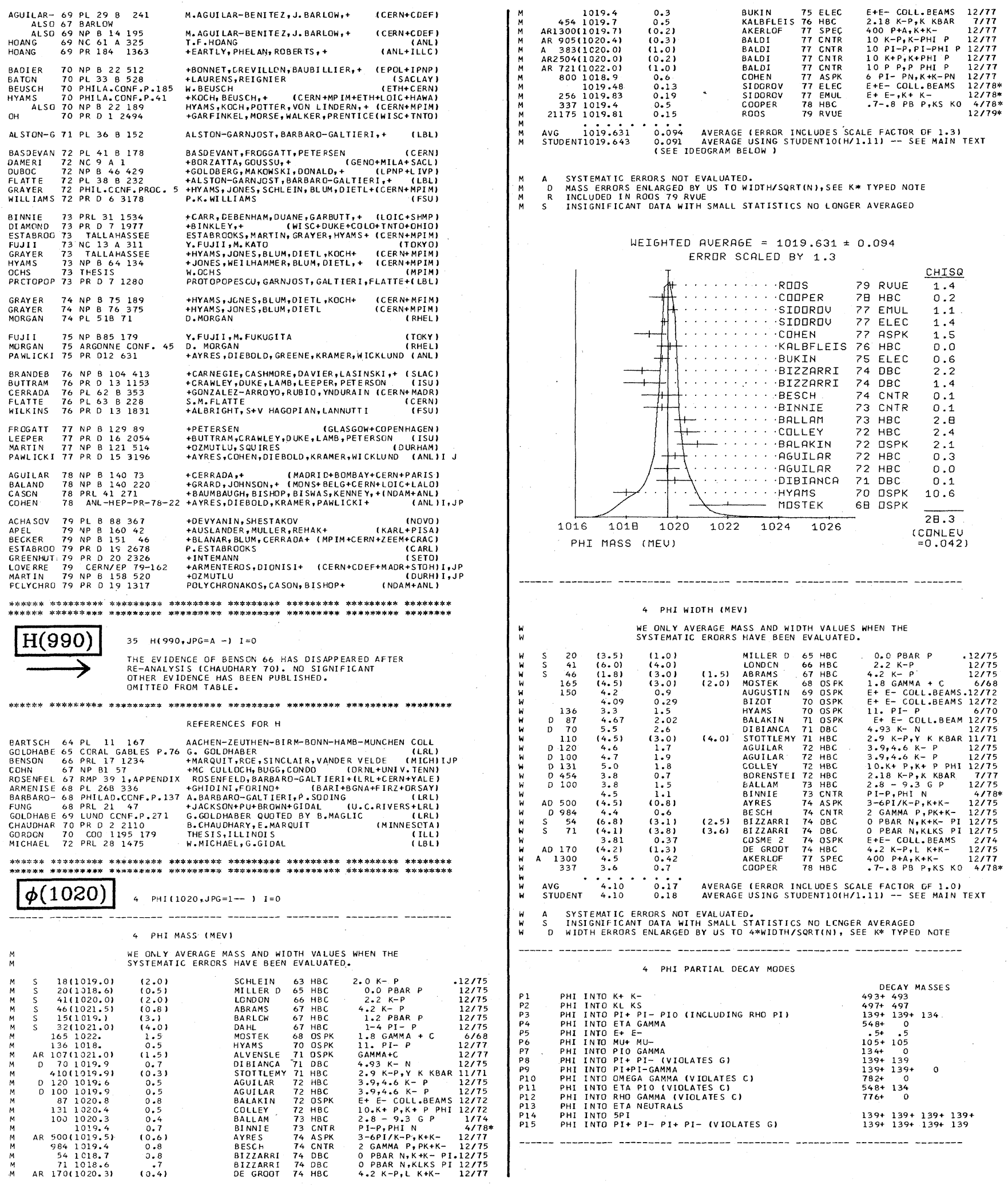




\section{Mesons} $\phi(1020)$
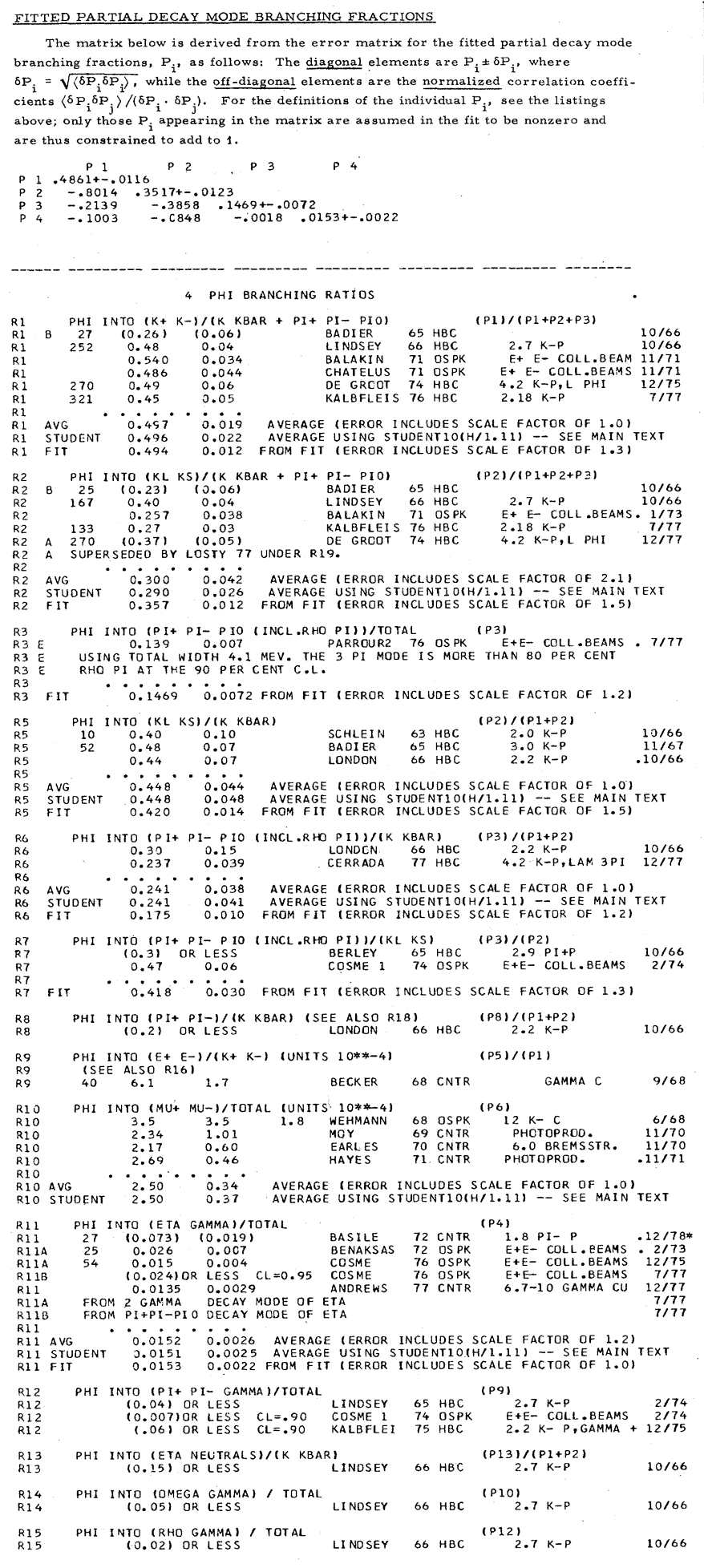

\section{Data Card Listings For notation, see key at front of Listings.}
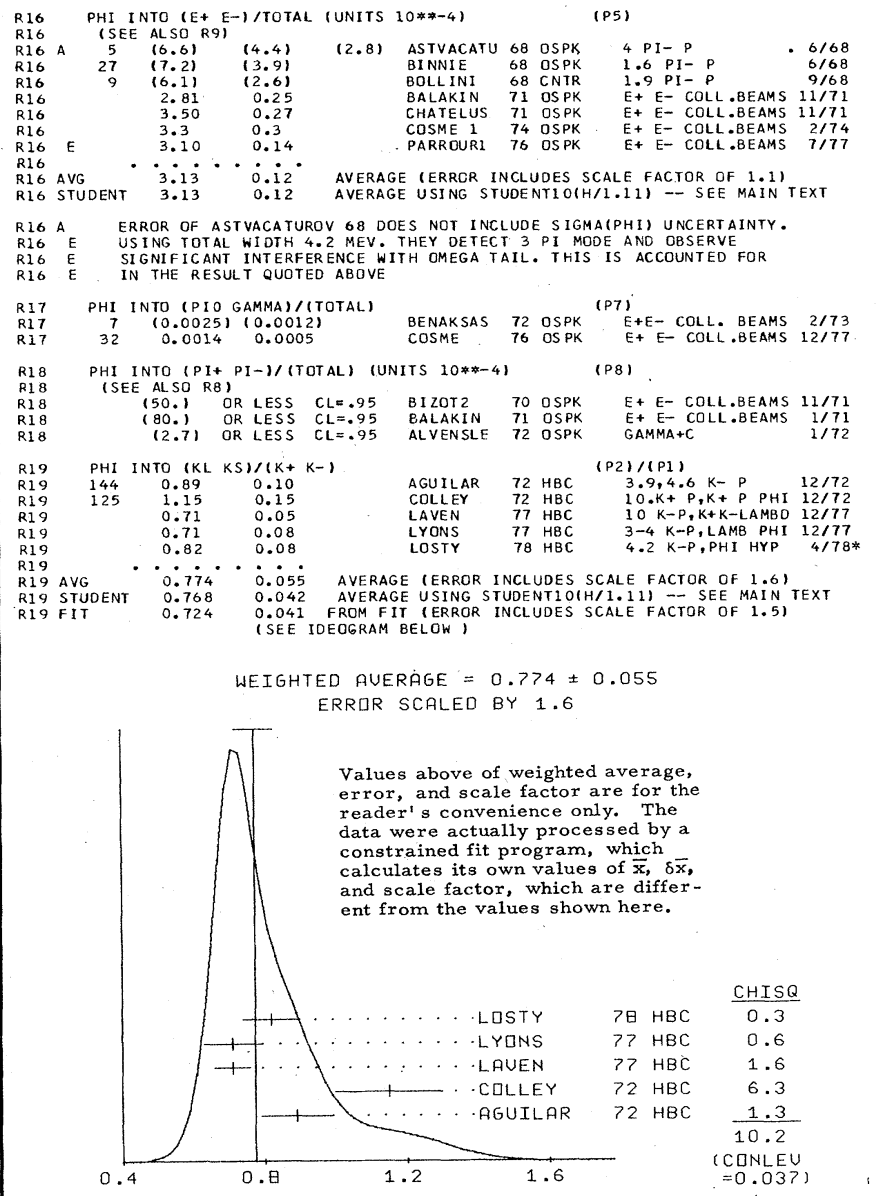

PHI INTO (KL KS $) /(K+K-)$

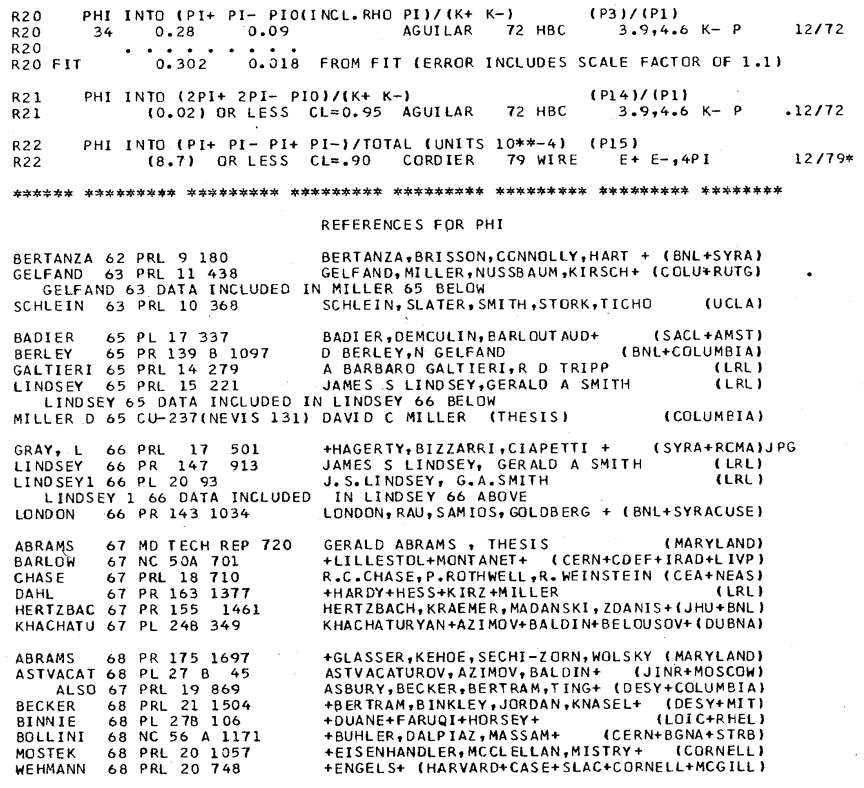




\section{Data Card Listings}

Mesons

For notation, see key at front of Listings. $\quad \mathrm{M}(1033-1040), \eta_{\mathrm{N}}(1080), \mathrm{M}(1150-1170), \mathrm{A}_{1}$

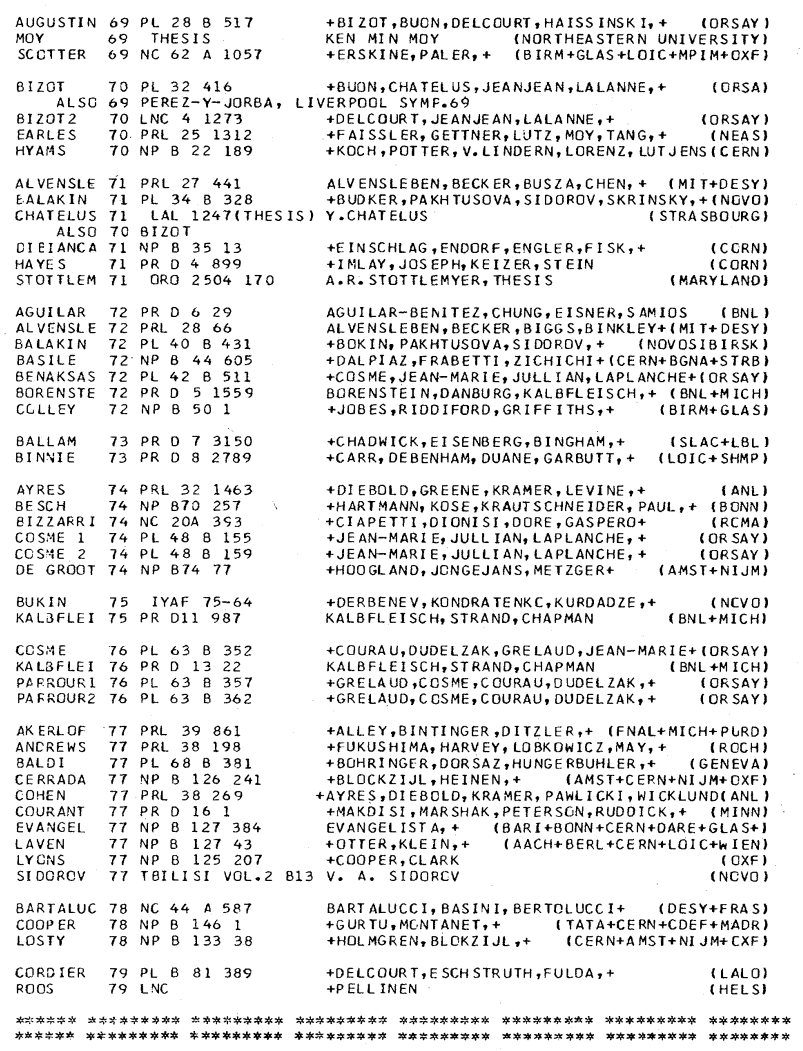

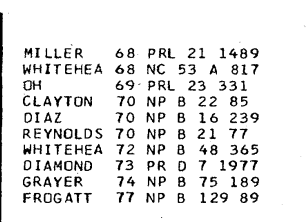

REFERENCES FCR ETA N

+GUTAY, JOHNSON, KENNEY + (PURDUE+NDAM+SLAC) C. WHITEHEAO + MASON, MUI RHEAD, RI GOPOULOS,+- (WISC+TNTO)
(LIPPATEN) +ALBRIGHT, BRADLEY, + (OHIO+F SU+MINN+CCLO) WHI TEHEA, AULD, + (AERE+RHEL+SHMP+LCUC) +BINKLEY,+
+HYAMS, JONES, BLUM, DI ETL, KOCH+ (CERN+MPIM)
+PETERSEN

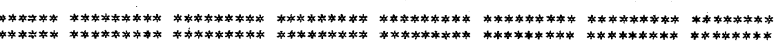

$\mathrm{M}(1150-1170)$ 68 THIS ENTRY LISTS REFERENCES TO PEAKS OF LOW
STATISTICAL SIGNIFICANCE IN THE 3 PI SYSSTEM BETWEEN THE AI AND THE A2, AS WELL AS A CLAIM T2. NOT CONFIRMED BY BUTTRAM 75. OMITTED FROM

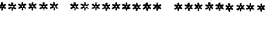
REFERENCES FOR M(1150-1170)

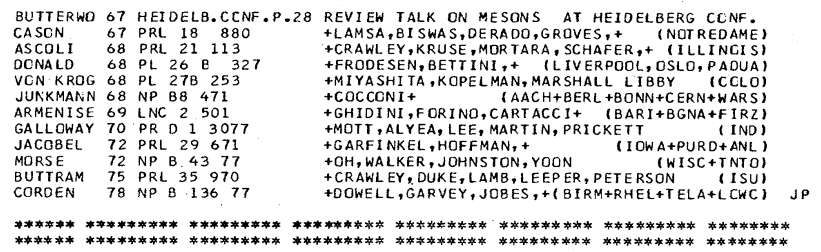

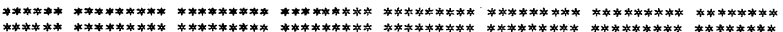

$A_{1}(1100-1300)$ 10 A1(1100-1300,JPG =1+-) $I=1$

The peak in the $(3 \pi)^{ \pm}$mass distribution near the $\rho \pi$ threshold was discovered by BELLINI 63 in very forward $\pi^{-}$scattering on carbon without nuclear break-up, thus coherent diffractive $\rho \pi$ production. Until 1977, all the significant observations of a $\rho^{0} \pi^{ \pm}$peak near $1100 \mathrm{MeV}$ were made in the reaction $\pi^{ \pm} \mathrm{N} \rightarrow(\pi \pi \pi)^{ \pm} \mathrm{N}$. At small momentum transfer, the diffraction-like mechanism without quantum number exchange in the $t$ channel contributes to this reaction. The dominant effect is a broad $\mathrm{J}^{\mathrm{P}}=1^{+}$enhancement in the $\mathrm{S}$-wave $\rho \pi$ system, its width being $\sim 300 \mathrm{MeV}$ (ANTIPOVI 73, OTTER 74, KRUSE 74, TABAK 74, THOMPSON 74, EMMSI 75, BALTAY 77, PERNEGR 78, ROBERTS 78, DAUM 80). The position of the maximum intensity falls in the range 1100 to $1300 \mathrm{MeV}$ and varies with $t$ (DAUM 80).

Most of these experiments have been partialwave analyzed by the method of ASCOLI 70. Assuming that, for a given momentum transfer $t$, the $3 \pi$ vertex is independent of the NN vertex, the $3 \pi$ vertex is composed, in the spirit of the nonunitary isobar model, of quasi-two-body $\pi \rho$ and $\pi \varepsilon$ amplitudes. The waves contributing to the diffractive $3 \pi$ final state are (at most) the $0^{-} \mathrm{P}, \mathrm{I}^{+} \mathrm{S}$, $I^{+} \mathrm{D}, 2^{-} \mathrm{P}, 3^{+} \mathrm{D}, \mathrm{I}^{-} \mathrm{P}$, and $2^{+} \mathrm{D} \rho \pi$ waves and the $\mathrm{C}^{-} \mathrm{S}$, $1^{+} \mathrm{P}$, and $2^{-} \mathrm{D} \varepsilon \pi$ waves. Here $\varepsilon$ stands for a pole simulating the non-resonant $\mathrm{J}^{\mathrm{P}}=0^{+} \pi \pi$ interaction in the 700 to $900 \mathrm{MeV}$ region [see the review of 


\section{Mesons}

$A_{1}(1100-1300)$

S-wave $\pi \pi$ interactions under $\varepsilon(1300)]$.

The results of these analyses have shown that the phase of the $1^{+} \mathrm{S}$ wave displays little variation relative to the $0^{-} \mathrm{S}(\varepsilon \pi), \mathrm{I}^{+} \mathrm{P}(\varepsilon \pi)$, and $2^{-} \mathrm{P}(\rho \pi)$ waves (ANTIPOV1 73, OTTER 74, TABAK 74, THOMPSON 74, BALTAY 77). As the $2^{+} \mathrm{D}$ wave exhibits a clear Breit-Wigner-like phase change in the $A_{2}$ region (ASCOLI 70, ANTIPOVI 73, OTTER 74, TABAK 74, THOMPSON 74, BALTAY 77), the above results have been interpreted to imply that no resonant $A_{1}$ is needed. Unitarity corrections to the isobar model did not change this conclusion (ASCOLI 75, AITCHISON 75).

More recent analyses, however, have provided new evidence for an $\mathrm{A}_{1}$ resonance. BOWLER 75 demonstrated that the small variation in the $1^{+} \mathrm{S}$ phase could be due to a phase difference between the Deck amplitude and the direct $A_{1}$ resonance production amplitude. This small phase variation also could be due to inelasticity, because of the coupling of $\rho \pi$ to the $\varepsilon \pi$ and $K^{*} \overline{\mathrm{K}}$ channels, or to rescattering (BRAYSHAW 76, LONGACRE 76,77). SCHULT 77 reanalyzed the ANTIPOV1 73 data using three-pionstate amplitudes which satisfy both unitarity and analyticity, and found a solution with considerably more phase variation than originally had been observed.

BASDEVANT 77 performed an analysis of the $\rho \pi$ waves exclusively, ignoring the $\varepsilon \pi$ waves as being meaningless in an isobar analysis since the $\varepsilon$ could not be considered a bona fide particle. Their full amplitude is properly analytic and unitary, and it includes: the Deck amplitude (resonant as well as background elastic $\rho \pi \rightarrow \rho \pi$ scattering), rescattering corrections, inelasticity due to the $\mathrm{K}^{*} \overline{\mathrm{K}}$ channel, and direct diffractive $A_{1}$ production. They take the $I^{+} \mathrm{S}$ phase from the difference of the known $\mathrm{A}_{2}$ phase and the observed $2^{+} \mathrm{D}-\mathrm{I}^{+} \mathrm{S}$ phase difference (ANTIPOVI 73). BASDEVANT 77 show that the ANTIPOVI 73 data are consistent with a resonance at $\mathrm{M}=1300 \pm 150 \mathrm{MeV}$, $\Gamma=400 \pm 100 \mathrm{MeV}$, and that the data are rather inconsistent with the hypothesis of no resonance.

New light has been shed on the existence of the $A_{1}$ by the PERNEGR 78 data on coherent $\pi$ scattering on nuclei. For the first time these data contain information on the $1^{+} \mathrm{S}-\mathrm{O}^{-} \mathrm{P}$ phase difference.

\section{Data Card Listings} For notation, see key at front of Listings.

Although this phase-shift analysis is ambiguous between two solutions, one solution exhibits a $1^{+} \mathrm{S}-\mathrm{O}^{-} \mathrm{P}$ phase increase of $90^{\circ}$ from threshold up to $1400 \mathrm{MeV}$, together with a peak in the $1^{+} \mathrm{S}$ intensity around $1100 \mathrm{MeV}$. The energy dependence of the $1^{+} \mathrm{S}-\mathrm{O}^{-} \mathrm{P}$ phase difference is in fact exactly as predicted by BASDEVANT 77 on the basis of the ANTIPOV1 73 data. Overwhelming confirmation now comes from the very large DAUM 80 experiment on a proton target. They find a unique and stable solution which exhibits not only the $1^{+} \mathrm{S}-0^{-} \mathrm{P}$ phase increase up to $1400 \mathrm{MeV}$, but, by comparing with the $A_{2}$ phase, they are able to show unambiguously that the $I^{+} \mathrm{S}, \mathrm{I}^{+} \mathrm{P}$, and $0^{-} \mathrm{S}$ phases all increase with mass, the forward motion of the $1^{+} \mathrm{S}$ phase being $\approx 80^{\circ}$ in the 1100 to $1500 \mathrm{MeV}$ region.

A long-standing problem of the $A_{1}$ has been its non-observation in non-diffractive processes (for a review of the situation up to 1976, see HABER 77). Here also the situation is completely changed due to new observations.

GAVILLET 77 have analyzed backwardly produced $3 \pi$ events in the reaction $\mathrm{K}^{-} \mathrm{p} \rightarrow \Sigma^{-} \pi^{+} \pi^{+} \pi^{-}$in sufficient number to project out the different partial waves. An $A_{1}$ peak seen in the total $3 \pi$ mass distribution can be attributed to the $1^{+} \mathrm{S}$ partial wave. The Breit-wigner parameters of the peak are $\mathrm{M}=1041 \pm 13 \mathrm{MeV}, \Gamma=230 \pm 50 \mathrm{MeV}$. The SU(3) assignment of an $A_{1}$ with these parameters to the $\mathrm{J}^{\mathrm{PC}}=\mathrm{I}^{++}$nonet together with the $\mathrm{Q}^{\prime} \mathrm{s}$, the D(1285), and the $\mathrm{E}(1420)$ is not completely satisfactory and may indicate that the experimental masses are far from the pole positions on the second sheet (MAZZUCATO 79, DIONISI 80). A possible confirmation of backward $A_{1}$ production by pions has been obtained by FERRER 78. The observed peak has the resonance parameters $M \cong 1050 \mathrm{MeV}, \Gamma \cong 200 \pm 50 \mathrm{MeV}$, but nothing is known about the partial-wave composition. The production cross sections at the two different beam momenta of FERRER 78 agree with limits set by earlier, less significant experiments (ANDERSON 69, ABASHIAN 75).

On the other hand, no evidence for the $A_{1}$ is found in the charge-exchange reactions $\pi^{+} \mathrm{n} \rightarrow \pi^{+} \pi^{-} \pi^{0} \mathrm{p}$ (EMMS2 75), $\pi^{+} p \rightarrow \pi^{+} \pi^{-} \pi^{0} \Delta^{++}$(WAGNER 75, BALTAY 77), $\pi^{-} \mathrm{p} \rightarrow \pi^{+} \pi^{-} \pi^{0} \mathrm{n}$ (CORDEN 78), or $\mathrm{K}^{-} \mathrm{p} \rightarrow \pi^{+} \pi^{-} \pi^{0} \Lambda^{0}$ (CERRADA 77). However, the number of partial waves 


\section{Data Card Listings \\ For notation, see key at front of Listings.}

Mesons

$A_{1}(1100-1300)$

is greater in charge exchange due to the two possible values of isospin, and thus the analysis is more complicated.

Other non-diffractive channels, such as $\bar{p} p$ annihilation, have not produced consistent results of sufficiently high statistical significance. At best, an $\mathrm{A}_{1}^{-}$shoulder has been seen in the $(3 \pi)^{-}$ spectrum from $\overline{\mathrm{p}} \rightarrow \mathrm{p}^{+} \pi^{-} \pi^{-} \pi^{0}$. However no $\mathrm{A}_{1}^{0}$ is observed. The effect is interpretable as interference between various resonance channels (KASPER 79). Finally, the semileptonic decay $\tau^{ \pm} \rightarrow A_{1}^{ \pm} \nu$ seems to have been discovered at PLUTO (ALEXANDER 78, WAGNER 79) and confirmed at SLAC-LBL (JAROS 78). The PLUTO $\pi^{+} \pi^{-} \pi^{ \pm}$mass distribution with a $\rho^{0} \pi^{ \pm}$ selection shows a peak centered at $\mathrm{M} \lesssim 1200 \mathrm{MeV}$, $\Gamma \sim 400$ to $500 \mathrm{MeV}$, very unlike phase space. The Dalitz plot distribution is consistent with $1^{+} \mathrm{S}$. However, with only 27 events in the plot, it is not possible to rule out all other possible waves.

To summarize, most of the data now seem to require the presence of an $A_{1}$ resonance, but the quantitative details are far from being determined exactly. BASDEVANT 78 used the data of ALEXANDER 78 and JAROS 78 to restrict the range of solutions for the $A_{1}$ resonance parameters obtained in their analysis of diffractive $A_{1}$ production (BASDEVANT 77). The values they obtain, when expressed as secondsheet pole parameters rather than as simple BreitWigner parameters are $\mathrm{M} \cong 1180 \pm 50 \mathrm{MeV}, \Gamma=400 \pm 50$ $\mathrm{MeV}$.

DAUM 80, fitting simultaneously the $1^{+} \mathrm{S}$ intensity and the phase relative to the $2^{+} \mathrm{D}$ wave above $1 \mathrm{GeV}$ with model amplitudes similar to the ones used by BOWLER 75 and BASDEVANT 77 , find for the $A_{1}$ parameters: $M \approx 1280 \mathrm{MeV}, \Gamma \approx 300 \mathrm{MeV}$. This is not in complete agreement with PERNEGR 78, GAVILLET 77, FERRER 78, ALEXANDER 78, and JAROS 78, who find the peak of the $1^{+} \mathrm{S}$ intensity around 1100 $\mathrm{MeV}$. Thus, if the $\mathrm{A}_{1}$ can finally be considered as a well established meson, the determination of its parameters is far from settled.

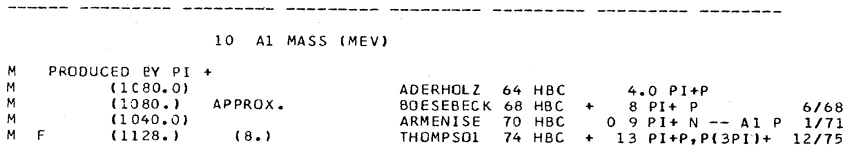

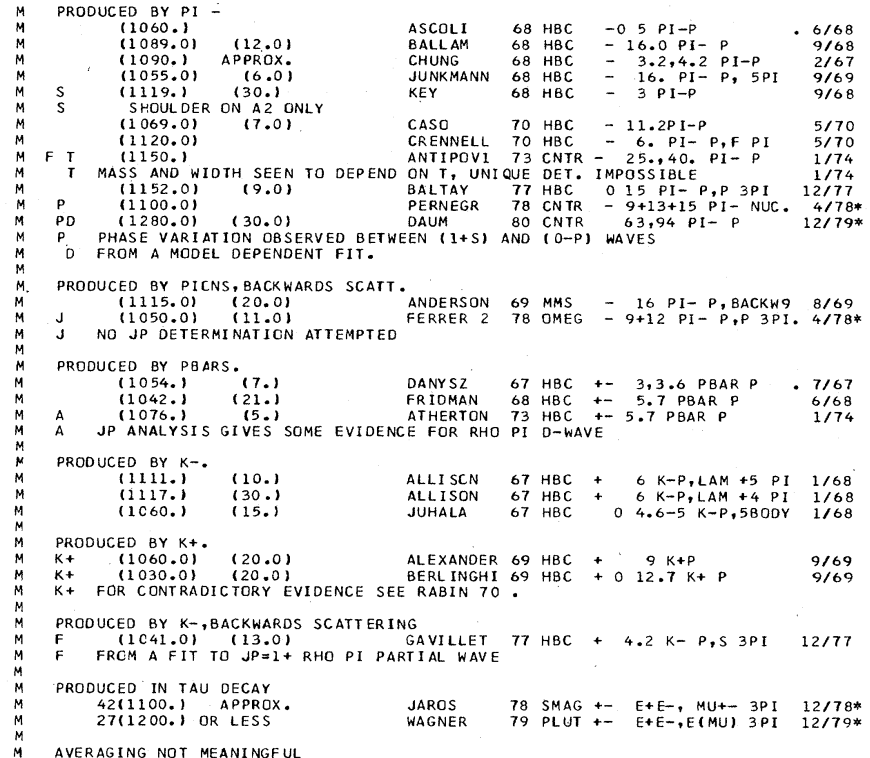

10 A1 WIDTH (MEV)

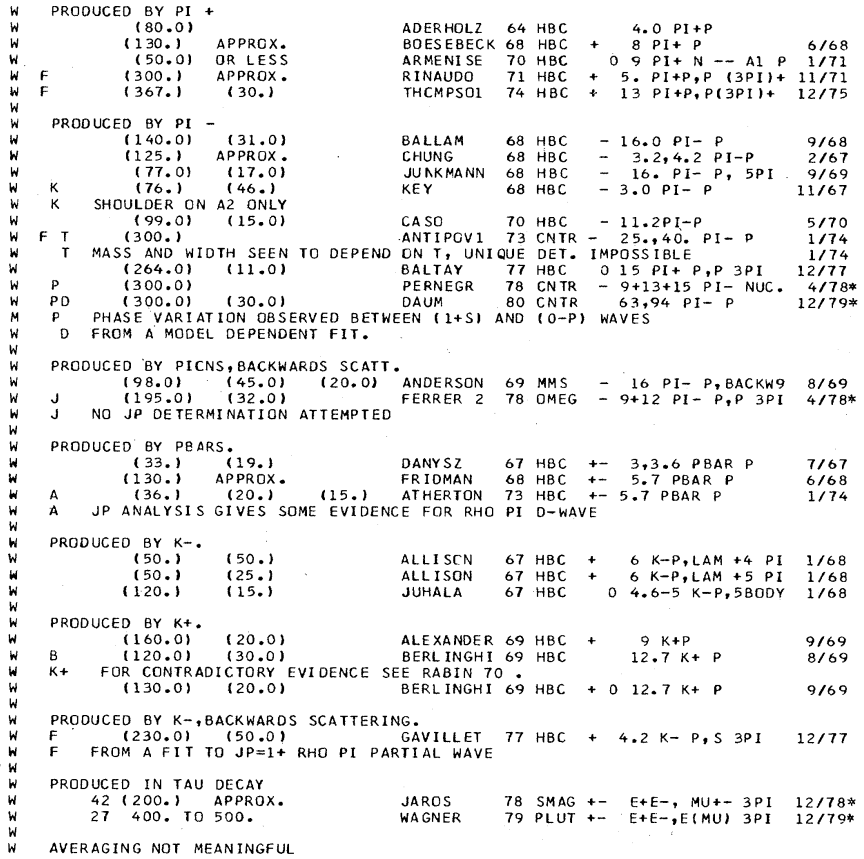

10 al PARTial DeCAY MCDES

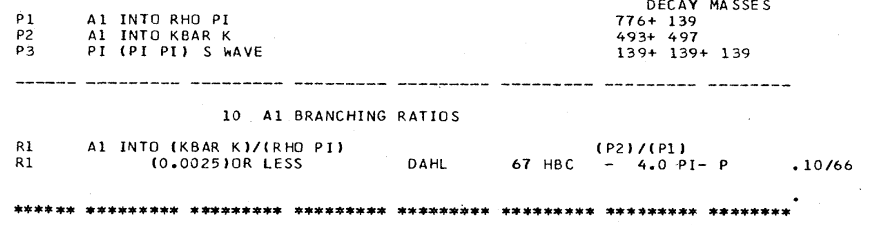


Mesons

$A_{1}(1100-1300), B(1235)$
Data Card Listings For notation, see key at front of Listings.

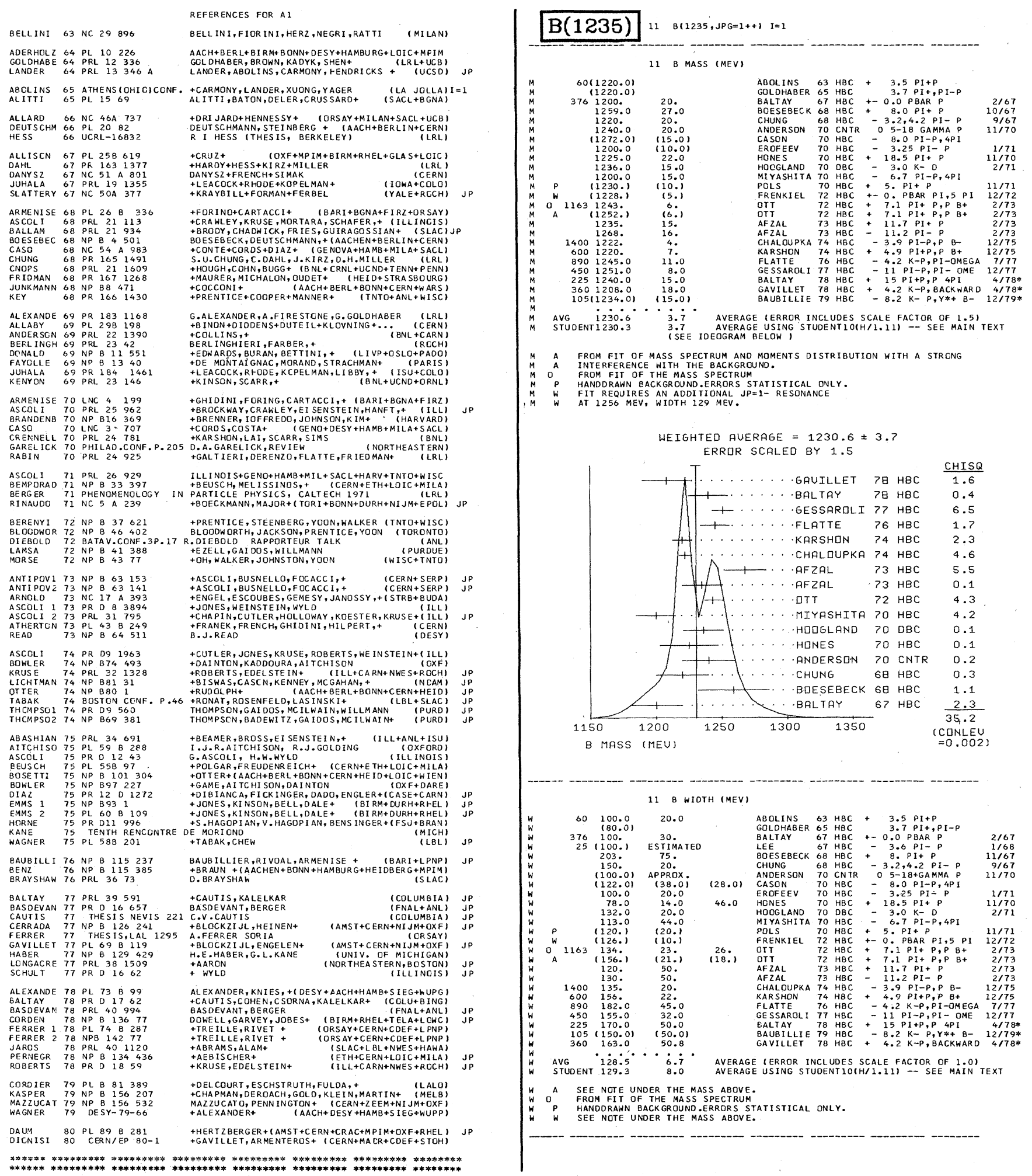

Rev. Mod. Phys., Vol. 52, No. 2, Part II, April 1980 
Data Card Listings

For notation, see key at front of Listings.

Mesons

$B(1235), \rho^{\prime}(1250), f(1270)$

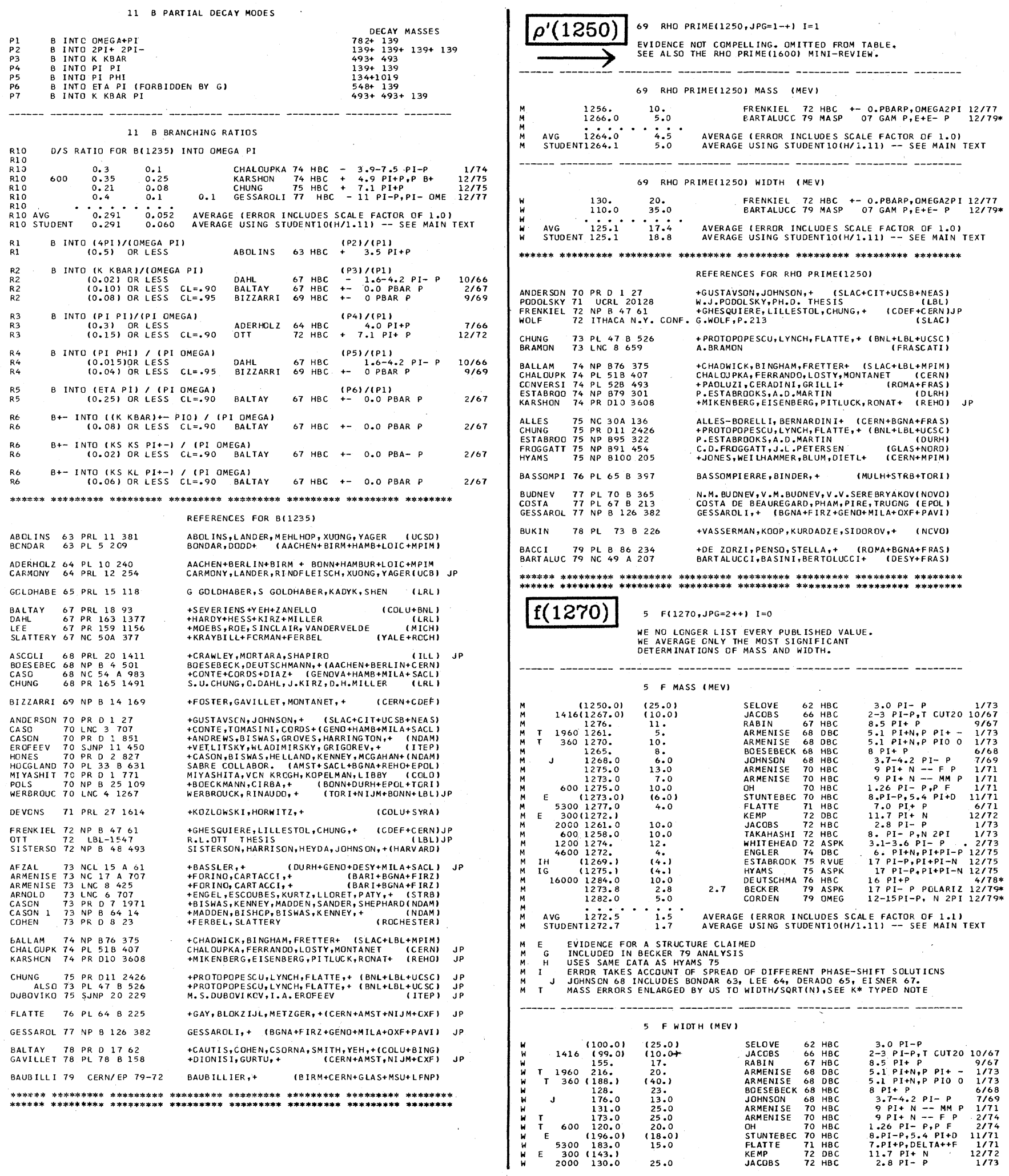


Mesons $\mathrm{f}(1270)$

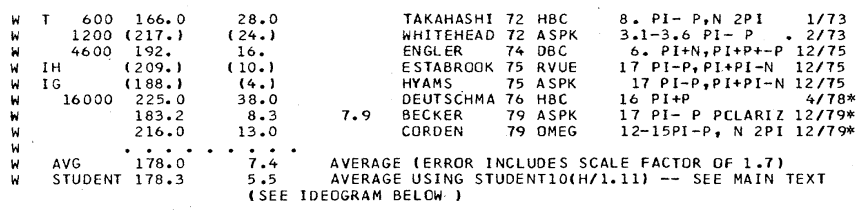

$W$
$W$
$W$

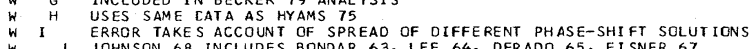

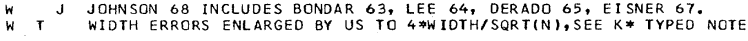
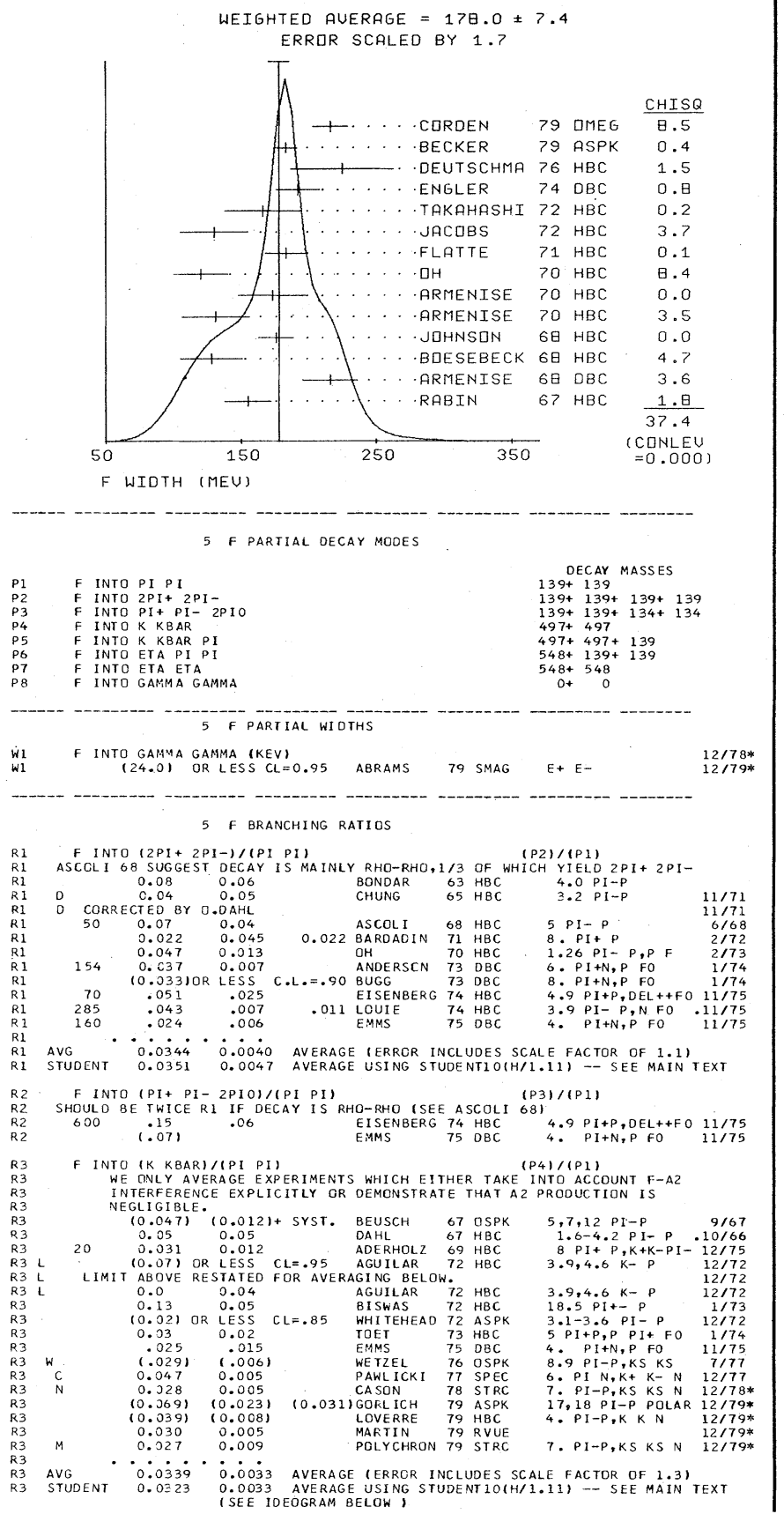

\section{Data Card Listings For notation, see key at front of Listings.}

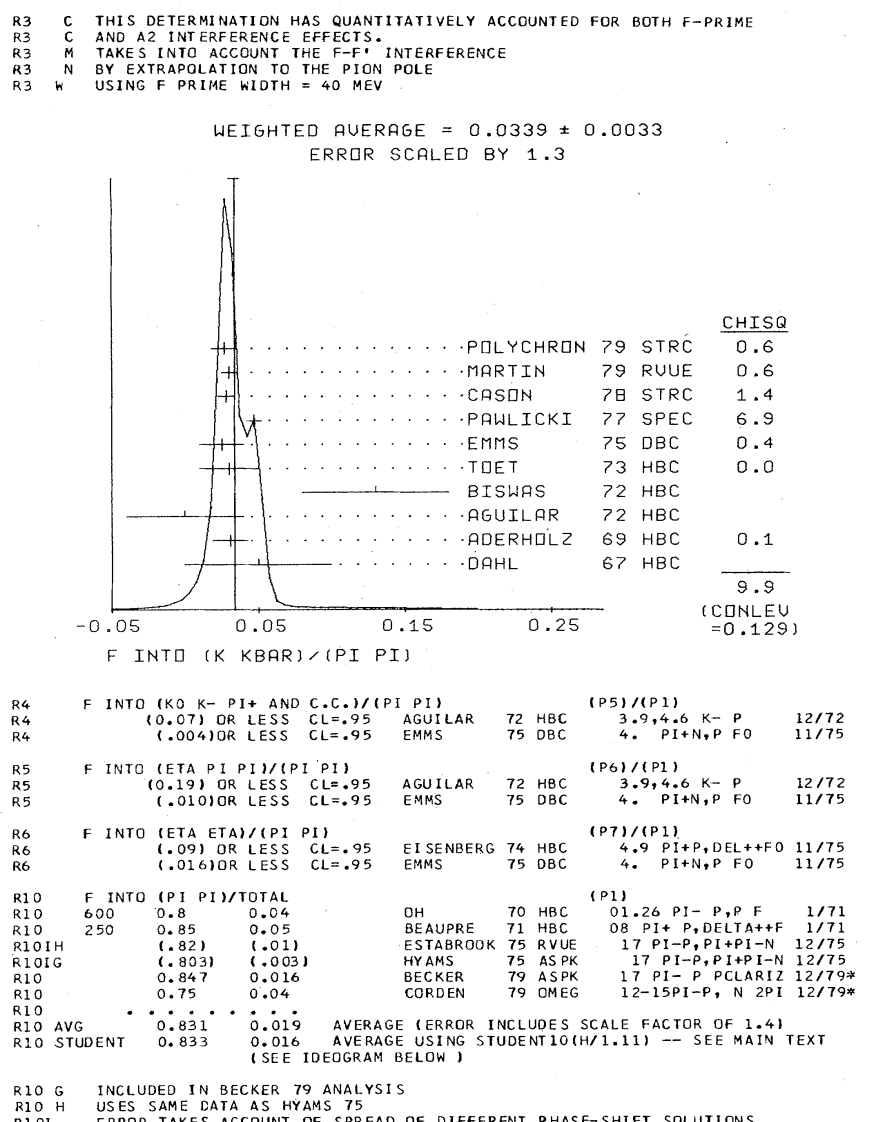

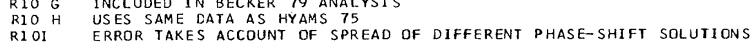

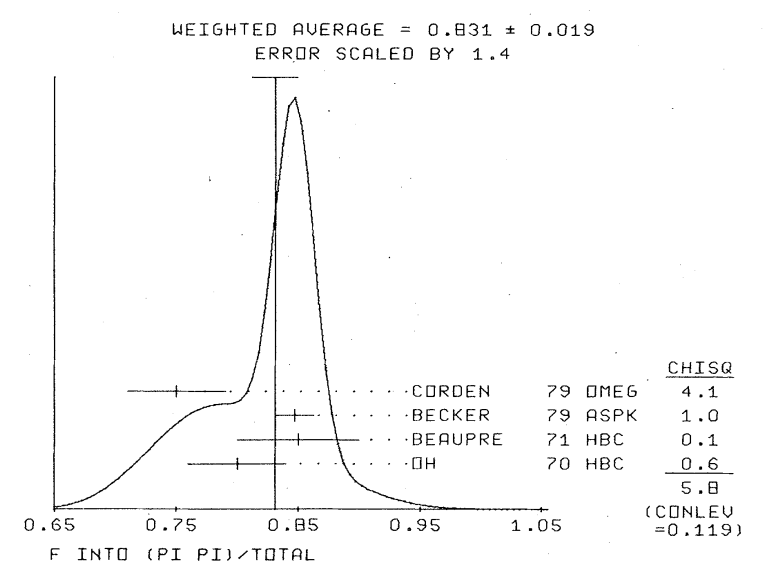

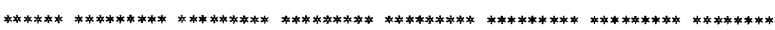

$\begin{array}{lllll}\text { SELOVE } & 62 & \text { PRL } & 9 & 272 \\ \text { BENDAR } & 63 & \text { PL } & 5 & 153\end{array}$

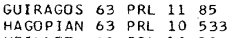
$\begin{array}{lllll}\text { ADERHOLZ } & 64 & \text { PL } & 10 & 240 \\ \text { BRUYANT } & 64 & \text { PL } 10 & 10 & 232\end{array}$

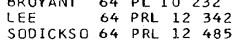
BARMIN 65 SJNP 1230

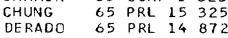

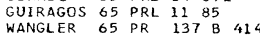

REFERENCES FOR F

SELOVE, HAGOPIAN, BRODY, BAKER, LE BOY (PENN)
BONDAR+ (AACHEN+BIRM+BONN+DESY+LOIC+MPIM)

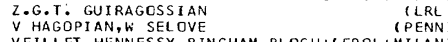
AACHEN-BERLIN-BERLIN-BONN-HAMBURG -LOIC-MPI IJ
BRUY ANT, GOLDBERG, HOL DER, FLEURY + (CER +EPOL) I LEE, ROE, SINCLAIR, VANDERVELDE +DOLGOLENKO, ELENSKY, EROFEEV+ (ITEP MOSCOW) JP CHUNG, DAHL, HARDY, HESS, JACOBS, KIRZ (LRL) DERADO, KENNEY, POIRIER, SHEPHARO (NOTRE DAME) Z G T GUIRAGOSSIAN 


\section{Data Card Listings}

Mesons
f(1270), $\eta(1275), D(1285)$

For notation, see key at front of Listings.
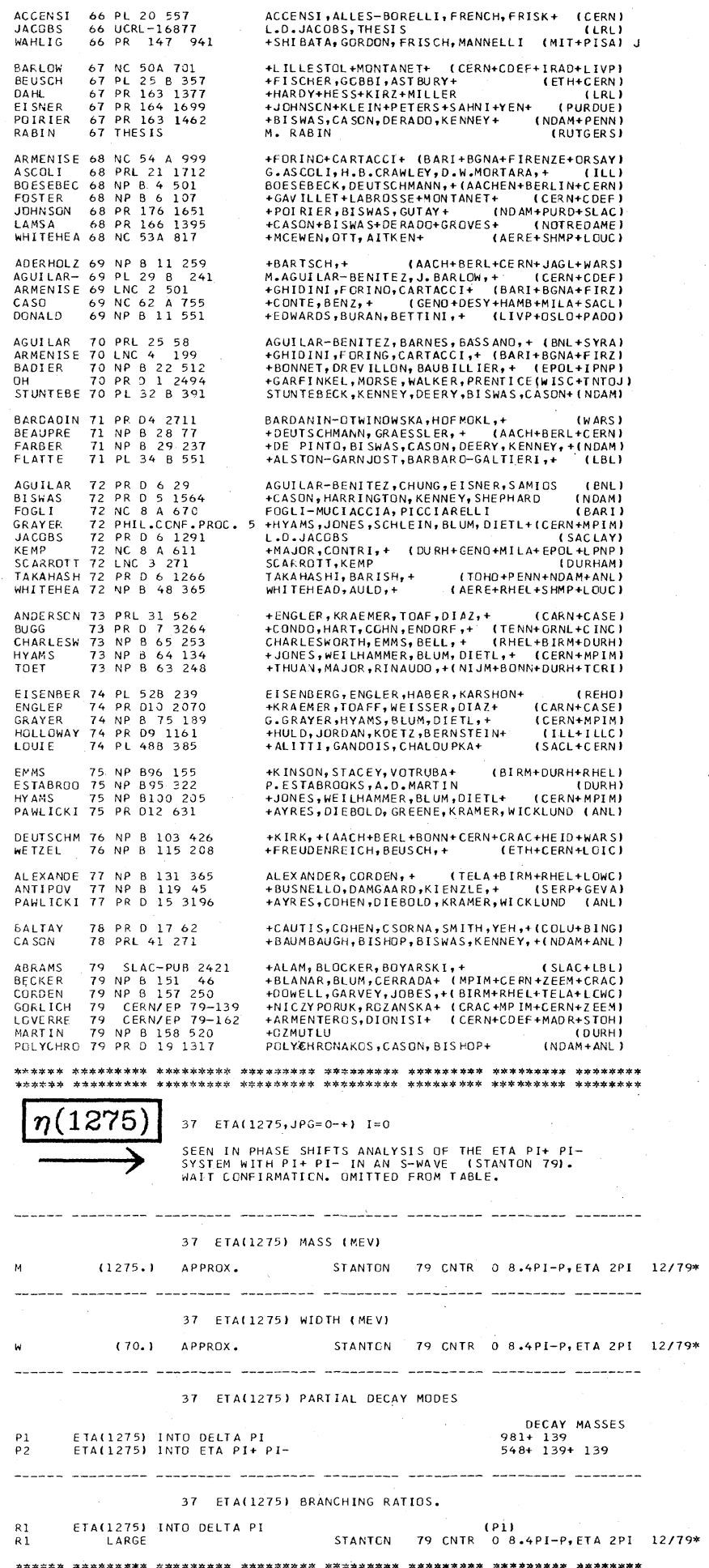

REFERENCES FOR ETA(1275)

STANTON 79 PRL 42346 +BROCKMAN, DANKOWYCH, + COSU+CARL +MCGI+TNTOI JP

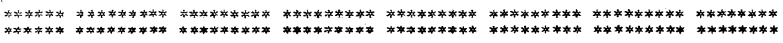

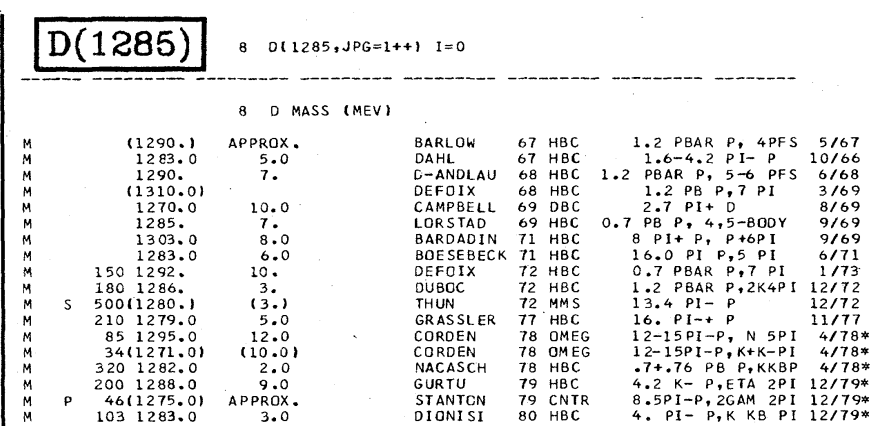

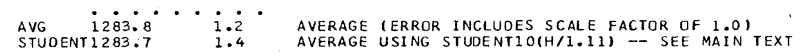

$\begin{array}{lll}M & P & \text { FROM PHASE SHIFT ANALYSIS OF ETA PI+PI - SYSTEM. } \\ M & S & \text { SEEN IN THE MISSING MASS SPECTRUM }\end{array}$

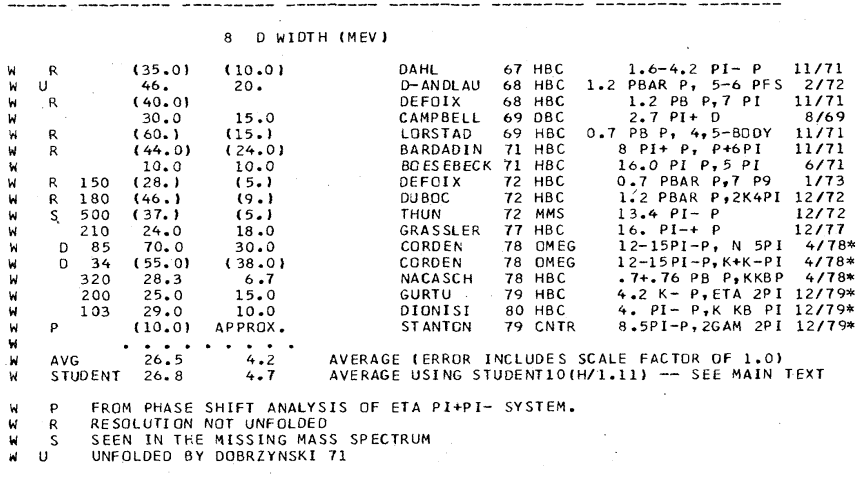

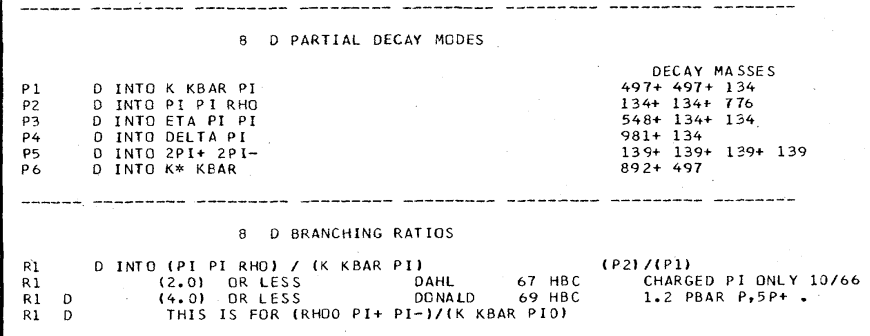

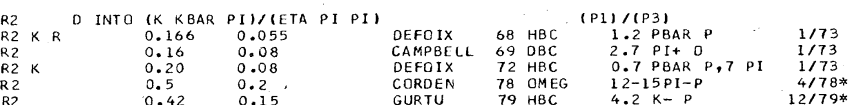

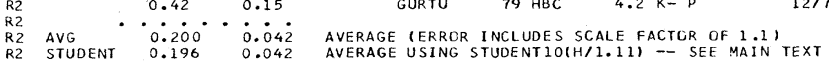

$\begin{array}{lll}R 2 & K & K \\ R 2 & K & \text { KAR SYSTEM CHARACTERIZED BY THE I I I THRESHCLO } \\ \text { R2 } & K\end{array}$

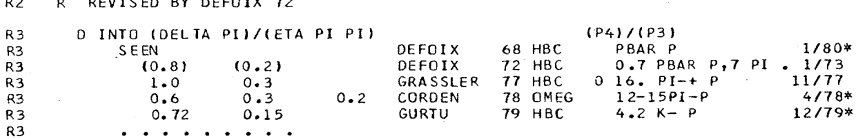

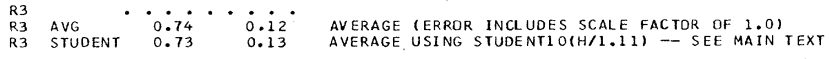

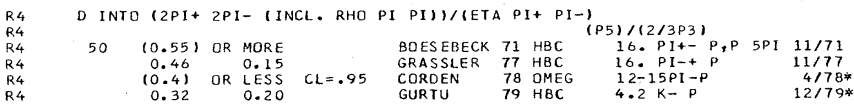

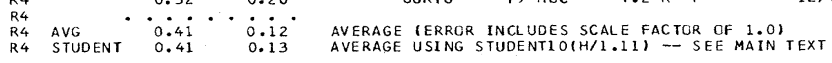

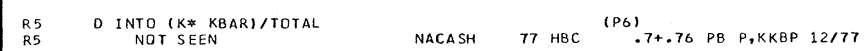

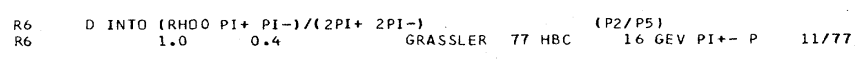




\section{Mesons}

$D(1285), \epsilon(1300)$

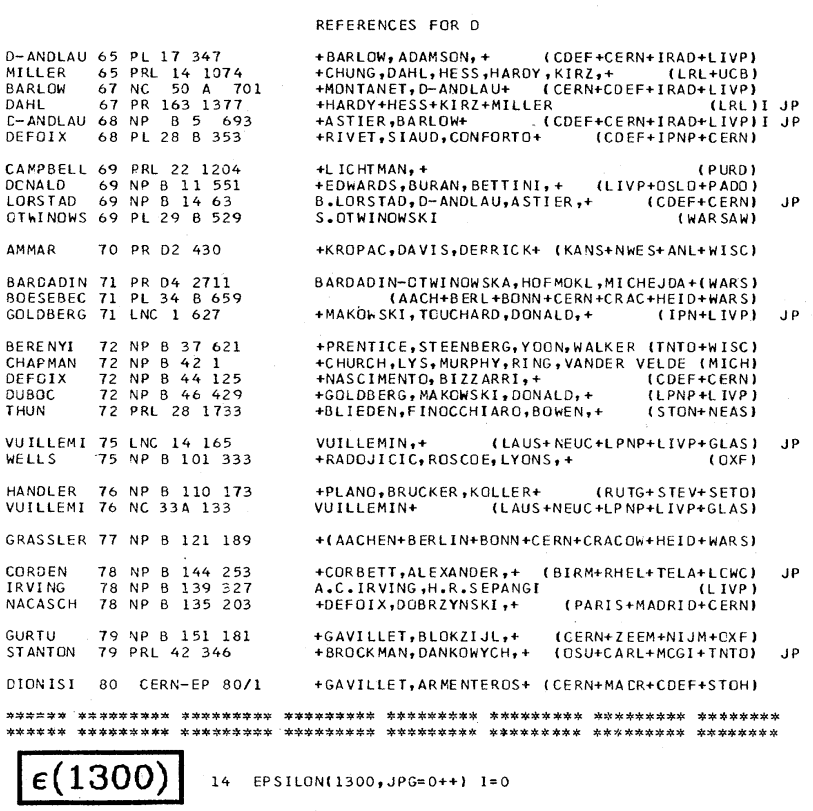

S-Wave $\pi \pi$ and $K \bar{K}$ Interactions

In this note we discuss information on the non-strange $I^{G} J^{P C}=0^{+} 0^{++}$partial wave ( $S$ wave) coupled to the $\pi \pi$ and $\mathrm{K} \overline{\mathrm{K}}$ systems.

Near the $\pi \pi$ threshold the $\mathrm{S}$ wave shows no resonant behavior. For a discussion of the relevant scattering lengths and various resonance-like kinematic effects, see our 1978 edition.

Up to the $\rho$ meson mass region, the phase shift $\delta_{0}^{0}$ is (qualitatively) uniquely determined: it rises monotonically and reaches $60^{\circ}$ to $70^{\circ}$ near $700 \mathrm{MeV}$ (SONDEREGGER 69, BATON 70, BAILLON 72, CARROLL 72, FRENKIEL 72, GAIDOS 72, PROTOPOPESCU 73, HYAMS 73, OCHS 73, ENGLER 74, ESTABROOKS 74,75, GRAYER 74).

In the early phase-shift analyses two solutions for $\delta_{0}^{0}$ were found (the "up-down ambiguity") in the 700 to $900 \mathrm{MeV}$ region. The "up" solution corresponds to an $\varepsilon$ resonance under the $\rho$ meson with mass and width similar to the $\rho$ meson, the $\varepsilon(800)$. The "down" solution is characterized by an approximately energy-independent phase shift of almost $90^{\circ}$, showing no resonant behavior. This ambiguity was considered resolved in favor of the "down" solution by the observation of a very rapid decrease in the modulus of the S-wave amplitude between $900 \mathrm{MeV}$ and the $\mathrm{k} \overline{\mathrm{K}}$ threshold, followed by a sharp drop in the elasticity. $\delta_{0}^{0}$ is $\sim 90^{\circ}$ at about $900 \mathrm{MeV}$ and reaches $\sim 180^{\circ}$ around $990 \mathrm{MeV}$ (FLATTE 72, GAIDOS 72, HYAMS 73,

\section{Data Card Listings For notation, see key at front of Listings.}

BINNIE 73, ENGLER 74). However, the region is complicated by the simultaneous presence of the $S$ * resonance and the opening of the $\mathrm{K} \overline{\mathrm{K}}$ channel, permitting almost discontinuous jumps from one solution to another.

Without polarization information, the reaction $\pi N \rightarrow \pi \pi N$ cannot be analyzed unambiguously due to the fact that there are more helicity amplitudes than observables (see, e.g., DONOHUE 75). Thus one is obljged to make some supplementary assumptions.

An amplitude analysis (ESTABROOKS 74) of the largest $\pi^{-} \mathrm{p}$ (unpolarized) $\rightarrow \pi^{+} \pi^{-} \mathrm{n}$ experiment (HYAMS 73, GRAYER 74) still finds both the "up" and the "down" solutions. This analysis assumes both spin coherence (the unnatural-parity-exchange, s-channel helicity amplitudes are nucleon spin-flip, i.e., no $A_{1}$-like exchange), and phase coherence (the $S$-wave amplitude and the unnatural-parity-exchange, meson helicity-zero P-wave amplitude have the same phase). These assumptions may tend to bias the results (MORGAN 74, DONOHUE 75,79 ).

The advent of $\pi^{-} p$ (polarized) $\rightarrow \pi^{+} \pi^{-} n$ data (BECKER 79) has made both the spin coherence and phase coherence assumptions unnecessary. Analyzing their data in a model-independent way, BECKER2 79 a].so find both the "up" and the "down" solutions. The reaction $\pi^{+} p \rightarrow \pi^{+} \pi^{-} \Delta^{++}$has been analyzed in the region 660 to $860 \mathrm{MeV}$ (OWENS 76, DONOHUE 79) and in the region 600 to $920 \mathrm{MeV}$ (GELFAND 78), using all the information carried by the $\Delta^{++}$decay. The conclusion from both analyses is that the $\varepsilon(800)$ of the "up" solution cannot be ruled out.

In a coupled-channel fit of various pole parametrizations to both $\pi \pi \rightarrow \pi \pi$ (ESTABROOKS 74) and $\pi \pi \rightarrow K \bar{K}$ data (CASON 76, PAWLICKI 77), ESTABROOKS 79 finds a pole located at 720 to $800 \mathrm{MeV}$ with a width of 800 to $1000 \mathrm{MeV}$. Note that the "down" solution of ESTABROOKS 74 was used as input to this analysis. Further indirect information comes from elastic $\pi \pi$ scattering in the crossed channel (ELVEKJAER 72, NIELSEN 70,72) in agreement with the "down" solution, but not with the "up" solution.

The only way to rule out the "up" solution at present is to study the $\pi^{0} \pi^{0}$ system, where the "up" solution predicts a $p$-meson-like bump unmasked by the $\rho$ meson. With the exception of one experiment (DAVID 77), all the $\pi^{0} \pi^{0}$ experiments agree that no 


\section{Data Card Listings \\ For notation, see key at front of Listings.}

Mesons

$\epsilon(1300)$

such bump is present and that the "down" solution describes the data well (DEINET 69, BENSINGER 71, APEL 72,79, BRAUN 73, SKUJA 73, RIESTER 75, BORREANI 79).

The region of elastic $\pi \pi$ scattering is known to extend to about $990 \mathrm{MeV}$, near the $\mathrm{K} \overrightarrow{\mathrm{K}}$ threshold (BATON 70, CARROLL 72, PROTOPOPESCU 73, HYAMS 73, OCHS 73). Beyond $1 \mathrm{GeV}$ we therefore have to consider the two channels $\pi \pi$ and $k \bar{K}$. In addition, the solutions have inherent ambiguities related to the Barrelet zeros of the amplitudes. Thus HYAMS 75 find four solutions in the region 1.0 to $1.8 \mathrm{GeV}$, ESTABROOKS 74 find eight solutions, and CORDEN 79, extending the $\pi \pi$ analysis to $2.08 \mathrm{GeV}$, find another eight solutions.

In the past many of these solutions have been ruled out by imposing continuity in various ways, as well as analyticity and unitarity (FROGGATT 75,77, COMMON 76, MARTIN 78).

Now that data on $\pi^{-} \mathrm{p}$ (polarized) $\rightarrow \pi^{+} \pi^{-} \mathrm{n}$ are available (BECKER 79), there is no need for such arguments. The model-independent partial-wave analysis of BECKER2 79 selects solution $\beta^{\prime}$ of MARTIN 78 and possibly solution $\beta$.

The $\beta$ and $\beta^{\prime}$ amplitudes describe the experimental moments in each bin without any explicit smoothing; they are analytic in $s$ and approximately analytic in $\cos \theta$. They take into account all waves up to $l=4$. The $\beta$ solution has a highly elastic $S$ wave, whereas the $S$ wave of solution $\beta^{\prime}$ is somewhat inelastic (MARTIN 78). The unique solution of FROGGATT 77, which has explicit smoothness built in and which takes account only of $l \leqslant 3$ waves, is rather similar to $\beta$. However, it has problems with unitarity, apparently because of the neglected G wave (MARTIN 78).

The $S$ wave is clearly resonant in the data of BECKER2 79. In the 1150 to $1400 \mathrm{MeV}$ region both the S-P and S-D phase differences show the presence of a broad resonance, and the intensity of the $S$ wave confirms this by exhibiting a peak at about $1300 \mathrm{MeV}$ with a width of about $300 \mathrm{MeV}$; see Fig. I(a).

The amplitude analysis of the $\pi^{-} p \rightarrow \pi^{+} \pi^{-{ }^{-}} n$ experiment of CORDEN 79 has two preferred solutions which are close to $\beta$ and $\beta^{\prime}$, giving some support for an $\varepsilon(1300)$. Also the $S$ wave in the $\pi^{0} \pi^{0}$ system
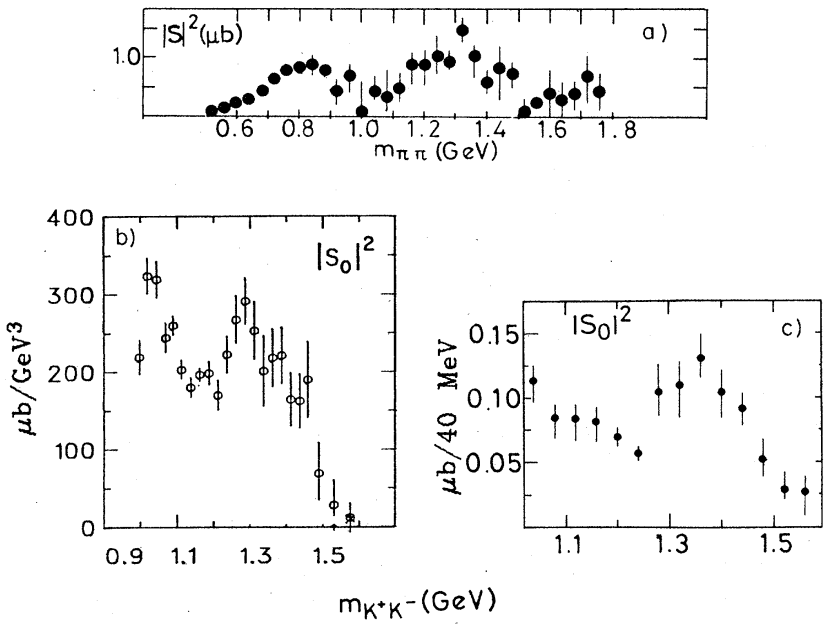

Fig. 1. (a) The absolute intensity (in $\mu \mathrm{b}$ ) of the $\pi^{+} \pi^{-}$. S wave in $40 \mathrm{MeV}$ bins (without dividing by the bin size), as given by the "down" solution of BECKER2 79. (b) Absolute intensity (in $\mu \mathrm{b} / \mathrm{GeV}^{3}$ ) of the $\mathrm{K}^{+} \mathrm{K}^{-} \mathrm{S}$ wave, as given by the favored solution of COHEN 78, for $|t|<0.08 \mathrm{GeV}^{2}$. (c) The absolute intensity (in $\mu \mathrm{b} / 40 \mathrm{MeV}$ bin) of the $\mathrm{K}^{+} \mathrm{K}^{-} \mathrm{S}$ wave, as given by the favored solution of GORLICH 79.

tends to confirm the $\varepsilon(1300)$ by staying near its unitarity limit around $1200 \mathrm{MeV}$ (APEL 79). Independent evidence for the $\varepsilon(1300)$ comes from studies of the $\mathrm{K} \vec{K}$ systems. In the reaction $\pi^{-} \mathrm{p} \rightarrow \mathrm{K}_{\mathrm{S}}^{0} \mathrm{~K}_{\mathrm{S}}^{0} \mathrm{n}$, the $\mathrm{S}$ wave exhibits a large intensity in the $1300 \mathrm{MeV}$ region (WETZEL 76, LOVERRE 79), with some evidence for a bump. Moreover, the $\left\langle\mathrm{Y}_{0}^{2}\right\rangle$ moment shows a large negative excursion indicating S-D interference (CASON 76, WETZEL 76, LOVERRE 79, POLYCHRONAKOS 79). The main problem is the isospin of the bump: if OPE were the only mechanism, $I=0$ would be assured. However, an $I=1$ non-OPE contribution in the same region cannot be excluded. Moreover, the $\mathrm{I}=1 \mathrm{~K}^{-} \mathrm{K}^{0}$ system does show some peaking (MARTIN 79), so one will possibly have to disentangle two resonances in the $\mathrm{K}_{\mathrm{S}}^{0} \mathrm{~K}_{\mathrm{S}}^{0}$ bump.

In agreement with this, the $\mathrm{K}^{+} \mathrm{K}^{-}$system produced in $\pi^{-} \mathrm{p}, \pi^{+} \mathrm{n}$, and $\pi^{-} \mathrm{p}$ (polarized) scattering clearly shows the $S$ wave peaking at $1300 \mathrm{MeV}$; again, both $I=0$ and $I=I$ may be present. While PAWLICKI 77, COHEN 78, and GORLICH 79 favor $I=0$, MARTIN 79 concludes that the isospin cannot be assigned unambiguously. The experiments disagree on the strength of the $\varepsilon(1300)$ coupling to $\mathrm{K} \bar{K}$. 


\section{Mesons $\epsilon(1300)$}

The ANL group (PAWLICKI 77, COHEN 78) find a relatively narrow $\varepsilon$ with a width $\sim 200 \mathrm{MeV}$ [see Fig. I(b)], whereas the GORLICH 79 peak is smaller and broader [see Fig. I(c)]. Part of the disagreement may be due to model-dependent assumptions in the ANL analysis. Note, however, that the S-wave amplitude and phase of the ANL experiment are impossible to fit with an $S^{*}$ and a narrow $\varepsilon$ (1300) (MARTIN 79). On the other hand, the ANL S-wave amplitude and phase are quite well described by an $\mathrm{S}^{*}$ and a broad $\varepsilon(1300)$, just as are the amplitude and phase of GORLICH 79 .

Thus in summary of the 1000 to $1400 \mathrm{MeV}$ region: the $\varepsilon(1300)$ exists, it is about $300 \mathrm{MeV}$ wide, and it couples to $\mathrm{K} \overline{\mathrm{K}}$ with a branching ratio of the order of 7\% (GORLICH 79, LOVERRE 79) or $\lesssim 10 \%$ (ESTABROOKS 79, GREENHUT 79). The elasticity of the $\beta^{\prime}$ solution (MARTIN 78) also seems to be of this order of magnitude.

Above the $\varepsilon(1300)$ resonance the phase shift has completed a full circle in the Argand plane, as witnessed by the almost vanishing amplitude near $1550 \mathrm{MeV}$ (BECKER2 79, GORLICH 79).

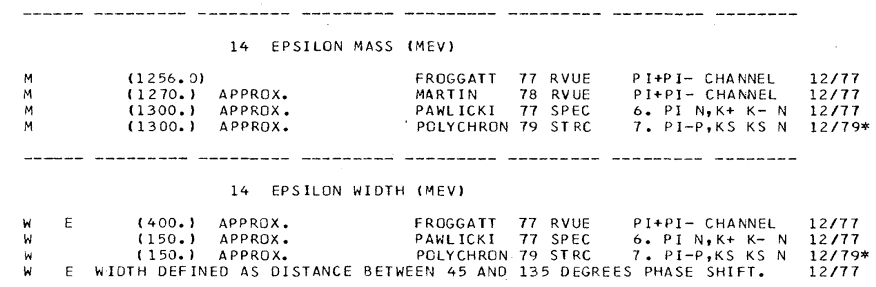

\begin{tabular}{|c|c|c|}
\hline & & REFE \\
\hline SAMIOS & 62 PRL 9139 & $(B N L+C U N Y+C C L U+K N T Y)$ \\
\hline $\begin{array}{l}\text { CKHINT } \\
\text { OTH } \\
\text { KZ }\end{array}$ & 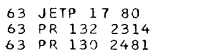 & 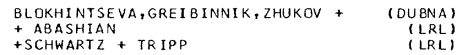 \\
\hline $\begin{array}{l}\text { ARISH } \\
\text { RANFOKD } \\
\text { EL FABR } \\
\text { ALHAS }\end{array}$ & 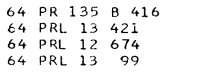 & 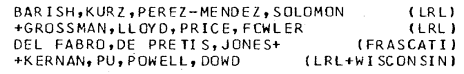 \\
\hline $\begin{array}{l}\text { BATCN } \\
\text { BIRGE } \\
\text { BROWN } \\
\text { DURAND }\end{array}$ & $\begin{array}{l}6.5 \text { NC } 361149 \\
65 \text { PR } 139 \text { B } 1600 \\
65 \text { CORAL GABLES } 219 \\
65 \text { PRL } 14329\end{array}$ & $\begin{array}{lr}\text { J.P. BATON, J.REGNIER } & \text { (SACLAY) } \\
\text { +ELLYGIDAL+KALMUS +CAMERINI + } & \text { (LRL+WISC) } \\
\text { BROWN+FAIER } & \text { (NORTHWESTERN) } \\
\text { L. DURAND AND Y.T. CHIU } & \text { (YALE) }\end{array}$ \\
\hline $\begin{array}{l}\text { COBS } \\
\text { PPELMAN } \\
\text { VELACEE }\end{array}$ & $\begin{array}{llll}66 & \text { PRL } & 16 & 669 \\
66 & \text { PL } & 22 & 1118 \\
66 & \text { PL } & 22 & 332\end{array}$ & $\begin{array}{lr}\text { +SELOVE } & \text { (LRL) } \\
\text { +ALLEN, GODDEN, MARSHALL + } & \text { (COLLRADO+1OWA) } \\
\text { LOVELACE, HEINZ, DCNNACHIE } & \text { (CERN) }\end{array}$ \\
\hline $\begin{array}{l}\text { ANDERSON } \\
\text { CLEGG } \\
\text { CORBETT } \\
\text { GUTAY } \\
\text { JOHNSCN } \\
\text { MALAMUD } \\
\text { WALKER } \\
\text { WALKER }\end{array}$ & 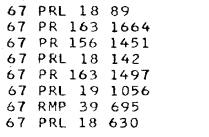 & 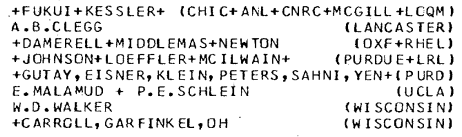 \\
\hline 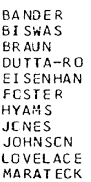 & 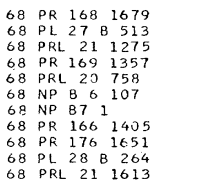 & 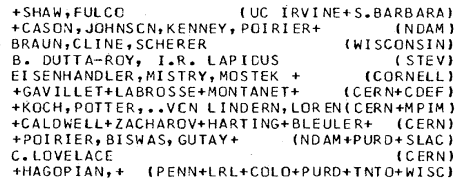 \\
\hline
\end{tabular}

\section{Data Card Listings For notation, see key at front of Listings.}

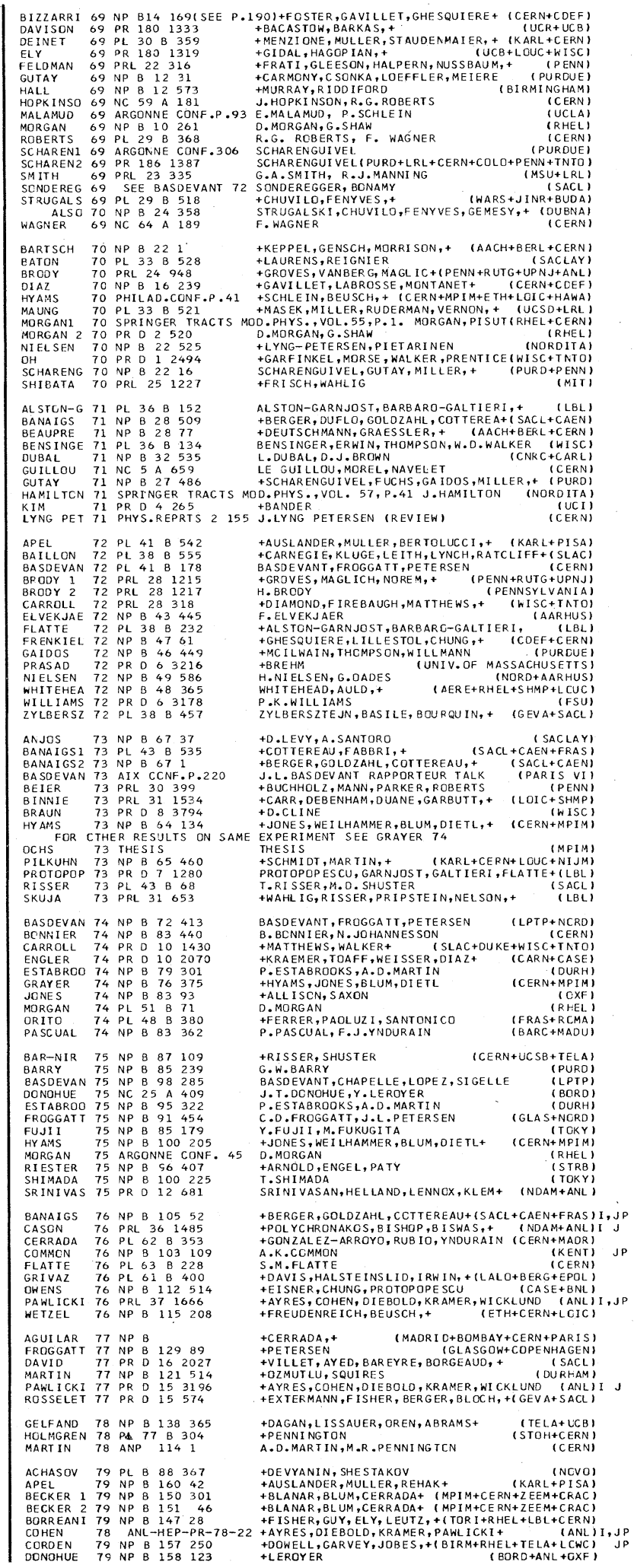




\section{Data Card Listings}

$\mathrm{A}_{2}(1310)$ 12 A2(1310, JPG 2+-1) I=1 WE LIST THE A2 AS AN ORDINARY BRE IT-WIGNER RESONANCE.
FOR DISCUSSICN OF THE REPORTEO SPLITTING, SEE OUR 72 AND APRIL 73 EDITION.

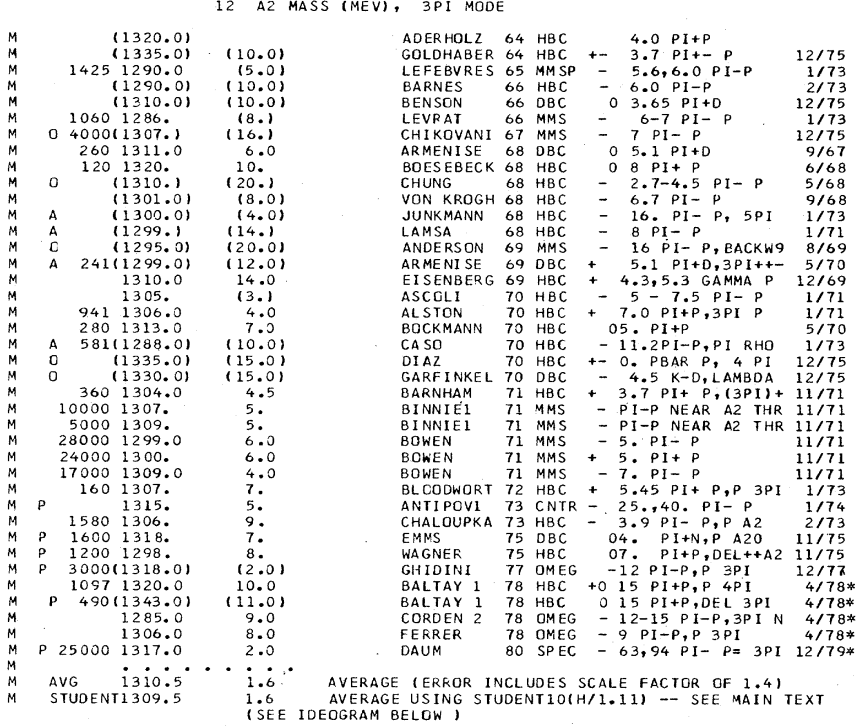

$M$ M CNLY EXPERIMENTS GIVING ERROR LESS THAN 15 MEV KEPT FOR AVERAGING $M$
$M$

12 A2 MASS (MEV), K KBAR MODE

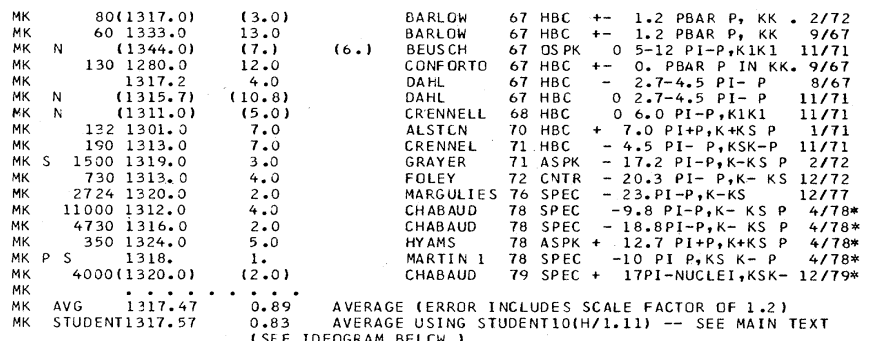

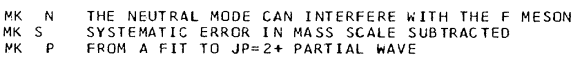

12. A2 MASS (MEV), ETA PI MODE

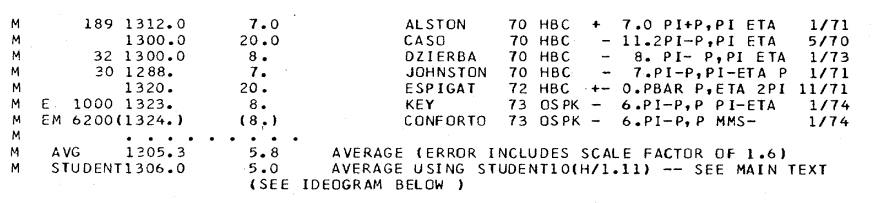

$M{ }_{M}{ }_{M}$ ERROR INCLUOES 5 MEV SYSTEMATIC MASS-SCALE ERROR
$M$ MISSING MASS WITH ENRICHED MMS $=E T A$ PI - ETA $=2$ GAMMA
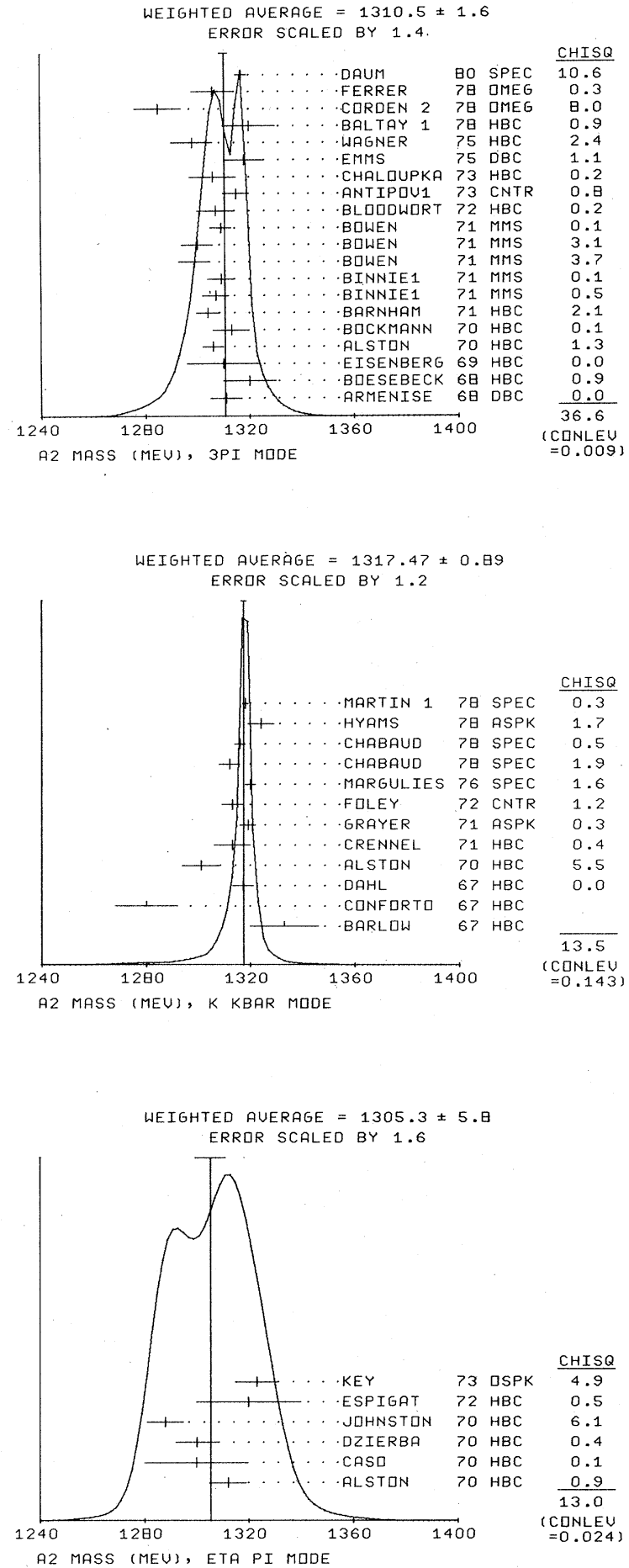
Mesons

$\mathrm{A}_{2}(1310)$
Data Card Listings For notation, see key at front of Listings.

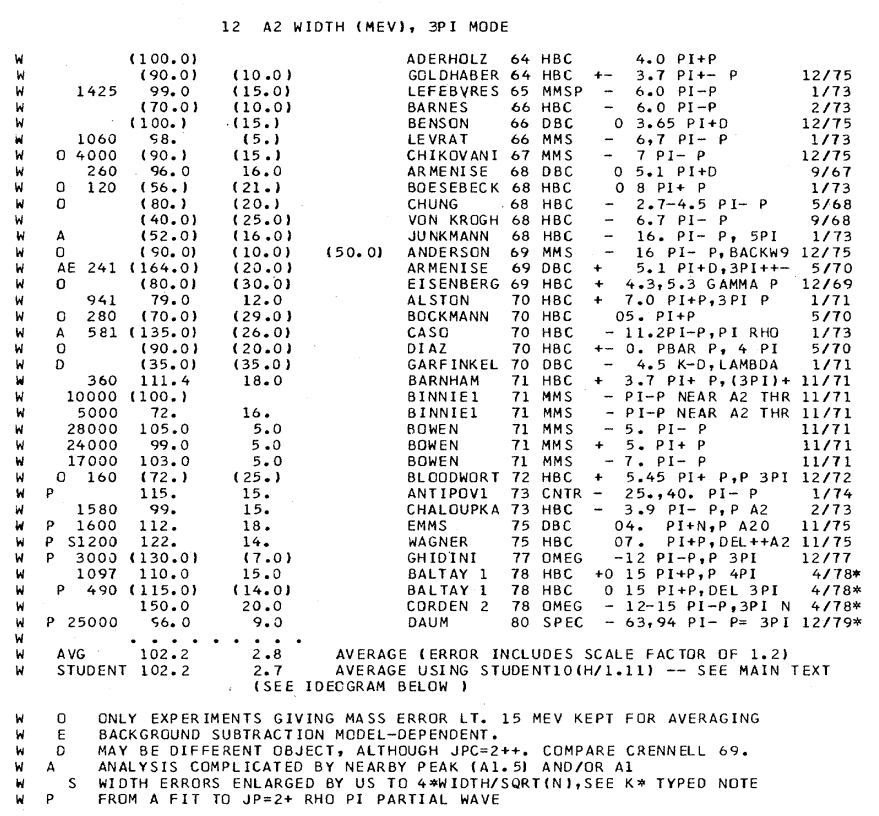

12 A2 WIDTH (MEV), K KBAR MODE

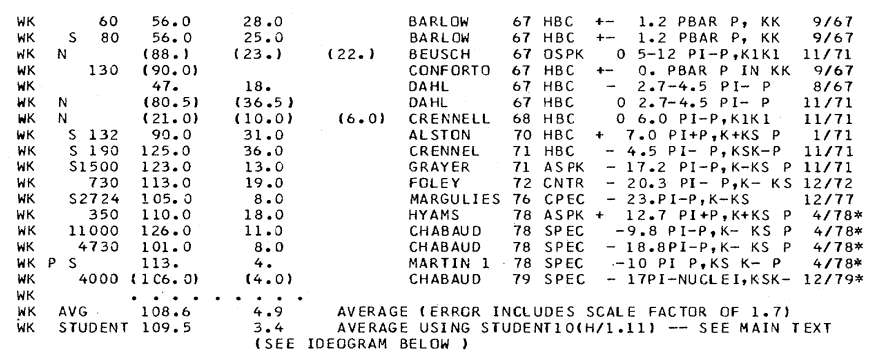

WK $P$ FROM A FIT TO JP=2+ PARTIAL WAVE.
WK N THE NEUTRAL MOOE CAN INTERFERE WITH THE F.MESON
WK S W WIDTH ERRORS ENLARGED BY US TO $4 *$ WIDTHSSORTINI,

WK N THE NEUTRAL MODE CAN INTERFERE WITH THE F. MESON
WK
S WIDTH ERRORS ENLARGED BY US TO 4 *WIDTH/SQRT(N), SEE K* TYPED NOTE

12 A2 WIDTH (MEV), ETA PI MODE

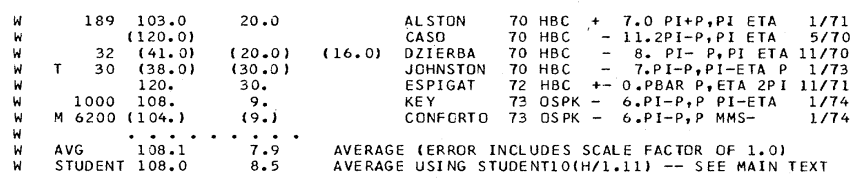

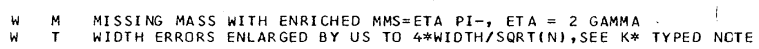

12 A2 PARTial DECAY MODES

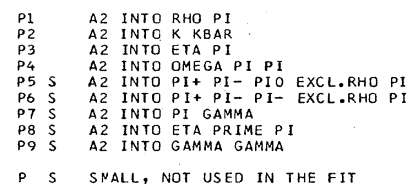

DECAY MASSES

$776+139$
$493+497$

$139+139+134$

$\begin{array}{rl}139+ & 0 \\ 957+139 & 0+13\end{array}$
$139+139+782$
$139+139+134$

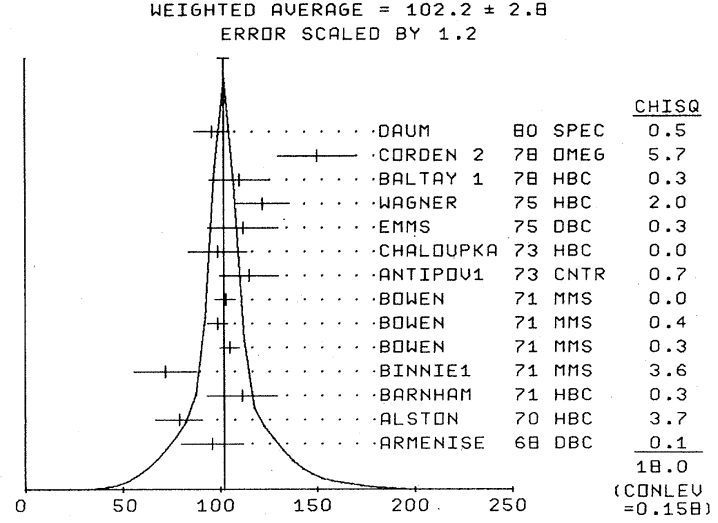

A2 WIDTH (MEU), 3PI MDDE

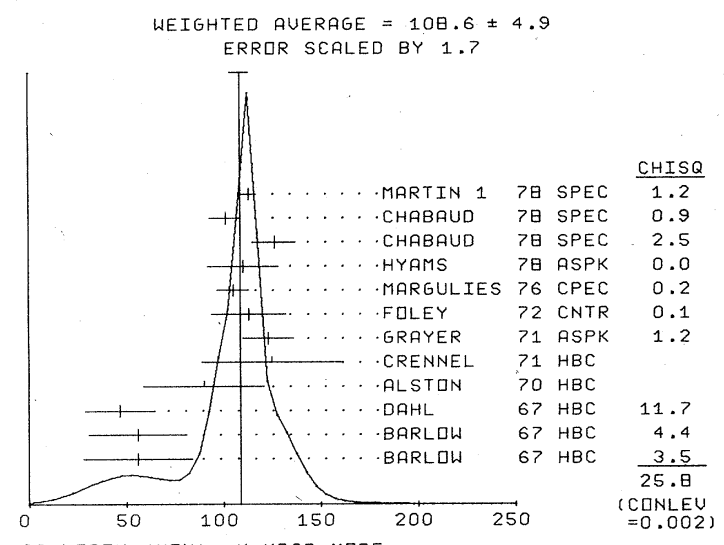

A2 WIDTH (MEU), K KBAR MDDE

\begin{tabular}{l} 
HISQ \\
\hline 0.5 \\
5.7 \\
0.3 \\
2.0 \\
0.3 \\
0.0 \\
0.7 \\
0.0 \\
0.4 \\
0.3 \\
3.6 \\
0.3 \\
3.7 \\
0.1 \\
\hline B.0 \\
NLEU \\
.15 B
\end{tabular}

ISQ

0.0

\section{FITTED PARTIAL DECAY MODE BRANCHING FRACTIONS}

The matrix below is derived from the error matrix for the fitted partial decay mode branching fractions, $P_{i}$, as follows: The diagonal elements are $P_{i} \pm \delta P_{i}$, where $\delta P_{i}=\sqrt{\left\langle\delta P_{i} \delta P_{i}\right\rangle}$, while the off-diagonal elements are the normalized correlation coefficients $\left\langle\delta \mathrm{P}_{i} \delta \mathrm{P}_{j}\right\rangle /\left(\delta \mathrm{P}_{\mathrm{i}} \cdot \delta \mathrm{P}_{j}\right)$. For the definitions of the individual $\mathrm{P}_{i}$, see the listings above; only those $P_{i}$ appearing in the matrix are assumed in the fit to be nonzero and are thus constrained to add to 1 .

$\begin{array}{ccccc}P & P 1 & P 2 & P 3 & P 4 \\ P & 1 & .7004+\cdots .0217 & P 2 \\ P & 2 & .1210 & .0479+-.0049 & \end{array}$

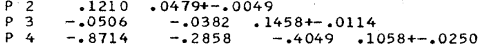

\section{A2 PARTIAL WIDTHS}

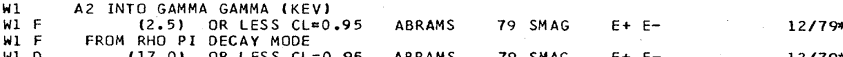

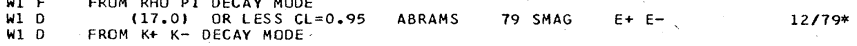




\section{Data Card Listings}

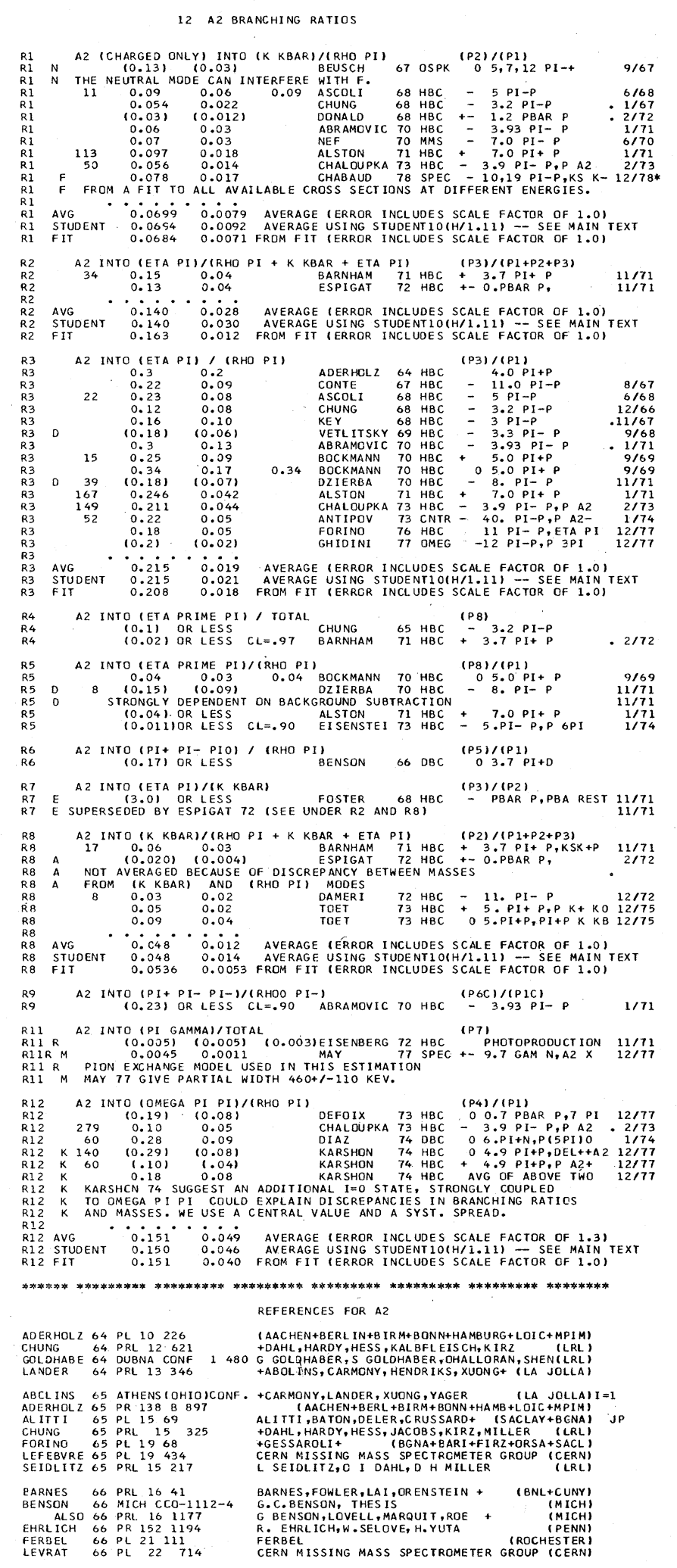

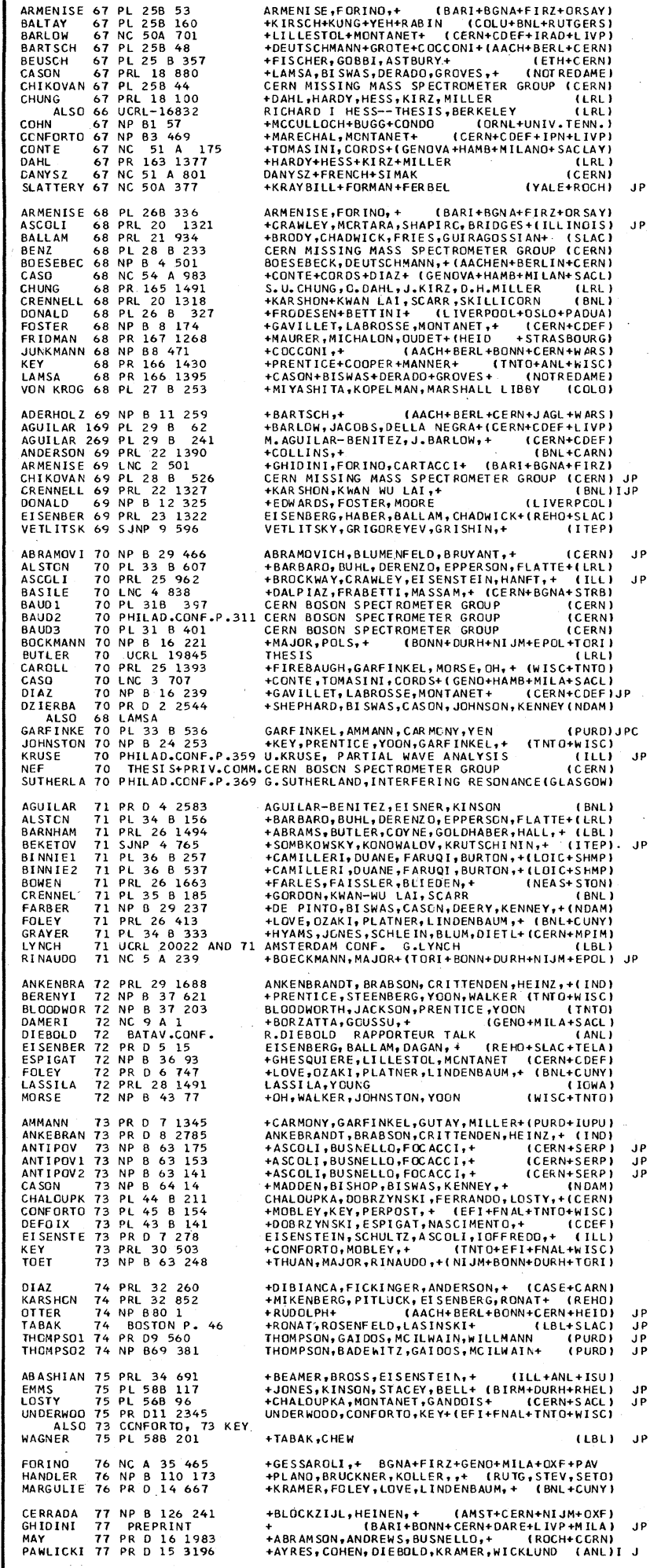




\section{Mesons}

$A_{2}(1310), E(1420), X(1410-1440), f^{\prime}(1515)$

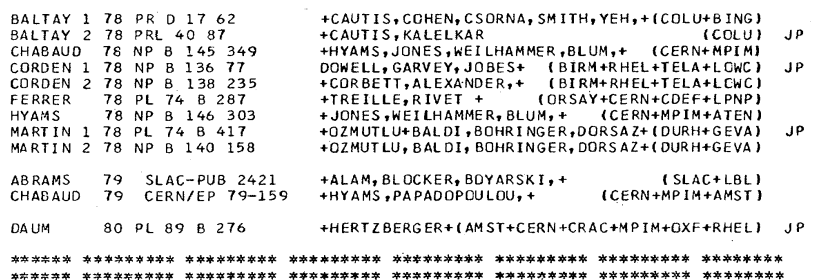

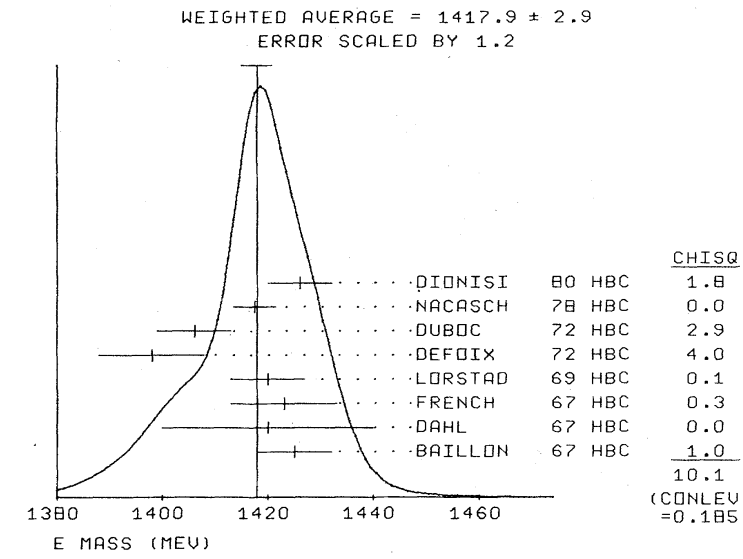

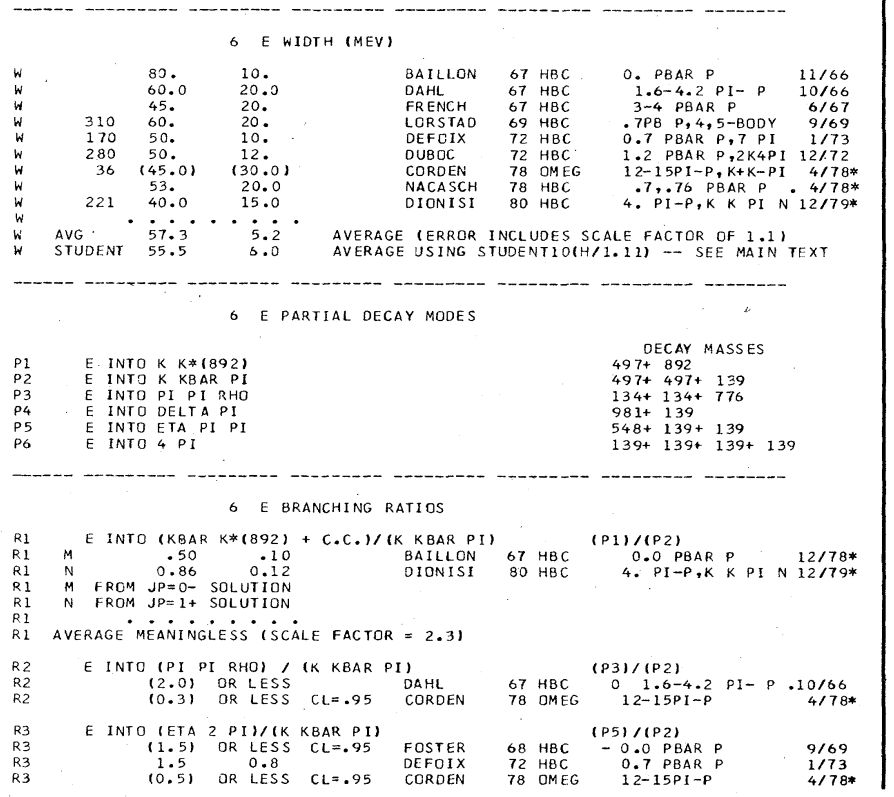

Data Card Listings For notation, see key at front of Listings.

$$
\mid
$$

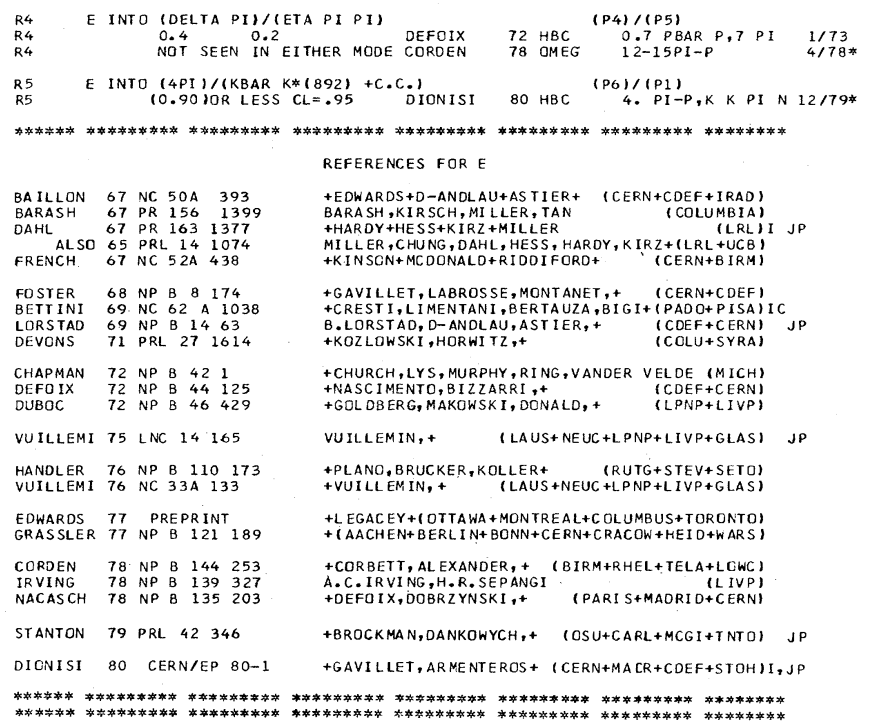

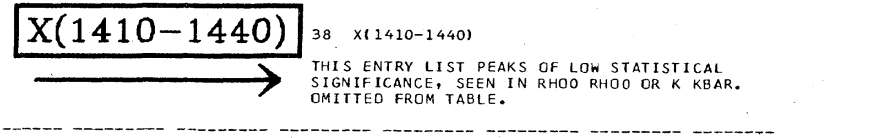$$
38 \times(1410-1440) \text { MASS (MEV) }
$$

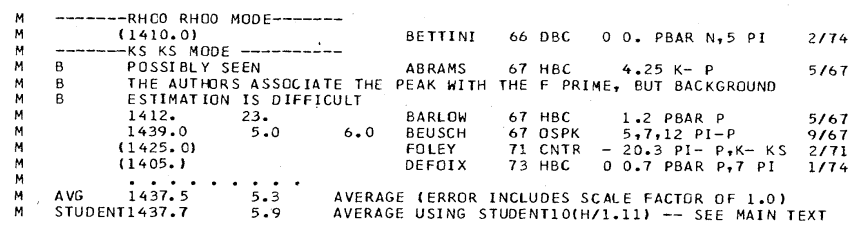

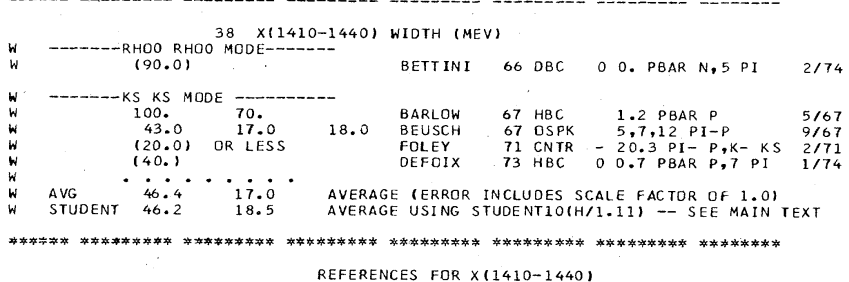

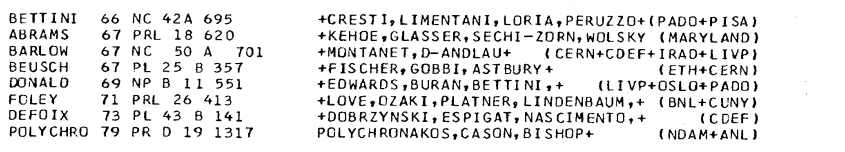

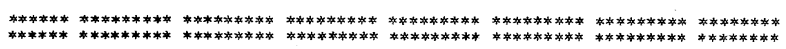$$
\mathrm{f}^{\prime}(1515) \quad 13 \quad \text { F PRIME(1515, JP G }=2++1 \text { I=0 }
$$

\begin{tabular}{cccccccc}
\multicolumn{7}{c}{ 13 F PRIME MASS IMEVI } \\
$M$ \\
$M$
\end{tabular}


Data Card Listings

For notation, see key at front of Listings.
Mesons $f^{\prime}(1515), F_{1}(1540), \rho^{\prime}(1600)$

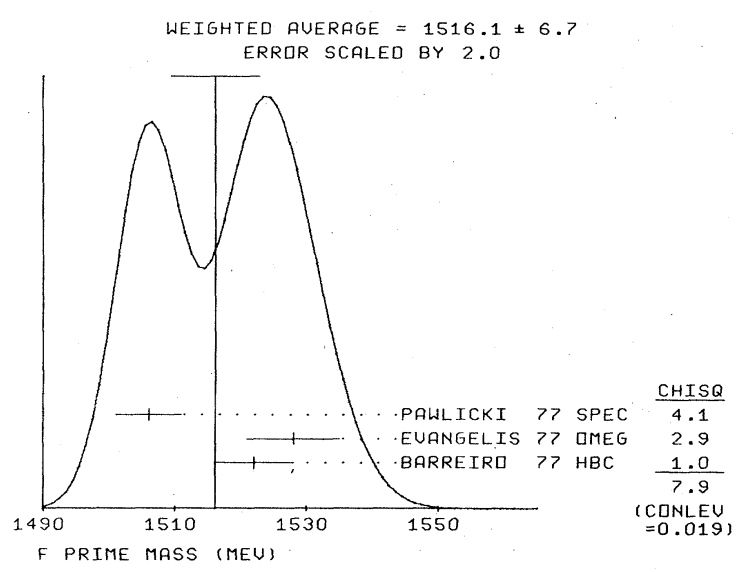

$M$ M WITH A PHASE SHIFT ANALYSIS
$M$ M MASS ERRORS ENLARGED BY US TC WIDTH/SQRTIN), SEE K* TYPED NCTE.

$M$ F FOM AN AMPLITUDE ANAL YSIS WHERE THE F PRIME WIDTH ANO
ELASTICI TY ARE IN COMPLETE DISAGREEMENT WITH VALUES

$M$
$M$
$M$

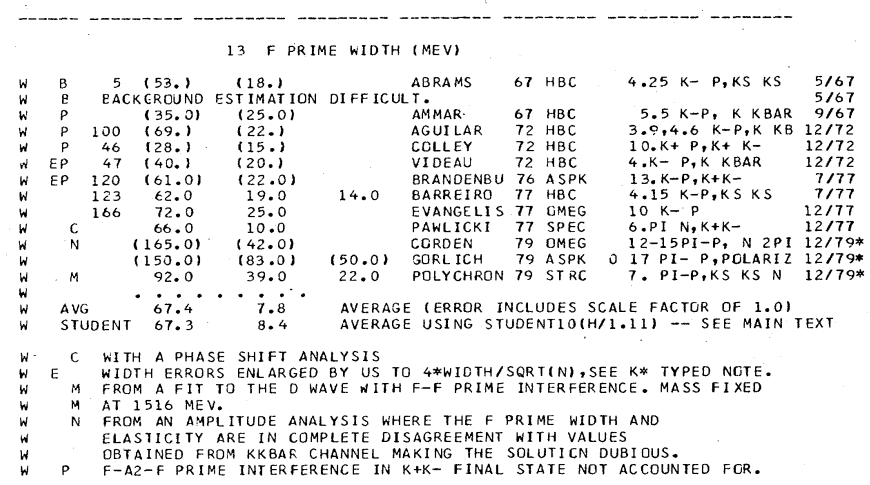

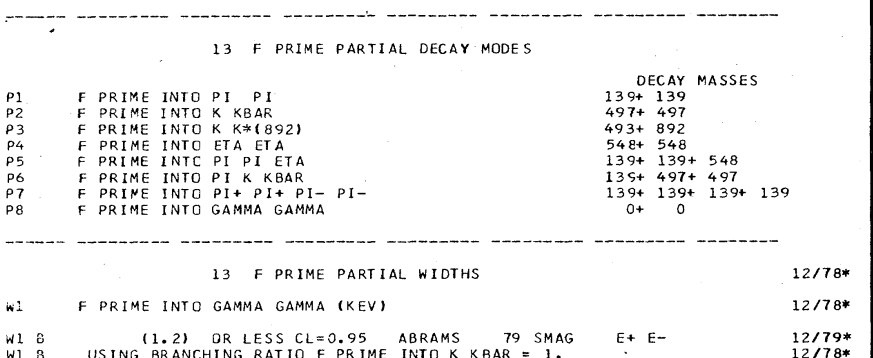

W1 8 USING BRANCHING RATIO F PRIME INTO K KBAR $=1$. $12 / 78 *$

13 F PRIME BRANCHING RATIOS

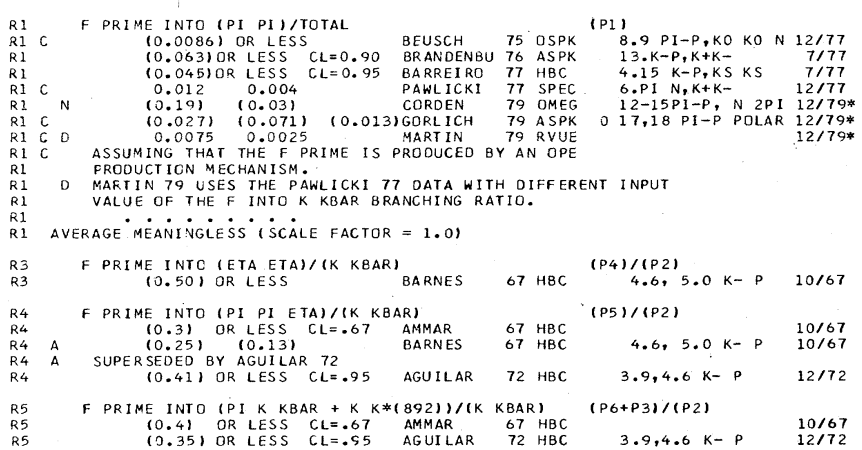

R6 F PRIME INTO (PI+ PI+ PI- PI-1/(K KBAR)
R6

REFERENCES FOR F PRIME

CRENNELL 66 PRL 161025 + KALBFLEISCH, LAI, SCARR, SCHUMANN + (BNL)I

ABRAMS 67 PRL 18620 TKEHOE, GLASSER, SECHI-ZORN, WOLSKY (MARYLAND)

BARNES 67 PRL 19 1071 TAVIS, HWANG, DAGAN, DERR ICK + (NWES+ANL) JP

$\begin{array}{llll}\text { ALIIII } 68 \text { PRL } 211705 & \text { +BARNES, CRENNELL, FLAMINIO, GOL DBERG, + (BNL) }\end{array}$

$\begin{array}{llllll}\text { LORSTAD } & 69 & \text { NP B } 14 & 63 & \text { B. LORSTAD, D-ANDLAU, ASTIER, + } & \text { (CDEF+CERN) } \\ \text { SCOTTER } & 69 & \text { NC } 62 \text { A } 1057 & \text { +ERSKINE, PALER, + } \\ \text { (BIRM +GLAS+LOIC+MPIM+OXF) }\end{array}$

AGUILAR 72 PR D 629

ILEAU $72 \mathrm{PL} 41$ B 213

BEUSCH 75 PL 60 B 101 + JOBES, RIDOIFORD, GRIFFITHS, + (BIRM+GLAS)

+BIRMAN, WEB SDALE, WETZEL (CERN+ETH)

BRANDE NBURG, CARNEG IE, CASHMORE, DAV I ER+ (SLAC)

+DIAZ, GAY, HEMINGWAY, + (CERN+AMST +NIJM+OXF) EVANGELISTA, + (BARI+BCNN+CERN+DARE+GLAS+)

+ AYRES, COHEN, DI EBOLD, KRAMER, WI ICKLUND (ANL) I JP

$\begin{array}{lllllll}\text { BARREIRO } & 77 & \text { NP } & 8 & 121 & 237 \\ \text { EVANGEL I } & 77 & \text { NP } & B & 127 & 384 \\ \text { PAEEN } & 77 & \text { PP } & \text { B } & 127 & 43\end{array}$

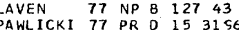

ABRAMS 79 SLAC-PUB 2421

+ALAM, BLOCKER, BOYARSK I ,

$(S L A C+L B L)$

COROEN 79 NP B 157250

GORLICH 79 CERN E P $79-139$
MART IN 79 NP B 158520

BLUM, CERRADA+ I MPIM+CERN+2EE +DOWELL, GARVEY, JOBES, + (BIRM+RHEL+TELA+LCWC) JP NICZY PORUK, ROZANSKA+ (CRAC+MP IM+CERN+ZEEM) OOZMUTLU
POLYCHRONAKOS, CASON, BISHOP. (OURH)

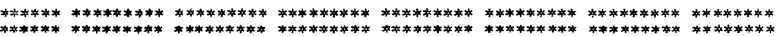

$F_{1}(1540)$ 47 F111540, JPG $=\quad, I=1$

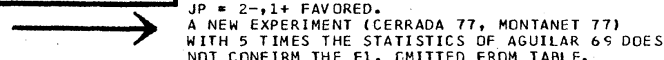

\section{FI MASS (MEV)}

$M$
$M$
$M$
$M$

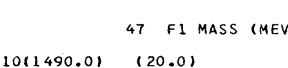

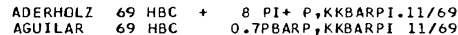

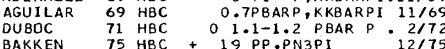

$25(1543.0) \quad 13.0$

DUBIOUS BACKGROUND SUBTRACTION

4.7 FI WIDTH (MEV)

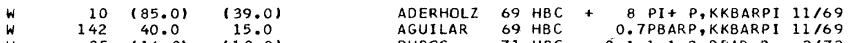

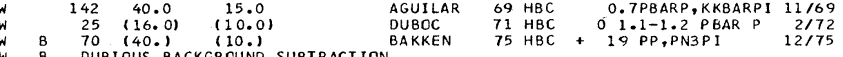

-

47 FI PARTIAL DECAY MODES

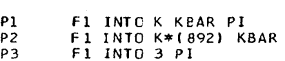

DECAY MASSES
$134+497+457$

$892+497+139$

REFERENCES FQR F

DDERHOL 2 G9 NP 8111259

+BARTSCH,+ (AACH+BERL+CERN+CRAC+WARS) -BARLOW, JACOBS, D-ANDLAu, ASTIER+ (CERN+CDEF)

CUBOC 71 PL 34 B 343 +GOLOBERG, MAKOWSKI, TOUCHARD, , (IPNP+LIVP)

$\begin{array}{llllllll}\text { CHAPMAN } & 72 & N P & \text { B } & 42 & 1 & \text { +CHURCH, LYS, MURPHY, RING, VANOER VELDE (MICH) } \\ \text { CUBOC } & 72 & \text { NP } & \text { B } & 46 & 429 & \text { +GOLDBERG, MAKOWSKI, DONALD, } & \text { (LPNP + IVIVI) }\end{array}$

BAKKEN 75 NP B90 227 +JACOBSEN, OLSSON, SKJEVLING COSLOIG=-

CERRADA 77 PREPR. BUDAPEST C +DIAZ, FERRANDO, GARZON+ (MADR+TATA+CERN +CDEF)
MONTANET 77 PRIVATE COMMUN. L. MONTANET
(CERN)

MINNAERT 78 NP B 13288 +BILLY,+ (BORD+LPNP+LAUS+NEUC+LIVP+GLAS) JP

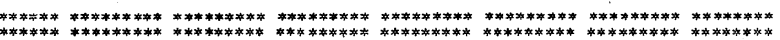

$\rho^{\prime}(1600)$ 65 RHO PRIME(1600,JPG $\left.=1-+\right) \quad 1=1$

The $\rho^{\prime}(1600)$ was first seen in the $\pi^{+} \pi^{+} \pi^{-} \pi^{-}$ system, or its $\rho^{0} \pi^{+} \pi^{-}$subsystem, in photoproduction (BINGHAM 72, DAVIER 73, SCHACHT 74, ALEXANDER 75, LEE 75, ATIYA 79, RICHARD 79) and in $e^{+} e^{-}$annihilation (BARBARINO 72, CONVERSI 74, CORDIER 79, COSME 79). The $\pi^{+} \pi^{-}$system in the $\rho^{0} \pi^{+} \pi^{-}$final state is apparently in an $S$ wave, although no $\varepsilon$ resonance at 


\section{Mesons $\rho^{\prime}(1600)$}

sufficiently low mass exists to make this final state a quasi-two-body $\rho \varepsilon$ system. For this reason all $\rho \varepsilon$ analyses have difficulties and all mass fits are strongly parametrization dependent (SMADJA 72, BUDNEV 77, CORDIER 79). Moreover, other mechanisms exist that can simulate a resonance peak in $\mathrm{e}^{+} \mathrm{e}^{-}$ annihilations to $4 \pi$ (HIRSCHFELD 74) and in photoproduction (SCHACHT 74).

Evidence for a $2 \pi$ decay mode has been looked for in phase-shift analyses of the $\pi^{-} p \rightarrow \pi^{+} \pi^{-} n$ reaction, as well as in photoproduction and $e^{+} e^{-}$ annihilations. The decay $\rho^{\prime} \rightarrow \pi^{+} \pi^{-}$has been reported in photoproduction (ATIYA 79, RICHARD 79) with a branching ratio of $16 \pm 5 \%$ (RICHARD 79). This information can now be used to distinguish between the various phase-shift solutions.

As noted in the mini-review on $S$-wave $\pi \pi$ interactions, the solutions denoted $\beta^{\prime}$ and $\beta$ of MARTIN 77 fit the data of BECKER 79 best, with a slight preference for solution $\beta^{\prime}$ in the $\rho^{\prime}$ region, 1.5 to $1.7 \mathrm{GeV}$. Both solutions require a $\rho^{\prime}$ at $1575 \mathrm{MeV}$. However, solution $\beta$ has a branching ratio to $2 \pi$ of $30 \%$, while $\beta^{\prime}$ has a branching ratio of only 15\%. Thus the photoproduction data also select $\beta^{\prime}$.

The unique solution of FROGGATT 77 and the solution B of CORDEN 79 are similar to solution $\beta$ (having, however, problems with the unitarity of the $S$ wave).

Further support for the $\rho^{\prime}(1600)$ comes from an analysis of the pion form factor (GENSINI 78). No $\rho^{\prime}(1250)$ is required in $e^{+} e^{-} \rightarrow \pi^{+} \pi^{-}$when the analysis is extended well outside the $\rho^{\prime}(1600)$ region. The $\rho^{\prime}(1250)$ resonance, claimed mainly by vector dominance arguments to explain the nucleon form factors, is also not found in any of the phaseshift solutions. The $\mathrm{J}^{\mathrm{P}}=1^{-}$partial wave of the $\omega \pi$ system is expected to contribute to the pion form factor (ROOS 75, COSTAI 77); it is indeed strong in the $1250 \mathrm{MeV}$ region, but does not exhibit a resonance behavior (CHUNG 73,75, CHALOUPKA 74, BUDNEV 77, GESSAROLI 77).

The $\rho^{\prime}(1600)$ is most explicitly seen in $e^{+} e^{-}$ annihilations into three or more hadrons (BACCI 79). Some support for a $\rho^{\prime}(1600)$ decay into $e^{+} e^{-}$has been claimed in $\bar{p}$ p annihilation (BASSOMPIERRE 76).

\section{Data Card Listings} For notation, see key at front of Listings.

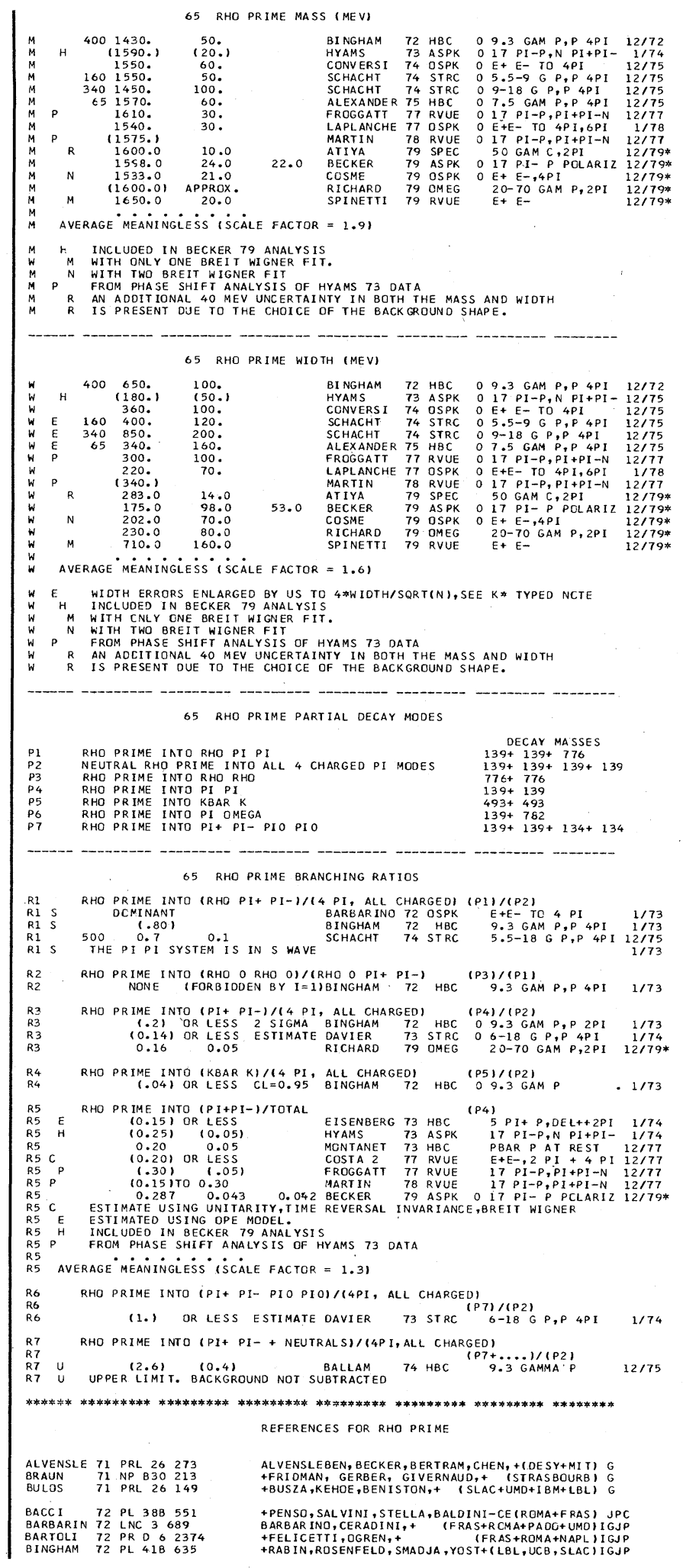




\section{Data Card Listings}

For notation, see key at front of Listings.

Mesons
$\rho^{\prime}(1600), A_{3}(1660)$

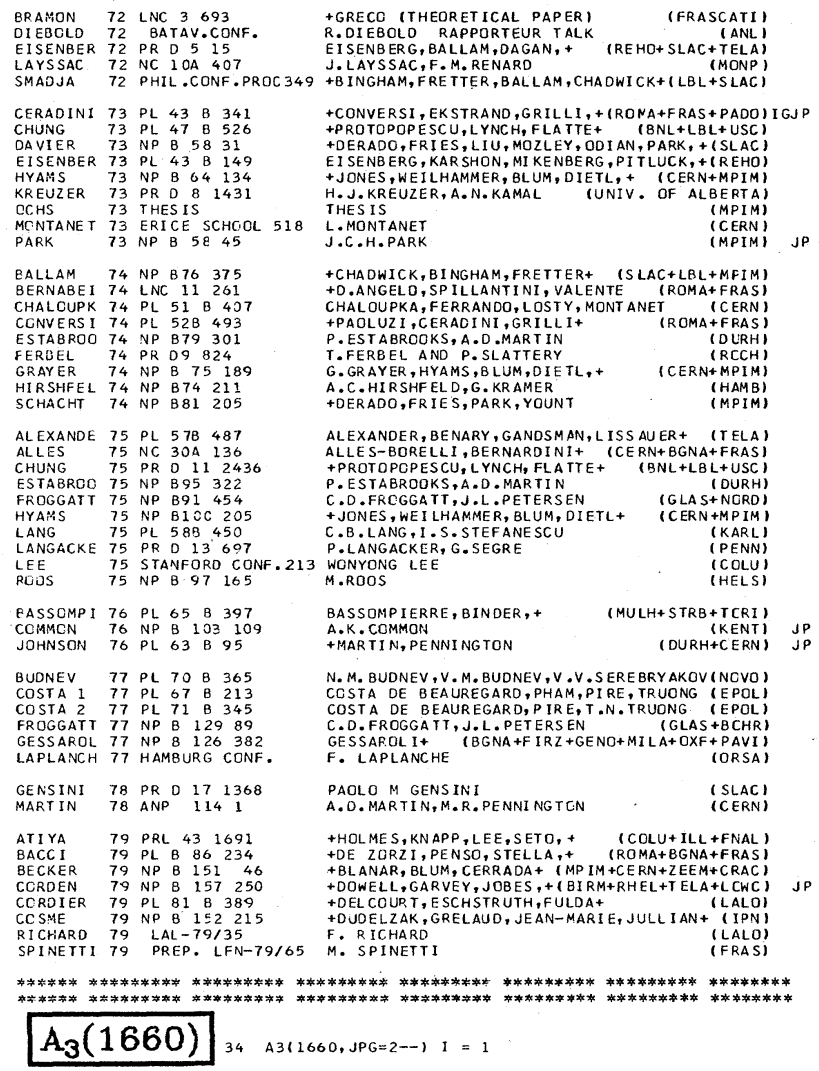

Evidence for the existence of the $A_{3}$ meson was previously confused due to its appearance near $f \pi$ threshold in the diffractive-like process $\pi N \rightarrow \pi \pi \pi N$, much like the $A_{1}$ meson. While everybody agreed that there was $a \approx 300 \mathrm{MeV}$ wide $\mathrm{f} \pi$ enhancement in the $\mathrm{J}^{\mathrm{P}} \mathrm{LM}=2$ - $\mathrm{SO}$ partial wave at about $1650 \mathrm{MeV}$, some claimed non-resonant status (ANTIPOVI 73, ASCOLII 73, BALTAY 77, GHIDINI 77), while others saw evidence for a resonance in the phase variation with respect to other partial waves (OTTER 74, THOMPSON 74).

In the non-diffractive charge-exchange reaction $\pi^{+} p \rightarrow \pi^{+} \pi^{-} \pi^{0} \Delta^{++}$(WAGNeR 75 , BALTAY 77 , CAUTIS 77) and in the hypercharge exchange reaction $\mathrm{K}^{-} \mathrm{p} \rightarrow$ $\pi^{+} \pi^{-} \pi^{0} \Lambda$ at $4.2 \mathrm{GeV} / \mathrm{C}$ (CERRADA 77), there is no evidence for $\mathrm{A}_{3}$ production.

Definitive proof for the resonant nature of the $A_{3}$ has been given by PERNEGR 78 using $60,0003 \pi$ events, diffractively produced by incident $\pi^{-}$on nuclei, and by DAUM 80 in an analysis of nearly 600,000 events of the reaction $\pi^{-} p \rightarrow \pi^{-} \pi^{-} \pi^{+} p$. A partial-wave analysis shows the $3 \pi$ system to be

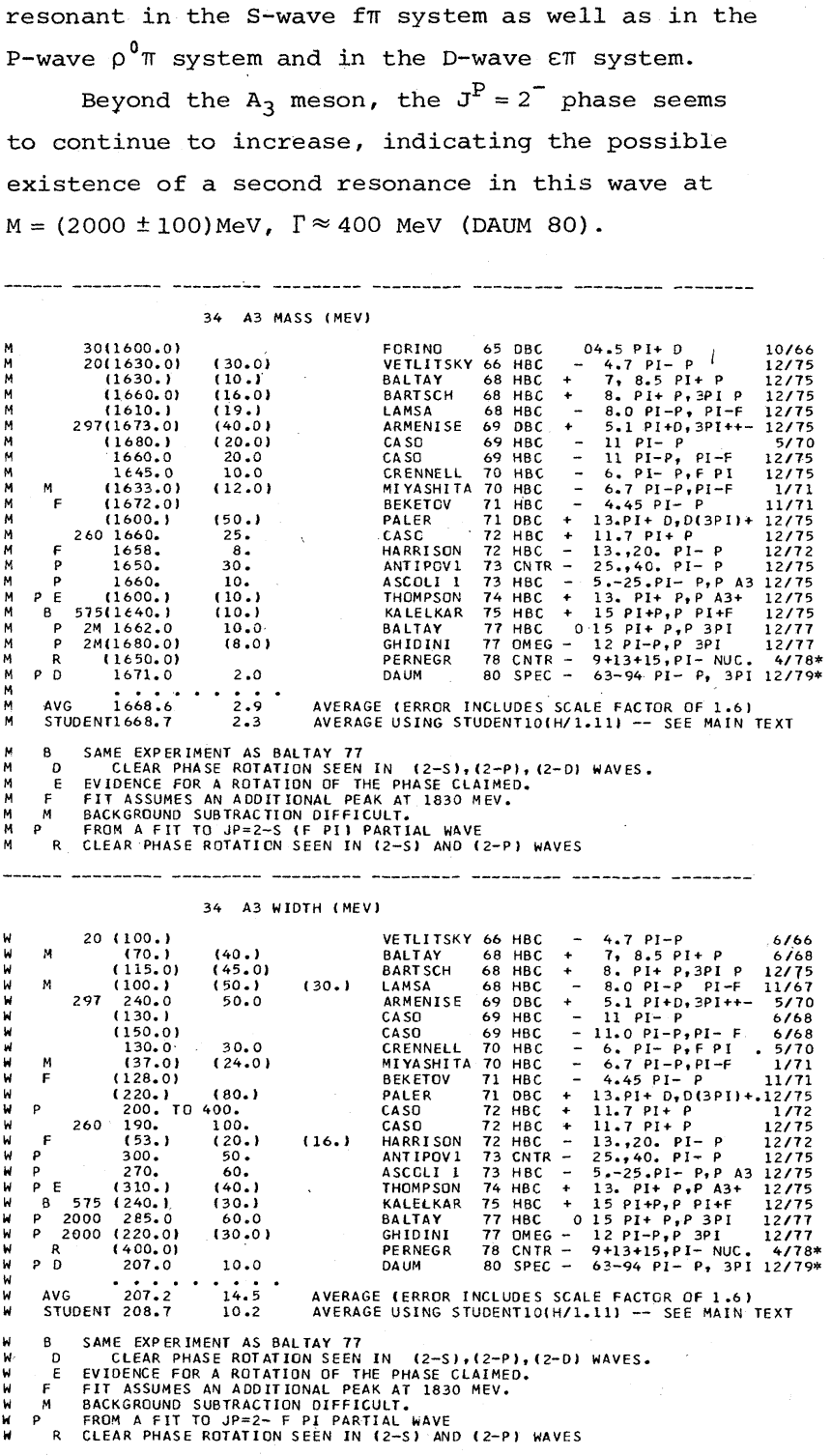

34 a3 PARTIAL DECAY MODES

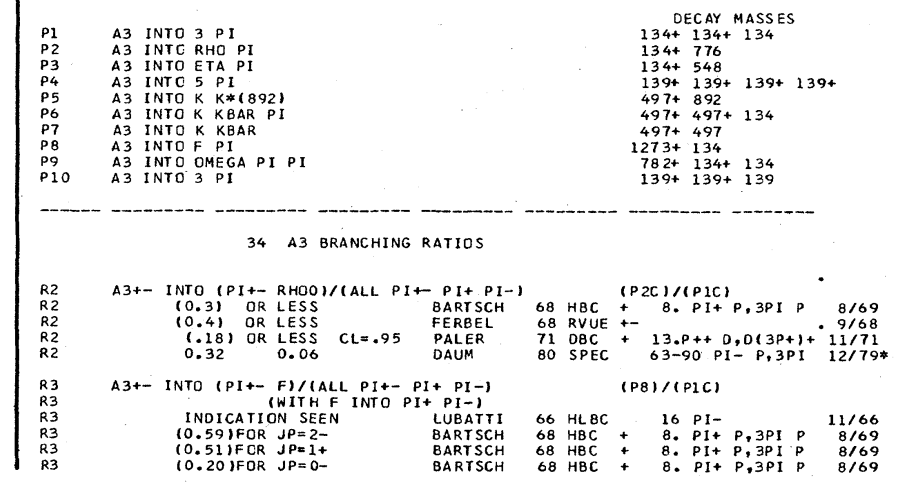


Mesons

$\mathrm{A}_{3}(1660), \omega(1670), \mathrm{g}(1700)$

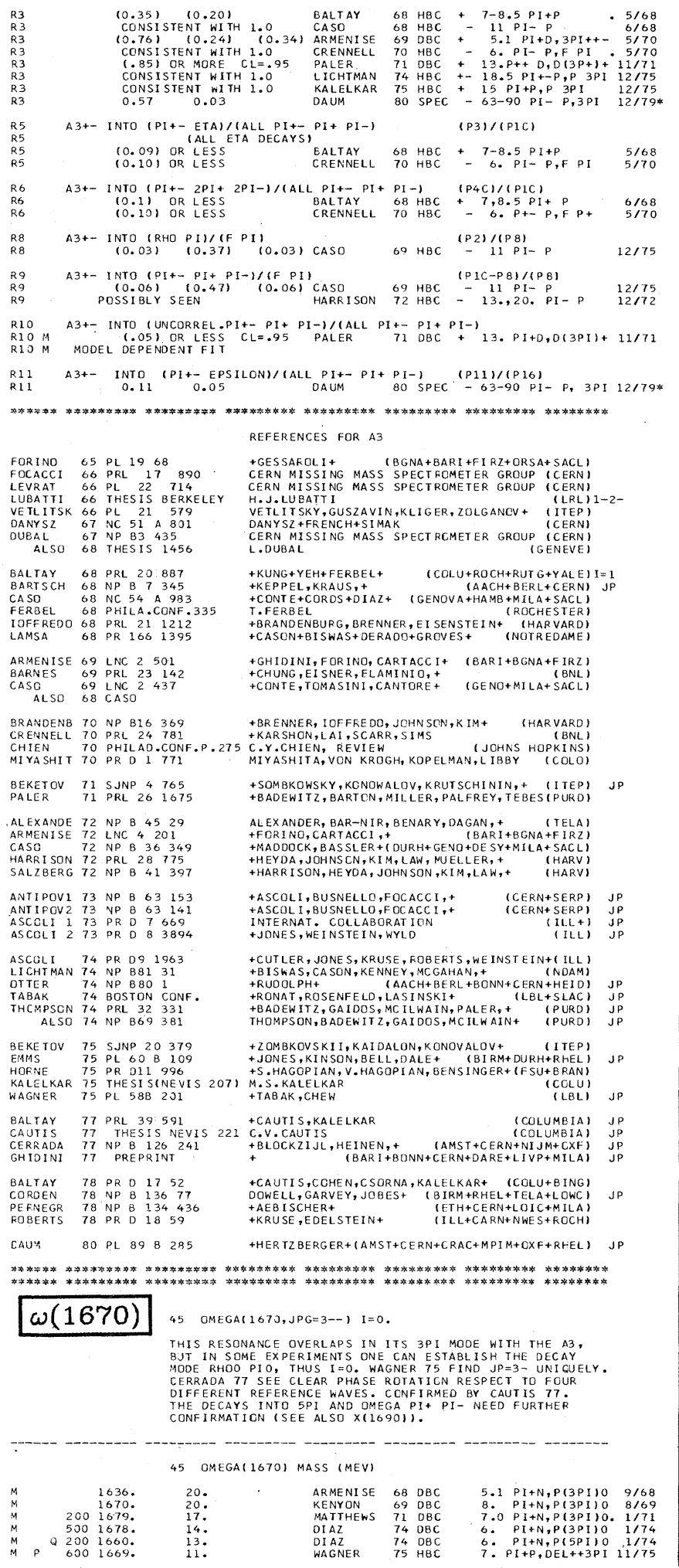

Data Card Listings

For notation, see key at front of Listings.

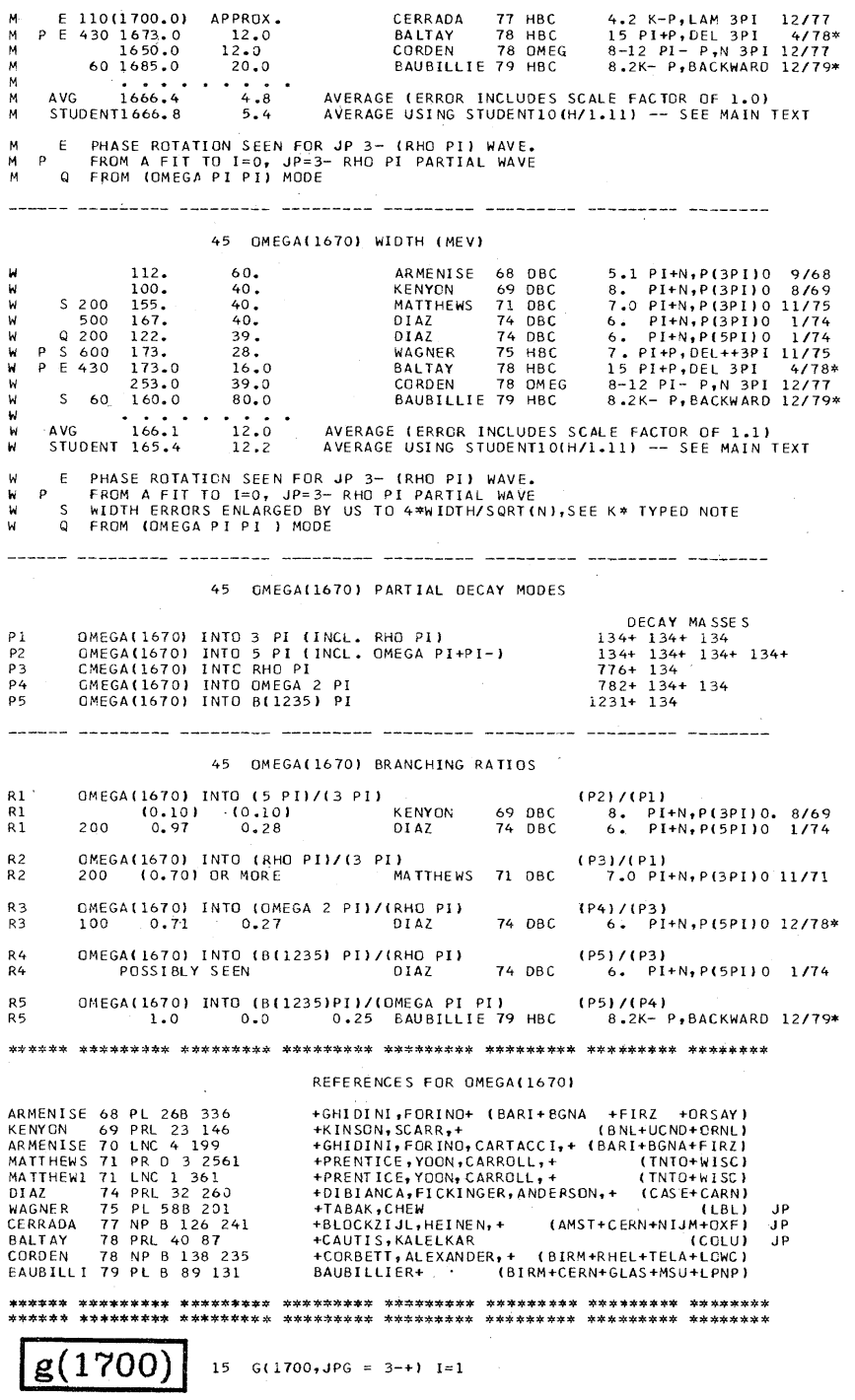

The $g$ meson is uniquely established near $1700 \mathrm{MeV}$ in the $\mathrm{I}^{G} \mathrm{~J}^{\mathrm{P}}=1^{+} 3^{-}$partial wave of the $\pi^{+} \pi^{-}$system (HYAMS 75 , MARTIN 378, BECKER 79 , CORDEN 79), in the $\mathrm{K}^{+} \mathrm{K}^{-}$system (GORLICH 79), and in the $\mathrm{K}^{-} \mathrm{K}^{0}$ system (MARTIN1,2 78). Its branching ratio into $\pi \pi$ is unanimously in the range 23-26\%, whereas determinations of the ratio $\mathrm{k} \overline{\mathrm{K}} / \pi \pi$ are

conflicting: $19.1 \%$ in a model-independent analysis of the $\mathrm{K}^{+} \mathrm{K}^{-}$system (GORLICH 79), but only 5.6\% in $\mathrm{K}^{-} \mathrm{K}^{0}$ (MARTIN1,2 78). It is clear from these

numbers, however, that the $g$ decays predominantly into channels other than $\pi \pi$ or $k \bar{k}$, such as $4 \pi$, $\omega \pi$, $\rho \pi \pi, A_{2} \pi$, and $\mathrm{k} \bar{k} \pi$. 


\section{Data Card Listings}

15 G MASS (MEV)

M WE AVERAge ONLY THE 2 PI AND KKBAR MODES WHICH haVE LARGe STATISTICS 2 PI MODE

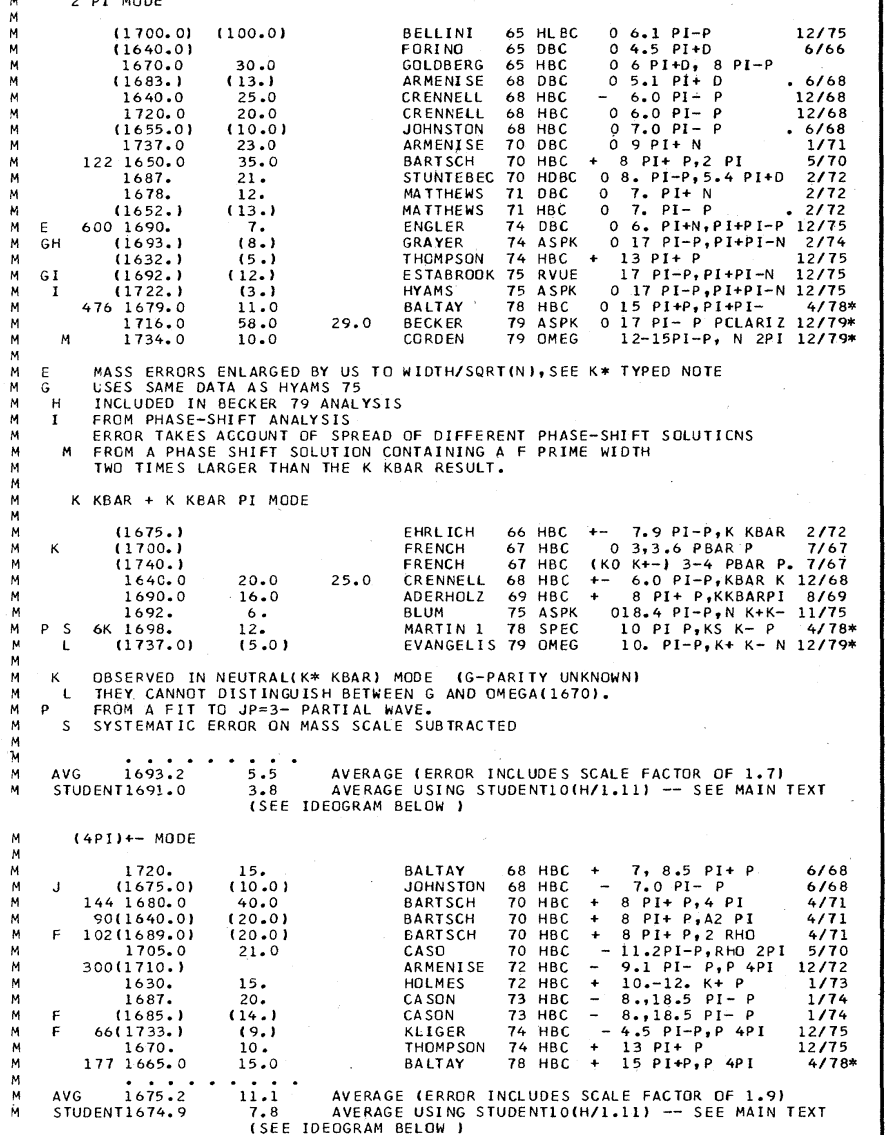

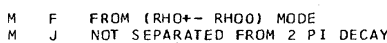

M. RHCO RHOO MODE

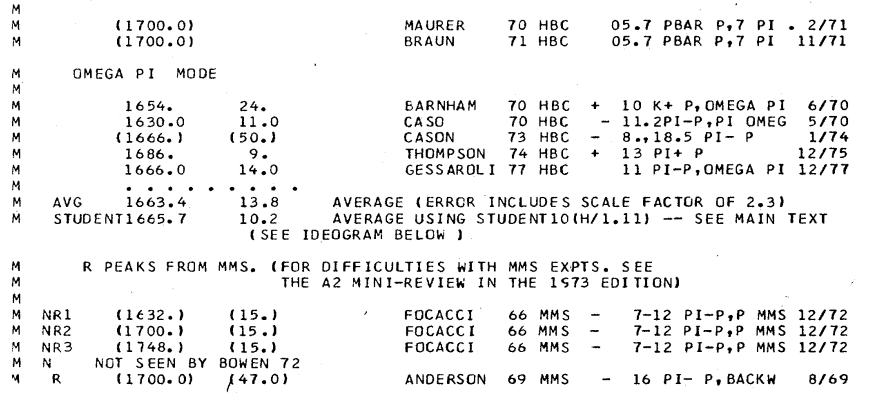

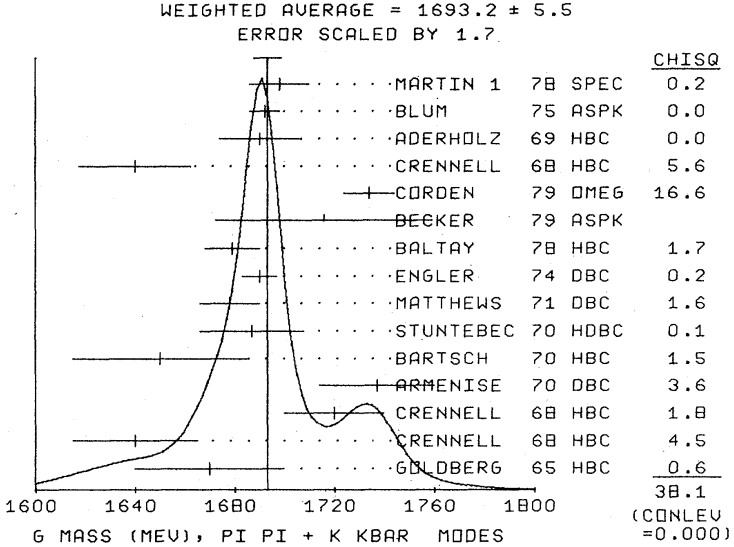
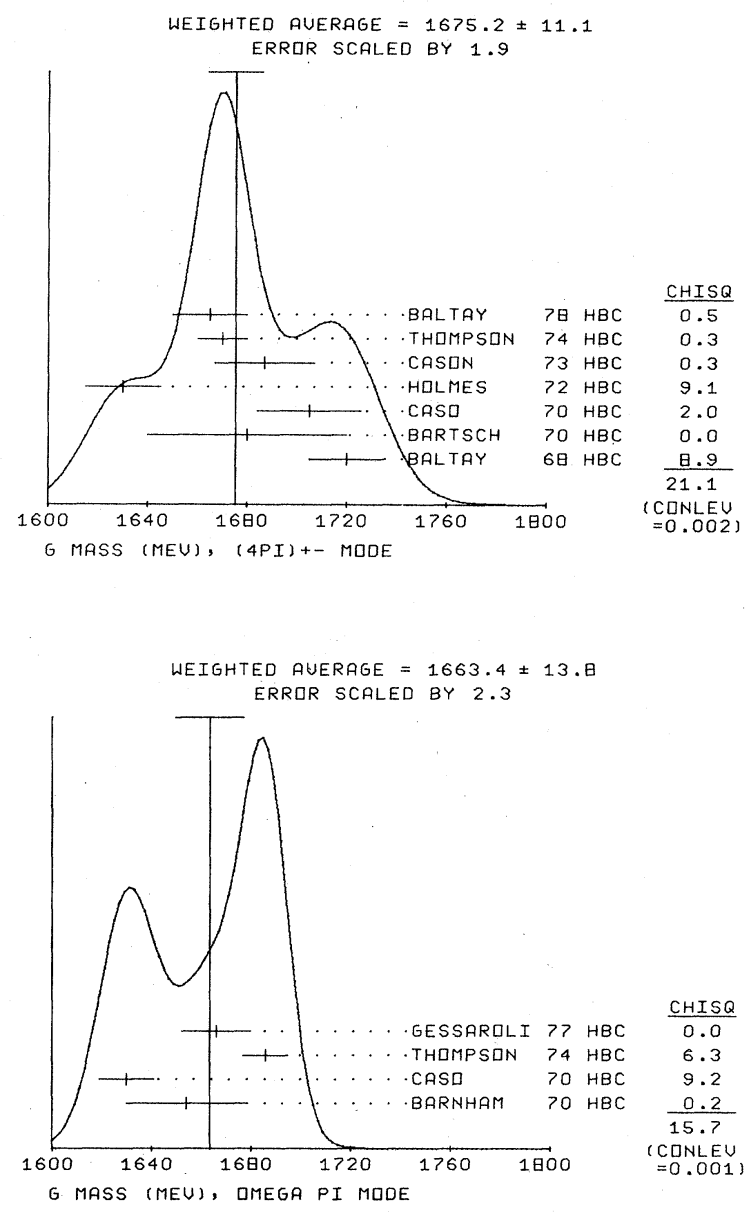
Mesons $\mathrm{g}(1700)$
Data Card Listings For notation, see key at front of Listings.

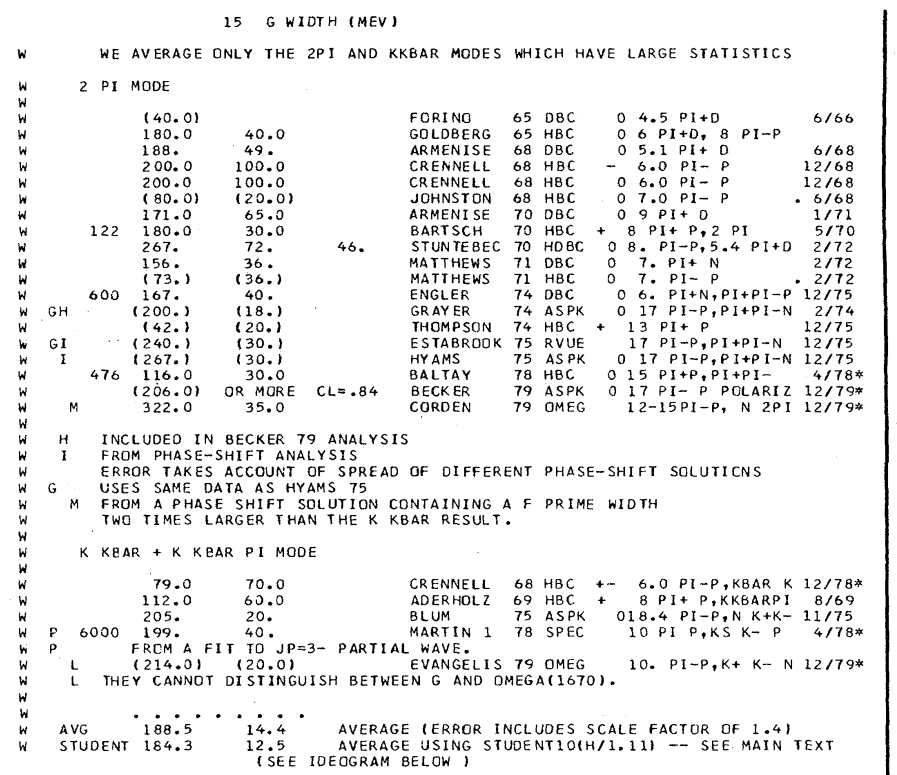

WEIGHTED AUERAGE $=1$ 日日. $5 \pm 14.4$

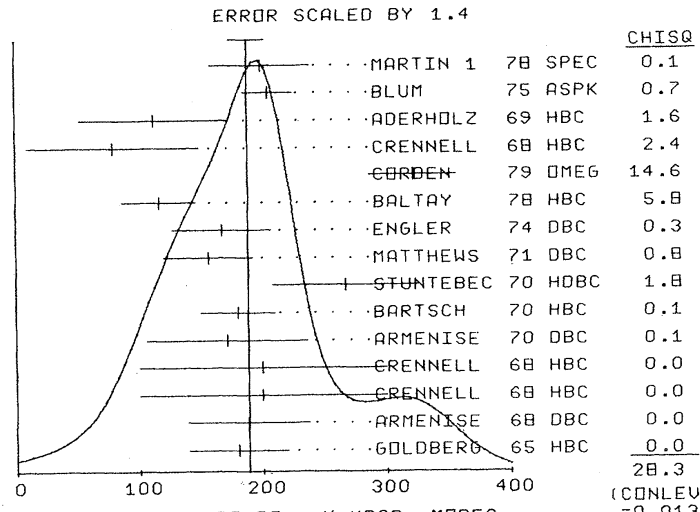

6 WIDTH (MEU), PI PI + K KBAR MDDES $\quad$ (CDNLEU

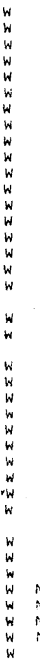
(4PI)+- MOOE

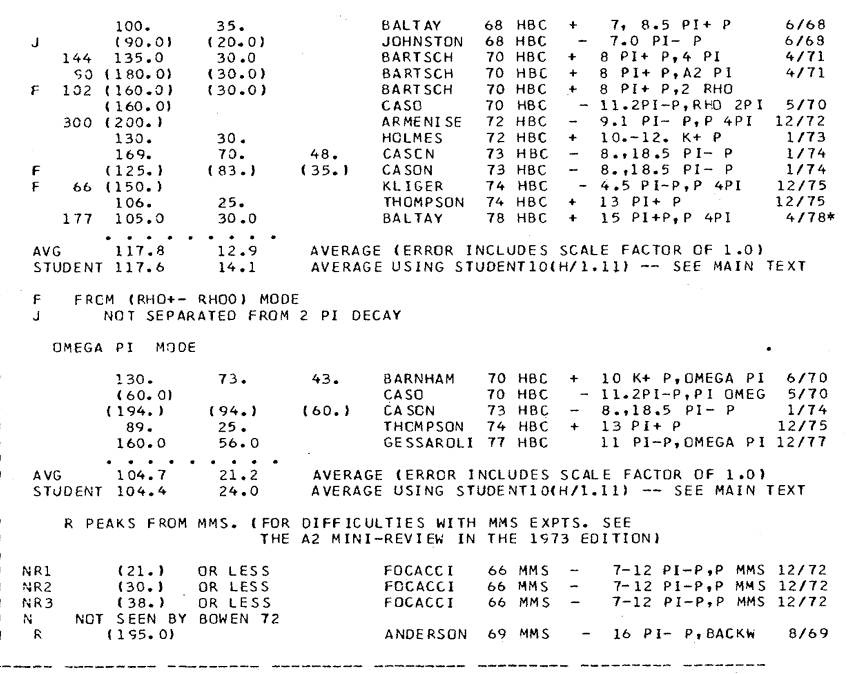

15 G PARTIAL DECAY MODES

\begin{tabular}{|c|c|c|}
\hline $\begin{array}{l}\text { P1 } \\
\text { P2 } \\
\text { P3 } \\
\text { P4 } \\
\text { P5 } \\
\text { P6 } \\
\text { P7 } \\
\text { P8 } \\
\text { P9 } \\
\text { P10 } \\
\text { P11 }\end{array}$ & 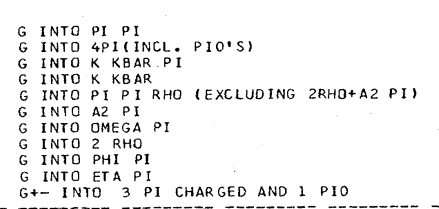 & $\begin{array}{l}\text { DECAY MASSES } \\
139+139 \\
139+139+139+139 \\
497+497+139 \\
497+497 \\
139+139+776 \\
1317+139 \\
139+782 \\
776+776 \\
1019+139 \\
548+139 \\
139+139+139+139\end{array}$ \\
\hline \multicolumn{3}{|c|}{ FITTED PARTLAL DECAY MODE BRANCHING FRACTION } \\
\hline \multicolumn{3}{|c|}{$\begin{array}{l}\text { The matrix below is derived from the error matrix for the fitted partial decay mode } \\
\text { branching fractions, } P_{i}, \text { as follows: The diagonal elements are } P_{i} \pm \delta P_{i} \text {, where } \\
\delta P_{i}=\sqrt{\left\langle\delta P_{i} \delta P_{i}\right\rangle} \text {, while the off-diagonal elements are the normalized correlation coeffi- } \\
\left.\text { cients }\left\langle\delta P_{i} \delta P_{j}\right\rangle / \delta \delta P_{i}, \delta P_{j}\right) \text {. For the definitions of the individual } P_{i}, \text { see the listings } \\
\text { above; only those } P_{i} \text { appearing in the matrix are assumed in the fit to be nonzero and } \\
\text { are thus constrained to add to } 1 \text {. }\end{array}$} \\
\hline & $\begin{array}{cccc}P 1 & P 2 & P 3 & P 4 \\
2401+-.0126 & P 2 C 9+-0160 \\
-.8706 & .7269+.0160 & \\
.1625 & -.5844 & .0239+-.0073 \\
.0584 & -.2517 & .0089 & .0151+-.0032\end{array}$ & \\
\hline
\end{tabular}

15 G BRANCHING RATIOS

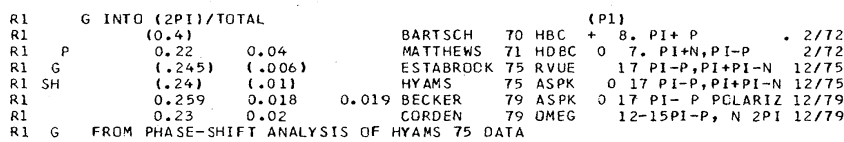

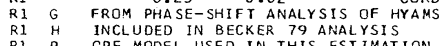

RI
RI

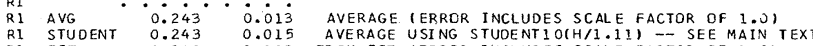

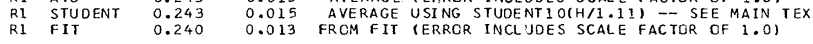

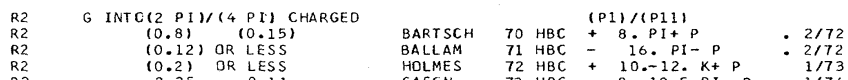

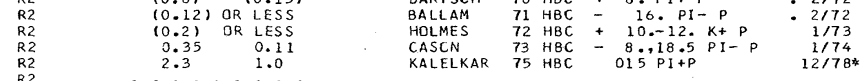

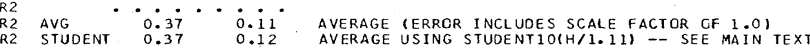

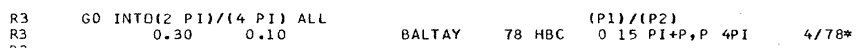

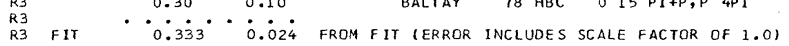

R4 G+ INTO (K KBAR)/(2PI) 0.03 CRENEEL 68 HBC $(P 4) /(P 1)$

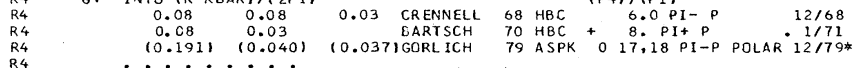

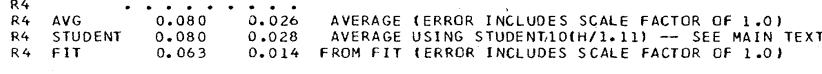

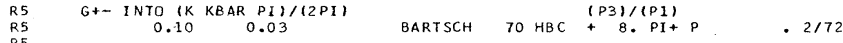

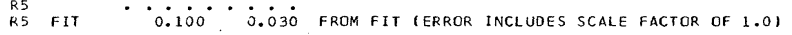

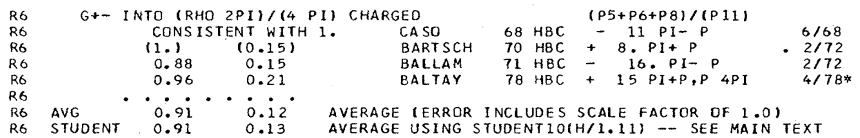

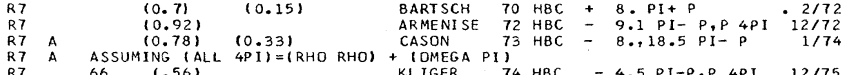

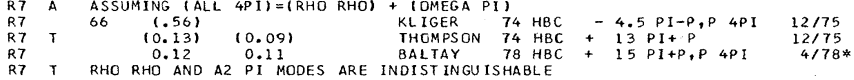

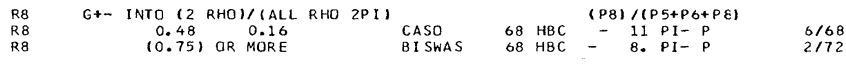

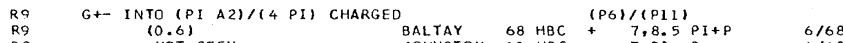

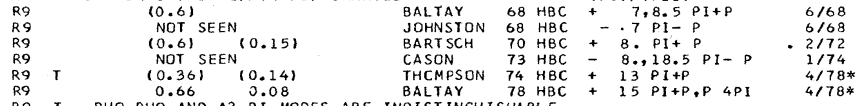

R9 T RHO RHO ANO A2 PI MODES ARE INOIST

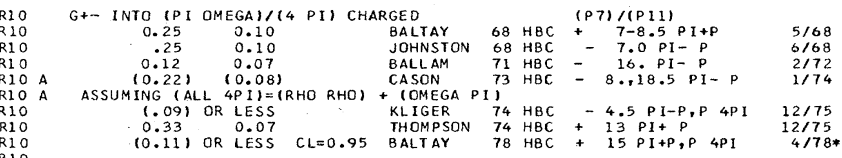
$\begin{array}{lll}\because .233 \\ 0.236 & 0.050 & 0.051\end{array}$ 


\section{Data Card Listings}

Mesons
$g(1700), X(1690), A_{4}(1900)$

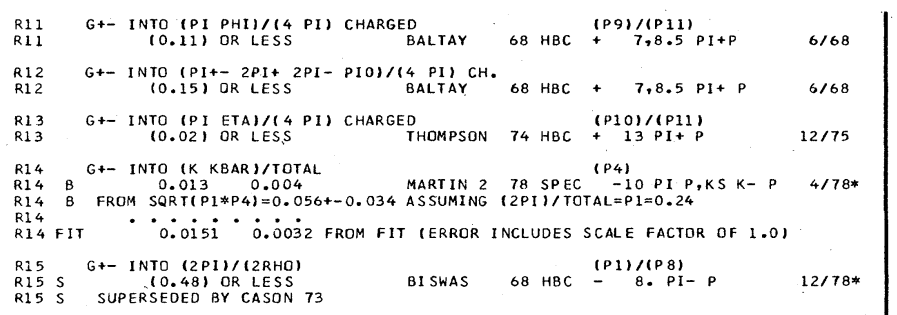

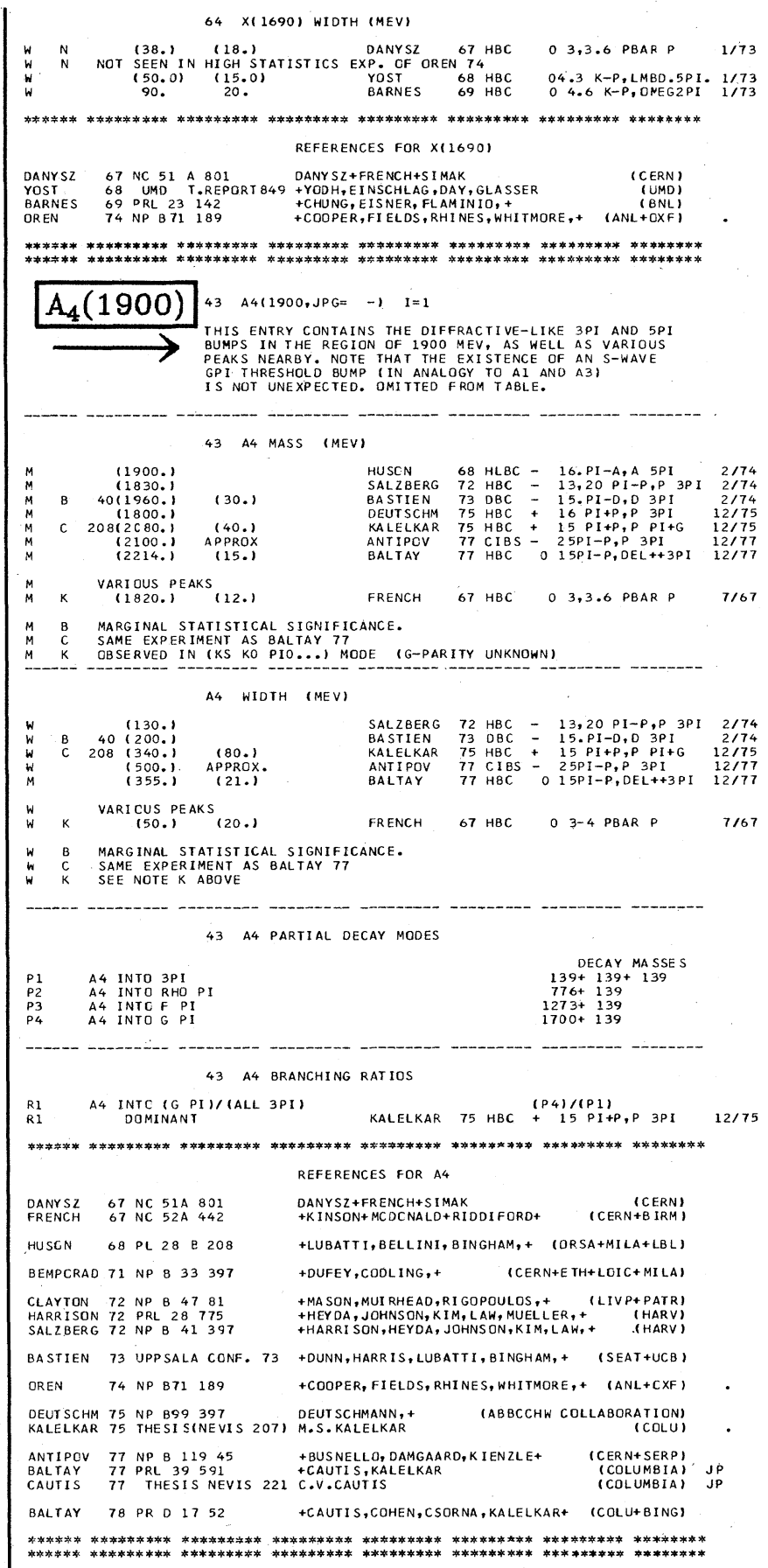

REFERENCES FOR $G$

$\begin{array}{llllll}\text { BELLINI } & 65 & N C & 40 & \text { A } & 548 \\ \text { DEUT SCHM } & 65 & \text { PL } & 18 & 351\end{array}$ BELLINI, OI CCRATO, DUIMIC, FIORINI (MILANO)
M.DEUTSCHMANN ET AL. (AACHEN+BERLIN+CERN) FORINOGESSAROLI + (BOLOGNA +OR SAY+SACLAY)

GOLDBERG 65 PL 17354

R. EHRLICH,W.SELOVE, H.YUTA (PENNSYLVANIA) CERN MISSING MASS SPECTROMETER GROUP (CERN)
CERN MISS ING MASS SP ECTROMETER GROUP (CERN)

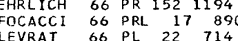

$\begin{array}{llllll}A B R A M S & 67 & P R L & 18 & 620 \\ \text { DANYSZ } & 67 & \text { PL } & 248 & 309\end{array}$ +KEHOE+GLASS ER+SECHI-ZORN+WOLSKY (MARYLANO) +FOCACCI +K IENZLE+LECHANOINE+LEVRAT+ (CERN) 1. DUBAL
$+K$ INSON+MCDONALO+RIDDIFORD+ (GENEVE)
(CERN+BIRM)

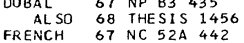

$\begin{array}{lllll}\text { ARMENISE } & 68 & \text { NC } & 54 & \text { A } 999 \\ \text { BALTAY } & 68 & \text { PRL } & 20 & 887\end{array}$ + FOR INO+CARTACCI + (BARI+ BGNA +FIRENZE+ORSAY) I
+KUNG+YEH+FERBEL+
(COLU+RDCH+RUTG+YALE) I $=1$ +CASON, DZI ERBA, GROVES, KENNEY, + CONTE+CORDS + DIAZ+ (GENOVA+HAMB+MILA+SACL) +KARSHON, LAI, SCARR, SKILLICORN (TORONTO+WISC) IJPP
+PRENTICE, STEENERG,YOON (BNL) $\begin{array}{llllll}\text { CASC } & 68 & \text { NC } & 54 & \text { A } & 983 \\ \text { CRENNELL } & 68 & \text { PL } & 28 & \text { B } & 136\end{array}$ $\begin{array}{llllll}A D E R H O L Z & 69 & \text { NP } & \text { B } & 11 & 259 \\ \text { ANDERSCN } & 69 & \text { PRL } & 22 & 1390\end{array}$ ARISH 69 PR $184 \quad 1375$

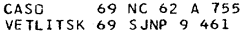
ARMENISE 70 LNC $4 \quad 199$ BARTSCH 70 NP $B 22109$ KRAMER 70 PRL 25396

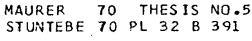
BALLAM 71 PR 0332606 +BARTSCH,+ (AACH+BERL+CERN+ JAGL+W ARS) SELOVE, BISWAS, CASON,+ (PENN+NDAM+ROCH) + CONTE, BENZ, + (GENO+DESY+HAMB+MILA+SACL)
+ GUZHAVIN, KL IGER, KOLGANOV, LEBEOEV+
(I TEP) +GHIDINI, FORINO, CARTACCI,+ (BARI + BGNATFIRZ) , JOBES, KENYON, PATHAK, RIDDIFORD IBIRM

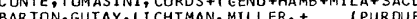
STUNTER BECK, KENNEY, DEERY, BISWAS, CASON + (NDAM)
STRASBORG) +CHADWICK, GUIRAGOSSIAN, JOHNSON, +
+FRIDMAN, GERBER, GIVERNAUD, KAHN,
(SIRB) +HYAMS, JONES, SCHLE IN, BLUM, + (CERN+MPIM) JP3
+PRENTICE, YOCN, CARROLL, +
(TNTO+WISCIJP3

+FORINO, CARTACCI, + (BARI +BGNA+FIRZ) (BARI+BGNA+FIRZ) JP

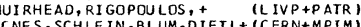
ARMENISE 72 LNC 420 BOWEN 72 PRL 29890

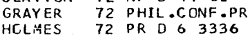

ARNOLO 73 LNC 6707

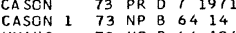

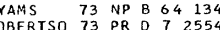
$\begin{array}{lllll}\text { DUBOVIKO } & 74 & \text { SJNP } & 1 S 568 \\ \text { ENGLER } & 74 & \text { PR } & 010 & 2070\end{array}$ $\begin{array}{llll}\text { ENGLER } & 74 & \text { NP B } 75 & 189 \\ \text { GRAYER } & 74 & 189 \\ \text { KLIGER } & 74 & \text { SINP } 19 & 428\end{array}$ $\begin{array}{lllll}\text { OREN } & 74 & \text { NP } & 871 & 189 \\ \text { THCMPSON } & 74 & \text { NP } & B 69 & 220\end{array}$ $\begin{array}{lllll}\text { BLUM } & 75 & \text { PL } & 578 & 403 \\ \text { ESTABROO } & 75 & \text { NPP } & 895 & 322 \\ \text { HYAMS } & 75 & \text { NP } & \text { B }\end{array}$ +CHABAUD, OIETL, GAREL ICK, GRAYER+ (CERN+MPIM) JP
P.ESTABROOKS, A. O.MARTIN

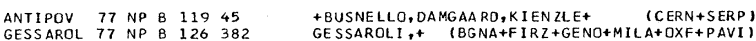
$\begin{array}{lllllll}\text { BALTAY } & 78 & P R & 0 & 1762 & 62 & \text { +CAUTIS, COHEN,C SORNA, SMITH, YEH, +(COLU+B ING) } \\ \text { FORINO } & 78 \text { NP B } 139413 & \text { +CARTACCI, } & \text { (BGNA+FIRZ GENO+MILA+OFF+PAVI) JP }\end{array}$

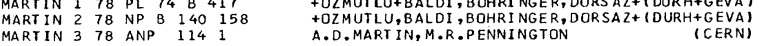
$\begin{array}{lrllllllll}\text { BECKER } & 79 & N P & B & 151 & 46 & \text { +BLANAR, BLUM, CERRADA+ (MPIM+CERN+ZEEM+CRAC) } \\ \text { CORDEN } & 79 & \text { NP } & 15 & 157 & 250 & \text { +DOWELL, GARVE, JOBES, + (BIRM+RHEL+TELA+LOWC) }\end{array}$

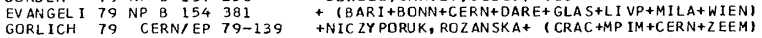

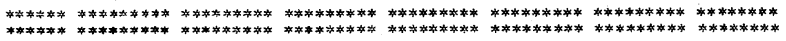

$\mathrm{X}(1690)$ 64 $\times(1690)$

THIS ENTRY CONTAINS CMEGA PI PI PEAKS AROUND $64 \times(1690)$ MASS (MEV)

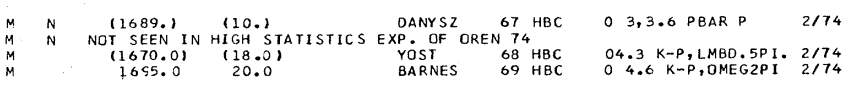


Mesons

$\mathrm{A}_{2}(1900), \mathrm{S}(1935)$

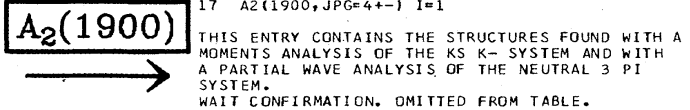

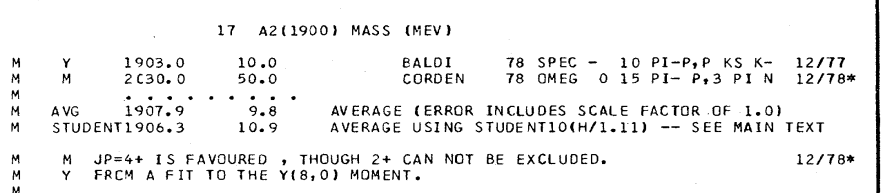

17 A2(1900) WIOTH (MEV)

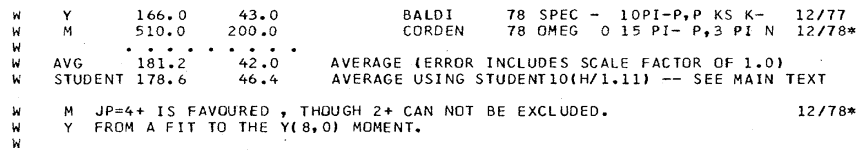

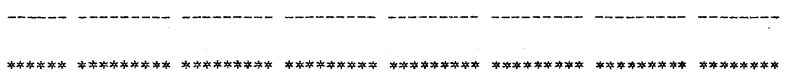

REFERENCES FOR AZ1 19001

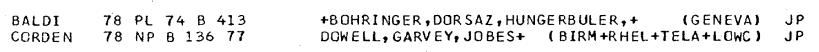

COROEN 78 NP B 13677 DOWELL, GARVEY, JOBES+ (BIRM+RHEL+TELA+LOWC) JP

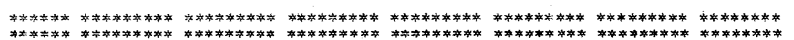

$\mathrm{S}(1935)$ 31 $511935, \mathrm{JPG}=$,

A narrow enhancement has been observed in the antiproton-proton total cross section, called the S(1935) (CARROL 74, CHALOUPKA 76, BRUCKNER 77). The three experiments are in reasonable agreement on the mass and width (see the Data Card Listings below) and on the size of the enhancement above background. However, CHALOUPKA 76 finds a large elasticity, whereas BRUCKNER 77 observes the enhancement mainly in the annihilation channels. SAKAMOTO 79 sees a narrow enhancement compatible with CARROLI 74, CHALOUPKA 76, and BRUCKNER 77, but of more limited statistical significance.

Considerable doubt has been cast on the existence of the $\mathrm{S}(1935)$ as a narrow state by new measurements of the antiproton-proton total cross section. KAMAE 80 see no effect at all. With much better statistics, HAMILTON 80 observes a broad enhancement at $1939 \pm 2 \mathrm{MeV}$, with a width of $22 \pm 6$ $\mathrm{MeV}$. The magnitude of the enhancement above background is $3.0 \pm 0.7 \mathrm{mb}$, compared with the $18_{-3}^{+6} \mathrm{mb}$ found by CARROLL 74. The dominant coupling seems to be to the annihilation channels.

No significant signal is observed for a narrow $\mathrm{S}(1935)$ in backward antiproton-proton elastic scattering (GARNJOST 79), nor in the charge-exchange cross section (GARNJOST 75, CHALOUPKA 76, HAMILTON $80)$.

\section{Data Card Listings} For notation, see key at front of Listings.

No evidence for the $\mathrm{S}(1935)$ has been reported in production experiments except by DAUM 79, who see a narrow enhancement at $1940 \mathrm{MeV}$ in an inclusive $\bar{p}$ p mass spectrum from proton-proton interactions at $93 \mathrm{GeV} / \mathrm{c}$. A $\bar{p} \mathrm{p}$ enhancement at the mass of the $\mathrm{S}$ (1935) and having a narrow width is also observed in photoproduction (RICHARD 79).

The absence of the $\mathrm{S}(1935)$ in the most recent antiproton-deuterium total cross-section measurements (ALBERI 79, HAMILTON 80) favors I=0 for a resonance with a width smaller than $20 \mathrm{MeV}$. The DEFOIX 80 data suggest that a large enhancement (width of the order of $80 \mathrm{MeV}$ ) at $1950 \mathrm{MeV}$ might be present in the $I=1$ five-pion annihilation channel.

The existence of a narrow $\mathrm{S}(1935)$ resonance is still open to conjecture, but it may be the case that two resonances, with $I=0$ and $I=1$, are present in the 1935-1950 MeV mass region, both having a relatively large coupling to the antiproton-proton channel. Spins of 2 to 4 are compatible with all experimental data, although HAMILTON 80 favors spin 0 or 1 .

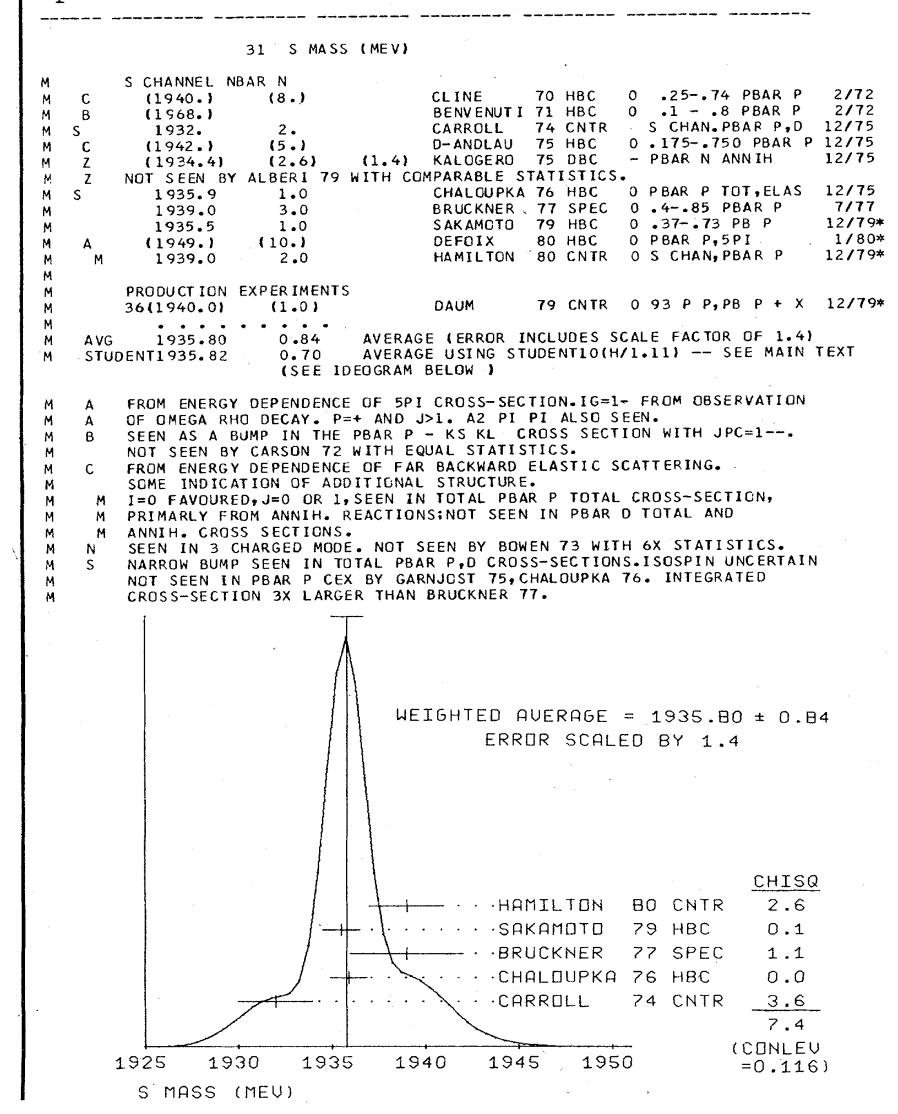




\section{Data Card Listings}

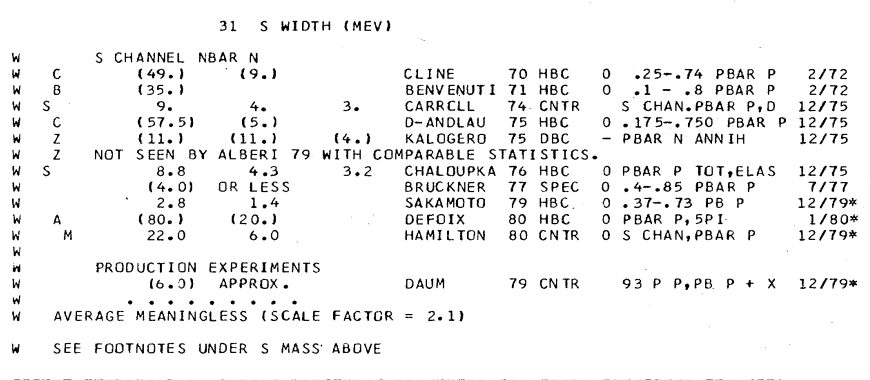

31 S PARTIAL DECAY MODES

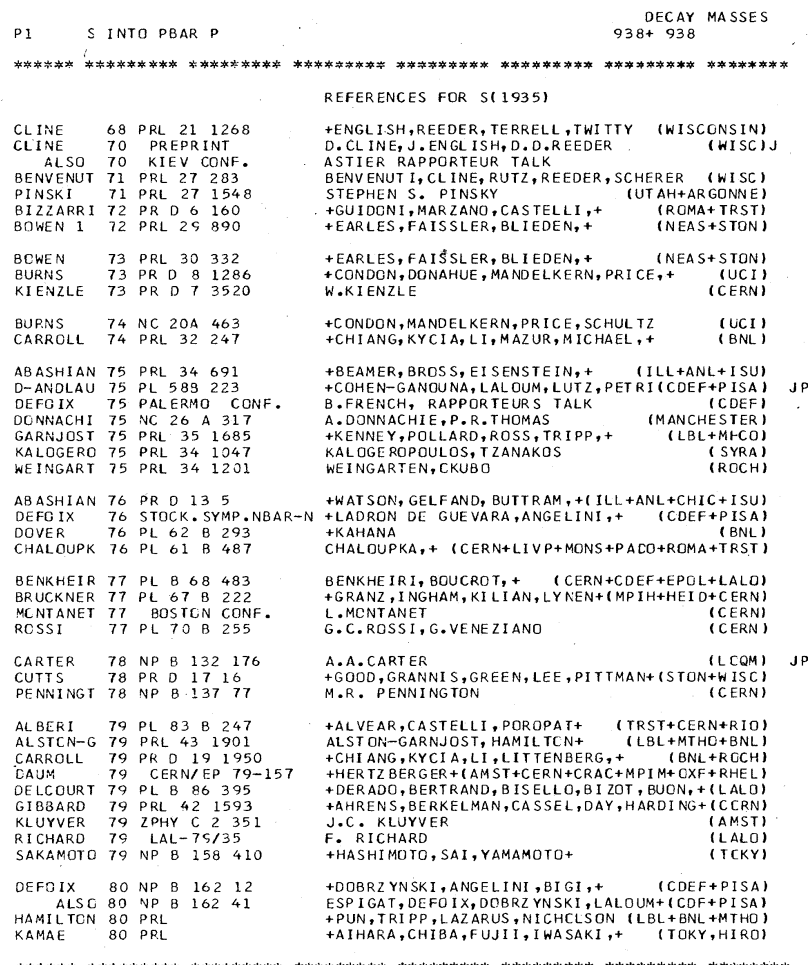

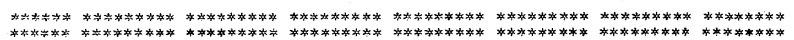

$\mathrm{h}(2040){ }_{16} \quad \mathrm{H}(2040, \mathrm{JPG}=4++) \quad \mathrm{I}=0$

APEL 75 AND BLUM 75 ESTABLISH JP AS $4+$ AND I=0.
ADDITIONAL EVIDENCE FOR THE H MESCN IS REPORTED

\section{IN W'AGNER 74.}

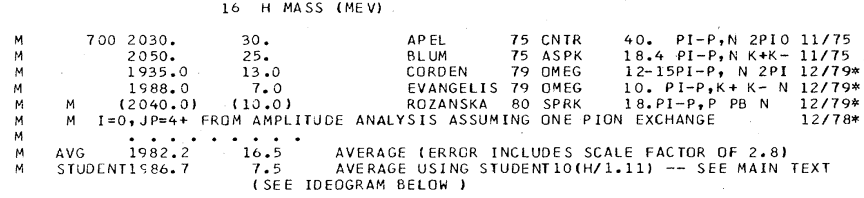

Rev. Mod. Phys., Vol. 52, No. 2, Part II, April 1980

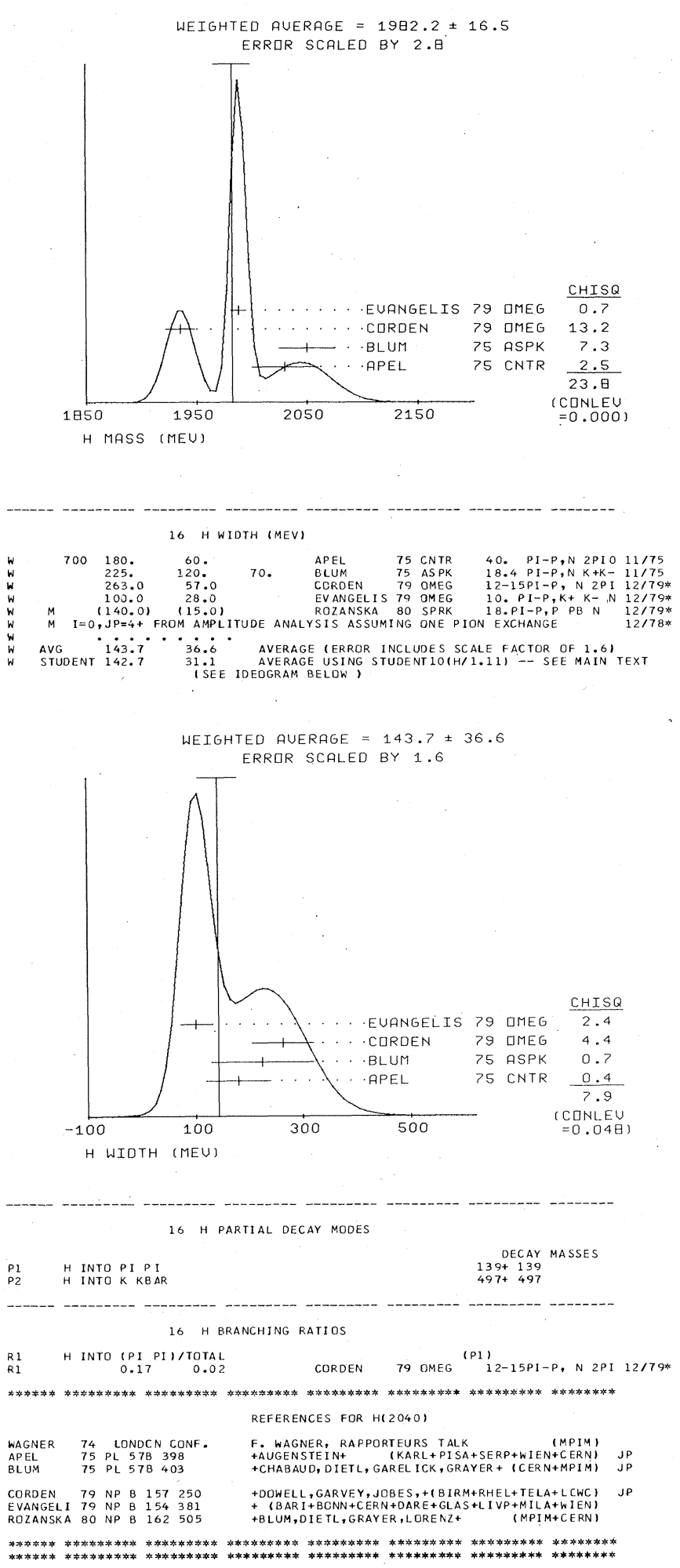




\section{Mesons}

\section{T AND U REGIONS, TO(2150), T1(2190)}

\section{Note on $T$ and $U$ Regions}

The observation of broad enhancements at 2190 and $2350 \mathrm{MeV}$ comes from pp total cross-section measurements (ABRAMS 67), ppp annihilation measurements (ALSPECTOR 73), $\bar{p}$ p elastic cross-section measurements (COUPLAND 77), and from $\bar{p} p$ chargeexchange cross-section measurements (CUTTS 78).

The comparison of $\bar{p} p$ and $\bar{p} d$ total cross sections (ABRAMS 67) suggests $I=1$ for the $2190 \mathrm{MeV}$ enhancement, called $T I$, whereas $I=0$ and $I=I$ are both present in the $2400 \mathrm{MeV}$ mass region, which we call UO and Ul, respectively.

Partial-wave analysis of $\overline{\mathrm{p} p}$ annihilation into $\pi^{+} \pi^{-}$(CARTER 77) and into $\pi^{0} \pi^{0}$ (DULUDE 78) have shown that resonances are formed in the $\pi \pi$ annihilation channels in the $2100-2500 \mathrm{MeV}$ mass region (no statistically significant data are available outside this mass region). The analysis of MARTIN 78 which combines the $\pi^{+} \pi^{-}$data of EISENHANDLER 75 and CARTER 77 and the $\pi^{0} \pi^{0}$ data of DULUDE 78 finds evidence for a $\mathrm{J}^{\mathrm{P}}=2^{+}, \mathrm{I}^{\mathrm{G}}=0^{+}$resonance near 2150 $\mathrm{MeV}$, called $\mathrm{TO}$, and for a $\mathrm{J}^{\mathrm{P}}=5^{-}, \mathrm{I}^{\mathrm{G}}=1^{+}$resonance near $2450 \mathrm{MeV}$, which may be too high in mass to be associated to the Ul bump observed in the $\overline{\mathrm{p}} \mathrm{p}$ total cross section. The $\pi \pi$ partial-wave analysis gives ambiguous results on the $I=1$ component in the $T$ region, favoring however, $\mathrm{J}^{\mathrm{P}}=\mathrm{I}^{-}$, and on both $\mathrm{I}=0$ and $I=1$ components in the $U(2350-2450 \mathrm{MeV})$ mass region, where resonances with spin three and four may be present.

Model-dependent partial-wave analyses of the $\bar{p} p$ system produced with incident pion beams and relying on one-pion-exchange mechanisms suggest the presence of resonances with spin 2, 3, 4, and 5 in the 2100 to $2500 \mathrm{MeV}$ mass region (EVANGELISTA 79, ROZANSKA 79).

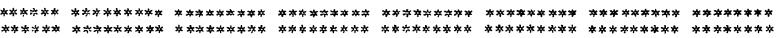

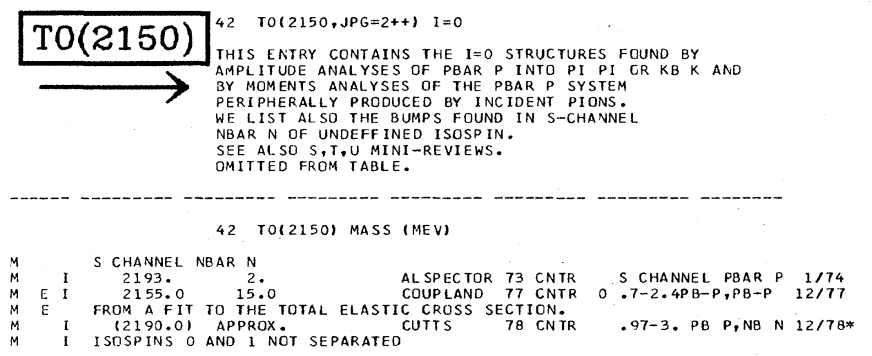

Data Card Listings For notation, see key at front of Listings. 
Data Card Listings For notation, see key at front of Listings.
Mesons T1(2190), X(2200), Uo(2350)

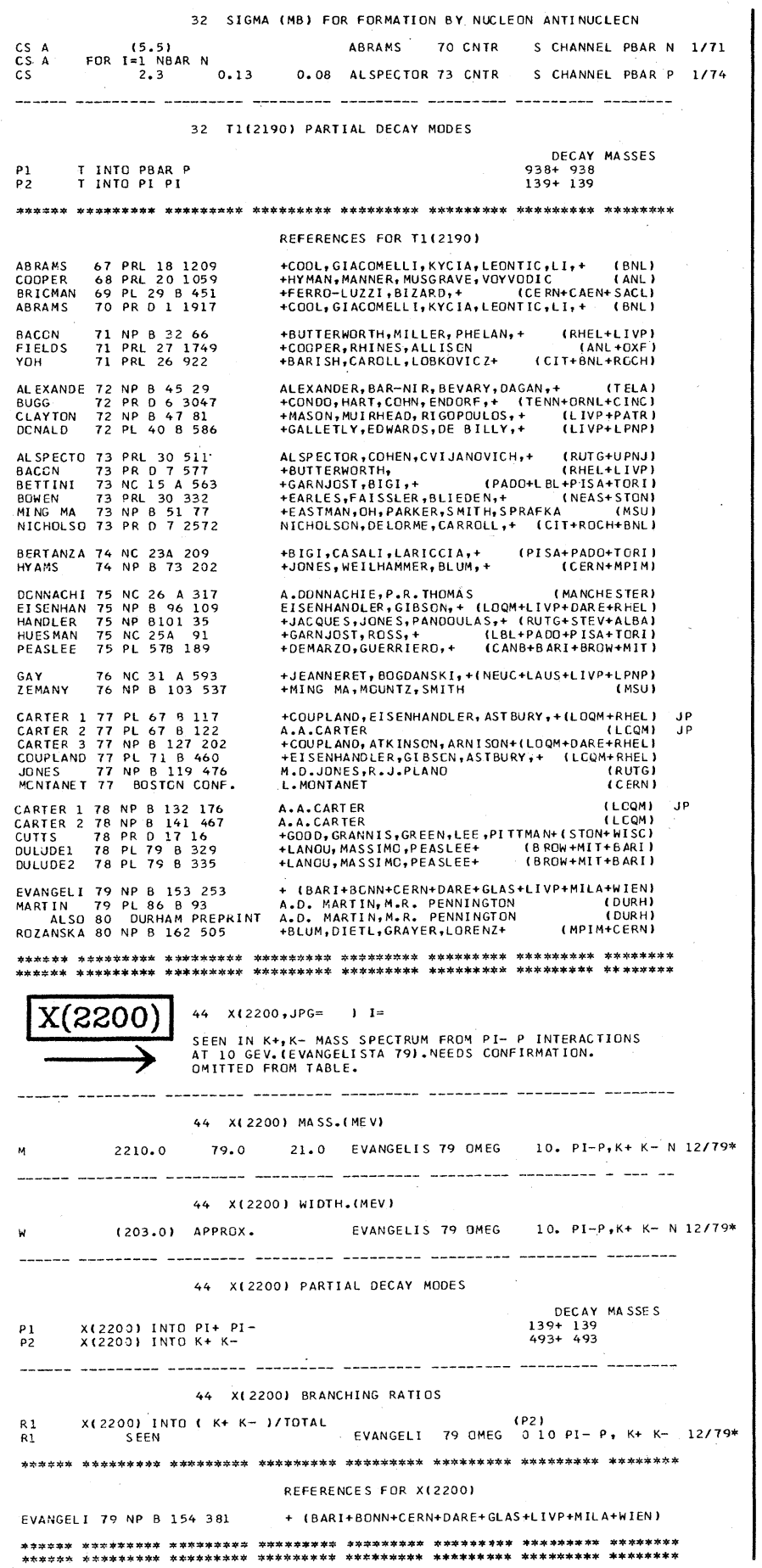

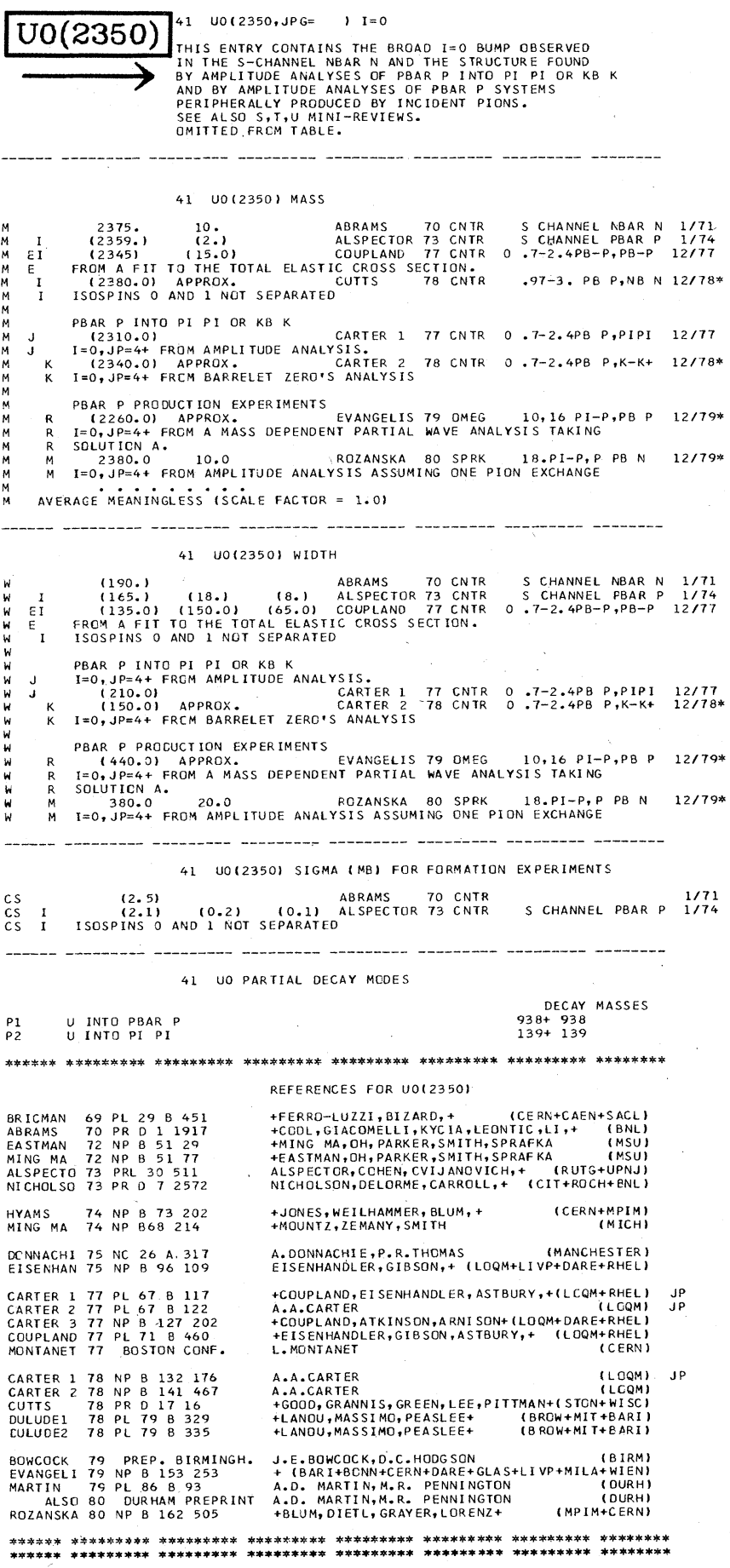


Mesons

U1(2400), $\overline{\mathrm{N}} \mathrm{N}(1400-3600)$

\section{For notation, see key at front of Listings.}

Data Card Listings

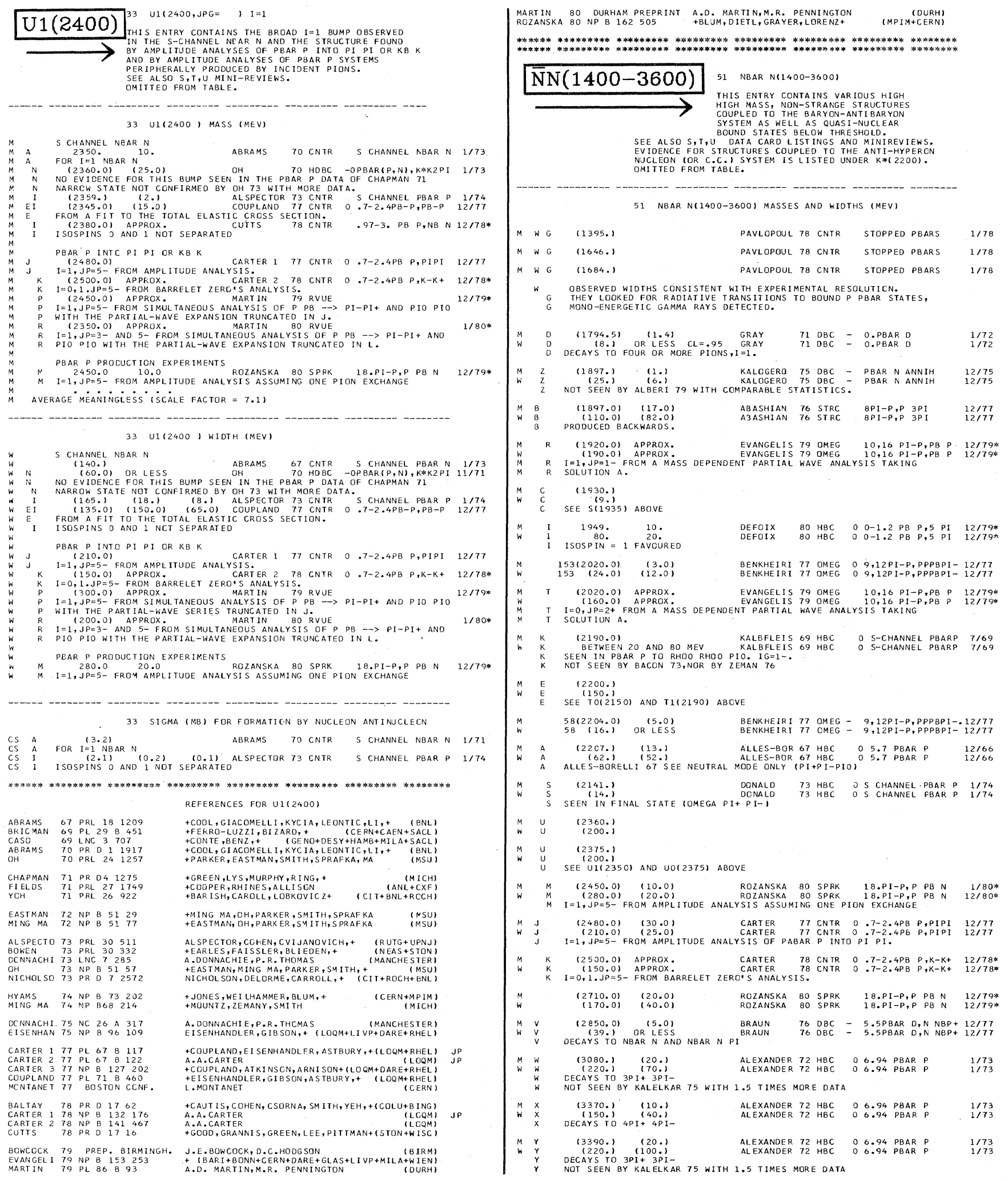




\section{Data Card Listings}

Mesons

For notation, see key at front of Listings.

$X(1900-3600), e^{+} e^{-}(1100-3100)$

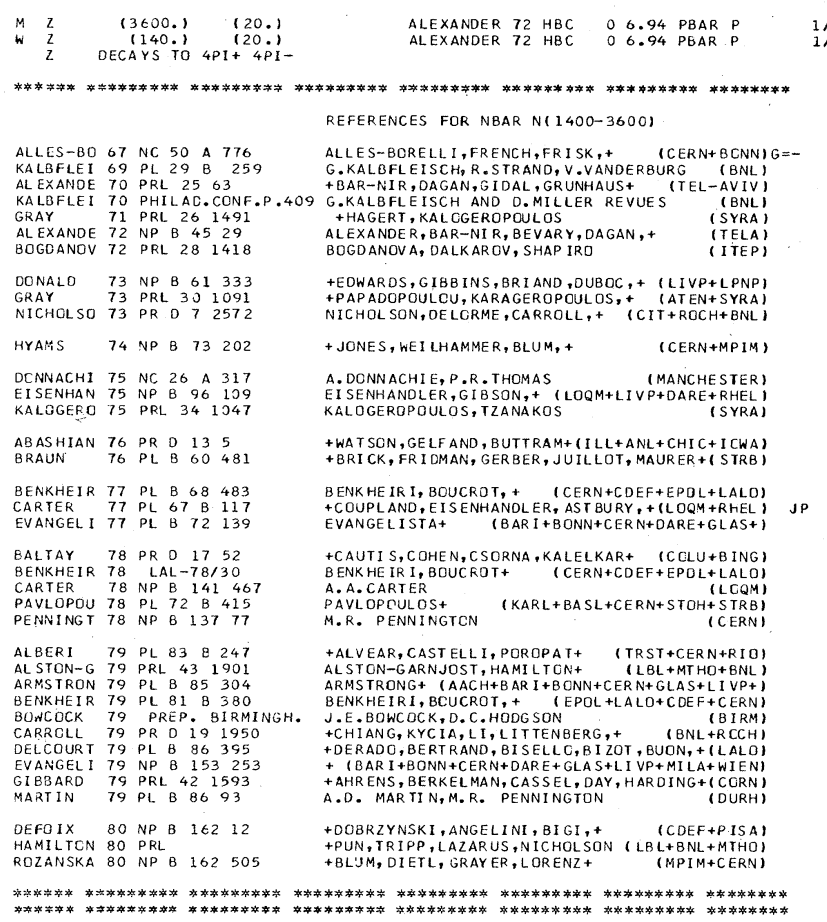

$\mathrm{X}(1900-3600)$ 46 $\times(1900-36001$

THIS ENTRY CONTAINS VARIOUS HIGH-MASS
NON-STRANGE PEAKS. OMITIED FROM TABLE.

The high mass region is covered nearly continuously by evidence for peaks of various widths and decay modes. As a satisfactory grouping into particles is not yet possible, we list all the $\mathrm{Y}=0$ bumps coupled neither to $\bar{N} \mathrm{~N}$ nor to $\mathrm{e}^{+} \mathrm{e}^{-}$, and having $M>1900 \mathrm{MeV}$, together, ordered by increasing mass. Note that ANTIPOV $72\left(\pi^{-} \mathrm{p} \rightarrow \mathrm{p} \mathrm{M^{- }}\right.$ at 25 and $40 \mathrm{GeV} / \mathrm{C})$ see no narrow bumps.

\section{X(1900-3600) MASSES AND WIDTHS (MEV)}

$\begin{array}{llllll}M & 100(1858 .) & 118 .) & & \text { THCNPSON } 74 \mathrm{HBC}+13 \mathrm{PI}+\mathrm{P}, 2 \mathrm{RHO} & 12 / 75 \\ W & 100(108 .) & 141 .) & (2.7 .) & \text { THOMPSON } 74 \mathrm{HBC}+13 \mathrm{PI}+\mathrm{P}, 2 \mathrm{RHO} & 12 / 75\end{array}$

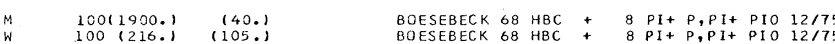

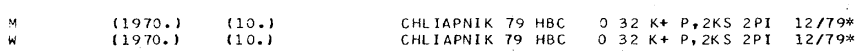

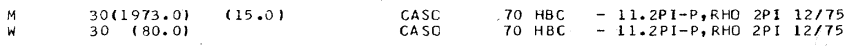

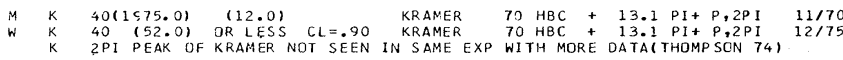

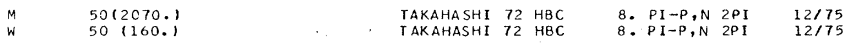

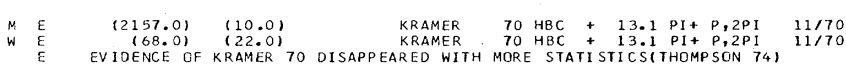

$M \quad(2190.01 \quad(10.0) \quad(2207.01(22.0) \quad$ CLAYTON 67 HBC +-2.5 PBAR, A2 +OMEGA $10 / 67$

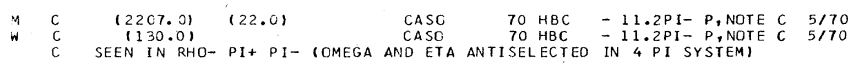

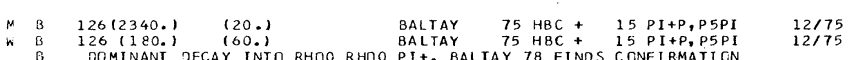

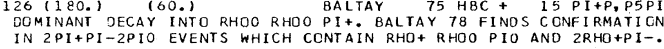

\begin{tabular}{|c|c|c|c|c|c|}
\hline $\begin{array}{r}\left(\begin{array}{rl}2500 \\
(87.0) \\
0\end{array}\right) \\
0\end{array}$ & $(32.0)$ & $\begin{array}{l}\text { ANDERSON } \\
\text { ANDERSON }\end{array}$ & $\begin{array}{l}69 \text { MMS } \\
69 \text { MMS }\end{array}$ & $\begin{array}{l}16 P_{1-}^{P}, B A C K W 9 \\
=16 \text { PI- P, BACKW }\end{array}$ & $\begin{array}{l}8 / 69 \\
8 / 69\end{array}$ \\
\hline $\begin{array}{l}550(2620 .) \\
550(85 .)\end{array}$ & $\left(\begin{array}{l}20 .) \\
(30 .)\end{array}\right.$ & $\begin{array}{l}\text { BAUD } \\
\text { BAUD }\end{array}$ & $\begin{array}{l}69 \text { MMS } \\
69 \text { MMS }\end{array}$ & $\begin{array}{l}8 \cdot-10 . \\
=8 .-10 \cdot P_{I-P}\end{array}$ & $\begin{array}{l}9 / 69 \\
9 / 69\end{array}$ \\
\hline $\begin{array}{r}(2676.01 \\
1150.01 \\
\text { SEEN IN RHO }\end{array}$ & $\begin{array}{l}(27.0) \\
0-\text { PI+ } P I-\text { IOMEGA A }\end{array}$ & $\begin{array}{c}\text { CASC } \\
\text { CASO } \\
\text { AND ETA ANTI }\end{array}$ & $\begin{array}{l}70 \mathrm{HBC} \\
70 \mathrm{HBC} \\
\text { TISELECTED }\end{array}$ & 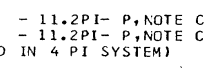 & $\begin{array}{l}5 / 70 \\
5 / 70\end{array}$ \\
\hline $\begin{array}{l}640(2800 .) \\
640(46 .)\end{array}$ & $\left(\begin{array}{l}20 .) \\
(10 .)\end{array}\right)$ & $\begin{array}{l}\text { BAUD } \\
\text { BAUD }\end{array}$ & $\begin{array}{l}69 \mathrm{MMS} \\
69 \mathrm{MMS}\end{array}$ & 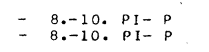 & $\begin{array}{l}9 / 69 \\
9 / 69\end{array}$ \\
\hline $\begin{array}{l}1552820.1 \\
15 \quad 150 . \\
\text { SEEN IN IK }\end{array}$ & $\begin{array}{l}(10.1) \\
10.0) \\
\text { KBAR PII PI) + MASS }\end{array}$ & $\begin{array}{l}\text { SABAU } \\
\text { SAAAU } \\
\text { DISTRIBUTIO }\end{array}$ & $\begin{array}{rl}71 & \mathrm{HBC} \\
71 & \mathrm{HBC}\end{array}$ & $\begin{array}{l}+\quad 8: p_{1+p} \\
+8: P 1+p\end{array}$ & $\begin{array}{l}11 / 71 \\
11 / 71\end{array}$ \\
\hline $\begin{array}{l}230(2880 .) \\
230(15 .)\end{array}$ & $\begin{array}{l}\text { I20.1 } \\
\text { OR LESS }\end{array}$ & $\begin{array}{l}\text { BAUD } \\
\text { BAUD }\end{array}$ & $\begin{array}{l}69 \mathrm{MMS} \\
69 \mathrm{MMS}\end{array}$ & $\begin{array}{l}=8 \cdot-10 . P_{1-} \\
=8 \cdot-10 .: P_{I-p}\end{array}$ & $\begin{array}{l}9 / 69 \\
9 / 69\end{array}$ \\
\hline $\begin{array}{l}43(3013.1 \\
43 \quad 10.1 \\
4.35 .0 . \\
\text { NOT SEEN BY }\end{array}$ & 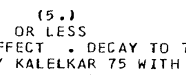 & $\begin{array}{l}\text { YOST } \\
\text { YOST } \\
\text { YIONS } \\
\text { SIONES }\end{array}$ & $\begin{array}{rl}71 & \mathrm{HBC} \\
71 & \mathrm{HBC} \\
\text { ORE } & \text { DATA }\end{array}$ & $\begin{array}{l}11 . P I+P, p(8 P I)+ \\
+11 . P 1+P, p(8 G I)\end{array}$ & $\frac{11}{5}$ \\
\hline $\begin{array}{r}(3025.0) \\
125.01\end{array}$ & $\begin{array}{c}(20.0) \\
\text { APPROX. }\end{array}$ & $\begin{array}{l}\text { BAUD } \\
\text { BAUD }\end{array}$ & $\begin{array}{l}70 \text { MMS } \\
70 \text { MMS }\end{array}$ & $\begin{array}{l}=10.5-13 P_{I-P} \\
=10.5-13 P_{1-}\end{array}$ & $\begin{array}{l}5 / 70 \\
5 / 70\end{array}$ \\
\hline $\begin{array}{r}(3075.0) \\
125.0)\end{array}$ & $\begin{array}{l}(20.0) \\
\text { APPRUX. }\end{array}$ & $\begin{array}{l}\text { BAUD } \\
\text { BAUD }\end{array}$ & $\begin{array}{l}70 \text { MM } \\
70 \mathrm{MMS}\end{array}$ & $\begin{array}{l}=10.5-13 \mathrm{PI}-\mathrm{P} \\
=10.5-13 \mathrm{PI}-\mathrm{P}\end{array}$ & $\begin{array}{l}70 \\
70\end{array}$ \\
\hline $\begin{array}{r}(3145.01 \\
(10.0)\end{array}$ & $\begin{array}{l}(20.0) \\
\text { OR LESS }\end{array}$ & $\begin{array}{l}\text { BAUD } \\
\text { BAUD }\end{array}$ & $\begin{array}{l}70 \text { MMS } \\
70 \text { MMS }\end{array}$ & 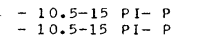 & 70 \\
\hline $\begin{array}{r}\left(\begin{array}{r}3475.0) \\
(30.0)\end{array}\right.\end{array}$ & $\begin{array}{c}(20.0) \\
\text { APPROX. }\end{array}$ & $\begin{array}{l}\text { BAUD } \\
\text { BAUD }\end{array}$ & $\begin{array}{l}70 \mathrm{MMS} \\
70 \mathrm{MMS}\end{array}$ & $\begin{array}{l}=14-15.5 \\
=141-15.5 \\
14 I-p\end{array}$ & 700 \\
\hline $\begin{array}{r}\left(\begin{array}{r}3535.0) \\
(30.0)\end{array}\right.\end{array}$ & $\begin{array}{l}(20.0) \\
\text { APPROX. }\end{array}$ & $\begin{array}{l}\text { BAUD } \\
\text { BAUD }\end{array}$ & $\begin{array}{l}70 \mathrm{MMS} \\
70 \mathrm{MMS}\end{array}$ & $\begin{array}{l}5 p_{I-p} \\
5 \text { PI } p\end{array}$ & $\begin{array}{l}5 / 70 \\
5770\end{array}$ \\
\hline
\end{tabular}

REFERENCES FOR $X(1900-3600)$

+MASON, MUIRHEAD, FILIPPAS+ LLIVERPOOL+ATHENS) BOES EBECK, DEUTSCHMANN, + (AACHEN+BERLIN+C ERN)
+ +COLLINS,
(BNL +CARN) CERN BOSCN SPECTROMETER GROUP
CERN BCSCN SPECTROMETER GROUP CERN BCSCN SPECTROMETER GROUP (CERN)
+CONTE, TOMASINI, COROS+ (GENO+HAMB +MILATSACN) +BARTON, GUTAY, LICHTMAN, MILLER, + (PUILDUE)
(PUR +URETSKY
+ MORRIS, AL BRIGHT, BRUCKER, LANNUTT I
(BUCH+ANL)
(FSU) (PURD) CAUTIS, COHEN, KALELKAR, PISELLO, + + COLU+B ING M. S. KALELKAR

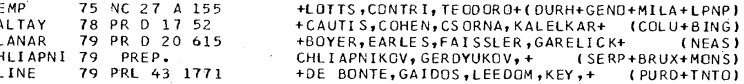

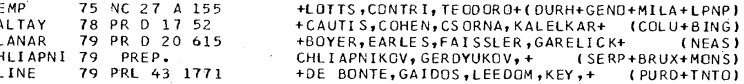

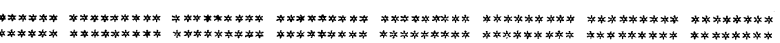
$\mathrm{e}^{+} \mathrm{e}^{-}(1100-3100)$

$E+E-11100-3100, J P G=1-1 \cdot I=$ THIS ENTRY CONTAINS NON-STRANGE
VECTOR MESONS COUPLED TO E+ E-CPHOTON VECTOR MESONS COUPLED TO E+ E-CPHOTON
BETWEEN PHI AND J/PSI MASS REGION.
SEE ILSO FHO PRIMECI250) SEE ALSO RHO PRIME(1250) AND RHO PRIMEI (1600) MINI-REV
OMITIED FROM TABLE.

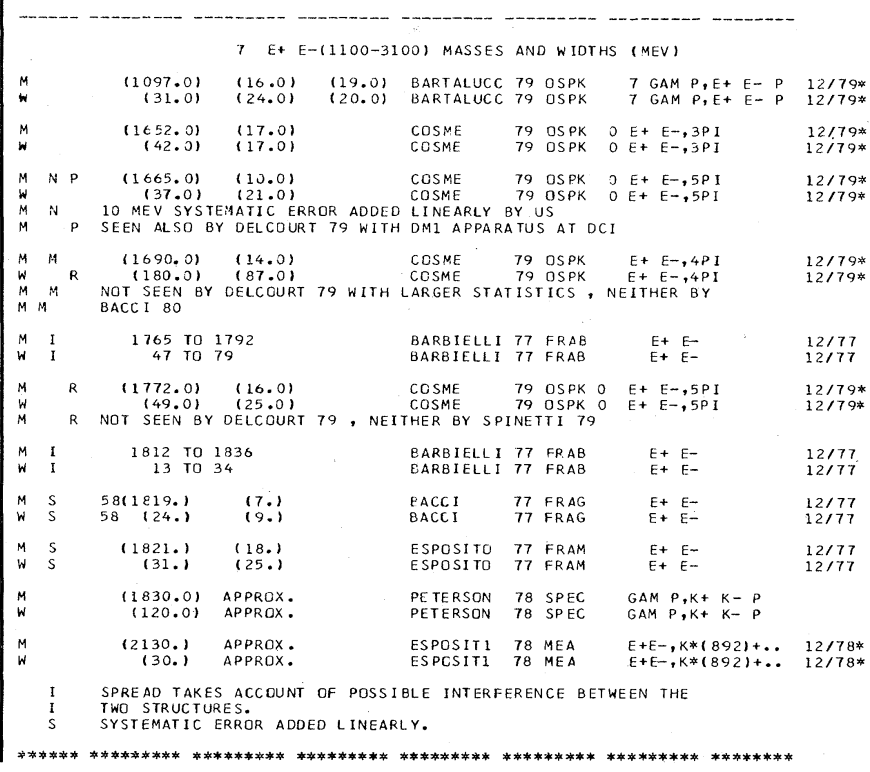




\section{Mesons}

\section{CHARMONIUM, X(2830), U(2980)}
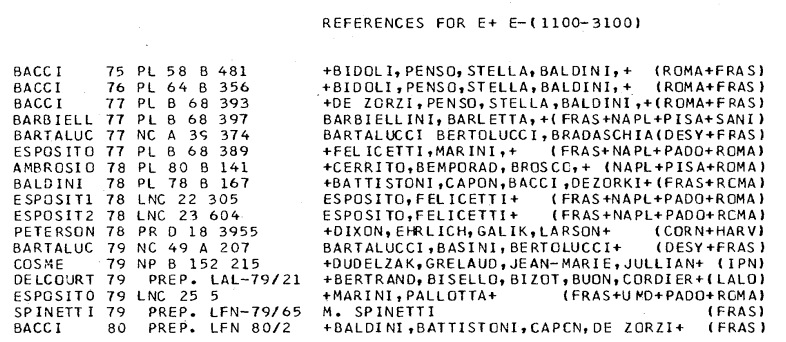

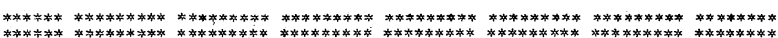

\section{The Charmonium System}

We group into this system those meson states commonly believed to consist of charmed-quarkcharmed-antiquark pairs. Since the discovery of the J/ $\psi(3100$ ) (AUBERT 74, AUGUSTINII 74) this family has increased to at least 13, of which we tabulate 9 as well established particles. Figure 1 shows the states of charmonium below the $\psi(3685)$, interpreted by the charmonium model, at the time of the 1979 Chicago Lepton-Photon Conference: 1) the $\mathrm{X}(2830)$ and $X(3455) \mathrm{O}^{-+}$candidates were not seen by the Crystal Ball Experiment (PARTRIDGE 79); 2) a new state, U(2980), had been discovered by examining the inclusive photon spectrum at the $\psi(3685)$ mass.

\section{Data Card Listings For notation, see key at front of Listings.}
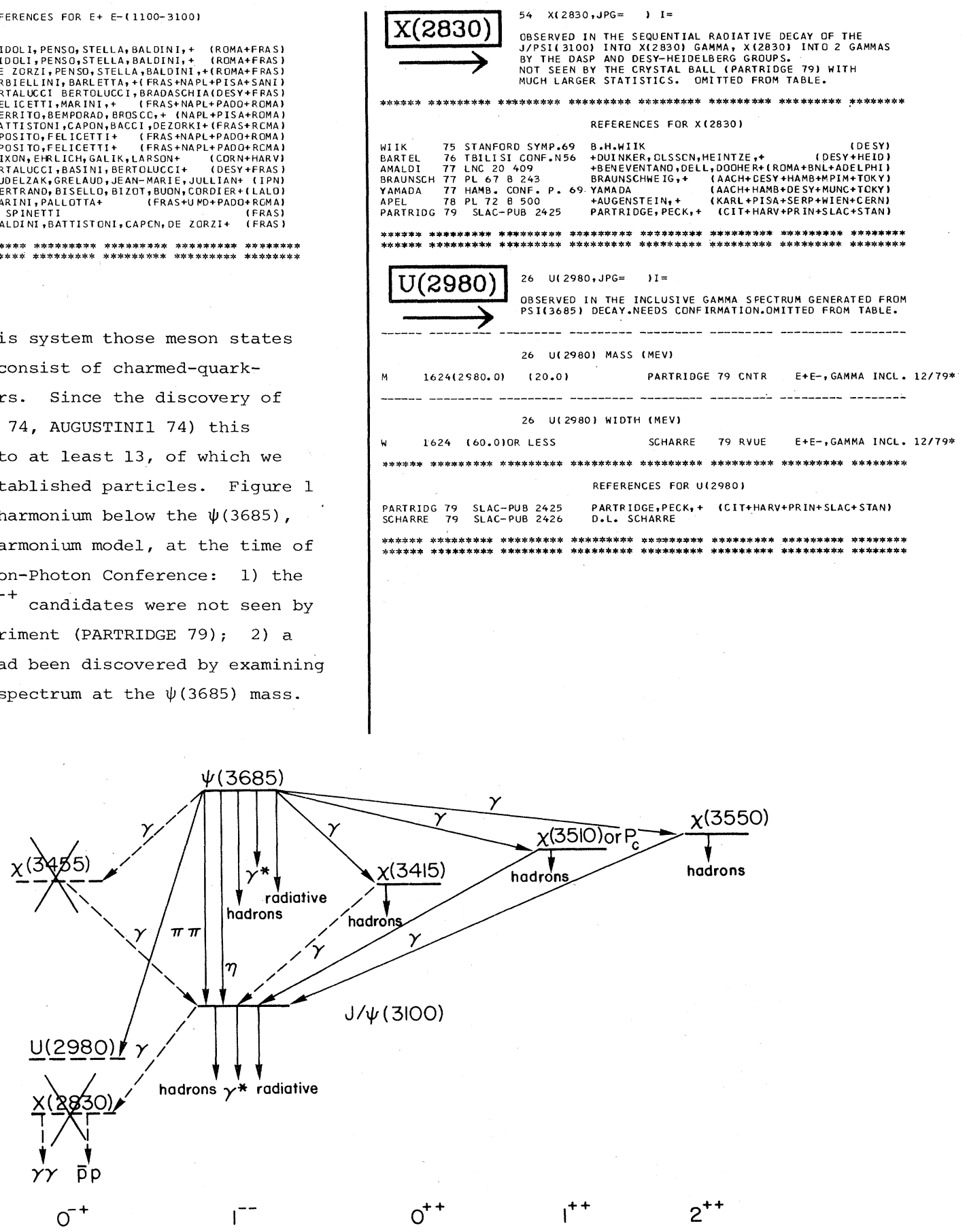

Fig. 1. The current state of knowledge of the charmonium system and transitions, as interpreted by the charmonium model. Uncertain states and transitions are indicated by dashed lines. JPC quantum number assignments are in some cases tentative, but all are at least consistent with experiment; see individual particle listings for discussion. The notation $\gamma^{*}$ refers to decay processes involving decays to $e^{+} e^{-}$and $\mu^{+} \mu^{-}$. The crosses correspond to the states not seen by PARTRIDGE 79.

Rev. Mod. Phys., Vol. 52, No. 2, Part II, April 1980 


\section{Data Card Listings \\ For notation, see key at front of Listings.}

Mesons

$\mathrm{J} / \psi(3100)$

$\mathrm{J} / \psi(3100)$ 70 J/PSII 3100, JPG $=1--1$ I=0

70 J/PSI(3100) MASS (MEV)

WE USE INDEPENDENT MEASUREMENTS OF THE J/P SI (3100) PERFORM A CONSTRAINED FIT.

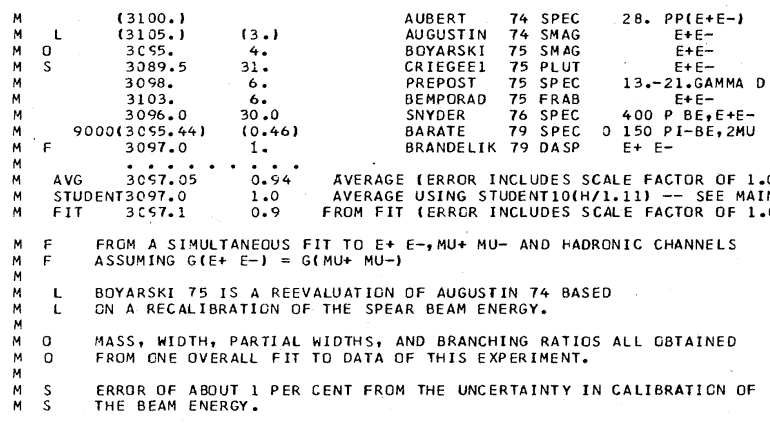

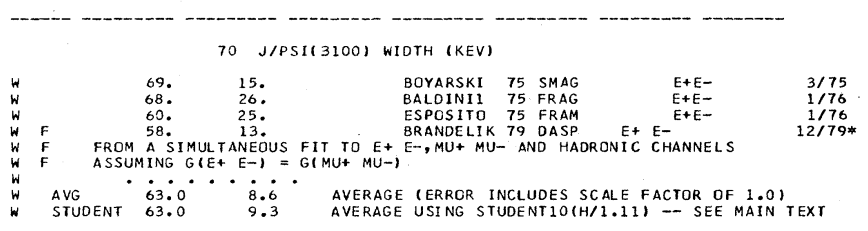

70 J/PSI 3100$)$ PARTIAL DECAY MODES

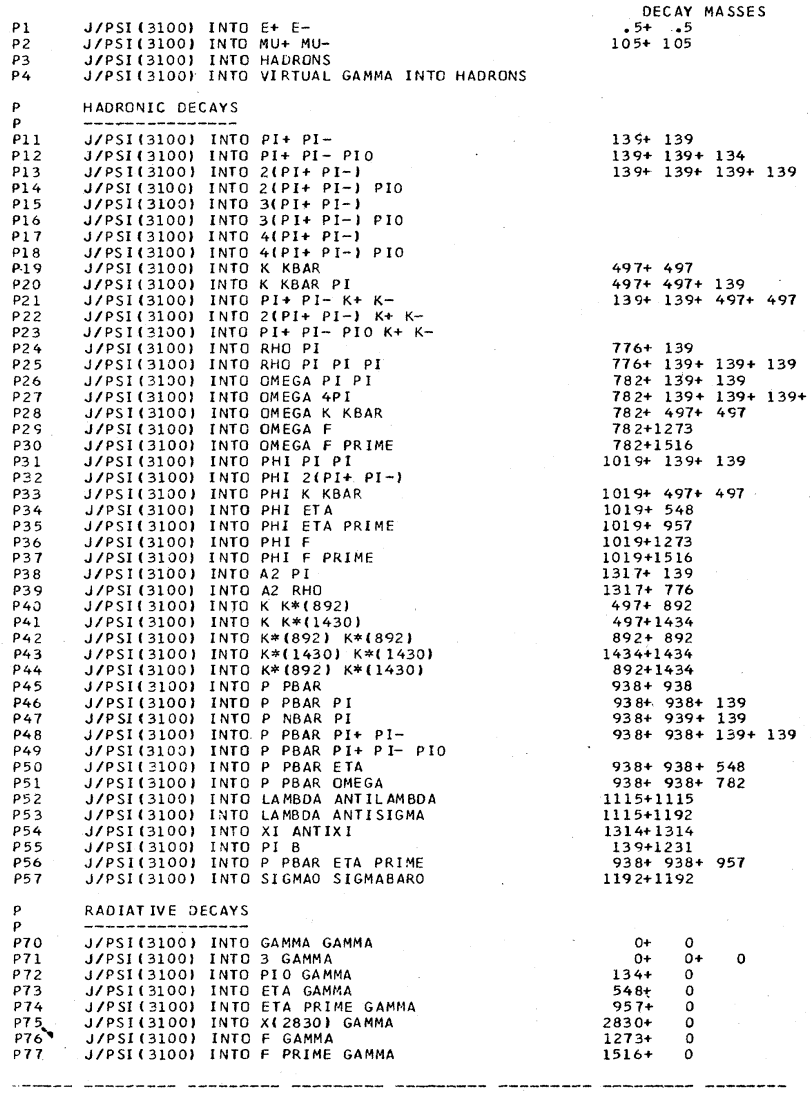

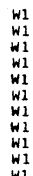

70 J/PSI(3100) PARTIAL WIDTHS (KEV)

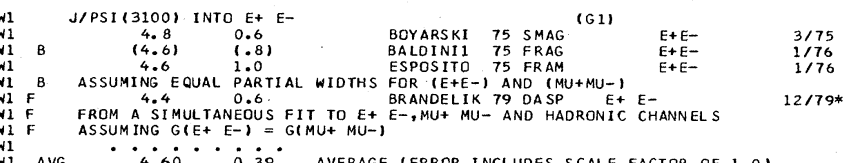

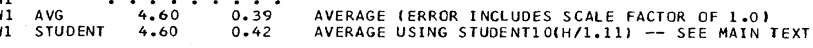

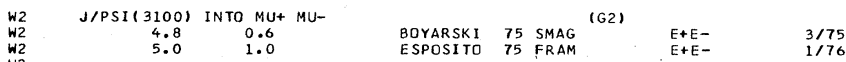

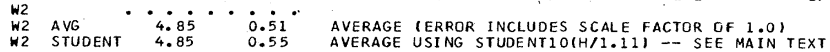

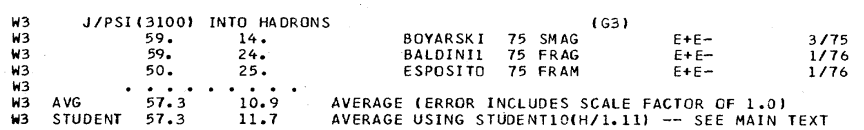

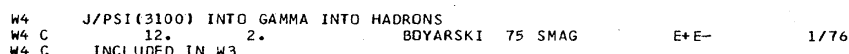

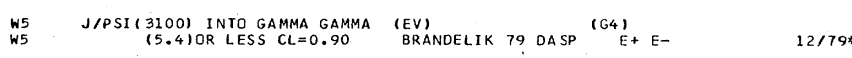

70 J/PSI(3100) BRANCHING RATIOS

FOR THE BRANCHING RATIOS R1 - RA, SEE ALSO THE PARTIAL
WIDTHS ABOVE, AND (PARTIAL WIDTHS)*RI BELOW.

WIDTHS ABOVE, AND (PARTIAL WTOTHS)*RI BELOW.

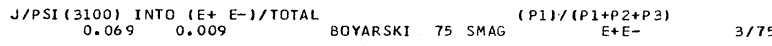

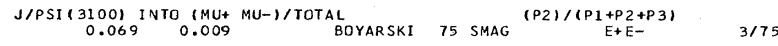

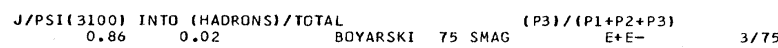

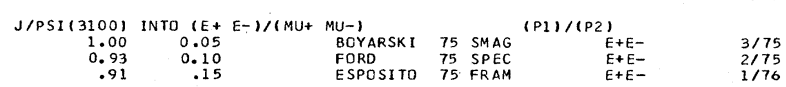

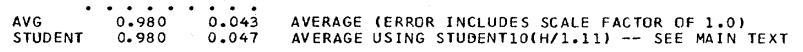

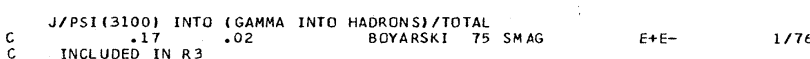

HADRONIC DECAYS

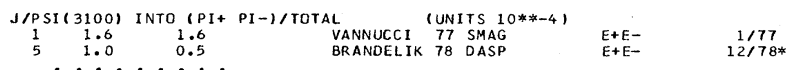

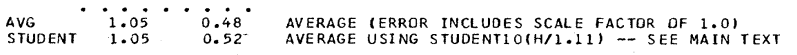

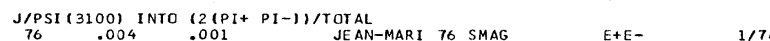

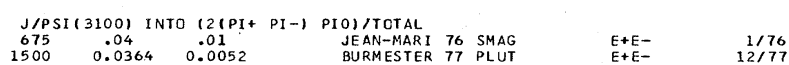

avG. - O.0372 0.0046 AVERAGE IERROR INCL UDES SCALE FAC TOR OF 1.01

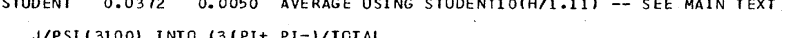

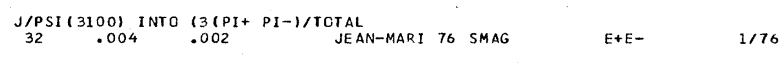

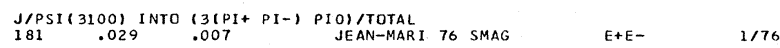

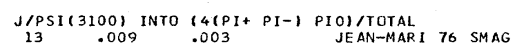

J/PSTI 3100$)$ INTO
205 P It $0.00720 .0023-K+K-1 /$ TOTAL
VANNUCCI 77 SMAG

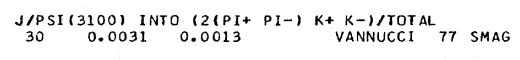

E+E-. $\quad 1 / 76$

E+E- $\quad 1 / 77$

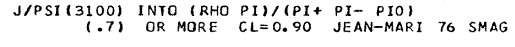

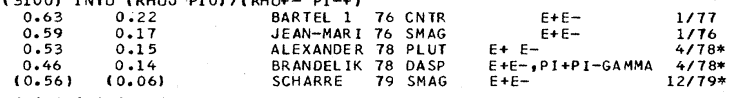

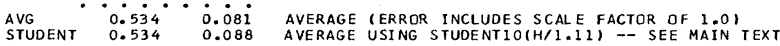

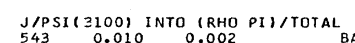

$\begin{array}{llllll}543 & 0.010 & 0.002 & \text { BARTEL } 1 & 76 & \text { CNTR } \\ 153 & 0.013 & 0.003 & \text { JEANMAR } & \text { CN SMAG }\end{array}$

$\begin{array}{ll}0.016 & 0.003 \\ 0.0163 & 0.004 \\ 0.013 & 0.0021 \\ 0.01321 & 0.00211\end{array}$

JEANMAR I 76 SMAG
ALEXANOER 78 PLUT
BRANDELIK 78 PASP

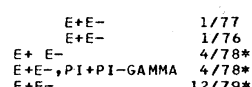

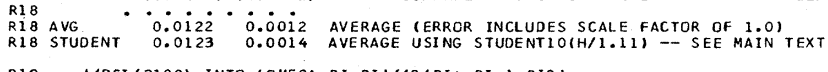

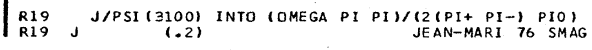

$E+E-\quad 1 / 76$ 
Mesons $\mathrm{J} / \psi(3100)$
Data Card Listings For notation, see key at front of Listings.

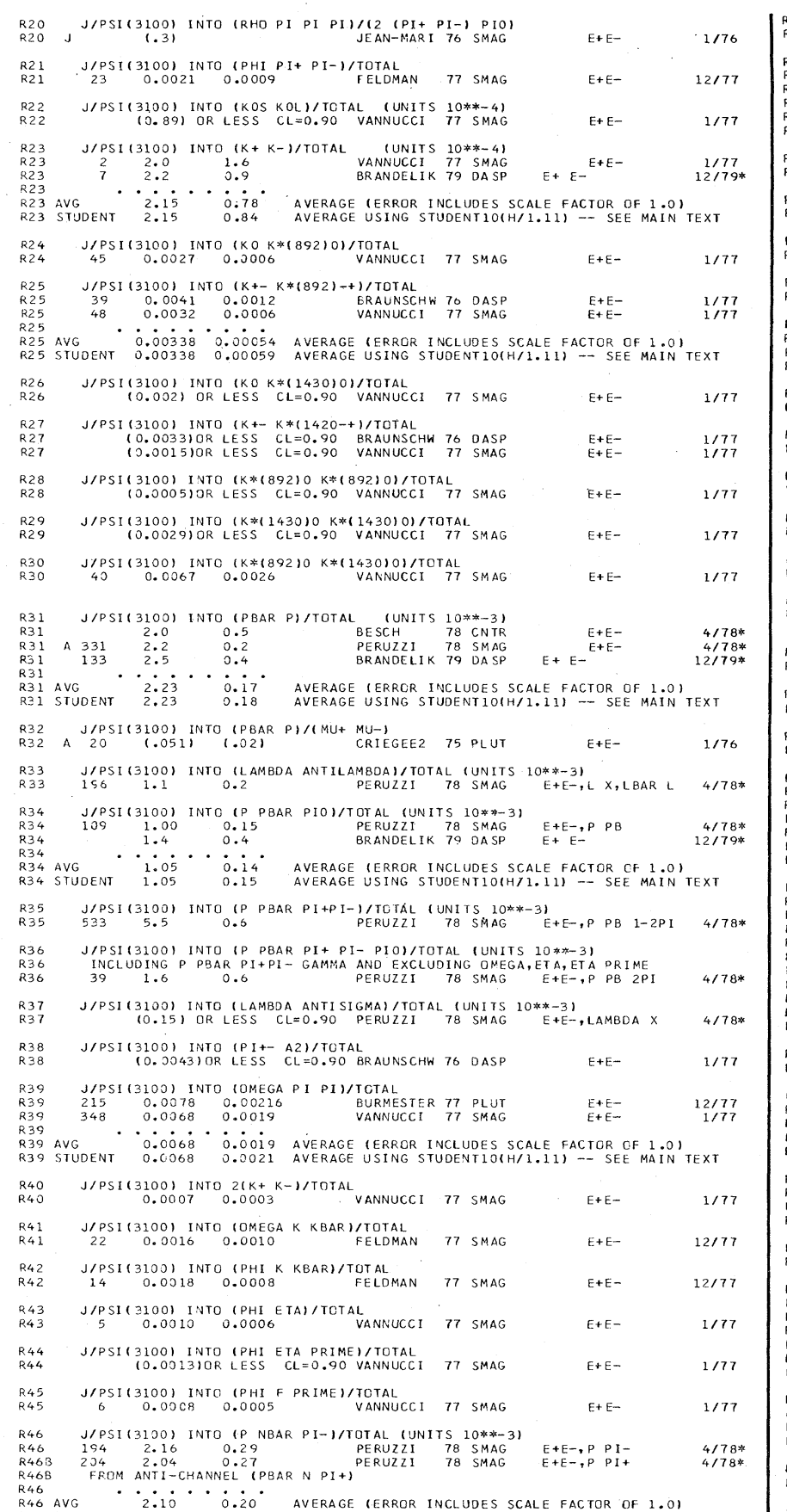

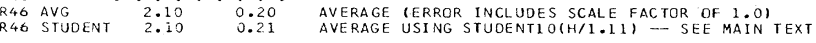

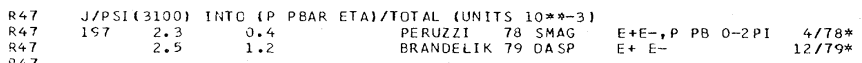

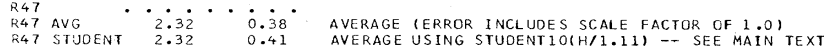

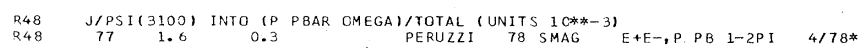

$\begin{array}{llllll}R 49 & \text { J/PSI } 31001 \text { INTO }(K E S \text { K+- PI-+1/TOTAL } \\ \text { RANNUCCI } & 77 \text { SMAG } & \text { E+E- } & 1 / 77\end{array}$

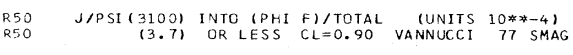

J/PSI (3100) INTO (PHI 2 (PI+PI-) )/TCTAL
$(0.0015)$ LER
CL=0.90 VANNUCCI 77 SMAG

J/PSI $(3100)$ INTO COMEGA F)/TOTAL
81000190.0008 VANNUCCI 77 SMAG

$E+E-\quad 1 / 77$

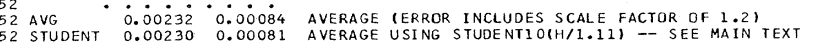

J/PSI (3100) INTO (OMEGA F PRINE)/TCTAL (UNITS 10**-4)
$(1.6)$ OR LESS CL $=0.90$ VANNUCCI 77 SMAG

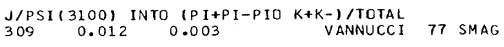

$E+E-\quad 1 / 77$

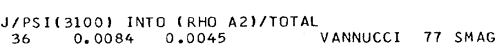

$E+E-\quad 1 / 77$

$E+E-\quad 1 / 77$

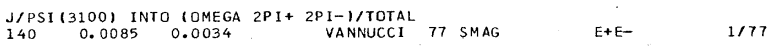

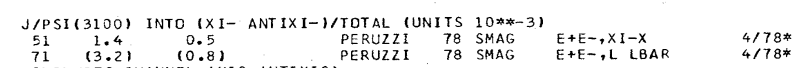

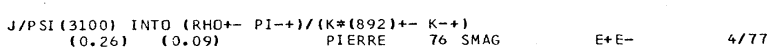

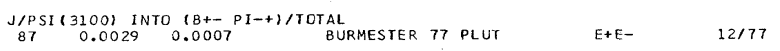

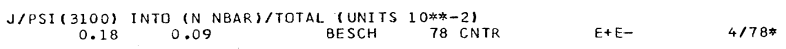

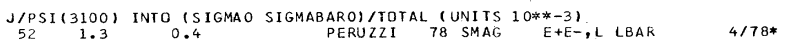

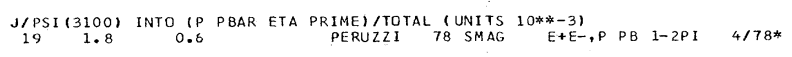

FINAL STATE $2(P 1+P I-P P I$
A ASSUMING ANGULAR DISTRIBUTION $(1,+\operatorname{COS}(T H E T A) * * 2)$

RADIATIVE DECAYS

J/PSI(3100) INTO 12 GAMMA)/TOTAL (UNITS $10 * *-3)$ (P4)
$10.5)$ DR LESS CL=0.90 BARTEL 77 CNTR

$J / P S I(3100)$ INTO (PIO GAMMA)/TOTAL (UNITS $10 * *-3)$
10
BRANDELIK 79 DASP

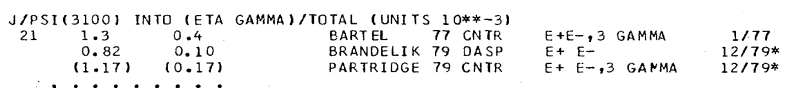

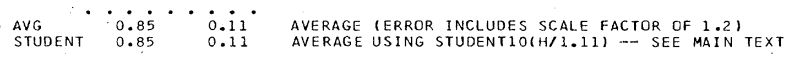
J/PSI(3100) INTO (ETA PRIME GAMMA)/TOTAL (UNITS 10**-3)
$(3.3)$ OR LESS CL=.90 BACCI

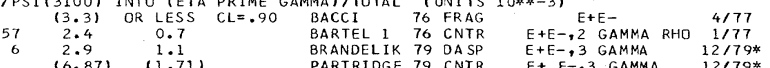

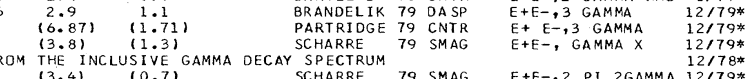

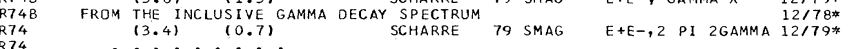

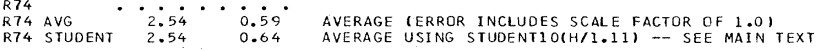

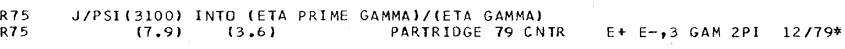

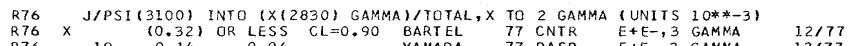

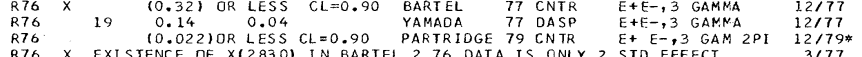
R78 J/PSI(3100) INTO (3 GAMMA)/TOTAL (UNITS 10**-3)

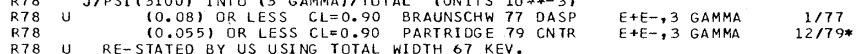

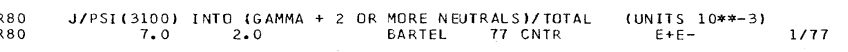

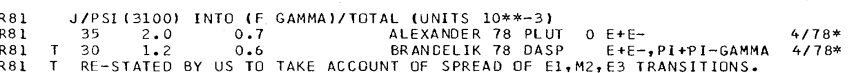

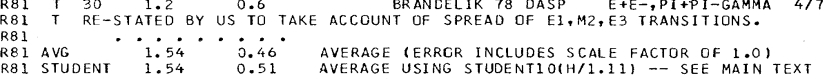

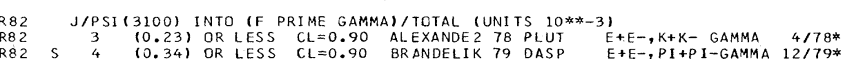

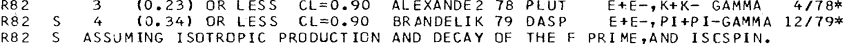
$R 84$
$R 84$ JPPSI (3100) INTO (P PBAR GAMMA)/TOTAL (UNITS 10 **-3) 70 J/PSI(3100) G(I)*G(E+E-)/G(TOTAL) (KEV) THIS COMBINATION OF A PARTIAL HIDTH WITH THE PAR IIAL WIDTH
INTO E+E-ANO WITH THE TOTAL WIDTH IS OBTA INED FROM THE INTEGRATED CROSS-SECTION INTD CHANNELII IN THE E+E- ANNIIILATICN.
WE ONLY LIST DATA NOT HAVING BEEN USED TO DETERMINE THE PARTIAL
WIDTH GII OR THE BRANCHING RATIG GIIITTOTAL. $G(E+E-) * G(E+E-) / G(T$ OTAL)
$(.32) \quad(.07)$

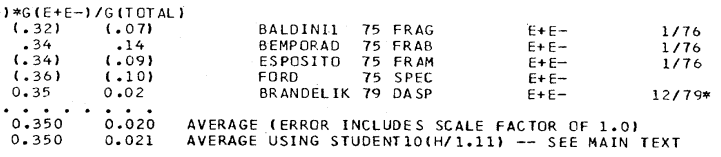




\section{Data Card Listings}

Mesons

\section{For notation, see key at front of Listings. $\mathrm{J} / \psi(3100), \chi(3415), \chi(3455), \mathbf{P}_{\mathrm{c}}$ or $\chi(3510)$}

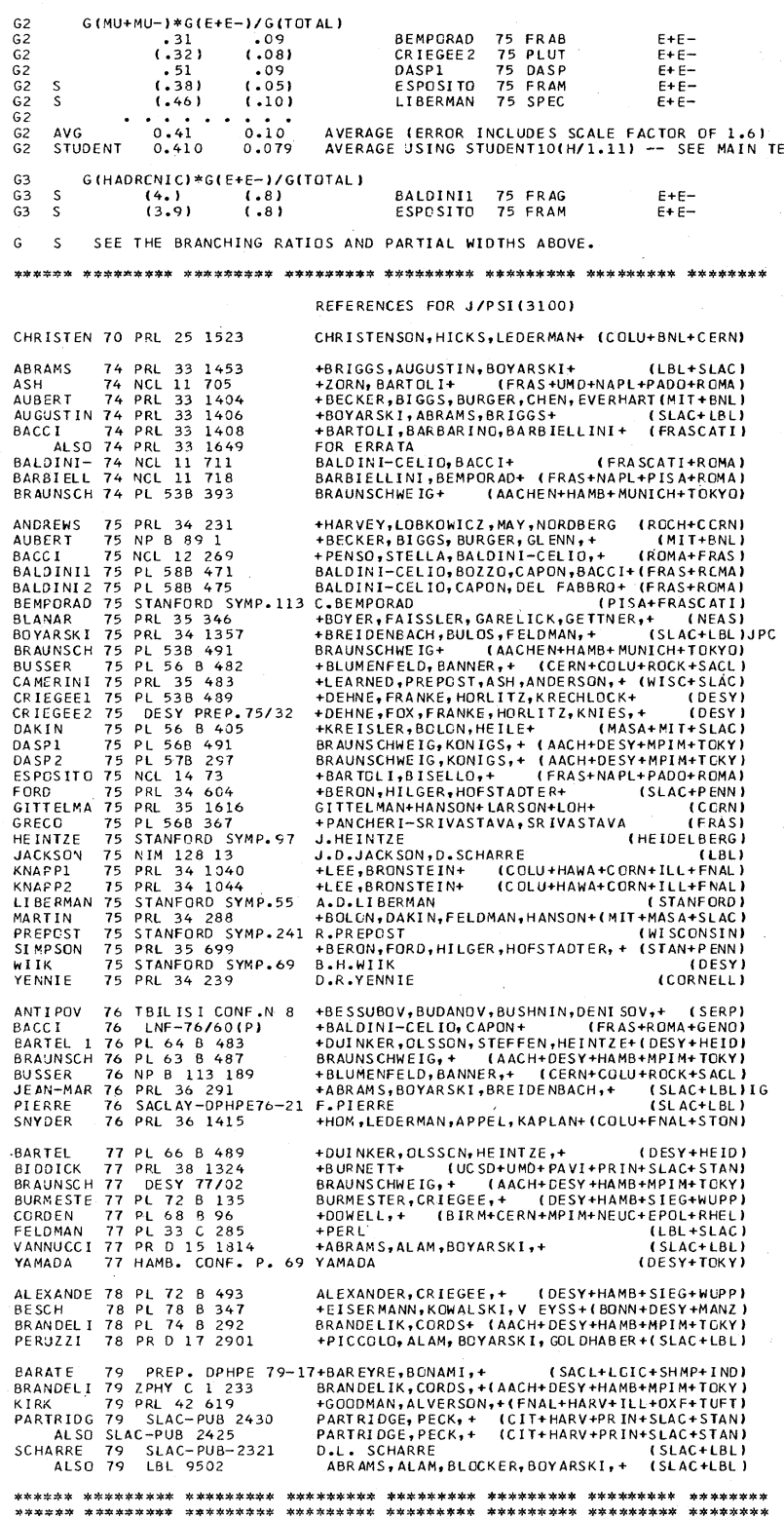

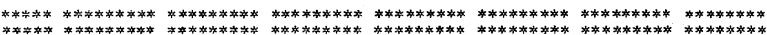

$\chi(3415)$ 56 ${ }^{2}$ CHI $\left.3415, \mathrm{JPG}=0++1\right)$ I=0

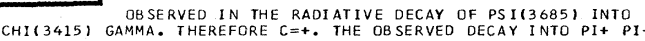

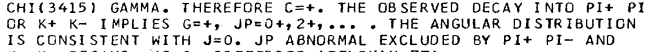

56 CHI(3415) MASS (MEV)

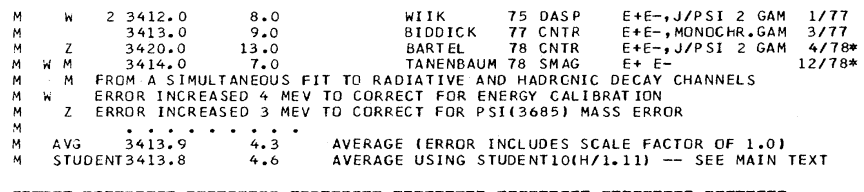

56 CHI(3415) PARTIAL deCAY MODES

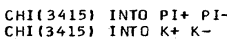

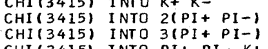

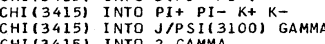

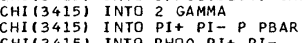

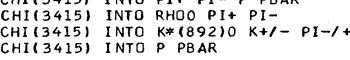

56 CHI(3415) BRANCHING RATIOS

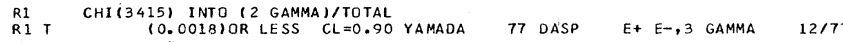

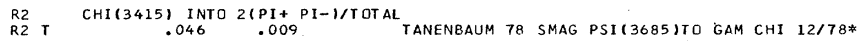

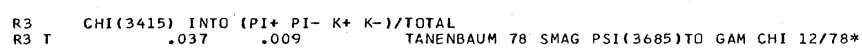

$R 4$
$R 4$

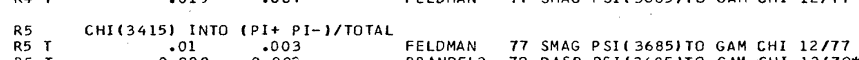

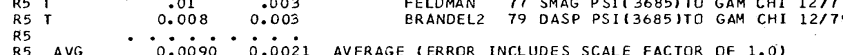

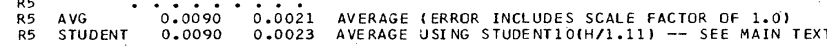

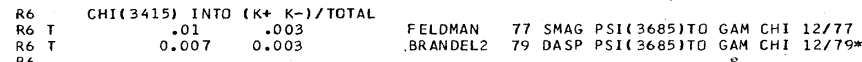

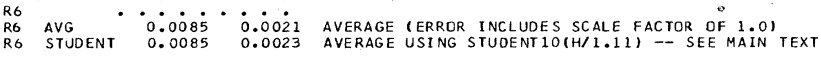

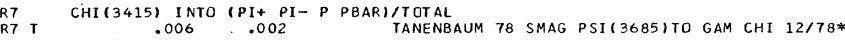

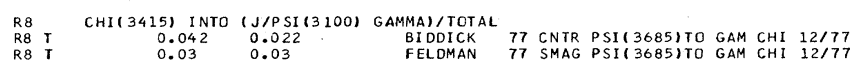

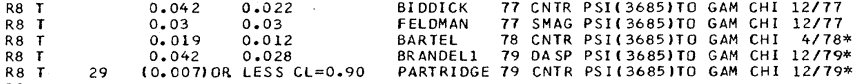

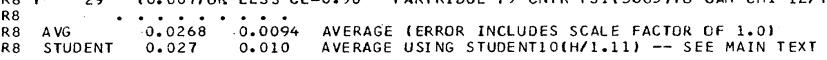

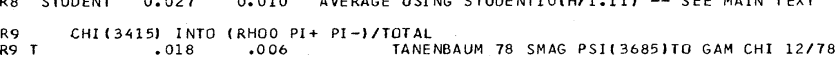

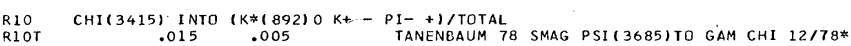

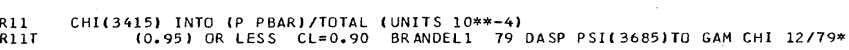

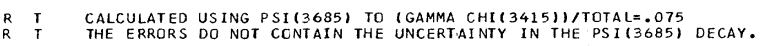

************************************************************************

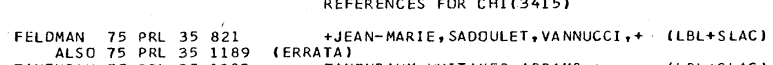

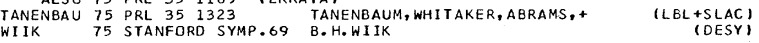

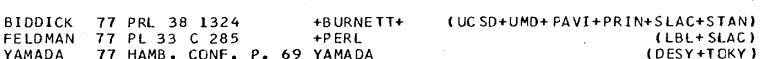

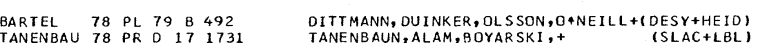

BRANDEL 1792 PHY C 1233 BRANOELIK, CCROS, + ( AACH+ CESY + HAMB +MPIM IMTCCKY)

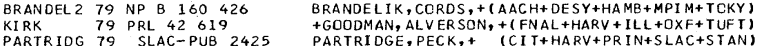

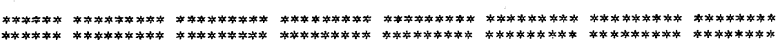

$X(3455)$ 58 CHII $3455, \mathrm{JPG}=$, I $=$

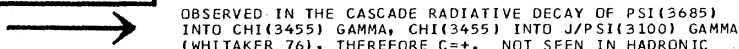

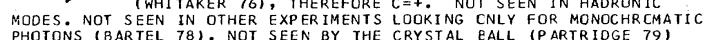
MODES. NOT SEEN IN OTHER EXPERIMENTS LOKING CNLY FOR MONOCHRCMA
PHOTONS BARTEL 78 . NOT SEEN BY THE CRSTAL EALL (PARTRIDGE 79)
WITH SUPER IOR STATISTICS. OMITTED FROM TABLE.

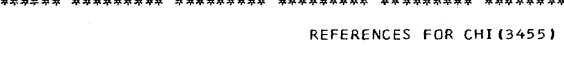

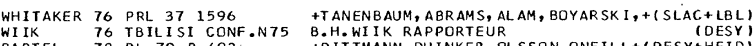

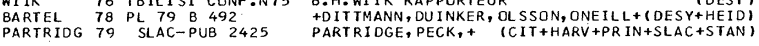

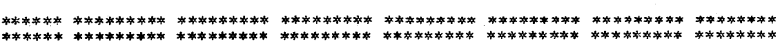

$P_{c}$ Or $\chi(3510)$ S5 PC OR CHI (3510,JPG = + +) I=O OBSERVED IN THE RADIATIVE SEQUENTIAL DECAY
OF THE PSII3685I INTO PC GAMMA, PC, INTO

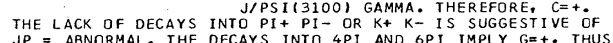

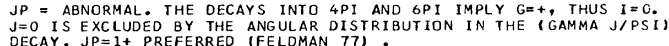




\section{Mesons}

$\mathrm{P}_{\mathrm{c}}$ or $\chi(3510), \chi(3550)$

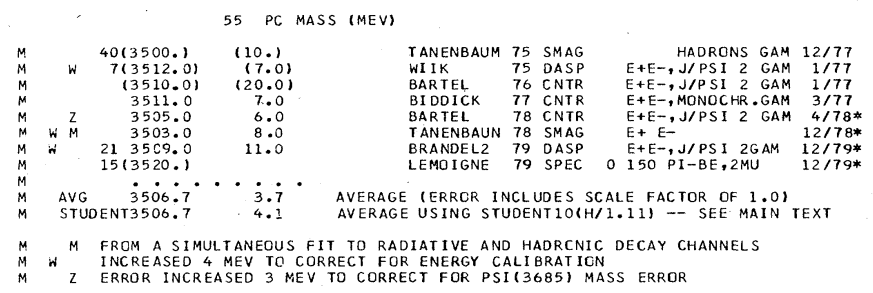

55 PC PARTial DeCAy modes

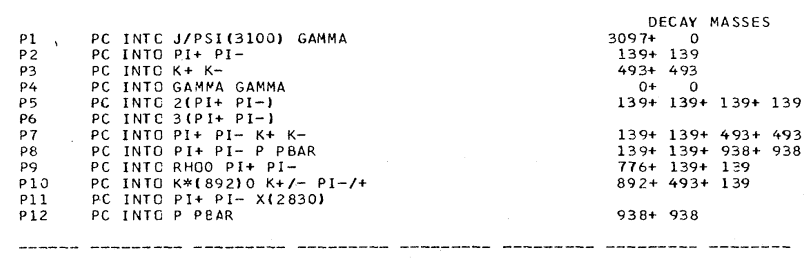

55 PC BRANCHING RATIOS
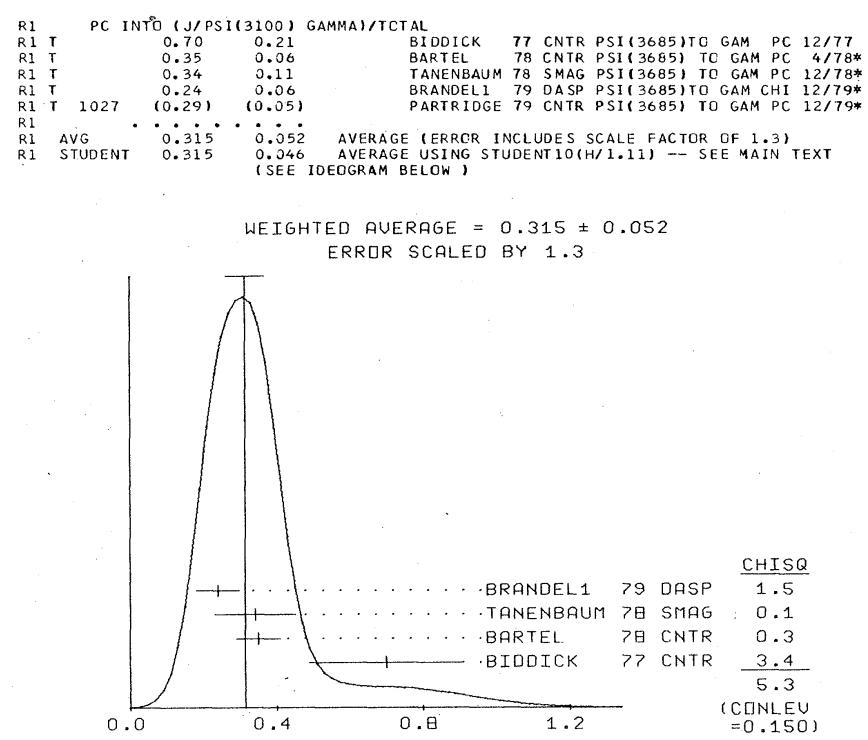

PC INTI (J/PSI(3100) GAMMA),TOTAL

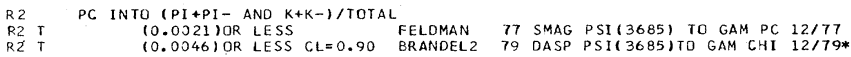

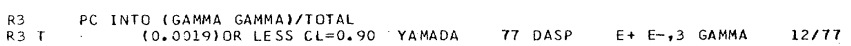

$R 4$
$R 4$ T PC INTC $2(P I+P I-) / T O T A L$
.020
R

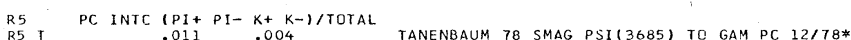

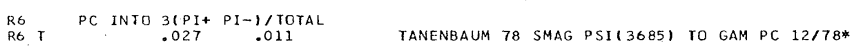

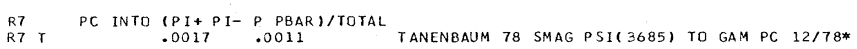

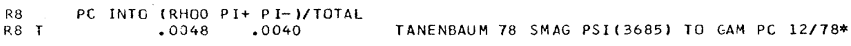

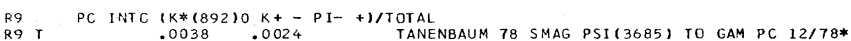

R11 PC INTC (P PBAR)/TOTAL
R11 T
$(0.0014) 0 R$ LESS CL $=0.90$ BRANDEL 2 79 DASP PSI(3685)TO GAM CHI 12/79*

$\begin{array}{llll}R & C & \text { THE VALUE IS NORMALIZED TO THE BRANCHI. RATIO O PSI(3685) } \\ R & C & \text { INTO (J/PSI(3100) ETA) TTOTAL. }\end{array}$

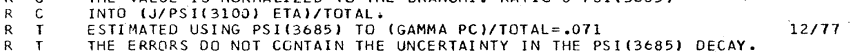

****************************************************************************4

\section{Data Card Listings For notation, see key at front of Listings.}

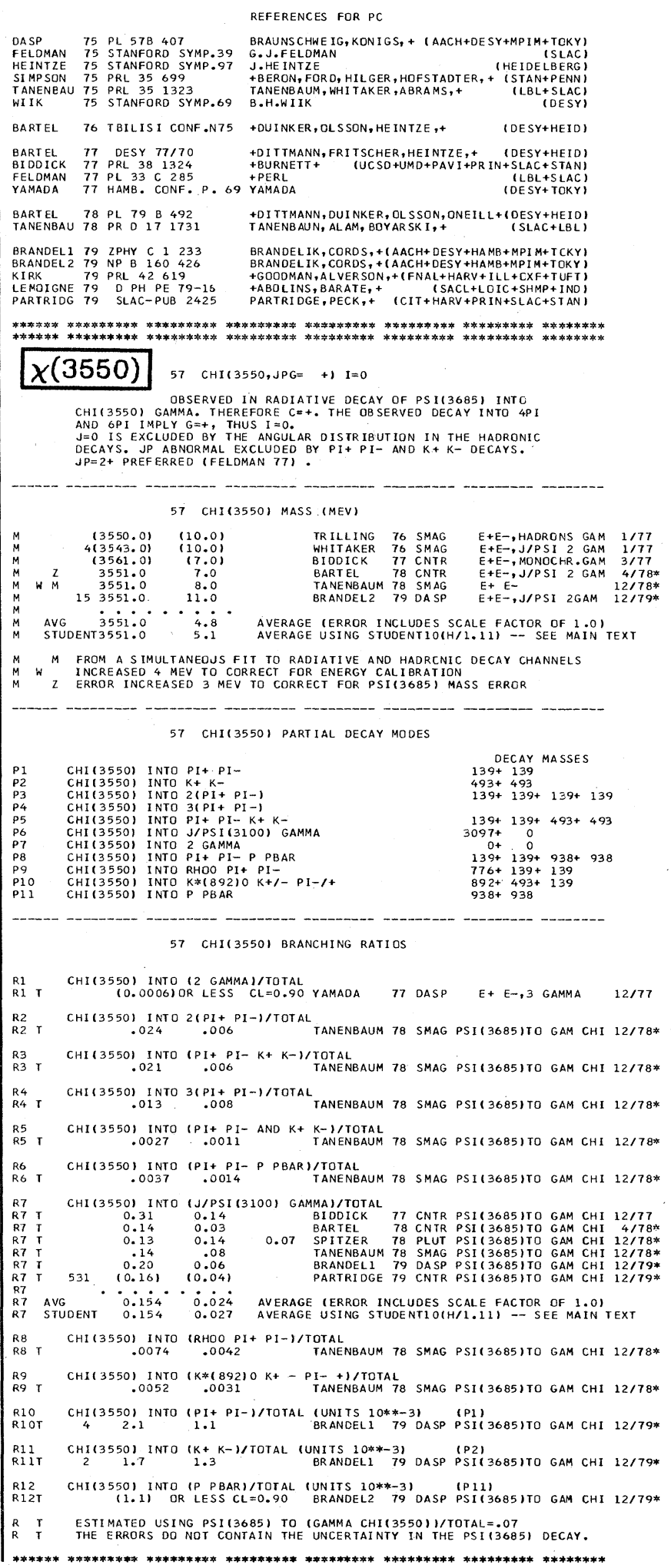

Rev. Mod. Phys., Vol. 52, No. 2, Part II, April 1980 


\section{Data Card Listings}

Mesons

For notation, see key at front of Listings.

$\chi(3550), \chi(3590), \psi(3685)$

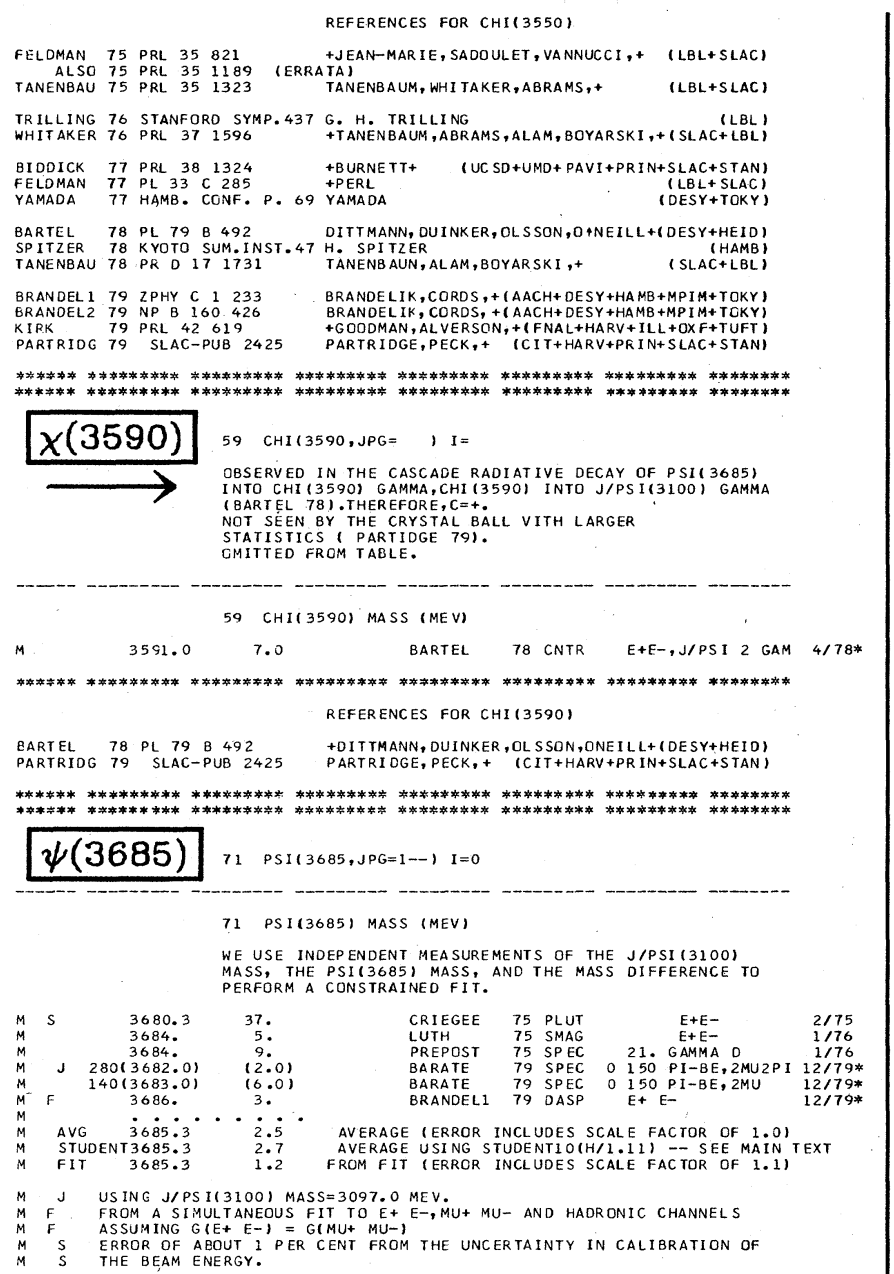

71 PSI(3685) - J/PSI(3100) MASS DIFFERENCE (MEV)

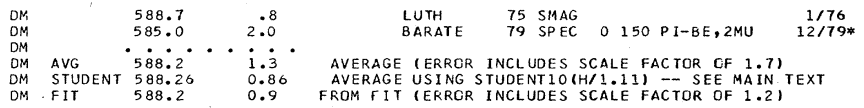

71 PSI(3685) WIDTH (KEV)

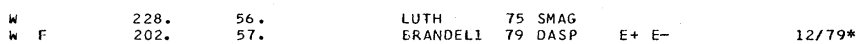

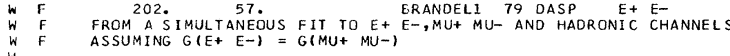

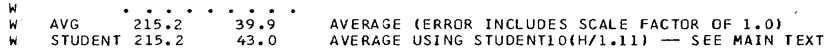

71 PSI $(3685)$ PART IAL DECAY MODES

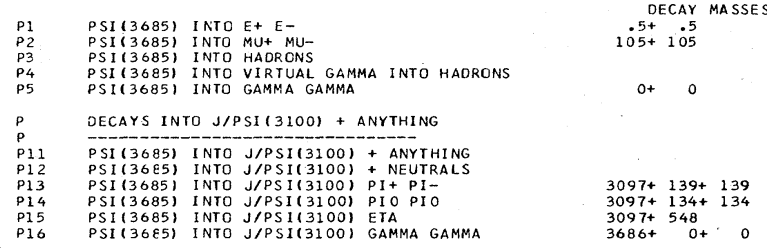

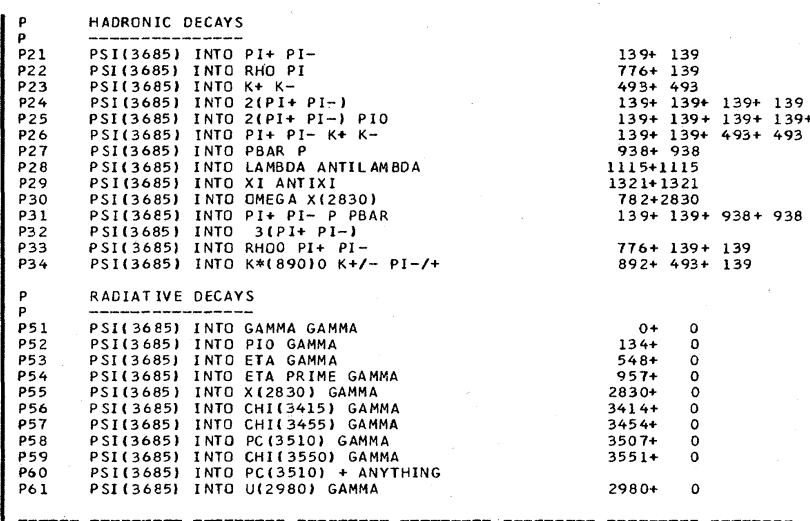

FITTED PARTIAL DECAY MODE BRANCHING FRACTIONS

The matrix below is derived from the error matrix for the fitted partial decay mode branching fractions, $P_{i}$, as follows: The diagonal elements are $P_{i} \pm \delta P_{i}$, where

$\delta P_{i}=\sqrt{\left\langle\delta P_{i} \delta P_{i}\right\rangle}$, while the off-diagonal elements are the normalized correlation coefficients $\left\langle\delta P_{i} \delta P_{j}\right\rangle /\left(\delta P_{i} \cdot \delta P_{j}\right)$. For the definitions of the individual $P_{i}$, see the listings above; only those $P_{i}$ appearing in the matrix are assumed in the fit to be nonzero and are thus constrained to add to 1 .

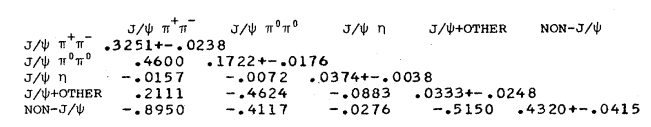

71 PSI(3685) PARTIAL WIDTHS (KEVI

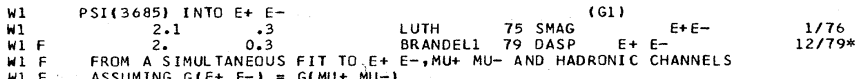

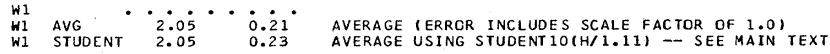

W3 PSI(3E85) INTO HADRONS
W3
224.

W5 PSI 3685$)$ INTO GAMMA GAMMA
W5

71 PSI(3685) BRANCHING RATIOS

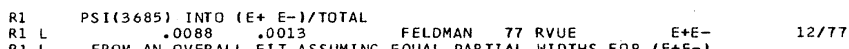

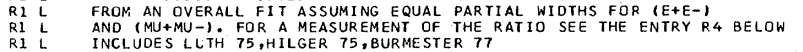

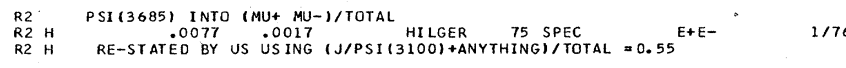

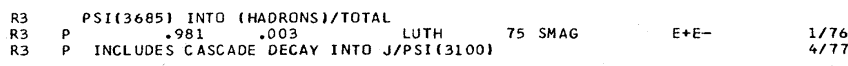

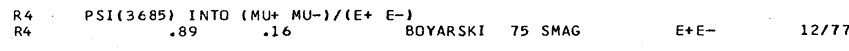

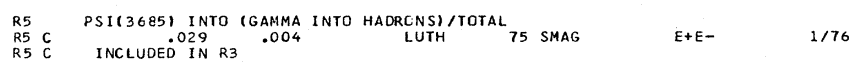

$R$ DECAYS INTO J/PSI(3100) + ANYTHING

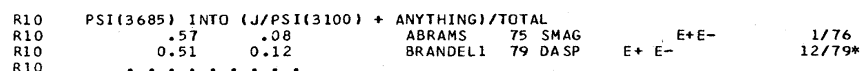

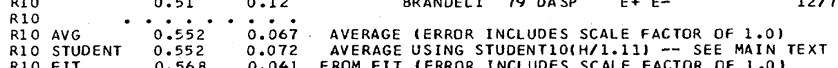
R1O FIT 0.5680 .041 FROM FIT IERROR INCLUDES SCALE FACTOR OF 1.01

$R 11$
$R 11$
$R 11$

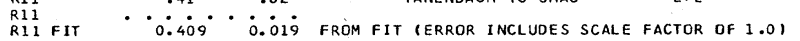

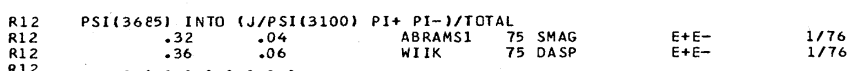

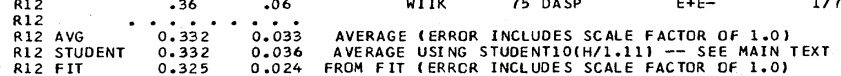


Mesons

$\psi(3685), \psi(3770)$
Data Card Listings For notation, see key at front of Listings.

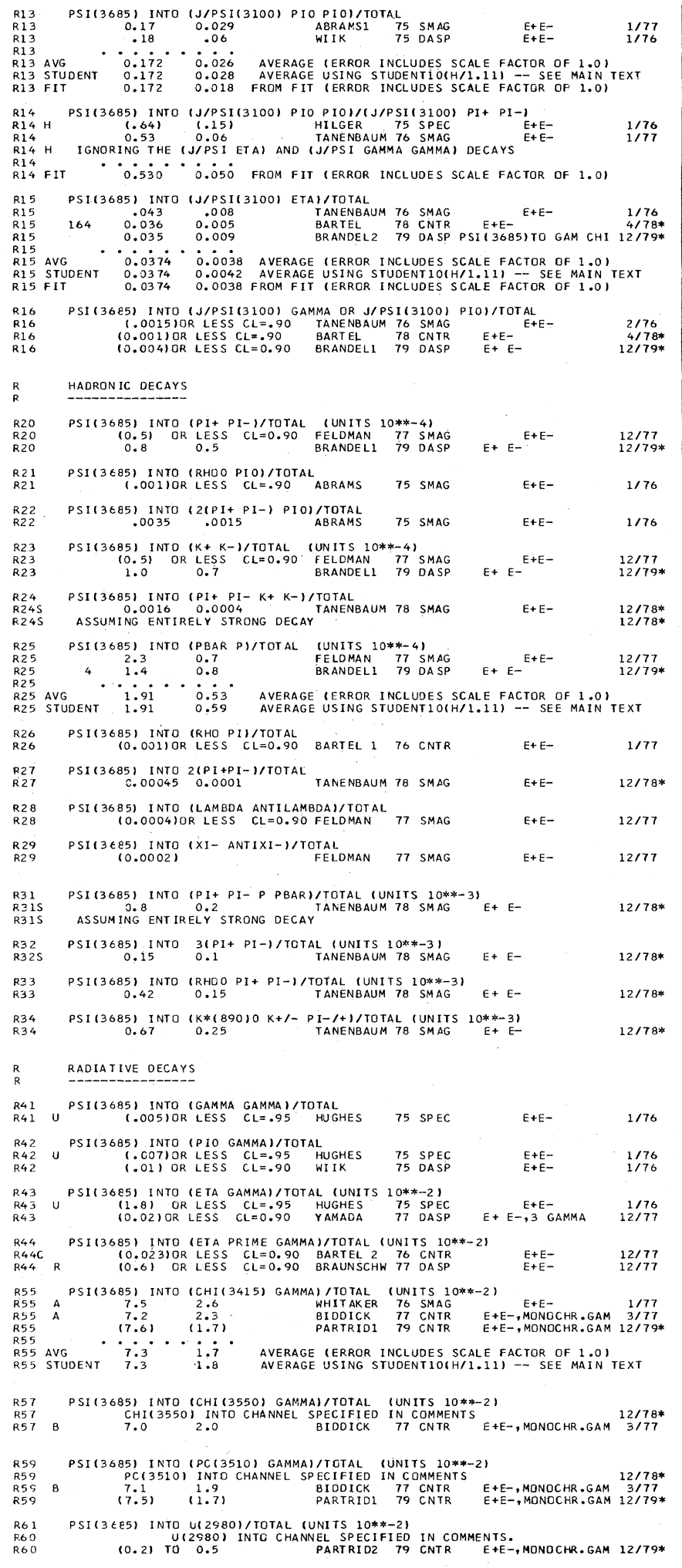

R63 3
R63 PSI(3685) INTO (CHI(3590) GAMMA)/TOTAL (UNITS 10**-2)
CHI(3590) INTO CHANNEL SPECIFIED IN COMMENTS

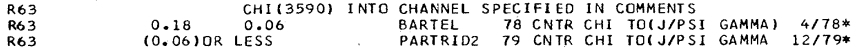

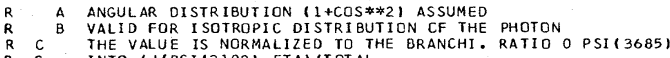

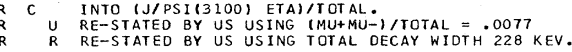

71 PSI(3685) G(I)*G(E+E-)/G(TOTAL) (KEV) THIS COMBINATION OF A PARTIAL WIDTH WITH THE PARTIAL WIOTH
INTO E+E- AND WITH THE TOTAL WIDTH IS OBTAINED FROM THE INTEGRATED CROSS-SECTION INTO CHANNEL(I) IN THE E+E- ANNIHILATICN.
WE ONLY IIST DATA NOT HAVING BEEN USED TO DETERMINE THE PARTIAL
WIDTH G(I) OR THE BRANCHING RATIO G(I)/TOTAL.

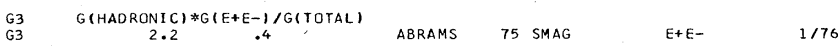

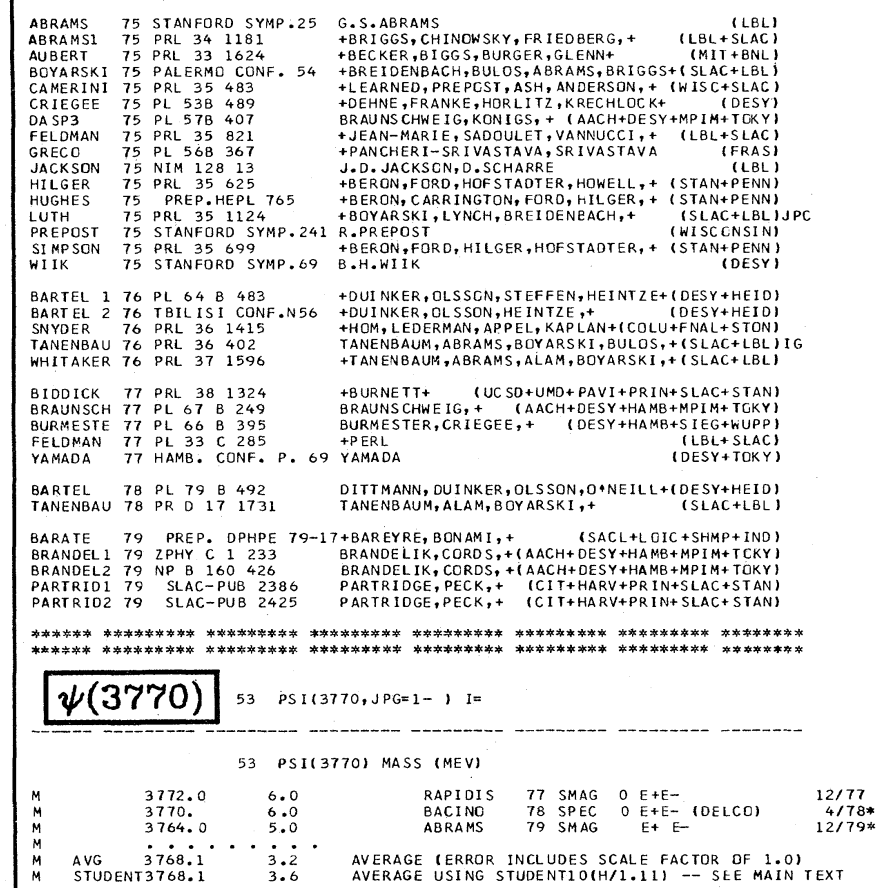

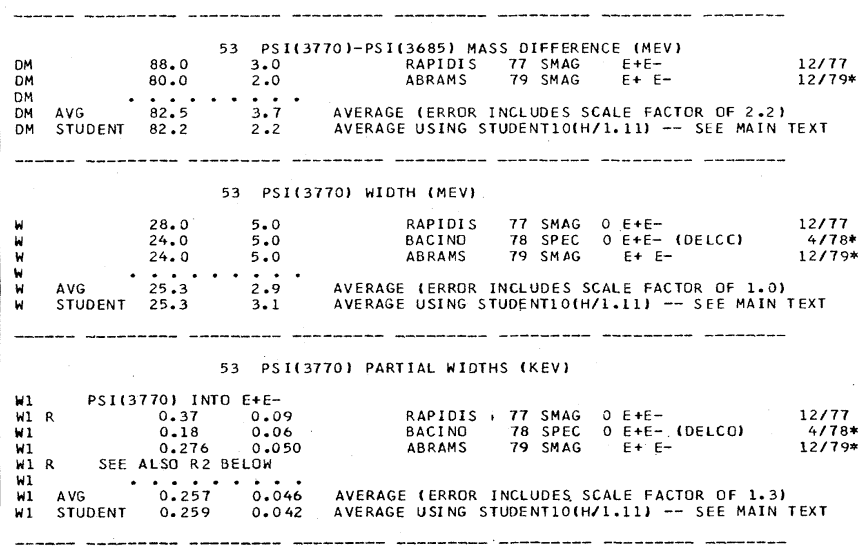


Data Card Listings

Mesons

For notation, see key at front of Listings. $\psi(3770), \psi(4030), \psi(4160), \psi(4415), \Upsilon(9460)$

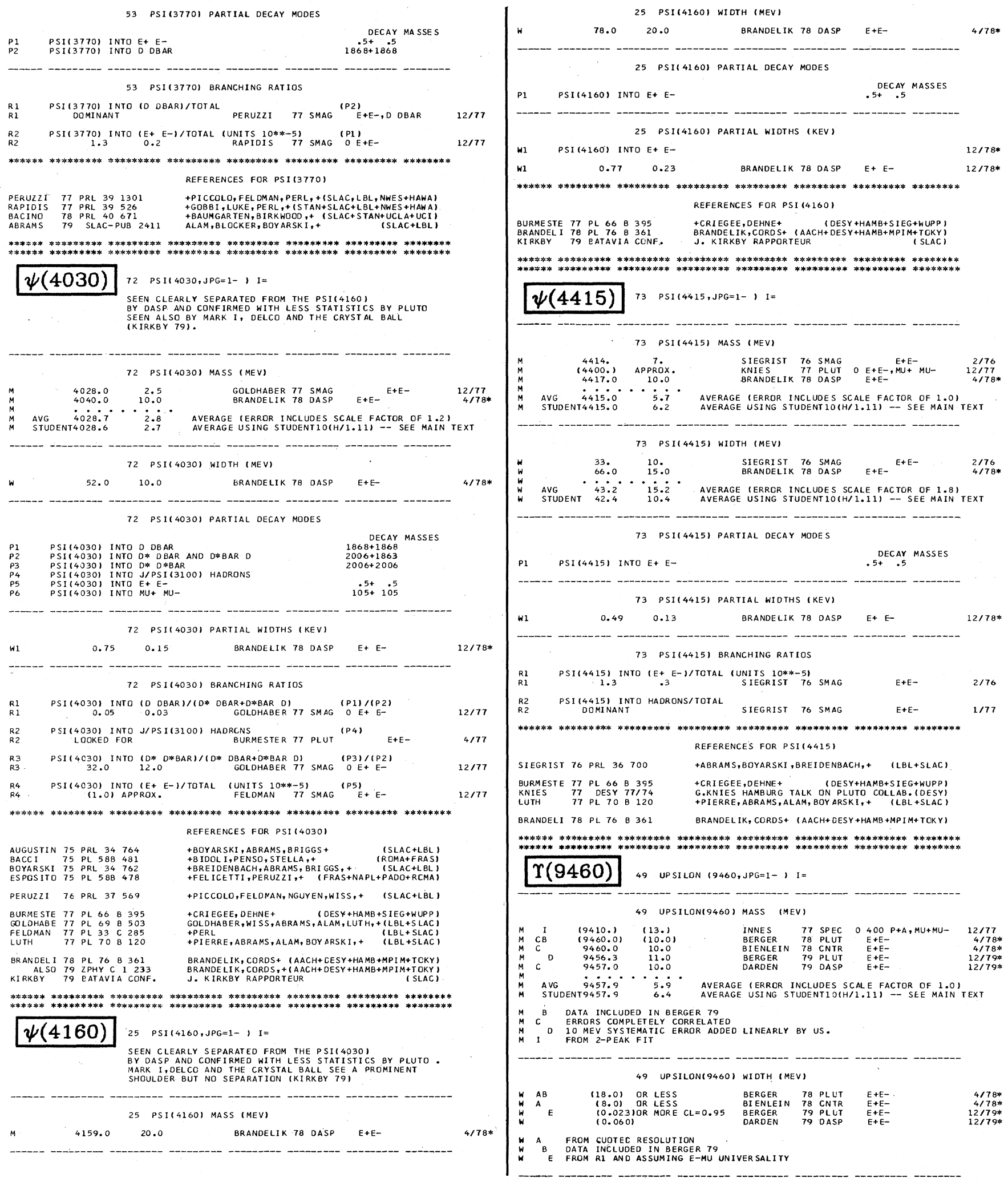


Mesons

$\Upsilon(9460), \Upsilon(10020), \Upsilon(10400), K^{ \pm}, K^{0}, K^{*}(892)$

49 UPSILON(9460) PARTIAL DECAY MODES

$\begin{array}{llll}\text { P1 UPSILCN(9460) INTO MU+ MU- } & \text { DECAY MASSES } \\ \text { P2 UPSILCN(9460) INTO E+ E- } & 105+105 \\ & \end{array}$

49 UPSILON(9460) PARTIAL WIOTHS (KEV)

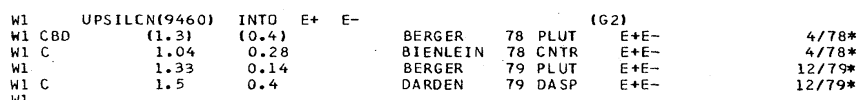

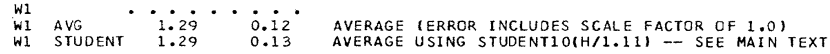

W2 B DATA INCLUDED IN BERGER 79

W1 C D ERRORS CORRELATED
WI DITH RADIATIVE CORRECTIONS IN

\section{UPSILON(9460) BRANCHING RATIOS}

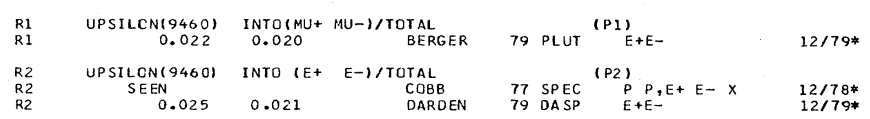
****************************************************************************1

$\begin{array}{llllll}\text { COBB } & 77 & P L & 72 & B & 273 \\ \text { HERB } & 77 & \text { PRL } 39 & 252 \\ \text { INNES } & 77 & \text { PRL } & 39 & 1240\end{array}$

REFERENGES FOR UPSILON $(9460)$

$\begin{array}{llllll}\text { BERGER } & 78 & \text { PL } & 76 & \text { B } & 243 \\ \text { BIENLEIN } 78 & \text { PL } & 78 & \text { B } & 360 \\ \text { DARDEN } & 78 & \text { PL } & 76 & \text { B } & 246\end{array}$

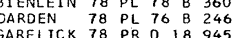

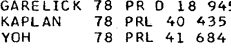

$\begin{array}{llllll}\text { ANGEL IS } & 79 & P C & 87 & \text { B } & 398 \\ \text { BADIER } & 79 & \text { PL } & 86 & 8 & 98\end{array}$

BERGER 79 ZPHY C 1343
DAROEN 79 PL 80 \& 419

+ IWATA, FAB JAN, GOLDBERG+ (BNL+CERN+SYRA+YALE) + HOM, LEDERMAN, APPEL, ITO,+ (COLU+FNAL+STON)
$+A P P E L, B R O W N, H E R B, H O M, F I S K+(C O L U+F N A L+S T O N)$

+ALEXANDER, DAUM, + (AACH+DESY +HAMB+SIEG + WUPG)

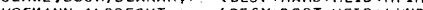
+GAUTHIER, HICKS, OLIVER, + (NEAS+WASH+TUFT) +APPEL, HERB, HCM, LEDERMAN,+ (STON+FNAL+CCLU)

+BESCH, BLUMENFELO,+ (CERN+CGLU+OXF+ROCK) +BOJCROT, BURGUN+ ( $S A C L+C E R N+C D E F+E P O L+L A L O)$ + ALE XANDER+
+ HOFMANN, ALBRECHT,
$+{ }^{+}$(DACH DESY+HAMB+SIEG+WUPG)

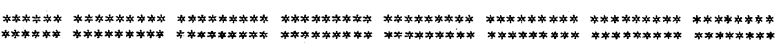

$\Upsilon(10020){ }_{52}$ UPSILON 10020, JPG $=1-, 1=$

52 UPSILON(10020) MASS (GEV)

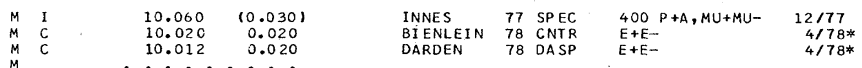

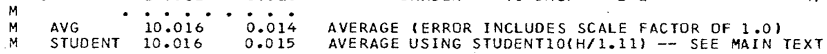

$\begin{array}{lll}M & C & \text { ERRORS COMPLETELY CORRELATED } \\ M & 1 & \text { FROM 2-PEAK FIT }\end{array}$

52 UPSILON(10020) WIDTH (MEV)

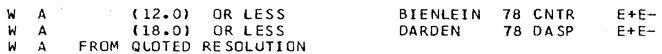

52 UPSILON (10020)-UPSILON( 9460 ) MASS DIFFERENCE (MEV)

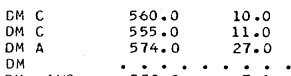

$\begin{array}{llllr}\text { BIENLEIN } & 78 & \text { CNTR } & \text { E+E- } & 4 / 78 * \\ \text { DARDEN } & 78 \text { DASP } & \text { E+E- } & 4 / 78 * \\ \text { UENO } & 79 \text { SPEC } & 400 \text { P PT, MU+MU- } & 12 / 79 *\end{array}$

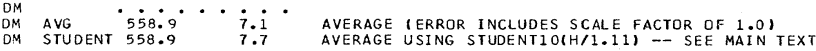

DM A FIXING THE UPSILON( 9460$)$ MASS AT 9460 MEV

52 UPSILON(10020) PARTIAL DECAY MODES

P1 UPSILON(10020) INTO MU+ MU-
P2 UPSILCN(10020) INTO E+ E-

OECAY MASSES
$105+105$
$5+105$

52 UPSILON(10020) PARTIAL WIDTHS (KEV)

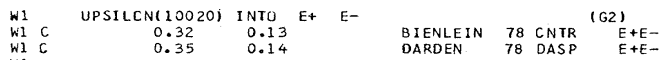
$4 / 78 *$

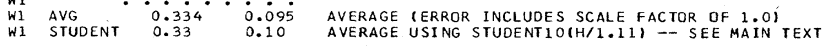

W1 C ERRORS COMPLETELY CORRELATED

52 UPSILON(10020) BRANCHING RATIOS

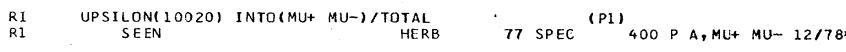

Data Card Listings For notation, see key at front of Listings.

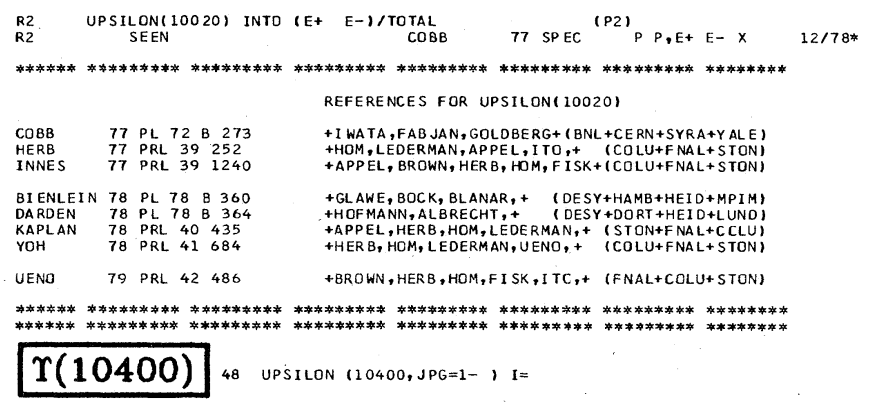

48 UPSILON(10400)-UPSILON( 9460$)$ MASS DIFFERENCE (MEV) DM A $550.0 \quad 30.0 \quad$ UENC 79 SPEC 400 P PT, MU+MU- $12 / 79 *$ DM A FIXING THE UPSILON 19460$)$ MASS AT 9460 MEV ANO THE
OM A UPSILON(10020)-UPSILON(9460) MASS DIFFERENCE AT 558 MEV.

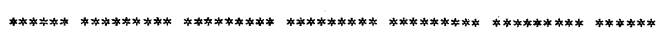

\begin{tabular}{|c|c|c|}
\hline & & REFERENCES FCR UPSILON(10400) \\
\hline $\begin{array}{l}\text { COBB } \\
\text { HERB } \\
\text { INNES }\end{array}$ & 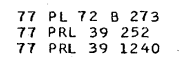 & 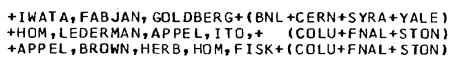 \\
\hline $\begin{array}{l}\text { KAPL AN } \\
\text { YOH }\end{array}$ & $\begin{array}{llll}78 & \text { PRL } & 40 & 435 \\
78 & \text { PRL } & 41 & 684\end{array}$ & $\begin{array}{l}\text { +APP EL, HERB, HOM, LEDERMAN,+ (STON+FNAL+COLU) } \\
\text { +HERB, HOM, LEDERMAN, UENO, }+ \text { (COLU+FNAL+STON) }\end{array}$ \\
\hline UENO & 79 PRL 42486 & +BROWN, HERB, HOM, FISK, ITD, + (FNAL+COLU+STON) \\
\hline
\end{tabular}

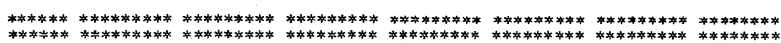

$\mathrm{K}^{ \pm} \quad 10$ CHARGED $K(494, \mathrm{JP}=0-1) \mathrm{I}=1 / 2$

SEE STABLE PARTICLE DATA CARD LISTINGS

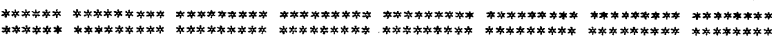

$\mathrm{K}^{0} 11$ NEUTRAL $K(498, \mathrm{JP}=0-) \mathrm{I}=1 / 2$

SEE STABLE PARTICLE DATA CARD LISTINGS

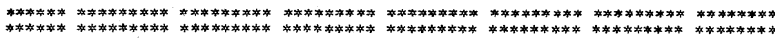

$\mathrm{K}^{*}(892)$

$18 K *(892, J P=1-) \quad I=1 / 2$

$18 K *(892)$ MASS (MEV)

$M$ CHARGED ONLY. THIS IS WHAT APPEARS ON MESON TABLE
$M$ C $100(898.0)$
(5.0)

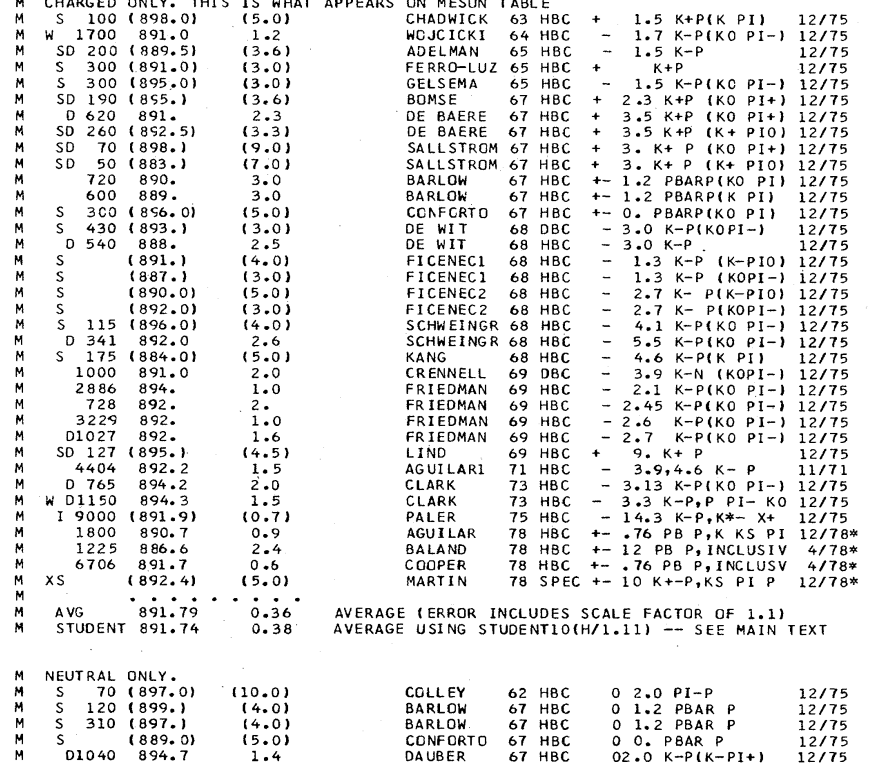




\section{Data Card Listings}

Mesons

\section{For notation, see key at front of Listings.}

$\mathrm{K}^{*}(892)$

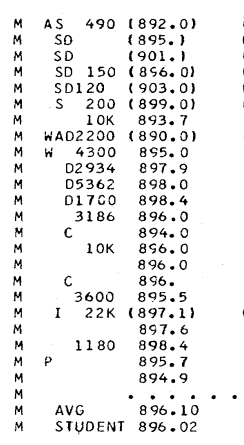

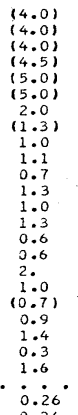
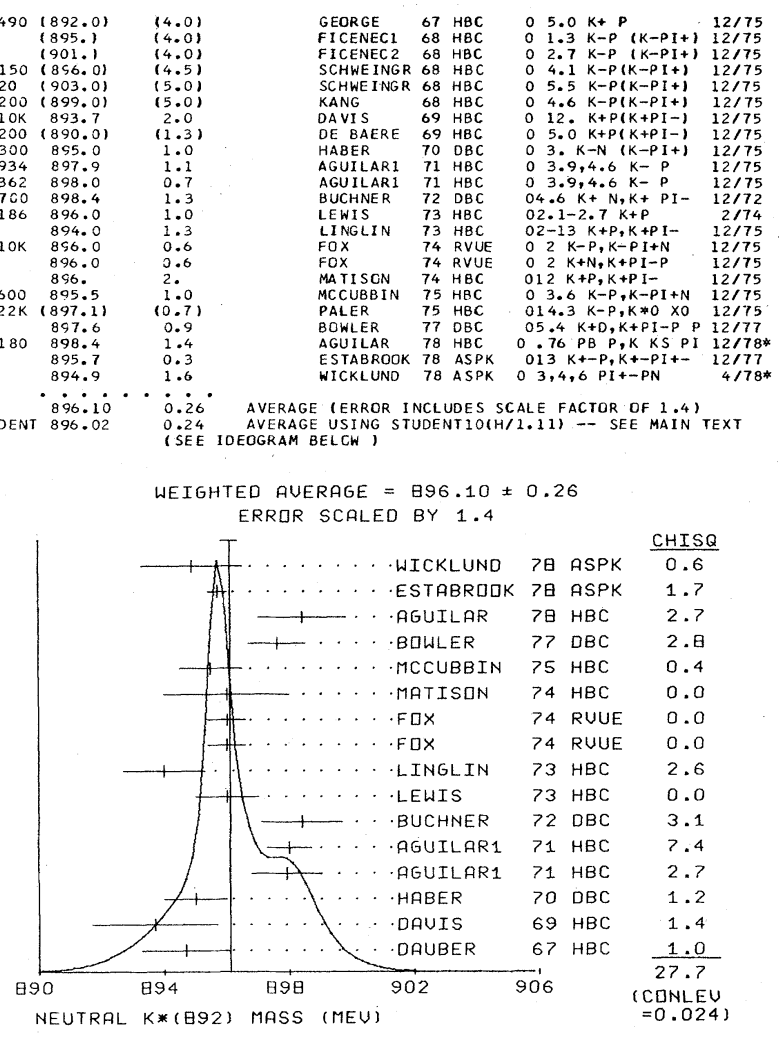

M A INCLUDED IN LINGLIN 73 WORLD K+P DST

$M$
$M$ C FROM POLE EXTRAPOLAT ION. US TO GAMMA/SQRT (N). SEE TYPED NDTE.

$M$
$M$

$M$
$M$ . DATA WITH MASS ERROR DF 3 MEV OR MORE NOT AVERAGED

M.-

Note on $K^{*}(892)$ Masses and Mass Differences

Unrealistically small errors are reported by some experiments. We use simple "realistic" tests for the minimum errors on the determination of mass and width from a sample of $\mathrm{N}$ events:

$$
\delta_{\min }(\mathrm{m})=\frac{\Gamma}{\sqrt{\mathrm{N}}}, \quad \delta_{\min }(\Gamma)=4 \frac{\Gamma}{\sqrt{\mathrm{N}}} .
$$

(For detailed discussion see the April 1971 edition of this note.) We consistently increase unrealistic errors before averaging.

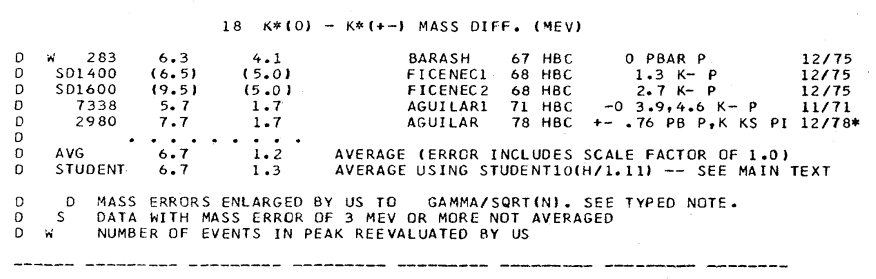

18 K* (892) WI DTH (MEV)

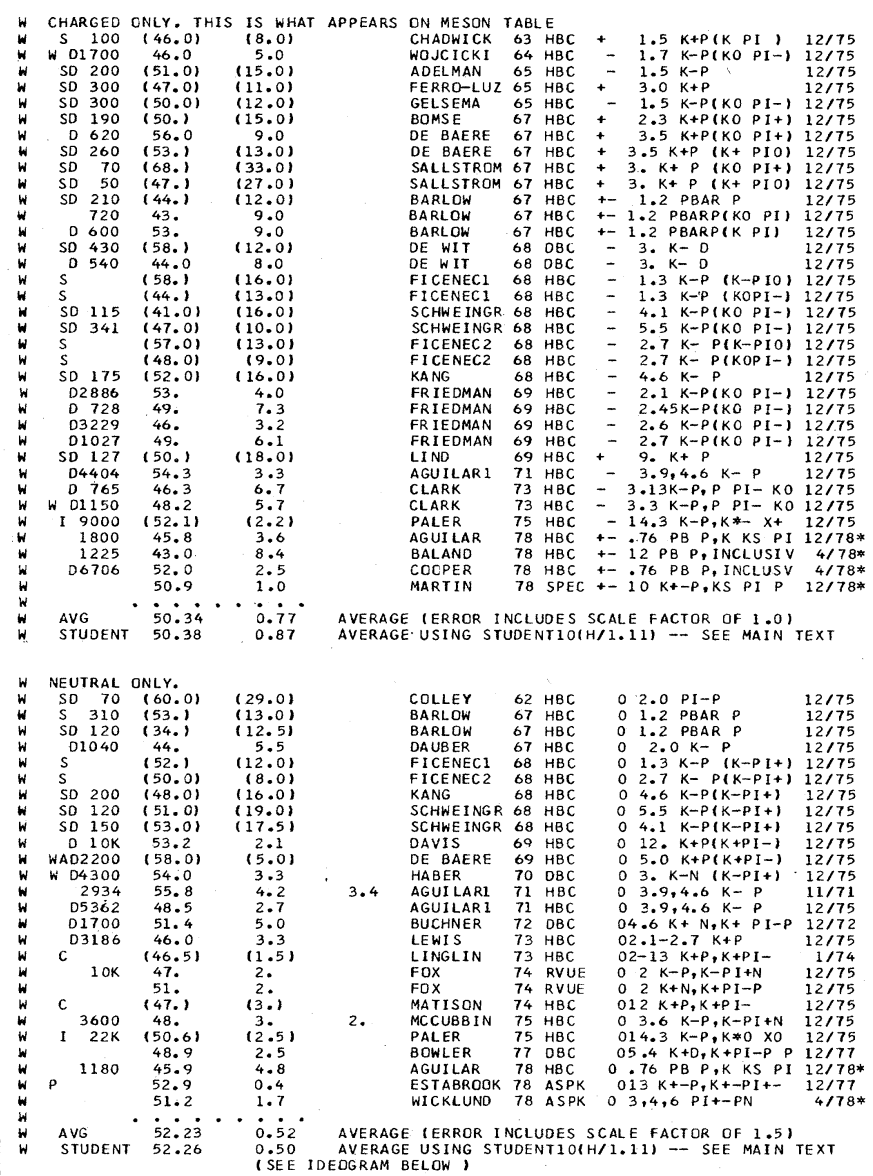

WEIGHTED AUERAGE $=52.23 \pm 0.52$

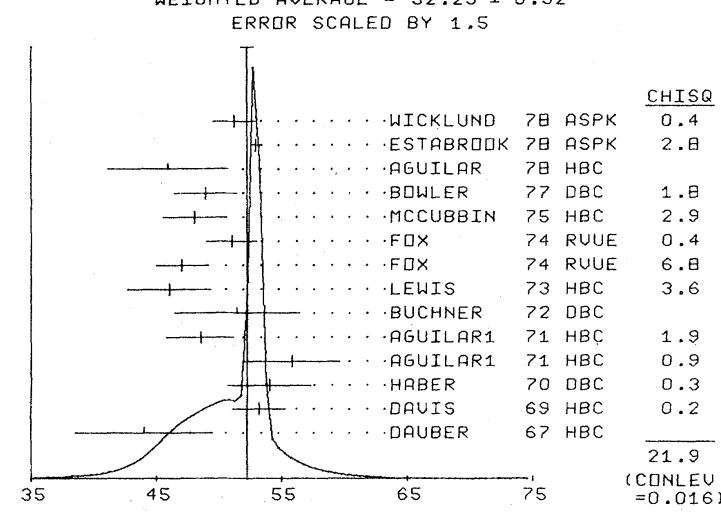

NEUTRAL K*(B92) WIDTH (MEU)

W A INCLUDED IN LINGLIN 73 WORLD K+P DST

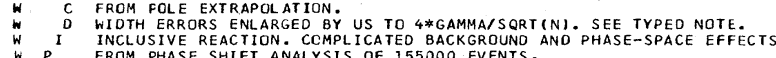
OATA HITH MASS ERROR OF 3 MEV OR MORE NOT AVERAGED
NUMBER OF EVENTS IN PEAK REEVALUATED BY US 
Mesons $\mathrm{K}^{*}(892), Q$

$18 K *(892)$ PARTIAL DECAY MODES

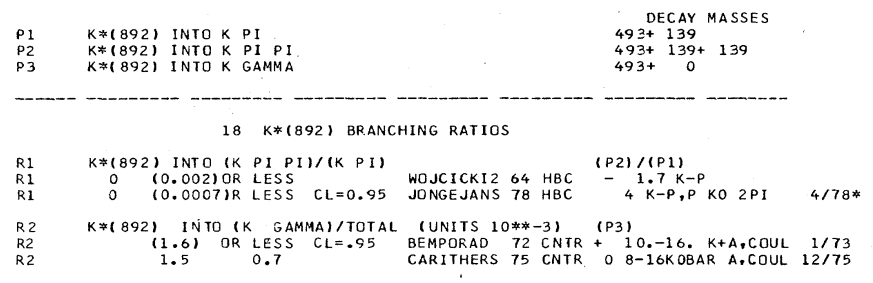

*************************************************************************1*

\begin{tabular}{|c|c|c|}
\hline & & NCES FDR $K *(892)$ \\
\hline $\begin{array}{l}\text { ALSTON } \\
\text { ALEXANDE } \\
\text { COLLEY }\end{array}$ & 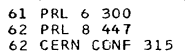 & $\begin{array}{l}\text { ALSTON, ALVAREZ, EBERHARD, GOOD, GRAZ IANO+(LRL) } \\
\text { ALEXANDER, KALBFL EISCH, MILLER,G SMITH (LRL) } \\
\text { D COLLEY, N GELFAND + } \\
\text { (COLUMB IA+RUTGERS) }\end{array}$ \\
\hline $\begin{array}{l}\text { CHAOWICK } \\
\text { GOLDHABE }\end{array}$ & $\begin{array}{l}63 \text { PL } 63 \text { C9 } \\
63 \text { ATHENS CONF } 92\end{array}$ & $\begin{array}{l}\text { CHADWICK, CRENNELL, DAVIES, BETTINI+ (OXF+PADO) } \\
\text { SULAMITH GOLOHABER (LRL) }\end{array}$ \\
\hline WoJCICKI & $\begin{array}{lllll}64 & \text { PR } & 135 & 8 & 484\end{array}$ & STANLEY G WGJCICKI \\
\hline $\begin{array}{l}\text { ADELMAN } \\
\text { FERRO-LU } \\
\text { FERRC-LU } \\
\text { GELSEMA } \\
\text { WANGLER }\end{array}$ & $\begin{array}{l}65 \text { ATHENS } 527 \\
65 \text { NC } 36 \text { 1101 } \\
65 \text { NC } 39417 \\
65 \text { THE SIS } \\
65 \text { PR } 137 \text { B } 414\end{array}$ & $\begin{array}{l}\text { STUART LEE ADELMAN } \\
\text { FERRO-LUZZI, GEORGE, HENRI, JONGE JANS (CAVENDISH) } \\
\text { FERRO-LUZZI, GEORGE, (COLOSCHMIDT-CLER+ (CERN) } \\
\text { E.S.GELSEMA (SEE ALSO PL 10 341) (AMSTERDAM) } \\
\text { WANGLER, ERWIN, WALKER } \\
\text { (WISCONSIN) }\end{array}$ \\
\hline $\begin{array}{l}\text { BARASH } \\
\text { BARLOW } \\
\text { BOMSE } \\
\text { CCNFORTO } \\
\text { DAUBER } \\
\text { DE BAERE } \\
\text { FRENCH } \\
\text { GEORGE } \\
\text { SALLSTRO }\end{array}$ & 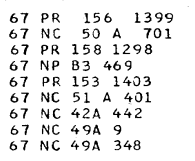 & 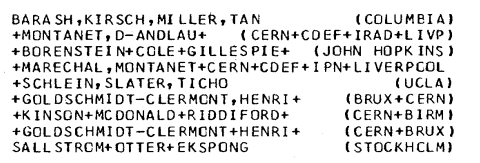 \\
\hline $\begin{array}{l}\text { DE HIT } \\
\text { FICENECI } \\
\text { FICENEC } 2 \\
\text { KANG } \\
\text { SCHWEING }\end{array}$ & 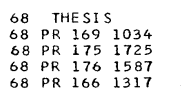 & $\begin{array}{lr}\text { S. DE WIT } & \text { (AMSTERCAM) } \\
\text { +HULSIZER+SWANSON+TROWER } & \text { (ILL) } \\
\text { FICENEC, GCRDCN, TROWER } & \text { (ILLINOIS) } \\
\text { Y.W.KANG } & \text { (ICWA) } \\
\text { SCHWEINGRIJBER, DERR ICK, FIELDS+ } & \text { (ANL+NWES) }\end{array}$ \\
\hline $\begin{array}{l}\text { CRENNELL } \\
\text { DAVIS } \\
\text { DE BAERE } \\
\text { FR IEDMAN } \\
\text { JUHALA } \\
\text { LING }\end{array}$ & 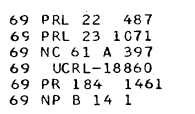 & 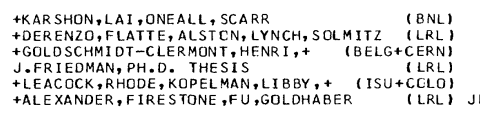 \\
\hline $\begin{array}{l}\text { ATHERTON } \\
\text { HABER }\end{array}$ & $\begin{array}{lllll}70 & N P & B & 1 \epsilon & 416 \\
70 & N P & B & 17 & 289\end{array}$ & $\begin{array}{l}\text { +FRANEK, FRENCH, FRISK, BEDNAR+ (CERN+PRAG) } \\
+ \text { +S HAPIRA, ALEXANDER+ } \\
\text { (REHO+SACL +BGNA+EPOL) }\end{array}$ \\
\hline $\begin{array}{l}\text { AGUILAR } \\
\text { AGUILARI } \\
\text { BARANAM } \\
\text { BUCHNER } \\
\text { CORDS } \\
\text { MERCER } \\
\text { YUTA }\end{array}$ & 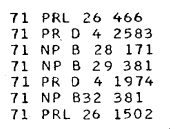 & 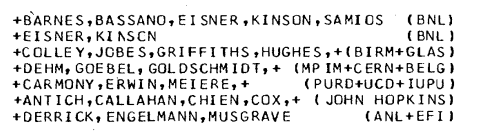 \\
\hline $\begin{array}{l}\text { ABRAMOVI } \\
\text { BINGHAM } \\
\text { BEMPCRAD } \\
\text { BRUNET } \\
\text { BUCHNER } \\
\text { CRENNELL } \\
\text { DEUTSCHM } \\
\text { ENGELMAN } \\
\text { ROUGE } \\
\text { TIECKE }\end{array}$ & 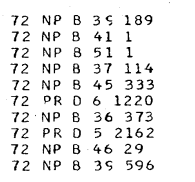 & 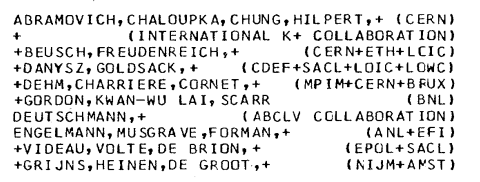 \\
\hline $\begin{array}{l}\text { BERTHON } \\
\text { CHARRIER } \\
\text { CLARK } \\
\text { LEWIS } \\
\text { LINGLIN } \\
\text { WALUCH. }\end{array}$ & $\begin{array}{llllll}73 & N P & B & 6 & 3 & 54 \\
73 & N P & 8 & 51 & 317 \\
73 & N P & 8 & 54 & 432 \\
73 & N P & 8 & 60 & 283 \\
73 & N P & B & 55 & 408 \\
73 & P R & 0 & 8 & 2837\end{array}$ & 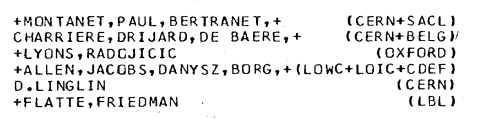 \\
\hline $\begin{array}{l}\text { FOX } \\
\text { MATISON }\end{array}$ & $\begin{array}{llll}74 & N P & B 80 & 403 \\
74 & \text { PR } & D 9 & 1872\end{array}$ & $\begin{array}{l}\text { G.C.FOX, M.L.GRISS } \\
\text { +GALTIERI, GARNJOST, FLATTE, FRIEDMAN,+ (CIT) }\end{array}$ \\
\hline $\begin{array}{l}\text { BR ANDENB } \\
\text { CARITHER } \\
\text { MCCUBBIN } \\
\text { PALER }\end{array}$ & $\begin{array}{lllll}75 & P L & 59 & 8 & 405 \\
75 & P R L & 35 & 349 \\
75 & N P & 886 & 13 \\
75 & N P & 896 & 1\end{array}$ & 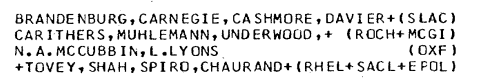 \\
\hline $\begin{array}{l}\text { KIRK } \\
\text { BOWLER }\end{array}$ & $\begin{array}{lllll}76 & N P & B & 116 & 99 \\
77 & \text { NP } & B & 126 & 31\end{array}$ & $\begin{array}{l}\text { +KLE IN, COUNI HAN, + (AACH+BERL+CERN+LOIC+WIEN) } \\
\text { +DAINTON, DRAKE, WILLIAMS } \\
\text { (OXFCRD) }\end{array}$ \\
\hline $\begin{array}{l}\text { AGUILAR } \\
\text { BALAND } \\
\text { BALOI } \\
\text { COOPER } \\
\text { ENGELEN } \\
\text { ESTABROC } \\
\text { ALSO } \\
\text { JCNGEJAN }\end{array}$ & $\begin{array}{llllll}78 & N P & B & 141 & 101 \\
78 & N P & B & 140 & 220 \\
78 & N P & 8 & 134 & 365 \\
78 & N P & B & 136 & 365 \\
78 & N P & B & 134 & 14 \\
78 & N P & B & 133 & 490 \\
78 & P R & D & 17 & 658 \\
78 & N P & B & 139 & 383\end{array}$ & 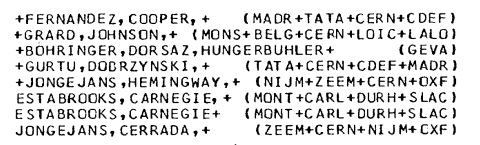 \\
\hline $\begin{array}{l}\text { MARTIN } \\
\text { WICKLUNO }\end{array}$ & $\begin{array}{l}78 \text { NP } 8134392 \\
78 \text { PRD } 171197\end{array}$ & $\begin{array}{l}\text { + SHI MADA, BALDI, BOHRINGER, DORSAZ+ (DURH+GEVA) } \\
\text { +AYRES, DIEBOLD, GREENE, KRAMER, PAWLICKI (ANL) }\end{array}$ \\
\hline
\end{tabular}

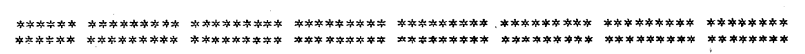

\section{Data Card Listings For notation, see key at front of Listings.}

Q REGION, $K \pi \pi(1200-1400)$

28 Q REGION $(1200-1400) \quad I=1 / 2$

The main effect in the $Q$ region is a broad bump in the $\mathrm{K} \pi \pi$ spectrum between 1200 and $1400 \mathrm{MeV}$ (not far above the $K^{*}(892) \pi$ threshold), produced by $\mathrm{K}$ beams without charge exchange. In particular, it has been observed in coherent $\mathrm{K}^{+} \mathrm{d}$ interactions (FIRESTONE 72) and in coherent interactions on heavy nuclei (BINGHAM 73). Throughout the entire region, $\mathrm{J}^{\mathrm{P}}=1^{+}$and $\mathrm{I}=1 / 2$. FIRESTONE1 72 observe a bump in the backward direction with a shape similar to that of the $Q$. The broad $Q$ peak does not have a simple Breit-wigner shape. It can be fitted at all energies by a superposition of two Breit-wigner amplitudes. Dalitz plot analyses of the interference between the $K^{*} \pi$ and $K \rho$ modes show the relative magnitude and relative phase of the two decay amplitudes varying with $\mathrm{K} \pi \pi$ mass. The $\mathrm{K} \rho$ mode has a maximum intensity below thàt of $K^{*} \pi$.

Partial-wave analyses have confirmed the rather complex situation in the $Q$ region (DEUTSCHMANN 74, ANTIPOV 75, OTTER 75,76, TOVEY 75, BRANDENBURG 76, BEUSCH 78, VERGEEST 79). The dominant states are $I^{+} \mathrm{S}\left(\mathrm{K}^{*} \pi\right)$, almost entirely $\mathrm{I}=1 / 2$ (VERGEEST 79), and $\mathrm{I}^{+} \mathrm{S}(\mathrm{K} \rho)$. Other important states are $\mathrm{J}^{\mathrm{P}}=0^{-}$ and $\mathrm{J}^{\mathrm{P}}=2^{+}$. The $\mathrm{K}^{*} \pi$ and $\mathrm{K} \rho$ modes are not produced coherently and have different polarization properties (BRANDENBURG 76, OTTER 76, VERGEEST 79). Whereas the $\mathrm{K} \rho$ mode approximately conserves s-channel helicity, the $\mathrm{K}^{*} \pi$ mode is approximately t-channel helicity conserving.

Experimentally, those data with sufficient statistics show the presence of a two-peak structure (OTTER 76, BRANDENBURG 76). BRANDENBURG $76 \mathrm{claim}$ to observe sufficient phase variation to warrant proposing the existence of two $\mathrm{J}^{\mathrm{P}}=\mathrm{I}^{+} \mathrm{K} \pi \pi$ resonances: $\mathrm{Q}_{1}$, with a mass around $1280 \mathrm{MeV}$, a width of the order of $150 \mathrm{MeV}$, and mainly coupled to the $\mathrm{K} \rho$ channel; and $Q_{2}$, with a mass around $1400 \mathrm{MeV}$, a width of the order of $150 \mathrm{MeV}$, and mainly coupled to the S-wave $K^{*} \pi$ channel (CARNEGIE 77). These results are experimentally confirmed by VERGEEST 79 and supported by the BOWLER 77 and BASDEVANT 79 analyses. 


\section{Data Card Listings \\ For notation, see key at front of Listings.}

Mesons

AACHEN 76 and WOHL 78 have shown some evidence for a $\mathrm{K} \omega$ decay of the $\mathrm{Q}_{1}$ in diffraction-like processes.

The $(\mathrm{K} \pi \pi)^{0}$ system produced in the chargeexchange reaction appears to have an important $\mathrm{J}^{\mathrm{P}}=1^{+}$contribution (OTTERl 75, VERGEEST 76). The $\mathrm{I}^{+}\left(\mathrm{K}^{*} \pi\right)$ and $\mathrm{I}^{+}(\mathrm{K} \rho)$ waves cannot be explained as decay products of a single resonance and the $K^{*} \pi$ wave behavior suggests a resonance contribution around $1400 \mathrm{MeV}$ (VERGEEST 76)

There are a number of claims for the observation of $K \pi \pi$ resonances in the $Q$ mass region in other non-diffractive processes (ARMENTEROS 64, CRENNEL 67,72, ASTIER 69, DAVIDSON 74, DORE 75). These data can be described in terms of a single resonance of characteristics consistent with those of the $Q_{1}$ (CONFORTO 77). A result from the hyperon-exchange reaction (GAVILLET 78) again shows the production of a $\mathrm{J}^{\mathrm{P}}=1^{+} \mathrm{K} \rho$ resonance with mass and width close to those of the $Q_{1}$. Neither ARMENTEROS 64 (nor a later analysis by ASTIER 69) nor GAVILLET 78 observe a $K^{*} \pi$ resonance compatible with the $\mathrm{Q}_{2}$. However the assignment to a $\mathrm{J}^{\mathrm{PC}}=1^{++} \mathrm{SU}(3)$ nonet of the $\mathrm{J}^{\mathrm{P}}=\mathrm{I}^{+}$ Ko resonance together with the $\mathrm{A}_{1}, \mathrm{D}(1285)$, and $E(1420)$ seems to support the existence of a $Q_{2}(1400)$ with parameters compatible with those of CARNEGIEl 77 (MAZZUCATO 79 and DIONISI 80).

Note that IRVING 80 discusses a model for non-diffractive $Q$ production in which the $\Omega_{2}$ is suppressed relative to the $Q_{1}$.

28 Q REGION MASS (MEV)

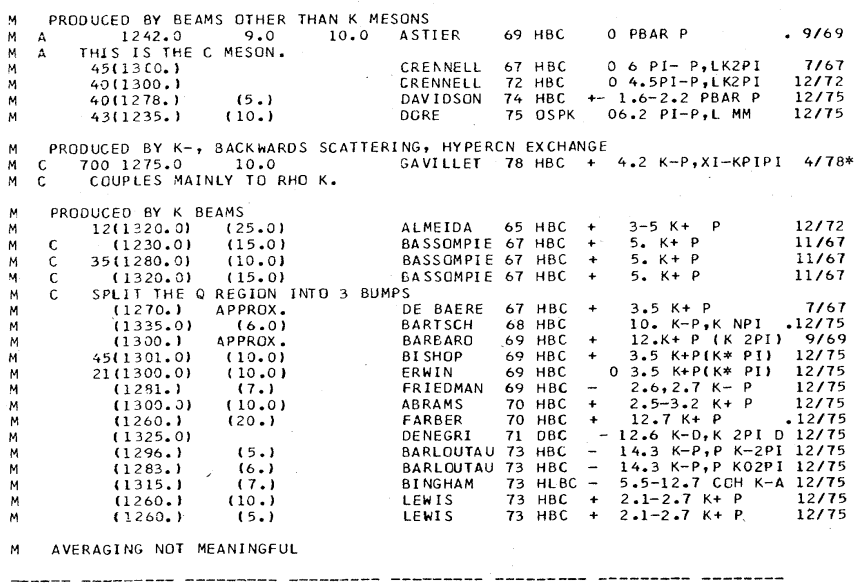

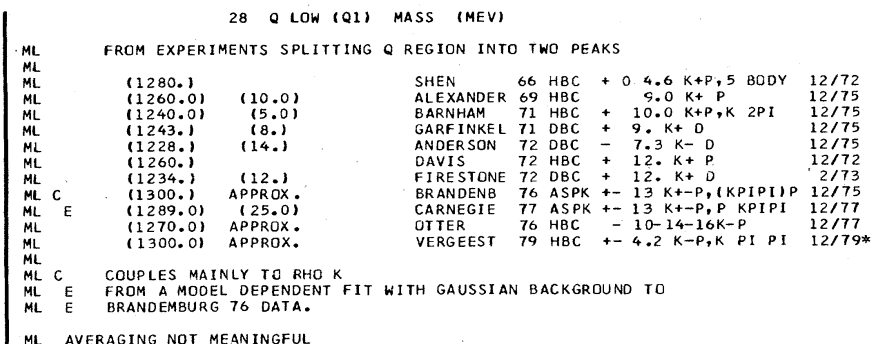

ML. AVERAGING NOT MEAN INGFUL

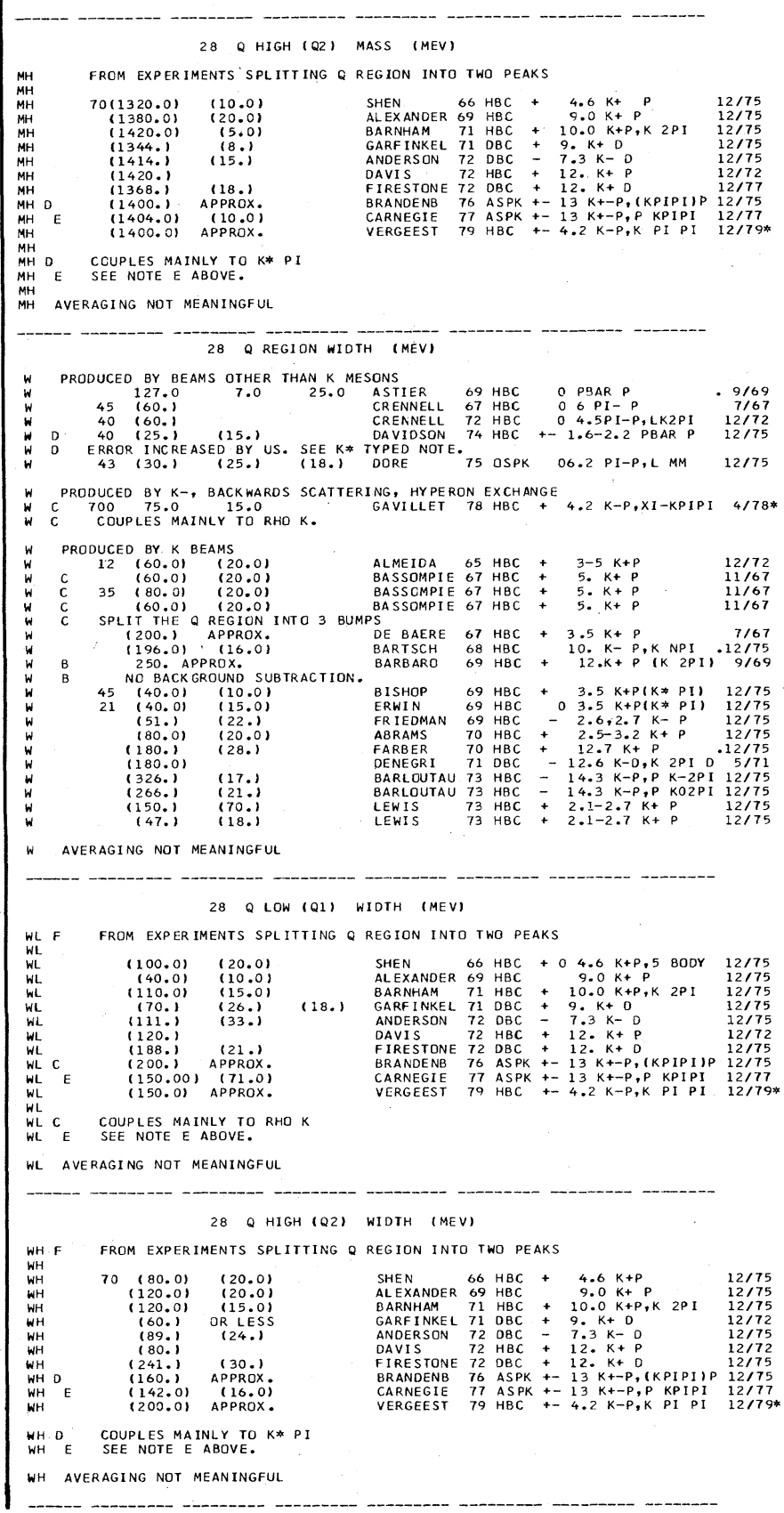




\section{Mesons \\ Q, $K^{\prime}(1400)$}

28 Q REgION PARTIAL DECAY MDDES

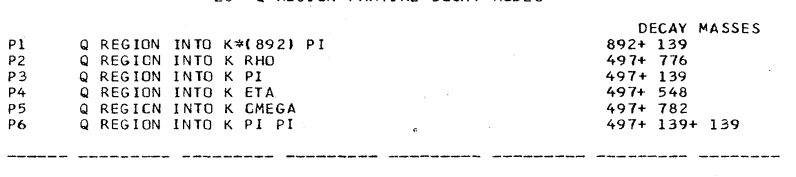

28 Q REGION PARTIAL WIOHTS (MEV)

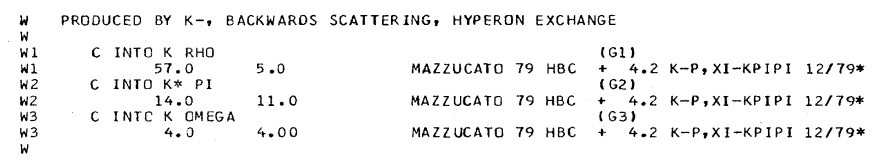

28 G LOW (Q1) PARTIAL WIOTHS (MEV

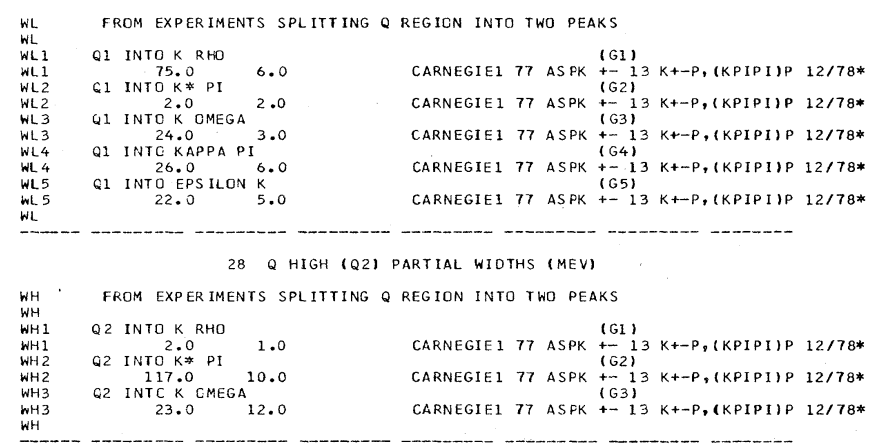

28 Q REGIGN BRANCHING RATIO

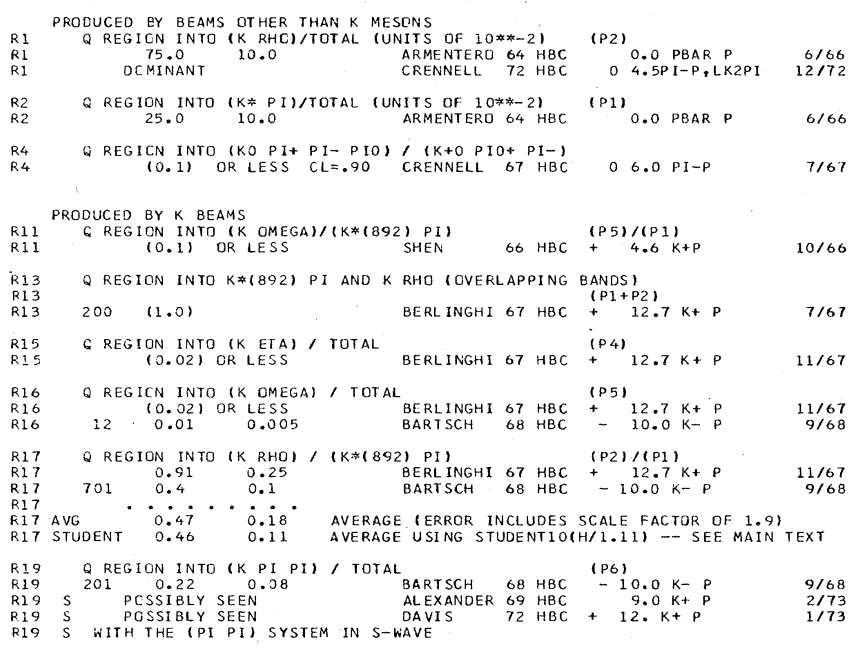

REFERENCES FOR Q REGION

ARMENTER 64 DUBNA CONF 1577 ARMENTEROS, EDWARDS, D-ANDLAU + (CERN+CDEF) ALSE 64 CUBNA CONF 1617 R ARMENTEROS (RAPPORTEUR)
AFMENTER 64 PL 9207 ARMENTEROS, EDWARDS, D-ANDLAU, - (CERN+CDEF) ALSO 66 PR 1451095 BARASH, KIRSCH, MILLER, TAN 109 (COLUMBIA)

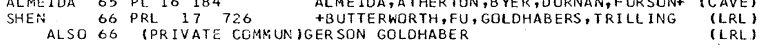

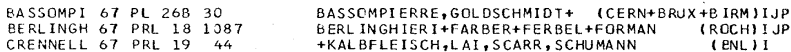
CRENNELL 67 PRL 1944 TKALBFLEISCH, LAI, SCARR, SCHUMANN (EN) II

ALSO PRIVATE COMMUN ICATION BY B. JONGEJANS
GOLOHABE 67 PRL 19976
G.GOLDHABER

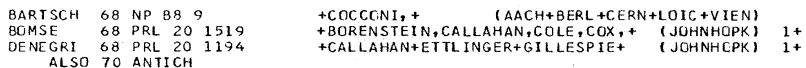

Data Card Listings For notation, see key at front of Listings.

ALEXANDE 69 NP B 13503

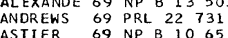

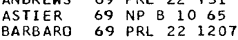

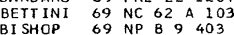
CHIEN 69 PL 298433

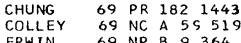

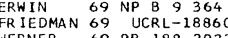
WERNER 69 PR 1882023 $\begin{array}{llllll}\text { ABRAMS } & 70 & \text { PR } & 0 & 1 & 2433 \\ \text { ANTICH } & 70 & \text { NP } & B & 20 & 201\end{array}$

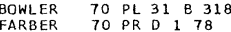
$\begin{array}{llllll}\text { BARNHAM } & 71 & \text { NP } & 825 & 49 \\ \text { DENEGRI } & 71 & \text { NP } & B & 28 & 13 \\ \text { FORMAN } & 71 & \text { PR } & 0 & 3 & 2610\end{array}$

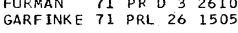
ANDERSON 72 PR D $6 \quad 1823$ BRANDENB 72 NP B 45397 CRENNELL 72 PR D 61220

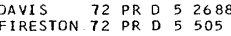

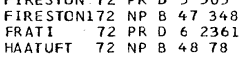
BARLOUTA 73 NP B 59374 DE JONGH 73 NP $B$ S 58110

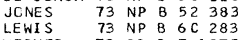
ANGELOPO 74 NC $20 A 49$ BOWLER 74 NP $874 \quad 493$

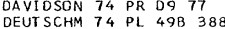
ANTIPOV 75 NP B $86 \quad 381$ $\begin{array}{llll}\text { DORE } & 75 & \text { LNC } 13 & 265 \\ \text { DREVILLO } & 75 & \text { PL } 55 \quad B \quad 245\end{array}$

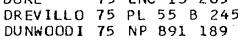

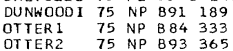
$\begin{array}{lllll}\text { OT TER3 } & 75 & \text { NP B } 96 & 29 \\ \text { TOVEY } & 75 & \text { NP } & \text { B95 } & 109\end{array}$

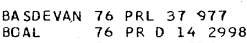

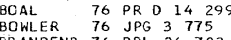
BRANDENB 76 PRL. 26703

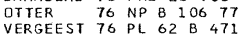
$\begin{array}{llllll}\text { CARNEGIE } 77 & \text { NP } & \text { B } & 127 & 509 \\ \text { CARNEGIE177 } & \text { PL } & \text { B } & 68 & 287\end{array}$ $\begin{array}{lll}\text { CHEN } & 77 & \text { ANL HEP PR } 77 \\ \text { CONFGRTO } 77 & R L-77-024 / A\end{array}$ $\begin{array}{llllll}\text { BEUSCH } & 78 & \text { PL } & 74 & 8 & 282 \\ \text { GAVILLET } & 78 & \text { PL. } 76 & 8 & 517\end{array}$

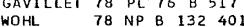
$\begin{array}{lllllll}\text { BASDEVAN } & 79 & \text { PR } & 0 & 19 & 246 \\ \text { MAZZUCAT } 79 & \text { NP } & \text { B } & 156 & 532\end{array}$

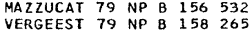
DIONISI 80 CERN/EP 80-1
IRVING 80 JPG 6153 G.ALEXANDER, FIRESTONE, GOL DHABER, + (LRL) + LACH, LUDLAM, SANOWEI SS, BERGER, + (Y YLE+LRL) BARB ARO-GALTIERI, DAVIS, FLATTE, + (LRL) +GOSHAW, ERWIN, WALKER
+MALAMUD, MELLLEMA, RUDNICK, SCHLEIN+ (WISC)
(UCLA) +EISNER +BAL L + +LUERS (BNL)
+ EASTWOOD + +WALKER, GOSHAW, WEINBERG (WI SC+PRIN+ VANDI J.FRIEDMAN, PH.D. THESIS
+ AMMAR, DAVI, KROPAC,YARGER, CHO, (NWES (LANL) $1+$ +EISENSTEIN, KIM, MARSHALL, O-HALLORAN, + (ILL)
+CARSON,CHIEN, COX, DENEGRI, ETTLINGER, + (JHU) +CARSON,CHIEN, COX, DENEGRI, ETTL INGER, ( IHU) $1+$
(OXFORD) $\begin{array}{lr}\text { M.G.BOWLER } \\ \text { FERBEL, SLATTERY, YUTA } & \text { (OXFCRD) } \\ \text { (RCCH) } & 1+\end{array}$ + COLLEY, GR IFFITHS, ALPER, + (BIRM+GLAS +CXF) +ANTIC, CALLAHAN, CAR SON, CHIEN, COX, + (JHU) $1+$ GARF INKEL, HOLLAND, CARMONY, LANDER+(PURO+UCD) $1+$ +FRANKLIN, GODDEN, KOPELMAN, LIBBY, TAN (COLO) BRANOENBURG, BRODY, JOHN SON, LE ITH, LOOS + ( SLAC) 作 +ALSTCN,BARBARD, FLAT TE, FRI EDM AN, LYNCH+ (LBL)
FIRESTONE, GOLOHABER, LI SSAUER, TRILLING (LBL) A.FIRESTONE +HALPERN, HARGIS, SNAPE, CARNAHAN, $+(P E N N+C$ INC)
+ARNOLD, HAGUENAUER, + (BERG +STRB+EPOL+ MADR) +DREVILLCN, SHAH, +
+FARWEL,
(SACL+EPOL +RHEL) JP + CORNET, CHARRIERE, + (BRUX+MONS +CERN+MPIM) G.T.JCNES + ALLEN, JACCBS, DANYSZ, BORG, + (LOWC+LOIC +CDEF)
+ SLATTERY,FERBEL
(ROCHESTER) ANGELOPOULOS, FILIPPAS+ ( ATHU+ATEN+LIVP+VIEN) JP +DAINTON, KADDCURA, AITCHISON .
(CXFAPMAN, GREEN, LYS, ROE SON
(MICH) OEUT SCHMANN, + (AACH+BERL+CERN+LOIC+VIEN) JP + ASCOLI, BUSNELLO, KIENZLE+ (SERP+CERN+ILL) JP + GUI DONI, LAAKSO, NARINI, CONFORTO+ (ROMA+RHEL)

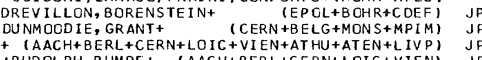
+RUDOLPH,RUMPF+ (AACH+BERL+CERN+LOIC+VIEN) JP + +RUDOLPH, SEYFERT + (AACH+BERL +CERN+LOIC+VIEN) I, JP
+HANSEN, BORENSTEIN,BORG+ (RHEL+EPOL+SACL)I, JP

BASOEVANT, BERGER (FNAL+ANL)

+EDWARDS, KAMAL, TORGESON (ALBERTA)
M.G. BOWLER
(OXFCRD)

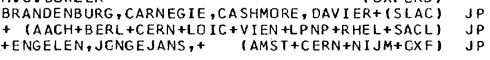
+ CASHMORE, DAVIER, DUNWODOIE,LAS INSK I+ (SLAC)
+CASHMORE, DUNWOODIE, LASINSKI,+
(SLAC) +FIELDS, RHINES (ANL)
B.CONFORTO AND G.CONFORTO (ROMA+RFEL) +BIRMAN, KONIGS, OTTER, + (CERN+AACH+ETH) JP +PALER, CHAURAND,

BA SDEVANT, BERGER (ANL) MAZZUCAT, PENNINGTON+ (CERN+ZEEM+NI JM+OXF)

+GAVILLET, ARMENT EROS + (CERN+MA DR +CDEF+STOH)

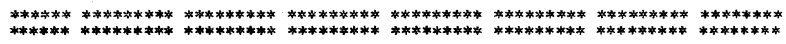
$\mathrm{K}^{\prime}(1400)$ 21 $\mathrm{K}$ PRIME(1400,JP=0-1 $\quad \mathrm{I}=1 / 2$

$\longrightarrow$ OBSERVED IN $K$ PI PI PARTIAL-WAVE ANALYSIS.

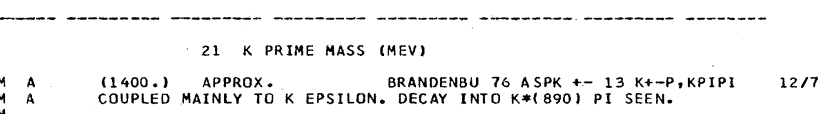
$\stackrel{M}{M}$

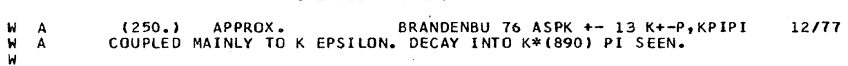
REFERENCES FOR K PRIME

BRANDENB 76 PRL 36 1239
AACHEN 77 PREP. AACHEN $41+$ BRANDENBURG, CARNEGIE, CASHMORE, DAVIER+(SLAC) JP
(AACHEN+BERLIN+CERN+LOIC+VIENNA) JP

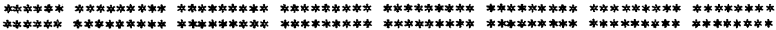




\section{Data Card Listings}

Mesons

For notation, see key at front of Listings.

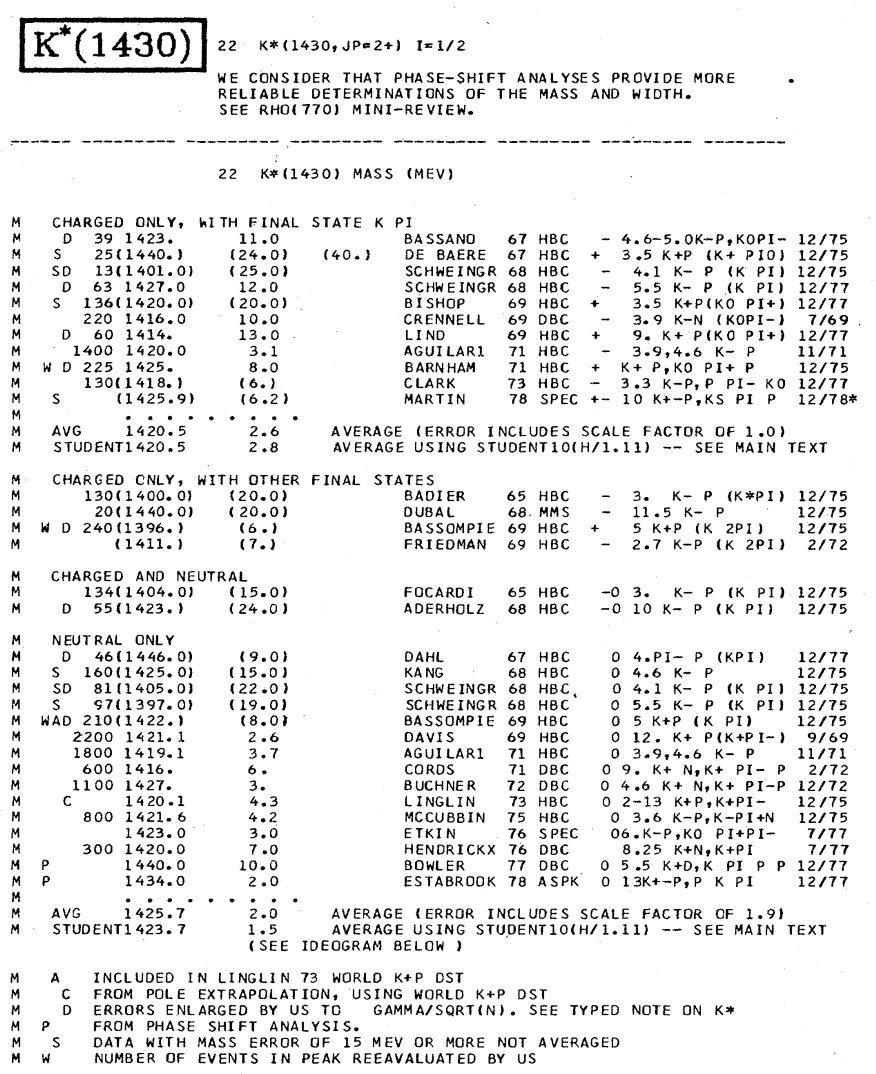

WEIGHTED AUERAGE $=1425.7 \pm 2.0$

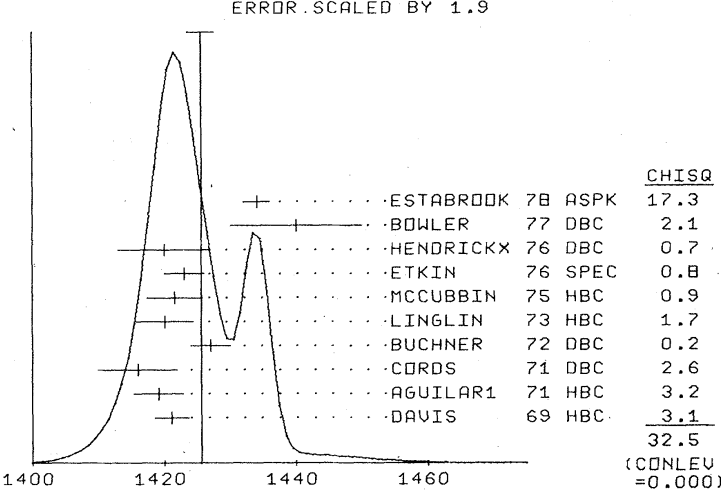

NEUTRAL K*(1430) MASS (MEU)

$22 K *(1430)$ WIDTH (MEV)

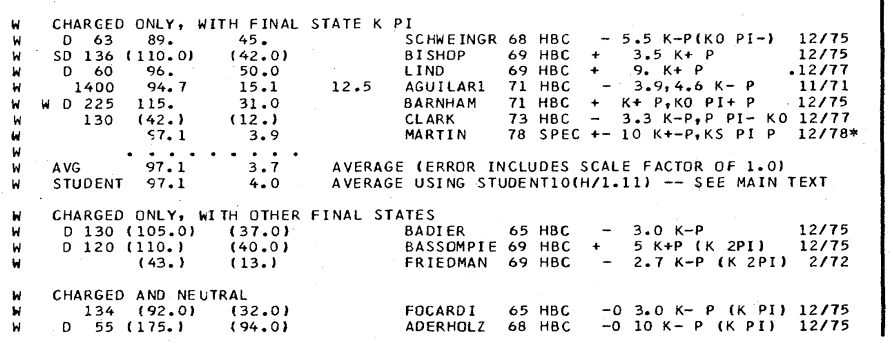

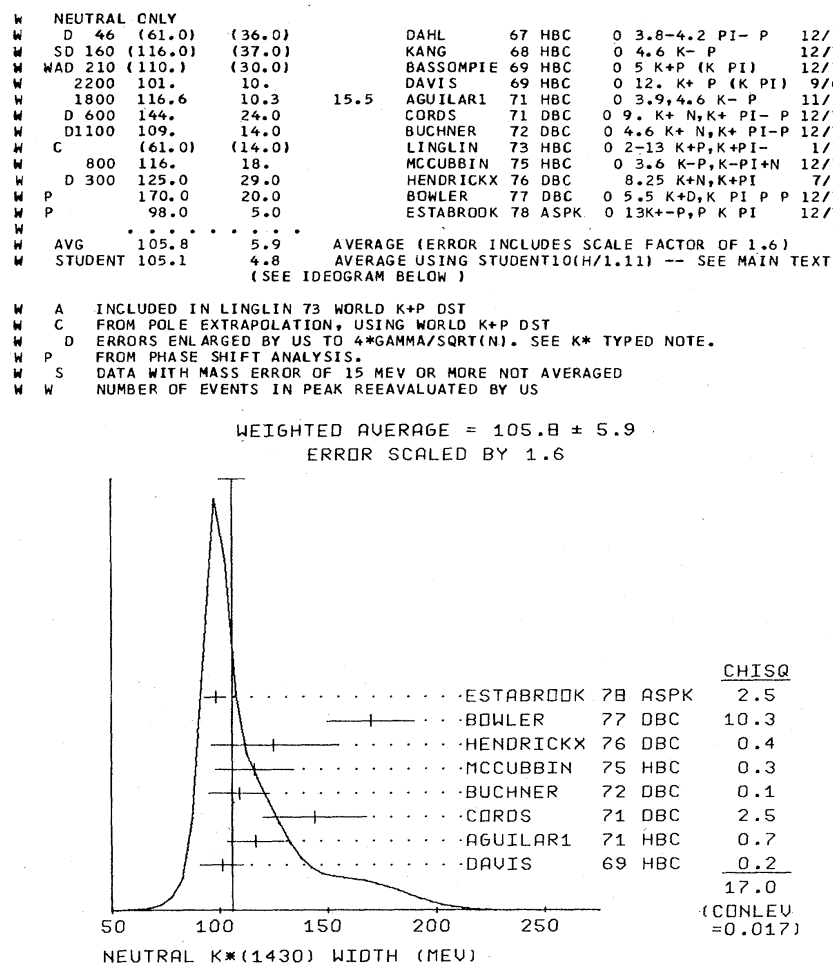

$22 K *(1430)$ PARTIAL DECAY MODES

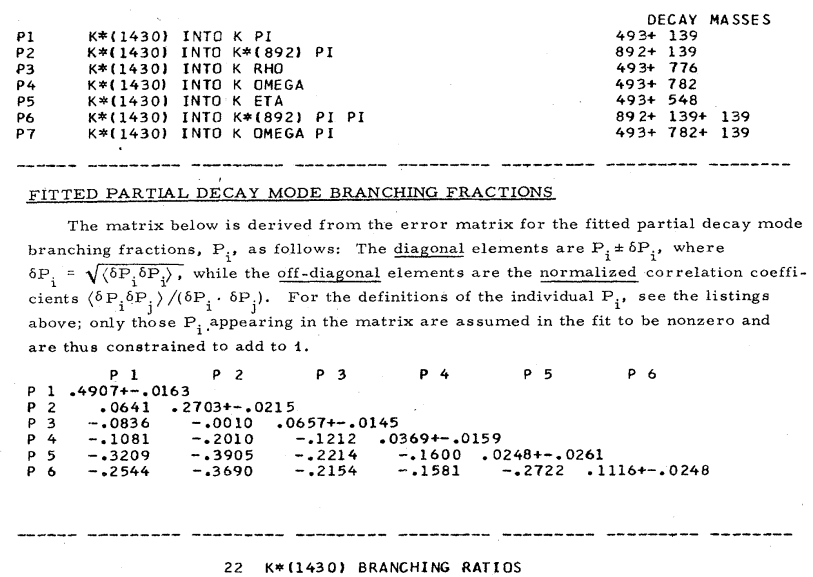

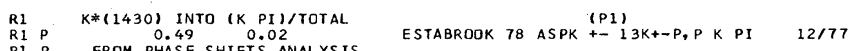

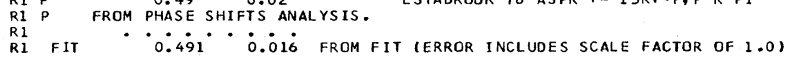

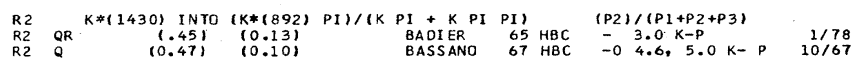

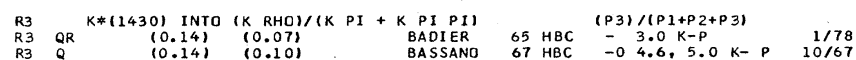
$R 4 \quad K *(1430)$ INTO $(K *(892) P I) /(K$ PI) 65 HBC $(P 2) /(P 1)$

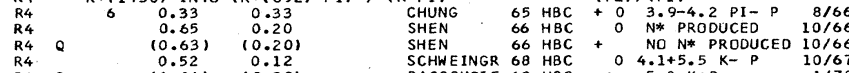

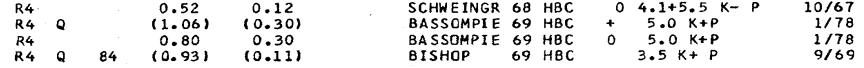


Mesons

$\mathrm{K}^{*}(1430), \kappa(1500)$
Data Card Listings For notation, see key at front of Listings.

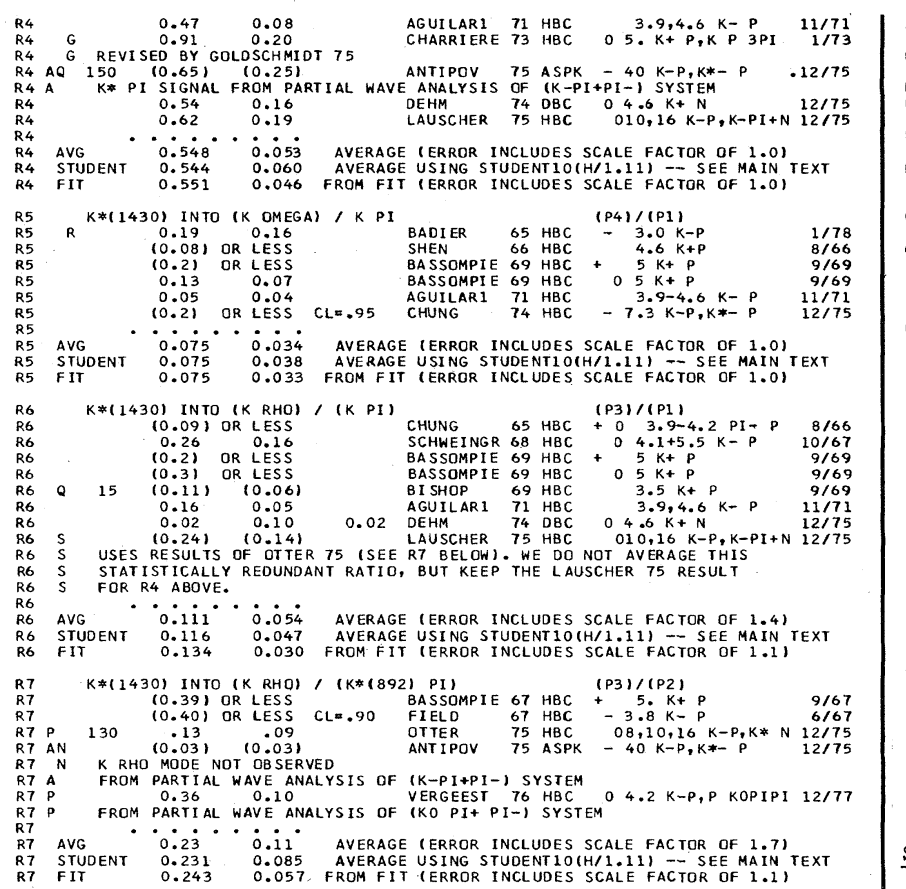

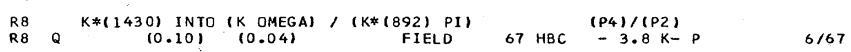
R9 $K *(1430)$ INTO (K ETA) / (K*(892) PI) $6 / 67$

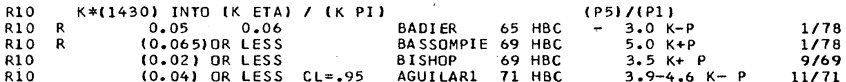
R10 FIT - $0.050^{\circ}$ - 0.054 FROM FIt (ERROR INCLUDES SCALE FaCtOR OF 1.0 )

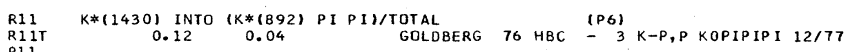

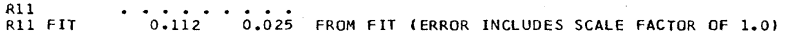

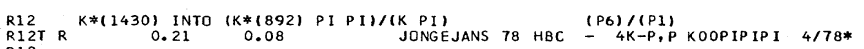
R12 FIT - O.227 - 0.053 FROM FIT (ERROR INCLUDES SCALE FaCtOR OF 1.01 R13 $K *(1430)$ INTO (K OMEGA PII/TOTAL (UNITS $10 * *-3)$ (P7)
R13 0 (0.72) OR LESS CL $=0.95$ JONGEJANS 78 HBC $4 \mathrm{~K}-\mathrm{P}, \mathrm{P}$ KO 4 P I

$R$
$R$ Q FOLLOWING SUGGESTICN BY AGUILAR 70, WE DO NOT MAKE USE OF MEASURE-

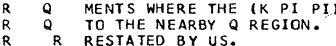

$\begin{array}{lll}R & R & \text { RESTATED BY US. } \\ R & T & \text { ASSUMING PI PI SYSTEM HAS ISO-SPIN 1, WHICH IS SUPPORTED BY } \\ R & T & \text { THE DATA }\end{array}$

************************************************************************1

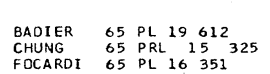

REFERENCES FOR K*(1430)

BADI ER, DEMOUL IN, GOLDBERG+ (EPOL+SACL +AMST)
+DAHL, HARDY, HESS, JACOBS, KIRZ, MILLER ILRL)

SHEN $\begin{aligned} & 66 \\ & \text { ALSO }\end{aligned} 66$ PRL 17.726 OCARDI, MI NGUZZI RANZI, SERRA+(BOLOGNA+SACL)

$\begin{array}{lllll}\text { BASSANO } & 67 & \text { PRL } & 19 & 968 \\ \text { BASSOMPI } & 67 & \text { PL } 268 & 30\end{array}$ $\begin{array}{llll}\text { CRENNELL } 67 & \text { PRL } 19 & 44 \\ \text { DAHL } & 67 & \text { PR } 163 & 1377\end{array}$

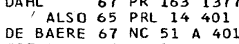

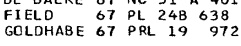

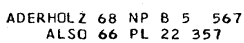

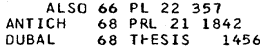

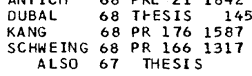
$\begin{array}{llll}\text { BASSOMPI } & 69 \text { NP } 813189 \\ \text { BISHOP } & 899 \text { NP } 899403\end{array}$

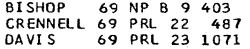
DE EAERE 69 NC 61 A 397
FRIEDMAN 69 UCRL 18860 ABRAMS 70 PR D 12433

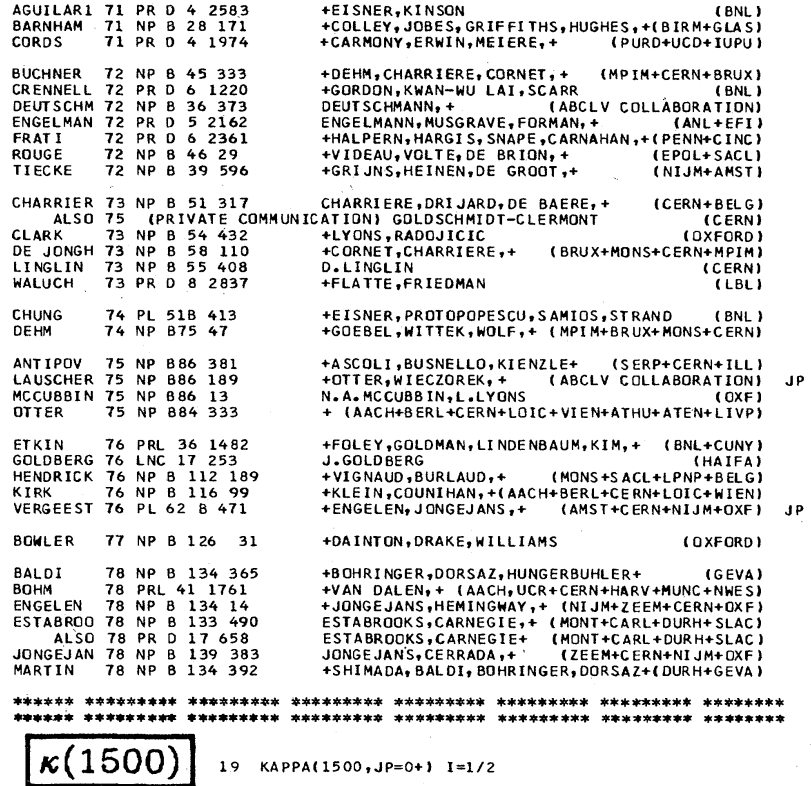

S-Wave $\mathrm{K} \pi$ Interactions

The $\mathrm{K} \pi$ interactions are reminiscent of the $\pi \pi$ interactions, apart from the inelastic thresholds, both for the leading $\mathrm{J}^{\mathrm{P}}=1^{-}, 2^{+}, 3^{-}$resonances and for the $S$ wave. The first inelastic $S$-wave thresholds are $\mathrm{K} \pi \pi \pi$ and $\mathrm{K} n$, neither of these channels being known to be important below $1400 \mathrm{MeV}$.

From the $\mathrm{K} \pi$ threshold to $\sim 1400 \mathrm{MeV}$, the phase shift $\delta_{0}^{1}$ of the $I\left(\mathrm{~J}^{\mathrm{P}}\right)=1 / 2\left(0^{+}\right)$wave is determined uniquely by the requirements of elastic unitarity. It grows monotonically, reaching $40^{\circ}$ at about $900 \mathrm{MeV}$, and $90^{\circ}$ at about $1350 \mathrm{MeV}$, being everywhere well described by an effective range formalism (MERCER 71, BINGHAM 72, FIRESTONE 71,72, MATISON 72,74, GALTIERI 73, YUTA 73, FOX 74, BAKER 75, LAUSCHER 75, BOWLER 77, ESTABROOKS 78); see Fig. 1. The ambiguous "up" solution in the region of the $\mathrm{K}^{*}(892)$ has been ruled out conclusively (MATISON 72,74, GALTIERI 73, BOWLER 77, ESTABROOKS 78). In the $1400 \mathrm{MeV}$ region the analysis becomes complicated due to the largeness of $\delta_{0}^{1}$, to the nearness of the $\mathrm{K}^{*}(1430)$ and the resulting strong $S-D$ interference, and to the opening of inelastic channels. Several groups have interpreted the slow passage of $\delta_{0}^{l}$ through $90^{\circ}$ as evidence for a resonance (FIRESTONE 71,72, FRATI 72, ROUGE 72, CORDS 73, LAUSCHER 75, MORGAN 75, ENGELEN 78), while others contended that $\delta_{0}^{1}$ was large but 


\section{Data Card Listings}

Mesons

For notation, see key at front of Listings.

non-resonant (AGUILAR 72, BUCHNER 72, CRENNELL 72, ENGELMANN 72, BAKER 75).

New features emerge as the phase-shift analysis is continued up to $1900 \mathrm{MeV}$ on a large-statistics experiment (ESTABROOKS 78). In the inelastic region where the ambiguities cannot be solved ESTABROOKS 78 find four solutions, in all of which the $S$ wave exhibits a rapid drop in the modulus of the amplitude near $1450 \mathrm{MeV}$; see Fig. 2. This behavior is confirmed with less statistics by BOWLER 77 and MARTIN 78, and a clear circular motion is seen in the Argand plot with a maximum speed in the region 1400-1500 MeV. Thus all four solutions provide evidence for an $\mathrm{S}$-wave $\mathrm{O}^{+} \mathrm{K} \pi$ resonance. The elasticity is greater than 0.8 in all but one solution. We call this resonance $K(1500)$ and enter it into the Table. ESTABROOKS 79 performs a coupled-channel fit to the mass dependence of the S-wave magnitude and phase using various parametrizations. All the fits result in a second-sheet pole very near the $\mathrm{K} \eta^{\prime}$ threshold with $\mathrm{M}=1480-1570$ $\mathrm{MeV}$ and $\Gamma=120-400 \mathrm{MeV}$, depending on which of the four solutions is used as input. No additional pole is required to explain the slow passage of $\delta_{0}^{1}$ through $90^{\circ}$ at about $1350 \mathrm{MeV}$. We note that this situation is reminiscent of the $\pi \pi$ system, where the "down" phase shift $\delta_{0}^{0}$ goes slowly through $90^{\circ}$ at about $850 \mathrm{MeV}$, far below the $\varepsilon(1300)$

resonance.

The present $0^{+}$nonet looks rather different from the one considered by MORGAN 75. The $\varepsilon$ and $k$ both have higher masses and smaller widths than before; the $\varepsilon$ in addition couples noticeably to $k \bar{k}$. Finally, we remark that two of the four solutions of ESTABROOKS 78 provide evidence for a second P-wave resonance with mass $\sim 1650 \mathrm{MeV}$, width $\sim 250 \mathrm{MeV}$, and elasticity $\sim 0.25$. This new state, $K^{*}$ '(1650), would, if confirmed, most probably be assigned in the quark model as a radial excitation, similarly to $\rho^{\prime}(1600)$.
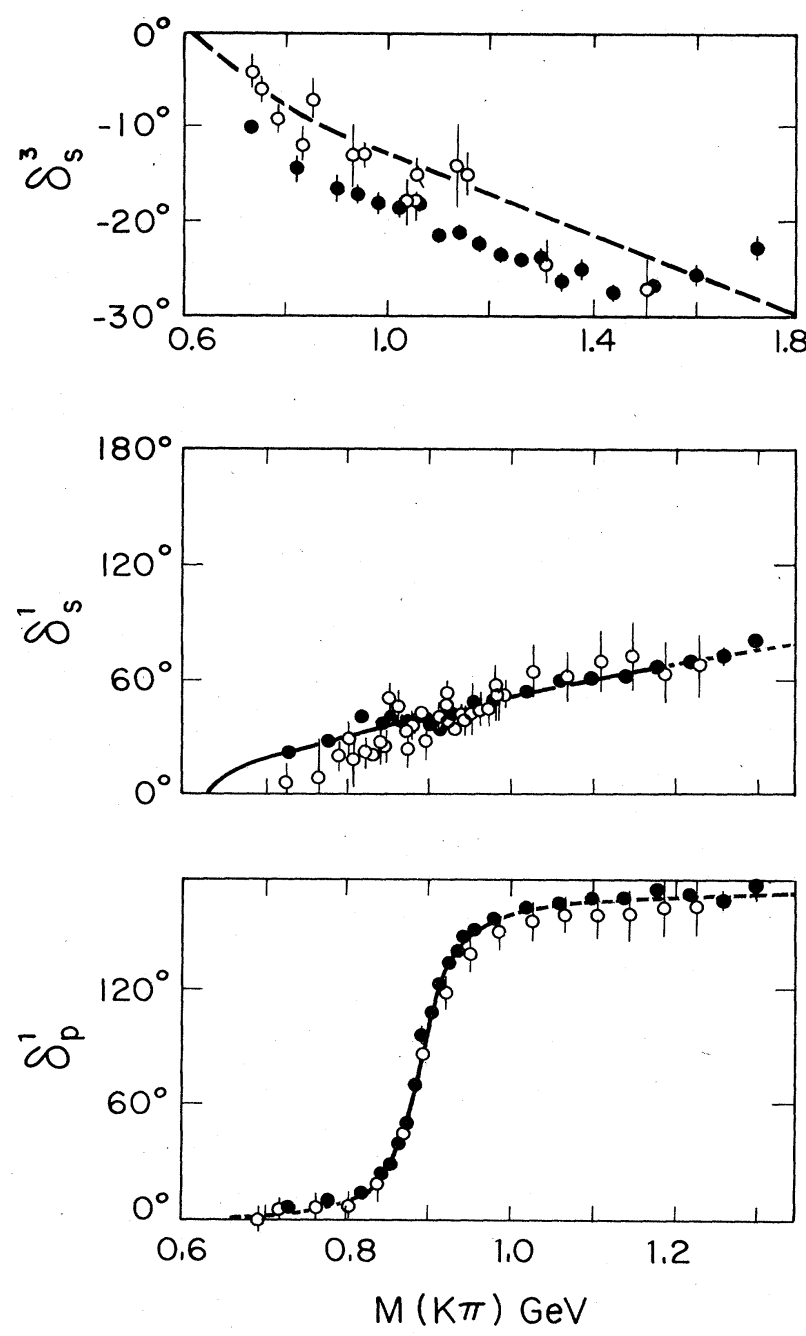

XBL $783-397$

Fig. 1. The solid points are the $\mathrm{K} \pi$ phase shifts calculated in a simultaneous analysis of the SLAC $13 \mathrm{GeV} / \mathrm{c}$ neutron and $\Delta$ recoil reactions. The curves represent the effective range or resonance fits of ESTABROOKS 78, except for the dashed curve on the $\delta_{s}^{3}$ plot which represents a constant cross section of $1.8 \mathrm{mb}$. The open circles are from MERCER 71, BINGHAM 72, BAKER 73, LINGLIN 73, and MATISON 74 . 
Mesons

$\kappa(1500), L(1580)$

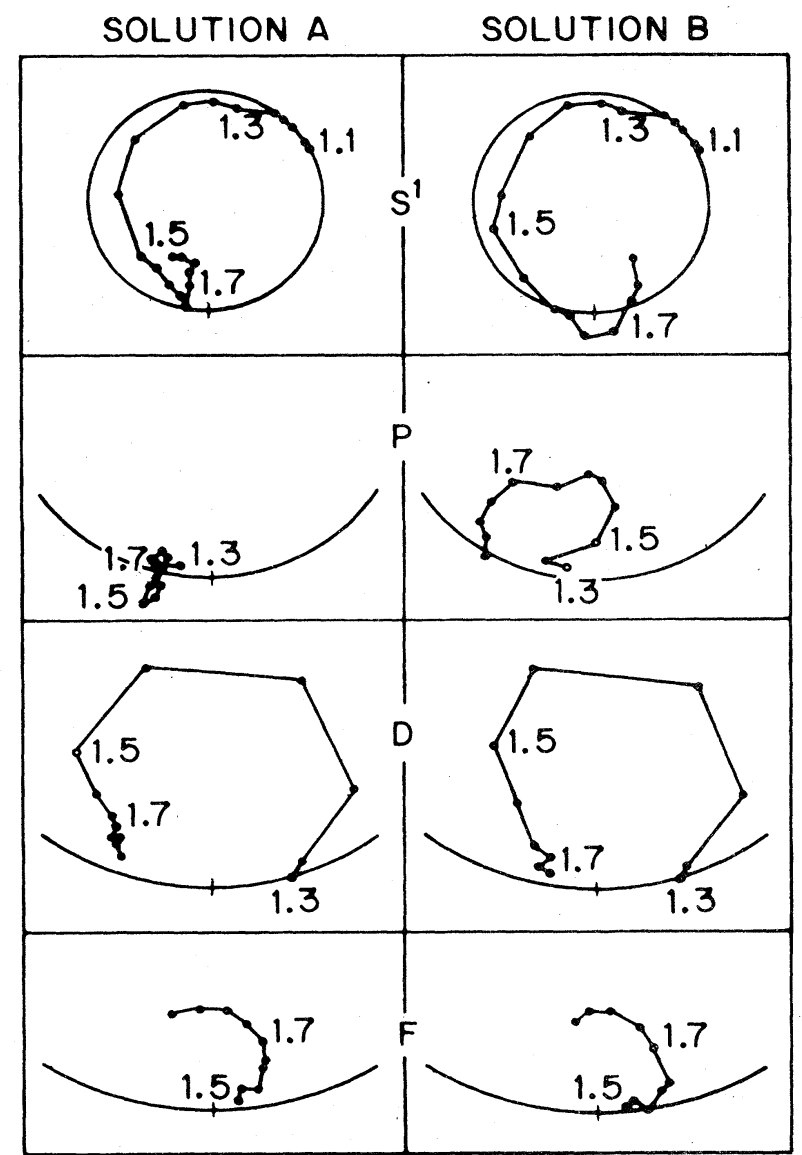

Data Card Listings For notation, see key at front of Listings.

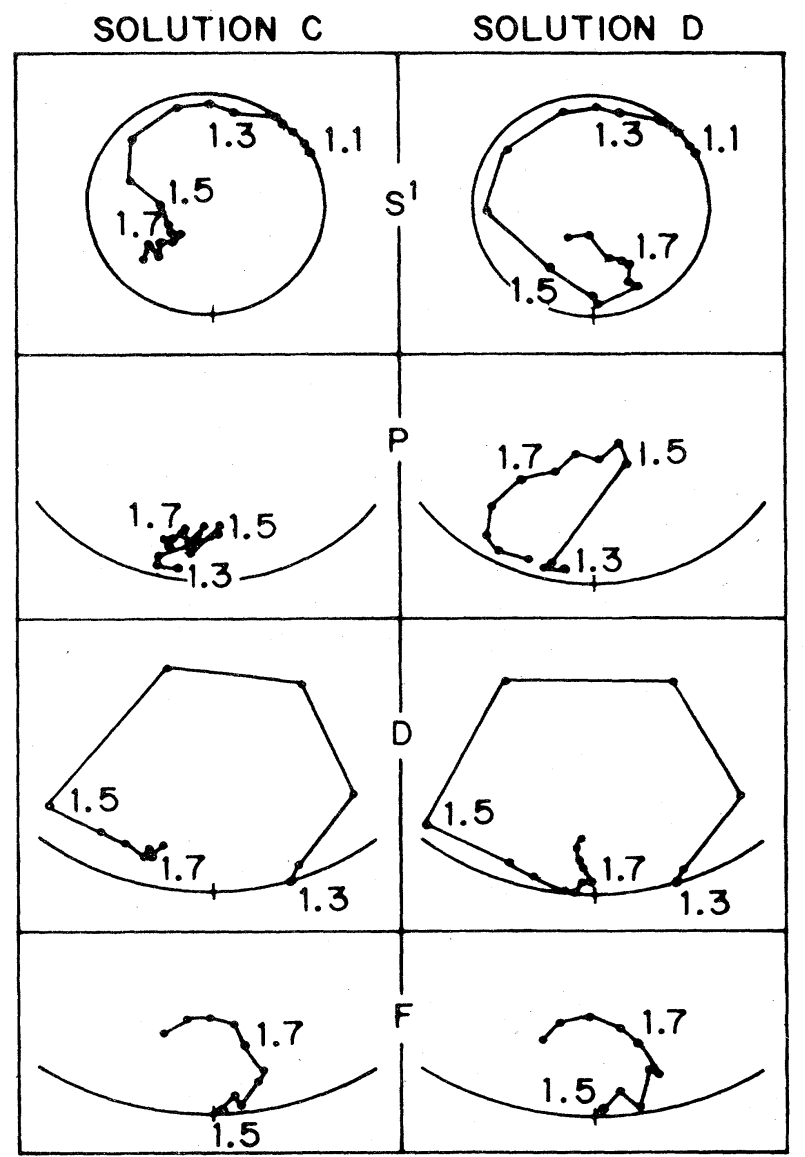

Fig. 2. Argand diagrams for the $\mathrm{K} \pi$ partial waves of ESTABROOKS 78 .

19 KÁPPA MASS (MEV)

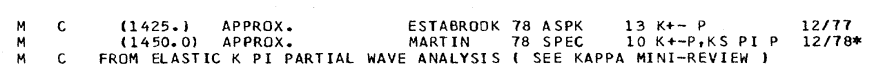
$M$ C FROM

19 KAPPA WIDTH (MEV)

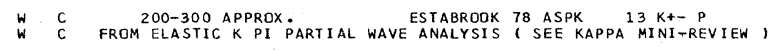

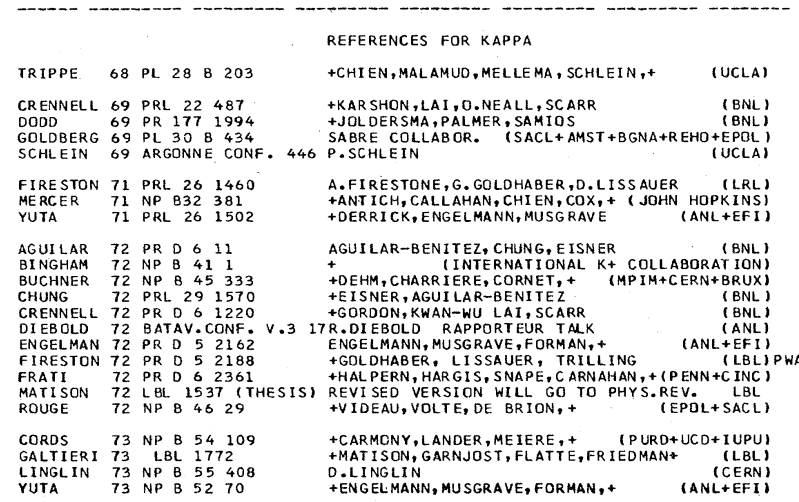

$\begin{array}{llllll}\text { FOX. } & 74 & \text { NP } & 880 & 403 \\ \text { MATISON } & 74 & \text { PR } & \text { D9 } & 1872\end{array}$ $\begin{array}{lllll}\text { MATISON } & 74 & \text { PR } & \text { D } 9 & 1872 \\ \text { MORGAN } & 74 & \text { PL } & 518 & 71\end{array}$ BAKER 75 NP 899211 $\begin{array}{lll}\text { LAUSCHER } 75 & \text { NP } 886 & 189 \\ \text { MORGAN } & 15 & 189 \\ \text { ARGONNE CONF. } 45\end{array}$

CHIEN 76 NP B 106355

$\begin{array}{llllllr}\text { BOWLER } & 77 & \text { NP } & 8 & 126 & 31 \\ \text { SPIRO } & 77 & \text { NP } & 8 & 125 & 162\end{array}$

BALOI 78 NP B 134365

$\begin{array}{lllllll}\text { ENGELEN } 78 & \text { NP } & \text { B } & 134 & 14 \\ \text { ESTABROO } & 78 & \text { NP } & \text { B } & 133 & 490\end{array}$ $\begin{array}{lllllll}\text { ESTABROO } & 78 & \text { NP } & \text { B } & 133 & 490 \\ \text { MARTIN } & 78 & \text { NP } & \text { B } & 134 & 392\end{array}$

$\begin{array}{llllll}\text { ESTABROO } & 79 & \text { PR } & \text { D } & 19 & 2678 \\ \text { VERGEEST } 79 & \text { NP } & \text { B } & 158 & 265\end{array}$

P.ESTABROOKS
+ (CARGELEN, KITTEL+
(NIJM+AMST+CERN + DXF)

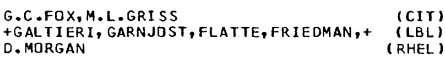

+BANER JEE, CAMPBELL, ALLEN, MARCH+ (LOIC+LOWC) DOTTER, WIECZOREK, + (ABCLV COLLABORATION)
(RHEL)

HFEIOCK, LUCAS, PEVSNER, ZOANIS (BALTIMCRE) +OAINTON, DRAKE, WI LLIAMS + (OXFORD)
+BARLOUTAUD, COMBER, PALER, + (SACL+RHEL+EPOL)

+BOHR INGER, DORSAZ, HUNGERBUHLER+ (GEVA) (NI DOHRINGER, DORSAZ+(DURH+GEVAI

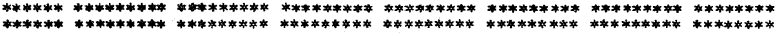

$L(1580)$ 39 Li $1580, J P=2-1 \quad I=1 / 2$

SEEN IN PARTIAL WAVE ANALYSIS OF THE K-PI+PI- SYSTEM NEED CONFIRMATION OMITTED FROM TABLE.

39 L(1580) MASS (MEV)

$79-10,14,16 \mathrm{~K}-\mathrm{P}$ - $12 / 79 *$

39 L(1580) WIDTH (MEV)

(110.) APPROX. OTTER 


\section{Data Card Listings}

For notation, see key at front of Listings.
39 L(1580) PARTIAL DECAY MODES

P1 L(1580) INTO K*(890) PI DECAY MASSES

-

39 L(1580) BRANCHING RATIOS

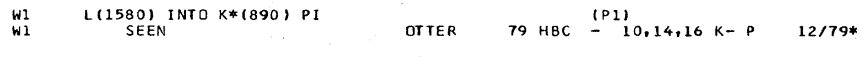

W2
W2
POSSI BLY SEEN

***********************************************************************1

REFERENCES FOR L(1580)

OTIER $79 \mathrm{NP}$ B 1471 TRUDOLPH, + (AACH+BERL+CERN+LOIC+WIEN) JP

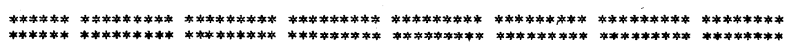

\begin{tabular}{|c|c|}
\hline$K^{*}(1650)$ & $\begin{array}{l}29 K *(1650, J P=1-1 \quad I=1 / 2 \\
\text { SEEN IN } K \text { PI PHASE SHIFTS ANALYSIS (ESTRABROOK 78). } \\
\text { WAIT CONFI RMATION.OMITTED FROM TABLE. }\end{array}$ \\
\hline & $29 K *(1650)$ MASS (MEV) \\
\hline$(1650)$. & ESTABROOK 78 ASPK O K+-P, K+-PI+- \\
\hline
\end{tabular}

W 250-300 APPROX. ESTABROOK 78 ASPK O K+-P,K+-PI+- 12/78*

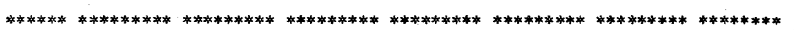
REFERENCES FOR $K *(1650)$

ESTABROO 78 NP B 133490 ESTABROOKS, CARNEGIE,+ (MONT+CARL+DURH+SLAC)

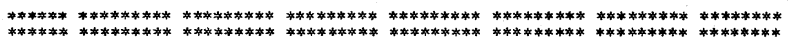

$\mathrm{K}_{\mathrm{N}}(1700){ }^{27} \mathrm{kN}(1700, \mathrm{JP}=1 \mathrm{I}=1 / 2$

$\longrightarrow \begin{gathered}\text { THIS ENTRY CONTAINS VARIOUS PEAKS IN STRANGE MESON } \\ \text { SYSTEMS RE PORTED IN IHE } 1700 \text { MEV REGIN. } \\ \text { EVIDENCE NOT COMPELLING, OMITTED FROM TABLE. }\end{gathered}$

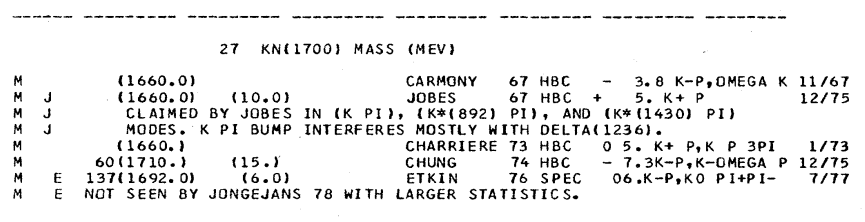

27 KN(1700) WIDTH (MEV)

$W$ J SEE NOTE J ABOVE $(20.0)$ JOBES $67 \mathrm{HBC}+5 . \mathrm{K}+\mathrm{P} \quad 12 / 75$

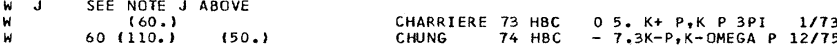

$W$
$W$
$W$

27 KN(1700) PARTIAL DECAY MOOES

\begin{tabular}{lll} 
P1 & KN(1700) INTO K PI & DECAY MASSES \\
P2 & KN(1700) INTO K PI PI & $493+139$ \\
P3 & KN(1700) INTO K*(892) PI & $493+139+139$ \\
P4 & KN(1700) INTO K $R$ RO & $892+139$ \\
P5 & KN(1700) INTO K*(1430) PI & $493+776$ \\
P6 & KN(1700) INTO K OMEGA & $734+139$ \\
& & $493+782$ \\
\hline
\end{tabular}

27 KN(1700) BRANCHING RATIOS

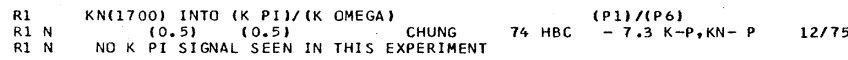

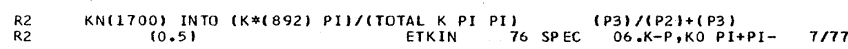
********************************************************************4

REFERENCES FOR KN(1700)

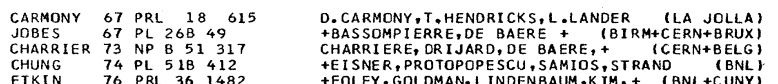

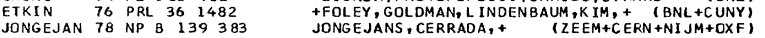

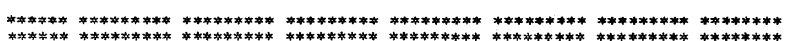

Mesons

$\mathrm{L}(1580), \mathrm{K}^{*}(1650), \mathrm{K}_{\mathrm{N}}(1700), \mathrm{L}(1770)$
$L(1770)$ 23 L11770, JP $=11=1 / 2$

The $L(1770)$ is seen as a bump in the diffractive-like process $\mathrm{KN} \rightarrow(\mathrm{K} \pi \pi) \mathrm{N}$. BARBARO 69 and FIRESTONE 72 find the decay is consistent with being entirely $\mathrm{K}^{*}(1430) \pi$, whereas AGUILAR 70, BARTSCH 70, COLLEY 71, and DENEGRI 71 present evidence for alternate decay modes. For a review see EISNER 74.

Partia1-wave analyses (DEUTSCHMANN 74, ANTIPOV 75, OTTER 75,79 ) have shown that the situation in the $L$ region is complicated with many unnatural parity waves contributing. The $2^{-} \mathrm{K}^{*}(1430) \pi \mathrm{S}$ wave is important, but cannot explain the whole $\mathrm{L}$ enhancement.

On the other hand, OTTER 79 propose the existence of a $2^{-}$resonance of mass $\approx 1580 \mathrm{MeV}$ and width $\approx 110 \mathrm{MeV}$ to explain the sharp rise of the $2^{-}$ $K^{*}(1430) \pi$ channel, the peak in the $2^{-} K^{*}(892) \pi$ partial wave, and the observed phase variation. Further confirmation is, however, needed before considering this an established resonance.

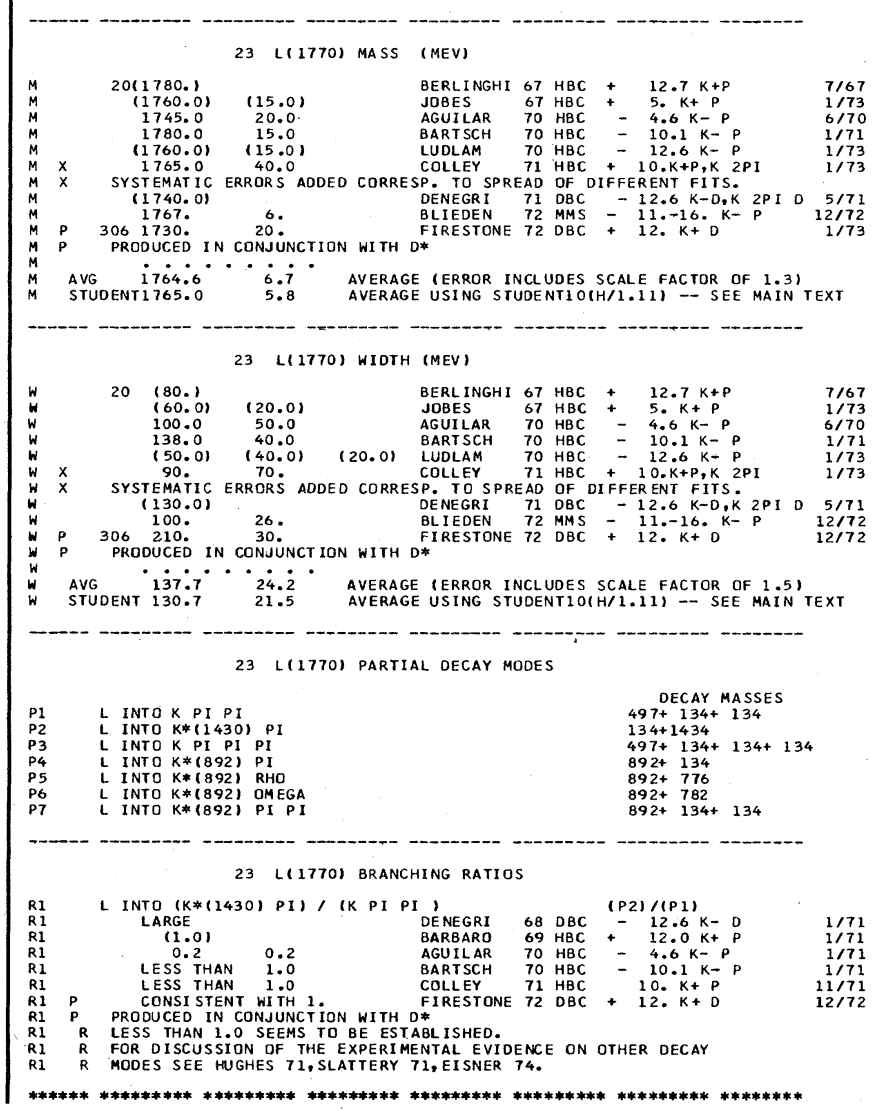




\section{Mesons $\mathrm{L}(1770), \mathrm{K}^{*}(1780)$}

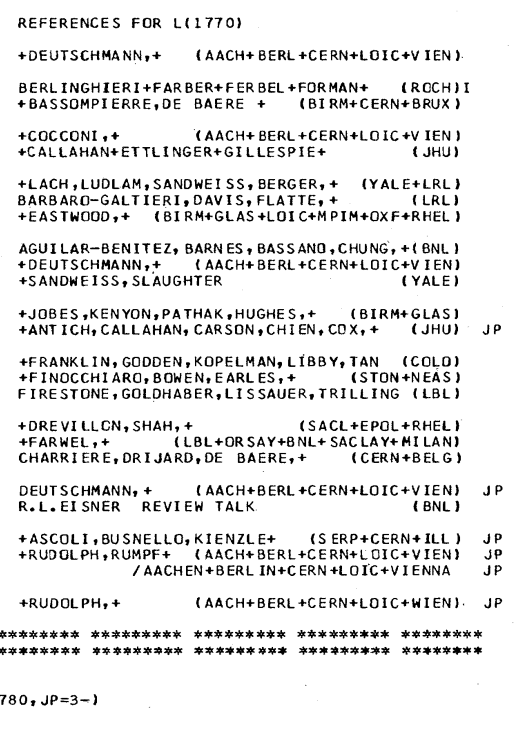

Evidence for $\mathrm{K}^{*}(1780)$ has been reported by a number of experiments which observe peaks of low statistical significance around $1800 \mathrm{MeV}$ in the mass spectra of $\mathrm{K} \pi$ and $\mathrm{K} \pi \mathrm{r}$ systems produced both with charge exchange (CARMONY 71, AGUILAR 73, SPIRO 76. CARMONY 77, GRASSLER 77) and without charge exchange (SPIRO 76). The large variation in the measured values of the mass (see the Data Card Listings) leads GRASSLER 77 to suggest that there may be further structure at higher mass (around $1850 \mathrm{MeV}$ ).

Additional evidence for the $\mathrm{K}^{*}(1780)$ has come from observations of structure in the charge-exchange $\mathrm{K} \pi$ angular distribution at $\sim 1800 \mathrm{MeV}$ (FIRESTONE 71, BRANDENBURG 76), which can be explained by a rapid rise of the $\mathrm{F}$-wave amplitude interfering strongly with other waves. This behavior has been interpreted by BRANDENBURG 76 as implying the existence of a resonance with $\mathrm{J}^{\mathrm{P}}=3^{-}, \mathrm{M} \sim 1780 \mathrm{MeV}$, and $\Gamma \sim 270 \mathrm{MeV}$. The existence of such a resonance has been confirmed by BALDI 76 and CHUNG 78. BALDI 76 analyze noncharge-exchange data and find significant signals at $\sim 1780 \mathrm{MeV}$ in all moments up to $\mathrm{L}=6$. A clear, statistically significant peak at $\sim 1786 \mathrm{MeV}$ is observed by CHUNG 78 in their charge-exchange $\mathrm{K} \pi$ mass spectrum. Both of these experiments obtain narrower widths than BRANDENBURG 76 . BALDI 76 finds a width of $135 \pm 22 \mathrm{MeV}$ and CHUNG 78 a width of $95 \pm 31 \mathrm{MeV}$.

There have been two phase-shift analyses of

\section{Data Card Listings For notation, see key at front of Listings.}

the $\mathrm{K} \pi$ system in this energy region. The energydependent analysis of BOWLER 77 supports the existence of a broad $\mathrm{J}^{\mathrm{P}}=3^{-}$resonance at $\sim 1760 \mathrm{MeV}$ with a width $\sim 300 \mathrm{MeV}$. The problem of the width has been partly resolved by the ESTABROOKS 78 analysis of the high-statistics spectrometer data of BRANDENBURG 76. ESTABROOKS 78 find four solutions (see the $\mathrm{K} \pi \mathrm{S}$-wave mini-review), all of which are compatible with the existence of an F-wave resonance at $\sim 1780 \mathrm{MeV}$ with a width $\sim 175 \mathrm{MeV}$ and

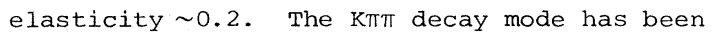
confirmed by BEUSCH 78 with good statistics and can be considered established.

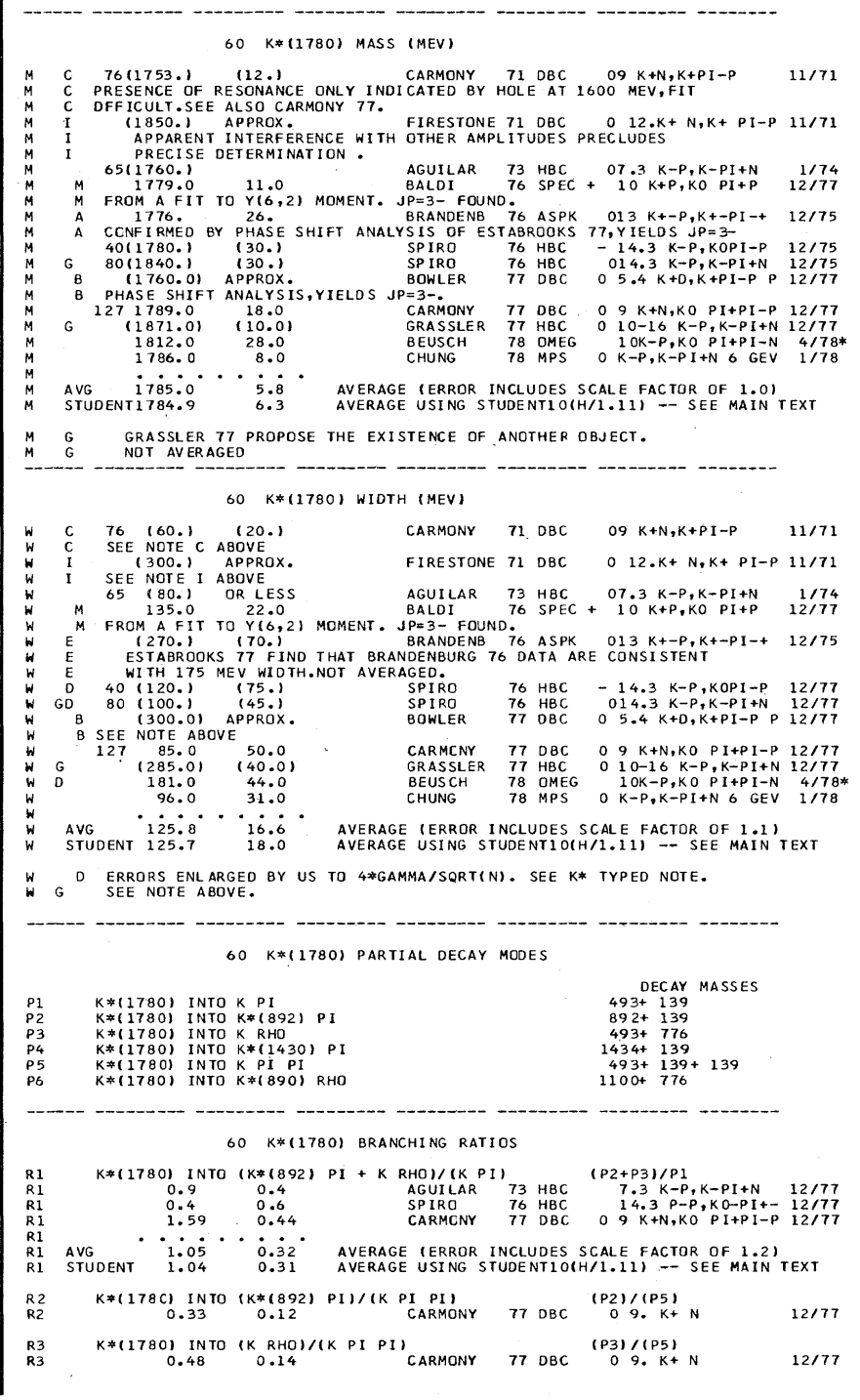


Data Card Listings

Mesons

For notation, see key at front of Listings. $\mathrm{K}^{*}(1780), \mathrm{K}^{*}(2200), \mathrm{I}(2600), \mathrm{D}^{ \pm}, \mathrm{D}^{0}, \mathrm{D}^{* \pm}, \mathrm{D}^{* 0}$

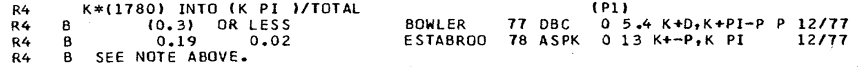

\begin{tabular}{|c|c|}
\hline & $\begin{array}{l}* * * * * * * * * * * * * * * * * * * * * \\
\text { REFERENCES FOR K*(1780) }\end{array}$ \\
\hline 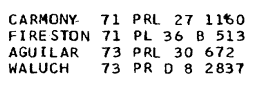 & 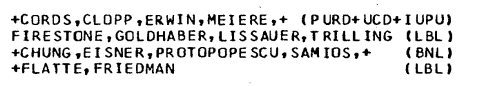 \\
\hline $\begin{array}{llllll}\text { BALDI } & 76 & \text { PL } & 63 & 8 & 344 \\
\text { BRANDENB } & 76 & \text { PL } & 60 & 8 & 478 \\
\text { SPIRO } & 76 & \text { PL } & 60 & \text { B } & 3789\end{array}$ & 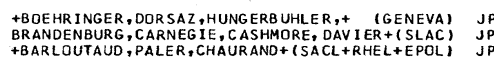 \\
\hline 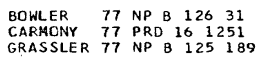 & 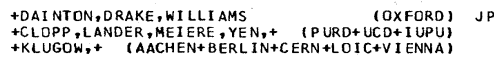 \\
\hline 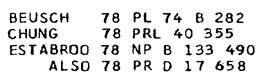 & 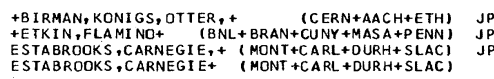 \\
\hline
\end{tabular}

$K^{*}(2200) 40 \quad K * 12200, J P=$,

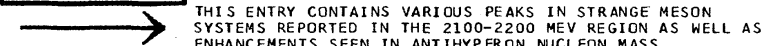
ENHANCENENTS SEEN IN ANT IHYP ERON NUCLEON MASS
SPECTRA. A MOMENTS ANALYSIS OF. THE CLELAND 80 DATA SPECTRA. A MOMENTS ANALYSIS OF. THE CLELAND 80 DATA
GIVE EVIDENCE FOR THO RESONANCES AT 2.3 AND 2.5 GEV

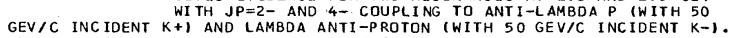

D

$$
40 \quad K * 12200) \text { MASS (MEV) }
$$

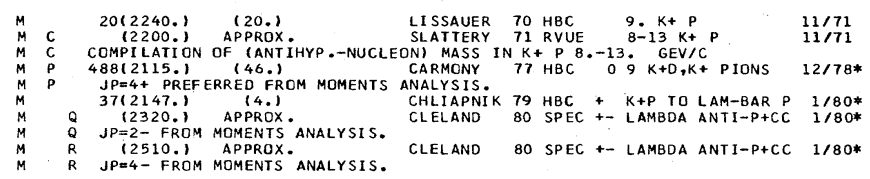

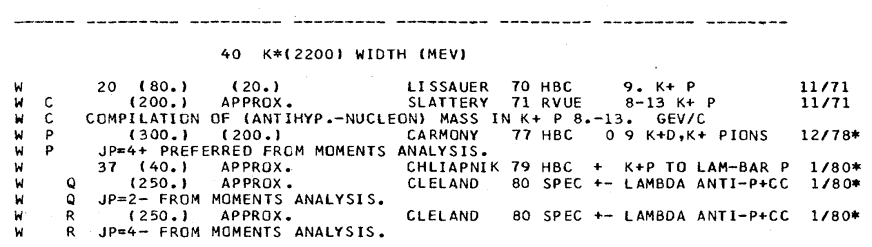

\section{*********************************************************************}

$\begin{array}{llll}\text { AL EXANOE } 68 \text { PRL } 20755 & 75 \\ \text { LISAUER } 70 \text { NP B } 18491 & \text { ALEXANOER, FIRESTONE, GOLOHABER, SHEN } & \text { (LRL) } \\ \text { TALEXANDER, F IRESTONE, GOLOHABER } & \text { (LBL) }\end{array}$

$\begin{array}{lll}\text { CARMONY } 71 & \text { PRL } 271160 & 1160 \\ \text { SLATTERY } 71 & \text { UR-875-332 (PREP) P.SLATY PRY, A REVI EW OF STRANGE MESONS(ROCH) }\end{array}$

CARMONY 77 PRD 161251 TLLOPP, LANDER, MEIERE, YEN, I (PURD+UCD+IUPU)

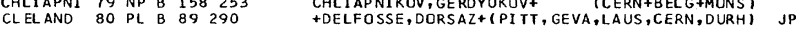

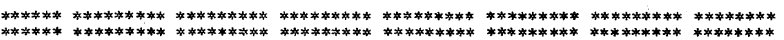

I(2600) $24 \quad 112600)$

$\longrightarrow \begin{gathered}\text { THIS ENTRY CONTAINS. High-MASS, NaRrow STRANGE PEAKS. } \\ \text { OMITTED FROM TABLE. }\end{gathered}$

$$
24 \text { I MASS (MEV) }
$$

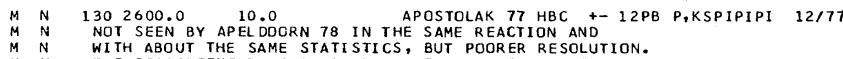

$M$
$M$

$M_{M}^{M}$ AL

$$
24 \text { I WIDTH (MEV) }
$$

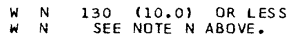

APOSTOLAK 77 HBC + $12 P B P, K S P I P I P I \quad 12 / 77$

$* * * * * * * * * * * * * * * * * * * * * * * * * * * * * * * * * * * * * * * * * * * * * * * * * * * * * * * * * * * * * * * * * * * *$

REFERENCES FOR I

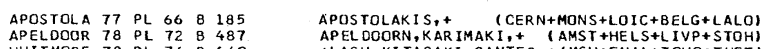

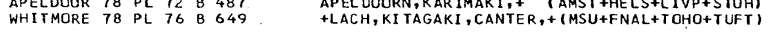

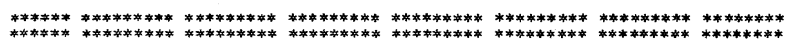

$\mathrm{C}= \pm 1$ MESON STATES

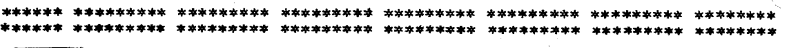

$D^{ \pm} \quad 31$ CHARGED D(1868,JP=0-) $I=1 / 2$

See stable particle data caro listings

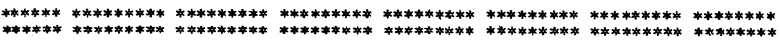

$D^{0} \quad 32$ NEUTRAL O(1863,JP=0-1) I=1/2

see stable particle data caro listings

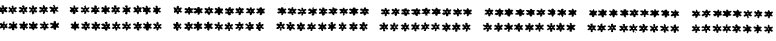

$\mathrm{D}^{* \pm}(2010)$ G2 CHARGED D*(2010, JP $\left.=1-\right)$ I $=1 / 2$

62 CHARGED D*(2010) MASS (MEV)

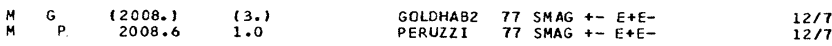

$M$
$M$ GROM SIMUL TANEUS FIT TO D*+, D*O, O+, AND DO, NOT INOEPENOENT OF

M P PEUZZ1 77 MASS NOT NDE PENDEN OF FELDMAN 77 MASS DIFFERENCE
$M$
$M$

62 (D*+1) - (DO) MASS DIFFERENCE (MEV)

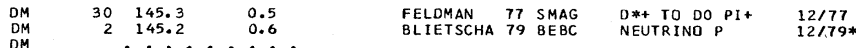

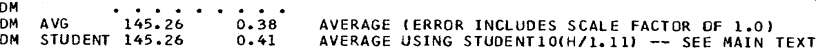

$62\left(D^{*+1}\right)-(0 * 0)$ MASS DIFFERENCE (MEV)

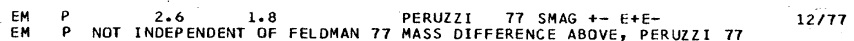

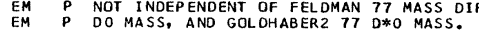

62 CHARGED D*(2010) WIDTH (MEV)

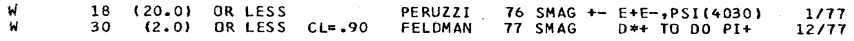

62 CHARGED D*(2010) PARTIAL DECAY MODES

P1 D*+12010) INTO DO PIt DECAY MASSES

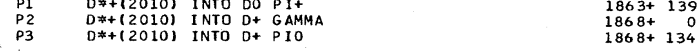

P D*-(2010) modes are charge conjugates of above modes

62 CHARGED D*(2010) BRANCHING RATIOS

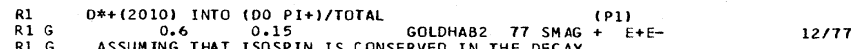

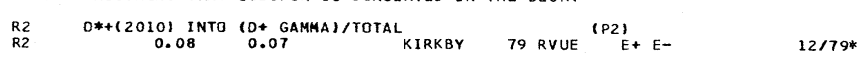

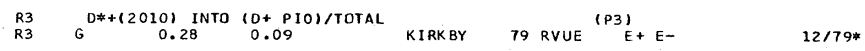

**************************************************************************

76 PRL 37569 +PICCOLO, FELDMAN, NGUYEN, WISS, (SLAC+LBL)

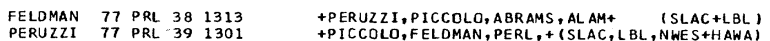

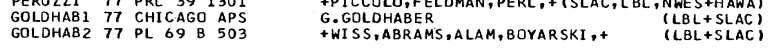

BLIETSCH 79 PL 86 B 108
KIRKBY 79 SLAC-PUB 2419

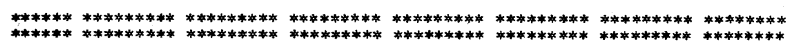

$D^{* 0}(2010)$ o1 neUtral $D *(2010$, JPF $1-1) \quad 1=1 / 2$ $\checkmark$ Cons ISTENT hith 1, VAlUe o RULED OUt ( NGUYEN 77 ).

61 Ne Utral D* (2010) MasS (MEV)

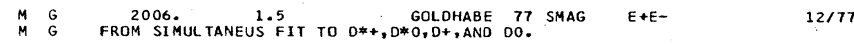

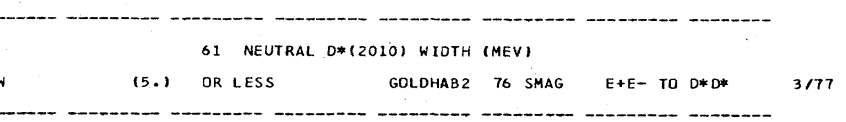




\section{Mesons}

$\mathrm{D}^{* 0}(2010), \mathrm{F}^{ \pm}, \mathrm{F}^{*}(2140)$, EXOTICS

61 NEUTRAL D*(2010) PARTIAL DECAY MODES

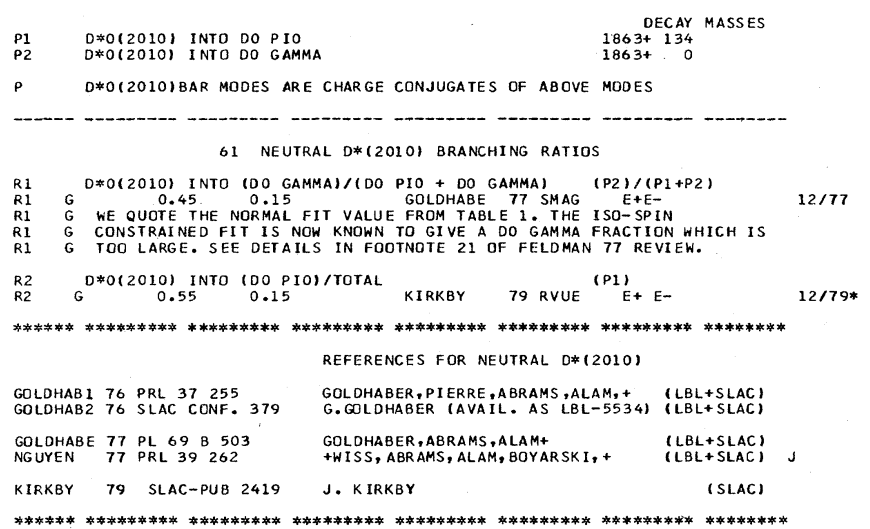

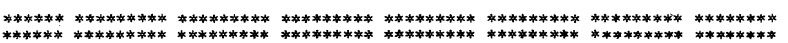

$\mathrm{F}^{ \pm}$
$\rightarrow$

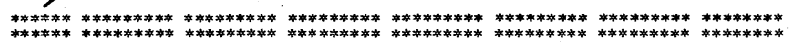

\begin{tabular}{|c|c|c|}
\hline$F^{*}(2140)$ & $\begin{array}{l}74 \text { F*(2140, JPG }=\quad, I= \\
\text { OMITTED FROM TABLE. }\end{array}$ & \\
\hline & 74 F* MASS (MEV) & \\
\hline 2140.0 & BRANOELIK 77 DASP +- E+E-, PI 3 GAMMA & $12 / 77$ \\
\hline
\end{tabular}

DM 110.46 .49 BRANDELIK 79 DASP +- E+E-,F GAMMA $12 / 79 *$

74 F* PARTIAL DECAY MODES

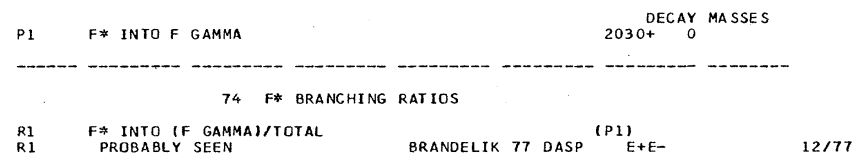

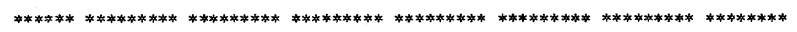

REFERENCES FOR F*(2140)

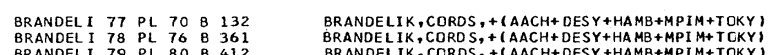

BRANDELIK, CORDS, + ( AACH+ OESY + HAMB +MPIM+ TOKY)

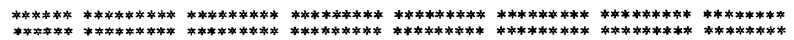

\section{Data Card Listings For notation, see key at front of Listings.}

EXOTIC MESON STATES

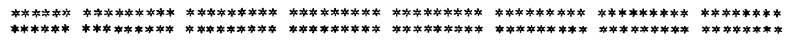

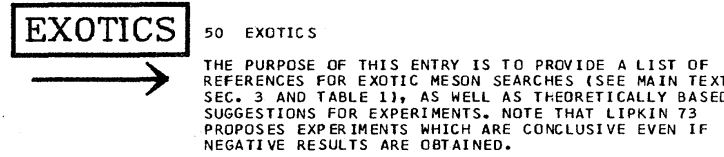

****************************************************************************)

REFERENCES FOR EXOTICS

REPORTS ON SEARCHES

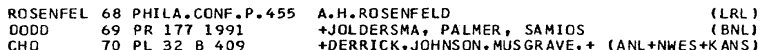

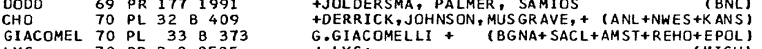

ROSNER $70 \mathrm{EXP}$. MESON SPECTROSCOPY, ED. C.BALTAY AND A.H.ROSENFELD, P.499
(WISCONSINI

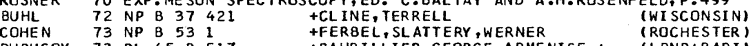

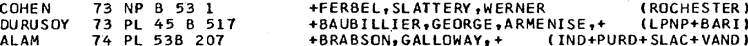

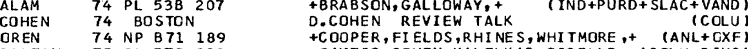

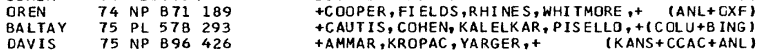

BRUNDIER 76 PL 64 B 107 BRUNDIERS, BRUN, FLURI, + (FREI BURG+SACL+ETH)

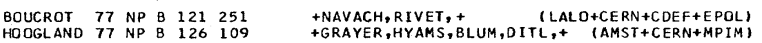

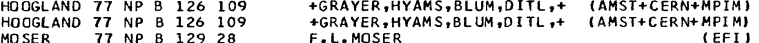

78 PRL 401685
ALAM 78 +BAGGETT, BAGLIN, BONAMY + (IND+PURD+SLAC+VAND)

LEMOIGNE 79 PRE. DPHPE 79-16 +ABOLINS, BARATE+ - (SACL+LOIC+SHMP+IND) SUGGESTIONS FOR SEARCHES

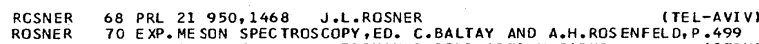

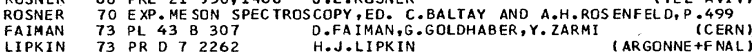

HOLMGREN 78 PL 77 \& 304 TPENNINGTON (STOH+CERN)
(STEN

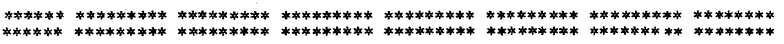




\section{Data Card Listings \\ For notation, see key at front of Listings.}

Note on $N^{\prime} s$ and $\Delta^{\prime} s$

I. Determination of Resonance Parameters

Values of masses, widths, and branching ratios are obtained mainly fxom partial-wave analyses. In addition to a few comprehensive partial-wave analyses, there are numerous others which are based on somewhat incomplete data or cover only a limited energy range. We also include some information from production and total cross-section experiments. This can be valuable in establishing the existence of high mass bumps, but at the lower energies these experiments have limited statistics compared to formation experiments and it is seldom clear which of several states at similar masses is being observed.

There are two main problems in obtaining reliable resonance parameters. First there is often disagreement as to just what the values of the partial-wave amplitudes are. This problem is obviously related to the quality and quantity of the data and to the procedures used to determine the amplitudes. Secondly, even if smooth curves were available for the amplitudes, there would still be some parametrization-dependent ambiguity in deciding what the resonance parameters should be. From a theoretical standpoint the most unambiguously defined resonance parameters are the pole position and residue, and it has been found in practice that, given sufficiently precise partial-wave amplitudes, these quantities can be extracted in a stable and parametrization-independent way, in spite of the fact that they require an extrapolation away from the physical region. This point has been discussed in detail with regard to the $\Delta(1232)$ in previous editions of this review. ${ }^{1,2}$ Although the bestdetermined pole parameters are those of $\Delta(1232)$, there are now a number of determinations for higher lying resonances which are included in the Data Card Listings. In most cases we specify pole parameters by giving the real and imaginary parts of the pole position and residue. It should be kept in mind that these real and imaginary parts tend to be highly correlated. For the residue, in particular, it is often the case that the absolute value is better determined than the phase. For further discussion see the corresponding references, e.g.,
NOGOVA 73, SPEARMAN 74, BALL 75, LICHTENBERG 75, VASAN 76, LONGACRE 77, ZIDELL 78, CUTKOSKY 79, and MIROSHNICHENKO 79 .

The following sections of this mini-review contain discussions of various new developments in experimental non-strange baryon spectroscopy. For a thorough discussion of earlier results see our 1978 edition $^{3}$ and the reviews of $\mathrm{K}$. Lanius ${ }^{4}$ and s. Ozaki. ${ }^{5}$

At the beginning of the Data Card Listings for $N^{\prime} s$ and $\Delta^{\prime} s$, we present a table giving our evaluation of the status of the $N$ and $\Delta$ resonances based on information contained in the Listings. In the Table of Particle properties, we do not quote values and errors for most parameters, but give only ranges for masses and widths in order to emphasize that in some cases these parameters are quite poorly determined. When in doubt about the reliability of a particular parameter, we choose the range quoted in the Tables to be conservatively large.

\section{References for Section I}

1. Particle Data Group, Rev. Mod. Phys. 43 , S114 (1971).

2. Particle Data Group, Phys. Lett. 39B, 103 (1972).

3. Particle Data Group, Phys. Lett. 75B, No. I (1978).

4. K. Lanius, in Proceedings of the 18th International Conference on High Energy Physics, (Tbilisi, 1976), Vol. I, pg.C45.

5. S. Ozaki, in Proceedings of the 19th International Conference on High Energy Physics, (Tokyo, 1978), pg.101.

For other references see the Data Card Listings.

\section{Two-Body Partial-Wave Analyses and New Resonances}

Several new partial-wave analyses have appeared, and older analyses have been published in final form, since our 1978 edition. ${ }^{1}$ In the $\pi N \rightarrow \pi N$ reactions we have the analyses of CUTKOSKY 79, HOEHLER 79, HENDRY 78, ZIDELL 78, and Chew. ${ }^{2}$ CUTKOSKY 79 analyzes $\pi \mathrm{N} \rightarrow \pi \mathrm{N}$ reactions in the mass range 1300$2150 \mathrm{MeV}$, and supersedes the analysis of CUTKOSKY 76 which concentrated on $I=3 / 2$ resonances in a narrower range. HOEHLER 79 is the published version 
Baryons

$N^{\prime} s$ and $\Delta^{\prime} s$
Data Card Listings For notation, see key at front of Listings.
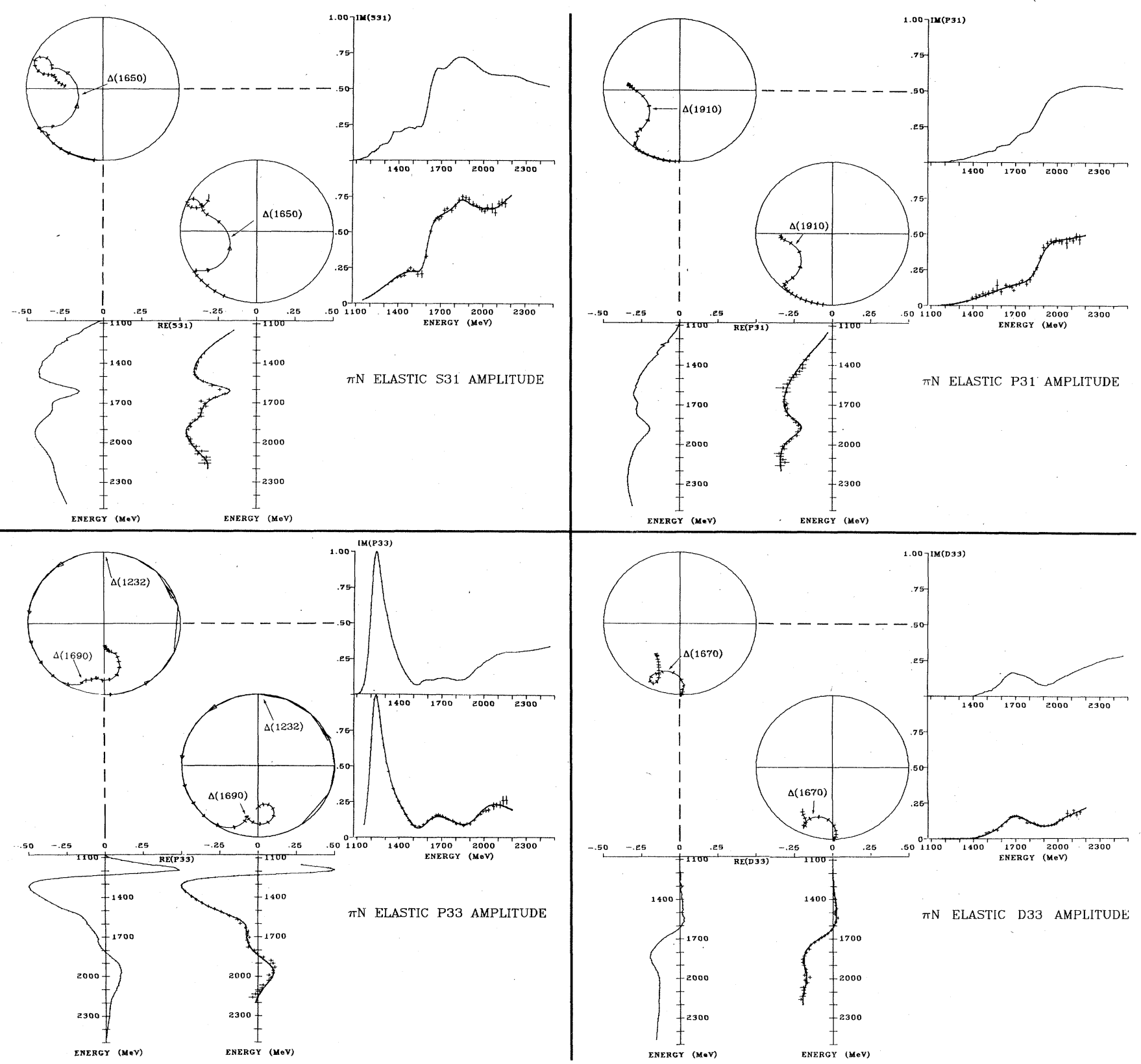

Fig. II.1. Amplitudes for $I=3 / 2 \pi \mathrm{N}$ elastic scattering in the $\mathrm{J}=1 / 2$ and $\mathrm{J}=3 / 2$ waves. The energy dependence of each amplitude is displayed by plotting its real and imaginary parts vs. energy, in alignment with the corresponding Argand plot. In addition, arrows are plotted on the Argand plots with bases positioned at integer multiples of $50 \mathrm{MeV}$ and a base-to-tip length of $5 \mathrm{MeV}$. All the energy axes run from elastic threshold to $2500 \mathrm{MeV}$. The established resonances in these waves are indicated on the Argand plots. The results of two different analyses are shown; the energy axes for the two analyses are aligned for ease of comparison. The lower Argand plot for each wave is from CuTKOSKY 79 (results of energy-independent fitting are shown as data points; the curves show an energy-dependent fit). The upper plot for each wave is from HOEHLER 79. 


\section{Data Card Listings}

Baryons

For notation, see key at front of Listings.

$N^{\prime} s$ and $\Delta^{\prime} s$
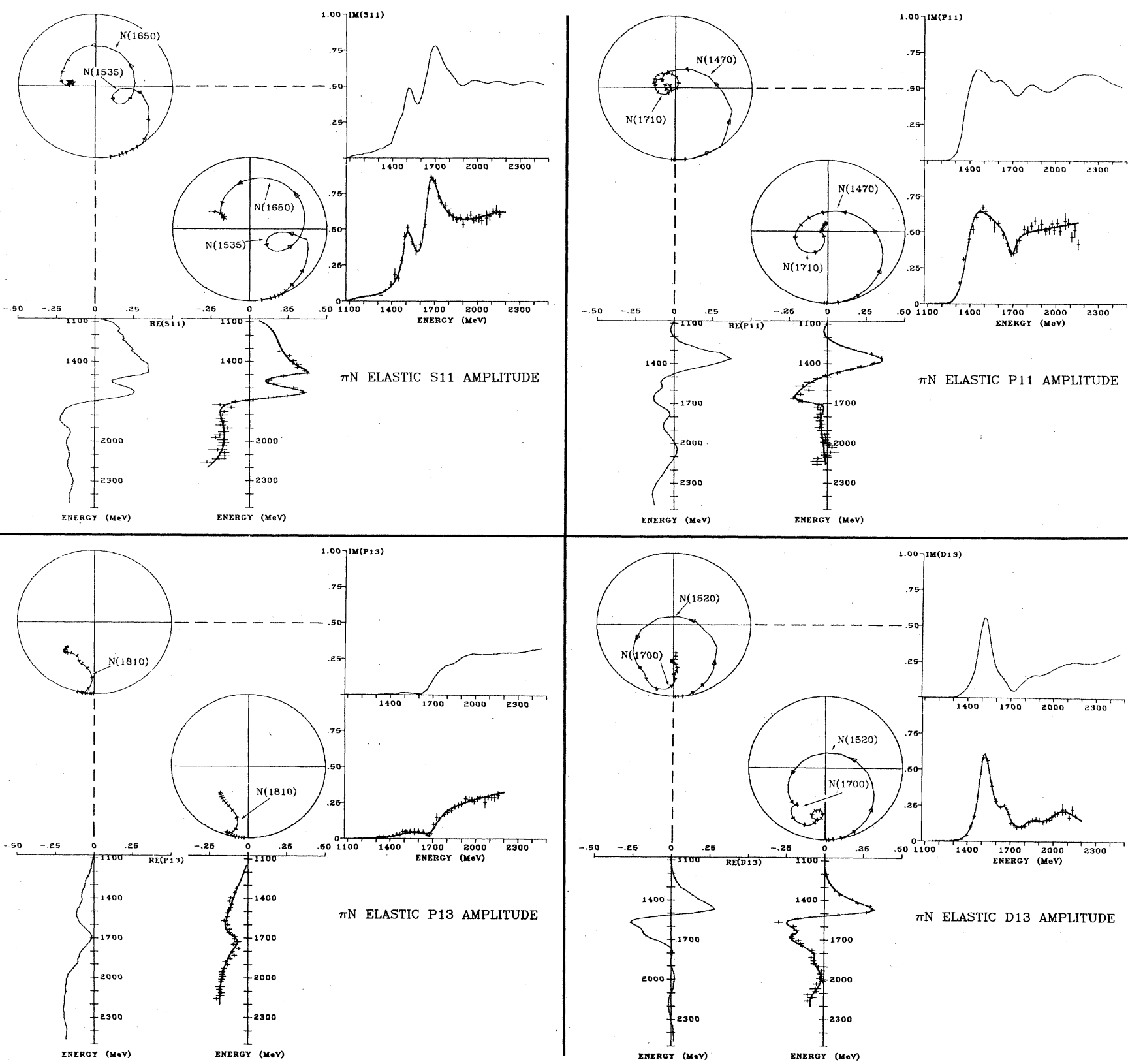

Fig. II.2. Amplitudes for $I=1 / 2 \pi \mathrm{N}$ elastic scattering in the $\mathrm{J}=1 / 2$ and $\mathrm{J}=3 / 2 \mathrm{waves}$. The energy dependence of each amplitude is displayed by plotting its real and imaginary parts vs. energy, in alignment with the corresponding Argand plot. In addition, arrows are plotted on the Argand plots with bases positioned at integer multiples of $50 \mathrm{MeV}$ and a base-to-tip length of $5 \mathrm{MeV}$. All the energy axes run from elastic threshold to $2500 \mathrm{MeV}$. The established resonances in these waves are indicated on the Argand plots. The results of two different analyses are shown; the energy axes for the two analyses are aligned for ease of comparison. The lower Argand plot for each wave is from CuTKOSKY 79 (results of energy-independent fitting are shown as data points; the curves show an energy-dependent fit). The upper plot for each wave is from HOEHLER 79. 


\section{Baryons}

$N^{\prime} s$ and $\Delta^{\prime} s$
Data Card Listings For notation, see key at front of Listings.
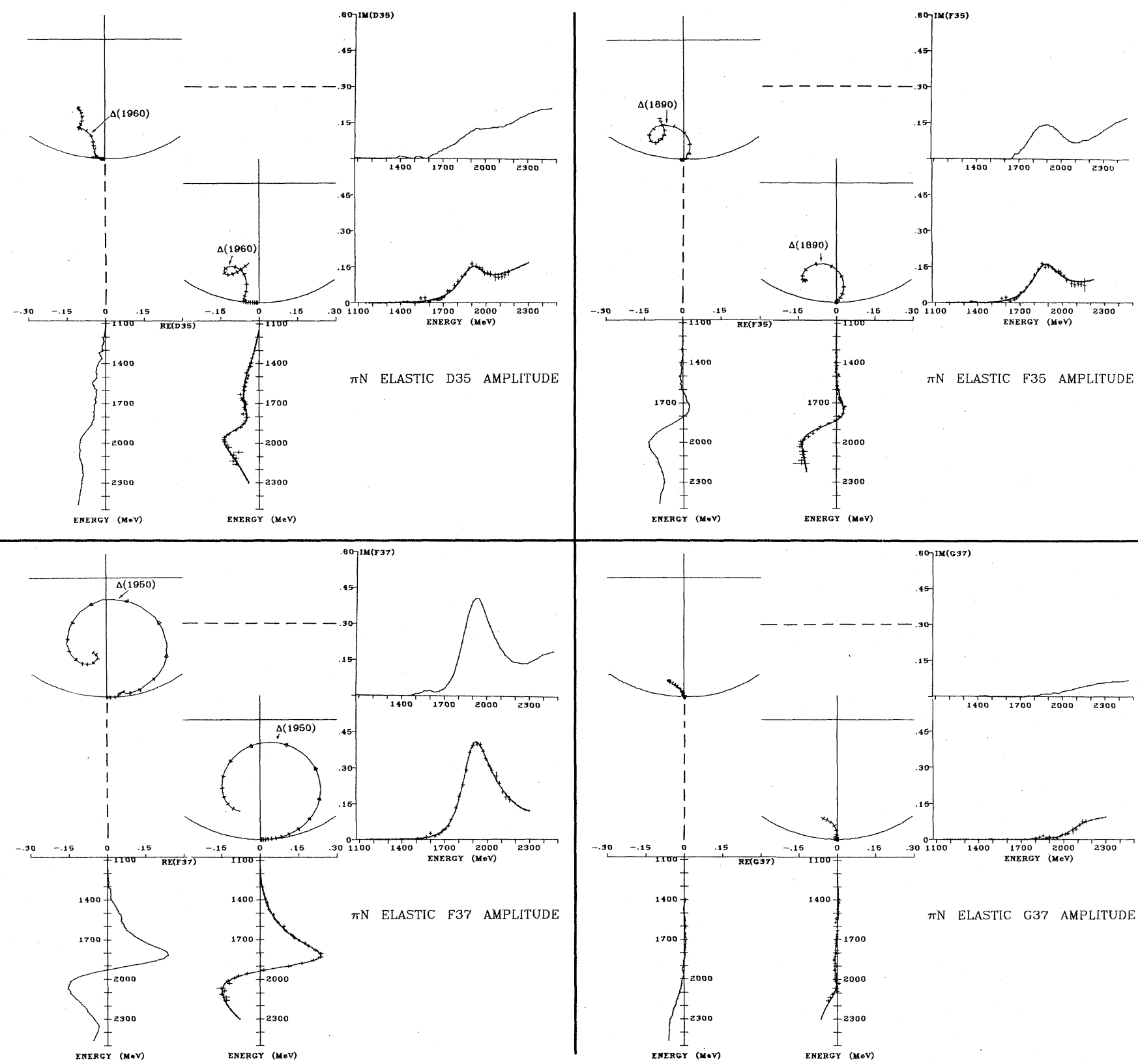

Fig. II.3. Amplitudes for $I=3 / 2 \pi N$ elastic scattering in the $\mathrm{J}=5 / 2$ and $\mathrm{J}=7 / 2$ waves. The energy dependence of each amplitude is displayed by plotting its real and imaginary parts vs. energy, in alignment with the corresponding Argand plot. In addition, arrows are plotted on the Argand plots with bases positioned at integer multiples of $50 \mathrm{MeV}$ and a base-to-tip length of $5 \mathrm{MeV}$. All the energy axes run from elastic threshold to $2500 \mathrm{MeV}$. The established resonances in these waves are indicated on the Argand plots. The results of two different analyses are shown; the energy axes for the two analyses are aligned for ease of comparison. The lower Argand plot for each wave is from CuTKosky 79 (results of energy-independent fitting are shown as data points; the curves show an energy-dependent fit). The upper plot for each wave is from HOEHLER 79. 


\section{Data Card Listings}
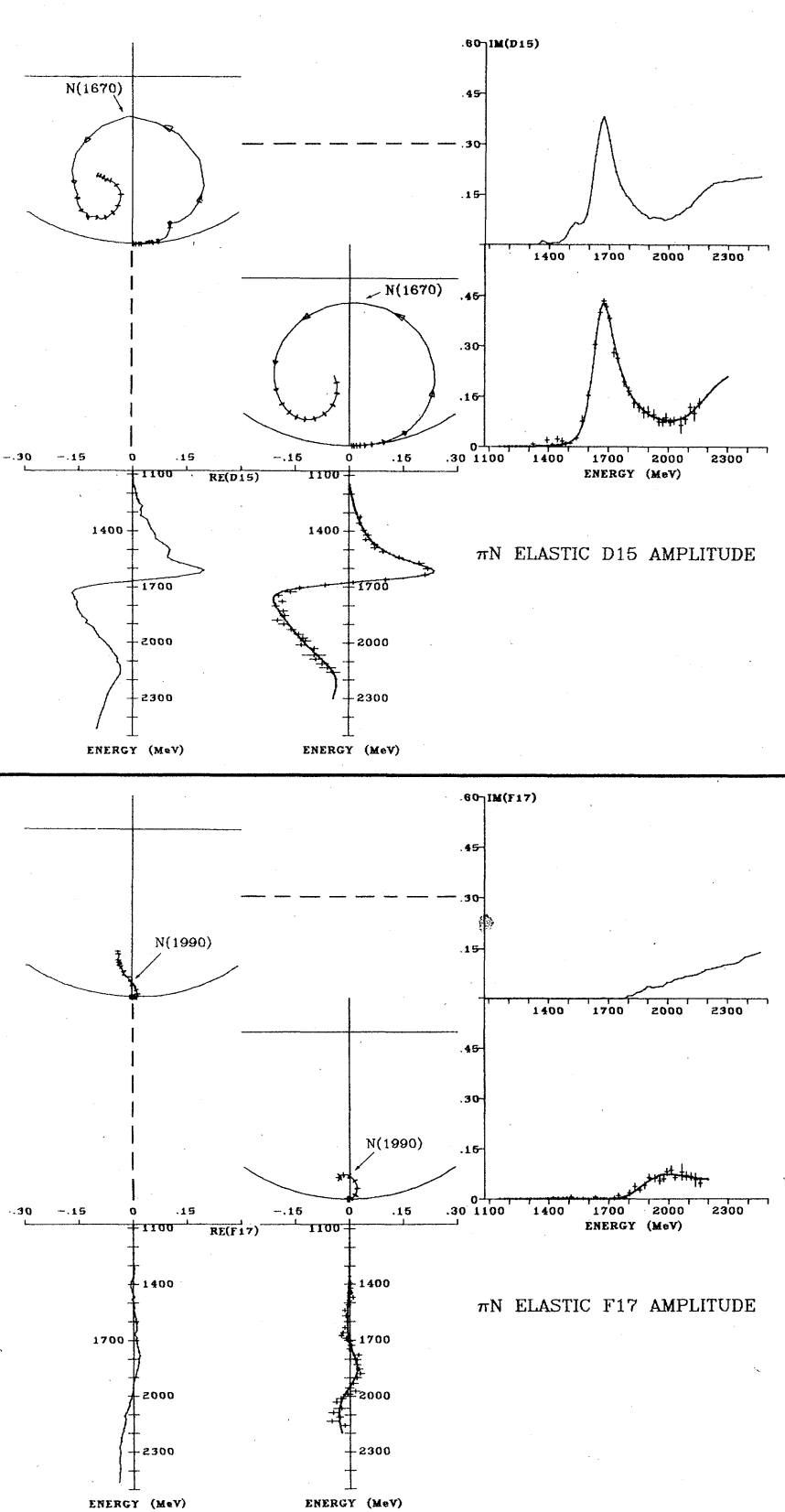
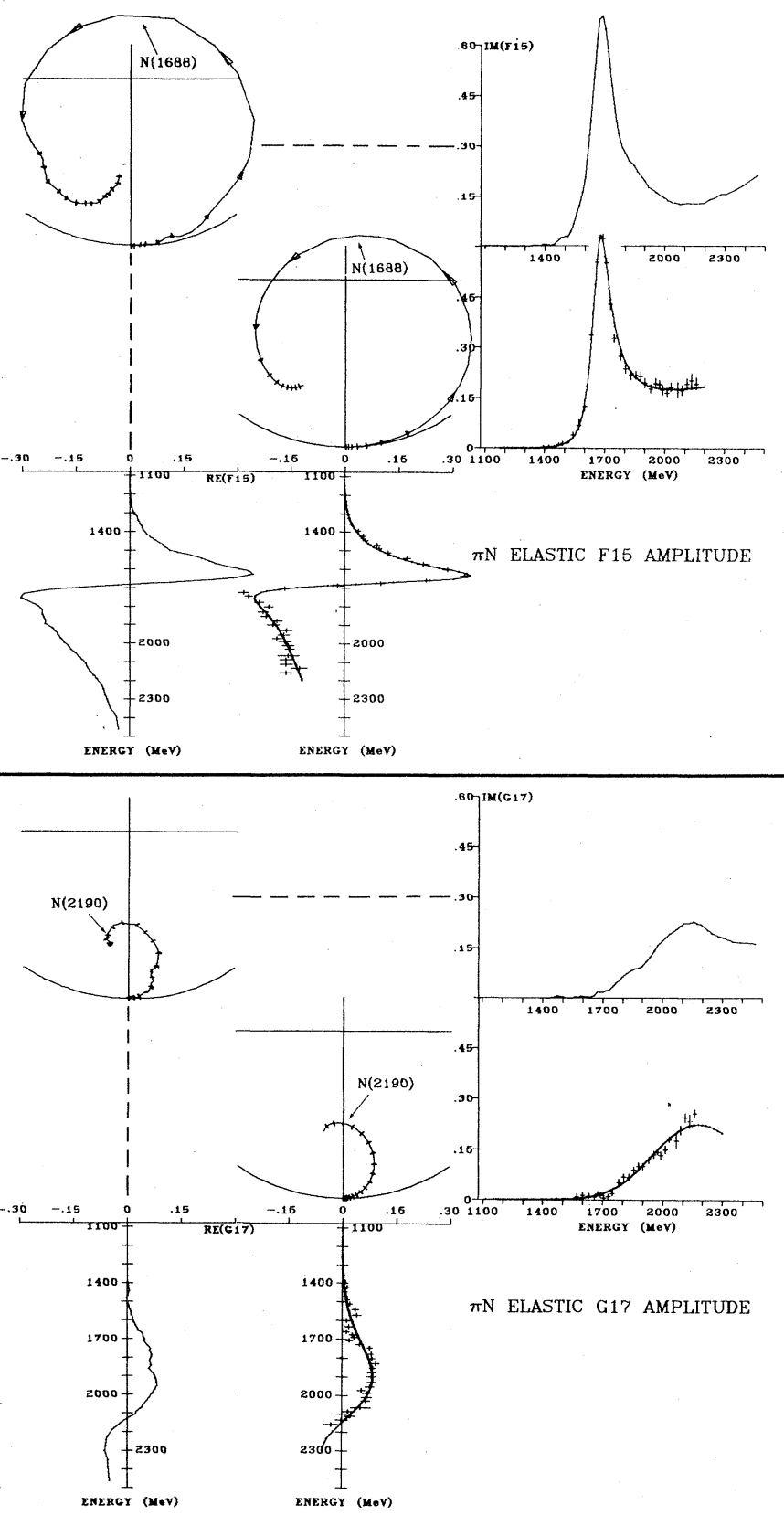

Fig. II.4. Amplitudes for $I=1 / 2 \pi N$ elastic scattering in the $\mathrm{J}=5 / 2$ and $\mathrm{J}=7 / 2$ waves. The energy dependence of each amplitude is displayed by plotting its real and imaginary parts vs. energy, in alignment with the corresponding Argand plot. In addition, arrows are plotted on the Argand plots with bases positioned at integer multiples of $50 \mathrm{MeV}$ and a base-to-tip length of $5 \mathrm{MeV}$. All the energy axes run from elastic threshold to $2500 \mathrm{MeV}$. The established resonances in these waves are indicated on the Argand plots. The results of two different analyses are shown; the energy axes for the two analyses are aligned for ease of comparison. The lower Argand plot for each wave is from CuTKOSKY 79 (results of energy-independent fitting are shown as data points; the curves show an energy-dependent fit). The upper plot for each wave is from HOEHLER 79. 
Baryons

$N^{\prime} s$ and $\Delta^{\prime} s$
Data Card Listings For notation, see key at front of Listings.

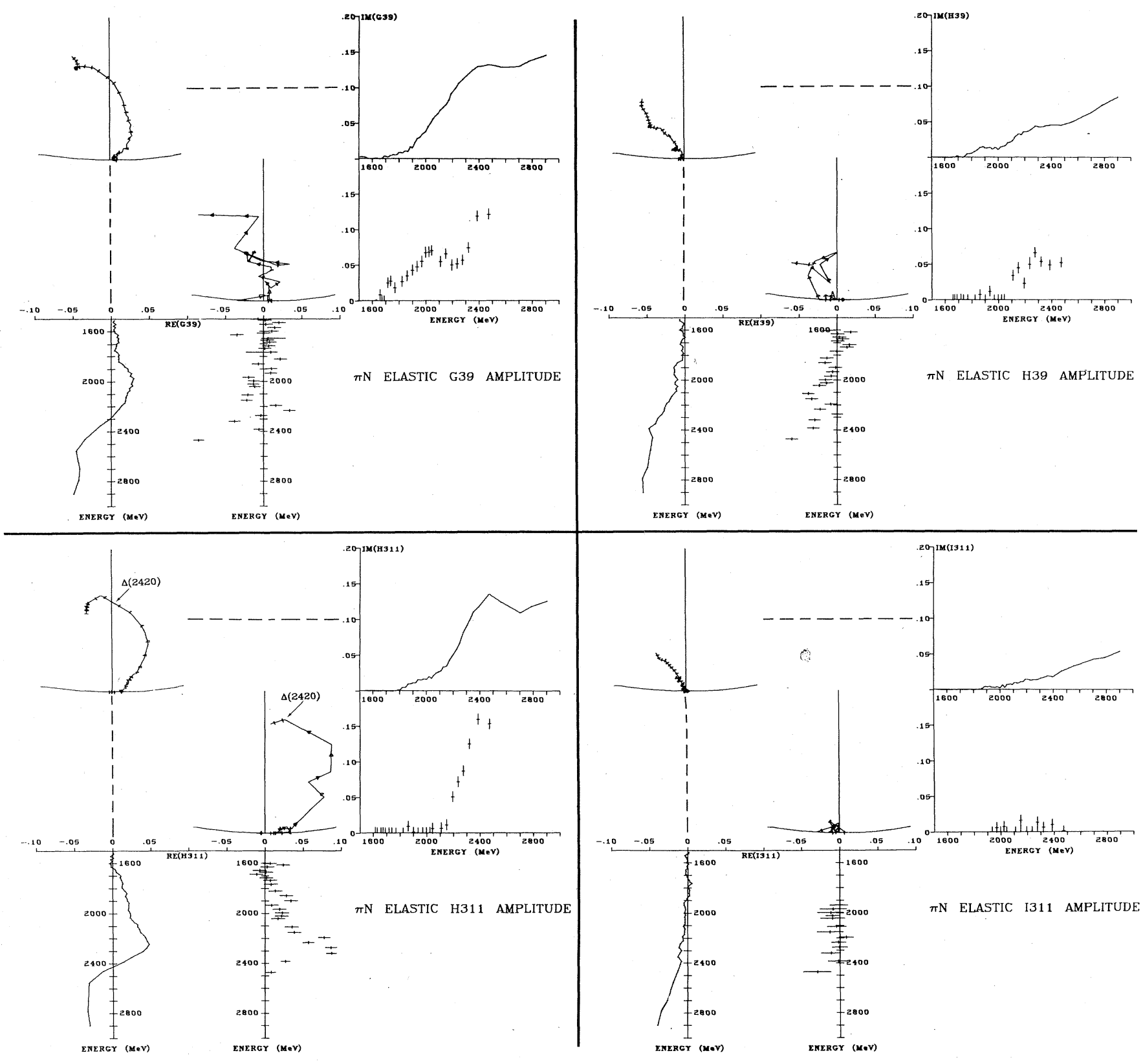

Fig. II.5. Amplitudes for $\mathrm{I}=3 / 2 \pi \mathrm{N}$ elastic scattering in the $\mathrm{J}=9 / 2$ and $\mathrm{J}=11 / 2 \mathrm{waves}$. The energy dependence of each amplitude is displayed by plotting its real and imaginary parts vs. energy, in alignment with the corresponding Argand plot. In addition, arrows are plotted on the Argand plots with bases positioned at integer multiples of $50 \mathrm{MeV}$ and a base-to-tip length of $5 \mathrm{MeV}$. All energy axes run from 1500 to 3000 $\mathrm{MeV}$. The established resonance in the $\mathrm{H}_{3} 11$ wave is indicated on its Argand plots. The results of two different analyses are shown; the energy axes for the two analyses are aligned for ease of comparison. The lower Argand plot for each wave is from AYED 76; the upper plot is from HOEHLER 79. 
Data Card Listings

For notation, see key at front of Listings.

Baryons

$N^{\prime}$ s and $\Delta^{\prime} s$
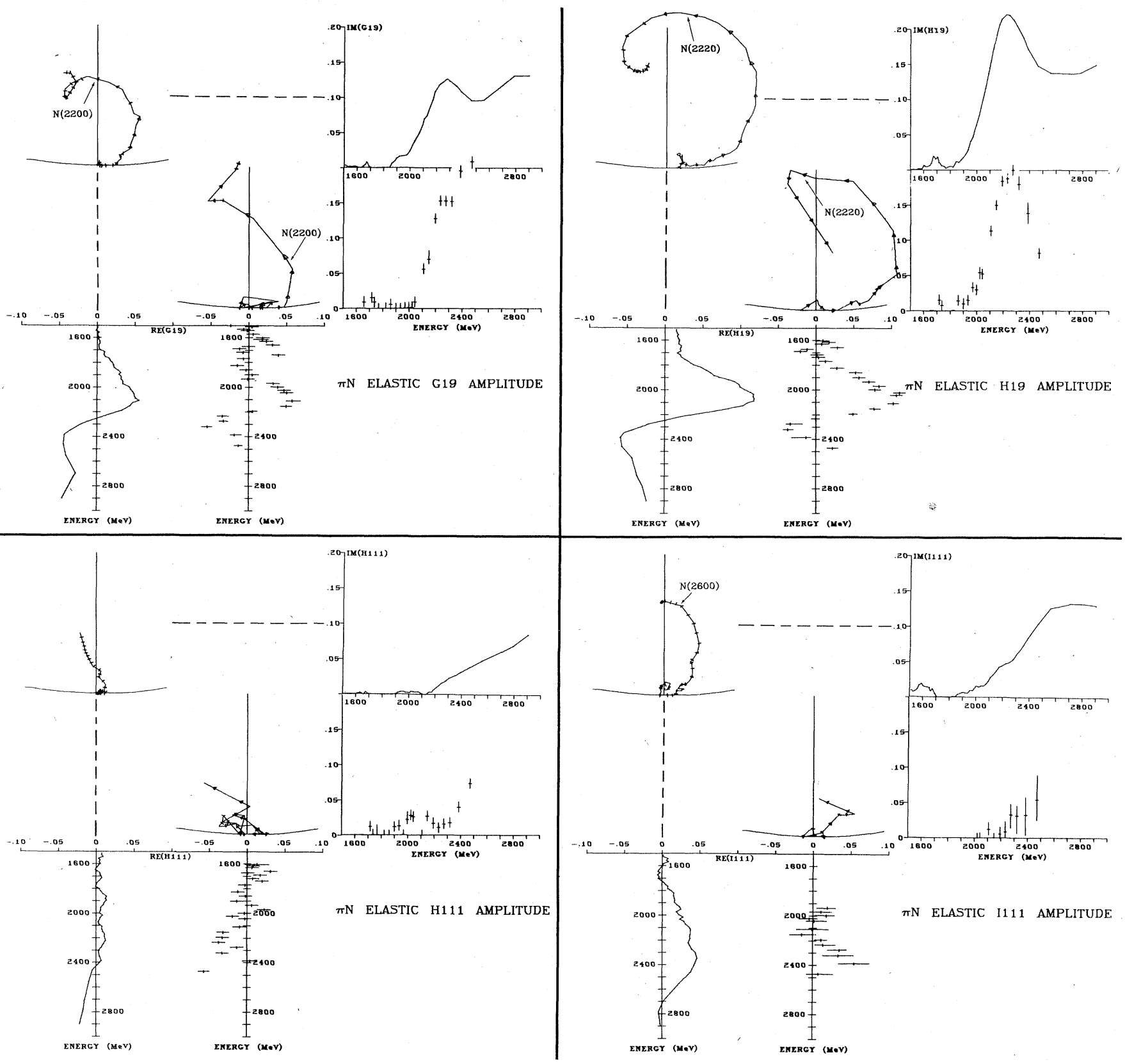

Fig. II.6. Amplitudes for $I=1 / 2 \pi N$ elastic scattering in the $J=9 / 2$ and $\mathrm{J}=11 / 2$ waves. The energy dependence of each amplitude is displayed by plotting its real and imaginary parts vs. energy, in alignment with the corresponding Argand plot. In addition, arrows are plotted on the Argand plots with bases positioned at integer multiples of $50 \mathrm{MeV}$ and a base-to-tip length of $5 \mathrm{MeV}$. All the energy axes run from 1500 to $3000 \mathrm{MeV}$. The established resonances in these waves are indicated on the Argand plots. The results of two different analyses are shown; the energy axes for the two analyses are aligned for ease of comparison. The lower Argand plot for each wave is from AYED 76; the upper plot is from HOEHLER 79. 


\section{Baryons $N^{\prime} s$ and $\Delta^{\prime} s$}

of the analysis made available to us by Pietarinen ${ }^{3}$ for our 1978 edition, and referred to there as PIETARINEN 78. This analysis covers a wide range, with claims for resonance masses as large as 3000 MeV. Both CUTKOSKY 79 and HOEHLER 79 make extensive use of analyticity in their parametrizations, but in quite different ways. The analysis of HENDRY 78 concentrates on the most peripheral high-spin, high-mass resonances, and extracts these using a diffractive + peripheral ansatz for the high energy behavior of the $\pi \mathrm{N} \rightarrow \pi \mathrm{N}$ amplitudes in the impactparameter representation. Resonances with spin as high as $21 / 2$ and mass as high as $4100 \mathrm{MeV}$ are reported. At lower energies ZIDELI 78 report new determinations of the $\Delta^{0}$ and $\Delta^{++}$pole positions, and $\mathrm{Chew}^{2}$ uses a Barrelet-zero technique to analyze $\pi^{+} p \rightarrow \pi^{+} p$ data below $2300 \mathrm{MeV}$.

Two analyses of inelastic reactions have also been reported. BAKER 79 analyzes $\pi^{-} p \rightarrow n n$ between 1500 and $2250 \mathrm{MeV}$, and SAXON 80 analyzes $\pi^{-} \mathrm{p} \rightarrow \mathrm{K}^{0} \Lambda$ below $2300 \mathrm{MeV}$. Both are energy-dependent analyses in which the existence (and sometimes the masses and widths) of $\mathrm{N}$ resonances are taken from $\pi N \rightarrow \pi N$ analyses, and the couplings to the inelastic reaction are determined.

In 1978 we added five new $N$ and $\Delta$ resonances to the Baryon Table (see Ref. 1). These have been strengthened by more recent results, and two have been promoted to 4-star status. This year we are adding one more nucleon resonance, the F17 N(1990). The results of CUTKOSKY 79 and HOEHLER 79 for this resonance are illustrated in Fig. II.4. It was also observed by BAKER 79, but not by SAXON 80 .

An important change within the Listings, which does not yet appear in the Table, is the further clarification of the P33 amplitude in the range 1500-2000 MeV. The $\Delta(1690)$ was added to the Table in 1978, and it seemed likely that there was another P33 resonance near $2000 \mathrm{MeV}$. Most of the evidence for this effect was contained in the rather confused listing for the $\Delta(2160)$. This year we have been able to extract the information relevant to the P33 partial wave from $\Delta(2160)$, combine it with newer information from CUTKOSKY 79 and HOEHLER 79, and list the new 2-star P33 resonance $\Delta(1960)$. Another important change in the Listings this year is the addition of many 1-star high-spin, high-mass

\section{Data Card Listings For notation, see key at front of Listings.}

resonances from the analyses of HOEHLER 79 and HENDRY 78.

\section{References for Section II}

1. Particle Data Group, Phys. Lett. 75B(1) (1978).

2. D. M. Chew, LBL-5306 and LBL-10075 (1979).

3. E. Pietarinen, private communication (1978).

For other references see the Data Card Listings.

\section{The $\pi \mathrm{N} \rightarrow \pi \pi \mathrm{N}$ Channel}

In general, the $2 \rightarrow 3$-body process, $a b \rightarrow c d e$, is described by the center-of-mass energy, $W$, three angles, $\alpha, \beta$, and $\gamma$, and two subenergies, $w_{c d}$ and $w_{\mathrm{de}}$. Thus, unlike $2 \rightarrow 2$-body reactions, fits at single values of $\mathrm{W}$ cannot be parametrized in terms of a set of constants without introducing some assumptions into the analysis. All fits to $\pi \mathrm{N} \rightarrow \pi \pi \mathrm{N}$ use the isobar model, which notes that almost all events for the reaction lie in bands for quasi-twobody processes in the Dalitz plot. It is therefore assumed that there is no pure three-body interaction and that the reaction proceeds by the formation of a two-body state which decays into three bodies. The basic form used is

$$
\begin{aligned}
T(\pi N \rightarrow \pi \pi N)=\sum & \left\{T_{\Delta \pi}^{J I L L^{\prime}}(w) B_{\Delta}\left(w_{\pi N}\right) x_{\Delta \pi}^{J I L L^{\prime}},\right. \\
& +T_{N^{*} \pi}^{J I L L^{\prime}}(W) B_{N^{*}}\left(w_{\pi N}\right) x_{N^{*} \pi}^{J I L L^{\prime}} \\
& +T_{\rho N}^{J I L L^{\prime}}(W) B_{\rho}\left(w_{\pi \pi}\right) x_{\rho N}^{J I L L^{\prime}} \\
& \left.+T_{\varepsilon N}^{J I L L^{\prime}}(W) B_{\varepsilon}\left(w_{\pi \pi}\right) x_{\varepsilon N}^{J I L L^{\prime}}\right\},
\end{aligned}
$$

where in present analyses $\Delta=\Delta(1232), N^{*}=N^{*}(1470)$, $\rho=\rho(770)$, and $\varepsilon$ is the S-wave, $I=0 \pi \pi$ enhancement (although not all the isobars may be included in the different analyses). Here, BW denotes the appropriate Breit-Wigner or corresponding two-body amplitude from $\pi N$ or $\pi \pi$ analyses, and $x$ is a welldefined function containing all the angular information. The decay of the resonances formed in the reaction is given by the partial-wave amplitudes, $\mathrm{T}_{\Delta \pi}^{\mathrm{JILL}}$, etc., with $\mathrm{J}$ giving the total angular momentum and I the total isospin of the state formed. I and L' are respectively the orbital angular momenta of the initial two-body and final quasi-two-body states. $\vec{J}=\vec{L}+\vec{S}=\vec{L} \cdot+\vec{S}^{\prime}$, with $S$ and $S^{\prime}$ as the initial and final total spins. In the 


\section{Data Card Listings For notation, see key at front of Listings.}

\section{Baryons N's and $\Delta^{\prime} s$}

case of the $\mathrm{\rho N}$ amplitude, it is necessary to add the suffix $25^{\prime}$, equal to 1 or 3 , to indicate the total $\rho \mathrm{N}$ spin. The partial-wave amplitudes are frequently denoted by $\Delta L L^{\prime} 2 I 2 J_{,} N^{*} L L^{\prime} 2 I 2 J$, $\rho_{2 S}, L$ L' $2 I 2 J$, and $\varepsilon L L^{\prime} 2 I$ IJ.

The Data Card Listings contain the results of four analyses.

LONGACRE 75 (LBL-SLAC) is an analysis using 200K events for $\pi^{-} p \rightarrow \pi^{-} \pi^{0} p, \pi^{-} \pi^{+} n$ and $\pi^{+} p \rightarrow \pi^{+} \pi^{0} p$ with $1300 \mathrm{MeV} \leqslant \mathrm{w} \leqslant 2000 \mathrm{MeV}$. Approximate unitarity constraints are imposed in the form of a simplified $\mathrm{K}$-matrix formalism that links the $\pi \pi N$ channel to the $\pi N$ channel. This gives smooth solutions and eliminates the overall phase ambiguity that can occur at each energy. The $\Delta \pi, \rho N$, and $\varepsilon N$ isobar states are included. Couplings and T-matrix pole positions are given for 14 resonances. A fuller description is given in the 1974 edition of Review of Particles Properties.

LONGACRE 77 (Saclay) is a coupled-channel analysis similar to LONGACRE 75 that fits $100 \mathrm{~K}$ events for $1380 \mathrm{MeV} \leqslant \mathrm{w} \leqslant 1740 \mathrm{MeV}$. The couplings and pole positions of 16 resonances are measured including those of the $\mathrm{P}_{13}(1540)$ and $\mathrm{P}_{31}$ (1550), which are suggested for the first time in this analysis and have not yet been seen in any other channel.

NOVOSELLER 78 (California Inst. of Technology) is an analysis of $\pi^{-} p \rightarrow \pi^{-} \pi^{0} p, \pi^{-} \pi^{+} n$ and $\pi^{+} p \rightarrow \pi^{+} \pi^{0} p$ for $1630 \mathrm{MeV} \leqslant \mathrm{W} \leqslant 1990 \mathrm{MeV}$, based on the LBL-SLAC energy-independent analysis. ${ }^{1}$ Again the $\Delta \pi, \rho N$, and $\varepsilon N$ isobar states are used, but the resonances are fitted by a simple Breit-Wigner rather than the K-matrix formalism of LONGACRE 75. This analysis considers the criticism made of earlier analyses that they ignore the effects of one-pion-exchange with $\pi \pi$ rescattering. This is used to calculate the higher partial waves, and it is concluded that it improves the fit for $\mathrm{W} \geqslant 1800 \mathrm{MeV}$ and helps eliminate the phase ambiguity. Another study of the importance of one-pion-exchange has been made by Aaron et al., ${ }^{2}$ who also find that it can give important corrections to the angular dependence. NOVOSELIER' 78 quotes two solutions, the second including the effects of $\pi$ exchange. They are given in the Data Card Listings as, respectively, fits to LONGACRE 75 and NOVOSELIER 78.
BARNHAM 79 (see also Ref. 3) is an analysis at Imperial College, London, of $44 \mathrm{~K}$ events for $\pi^{+} \mathrm{p} \rightarrow$ $\pi^{+} \pi^{0} \mathrm{p}, \pi^{+} \pi^{+} \mathrm{n}$ for $1440 \mathrm{MeV} \leqslant \mathrm{w} \leqslant 1700 \mathrm{MeV}$, thus concentrating on the $\Delta$ resonances and using data not available to the other analyses. It considers that the reaction proceeds by the $\pi \Delta, \rho N$, and $\pi N^{*}(1470)$ isobar states, the last one being necessary to account for the difference between the $\pi^{+} \pi^{0} p$ and $\pi^{+} \pi^{+} n$ cross sections. Also included is the effect of one-pion-exchange leading to the $S$-wave $\pi \pi$ state with $I=2$. The phase ambiguity is resolved by requiring the $\pi \Delta$ amplitude for the $\mathrm{D}_{33}(1670)$ to have a Breit-Wigner phase. The parameters of four resonances are evaluated, including the $\mathrm{P}_{31}(1550)$, but since some of the data used are also used by LONGACRE 77 it is not clear that the existence of this resonance is confirmed.

It is difficult to assess the accuracy with which the couplings of the resonances to the final isobar states are known, but those that are indicated in the Data Card Listings as being considered well determined in LONGACRE 77 do, in general, at least agree in sign with the values from other analyses, although some of the $\rho_{3}$ couplings have not been measured elsewhere. The group at Imperial College also claim to get a clear measurement of the signs of $\rho_{1}$ SS31, $\rho_{1}$ DD 33 , and $N^{*}$ PP 33 .

All existing isobar models can be criticized because they neglect possible subenergy dependence of the partial-wave amplitudes and because it can be shown ${ }^{4}$ that this is not consistent with unitarity. This problem has been studied by Aitchison and Brehm, ${ }^{5}$ who derive an isobar expansion that is consistent with Bose symmetry and with subenergy analyticity and unitarity. The resulting coupled integral equations are suitable for both dynamical and phenomenological studies of $\pi \mathrm{N} \rightarrow \pi \pi \mathrm{N}$. They estimate the subenergy corrections to the isobar model and conclude that such corrections may not be significant for existing isobar fits but could become important with improved experimental data. ${ }^{6}$ A rough estimate of these corrections has also been made by the Imperial College group, ${ }^{3}$ who find that they are small.

\section{References for Section III}

1. D.J.Herndon, R.Iongacre, L.R.Miller, A.H.Rosen- 


\section{Baryons}

$N^{\prime} s$ and $\Delta^{\prime} s$

feld, G.Smadja, P.söding, R.J.Cashmore, and D.W.G.S.Leith, Phys. Rev. D11, 3183 (1975).

2. R.Aaron, R.D.Amado, R.A.Arndt, Y.Goradia, D.C.Teplitz, and V.L.Teplitz, Phys. Rev. D16, 50 (1977)

3. K.W.J.Barnham, in Proceedings of the Topical Conference on Baryon Resonances, Oxford, 1976, edited by R.T.Ross and D.H.Saxon, pg.109.

4. R.Aaron and R.D.Amado, Phys. Rev. Lett. 31 , 1157 (1973).

5. I.J.R.Aitchison and J.J.Brehm, Phys. Rev. D20, 1119 (1979).

6. I.J.R.Aitchison and J.J.Brehm, Phys. Rev. D2O, 1131 (1979).

IV. Photon Couplings

IVa. Photoproduction

The couplings of $\gamma_{N}$ to $N^{*}$ and $\Delta$ resonances can be studied in any formation process in which the coupling to the final strong decay channel is well known. In practice, this limits the sources of such couplings to the analysis of single-pion photoproduction, for which the final state has been extensively studied in the phase-shift analysis of elastic $\pi N$ scattering. There are also more experimental data for single-pion photoproduction than for any other photoproduction reactions. All analyses rely heavily on information from $\pi \mathrm{N}$ elastic phaseshift analyses for values of the masses and widths of the resonances. These are fitted in only a few photoproduction analyses, and even in these it is necessary to rely on the $\pi N$ phase-shift analyses for a prior knowledge of the existence of a resonance and for starting values for its mass and width. However, the photoproduction results are of interest since they give information for the resonance states with charge of +1 .

The most important analyses of single-pion photoproduction are reviewed below. The formalism has been previously described ${ }^{1}$ and readers are referred there for additional information. There are three basic methods of analysis. All have had to cope with the difficulty of having four independent complex spin amplitudes at any energy and production angle, and of having only up to four independent experimental measurements. The recent measurements of the $G$ and $H$ observables ${ }^{2}$ have not

\section{Data Card Listings For notation, see key at front of Listings.}

yet been used in any analysis.

(a) Simple Isobar Model

This is the simplest form of energy-dependent analysis. The partial waves are parametrized as a smooth background to which Breit-wigner resonant structure is added. Usually, the electric, but not magnetic, Born terms are included explicitly to reproduce the forward peak in charged-pion production. This method is sufficiently flexible to give excellent fits to the experimental data, but there are, in principle, difficulties concerning the uniqueness of the solution due to the large number of partial waves that are involved. This is overcome by the form of the parametrization, but it is not clear how this may bias the solution. The most extensive analysis of this type is METCALF 74, which is an extension of the earlier Walker analysis. ${ }^{3}$ It fits $\gamma_{p} \rightarrow \pi^{+} \mathrm{n}, \pi^{0} \mathrm{p}$ and $\gamma_{\mathrm{n}} \rightarrow \pi^{-} \mathrm{p}$ from the first to the fourth resonance region. FELLER 76 fits only $\gamma_{p} \rightarrow \pi^{+} \mathrm{n}$ and $\pi^{0} \mathrm{p}$ from the first to the third resonance region but uses data not available to the earlier analysis. Other isobar analyses (ROSSI 73, HEMMI1 73, HEMMI2 73, BENEVENTANO 74, and KRIVETS $74^{4}$ ) have been made on a significantly smaller scale using small and sometimes restricted data sets.

(b) Fixed-t Dispersion Relations (FTDR)

This technique uses the apparent resonant dominance of the photoproduction amplitudes to get a relatively simple parametrization of their imaginary parts. Fixed-t dispersion relations are used to calculate the real parts without the introduction of other free parameters, or, in some cases, with only a relatively small number of additional parameters. This significantly reduces the possibility of multiple solutions and automatically satisfies the requirements of analyticity. However, the method is relatively inflexible compared to the isobar model, giving poorer fits. Also, as has been described in NOELLE 78 and elsewhere, ${ }^{5}$ the divergence of the partial-wave expansions for the dispersion integrals does not allow the use of experimental data at all angles above about the third resonance region. Not all analyses include the constraints of unitarity and time-reversal invariance as given 


\section{Data Card Listings \\ For notation, see key at front of Listings.}

Baryons

$N^{\prime} s$ and $\Delta^{\prime} s$

by Watson's theorem. ${ }^{6}$

FTDR analyses have been made by groups at Berkeley (MOORHOUSE 73, KNIES 74, and MOORHOUSE 74), at Lancaster (DEVENISH 73, DEVENISH2 74), at Glasgow (CRAWFORD 75, BARBOUR 76, and BARBOUR 78) and at Yerevan (AZNAURYAN 77). NOELLE 78 is a hybrid analysis incorporating FTDR in a coupled-channel isobar model.

(c) Energy-Independent Analysis

These evaluate the partial waves by making independent fits over a range of essentially single energies, and are thus the least biased of all the techniques employed. It is necessary to use Watson's theorem to fix the complex phases of the partial waves in order to get a unique solution. Due to inelasticity, this becomes difficult above the first resonance region, and only BERENDS 77 extends into the second resonance region. This analysis suggests in particular that the $A_{3 / 2}^{\mathrm{P}}$ coupling for the $D_{13}^{\prime}(1520)$ from the energy-dependent analyses is too large by a factor of almost two due to the omission of nonresonant background.

\section{New Analyses in the Data Card Listings}

AZNAURYAN 77 is an FTDR analysis of $\gamma_{p} \rightarrow \pi^{0} p$ from threshold to a laboratory photon energy of 1.2 GeV. NOELLE 78, as described, is a hybrid isobar and FTDR analysis of the first and second resonance regions. BARBOUR 78 is an FTDR analysis that combines a partial-wave analysis for center-of-mass energies, W, up to $2.5 \mathrm{GeV}$ with an amplitude analysis at higher energies to reduce the uncertainty in the FTDR from the high energy parts of the dispersion integrals. Data at all accelerator energies are fitted, and, as in the other Glasgow analyses, the resonance masses and widths are evaluated with the couplings. MIROSHNICHENKO 79 is based on an earlier energy-independent analysis ${ }^{7}$ and measures the pole position of the $\Delta^{+}, \mathrm{P}_{33}(1232)$, resonance.

Resonance Couplings and Exrors

in the Data Card Listings

The Data Card Listings give the results of all recent and extensive analyses. If no error is given, only a unique result has been quoted. The Berkeley analyses and CRAWFORD 75 give for the errors the spread of solutions around a central value. The

TABLE IV.1. The average of the couplings from MOORHOUSE 74, KNIES 74, METCALF 74, DEVENISH2 74, FELLER 76, BERENDS 77, and BARBOUR 78. The errors take into account both statistical errors and the variation of values over the analyses. Where no error is shown, it is considered that there are too few analyses to make a reliable estimate.

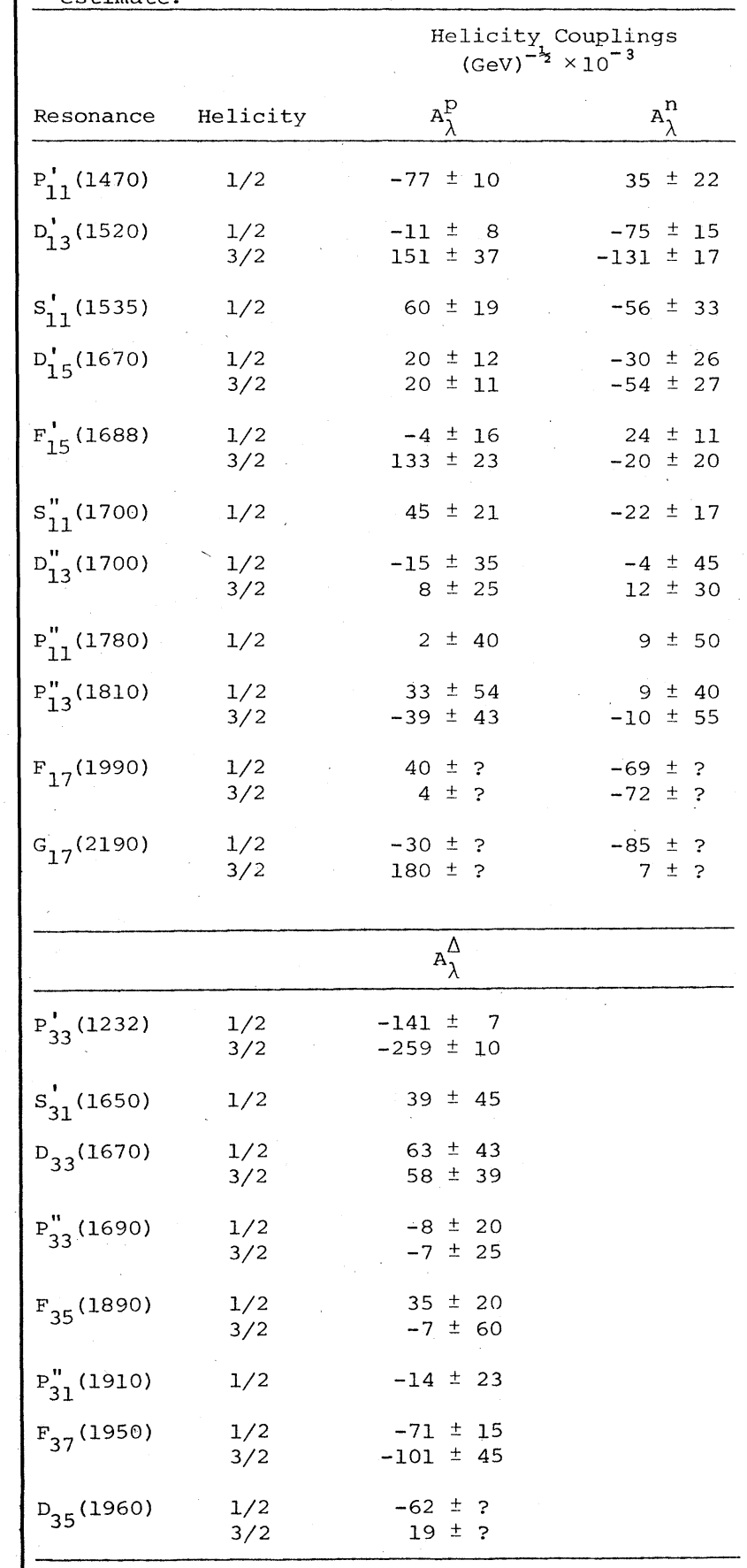




\section{Baryons $N^{\prime} s$ and $\Delta^{\prime} s$}

Lancaster group give as an error the change of value for each coupling that is required to increase the "best possible" $\chi^{2}$ by 1\%. METCALF 74, FELLER 76, and AZNAURYAN 77 quote similar errors. In BARBOUR 78, the point of view is taken that the systematic variations due to the different methods of analyses are at least as significant as the purely statistical errors that are usually given. Thus, the errors quoted are obtained by comparison with other analyses as well as from the random variation of the parameters over a number of fits.

In the compilation of couplings given in Table IV.1, the errors given are calculated in a similar manner from both the statistical errors quoted in the analyses used and from the spread of results over the analyses.

\section{References for Section IVa}

1. Particle Data Group, Rev. Mod. Phys. 48, S157 (1976).

2. P.J.Bussey et al., Daresbury preprint DL/P290E (1979).

3. R.L.Walker, Phys. Rev. 182, 1729 (1969).

4. A.G.Krivets et al., Sov. J. Nucl. Phys. 20 430 (1975).

5. R.C.E.Devenish, D.H.Lyth, and W.A.Rankin, Daresbury report DNPL/PI09 (1972).

6. K.M.Watson, Phys. Rev. 95, 228 (1954).

7. I.I.Miroshnichenko et al., Sov. J. Nucl. Phys. 26,52 (1977).

IVb. Electroproduction in the Resonance Region (by F. Foster, Lancaster University, April 1978) Both the quantity and quality of the data continue to improve in this interesting but somewhat unfashionable corner of the $\nu, Q^{2}$ plane. Most experiments now use the coincidence technique: detecting the scattered electron to fix the energy and momentum transfer, together with one of the final state hadrons $\left(p, \pi^{ \pm}\right)$. By this method the virtual photoproduction differential cross sections for particular exclusive channels can be determined.

At the date of the 1976 Review many excellent data sets were already available. These included $\pi^{0}$ production in the first resonance region from DESY, ${ }^{1,2}$ the Lancaster-Manchester group at Daresbury, ${ }^{3}$ and Bonn University. ${ }^{4}$ There was

\section{Data Card Listings For notation, see key at front of Listings.}

also good $\pi^{0}$ data in the second resonance region ${ }^{5}$ and forward $\pi^{+}$data in the second and third resonance regions from Lancaster-Manchester. ${ }^{6}$ Much interest was generated by the $n$ data from Daresbury, DESY, and Bonn, which is a unique indicator for the $S_{11}^{\prime}$ (1535) resonance. ${ }^{7-9}$ The total cross-section measurements from DESY, ${ }^{10}$ Bonn, ${ }^{11}$ and Stanford ${ }^{12}$ gave essential information on the $Q^{2}$ variation of the resonance "peak" heights in relation to the background, and demonstrated that the longitudinal-to-transverse ratio $\sigma_{L} / \sigma_{T}$ was everywhere very close to zero.

During the past two years much new information has become available. In particular we have the detailed data sets from DESY on single $\pi^{+}$and $\pi^{0}$ production in the second and third resonance regions ${ }^{13,14}$ at $Q^{2}$ values of 0.6 and $1.0 \mathrm{GeV}^{2}$, and new $n$ data near the $S_{i 1}$ (1535) from DESY ${ }^{15,16}$ at 0.6 , $1.0,2.0$, and $3.0 \mathrm{GeV}^{\frac{1}{2}}$ and Bonn ${ }^{17}$ at $Q^{2}$ of $0.4 \mathrm{GeV}^{2}$. By changing the virtual photon polarization, both groups have succeeded in measuring the longitudinal excitation of the $S_{11}$ resonance with the results: $\sigma_{\mathrm{I}} / \sigma_{\mathrm{T}}=0.15 \pm 0.18\left(Q^{2}=0.6\right),{ }^{15}-0.06 \pm 0.16\left(Q^{2}=\right.$ $1.10),{ }^{16} 0.16 \pm 0.10\left(Q^{2}=0.4\right) .{ }^{17}$ The LancasterManchester group has taken a large amount of data on $\pi^{-}$and $\pi^{+}$production from a deuterium target over the second and third resonance regions at $Q^{2}$ values 0.5 and $1.0 \mathrm{GeV}^{2}$. From this data it will be possible to extract the differential cross sections $\gamma_{\mathrm{v}} \mathrm{n} \rightarrow \mathrm{p} \pi^{-}$, which are essential to the understanding of the multipole couplings to the isospin-1/2 resonances. Preliminary data were available at the Hamburg conference ${ }^{18}$ for the $\pi^{-} / \pi^{+}$ratio at $Q^{2}=0.5$ $\mathrm{GeV}^{2}$ in the forward direction, and estimates were presented of the neutral $D_{13}^{\prime}(1520)$ multipole couplings. ${ }^{19}$

Theoretical analyses of the data sets to extract resonance multipole couplings necessarily rely on fixed-t dispersion-relation calculations which relate the real (background) and imaginary (resonant) parts of these matrix elements. R. C. E. Devenish and D. H. Lyth ${ }^{20}$ incorporated the constraints into an energy-dependent fitting procedure and produced the first estimates of second and third resonance multipoles up to $Q^{2}=1.5 \mathrm{GeV}^{2}$ using the Lancaster-Manchester ${ }^{5,6}$ and preliminary DESY $\pi^{0}, \pi^{+}$, and $n$ data together with 


\section{Data Card Listings}

For notation, see key at front of Listings.

total cross-section measurements. Since then, Gayler 21 has used the final DESY data in the same fitting routines and has produced improved results on the couplings to the $S_{11}^{\prime}(1535), D_{13}^{\prime}$ (1520), $\mathrm{F}_{15}^{\prime}$ (1688), and $\mathrm{P}_{11}^{\prime}(1470)$ resonances.

The present status of the couplings to the more prominent resonant states may be summarized as follows :

$\underline{\mathrm{P}}_{33}^{\prime}$ (1232): There is no change from previous reviews. However, Gayler remarks ${ }^{21}$ that while the resonance appears to be a dominantly magnetic (quark spin flip) excitation, the background, which forms an increasing fraction of the single-pion cross section as $Q^{2}$ increases, is dominantly helicity-1/2 in the $\gamma_{\mathrm{v}} \mathrm{p}$ system. This is to be expected on the basis of the quark parton model and duality.

$D_{13}^{\prime}(1520):$ It is firmly established $6,13,14,21$ that the transverse helicity-l/2 excitation increases rapidly as $\mathrm{O}^{2}$ increases from 0 to 1.0 $\mathrm{GeV}^{2}$. The rate of increase observed depends on the details of the fitting procedures used, but is consistent with the magnetic coupling falling slowly and the electric coupling falling like a "dipole," almost as one would expect from a naive harmonic oscillator quark model. 22

$S_{11}^{\prime}(1535):$ The new data ${ }^{14-17}$ establish beyond doubt that the excitation of this resonance falls less rapidly than its $\mathrm{SU}(6)$ partner $\mathrm{D}_{13}^{\prime}(1520)$ and the excitation is dominantly transverse. At $Q^{2}=0$, contributions of the $D_{13}$ and $S_{11}$ are in the ratio $4: 1$, while at $Q^{2}=3.0 \mathrm{GeV}^{2}$ the ratio becomes an amazing $1: 2$. Thus the second resonant peak in total cross-section measurements at high $Q^{2}$ is dominated by the $S_{11}^{\prime}(1535)$ - this may account for possible small changes in the observed shape as $\mathrm{Q}^{2}$ increases.

$P_{11}^{\prime}(1470):$ The situation here is fluid; although there are no clear signals for this resonance, some analyses ${ }^{21}$ do require a significant contribution at $\mathrm{Q}^{2}=1.0 \mathrm{GeV}^{2}$. New $\pi^{0}$ and $\pi^{+}$data from Lancaster-Manchester below the second resonance and from DESY at high $Q^{2}$ may clarify the position.

$F_{15}^{\prime}$ (1688): Here again the helicity-1/2 to helicity $-3 / 2$ ratio is observed to increase with $Q^{2}$ as we expect from the quark model.
Some progress has been made in understanding the phenomenology of resonance electroproduction within the framework of $\mathrm{SU}(6)_{\mathrm{W}}$ symmetry. ${ }^{23}$ Cashmore et al. ${ }^{24}$ for example, have shown that radiative transitions between the nucleon and members of the $\left\{70,1^{-}\right\}$multiplet are consistent with only three independent amplitudes, corresponding to quark orbit flip, spin flip, and simultaneous spin-orbit excitation. (Note that the spin-orbit term is normally neglected in "naive" quark model calculations. ${ }^{22}$ ) It is necessary to find out if this relatively simple structure persists as $Q^{2}$ increases and, if it does, to determine the $Q^{2}$ variation of the three amplitudes. Using mainly the amplitudes connecting the proton and the charged $\mathrm{D}_{13}$ and $\mathrm{s}_{11}$ resonances, Foster ${ }^{25}$ and Alcock et al. ${ }^{26}$ have shown that all three excitation terms are necessary to describe electroproduction at $Q^{2}$ values up to 1 $\mathrm{GeV}^{2}$. The orbit flip term falls rapidly, while the spin flip term remains almost constant as $\Omega^{2}$ increases (just like the simple quark model predictions), causing the observed helicity changeover. The spin-orbit term has an intermediate variation with $Q^{2}$ and appears to be influential in causing the $\mathrm{S}_{11}$ to dominate the $\mathrm{D}_{13}$ at $\mathrm{Q}^{2}$ values above $0.5 \mathrm{GeV}^{2}$. To be sure that the three terms are sufficient to describe electroproduction of the $\left\{70,1^{-}\right\}$multiplet we will have to await accurate determination of more charged resonance multipoles or some measurements of the neutral isospin-1/2 resonance multipoles. The preliminary data from Lancaster-Manchester ${ }^{18,19}$ on the process $\gamma_{\mathrm{V}} \mathrm{n} \rightarrow \pi^{-} \mathrm{p}$ near the second resonance give results for $\mathrm{M}_{2-}^{\mathrm{n}}$ and $\mathrm{E}_{2-}^{\mathrm{n}}$ which are in fair agreement with the $\mathrm{SU}(6) \mathrm{W}$ scheme. 25

\section{References for section IVb}

1. W. Albrecht et al., Nuc1. Phys. B25, 1 (1970).

2. J.C. Adlex et al., Nucl. Phys. B46, 573 (1972).

3. R. Siddle et al., Nucl. Phys. B35, 93 (1971).

4. K. Bätzner et al., Nucl. Phys. B76, 1 (1974).

5. W. J. Shuttleworth et al., Nucl. Phys. B45, 428 (1972).

6. E. Evangelides et al., Nucl. Phys. B71, 381 (1974).

7. P. S. Kummer et al., Phys. Rev. Lett. 30, 873 (1973). 


\section{Baryons} $N^{\prime} s$ and $\Delta^{\prime} s$

8. J. C. Adler et al., Nucl. Phys. B91, 386 (1975).

9. U. Beck et al., Phys. Lett. 51B, 103 (1974).

10. F. W. Brasse et al., Nucl. Phys. B110, 413 (1976).

11. M. Kobberling et al., Nucl. Phys. B82, 201 (1974).

12. S. Stein et al., Phys. Rev. D12, 1884 (1975).

13. J. C. Adler et al., Nucl. Phys. B99, 1 (1975).

14. J. C. Adler et al., Nuc1. Phys. B105, 253 (1976).

15. F. W. Brasse et al., DESY preprint $77 / 73$.

16. F.W. Brasse et al., DESY preprint 77/?.

17. H. Breuker et al., Bonn preprint BONN-1R-77-11.

18. J. Wright et al., Contribution to 1977 Hamburg Symposium, DL/P276.

19. R. Marshall, Invited talk at 1977 Hamburg Symposium.

20. R. C. E. Devenish and D. H. Lyth, Nucl. Phys. B93, 109 (1975).

21. J. Gayler, Proc. Oxford Conference on Baryon Spectroscopy, edited by R. Ross, D. Saxon, 1976; also DESY $76 / 42$.

22. E.g., F. Ravndal, Phys. Rev. D4, 1466 (1971); also F. Foster, Proc. XVII Internat'1. Conf. London (1974), edited by J.R. Smith II, p.163.

23. A. J.G. Hey, Proc. Oxford Conf. on Baryon Spectroscopy, edited by R. Ross, D. Saxon, 1976.

24. R. J. Cashmore et al., Nucl. Phys. B98, 337 (1975).

25. F. Foster, Contribution to 1977 Hamburg Symposium; also Ref. 19.

26. J. W. Alcock, W. N. Cottingham, and A. C. Davis, Phys. Lett. 69B, 457 (1977).

\section{Production Experiments}

It is difficult to draw firm conclusions about $\mathrm{N}$ and $\Delta$ resonance properties from production experiments because each prominent bump seen in production is generally a coherent superposition of several resonances plus non-resonant background. However, production and formation experiments are clearly closely related, and we give parameters obtained from production experiments in the Listings, although they are not used in the Tables. This section contains a brief review of the main results of recent $\mathrm{N}$ and $\Delta$ production experiments. We concentrate on diffractive production of $\pi \mathrm{N}$ and $\pi \pi \mathrm{N}$ systems as this is where most of the recent experimental activity has been.

Data on the exclusive channels NN $\rightarrow$ NNT and
NNTT at high energy are now available, both from FNAL (BIEL 78) and the CERN ISR (WEBB 75, DEKERRET 76, and GOGGI 79). Double diffraction dissociation reactions have also been studied at the ISR (GOGGIl 78). The diffractively produced, $\pi N$ and $\pi \pi N$ systems have mass shapes which are remarkably similar to those observed at lower momenta.

The low mass $\pi N$ system is dominated by a broad bump peaking around $1.35 \mathrm{GeV}$, on which may be superimposed other structures at 1.5 and $1.7 \mathrm{GeV}$. The $1.35 \mathrm{GeV}$ bump is produced more peripherally than those at 1.5 and $1.7 \mathrm{GeV}$ (DEKERRET 76, HARRIS 77, BIEL 78, and CHADWICK 78). The two higher mass peaks are probably associated with the $D_{13}^{\prime}(1520)$ and $F_{15}^{\prime}$ (1688), respectívely.

There is mounting evidence for an appreciable $\mathrm{J}^{\mathrm{P}}=1 / 2^{-}$component in low mass $\mathrm{N} \rightarrow \mathrm{N} \pi$ diffractive dissociation. This is in violation of the GribovMorrison rule which would allow only $1 / 2^{+}, 3 / 2^{-}$, $5 / 2^{+}$, etc. The reactions $\pi^{ \pm} \mathrm{p} \rightarrow \pi^{ \pm}\left(\mathrm{p} \pi^{0}\right)$ and $\pi^{ \pm}\left(\mathrm{n} \pi^{+}\right)$ have been analyzed by OCHS 75 at $14 \mathrm{GeV} / \mathrm{c}$ and OTTER 77 at $16 \mathrm{GeV} / \mathrm{c}$ [who included data on $\pi^{ \pm} \mathrm{p} \rightarrow$ $\left.\pi^{0}\left(\mathrm{p}^{ \pm}\right)\right]$. In each case the data were fitted with coherent sums of diffractive and $\Delta$-production amplitudes. Both analyses concluded that there is significant diffractive production of $1 / 2^{-}$ background for $\pi \mathrm{N}$ masses below $1.3-1.4 \mathrm{GeV}$. Moreover, the diffractively produced $\mathrm{mN}$ system could be described completely by $1 / 2^{-}$and $3 / 2^{+}$ waves only (i.e., both Gribov-Morrison violating), although a sizeable $3 / 2^{-}$contribution could not be excluded. SOTIRIOU 75 also analyzed $\pi^{+} p \rightarrow \pi^{+}\left(n \pi^{+}\right)$at $16 \mathrm{GeV} / \mathrm{c}$ for $\mathrm{n}^{+}$masses below $2 \mathrm{GeV}$. In addition to enhancements associated with known resonances, a $200-\mathrm{MeV}$ broad bump at $1.35 \mathrm{GeV}$ was found. The $\mathrm{J}^{\mathrm{P}}$ determination at low mass was not completely unambiguous, but the bump appears to be predominantly $1 / 2^{-}$below $1.35 \mathrm{GeV}$, and predominantly $1 / 2^{+}$ above. RUSHBROOKE 76 analyzed data on $n p \rightarrow\left(p \pi^{-}\right) p$ fxom 9 to $24 \mathrm{GeV} / \mathrm{c}$. They used a Deck-plus-resonances parametrization and found broad resonance signals in both the $1 / 2^{-}$and $1 / 2^{+}$waves at about $1.4 \mathrm{GeV}$, as well as a large $1 / 2^{-}$Deck contribution peaking below $1.3 \mathrm{GeV}$. In STRACHMAN 75 the $\mathrm{t}$-channel isospin-0 and isospin-1 parts of $\overline{\mathrm{N} N} \rightarrow \overline{\mathrm{N}}(\mathrm{N} \pi)$ at $5.7 \mathrm{GeV} / \mathrm{C}$ were separated and the $I_{t}=1 \mathrm{~N} \pi$ mass spectrum found to contain known resonance peaks, while the $I_{t}=0$ 


\section{Data Card Listings \\ For notation, see key at front of Listings.}

Baryons

$N^{\prime} s$ and $\Delta^{\prime} s$ spectrum had only a broad bump centered at $1.35 \mathrm{GeV}$.

The low mass $\pi \pi N$ system exhibits a broad

enhancement below $2 \mathrm{GeV}$ on which subsidiary peaks are superimposed at around 1.5 and $1.7 \mathrm{GeV}$. The production characteristics are consistent with diffraction.

Partial-wave analyses of diffractively produced $\pi \pi N$ systems have recently been carried out by CARNEY 76, BACON 77, HEINEN 77, IDSCHOK 78, and OTTER 78. All these analyses use bubble chamber data in the medium energy range. CARNEY 76 use a compilation of data on $\mathrm{K}^{+} \mathrm{p} \rightarrow \mathrm{K}^{+}\left(\mathrm{p} \pi^{+} \pi^{-}\right)$between 7.3 and $16 \mathrm{GeV} / \mathrm{C}$. The reaction $\mathrm{K}^{-} \mathrm{p} \rightarrow \mathrm{K}^{-}\left(\mathrm{p} \pi^{+} \pi^{-}\right)$is studied by HEINEN 77 at $4.2 \mathrm{GeV} / \mathrm{C}$ and by OTTER 78 at 10, 14.3, and $16 \mathrm{GeV} / \mathrm{C}$. BACON 77 analyze the 10 and $16 \mathrm{GeV} / \mathrm{C}$ data of OTTER 78 together with $\pi^{ \pm} p \rightarrow \pi^{ \pm}\left(p \pi^{+} \pi^{-}\right)$at 8, 16, and $23 \mathrm{GeV} / \mathrm{c}$. IDSCHOK 78 study $\mathrm{pp} \rightarrow \mathrm{p}\left(\mathrm{p} \pi^{+} \pi^{-}\right)$ at 12 and $24 \mathrm{GeV} / \mathrm{c}$.

All the analyses agree that the low mass $\pi \pi N$ system can be adequately described assuming a dominant contribution from partial waves having spin-parities $\mathrm{J}^{\mathrm{F}}=1 / 2^{+}, 3 / 2^{-}$, and $5 / 2^{+}$, and having $\Delta \pi, p \varepsilon$, and, to a less extent, p p decay modes only. CARNEY 76 require $\mathrm{J} \geqslant 7 / 2$ above about $1.7 \mathrm{GeV}$. BACON 77 cannot rule out alternative solutions completely in terms of partial waves in the series $\mathrm{J}^{\mathrm{P}}=1 / 2^{-}, 3 / 2^{+}, 5 / 2^{-}$, etc., although this conclusion is not supported by CARNEY 76. OTTER 78 find evidence for a small contribution (of order 20\%) from $5 / 2^{-}(l=2) \Delta \pi$ in the mass region $1.60-1.75 \mathrm{GeV}$ in addition to partial waves satisfying the GribovMorrison rule. The shape of the $5 / 2^{-}$enhancement is consistent with a Breit-wigner form $(M=1.67 \mathrm{GeV}$, $\Gamma=0.15 \mathrm{GeV}$ ) and is interpreted as evidence for production of $\mathrm{D}_{15}^{\prime}(1670)$.

It seems clear that the enhancement at $1.5 \mathrm{GeV}$ is predominantly $3 / 2^{-}(l=0) \Delta \pi$, although other partial waves such as $1 / 2^{+}(l=0) p \varepsilon$ are contributing in this region. No evidence for a resonant nature of the $1.5 \mathrm{GeV}$ enhancement could be obtained from studies of relative phases (BACON 77, HEINEN 77, IDSCHOK 78, and OTTER 78).

The situation concerning the peak at $1.7 \mathrm{GeV}$ is more complex in that the different analyses come to different conclusions as to the detailed spinparity decomposition. However, all analyses do agree that $5 / 2^{+}$is not the only contribution, and thus the enhancement cannot be completely associated with the $\mathrm{F}_{15}^{\prime}$ (1688). CARNEY 76 are unable to conclusively identify all the waves contributing to the enhancement, but both $3 / 2^{-}(l=1) \mathrm{p} \varepsilon$ and $3 / 2^{-}(l=2) \Delta \pi$ are strong. On the other hand, BACON 77 find that $1 / 2^{+}(l=0) p \varepsilon$ is important in the $1.7 \mathrm{GeV}$ region, together with $3 / 2^{-}(l=1) p \varepsilon, 3 / 2^{-}(l=2) \Delta \pi$, and $5 / 2^{+}(\ell=1) \Delta \pi$. HEINEN 77 also find that spin-parity $1 / 2^{+}$contributes, but that the decay mode is $(l=1) \Delta \pi$. In addition, $3 / 2^{-}(l=1) p \varepsilon$ and $5 / 2^{+}(l=2) p \varepsilon$ are present. Spin-parity $5 / 2^{+}$is the most important contribution to the data of IDSCHOK 78 above 1.6 $\mathrm{GeV}$, but $3 / 2^{-}(l=1) p \varepsilon$ is also significant. No partial wave exhibits a phase variation. OTTER 78 also conclude that the $1.7 \mathrm{GeV}$ enhancement is composed of several partial waves with spin-parities $1 / 2^{+}$(or $1 / 2^{-}$- the analysis cannot distinguish the two possibilities in this region), $3 / 2^{-}$, and $5 / 2^{+}$ all contributing, as well as $5 / 2^{-}(\ell=2) \Delta \pi$.

The production of the different spin-parity states depends very differently on four-momentum transfer (IDSCHOK 78). Thus the apparent disagreement concerning the exact nature of the $1.7 \mathrm{GeV}$ enhancement could be due in part to the different regions of four-momentum transfer used by the various analyses.

It is interesting to note that the GribovMorrison rule appears to be reasonably well satisfied for diffractively produced $\pi \pi N$ systems, in contrast to the situation for $\pi N$ diffraction dissociation. This could be connected with the fact that the Deck mechanism is expected to be important at low mass and that the major contribution is to $S$-wave states. Thus s-wave $\Delta \pi\left(\mathrm{J}^{\mathrm{P}}=3 / 2^{-}\right)$ and $p \varepsilon\left(1 / 2^{+}\right)$both give rise to spin-parities in the "allowed" series, whereas $s$-wave $\pi \mathrm{N}\left(1 / 2^{-}\right)$does not. A similar situation occurs in three-meson diffractive production, where the "allowed" series is dominant.

\section{References for Section V}

See the Data Card Listings. 


\section{Baryons}

$N^{\prime} s$ and $\Delta^{\prime} s, p, n, N(1470)$

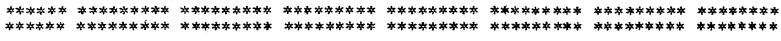

STATUS OF N* RESONANCES
THOSE WITH AN OVERALL STATUS OF *** OR $\$ * * *$ ARE INCLUDEO IN THE MAIN BARYON
TABLE. THE OTHERS AWAIT CONF IRMATION.

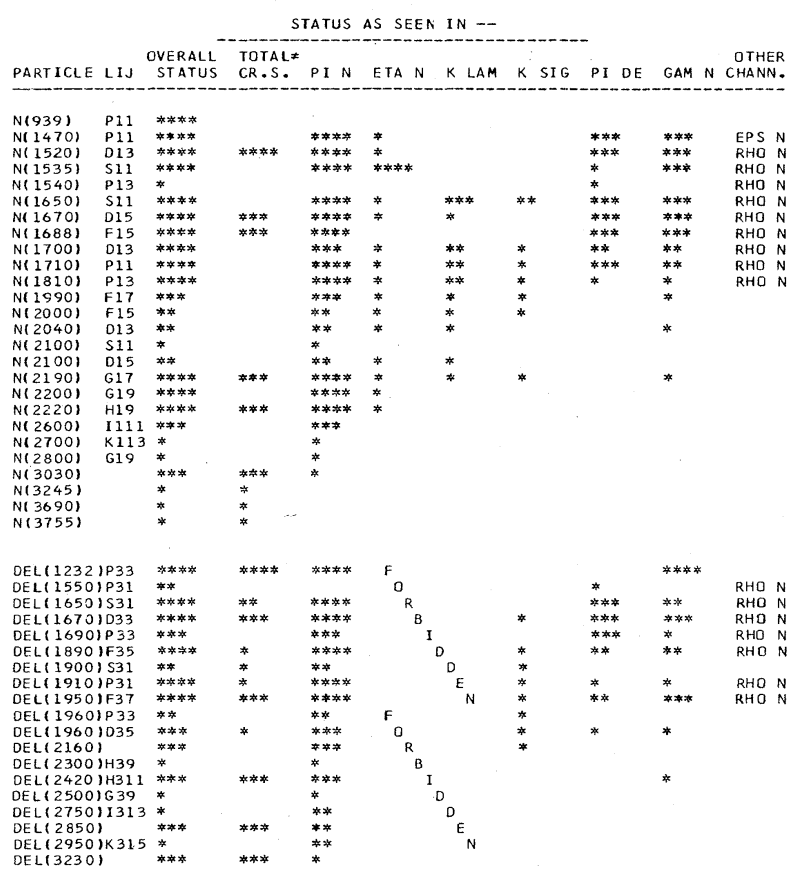

*\#\#*\# GOOD, CLEAR, AND UNMISTAKABLE.
**** GOOD, BUT IN NEED OF CLAR IFICATION OR NOT ABSOLUTELY CERTAIN.

* * NEESS CONFTRMATION.

* WEAK. Citributed to the state closest to hHere the cross section peaks.

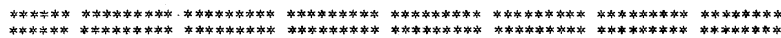
$\mathrm{S}=0 \mathrm{I}=1 / 2$ NUCLEON STATES $(\mathrm{N})$

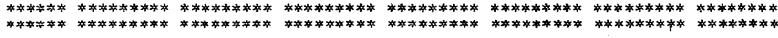

D 16 PROTON(938, J=1/2) $\quad I=1 / 2$

SEE STABLE PARTICle DatA CARD LISTINGS

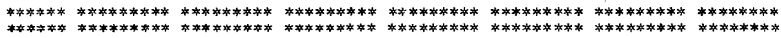

II 17 NEUTRON(939, JE1/2) $I=1 / 2$

SeE STABle Particle data CARd LISTINGS

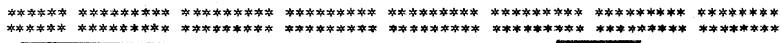

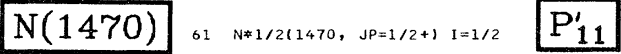

MASS AND WIDTH ARE BEST DETERMINED FROM PARTIAL WAVE ANALYSES. WE LIST PRODUCTION EXPERIMENTS SEPARATELY AYED 76 CLAIMS TWO P1I STATES IN THE 1500 MEV REGION.

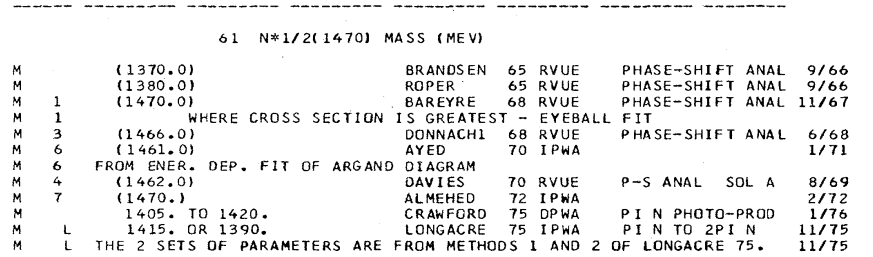

Data Card Listings For notation, see key at front of Listings.

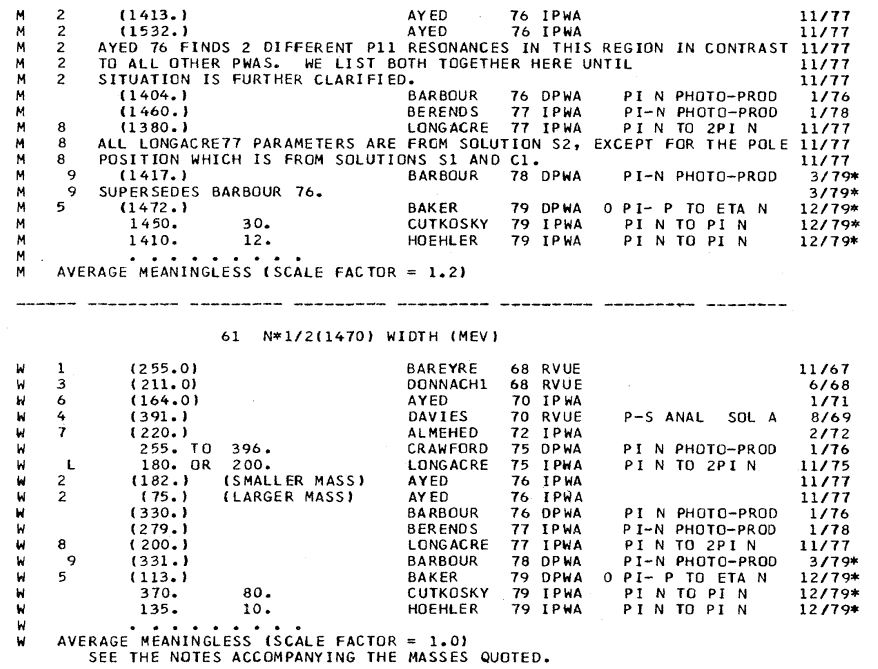

-

\begin{tabular}{|c|c|c|c|c|c|}
\hline $\begin{array}{ll}R E & \\
R E & \\
R E & 8 \\
R E & \end{array}$ & $\begin{array}{l}(1375 .) \\
(1381 .) \\
1360.0 \mathrm{DR} \\
(1369 .)\end{array}$ & $\begin{array}{l}(5 .) \\
1333 .\end{array}$ & $\begin{array}{lll}\text { LEE } & 73 & \\
\text { LONGACRE } & 75 & \text { IPWA } \\
\text { LONGACRE } & 77 & \text { IPWA } \\
\text { CUTKDSKY } & 79 & \text { IPWA }\end{array}$ & $\begin{array}{l}\text { FIT TO ALMEHEO72 } \\
\text { PI N TO } 2 P I N \\
\text { PI N TO } 2 \text { I I N } \\
\text { PI N TO PI N }\end{array}$ & $\begin{array}{l}1 / 74 \\
11 / 75 \\
11 / 77 \\
12 / 79 *\end{array}$ \\
\hline & & $61 \quad N * 1 / 2(1470)$ & -2*IMAG PART OF PCLE $P$ & POS ITION (MEV) & $1 / 74$ \\
\hline & $\begin{array}{l}(216 .) \\
(209 .) \\
1167 .) \text { OR } \\
(178 .)\end{array}$ & $\begin{array}{l}(10.1 \\
234 .\end{array}$ & $\begin{array}{lll}\text { LEE } & 73 & \\
\text { LONGACRE } & 75 & \text { IPWA } \\
\text { LONGACRE } & 77 & \text { IPWA } \\
\text { CUTKOSKY } & 79 & \text { I PWA }\end{array}$ & $\begin{array}{l}\text { FIT } \text { TO ALMEHED72. } \\
\text { PI N TO } 2 \text { PI N } \\
\text { PI N TO } 2 \text { PI N } \\
\text { PI N TO PI N }\end{array}$ & $\begin{array}{l}1 / 74 \\
11 / 75 \\
11 / 77 \\
12 / 79 *\end{array}$ \\
\hline RER & $(-9)$. & $61 \quad N * 1 / 2(1470)$ & $\begin{array}{l}\text { REAL PART OF ELASTIC P } \\
\text { CUTKOSKY } 79 \text { IPWA }\end{array}$ & $\begin{array}{l}\text { POLE RESIDUE (MEV) } \\
\text { PI N TO PI N }\end{array}$ & $12 / 79 *$ \\
\hline IMR & $(-48)$. & $61 \quad N * 1 / 2(1470)$ & $\begin{array}{l}\text { IMAG PART OF ELASTIC P } \\
\text { CUTKOSKY } 79 \text { IPWA }\end{array}$ & $\begin{array}{l}\text { POLE RESIDUE (MEV) } \\
\text { PI N TO PI N }\end{array}$ & $12 / 79 *$ \\
\hline$A B S$ & (74.) & $\begin{array}{l}\text { 61. } N * 1 / 2(1470) \\
(5 .)\end{array}$ & $\begin{array}{c}\text { ABSOLUTE VALUE OF POLE } \\
\text { LEE } 73\end{array}$ & $\begin{array}{l}\text { E RESIOUE (MEV) } \\
\text { FIT TO ALMEHEDT2 }\end{array}$ & $\begin{array}{l}1 / 74 \\
1 / 74\end{array}$ \\
\hline PH & $(-1.4)$ & $61 \mathrm{~N} * 1 / 2(1470)$ & $\begin{array}{l}\text { PHASE OF POLE RESIDUE } \\
\text { LEE }\end{array}$ & $\begin{array}{l}\text { (RADIANS) } \\
\text { FIT TO ALMEHEDT } 2\end{array}$ & $\begin{array}{l}1 / 74 \\
1 / 74\end{array}$ \\
\hline
\end{tabular}

61 N*1/2(1470) PARTIAL DECAY MODES
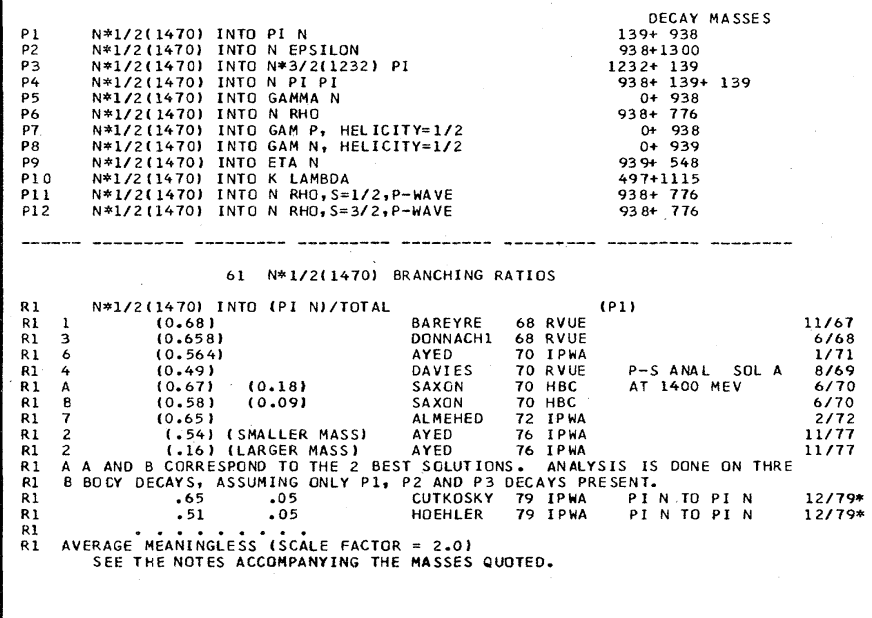


\section{Data Card Listings}

Baryons

For notation, see key at front of Listings.

$\mathrm{N}(1470)$
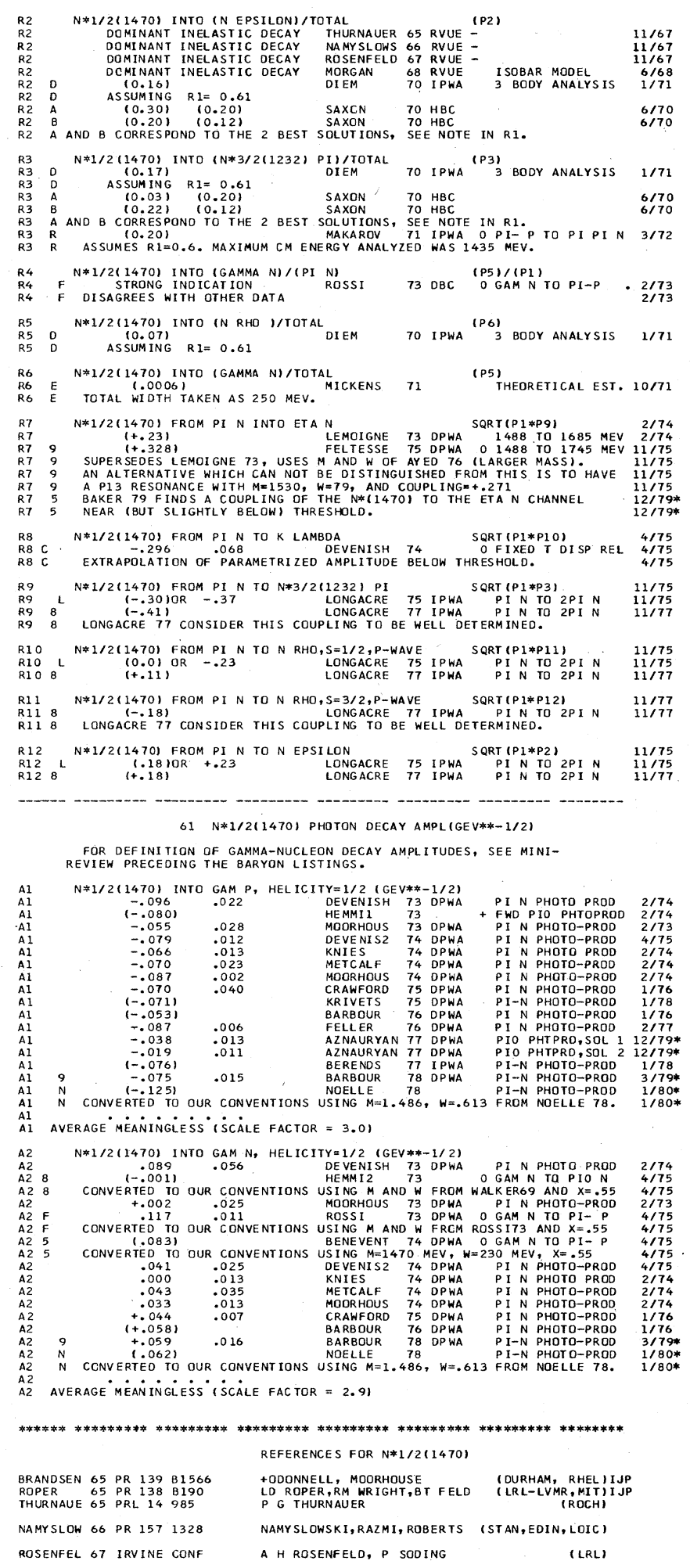

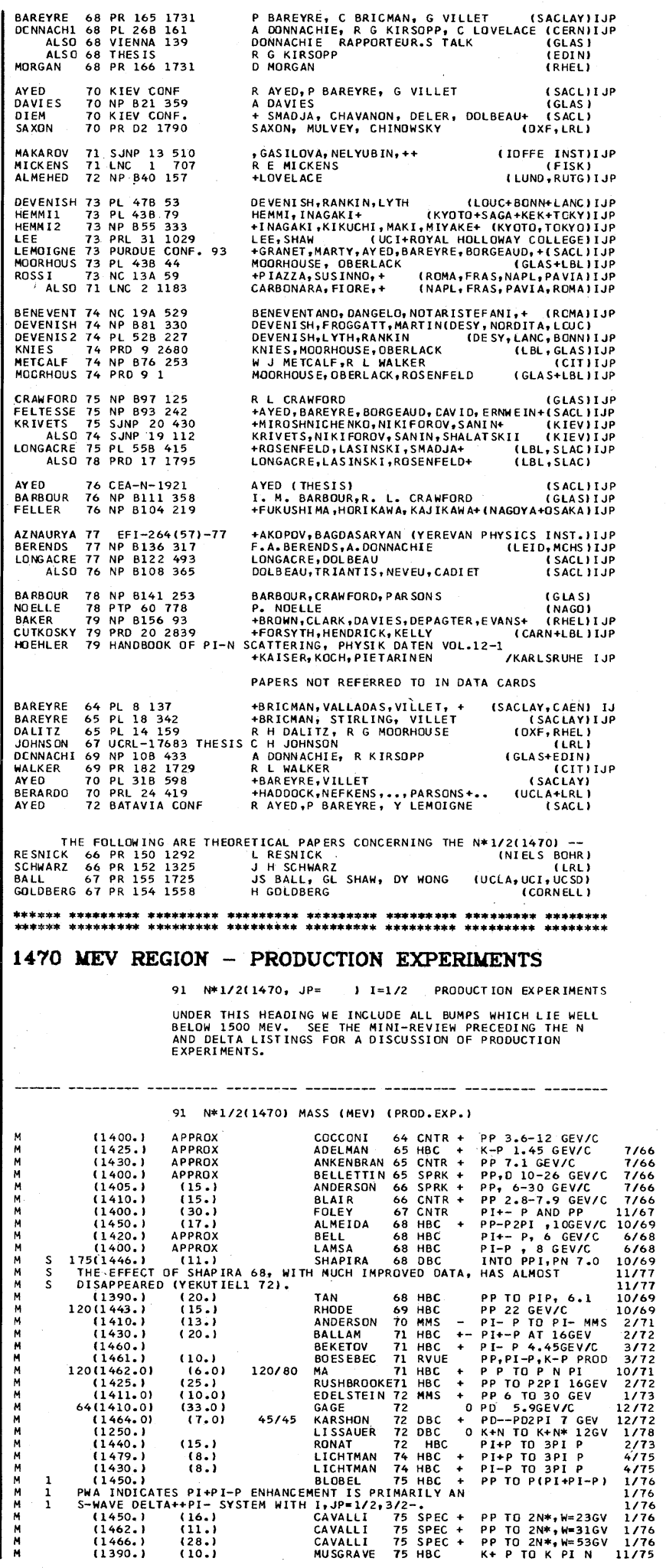




\section{Baryons \\ $\mathrm{N}(1470), \mathrm{N}(1520)$}

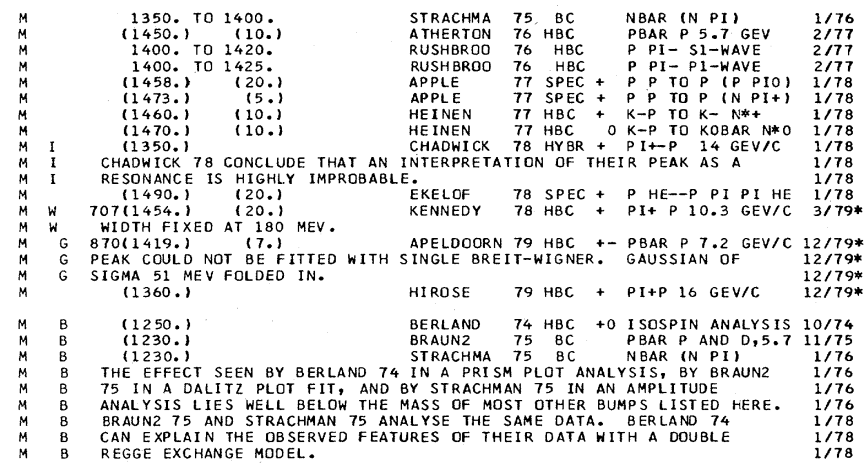

$91 N * 1 / 2(1470)$ WIDTH (MEV) (PROD. EXP.)

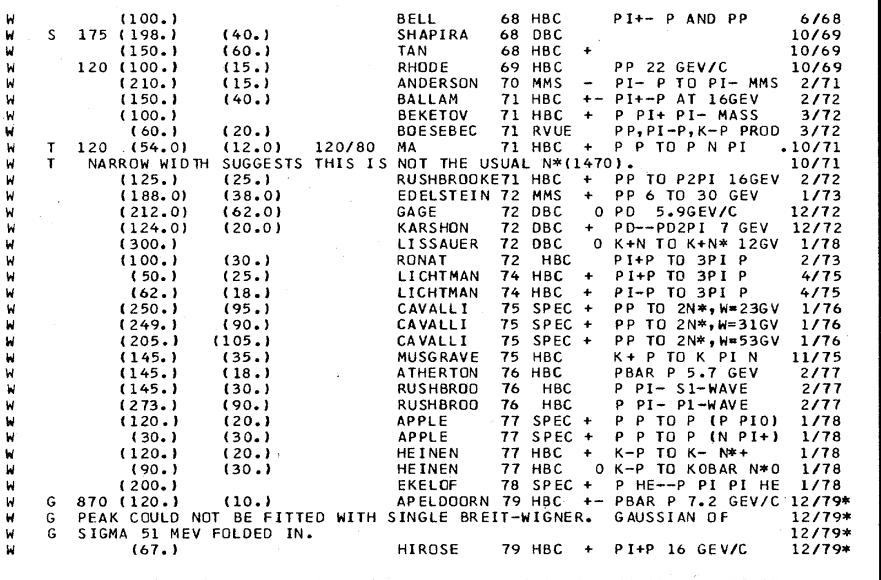

$91 N * 1 / 2(1470)$ PARTIAL DECAY MODES (PROD. EXP.)

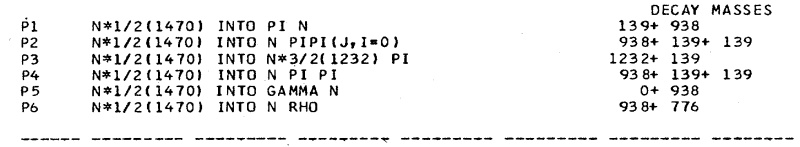

91 N*1/2(1470) BRANCHING RATIOS (PROD. EXP.)

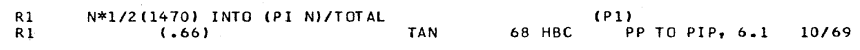

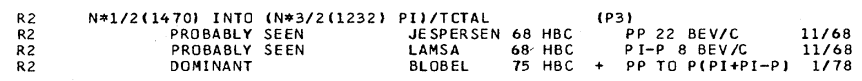

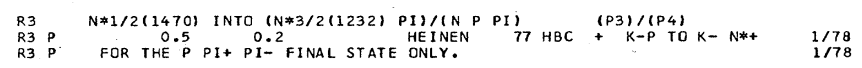

**************************************************************************1

\begin{tabular}{|c|c|c|}
\hline & & \\
\hline $\begin{array}{l}\text { COCCONI } \\
\text { ADELMAN } \\
\text { ANKE NBRA } \\
\text { BELLETT } \\
\text { ANDERSON } \\
\text { BLAIR }\end{array}$ & $\begin{array}{llll}64 & P L & 8 & 134 \\
65 & P R L & 14 & 1043 \\
65 & N C & 35 & 1052 \\
65 & P L & 18 & 167 \\
66 & P R L & 16 & 855 \\
66 & P R L & 17 & 789\end{array}$ & 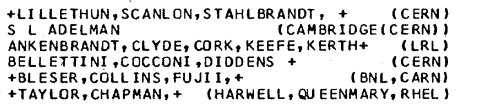 \\
\hline $\begin{array}{l}\text { FOLEY } \\
\text { ALMEIDA } \\
\text { BELL } \\
\text { JE SPERSE } \\
\text { LAMSA } \\
\text { SHAPIRA } \\
\text { TAN } \\
\text { RHODE } \\
\text { ANDERSON }\end{array}$ & 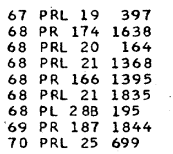 & 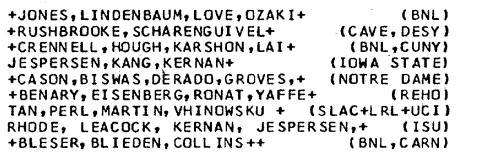 \\
\hline $\begin{array}{l}\text { BALLAM } \\
\text { BEKETOV } \\
\text { BOESEBEC } \\
\text { MA } \\
\text { RUSHBROO }\end{array}$ & 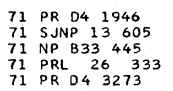 & 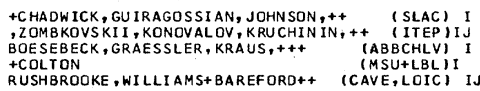 \\
\hline
\end{tabular}

\section{Data Card Listings For notation, see key at front of Listings.}

EDELSTEIN, CARRIGAN, HI EN, MCMAHON, + (CARN+BNL)
W GGGE, COL TON, W CHINOWSKI
TFIRESTONE, GINESTET, GOL DHAB , TR ILLING(LBL) TIRESTONE, GINESTET, GOL DHABER, TR ILLING (LBL) I JP
+YEKUTIELI, YAFFE, SHAPIRA, RONAT,
TE IREHO) I $\begin{array}{ll}\text { +EISENBER, L Y YONS, SHAPIRA, TOAFF+ } & \text { (REHO) } \\ \text { YEKUTIELI, YAFFE, SHAPI RA, RONAT + } & \text { (REHO) }\end{array}$ BERLAND, HABER, HODOUS, HULSIZER,
BERLAN, HABER, HODOUS, HULSIZER;
(MIT) I
(MIT)

BERLAND 74 NP $B 7593$ $\begin{array}{llll}\text { LICHTMAN } 74 & \text { NP } & \text { B } 81 & 31\end{array}$ \begin{tabular}{lllll} 
BLOBEL & 75 & NP & 897 & 201 \\
BRAUN2 & 75 & NP & 895 & 503 \\
\hline
\end{tabular} $\begin{array}{rlll}\text { CAVALLI } 75 & \text { LNC } 14 & 353 \\ \text { ALSO } 75 & \text { LNC } 14 & 345+359\end{array}$ MUSGRAVE 75 NP 887365
STRACHMA 75 NP $B 988120$
ALSO 76 NP B107 330 ATHERTON 76 NP B103 381

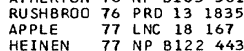
CHAOWICK 78 PRD $17 \quad 1713$ KENNEEY 78 PRD 172888
APELDOOR 79 NP B 156111 APEL DOOR 79 NP B156 111
HIROSE 79 NC $50 A 120$

GELLERT 66 PRL 17884 $\begin{array}{llllll}\text { CLEGG } & 69 & \text { NP } & 813 & 222 \\ \text { ALEXANDE } & 73 & \text { NP } & 852 & 221\end{array}$ $\begin{array}{lllll} & \\ \text { ANSORGE } & 73 & \text { NP } & 863 & 93 \\ \text { BEAUPRE } & 73 & \text { NP } & 866 & 93 \\ \text { SEO }\end{array}$

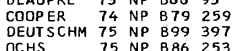
$\begin{array}{lllll}\text { CARNEY } & 76 & \text { NP } & B 110 & 248 \\ \text { DEKERRET } & 76 & \text { PL } & 63 B & 477,483\end{array}$

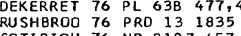
SOTIRIOU 76 NP B107 757
BACON 77 NC $42 A 431$

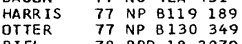
$\begin{array}{lllll}\text { BIEL } & 78 & P R D & 18 & 3079 \\ \text { ALSO } 76 & \text { PRL } & 36 & 504,507\end{array}$

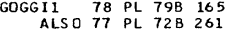
GOGG 12 78 78 B 143365
IDSCHOK 78 NC $48 A 395$

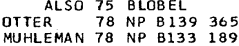

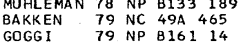
+ESKREYS, FESEFELDT, FRANZ+ (BONN+HAMB +MFIM) IJP CAVALLI-SFORZA,CONTAt , SCHIBYt STBRB,
(PAVIIAPRP IN) CAVALL-SFORZA, CONAT
TPEETERS, SCREINER, WHITMORE, YUTA (PAVIA+PRIN)
(ANL) TPEEER, STREATMA, BRAUN, GERBER, MAURER+ (LPNP+STRB) I
STRACCMANAN
(LPNP) I ATHERTON, FRENCH, SKURA, BOHM+ (CERN+PRAG)
RUSHRBOOKE, RAJA, ANSORGE, CARTER, NEALE (CAVE) JP RUSHRBOOKE, RAJA, ANSORGE, CARTER, NEALE (CAVE) JP
+ASH, CHENG, COYNE,GROSSMAN+
(PRIN+PAVIA)
ENGELEN, KITTEL, METZGER+
(NI JM+AMST+CERN) IJP +CARROLL, CHALOUPKA,BALLAM+ (SLAC+CIT+LBL) IJJP + ZEMANY, BEAUFAYS, KEY, LUSTE, PRENT ICE+ (TNTO) VAN APELDOORN, HAR TING, HOLTHUIZEN+
+KANAI, KITAMURA, KOBAYASHI
(TOKST)

PAPERS NOT REFERREO TO IN OATA CARDS +SMI TH, WDJCICKI, COLTON, SCHLEIN + (LRL, UCLA) +THOMPSON, ROBERT SON, OH, LEE, HARTUNG, + (WISC) CLEGG
ALEXANDER, BENARY + (TEL-AVIV+HEI DEL BERG+DESY)

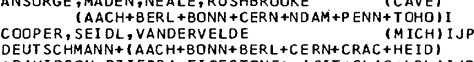
+DAVIDSON, DZIERBA, FI RESTONE+ ( CIT+SLAC+LBL) IJ +COLLEY, JONES, KENYON+ (BIRM+BRUX+CERN+MCNS) I JP RUSHBRDOKE, RAJA, ANSORGE, CARTER, NEALE (CAVE) I IJP O. SOTIRIOU RA, ANSORGE, ,CARTER, NEALE (IAVE)IJP
+BARNHAM, DORNAN, EASON, PCLLOCK+ (LEN) IJP
(LCICIIJP +LUBATT I, MOR IYA ESO (ROCH+NWES +FNAL+SLAC) BIEL, BLESER, FERBEL+ (ROCH+FNAL+SLAC+NWES) GOGGI, MANTOVANI, CAVALLI-SFORZA+ (CERN+PAVI) GOGG
+CAVALI-SFORZA,CONTA+
+SCHRODER, BLOBEL, FRANZ+ (BONN+HAMB+MPIM) I JP

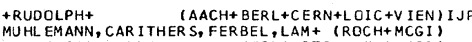
+CONTA, FRA TERNALI, LIVAN+ (CERN+PAVI + IRST)

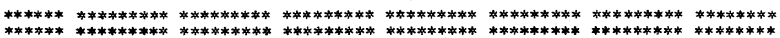

$\mathrm{N}(1520)$ 62 ${ }^{*}{ }^{* 1 / 2\left(1520, J_{P} 3 / 2-1\right.} \mathrm{I}=1 / 2 \quad \mathrm{D}_{13}^{\prime}$ THE EXISTENCE OF THIS RESONANCE IS WELL ESTABLISHEO.

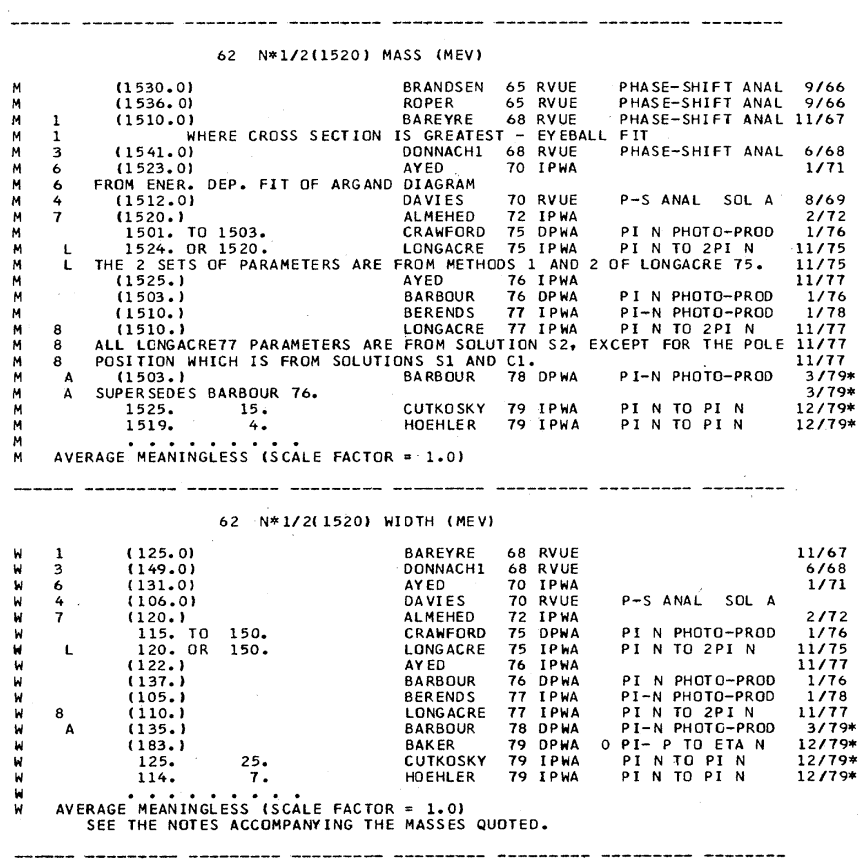




\section{Data Card Listings}

Baryons

For notation, see key at front of Listings.

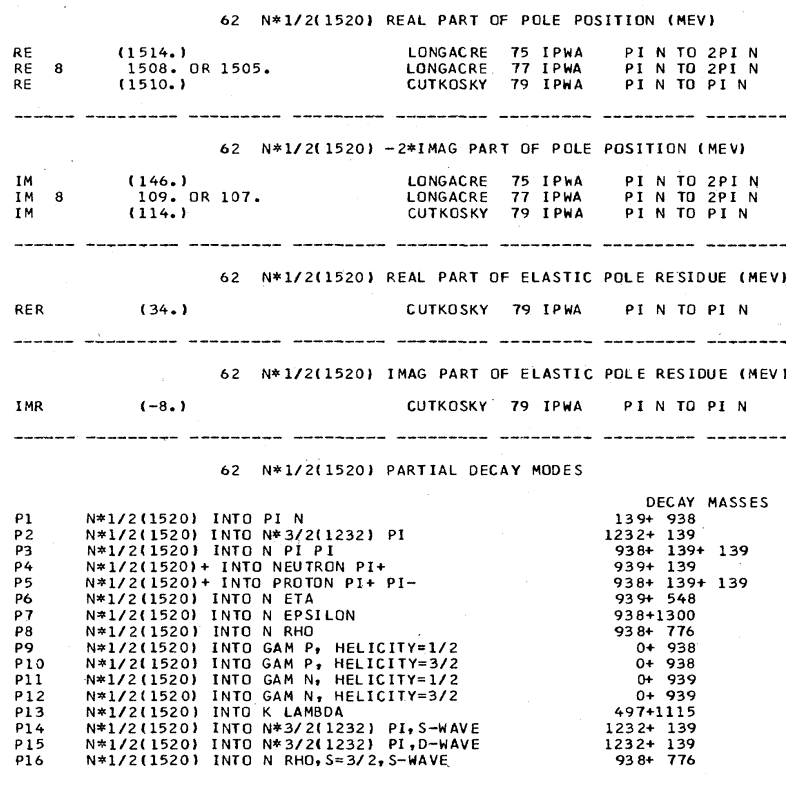

$62 N * 1 / 2(1520)$ BRANCHING RATIOS

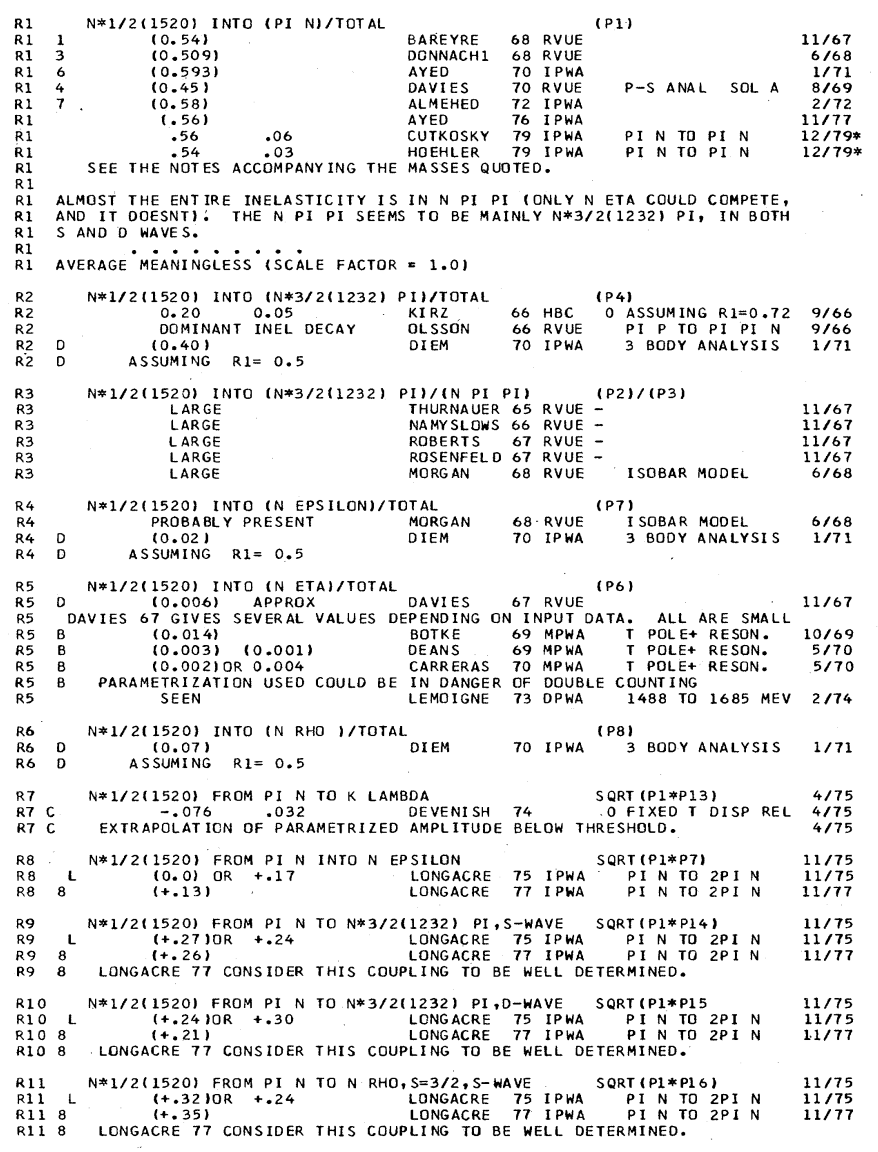

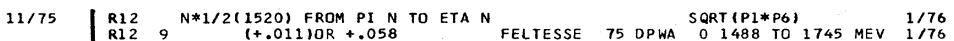

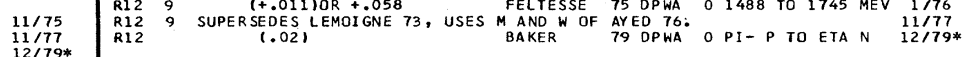
$62 N * 1 / 2(1520)$ PHOTON DECAY AMPLIGEV**-1/2) FOR DEFINITION OF GAMMA-NUCLEON DECAY AMPL ITUDES, SEE MINI-

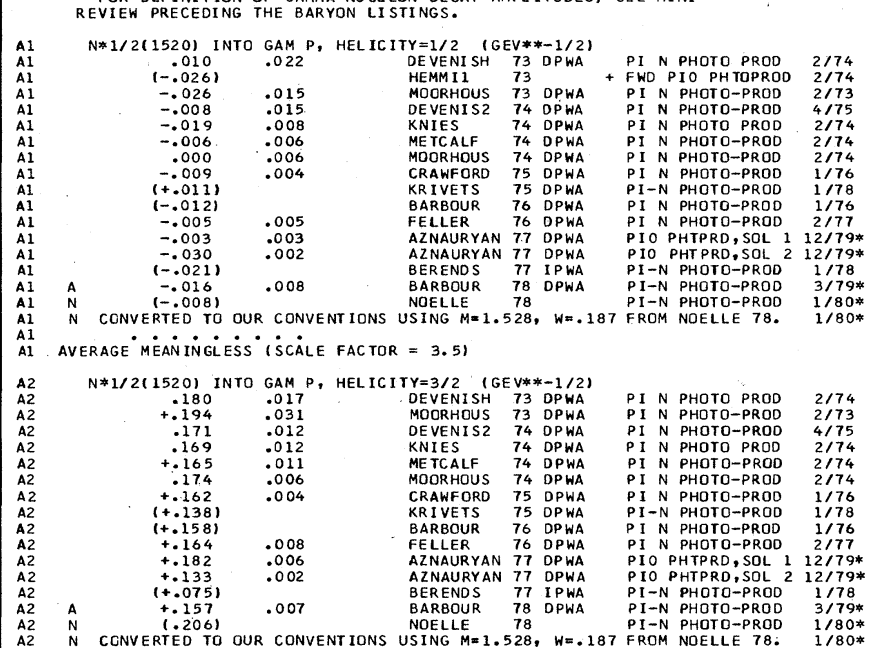
A2 AVERAGE MEÁ INGLESS (SSCALE FAC TOR $=4.11$

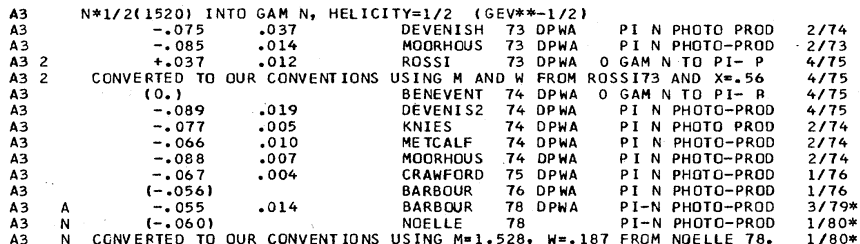

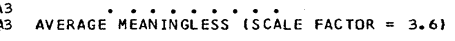

A4 $N * 1 / 2(1520)$ INTO GAM N, HELICI IY $=3 / 2$ (GEV**-1/2)

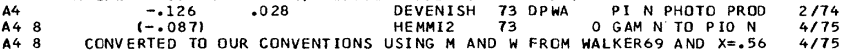

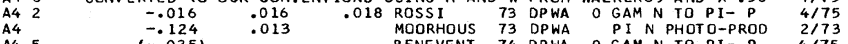

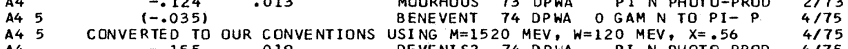

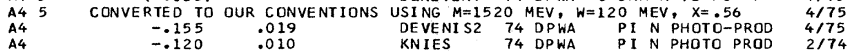

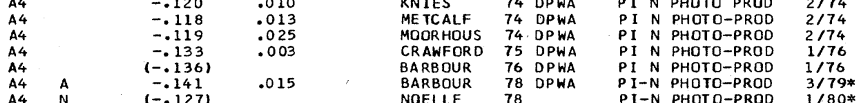

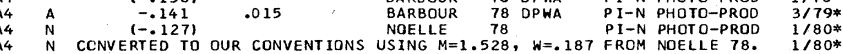

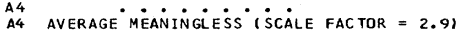

\begin{tabular}{|c|c|c|c|}
\hline \multicolumn{4}{|c|}{ REFERENCES FOR Nं*1/2(1520) } \\
\hline $\begin{array}{l}\text { RANDSEN } \\
\text { PPER } \\
\text { HURNAUEE }\end{array}$ & $\begin{array}{l}\text { SEE A PREVIOUS } \\
65 \text { PR } 13981566 \\
65 \text { PR } 138 \text { B } 190 \\
65 \text { PRL } 14985\end{array}$ & $\begin{array}{l}\text { EDITION (RMP 37, 633, 1965) FOR E } \\
\text { +ODONNELL, MOORHOUSE } \\
\text { LO ROPER,RM WRIGHT,BT FELD } \\
\text { P G THURNAUER }\end{array}$ & $\begin{array}{l}\text { EARLIER REFERENCES. } \\
\text { (DURHAM, RHEL) IJPP } \\
\text { (LRL-LVMR, MIIIJJP } \\
\text { (ROCH) }\end{array}$ \\
\hline $\begin{array}{l}\text { KIRZ } \\
\text { NUMBE } \\
\text { NAMYSLOW } \\
\text { CLSSCN }\end{array}$ & $\begin{array}{l}\text { 66 PRIVATE COMM } \\
\text { ER EXTRACTED FROM } \\
66 \text { PR } 1571328 \\
66 \text { PR } 1451309\end{array}$ & $\begin{array}{l}J \text { KIRZ } \\
\text { DATA DISCUSSED IN KIRZ } 63 \text {. Z } 63 \\
\text { NAMYSLOWSKI,RAZMI, ROBERTS } \\
M G \text { OLSSON, G B YDDH }\end{array}$ & $\begin{array}{r}\text { (LRL) } \\
\text { (SSTAN, EDIN, LCIC) } \\
\text { (WI SC, UMD) }\end{array}$ \\
\hline $\begin{array}{l}\text { JVIES } \\
\text { OBERTS } \\
\text { OBEREL } \\
\text { OSENFEL }\end{array}$ & 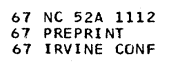 & $\begin{array}{l}\text { A } T \text { DAVIES, R G MOORHOUSE } \\
R \text { G ROBERTS }{ }^{\circ} \text { ROORH } \\
\text { A H ROSENFELD, P SODING }\end{array}$ & $\begin{array}{r}\text { (GLASGOW, RHEL) } \\
\text { (DURHAM) } \\
\text { (LRL) }\end{array}$ \\
\hline $\begin{array}{l}\text { BAREYRE } \\
\text { OONNACH1 } \\
\text { ALSO } \\
\text { ALSO } \\
\text { MORGAN }\end{array}$ & $\begin{array}{llll}68 & P R & 165 & 1731 \\
68 & P L & 268 & 161 \\
68 & \text { VIENNA } & 139 \\
68 & \text { THESIS } & \\
68 & \text { PR } & 166 & 1731\end{array}$ & $\begin{array}{l}\text { P BAREYRE, } C \text { BRICMAN, G VILL } \\
\text { A DONACHIE, R G KIRSOPP, C } \\
\text { DONNACHIE RAPPDRTEUR .S TALK } \\
\text { R G KIRSOPP } \\
\text { D MORGAN }\end{array}$ & $\begin{array}{l}\text { LEET (SACLAY)IJJP } \\
\text { ELOVELACE (IERN)IIJP } \\
\text { (GLAS) } \\
\text { (EDIN) } \\
\text { (EHEL) }\end{array}$ \\
\hline $\begin{array}{l}\text { BOTKE } \\
\text { DEANS }\end{array}$ & $\begin{array}{llll}69 & P R & 180 & 1417 \\
69 & P R & 185 & 1797\end{array}$ & $\begin{array}{l}\text { J C BOTKE } \\
\text { S DEANS, J WOOTEN }\end{array}$ & $\begin{array}{l}\text { (UCSB) } \\
\text { (UNIV S FLOR IDA) }\end{array}$ \\
\hline $\begin{array}{l}\text { AYED } \\
\text { CARRERAS } \\
\text { DAVIES } \\
\text { DIEM } \\
\text { ALMEHED }\end{array}$ & $\begin{array}{l}70 \text { KIEV CONF } \\
70 \text { NP } 16 B 35 \\
70 \text { NP B } 31359 \\
70 \text { KIEV CONF. } \\
72 \text { NP B } 40157\end{array}$ & $\begin{array}{l}\text { R AYED, P BAREYRE, G VILLET } \\
\text { B CARRERAS, A DONNACHIE } \\
\text { A DAVIES S CHAVAN, DEL ER, } \\
+ \text { SMADJA, CHAVANON, } \\
\text { +LOVELACEE }\end{array}$ & $\begin{array}{l}\text { (SACL) IJP } \\
\text { (DARE,MCHS) } \\
\text { DOLLEAS) } \\
\text { (GLAU+ (SACL) } \\
\text { (LUND, RUTG) IJPP }\end{array}$ \\
\hline
\end{tabular}




\section{Baryons $\mathrm{N}(1520), \mathrm{N}(1535)$}

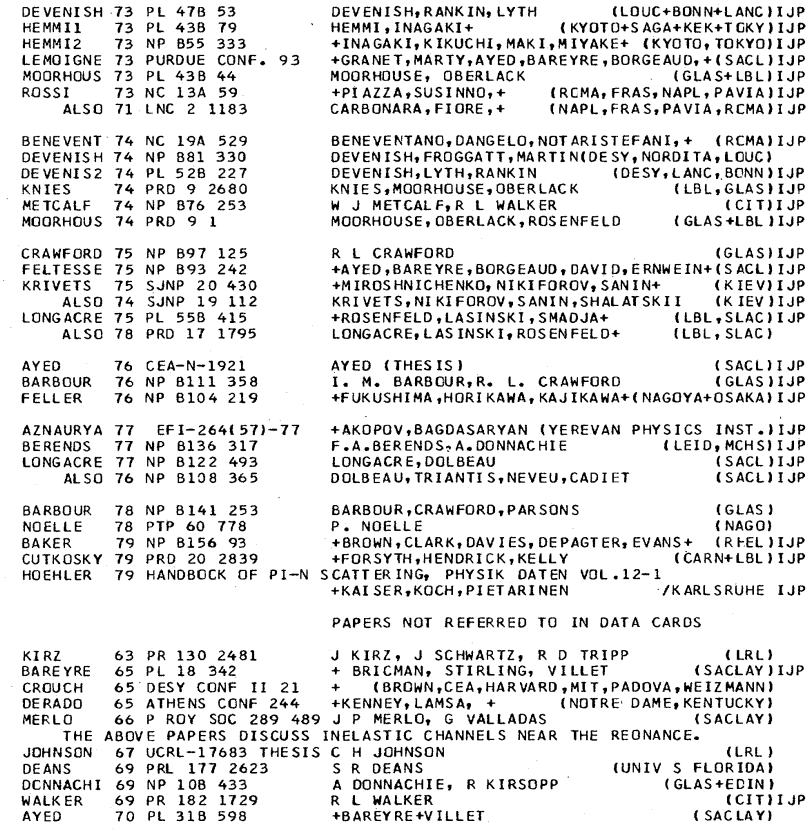

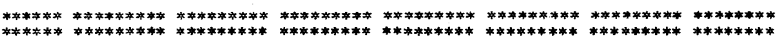
1520 MEV REGION - PRODUCTION EXPERIMENTS

$8 N * 1 / 2(1520, J P=\quad, I=1 / 2 \quad$ PRODUCTION EXPERIMENTS SEE THE MINI-REVIEW PRECEDING THE N AND OELTA LISTINGS
FOR A DISCUSSION OF PRODUCTION EXPERIMENTS.

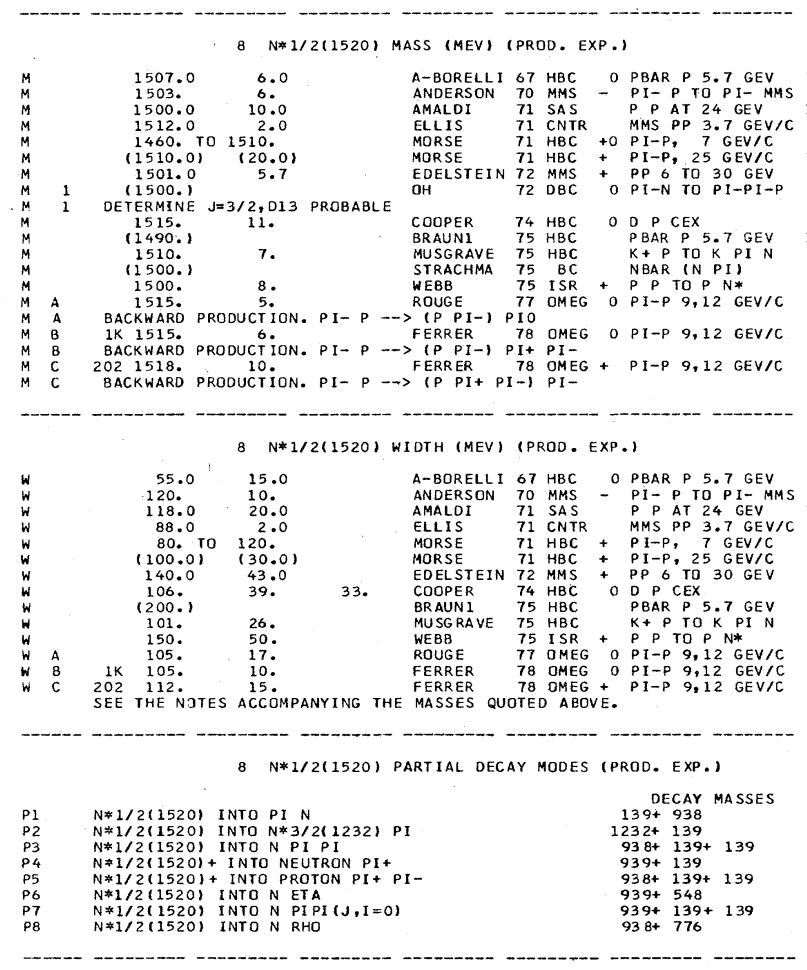

Data Card Listings For notation, see key at front of Listings. 


\section{Data Card Listings}

Baryons

For notation, see key at front of Listings.

$\mathrm{N}(1535)$

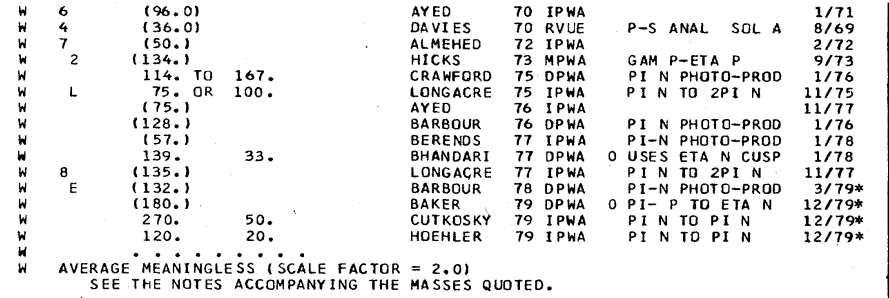

SEE THE NOTES ACCOMPANY ING THE MASSES QUOTED.

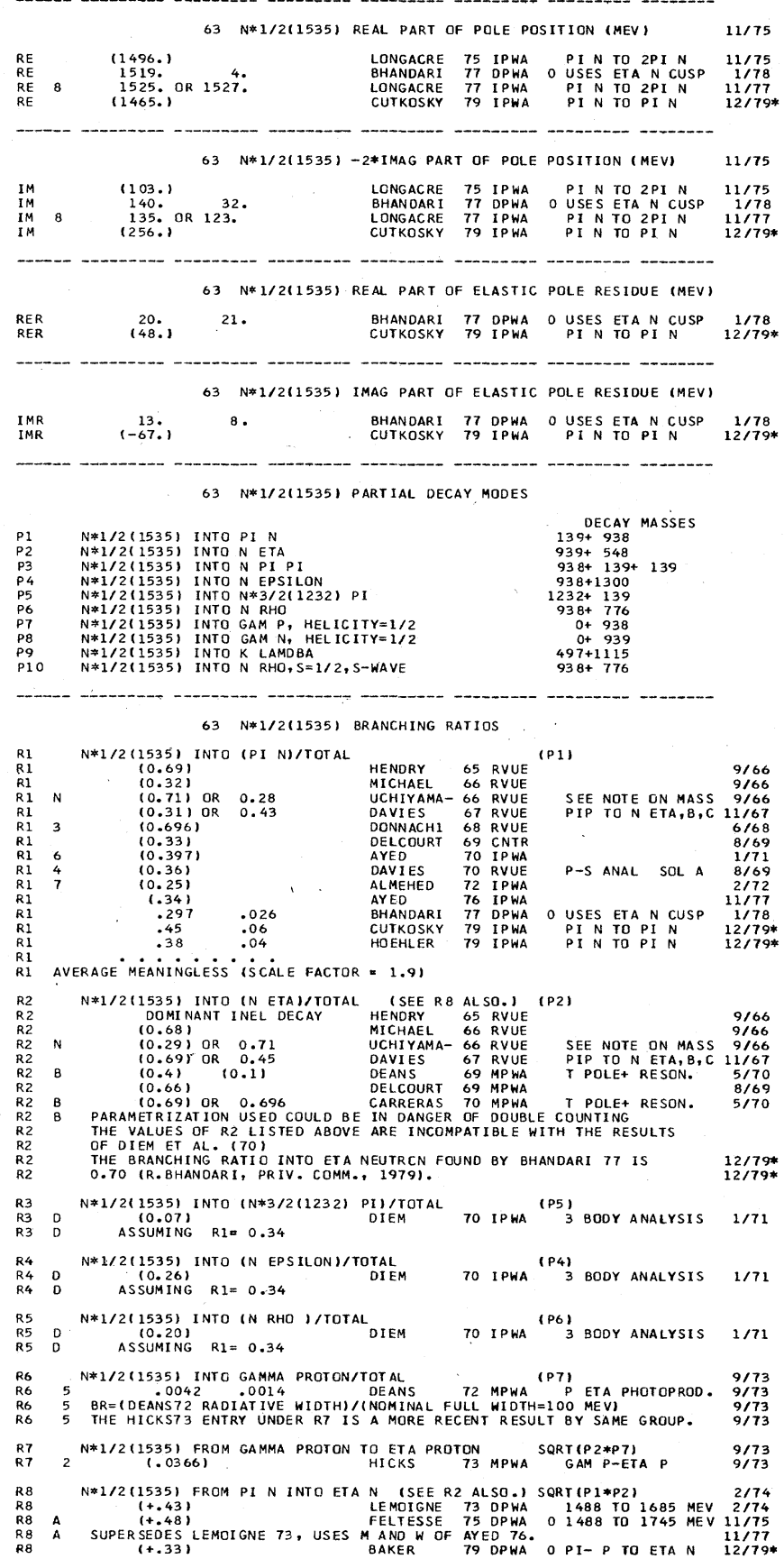

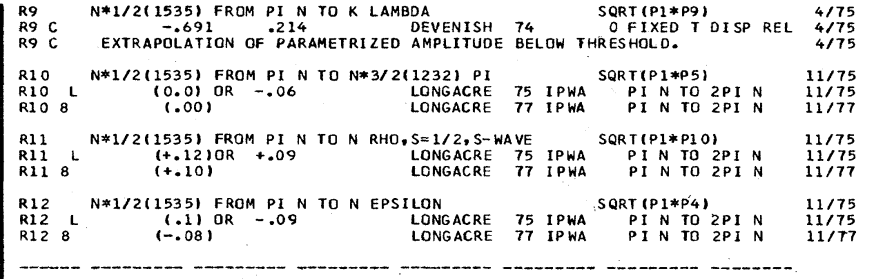

$63 N * 1 / 2(1535)$ PHOTON DECAY AMPL(GEV**-1/2)

FOR DEFINI TION OF GAMMA-NUCLÉN DECAY AMPLI ITUDES, SEE MINI-
REVIEW PRECEOING THE AARYON LISTINGS.

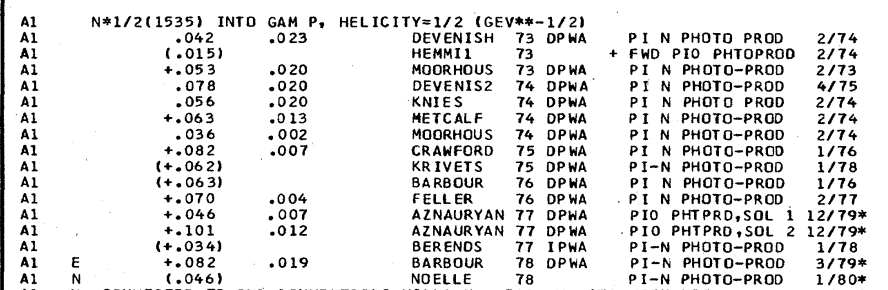

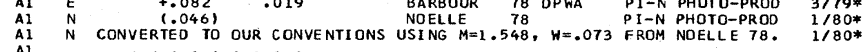

A1 AVERAGE MEÁNI

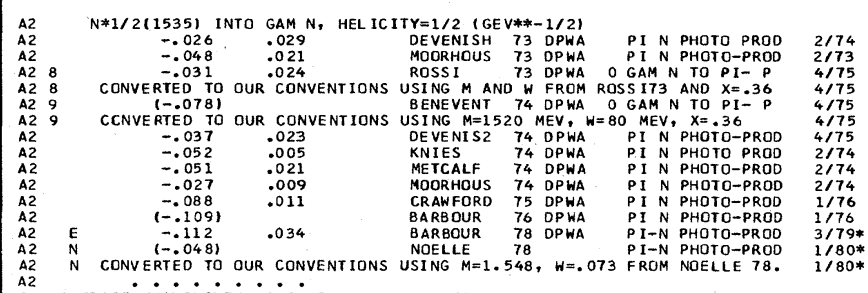

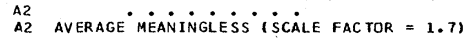

H**

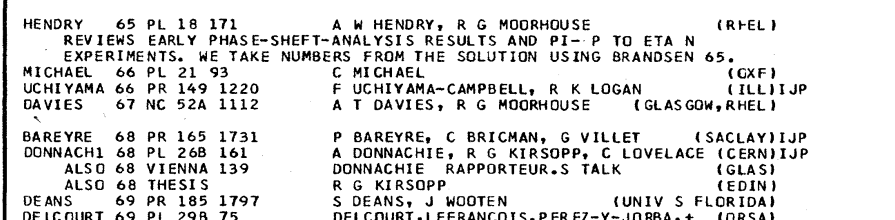

DEANS 69 PR 1851797
DE LCOURT 69 PL 29875

AYED
CARREAS
70
CARE

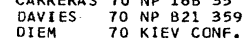

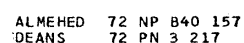

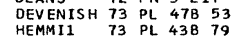

HICKS 73 PRD 72014
LE MOIGE 73 PUROUE CONF. 93

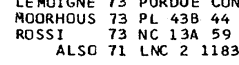

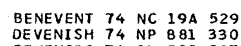

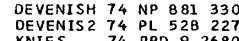

KNIES 74 PRD 92680
METCALF 74 NP 76253
MOORHOUS 74 PRD 91
COR 253

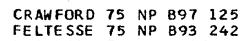

KR IVETS 75 SINP 20430
ALSO 74 SSNP 19.112

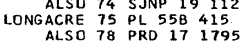

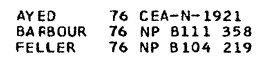

R AYED, P BAREYRE, G VILLET
B CARRERAS. A DONNACHIE (SACL)IJ JP
(DARE, MCHS)

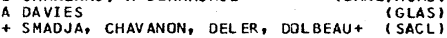

+L OVELACE
+JACOBS, LYONS, HICKS IU S FL TLUNDA RUTGGIIJP
TAMACARN)

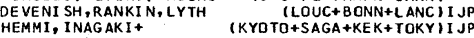

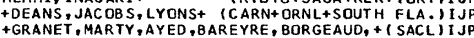

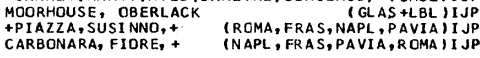
BENEVENTANO, DANGELO, NOTARI STEF ANI, , (RCMA) I JP
DEVENISH, FROGGAT, MART INIDESY, NOROI TA, LOUC)
DE

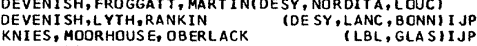
WJ METCALF, R W HALKER
MOORHOUSE, OBERLACK, ROSENFELD IGLASTIIJP

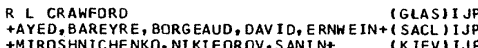
KR IVETS, NIEIFOROV, SANIN, SHALAT SKII (KIEVIIJP

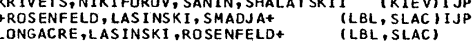

Z2NAUVYA 77 EFI-2641571-77 AAKOPOV,BAGDASARYAN (Y EREVAN PHYSICS INST. IJIP

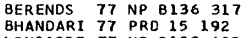
$\begin{aligned} & \\ & \text { ONGACRE } 77 \text { NP } 8122493 \\ & \text { ALSO } 76 \text { NP B108 } 365\end{aligned}$

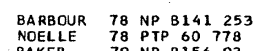

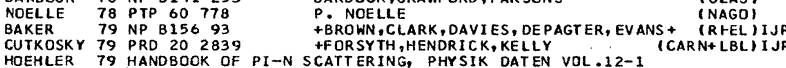




\section{Baryons \\ N(1535), N(1540), N(1650)}

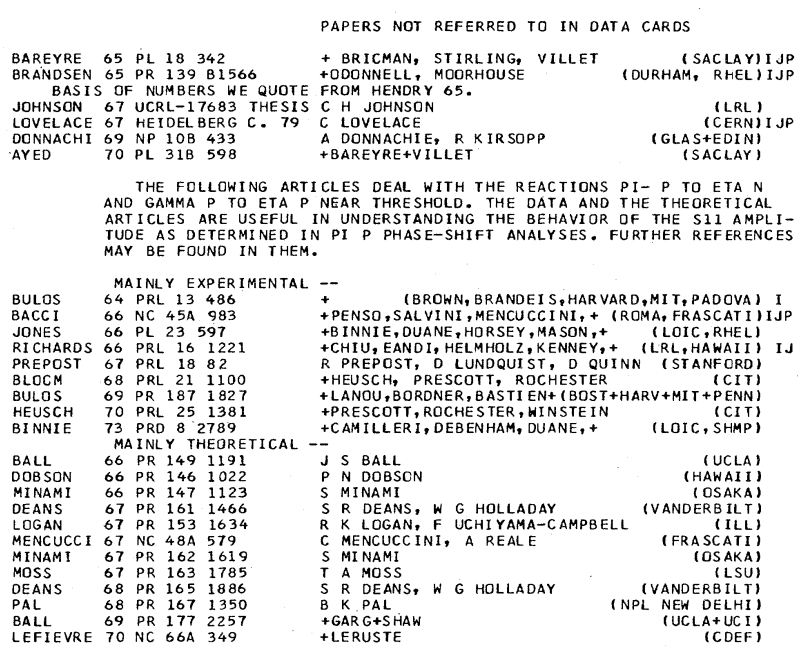

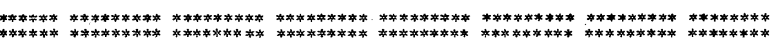

$109 \mathrm{~N} * 1 / 2(1540)$ MASS (MEV) $\quad 11 / 77$

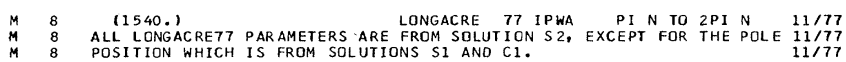

109 N*1/2(1540) WIDTH (MEV)

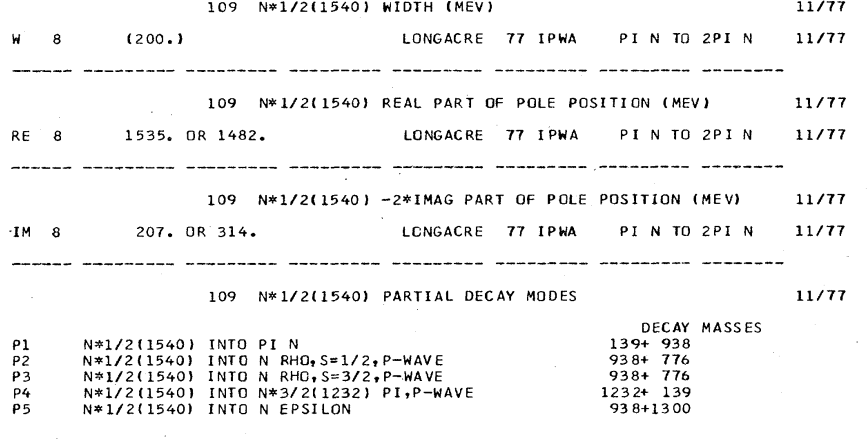

$109 \quad N * 1 / 2(1540)$ BRANCHING RATIOS $11 / 77$

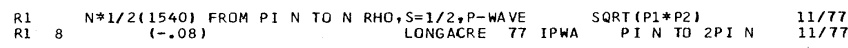

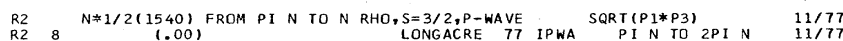

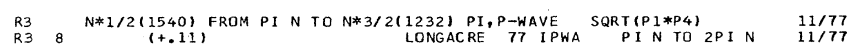

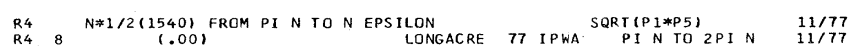

\#*************************************************************************1

REFERENCES FOR $N * 1 / 2(1540)$

LONGACRE 77 NP $B 122493$
ALSO 76 NP B108 365

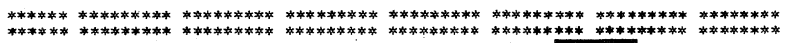

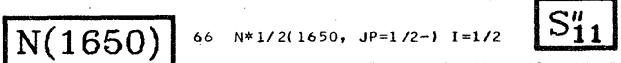

the existence of this resonance is Well established.

Data Card Listings For notation, see key at front of Listings.

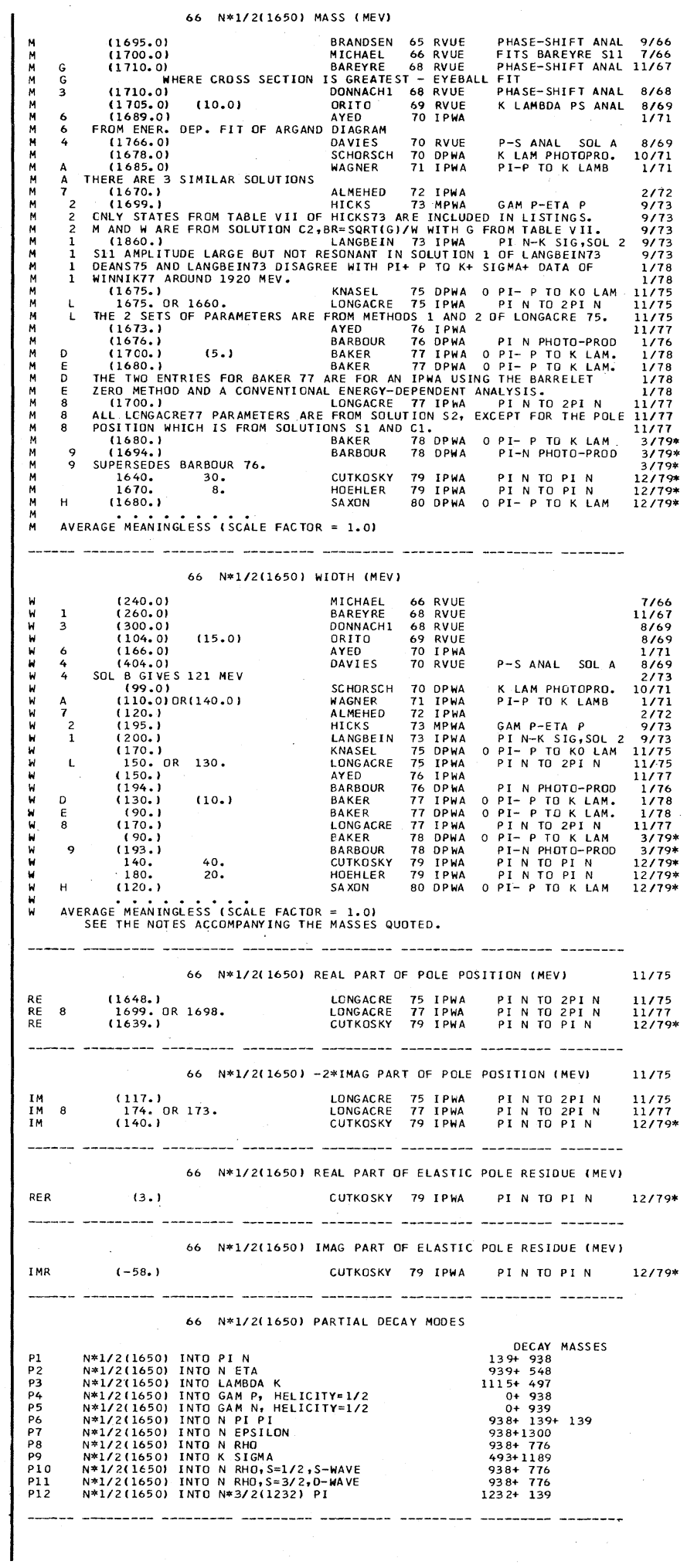




\section{Data Card Listings}

For notation, see key at front of Listings.

Baryons

$\mathrm{N}(1650), \mathrm{N}(1670)$

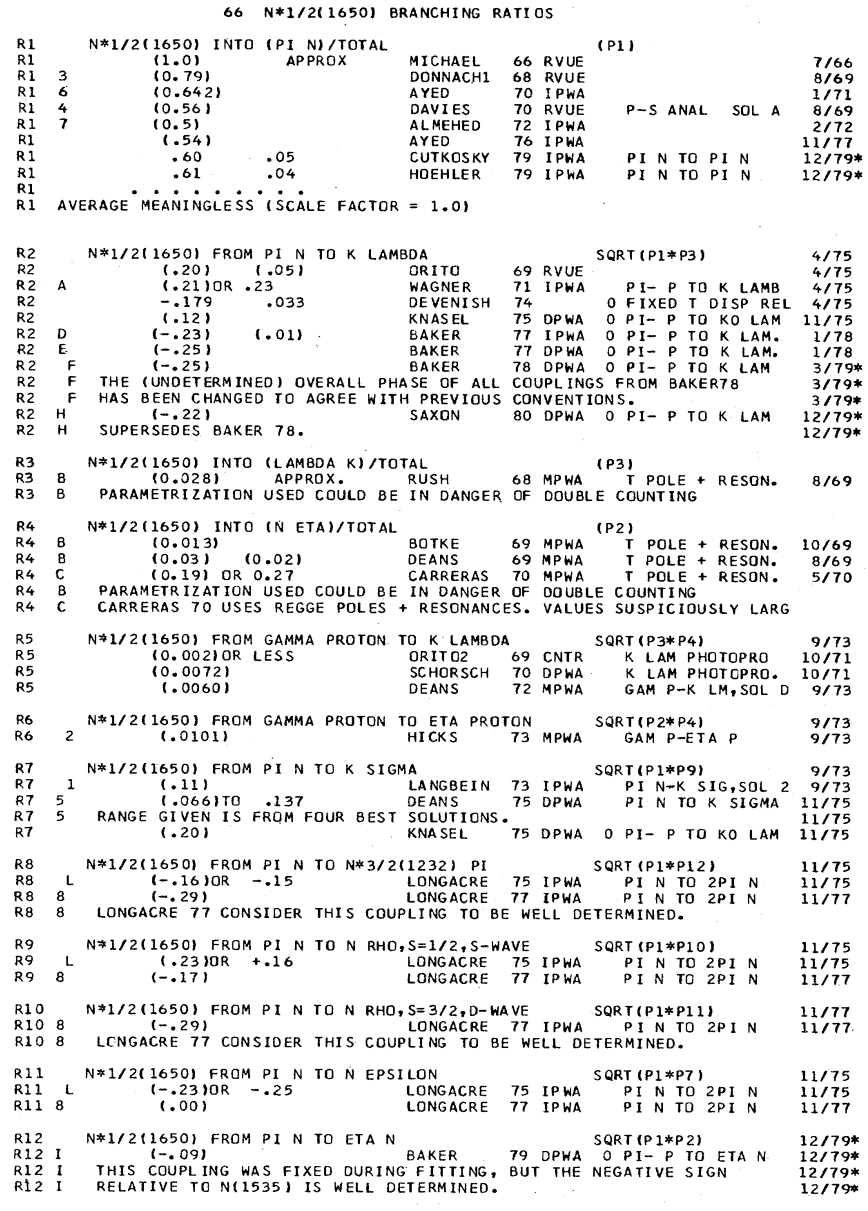

$66 N * 1 / 2(1650)$ PHOTON DECAY AMPL (GEV**-1/2)

FOR DEFINITION OF GAMMA-NUCLEON DECAY AMPLITUDES, SEE MINI-
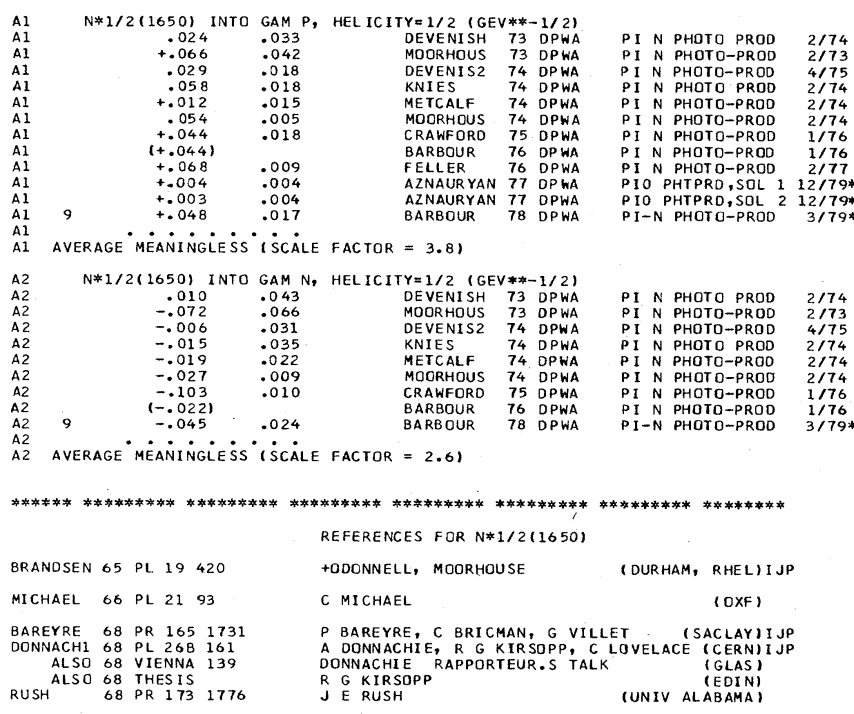
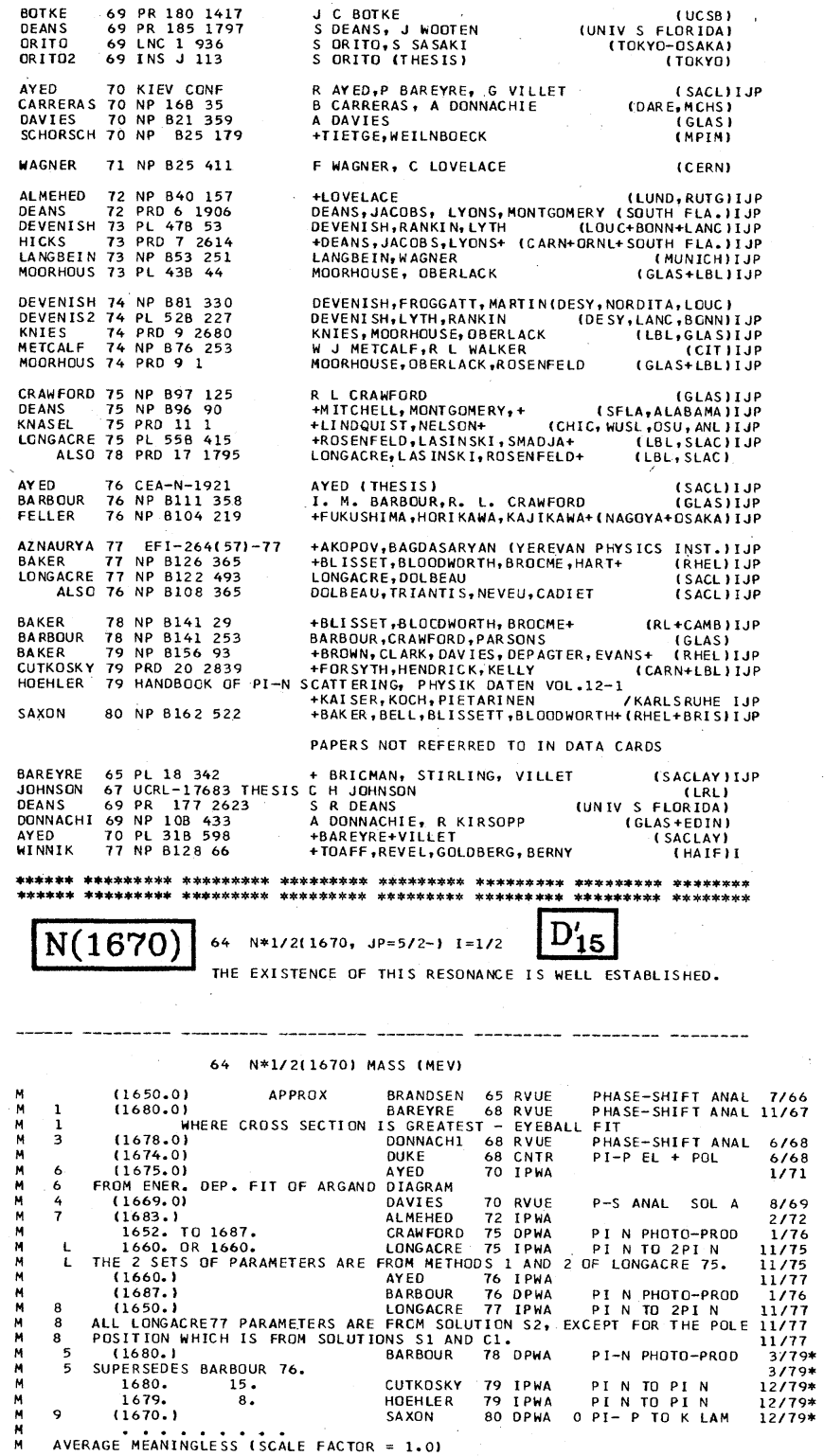

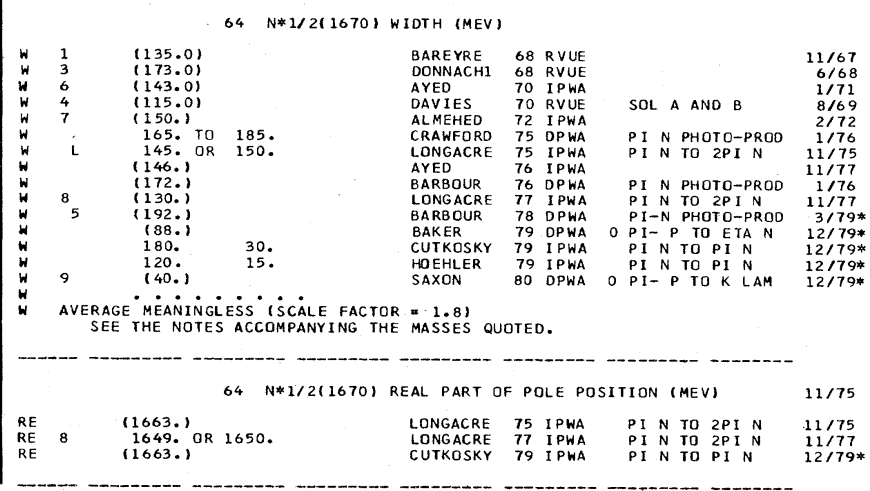




\section{Baryons $\mathrm{N}(1670)$}

\section{Data Card Listings} For notation, see key at front of Listings.

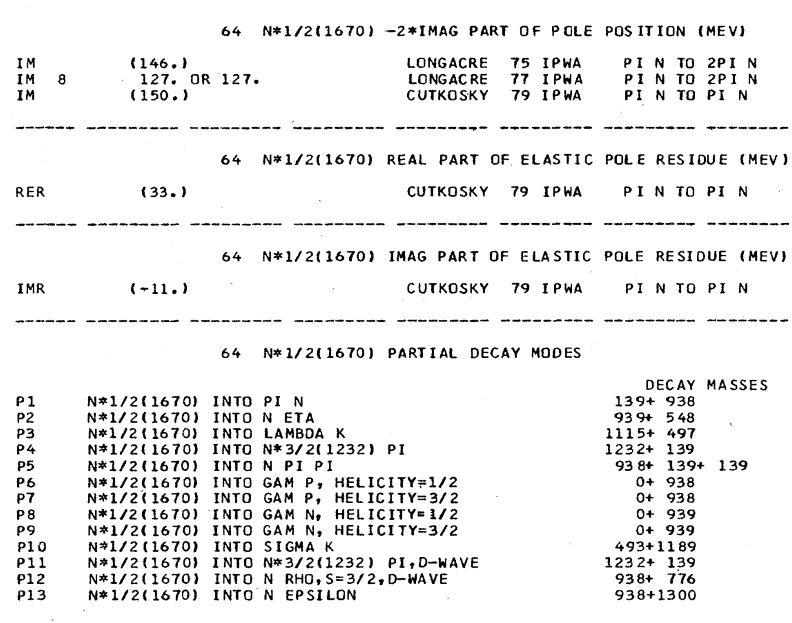

$64 N * 1 / 2(1670)$ BRANCHING RATIOS

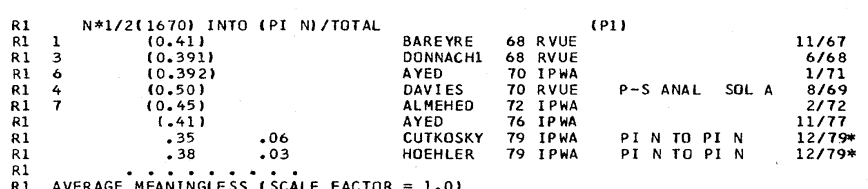

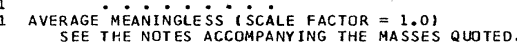

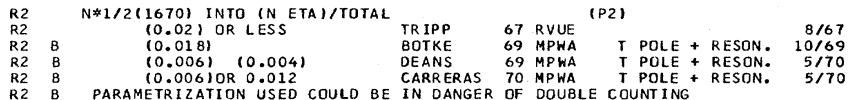

R3
R3 $* 1 / 2(1670)$ INTO (LAMBOA K) $1 /$ TOTAL
TRIPP

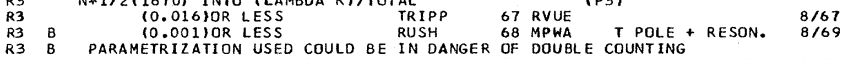
$R 4 \quad N * 1 / 2(1670)$ INTO $(N * 3 / 2(1232)$ PI)/TOTAL (P4)

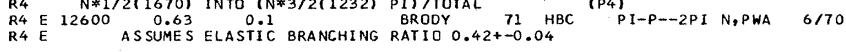

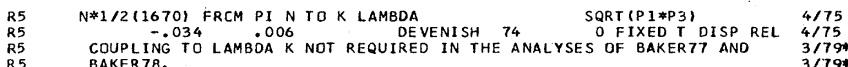

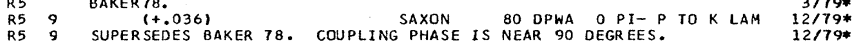

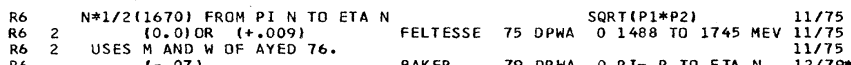

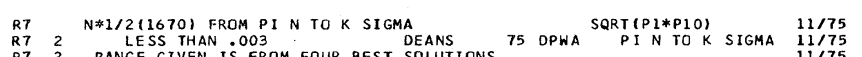

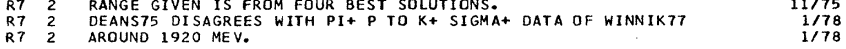

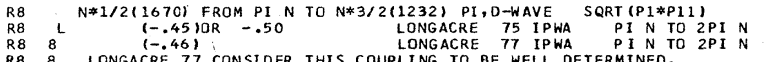
$R 8$ \& LONGACRE 77 CONSIDER THIS COUPLNG TO BE WELL DETERMINED
R8
NOVOSELLE 78 IPWA PI N TO 2 PI N R8 . N 8 N FIT TO LONGACRE 75 IPWA.

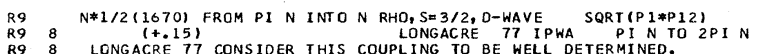
R10
R10 SEE NCTE PRECEDING THE N*1/2(1688) INELASTIC DECAY MODE MEASUREMENTS. $64 N * 1 / 2(1670)$ PHOTON DECAY AMPL (GEV**-1/2) FOR DEFINI IION OF GAMMA-NUCLEON DECAY AMPLITUDES, SEE MINI-
REVIEW PRECEDING THE BARYON LISTINGS.

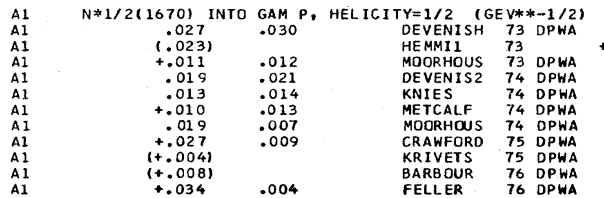

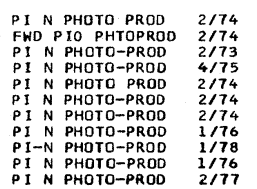

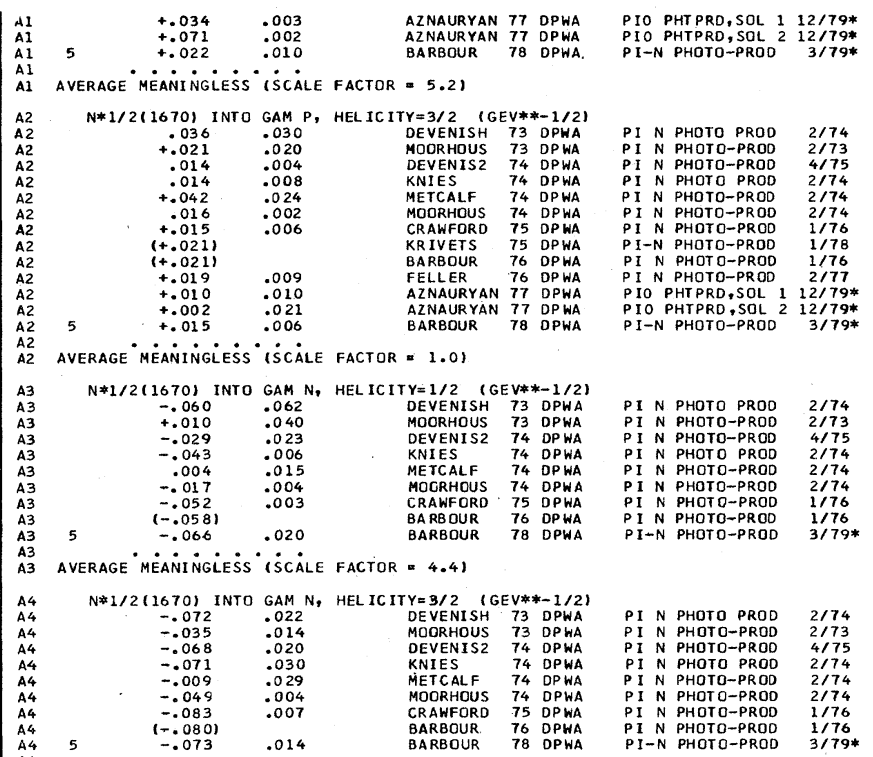

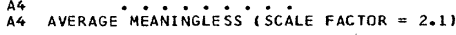

BRANDSEN 65 PL 19420 +ODONNELL, MOORHOUSE (DURHAM, RHELIIJP TRIPP 67 NP B3 10 + LEITH, + (LRL, SLAC, CERN, HEID, SACLAY) $\begin{array}{lllllll}\text { BAREYRE } & 68 & \text { PR } 165 & 1731 & \text { P BAREYRE, C BRICMAN, G VILLET } & \text { (SACLAY) IJPP } \\ \text { DCNNACH1 } & 68 & \text { PL } 268 \text { 161 } & \text { A DONACHIE, R G K IRSOPP, C LOVELACE (CERN)IJP }\end{array}$

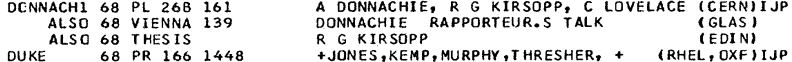

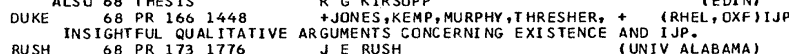

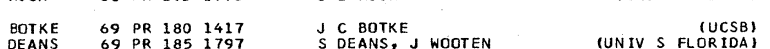
$\begin{array}{lllc}\text { AYEO } & 70 \mathrm{KIEV} \text { CONF } & \text { R AYED, P BAREYRE, G VILLET } & \text { (SACL) I JP } \\ \text { CARRERAS } 700 \text { NP } 16835 & \text { B } 35 \text { CARRERAS, A DONNACHIE } & \text { (DARE, MCHS) } \\ \text { DAVIES } 70 \text { NP B21 } 359 & \text { A DAVIES } & \text { (GLAS) }\end{array}$ $\begin{array}{lllllll}\text { BRODY } & 71 & \text { PL } & 34 B & 665 & \text { +CASHMORE+ .. +HERNDON+.. } & \text { (SLAC+LRL) } \\ \text { WAGNER } & 71 & \text { NP B25 } & 411 & \text { F HAGNER, } & \text { (CERN) }\end{array}$ ALMEHED 72 NP 840157 HOVELACE (LUND, RUTGIIJP

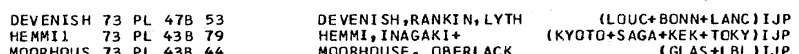
DEVENISH 74 NP 881330 DEVENISH, FROGGATT, MART INCDESY, NORDITA, L OUCI,

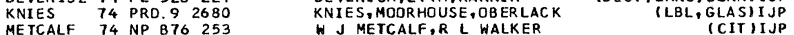
METCALF $74 \mathrm{NP} B 76253$
MOORHOUS 74 PRO 91

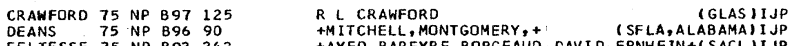

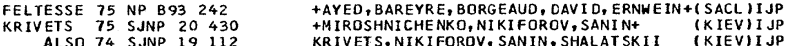

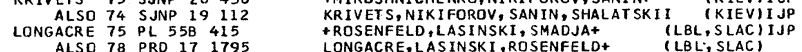

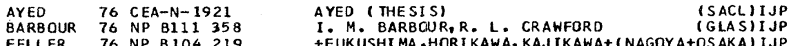
AZNAURYA 77 EFI-264(57)-77 +AKOPOV, BAGDASARYAN (YEREVAN PHYSICS INST. IIJP
LONGACRE 77 NP B 122493 LONGACRE, DOLBEAU LONGACRE 77 NP 8122493
ALSO 76 NP B108 365 $\begin{array}{lllll}\text { BARBOUR } 78 \text { NP B141 } 253 & \text { BARBOUR, CRAWFORD, PAR SONS } & \text { (GLAS) } \\ \text { NOVOSELL } 78 \text { NP } 8137509 & \text { D. E. NOVOSELLER } & \text { (CAL TECHIIJP } \\ \text { ALSO } 78 \text { NP B } 137445 & \text { D. E. NOVOSELLER } & \text { (CAL TECHIIJP }\end{array}$ $\begin{array}{llll}\text { BAKER } & 79 \text { NP } 815693 & \text { +BROWN, CLARK, DAVIES, DEPAGTER, EVANS + (RHEL) IJP } \\ \text { CUTKOSKY } 79 \text { PRD } 202839 & \text { TFOSYTH, HENDICK, KELLY } & \text { (CARN+ LBLIIJP }\end{array}$

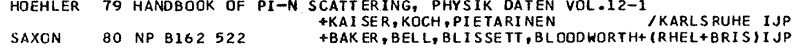

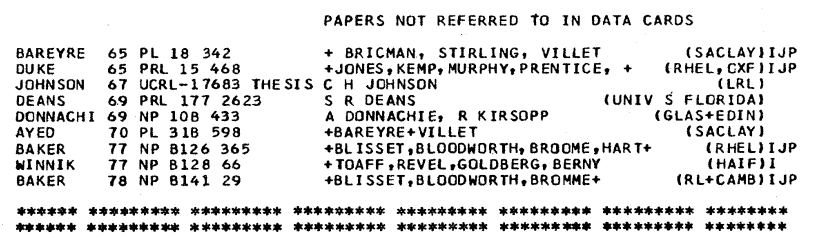




\section{Data Card Listings}

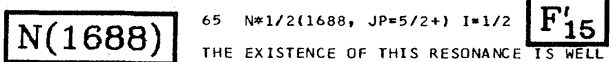

\begin{tabular}{llllll}
\multicolumn{7}{c}{$c$} \\
$M$
\end{tabular}

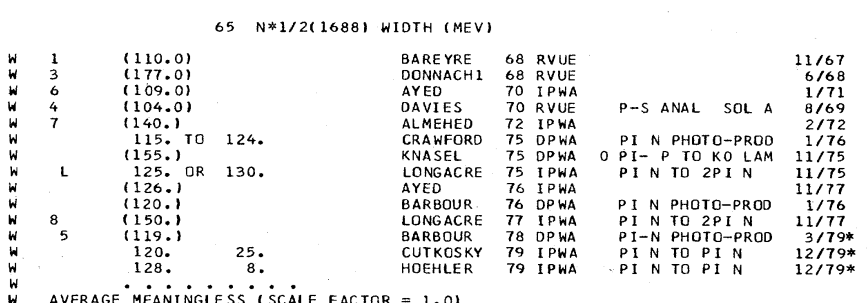

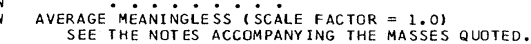

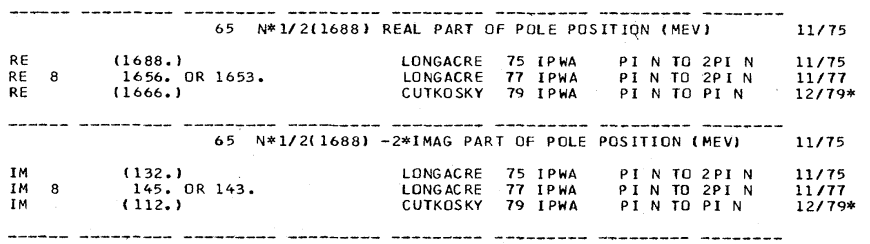

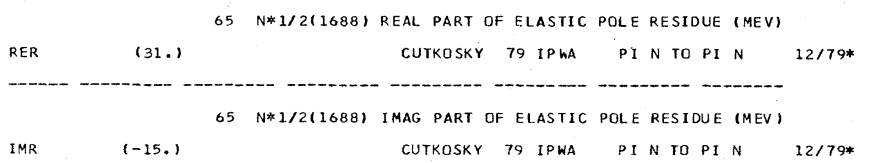

$65 N * 1 / 2(1688)$ PARTIAL DECAY MODES

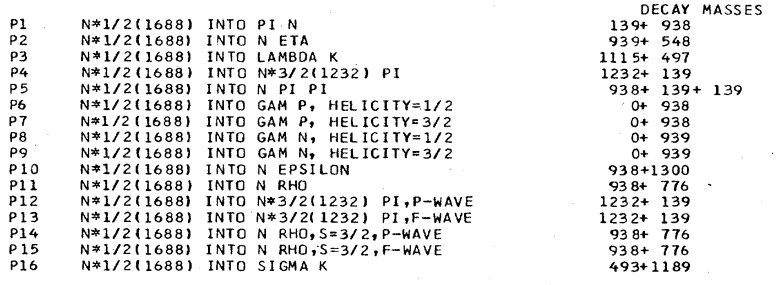

65 N*1/2(1688) BRANCHING RATIOS

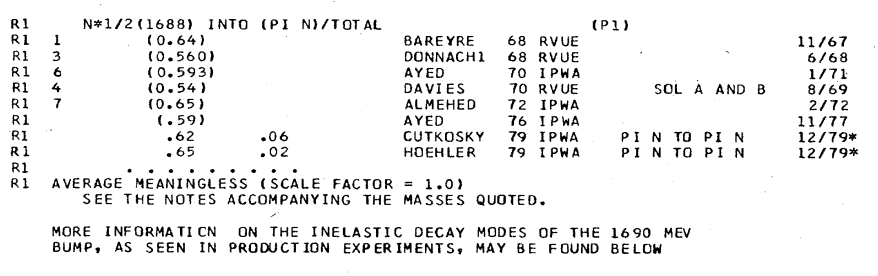

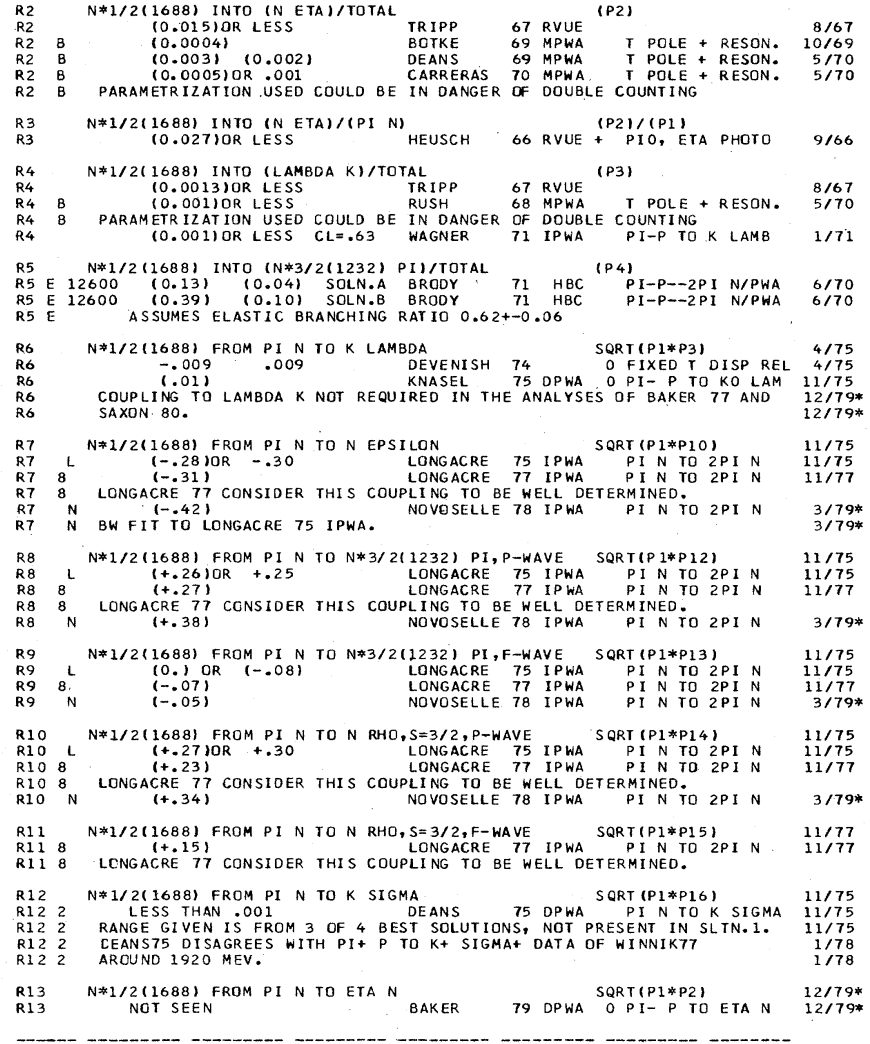
$65 N * 1 / 2(1688)$ PHOTON DECAY AMPL (GEV**-1/2)

FOR DEFINI YION OF GAMMA-NUCLEON DECAY AMPL ITUDES, SEE MINI-
REVIEW PRECEDING IHE BARON LISTNGS.

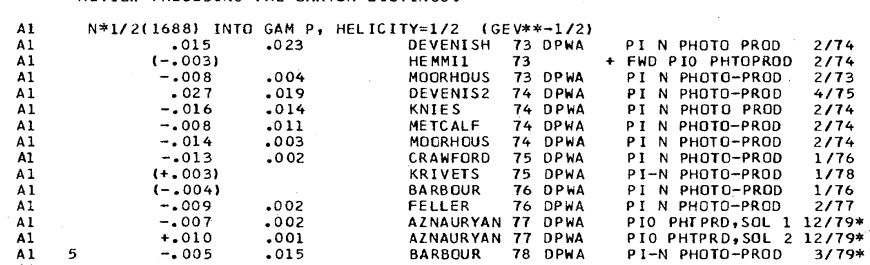

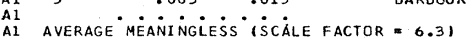

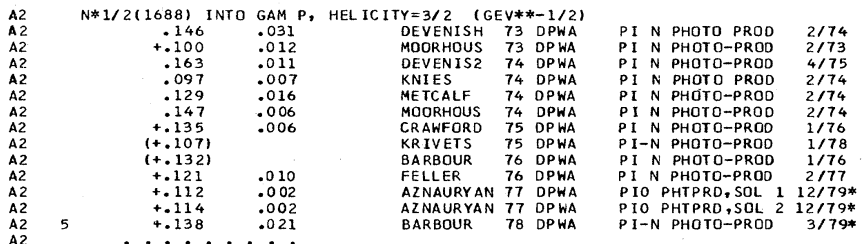

A2 average MeÁningless is is CAle factor $=3.11$

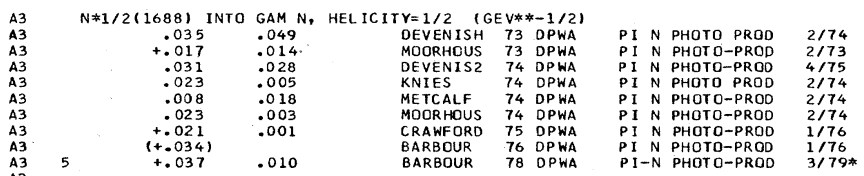

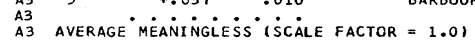




\section{Baryons \\ $\mathrm{N}(1688), \mathrm{N}(1700)$}

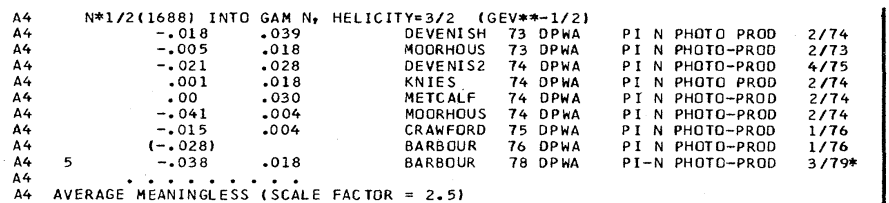

REFERENCES FOR $N * 1 / 2(1688$

SEE A PREVIOUS EDITION (RMP 37, 633, 1965 ) FOR EARLIER REFERENCES.

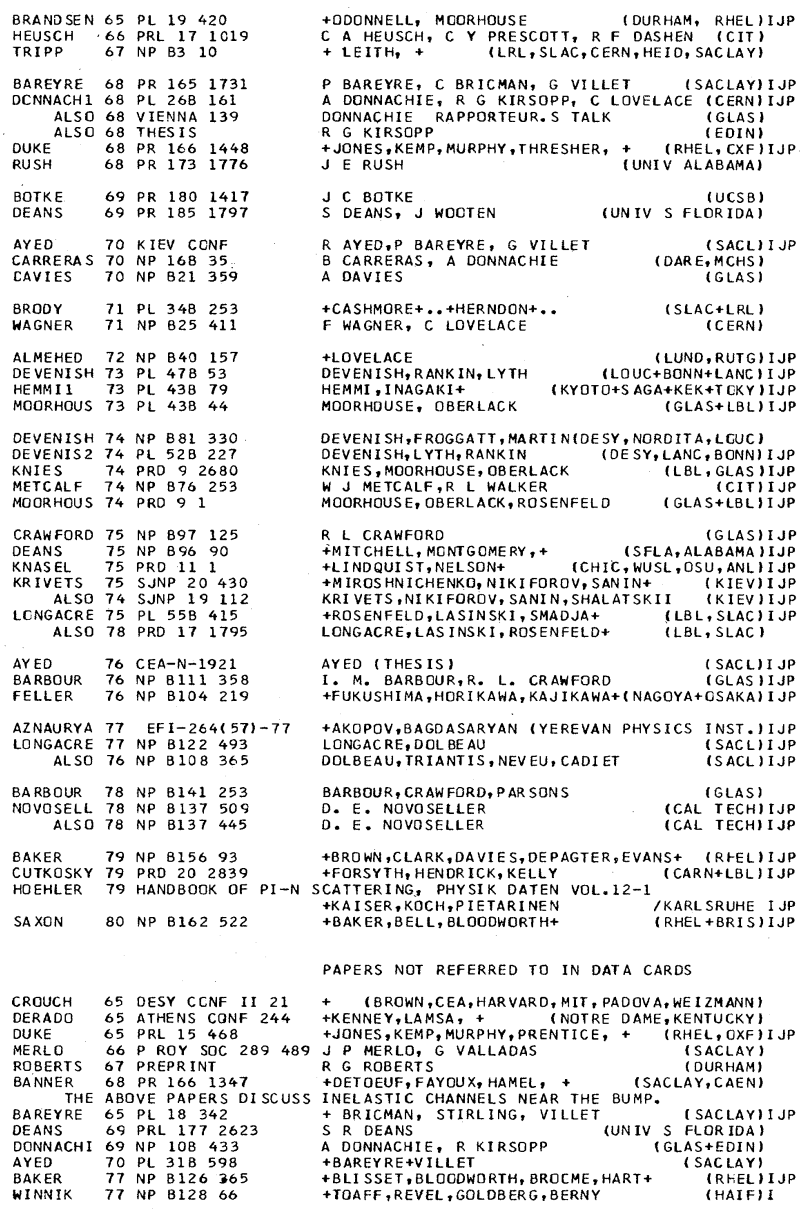

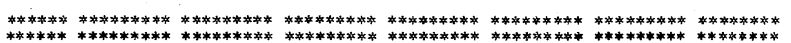

$\mathrm{N}(1700){ }_{18} \quad \mathrm{N*1/2(1700,JP=3/2-1)} \mathrm{I=1/2} \quad \mathrm{D}_{13}^{\prime \prime}$

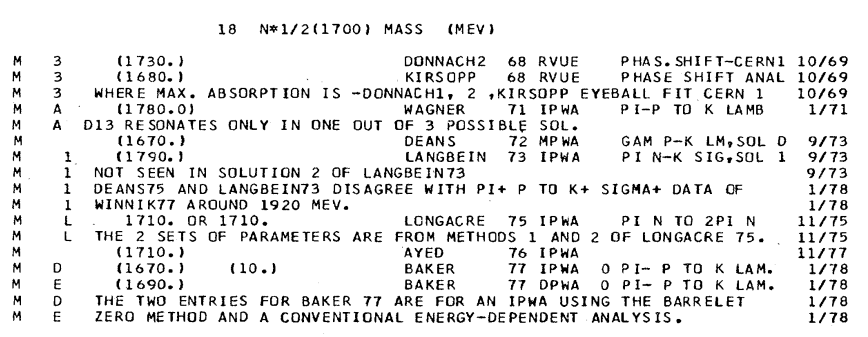

\section{Data Card Listings} For notation, see key at front of Listings.

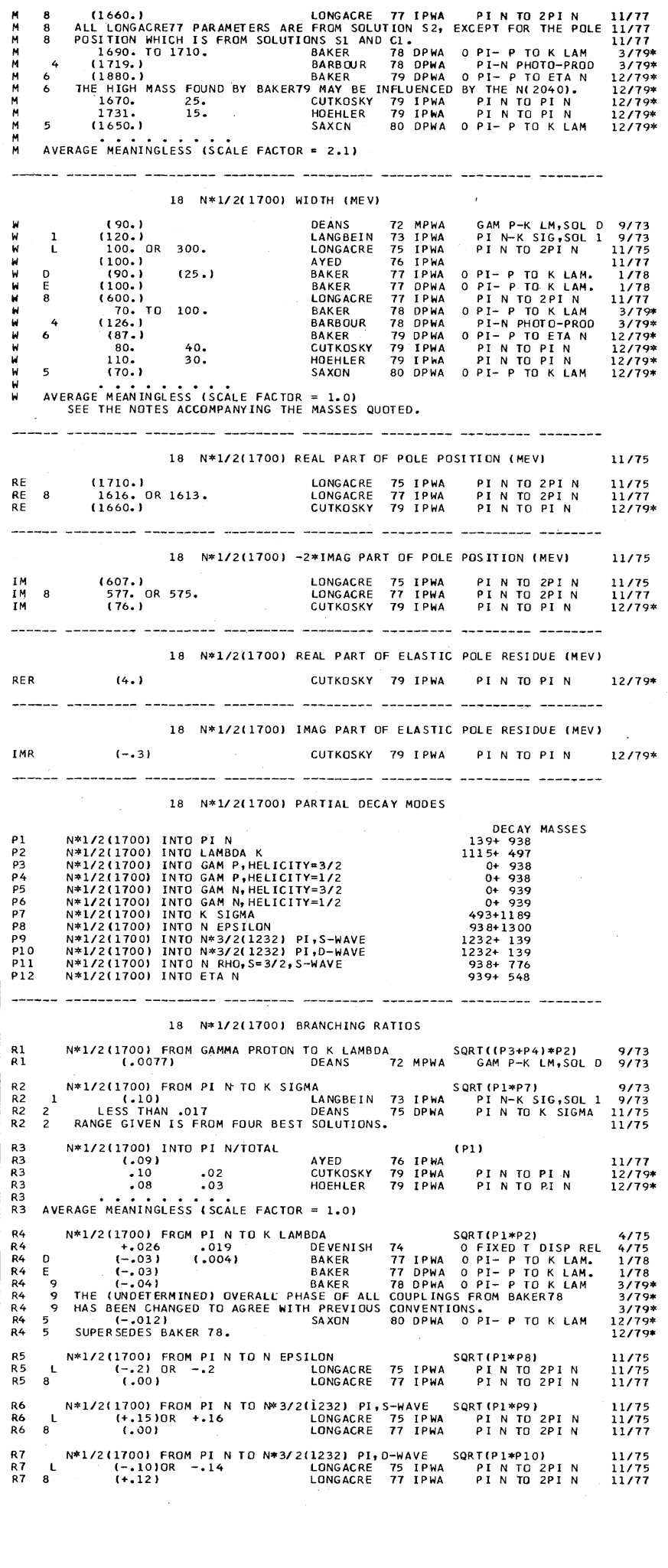




\section{Data Card Listings}

Baryons

For notation, see key at front of Listings.

$\mathrm{N}(1700)$

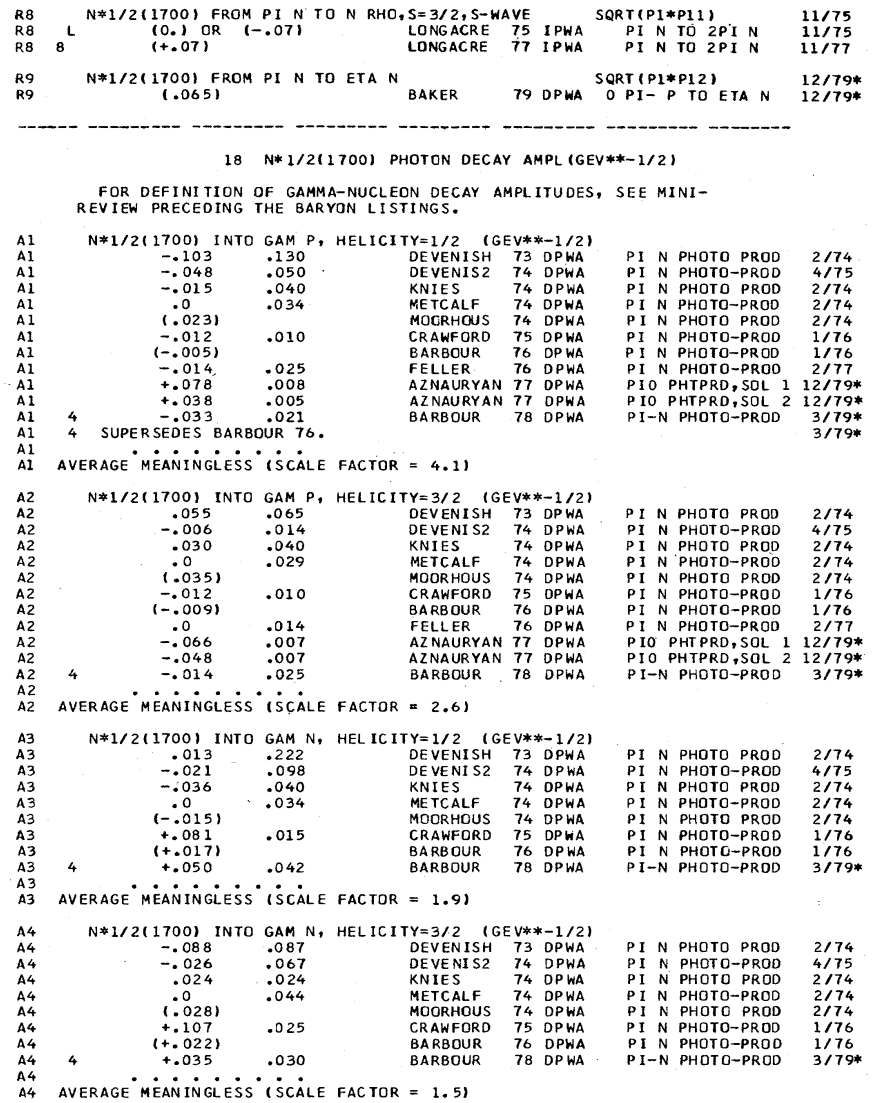

\begin{tabular}{|c|c|c|}
\hline ****** & $* * * * * *$ & $\begin{array}{l}* * * * * * * * * * * * * * * * * * * * * * * * * * * * * * * * * * * * * * * * * \\
\text { REFERENCES FOR } N * 1 / 2(1700)\end{array}$ \\
\hline $\begin{array}{l}\text { DONNACH2 } \\
\text { KIRSOPP } \\
\text { WAGNER } \\
\text { DEANS } \\
\text { DEVENISH } \\
\text { LANGBEIN }\end{array}$ & 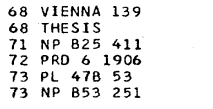 & 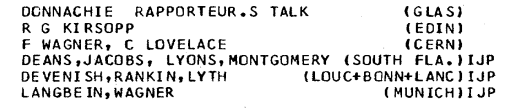 \\
\hline $\begin{array}{l}\text { DEVENISH } \\
\text { DEVENIS2 } \\
\text { KNIES } \\
\text { METCALF } \\
\text { MOORHOUS }\end{array}$ & $\begin{array}{l}74 \text { NP } B 81330 \\
74 \text { PL } 52 B 227 \\
74 \text { PRD } 92680 \\
74 \text { NP } B 76 \quad 253 \\
74 \text { PRD } 91\end{array}$ & 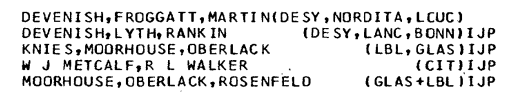 \\
\hline $\begin{array}{l}\text { CRAWFORD } \\
\text { DEANS } \\
\text { LONGACRE } \\
\text { ALSO }\end{array}$ & 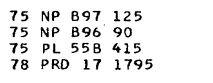 & 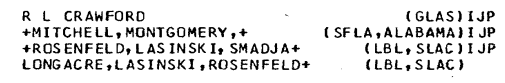 \\
\hline $\begin{array}{l}\text { AYED } \\
\text { BARBOUR } \\
\text { FELLLER }\end{array}$ & $\begin{array}{l}76 \text { CEA-N-1921 } \\
76 \text { NPB B } 111358 \\
76 \text { NPB104 } 219\end{array}$ & $\begin{array}{lr}\text { AYED (THESIS) } & \text { (SACL) IJP } \\
\text { I. M. BARBOUR,R. LL. CRAWFORD } & \text { (GLAS) IIJP } \\
\text { +FUKUSHIMA, HORIKAWA, KAJIKAWA+ (NAGOYA+OSAKA) IJP }\end{array}$ \\
\hline $\begin{array}{l}\text { AZNAURY A } \\
\text { BAKER } \\
\text { LONGACRE } \\
\text { ALSO }\end{array}$ & $\begin{array}{l}77 \text { EFI }-264(57)-77 \\
77 \text { NP B } 126365 \\
77 \text { NP B B } 322493 \\
76 \text { NP B B } 108365\end{array}$ & 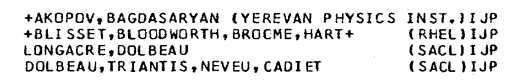 \\
\hline $\begin{array}{l}\text { BAKER } \\
\text { BARBOUR }\end{array}$ & $\begin{array}{lllll}78 & N P & B 141 & 29 \\
78 & N P & B & 141 & 253\end{array}$ & $\begin{array}{l}\text { +BLI ISSET, BLOOOWWORTH, BROOME+ } \\
\text { BARBOUR, CRAWFORD, PARSONS }\end{array}$ \\
\hline $\begin{array}{l}\text { BAKER } \\
\text { CUTKOSKY } \\
\text { HOEHLER }\end{array}$ & $\begin{array}{l}79 \text { NP } 815693 \\
79 \text { PRD } 202839 \\
79 \text { HANDBDOK OF PI }\end{array}$ & 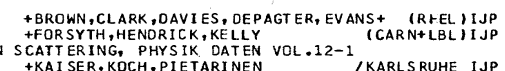 \\
\hline SAXON & 80 NP B162 522 & $\begin{array}{l}\text { +BAKER, BELL, BLISSETT, BLOODWORTH+(RHEL+BRIS)IJP } \\
\text { PAPERS NOT REFERRED TO IN DATA CARDS }\end{array}$ \\
\hline $\begin{array}{l}\text { HERNOON } \\
\text { WINNIK }\end{array}$ & $\begin{array}{l}72 \text { L BL } 1065 \\
77 \text { NP B128 } 66\end{array}$ & 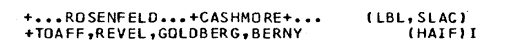 \\
\hline
\end{tabular}

\section{MEV REGION - PRODUCTION EXPERIMENTS}

$20 N * 1 / 211700, J P=\quad, I=1 / 2$ PROOUCTION EXPERIMENTS SEE THE MINI-REVIEW PRECEDING THE N AND DELTA LISTINGS
FOR DI SCUSSION OF PRODUCTION EXPRIMENTS.

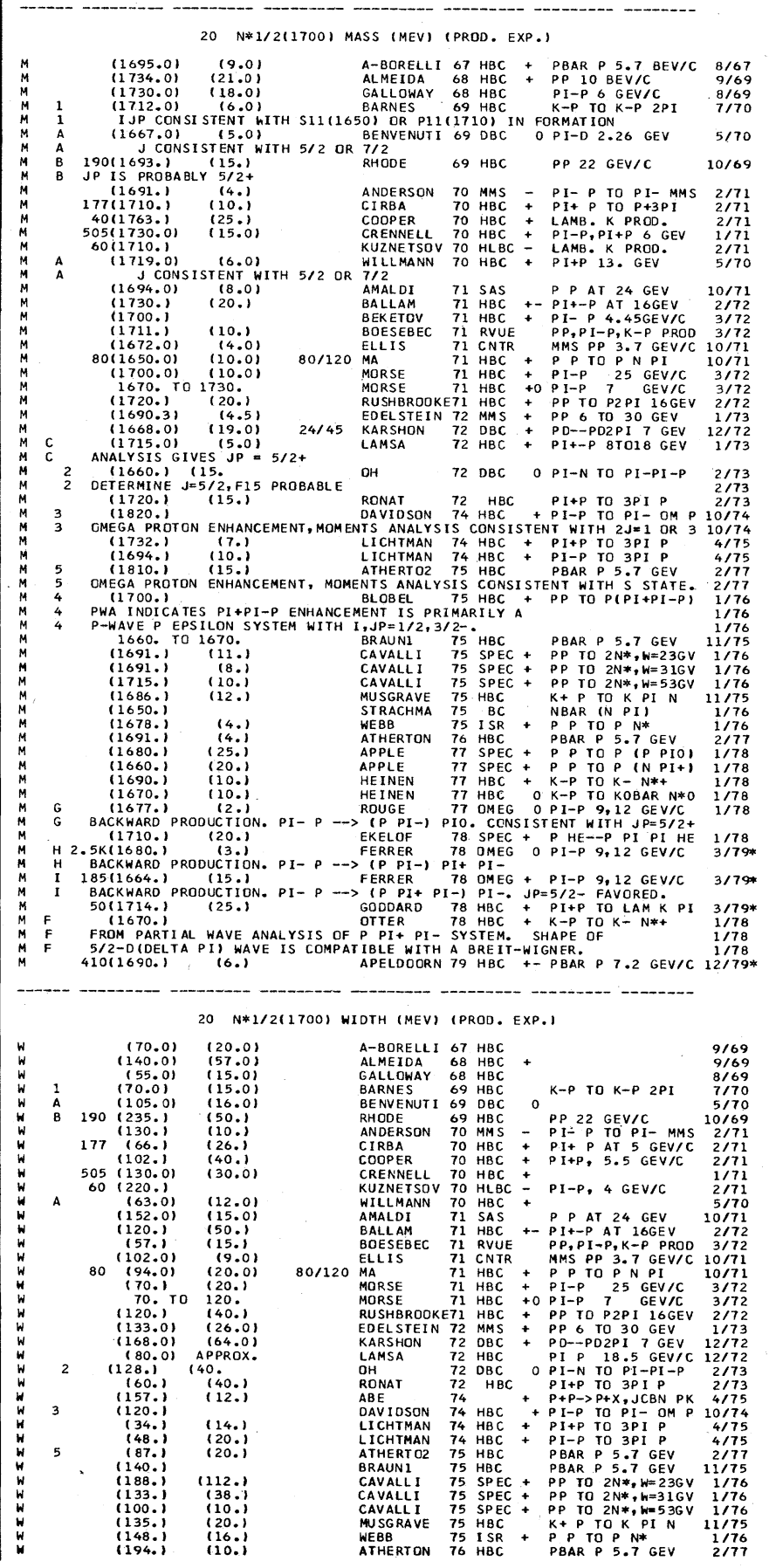




\section{Baryons}

$\mathrm{N}(1700), \mathrm{N}(1710)$

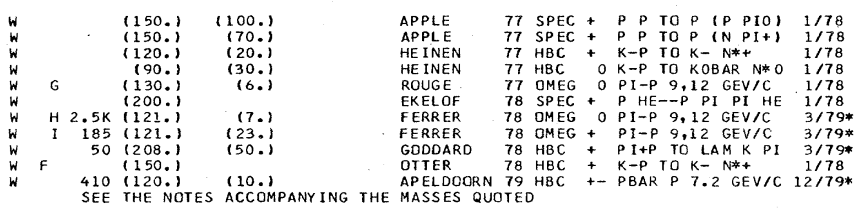

$20 N * 1 / 2(1700)$ PARTIAL DECAY MODES (PROD. EXP.)
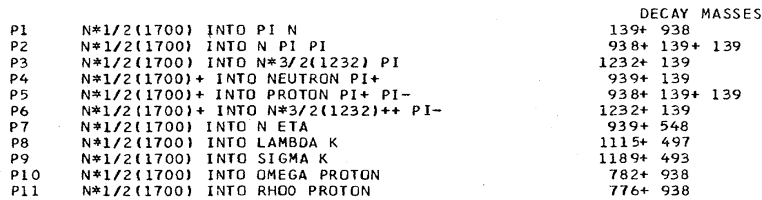

20 N*1/2(1700) BRANCHING RATIOS (PROD. EXP.)

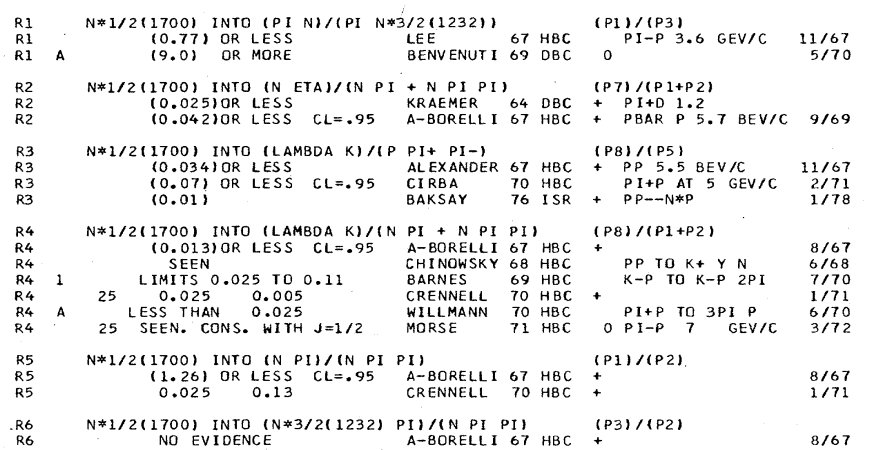

R6
R6 NO EVIDENCE
SEE MERLO 66 FOR A REVIEW.

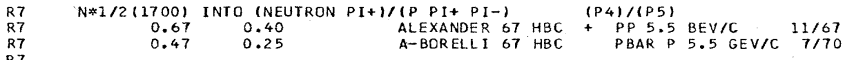

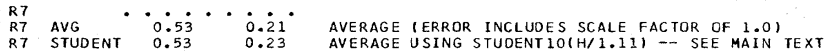

R8 $N * 1 / 2(1700)$ INTO $\left(N * 3 / 2(1232)++P I-1 / 1 P P+P_{I}-\right)(P 6) /(P 5)$

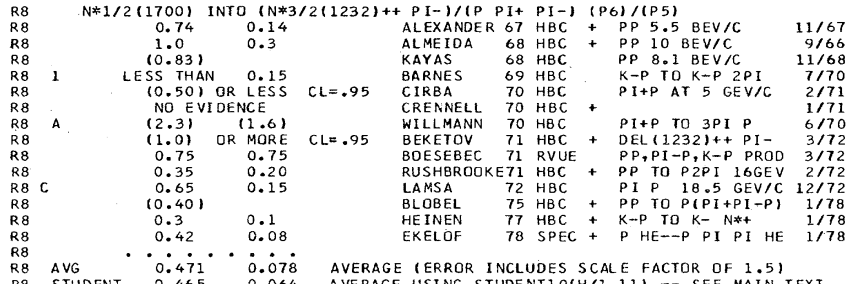

$\begin{array}{lllll}\text { R8 AVG } & 0.471 & 0.078 & \text { AVERAGE } \\ \text { R8 ERROR INCLUDES SCALE FACTOR OF } 1.51 \\ \text { STUDENT } & 0.465 & 0.064 & \text { AVERAGE USING STUDENT1O(H/1.11) - SEE MAIN TEXT }\end{array}$

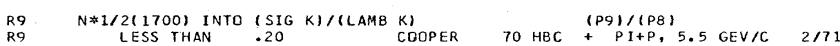

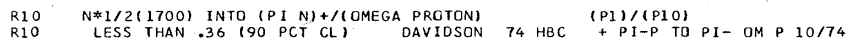

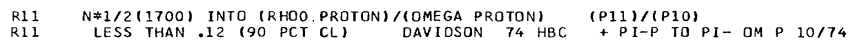
SEE THE NOTES aCCOMPANYING THE MaSSES QUOTED

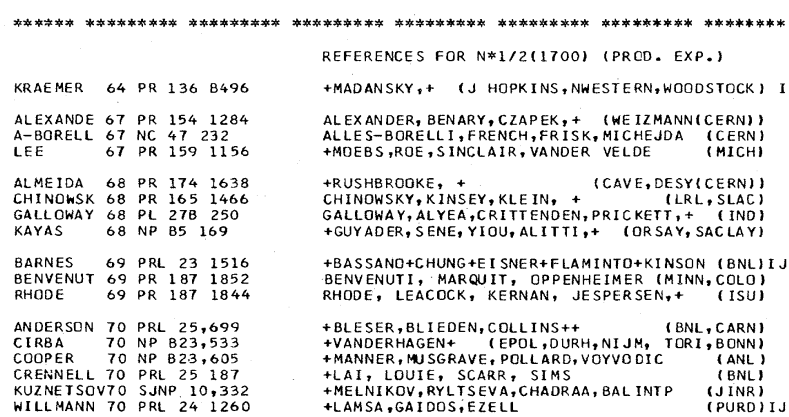

Data Card Listings For notation, see key at front of Listings.

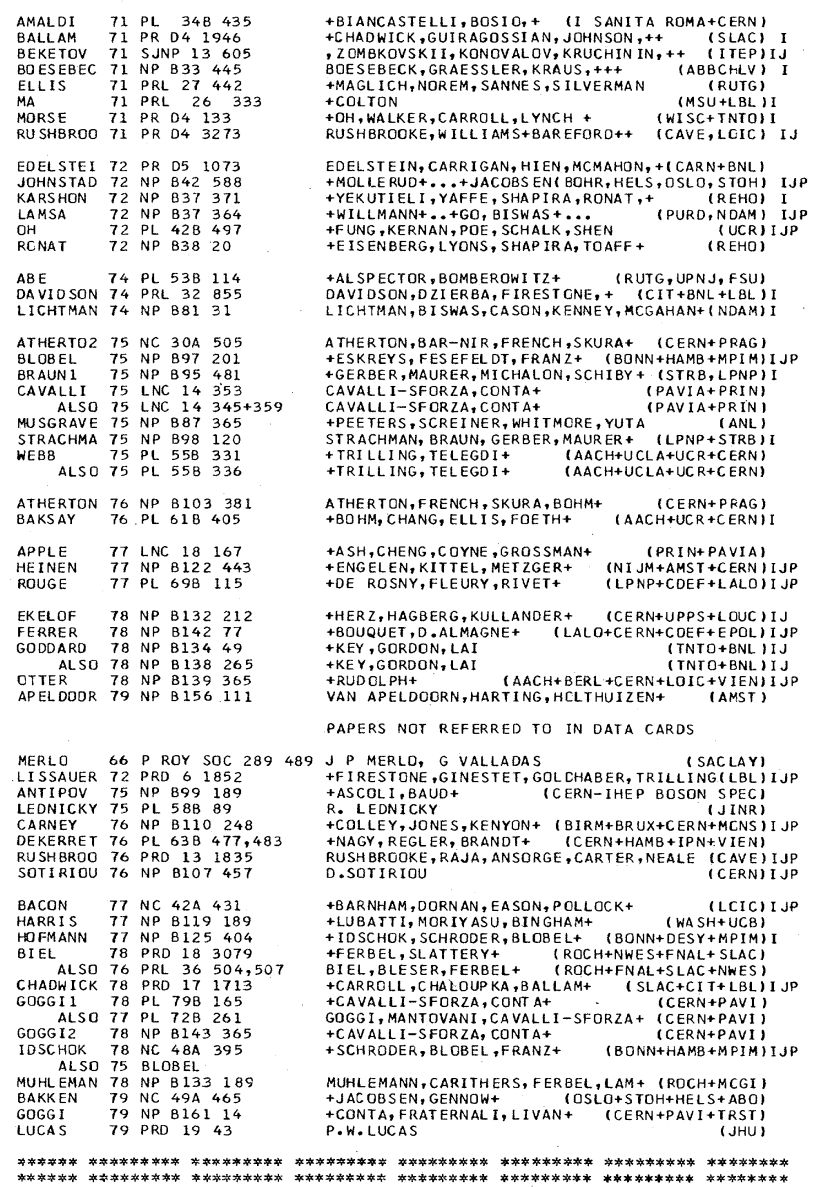

$\mathrm{N}(17 \mathrm{10}){ }_{14}{ }^{\mathrm{N} * 1 / 2(1710, j \mathrm{j}=1 / 2+1 \mathrm{i}=1 / 2} \mathrm{P}_{11}^{\prime \prime}$

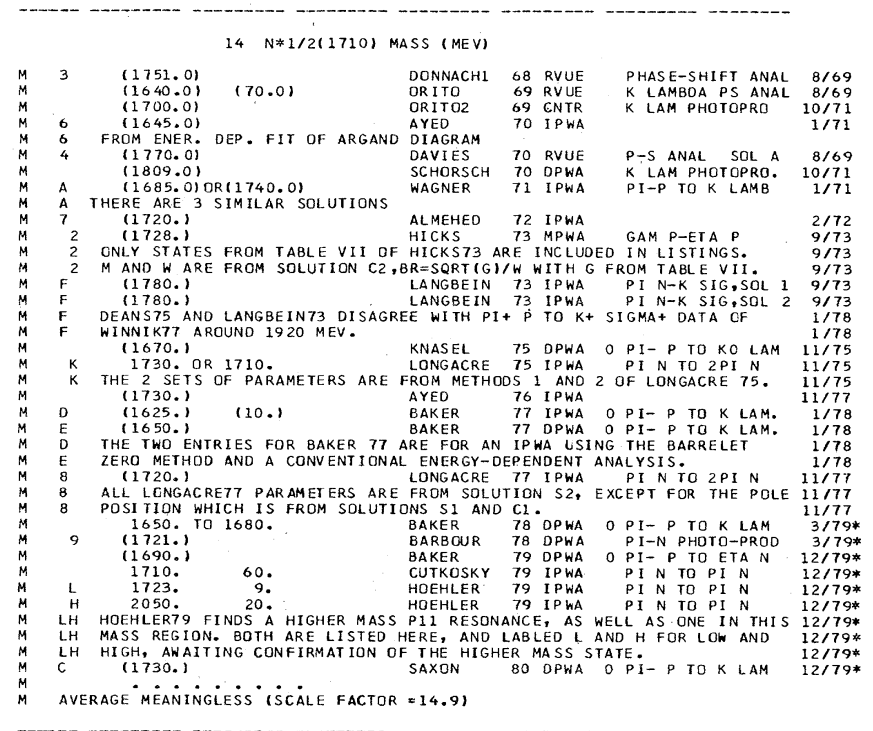




\section{Data Card Listings}

\section{Baryons}

\section{For notation, see key at front of Listings.}

$14 N * 1 / 2(1710)$ WIDTH (MEV)

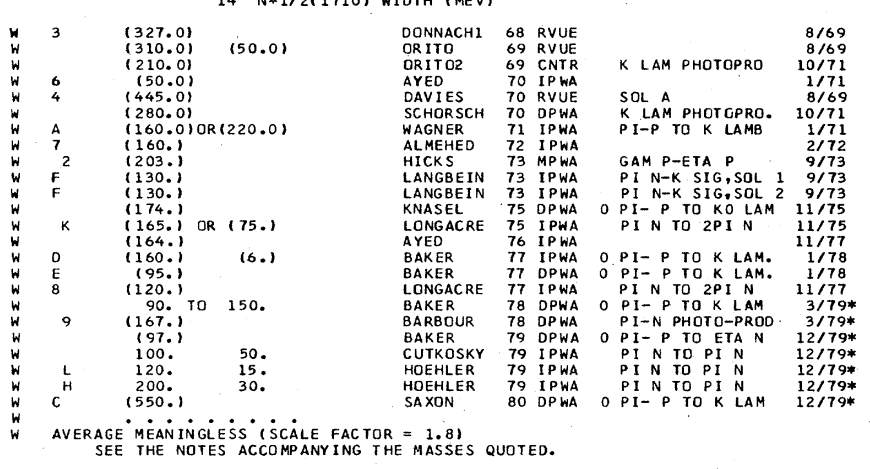

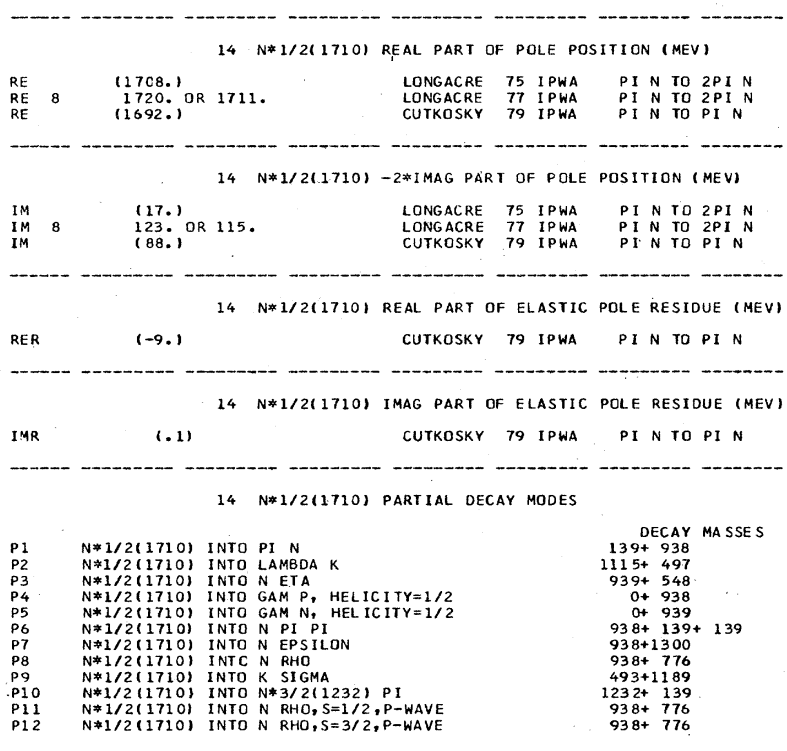

$14 N * 1 / 2(1710)$ BRANCHING RATIOS

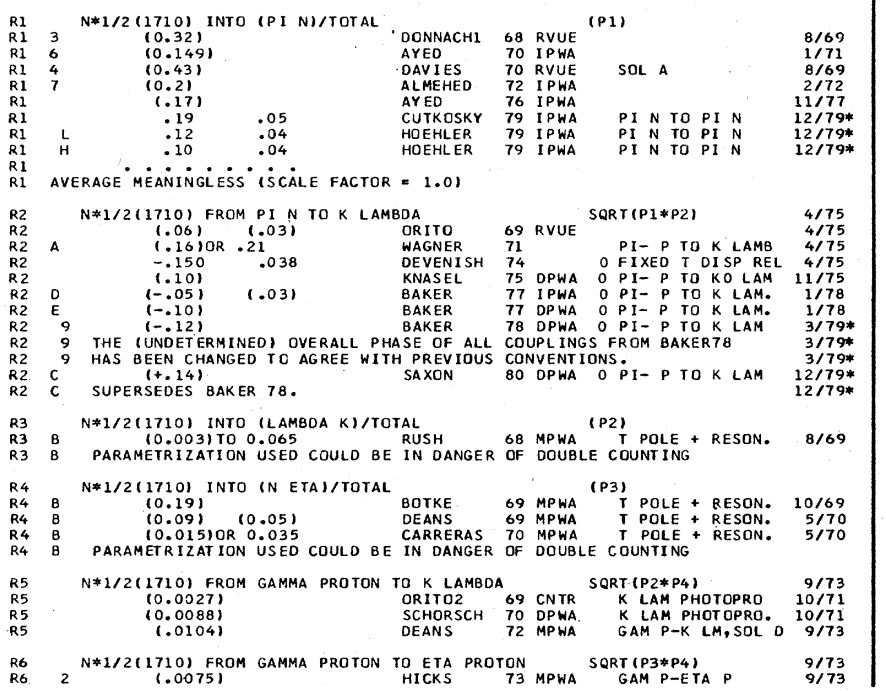

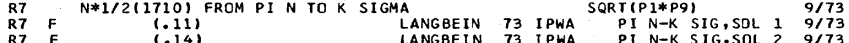

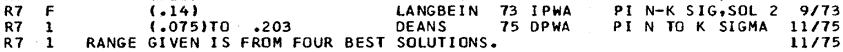

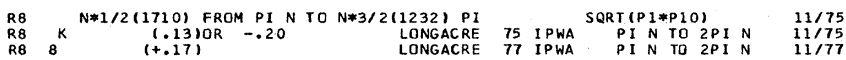

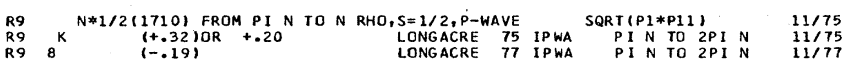

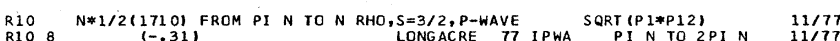

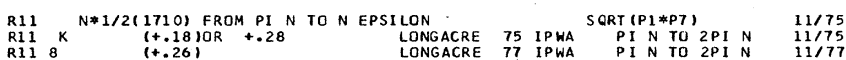

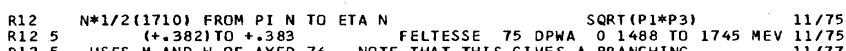

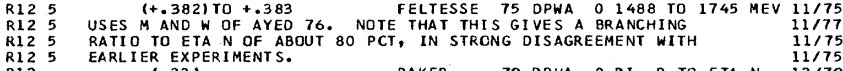
BAKER 79 DPHA FOR DEFINITION OF GAMMA-NUCLEON DECAY AMPLITUDES, SEE MINI-
REVIEW PRECEDING THE BARYON LISTINGS.

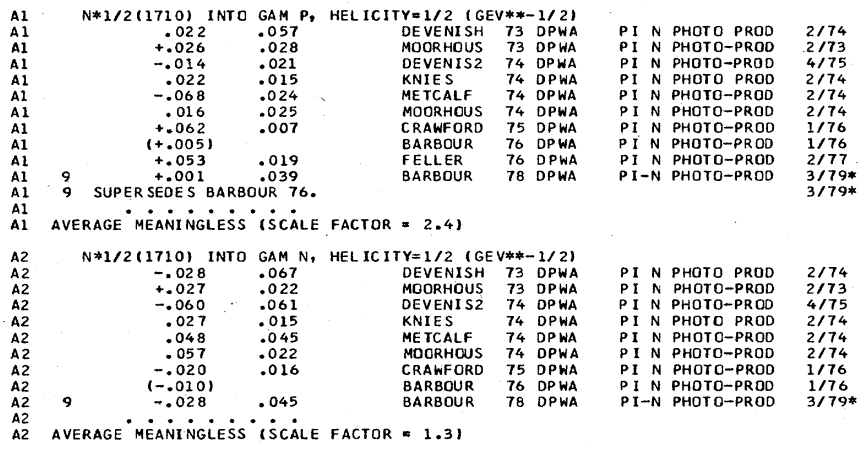

$\begin{array}{rllll}\text { DONNACH1 } & 68 & \text { PL } 268 & 161 \\ \text { ALSO } & 68 & \text { VIENNA } & 139\end{array}$

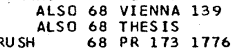
$\begin{array}{llllll}\text { BOTKE } & 69 & \text { PR } & 180 & 1417 \\ \text { DEANS } & 69 & \text { PR } & 185 & 1797\end{array}$ $\begin{array}{llll}\text { DEANS } & 69 & \text { PR } & 185 \\ \text { ORIIT } & 69 & \text { LNC } 1 & 936 \\ \text { CRITO2 } & 69 & \text { INS J } & 113\end{array}$ $\begin{array}{lll}\text { AYED } & 70 \mathrm{KIEV} \text { CONF } \\ \text { CARRERAS } 70 \mathrm{NP} 16 \mathrm{~B} 35\end{array}$ $\begin{array}{lllll}\text { CARERA } & 70 & \text { NP } & 16 B & 35 \\ \text { DAVIES } & 70 & \text { NP } & 359 \\ \text { SCHORSCH } & 70 & \text { NP } & \text { B } 25 & 179\end{array}$ WAGNER 71 NP $B 25411$ $\begin{array}{llllll}\text { ALMEHED } & 72 & \text { NP } & 8 & 40 & 157 \\ \text { DEANS } & 72 & \text { PRD } & 6 & 1906\end{array}$ DEVENISH 73 PL 47853

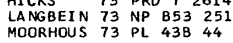
$\begin{array}{lllll}\text { DEVENISH } & 74 & \text { NP } & B 81 & 330 \\ \text { DEVENIS } & 74 & \text { PL } & 528 & 227\end{array}$ DEVENS 74 PRD 92680
KNIES
METCALF 74 NP 876253
MOORHOUS 74 PRD 91 $\begin{array}{lllll}\text { CRAWFORD } 75 & \text { NP } & 897 & 125 \\ \text { DEANS } & 75 & \text { NP } & \text { B96 } & 90\end{array}$ $\begin{array}{lllll}\text { DEANS } & 75 & \text { NP } & B 96 & 90 \\ \text { FELTESSE } & 75 & \text { NP } & 893 & 242\end{array}$ $\begin{array}{lllll}\text { KNASEL } & 75 & \text { PRO } 11 & 1 \\ \text { LONGACRE } & 75 & \text { PL } 558 & 415\end{array}$ ALSO 78 PRD 171795 $\begin{array}{llll}\text { AYED } & 76 & \text { CEA-N-1921 } \\ \text { BARBOUR } & 76 & \text { NP B B111 } 358\end{array}$ $\begin{array}{lllll}\text { FELLER } & 76 & \text { NP } & B 104 & 219 \\ \text { BAKER } & 77 & \text { NP } & \text { B126 } & 365\end{array}$

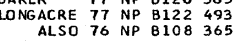
$\begin{array}{lllll}\text { BAKER } & 78 & \text { NP } & B 141 & 29 \\ \text { BARBOUR } & 78 & \text { NP } & B 141 & 253\end{array}$ $\begin{array}{llll}\text { BAKER } & 79 & \text { NP B } 156 \quad 53 \\ \text { CUTKOSKY } 79 & \text { PRD } 202839\end{array}$ CUTKOSKY 79 PRD 202839
HOEHLER 79 HANDBOOK OF SAXON 80 NP 8162522

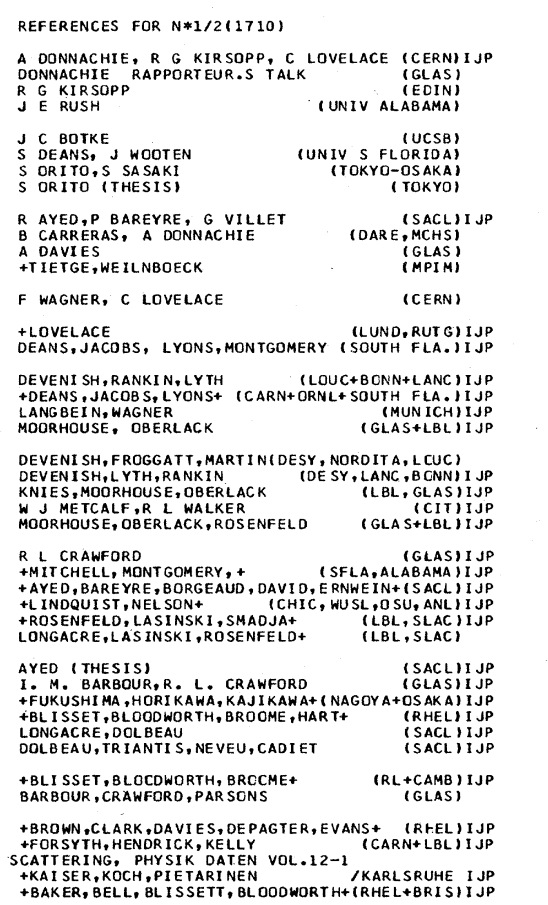


Baryons $\mathrm{N}(1710), \mathrm{N}(1810)$

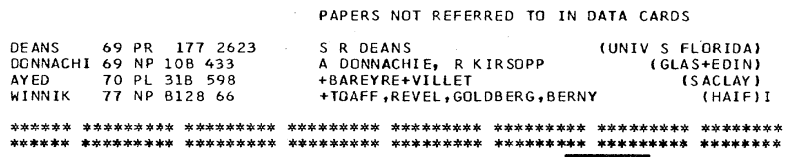

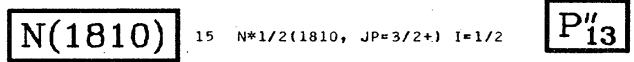

THE EXISTENCE OF THIS RESONANCE IS WELL ESTABLISHED.

\section{Data Card Listings For notation, see key at front of Listings.}

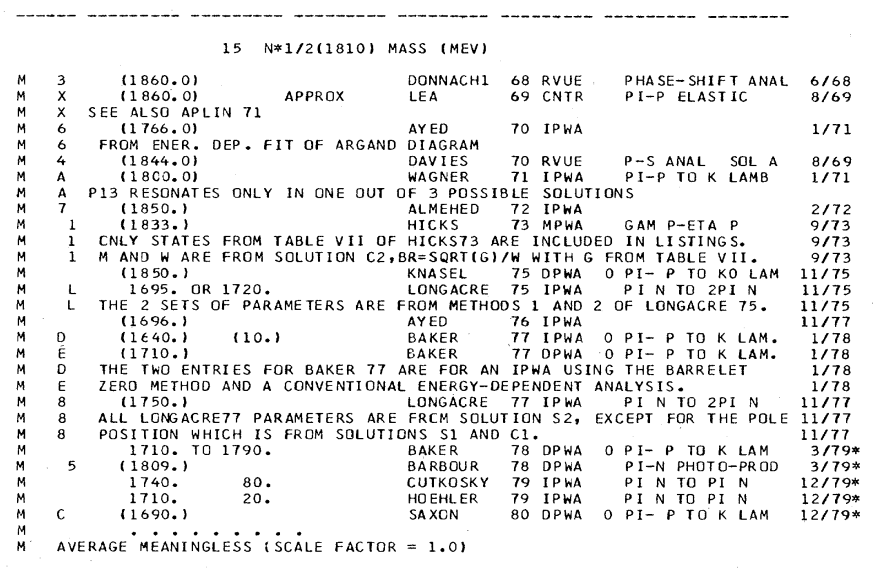

\section{$15 N * 1 / 2(1810)$ WIDTH (MEV)}

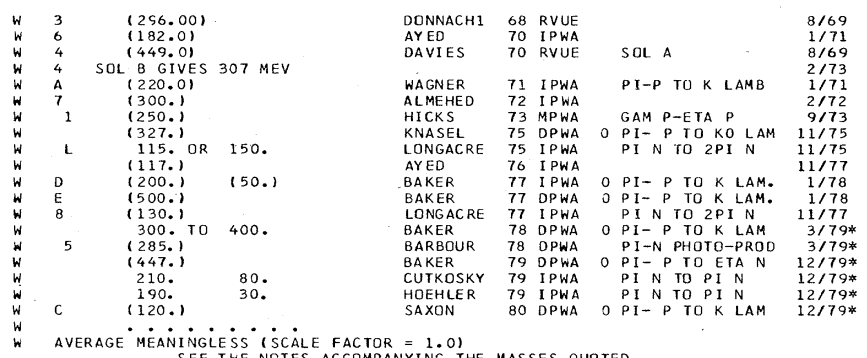

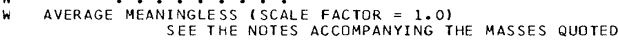

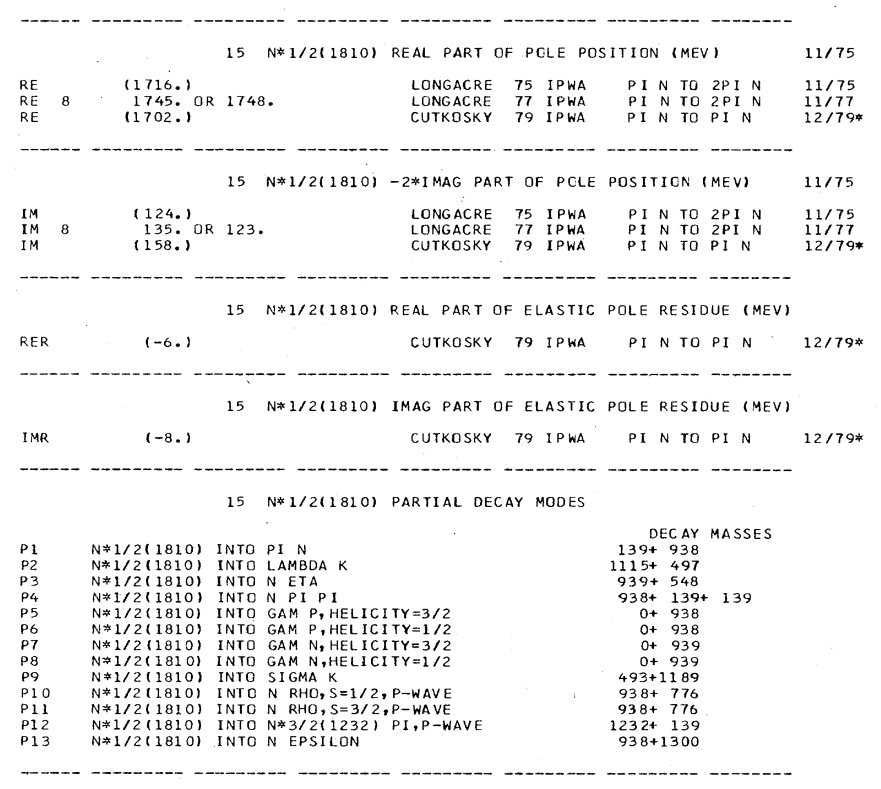

$15 N * 1 / 2(1810)$ BRANCHING RATIOS

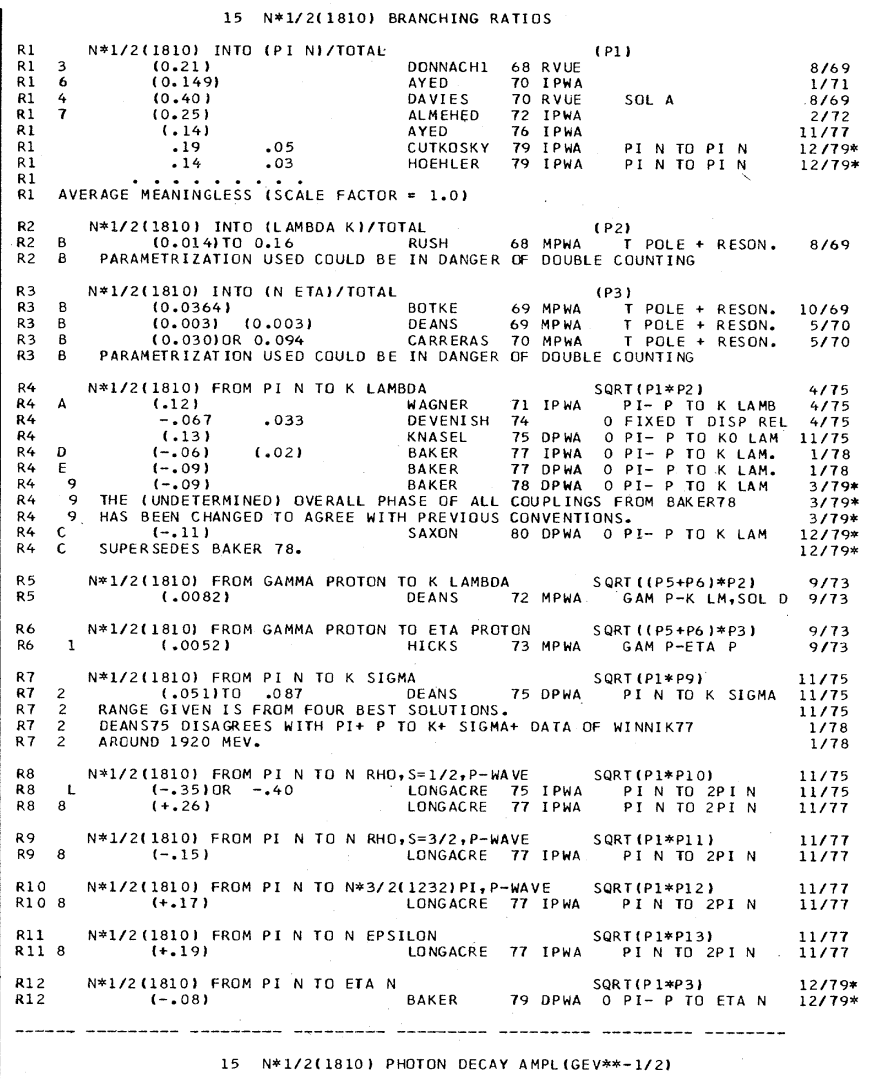

FOR DEFINITION OF GAMMA-NUCLEON DECAY AMPLITUDES, SEE MINI-
REVIEW PRECEDING THE BARYON LISTINGS.

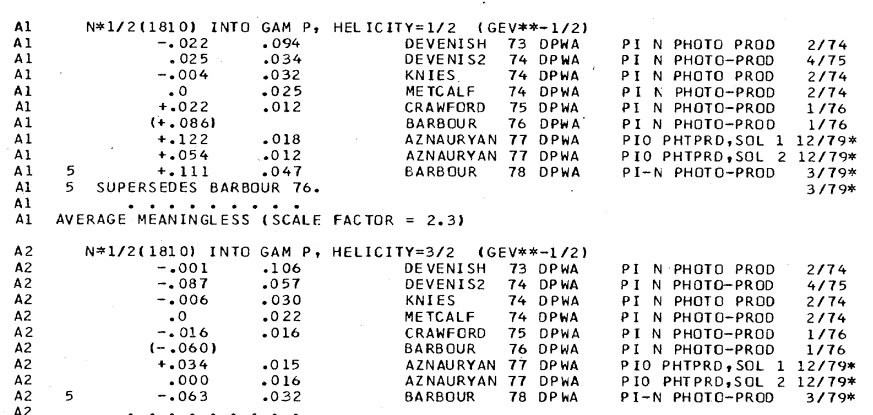

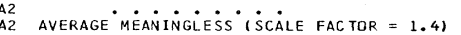

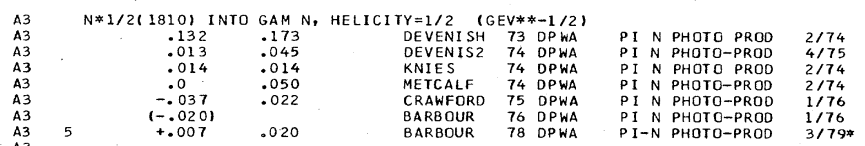

A3 AVERAGE MEANINGGLESS ('SCALE FACTOR $=1.01$

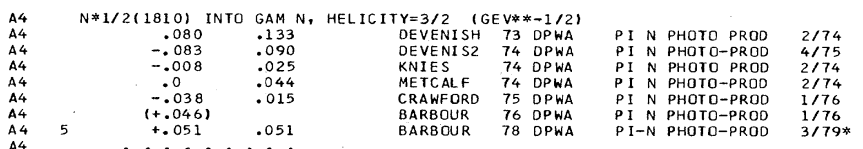

A4
A4 AVERAGE MEANINGLESS 


\section{Data Card Listings}

For notation, see key at front of Listings.

Baryons

$\mathrm{N}(1810), \mathrm{N}(1990)$

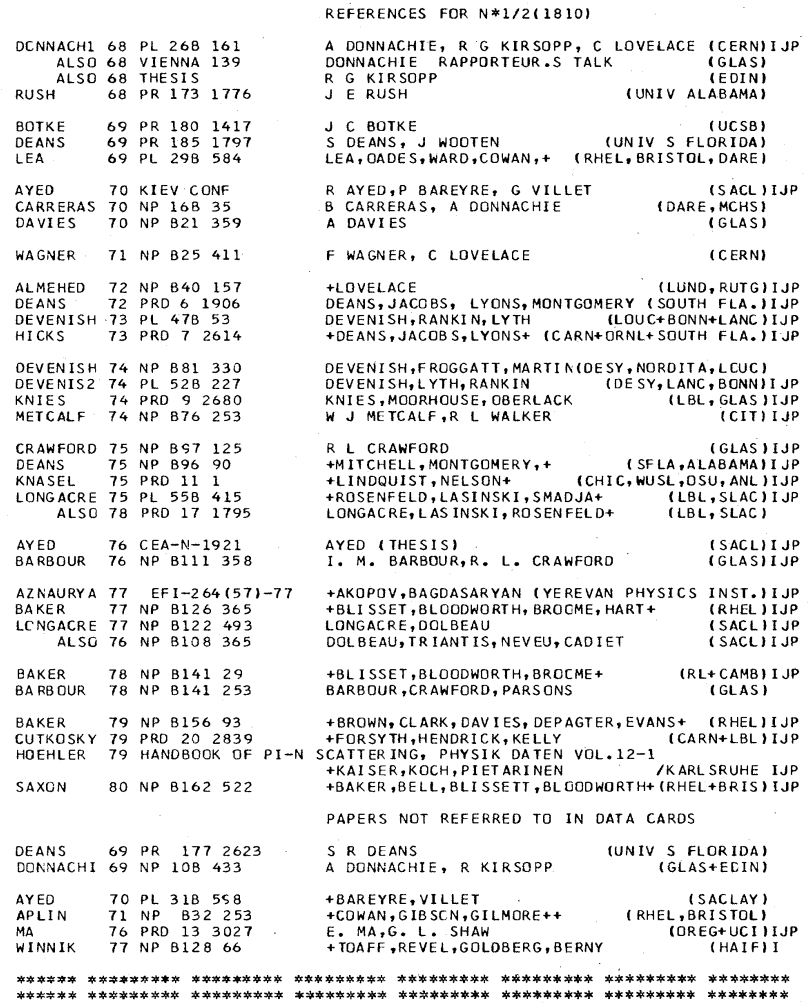

$\mathrm{N}\left(\mathbf{1 9 9 0 )}{ }^{17} \quad \mathrm{~N}^{\mathrm{N} 1 / 2(1990,}, \mathrm{JP}=7 / 2+1\right) \quad \mathrm{I}=1 / 2 \quad \mathrm{~F}_{17}$

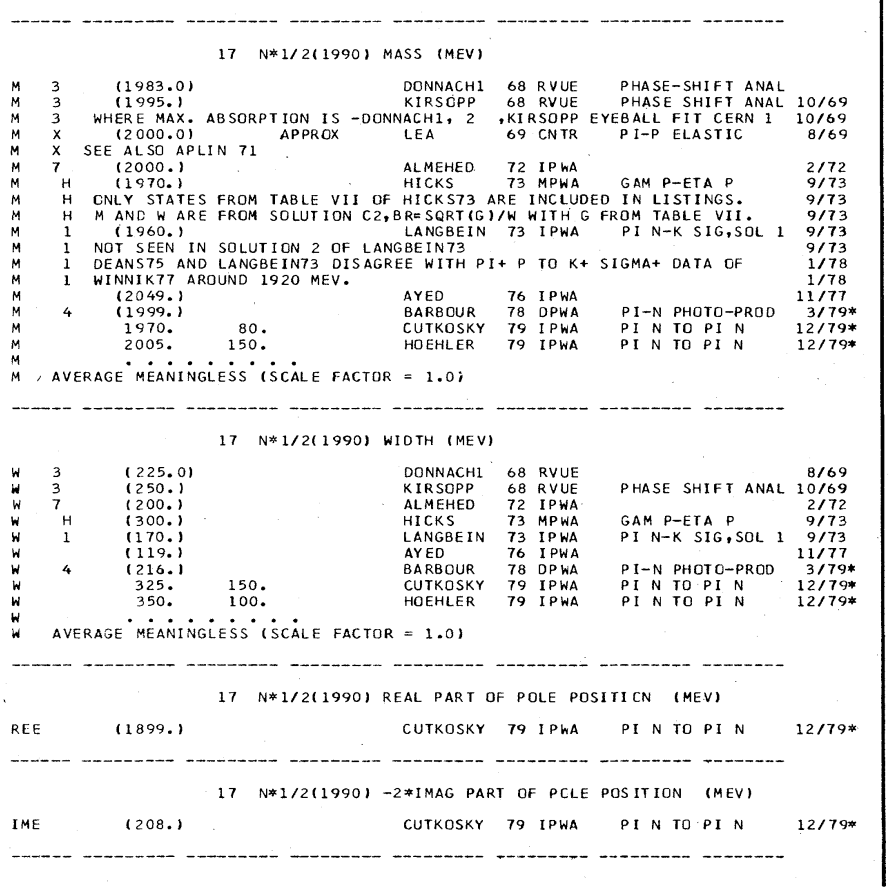

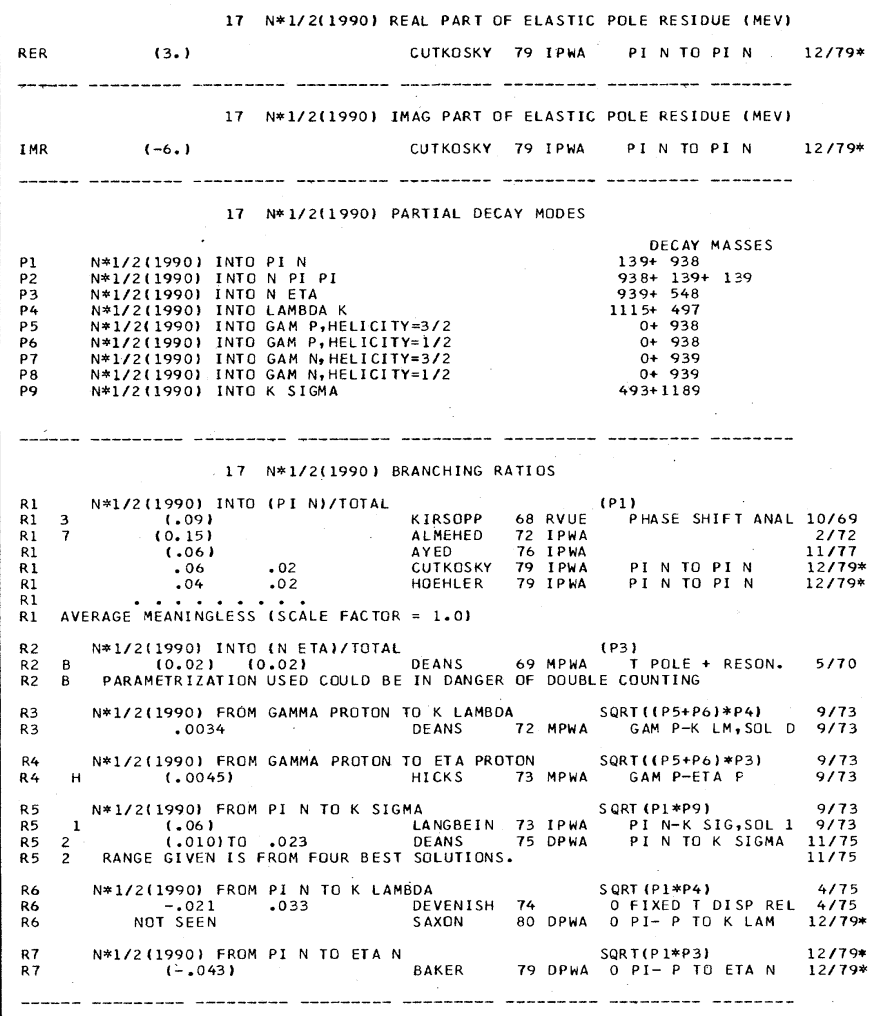

17 N*1/2(1990) PHOTON DECAY AMPL (GEV**-1/2)

FOR DEFINITION OF GAMMA-NUCLEON DECAY AMPLITUDES, SEE MINI-
REVIEW PRECEOING THE BARYON LISTINGS.

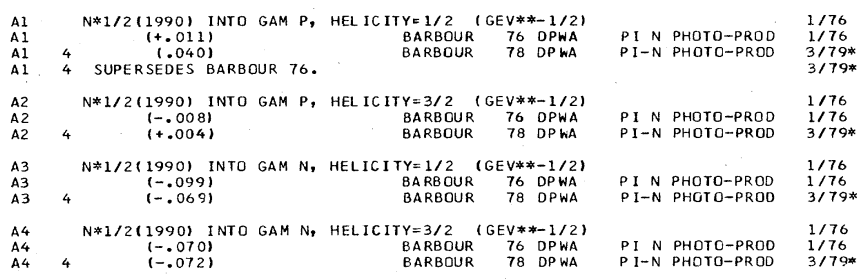
***********************************************************************1 REFERENCES FOR $N * 1 / 2(1990)$

$\begin{array}{lllll}\text { DONNACH1 } & 68 & \text { PL } 268 & 161 \\ \text { KIRSOPP } & 68 & \text { THESIS } & \end{array}$ A DONNACHIE, R G KIRSOPP, C LOVELACE (CERN) (EDIJP S DEANS, J WOOTEN
LEA, OADES, WARD, COWAN, + (RHEL, BRISTOLL, DARA)

$\begin{array}{llllll}\text { DEANS } & 69 & \text { PR } & 185 & 1797 \\ \text { LEA } & 69 & \text { PL } & 298 & 584\end{array}$

\begin{tabular}{lllll} 
ALMEHEO & 72 & NP & B 40 & 157 \\
\hline DEANS & 72 & PRD & 6 & 1906
\end{tabular}

\begin{tabular}{lllll} 
DEANS & 72 & PRD & 6 & 1906 \\
HICKS & 73 & PRD 7 & 2614 \\
\hline
\end{tabular} LEA, OADES, WARD, COWAN, T IRHEL, DRISTOL, DAREY (RUTG) IJP
DEANELACE
DEAS, SACOBS, LYONS, MONTGOMERY (SOUTH FLA.) IJJP +DEANS, JACOBS, LYONS+ (CARN+ORNL+SOUTH FLA.) IJP
(MANGBEIN, WAGNER
(MUNIIJP DEVENISH, FROGGATT, MARTINCDESY, NORDITA, LCUC)
+MITCHELL, MCNTGOMERY, +
(SFLA, ALABAMA) I JP $\begin{array}{lllll}\text { DEVENISH } & 74 & \text { NP } & 881 & 330 \\ \text { DEANS } & 75 & \text { NP } & 896 & 90\end{array}$ $\begin{array}{llll}\text { AYED } & 76 & \text { CEA-N-1921 } \\ \text { BARBOUR } & 76 & \text { NP } 8111 & 358\end{array}$ BARBOUR 78 NP B141 253 AYED (THES IS)
I. M. BARBOUR,R. L. CRAWFORD BARBOUR, CRAWFORD, PARSONS (GLAS)

$\begin{array}{lllll}\text { BAKER } & 79 & \text { NP } & B 156 & 93 \\ \text { CUTKOSKY } 79 & \text { PRD } 20 \quad 2839\end{array}$ +BROWN, CLARK, DAV IES, DEPAGTER, EVANS+ (RHEL)IJP
+FORSYTH, HENDRICK, KELLY
(CARN+LBL) IJP SCATIER ING, PHY SIK DA TEN VOL. 12-1
IKARL SRUHE IJP +KAISER, KOCH, PIETARINEN
+BAKER, BELL, BLI SSETT, BLCOOWORTH+ (RARLL +BRIS) IJP PAPERS NOT REFERREO TO IN DATA CARDS

SAXCN $\quad 80$ NP B 162522

$\begin{array}{lllll}\text { DEANS } & 69 & \text { PR } & 177 & 2623 \\ \text { AYED } & 70 & \text { PL } & 31 B & 598\end{array}$ $S R$ DEANS (UNIV S FLORIDA)

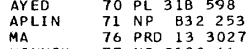
+BAREYRE, VILLET
+ COWAN, GIBSON, GILMORE++ (RHEL, BRISTOL) E. MA,G. L. SHAW
+TOAFF, REVEL, GOLDBERG, BERNY (OREG+UCI) IJP
(HAIFII 
Baryons

$\mathrm{N}(2000), \mathrm{N}(2040)$

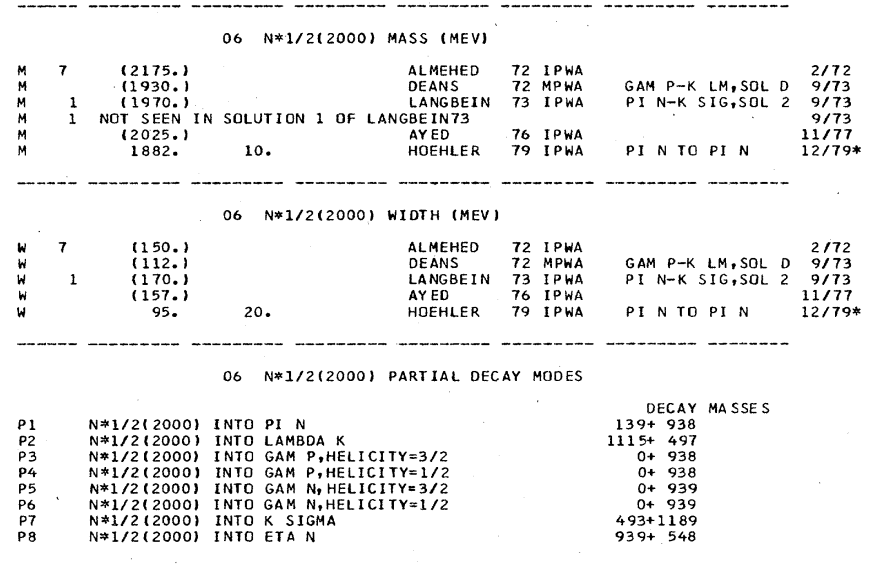

O6 N*1/2(2000) BRANCHING RATIOS

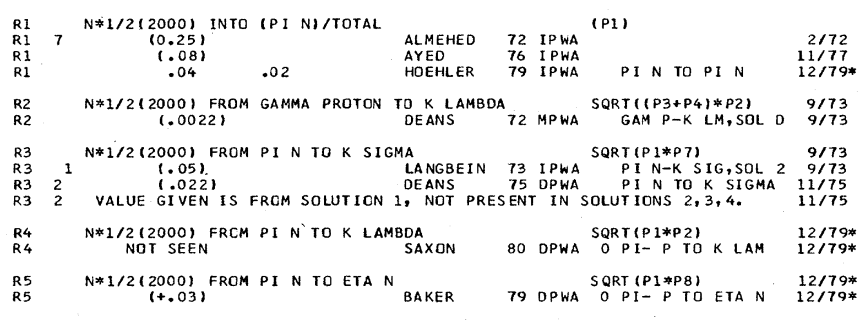
***********************************************************************1* REFERENCES FOR $N * 1 / 2(2000)$

$\begin{array}{lllll}\text { ALMEHED } & 72 & \text { NP } & B 40 & 157 \\ \text { DEANS } & 72 & \text { PRD } 6 & 1906\end{array}$

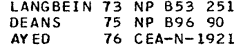
$\begin{array}{lll}\text { BAKER } & 79 \text { NP } 815693 \\ \text { HOEHLER } & 79 & \text { HANOBOOK OF PI }\end{array}$ SAXON $\quad 80$ NPB162 522 (LOVELACE
DEANS, JACOBS, LYCNS, MONTGOMERY (SOUTH FLA.) IJ JP
LANGBEIN, WAGNER LANGBEIN, WAGNER
+MITCHELL, MCNTGOMERY, +
AYED (THESIS) +BROWN, CLARK, DAVIES, DEPAGTER, EVANS* (RHEL)IJP SCATTER ING, PHYSIK DA TEN VOL. I2-1
+KAISER,KOCH,PIETARINEN +BAKER, BELL, BLI SSETT, BL CODHORTH+ (RHEL+BRIS) IJP PAPERS NOT REFERRED TO IN DATA CARDS

MA $\quad 76$ PRD $13 \quad 3027$ E. MA,G. L. SHAW (OREG+UC IIIJP

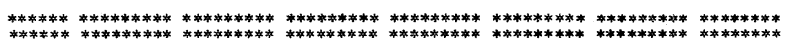

\section{$\mathrm{N}(2040)$} $16{ }^{N * 1 / 2(2040, J P=3 / 2-)} I=1 / 2 \quad D_{13}^{\prime \prime \prime}$ THERE ARE INDICATIONS OF 1 OR 2 RESONANC ES IN THIS WAVE WITH MASSES BETWEEN 1800 AND 2200 MEV THE EVIDENCE
IN THE PI N CHANNEL IS RATHER STRONG ISEE CUTKOSKY 79 AND HOEHLER 79) BUT IS NOT YET CONCLUSIVE ENOUGH FOR

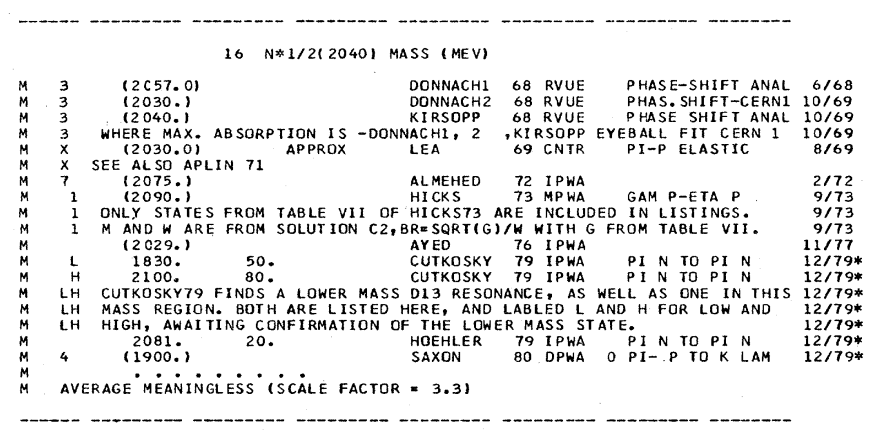

\section{Data Card Listings For notation, see key at front of Listings.}

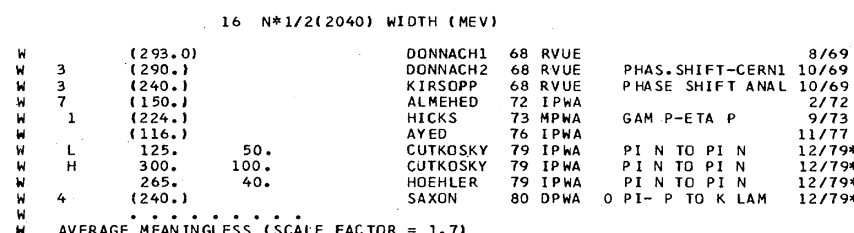

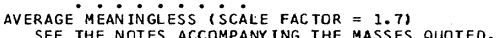

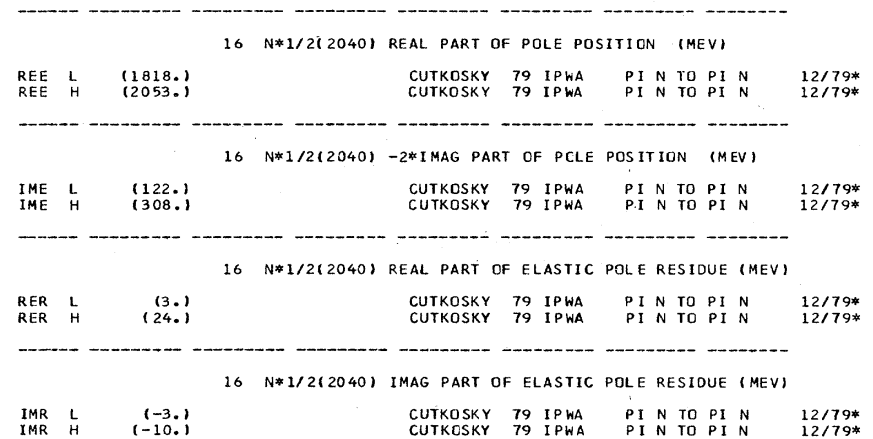

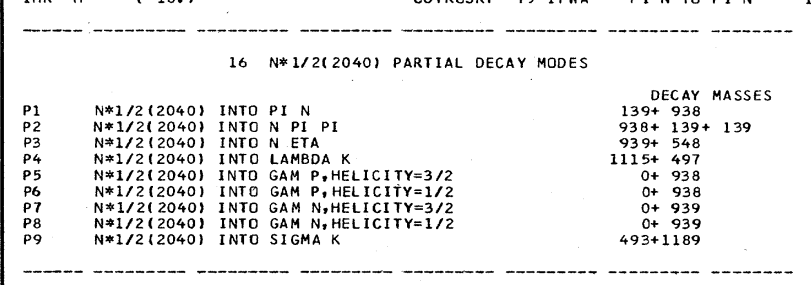

$16 N * 1 / 2(2040)$ BRANCHING RATIOS

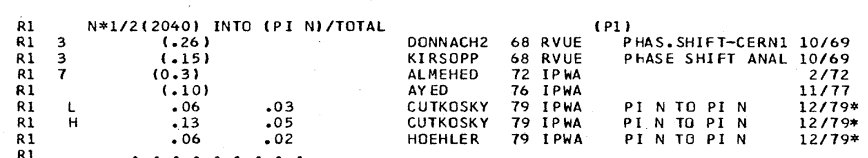

R1 AVERAGE MEANíni NGLESS iscale FACTOR $=1.01$

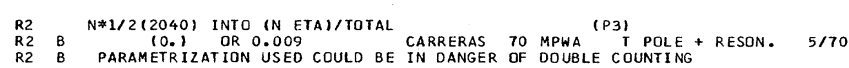

$R 3$
$R 3$
$R 3 * 1 / 2(2040)$ FROM GAMMA PROTON TOK K LAMBDA
R3
(.0070)

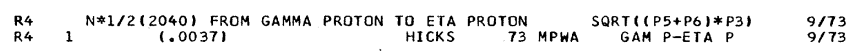

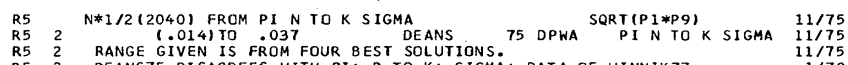

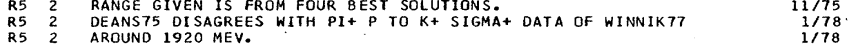

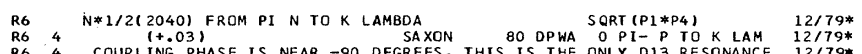

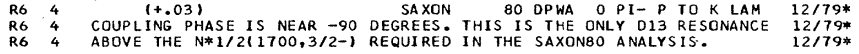

R7 N*1/2(2040) FROM PI N TO ETA N
RT NOT SEEN

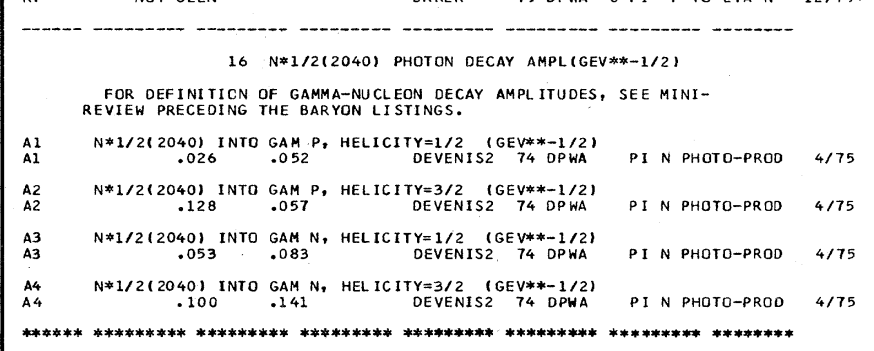




\section{Data Card Listings}

\section{For notation, see key at front of Listings.}

\section{Baryons $\mathrm{N}(2040), \mathrm{N}(2100), \mathrm{N}(2190)$}

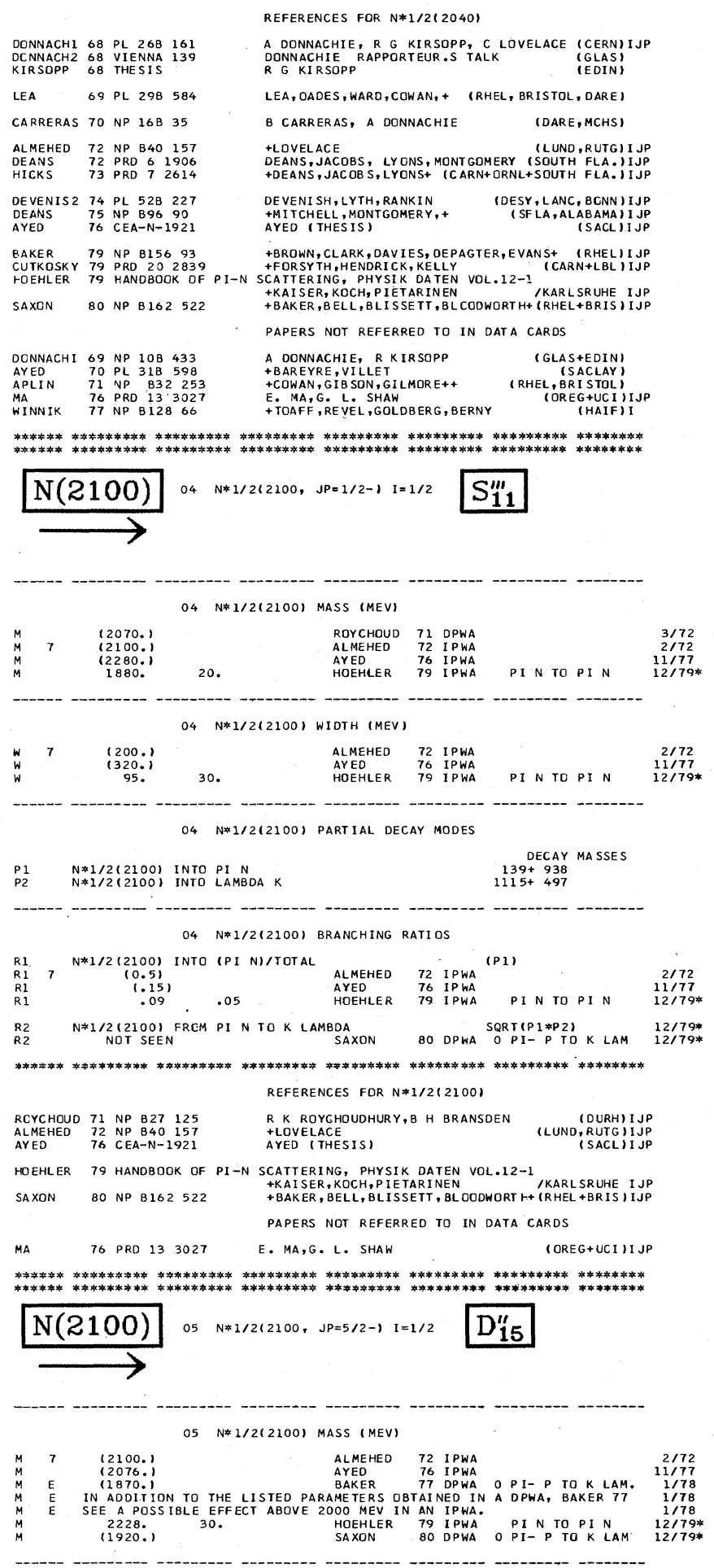

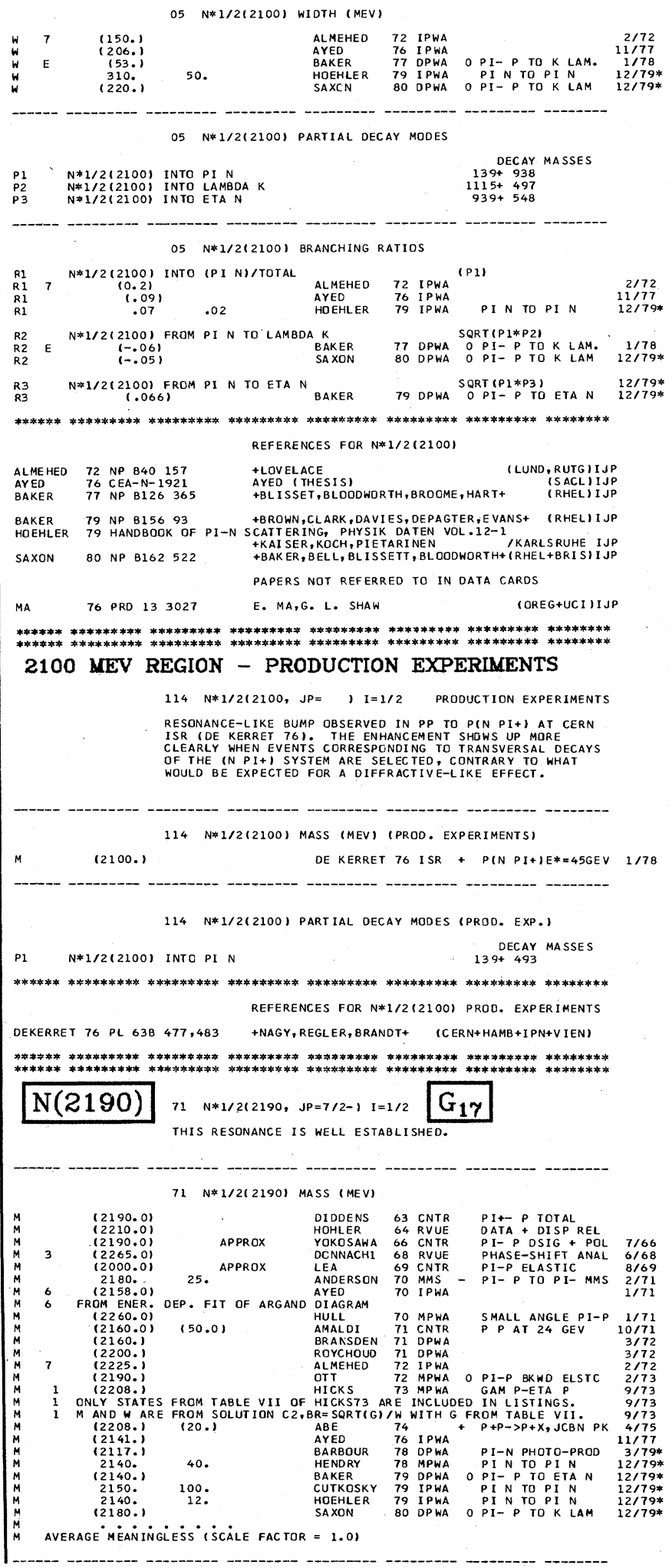


Baryons

$\mathrm{N}(2190), \mathrm{N}(2200)$

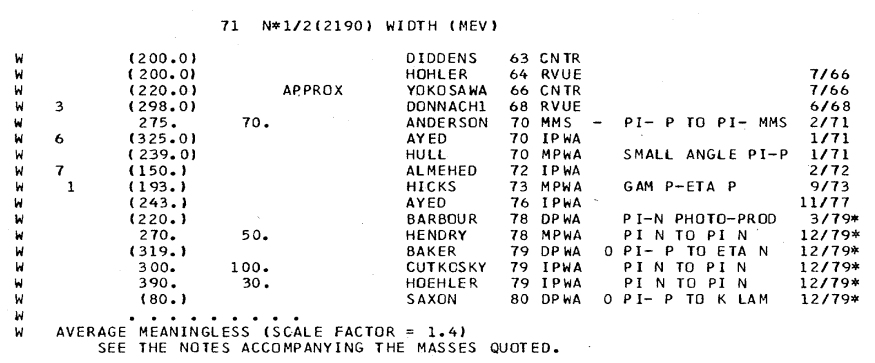

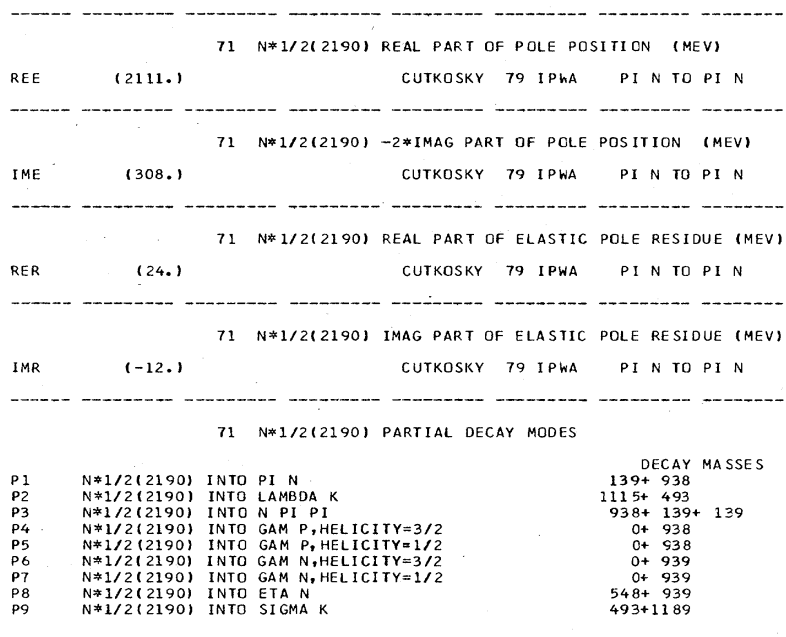

71 N*1/212190) BRANCHING RATIOS

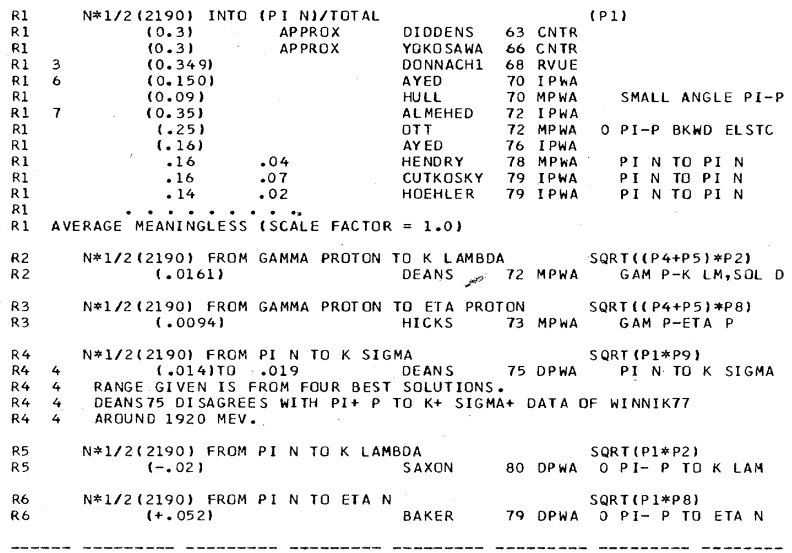

$71 N * 1 / 2(2190)$ PHOTON DECAY AMPL (GEV**-1/2)

FOR DEFINITION OF GAMMA-NUCLEON DECAY AMPLITUDES, SEE MINI-

A1 N*1/2(2190) INTO GAM P, HELICITY=1/2 (GEV**-1/2)
A1
$(-.030)$

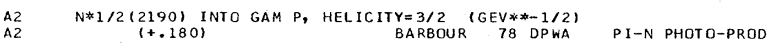

A3 N*1/2(2190) INTO GAM N, HELICITY $=1 / 2$ (GEV**-1/2)

A4 N*1/2(2190) INTO GAM N, HELICITY $=3 / 2$ (GEV**-1/2)

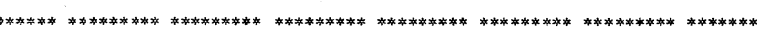

REFERENCES FOR $N * 1 / 2(2190)$

$\begin{array}{lllllll}\text { DIDDENS } & 63 & \text { PRL } 10262 & \text { +JENKINS, KYCIA, RILEY } & \text { (BNL) } \\ \text { HOHLER } & 64 \text { PL } 12 & 149 & \text { G } & \text { GOHLER, J GIESECKE } & \text { (KARLSRUHE) } \\ \text { YOKOSAWA } & 66 & \text { PRL } 16714 & \text { +SUWA, HILL, ESTERLING, BOOTH } & \text { (ANL, CHIC) JP }\end{array}$

\section{Data Card Listings For notation, see key at front of Listings.}

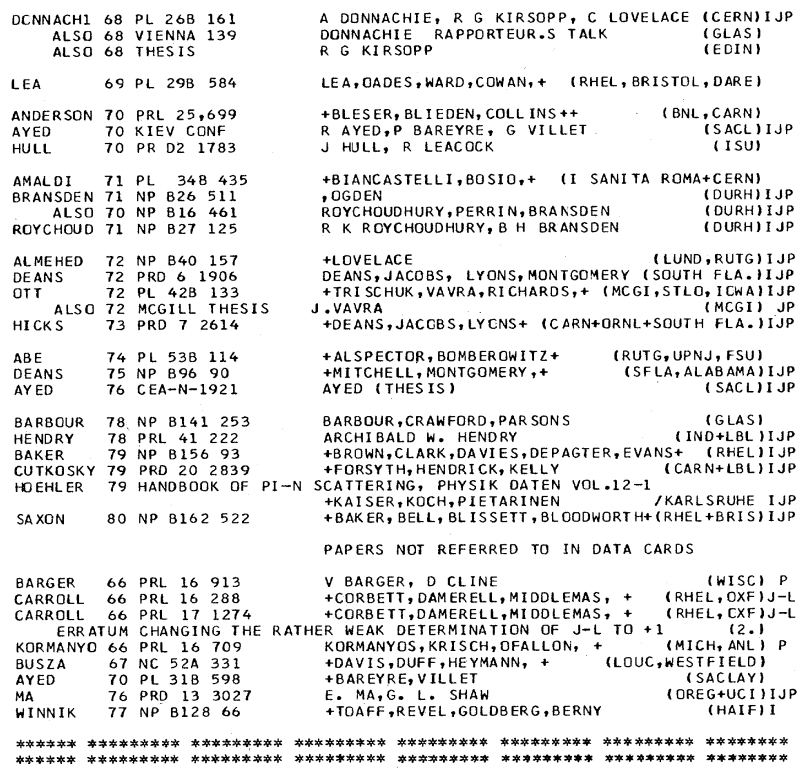

$\mathrm{N}(2200){ }_{113}{ }_{\mathrm{N} * 1 / 2(2200, J P=9 / 2-1} \mathrm{I}=1 / 2 \quad \mathrm{G}_{19}^{\prime}$

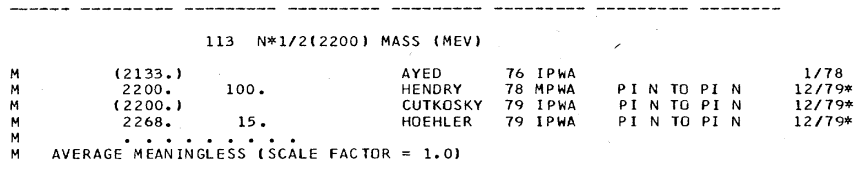

$113 N * 1 / 2(2200)$ WIDTH (MEV)

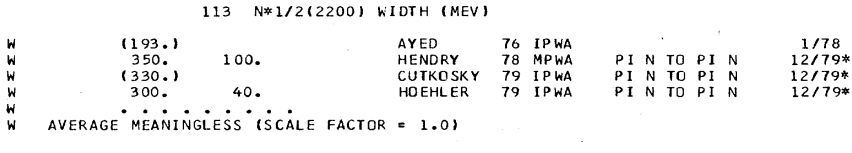
113 N*1/2(2200) REAL PART OF POLE POSITION (MEV)

REE (2169.) CUTKOSKY 79 I PWA PI N TO PI N 12/79*

113
IME
N*1/212200) -2*IMAG PART OF POLE POSIIION IMEV)
CUTKOSKY 79 IPHA PI N TO PI N

IME 1290.1 CUTKOSKY 79 IP WA PI N TO PI N 12/79*

113 N*1/2(2200) REAL PART OF ELASTIC POLE RESIDUE (MEV)

RER 115.1 CUTKOSKY 79 IPWA PIN TO PIN $12 / 79 *$

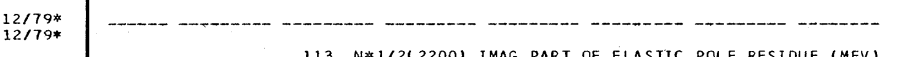

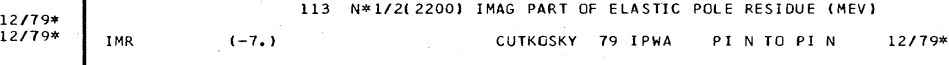

$113 \quad N * 1 / 2(2200)$ PARTIAL DECAY MODES

N*1/2(2200) INTO PI N DECAY MASSES

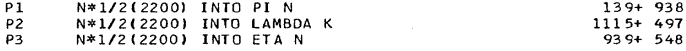

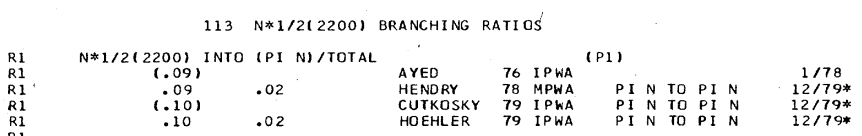

R1 AVERAGE MEANINGGLESS iscale FACTOR $=1.01$

$R 2$
$R 2$
$R 21 / 2122001$ FROM PI N TO K LAMBDA
NOT SEEN

R3 N*1/2(2200) FROM PI N TO ETA N
R3
$(-.043)$ 
Data Card Listings

For notation, see key at front of Listings.

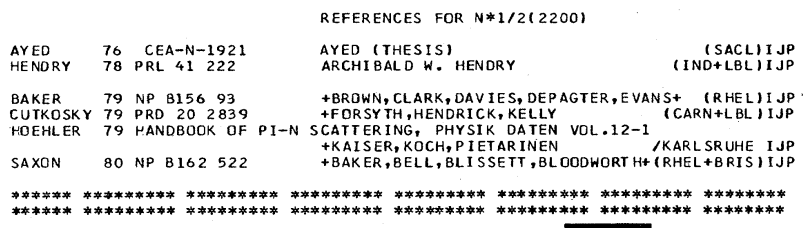

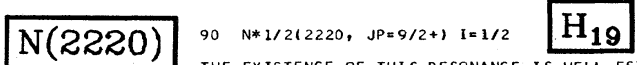

THE EXISTENCE OF THIS RESONANCE IS WELL ESTABLISHED.

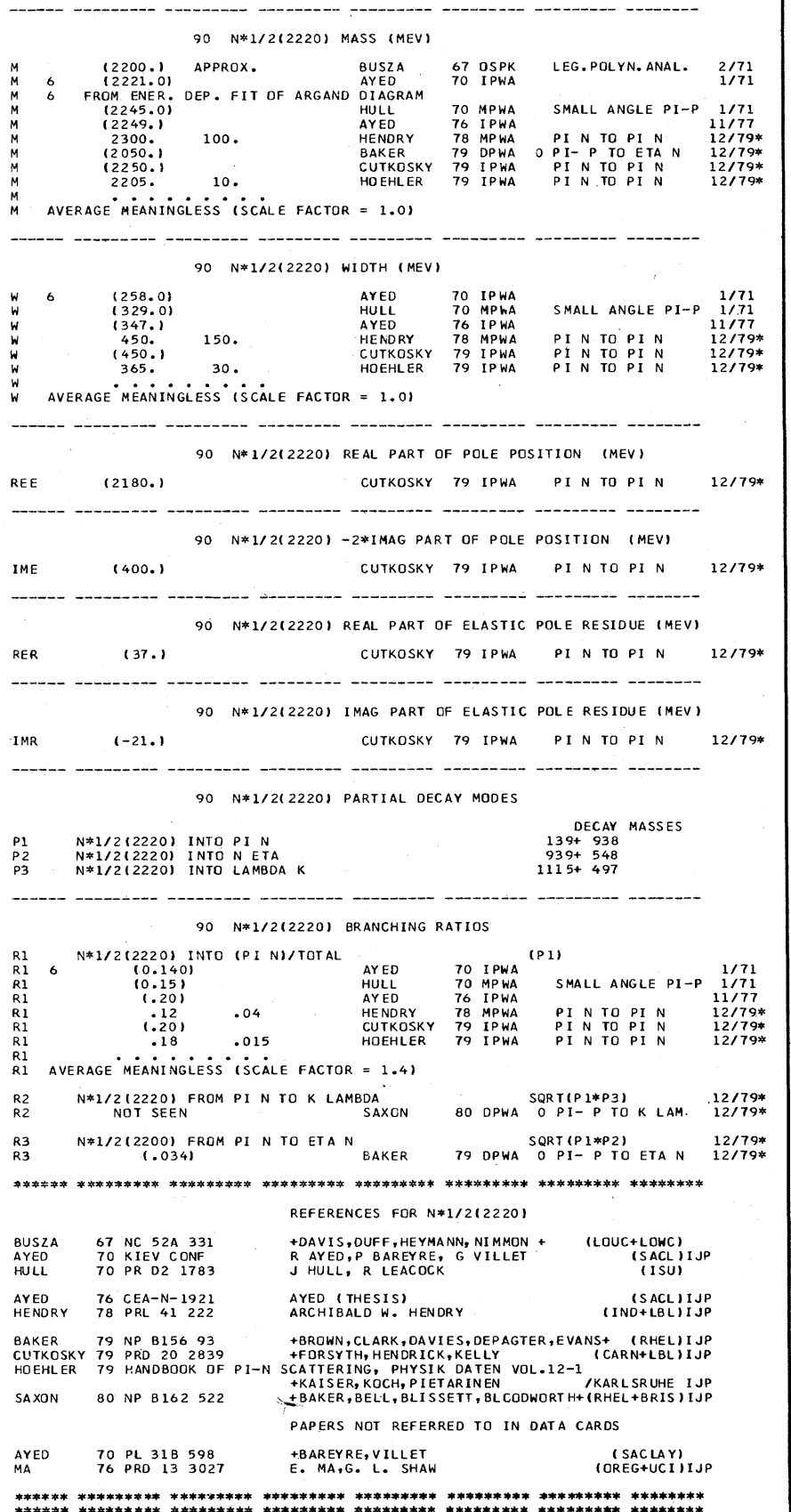

2200 MEV REGION - PRODUCTION EXPERIMENTS

$111 N * 1 / 2(2200, J P=2) \quad I=1 / 2$ PRODUCTION EXPERIMENTS WE LIST HERE BUMPS OBSERVED IN THE RANGE 1900-2500 MEV.

$111 N * 1 / 2(2200)$ MASS (MEV) (PROD. EXP.)

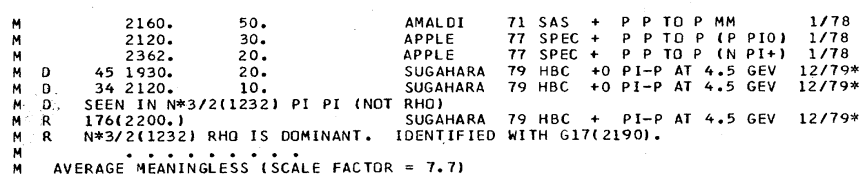

(NOT RHO)

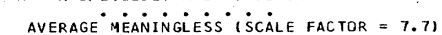

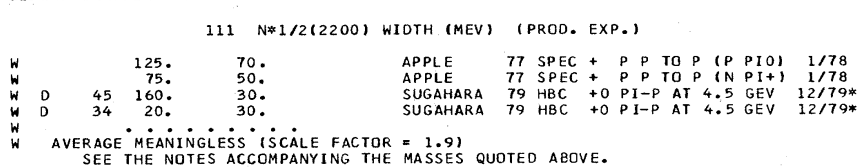

************************************************************************ REFERENCES FOR $N * 1 / 2(2200)$

AMALDI $71 \mathrm{PL} 348435$
ABIANCASTELLI, BOSIO, MATTHIAE+ (SANI+CERN) +ASH, CHENG, COYNE, GROSSMAN+ ONA (PR IN+PAVIA)
+ SUZUKI, FUKAWA, KABE, KICHIMI, OCHIAI+ (KEK)

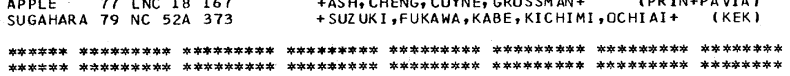

>2500 MEV REGION - FORMATION EXPERIMENTS

$128 \quad \mathrm{~N} * 1 / 2(>2500) \quad I=1 / 2$

WE LIST HERE II $1 / 2$ RESONANCES WITH MASS GREATER THAN
ABOUT 2.5 GEV WHICH HAVE BEEN SEEN IN A SINGLE PARTIAL WAVE ANALYSIS ONLY. ALL RESONANCES WHICH HAVE BEEN
OBSERVED IN II ANALYSIS AT ABOUT THE SAME MASS ARE
GIVEN A SEPARATE LISTING WITH THE APPROPRIATE QUANTUM
NUMBERS. GIVEN A
NUMBERS.

$128 * 1 / 2(>2500)$ MASS (MEV)

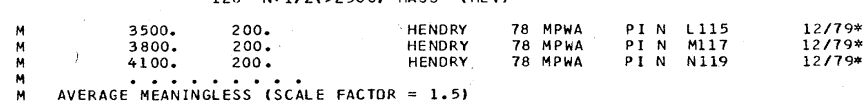

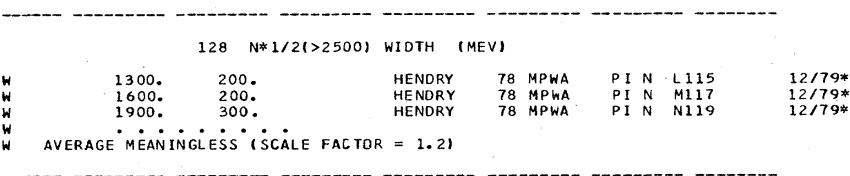

$128 \quad N * 1 / 21>2500)$ PARTIAL DECAY MODES

$\begin{array}{ccc}\text { P1 } & N * 1 / 21>2500) \text { INTO PI N } & \text { DECAY MASSES } \\ 139+938 & \\ 128 \quad N * 1 / 2(>2500) & \text { BRANCHING RATIOS }\end{array}$

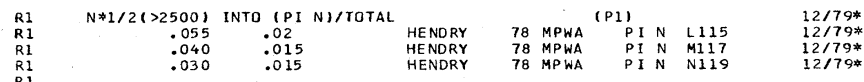

R1 AVERAGe MEANingless iscale FaCtor $=1.01$

$* * * * * * * * * * * * * * * * * * * * * * * * * * * * * * * * * * * * * * * * * * * * * * * * * * * * * * * * * * * * * * * * * * *$
REFERENCES FOR N*1/21>2500)

HENORY 78 PRL 41222 ARCHIBALD W. HENDRY (IND+LBLIIJP

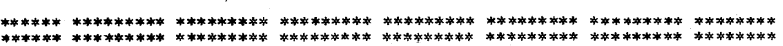

$N(2600){ }^{120}{ }^{N * 1 / 2(2600, J P=11 / 2-1 \quad I=1 / 2} I_{111}$

$120 \quad N * 1 / 2(2600)$ MASS (MEV)

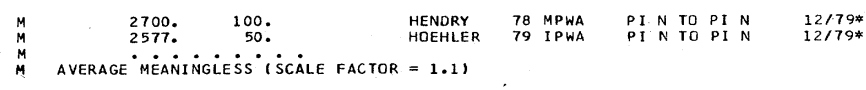




\section{Baryons}

Data Card Listings $\mathrm{N}(2600), \mathrm{N}(2650), \mathrm{N}(2700), \mathrm{N}(2800), \mathrm{N}(3030)$ For notation, see key at front of Listings.

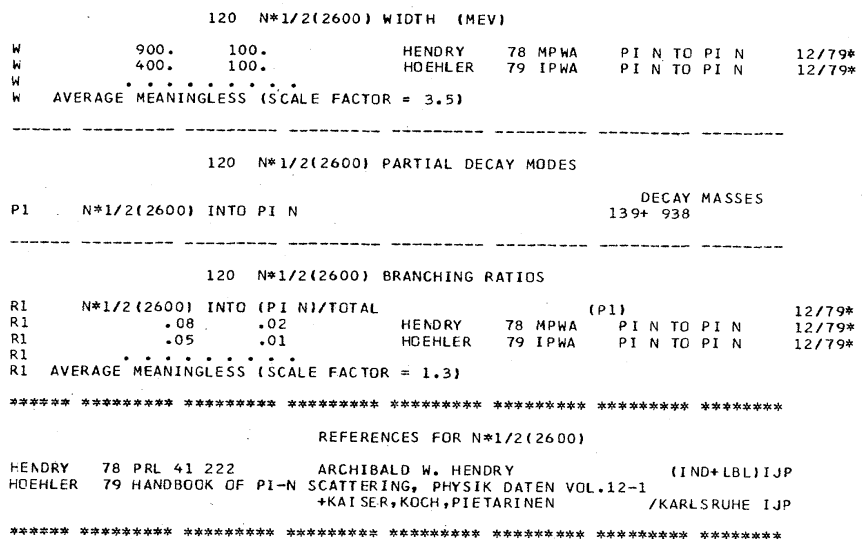

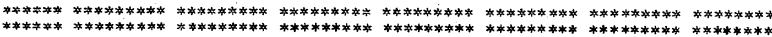

2650 MEV REGION - MISCELLANEOUS EXPERIMENTS

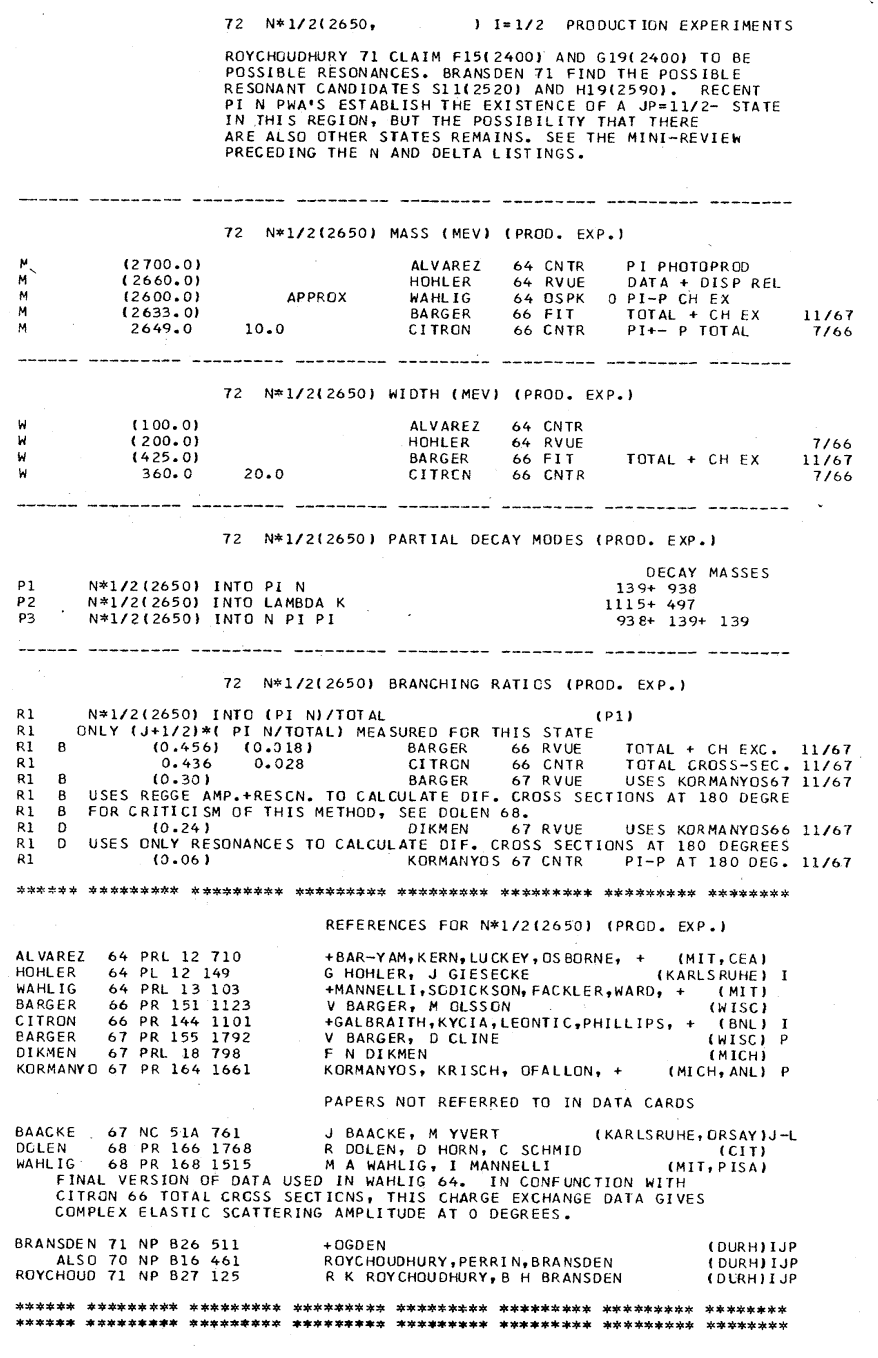

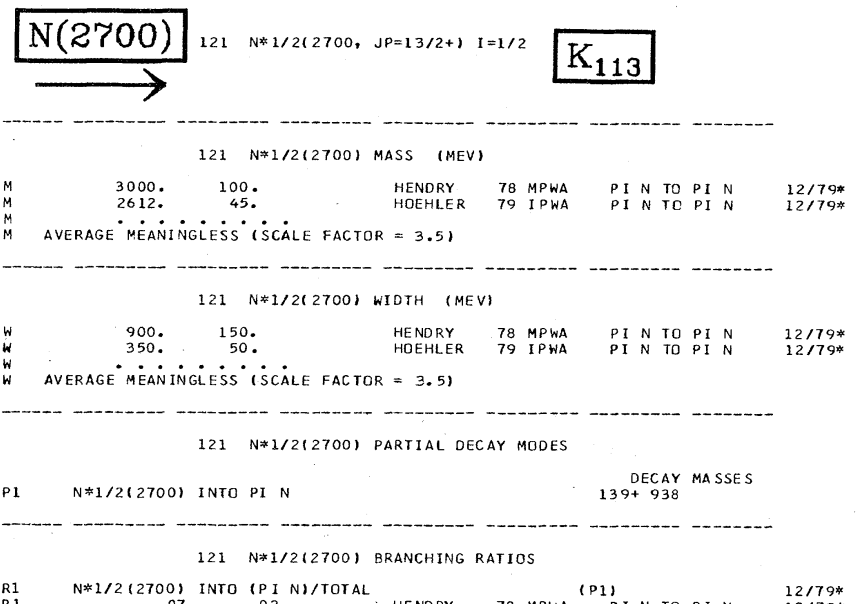

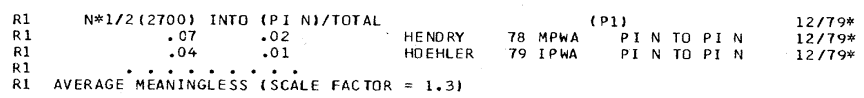

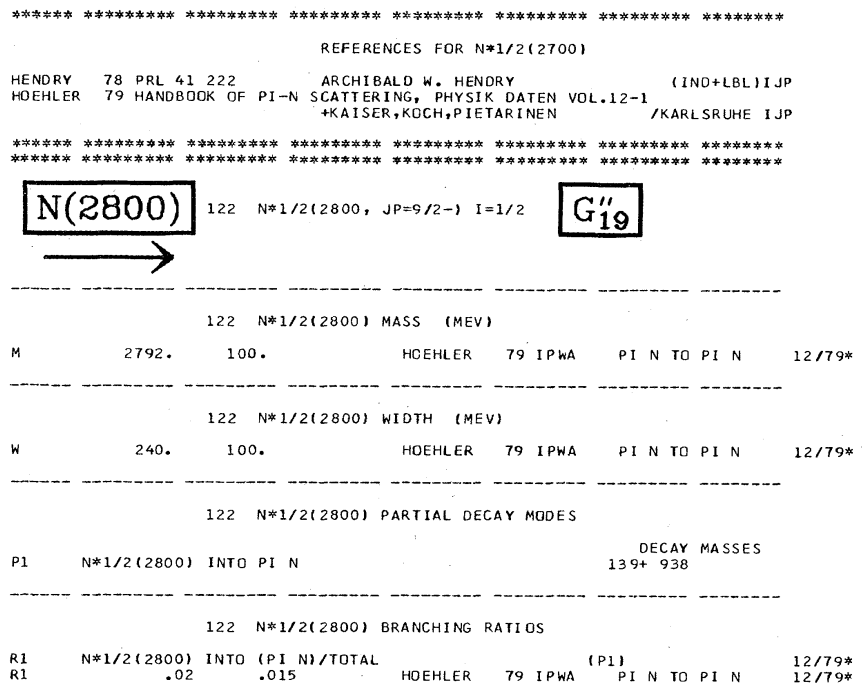
$* * * * * * * * * * * * * * * * * * * * * * * * * * * * * * * * * * * * * * * * * * * * * * * * * * * * * * *$
REFERENCES FOR $N * 1 / 2(2800)$

HOEHLER 79 HANOBOOK OF PI-N SCATTERING, PHYSIK DATEN VOL.12-1
+KAISER, KOCH, PIETARINEN

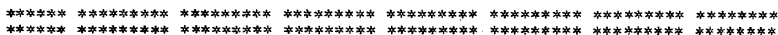
$\begin{aligned} & \mathrm{N}(3030) \\ & \text { BUMPS }\end{aligned} 73 \quad \mathrm{~N} * 1 / 213030, \mathrm{JP}=\quad, \mathrm{I}=1 / 2 \quad$ PRODUCT ION EXPERIMENTS

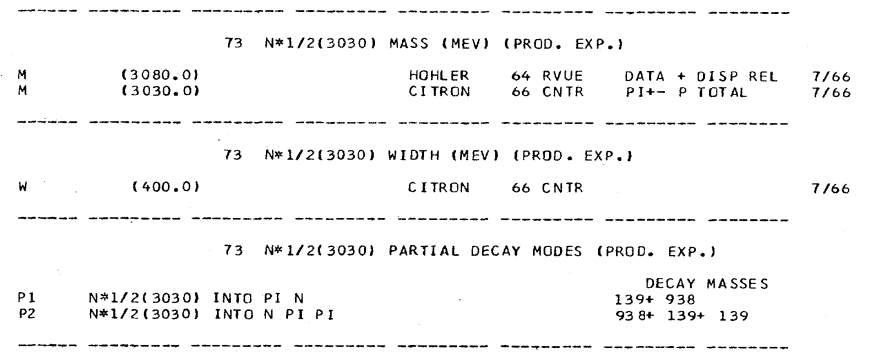




\section{Data Card Listings For notation, see key at front of Listings.}

Baryons

73 N*1/2(3030) BRANCHING RATIOS (PROD. EXP.)

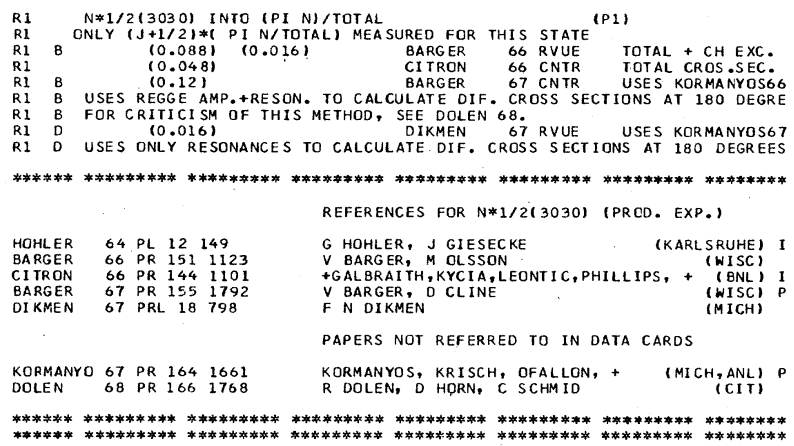
$\mathrm{N}_{2}(3245) 7^{74} \quad \mathrm{~N} * 1213245$, JP $=1 \quad$ PRODUCTION EXPERIMENTS BUMPS EXISTENCE NOT CONCLUSIVELY ESTABLISHED. ILSPIN

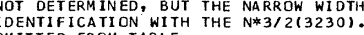

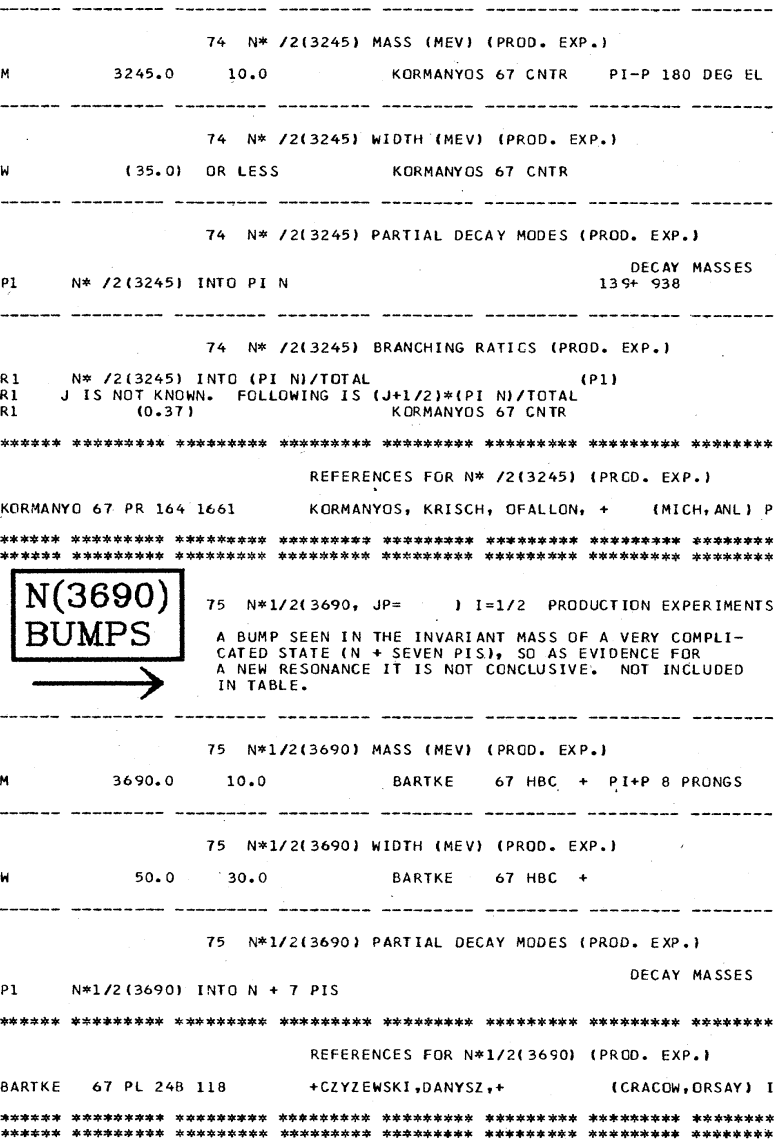

\section{$N_{P}(3245), N(3690), N_{P}(3755), \Delta(1232)$}

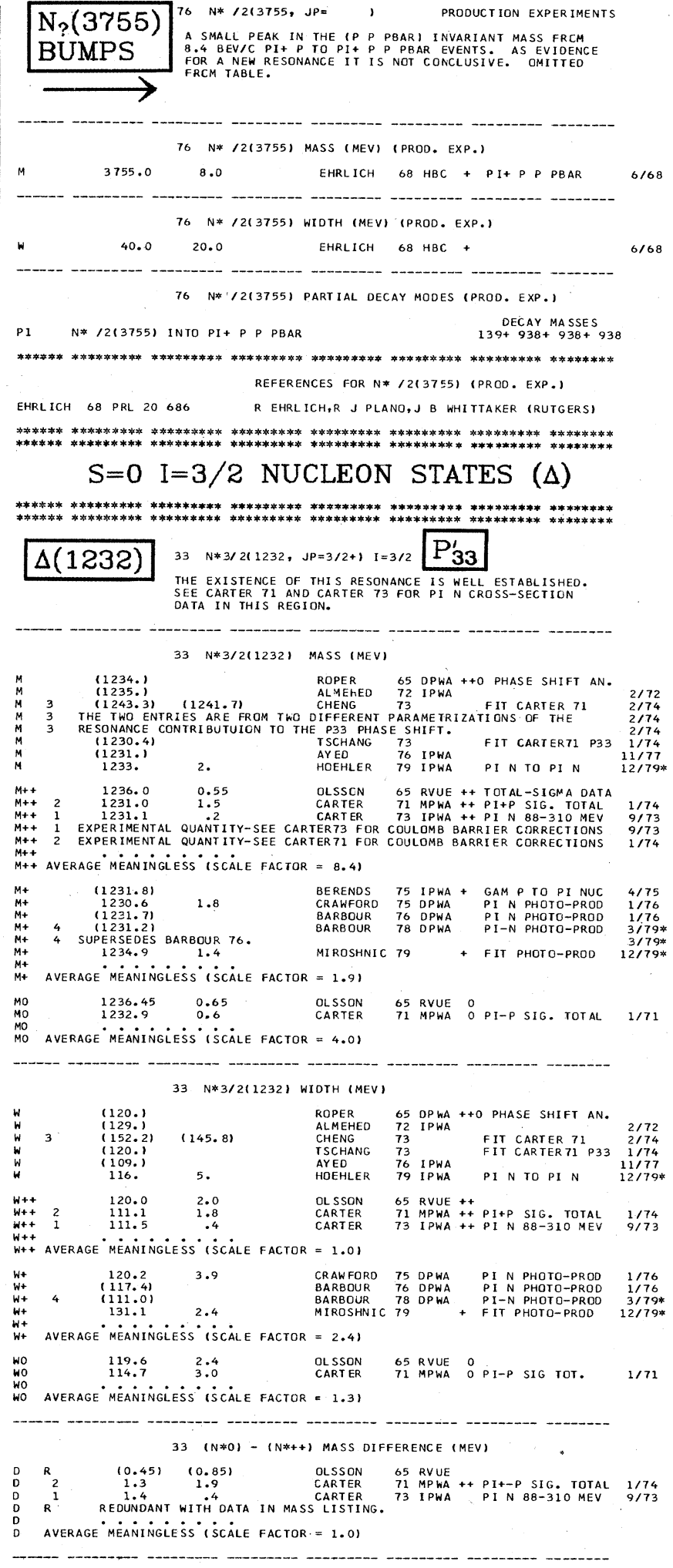




\section{Baryons $\Delta(1232)$}

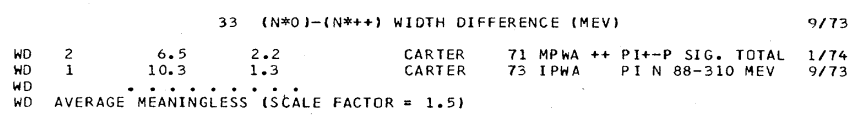
$33 N * 3 / 2(1232)$ REAL PART OF POLE POSITICN (MEV)

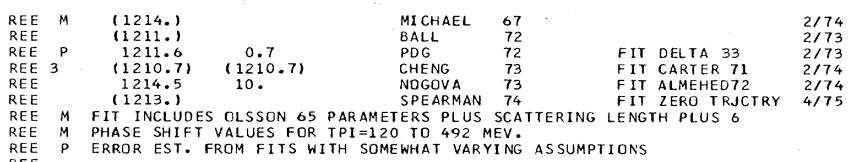

REE AVERAGE MEANI INGLE SS i SCALE FAC TOR $=1.01$

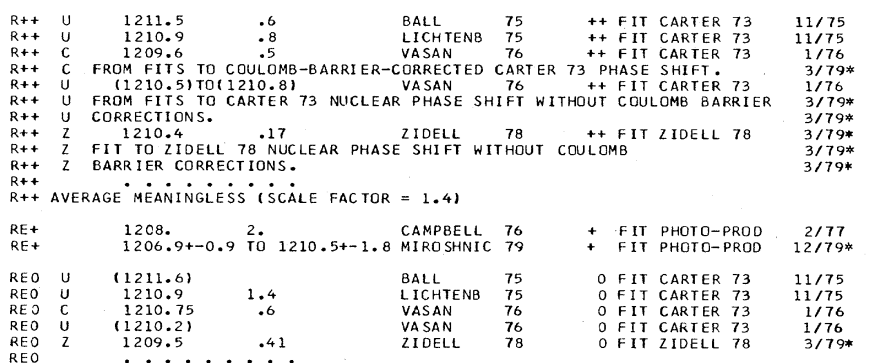

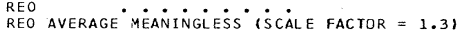

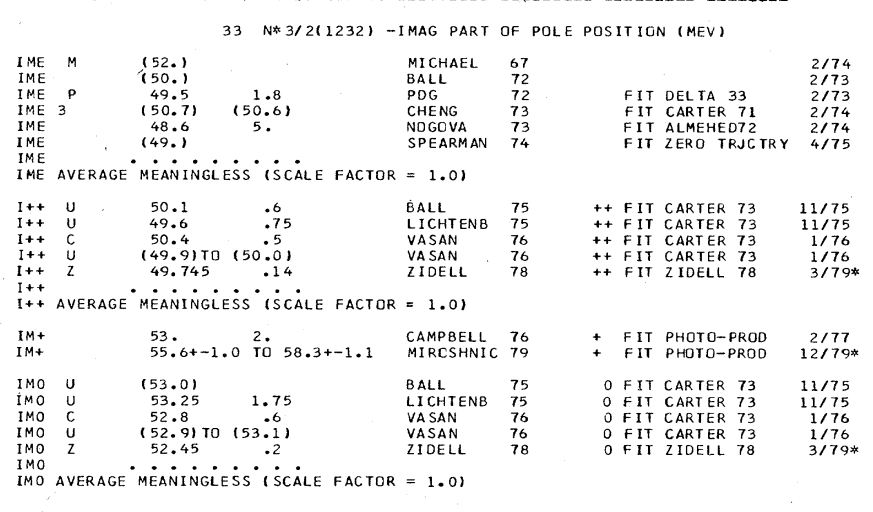

\begin{tabular}{|c|c|c|c|c|c|}
\hline$A B S$ & (53.) & BALL & 73 & FIT DELTA 33 & $9 / 73$ \\
\hline $\begin{array}{l}A++ \\
A+t \\
A+t\end{array}$ & $(52.4)$ To $(53.2)$ & VASAN & 76 & +t FIT CARTER 73 & $1 / 76$ \\
\hline $\begin{array}{ll}A B O & C \\
A B O & U\end{array}$ & $\begin{array}{l}(54.8) \text { to }(55.0) \\
(55.2) \text { To }(55.3)\end{array}$ & 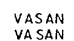 & $\begin{array}{l}76 \\
76\end{array}$ & $\begin{array}{l}+ \text { FII CARTER } 73 \\
++ \text { FII CARIER } 73 \\
++\end{array}$ & $\begin{array}{l}1 / 76 \\
1 / 76\end{array}$ \\
\hline
\end{tabular}

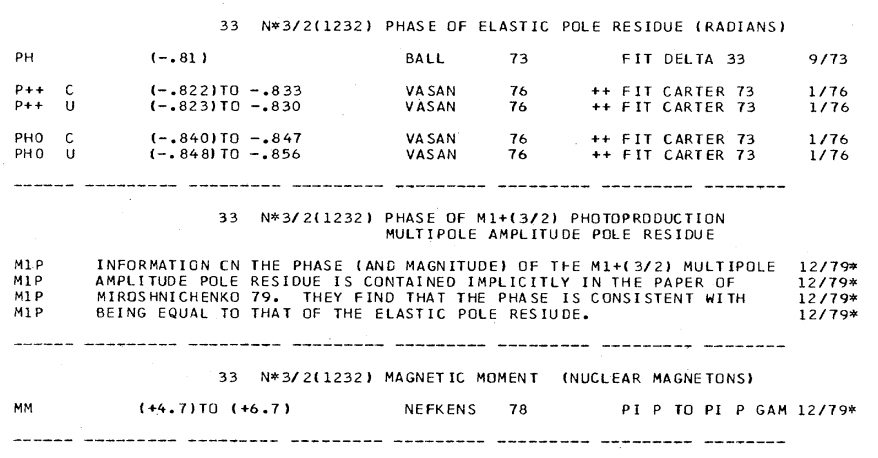

\author{
Data Card Listings \\ For notation, see key at front of Listings.
}

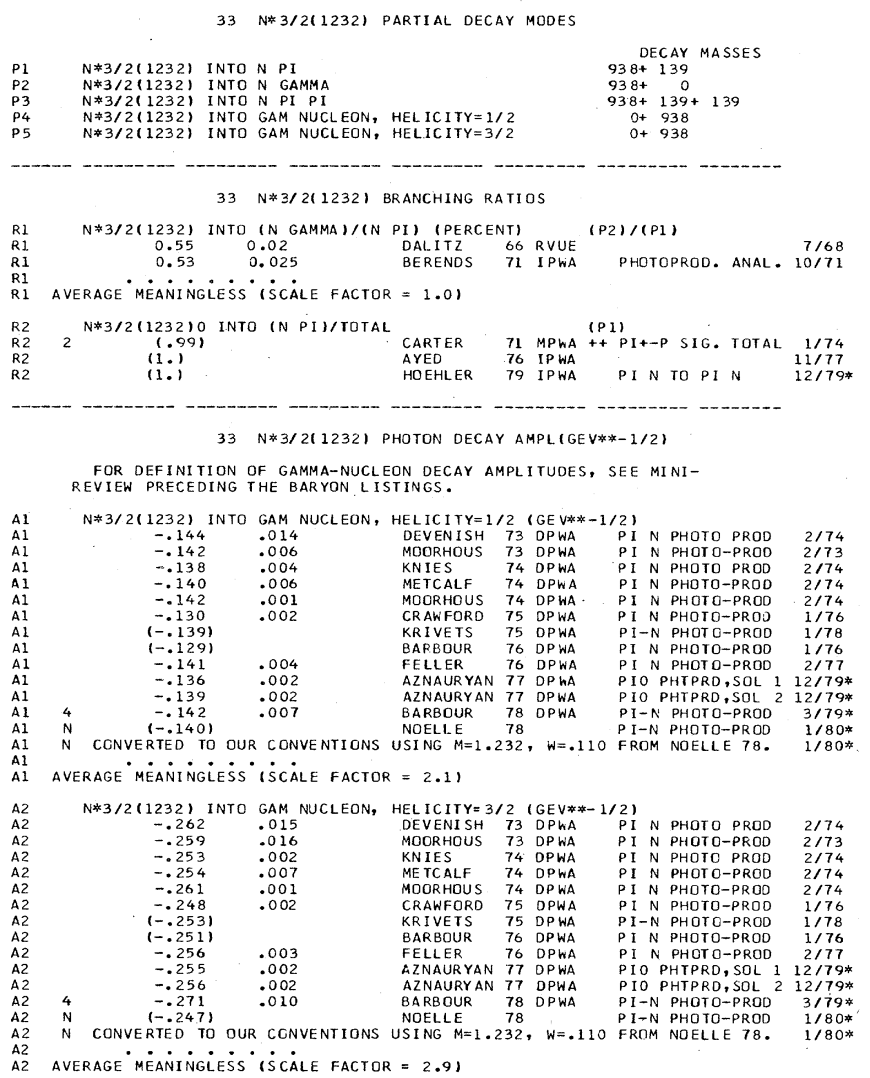

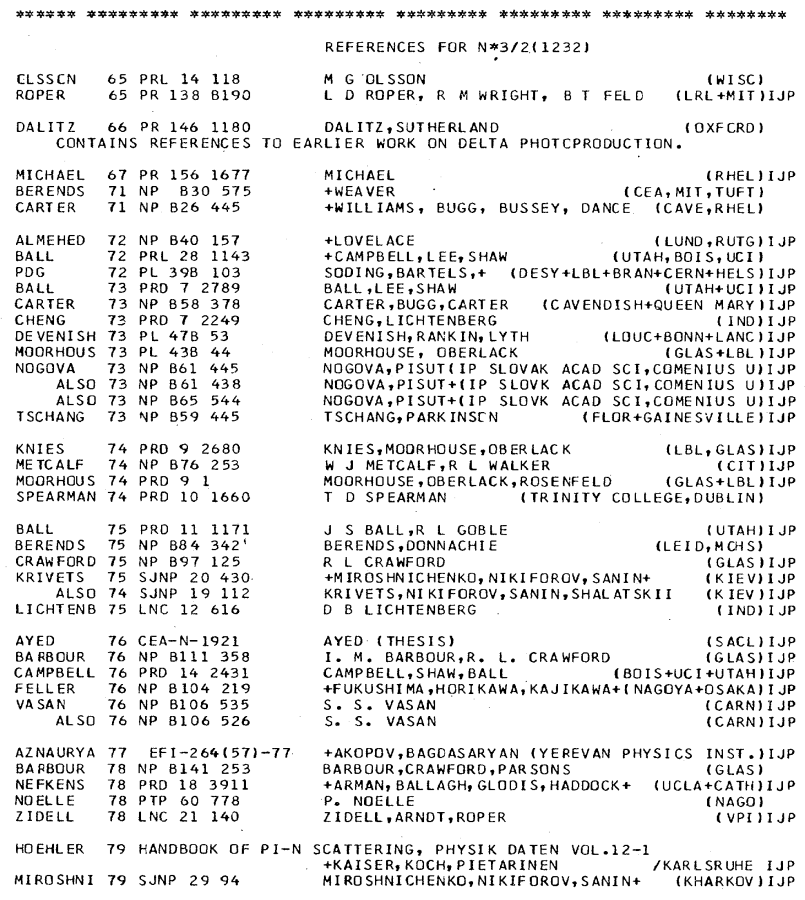




\section{Data Card Listings}

\section{For notation, see key at front of Listings.}

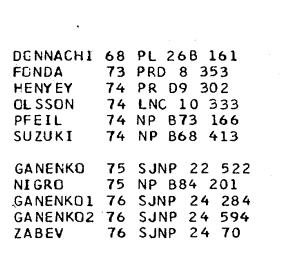

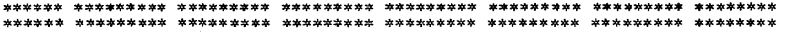

\section{MEV REGION - PRODUCTION EXPERIMENTS}

$81 N * 3 / 2(1232, J P=3 / 2+) \quad I=3 / 2$ PRODUCTION EXPERIMENTS SEE THE MINI-REVIEW PRECEDING THE N AND OELTA LISTINGS
FOR A DISCUSSION OF PRODUCTION EXPERIMENTS.

$81 N * 3 / 2(1232)$ MASS (MEV) (PROD. EXP.)

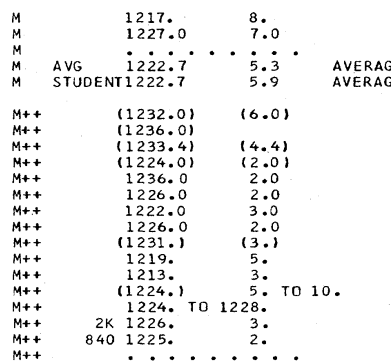

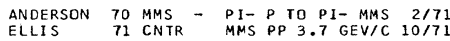

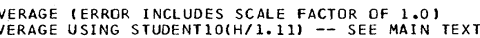
$\begin{array}{llll}\text { FERRO-LUZ } & 65 & \mathrm{HBC} & ++ \\ \text { DEANS } & 66 & K \text { PVUE } & \text { TO KO PI+P TOTAL PI+ } \\ \text { DEANS } & 7 / 66\end{array}$ $66 \mathrm{DBC}++$ D D TO NN(NN) PI $7 / 66$

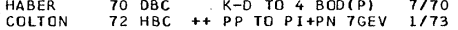
$72 \mathrm{HBC}++\mathrm{TO} P \mathrm{P}+\mathrm{PI}-\mathrm{PP}$

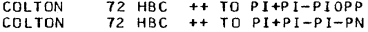

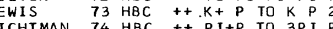
ICHTMAN $74 \mathrm{HBC}++$ PI-P TO $3 P$ I $P$

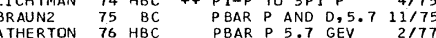

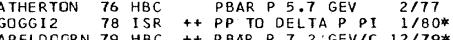

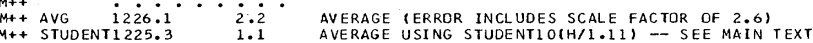

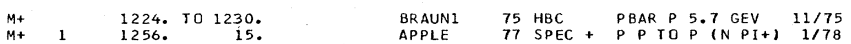

MO
MO
MO
$M-$

(1231. 11 . $\begin{array}{cc}1241.31 & 15.11 \\ 1239.0 & 5.0\end{array}$

\section{COOPER}

BRAUN1

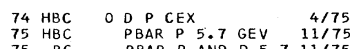
$\begin{array}{lll}66 \mathrm{DBC} & \\ \text { GIDAL } & & 7 / 66 \\ \text { COLTON } & 72 \mathrm{HBC} & - \\ & \end{array}$

$81(N *-)-(N *++)$ MASS DIFFERENCE (MEV) (PROD. EXP.) GIDAL $\quad 66$ DBC

$81 N * 3 / 211232$ ) WIDTH (MEV) (PROD. EXP.)

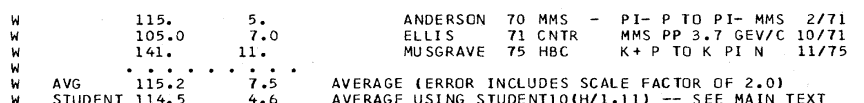

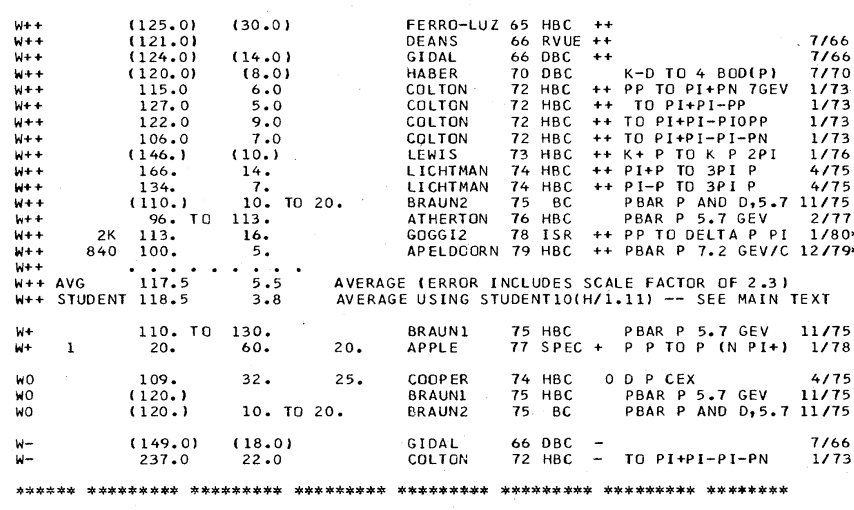

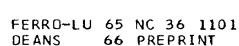
REFERENCES FOR $N * 3 / 2(1232)$ (PRCD. EXP.) $\begin{array}{lll}\text { DEANS } & 66 & \text { PREPRINT } \\ \text { GIDAL } & 66 & \text { PR } 141.1261\end{array}$ $\begin{array}{lllll}\text { ANDERSON } & 70 & \text { PRL } 25 & 699 \\ \text { HABER } & 70 & \text { NP } 178 & 289\end{array}$ $\begin{array}{lllll}\text { HABER } & 70 & \mathrm{NP} & 178 & 289 \\ \text { ELLIS } & 71 & \text { PRL. } & 27 & 442\end{array}$ (CERN)
FERRO-LUZZI, GEORGE, ${ }^{+}$
S R DEANS, WG HOLLADAY
G GIDAL, KERNAN, S KIM (BNL,CARN)

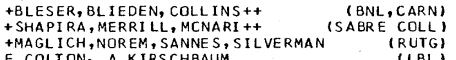
+MAGLICH, NOREM, SANNES, SILVERMAN (RUTG)
E COLTON, A KRSCHBAUM
+ALLEN, JACOBS, DANYSZ, I SLAM+ (LOWC+LOIC+CCEF)

\section{Baryons $\Delta(1232), \Delta(1550), \Delta(1650)$}

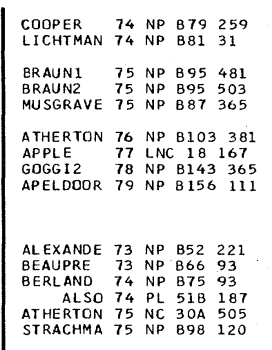
COOPER, SEI DL, VANDERVELDE (MICH)
LICHTMAN, BI SWAS, CA SON, KENNEY, MCGAHAN+ (NDAM) I +GERBER, MAURER, MICHALON, SCHIBY+ (STRB, LPNP) I
TGERBER, MAURER, MICHALON, SCHIBY+ (STRB, LPNP) I PEETERS, SCRE I NER, WHITMORE, YUTA A ATHERTON, FRENCH, SKURA, BOHM+
(CERN + PRAG)
+ SS, CHENG, COYNE, GROSSMAN+ (CERN+PAVI

PAPERS NOT REFERRED TO IN DATA CARDS

ALEX ANDER, BENARY +(TEL-AVIV+HEI DELBERG+DESY)

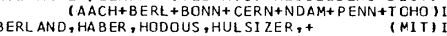
BERLAND, HABER, HODOUUS, HUL SI ZER, , $\begin{array}{ll}\text { ATHERTON,BAR-NIR, FRENCH, SKURAt } & \text { (CERN+PRAG) I } \\ \text { STRACHMAN, BRAUN, GERBER, MAURER+ } & \text { (LPNP+STRB)I }\end{array}$

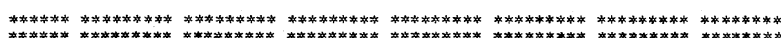

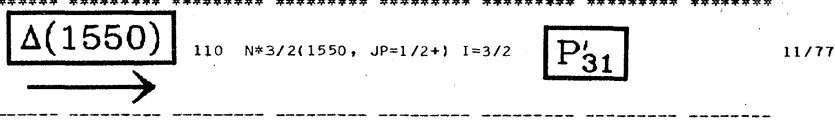
110. $N * 3 / 2(1550)$ MASS (MEV) $11 / 77$

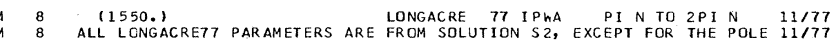

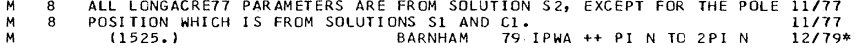
$110 \quad N^{*} 3 / 2(1550)$ WIDTH (MEV) $11 / 77$

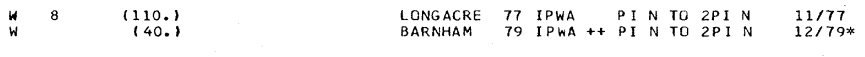
110 N*3/2(1550) REAL PART OF POLE POSITION (MEV) 11/77 RE 8 1554. OR 1553. LONGACRE 77 IPWA PIN TO $2 P I N$ N $11 / 77$ 110 N*3/2(1550) -2*IMAG PART OF POLE POSITION (MEV) $11 / 77$ IM 8 105. OR 104. LONGACRE 77 I PWA PIN TO $2 P$ I N $11 / 77$ $110 N * 3 / 211550)$ PARTIAL DECAY MODES $11 / 77$ $\stackrel{\substack{p \\ p}}{p}$

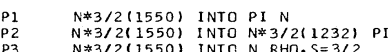
DECAY MASSES
$139+938$ $139+938$
$1232+139$
$938+776$ $110 N * 3 / 2(1550)$ BRANCHING RATIOS

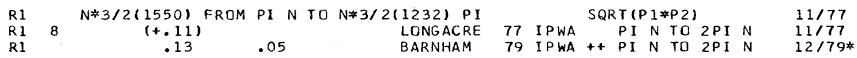

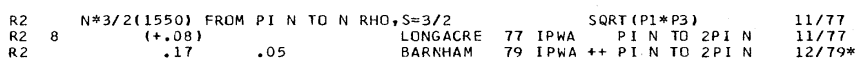
****************************************************************************1

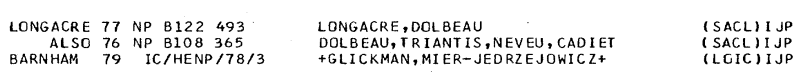

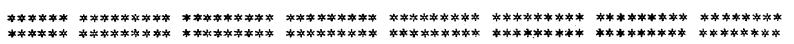
$\Delta(1650){ }_{82}{ }^{N * 3 / 2(1650, J P=1 / 2-1} \mathrm{I}=3 / 2 \quad S_{31}^{\prime}$ $82 N * 3 / 2(1650)$ MASS (MEV)

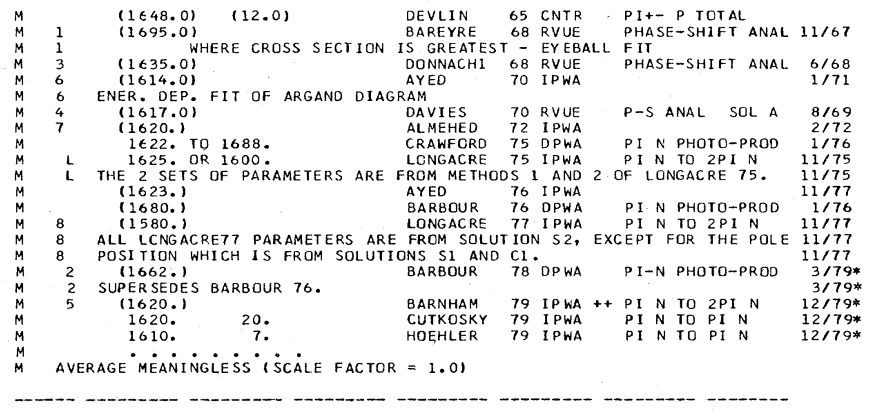




\section{Baryons $\Delta(1650), \Delta(1670)$}
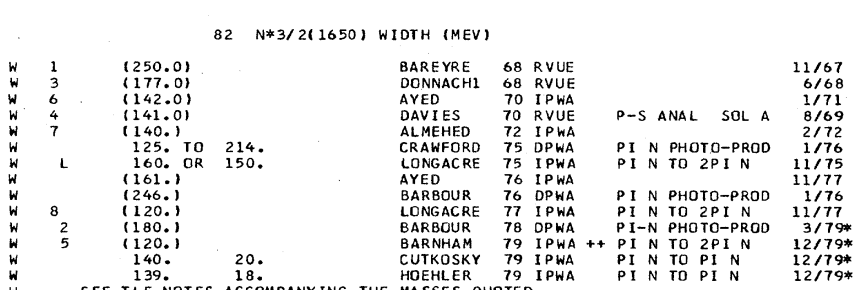

AVERAGE MEANi MGLLE SS $^{\circ}$ i SCALE FACTOR $=1.0$ )

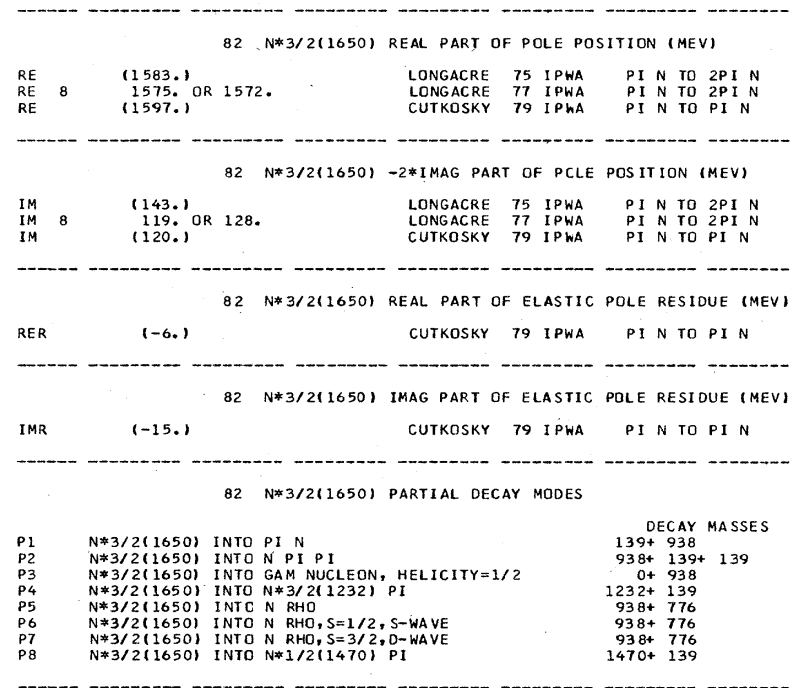

$82 N * 3 / 2(1650)$ BRANCHING RATIOS

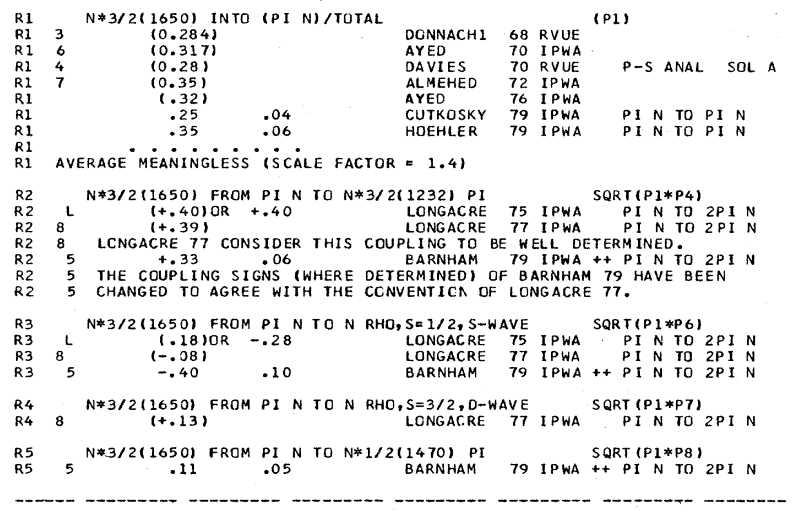

$82 N * 3 / 2(1650)$ PHOTON DECAY AMPL (GEV**-1/2) FOR DEFINITION OF GAMMA-NUCLEON DECAY AMPL ITUDES, SEE MINI-
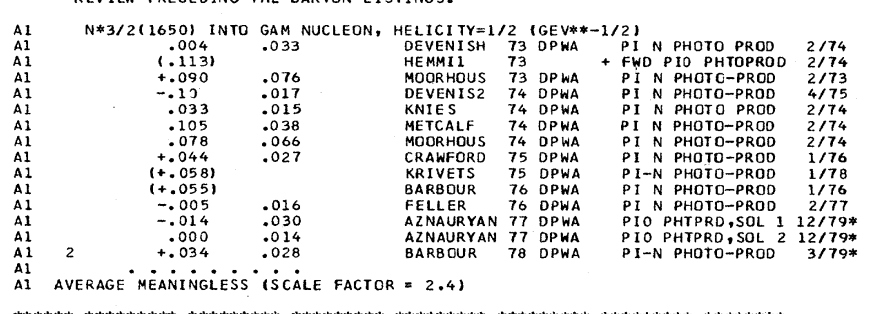

$6 / 68$
$1 / 171$
187169
27172
11777
127797
$12 / 790 *$ $11 / 75$
11775
11177 $11 / 77$
$12 / 79 *$
$12 / 779 *$ $12 / 79 *$ $11 / 75$
$11 / 75$
$11 / 77$
$12 / 79 *$ ${ }_{11 / 77}^{11 / 77}$ $12 / 79 *$

\section{Data Card Listings For notation, see key at front of Listings.}

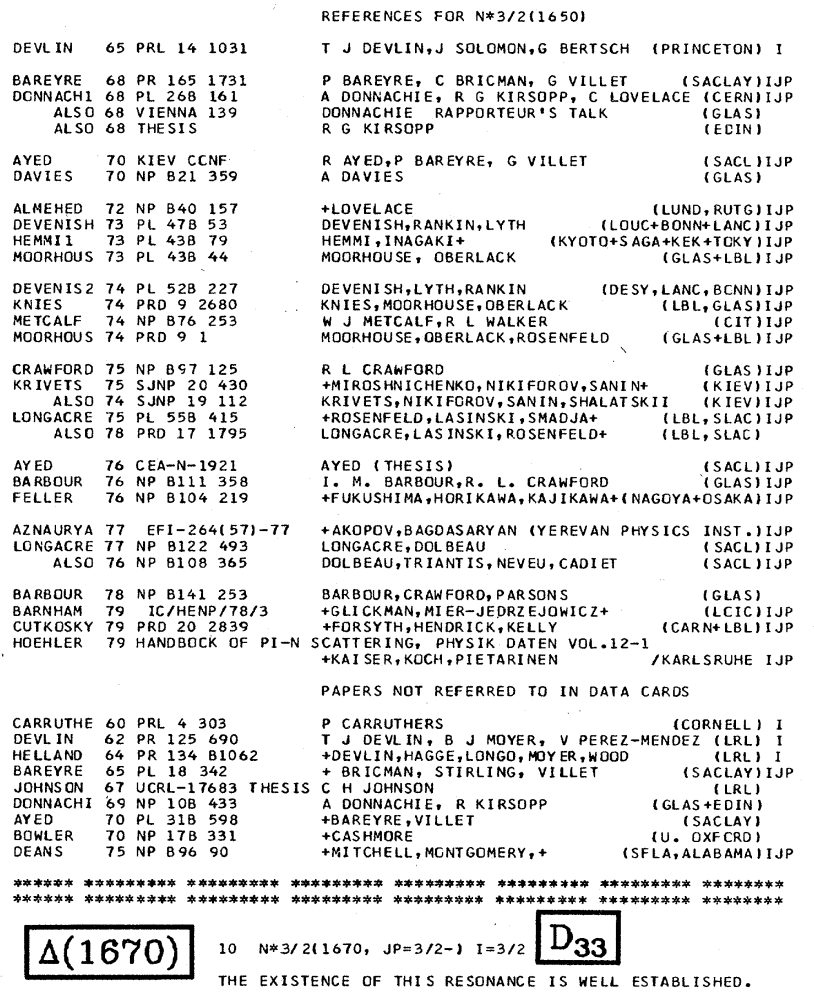

$10 N * 3 / 2(1670)$ MASS (MEV)

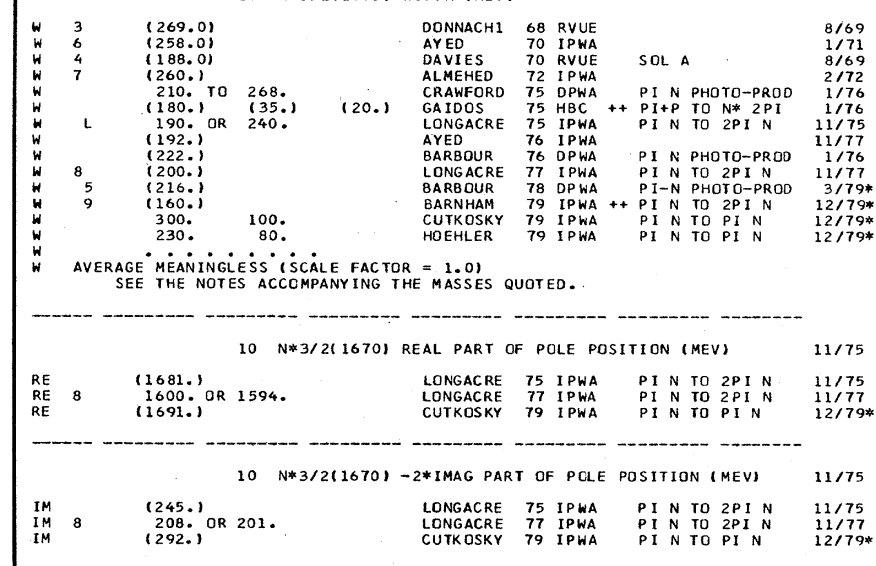




\title{
Data Card Listings
}

\author{
For notation, see key at front of Listings.
}

\section{Baryons $\Delta(1670), \Delta(1690)$}

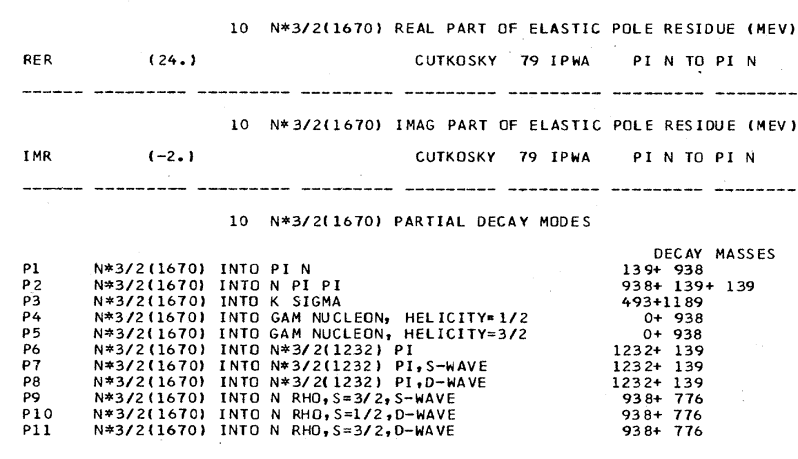

$10 N * 3 / 2(1670)$ BRANCHING RATIOS

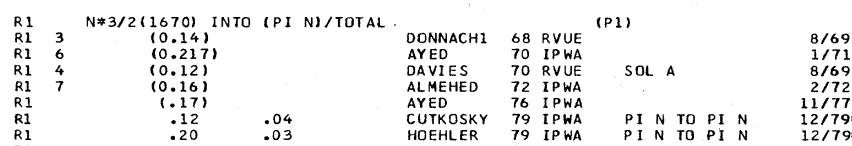
R1 AVERAGE MEANINGLESS iSCALE FACTOR $=1.61$

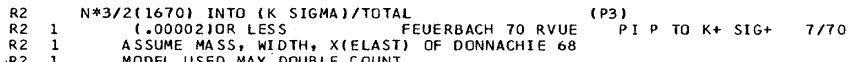

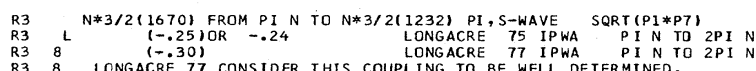

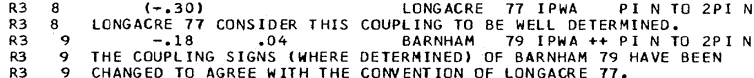

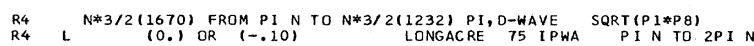

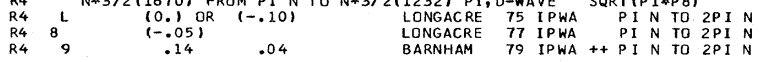

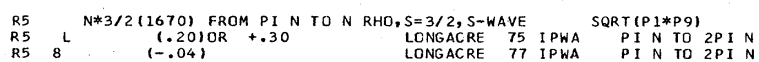
R6
R6 $2 * 3 / 2(1670)$ FROM PI N TO K SIGMA
DEANS 75 DPWA SQRT PII $N$ PP3)

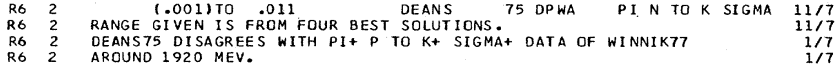

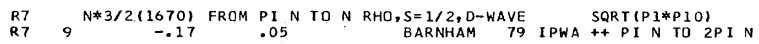

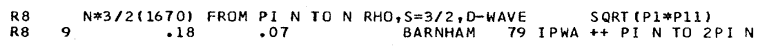
$10 N * 3 / 2(1670)$ PHOTCN DECAY AMPL (GEV**-1/2) FOR OEFINITION OF GAMMA-NUCLEON DECAY AMPL ITUDES, SEE MINI-

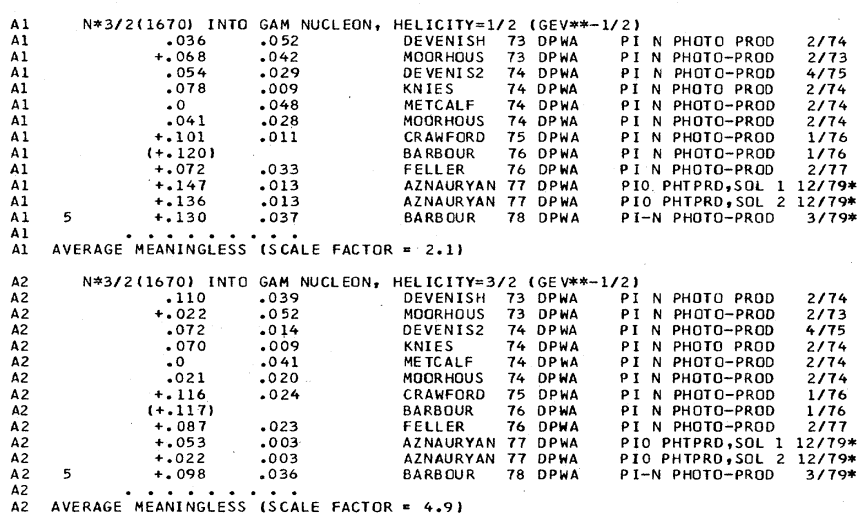
********************************************************************* $\begin{array}{rllll}\text { DONNACH1 } & 68 & \text { PL } & 268 & 161 \\ \text { ALSO } & 68 & \text { VIENNA } & 139\end{array}$ REFERENCES FOR $N * 3 / 211670$ ALSO 68 VIENNA 13
ALSO 68 THESIS

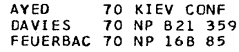
A DONNACHIE, R G KIRSOPP, C LOVELACE (CERN) IJPP DONNACHIE RAPPORTEUR'S TALK
R G KIRSOPP
(ELIN) R AYED, P BAREYRE, G VILLET

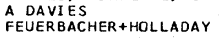

${ }_{11 / 775}^{11 / 75}$

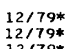
${ }_{111775}^{11 / 75}$

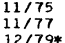
$11 / 75$

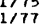
$1 / 75$
1175
1175
1178
$1 / 78$ ${ }_{12,79 *}^{12 / 79 *}$ $\frac{12 / 79 *}{12 / 79 *}$

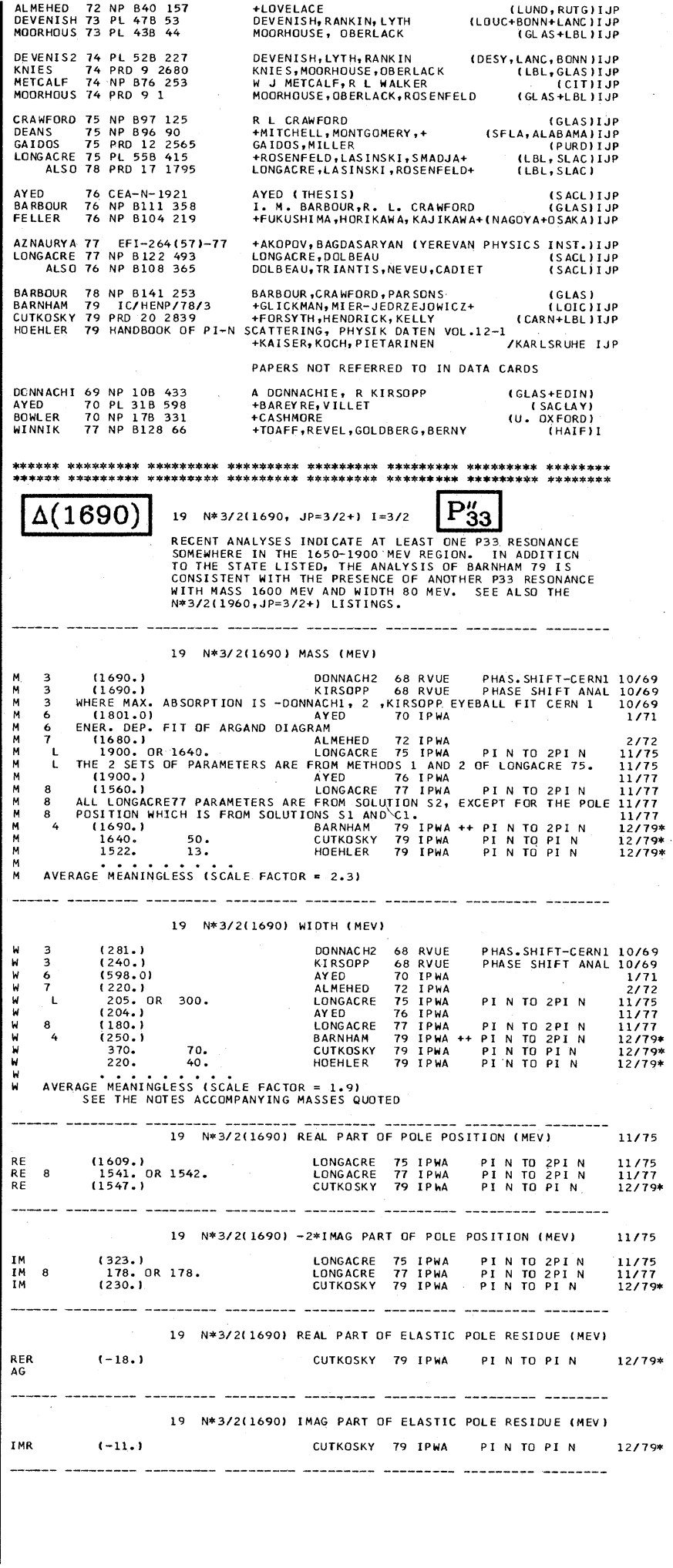


Baryons

$\Delta(1690), \Delta(1890)$

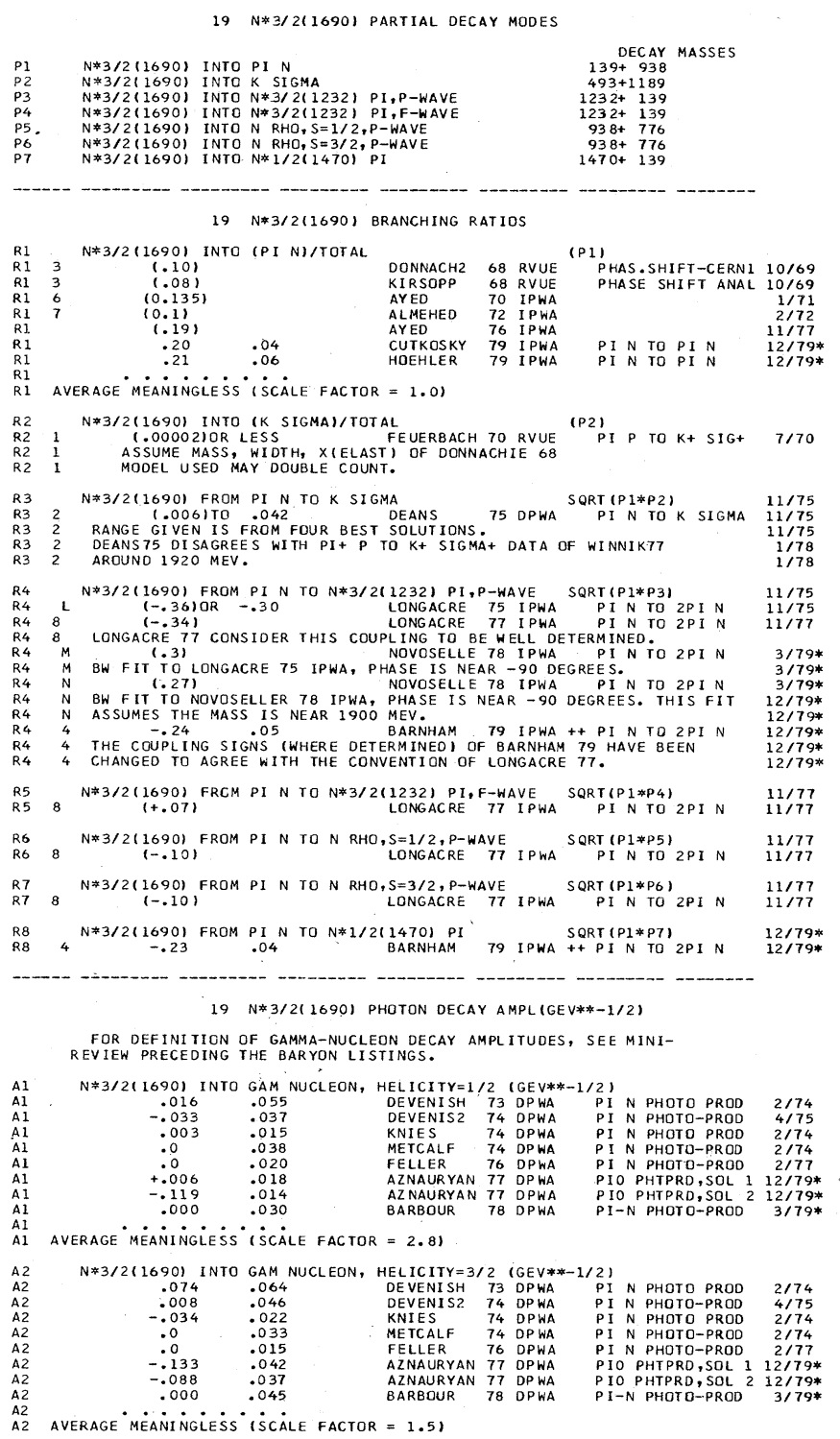

\section{Data Card Listings} For notation, see key at front of Listings.

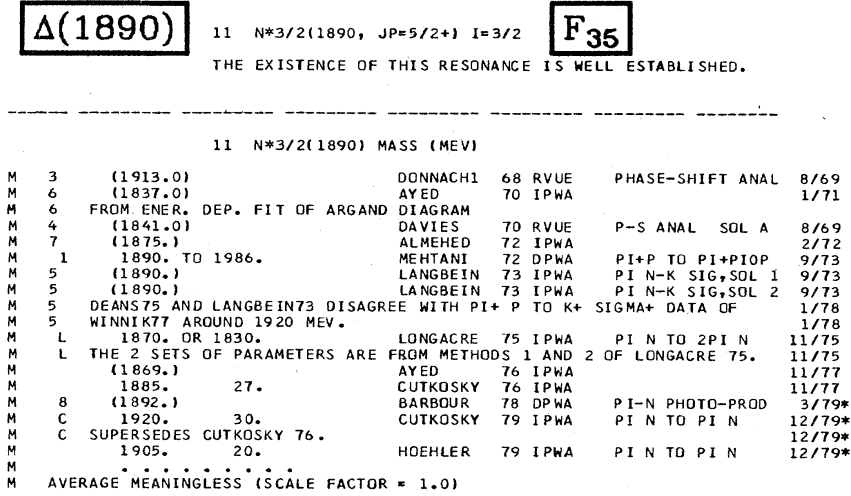

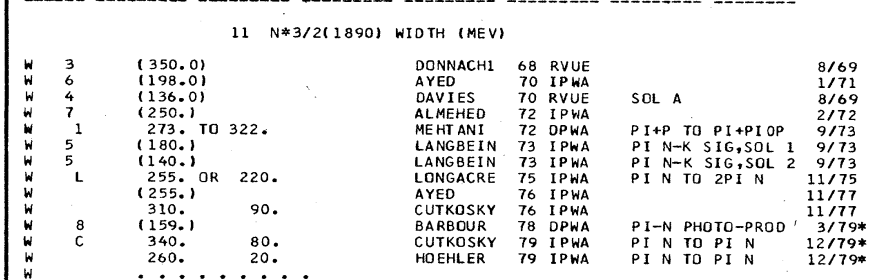

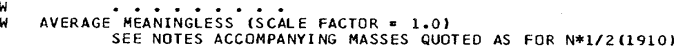

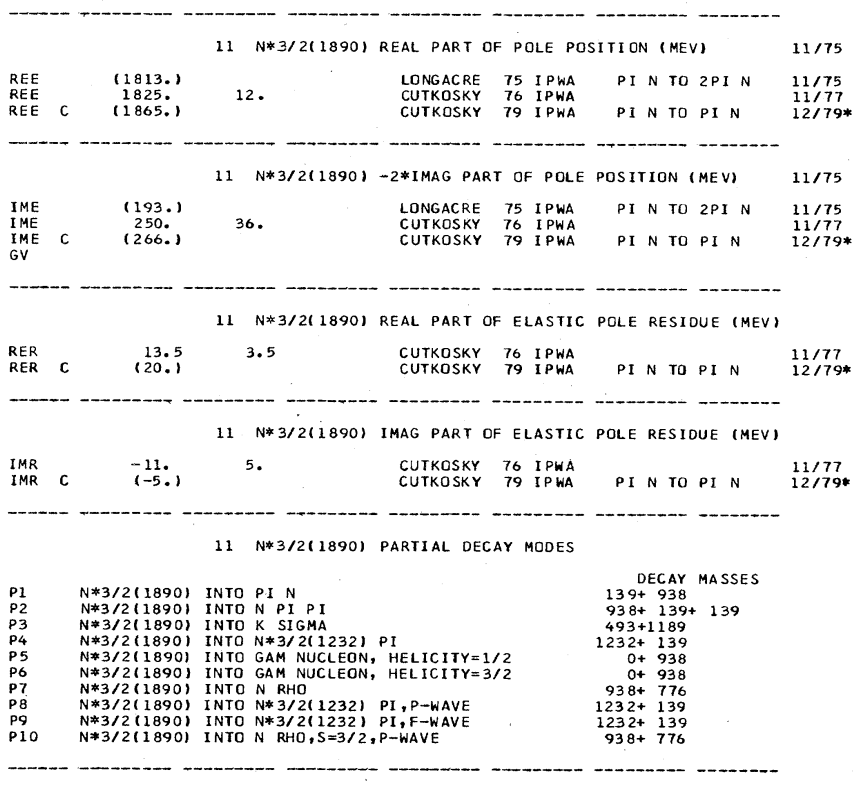




\section{Data Card Listings}

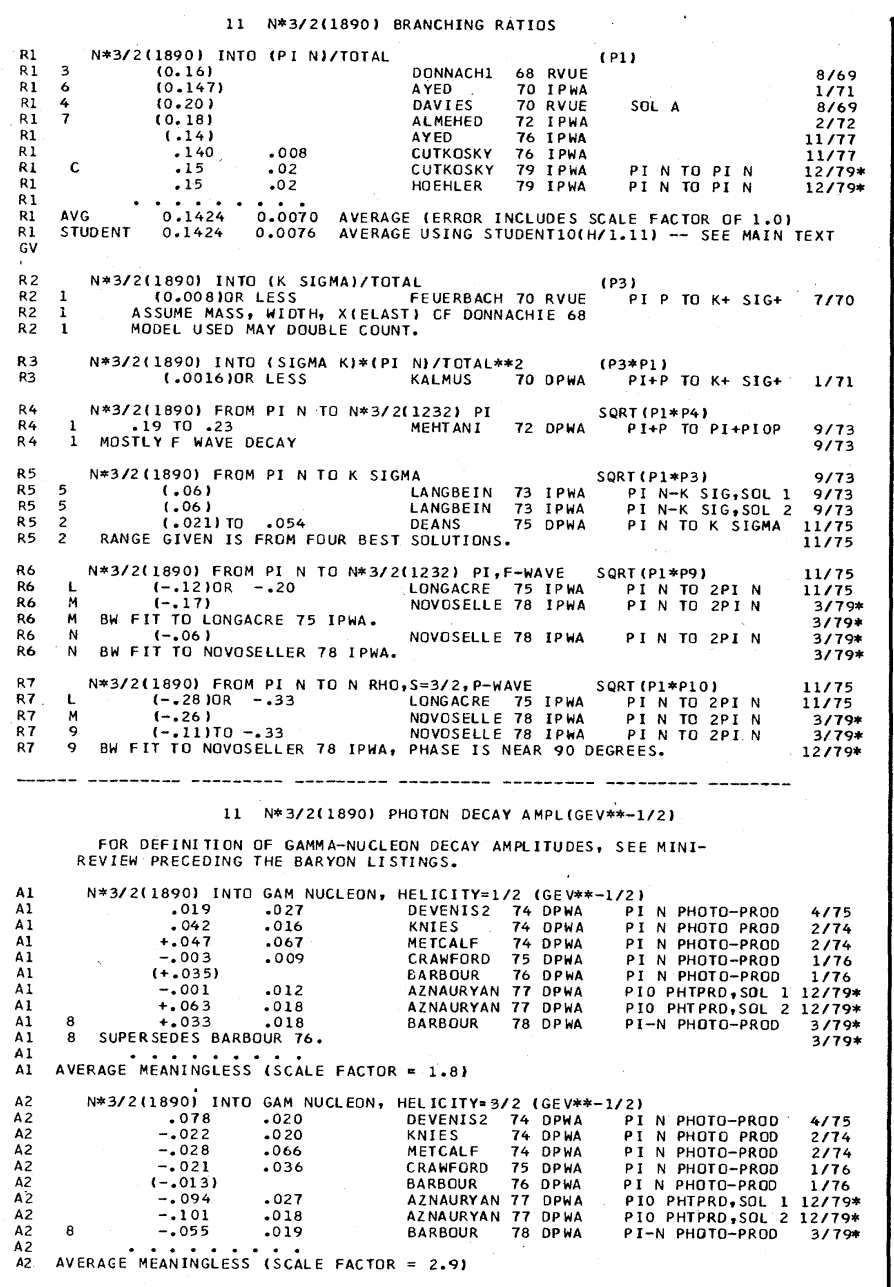

*********************************************************************1* $\begin{array}{rllll}\text { DCNNACH1 } & 68 & \text { PL } & 26 B & 161 \\ \text { ALSO } & 68 & \text { VIENNA } & 139 \\ \text { ALSO } & 68 & \text { THESIS } & 139\end{array}$

$\begin{array}{lll}\text { AYED } & 70 \mathrm{KIEV} \text { CONF } \\ \text { DAVIES } & 70 \mathrm{NP} B 21359\end{array}$ $\begin{array}{lllll}\text { DAVIES } & 70 & \text { NP } & B 21 & 359 \\ \text { FEUERBAC } & 70 & \text { NP } & 168 & 85 \\ \text { KALMUS } & 70 & \text { PR } & \text { D2 } & 1824\end{array}$

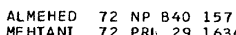

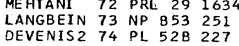

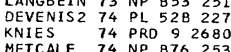
CRAWFORO 75 NP 897125

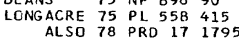
$\begin{array}{lll}\text { AYED } & 76 & \text { CEA-N-1921 } \\ \text { BA FBOUR } & 76 & \text { NP B11 } 11258 \\ \text { CUTKOSKY } 76 \text { PRL } 37645\end{array}$ $\begin{array}{ccc}\text { CUTKOSKY } 76 & \text { PRL } 37 & 645 \\ \text { ALSO } 76 & \text { OXFORD CONF. } & 49 \\ \text { AZNAURYA } 77 & \text { EFI }-264(57)-7\end{array}$ $\begin{array}{rlllll}\text { BAFBOUR } & 78 & \text { NP } & B 141 & 253 \\ \text { NOVOSELL } 78 & \text { NP } & B 137 & 509 \\ \text { ALSO } 78 & \text { NP } & B 137 & 445\end{array}$ REFERENCES FOR $N * 3 / 2(1890$

A DONNACHIE, R G KIRSOPP, C LOVELACE (CERN) IJP
DONNACHIE RAPPORTEUR'S TALK DONNACHE RAPPORTEUR'S TALK
$R G$ KIRSOPP
(GLAS)

$R$ AYED, P BAREYRE, G VILLET
A DAVIES G KALMUS, G BORREANI, J LOUIE (VANDERBILT) HOVELACE
+FUNG, KERNAN, SCHALK, + (LUND, RUTGIIJP DEVENISH, LYTH, RANKIN
KNIES, MOORHOUSE, OBERLACK
(OESY, LANC, BONNIIJP
(LBL, GLAS)IJP KNIES, MOORHOUSE, OBERLACK
$W J$ METCALF, R L WALKER $R$ L CRAWFORD
+MIICHELL, MONTGOMERY ,+
(SFLAS IIJP +ROS ENFELD, LASINSKI, SMADJA+
LONGACRE, LASINSKI, ROSENFELD+ AYED (THESIS)
(SACL) IJPP CUITOS KY, HENDRICK, KELLY
CUTKOSKY, HENORICK, CHAO+
(CARN+LBL) IJP CUTKOSKY, HENDRICK, CHAO+ (CARN+LBL+BRISIIJP
+AKOPOV, BAGDASARYAN (YEREVAN PHYSICS INST.IIJP BARBOUR, CRAWFORD, PAR SONS D. E. NOVOSELLER (CAL 'GLAS) CUTKOSKY 79 PRD 202839
HOEHLER 79 HANDBOCK OF PI-N SCATIER ING, PHYSICK IK OATEN VOL.12-1 (CARN+LBLIIJP +KAI SER, KOCH, PIETARINEN TINARLSRUHE IJP
PAPERS NOT REFERRED TO IN DATA CAROS $\begin{array}{llllll}\text { AYED } & 70 & \text { PL } 31 B 598 & \text { +BAREYRE+VILLET } & \text { (SACLAY) } \\ \text { WINNIK } & 77 & \text { NP B } 12866 & \text { +TOAFF, REVEL, GOLDBERG, BERNY } & \text { (HAIF) }\end{array}$

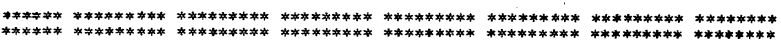

$\Delta(1900)$ 30 N*3/211900, JP=1/2-1 I*3/2 $S_{31}^{\prime \prime}$ THIS EFFECT IS SEEN IN TWO CHANNELS. $30 \quad N * 3 / 2(1900)$ MASS (MEV) $9 / 73$

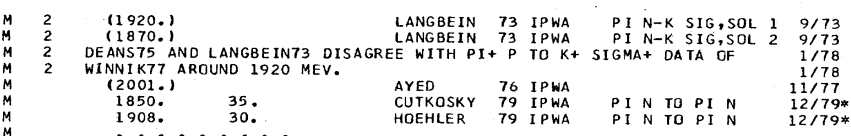

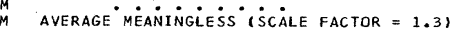

\begin{tabular}{|c|c|c|c|c|c|c|}
\hline & & $30 N * 3 / 2(1900)$ & WIDTH (MEV) & & & $9 / 73$ \\
\hline $\begin{array}{ll}w & 2 \\
W & 2 \\
W & \\
W & \end{array}$ & $\begin{array}{l}(140 .) \\
1160:) \\
1307:) \\
130: \\
140 .\end{array}$ & $\begin{array}{l}40 . \\
40 .\end{array}$ & $\begin{array}{l}\text { LANGBEIN } \\
\text { LANGEIN } \\
\text { AYEDEIN } \\
\text { CUTKOSKY } \\
\text { HOEHLER }\end{array}$ & 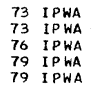 & $\begin{array}{l}\text { PI N-K SIG,SOL } 1 \\
\text { PI N-K SIG;SOL } \\
\text { PI N TO PI } \\
\text { PI N TO PI N } \\
\text { PI N TO PI N }\end{array}$ & $\begin{array}{r}9 / 73 \\
9973 \\
11 / 77 \\
12279 \% \\
12 / 79 *\end{array}$ \\
\hline
\end{tabular}

average Méaningiess iscale factor $=1.01$

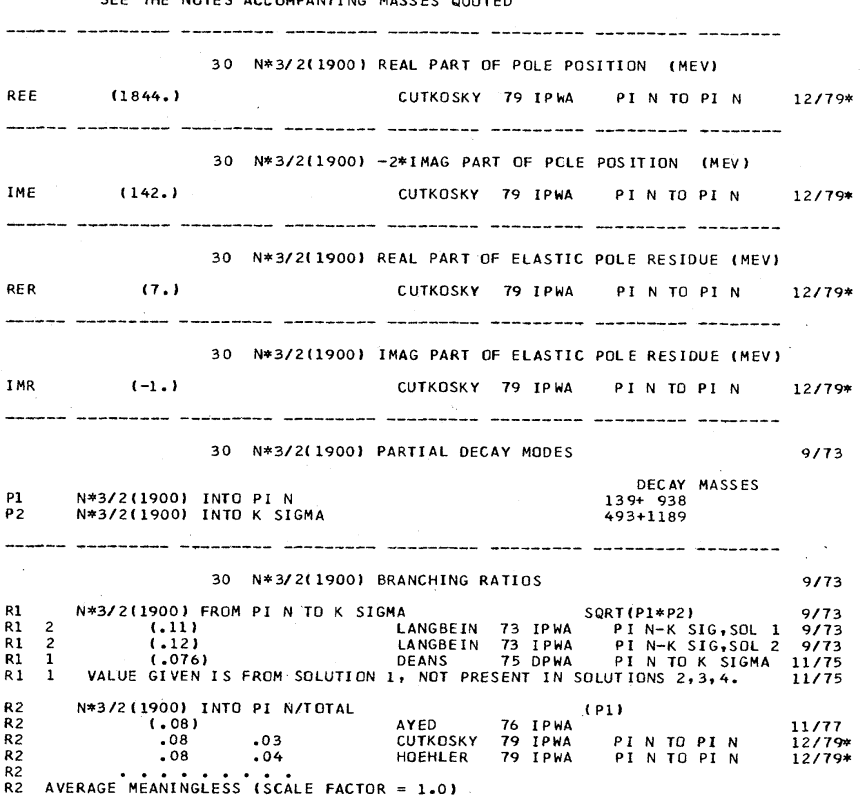

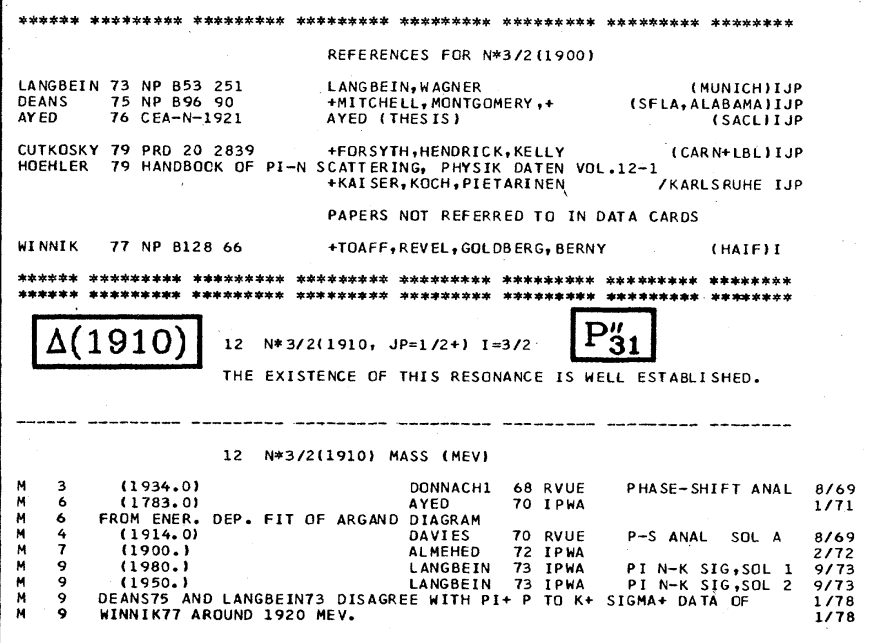




\section{Baryons}

$\Delta(1910), \Delta(1950)$

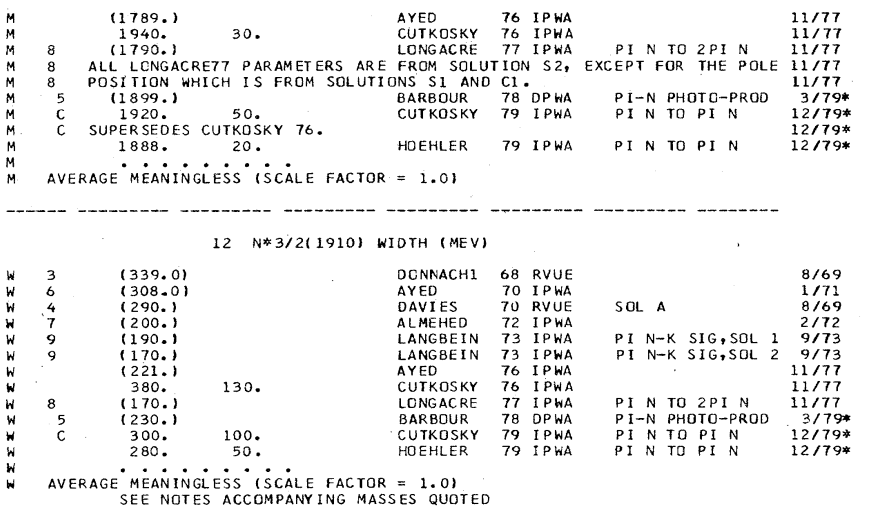

\begin{tabular}{|c|c|c|c|c|c|}
\hline $\begin{array}{ll}\text { REE } \\
\text { REE } & 8 \\
\text { REE } & \text { C }\end{array}$ & 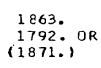 & $\begin{array}{r}15 . \\
1801:\end{array}$ & $\begin{array}{lll}\text { CUTKOSKKY } & 76 & \text { I PWA } \\
\text { LONGACRE } & \text { IPWA } \\
\text { CUTKOSKY } & 79 & \text { IPWA }\end{array}$ & 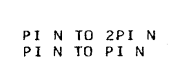 & $\begin{array}{l}11 / 77 \\
11177 \\
12 / 79 *\end{array}$ \\
\hline & & $12 \mathrm{N*}$ & -2*1MAg part of pole & POSITION IMEVI & \\
\hline $\begin{array}{l}\text { IME } \\
\text { IME } \\
\text { IME }\end{array}$ & $\begin{array}{l}250 . \\
172.0 R \\
(200 .)^{\circ R}\end{array}$ & 165. & $\begin{array}{lll}\text { CUTKOSKKY } & 76 & \text { IP } \\
\text { LONGACRA } & 77 & \text { IPWA } \\
\text { LUUTKOSKY } & 79 & \text { IPWA }\end{array}$ & 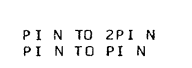 & $\begin{array}{l}11 / 77 \\
11277 \\
12 / 79 *\end{array}$ \\
\hline & & $12 N *$ & REAL PART OF ELASTIC & POLE RESIDUE (MEV) & \\
\hline $\begin{array}{lll}\substack{R \in R \\
R \in R} & C\end{array}$ & $(-4.6)$ & 5. & 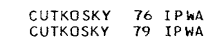 & PIN то PIN & $\begin{array}{l}11 / 77 \\
12 / 79 *\end{array}$ \\
\hline $\begin{array}{l}\operatorname{IMR} \\
\operatorname{IMR}\end{array}$ & $(-278)$ & $12 \mathrm{N*}$ & $\begin{array}{c}\text { IMAG PART OF ELASTIC } \\
\text { CUTKOSKY } 76 \text { IPWA } \\
\text { CUTKOSKY } 79 \text { IP }\end{array}$ & $\begin{array}{l}\text { POLE RESIDUE (MEV) } \\
\text { PI N TO PI N }\end{array}$ & $\begin{array}{l}11 / 77 \\
12 / 79 *\end{array}$ \\
\hline
\end{tabular}

$12 N * 3 / 2(1910)$ PARTIAL DECAY MODES

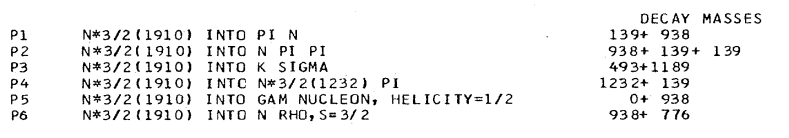

$$
12 \text { N*3/2(1910) BRANCHING RATIOS }
$$

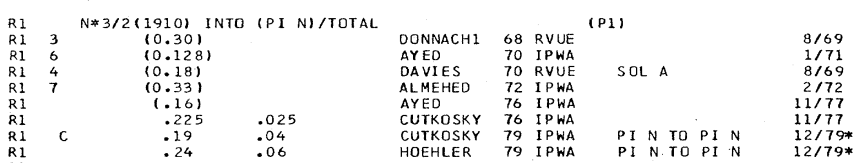

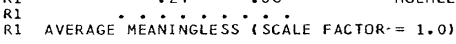

$R 2$
$R 2$ 1 N*3/2(1910) INTC (K SIGMA)/TOTAL
$(0.008) 0 R$ LESS

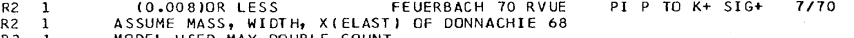
R2 1 MODEL USED MAY DOUBLE COUNT.

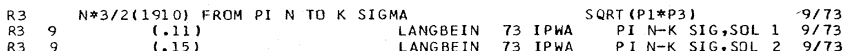

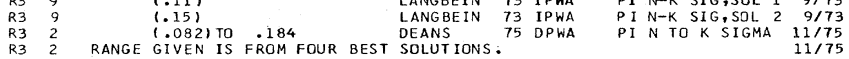
$R 4$
$R 48$ $\begin{aligned} & N * 3 / 2(1910) \text { FROM PI N TO N*3/2(1232) PI } \\ & \text { LONGACRE } 77 \text { I PWA }\end{aligned}$

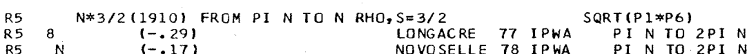
R5 $N$ EVIDENCE FOR THIS COUPLING IS WEAK, SEE NOVOSELLER 78 . THIS

\section{Data Card Listings For notation, see key at front of Listings.}

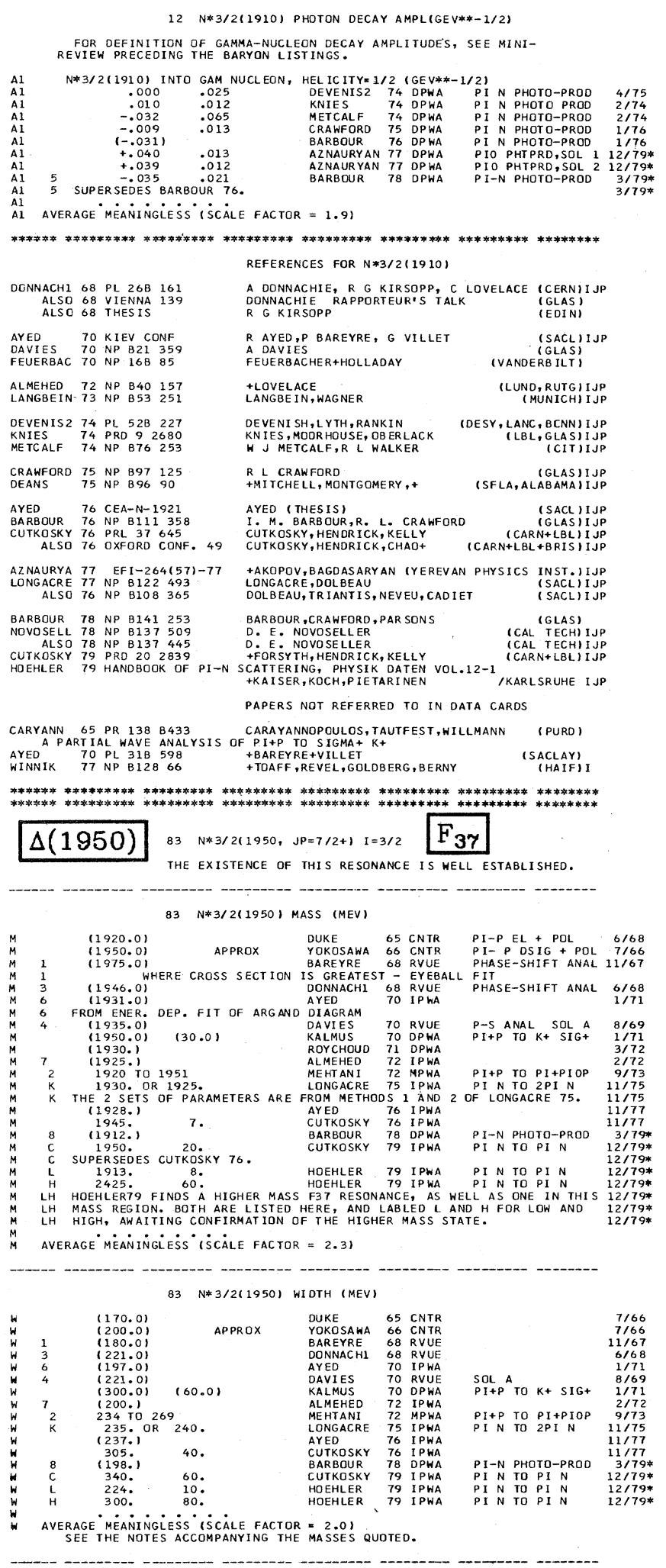




\section{Data Card Listings}

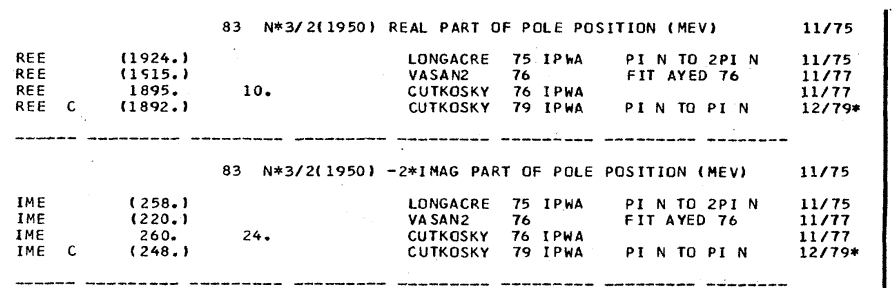

\begin{tabular}{|c|c|c|c|c|}
\hline$R$ & $(43)$. & $\begin{array}{c}83 \quad N * 3 / 2(1950) \\
6 .\end{array}$ & $\begin{array}{l}\text { REAL PART OF ELASTIC } \\
\text { CUTKOSKY } 76 \text { IPWA } \\
\text { CUTKOSKY } 79 \text { I PWA }\end{array}$ & $\begin{array}{l}\text { C POLE RESIDUE IMEV } \\
\text { PI N TO PI N }\end{array}$ \\
\hline $\begin{array}{l}\text { IMR } \\
\text { IMR }\end{array}$ & $\begin{array}{l}-25 \\
(-24 .)\end{array}$ & $\begin{array}{l}83 N * 3 / 2(1950) \\
9 .\end{array}$ & $\begin{array}{l}\text { IMAG PART OF ELASTIC } \\
\text { CUTKOSKY } 76 \text { IPWA } \\
\text { CUTKOSKY } 79 \text { IPWA }\end{array}$ & $\begin{array}{l}\text { POLE RESIDUE IM } \\
\text { PI N TO PI N }\end{array}$ \\
\hline $\begin{array}{l}\text { P1 } \\
\text { P2 } \\
\text { P3 } \\
\text { P4 } \\
\text { P5 } \\
\text { P6 } \\
P 7 \\
\text { P8 } \\
\text { P9 } \\
\text { P110 } \\
P 11 \\
\text { P11 } \\
\text { P13 }\end{array}$ & $\begin{array}{l}N * 3 / 2(1950) \\
N * 3 / 2(1950) \\
N * 3 / 2(1950) \\
N * 3 / 21950) \\
N * 3 / 2(1950) \\
N * 3 / 21950) \\
N * 3 / 2(1950) \\
N * 3 / 2(1950) \\
N * 3 / 2(1950) \\
N * 3 / 2(1950) \\
N * 3 / 2(1950) \\
N * 3 / 2(1950) \\
N * 3 / 2(1950)\end{array}$ & 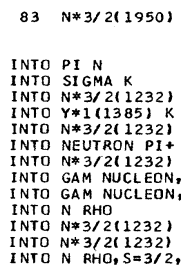 & $\begin{array}{l}\text { PI } \\
\text { PI } \\
\text { RHO } \\
\text { PI+ } \\
\text { PI PI (NOT RHO) } \\
\text {, HELIIIY }=1 / 2 \\
\text { HELICI TY }=3 / 2 \\
\text { PI, F-WAVE } \\
\text { PI, H-WAVE } \\
\text { PF-WAVE }\end{array}$ & $\begin{array}{l}\text { DECAY MASSE } \\
139+938 \\
1189+493 \\
1232+139 \\
1384+493 \\
1232+776 \\
939+139+139 \\
1232+139+139 \\
0+938 \\
0+938 \\
938+776 \\
1232+139 \\
1232+139 \\
938+776\end{array}$ \\
\hline
\end{tabular}

$83 N * 3 / 2(1950)$ BRANCHING RATIOS

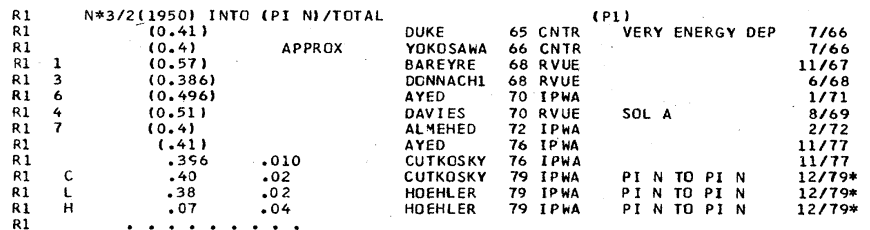

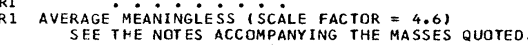

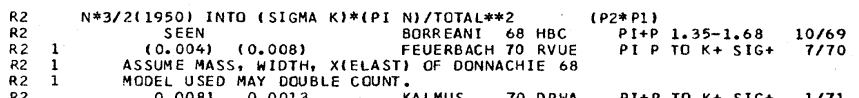

$R 3$
$R 3$ $2 \begin{array}{ll}N * 3 / 2(1950) \text { FROM PI N TO N*3/2(1232) PI } \\ \text { R3 }\end{array}$

R4 N*3/2(1950) FROM PI N TO SIGMAK SQRT(P1*P2).

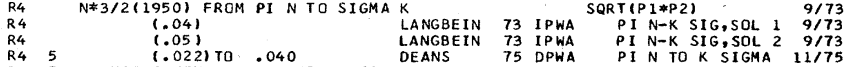

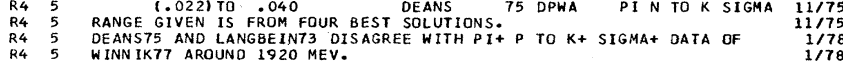

$\begin{aligned} & R 5 \\ & R 5\end{aligned} \quad N * 3 / 2(1950)$ FROM PI N TO N*3/2(1232) PI, F-WAWE SORT (P1*P11)

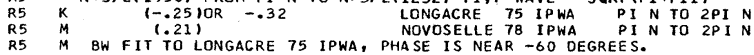

$R 5$
$R 5$ N BW FIT TO NOVOSELLER 78 I PWA, PHASE IS NEAR -60 DEGREES.

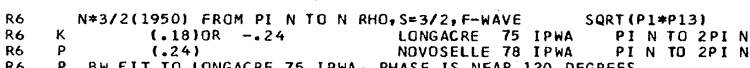

R6
R6
R6

MORE INFORMATION ON INEL ASTIC DECAY MODES OF BUMPS, SEEN IN PRODUCTION
EXPERIMENTS ARCUND 1950 MEV, MAY BE FOUND IN THE NEXT ENTRY

$$
83 N * 3 / 2(1950) \text { PHOTON DECAY AMPL (GEV**-1/2) }
$$

FOR DEF INITION OF GAMMA-NUCLEON DECAY AMPLITUDES, SEE MINIERECEDING
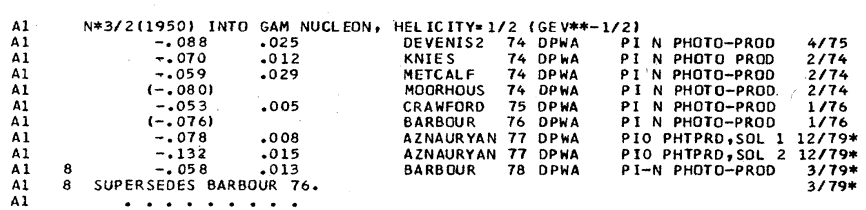

$11 / 75$
$11 / 75$
$3 / 79 *$
$3 / 79 *$
$3179 *$
$3 / 79 *$
$11 / 75$
$11 / 75$
$3179 \%$
$3 / 79 \%$
$3179 \%$
$3 / 79 *$

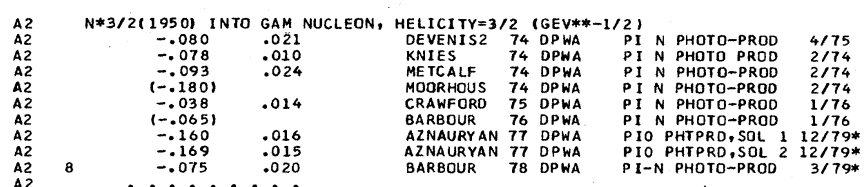

A2 average méaninglés is iscale factor $=3.21$

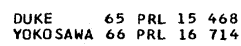
REFERENCES FOR $N * 3 / 2(1950)$

BAREYRE 68 PR 1651731 +JONES, KEMP, MURPHY, PRENTICE, +
+SUWA, HILL, ESTERLING, BOOTH

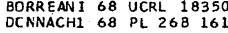
ALSO 68 VIENNA 139
ALSO 68 THESIS $\begin{array}{llll}\text { AYEO } & 70 & \text { KIEV } & \text { CONF } \\ \text { DAVIES } 70 & \text { NP } & \text { B21 } & 359 \\ \text { FEUERBAC } 70 & \text { NP } 168 & 85 \\ \text { KALAUS } & 70 & \text { PR } & \text { D2 } 1824\end{array}$ ROYCHOUD 71 NP 827125 $\begin{array}{lllll}\text { ALMEHED } & 72 & \text { NP } & 840 & 157 \\ \text { MEHTANI } & 72 & \text { PRL } 29 & 1634 \\ \text { LANGBEIN } & 73 & \text { NP } & \text { B53 } & 251\end{array}$ DE VENIS2 74 PL $528 \quad 227$

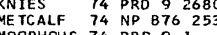

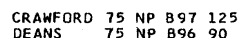

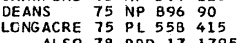
$\begin{array}{llll}\text { AYED } & 76 & \text { CEA-N-1921 } \\ \text { BARBOUR } & 76 & \text { NP B111 } 358\end{array}$ ALSO 76 OXFORD CONF. 49
VASAN2 76 NP B106 526 AZNAURYA 77 EFI-264(57)-77 NOVOSELL 78 NP B137 509 P BAREYRE, C BRICMAN, G VILLLT (SACLAY)IJJP A DONNACHIE, R G KIRSOPP, C LOVELACE (CERNIIJP DONNACHIE RAPPORTEUR'S TALK
$R G$ KIRSOPP
(GLAS) R AYEO, P BAREYRE, G VILLET FEUERBACHER+HOLLADAY R K ROYCHOUDHURY, B H BRANSOEN +LOVELACE
+FUNG, KERNAN, SCHALK, +
LANGBE IN, WAGNER (GLAS)
ERB ILT) (LUNO, RUTG)IJP DEVENI SH, LYTH, RANKIN KNIES,MOORHOUSE, OBERLACK LANC, BONN) IJPP WESMCALF, R L WALKER
WO METCALF
MOORHOUSE, OBERLACK, ROSENFELD $R$ L CRAWFORD
+MITCHEL, MONTGOMERY,
TOSLAS) IJPP +ROSENFELD, LAS INSKI, SMADJA+
LONGACRE, LASINSKI, ROSENFELO+
(LBL, SLAC)IJ AYED (THESIS)

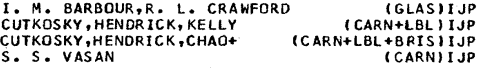
+AKOPOV, BAGDASARYAN (YEREVAN PHYSICS INST. II JP
BARBOUR, CRAWFORD, PARSONS BARBOUR, CRAWFORD, PARSONS
O. E. NOVOSELLER (CGL TECH) IJP
(CAL TECH) IJP CUTKOSKY 79 PRD 202839 FFORSYTH, HENDR ICK, KELLY (CARN+LBL)IJP
CCATTER ING. PHYSIK DATEN VOL.12-1 (CAR + TKAISER, KOCH, PIETARINEN PAPERS NOT REFERRED TO IN DATA CARDS

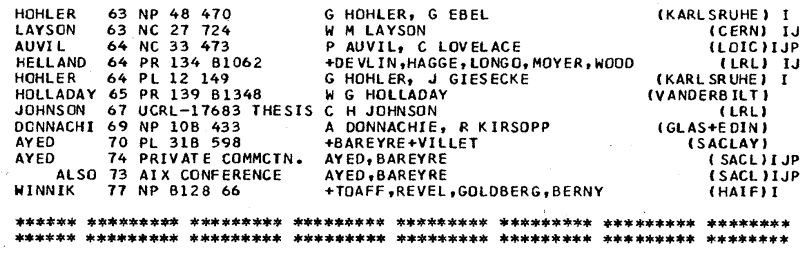

1950 MEV REGION - PRODUCTION AND $\sigma_{\text {TOTAI EXP'TS }}$

$70 N * 3 / 211950, J P=\quad, I=3 / 2 \quad$ PRODUCTION EXPERIMENTS SEE THE MINI-REVIEW PRECEDING THE N AND DELTA LISTINGS
FOR A DISCUSSION OF PRODUCTION EXPER IMENTS $70 N * 3 / 2(1950)$ MASS (MEV) (PRDD. EXP.)

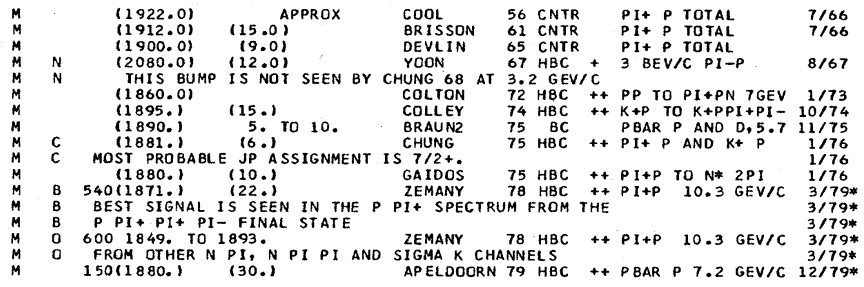
70 N*3/2(1950) WIOTH (MEV) (PROD. EXP.)

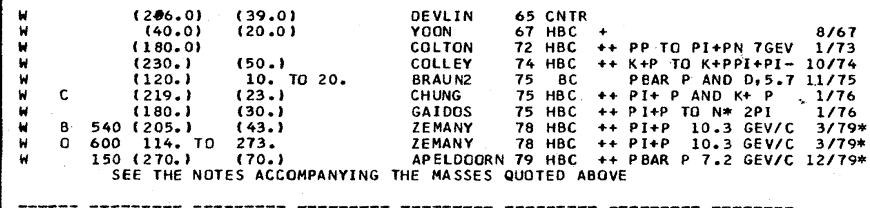




\section{Baryons $\Delta(1950), \Delta(1960)$}

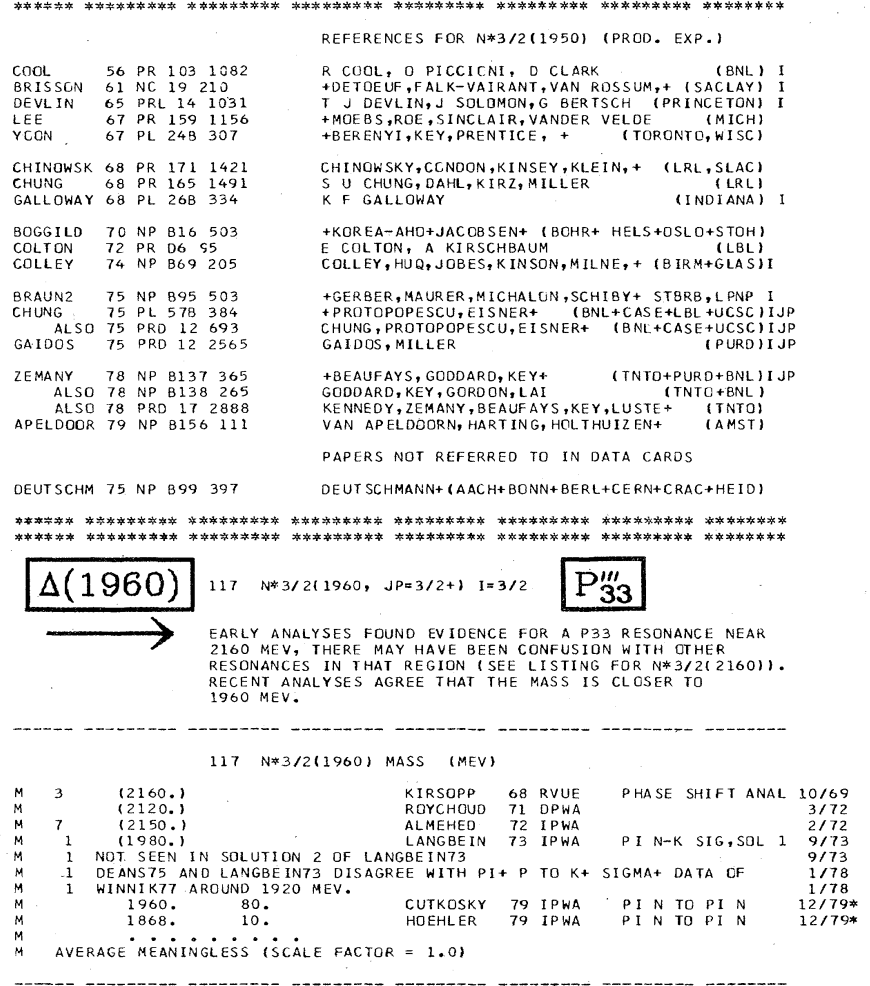

$70 N * 3 / 2(1950)$ PARTIAL DECAY MODES (PROD. EXP.)

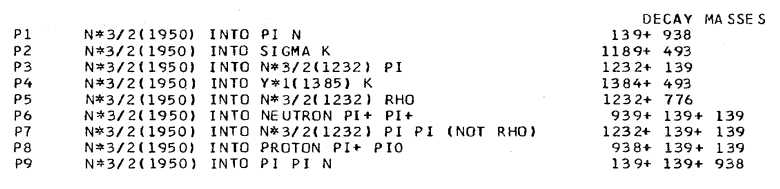

$70 N * 3 / 2(1950)$ BRANCHING RATIOS (PROD. EXP.)

\begin{tabular}{|c|c|c|c|c|c|}
\hline & 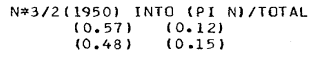 & $\begin{array}{l}\text { DEVLIN } \\
\text { ZEMANY }\end{array}$ & $\begin{array}{l}65 \text { CNTR } \\
78 \text { HBC }\end{array}$ & $\begin{array}{l}\left(P_{1}\right) \\
++P_{I}+P \\
10 .\end{array}$ & 11 \\
\hline & $\begin{array}{r}3 / 2(1950) \text { INTO } \\
0.059\end{array}$ & & $68 \mathrm{HBC}$ & 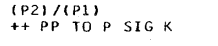 & It \\
\hline & $\begin{array}{l}\text { *3/2(1950) INTO N*3/2(1232) PI } \\
\text { SEEN } \\
\text { SEEN }\end{array}$ & $\begin{array}{l}\text { II PI } \\
\text { CHIN } \\
\text { BOGG }\end{array}$ & $\begin{array}{l}\text { RHOI } \\
68 \text { HBC } \\
70 \mathrm{HBC}\end{array}$ & $\begin{array}{l}(P 7) \\
++P P \text { TO } \\
P P \text { TC }\end{array}$ & $\begin{array}{r}11 / 68 \\
6 / 70\end{array}$ \\
\hline & $\begin{array}{c}N 3 / 2(1950) \text { INTO (PI } N) /(N * 3 / 21 \\
(0.55) \text { OR LESS }\end{array}$ & $\begin{array}{ll}2(1232) \\
\text { LEE }\end{array}$ & $67 \mathrm{HBC}$ & $\begin{array}{l}(P 1) /(P 3) \\
P I-P 3.63 \mathrm{BEV} / \mathrm{C}\end{array}$ & 1) \\
\hline & $N * 3 / 2(1950)$ INTO (IPI N)*(NEUTR & & & & \\
\hline & 0.013 & GALLOWAY & $68 R$ & To & 61 \\
\hline & ${ }_{5}^{I N T O}(Y * 1(1385) \mathrm{k}) /$ & $\begin{array}{l}\text { /(PI N) } \\
\text { CHINOWSKY }\end{array}$ & $68 \mathrm{HBC}$ & P1) & \\
\hline & $\begin{array}{l}\text { (1232) R } \\
\text { N FOR UN }\end{array}$ & $\begin{array}{c}\mathrm{RH} \\
\mathrm{C} \\
\mathrm{NS}\end{array}$ & & $\begin{array}{l}1 P 5 \\
++ \\
\text { FAC }\end{array}$ & $11 /$ \\
\hline & $\begin{array}{l}N * 3 / 2(1950) \text { INTO }(N * 3 / 2(1232) R \\
\text { SEEN SEEN } \\
\text { NOT SEEN }\end{array}$ & $\begin{aligned} R H O 1 \\
\text { YOC } \\
\text { BOC }\end{aligned}$ & $\begin{array}{l}67 \mathrm{HBC} \\
70 \mathrm{HBC}\end{array}$ & & $6 / 70$ \\
\hline & $O N P I+P I$ & $\begin{array}{l}\text { IOS } \\
\text { ZE }\end{array}$ & $78 \mathrm{HE}-2 \mathrm{rar}$ & ItP 10.3 GEV/C & \\
\hline & TRON PI+ P & $\begin{aligned} P I+ \\
Z E\end{aligned}$ & $78 \mathrm{HBC}$ & $10.3 \mathrm{GEV} / \mathrm{C}$ & 11 \\
\hline & $\begin{array}{l}\text { *3/2(1950) INTO (S IGMA K)/TOTA } \\
(0.03)(0.01)\end{array}$ & ZEN & $78 \mathrm{H}$ & I+P $10.3 \mathrm{GEV} / \mathrm{C}$ & \\
\hline 2 & 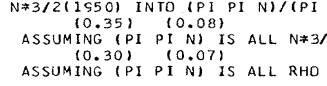 & $\begin{array}{l}N+P 1 P_{1} \\
\text { GAIDOS } \\
3212321 P I \\
\text { GAIDOS } \\
\text { PI }\end{array}$ & $\begin{array}{l}N 1 \\
75 \\
1, \\
75\end{array}$ & $\begin{array}{l}(P 9) /(P 1+P 9) \\
++P I+P \text { TO } N * 2 P I \\
\text { AGREE WITH DATA } \\
++P I+P \text { TO N*2PI }\end{array}$ & $\begin{array}{l}1 / 78 \\
1 / 78 \\
1 / 78\end{array}$ \\
\hline
\end{tabular}

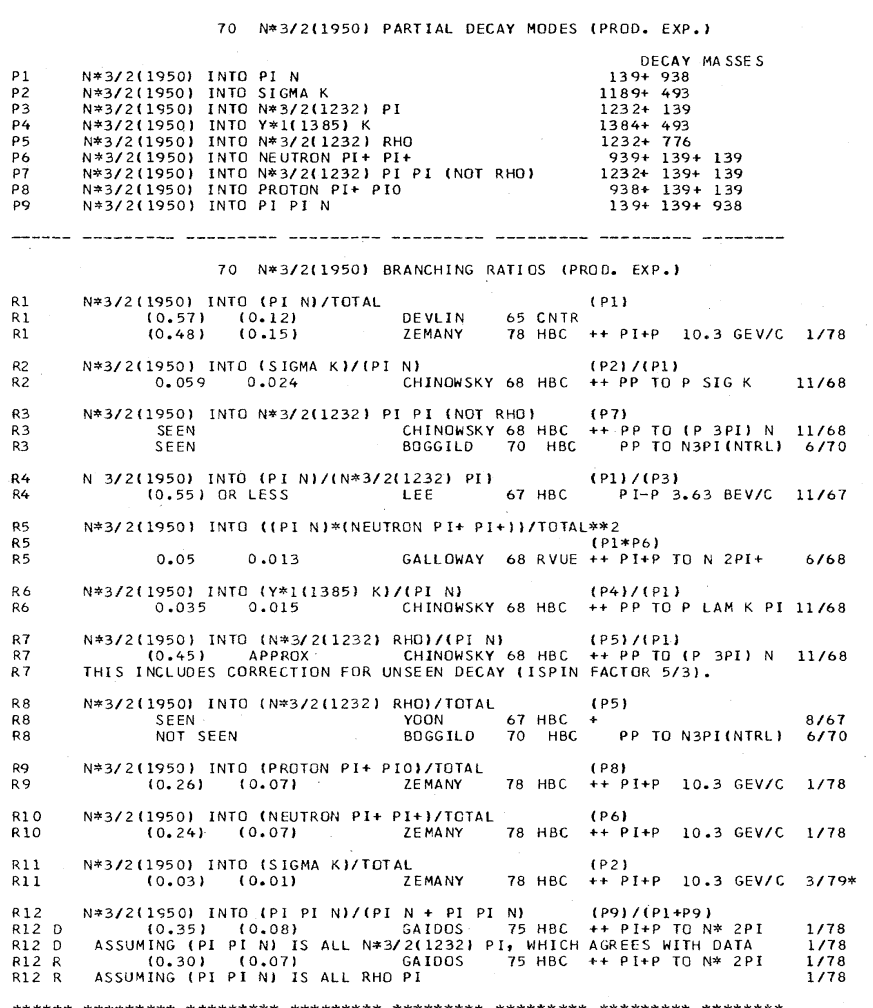

Data Card Listings For notation, see key at front of Listings.

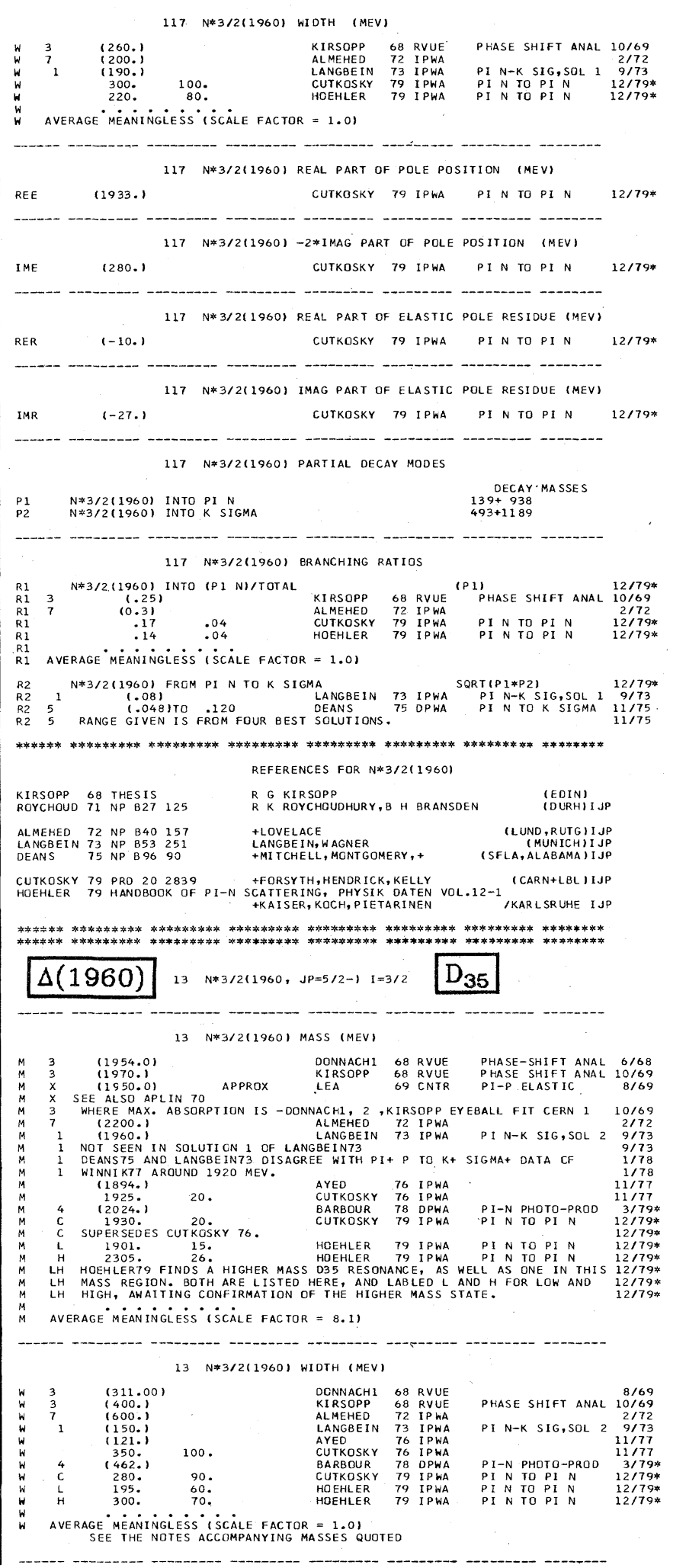


Data Card Listings

For notation, see key at front of Listings.

Baryons

$\Delta(1960), \Delta(2160)$

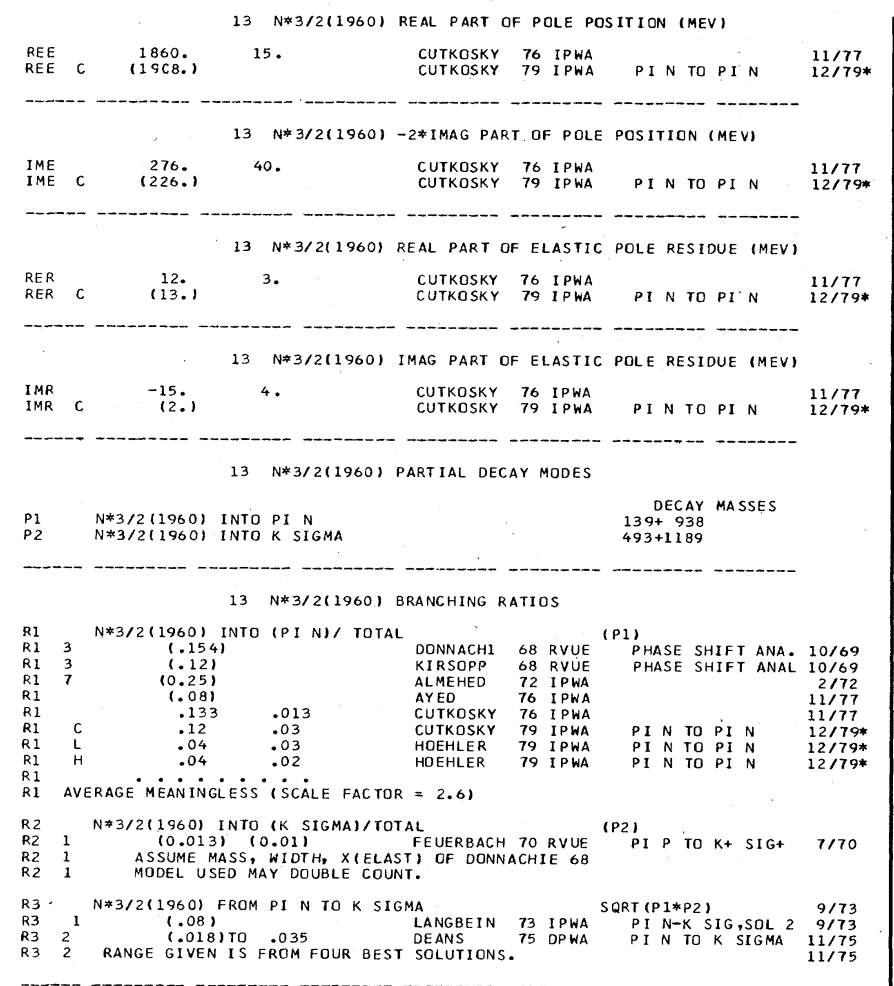

$13 N * 3 / 2(1960)$ PHOTON DECAY AMPL (GEV**-1/2)

FOR DEFINITION OF GAMMA-NUCLEON DECAY AMPLITUDES, SEE MINI-

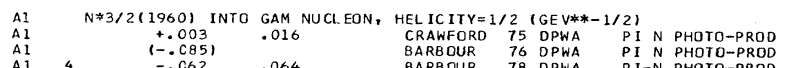

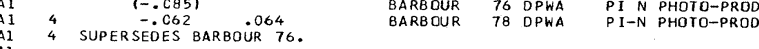

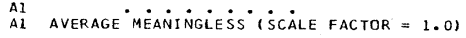

A2
A2

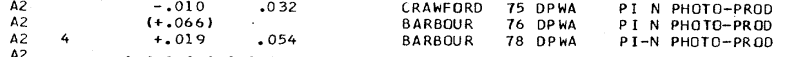

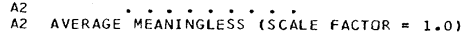

*********************************************************************1*

REFERENCES FOR $N * 3 / 2(1960)$

LEA $\quad 69$ PL 298584

$\begin{array}{lllll}\text { FEUERBAC } 70 & N P & 16 B & 85 \\ \text { ALMEHED } 72 & \text { NP } & B 40 & 157\end{array}$

LANGBEIN 73 NP $B 53251$

$\begin{array}{llllll}\text { CRAWFORD } & 75 & \text { NP } & \text { B97 } & 125 \\ \text { DEANS } & 75 & \text { NP } & \text { B } 96 & 90\end{array}$

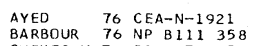

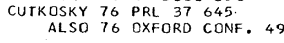

(CERN)
(EDIN)

LEA, OADES, WARD, COWAN,+ (RHEL, BRISTOL, DARE)
(VEUERBACRBILT)

FEUERBACHER+HOLLADAY
+ LOVELACE

LANGBEIN, WAGNER (MUNICH) IJP

R L CRAWFORD
$+M I T C H E L L, M C N T G O M E R Y,+$

AYED (THESIS)
I. M. BARBOUR, R. L. CRAWFORD. (SACL)IJPP
(GLA) IIJP

(CARN+BL)IJP
CUTKOSKY, HENORICK, KELLY
CUTKOSKY, HENDICK, CHAO+ (CARN+LBL+BRIS)IJP

$\begin{array}{llll}\text { BARBCUR } & 78 . N P & B 141 & 253 \\ \text { CUTKOSKY } 79 \text { PRD } 20 \quad 2839\end{array}$

BARBOUR, CRAWFORD, PAR SONS IGLAS)

$\begin{aligned} & \text { HFORSYTH, HENDRICK, KELLY } \text { (CARN+LBL) IJP } \\ & \text { HOEHLER } 79 \text { HANDBOOK OF PI-N SCATTER ING, PHYSIK OATEN VOL.12-1 } \\ & \text { +KAISER, KOCH, PIETARINEN }\end{aligned}$ PAPERS NOT REFERRED TO IN DATA CARDS

$\begin{array}{lllll}\text { DCNNACHI } & 69 & \text { NP } & 10 B & 433 \\ \text { AYED } & 70 & \text { PL } & 318 & 598\end{array}$

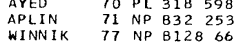

A DONNACHIE, R KIRSOPP

(GLAS+EDIN)

(RHEL, BRISTOL)
+ COWAN,GIBSCN, GILMORE++
+ (HAIF) I
$\Delta(2160), N_{N * 3 / 212160,}, I=3 / 2$

EARLY ANALYSES FOUND EVIDENCE FOR A RESONANCE RESULTS ARE NOW INCLUDED WITH THE LISTING FOR N*3/2(1960, JPE $3 / 2+)$. IN ADDI ION, ROYC HOUOHURY 71

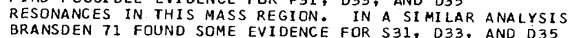

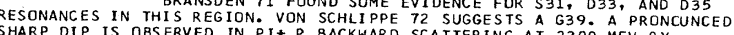

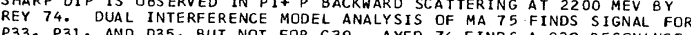

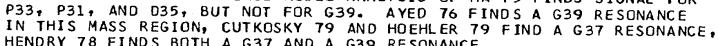

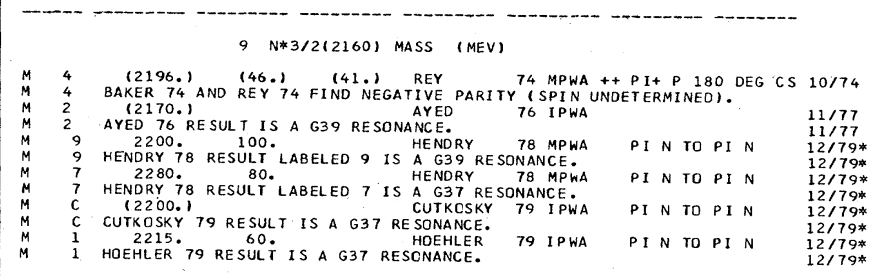

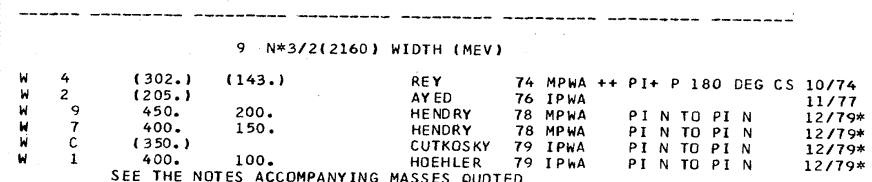

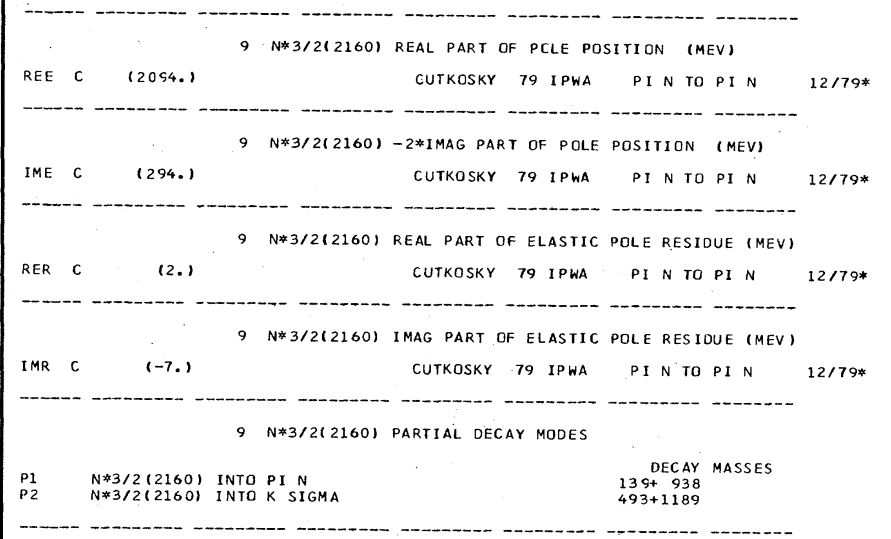

$9 N * 3 / 2(2160)$ BRANCHING RATIOS

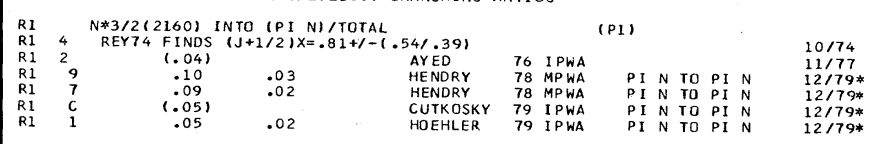

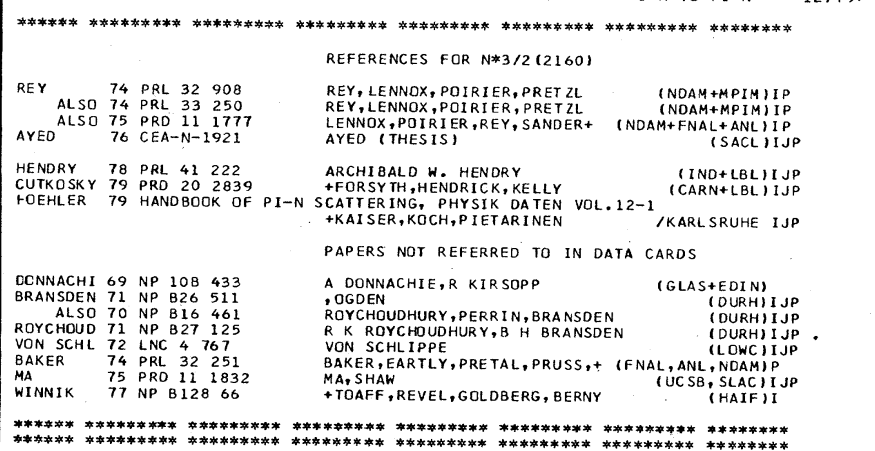




\section{Baryons $\Delta(2300), \Delta(2420), \Delta(2500)$}

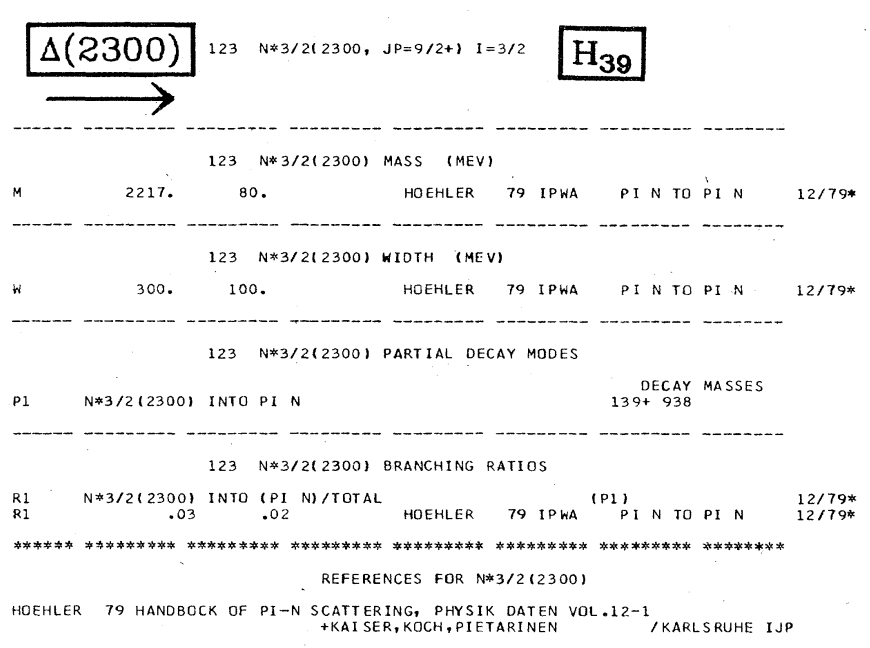

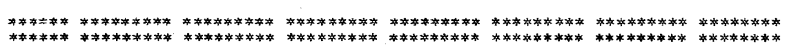
$\Delta(2420){ }_{84} \quad N^{N} * 3 / 2\left(2420, J^{p=11 / 2+1} \quad \mathrm{I}=3 / 2 \quad \mathrm{H}_{311}\right.$ BOTH ROYCHOUOHURY 71 ANO BRANSDEN 71 SEE A POSSIBLE
RESSONANT F35 IN THIS MASS REGION. IN ADOITION BRANSOEN 71 FIND A RESONANT P33 AT 2600 MEV.

$84 N * 3 / 2(2420)$ MASS (MEV)

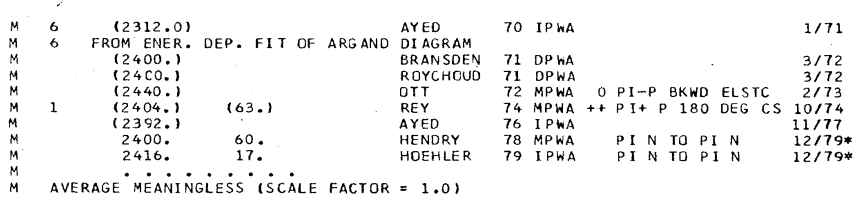
ARAge MEANI NGLESS iscale FACTOR $=1.01$

$84 N * 3 / 2(2420)$ WIDTH (MEV)

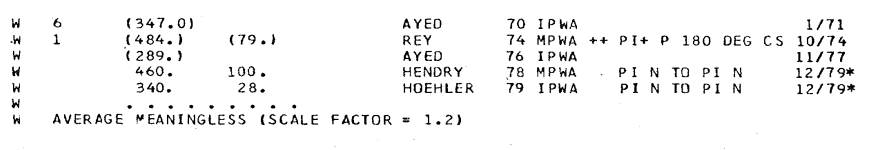
$84 N * 3 / 212420)$ PARTIAL DECAY MODES

$\begin{array}{lll}P 1 & N * 3 / 2(2420) \\ P 2 & \text { INTO PI N } \\ \text { N*3/2(2420) INTO SIGMA K }\end{array}$ DECAY MASSES $84 \quad N * 3 / 2(2420)$ BRANCHING RATIOS

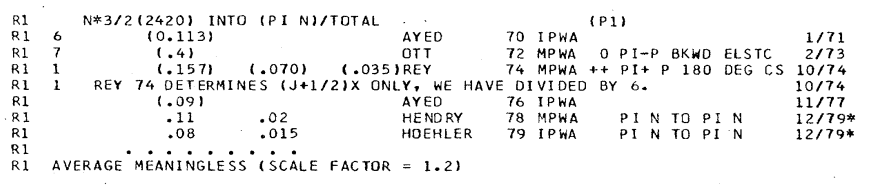
**************************************************************************

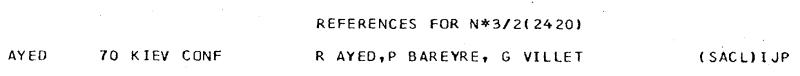

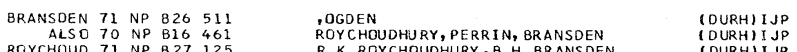

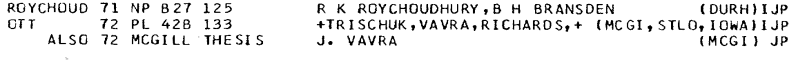

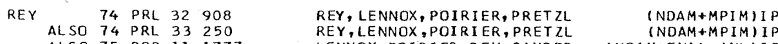

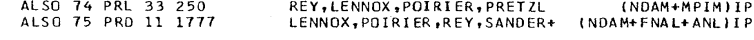

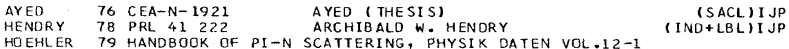
HENDRY 78 PRL 41222 ARCHIBALO W. HENDRY
HOEHLER 79 HANDBOOK OF PI-N SCATTERING, PHSIK DATEN VOL.12-1 (IND+LBLIIJP
HKAISER, KOCH, PIETARINEN
/KARLSRUHE IJP

\section{Data Card Listings For notation, see key at front of Listings.}

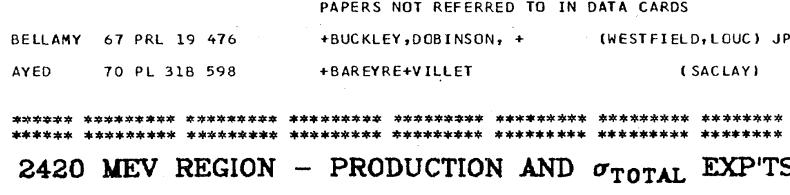

$69 N * 3 / 212420, J P=\quad I I=3 / 2$ PRODUCTION EXPER IMENTS

SEE THE MINI-REVIEW PRECEDING THE N AND DELTA LISTINGS
FOR A OISCUSSION OF PROOUCTION EXPERIMENTS.

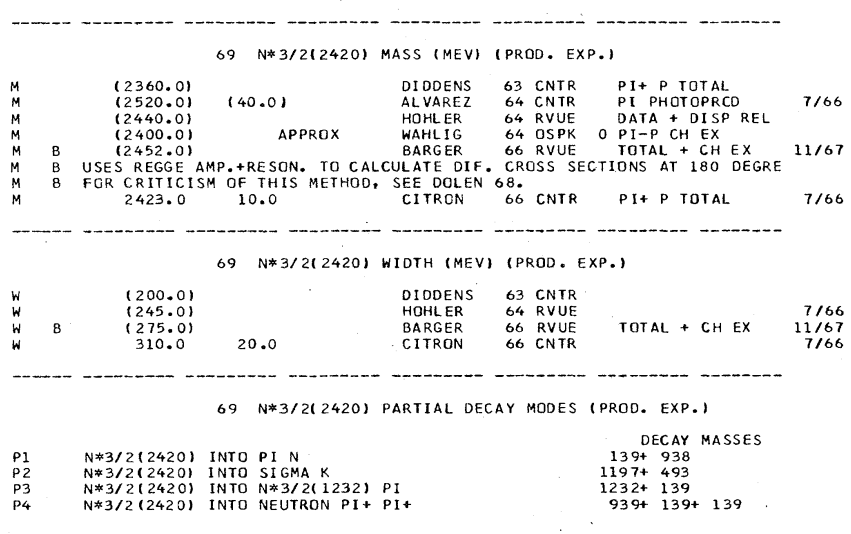

69 N*3/212420) BRANCHING RATIOS. (PROD. EXP.)

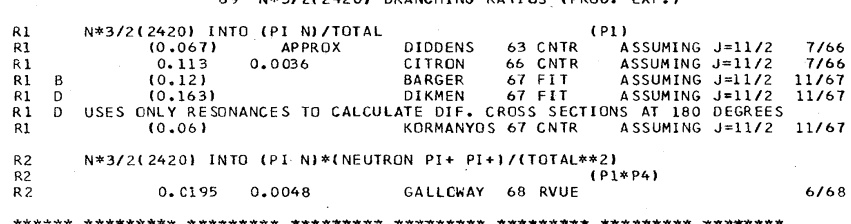

******************************************************************************

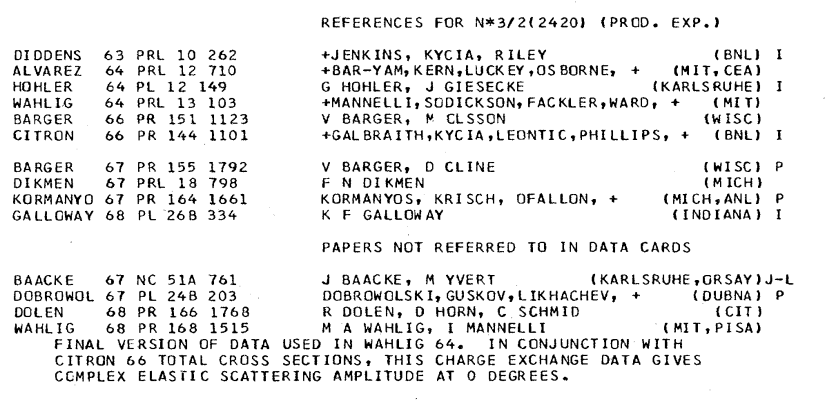

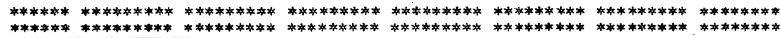

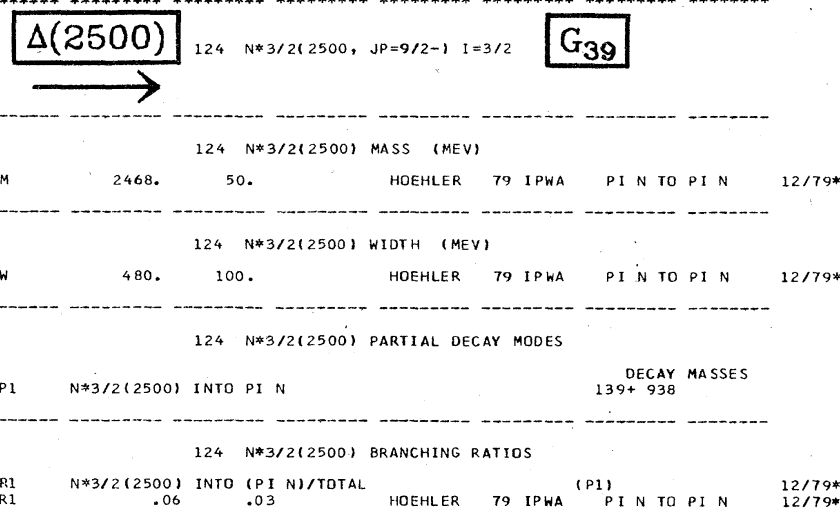


Data Card Listings

Baryons

For notation, see key at front of Listings. $\Delta(2500), \Delta(>2500), \Delta(2750), \Delta(2850), \Delta(2950)$

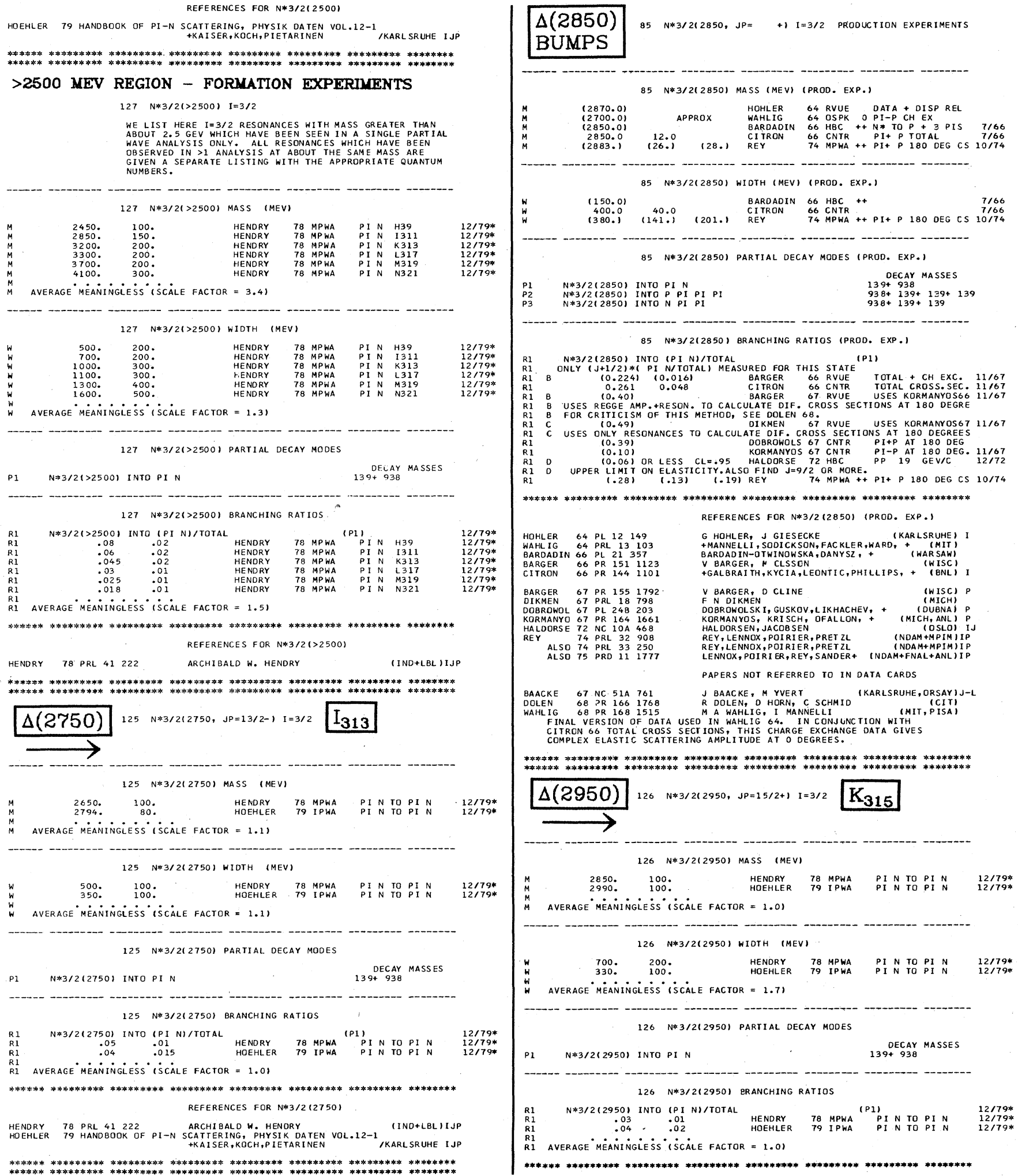




\section{Baryons}

\section{$\Delta(3230)$, EXOTIC NUCLEONS, $Z^{*} s, Z_{0}(1780)$}

$86 N * 3 / 2(3230)$ MASS (MEV) (PROD. EXP.)

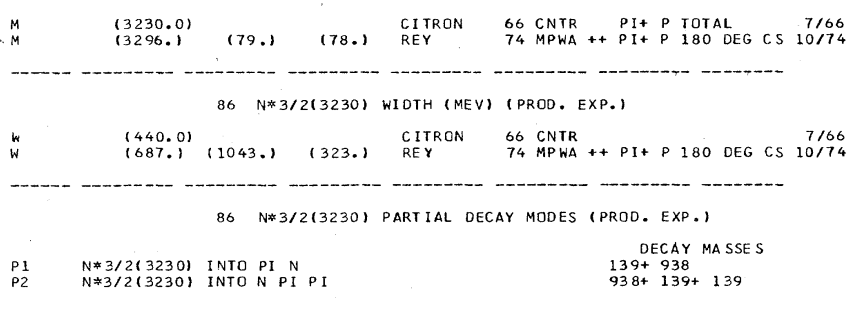

$86 \quad N * 3 / 2(3230)$ BRANCHING RATIOS

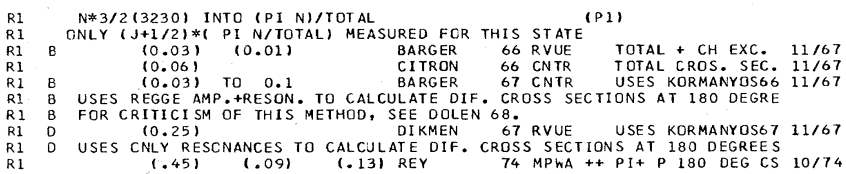
**************************************************************************1 REFERENCES FOR $N * 3 / 2(3230)$ (PROD. EXP.)

$\begin{array}{lllllll}\text { BARGER } & 66 & P R & 151 & 1123 & \text { V BARGER, M OLSSON } \\ \text { CITRON } & 66 & \text { PR } 1441101 & \text { +GALBRAITH,KYCIA,LEONIIC, PHILLIPS, + (WISC) } \\ \text { (BNL) }\end{array}$

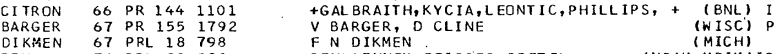

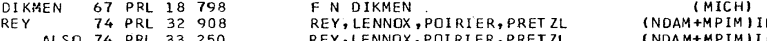
$\begin{array}{lll}\text { ALSO } 74 \text { PRL } 33250 & \text { REY, LENNOX, POIRIER, PRETZL } & \text { (NDAM+MPIMIIP } \\ \text { ALSO } 75 \text { PRD } 111777 & \text { LENNOX, POIRIER, REY, SANDER+ } & \text { (NDAM+FNAL+ANLIIP }\end{array}$ PAPERS NOT REFERRED TO IN DATA CARDS $\begin{array}{llll}\text { KGRMANYO } 67 \text { PR } 1641661 & \text { KORMANYOS, KRISCH, OFALLON, } \\ \text { DOLEN } & 68 \text { PR } 1661768 & \text { R DOLEN, D HORN, C SCHMID } & \text { (MICH, ANL) } \\ \text { (CIT) }\end{array}$

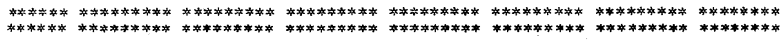
EXOTIC NUCLEONS - 1640 MEV REGION

EXOTIC NUCLECNS

THIS IS NOT A COMPLETE LIST. WE WILL TABULATE EXOTICS FROM NOW ON

EX(1640, JP $=\quad, I=5 / 2$

THIS IS NOT A COMPLETE LIST. WE TABULATE

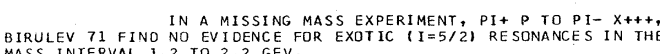
MASS INTERVAL 1.2 . TO 2.2 GEV.

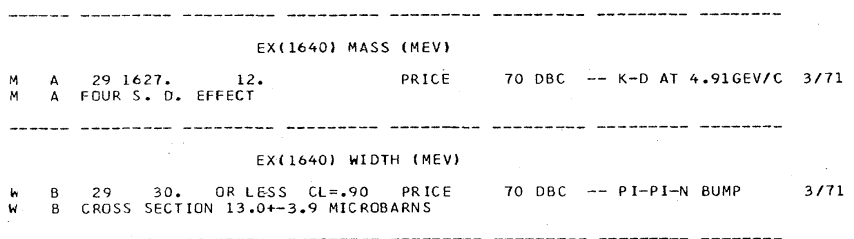

EX(1640) CROSS SECTION LIMITS (MICROBARN)

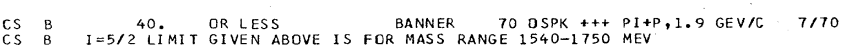
**************************************************************************1* REFERENCES FOR EX(1640)

$\begin{array}{lll}\text { BANNER } & 70 \mathrm{NP} 815205 & \text { +CHEZE, HAMEL, TEIGER, ZACCONE + } \\ \text { PRICE } & 70 \mathrm{PL} 338,533 & \text { (SACLAY) }\end{array}$

PAPERS NOT REFERRED TO IN DATA CARDS

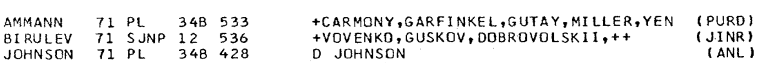

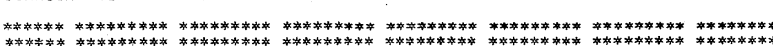

\section{Data Card Listings For notation, see key at front of Listings.}

Note on the $S=+1$ Baryon System

The evidence for $\mathrm{S}=+1$ baryon's was thoroughly reviewed in our 1976 edition. $^{1}$ More recent measurements, including completed experiments and experiments in progress, have been reviewed by Kelly. ${ }^{2}$ Most of the new data which have recently been becoming available have not yet been subjected to partial-wave analysis, and the whole $z^{*}$ question may be clarified when this is done. In the interim two analyses have been reported by ARNDT 78 and GIACOMELII 76. ARNDT 78 is an energy-dependent analysis of $\mathrm{K}^{+} \mathrm{p}$ elastic scattering below $2 \mathrm{GeV} / \mathrm{c}$. Although seven resonance poles are found in various waves, only the $\mathrm{P}_{13}$ pole at $(1796-101 \mathrm{i}) \mathrm{MeV}$ is considered to be a strong $\mathrm{z}_{1}^{*}$ candidate, and only this pole is entered in the Data Card Listings below. No information is given on the pole residue and its uncertainty. GIACOMELLIl 76 searched for a $z_{1}^{*}$ decaying into $k \Delta$, but found no evidence for such an effect. The evidence for the existence of $\mathrm{Z}^{*}$ 's thus remains inconclusive.

\section{References}

1. Particle Data Group, Rev. Mod. Phys. 48, S188 (1976).

2. R. L. Kelly, in Proceedings of the Meeting on Exotic Resonances (HUPD-7813), eds. I. Endo et al., Hiroshima, 1978.

See the Data Card Listings for other references.

\section{$\mathrm{S}=1$ I=0 EXOTIC STATES $\left(\mathrm{Z}_{0}\right)$}

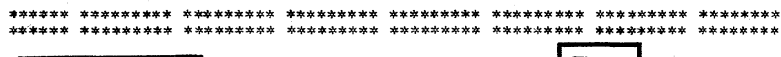

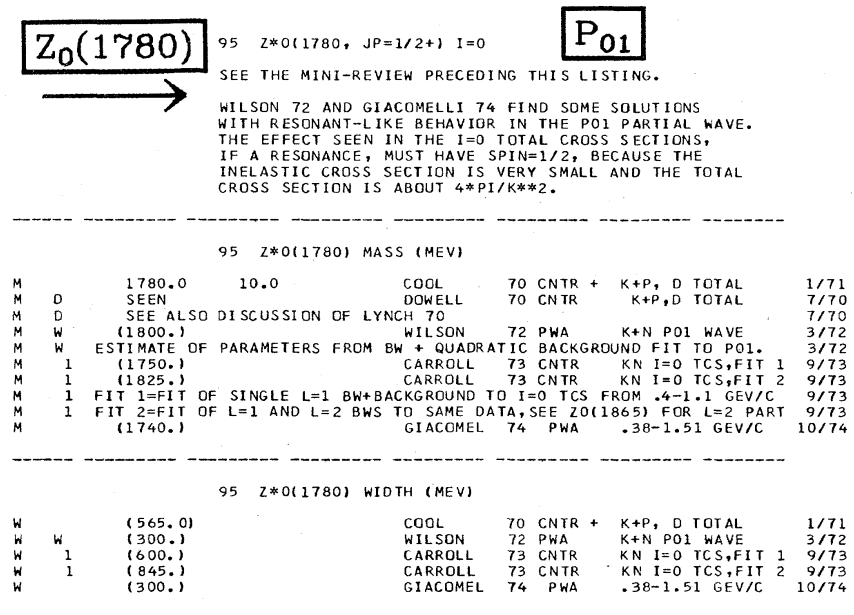




\title{
Data Card Listings
}

\author{
Baryons \\ $z_{0}(1780), z_{0}(1865), z_{1}(1900)$
}

For notation, see key at front of Listings.

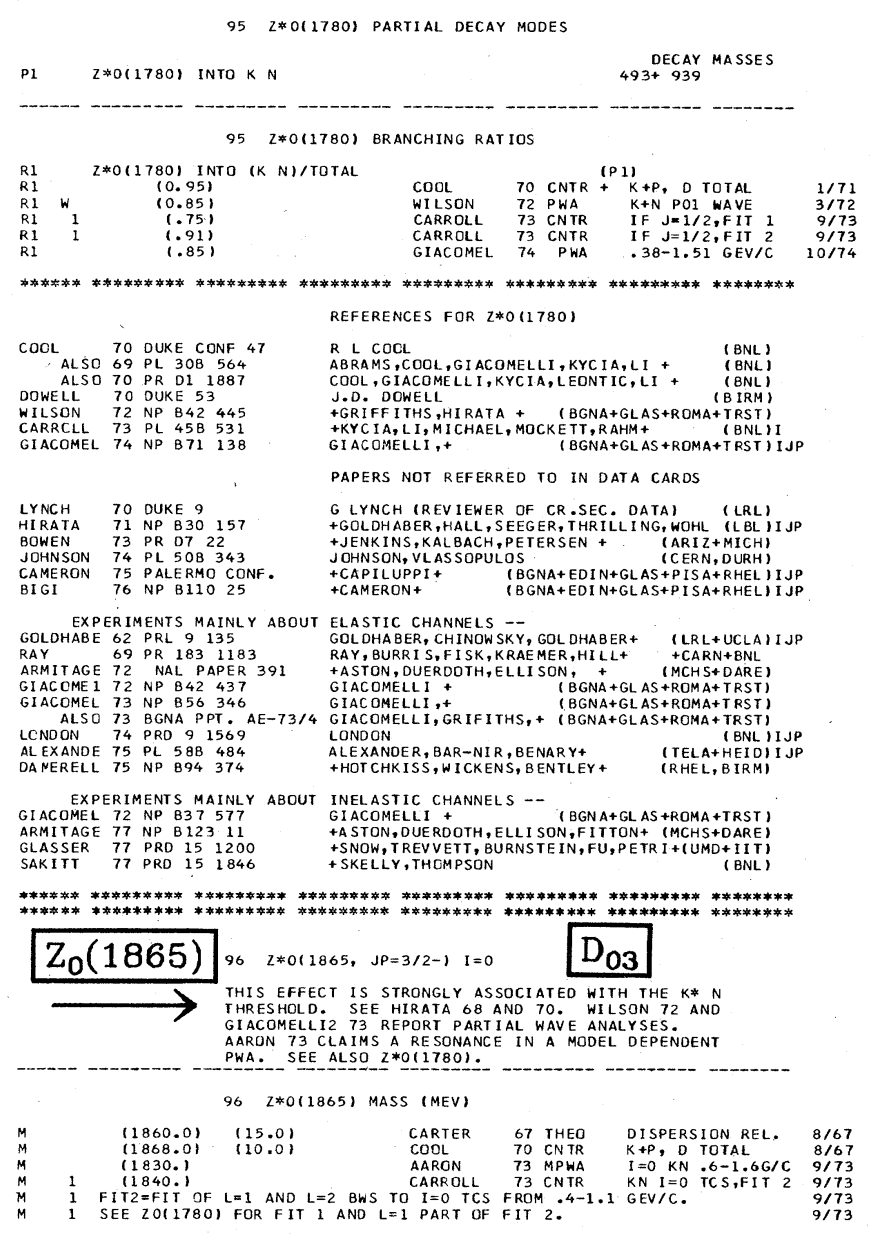

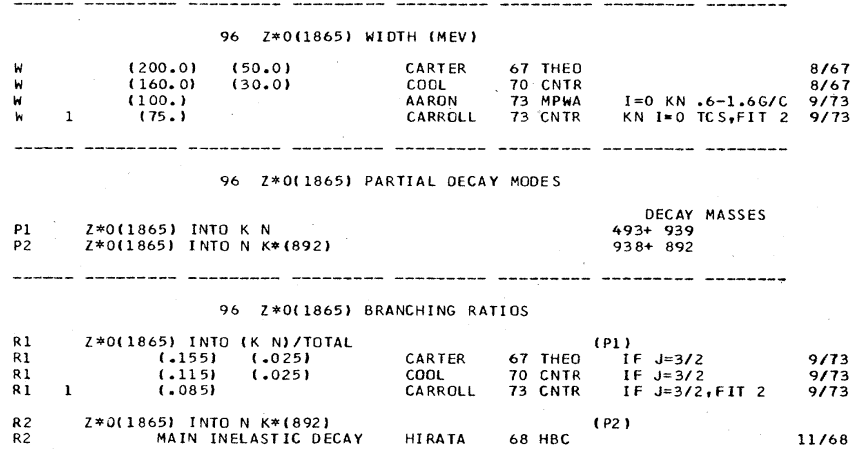

***************************************************************************

$\begin{array}{llll}\text { CARTER } & 67 & \text { PRL } 18 & 801 \\ \text { HIRATA } & 68 & \text { PRL } 21 & 1485 \\ \text { COOL } & 70 & \text { PR D } 11887\end{array}$

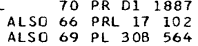

$\begin{array}{lllll}\text { AARON } & 73 & \text { PRD } & 7 & 1401 \\ \text { CARROLL } & 73 & \text { PL } & 458 & 531\end{array}$

$\begin{array}{llll}\text { HIRATA } & 70 & \text { DUKE } & 429 \\ \text { AARCN } & 71 & \text { PRL } 26 & 407 \\ \text { HIRATA-1 } & 71 & \text { NP } B 33 & 445\end{array}$

HIRATA-1 71 NP $B 33 \quad 445$
GIACOMEL 72 NPP 837577
WILSON 72 NP $B 42 \quad 445$

REFERENCES FOR $Z * 0(1865)$

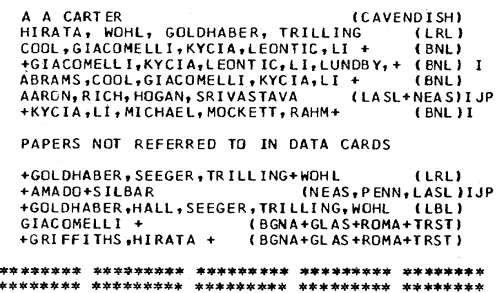

$\mathrm{S}=1$ I=1 EXOTIC STATES $\left(\mathrm{Z}_{1}\right)$

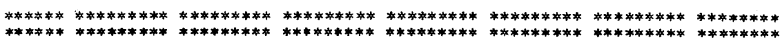

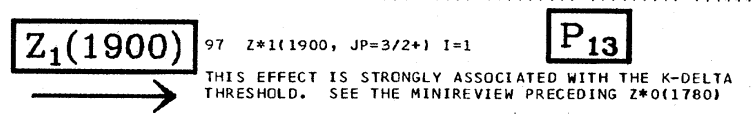

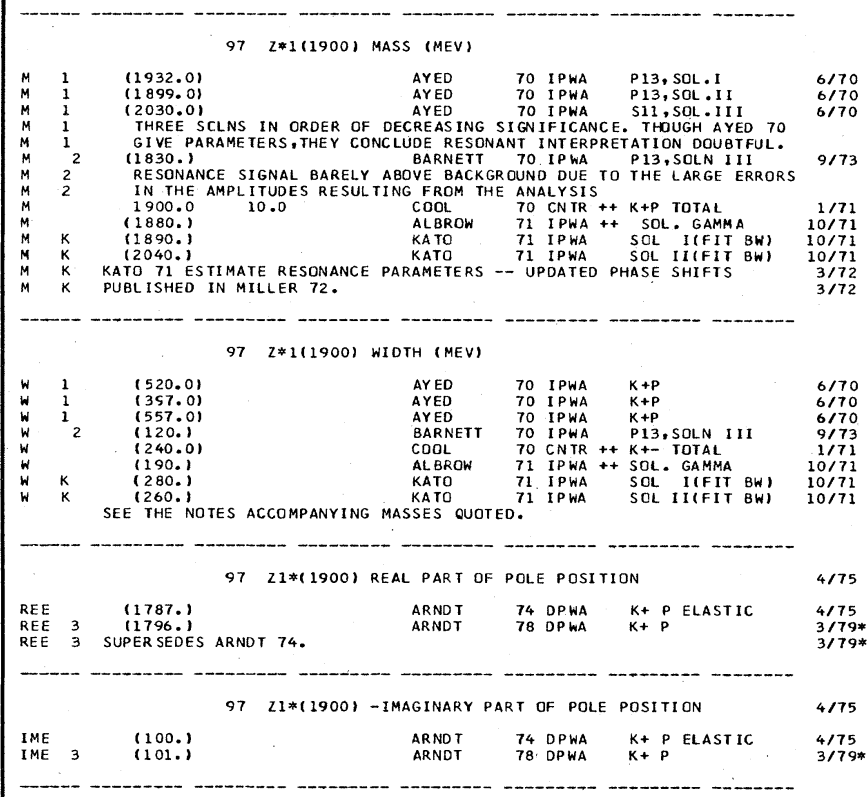

$972 * 1(1900)$ PARTIAL DECAY MODES

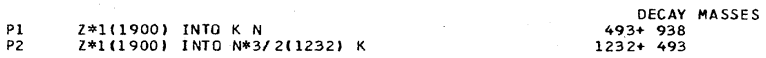

$97 Z$ Z1(1900) BRANCHING RATIOS

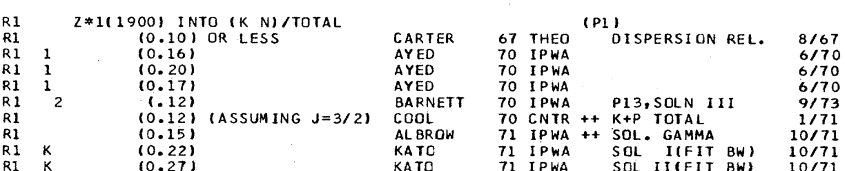

SEE NOTES ACCompanying the masses Quoteo.

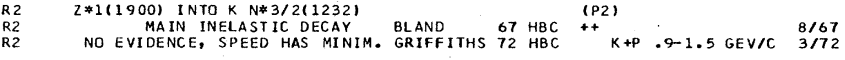

*********************************************************************

REFERENCES FOR Z*1(1900)

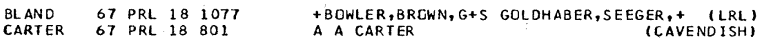

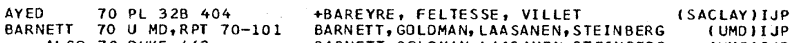

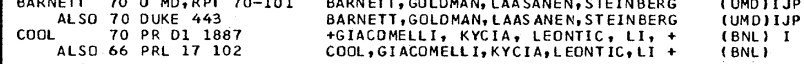

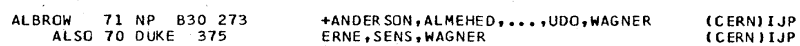

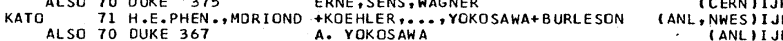

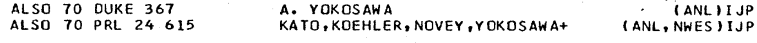

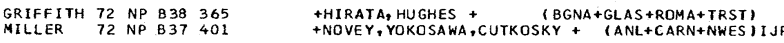

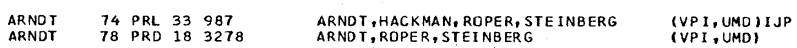

PAPERS NOT REFFERED TO IN $2 * 1$ DATA CARDS

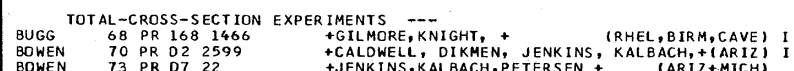

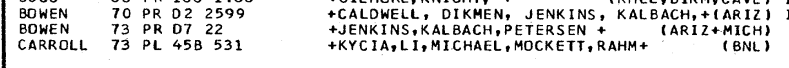




\section{Baryons}

$Z_{1}(1900), Z_{1}(2150), Z_{1}(2500), \Lambda^{\prime} s$ and $\Sigma^{\prime} s$

\section{Data Card Listings} For notation, see key at front of Listings.

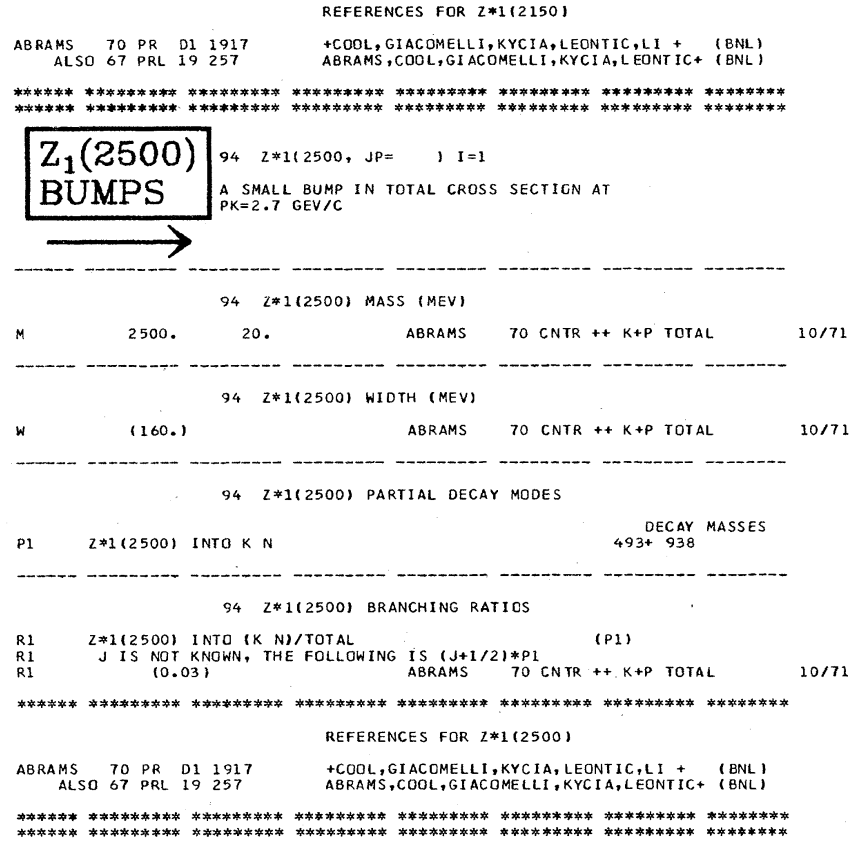

\section{$\mathrm{Z}_{1}$ CROSS SECTION LIMITS}

SEE MINIREVIEH PRECEDING $Z * 0$

CS UNITS MICROBARNS

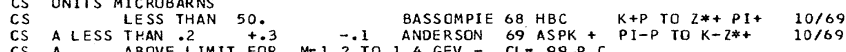

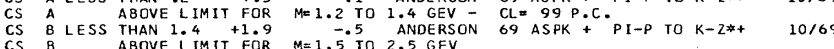
\#****************************************************************************1 REFERENCES FOR $Z * 1$ CROSS SECTION LIMITS

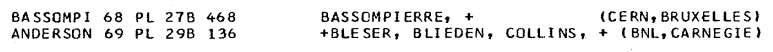

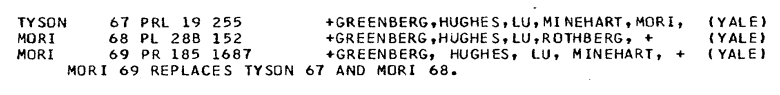

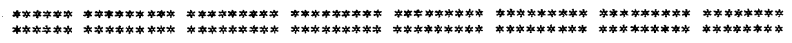

Note on $\Lambda^{\prime} s$ and $\sum$ 's

The number of confirmed resonances is still increasing, but very slowly; in 1978, we added three more states to the $\mathrm{Y}^{*}$ portion of the Baryon Table, and there has been no further increase this year. There remains, however, a large number of proposed, but unconfirmed resonances, and some of the states we enter in the Data Card Listings may really be more than one resonance.

All the $\mathrm{Y}^{*}$ 's proposed in the last few years are only weakly coupled to their two-body decay channels $\overline{\mathrm{K}} \mathrm{N}, \Lambda \pi$, and $\Sigma \pi$. For this reason they appear as very small peaks or make no appearance at all in invariant mass distributions. Rather, 


\section{Data Card Listings}

\section{For notation, see key at front of Listings.}

Baryons

$\Lambda$ 's and $\Sigma$ 's when the two-body reactions $\overline{\mathrm{K} N} \rightarrow \overline{\mathrm{K}} \mathrm{N}, \overline{\mathrm{K}} \mathrm{N} \rightarrow \Lambda \pi$, and

$\overline{\mathrm{KN}} \rightarrow \Sigma \pi$ are partial-wave analyzed, some of the amplitudes are found to traverse resonance-like counterclockwise circles. The results of partialwave analysis give $\mathrm{J}^{\mathrm{P}}$ information, whereas a peak seen in an invariant mass distribution or a total cross section often cannot be analyzed for its quantum numbers. We will keep information coming from formation experiments and from production experiments separate whenever necessary.

\section{Formation Experiments}

Partial-wave analyses have been performed mainly for the channels $\overline{\mathrm{K}}, \Lambda \pi$, and $\sum \pi$; a few results exist also for $\Xi K, \Lambda \omega$, and some quasi-twobody channels. With a few exceptions (e.g., BAILLON 75 and VANHORN 75 for the $\Lambda \pi$ channel), the great majority of the analyses done so far cover rather narrow energy ranges, usually corresponding to a single bubble chamber experiment. A disturbing feature that appears when examining the partial waves obtained in such analyses is that they do not always join smoothly with the partial waves given in analyses done for the same channel over a different energy range.

More ambitious analyses treat all channels simultaneously so that unitarity constraints are automatically obeyed and the resonances appear with the same masses and widths in the different channels. The multi-channel analyses done prior to 1974 (KIM 71, LANGBEIN 72, and LEA 73) included the three two-body channels $\overline{\mathrm{K} N}, \Lambda \pi$, and $\Sigma \pi$, and were carried out in the mass range 1.5 to $1.9 \mathrm{GeV}$. This is the mass range of a particular bubble chamber experiment (ARMENTEROS 68), the only one which at that time had relatively good statistical accuracy.

In recent years, additional experimental results have been obtained. Bubble chamber experiments now exist with better statistics in the mass range already considered (HEMINGWAY 75, RLIC 77) and with somewhat lower statistical accuracy up to a mass of $2.5 \mathrm{GeV}$ (BELLEFON1 75, 2 75, 77, and 78). However, the most important recent contributions to this field, for the $\bar{K} N$ channel at least, are from electronic counter experiments. These provide results which are difficult if not impossible to get in a conventional bubble chamber experiment.
They include high-statistics measurement of the $\mathrm{K}^{-} \mathrm{p} \rightarrow \overline{\mathrm{K}}^{0} \mathrm{n}$ total $^{1}$ and differential cross section ${ }^{2}$ at low energies, $K^{-} \mathrm{p}$ elastic polarization measurements, ${ }^{3}$ and $\mathrm{K}^{-} \mathrm{n}$ elastic angular distributions (DECLAIS 77 and Ref. 4).

We may hope that improved partial-wave analyses over a wide energy range will be performed in the near future in order to disentangle the rather unsatisfactory present situation. Even though the unconfirmed resonances (one- and twostar states in Table 1) are often "seen" in several analyses with more or less compatible parameters, the corresponding partial-wave behavior is often very different in each of these analyses. Thus the confidence one has in the existence of these resonances is rather weak.

The three more recent analyses are discussed below. Two of them are multi-channel analyses, fitting data from the three channels, $\overline{\mathrm{K}} N, \Lambda \pi$, and $\sum \pi$, and covering a wide mass range.

a) In the analysis of the Rutherford LaboratoryImperial College collaboration (RLIC 77) the mass range extends from 1480 to $2170 \mathrm{MeV}$. The data used have been carefully selected in order to eliminate inconsistencies (usually the older and statistically less accurate points have been rejected). Angular distributions were directly used in the fit except when the quality of the data was such that no loss of information occurred by using Legendre coefficients $\left(\mathrm{e} . \mathrm{g}, \mathrm{K}^{-} \mathrm{p} \rightarrow \Sigma^{0} \pi^{0}\right)$. In this work, a conventional energy-dependent analysis is performed first for each of the three channels ( $\overline{\mathrm{K}} \mathrm{N}, \Lambda \pi$, and $\Sigma \pi$ ). As usual, the presence of a resonance in a partial wave is detected by comparing the goodness of the fit when this wave is parametrized as a smooth background to the alternative fit when a Breitwigner is added to the background. The three separate fits are then considered together in order to obtain a real multi-channel analysis. Internal consistency requires that the masses and widths of the resonances be the same in each of the three channels. The final fit has been done with these resonance parameters fixed and equal to a "weighted average" of the three values.

Some suspected resonances are confirmed by this analysis, but many other reported "resonance 


\section{Baryons}

$\Lambda$ 's and $\Sigma$ 's
Data Card Listings For notation, see key at front of Listings.
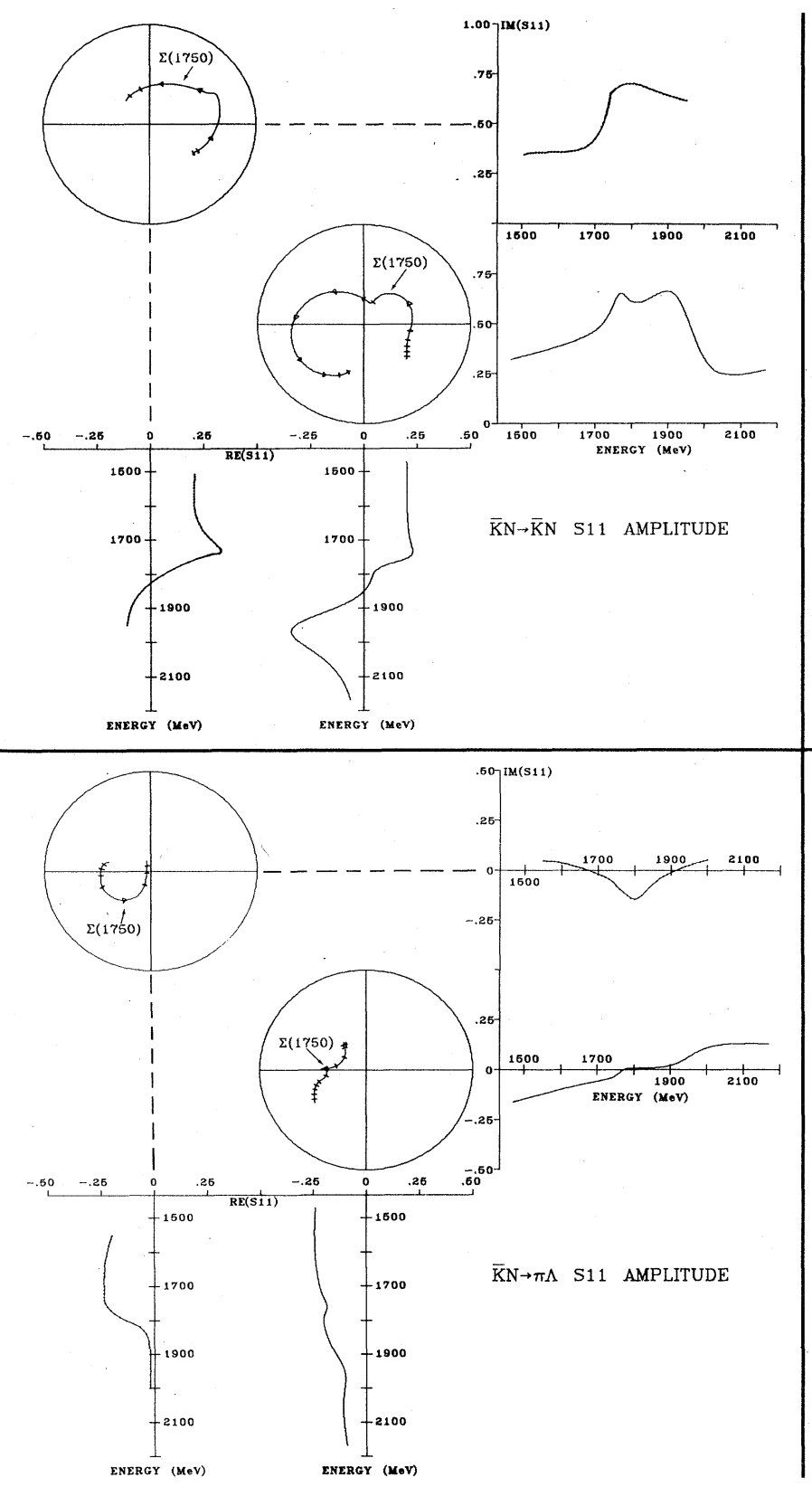

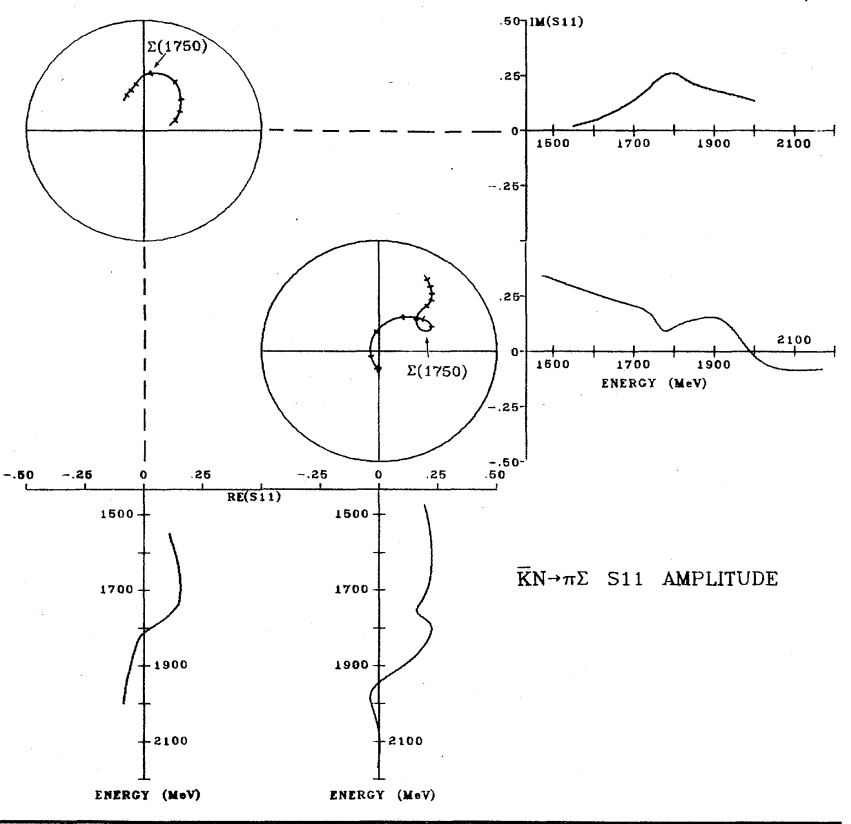

Fig. 1. Amplitudes for $\overline{\mathrm{K} N}$ scattering in the $\mathrm{S}_{11}$ partial wave. The energy dependence of each amplitude is displayed by plotting its real and imaginary parts versus energy, in alignment with the corresponding Argand plot. In addition, arrows with bases positioned at integral multiples of $50 \mathrm{MeV}$ and a base-to-tip length of $5 \mathrm{MeV}$ are plotted on the Argand plots, and the only established resonance in this wave, the $\Sigma(1750)$, is indicated. The results of two different analyses are shown for each channel; the energy axes all run from elastic threshold to $2200 \mathrm{MeV}$ and are aligned for ease of comparison. The lower plot for each channel is from RLIC 77 . The upper plot is from ALSTON 78 for the elastic channel and from MARTIN 77 for the inelastic channels. 


\section{Data Card Listings}

For notation, see key at front of Listings.

Baryons

$\Lambda^{\prime}$ 's and $\Sigma^{\prime}$ 's
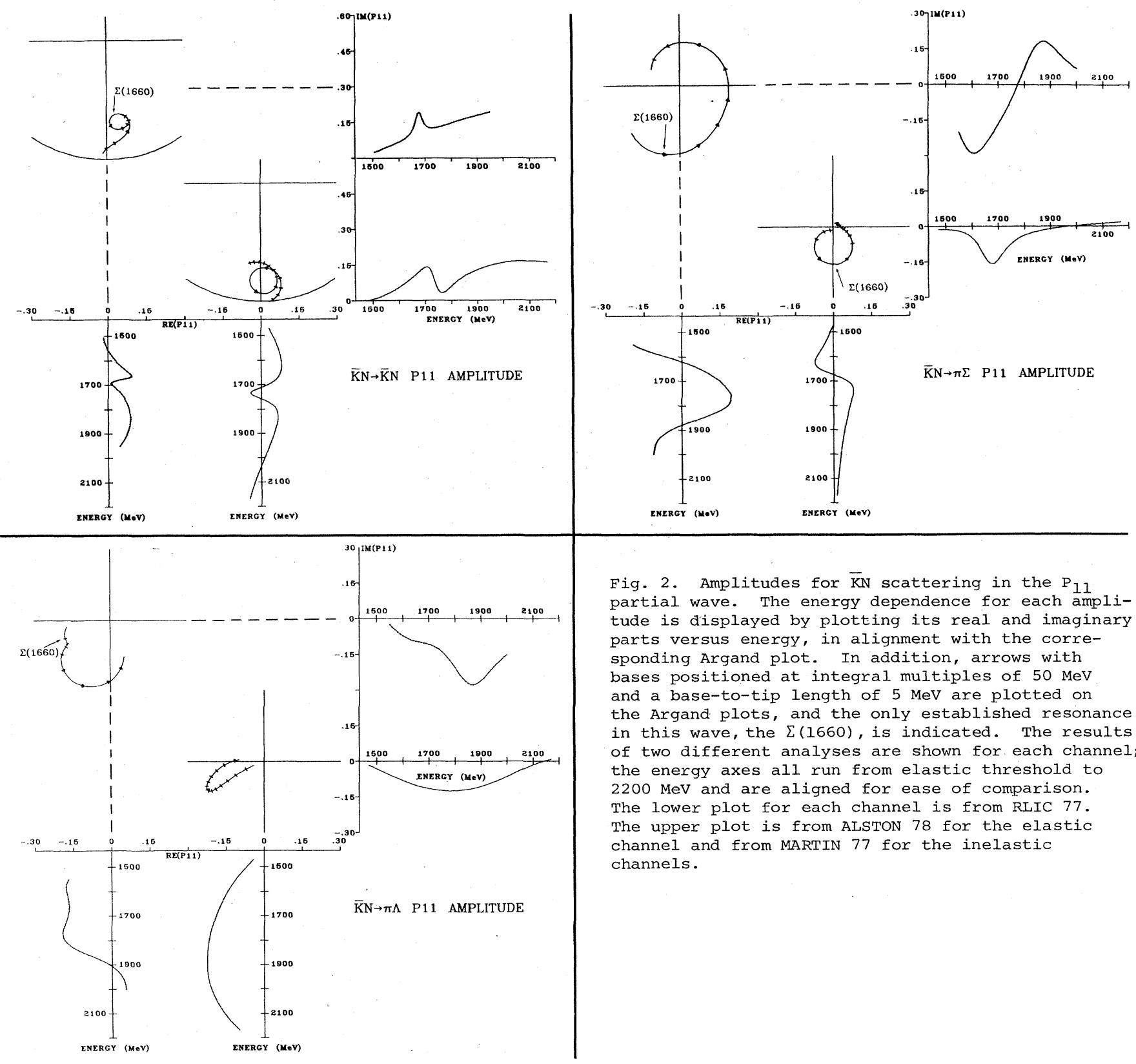

Fig. 2. Amplitudes for $\overline{\mathrm{K}} \mathrm{N}$ scattering in the $\mathrm{P}_{11}$ partial wave. The energy dependence for each amplitude is displayed by plotting its real and imaginary parts versus energy, in alignment with the corresponding Argand plot. In addition, arrows with bases positioned at integral multiples of $50 \mathrm{MeV}$ and a base-to-tip length of $5 \mathrm{MeV}$ are plotted on the Argand plots, and the only established resonance in this wave, the $\Sigma(1660)$, is indicated. The results of two different analyses are shown for each channel; the energy axes all run from elastic threshold to $2200 \mathrm{MeV}$ and are aligned for ease of comparison. The lower plot for each channel is from RLIC 77. The upper plot is from ALSTON 78 for the elastic channel and from MARTIN 77 for the inelastic channels. 


\section{Baryons}

$\Lambda$ 's and $\Sigma$ 's
Data Card Listings For notation, see key at front of Listings.
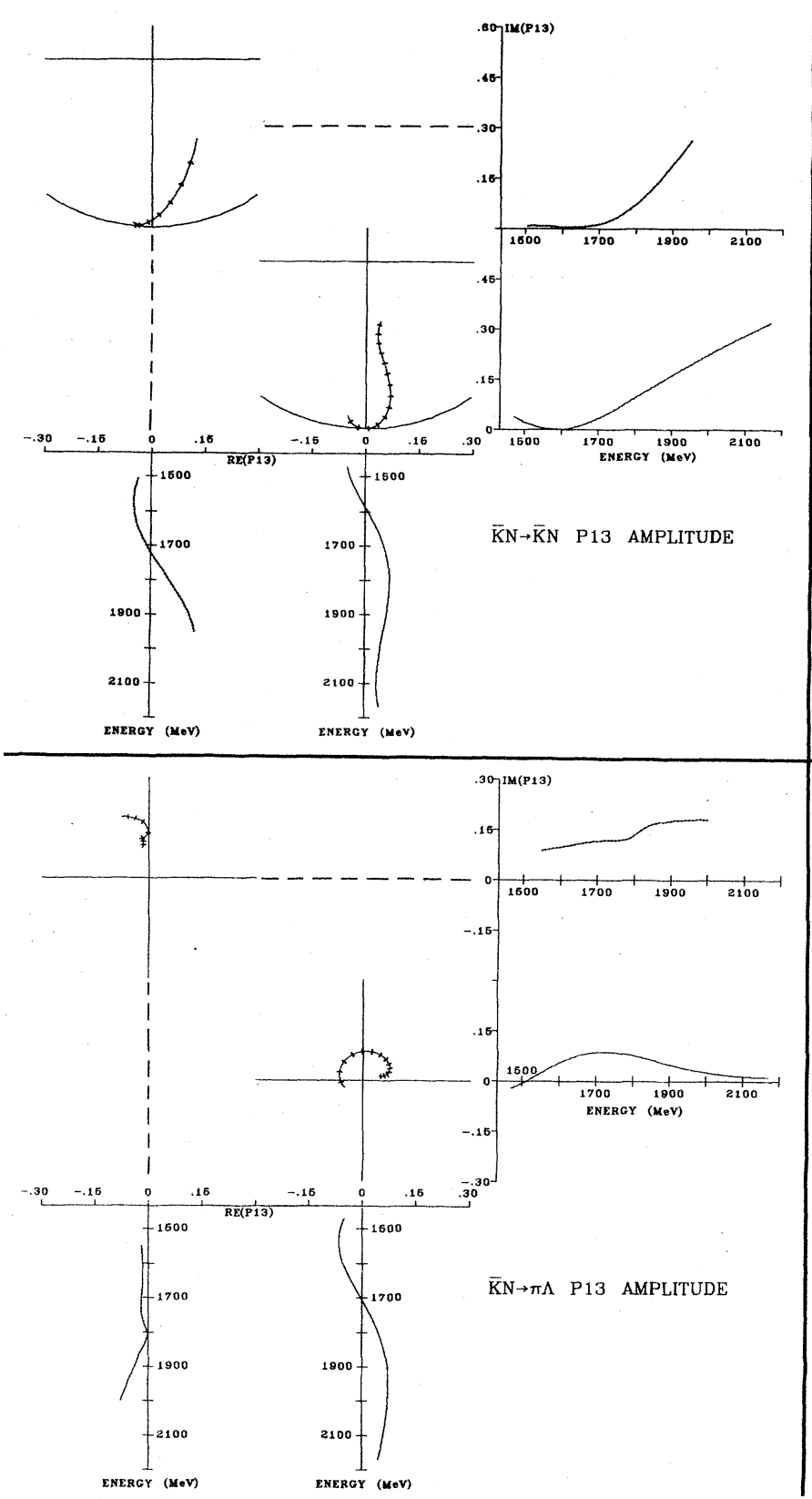

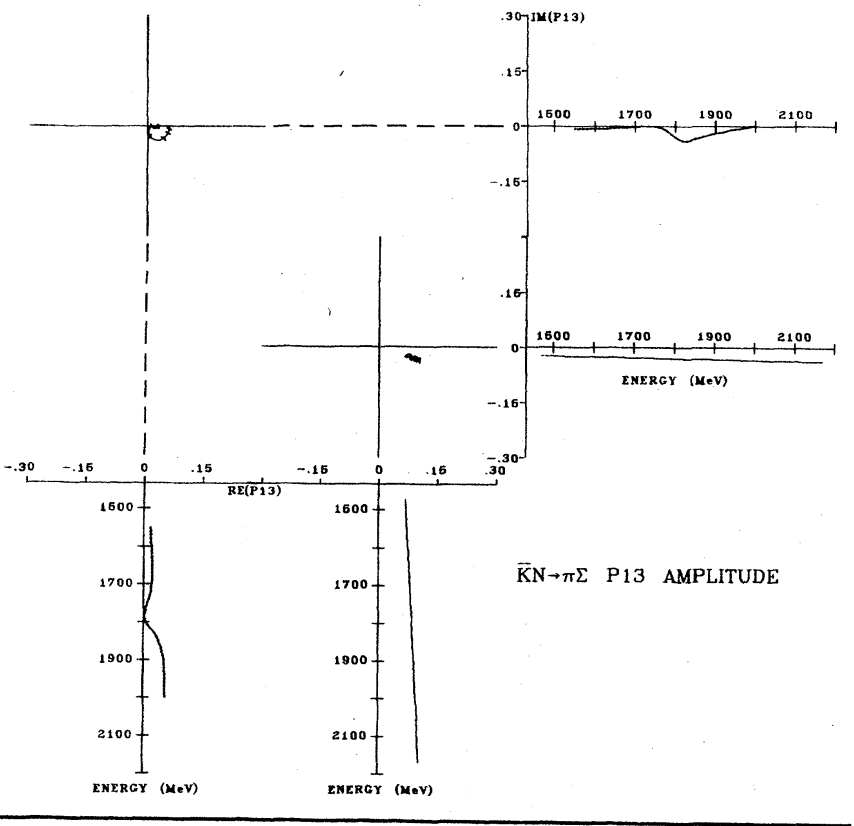

Fig. 3. Amplitudes for $\overline{\mathrm{K} N}$ scattering in the $\mathrm{P}_{13}$ partial wave. The energy dependence of each amplitude is displayed by plotting its real and imaginary parts versus energy, in alignment with the corresponding Argand plot. In addition, arrows with bases positioned at integral multiples of $50 \mathrm{MeV}$ and a base-to-tip length of $5 \mathrm{MeV}$ are plotted on the Argand plots. The only established resonance in this wave, the $\sum(1385)$, lies below elastic threshold and is not shown. The results of two different analyses are shown for each channel; the energy axes all run from elastic threshold to $2200 \mathrm{MeV}$ and are aligned for ease of comparison. The lower plot for each channel is from RLIC 77. The upper plot is from ALSTON 78 for the elastic channel and from MARTIN 77 for the inelastic channels. 


\section{Data Card Listings}

For notation, see key at front of Listings.

Baryons

$\Lambda$ 's and $\Sigma$ 's

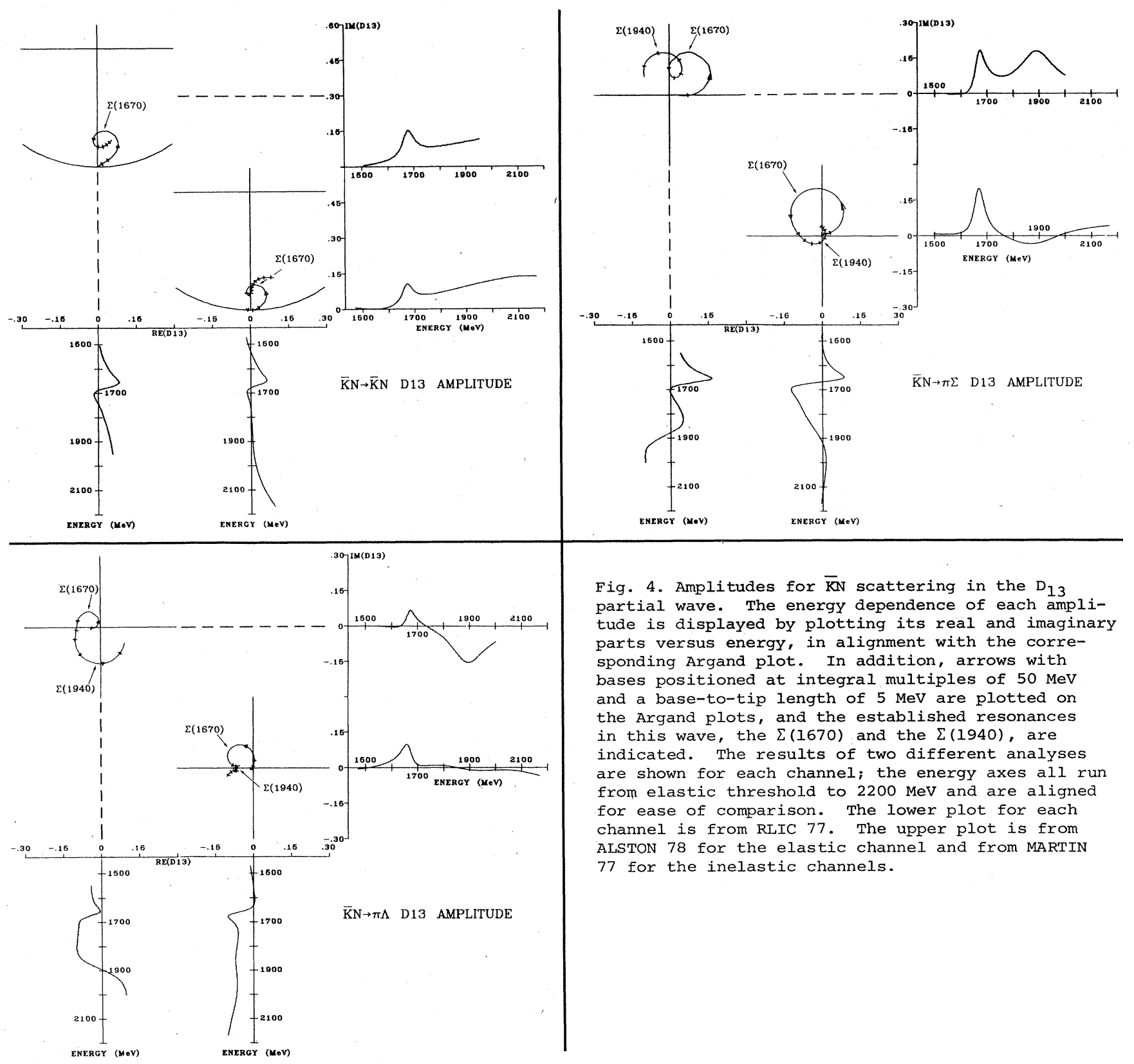




\section{Baryons}

$\Lambda$ 's and $\Sigma$ 's
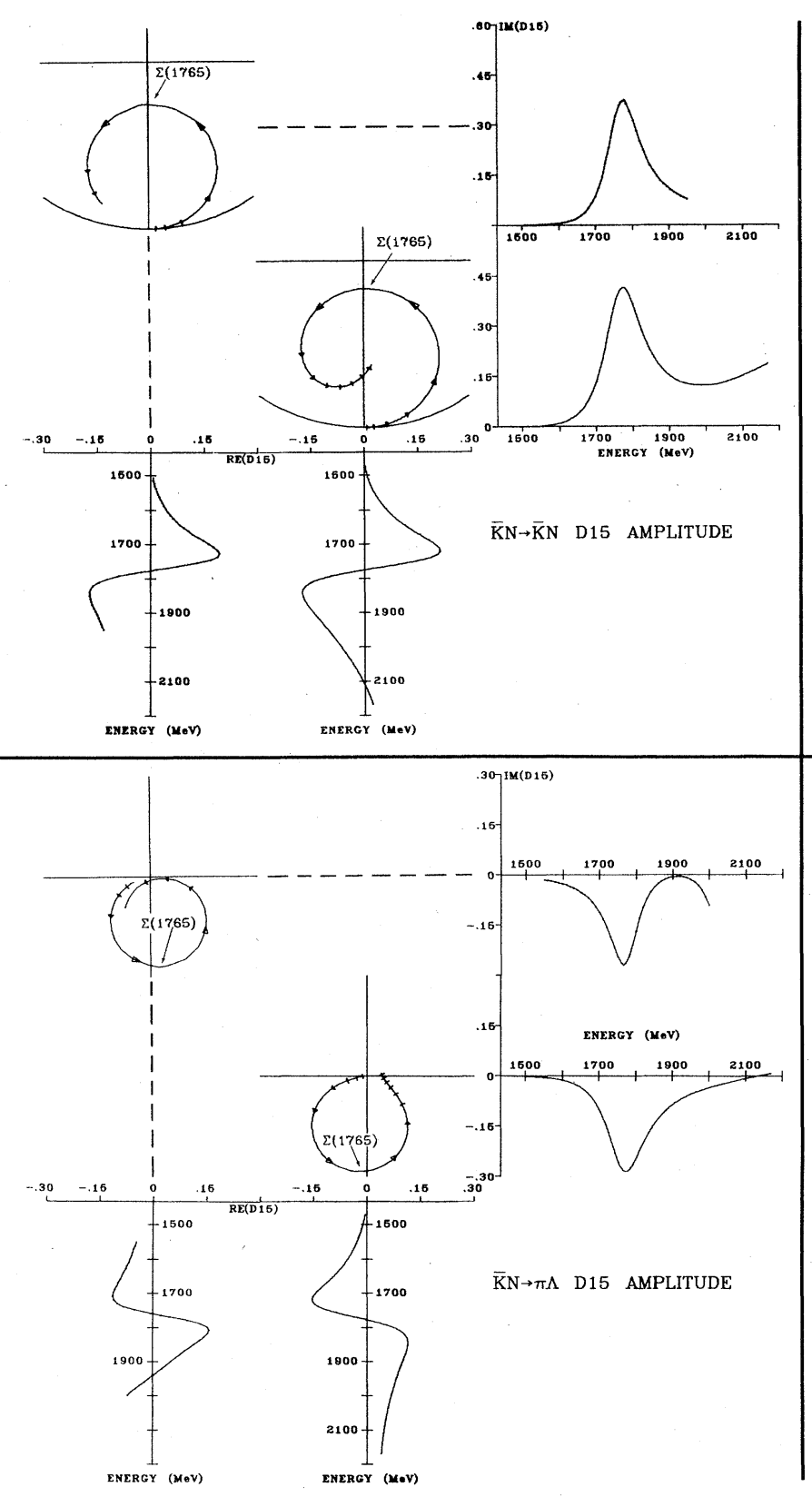

Data Card Listings For notation, see key at front of Listings.

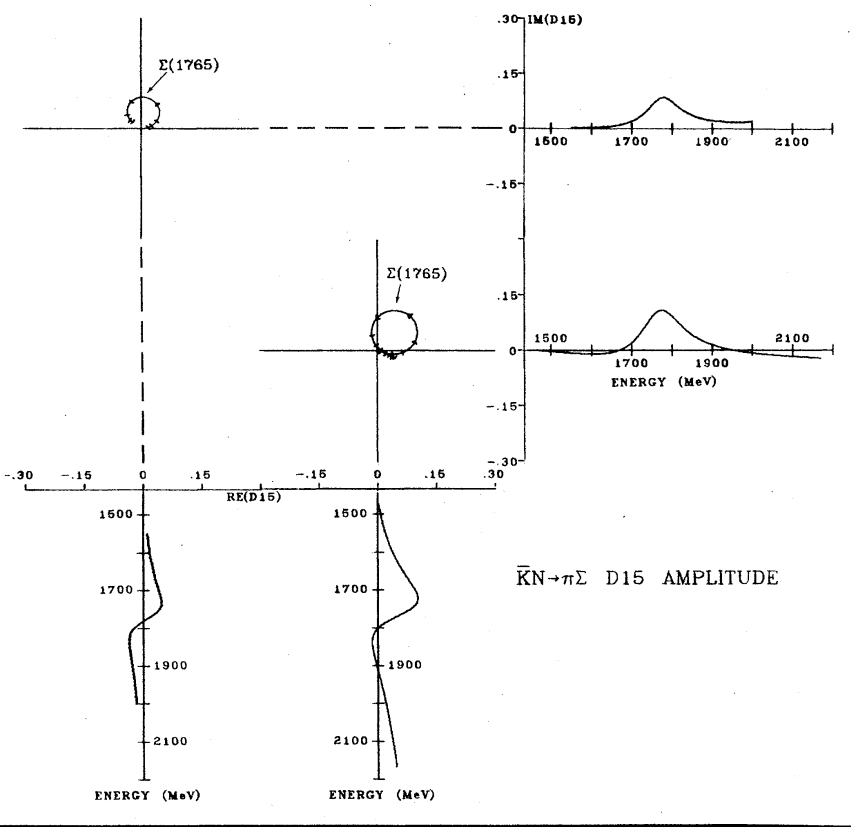

Fig. 5. Amplitudes for $\overline{\mathrm{K} N}$ scattering in the $\mathrm{D}_{15}$ partial wave. The energy dependence of each amplitude is displayed by plotting its real and imaginary. parts versus energy, in alignment with the corresponding Argand plot. In addition, arrows with bases positioned at integral multiples of $50 \mathrm{MeV}$ and a base-to-tip length of $5 \mathrm{MeV}$ are plotted on the Argand plots, and the only established resonance in this wave, the $\Sigma(1765)$, is indicated. The results of two different analyses are shown for each channel; the energy axes all run from elastic threshold to $2200 \mathrm{MeV}$ and are aligned for ease of comparison. The lower plot for each channel is from RLIC 77. The upper plot is from ALSTON 78 for the elastic channel and from MARTIN 77 for the inelastic channels. 


\section{Data Card Listings}

For notation, see key at front of Listings.

Baryons

$\Lambda$ 's and $\Sigma$ 's

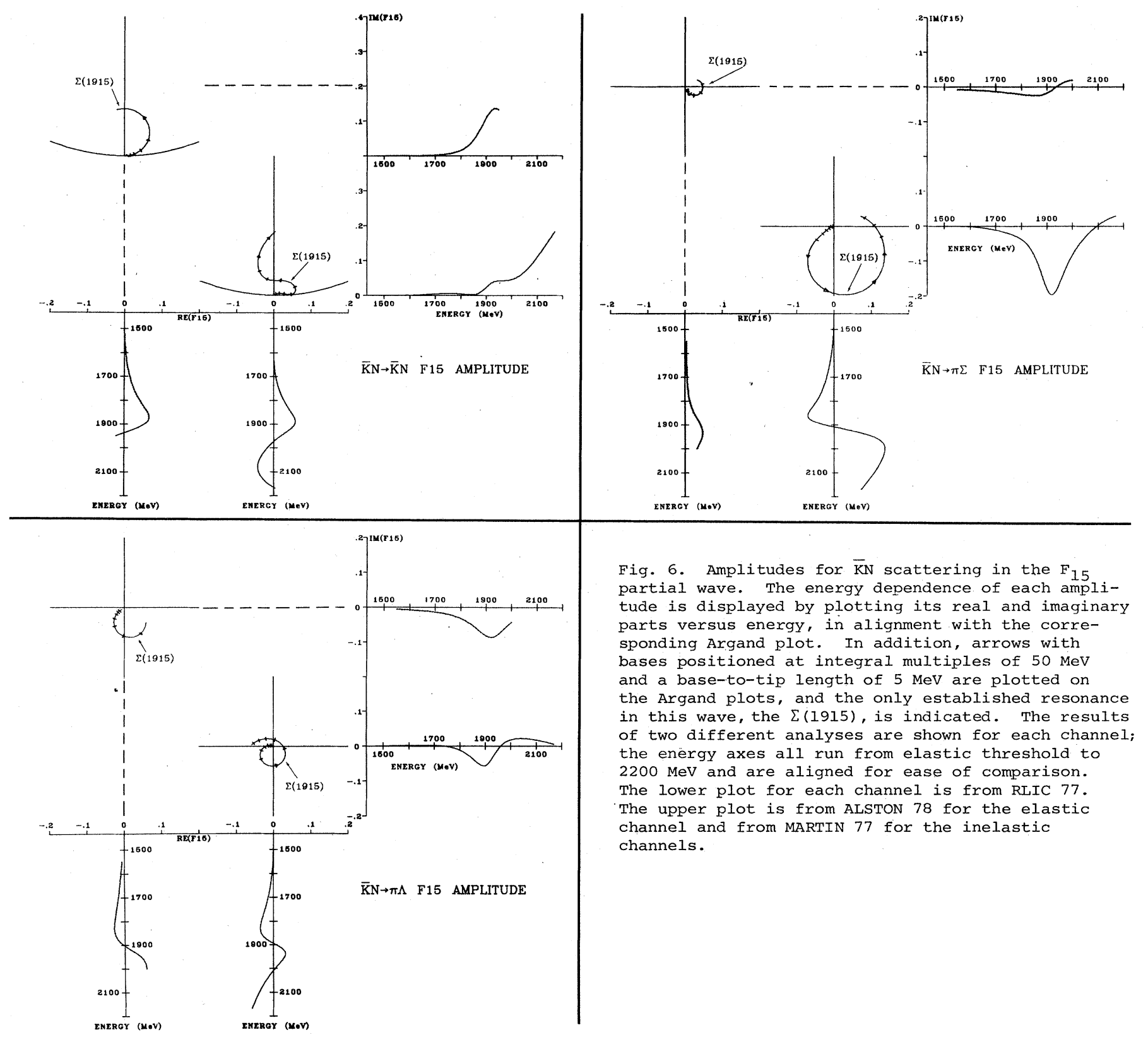




\section{Baryons}

$\Lambda^{\prime} \mathrm{s}$ and $\Sigma$ 's
Data Card Listings For notation, see key at front of Listings.

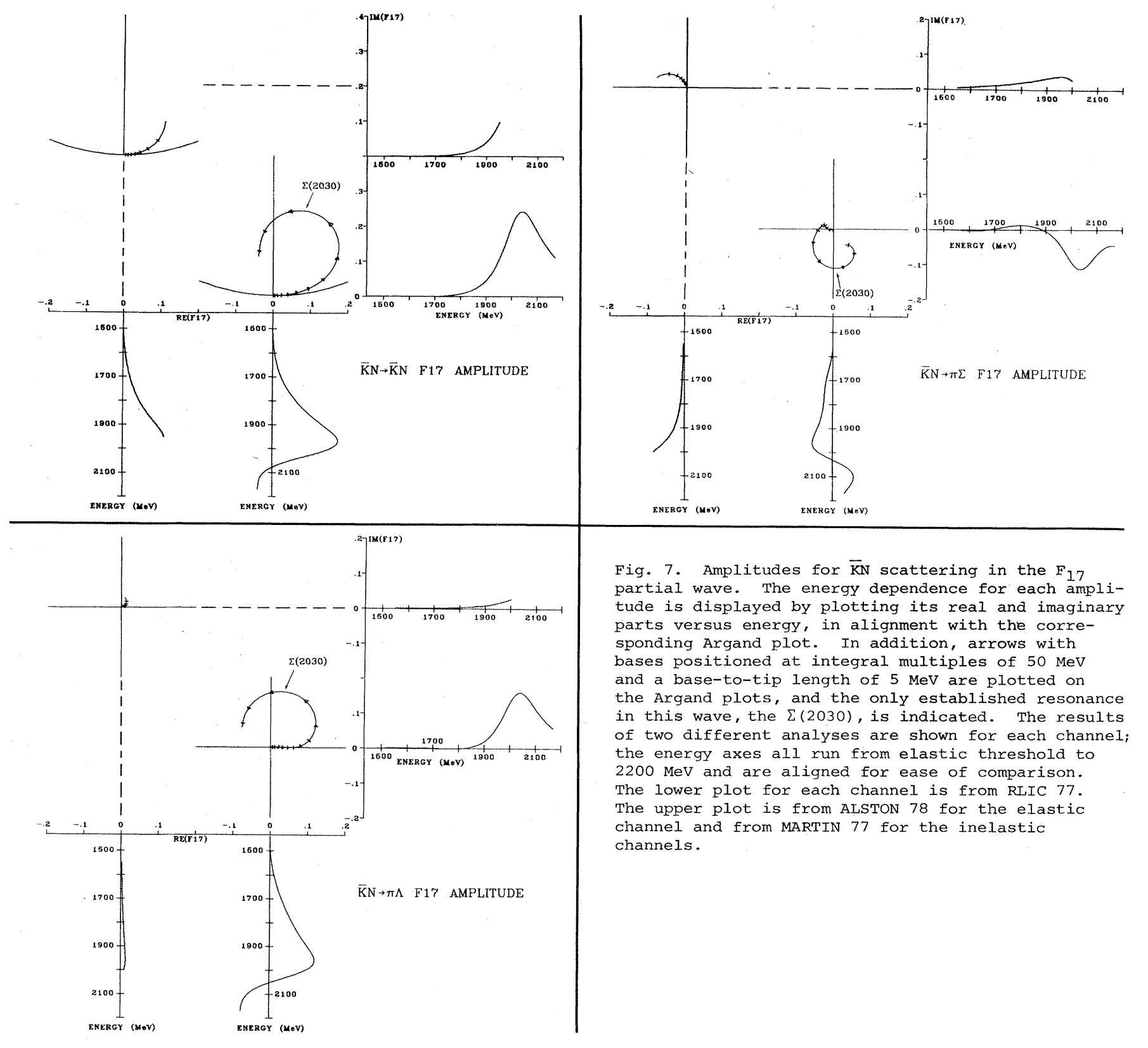


Data Card Listings

For notation, see key at front of Listings.

Baryons

$\Lambda$ 's and $\Sigma^{\prime}$ 's
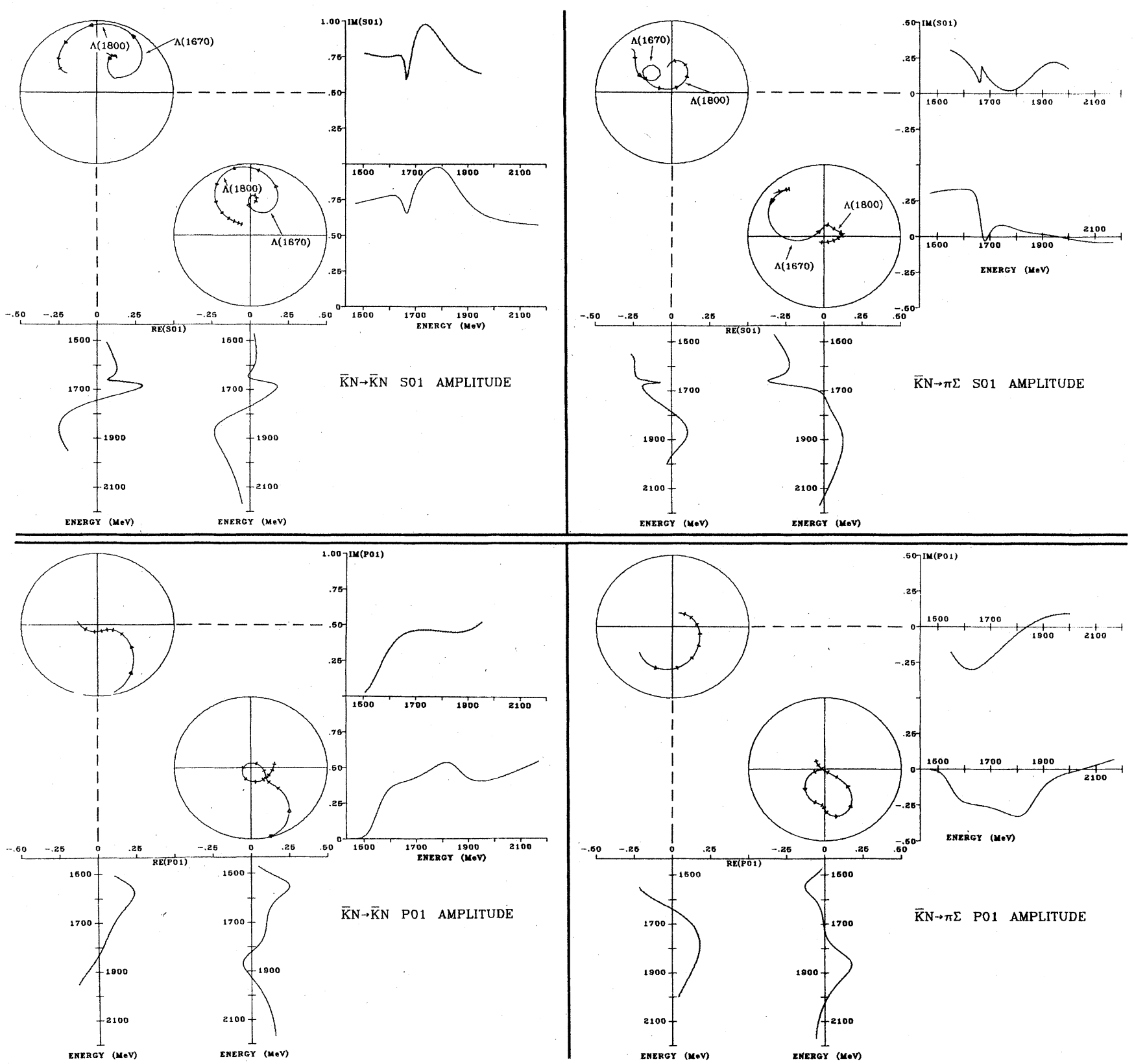

Fig. 8. Amplitudes for $\overline{\mathrm{K} N}$ scattering in the $\mathrm{S}_{\mathrm{Ol}}$ and $\mathrm{P}_{\mathrm{Ol}}$ partial waves. The energy dependence of each amplitude is displayed by plotting its real and imaginary parts versus energy, in alignment with the corresponding Argand plot. In addition, arrows with bases positioned at integral multiples of 50 Mev and a base-to-tip length of $5 \mathrm{MeV}$ are plotted on the Argand plots, and the established resonances $\Lambda$ (1670) and $\Lambda(1800)$ are indicated. The only other established resonance in these waves is the $\Lambda$ (1405), which lies below elastic threshold in the $S_{01}$ wave and is not shown. The results of two different analyses are shown for each channel; the energy axes all run from elastic threshold to $2200 \mathrm{MeV}$ and are aligned for ease of comparison. The lower plot for each channel is from RIIC 77 . The upper plot is from ALSTON 78 for the elastic channel and from MARTIN 77 for the $\pi \Sigma$ channel. 


\section{Baryons}

$\Lambda$ 's and $\Sigma$ 's
Data Card Listings

For notation, see key at front of Listings.
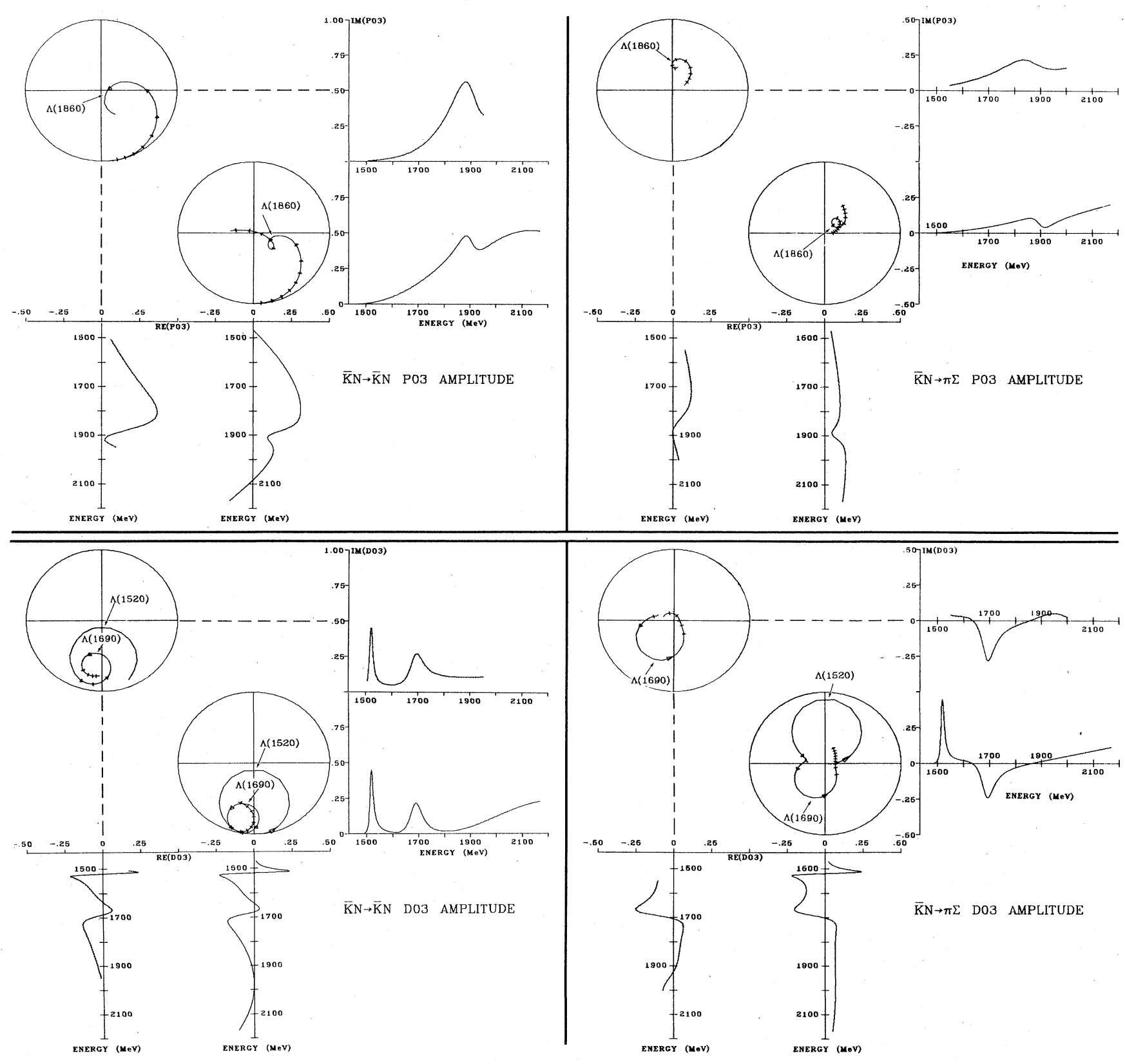

Fig. 9. Amplitudes for $\overline{\mathrm{K} N}$ scattering in the $\mathrm{P}_{03}$ and $\mathrm{D}_{\mathrm{O} 3}$ partial waves. The energy dependence of each amplitude is displayed by plotting its real and imaginary parts versus energy, in alignment with the corresponding Argand plot. In addition, arrows with bases positioned at integral multiples of 50 MeV and a base-to-tip length of $5 \mathrm{MeV}$ are plotted on the Argand plots, and the established resonances $\Lambda$ (1520) $\Lambda(1690)$, and $\Lambda$ (1860) are indicated. The results of two different analyses are shown for each channel; the energy axes all run from elastic threshold to $2200 \mathrm{MeV}$ and are aligned for ease of comparison. The lower plot for each channel is from RLIC 77. The upper plot is from ALSTON 78 for the elastic channel and from MARTIN 77 for the $\pi \sum$ channel. 
Data Card Listings

For notation, see key at front of Listings.

Baryons

$\Lambda$ 's and $\Sigma$ 's
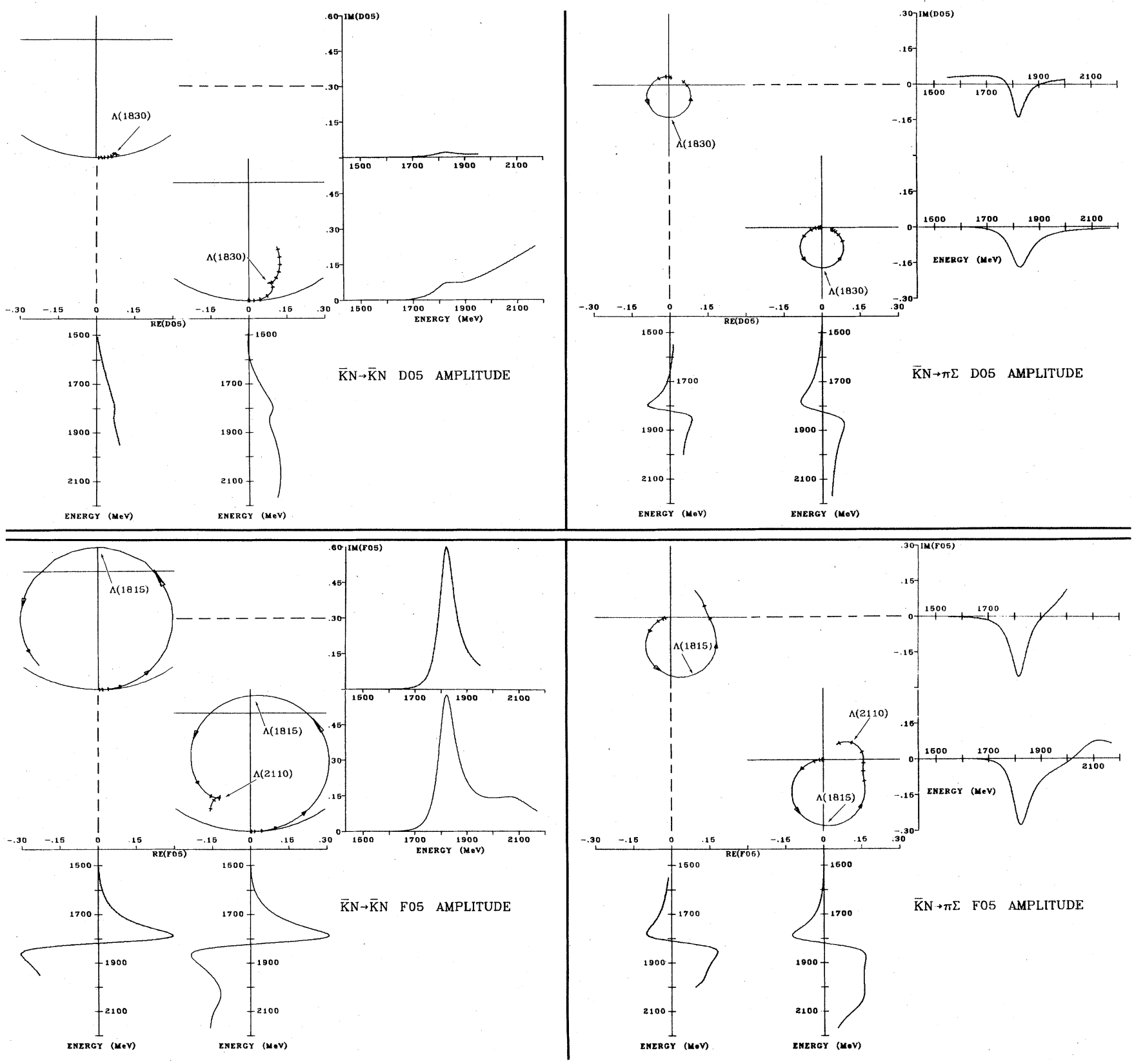

Fig. 10. Amplitudes for $\overline{\mathrm{KN}}$ scattering in the $\mathrm{D}_{05}$ and $\mathrm{F}_{05}$ partial waves. The energy dependence of each amplitude is displayed by plotting its real and imaginary parts versus energy, in alignment with the corresponding Argand plot. In addition, arrows with bases positioned at integral multiples of 50 Mev and a base-to-tip length of $5 \mathrm{MeV}$ are plotted on the Argand plots, and the established resonances $\Lambda$ (1815) $\Lambda(1830)$, and $\Lambda(2110)$ are indicated. The results of two different analyses are shown for each channel; the energy axes all run from elastic threshold to $2200 \mathrm{MeV}$ and are aligned for ease of comparison. The lower plot for each channel is from RLIC 77. The upper plot is from ALSTON 78 for the elastic channel and from MARTIN 77 for the $\pi \sum$ channel. 


\section{Baryons}

\section{$\Lambda$ 's and $\Sigma$ 's}

Data Card Listings For notation, see key at front of Listings.
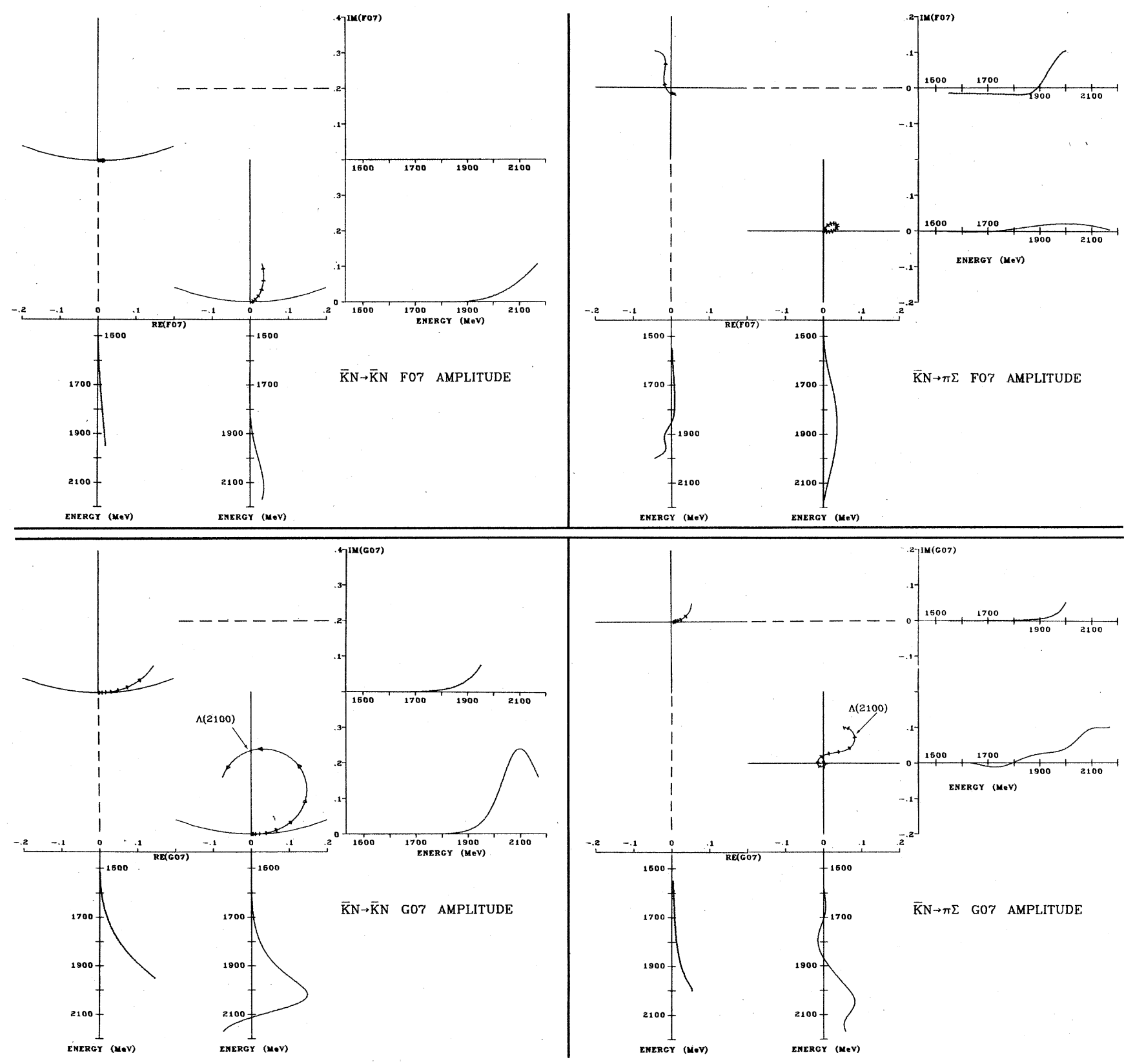

Fig. 11. Amplitudes for $\overline{\mathrm{KN}}$ scattering in the $\mathrm{F}_{07}$ and $\mathrm{G}_{07}$ partial waves. The energy dependence of each amplitude is displayed by plotting its real and imaginary parts versus energy, in alignment with the corresponding Argand plot. In addition, arrows with bases positioned at integral multiples of $50 \mathrm{MeV}$ and a base-to-tip length of $5 \mathrm{MeV}$ are plotted on the Argand plots, and the established resonance $\Lambda$ (2100) is indicated. The results of two different analyses are shown for each channel; the energy axes all run from elastic threshold to $2200 \mathrm{MeV}$ and are aligned for ease of comparison. The lower plot for each channel is from RLIC 77. The upper plot is from ALSTON 78 for the elastic channel and from MARTIN 77 for the $\pi \Sigma$ channel. 


\section{Data Card Listings \\ For notation, see key at front of Listings.}

\section{Baryons}

$\Lambda$ 's and $\Sigma^{\prime} s$

effects" are not found and new possible resonances are proposed. The situation in particular for the low partial waves and for low energy is still very confused.

The same group has also published analyses for the main quasi-two-body channels, namely $\Lambda$ (1520) $\pi$, $\Sigma(1385) \pi$, and $\overline{N K}^{*}(892)$ (CAMERON 77, CAMERON 78, and CAMERON2 78). The statistical accuracy of the data here is lower than for the real two-body channels, and only constrained energy-dependent fits have been made, with most of the well-known resonances being included with fixed parameters. Only one new resonant structure, not observed in real two-body analyses, is suggested, and this is seen in the $\mathrm{NK}^{*}$ channel only. Many new couplings of known resonances to quasi-two-body channels were found.

b) The analysis of B. Martin and M. Pidcock (MARTIN 77) is a multi-channel energy-dependent partial-wave analysis with parametrized K-matrix elements. The mass range covered is 1.54 to $\sim 2 \mathrm{GeV}$. Here the 3 channels $\overline{\mathrm{KN}}, \Lambda \pi$, and $\sum \pi$ are always considered simultaneously, a fictitious channel being introduced to account for the global effect of the remaining final states. The $\Lambda \eta$ and $\Sigma \eta$ channels have thresholds within the above mass range and they may induce a "cusp effect". In order not to exclude such a possibility, an additional channel, opening up at the right energy, is also included for the $S$ waves. (It is known that the inelasticities in the $S$ waves are largely due to the production of the $n$ meson, and a similar effect is seen in S-wave $\pi \mathrm{N}$ elastic scattering.) These additional channels, for which no data are fitted, have large cross sections, so it is not clear if such a multi-channel analysis really imposes more stringent unitarity constraints than those already contained in single-channel fits. For this analysis also, a careful selection of the available data has been made. The number of data points which have been fitted amounts to about 12,000. Some of the experimental points have been renormalized in order to suppress unphysical discontinuities in the data. The various channels or types of data may have very different numbers of data points; it would be possible to have a good overall $X^{2}$ with some pieces of data being badly fitted. This was prevented by introducing weights for the various types of data so that each type is reasonably well fitted.

Resonances can appear as poles of the K-matrix, but the K-matrix parameters thus deduced are difficult to compare to those obtained in the more conventional analyses. Two other methods in which the resonance parameters are calculated from the poles of the T-matrix are also given. We list the results of these two methods in the Data Card Listings. It should be noted that no claim for uniqueness of the proposed solution is made; in fact, another solution with almost the same $\chi^{2} / \mathrm{N}$ was presented at the oxford Conference. ${ }^{5}$ This latter solution has not been retained as it was not in agreement with present ideas about the low energy behavior of some waves.

c) The single-channel $\overline{\mathrm{KN}}$ analysis of ALSTON 78 is worth mentioning because it includes a large amount of new data ${ }^{1-3}$ not used by the two analyses mentioned above. It is a conventional energydependent analysis, covering the mass range 1.5 to $1.94 \mathrm{GeV}$, which uses a unitary background parametrization expressed in terms of scattering lengths. The cusp effects observed at the $\Lambda \eta$ and $\Sigma \eta$ thresholds are included by the introduction of a square-root singularity in the energy variation of the S-wave resonance widths. All the confirmed states are observed, but most of the less well established resonances (one and two stars in Table I) are neither observed nor required.

d) Other analyses: Preliminary results of a $\overline{\mathrm{KN}}$ single-channel analysis by Hansen et al. have been reported. They incorporate more theoretical ingredients than any of the analyses done so far. We have not yet included these results in the Listings. In this energy-independent analysis, invariant amplitudes at fixed $t$ are used as supplementary constraints in the fit. These fixed-t amplitudes themselves are computed using dispersion relations for which not only experimental data but also a first estimate of the partial waves is needed. The method requires that the process of estimating the fixed-t amplitudes and then the partial-wave amplitudes be iterated a few times until full consistency is obtained. This kind of analysis may eventually provide us with more 


\section{Baryons $\Lambda$ 's and $\Sigma$ 's}

reliable partial-wave amplitudes which satisfy fixed-t analyticity and crossing.

In the Listings, the resonance parameters obtained in the latest single-channel analyses are given. These usually cover a limited mass range, but include new data not yet incorporated in the multi-channel analyses described above. In particular, the Saclay-Collège de France collaboration has now published three separate energy-dependent analyses for the channels $\overline{\mathrm{K}} \mathrm{N}, \Lambda \pi$, and $\sum \pi$ extending up to a mass of $2.5 \mathrm{GeV}$ (BELLEFON 78, 76, and 77, respectively). They indicate that the bumps seen in total cross sections at these higher energies are made up of many resonant states.

For a brief description of the older analyses we refer to the previous editions of this compilation.

\section{Production Experiments}

Production experiments are often difficult to analyze for the same reasons as mentioned in the preceding Note on $\mathrm{N}^{\prime} \mathrm{s}$ and $\Delta^{\prime} \mathrm{s}$. I=0 states can only be studied when there is no $I=1$ state at a similar mass. In the Baryon Table we only use results from production experiments for the lower mass states. $\Sigma(1385)$ and $\Lambda$ (1405) lie below the $\bar{K} N$ threshold. Production and formation experiments agree quite well in the case of $\Lambda(1520)$, and thus they have been combined for this state. There is some disagreement between the two types of experiment in the 1600-to-1700 MeV region. See the $\Sigma(1620)$ and $\Sigma(1670)$ mini-reviews for details.

\section{Figures}

Argand plots of fifteen $S=-1$ partial waves are shown in Figs. 1 through 11 . The analyses shown were picked largely for illustrative purposes rather than on the basis of our judgment of their quality; for the $\overline{\mathrm{K} N}$ channel, we chose to show the amplitudes obtained by RLIC 77 and ALSTON 78, and for the $\Lambda \pi$ and $\Sigma \pi$ channels those from RLIC 77 and MARTIN 77.

\section{Errors on Masses and Widths}

Often the quoted errors in partial-wave analyses are only statistical, and the values of masses and widths can change by more than these errors when a new parametrization is used. For this reason we report the values of $M, \Gamma$, and $x_{i}$

\section{Data Card Listings For notation, see key at front of Listings.}

obtained by different authors even if they analyze the same data. The spread of these masses and widths is certainly a better estimate of the uncertainties than the statistical errors. Sometimes the errors quoted are obtained by the inspection of various fits done with different hypotheses (see, for example, BERTHON 70, GALTIERI 70, VANHORN 75, RLIC 76). For three states, $\Lambda$ (1520), $\Lambda(1815)$, and $\sum(1765)$, there are enough data available to perform an overall fit of the various $x_{i}$ of the type discussed in the main text (Sec.VII B). In this case we are forced to use the errors, however small they may be, but we warn the reader that the final errors are not to be taken seriously. In the Baryon Table we choose not to give errors on masses and total widths determined primarily by partial-wave analyses, but, whenever necessary, to show a range of values. As for the branching ratios, we use the errors when needed to perform an overall fit, but we caution the reader.

\section{Conclusions}

Table $I$ is an attempt to evaluate the status of the various $\mathrm{Y}^{*} \mathrm{~s}$. The evaluations are of course partly subjective. A blank indicates that there is no corresponding evidence at all. This may mean either that the relevant couplings are small or that the resonance does not really exist. The Baryon Table includes only the well established resonances. It seems clear, however, that whereas any particular one of the questionable resonances may disappear with the next analysis, there are probably many new resonances underlying those already established.

\section{References}

1. M. Alston-Garnjost et al., Phys. Rev. D17, 2216 (1978).

2. M. Alston-Garnjost et al., Phys. Rev. D17, 2226 (1978).

3. R. D. Ehrlich et al., Phys. Lett. 71B, 455 (1977).

4. C. Damerell et al., Nucl. Phys. B129, 397 (1977).

5. B. R. Martin, in proceedings of the Topical Conference on Baryon Resonances (Oxford, 1976), edited by R. T. Ross and D. H. Saxon, pg. 285.

6. P.N. Hansen et al., in Oxford Conference Proceedings (ibid.), pg. 275.

For other references, see the Data Card Listings. 


\section{Data Card Listings}

Baryons

For notation, see key at front of Listings.

$\Lambda^{\prime} \mathrm{s}$ and $\Sigma^{\prime} \mathrm{s}, \Lambda, \Lambda(1330), \Lambda(1405)$

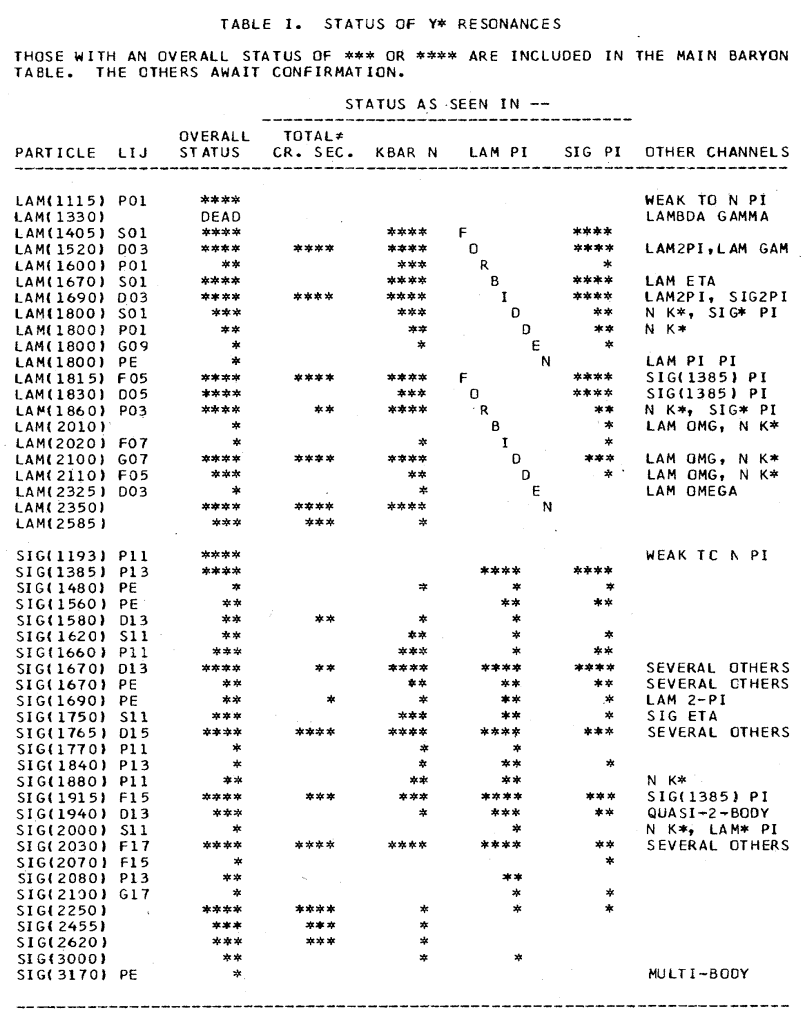

**** GOOD, CLEAR, AND UNM ISTAKABLE.
*** GOOD, BUT IN NEED OF CLARIFICATION CR NOT ABSOLUTELY CERTAIN. * NEEDS. CONFIRMATION.

* attributed to the state closest to where the cross section peaks.

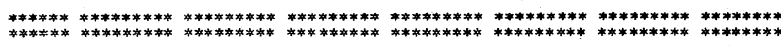
$\mathrm{S}=-1 \mathrm{I}=0$ HYPERON STATES $(\Lambda)$.

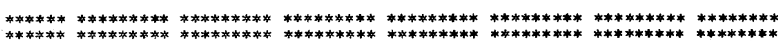

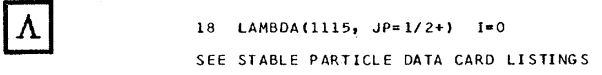

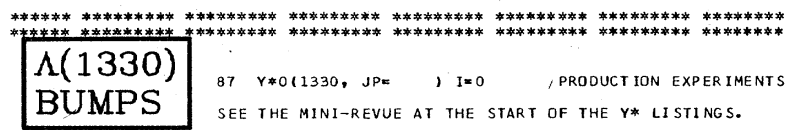

A PEAK WAS SEEN NEAR 1330 MEV IN THE LAMBDA GAMMA
SPECTRUM IN THREE PI PROPANE EXPERIMENS (YUNG-CHANG
64. BUBELEV O7, AND BOZOKI 68). ALL MORE RECENT

RESULTS INDICATE THAT THERE IS NO RESONANACE NEAR THIS MASS VALUE.
IS THELV 6 , AND BOZOK 681 . ALL MORE RECENT

************************************************************************** REFERENCES FOR $Y * 0(1330)$ (PROD. EXP.)

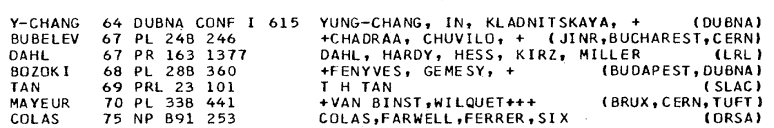

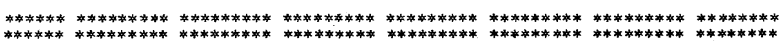

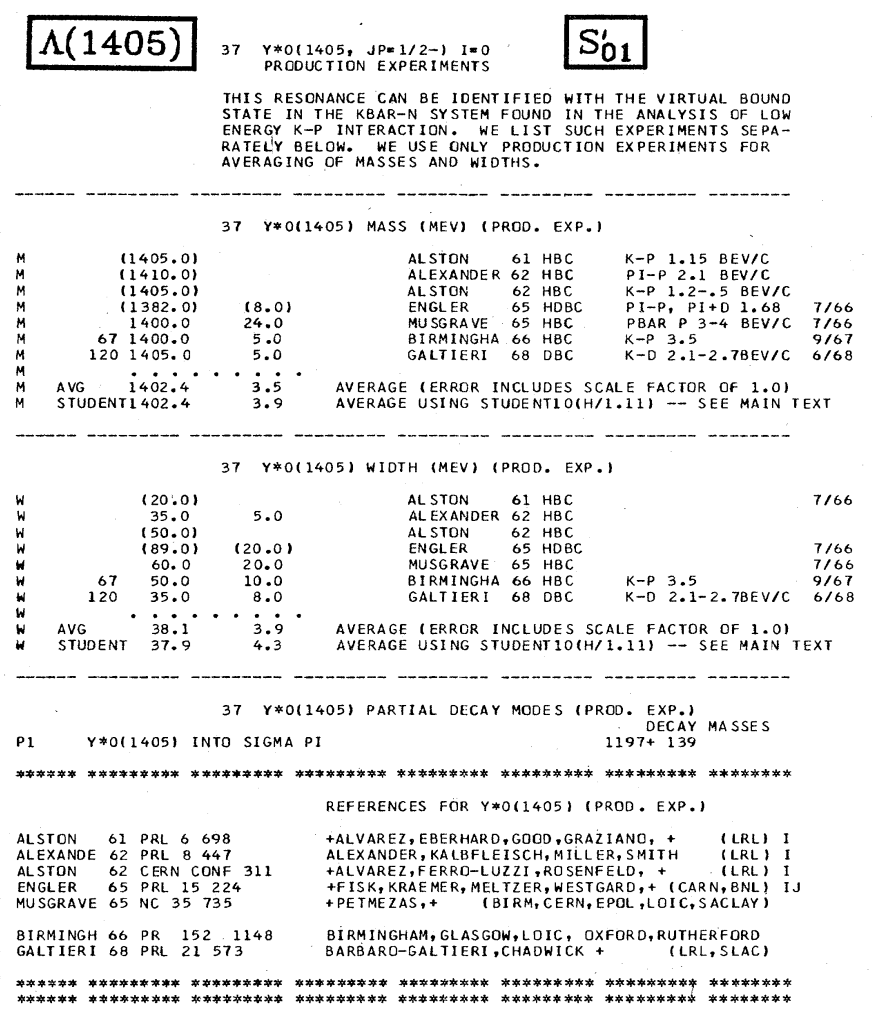

1405 MEV REGION: EXTRAPOLATIONS BELOW THRESHOLD

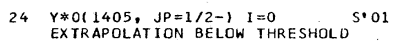

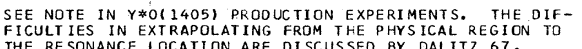

THE QUESTION ON WHETHER Y*(1405) IS A KBAR-N BOUND
STATE OR A CDO POLE IDALITZ 70, RAJASKARAN 72 HAS BEEN INVESIGATED

BY CLINE 71, MARTIN 71 , GALTIERI 72, AND DOBSON 72 . THE
PAPERS CONCLUDE THAT THE DATA CANNOT TELL THE DIFFERENCE.

THE (N KBARI/(PI SIGMA) COUPLING RATIO IS DISCUSSED BY DADES 77.

$$
24 Y * 0(1405) \text { MASS (MEV) }
$$

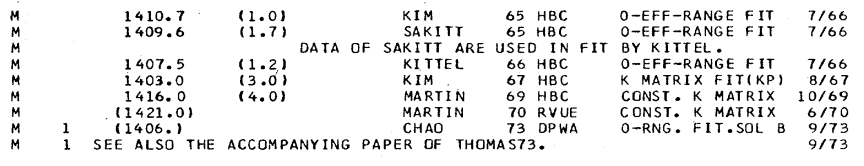

\begin{tabular}{|c|c|c|}
\hline & & REFERENCES FOR $Y * 0(1405)$ (FROM EXTRAPOLATIONS) \\
\hline \begin{tabular}{l} 
KIM \\
SAKITT \\
KIITEL \\
KIM \\
MARIIN \\
MARTIN \\
CHAO \\
\multicolumn{1}{|l}{ ALSO }
\end{tabular} & $\begin{array}{llll}65 & P R L & 14 & 29 \\
65 & P R & 139 & B 719 \\
66 & P L & 21 & 349 \\
67 & P R L & 19 & 1074 \\
69 & P R & 183 & 1352 \\
70 & \text { NP } & 816 & 479 \\
73 & \text { NP } & 856 & 46 \\
73 & \text { NP } & 856 & 15\end{array}$ & 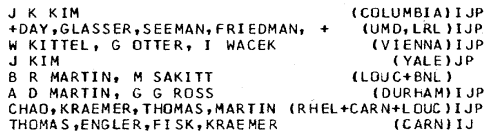 \\
\hline
\end{tabular}

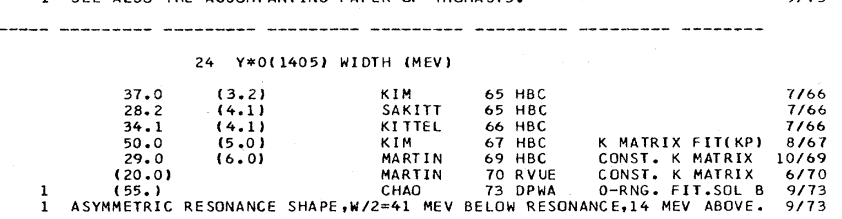




\section{Baryons $\Lambda(1405), \Lambda(1520)$}
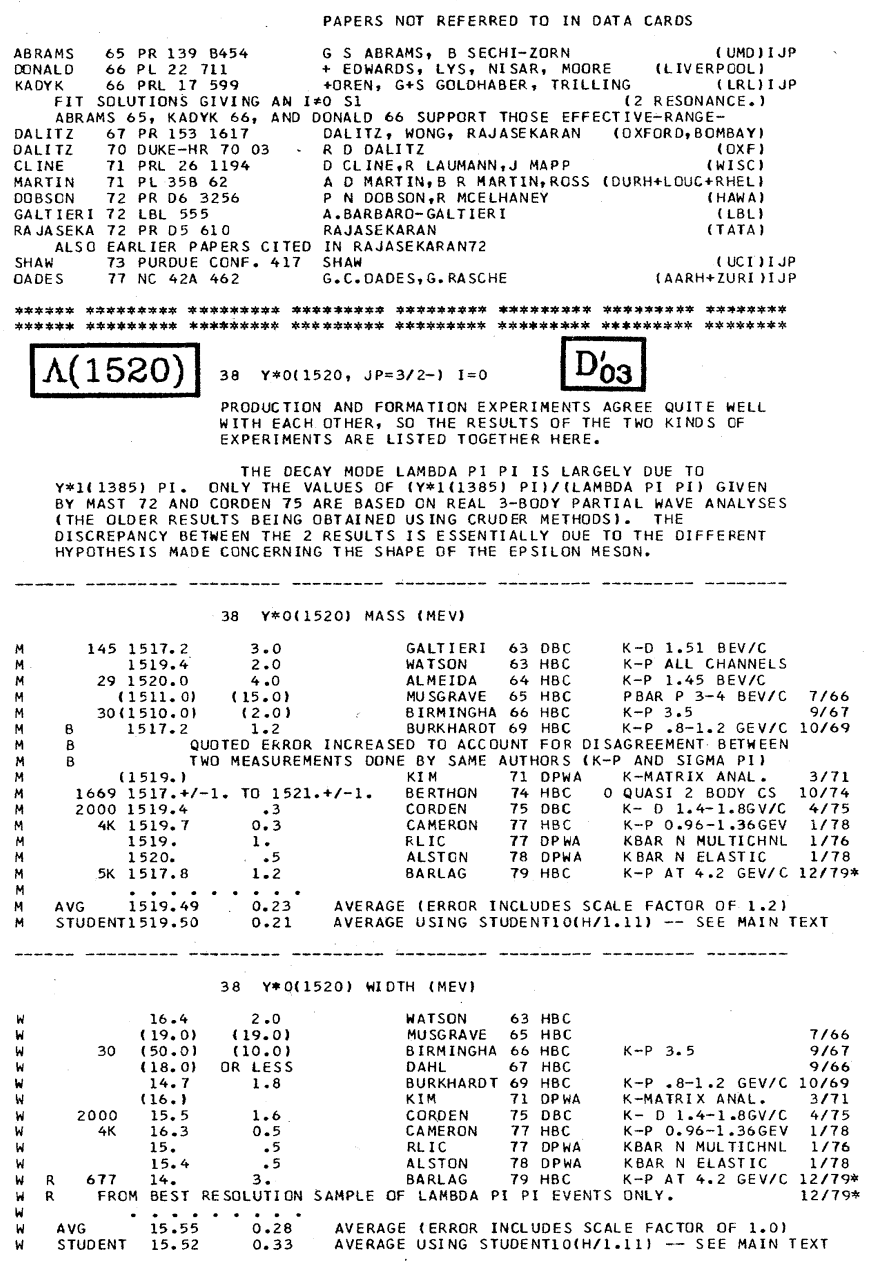

$38 Y * 0(1520)$ PARTIAL DECAY MODES

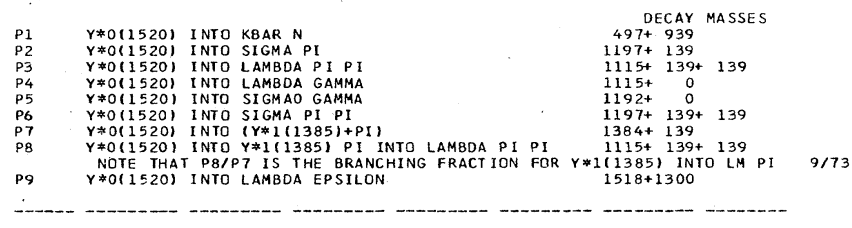

\section{FITTED PARTIAL DECAY MODE BRANCHING FRACTIONS}

The matrix below is derived from the error matrix for the fitted partial decay mode branching fractions, $P_{i}$, as follows: The diagonal elements are $P_{i} \pm \delta P_{i}$, where

$\delta P_{i}=\sqrt{\left\langle\delta P_{i} \delta P_{i}\right\rangle}$, while the off-diagonal elements are the normalized correlation coefficients $\left\langle\delta \mathrm{P}_{i} \delta \mathrm{P}_{j}\right\rangle /\left(\delta \mathrm{P}_{i} \cdot \delta \mathrm{P}_{j}\right\rangle$. For the definitions of the individual $\mathrm{P}_{i}$, see the listings above; only those $\mathrm{P}_{\mathrm{i}}$ appearing in the matrix are as sumed in the fit to be nonzero and are thus constrain $P_{1}$ to add to 1 .

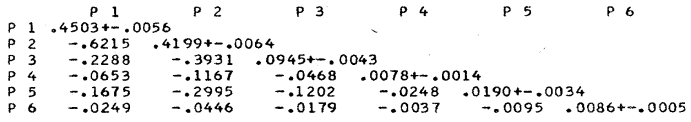

Data Card Listings For notation, see key at front of Listings.
$38 Y$ Y*O(1520) BRANCHING RATIOS

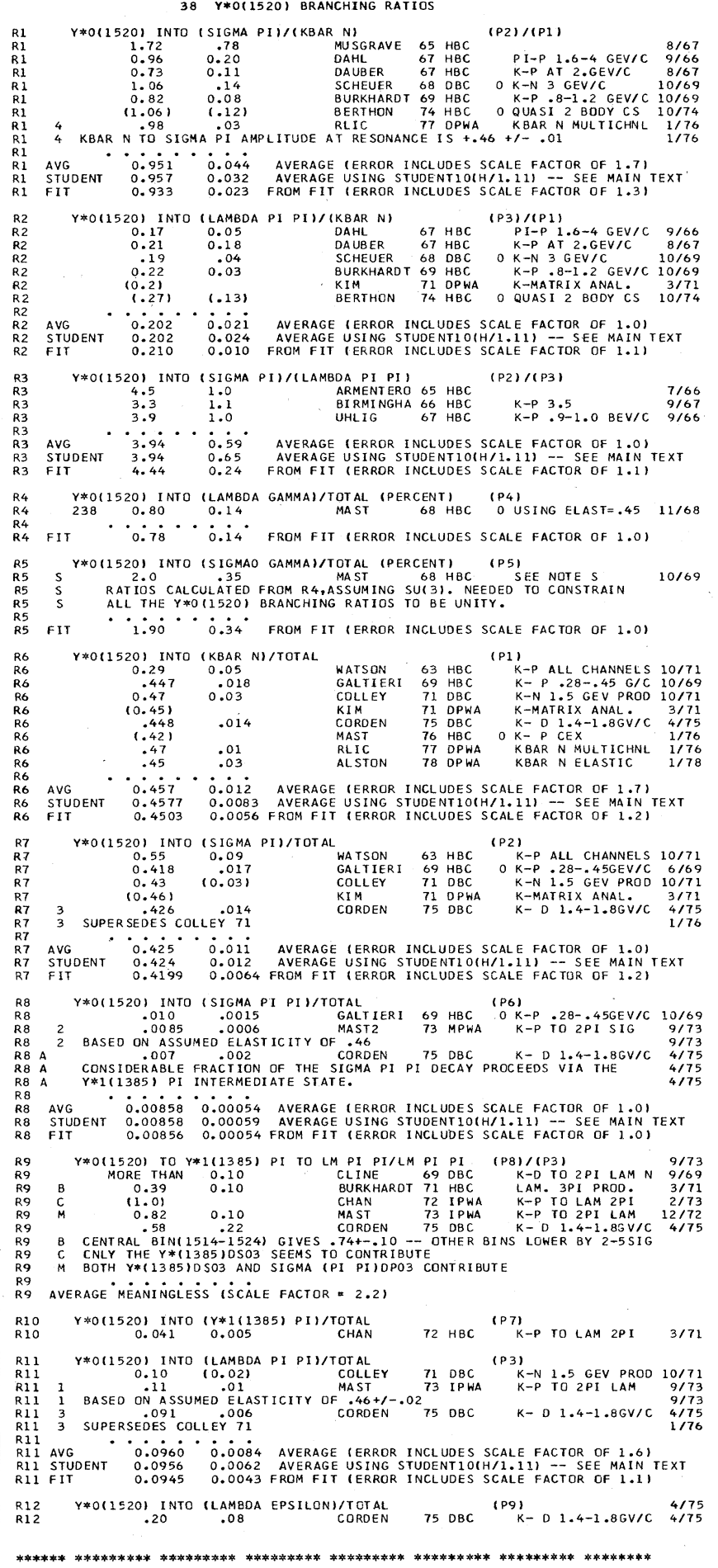




\section{Data Card Listings}

For notation, see key at front of Listings.

Baryons $\Lambda(1520), \Lambda(1600), \Lambda(1670)$

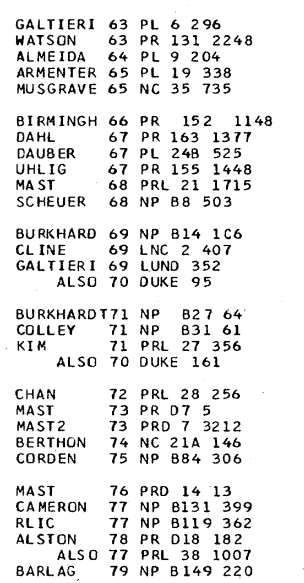

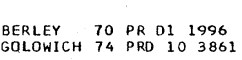

REFERENCES FOR Y*O(1520) A BARBARO-GALTIERI, A HUSSAIN, RD TR IPP (LRL)
$M$ B WATSON, M FERRO-LUZZI, R D TRIPP (LRL)I JP $S P$ ALMEIDA, G R LYNCH
ARMENTEROS, F-LUZ I T
(CERN) +PETMEZAS,+ (BIRM, CERN, EPOL, LOIC, SACLAY) BIRMINGHAM, GLASGOW, I. C., OXFORD, RUTHERFORD
DAHL, HAROY, HESS, KIRZ, MILLER
(LRL) + MALAMUD, SCHLEIN, SLATER, STORK +
+CHARLTLN, CONDON, GLASSER, YODH, +
+UMD, NRLI) MAST, ALSTON, BANGERTER, GALTIERI+
SABRE COLLAB. (LRL)
(SACL+AMST+BGNA+REHO+EPOL) +FILTHUTH+KLUGE+.. (HEID+EFI +CERN+SACLAY) +LAUMANN+MAPP
BARBARO-GALTIERI, BANGER TER, MAST, TRIPP (WISC)
RD TRIPP
(LRL) +F ILTHUTH, KLUGE, OBERLACK++ (HEID+CERN+SACL)
+ +COX,EASTWOOD, FRY .. (BIRM+ED IN+GLAS+LCIC) J.K KIM
J. K. KIM +BUT. -SHAFER, HERTZBACH, KOFLER++ (MASA, YALE)
+ALSTONGARNJOST, BANGRTER,
+BANGERTER, ALSTON-GARNJOST,
(LBL) IJJP BERTHON, TRISTRAM, +
CORDEN, COX, OARTNELL, KENYON, ONEALE, + +STRB)
(BIRM) MAST, ALSTON-GARNJOST, BANGERTER+ (LBL)
+FRANEK, GOPAL, KALMUS, MC PHERSON+ (RHEL+LEIC) IIJP GOPAL, ROSS, VAN HORN, MC PHERS ON+ (LOIC+RHEL) IJJ

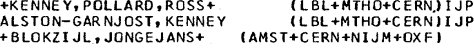
PAPERS NOT REFERRED TO IN DATA CARDS PYAM IN, KOFLER, MANN, MEI SNER+ (BNL, MASA, YALE) I JP
EUGEN GOLOWICH

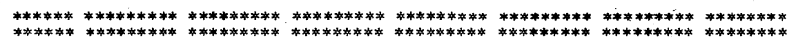

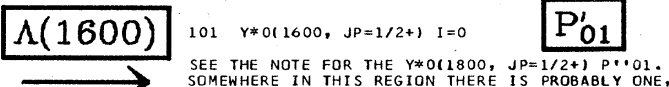
SOMEWHERE IN THIS REGION THE,
AND PERHAPS TWO, POI STATES.

$101 Y * 0(1600)$ MASS (MEV)

$M$
$M$
$M$
$M$
$M$
$M$
$M$
$M$ $\begin{array}{cc}M & M \\ M & M \\ M & M \\ M & M \\ M & M \\ M \\ M \\ M \\ M\end{array}$

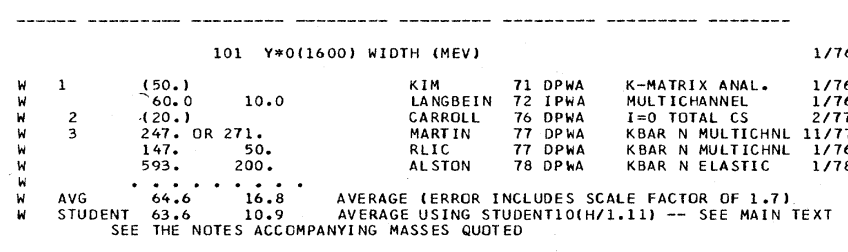

101 Y*0(1600) PARTIAL DECAY MODES

$\begin{array}{lll}P 1 & Y * 0(1600) \\ P 2 & \text { INTO KBAR N } \\ \text { P2 } & \text { K1600) INTO SIGMA P1 }\end{array}$

DECAY MASSES KIM 71 DPWA

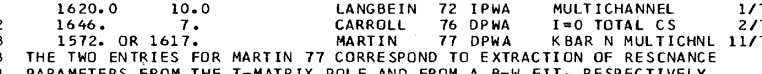
THE TWO ENTRIES FOR MARTIN 77 CORRE SPOND TO EXTRACTION OF RESCNANCE
PARAMETERS FROM THE T-MATRIX POLE AND FROM A B-W FIT, RESPECTIVELY.

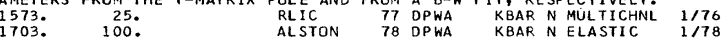
RAGE MEÁNiNGLiess ${ }^{\circ}$ i SCALE FaC TOR $=2.31$

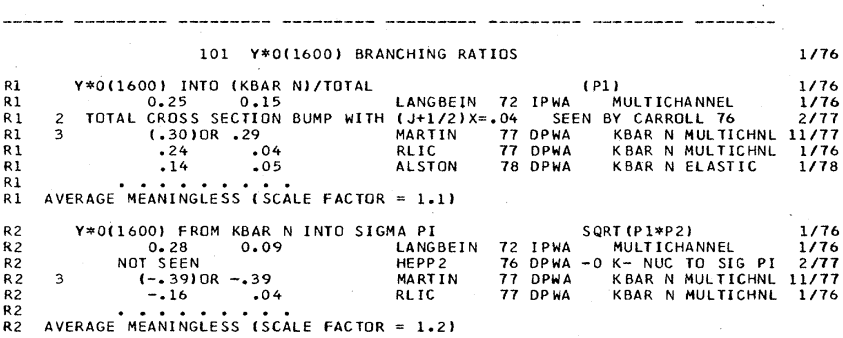

R2 AVERAGE MEANINGLESS (SCALE FACTOR $=1.2$ )

\begin{tabular}{|c|c|c|c|}
\hline & & REFERENCES FOR $Y * 0(1600)$ & \\
\hline KIM ALSO & $\begin{array}{l}71 \text { PRL } 27356 \\
70 \text { DUKE } 161\end{array}$ & $\begin{array}{l}J . k K I M \\
\text { J. } k . K I M\end{array}$ & $\begin{array}{l}(\text { HARV)IJP } \\
\text { (HARVIIJP }\end{array}$ \\
\hline LANGBEIN & 72 NP $847 \quad 477$ & +WAGNER & (MPIM) I JP \\
\hline $\begin{array}{l}\text { CARROLL } \\
\text { HEPP2 }\end{array}$ & $\begin{array}{lllll}76 & \text { PRL } & 37 & 806 \\
76 & \text { PL } & 65 & 58 & 487\end{array}$ & $\begin{array}{l}\text { +CHIANG, KYCIA, LI, MAZ UR, MIC HAEL + } \\
\text { +BRAUN, GR IMM, STROBELE, THOL + (CERRN, }\end{array}$ & $\begin{array}{l}(B N L) I \\
I D, M P I M) I J P\end{array}$ \\
\hline
\end{tabular}

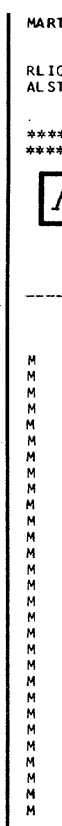

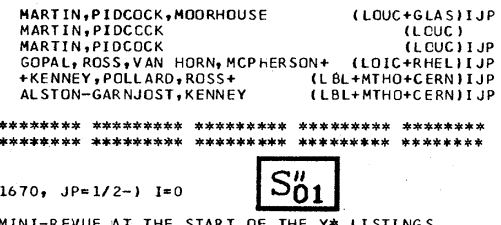

SEE THE MINI-REVUE AT THE START OF THE Y* LISTINGS. THIS RESONANCE IS WELL ESTABLISHED.

$40 \quad \gamma * 0(1670)$ MASS (MEV)

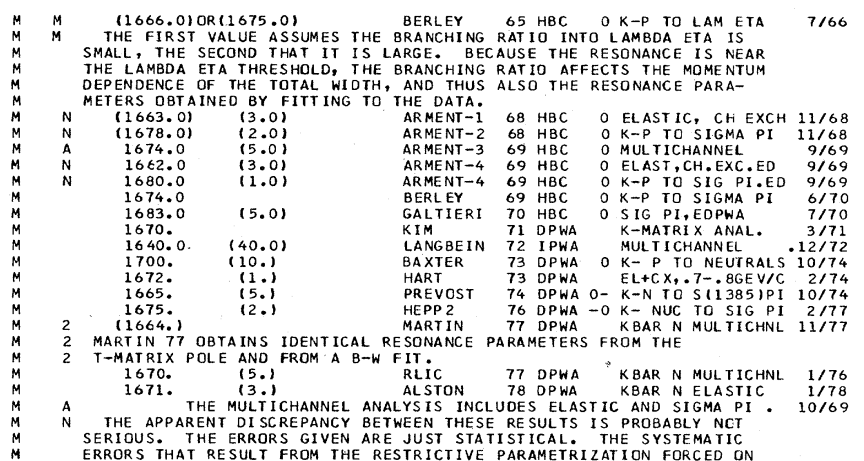
SERIOUS. THE ERRORS GIVEN ARE JUST STATISTICAL. THE SYSTEMATIC
ERRORS THAT RESULT FROM THE RESTRICTIVE PARAMETRIZATION FORCED ON THE PARTI AL-WAVE AMPLITUDES ARE NOT INCLUDED, AND CAN BE LARGE. $40 Y * 0(1670)$ WIOTH (MEV)

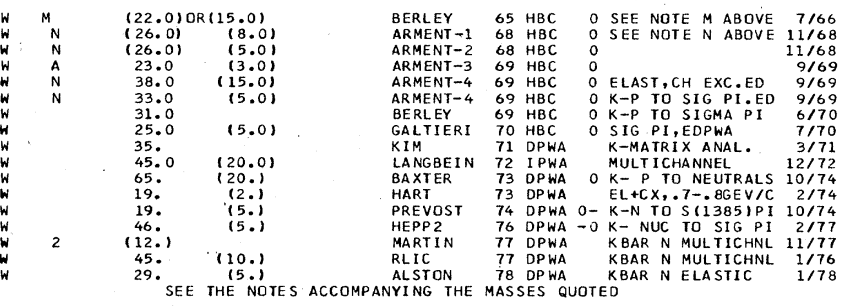

40 \% $0(1670)$ PARTIAL DECAY MODES

$\begin{array}{ll}P_{1} & Y * 0(1670) \text { INTO KBAR N } \\ P_{2} & Y * 0(1670) \text { INTO LAMBDA ETA } \\ P_{3} & Y * 0(1670) \text { INTO SIGMA PI } \\ \text { P4 } & Y * 01670)\end{array}$

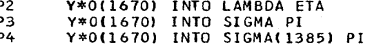

DECAY MASSES
$497+939$

$497+939$
$1115+548$

$1189+139$
$139+1384$

$40 \gamma$ *0(1670) BRANCHING RATIO

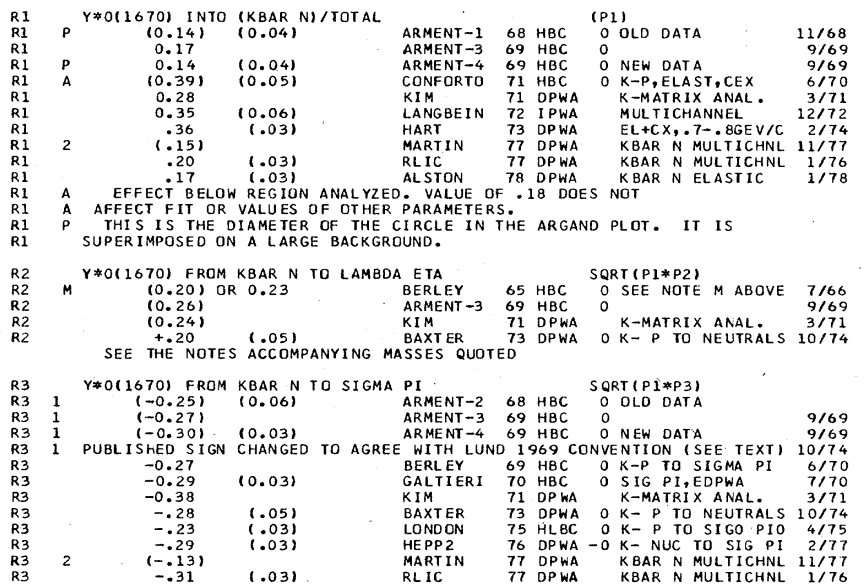




\section{Baryons}

$\Lambda(1670), \Lambda(1690), \Lambda(1800)$
Data Card Listings For notation, see key at front of Listings.

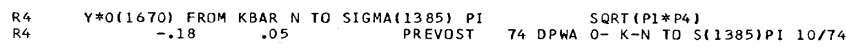

\begin{tabular}{|c|c|}
\hline & REFERENCES FOR $Y * 0(1670)$ \\
\hline $\begin{array}{lllll}\text { BERLEY } & 65 & \text { PRL } & 15 & 641 \\
\text { ARMENT-1 } & 68 & N P & B 8 & 195 \\
\text { ARMENT-2 } & 68 & \text { NP } & 88 & 223\end{array}$ & $\begin{array}{l}\text { +CONNOLLY, HART, RAUM, STONEHILL, + (BNL) IJPP } \\
\text { ARMENTEROS, BAILLON, + (CERN, HEIDEL, SACLAY) IJP } \\
\text { ARMENTEROS, BAILLON, + (CERN, HEIDEL, SACLAY IIJP }\end{array}$ \\
\hline $\begin{array}{l}\text { ARMENT-3 } 69 \text { LUND PAPER } 229 \\
\text { VALUES ARE QUDTED IN LEVI } \\
\text { ARMENT-4 } 69 \text { N } P \text { P B } 14 \quad 91 \\
\text { BERLEY } 69 \text { PL } 308 \quad 430 \\
\text { GALTIERI } 70 \text { DUKE } 173\end{array}$ & $\begin{array}{l}\text { ARMENTEROS, BAILLON, + (CERN, HEIDEL, SACLAY)IJP } \\
\text { SEIII G9. } \\
\text { ARMENTERDS, BAILLON, + (CERN, HEIDEL, SACLAY) IJP } \\
\text { + HART, RAHM, WILLIS, YAMAMOTO } \\
\text { A. BARBARO GALTIERI } \\
\text { (BNL) IJPP }\end{array}$ \\
\hline 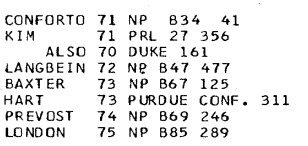 & 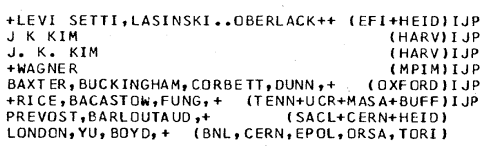 \\
\hline 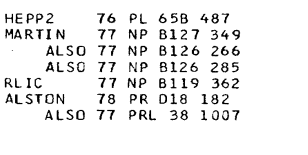 & 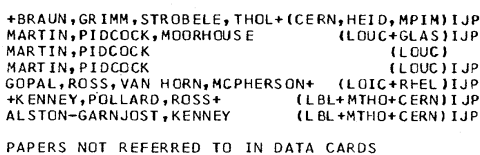 \\
\hline $\begin{array}{l}\text { BIRMINGH } 66 \text { PR } 1521148 \\
\text { LEVI IETT } 69 \text { LUND } 339\end{array}$ & $\begin{array}{l}\text { (BIRMINGHAM, GLASGOW, LOIC, OXF ORD, RUTHERFD) } \\
\text { RLEVI SETTI (RAPPORTEUR) } \\
\text { (CHICAGOO) }\end{array}$ \\
\hline
\end{tabular}

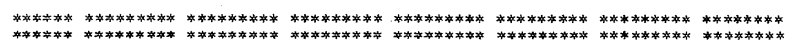

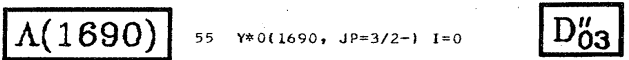

SEE THE MINI-REVUE AT THE START OF THE Y* LISIINGS.

THIS RESONANCE IS WELL ESTABLISHED.

$55 \%$ Y 11690$)$ MASS (MEV)
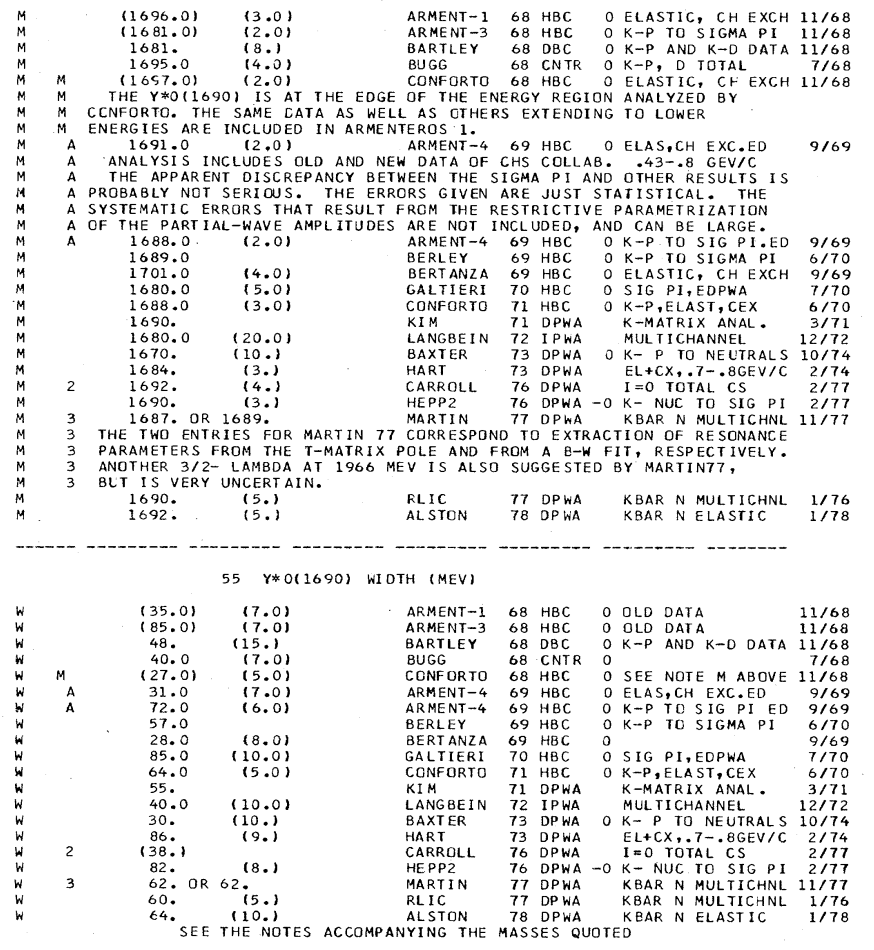

$55 Y * 0(1690)$ PARTIAL DECAY MODES
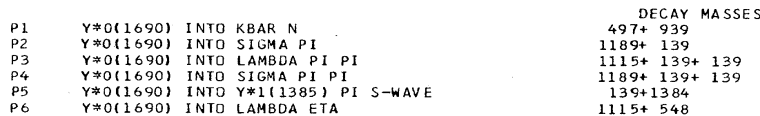

$55 Y$ Y $(1690)$ BRANCHING RATIOS

THE SUM OF ALL THE QUOTED BRANCHING RATIOS IS MORE THAN 1.0 . THE TWO-BODY RATIOS ARE FROM PART IAL WAVE ANALYSES, AND THUS PROBABLY AR
MORE REL ABLE THAN THE THREE-BDOY RATIOS, WHICH ARE DETERMINED FRCM
BUMPS IN CROSS SECTIONS. OF THE LATTER, THE SIGMA PI PI BUMP LOCKS BUPPS IN CROSS SECTIONS. OF THE LATTER, THE SIGMA PI PI BUMP LOCKS
MORE SIGNIFICANT (THE ERROR GIVEN FOR THE LAMEA PI PI RAT IO LOOKS UNREASONABLY SMALL). HARDLY ANY OF THE SIGMA PI PI DECAY CAN BE VIA
Y*1(1385), FOR THEN NINE TIMES AS MUCH LAMBDA PI PI DECAY WOULD BE

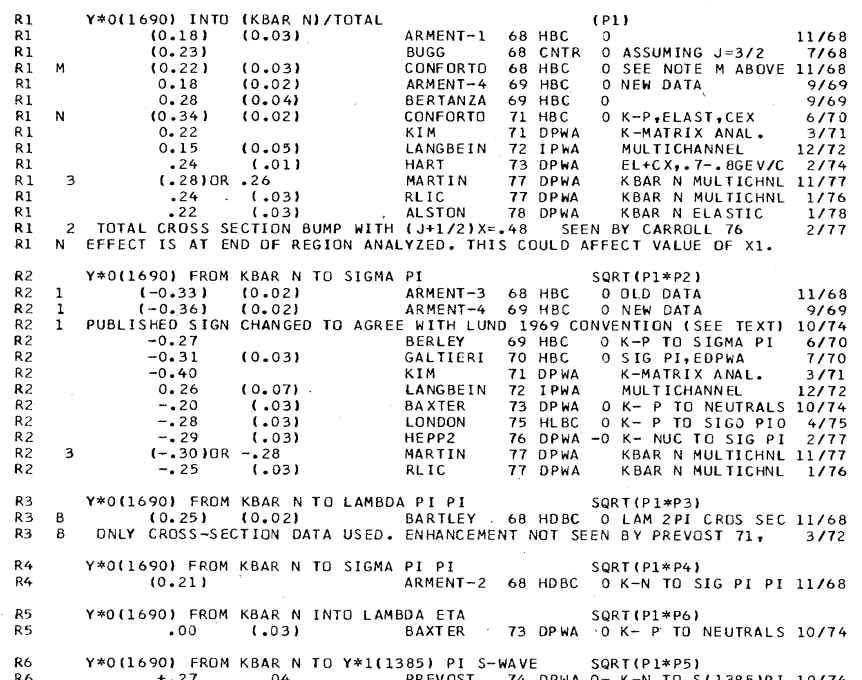

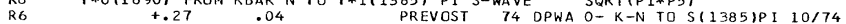

REFERENCES FOR Y*0(1690)

$\begin{array}{lllll}\text { ARMENT- } & 68 & \text { NP } & 88 & 195 \\ \text { ARMENT-2 } & 68 & \text { NP } & 88 & 216 \\ \text { ARMENT-3 } & 68 & \text { NP } & \text { B } & 223\end{array}$

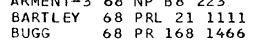

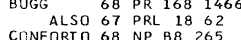
ARMENTEROS, BAILLON, + ICERN, HEIDEL, SACLAYIIJP ARMENTEROS, BAILLON, + (CERN, HEIDEL, SACLAYI I (BIRM, CAVE, RHEL) (BIRM, CAVE, RHEL) I
DAVIES, DOWELL, + $\begin{array}{lllll}\text { ARMENT- } & 69 & \text { NP } & 814 & 91 \\ \text { BERLEY } & 69 & P L & 308 & 430 \\ \text { BERTANZA } & 69 & P R & 177 & 2036\end{array}$ ARMENTEROS, BAILLON, + (CERN, HEI DEL, SACLAY) IJP
P HART, RAHM, WLLIS, YAMAMOTO
+ HIGI, CARRARA, CASALI, +
+PISA, BNL, YANL) IJP IJP GALTIERI 70 DUKE 173
CONFORTO 71 NP $\begin{array}{lll} & \\ \text { KIM ALSO } 71 & \text { PRL } 27356 \\ \text { DUKE } 161\end{array}$

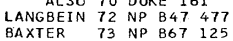
$\begin{array}{lll}\text { HART } & 73 & \text { PURDUE CONF. } 311 \\ \text { PREVOST } & 74 & \text { NP } \\ \text { PR9 } & 246\end{array}$ $\begin{array}{lllll}\text { LONOON } & 75 & \text { NP } & B 85 & 289 \\ \text { CARROLL } & 76 & \text { PRL } & 37 & 806 \\ \text { HEPP2 } & 76 & \text { PL } & 65 B & 487\end{array}$ A. BARBARO GALTIER I
+ LEVII SETTI, LASI NSKI .. OBBERLACK++ (EFI I HEIO) IJJP J K KIM
J K K KIM +WAGNER
BAXIER, BUCKINGHAM, CORBETT, DUNN, + (MPIM) IJP
(OXFORO)IJP +RICE, BACASTOW FUNG, + (TENN+UCR+MASA+BUFF) IJP
PREVOST, BARLOUTAUD,
(SACL+CERN+HEID) LONDON, YU, BOYD, + (BNL, , CERN, EPCL, ORSA, TCRI) +CHIANG, KYCIA, LI, MAZUR, MICHAELL, ORSA, (BNL II
+ BRAUN, GRI MM, STROBELE, THOL + (CERN, HEID, MPIM) IJP MARTIN 77 NP B127 349

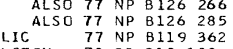
MART IN, PIDCOCK, MOORHOUSE (LDUC+GLAS)IJPP MARTIN, PIDCOCK
MARTIN, PIDCOCK
(LOUC) MARTIN, PIDCOCK
GDPAL, ROSS, VAN HORN, MCPHERSON+ (LOUCIIJP

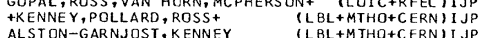

PAPERS NOT REFERRED TO IN DATA CARDS $\begin{array}{llllll}\text { ALSO } & 78 & \text { PR } & 118 & 182 \\ \text { ALST } & \text { PRL } & 38 & 1007\end{array}$

PREVOST 71 aMSTERDAM CONF + CHS COLLABORATION (CERN+HEID+SACL)

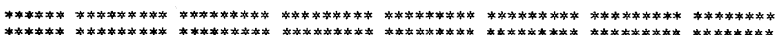
$\Lambda(1800){ }_{36}^{36} \gamma^{*} 0\left(1800\right.$, pp=1/2-1 $1=0 \quad S_{01}^{\prime \prime \prime}$ THE SO 1 AMPLITUDE SHOWS A RATHER CLEAR SECOND RESCNANCE
BEHAVIDR IN THE $1700-1900$ MEV REG ION. THERE ARE WIDE DI SAGREEMENTS AMON

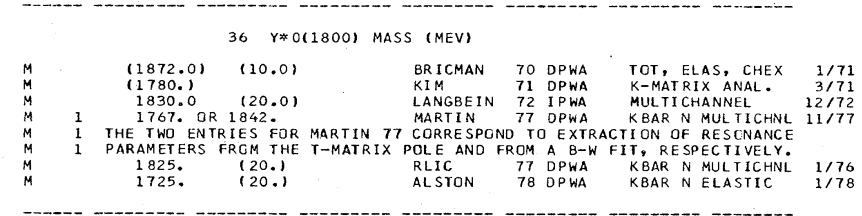




\section{Data Card Listings}

Baryons

For notation, see key at front of Listings. $\Lambda(1800)$

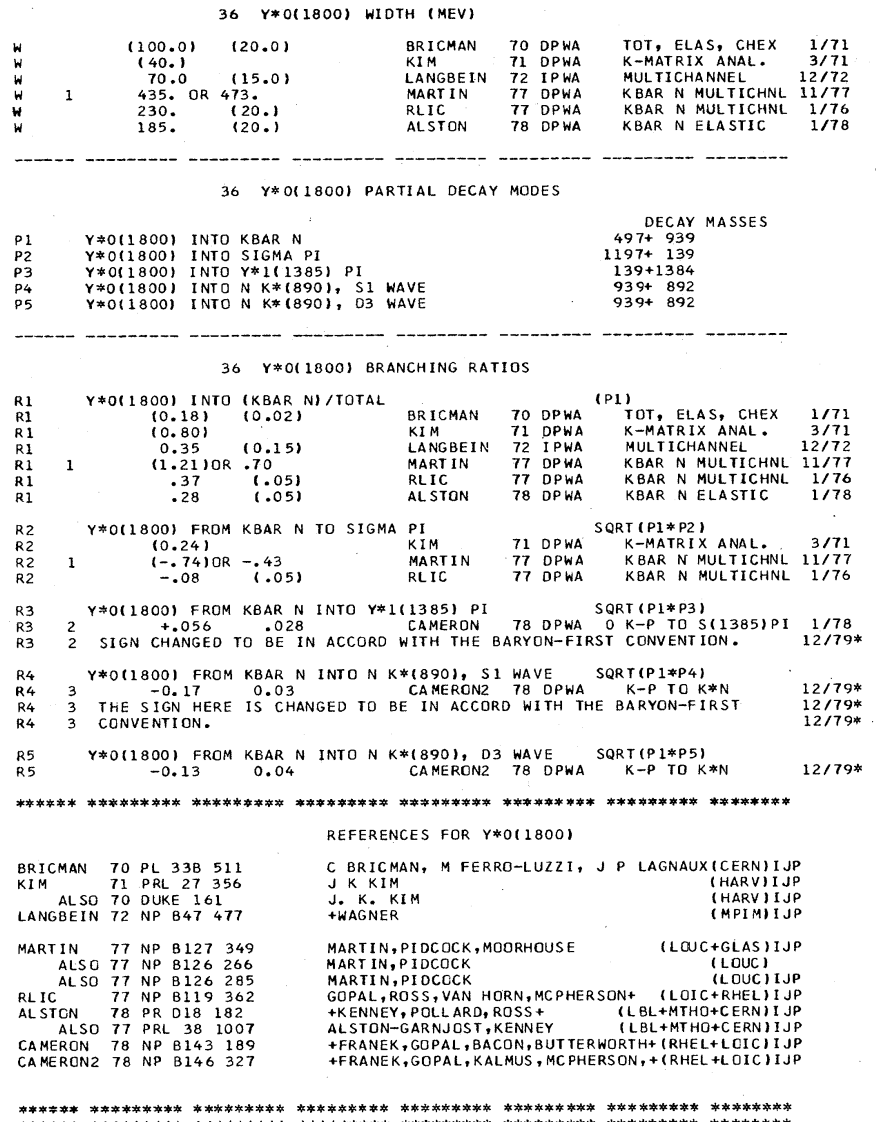

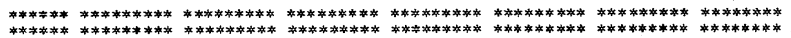

$\Lambda(1800){ }_{77}{ }_{Y * 0(1800, J P=1 / 2+1} \quad I=0 \quad P_{\text {O1 }}^{\prime \prime}$

SEe the Mini-Review at the start of the Y* Listings.

THE EVIDENCE FOR THIS STATE IS SOMEHHAT CONFUSED. IT

WAS FIRST SUGGESTED IN A PARTIAL WAVE ANALSIS OF
KBAR N DATA BY THE BEHAVIOUR OF THE POA AMPLIIUDE

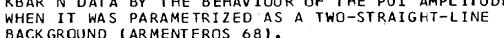

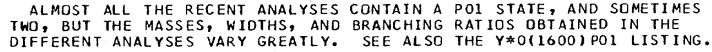

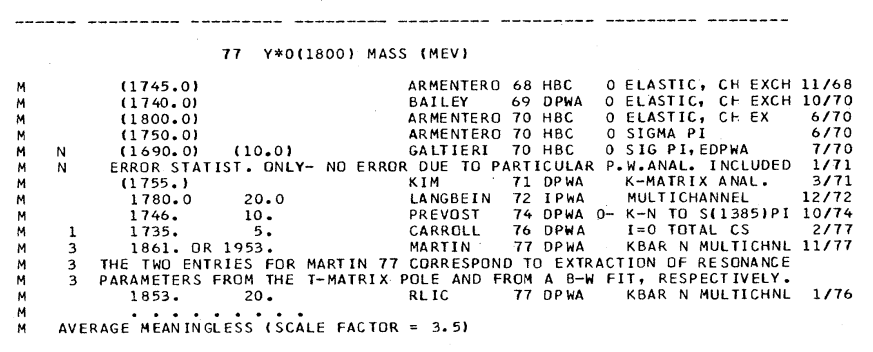

77 Y*0(1800) WIDTH (MEV)

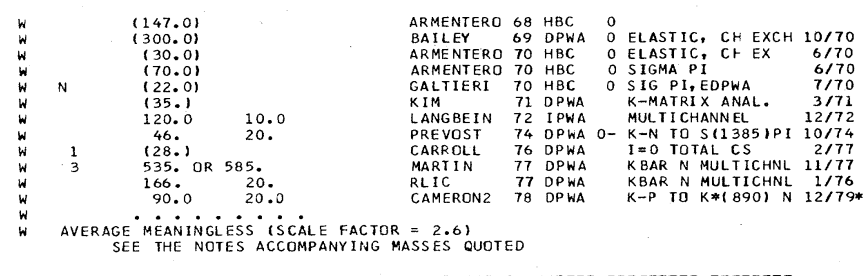

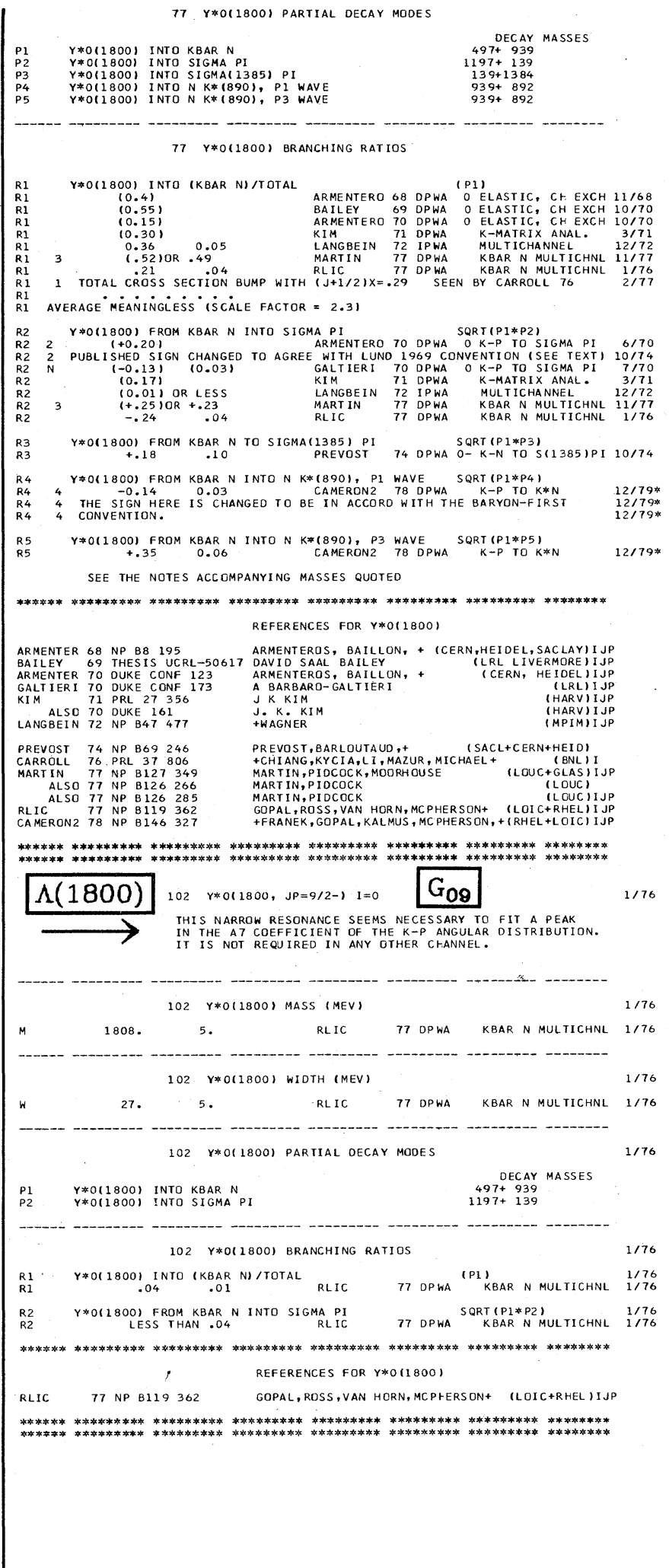


Baryons

$\Lambda(1800), \Lambda(1815)$
$\Lambda(1800) 119 \quad \gamma * 011800, J P=\quad$ I I=? PRODUCTION EXPER IMENTS BUMPS LOCKMAN 78 OBSERVE A 5 STD OEV. ENHANCEMENT IN THE PP $\rightarrow$ LAMBDA PIt PI- + ANYTHING IN A CERN ISR

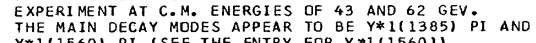

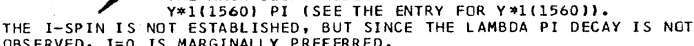

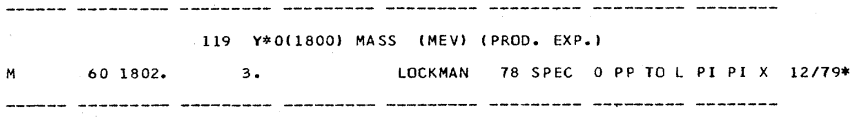

119 Y*O(1800) WIDTH (MEV) (PROD. EXP.)

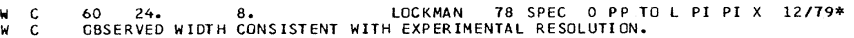
119 Y*o(1800) partial deCAY MODES (PROD. EXP.

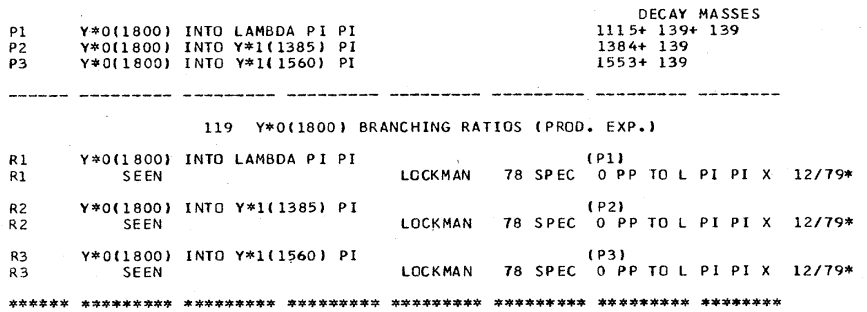

REFERENCES FOR $Y * O(1800)$ (PROD. EXP.)

LOCKMAN 78 CEN DPHPE 78-01 TMEYER, RANOER, POSTER, SCHLEIN+ (UCLA+SACL)

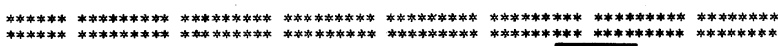
$\Lambda(1815)$ 39 $\quad$ Y*0(1815, JP $=5 / 2+1$ I=0 $\quad F_{05}^{\prime}$ SEE THE MIN1-REVIEW AT THE START OF THE Y* LISTINGS. THIS STATE IS WELL ESTABLISHED. MOST OF THE QUOTED ER-
RORS ARE STATISTICAL ONLY. THE SY STEMATIC ERRORS DUE TO NOT INCLUDED. FOR THIS REASON WE DO NGT CALCULATE WEIGHTED AVERAGES FOR MASS AND WIDTH

$39 \quad \mathrm{Y} * 0(1815$ ) MASS (MEV)
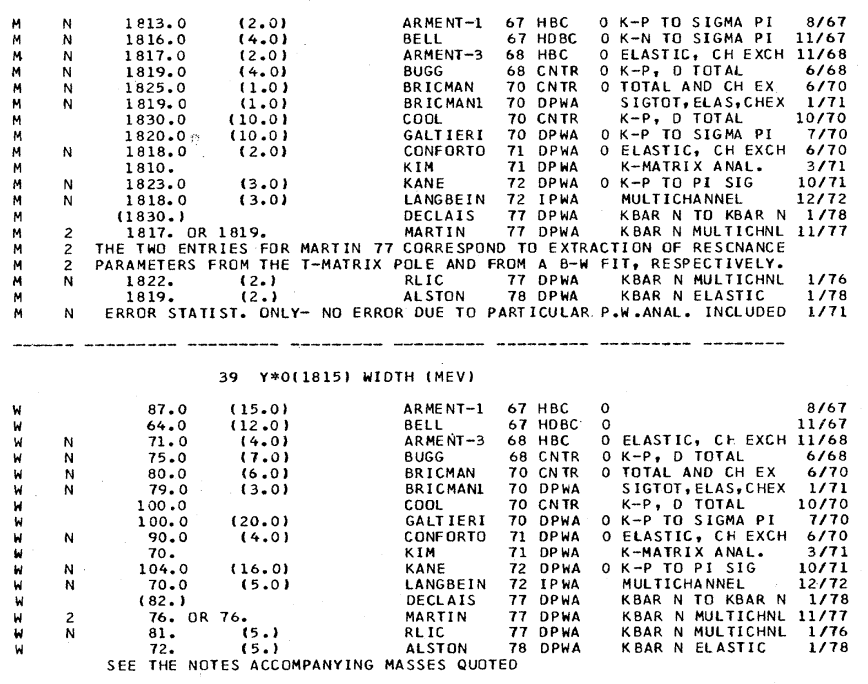

39 Y*011815) PARTIAL DECAY MODES

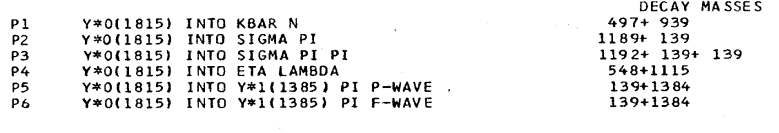

Data Card Listings For notation, see key at front of Listings.

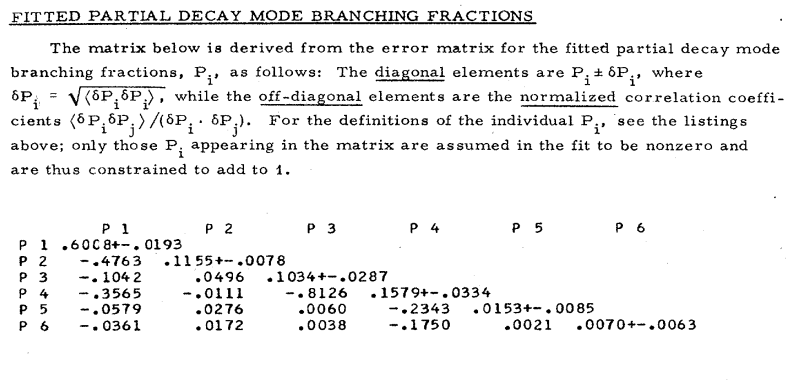

$39 Y * 0(1815)$ BRANCHING RATIOS

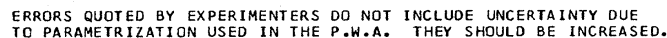

$Y * 0(1815)$ INTO (KBAR NI/TOTAL

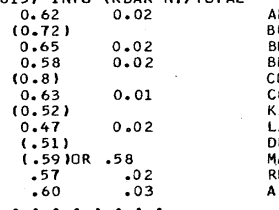

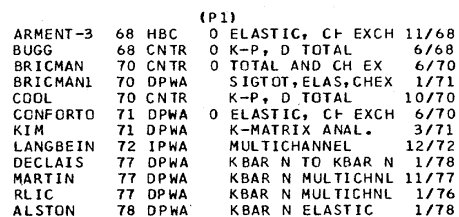

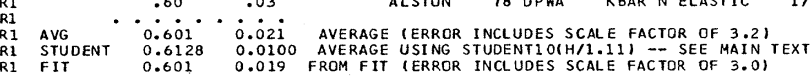

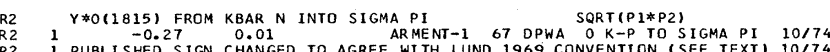

2 I PUBLISHED SIGN CHANGED TO AGREE WITH LUND 1969 CONVENTION (SEE TEXT) 10/74

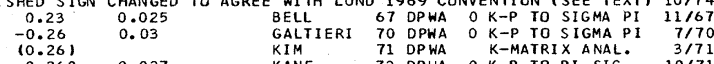

$\begin{array}{ll}-0.268 & 0.027 \\ -0.25 & 0.03 \\ 0.25108 & -.25\end{array}$

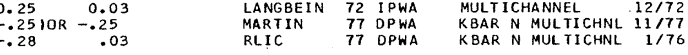

AVG MOD $\because 2645^{\circ} \cdot 0.0078$ AVERAGE IERROR INCLUDES SCALE FAC TOR OF 1.01

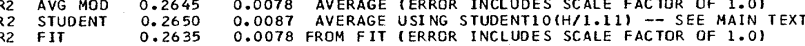

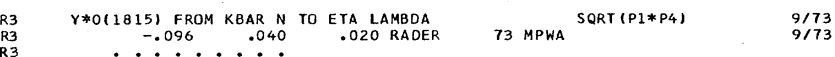

R3 FIT $\because 0.096^{\circ} \cdot 0.027$ FROM FIT (ERROR INCLUDES SCALE FACTOR OF 1.0 )

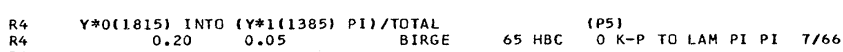

$R 4$
$R 4$ FIT $\because 0.103 \cdot 0.029$ FROM FIT (ERROR INCLUDES SCALE FACTOR OF 1.5 )

R5
R5 P
POO(1815) INTO (SIGMA PI PI)/TOTAL
NO CLEAR SIGNAL PI

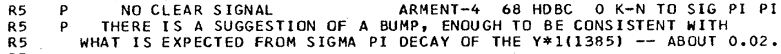

R5 FIT - O.158 0.033 FROM FIT

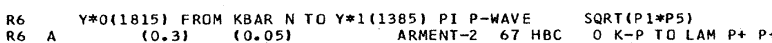

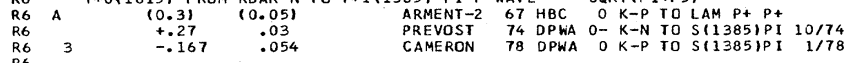

R6 AVG MOO $0.246^{\circ} \cdot \dot{0} 04^{\circ}$ aVERAGE (ERROR INCLUDES SCALE FaC TOR OF 1.7 )

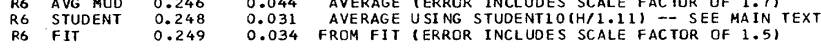

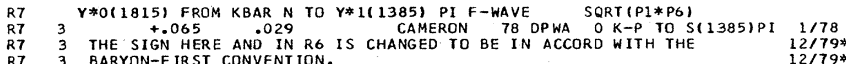

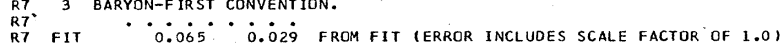

\#*****************************************************************1 REFERENCES FOR $Y * 0(1815)$

BIRGE 65 ATHENS CONF 296 +ELY, KALMUS, KERNAN, LOUI E, SAHOURIA, + ILRLI JP ARMENT-1 67 PL 248198
ARMENT-2 67 ZEIT PHY 202486 ARMENTEROS, F LUZZI, + (CERN,HEIDEL, SACLAYIIJP
ARMENTEROS, F LUZZI, + (CERN, HEIDEL, SACLAYIIJP BELL 67 PRL 19936
ARMENT-3 68 NP B8 195

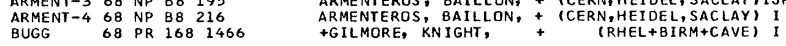

BRICMAN 70 PL 318152 TFERRO LUZZI, PERREAU, + (CERN, CAEN, SACLAY)

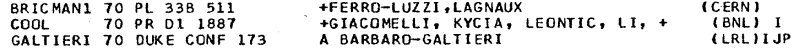

CCNFORTO 71 NP 83441
KIM 71 PRL 27356

ALSO 70 OUKE 161

(HARV)IJP

+ F KANERE

(MPIM)IJP 


\section{Data Card Listings}

For notation, see key at front of Listings.
Baryons $\Lambda(1815), \Lambda(1830), \Lambda(1860)$
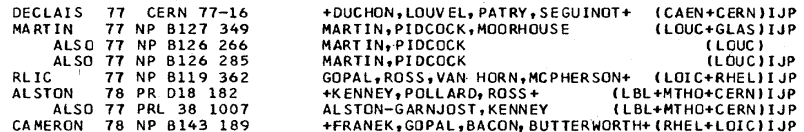

PAPERS NOT REFERRED TO IN DATA CARDS

THE FOLLOWING PAPERS ARE NOW OF ONLY HISTORICAL INTEREST -

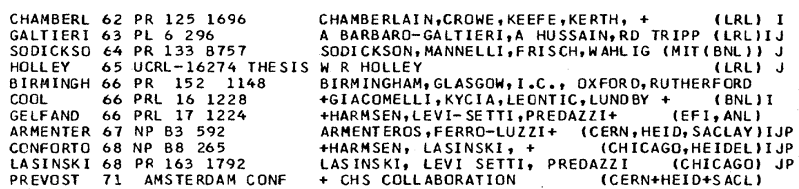

LASINSKI 68 PR 1631792 LASINS KI, LEVI SETII, PREDAZZI I (CHICAGO) JP
PREVOST 71 AMSTERDAM CONF + CHS COLLABORATION
(CERN+HEID+SACL)

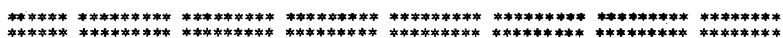

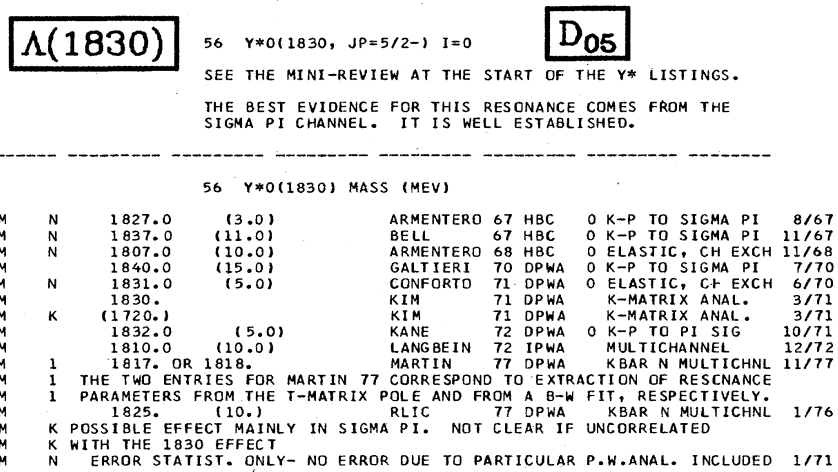

56 Y*0(1830) WIOTH (MEV)

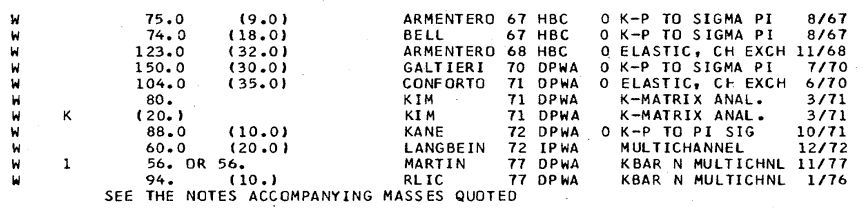

$56 \quad Y * 0(1830)$ PARTIAL DECAY MOOES

$\begin{array}{lll}P 1 & Y * 0(1830) \text { INTO KBAR N } & \text { DECAY MASSES } \\ \text { P2 } & Y * 0(1830) \text { INTO SIGMAPI } & 497+939 \\ \text { P3 } & Y * 0(1830) \text { INTO Y*111385) PI D-WAVE } & 1189+139 \\ \text { P4 } & Y * 0(1830) \text { INTO ETA LAMBDA } & 139+1384 \\ & & 548+1115\end{array}$

56 Y*0(1830) BRANCHING RATIOS
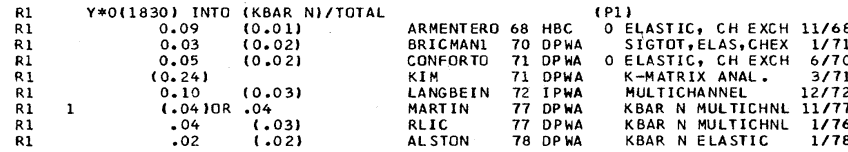

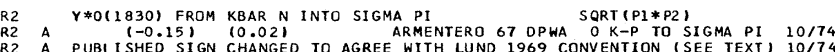

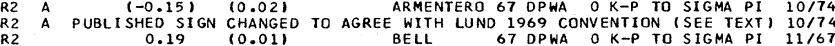

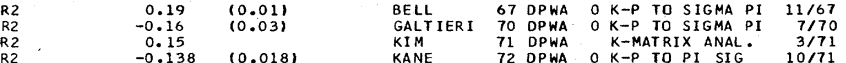

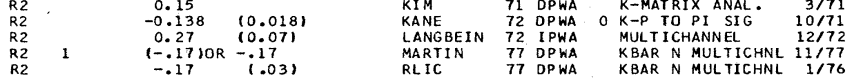

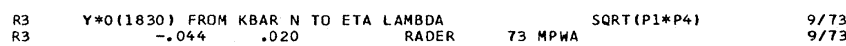

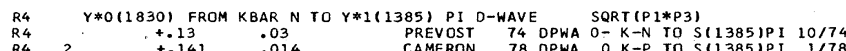

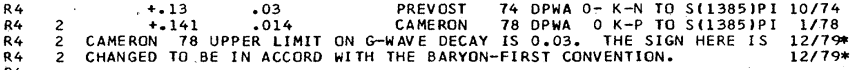
R4 AVERAGE MEANi NGLiESS i SCALL FACTOR $=1.01$

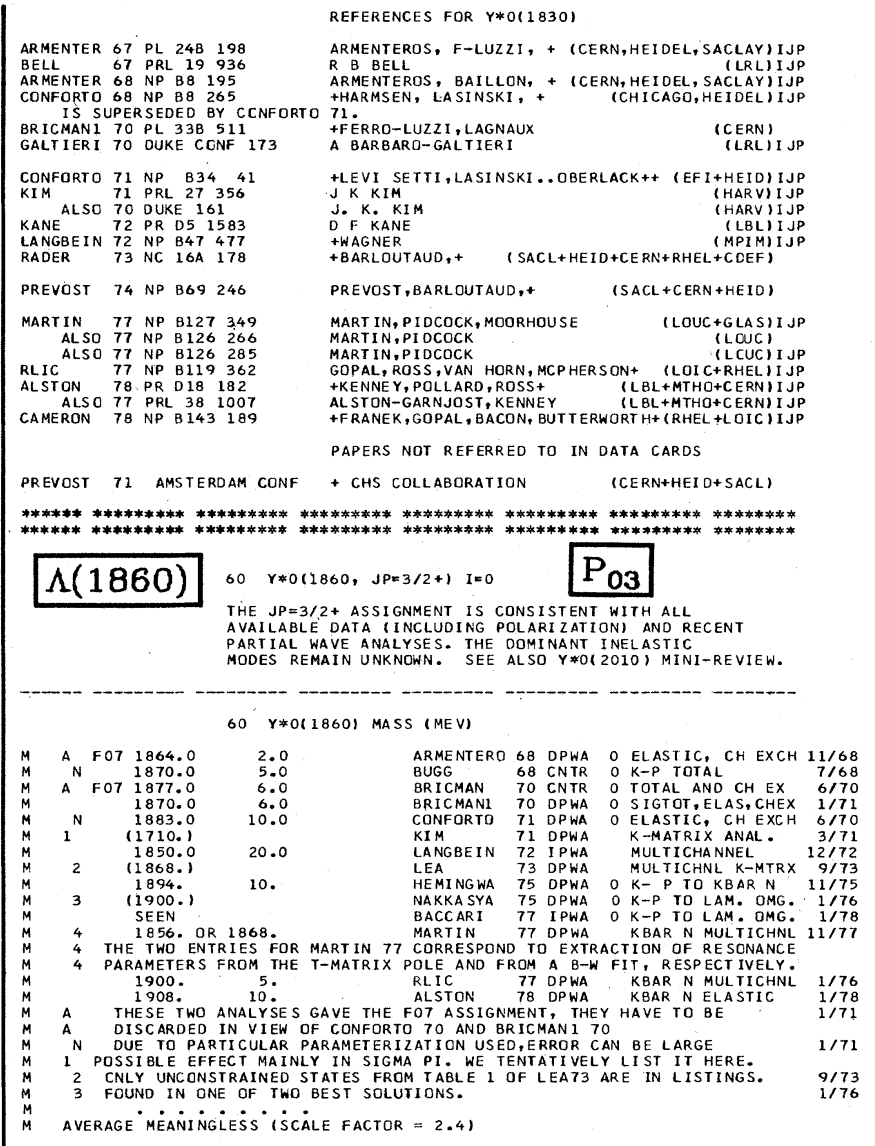

$60 \mathrm{Y} * 0(1860)$ WIOTH (MEV)

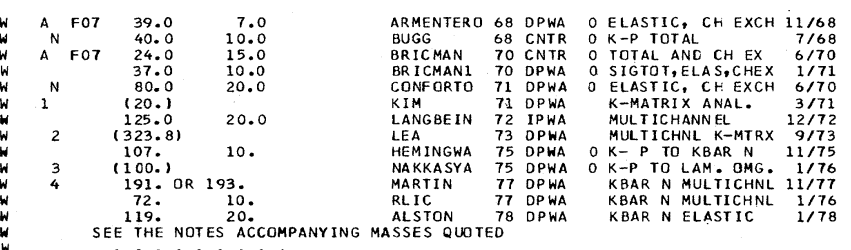

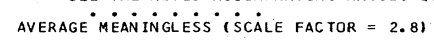

60 Y*0(1860) PARTIAL DECAY MODES

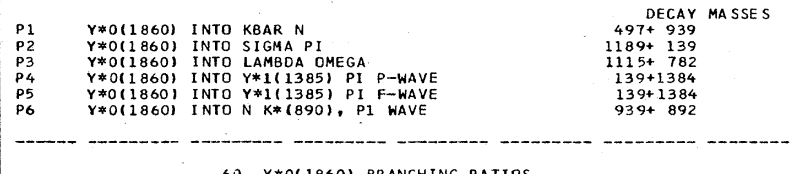

$60 \%$ (1860) BRANCHING RATIOS

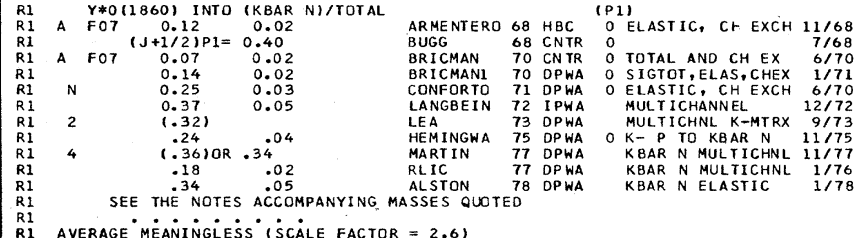




\section{Baryons}

$\Lambda(1860), \Lambda(2010), \Lambda(2020), \Lambda(2100)$

\section{Data Card Listings For notation, see key at front of Listings.}

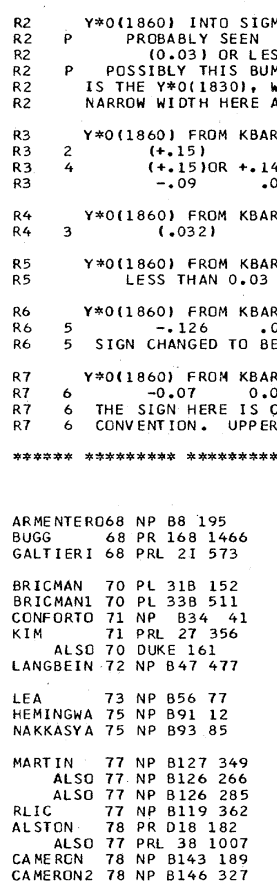

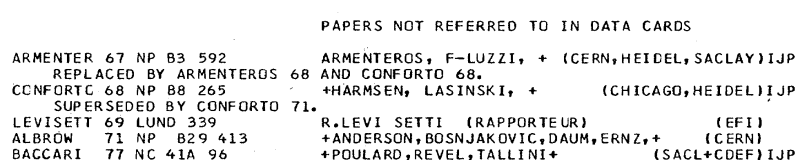

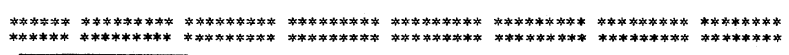

$\Lambda(2010)$ 89 $\quad$ Y*0<2010, II=0

SeE tre mini-review at the start of the Y* Listings. WE LIST HERE ALL THE AMB IGUOUS RESONANCE POSSIBLI TIES WITH A MASS AROUND 2 GEV THE PROPOSED QUANTMM NUMBERS
ARE D3 (GALIIERITO IN SIGMA PI), D3+F5, P3+05, OR

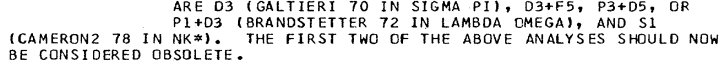

$$
89 \text { Y*0(2010) MASS (MEV) }
$$

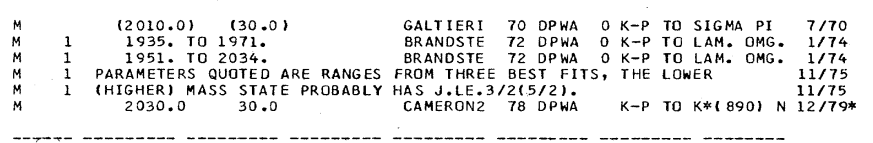

89 Y*0(2010) WIDTH (MEV)

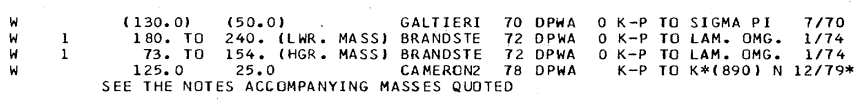

89 Y*0(2010) PARTIAL DECAY MODES

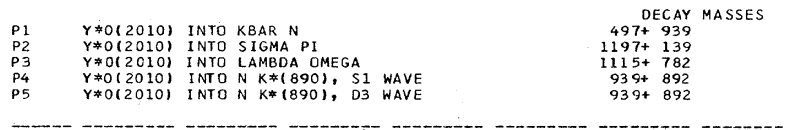

$89 Y * 0(2010)$ BRANCHING RATIOS

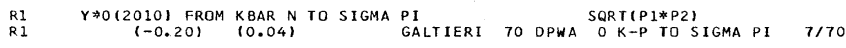

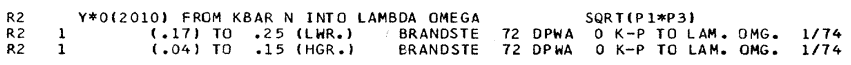

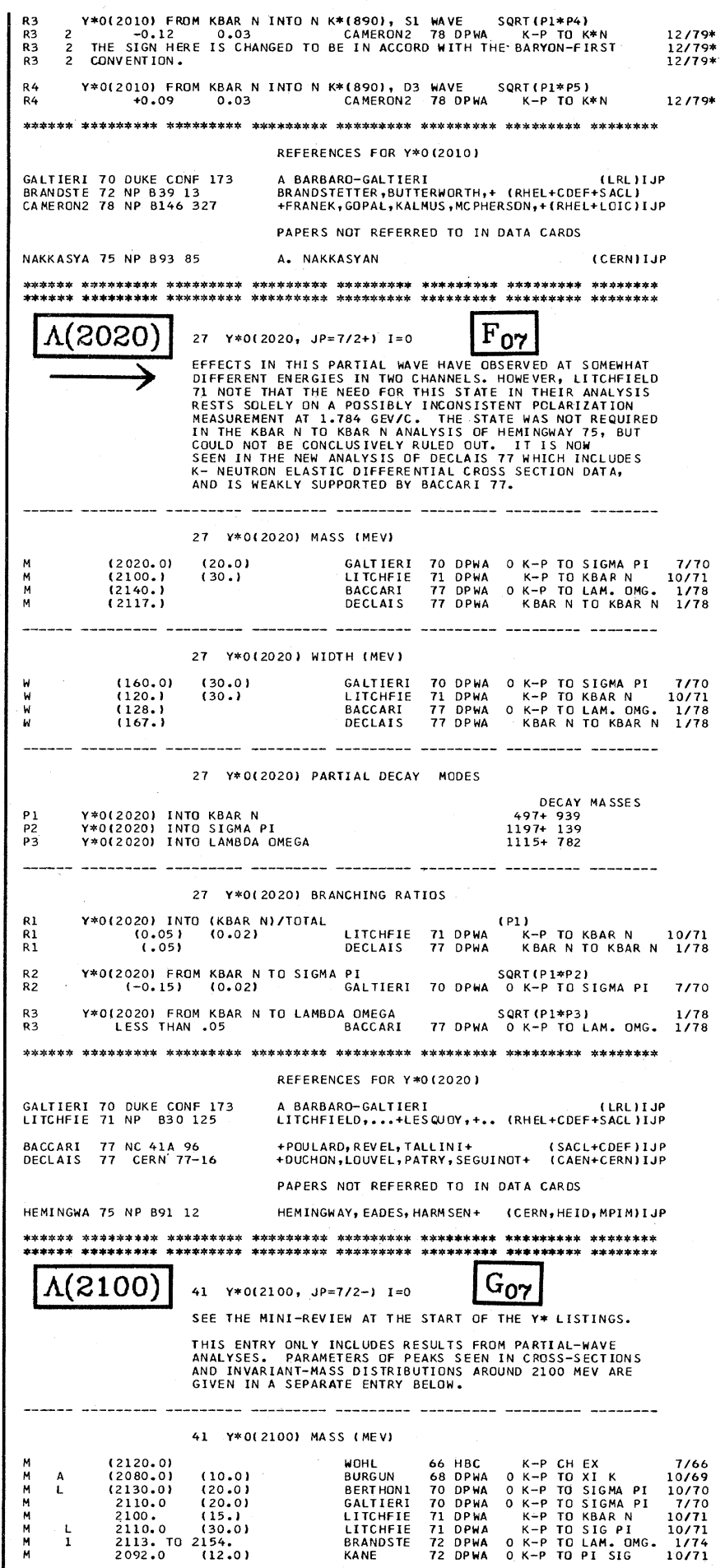




\section{Data Card Listings}

\section{Baryons $\Lambda(2100), \Lambda(2110)$}

For notation, see key at front of Listings.
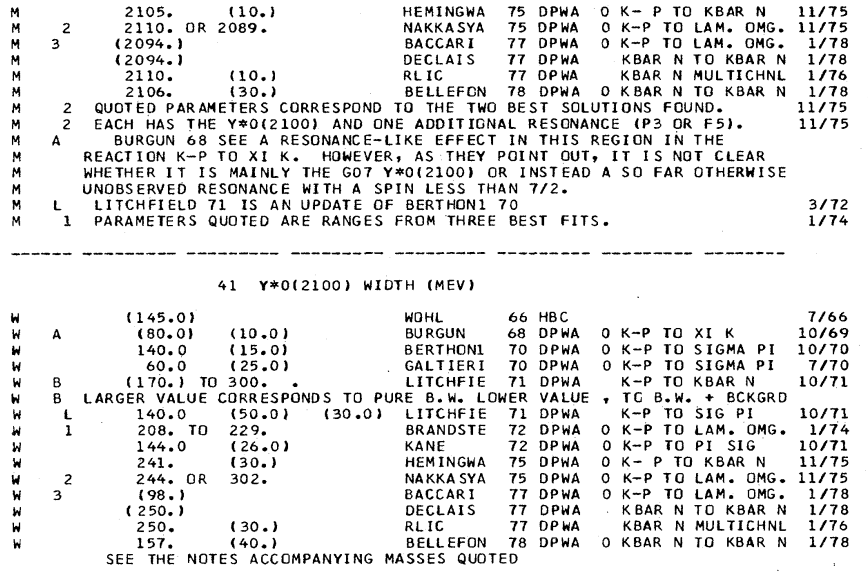

$41 Y * 0(2100)$ PARTIAL DECAY MODES

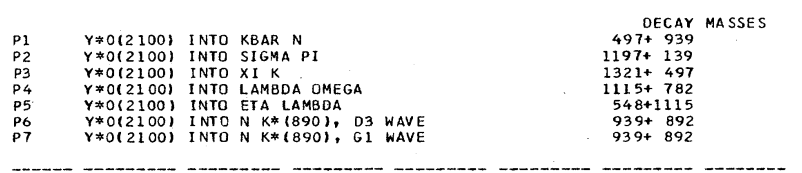

$41 Y * 0(2100)$ BRANCHING RATIOS

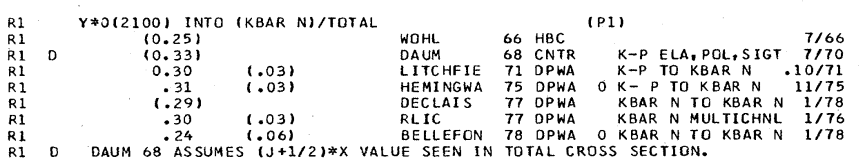

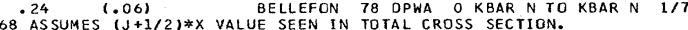

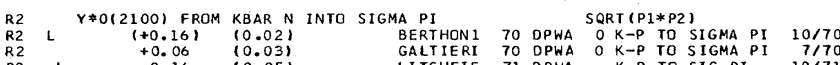

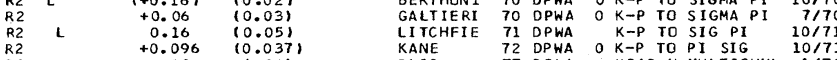

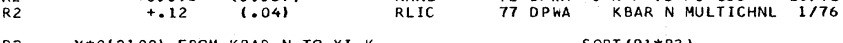

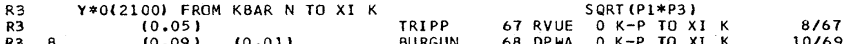

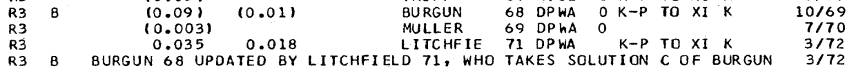

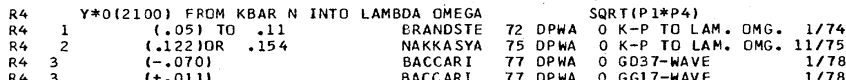

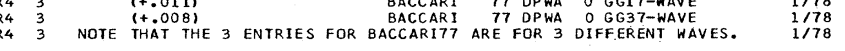

$\begin{array}{lcllll}R 5 & * 0(2100) \text { FROM KBAR N TO ETA LAMBDA } & & \text { SQRT(P1*P5) } & 9 / 73 \\ \text { R5 } & -.050 \quad .020 & \text { RADER } & 73 \text { MPWA } & 9 / 73\end{array}$

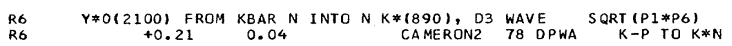

R7 Y*0(2100) FROM KBAR N INTON K*(890), G1 WAVE SQRT (P1*P7),

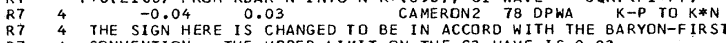
CONVENTION. THE UPPER LIMIT ON THE G3 WAVE

*****************************************************************

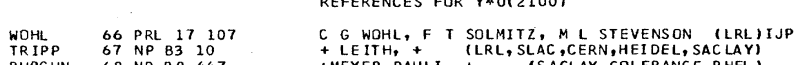
$\begin{array}{lll}\text { BURGUN } & 68 \text { NP B } 8.447 & \text { TMEYER, PAULI, } \\ \text { DAUM } & 68 \text { NP } 87.19 & \text { (SACLAY, COLFRANCE, RHEL) }\end{array}$ DAUM CONFIRMS THE SPIN-PARITY ASS TGNMENT.
MULLER O9 THESIS, UCRL 19372 R A MULLER

(LRL)

BERTHON1 70 NP B24 417
GALTIERI 70 DUKE CONF 173
LITCHFIE 71 NP B30 125

+VRANA, BUTTERWORTH, + (COEF, RHEL, SACLAY)IJP

$\begin{array}{lllll}\text { BRANOSTE } & 72 & \text { NP } & 839 & 13 \\ \text { KANE } & 72 & \text { PR } & 05 & 1583\end{array}$

CITCHFIELD, ...+LESQUOY, +... (RHEL+CDEF+ SACL)I JP

BRANOSTETTER+... + TALLINI (RHEL, CDEF, SACL) IJ I I I I
D F KANE

+Barloutaud, , (SACl+HEID+CERN+RHEL+CDEF)

$\begin{array}{lllll}\text { HEMINGWA } & 75 & \text { NP } & B 91 & 12 \\ \text { NAKKASYA } & 75 & \text { NP } & 893 & 85\end{array}$

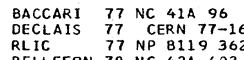

$\begin{array}{lllll}\text { RLIC } & 77 & \text { NP } & 8119 & 362 \\ \text { BELLEFN } 78 & \text { NC } & 42 A & 403 \\ \text { CAMERON2 } & 78 & \text { NP } & \text { B146 } 327\end{array}$

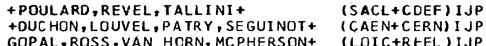

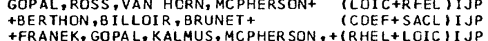

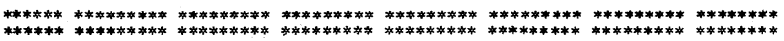

$\Lambda(2110){ }_{35}{ }^{2}{ }^{\prime} 0(2110, j p=5 / 2+1) \quad \mathrm{I}=0 \quad \mathrm{~F}_{05}^{\prime \prime}$

BERTHON1 70 FIND EITHER FOS OR DOS POSS IBLE IN
THE SIG PI CHANNEL, WITH FO5 SLIGHTLY PREFERRED. THE SIG PI CHANNEL, WITH FO5 SLIGHTLY PREFERRED.
IN THE KBAR N CHANNEL, LITCHFIELDTI ISAME GROUP FIND DNLY DO5. AS USUAL, THE STATISTICS ARE MUCH
BETIER IN THE ELASTIC CHANEL. BETTER IN THE ELASTIC CHANNEL. THE UNUSUALLY BROAD
ALTHOUGH KANE 72 FINDS AN FOS EFFECT; THE UNST WIDTH MAY INVALIDATE A RESONANT INTERPRETATION. HOWEVER RLY C 77
BELLEFON 77 , AND BELLEON 78 ALSO FIND AN FO5. THE EVIDENCE FOR FO5 FROM THE LAMBDA OMEGA ANALYSES, NAKKASYAN 75 AND BACCARI 77 IS QUITE WEAK, BUT THEY GIVE NO EVIDENCE IN FAVOR OF D05. THE
WEIGHT OF THE EVIDENCE IS THUS IN FAVOR OF FOS. SEE ALSO THE $\checkmark * 0(2010)$ MINI-REVIEW.

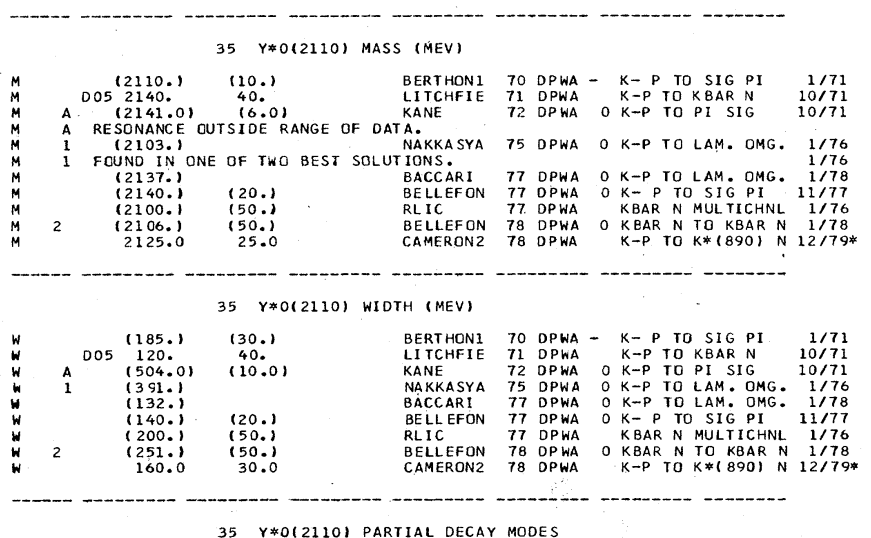

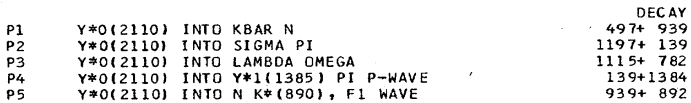

$35 Y * O(2110)$ BRANCMING RATIOS

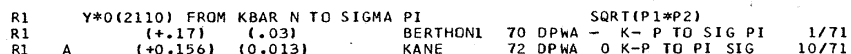

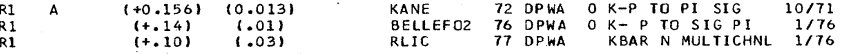

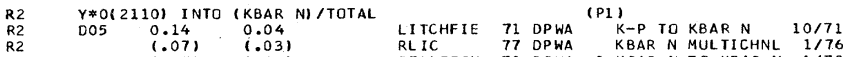

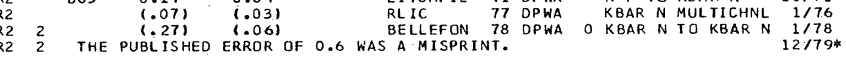

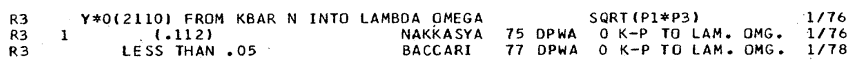

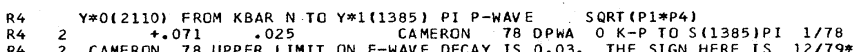

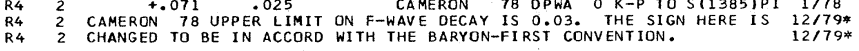

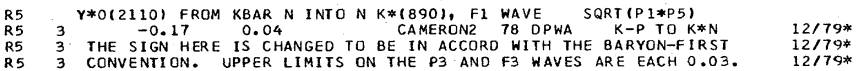
******************************************************************** REFERENCES FOR $Y * 0(2110)$

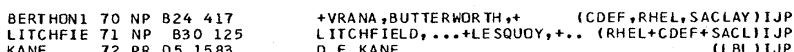
NAKKASYA 75 NP B93 85
(CERN)IJP

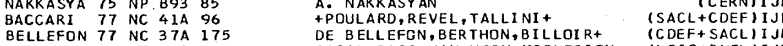

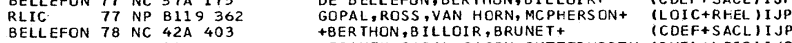

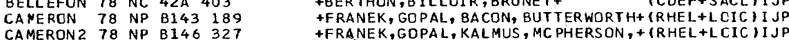




\section{Baryons}

$\Lambda(2110), \Lambda(2325), \Lambda(2350)$

2100 MEV REGION - PRODUCTION AND $\sigma_{\text {TOTAL EXP'TS }}$ $25 \mathrm{Y} * 012100, J P=, I=0 \quad$ PRODUCT ION EXPERIMENTS SEE THE MiNI-REviEW at THE START OF THE Y* Listings.

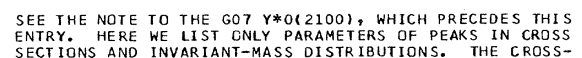
SECTION PEAKS ARE AT LEAST DOMINANTLY ASSOCIATED WITH THE Y*OL 2100$)$.
BUT MAY CONTAIN A SMALL CONTIBUTION FROM THE SUGGESTED BUT NOT ESTABTESONANCES IN THIS REGION.

25 Y*O(2100) MASS (MEV) (PROD. EXP.)

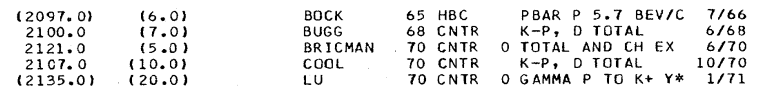
25 Y*0(2100) WIDTH (MEV) (PROD. EXP.)

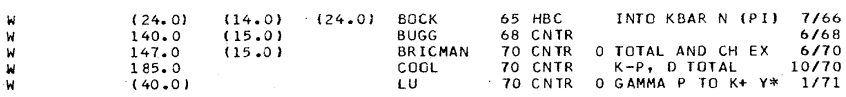

25 Y*0(2100) PARTIAL DECAY MODES (PROO. EXP.)

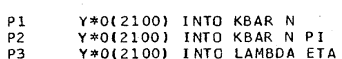

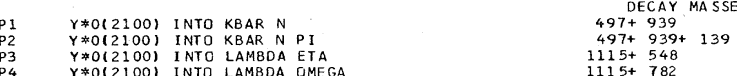

$R 1$
$R 1$
$R 1$
$R 1$
$R 1$
$R 2$
$R 2$
$R 2$
$R 3$
$R 3$
$R 3$
$R 4$
$R 4$

25 Y*O(2100) BRANCHING RATIOS (PROD. EXP.)

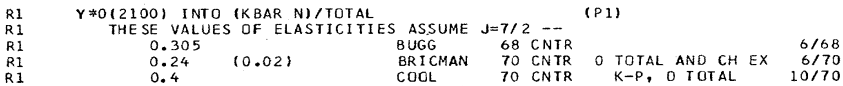

R2
R2 *012100) $\begin{aligned} & \text { INTO KBAR N PI } \\ & \text { SEEN }\end{aligned}$

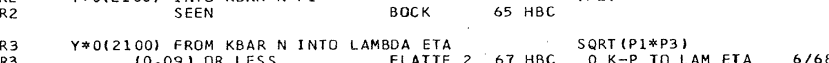

R3
$R 30(2100)$ FROM KBAR N INTO LAMBDA ETA
$0.09)$ FR LESS

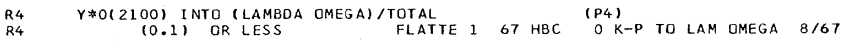
************************************************************************1 REFERENCES FOR Y*0(2100) (PROD. EXP.)

+ CDOPER, FR ENCH, KINSON, + (CERN, SACLAY)

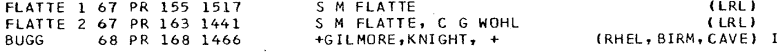

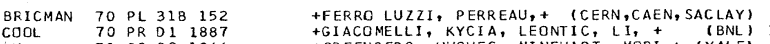

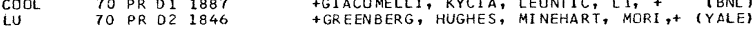
PAPERS NOT REFERRED TO IN DATA CARDS

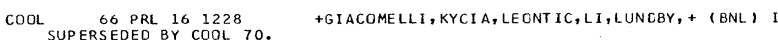

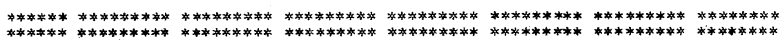

$\Lambda(2325){ }_{112} \quad Y * 0\left(2325, j \rho=3 / 2-1 \quad\right.$ I $=0 \quad D_{03}^{\prime \prime}$

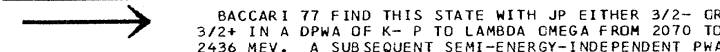
FROM THRES HOLD TO 2436 MEV SELECTS $3 / 2-$ S. SELEFCN 78 (SAME GROP) ALSO SEE THS SATE IN A
PWA OF K- P ELASTIC AND CHARGEEXXHANGE DATA IN THE SAME ENERGY RANGE, ANO FIND JPE $3 / 2-$ OR $3 / 2+$ OF MODEL DEPENDENT CONSIDERATIONS.

\section{$112 \quad Y * 0(2325)$ MASS (MEV)}

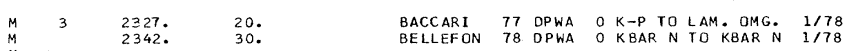
M average Meaningiés is iscale factor $=1.01$

112 Y*O(2325) WIDTH (MEV)

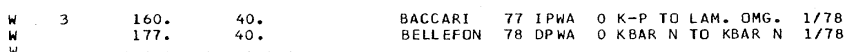

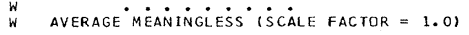

$112 Y * 0(2325)$ PARTIAL DECAY MODES

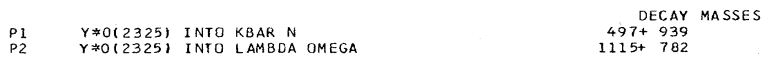

Data Card Listings For notation, see key at front of Listings.
$112 \gamma * 0(2325)$ BRANCHING RATIOS

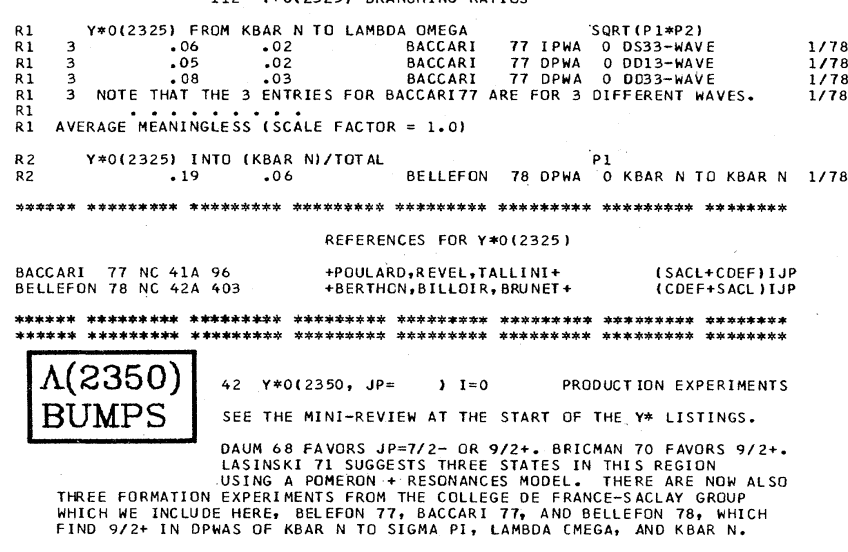

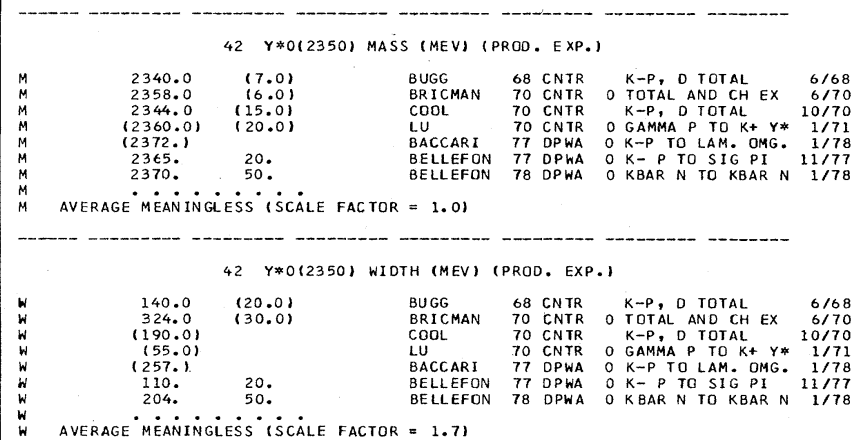

$42 Y * 0(2350)$ PARTIAL DECAY MODES (PROD. EXP.)

\begin{tabular}{llll} 
& & \\
P1 & $Y * 0(2350)$ INTO KBAR N & DECAY MASSES \\
P2 & $Y * 0(2350)$ INTO SIGMA PI & $497+939$ \\
P3 & $Y * 0(2350)$ INTO LAMBDA OMEGA & $1197+739$ \\
& & $1115+782$ \\
\hline
\end{tabular} $42^{\circ} Y$ Y $(2350)$ BRANCHING RATIOS (PROD. EXP.)

$\begin{array}{llllll}R 1 & Y * 0(2350) \\ R 1 & .12 & .04 & .12 & \end{array}$

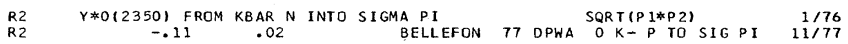

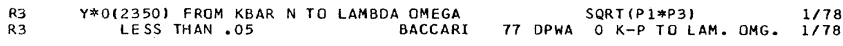
$R 4$
$R * 0(2350)$ INTO IKBAR NI/TOTAL
$R 4$
$R 4$

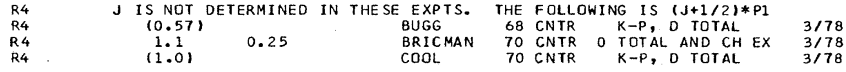
******************************************************************************1 $\begin{array}{lll}\text { BUGG } & 68 \text { PR } 1681466 & \text { +GILMORE, KNIGHT, + } \\ \text { DAUM } & 68 \text { NP B } 19 & \text { +ERNE, LAGNAUX, SENS, STEUER, UDO BRM, CAVE) I I } \\ \text { (CERN) JP }\end{array}$

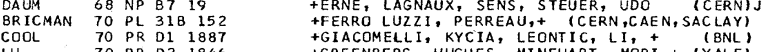
+GREENBERG, HUGHES, MINEHART, MORI, + (YALE)

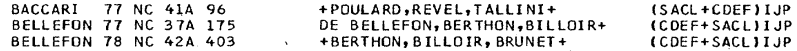
PAPERS NOT REFERRED TO IN OATA CARDS
COOL 66 PRL 161228 +GIACOMELLI, KYCIA, LE ONT IC, LI, LUNDBY, + (BNL) I

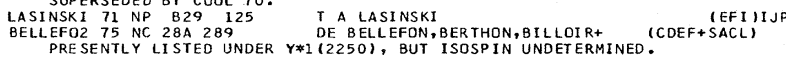

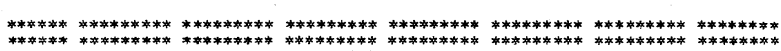




\section{Data Card Listings} For notation, see key at front of Listings.
Baryons $\Lambda(2585), \Sigma^{+}, \Sigma^{-}, \Sigma^{0}, \Sigma(1385)$

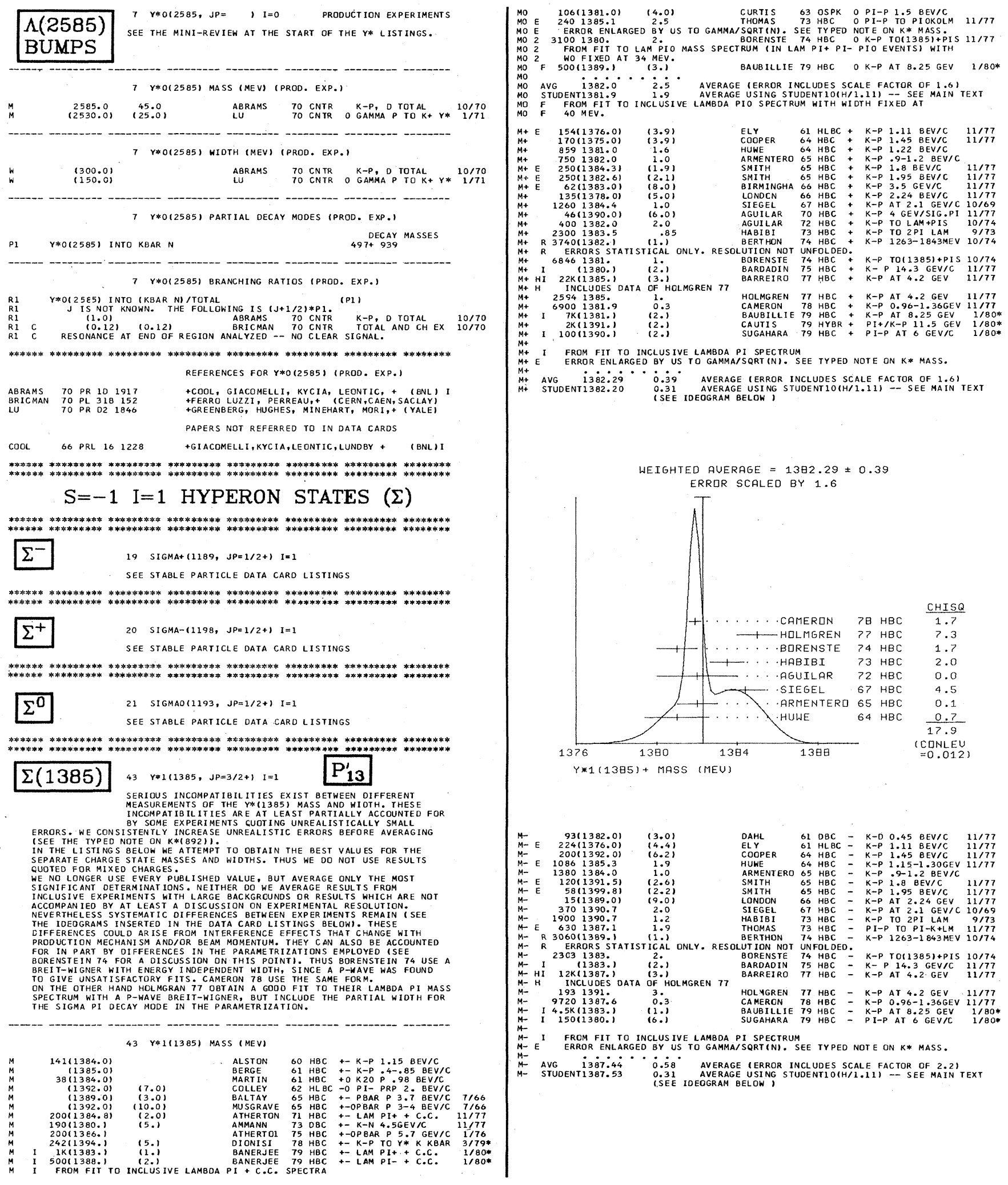




\section{Baryons $\Sigma(1385)$}

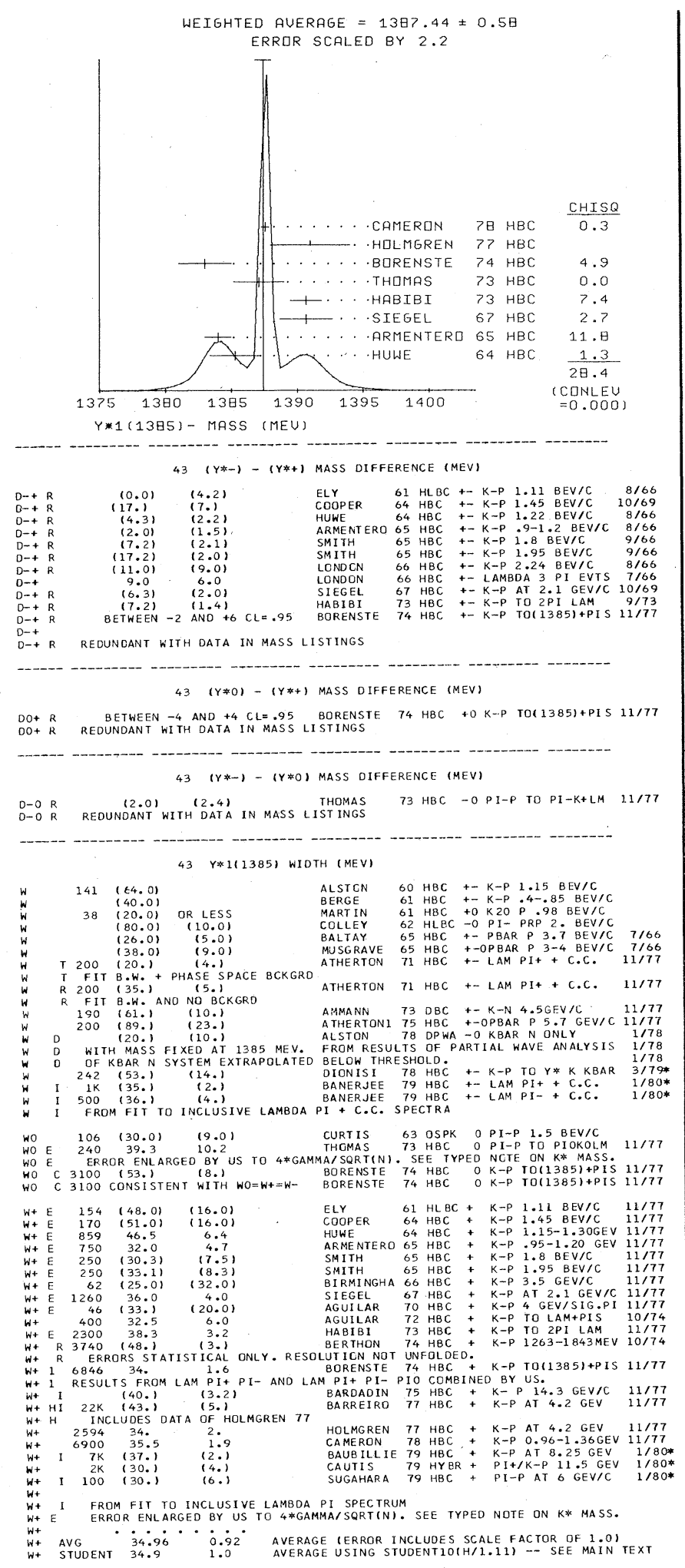

\section{Data Card Listings For notation, see key at front of Listings.}

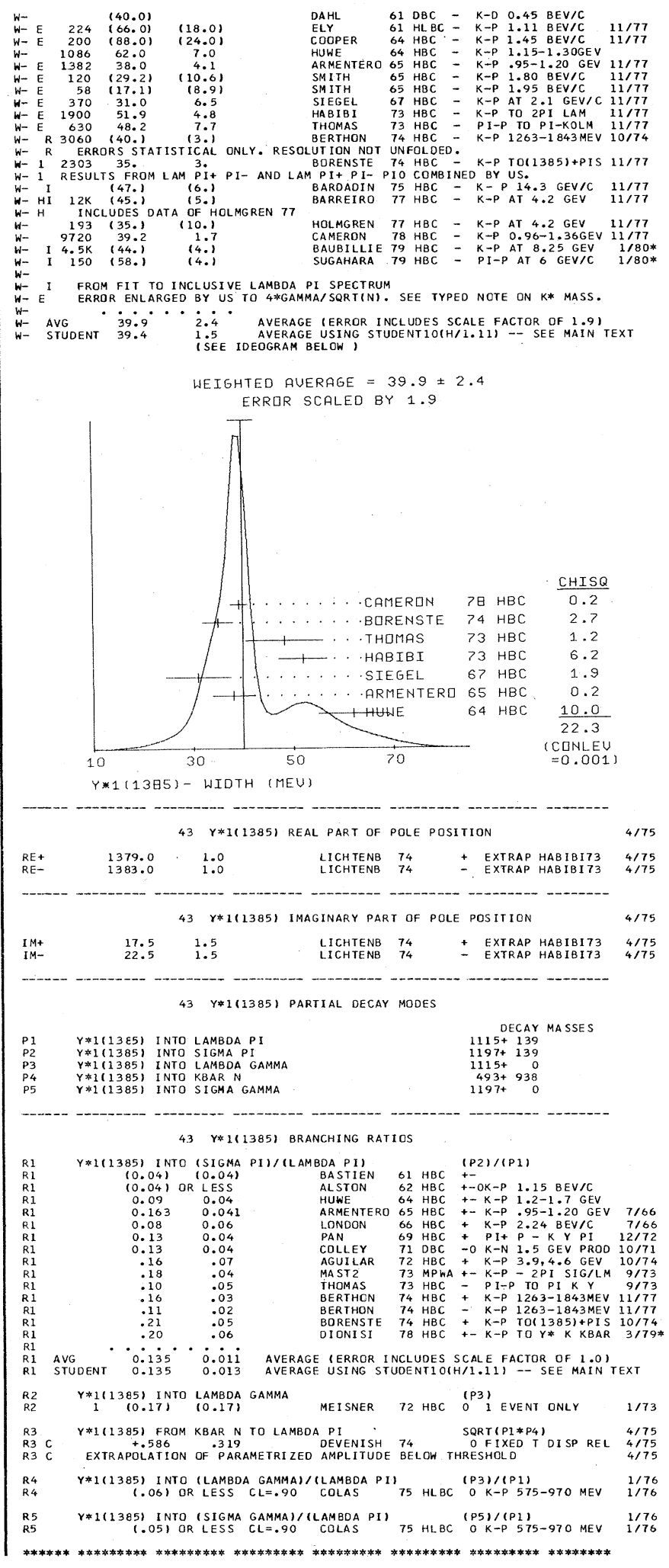




\section{Data Card Listings}

For notation, see key at front of Listings.

Baryons

$\Sigma(1385), \Sigma(1480), \Sigma(1560), \Sigma(1580)$

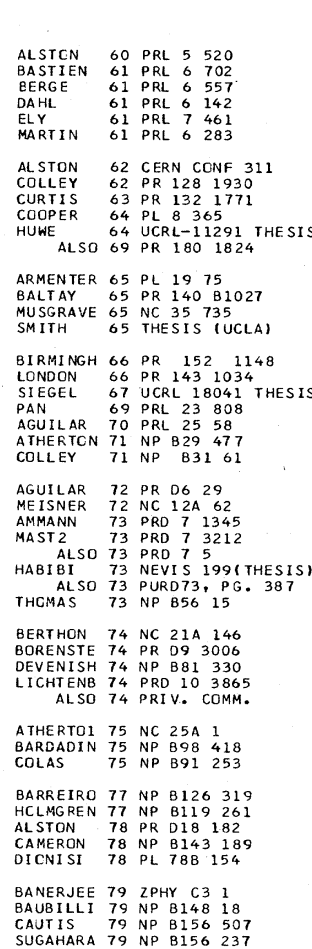

REFERENCES FOR $Y * 1(1385)$

+ALVAREZ, EBERHARD, GOOD, GRAZIANO, +
P BASTIEN, (LRL FERRO-LUZZI, A H ROSENFELD (LRL) +BASIIEN,DAHL, FERRO-LUZZI, KIRZ, +
+ HORWITZ,MILLER, MURR AY, WHITE
(LRL) (LRL)
FUNG, GI DAL, PAN, POWE LL, WHITE
FEIPUNER, CHINOWSKY, SHIVELY, +

ALVAREZ, FERRO-LUंZZI, ROSENFELD, + (LRL)

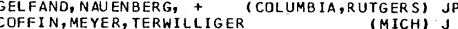
FFILTHUTH, FR IDMAN, MALAMUD, +
(CERN, ILAST) JP
(IRL) JPWE (CERN, HEI DEL, SACLAY)
ARMENTEROS, + +SANDWE ISS, TAFT, C ULWICK, KOPP, + (YALE, BNL)
PPEMEEZAS, +
(BIRM, CERN, EPOL, LOIC, SACLAY) BIRMINGHAM, GLASGOW, I.C., OXFORO, RUTHERFORO $M$ SIEGEL (LRL)
(PENN) BARNES, BASSANO, CHUNG, EI SNER, $+(B N L$, SYRA)
SY +COX,EASTWOOD, FRY+... (BIRM+EDIN+GLAS +LOIC) AGUI LAR-BENITEZ, CHUNG, EISNER, SAMIOS (BNL) CARMONY, GARFINKEL, GUTAY, + (PURD+IUPU) +BANGERTER, ALSTON-GARNJCST,+
MAST, BANGERTER, ALSTON-GARNJOST,+
(LBL) IJPP MAST, BANGERTER, ALSTON-GARNJOST,+ (COLUMBIA)
M.HABBI BALTAY, BRIDGEWATER, CODPER, + (COLUMBIA B ING)
THOMAS, ENGLER, FI SK, KRAEMER (CARN) JP
(CARN) BERTHON, TRISTRAM, + (CDEF+RHEL+ SACL+ STRB) BORENSTEIN, KAL BFLE ISCH, STRAND, + (BNL+MICH
DEVENI SH, FROGGATT, MART INIDESY, NORD ITA, LCUC DEVENI SH, FROGGATT, MARTIN(DESY, NORDITA, LCUC)
D B LICHTENBERG (INDIANA UNIVERSITY) $\begin{array}{lr}\text { ATHERTON, BAR-NIR, FRENCH } & \text { (CERN) } \\ \text { BARDADINOOWWINNSKA+ } & \text { (SACL+EPOL+RHEL) } \\ \text { COLAS, FAR WELL, FERRER, SI X } & \text { (ORSA) }\end{array}$ +BERGE, GANGULI, BLOKZI JL+
+AGUILARGENITEZ,KLYYVER+ A STON-GARN +FRANEK, GOPAL, BACON, BUT TERWORTH+ (RHEL+LCIC)
+ARMENTEROS, DIAZ
(CERN+AMST+NIJM+OXF) +GANGULI, MALHOTRA, RAGHA VAN, SUDHAKAR (TATA)
BAUBILLIER+
(BI RM+CERN+GLAS+MSU+LPNP) B BALLAM, BOUCHEZ, CARROLL, CHADWICK+ (SLAC)

PAPERS NDT REFERRED to IN DATA CARDS

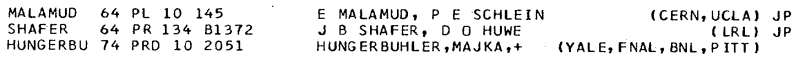

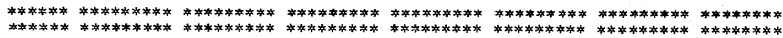
$\sum(1480) \quad 23 \quad Y * 1(1480, J P=\quad, I=1 \quad$ PRODUCTION EXPERIMENTS BUMPS see the mini-revue at the start of the $Y *$ Listings. PEAKS ARE SEEN IN LAMBDA PI AND SIGMA. PI SPECTRA IN
THE REACTION P IIP TO K+ PI Y AT 1.7 GEVIC. ALSO THE
$Y$ POLARIZATION OSCILLATES IN THE SAME REGION. SEE MILLER 70 FOR A OISCUSSION OF THIS STATE. HE SUGGESTS A POS-
SIBLE ALTERNATE EXPLANAT ION IN TERMS OF A REFLECTION OF N*1/21 167010 DECAY TO LAMBDA K. HOWEVER, SUCH AN EXPLANATION FOR THE K+ SIGMA+ PIO CHANNEL SEEMS UNLIKELY ISEE PAN 701 IN TERMS OF KNOWN N*3/21 16901
DECAY INTO SIGMA K. IN ADDITION SUCH REFLECTIONS WOULD TO ACCOUNT FOR THE OSCILLATION OF THE Y POLARI ZATION IN THE 1480 MANSON 71, WI TH FEWER DATA THAN PAN 70, CAN NEITHER CONFIRM NOR HANSON EXISTENE OF THIS STATE. MAST 75 SEES NO STRUC TURE IN THIS
DASS THEGION IN K- P TO LAMBDA PIO.

$23 r * 1(1480)$ MASS (MEV) (PROD. EXP.)

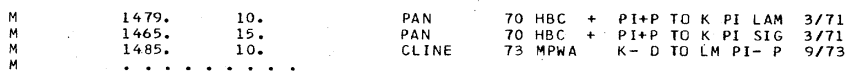
M AVERAge Méaningiess $i$ iscale Factor $=1.01$

$23 Y * 1(1480)$ WIDTH (MEV) (PROD. EXP.)

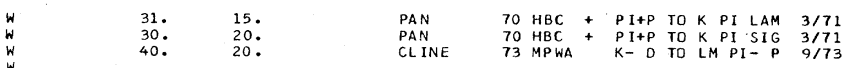

W Average méaninglés iscile factor $=1.01$

23 Y*1(1480) PARTIAL DECAY MODES (PROD. EXP.)

$\begin{array}{ll}P 1 & Y * 1(1480) \text { INTO KBAR N } \\ \text { P2 } & Y * 1(1480) \text { INTO LAMBDA P } \\ \text { P3 } & Y * 1(1480) \text { INTO SIGMA PI }\end{array}$

DECAY MASSE
$497+939$

$1115+139$
$1189+139$

$23 \gamma * 1(1480)$ BRANCHING RATIOS (PROD. EXP.)

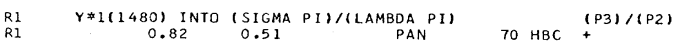

$R 2$
$R 2$$\quad \begin{array}{lll}0.36 \\ \text { R2 }\end{array}$

\section{R3
R3 \\ REFERENCES FOR $Y * 111480)$ (PROD. EXP.) \\ PAN 70 PR 02,49
CLINE
73 LNC 6205 \\ PAPERS NOT REFÉRREO to IN DATA CARDS

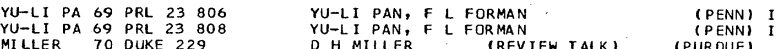 \\ $\begin{array}{llll}\text { HANSON } & 71 \text { PR D4 } 1296 & \text { TKALMUS, LOUIE } \\ \text { HAST } & 75 \text { PRD } 113078 & \text { TALSTON-GARNJOST, BANGERTER+ } & \text { (LEL) I } \\ \text { MAST } & \text { (LBL) }\end{array}$

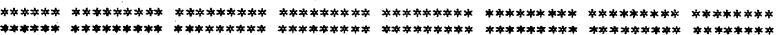 \\ $\Sigma(1560)$ 80 $\quad \mathrm{F} * 111560$, JP $=\quad$ I I=1 PRODUCT ION EXPER IMENTS \\ BUMPS THIS ENTRY LISTS PEAKS REPORTED IN MASS SPECTRA AROUNO \\ $\longrightarrow$ RELATED. 78 OBSERVE A 6 STD. DEV. ENHANCEMENT AT 1553 \\ MEV IN THE CHARGED ILAMBDA SIGMA PI MASS SPECT TRA

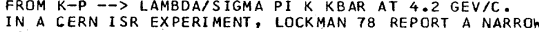

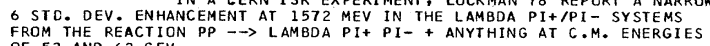 \\ THES ENHANEMENTS ARE UNL ITELY TO BE ASSOCIATED WITH THE Y*1115801 \\ IHICH HAS NOT BEEN COSF IRMED BY SEVERAL RECENT EXPERIMENTS - SEE \\ CARROLL 76 OBS ERVE A BUMP AT 1550 MEV LAS WELL AS AT 1580 MEVI IN THE \\ KN IU TOTAL CROSS SECTION, BUT UNCERTAINTIES IN CROSS SECTION
MEASREMEETS OUTSIDE THE MASS RANGE OF THE EXPERIMENT PRECLUDE \\ IN NEED OF CONFIRMATION. DMITTED FROM TABLES.}

$80 Y * 1(1560)$ MASS (MEV) (PROD. EXP.)

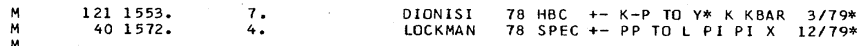
M. average méaningiess iscale factor $=2.41$

$80 Y * 1(1560)$ WIDTH (MEV) (PROD. EXP.)

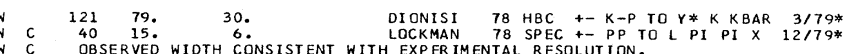

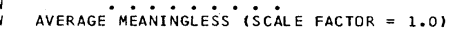

80 Y $1(1560)$ PARTIAL DECAY MODES (PROD. EXP.)

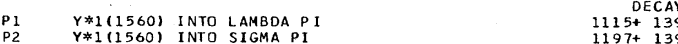

SO $Y * 1(1560)$ BRANCHING RATIOS (PROD. EXP.)

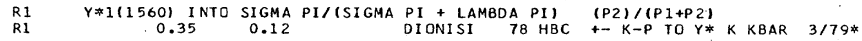

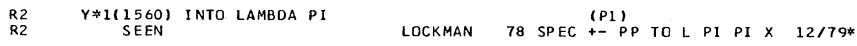

\#*********************************************************************1

REFERENCES FOR $Y * 1(1560)$ (PROD. EXP.)

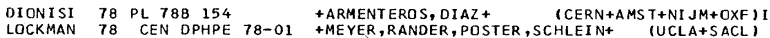

PAPERS NOT REFERRED TO IN DATA CARDS

+CHIANG, KYCIA, LI, MAZUR, MICHAEL+ (BNL)I

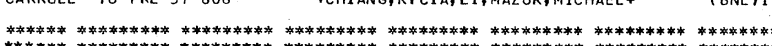

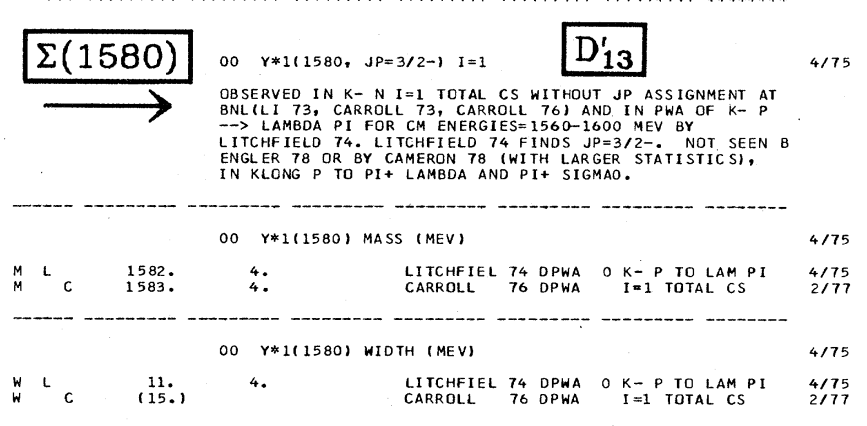




\section{Baryons $\Sigma(1580), \Sigma(1620)$}

OO Y*1(1580) PARTIAL DECAY MOOES

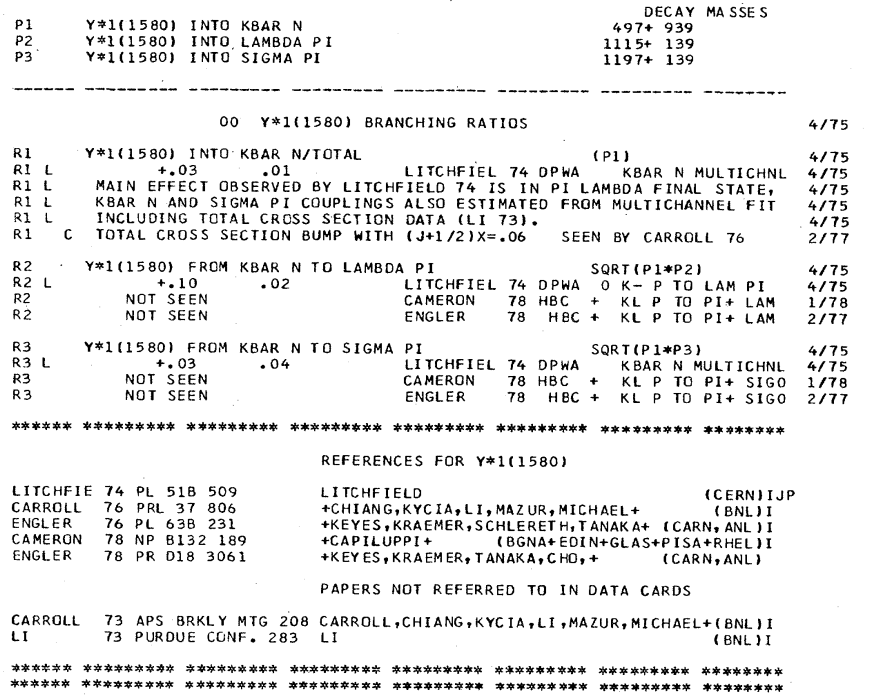

Note on $\Sigma(1620)$

This state was first suggested by the BNL-CCNY collaboration (CRENNELL 68) who presented evidence for it in the reaction $\mathrm{K}^{-} \mathrm{n} \rightarrow \sum(1620)^{ \pm} \pi^{\mp} \pi^{-}$with $\Sigma(1620)^{ \pm}$decaying into $\Lambda \pi^{ \pm}$. Since then there have been conflicting reports about this state (or states).

\section{Total Cross-Section Experiment}

A measurement of the $\overline{K^{-} \mathrm{p}}$ and $\mathrm{K}^{-} \mathrm{d}$ total cross sections in the 0.4 to $1.1 \mathrm{GeV} / \mathrm{C}$ range has been reported by the BNL group (CARROLL 76). Three narrow $(10-15 \mathrm{MeV}$ wide) bumps in the $\mathrm{I}=1 \mathrm{~K}$ N cross section are seen at 1583, 1608, and $1633 \mathrm{MeV}$.

\section{Formation Experiments}

There is evidence from several partial-wave analyses for one or two fairly narrow states within $\sim 50 \mathrm{MeV}$ of the effect seen in production; see the entries for $\sum\left(1580,3 / 2^{-}\right), \sum\left(1620,1 / 2^{-}\right)$, and $\Sigma(1660$, $\left.1 / 2^{+}\right)$. Note however that the various analyses do not agree on the widths and branching ratios of these states.

\section{Production Experiments}

A good review of the production experiments has been given by MILLER 70. There has been no new evidence from production experiments since 1970. The existing evidence is only in the $\Lambda \pi$ channel. The BNL-CCNY collaboration (CRENNELL 69) claimed
Data Card Listings For notation, see key at front of Listings.
$4 / 75$

the effect in the $\Lambda \pi$ channel with no evidence seen in $\overline{\mathrm{K}} \mathrm{N}$ or $\overline{\mathrm{K}} \mathrm{N} T$. SABRE 70 studied the same reaction at $3.0 \mathrm{GeV} / \mathrm{c}$ with comparable statistics and did not see any evidence for it in the $\Lambda \pi$ channel; on the contrary, they believed it to be a spurious peak resulting from misidentified $\Sigma^{0}$ from the production of $\Sigma(1670)$ decaying into $\Sigma^{0} \pi^{+}$. AMMANN 70 studied the same reaction at $4.5 \mathrm{GeV} / \mathrm{c}$ and reported a state at $1640 \mathrm{MeV}$, again decaying only into $\Lambda \pi$ (no evidence seen in $\Sigma \pi$ or $\overline{\mathrm{K} N}$ channels). Upper limits on production cross sections for a $25 \mathrm{GeV} / \mathrm{c} \Sigma^{-}$beam are reported by HUNGERBUHLER 74 .

In conclusion, for understanding of the $\Sigma(1620)$ we probably have to wait for more data and for a more complete understanding of the entire mass region from 1600 to $1700 \mathrm{MeV}$. The closeness of the $\Sigma(1620)$ mass to $1670 \mathrm{MeV}$ is suggestive that this effect may be related to what goes on in that region (see the "Note on $\Sigma(1670)$ " below).

$$
\stackrel{\Sigma(1620)}{\longrightarrow}
$$

$32 \gamma * 1\left(1620, J P=1 / 2-1\right.$ I=1 $S_{11}^{\prime}$

THE S11 STATE AT 1697 MEV REPPRTED BY VANHORN 75 IS

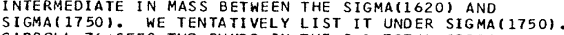

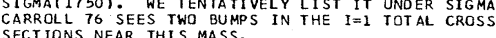

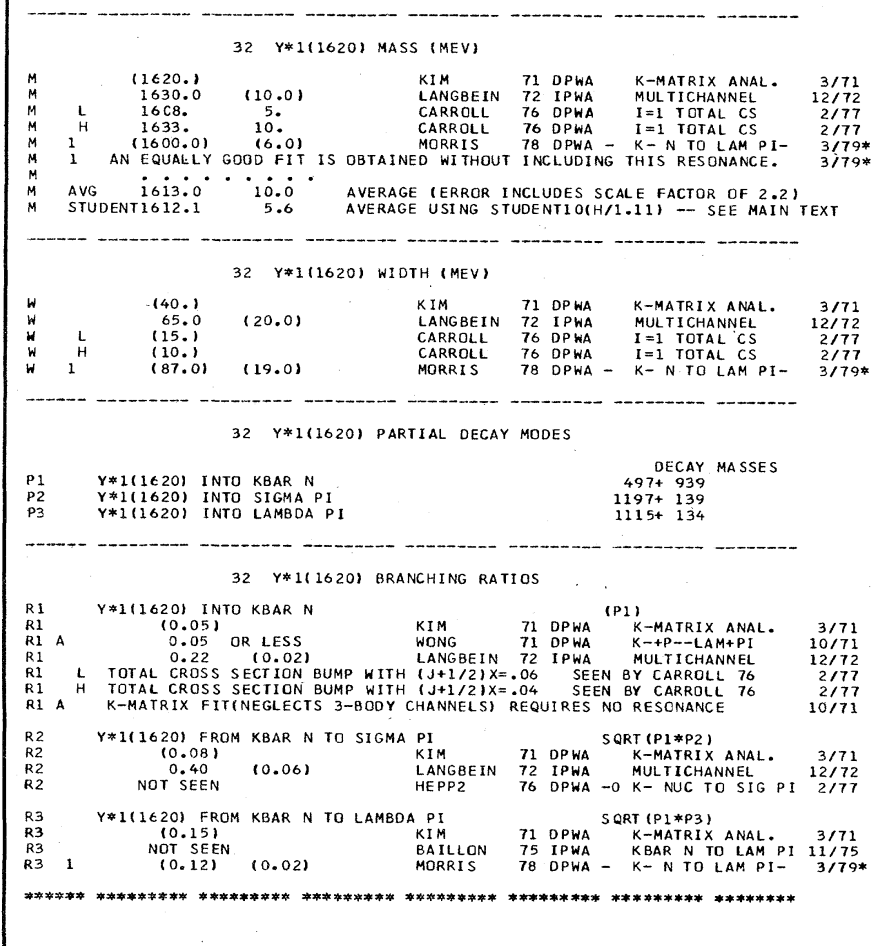


Data Card Listings

For notation, see key at front of Listings.

\section{Baryons \\ $\Sigma(1620), \Sigma(1660)$}

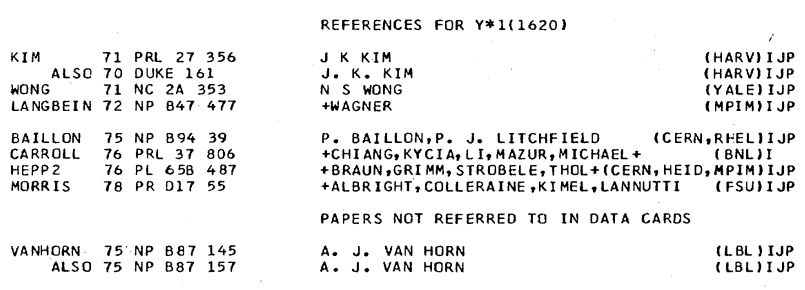

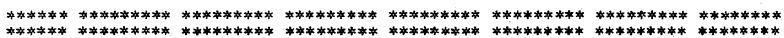

1620 MEV REGION - PRODUCTION EXPERIMENTS

$78 \mathrm{Y} * 1(1620, \mathrm{JP}=, \mathrm{I}=1 \quad$ PRODUCTION EXPERIMENTS SEe the Mini-Revue at thė StaRt of THE Y* Listings. THIS RESONANCE NEEOS CCNFIRMATION. THE RESULTS OF
CRENNELL G9 AT 3.9 GEV IC ARE NOT CONFIRMED BY THE SABRE COLLABORATION AT 3.0 GEV/C (SABRE 70). HOWEVER IN AN EXPER IMENT AT
4.5 GEV/C. AMMANN 70 SEE A PEAK AT 1642 MEV WHICH ON THE BAS IS OF 4.5 GEV/C, AMMANN 70 SEE A PEAK AT 1642 MEV WHICH ON THE BAS IS OF
BRANCHING RATIOS THEY DO NOT ASSOCIATE WITH THE Y*1(1670). SEE MILLER
70 FOR A REVIEW OF THESE CONFLICTS.

78 Y*1(1620) MASS (MEV) (PROD. EXP.)

$M \quad N \quad(1616.0) \quad(8.0) \quad$ CRENNELL 68 OBC $+-K-D 3.9$ BEV/C $11 / 68$

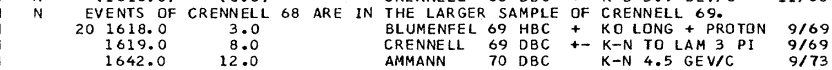

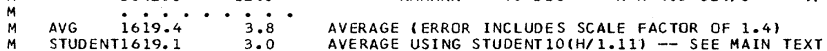

78 Y*1(1620) WIDTH (MEV) (PROD. EXP.)

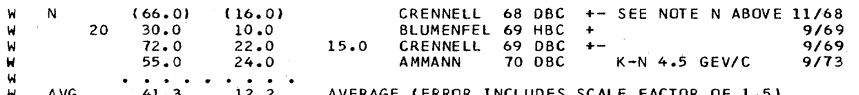

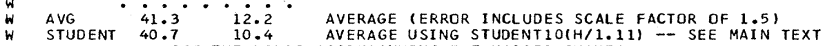
SEE THE NOTES ACCOMPANYING THE MASSES QUOTED -- SEE MAIN TEXT

78 Y*111620) PARTIAL DECAY MODES (PROD. EXP.

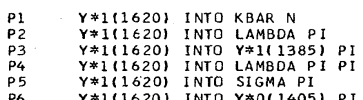

DECAY MASSES

F*11(1620) INTO Y*111385)

$Y * 1(1620)$ INTO $Y * 0(1405)$ P

$78 Y * 1(1620)$ BRANCHING RATIOS (PROD. EXP.)

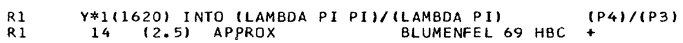

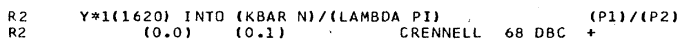

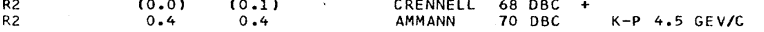

R3 $Y * 1(1620)$ INTO LAMBDA PI
RARGE

$R 4 \quad Y * 1(1620)$ INTO $(Y * 1(1385) P 1) /(L A M B D A P 1) \quad$ (P3)/(P2)

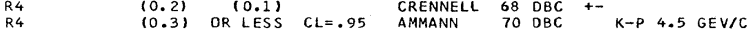

R5
R5

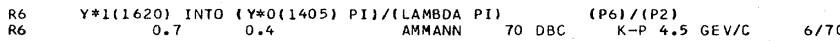

\#*****************************************************************1 REFERENCES FOR $Y * 1(1620)$ (PROD. EXP.)

$\begin{array}{ll}\text { CRENNELL } 68 \text { PRL } 21648 & \text { +DELANEY, FLAMINIO, KARSHON, + (BNL, CUNY) } \\ \text { BLUAAENFE } 69 \text { PL } 29858 & 58 \text { BLUMENFELD, KALBFLEISCH }\end{array}$ CRENNELL O9 LUND PAPER 183 TKARSHON, LAI, ONEIL, SCARR, + (BNL, (BUNY)

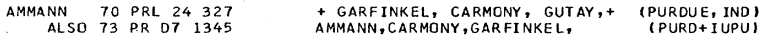
PAPERS NOT REFERRED TO IN DATA CARDS

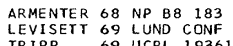
$\begin{array}{lll}\text { TRIPP } & 69 & \text { UCRL } 1936 \\ \text { AR MENTER } 70 \text { DUKE } 123\end{array}$ ARMËNTEROS, BAILLON +
$R$ LEVI SETYII (RAPPORTEUR)
(CERN+HEID+SACL)
EFINS

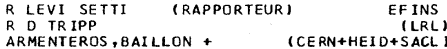

$6 / 70$ $11 / 68$ $11 / 68$
$6 / 70$ 73 170

MILLER 70 DUKE 229 D H MILLER (REVIEH TALK) (PURDUE) SABRE COLLAB. RE (SACL, AMST, BGNA, REHO, EPOL)
HUNGERBULLER, MAJKA, (Y (YLE, FAAL, BNL, PITT)

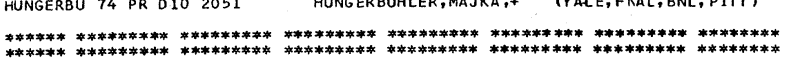

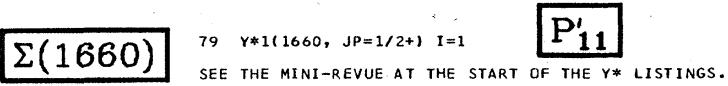

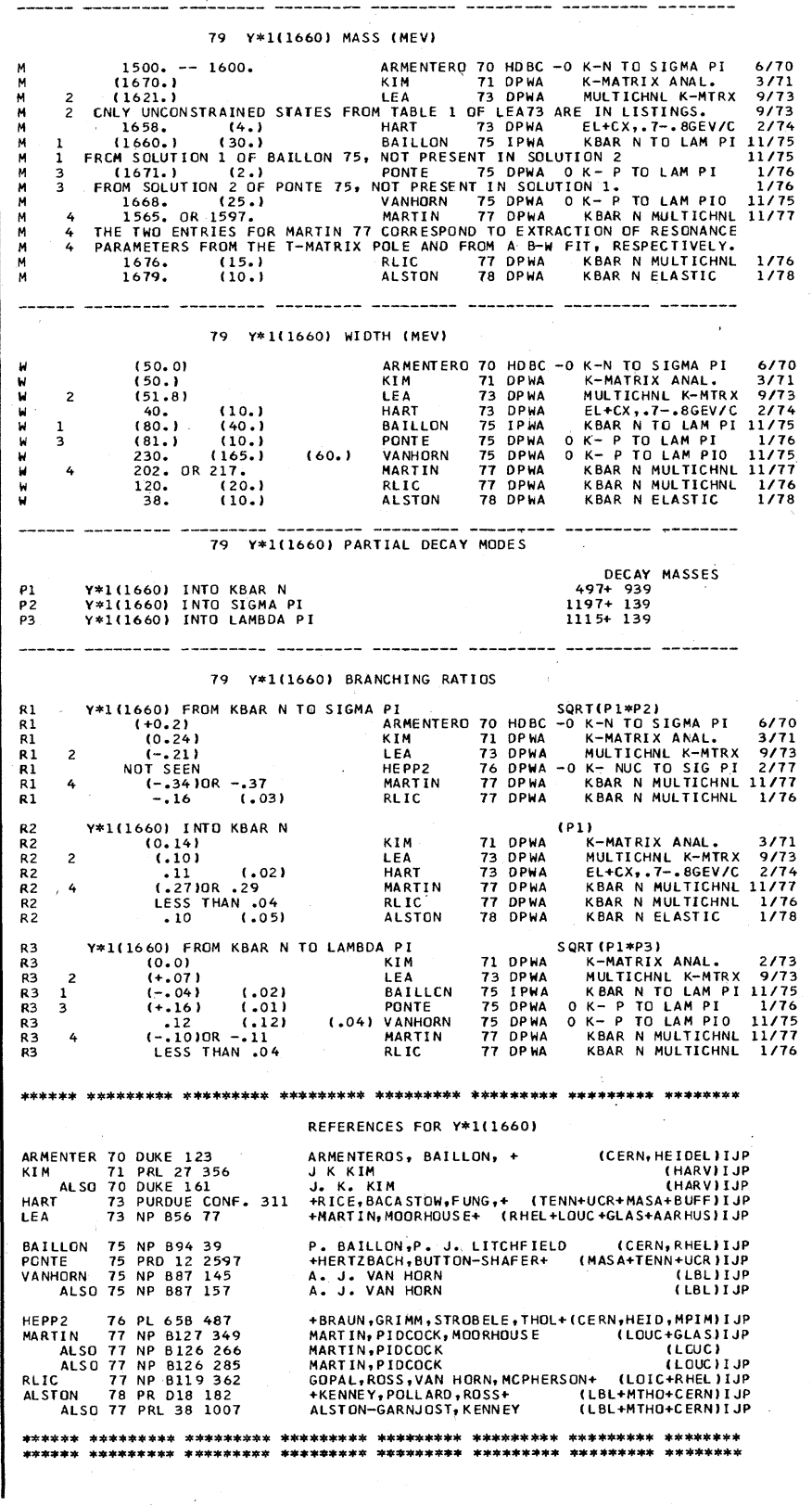




\section{Baryons $\Sigma(1670)$}

\section{Note on $\Sigma(1670)$}

\section{Production Experiments}

The measured $\Sigma \pi / \Sigma \pi \pi$ branching ratio for produced $\Sigma(1670)$ 's is strongly dependent on momentum transfer. This was first discovered by EBERHARD 69, who suggested the existence of two $\mathrm{Y}_{1}^{*}$ 's with the same mass and quantum numbers; one object with a large $\Sigma \pi \pi$ [mainly $\Lambda(1405) \pi$ ] decay mode produced peripherally, and another one with a large $\Sigma \pi$ decay mode produced at larger angles. This observation has been confirmed by AGUILAR-BENITEZ 70, ASPELL 74, ESTES 74, and TIMMERMANS 76. When determined, the most likely quantum numbers are $3 / 2^{-}$[for both $\sum \pi$ and $\Lambda(1405) \pi]$. There is also the possibility of a third $Y_{1}^{*}$ state, referred to as $\Sigma(1690)$ in the Data Card Listings, with a large $\Lambda \pi / \Sigma \pi$ branching ratio and somewhat larger mass. The large branching ratio is the main justification for this hypothesis and needs confirmation. These problems have been reviewed by EBERHARD 73 and MILLER 70 .

\section{Formation Experiments}

Two states are also observed near this mass in formation. One of these, the $\sum\left(1670,3 / 2^{-}\right)$, has the same quantum numbers as those observed in production and a large $\sum \pi / \sum \pi \pi$ branching ratio. It may well correspond to the produced $\sum(1670)$ seen at larger angles. (See TIMMERMANS 76 on this point.) The other state, the $\sum\left(1660,1 / 2^{+}\right)$, has different quantum numbers from those seen in production, and its $\sum \pi / \sum \pi \pi$ branching ratio is unknown. Thus its relation to the produced $\sum(1670)$ remains obscure. (See also the "Note on $\sum(1620) "$ above.)

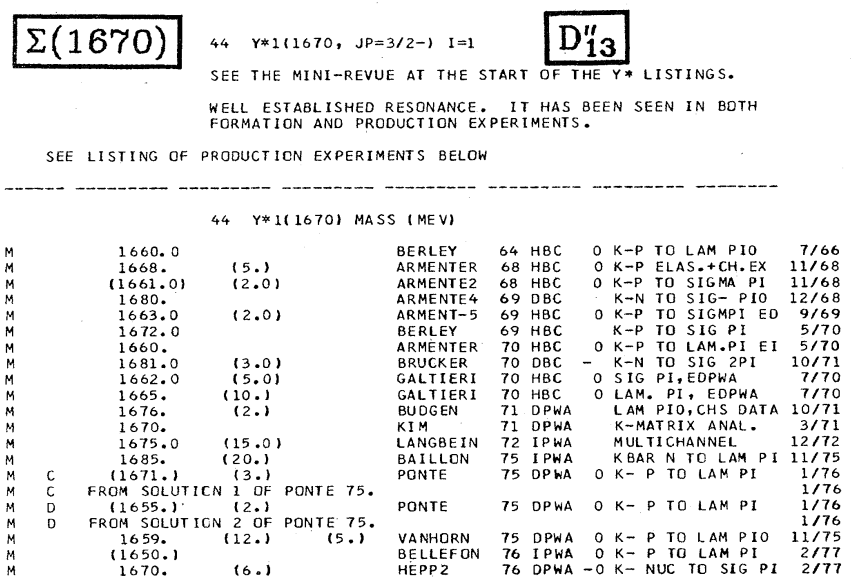

\section{Data Card Listings For notation, see key at front of Listings.}




\section{Dạta Card Listings}

For notation, see key at front of Listings.

Baryons

$\Sigma(1670)$

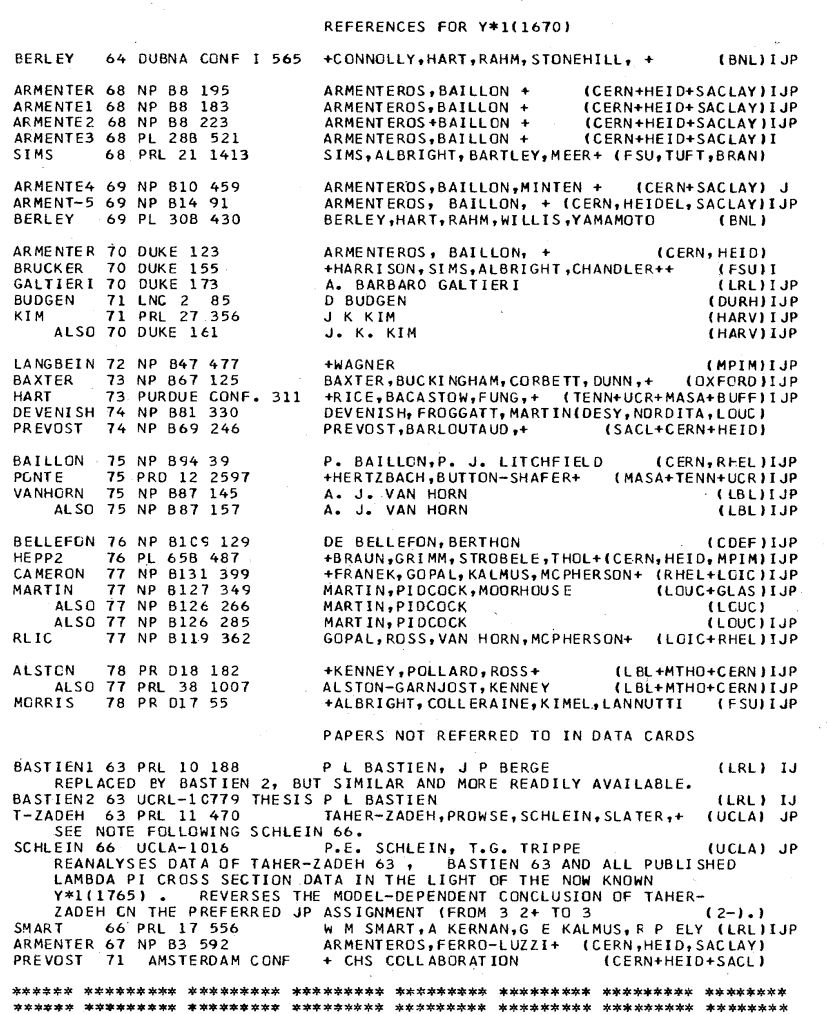

$\Sigma(1670)$

BUMPS

$51 \gamma * 1(1670, J P=\mid I=1$ PROD. AND CROSS SECT. EXPS.

SEE NOTE PRECEDING Y*1(1670)

PROBABLY THERE ARE TWO STATES AT SAME MASS WITH SAME
QUANTMM NUMBERS. ONE DECAYING INTO SIGMA PI AND LAMBDA
PI, THE OT HER INTO Y YO (1405) PI,

$51 Y * 1(1670)$ MASS (MEV) (PROD. EXP.)

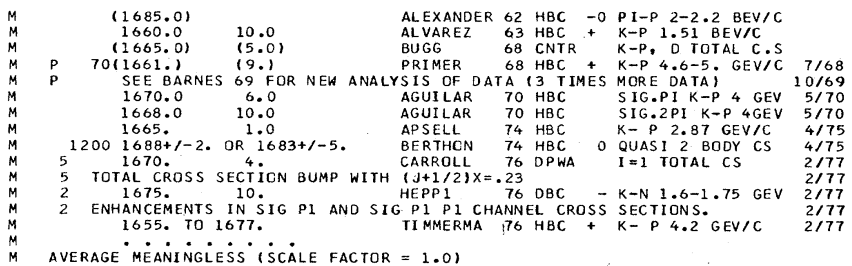

average Méaníngle śs iscalle factor $=1.0$

$51 Y * 1(1670)$ MASS (MEV) (PROD. EXP.)

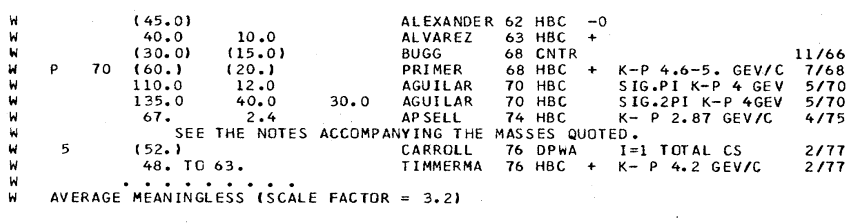

51 Y*1(1670) PARTIAL DECAY MODES (PRDD. EXP.)

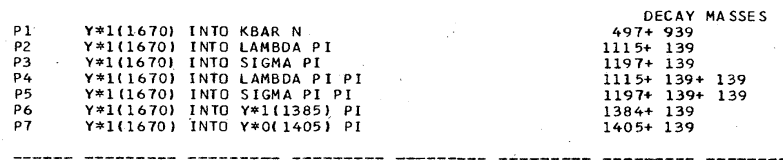

51 Y*1(1670) BRANCHING RATIOS (PROD. EXP.)

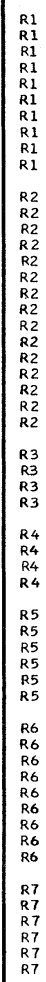

Y*1(1670) INTO (KBAR N)/ SSIGMA PI)

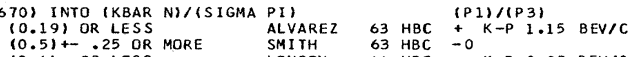

$\begin{array}{lll}(0.6) \text { OR LESS } & \text { LONDON } 63 \mathrm{HBC}+0 \\ (0.025) & 66 \mathrm{HBC}+\mathrm{K}-\mathrm{P} 2.25 \mathrm{BEV} / \mathrm{C}\end{array}$

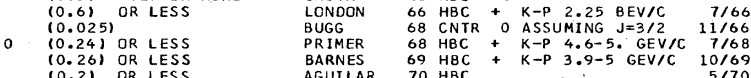

$\begin{array}{llll} & \text { BARNES } & 69 \mathrm{HBC}+\mathrm{H}-\mathrm{P} 3.9-5 \mathrm{GEV} / \mathrm{C} & 10 / 69 \\ 10.21 & \text { DR LESS } & \text { AGUILAR } 70 \mathrm{HBC} & \\ 10.101 & \text { OR LESS } & \text { BERTHON } 74 \mathrm{HBC} \text { O QUASI } 2 \text { BODY CS } & 4775\end{array}$

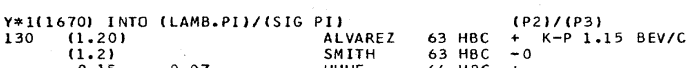

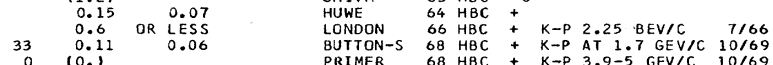

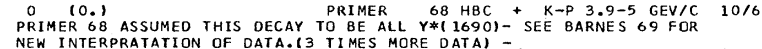

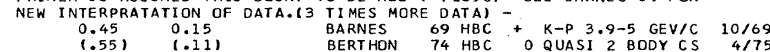

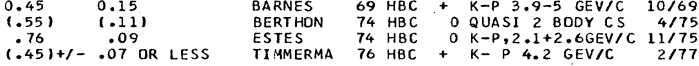

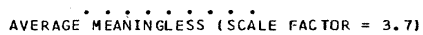

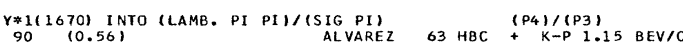

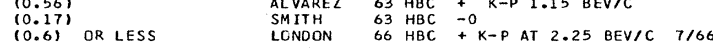

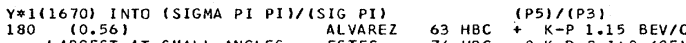

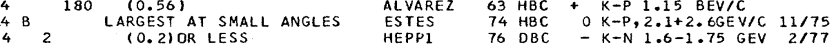

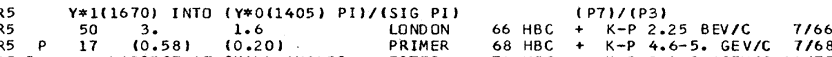

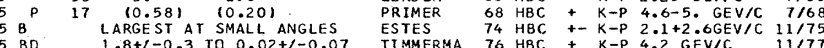

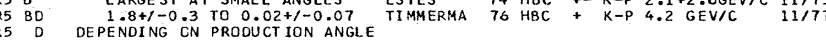

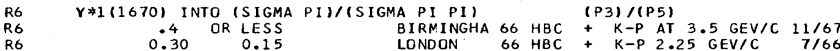

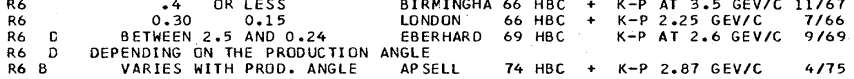

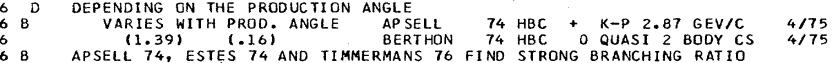

$\begin{array}{lll}R 6 & \text { APSELL 74, ESTES } 74 \text { AND TIMMERMANS } 76 \text { FIND STRONG BRANCHING RATIO } \\ \text { R6 } & B & \text { DEPENDENCE ON PRODUCTION ANGLE, AS IN EARLIER PROD. EXPERIMENTS. }\end{array}$

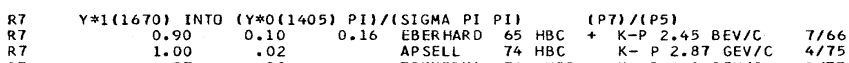

TIMMERMA 76 HBC K-P 4.2 GEV/C $2 / 77$

average MeAN INGLiess (SCALE FaC TOR $=1.01$

$Y * 1(1670)$ INTO (Y*0(1405) PI)/(Y*1(1385) P1)
$(0.8)$ DBERHARD
OLES

$Y * 11670)$ INTO (LAMBDA PI PI)/(SIGMA PI PII
0.35

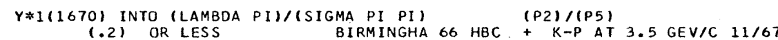

$Y * 1(1670)$ INTO (LAMBDA PI)/(LAMBDA PI + SIG PI) (P2)/(P2+P3)
$(0.6)$ OR LESS

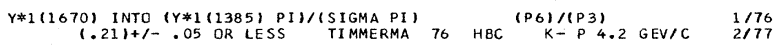

$51 Y * 1(1670)$ QUANTUM NUMBER DETERMINATI ON (PRDD. EXP.)

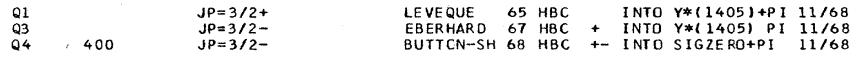

**********************************************************************

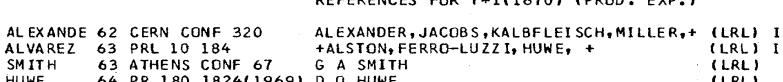

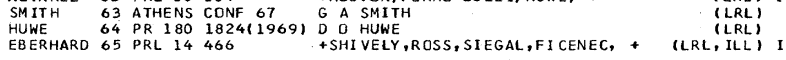

$\begin{array}{lll}\text { BIRMINGH } 66 \text { PR } 152 & 1148 & \text { BIRMINGHAM, GLASGOW, I. C. Y OXFOR D, RUTHERFCRD } \\ \text { LONDON } 66 \text { PR } 1431034 & \text { +RAU, SAMIOS,YMMAMOTO,GOLOBERG, }+ \text { (BNL, SYRA) }\end{array}$

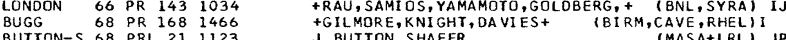

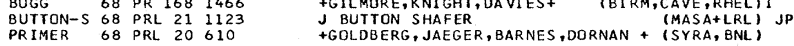

$\begin{array}{lllll}\text { BARNES } & 69 \mathrm{BNL} & 13823 & \text { +CHUNG, EISNER, FLAMINIO+ } & \text { (BNL, SYRA) } \\ \text { EBERHARD } & 69 & \text { PRL } 22 & 200 & \text { +FRIEDMAN, PRIPSTEIN, ROSS }\end{array}$

+ BARNES, Bassano, chung EISNE

$\begin{array}{llll}\text { APSELL } & 74 & \text { PRD } 101419 & \text { APSELL,FORD, GOUREVITCH+ (BRAN, UMD, SYRA, TUFT) } \\ \text { BER THON } & 74 \text { NC } 211 \text { IA } 146 & \text { BERTHN, TRISTRAM, } & \text { (CDEF+RHEL +SACL + STRB) }\end{array}$

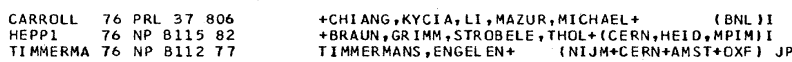

PAPERS NOT REFERRED TO IN DATA CARDS

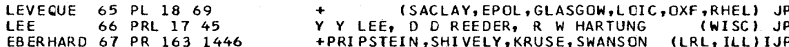

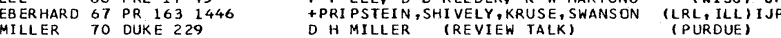

EBERHARD 73 PURDUE CONF. 247 EBERHARD
HUNGERBU 74 PRD 10 2051
HUNGERBUHLER, MAJKA, + (YALE, FNAL, BNL, PITT) IJP

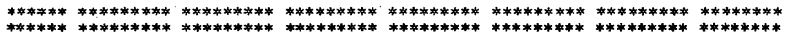




\section{Baryons \\ $\Sigma(1690), \Sigma(1750)$}

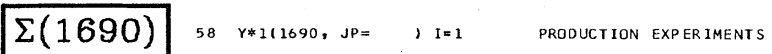

BUMPS SEe the Mini-revue at the start of the Y* Listings.

SEE NOTE PRECEDING Y*1(1670) LISIINGS, SEEN IN PRO.
EXPERIMENTS ONLY, MAIN DECAY MODE IS LAMBDA PI.

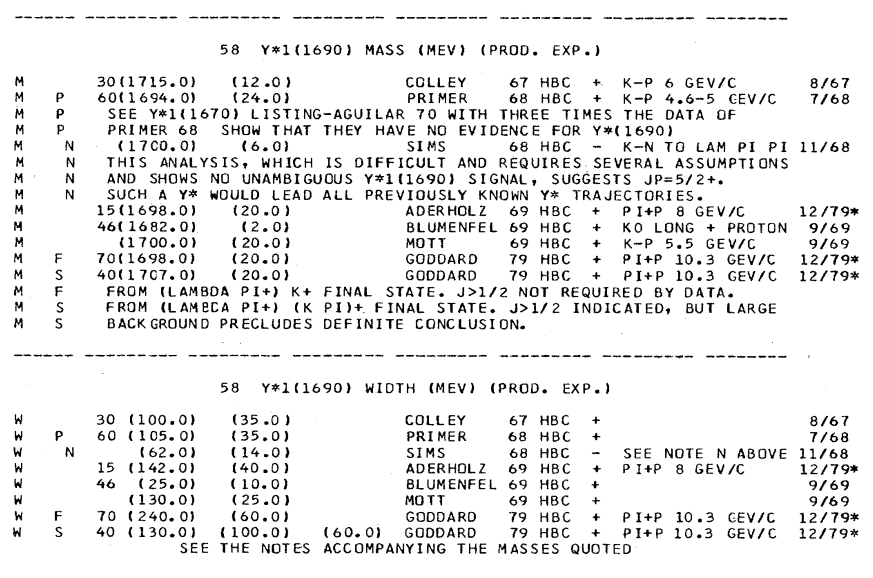

58 Y $1(1690)$ PARTIAL DECAY MODES (PROD. EXP.)

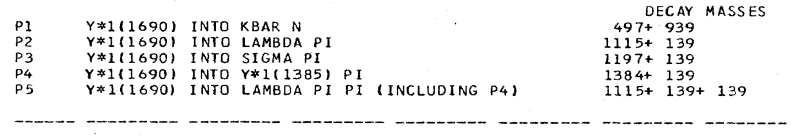

$58 Y * 1(1690)$ ERANCHING RATIOS (PROD. EXP.)

$R$
$R$
$R$
$R$
$R$
$R 2$
$R 2$
$R 2$
$R 2$
$R 3$
$R$
$R 4$
$R$
$R$
$R 4$
$R 4$
$R 4$
$R 5$
$R 5$

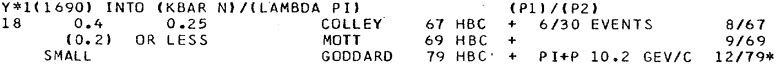

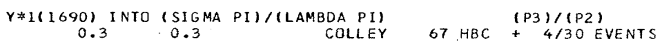

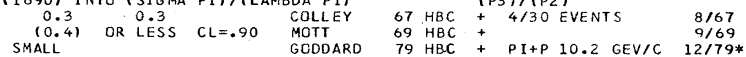
$Y * 1(1690)$ INTO (Y*1(1385) PI)/CLAMBDA PI)
$(0.5)$ OR LESS

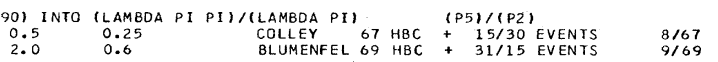

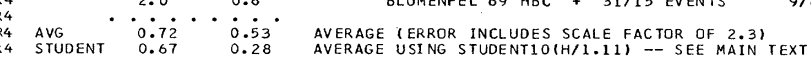

R5 $5 * 111690)$ INTO ( $* 1$ (1385) PI)/(LAMBDA PI PI) (P4)/(P5)

$\begin{array}{llllll}R & \text { SMALL } & \text { COLLEY } & 67 & \mathrm{HBC} & \pm \\ R 5 & \text { LARGE } & \text { SIMS } & 68 \mathrm{HBC} & \mathrm{H}-\mathrm{N} \text { TO L2PI } & 8 / 67 \\ \text { R5 } & 11 / 68\end{array}$

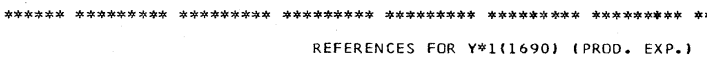

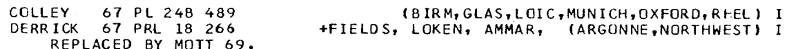

$\begin{array}{lllll}\text { PRIMER } & 68 & \text { PRL } & 20 & 610 \\ \text { SIMS } & 68 & \text { PRL } & 21 & 1413\end{array}$

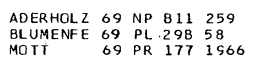

GODDARD 79 PR 0191350

+GOLDBERG, JAEGER, BARNES, + (SYRACUSE, BNL) I
+ALBRIGHT, +
(FSU,TUFTS, BRANDEIS) I

+BARTSCH, SCHUL TE+ (AACH+ BERL+CERN+CRAC+W ARS ) I

B J BLUMENFELD, G R KALBFLE ISCH
+ AMMAR, DAVIS, KROPAC, + (NORTHWEST, ARGONNE) I

+KEY, LUSTE, PRENTICE, YOON, GORDON+ (TNTO+ BNL) I, J

PAPERS NOT REFERRED TO IN DATA CARDS

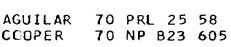

AGUILAR-BENITEZ, BARNES, BASSANO+ (BNL+SYRA)

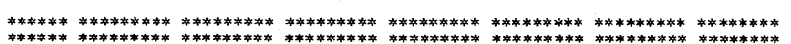

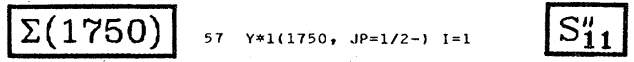

THERE IS EVIDENCE FOR THIS STATE IN MANY P.AR IIAL-
WAVE ANALYSES, BUT WITH RATHER WIDE VARIATIONS IN

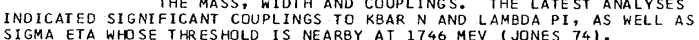

57 Y $* 1(1750)$ MASS (MEV)

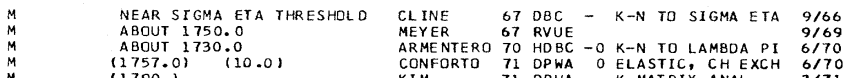

Data Card Listings For notation, see key at front of Listings.

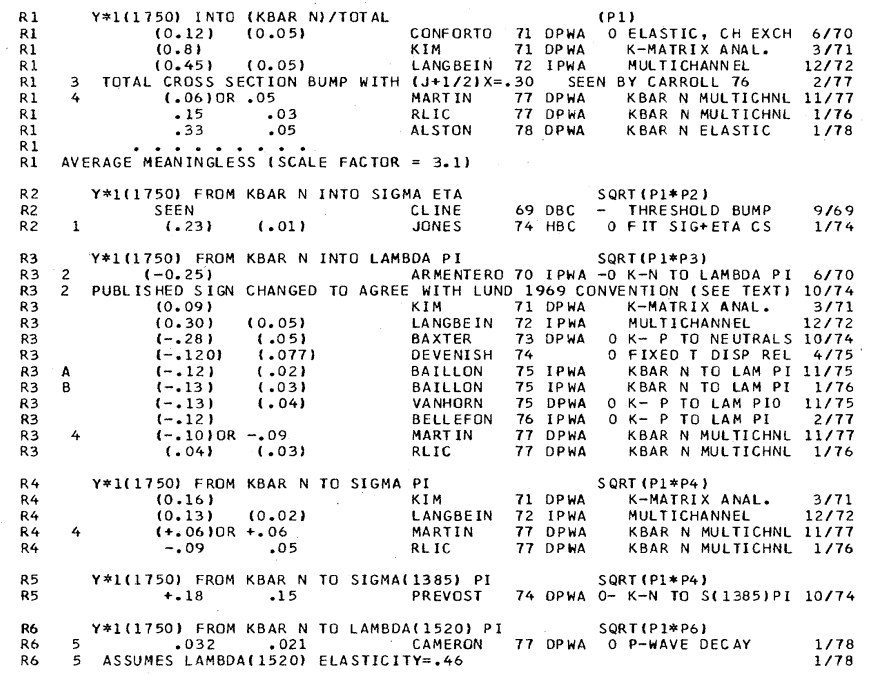

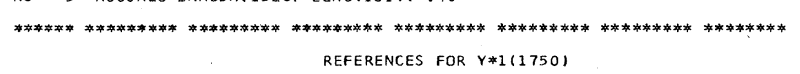

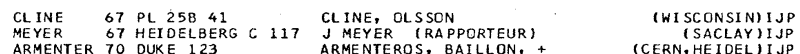

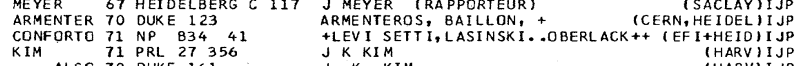

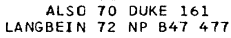
$\begin{array}{lllll}\text { BAXTER } & 73 & \text { NP } & 867 & 125 \\ \text { CHU } & 74 & \text { NC } & 204 & 35\end{array}$

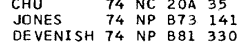
$\begin{array}{llll}\text { OEVENISH } 74 & \text { NP } & 881 & 330 \\ \text { PREVOST } 74 & \text { NP } & 869 & 246\end{array}$ $\begin{array}{ccccc}\text { BAILLCN } & 75 & \text { NP } & 894 & 39 \\ \text { VANHORN } & 75 & \text { NP } & 887 & 145 \\ \text { ALSO } & 75 & \text { NP } & 887 & 157\end{array}$

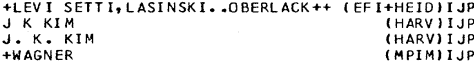
BAXT ER, BUCKINGHAM, CORBE TT, DUNN, + IOXFORDII JP DEVENISH, FROGGAT, MARTIN IDESY, NORDI IA, LOUC)
PREVOST, BARLOUTAUD, +
(SACL+CERN+HEID)

P. BAILLON, P. J. LITCHFIELD ICERN, RHEL)IJJP
A. ILBLIIJP JONES 


\section{Data Card Listings}

For notation, see key at front of Listings.

\section{Baryons} $\Sigma(1750), \Sigma(1765)$
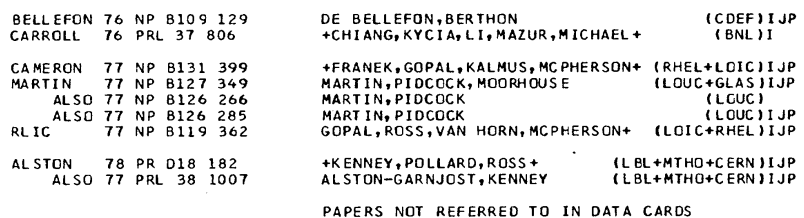

FERRO-LU 66 BERKELLEY CONF 183 M FERRO LUZZI (RAPPORTEUR) I (CERN)

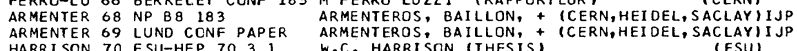

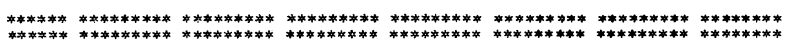
$\sum(1765){ }_{45}^{4}+1\left(1765, J P=5 / 2-1\right.$ I $=1 \quad D_{15}$ SEE THE MINI-REVIEW AT THE START OF THE Y* ListingS.
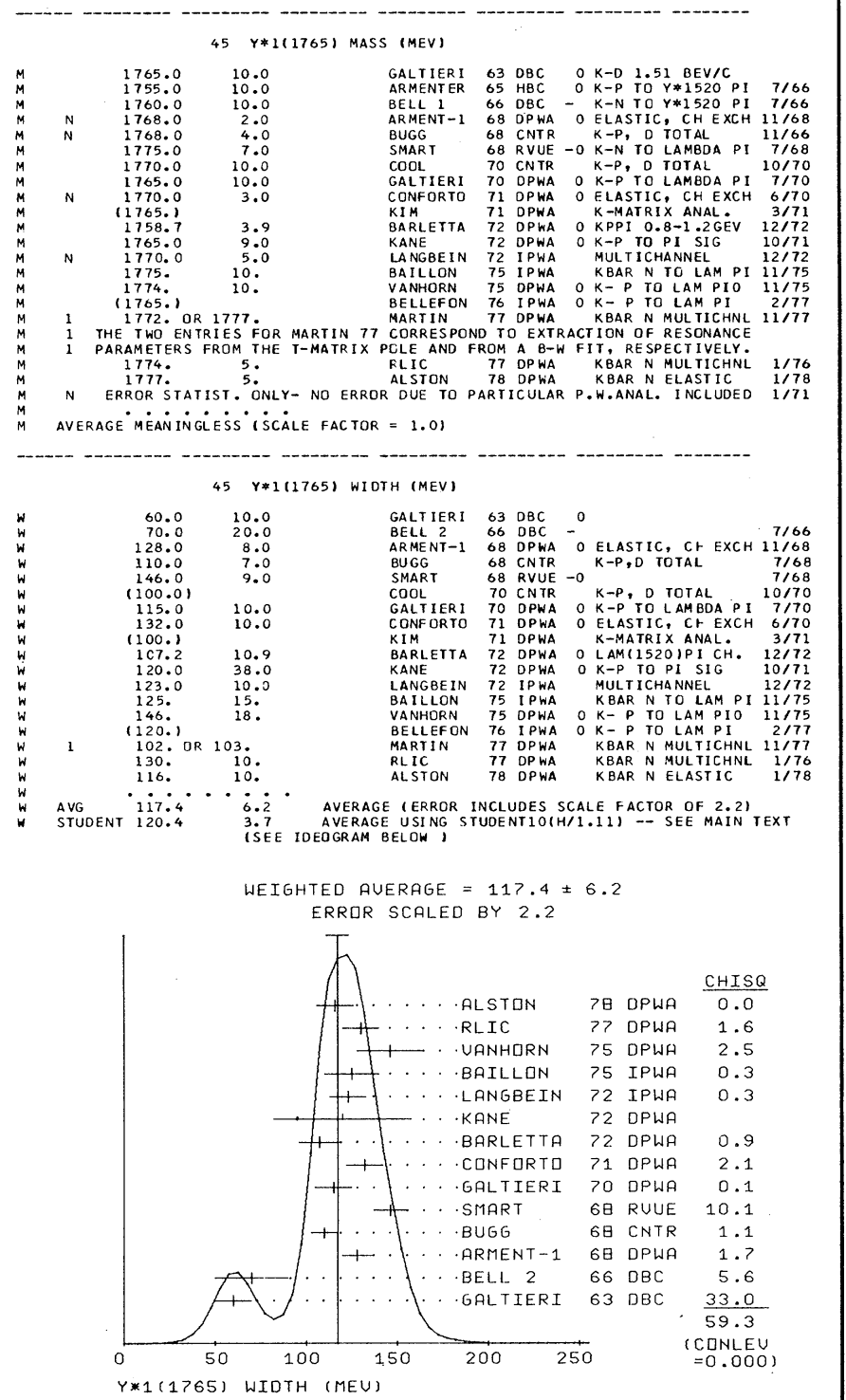

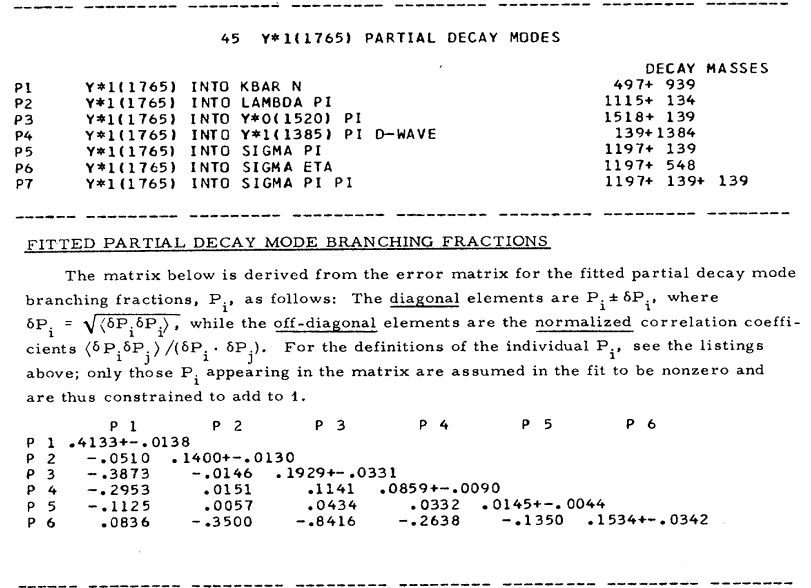

$45 \%$ 1(1765) BRANCHING RATIOS

ERRORS QUOTED BY EXPERIMENT ERS DO NOT INCLUDE UNCERTA INTY DUE
TO PARAMETRIZATION USED IN THE P. W.A. THEY SHCULD BE INCREASED.

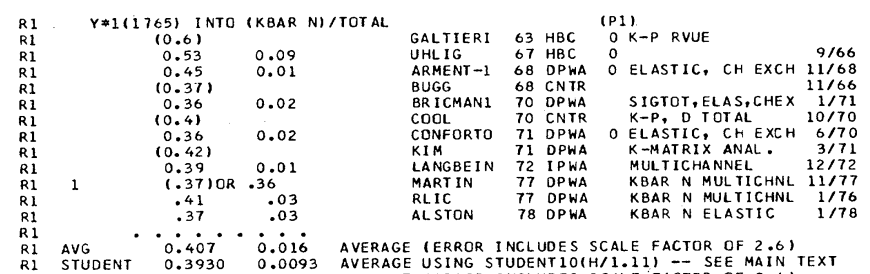

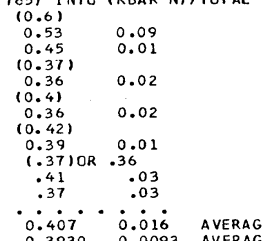

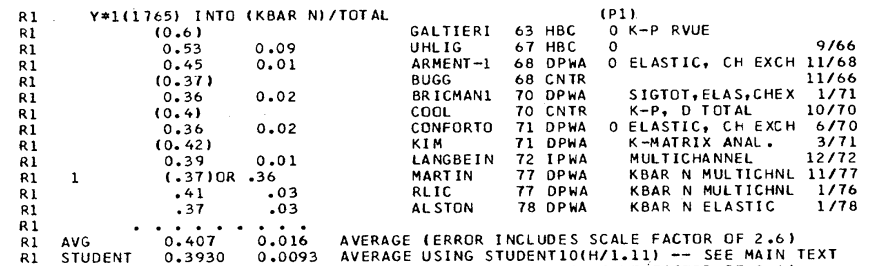

$\begin{array}{llllll}R 1 & \text { AVG } & 0.407 & 0.016 & \text { AVERAGE (ERROR INCLUDES SCALE FACTOR OF } 2.6 \text { ) } \\ \text { R1 } & \text { STUDENT } & 0.3930 & 0.0093 & \text { AVERAGE USING STUDENT DOCH1.11) - SEE MAIN TEX } \\ \text { R1 } & \text { FIT } & 0.413 & 0.014 & \text { FROM FIT (ERROR INCLUDES SCALE FACTOR OF } 2.4 \text { ) }\end{array}$

WEIGHTED AUERAGE $=0.407 \pm 0.016$

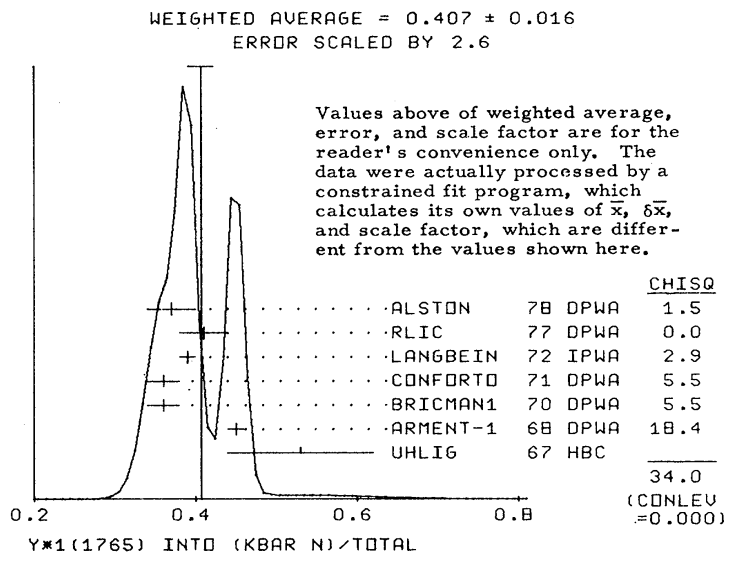

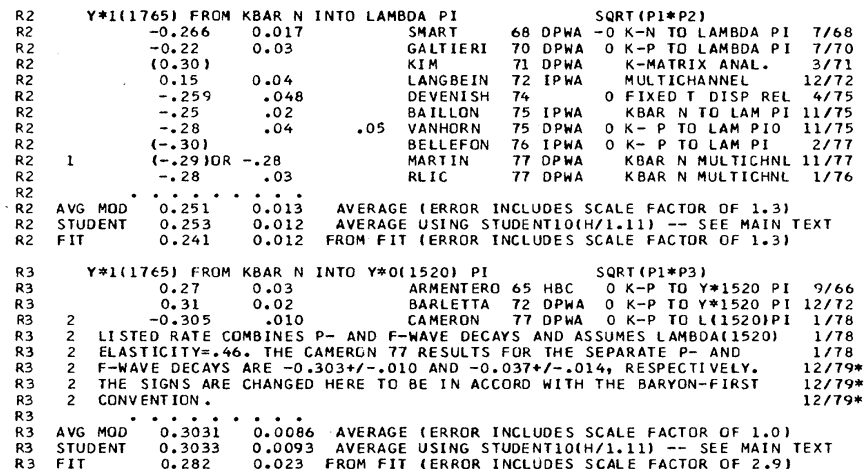




\section{Baryons \\ $\Sigma(1765), \Sigma(1770), \Sigma(1840)$}

\section{Data Card Listings For notation, see key at front of Listings.}

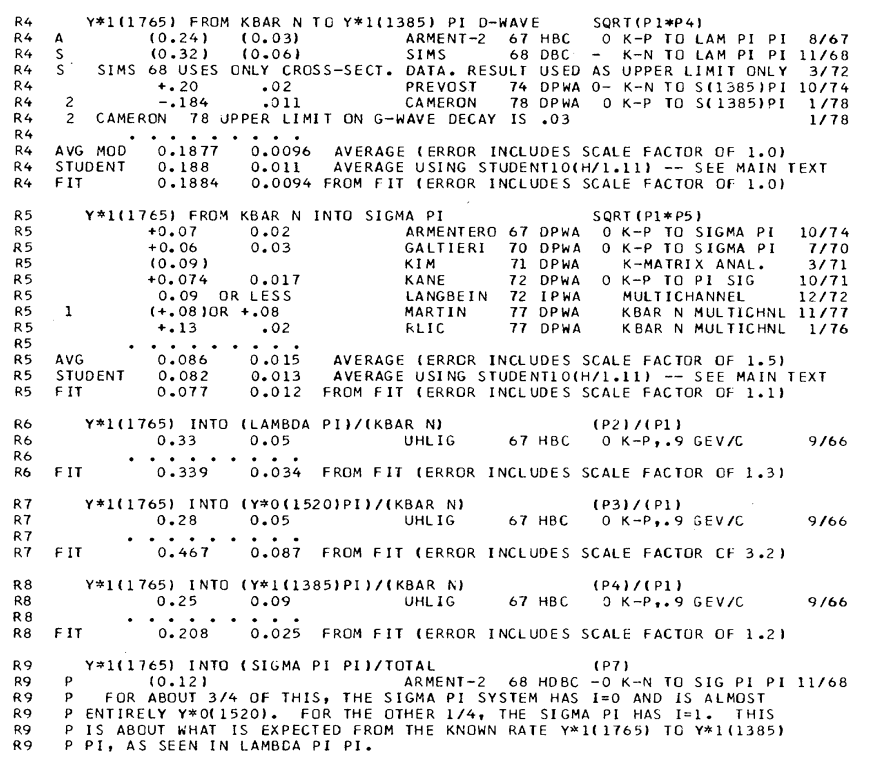

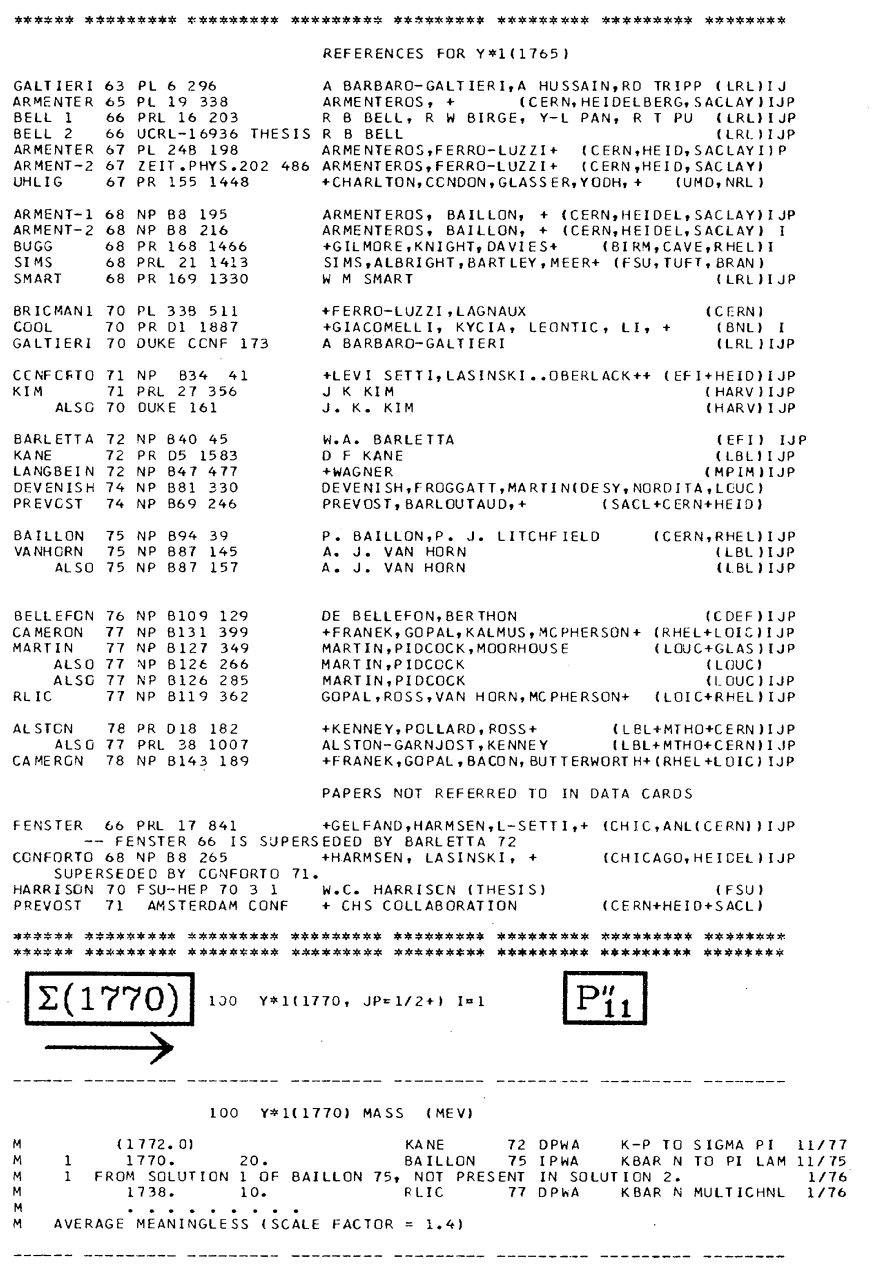

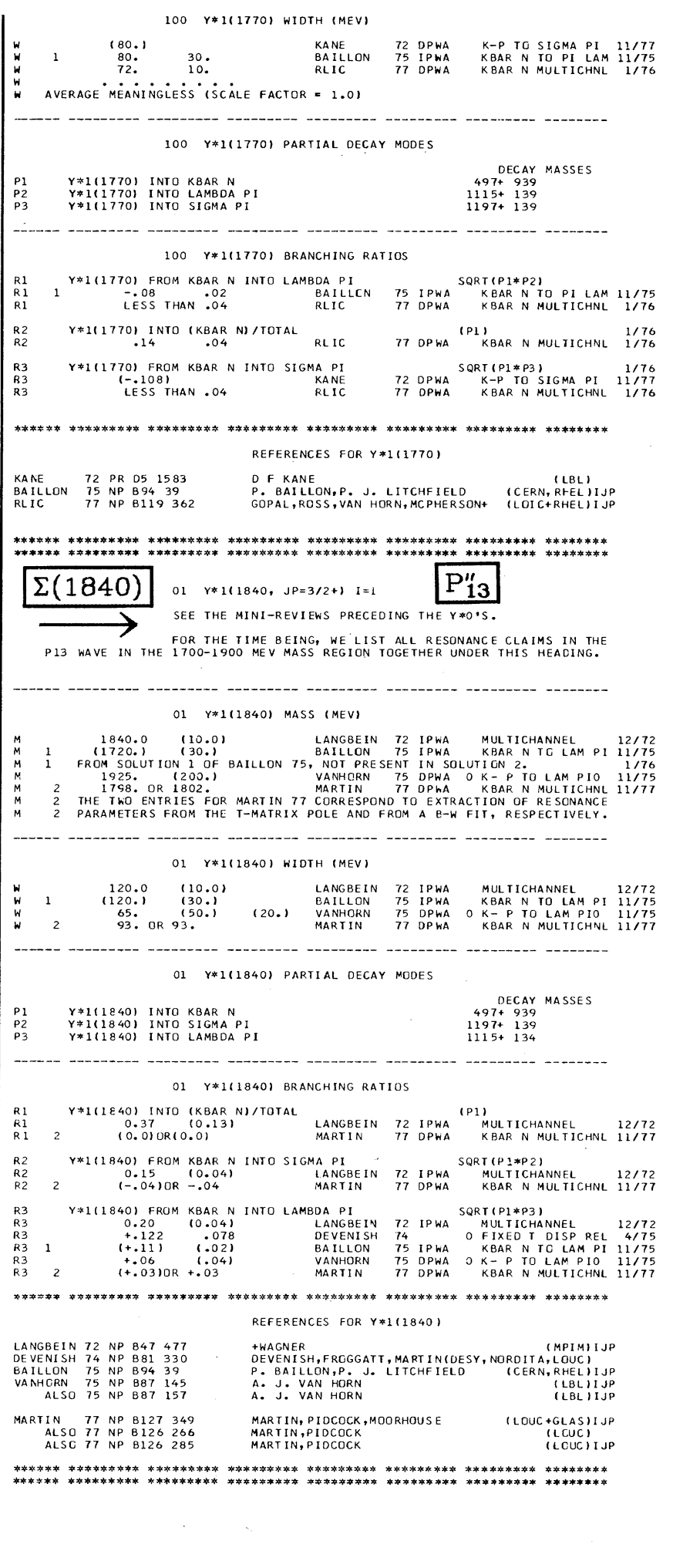


Data Card Listings

\section{Baryons \\ $\Sigma(1880), \Sigma(1915)$}

For notation, see key at front of Listings.

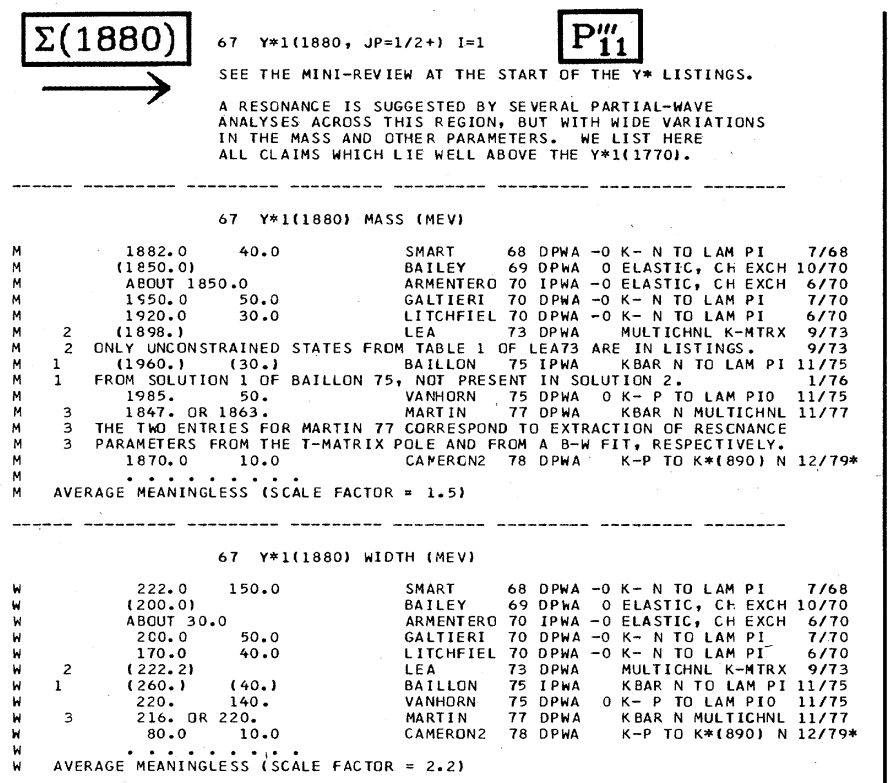

$67 Y * 1(1880)$ PARTIAL DECAY MODES

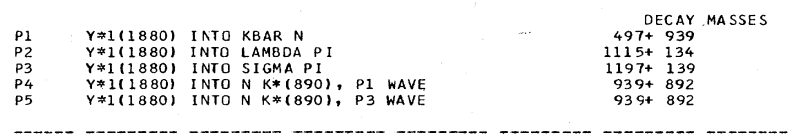

$67 \%$ Y(1880) BRANCHING RATIOS

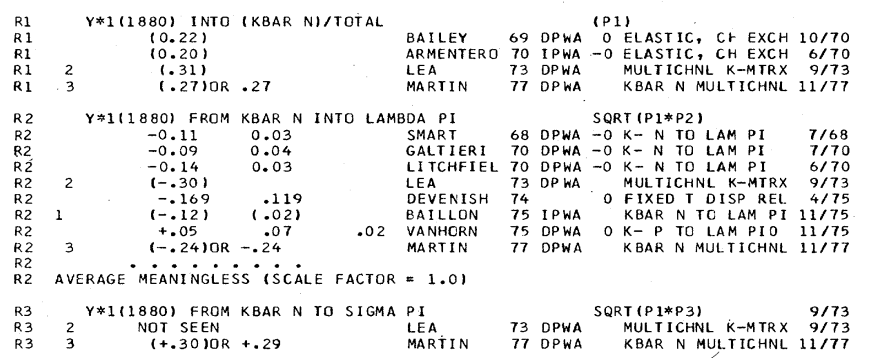

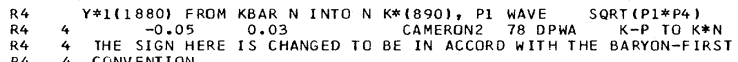
$\begin{array}{llll}R 5 & Y * 1(1880) \text { FROM KBAR N INTO N K*(890), P3 WAVE } \\ \text { R5 } & +0.11 & 0.03 & \text { CAMERON2 } \\ 78 \text { DPWA } & \text { SQRT (P1*P5) } \\ K \rightarrow P \text { TO K*N } & 12 / 79 *\end{array}$ \#************************************************************************

REFERENCES FOR $Y * 1(1880)$

$\begin{array}{llll}\text { SMART } & 68 \text { PR } 1691330 & \text { W M SMART } & \text { (LRL)IJP } \\ \text { BAILEY } & 69 \text { THESIS UCRL-50617 DAVID SAAL BAILEY } & \text { (LRL LIVERMRE)IJJP }\end{array}$

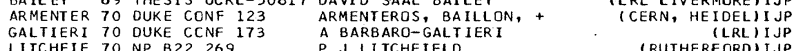

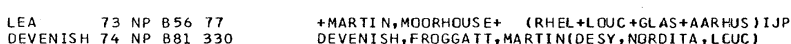
BAILLON 75 NP 89439
VANHORN 75 NP B87 145

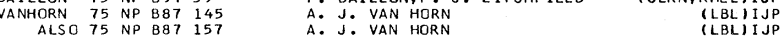
$\begin{array}{cccc}\text { MART IN } 77 \text { NP } 8127349 & \text { MARTIN,PIDCOCK, MOORHOUSE } & \text { (LOUC+GLAS) IJP } \\ \text { ALSO } 77 \text { NP B126 } 266 & \text { MART IN,PIDCOCK } & \text { (LOUC) }\end{array}$

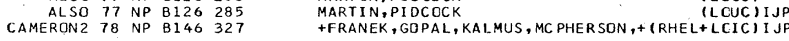

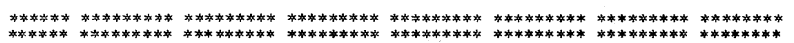

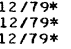

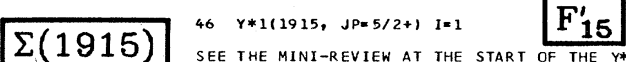

THE Y* LISTINGS. THIS RESONANCE WAS FIRST SEEN IN THE TOTAL-CROSS-SEC-
TION MEASUREMENTS OF COOL 66. IN THIS ENTRY, HOWEVER,

WEE LIST ONLY THE RESULTS FROM PARTIAL-WAVE ANALYSES.
SEE THE NEXT ENTRY FOR. THE PARAMETERS OF PEAKS SEEN AROUND. $1900-1950$

MEV IN CROS SECTIONS AND INVARIANT-MASS DISTR IBUTIONS. WE MAKE THIS
SEPARATION BECAUSE ONLY THE PARTIAL-WAVE ANALYSES ISOLATE THE FIS WAVE. SEE ALSO THE NOTE TO THE NEXT ENTRY

$46 \quad Y * 1(1915)$ MASS (MEV)

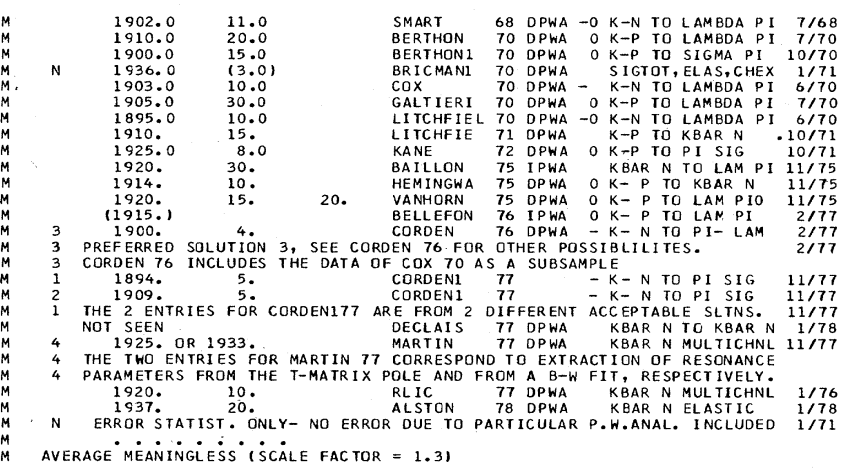
$46 Y * 1(1915)$ WIDTH (MEV)

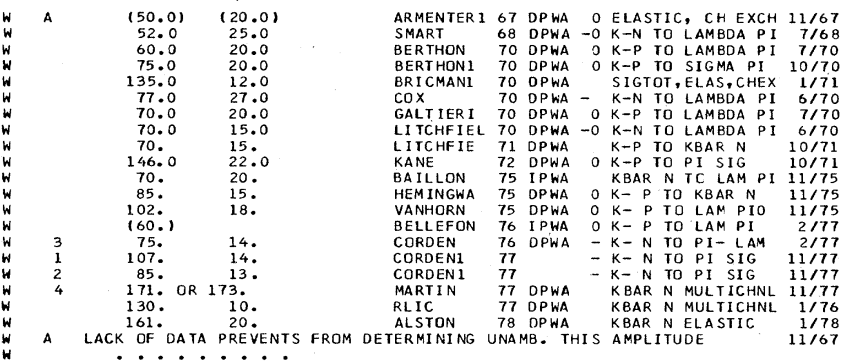

average Méaningie SS 1 iscale factor $=1.91$

\section{$46 \quad Y * 1(1915)$ PARTIAL DECAY MODES}

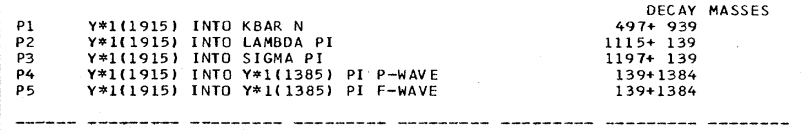

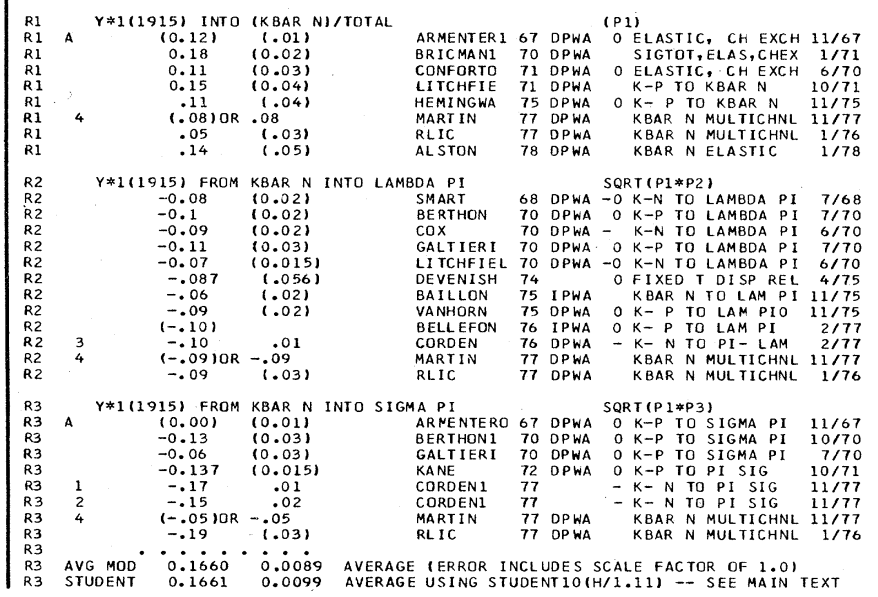


Baryons $\Sigma(1915), \Sigma(1940)$
Data Card Listings For notation, see key at front of Listings.

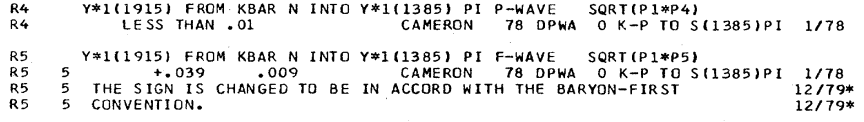
************************************************************************* REFERENCES FOR $Y * 111915$

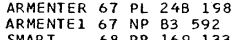

BERTHON $70 \mathrm{NP} B 20476$ BRICMAN1 $70 \mathrm{PL} 33 \mathrm{~B} 521$

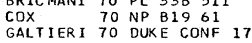

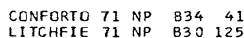

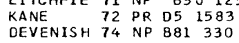

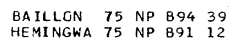

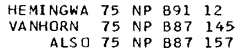
BELLEFON 76 NP 8105129
CORDEN 76 NP B104382 CORDEN1 77 NP B12561 DECLAIS 77 CERN 77-1

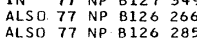
$\begin{aligned} \text { ALSO } & 77 \text { NP. B126 } 285 \\ \text { RLIC } & 77 \text { NP } 8119362\end{aligned}$ ALS ALS 77 PRL 381007
CAMERON 78 NP $B 143189$ ARMENTEROS, FERRO-LUZZI+ ICERN, HEID, SACLAY ARMENTEROS, FERRO-LUZZI+ (CERN, HEID, SACLAY)
(LRL)IJJP +RANGAN, VRANA, + ICOL FRANCE, RHEL, SACLAYIIJP +VRANA, BUTTERWORTH, + ICDEF, RHEL, SACLAYYIJ

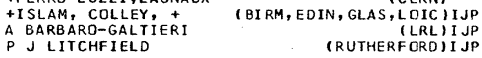

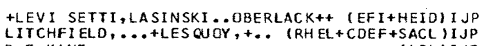

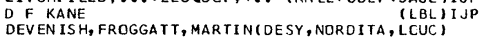

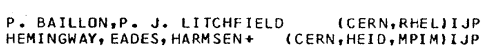

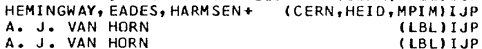
DE BELLEFON, BERTHON
(CDEE) IJP +COX, KENYON, ONEALE, STUBBS, SUMOROKK (BIRM) I JP

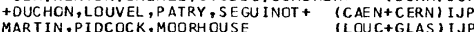
MAR IN,P IDCCCK
MARTIN PIDCCK
(LOOC)
(LOUC)

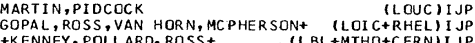
ALSTON-GARNJOST, KENNEY TFRANEK, GOPAL, BACON, BUT TERHORTH (RHEL+LOICIIJP PAPERS NOT REFERRED TO IN DATA CARDS

SMART 66 PRL 17556
SUPERSEDED BY SMART 68.

W M SMART, A KERNAN, G E KaLMUS, R P ELY (LRL)IJP CCNF ORT 68 NP BB 265 265 68.
SUPERSEDED BY CONFORTO 71.

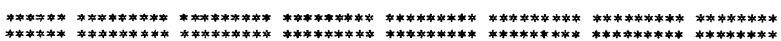
1915 MEV REGION - PRODUCTION AND $\sigma_{\text {TOTAI EXP'TS }}$ $29 \quad Y * 111915, J P=\quad I I=1 \quad$ PRODUCTION EXPERIMENTS SEE THE MINI-REVIEW at THE START OF THE $Y *$ LISTINGS. SEE THE NOTES TO THE Y*1(1915) AND Y*1(1940), WHICH IMMEDIATEL Y PRECEDE AND FOLL OW THIS ENTRY. HERE WE
LIST ONLY PARAMETERS OF PEAKS SEEN IN CROSS SECTIONS AND INVARIANT-MASS DISTRIBUTIONS. YHE CROSS SECT ION PEAKS ARE ALMOST
CERTAINLY ASSOCIATED WITH THE FIS Y $1(1915)$ SEEN IN PARTIAL-WAVE CERTAINLY ASSOCIATED WITH THE F15 Y*111915) SEEN IN PARTIAL-WAVE
ANALYSES. THE INVARIANT-MASS PEAKS SEEM MORE LIKELY TO BE ASSOCIATED WNALYSES. THE INVART

$29 Y * 1(1915)$ MASS (MEV) (PROD. EXP.)

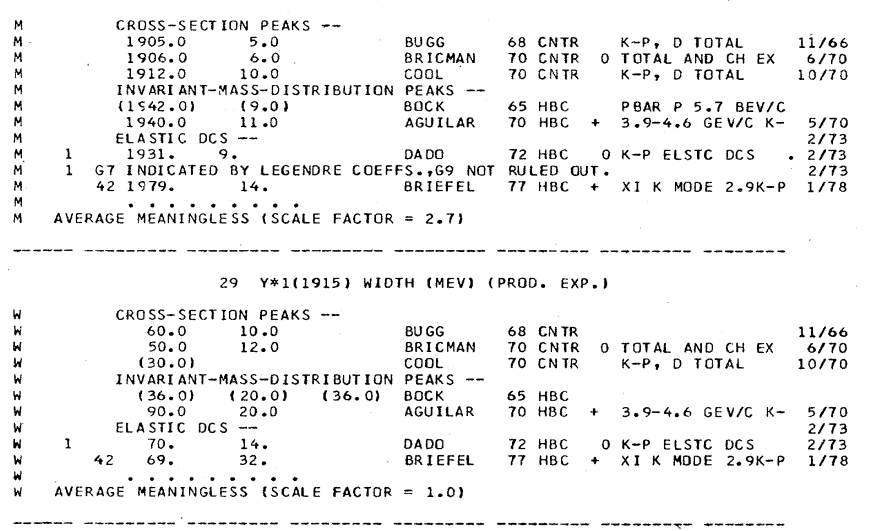

$29 Y * 1(1915)$ PARTIAL DECAY MODES (PROD. EXP.)

$\begin{array}{ll}P_{1} & Y * 1(1915) \text { INTO KBAR N } \\ P_{2} & Y * 1(1915) \text { INTO LAMBDA PI } \\ P_{3} & Y * 1(1915) \text { INTO SIGMA PI } \\ P_{4} & Y * 1(1915) \text { INTO XI K }\end{array}$

DECAY MASSES

$497+939$
$1115+134$
$1197+139$

$1197+139$
$29 Y * 1(1915)$ BRANCHING RATIOS (PROD. EXP.)

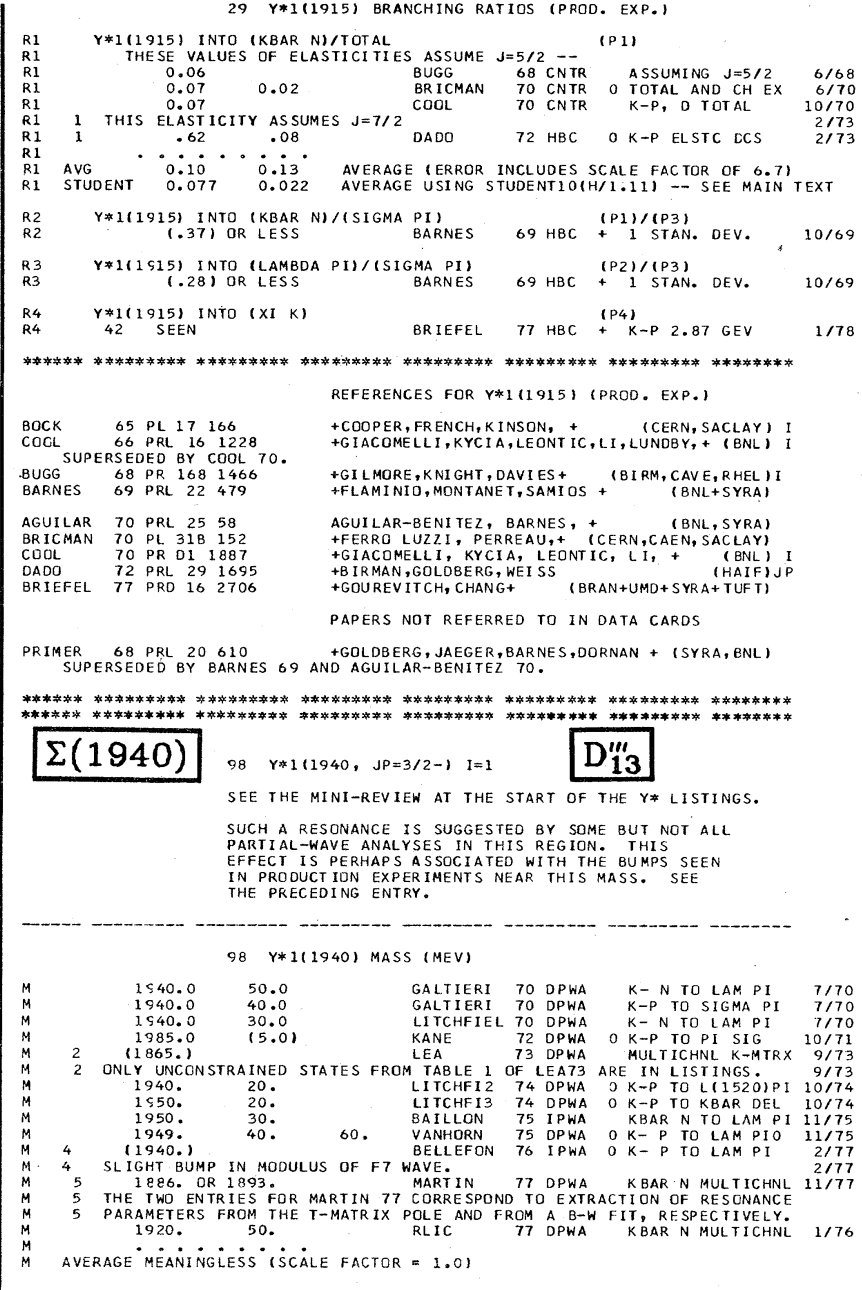

$98 \quad$ \$1(1940) WIDTH (MEV)

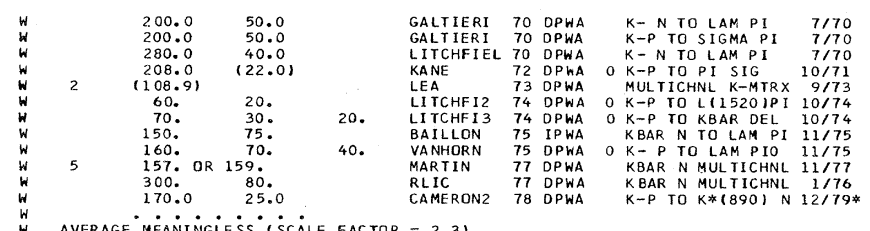

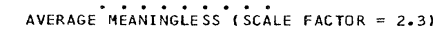

$98 Y * 1(1940)$ PART IAL DECAY MODES

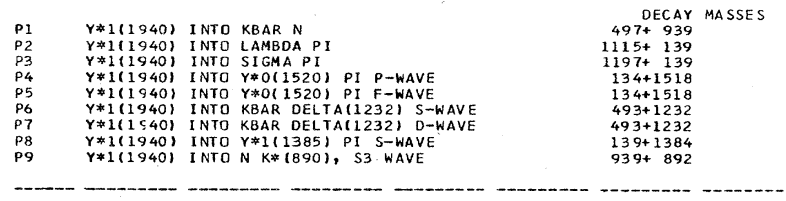




\section{Data Card Listings}

Baryons
$\Sigma(1940), \Sigma(2000), \Sigma(2030)$

For notation, see key at front of Listings.

$\Sigma(1940), \Sigma(2000), \Sigma(2030)$

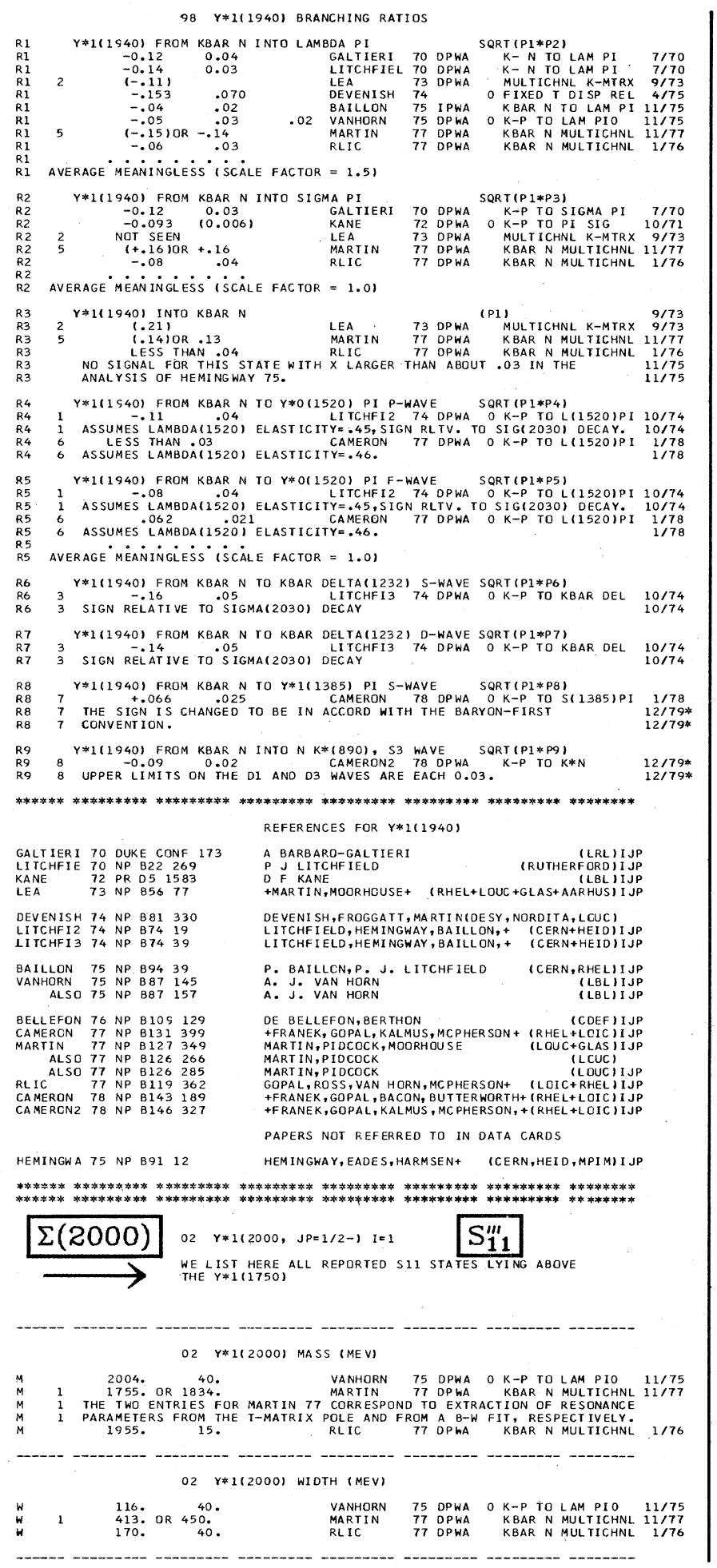

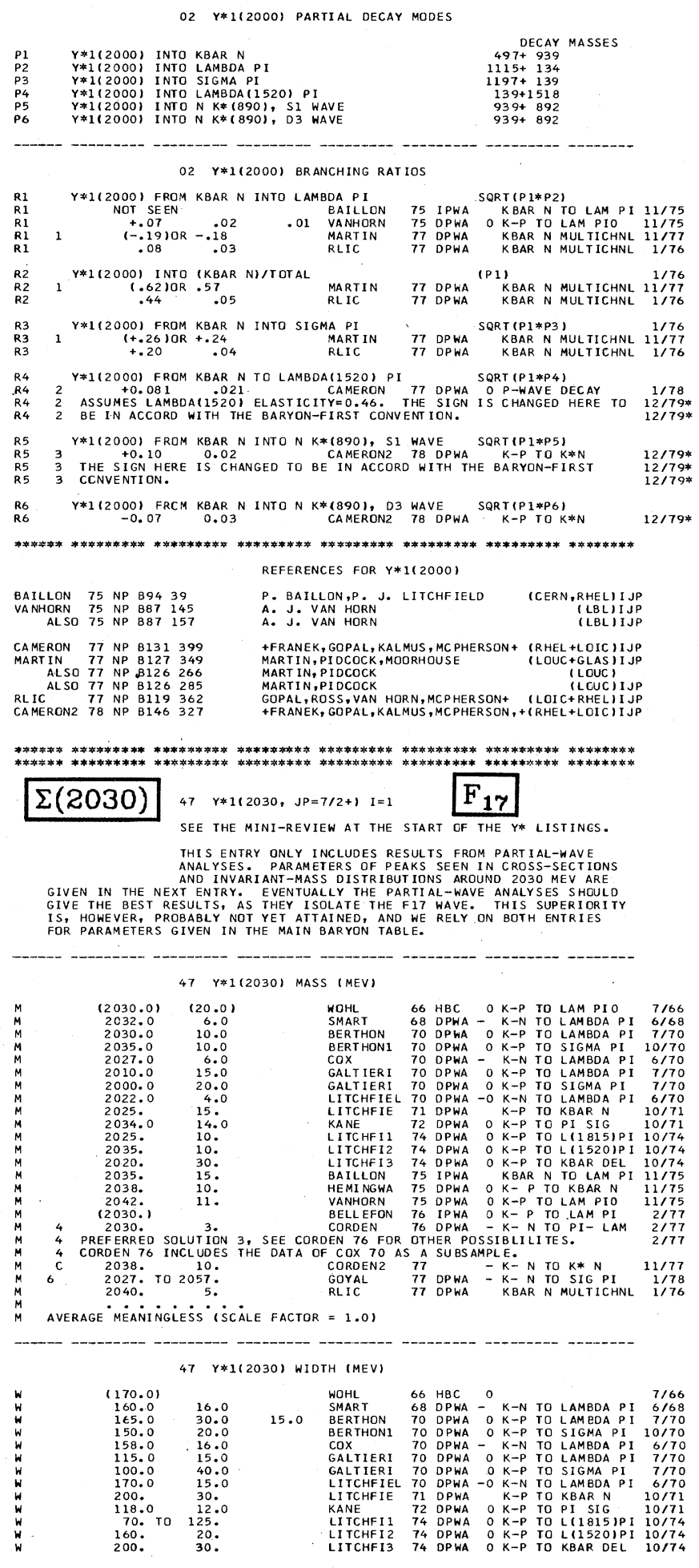




\section{Baryons $\Sigma(2030)$}

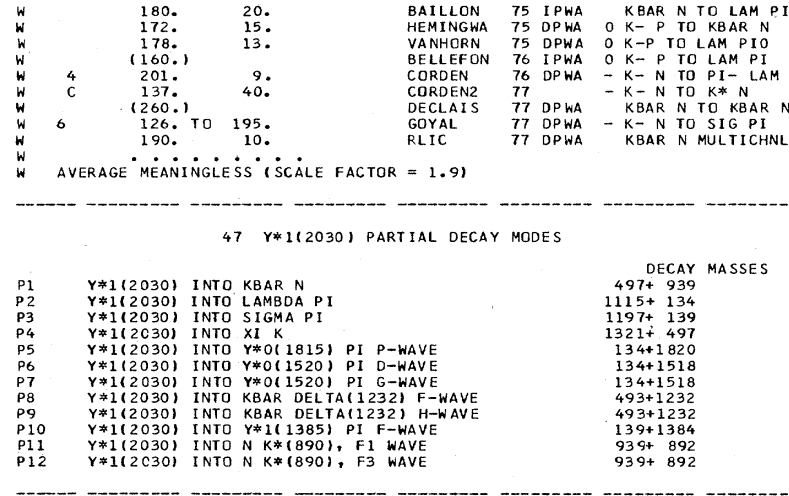

47 Y*1(2030) BRANCHING RATIOS

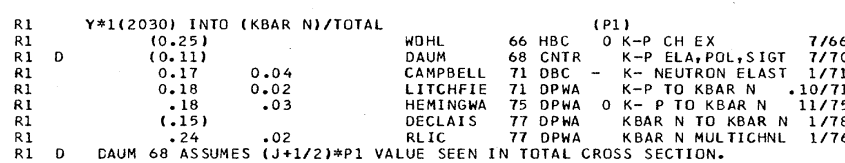

R1 AVERAGE MEÁAINGLESS (SCALE FACTOR $=1.4$ )

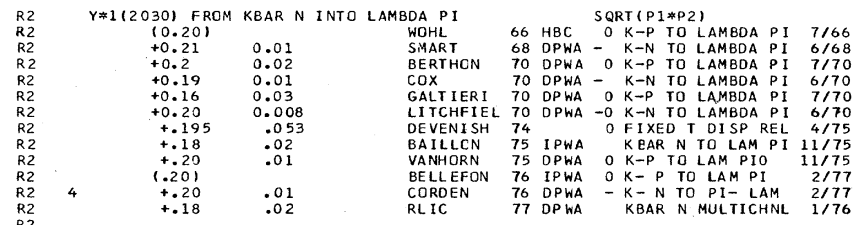

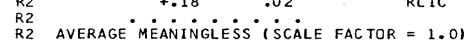

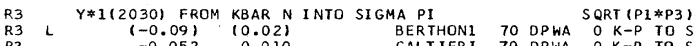

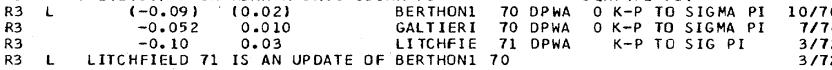

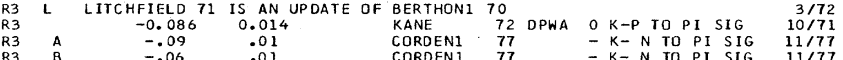

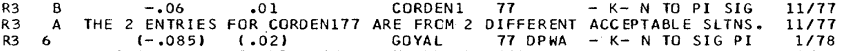

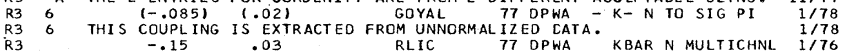

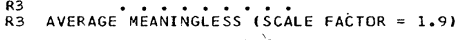

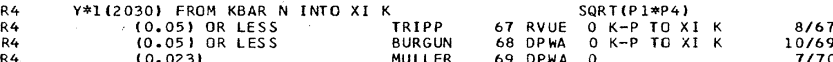

5 Y*1(2030) FROM KBAR N TO Y*O(1815) PI P-HAVE SQRT(P $1 * P 5)$

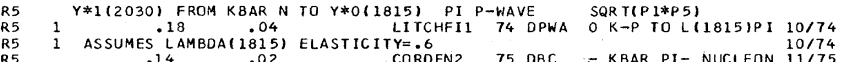

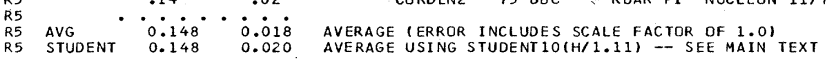

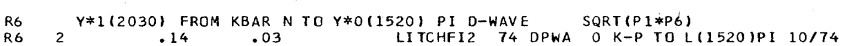

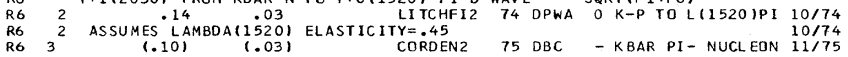

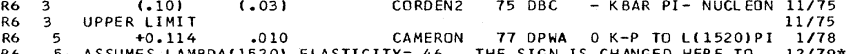

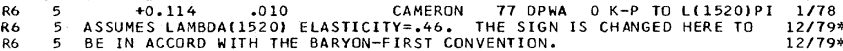

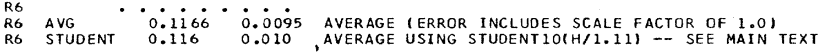

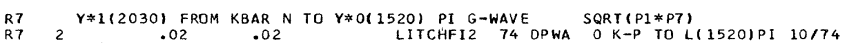

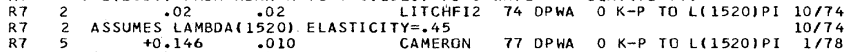

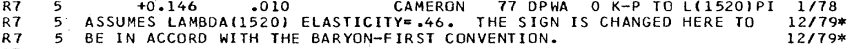

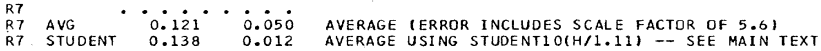

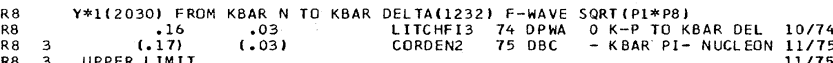

R9 $Y * 1(2030)$ FROM KBAR $N$ TO KBAR DELTA 1232$)$ H-WAVE SQRT $(P 1$ P 1 PQ)

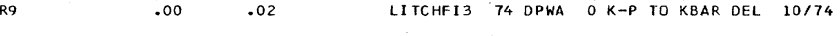

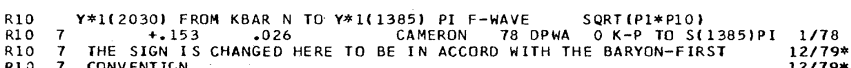

Data Card Listings For notation, see key at front of Listings.

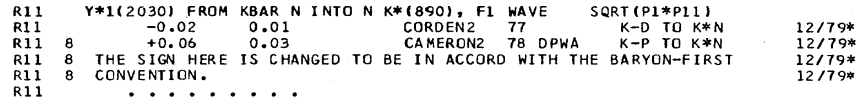

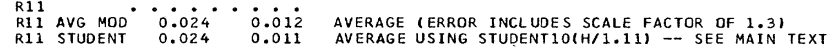

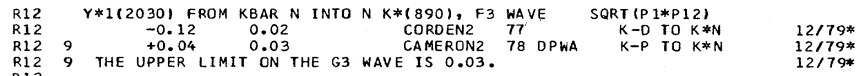

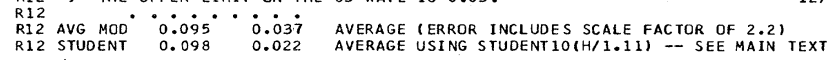

R12 STUDENT 0.0980 .022 AVERAGE USING STUDENT1O(H/1.11) -- SEE MAIN

REFERENCES FOR $Y * 1(2030)$

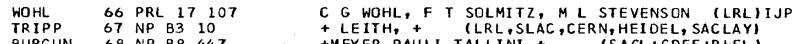

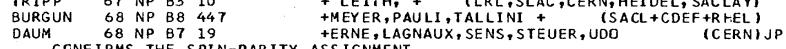

$\begin{array}{ll}\text { CONF IRMS THE SPIN-PARITY ASS IGNMENT. } & \\ \text { SMAR } & 8 \text { PR } 1691336 \\ \text { MULLER } 69 \text { THES IS, UCRL } 19372 \text { R A MMART } & \text { (LRLIIJP } \\ \text { (LRL) }\end{array}$

$\begin{array}{lllll}\text { BERTHON } & 70 & \text { NP } & \text { B } 20 & 476 \\ \text { BERTHON1 } & 70 & \text { NP } & \text { B24 } & 417\end{array}$

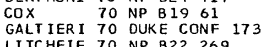

+RANGAN, VRANA, + +COL FRANCE, RHEL, SACLAYIIJP
+VRANA, BUTTERHORTH, + ICDEF, RHEL, SAC LAYIIJP

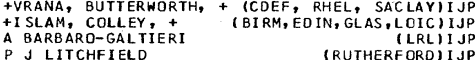

$\begin{array}{lllll}\text { CAMPBELL } & 71 & \text { NP } & 825 & 75 \\ \text { ITCHFIE } & 71 & \text { NP } & B 30 & 125 \\ \text { KANE } & 72 & \text { PR } & D 5 & 1583\end{array}$

+MORTON, NEGUS, GOYAL, MILLER (GLAS, LOIC) IJP

DEVENISH 74 NP B 81330

LITCHFIELD,...+LES QUOY,..+ (RHEL +CDEF+SACL IIJP
(LBL) IJP

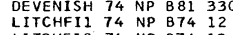

DEVENI SH, FROGGATT, MARTIN IDESY, NORDITA, LCUC,

$\begin{array}{lllll}\text { LITCHFI } & 74 & \text { NP } & B 74 & 19 \\ \text { LITCHFI3 } & 74 & \text { NP } & 874 & 39\end{array}$

$\begin{array}{lllll}\text { BAILLON } & 75 & \text { NP } & 8 \$ 4 & 39 \\ \text { CORDEN2 } & 75 & \text { NP } & 892 & 365\end{array}$

$\begin{array}{lllll}\text { HEMINGWA } 75 & \text { NP } & 891 & 12 \\ \text { VANHORN } & 75 & \text { NP } & 887 & 145\end{array}$

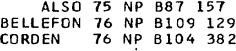

CAMERON 77 NP B 131399

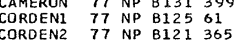

DECLAIS 77 CERN $77-16$

$\begin{array}{lllll}\text { GOYAL } & 77 & \text { PRD } & 16 & 2746 \\ \text { RLIC } & 77 & \text { NP } & \text { B } 119 & 362 \\ \text { CAMERON } & 78 & \text { NP } & \text { B143 } & 189\end{array}$

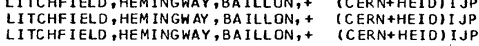

P. BAILLON, P. J. LITCHFIELD (CERN, RHELIIJP
+COX,DARTNELL, KENYON, ONEALE, SUMOROK+ (BIRM) IJP +COX, DARTNELL, KENYON, ONEALE, SUMORDK+ (BIRM) IJP
HEMINGWAY, EADES, HARM SEN + (CERN, HEID, MPIM) I JP

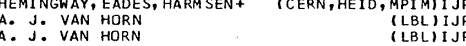
DE BELLEFON, BERTHON
+ COX,DARTNELL, KENYON, ONEALE, SUMOROK+ (CDEF)IJPP
(BIRM) IJP COX,DARTNELL, KENYN , ONEALE, SUMOROK+ ICBRFMIIJP +FRANEK, GOPAL, KALMUS, MC PHERSON+
+ +COX, RENYON, ONEALE, STUBBS, SUMOROK +LOICIIJP
(BIRMIIJP + COX, KENYON, ONEALE, STUBBS, SUMOROK+ (BIRM) IJP D.P.GOYAL, A.V.SODHI SEGUINOT + (DELAE UNIV.)IJP GOPAL, ROSS, VAN HORN, MCPHERS ON+ (LOIC+RHEL IIJP +FRANEK, GOPAL, BACON, BUT TERWORTH+ (RHEL+LCIC) I IJP
+FRANEK, GOPAL, KALMUS, MCPHER SON, + (RHEL+LOIC) I JP

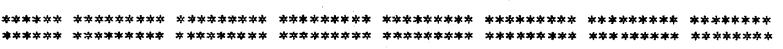

2030 MEV REGION - PRODUCTION AND $\sigma_{\text {TOTAL EXP'TS }}$

$28 Y * 112030$, JPE J IN1 PRODUCT ION EXPERIMENTS

SEE THE MINI-REVIEW AT THE START OF THE Y* Listings.

SEE THE NOIE TO THE F17 Y*1(2030), WHICH PRECEDES THIS
ENTRY. HERE WE LIST ONLY PARAMETERS OF PEAKS IN CROSS

SECT IONS AND INVARIANT-MASS DISTRIBUTIONS. THE CROSS-

SECTION PEAKS ARE AT LEAST DOMINANTLY ASSOCIATED WITH THE Y*1120301,
BUT, MAY CONTAIN A SMALL CONTRIBUTION FROM THE SUGGESTED BUT NOT ESTAB-

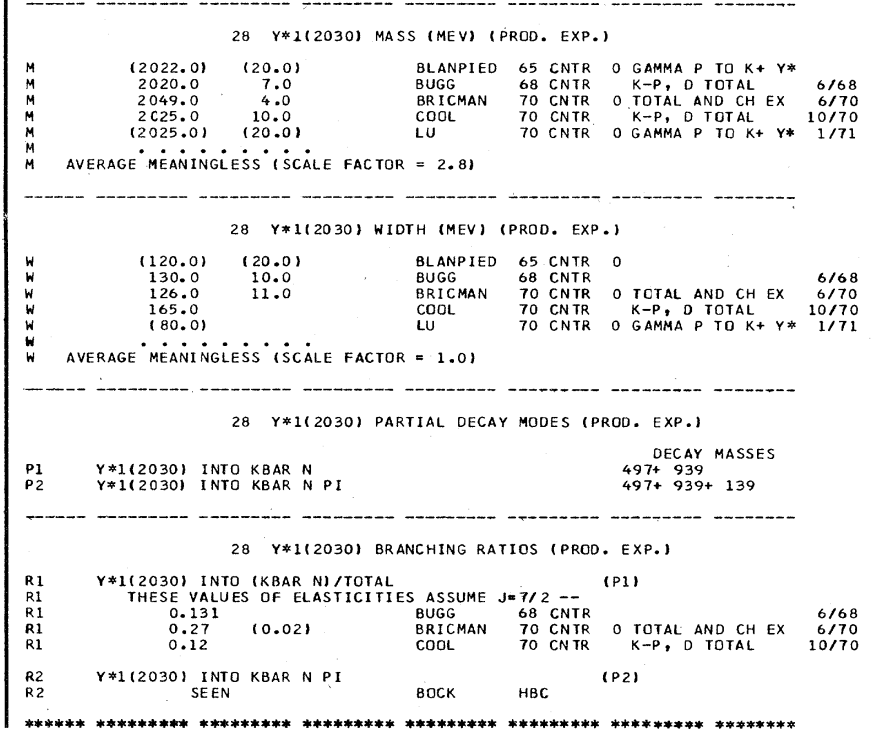

Rev. Mod. Phys., Vol. 52, No. 2, Part II, April 1980 


\section{Data Card Listings}

Baryons

For notation, see key at front of Listings. $\Sigma(2030), \Sigma(2070), \Sigma(2080), \Sigma(2100), \Sigma(2250)$

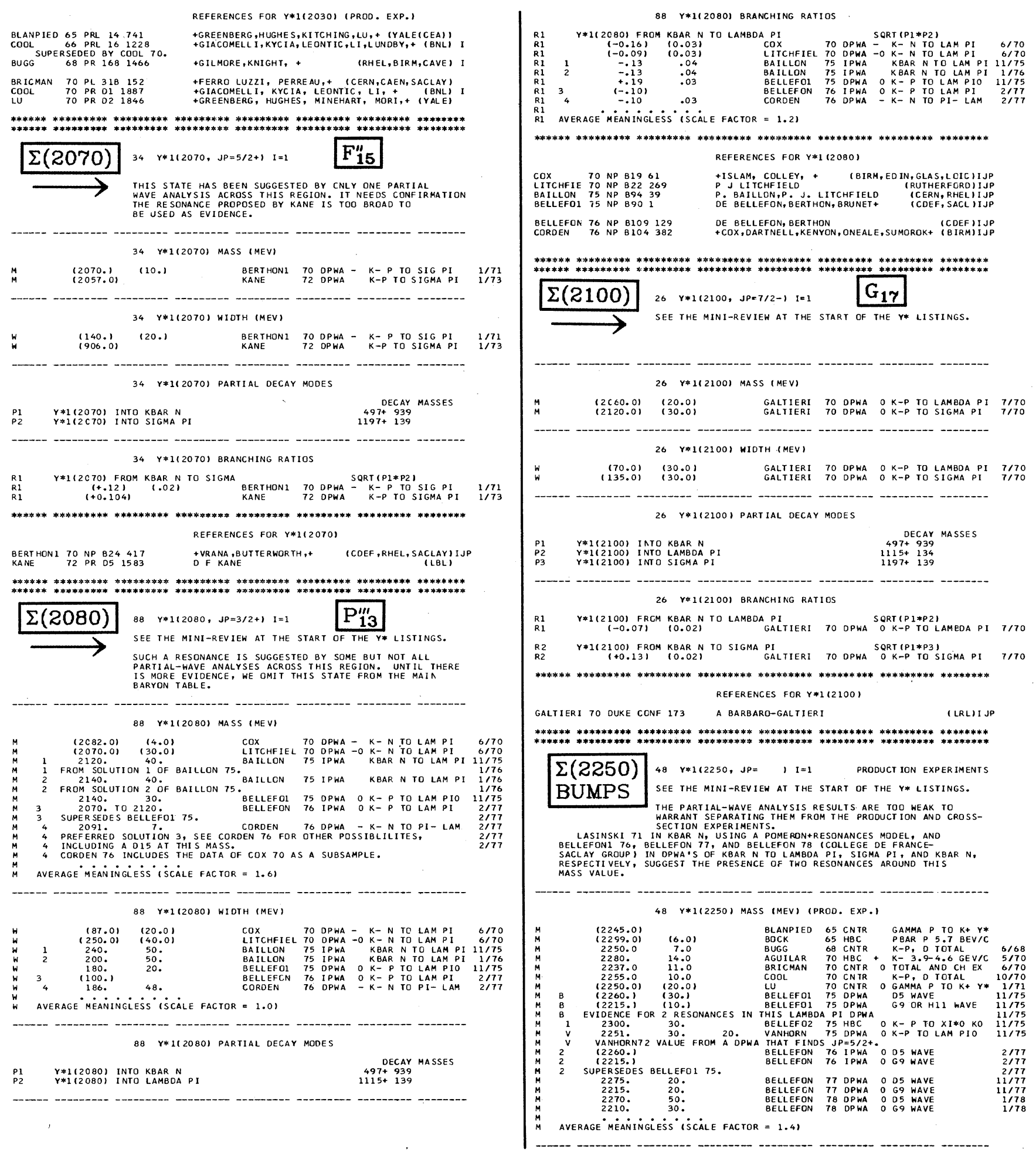




\section{Baryons \\ $\Sigma(2250), \Sigma(2455), \Sigma(2620)$}

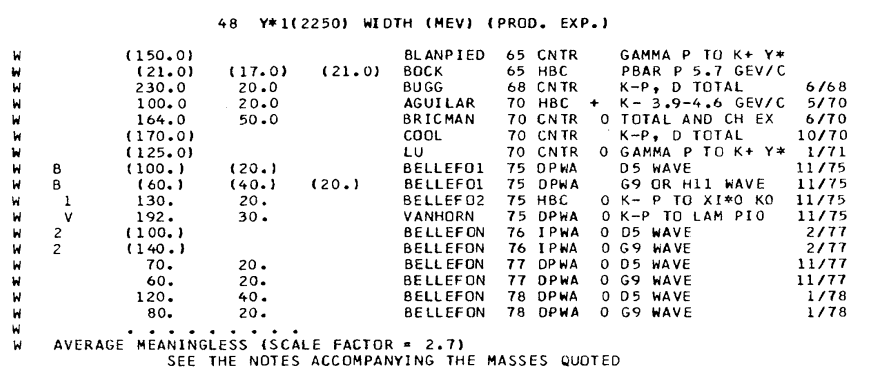

48 Y*1(2250) PART IAL DECAY MODES (PROD. EXP.)

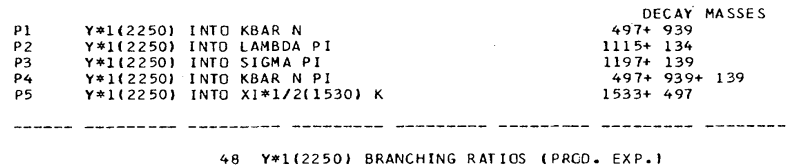

$48 \quad Y * 1(2250)$ BRANCHING RATIOS (PRGD. EXP.)

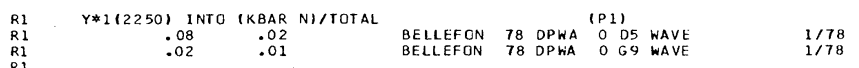

R1 AVERAGE MEÁANI NGGLE SS 1 SCALE FACTOR $=2.71$

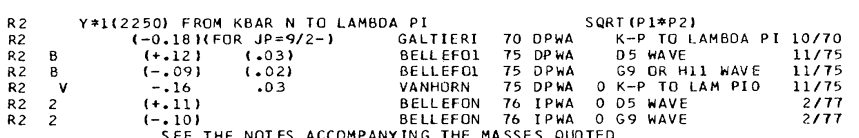

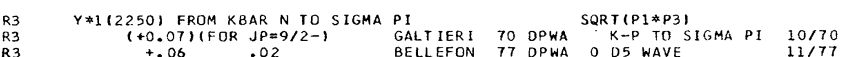

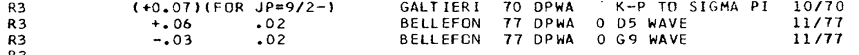

R3 AVERAGE MÉAN INGLE RS $^{\circ}$ i SCALE FAC TOR $=1.11$

R4 $Y * 1(2250)$ INTO (KBAR N)/(ISIGMA PI)
R4 10.18$)$ OR LESS

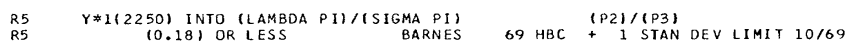

R6 $Y * 1(2250)$ FROM $K-P$ TO $X I * 1 / 2(1530) 0$ KO 75 HBC $\begin{gathered}\text { SQRT } \\ R-P 1 * P 5)\end{gathered}$

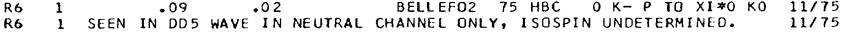

R7
$R 7 * 1(2250)$ INTO (KBAR N)/TOTAL
$R 7$
$R 7$

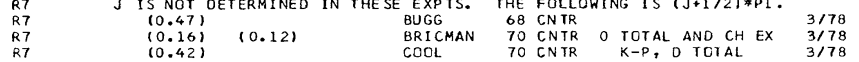

\#**********************************************************************1

REFERENCES FOR Y*1(2250) (PROD. EXP.)
BLANPIEO 65 PRL 14,741 TGRENBERG, HUGHES,KITCHING, + (YALE(CEA)

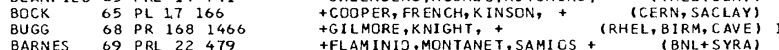

$\begin{array}{lllll}\text { AGUILAR } & 70 & \text { PRL } & 25 & 58 \\ \text { BRICMAN } & 70 & \text { PL } & 318 & 152\end{array}$

$\begin{array}{lll}\text { CCOL } & 70 \text { PR D1 } 1887 \\ \text { GALTIERI } & 70 & \text { DUKE CONF } \\ \text { LU } & 70 & \text { PR D2 } 1843\end{array}$

AGUILAR-BENITEZ, BARNES, + (BNL, SYRA)

+FERRO LUZZI, PERREAU, (CERN, CAEN, SACLAY)
GIACOMELLI, KYCIA, LEONTIC, LI, + (BNL) I

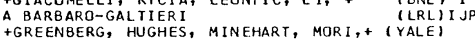

$\begin{array}{lllll}\text { BELLEFO1 } & 75 & \text { NP } & \text { B90 } & 1 \\ \text { BELLEFO2 } & 75 & \text { NC } & 28 A & 289\end{array}$

$\begin{array}{ccccc}B E L L E F O 2 & 75 & N C & 284 & 289 \\ \text { VANHORN } & 75 & \text { NP } & B 87 & 145 \\ \text { ALSO } & 75 & \text { NP } & 887 & 157\end{array}$

$\begin{array}{ll}\text { DE BELLEFO2, BERTHON, BRUNET+ } & \text { (CDEF+SACL)IJ JP } \\ \text { DE BELLEFD2, BERTHCN, BILLOIR+ } & \text { (CDEF, SACL) }\end{array}$

$\begin{array}{lr}\text { DE BELEFO2,BERTHON, BILLLIR+ } & \text { (CDEF, SACL) } \\ \text { A. J. VAN HCRN } & \text { (LBLIIJP } \\ \text { A. J. VAN HORN } & \text { (LBLIIJP }\end{array}$

BELLEFON 76 NP $B 10 S 129$
BELLEFN 77 NC $374 \quad 175$

DE BELLEFON, BER THON
DE BELEFON,BERTHON, BILLOIR+ (COEF)IJP
OCOEF+SAL) IJP

PAPERS NOT REFERRED TO IN DATA CARDS

$\begin{array}{ll}66 \text { PRL } 161228 & \text { +GIACOMELL I, KYCIA, LEONT IC, LI, LUNOBY, + (BNL) I } \\ \text { SUPEREOEO BY COOL } 70 . & \text { SCHLEIN, SLATER, STORK, TICHO (UCLAILRLI) I }\end{array}$ DAUBER 66 PL $23 \quad 154$

+SCHLEIN, SLATER, STORK, TICHO IUCLAILRLI) J
HAVIOR IN SIGMA- PI+, BUT APPEARS

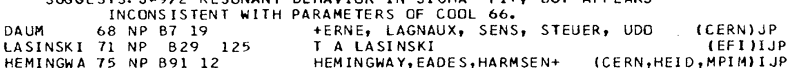

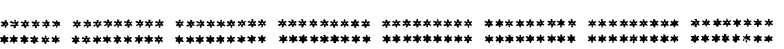

Data Card Listings For notation, see key at front of Listings.

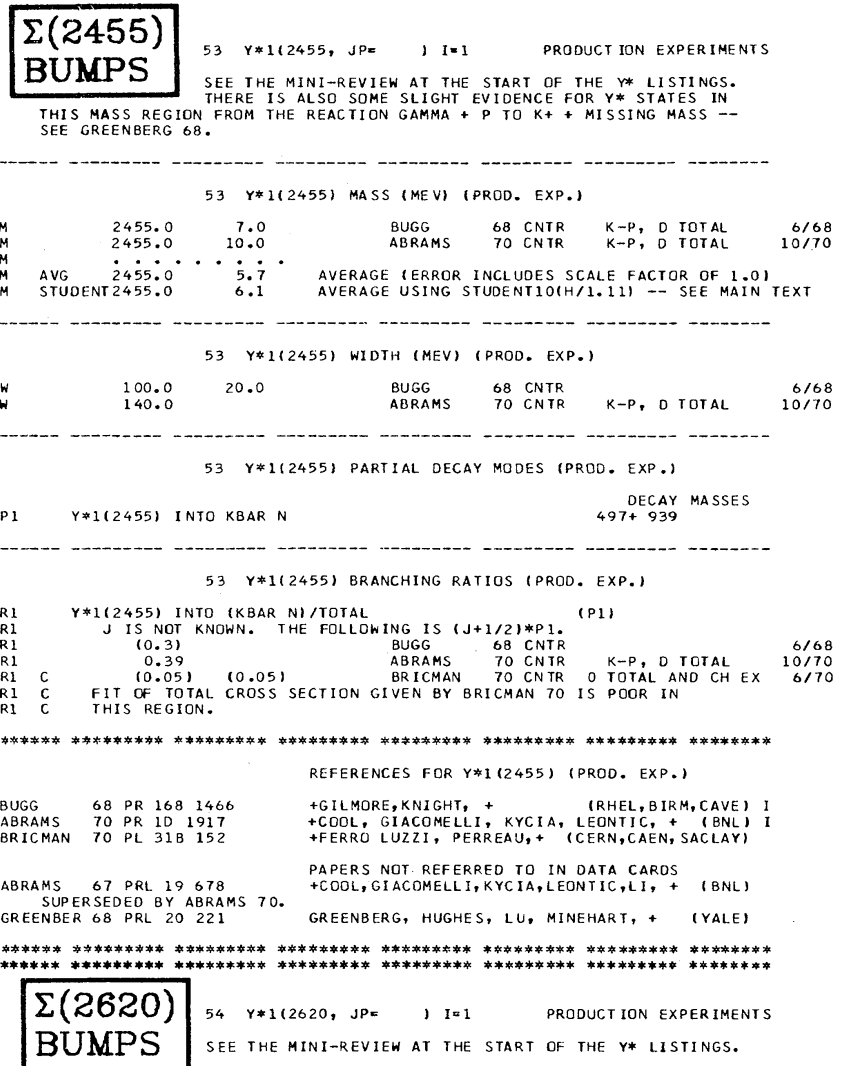

M

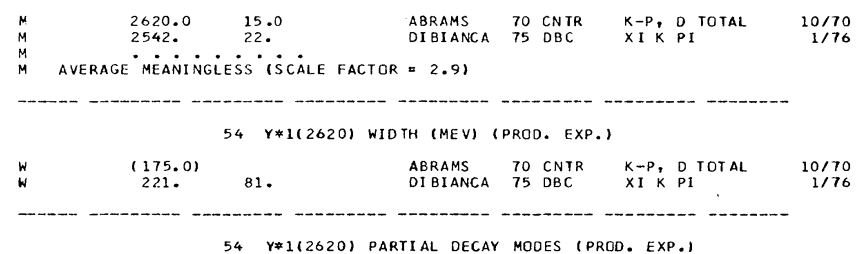

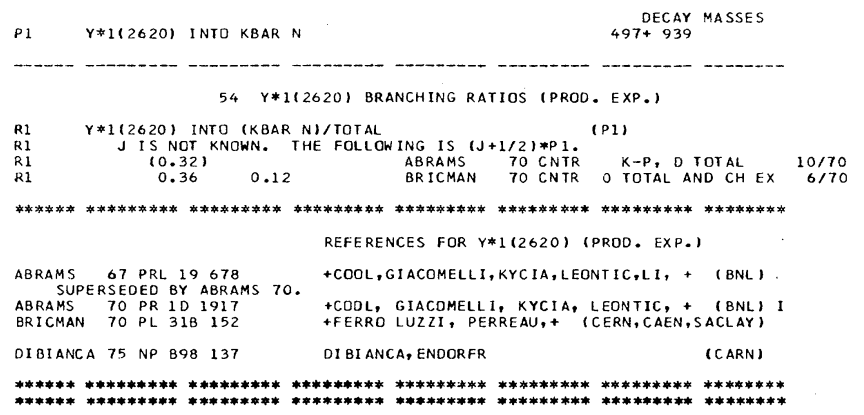


Data Card Listings

For notation, see key at front of Listings.

\section{Baryons} $\Sigma(3000), \Sigma(3170)$, EXOTIC HYPERONS, $\Xi ' s$

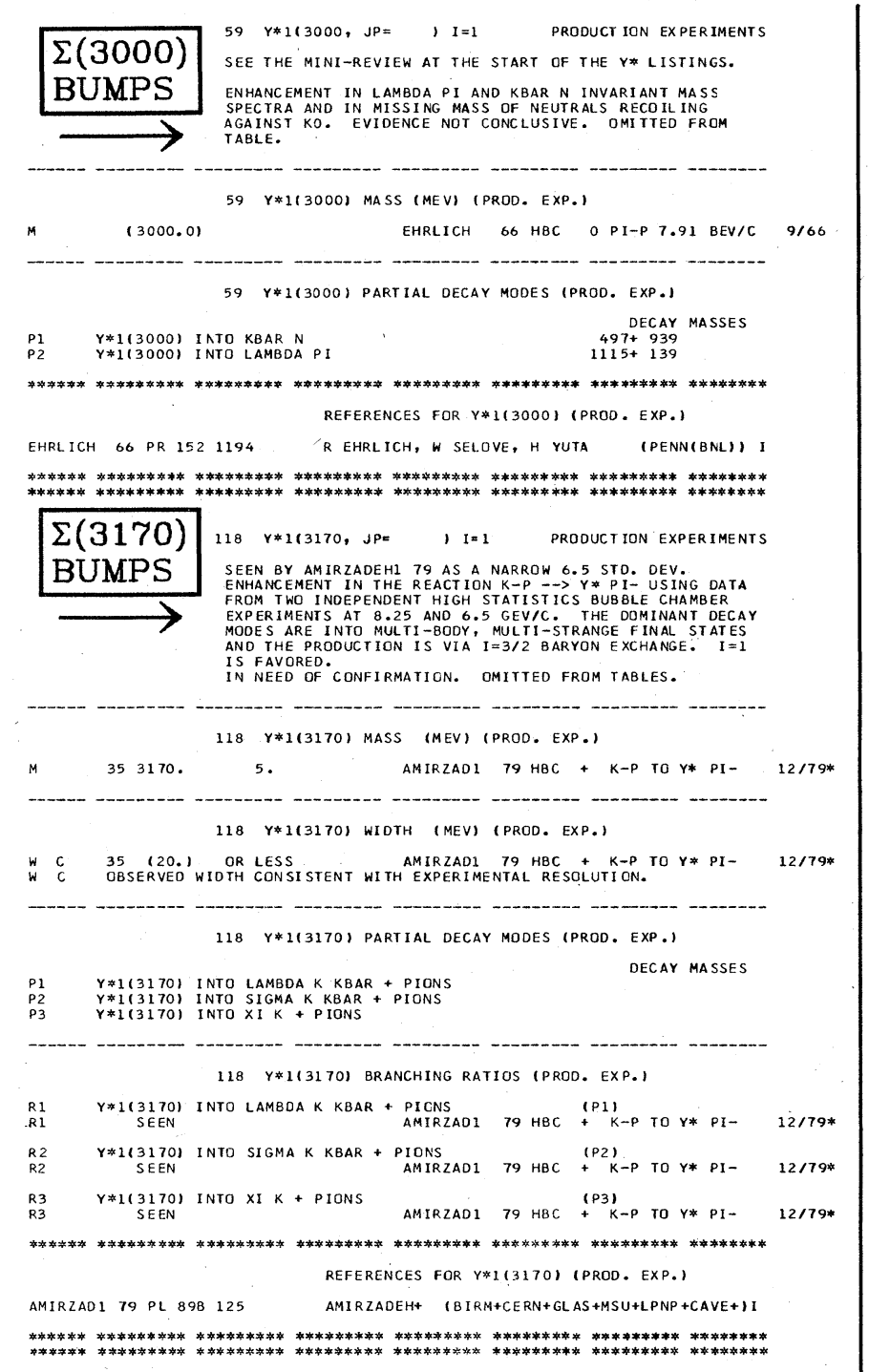

EXOTIC HYPERON CROSS SECTION LIMTS

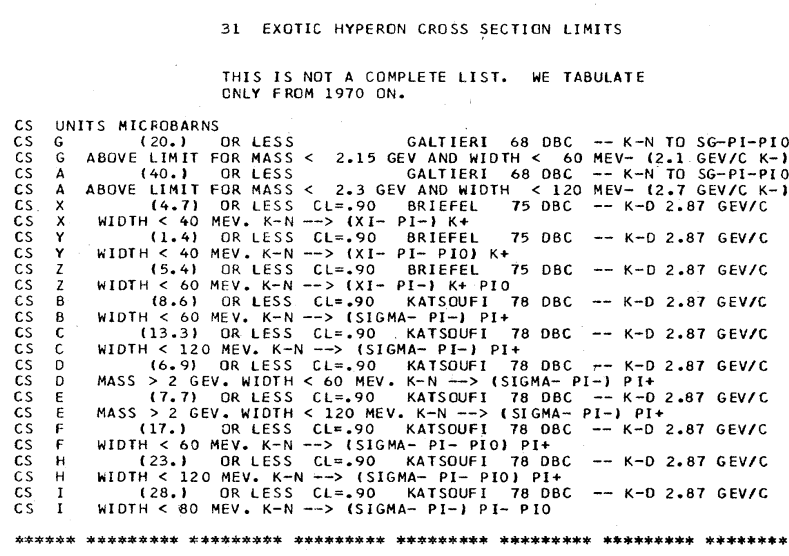

AT BARBaro-gaLIIERI CHAOH

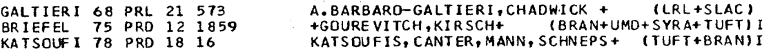

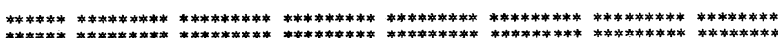
Note on $\Xi$ Resonances

The $\Xi$ resonance situation has long been unsettled. This is mainly because: (1) $\Xi^{*}$ 's can only be produced as part of a final state, $\mathrm{K}^{-} \mathrm{p} \rightarrow \Xi^{*}+$ others, where the analysis is more complicated than if direct formation were possible; (2) they are so-produced with small cross sections (typically a few $\mu \mathrm{b}$ ); and (3) the final states are in general topologically complicated and difficult to study with purely electronic techniques. Thus over the years our knowledge of $\Xi^{*}$ spectroscopy has come wholly from bubble chamber experiments, where the number of events available are small.

Until fairly recently only the $\Xi(1530)$ could be considered as really well established. However, the 1978 edition of this review ${ }^{1}$ saw a significant improvement in our understanding of the $\Xi^{*}$ spectrum with the data of GAY 76 and HEMINGWAY 77. The $\Xi(1820)$ and $\Xi(2030)$ were definitely established as narrow states (with widths $\sim 20 \mathrm{MeV}$ ), and the spin of the $\Xi(1820)$ was determined to be $3 / 2$ (TEODORO 77).

As far as the other $\Xi^{*}$ states are concerned, the situation continues much as before, although there is some evidence for a new $\Xi$ (2370) (AMIRZADEH2 79). There is probably at least one other state in the 1850-2000 MeV region and there are indications of several others above $2000 \mathrm{MeV}$. Indeed, numerous states are predicted to exist below $2500 \mathrm{MeV}$ and the broad $\Xi(1940)$ could well be a mixture of several. ${ }^{2}$ Thus for the time being, we are still forced to group together rather disparate observations and await more new results. The disagreement among various experiments is indicated by means of ideograms in the Data Card Listings.

More new results may shortly be forthcoming from two large bubble chamber experiments currently in progress (MORRIS 75, AMIRZADEH2 79). In addition, future experiments with the MPS at BNL and with hyperon beams at both FNAL and $\mathrm{CERN}^{3}$ may further clarify the situation.

The table following this note gives our evaluation of the status of the $\Xi$ resonances, based on data available at this time. 


\section{Baryons}

$\Xi^{\prime} s, \Xi^{-}, \Xi^{0}, \Xi(1530)$

\section{References}

1. Particle Data Group, Phys. Lett. 75B, 1 (1978).

2. R.J.Hemingway, Proc. of the Topical Conference on Baryon Resonances, Oxford, 1976, edited by R.T.Ross and D.H.Saxon (Science Research Council, Chilton).

3. M. Bourquin et al., Nucl. Phys. B153, 13 (1979). For other references see the Data Card Listings.

STATUS OF XI* RESONANCES
THOSE WITH AN OVERALL STATUS OF *** CR ***** ARE INCLUDED IN THE MAIN BARYON

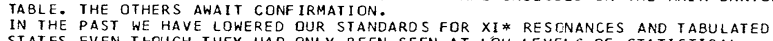

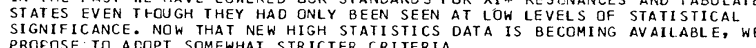

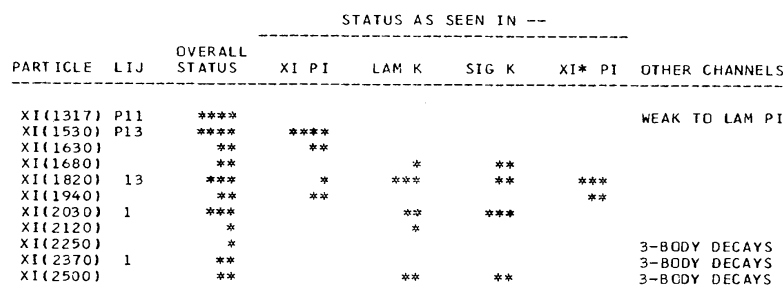

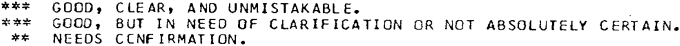
\# WEAK.

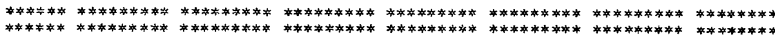
$S=-2 I=1 / 2$ HYPERON STATES (芯)

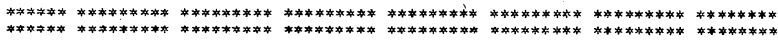

$22 \quad x I-(1321, J P=1 / 2) I=1 / 2$

SeE stable particle data card Listings

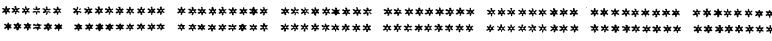

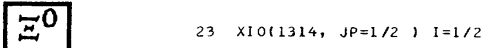

see stable particle data caro I. IStings

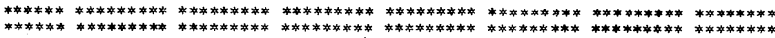

\begin{tabular}{|c|c|c|}
\hline$\Xi(1530)$ & $49 X I * 1 / 2(1530, J P=3 / 2+1) \quad I=1 / 2$ & $P_{13}$ \\
\hline
\end{tabular}

WE DO NOT USE DET ERMINATIONS OF THE MASS AND THE WIOTH OF THIS
STATE UNLESS THEY ARE ACCOMPANIED BY SCME DISUSSION OOF SYSTEMTICS SATE UNLESS THEY DE
AND RESOLUTION.

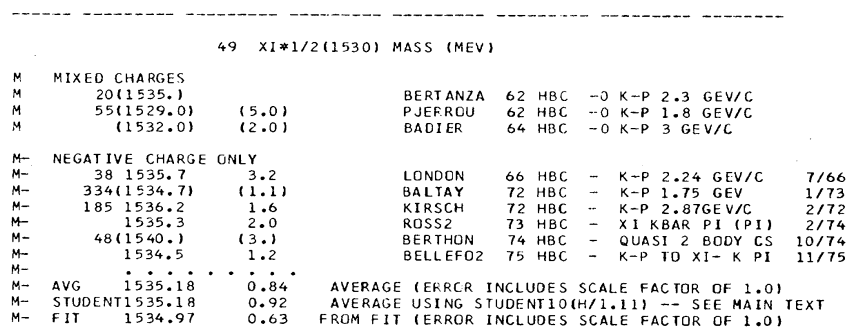

Rev. Mod. Phys., Vol. 52, No. 2, Part II, April 1980
Data Card Listings For notation, see key at front of Listings.

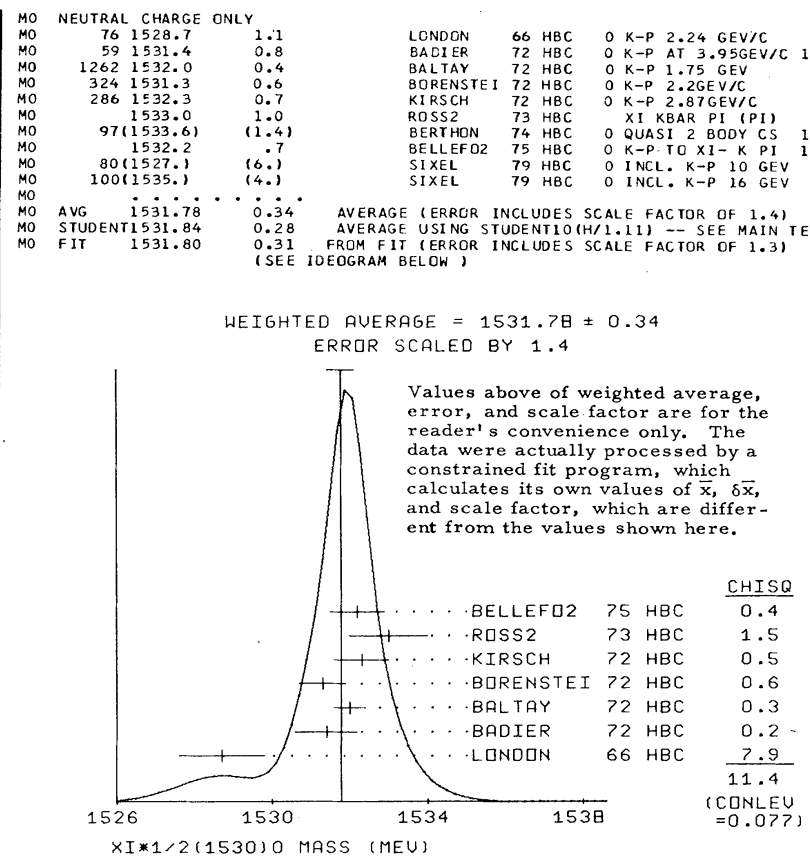

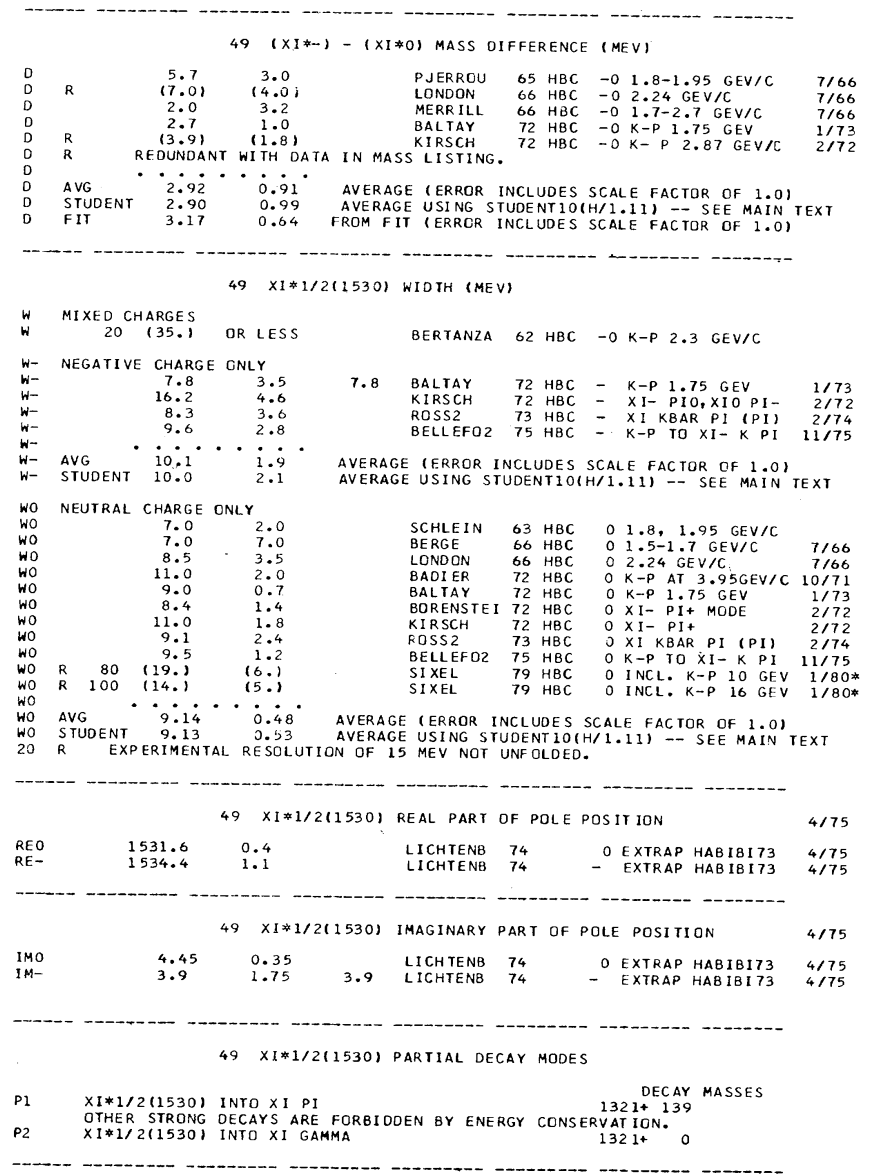




\section{Data Card Listings}

For notation, see key at front of Listings.

\section{Baryons $\Xi(1530), \Xi(1630), \Xi(1680), \Xi(1820)$}

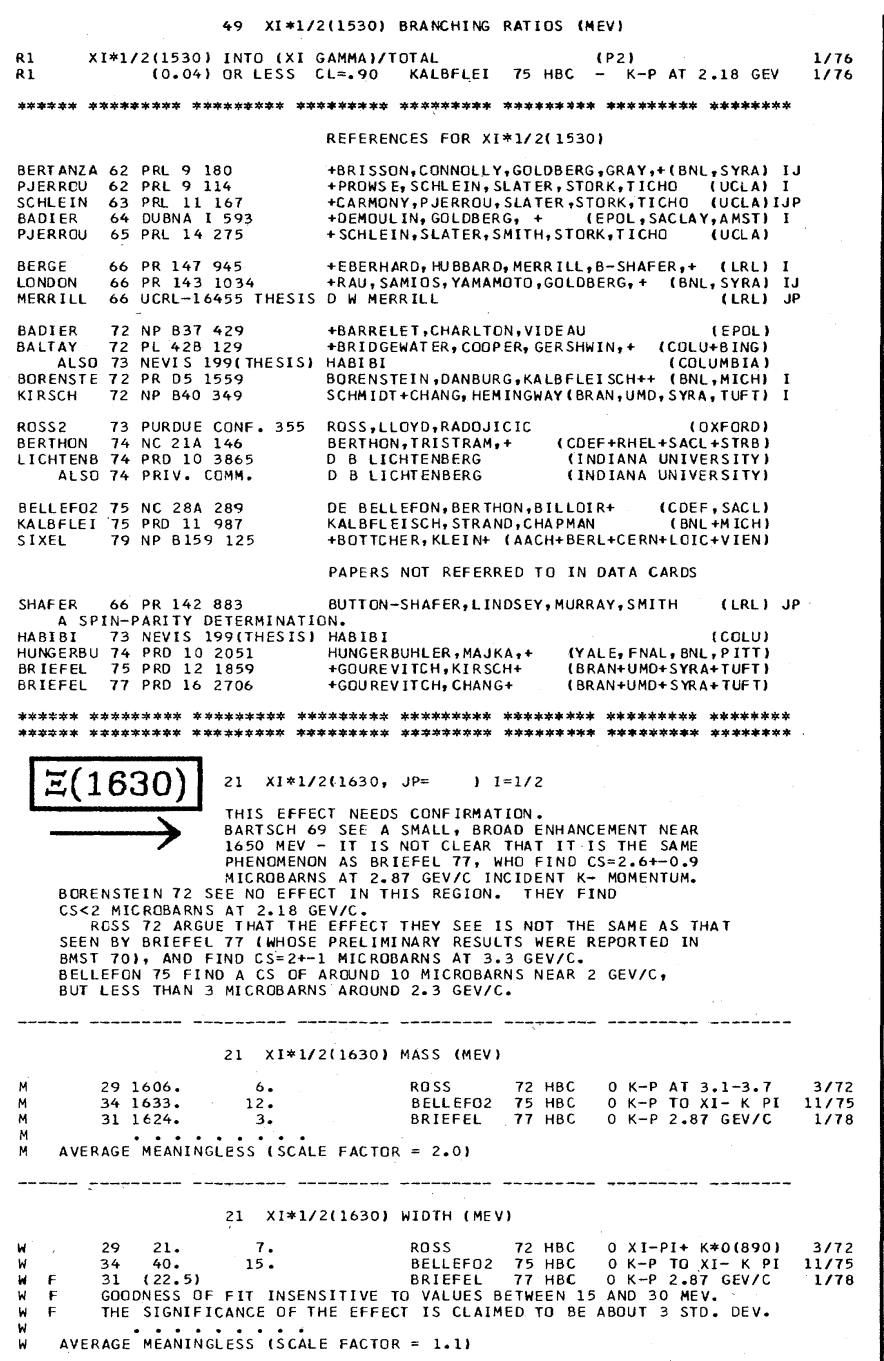

$21 X I * 1 / 2(1630)$ PARTIAL DECAY MODES

$P_{1} X I * 1 / 2(1630)$ INTO $X I P I \quad$ DECAY MASSES SEEN IN K- $P$ TO $X I-P I+$ Ko AND XI- PIO K+.

**********************************************************************1 REFERENCES FOR $X I * 1 / 2(1630)$

$\begin{array}{llllll}\text { ROSS } & 72 & \text { PL } 388177 & \text { BBURAN, LLOYD, MULVEY, RADCJICIC } & \text { (OXF) } \\ \text { BELLEFO2 } & 75 & \text { NC } 28 A & 289 & \text { DE BELLEFON, BERTHON, BILLOIR+ } & \text { (CDEF, SACL) }\end{array}$

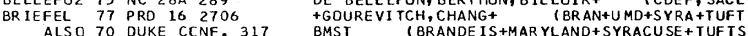
PAPERS NOT REFERRED TO IN DATA CARDS APSELL 69 PRL $23884+$ + (BRANDEIS, MARYLAND, SYRACUSE, TUFTS) SUPERSEDED BY BMST 70.
BARTSCH 69 PL 28843970 (AACHEN, BERLIN, CERN, LOIC, VIENNA)
KALBFLEI 70 DUKE CONF 331 G R KALAFLEISCH I

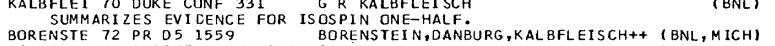
SCHMIDT (BRANDEISI $\begin{array}{llll}\text { HUNGERBU } 74 & \text { PRD } 102051 & \text { HUNG ERBUHLER, MAJKA,+ } & \text { (YALE, FNAL, BNL, PITT } \\ \text { BR IEFEL 75 PRD } 121859 & \text { +GOUREVITCH, KIRSCH+ } & \text { CBRAN+UMD+SYRA+TUFT }\end{array}$

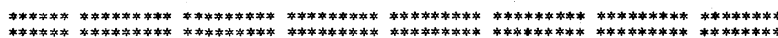

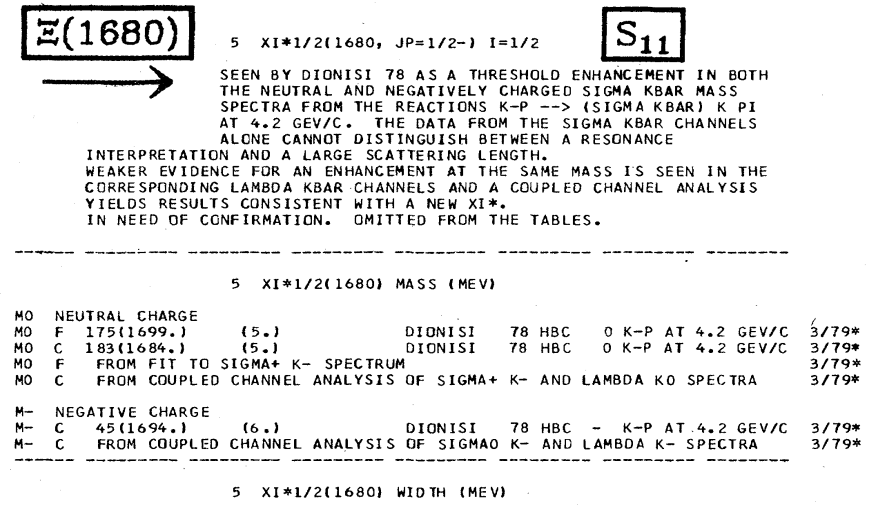

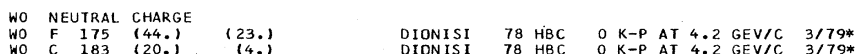

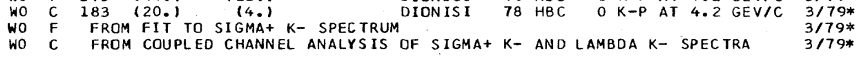

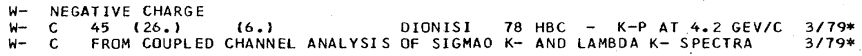

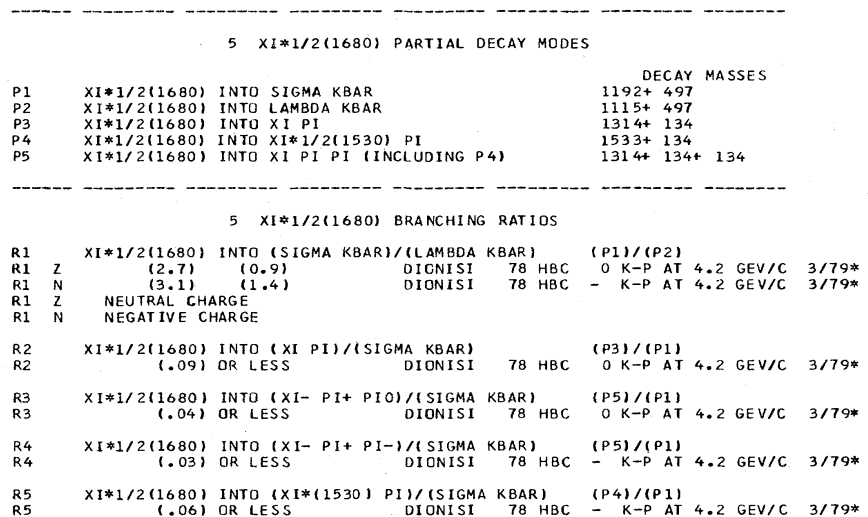
************************************************************************ REFERENCES FOR XI*1/2(1680)
DICNISI 78 PL $80 B 145 \quad$ +DIAZ, ARMENTEROS+ (CERN+AMST+NI JM+ OXF) II, JP

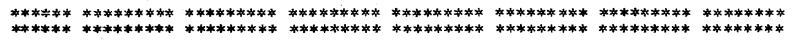
$\Xi(1820)$ 50 $\quad x_{1} * 1 / 2(1820$, JPE $3 / 2,1=1 / 2$ WE LIST HERE EVERYTHING REPORTED IN THE MASS RANGE
$1750-1875$ MEV. THE CLEAREST EVIDENCE FOR THIS STATE IS THAT OF GAY 76, WHO SEE AN 8 STD. DEV. ENHANCEMENT IN
LAMDA K-, AS WELL AS ENHANCEMENTS IN XI *(1680) PI, IS NARROW (WIDTH $21+-7$ MEV), IN CONTRAST TO RESULTS FROM EARLIER LESS
SIGNIFICANT DATA WHERE WIDTHS OF UP TO 100 MEV HAVE BEEN REPORED (SEE CHANNEL. IT IS POSSIBLE THAT THE XI PI ENHANCEMENTS SEEN IN THIS MASS REGION BY SOME EXPERIMENTS AT LOWER MOMENTUM ARE AT LEAST PARTIALLY DUE TO THE XI* EXPER IMENTS BEING FORCED TO ADD SEVERAL DIFFERENT CHANNELS TO OVERCOME POOR STATISTICS (CRENNELL 70, BADER 711 .
A SPIN PARITY ANAY
IT TOSIS OF THE GAY 76 DATA BUT WITH MORE STATISTICS

$50 x 1 * 1 / 2(1820)$ MASS (MEV)

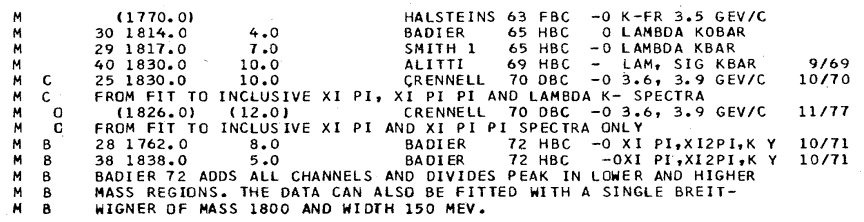




\section{Baryons $\Xi(1820)$}
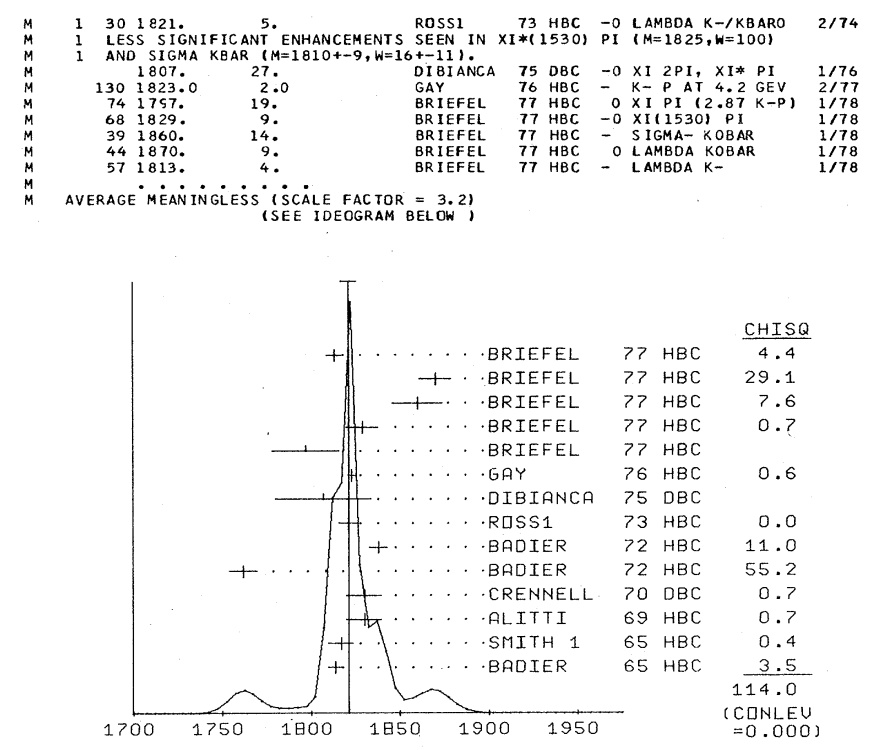

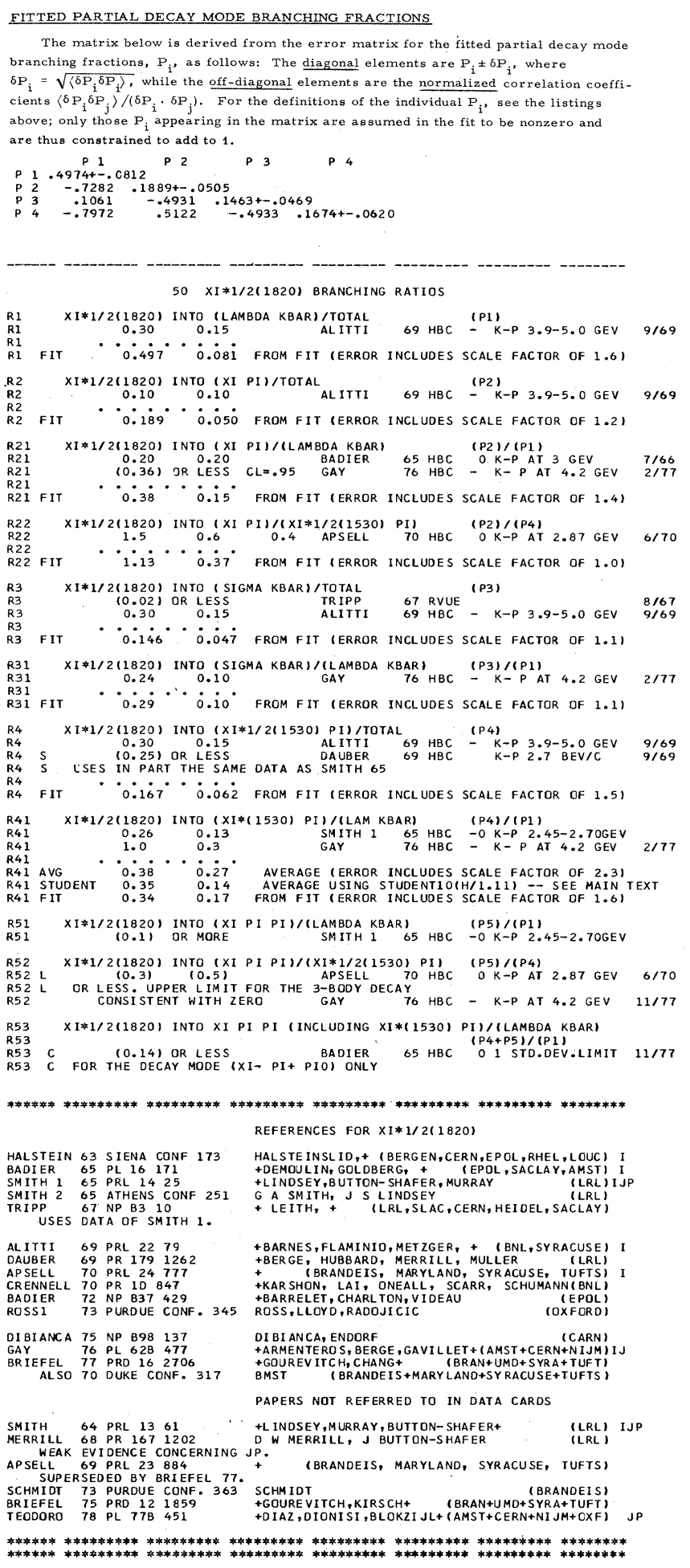

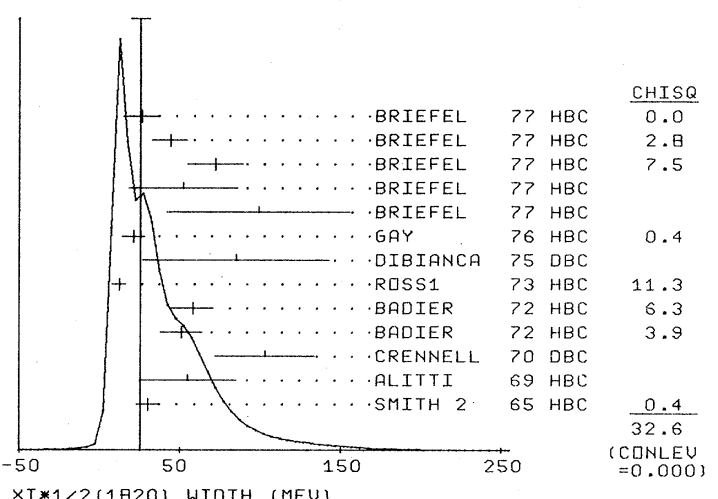

$50 X I * 1 / 2(1820)$ PARTIAL. DECAY MODES

$X I * 1 / 2(1 \theta 20)$ WIDTH (MEU)
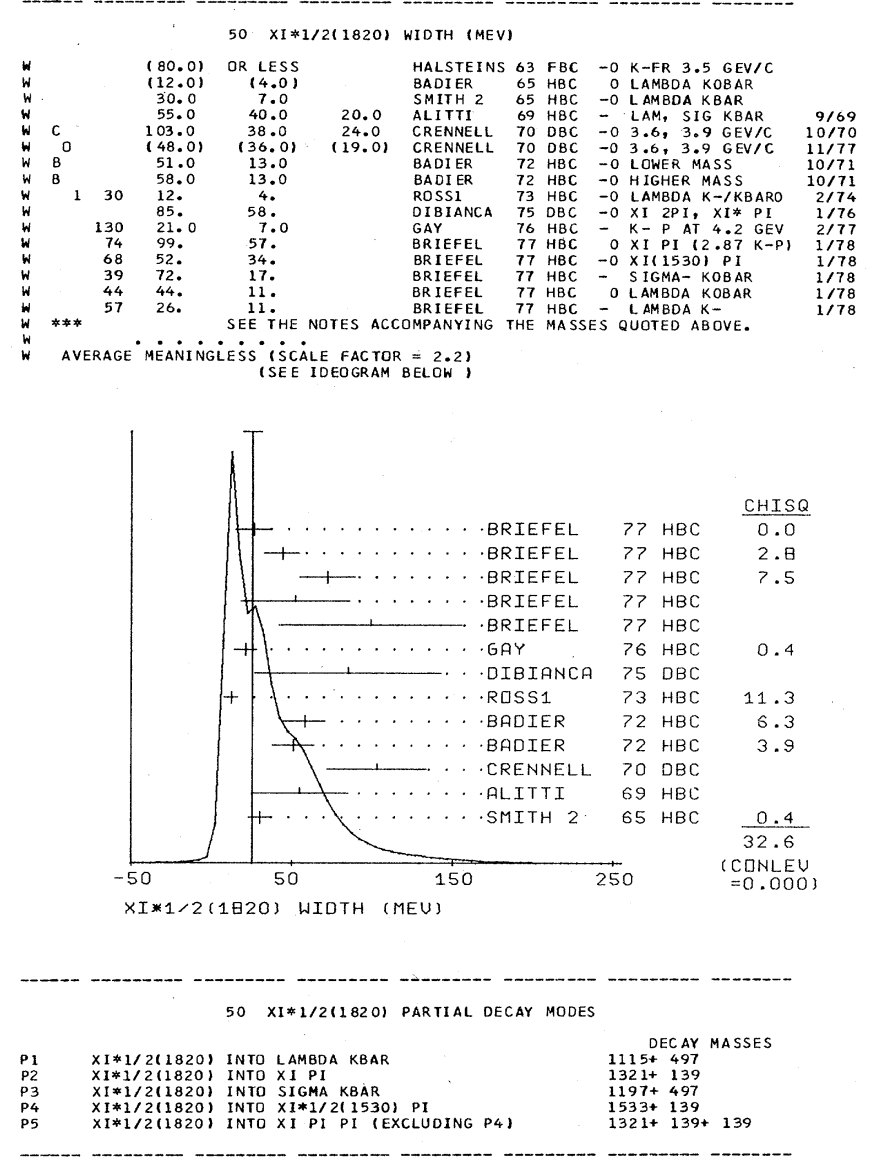

\section{Data Card Listings
at front of Listings. \\ For notation, see key at front of Listings.}

$$
\begin{aligned}
& \text { FI } \\
& \text { br } \\
& 6 \mathrm{P} \\
& \mathrm{ci} \\
& \mathrm{ab} \\
& \mathrm{ar} \\
& \mathrm{ar}
\end{aligned}
$$

$\mid$

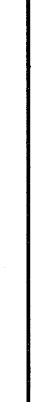




\section{Data Card Listings}

For notation, see key at front of Listings.

\section{Baryons $\Xi(1940), \Xi(2030)$}
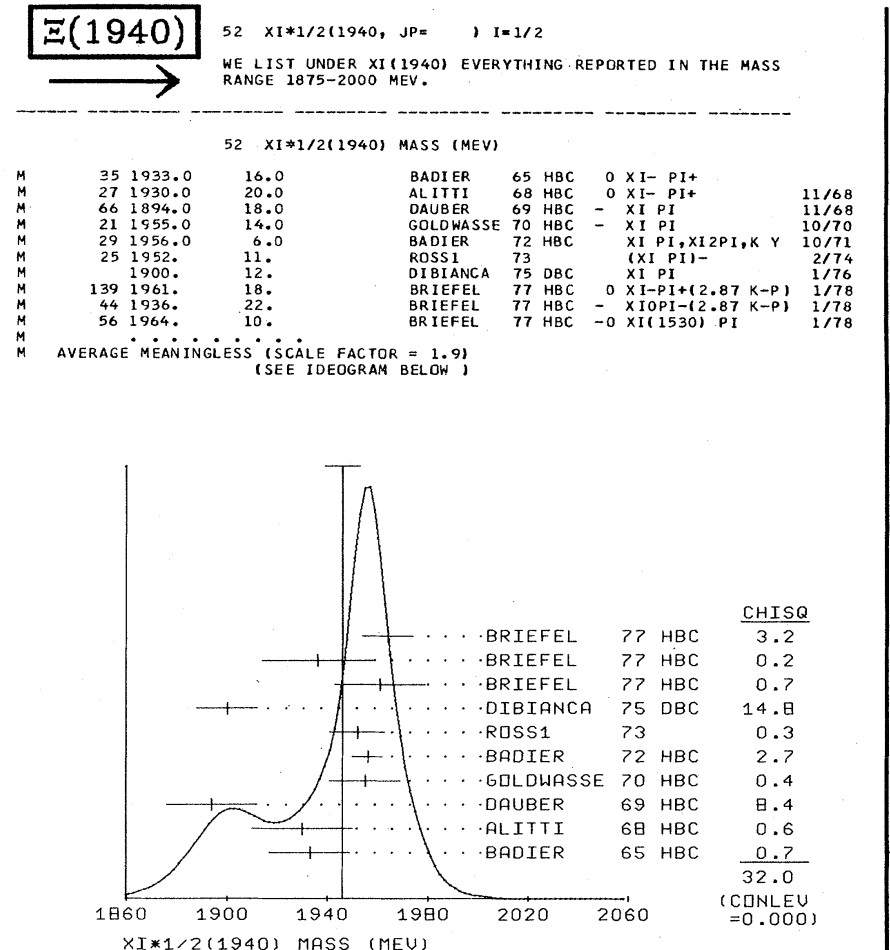

$52 \times 1 * 1 / 2(1940)$ WIOTH (MEV)
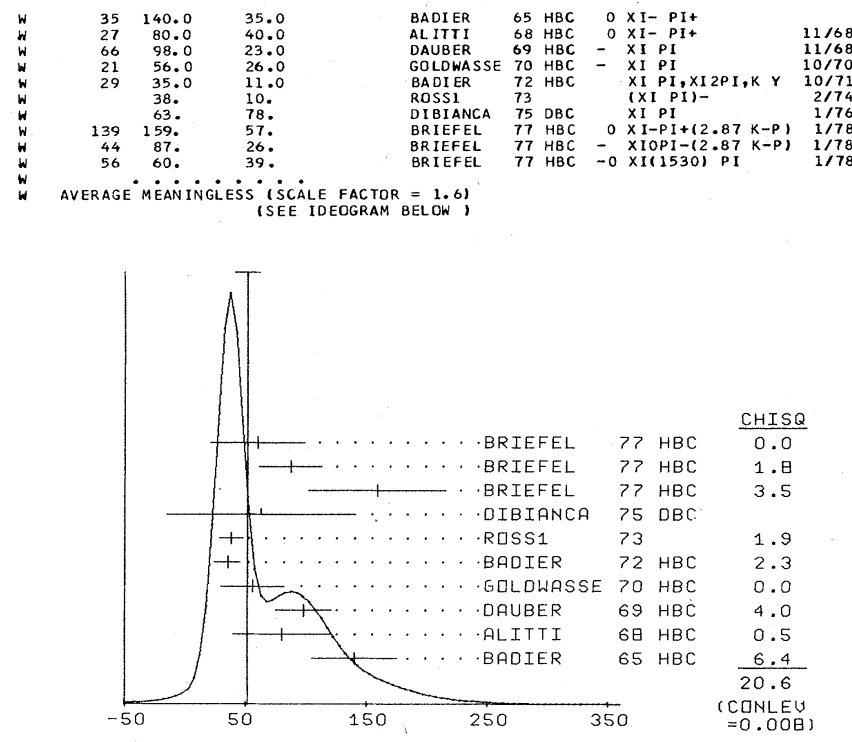

$52 X I * 1 / 2(1940)$ PARTIAL DECAY MODES

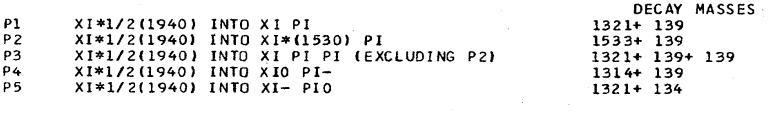

$52 X 1 * 1 / 2(1940)$ BRANCHING RATIOS

THE XI(1940) IS SEEN MAINLY IN XI PI AND SOME IN XI(1530) PI. IT
HAS BEEN LOOKEO FOR IN OTHER CHANNELS BUT NOT SEEN.

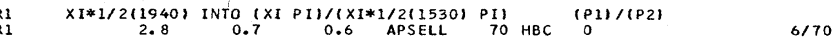

$\begin{array}{lcccc}R 2 & X I * 1 / 2(1940) & \text { INTO } \\ R 2 & 0.0 & 0.3 & (X I & P I\end{array}$

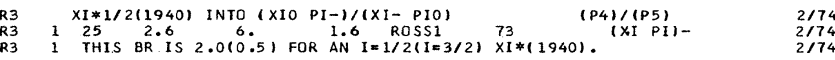

*******************************************************************1 REFERENCES FOR $X I * 1 / 2(1940)$

AADIER $\quad 65$ PL $16 \quad 171$

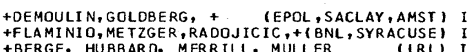
+BERGE, HUBBARD, MERRILL, MULLER (LRL) E L GOLOWASER, P F SCHULTZ SYRACUSE, TUFTS)
(ILLINOIS) PBARELET, CHARLTON,VIDEAU
ROSS, LLOYD, RADOJICIC (EPOL))
(OXFORD) $\begin{array}{lll}\text { BADIER } & 72 & \text { NP B B } 37,429 \\ \text { MOSS1 } & 73 & \text { PURDUE CONF. } 345\end{array}$ DI BIANCA 75 NP $898 \quad 137$ +GOUREVITCH, CHANG+ (BRAN+UMD+SYRA+TUFT)
BMST (BRANDE IS +MARYLAND+SYRACUSE+TUFTS) PAPERS NOT REFERRED TO IN DATA CARDS

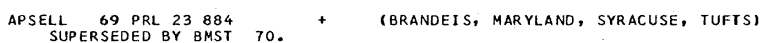
SCHMIDT 73 PURDUE CONF. 363 SCHMIDT
BRIEFEL 75 PRD 12 (BRANDEIS)

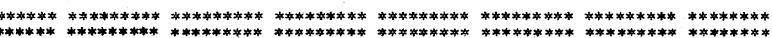
$\Xi(2030)$

$68 X I * 1 / 2(2030, J P=5 / 2$ OR GREATER) $I=1 / 2$ THE EVIDENCE FOR THIS STATE HAS BEEN MUCH IMPROVED IN SIGMA KBAR AND A WEAKER COUPLING TO LAMBDA KBAR. ALITTI 68 AND HEMINGWAY 77 OBSERVE NO SI GNALS IN THE $X I$ PI PI IOR XI*(1530) PI) CHANNEL, IN CONTRAST TO OIBIANCA 75. THE
DECAY INTO LAMBDA/SIGMA KBAR PI REPORTED BY BARTSCH 69 IS ALSO NOT A MOMENTS ANALYS IS OF THE HEMINGWAY 77 DATA INDICA TES THAT THE SPIN
IS GREATER THAN OR EQUAL TO $5 / 2$ AT A LEVEL OF 3 STD. DEVIATIONS.

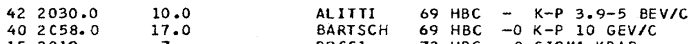
ROSS1 $73 \mathrm{HBC}$-O SIGMA KBAR

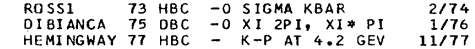
average MEANingLess iscale FaCTOR = 1.5 ,

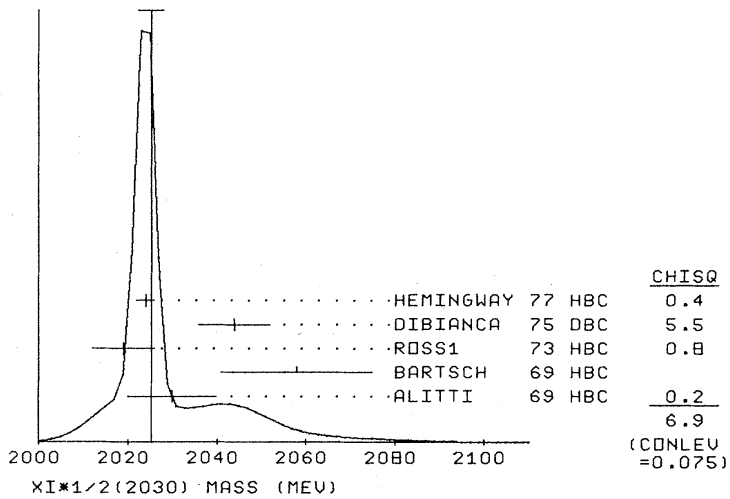

$68 X I * 1 / 2(2030)$ WIOTH (MEV)

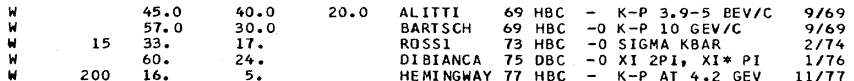
* average Méaningiess iscille factor = 1.21 
Baryons

$\Xi(2030), \Xi(2120), \Xi(2250), \Xi(2370)$

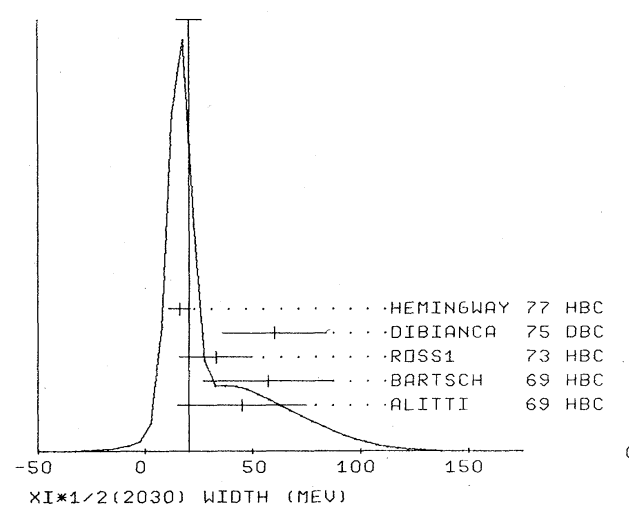

$\frac{C H I S Q}{0 . B}$

$0 . \mathrm{B}$

2.7

1.5

$\frac{0.7}{6.2}$

(CONLEU
$=0.184$ )

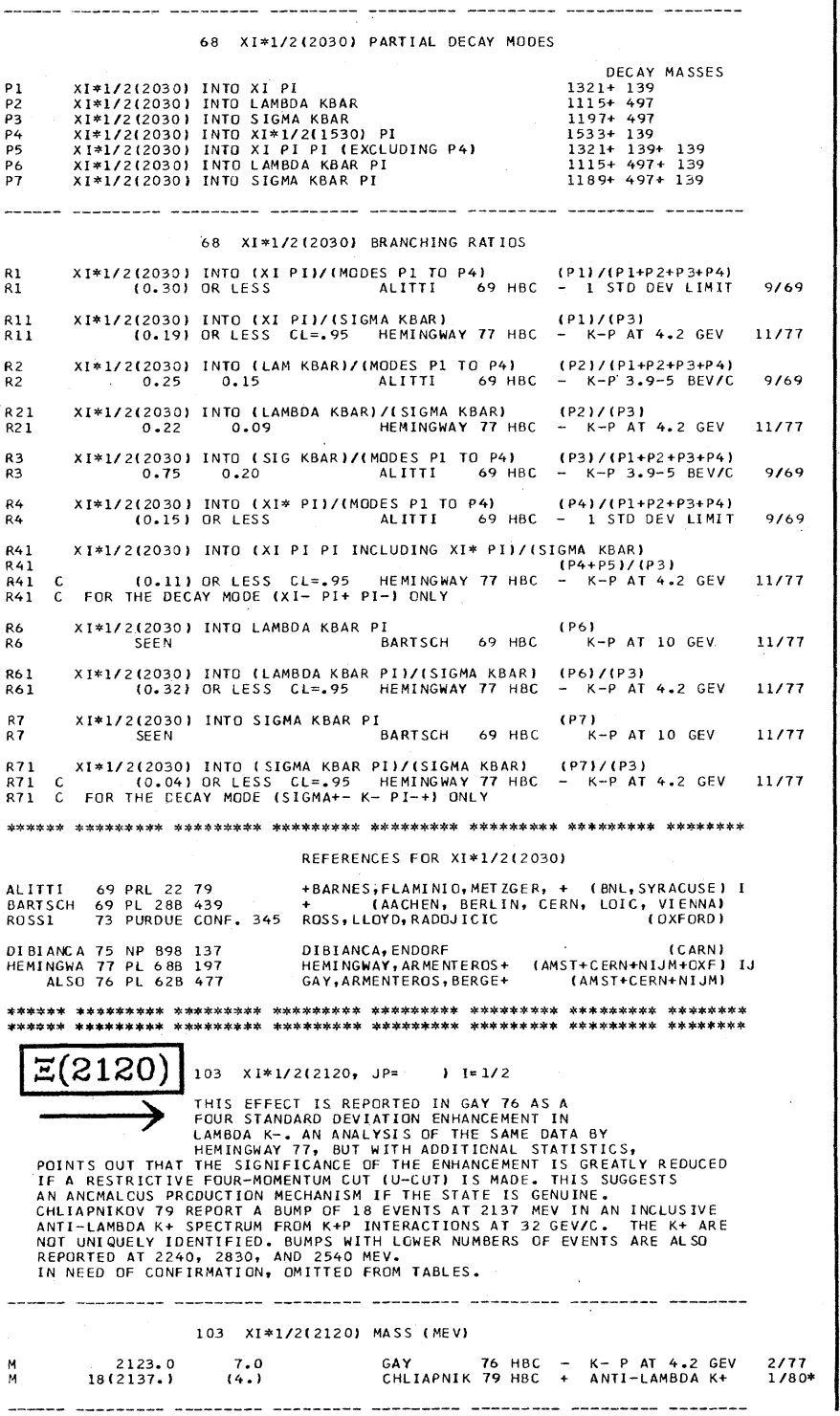

Data Card Listings For notation, see key at front of Listings.

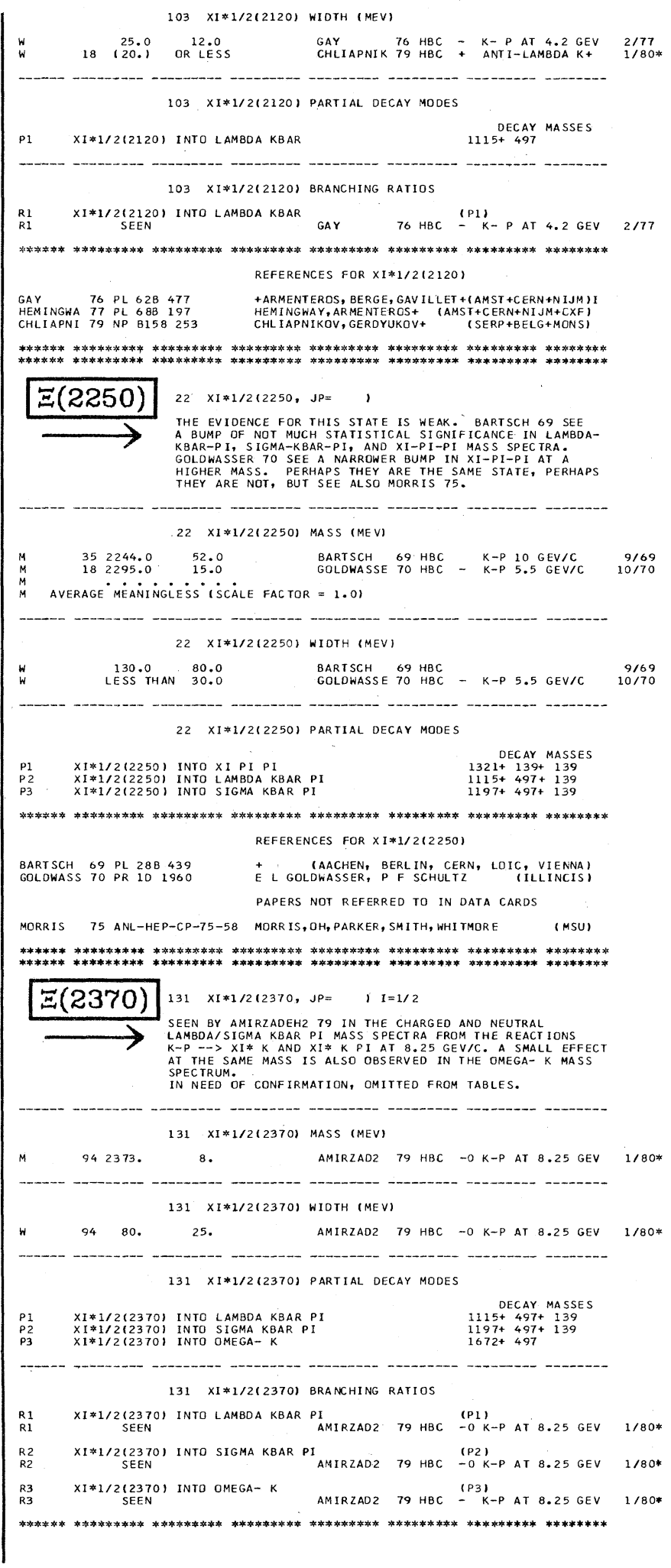




\section{Data Card Listings}

\section{For notation, see key at front of Listings.}

\section{Baryons $\Xi(2500), \Omega^{-}, \Lambda_{c}^{+}, \Sigma_{c}(2430)$, DIBARYONS}

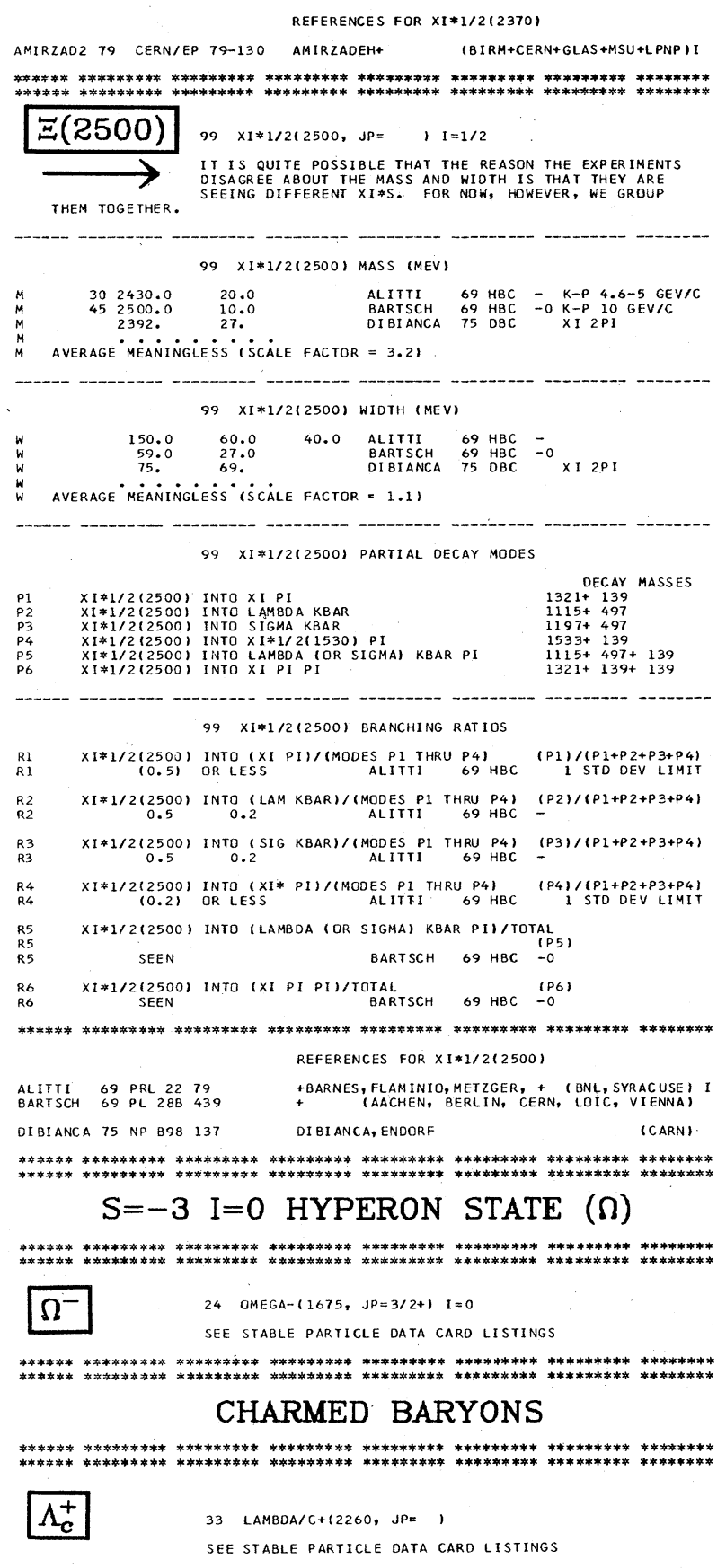

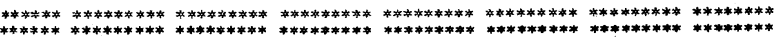

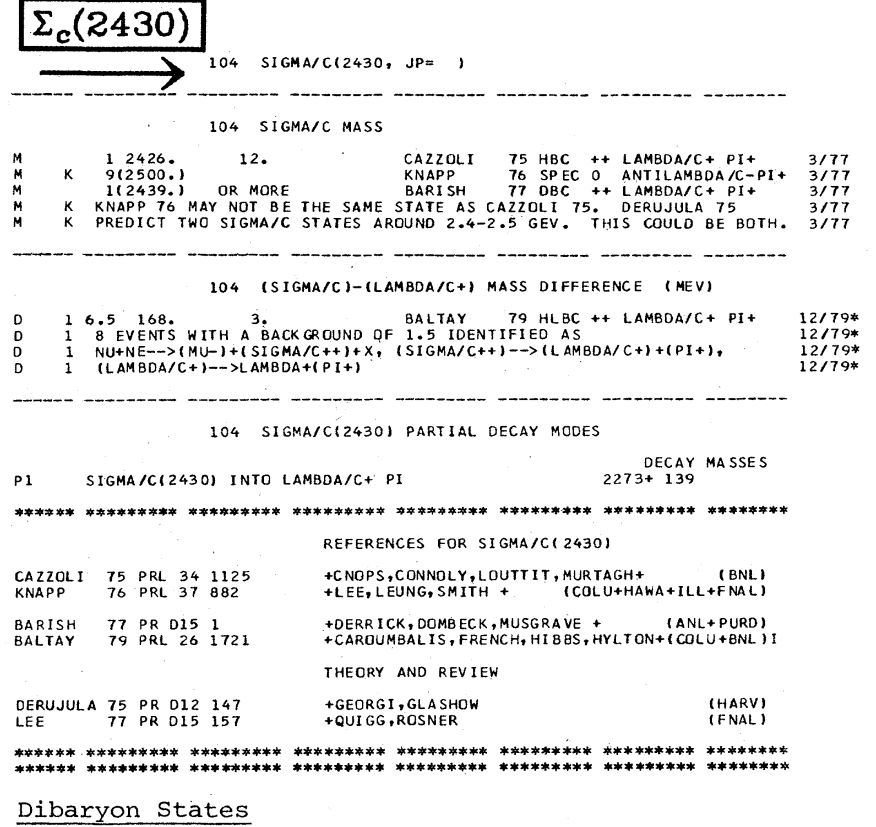

Dibaryon resonances have been predicted theoretically $\mathrm{y}^{1-4}$ and claimed experimentally, but although considerable evidence for them has been published, their existence remains controversial. Problems with the pp data have been pointed out by Bugg. ${ }^{5}$ Either the $\Delta\left(\sigma_{L}\right)$ data and elastic data at $I$ and 1.1 GeV/c are inconsistent, or accepted ideas about the mechanism of the inelastic channels are wrong.

Most significant evidence is included in the Listings, but there may be omissions, especially in earlier work. We have not included evidence on nuclear properties, hypernuclei, $d^{*} ' s$, or $\Delta^{\prime} s$ bound within the deuteron - though these may be related to effects observed in the search for dibaryon resonances. We have also omitted most data on low energy $\pi^{+} \mathrm{d} \rightarrow \mathrm{pp}$. There is a large amount of literature on this reaction which we did not have time to review adequately. Most of these experiments are addressed to the question of whether there is a resonance associated with the $\mathrm{N} \Delta$ threshold.

The Listings are grouped by strangeness.

\section{References}

1. R. J. Oakes, Phys. Rev. 131, 2239 (1963).

2. R. Dyson and N. Xuong, Phys. Rev. Lett. 13, 815 (1964). 


\section{Baryons DIBARYONS}

3. R. L. Jaffe, Phys. Rev. Lett. 38, 195 (1977).

4. J. J. de Swart, Nijmigen preprint THEF-NYM-79-16.

5. D. V. Bugg, J. Phys. G5, 1349 (1979).

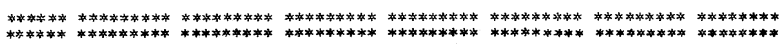

\section{DIBARYONS}

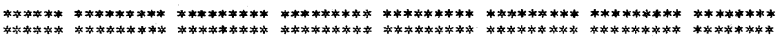
$S=0$
$\longrightarrow$$\quad 106$ baryon number 2, strangeness o states

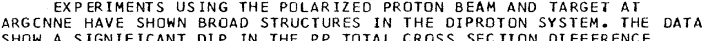

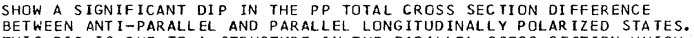

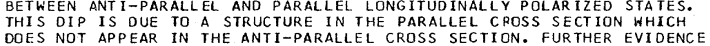
DOES NOT APPEAR IN THE ANTI-PARALLEL CROSS SECTION. FURTHER EVIDENCE
IS THE STRUCTURE IN THE ENERGY DEPENOENCE OF THE PP ELASTIC SCATTERING

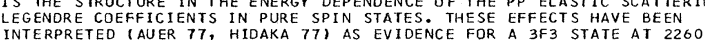

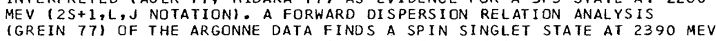
AND A SPIN TRIPLET STATE AT 2320 MEV, HITH HDTHS OF ABOUT 100 MEV AAN 290 MEE, RESPECTIVELY. (THE DISPERS ITN RELAATON ANALYSIS CANNO

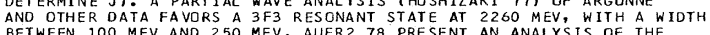

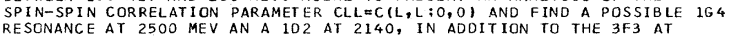
RESONANCE AT 2500 MEV AN A 102 AT 2140 , IN ADOITION TO THE 3 FH 3 AT
2260 . 2260 MI NAMI 78 HAS PRESENTED CRITICISM DF THE ANALYSIS OF BOTH
HIDAKA 77 ANO HOSHIZAKI 77, AND SUGGESTS THE OBSERVED EFFECTS ARE

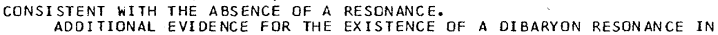
THE 2350 MEV REGION COMES FROM A PHOTODI IINTEGRA IION EXPERI MENT
(KAMAE T7 AND IKEDA 79), WHERE AN ANOMALY IN THE POLARIZATION IS CONSISTENT WI TH AN I I O J J 3 STATE.
UEOA 78 SUGGESTS THE 3 F3 STATE IS A (PIN N) SYSTEM WITH THE PI N S SYTEMS IN THE DELTEA3 RESONANCE. RE SONANCES AT 2140,2260 ,

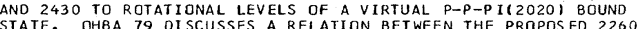

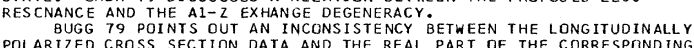
POLARIZED CROSS SECTION DATA AND THE REAL PART OF THE CORRESPONDING
FORWARD AMPLITUOE BELOW THE REGION OF THE RESONANGES CLAIMED BY THEF ARGONNE GROUP. WANTANBE 79 CALCULATES LARGER THAN-EXPECTED CORRECT ION
FRCM COULOMB-NUCLEAR INTERFERENCE TO PURE SPIN STATE CROSS SECTICNS. IN SPITE OF THE PROGRESS MADE BY RECENT WORK, MORE EXPERIMENTAL CAN BE CONS IDERED CONF IRMED. IT IS UNLIKELY THAT THE DIPROTON(S) CAN BEE FIRMLY ESTABLI SHED UNT IL MUCH MORE INFORMATION ON ANGULAR

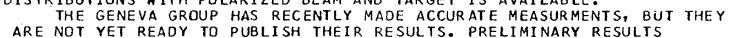
PRESENTED AT THE 1979 CONFERENCE ON HIGH ENERRY PHYSICS AND MEASUREMENTS FROM ARGONNE.

$1068=2$, S=0 STATES - CROSS SECTICN

THIS SECTION WE USE THE FOLLOWING ABBREVIATIONS FOR MEASURED QUANTITIES.

LP W THE ENERGY DEPENDENCE OF THE P P TORAL CROSS SECTION
DIFFERENCE BETWEEN ANT I-PARRALLL AND PARALLEL

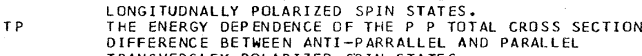
TRANSVERSLEY POLARIZED SPIN STATES.
THE ENERGY DEPENDENCE OF THE LEGENDRE COEFFICIENTS FOR

LEG

CLL
PWA THE CORRELATION PARAMTER CIL,L:O,0) FOR P P SCATTERING
PATIAL WAVE ANALSIS FOR P P SCATTERING.

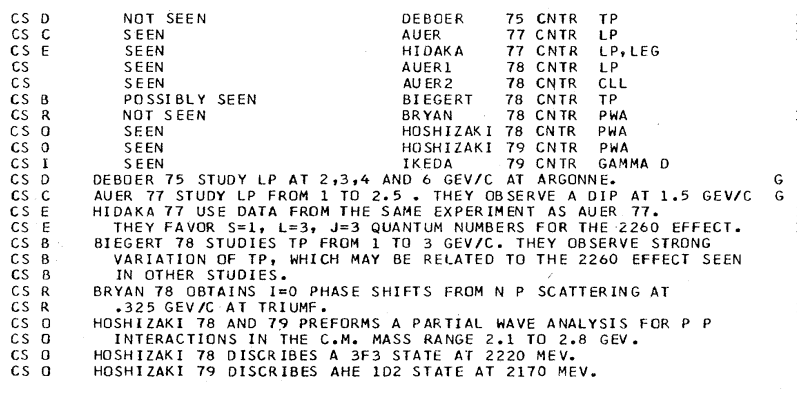

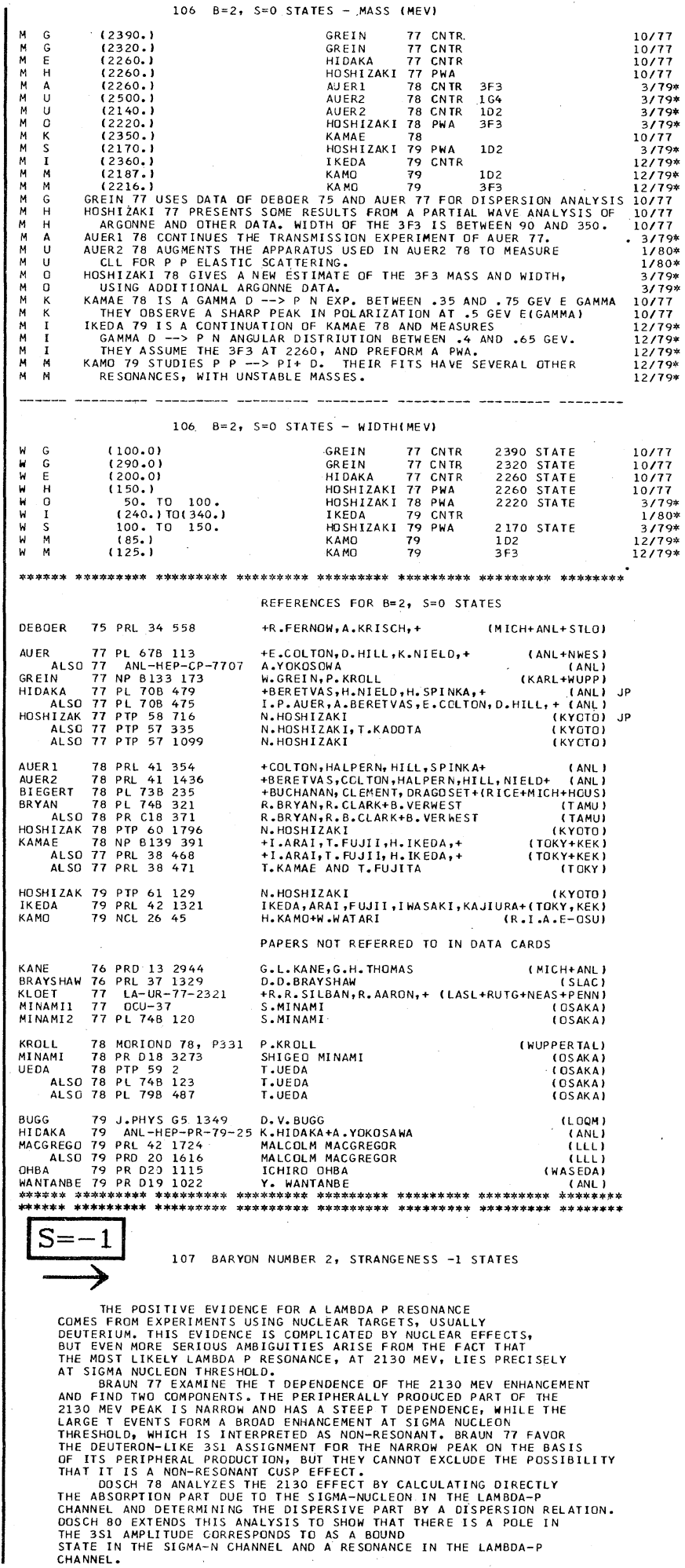

Data Card Listings For notation, see key at front of Listings. 


\section{Data Card Listings}

For notation, see key at front of Listings.

\section{Baryons} DIBARYONS

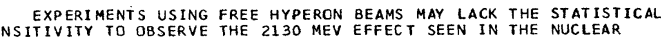
TARGET DATA.

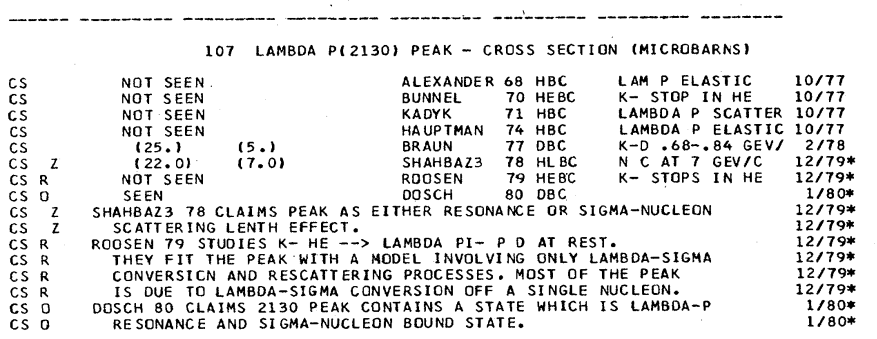

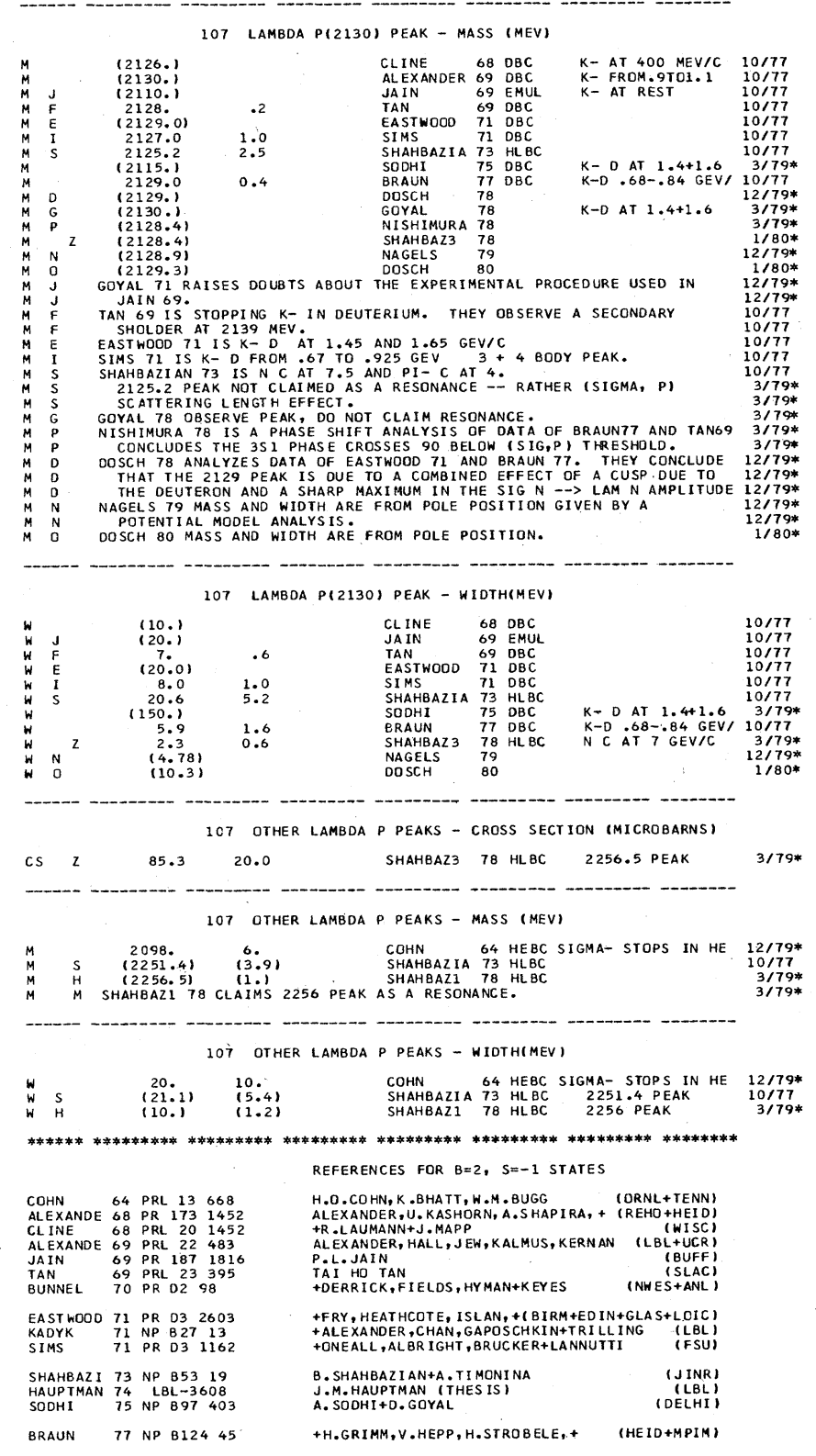

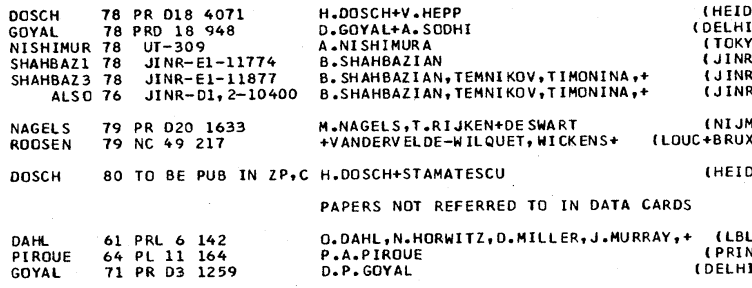

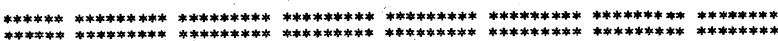

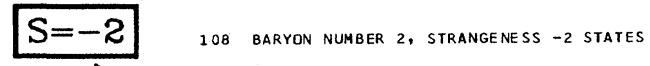

EXPERI MENTAL EVIDENCE FOR RESONANCES IN THE STRANGNESS $=-2$
DIBARYON SYSTEMS IS NOT VERY STRONG. THOUGH RESONANCES ARE PREDICTED

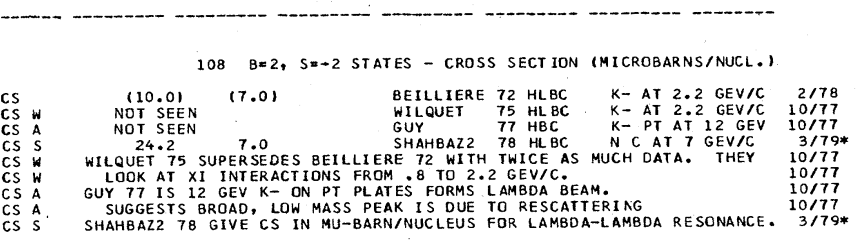

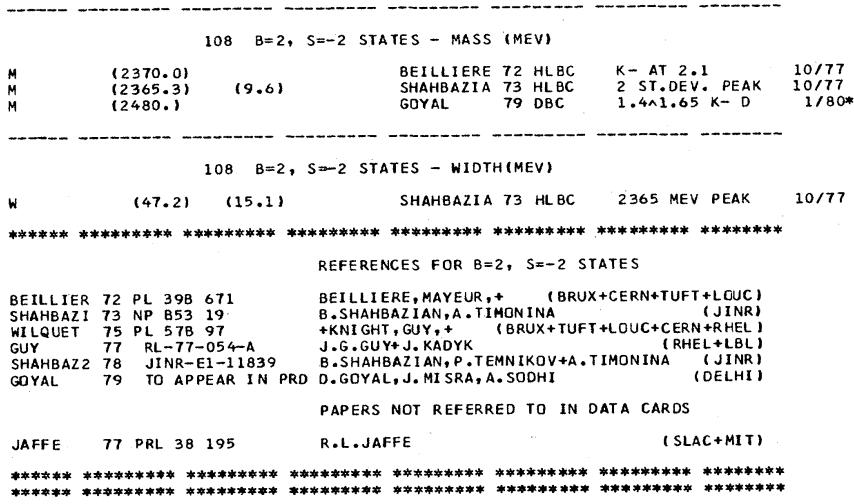

115 BaRYON NUMBER $>=2$, STRANGENESS -2 STATES

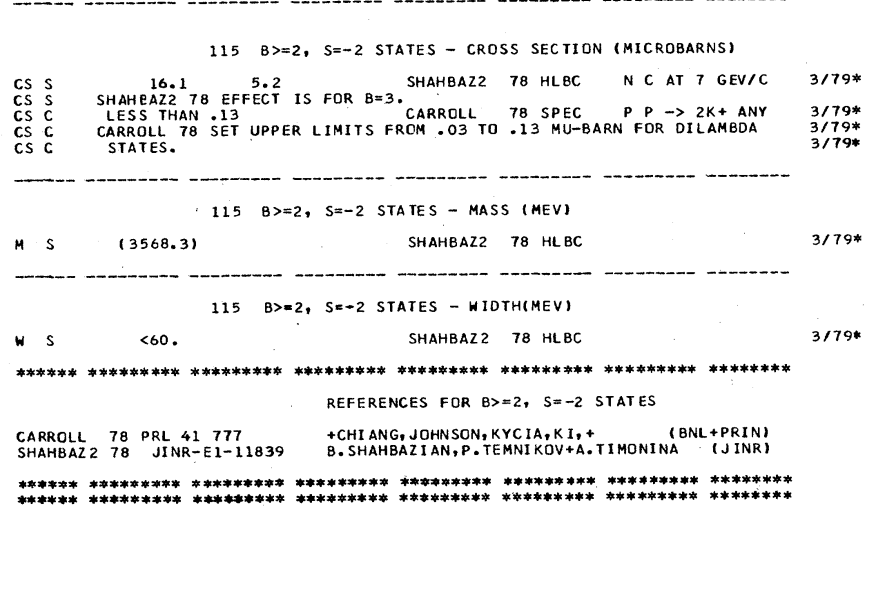


Appendix I

TEST OF $\Delta \mathrm{I}=1 / 2$ RULE FOR $K$ DECAYS

The quantities of interest for making tests of theoretical predictions regarding the $\Delta I=1 / 2$ rule for $K$ decay are usually partial decay rates for single channels or special sums of channels. It is not possible to compute the errors on sums, differences, and ratios of partial decay rates from the information given in the Table of Stable Particles because of the presence of off-diagonal terms in the error matrix. For this reason we give some of these quantities in Table I. Throughout this Appendix, italics are used to indicate that a quantity has changed by more than one (old) standard deviation since our previous edition, and $S$ gives the scale factor included in the quoted error because of inconsistencies in the data (see footnote at end of Stable Particle Table for definition of $S$ ).

Table I. $(000)$ and $(+-0)$ refer to the sign of the pions into which the $K_{L}$ decays.

$\left\{\begin{array}{lll}\Gamma_{\mathrm{K}_{\ell 3}^{+}}=\Gamma_{\mathrm{K}_{\mathrm{e} 3}^{+}}+\Gamma_{\mathrm{K}_{\mu 3}^{+}} & =(6.484 \pm 0.089) 10^{6} & \mathrm{sec}^{-1} \\ \Gamma_{\mathrm{K}_{\mu 3}^{+}} / \Gamma_{\mathrm{K}_{\mathrm{e} 3}^{+}} & =0.663 \pm 0.018 & \mathrm{~S}=1.7^{*} \\ \Gamma_{\mathrm{K}_{\tau}^{+} / \Gamma_{\mathrm{K}_{\tau^{+}}^{+}}} & =3.226 \pm 0.082 \\ \Gamma_{\mathrm{K}_{t 3}^{0}}=\Gamma_{\mathrm{K}_{\mathrm{e} 3}^{0}}+\Gamma_{\mathrm{K}_{\mu 3}^{0}} & =(12.70 \pm 0.15) 10^{6} & \mathrm{sec}^{-1} \mathrm{~S}=1.1^{*} \\ \Gamma_{\mathrm{K}_{\mu 3}^{0} / \Gamma_{\mathrm{K}_{\mathrm{e} 3}^{0}}} & =0.695 \pm 0.017 & \\ \Gamma_{\mathrm{K}^{0}(000)} / \Gamma_{\mathrm{K}^{0}(+-0)} & =1.733 \pm 0.076 & \mathrm{~S}=1.3^{*}\end{array}\right.$

1. Leptonic decay rates

The $\Gamma_{K}$ rates are useful in testing the leptonic $\Delta I=1 / 2$ rule in the way suggested by Trilling. ${ }^{1}$ The predictions are

$\Gamma_{K_{\ell 3}^{0}} / 2 \Gamma_{K_{\ell 3}^{+}}=1.012$, a phase-space factor, ${ }^{2}$

and

$\Gamma_{K_{\mu 3}^{0}} / \Gamma_{K_{e 3}^{0}}=\Gamma_{K_{\mu 3}^{+}} / \Gamma_{K_{e 3}^{+}}$.

From Table 1, and

$\Gamma_{K_{t 3}^{0}} / 2 \Gamma_{K_{t 3}^{+}}=0.979 \pm 0.018$

$\frac{\Gamma_{K_{\mu 3}^{0}}}{\Gamma_{K_{e 3}^{0}}}\left[\frac{\Gamma_{K_{\mu 3}^{+}}}{\Gamma_{K_{e 3}^{+}}}\right]^{-1}=1.048 \pm 0.038$.
These results seem to show a less than $2 \sigma$ disagreement with the predictions, but the errors should be regarded with caution in view of the internal disagreements in the data. (Note the ideograms in the Data Listings for the charged $\mathrm{K}$ meson.)

\section{Three-pion decays}

We follow here the tests done by Mast et al. ${ }^{3}$ based on the general analysis of $K$ decays suggested by Zemach. ${ }^{4}$ Both decay rates $(\Gamma)$ and slopes ( $g$, the energy dependence of the Dalitz plot distributions) are used. The $\Delta I=1 / 2$ rule predicts that the following test quantities are all equal to zero:

$$
\begin{aligned}
& \text { Test } 1=\frac{2}{3} \frac{\Gamma_{K^{0}(000)}}{\phi_{1}}\left[\frac{\Gamma_{K^{0}(+-0)}}{\phi_{2}}\right]^{-1}-1, \\
& \text { Test } 2=\frac{1}{4} \frac{\Gamma_{K_{\tau}^{+}}}{\phi_{3}}\left[\frac{\Gamma_{K_{\tau^{+}}^{+}}}{\phi_{4}}\right]^{-1}-1, \\
& \text { Test } 3=\frac{1}{2} \frac{\Gamma_{K_{\tau}^{+}}}{\phi_{3}}\left[\frac{\Gamma_{K^{0}(+-0)}}{\phi_{2}}\right]^{-1}-1, \\
& \text { Test } 4=\frac{1}{2} g_{K_{\tau^{+}}^{+}+g_{K_{\tau}^{+}},} \\
& \text {Test } 5=g_{K^{0}(+-0)}+g_{K_{\tau}^{+}}-\frac{1}{2} g_{K_{\tau^{+}}^{+}} .
\end{aligned}
$$

The $\phi_{i}$ are phase-space factors which have been calculated as described in Mast et al. ${ }^{3}$ by use of a relativistic formulation and the masses and slopes from this edition. The factors labeled UDP are the relative areas of the Dalitz plots, assuming a uniform distribution. The NUDP include the observed slopes (see below). The CNUDP have been calculated by including the final-state Coulomb interaction

The values are:

\begin{tabular}{llll} 
& \multicolumn{3}{c}{ Method } \\
$\phi_{1}(000)=$ & $\frac{\text { UDP }}{1.490}$ & $\frac{\text { NUDP }}{1.490}$ & $\frac{\text { CNUDP }}{1.444}$ \\
$\phi_{2}(+-0)=$ & 1.221 & 1.303 & 1.287 \\
$\phi_{3}(++-)=$ & 1.000 & 1.000 & 1.000 \\
$\phi_{4}(+00)=$ & 1.247 & 1.173 & 1.137
\end{tabular}

For convenience, we repeat the slope parameters tabulated in the Stable Particle Table. They are as follows:

$\begin{array}{lll}\mathrm{g}_{\mathrm{K}_{\tau}^{+}} & =-0.215 \pm 0.004 & \mathrm{~S}=1.4^{*} \\ \mathrm{~g}_{\mathrm{K}_{\tau}^{-}} & =-0.217 \pm 0.007 & \mathrm{~S}=2.5^{*} \\ \bar{g}_{\mathrm{K}_{\tau}^{ \pm}} & =-0.215 \pm 0.003 & \\ \mathrm{~g}_{\mathrm{K}_{\tau^{\prime}}^{+}} & =0.607 \pm 0.030 & \mathrm{~S}=1.3^{*} \\ \mathrm{~g}_{\mathrm{K}^{0}(+-0)} & =0.670 \pm 0.014 & \mathrm{~S}=1.6^{*}\end{array}$


A difference in the $\tau^{+}$and $\tau^{-}$slopes would be an indication of CP violation in this decay. Since no difference is observed at this time, we average he two and use this value in Test 4 and Test 5 .

We use the CNUDP factors and the rates and slopes reported in this edition to compute the five test quantities which the $\Delta I=1 / 2$ rule predicts to be zero. The results are:

$\begin{array}{lr}\text { Test } 1= & 0.030 \pm 0.045 \\ \text { Test } Z= & -0.083 \pm 0.023 \\ \text { Test } 3= & 0.216 \pm 0.020 \\ \text { Test } 4=0.088 \pm 0.016\end{array}$

Test $5=0.152 \pm 0.021$

- The three-pion final state can be in isospin states $I=$ $1,2,3$. Tests 1 and 2 test the existence of isospin $I=3$ in the final state. Since the rate tests (Tests 1,2 , and 3 ) could differ from zero by as much as 0.1 owing to the mass differences and the occurrence of big slopes ${ }^{5}$, no evidence for $I=3$ is found. Test 4 is related to the $I=2$ amplitude in the final state and indicates the presence of $I=2$. Tests 3 and 5 give information on the $\Delta I=3 / 2$ part of the $I=1$ amplitude relative to the $\Delta I=1 / 2$ part Both tests indicate the presence of $\Delta I=3 / 2$.

\section{References}

1. G. Trilling, K-Meson Decays, UCRL-16473, (updated from Argonne Conference Proceedings, 1965, p. 115).

2. N. Brene (CERN), private communication. In our Jan. 1968 edition we had erroneously used 1.04

3. T. S. Mast, L. K. Gershwin, M. Alston-Garnjost, R. 0. Bangerter, A. Barbaro-Galtieri, J. J. Murray, F. T. Solmitz, and R. D. Tripp, Phys. Rev. 183, 1200 (1969).

4. C. Zemach, Phys. Rev. 133, B1201 (1964).

5. C. Bouchiat and M. Veltman, Topical Conference on Weak Interactions, CERN 69-7 (1969), p. 225.
Appendix II

TEST OF $\triangle I=1 / 2$ RULE FOR HYPERON DECAYS

$$
\begin{aligned}
& \text { O. E. Overseth } \\
& \text { University of Michigan }
\end{aligned}
$$

1. Nonleptonic decay Amplitudes

In this edition we again use the new convention for the amplitudes A and B adopted in 1973. Some theorists have suggested that dimensionless amplitudes are more useful to them than the ones appearing in the literature. Berge ${ }^{1}$ used a convention with $A$ and $B$ in units of $\sec ^{-1 / 2}$. Samios ${ }^{2}$ used a convention which gave $A$ and $B$ in units of $(\mathrm{MeV}-\mathrm{sec})^{-1 / 2}$. Following is the convention suggested by Jackson ${ }^{3}$, which gives dimensionless $A$ and $B$.

The effective Lagrangian density for nonleptonic hyperon decays $\left(B_{1} \rightarrow B_{2}+\pi\right)$ can be written

$$
L_{\text {eff }}=\mathrm{G} \mu_{\mathrm{c}}^{2}\left[\bar{\psi}_{2}\left(\mathrm{~A}+\mathrm{B} \gamma_{5}\right) \psi_{1}\right] \phi_{\pi} \text {. }
$$

where $G=10^{-5} \mathrm{~m}_{\mathrm{p}}^{-2}$ is a coupling constant characteristic of first-order weak decays, $\mu_{c}$ is the charged pion mass, and $A$ and $B$ are dimensionless complex numbers giving the relative amplitudes of the parity-violating and parity-conserving decays, respectively. The matrix $\gamma_{5}$ is to be taken in the Pauli form, $\gamma_{5}=\left(\begin{array}{rr}0 & -I \\ -I\end{array}\right)$. The invariant amplitude for the decay is

$$
M=\mathrm{G} \mu_{\mathrm{c}}^{2}\left[\overline{\mathrm{u}}(\mathrm{p})\left(\mathrm{A}+\mathrm{B} \gamma_{5}\right) \mathrm{u}(\mathrm{P})\right],
$$

where $P$ is the 4-momentum of the decaying hyperon of mass $M$ and $p$ is the 4-momentum of the baryon decay product of mass $m$. With the normalization convention, $\bar{u}_{i} u_{i}=2 m_{i}$, the Pauli form of the matrix element in the rest frame of the decaying hyperon is

$$
M=\mathrm{G} \mu_{\mathrm{c}}^{2}\left\langle x_{2}|\sqrt{2 \mathrm{M}(\mathrm{E}+\mathrm{m}) \mathrm{A}}+\sqrt{2 \mathrm{M}(\mathrm{E}-\mathrm{m})} \mathrm{B} \vec{\sigma} \cdot \hat{\mathrm{q}}| x_{1}\right\rangle,
$$

where $E$ is the total energy of the final baryon and $\hat{q}$ is a unit vector in the direction of motion of the final baryon. Comparison with Sec. VI D of the text shows that the amplitudes $s$ and $p$ defined there are proportional to $A$ and $B$ :

$$
\frac{p}{s}=\left(\frac{E-m}{E+m}\right)^{1 / 2} \frac{B}{A}=\left[\frac{(M-m)^{2}-\mu^{2}}{(M+m)^{2}-\mu^{2}}\right]^{1 / 2} \frac{B}{A} .
$$

Here $\mu$ is the mass of the pion entering the decay. The parameters $\alpha, \beta$, and $\gamma$ can therefore be expressed in terms of $A$ and $B$, rather than $s$ and $p$, if desired.

The decay rate for $B_{1} \rightarrow B_{2}+\pi$ is

$$
\Gamma=\frac{G^{2} \mu_{c}^{4}}{8 \pi} q\left\{\left[\frac{(M+m)^{2}-\mu^{2}}{M^{2}}\right]|A|^{2}+\left[\frac{(M-m)^{2}-\mu^{2}}{M^{2}}\right]|B|^{2}\right\}
$$


where $q$ is the c.m. momentum of the decay products. For reference, the dimensionless constant in this expression has the value $\left(G^{2} \mu_{c}^{4} / 8 \pi\right)=1.9488 \times 10^{-15}$

Table I summarizes the amplitudes $A$ and $B$ for the nonleptonic decays of the $\Lambda, \Sigma$, and $\Xi$ hyperons. These amplitudes have been calculated by using the experimental data for mean lives, branching ratios, and the decay asymmetry a given in the Stable Particle Table of this Review. Time-reversal invariance is assumed and final-state interactions are neglected, so $A$ and $B$ are taken to be relatively real. The subscript on the hyperon refers to the sign of the decaying pion. The statistical correlation coefficient

$$
C_{A B}=\frac{\langle\Delta A \Delta B\rangle}{\sqrt{\left\langle\Delta A^{2}\right\rangle\left\langle\Delta B^{2}\right\rangle}}
$$

is also given. The absolute signs of $A$ and $B$ have been assigned, using the following convention. Taking $A\left(\Lambda^{0}\right)$ as positive, the other $S$-wave decay amplitudes are chosen to give an approximate fit to the triangular relationships

$$
\sqrt{2} A\left(\Sigma_{0}^{+}\right)+\mathbf{A}\left(\Sigma_{+}^{+}\right)=\mathbf{A}\left(\Sigma_{-}^{-}\right) \text {and } \sqrt{3} \mathbf{A}\left(\Sigma_{0}^{+}\right)+\mathbf{A}\left(\Lambda_{-}^{0}\right)=2 \mathrm{~A}\left(\Sigma_{-}^{-}\right) \text {. }
$$

The signs of the $B$ amplitudes relative to those of the corresponding A amplitudes are determined by the sion of the appropriate a decay parameter.

\begin{tabular}{lrrr}
\hline \multicolumn{4}{c}{ Table $\mathrm{I}$} \\
\hline$M \rightarrow \mathrm{m}+\mu$ & \multicolumn{1}{c}{$\mathrm{A}$} & \multicolumn{1}{c}{$\mathrm{B}$} & \multicolumn{1}{c}{$\mathrm{C}_{\mathrm{AB}}$} \\
$\Lambda \Lambda_{0} \rightarrow \mathrm{p}+\pi^{-}$ & $1.47 \pm 0.01$ & $9.98 \pm 0.24$ & -0.289 \\
$\Lambda_{0} \rightarrow \mathrm{n}+\pi^{0}$ & $-1.07 \pm 0.01$ & $-7.14 \pm 0.56$ & -0.741 \\
$\Sigma_{+}^{+} \rightarrow \mathrm{n}+\pi^{+}$ & $0.06 \pm 0.01$ & $19.07 \pm 0.07$ & -0.038 \\
$\Sigma_{0}^{+} \rightarrow \mathrm{p}+\pi^{0}$ & $1.48 \pm 0.05$ & $-12.04 \pm 0.58$ & 0.982 \\
$\Sigma_{-} \rightarrow \mathrm{n}+\pi^{-}$ & $1.93 \pm 0.01$ & $-0.65 \pm 0.07$ & 0.003 \\
$\Xi_{0}^{0} \rightarrow \Lambda+\pi^{0}$ & $1.54 \pm 0.03$ & $-6.43 \pm 0.66$ & 0.188 \\
$\Xi_{-} \rightarrow \Lambda+\pi^{-}$ & $2.04 \pm 0.01$ & $-6.93 \pm 0.31$ & 0.268 \\
\hline
\end{tabular}

\section{Tests of the $\Delta I=1 / 2$ Rule}

(a) $\wedge$ Decay

For $\Lambda$ decay the $\Delta I=1 / 2$ rule predicts that $\Gamma_{0} / \Gamma_{-}=0.50$ and $a_{0}=a_{-}$. In order to determine the magnitude of possible $\Delta I=3 / 2$ amplitudes present we write the linear expressions ${ }^{4}$ for the $\Delta I=3 / 2 A-$ and $B-w a v e$ amplitudes in terms of $\Delta a$, where $\Delta a$ is the measured value of $a_{0} / a_{-}$minus the predicted value, and in terms of $\Delta \Gamma$ similarly defined. Evaluating these we find

$$
\begin{aligned}
& \Delta a=-1.54\left(\mathrm{~A}_{3} / \mathrm{A}_{1}\right)+1.61\left(\mathrm{~B}_{3} / \mathrm{B}_{1}\right), \\
& \Delta \Gamma=1.84\left(\mathrm{~A}_{3} / \mathrm{A}_{1}\right)+0.25\left(\mathrm{~B}_{3} / \mathrm{B}_{1}\right) .
\end{aligned}
$$

Here the $\Delta I=3 / 2$ amplitudes are expressed relative to the $\Delta I=1 / 2$ amplitudes. The numerical values of the coefficients depend on the ratio $B / A$. The uncertainties in the coefficients are small compared to the uncertainties in $\Delta a$ and $\Delta r$. Final-state $\pi N$ interactions have been included in these relations but have a very small effect. From the Stable Particle Table,

$$
\Delta a=0.006 \pm 0.066, \quad \Delta \Gamma=0.058 \pm 0.012 \text {, }
$$

and hence

and

$$
\left(A_{3} / A_{1}\right)=0.027 \pm 0.008
$$

$$
\left(B_{3} / B_{1}\right)=0.030 \pm 0.037 \text {. }
$$

The possible $3 \% \Delta \mathrm{I}=3 / 2$-wave amplitude is due to the disagreement of decay rates with prediction. At this level the results are sensitive to electromagnetic corrections. However, in $\Lambda$ decay the phase space correction and the other radiative corrections appear to be about equal in magnitude and have opposite signs, 5,6 and hence cancel each other in the correction to the decay rates.

(b) $\equiv$ Decay

The analysis for $\equiv$ decay is very similar to that for $\Lambda$ decay. If the $\Delta I=1 / 2$ rule is valid, $\Gamma_{0}\left(\Xi^{0}\right) / \Gamma_{-}\left(\Xi^{-}\right)=0.50$ and $a_{0}=a_{-}$. For this case the expressions linear in $\Delta I=3 / 2 A-$ and $B-$ wave amplitudes are 4

$$
\begin{aligned}
& \Delta a=1.37\left(A_{3} / A_{1}\right)-1.37\left(B_{3} / B_{1}\right), \\
& \Delta \Gamma=-1.44\left(A_{3} / A_{1}\right)-0.06\left(B_{3} / B_{1}\right) .
\end{aligned}
$$

From the Stable Particle Table,

$$
\Delta a=0.18 \pm 0.12, \quad \Delta \Gamma=0.066 \pm 0.020,
$$

and we find

and

$$
\begin{aligned}
& \left(A_{3} / A_{1}\right)=-0.038 \pm 0.014 \\
& \left(B_{3} / B_{1}\right)=-0.17 \pm 0.09 .
\end{aligned}
$$

(c) $\sum$ Decay

The traditional test of the $\Delta I=1 / 2$ rule in $\Sigma$ decay is that the amplitudes satisfy the relationship

$$
\sqrt{2} \Sigma_{0}^{+}+\Sigma_{+}^{+}-\Sigma_{-}^{-}=0 \text {. }
$$

Graphically this is equivalent to closing the $\Sigma$ triangle when the amplitudes are plotted on $A, B$ axes. Including $\Delta I \geq 3 / 2$ amplitudes in $\Sigma$ decay analysis, the " $\Sigma$ triangle" relationship becomes

$$
\sqrt{2} A_{0}+A_{+}-A_{-}=-3 \sqrt{2 / 5} A_{3}+\frac{2}{\sqrt{15}} A_{5} \text {. }
$$


where $A_{3}$ and $A_{5}$ are $\Delta I=3 / 2$ and $\Delta I=5 / 2$ amplitudes, respectively. There is a similar equation for the $B$ amplitudes. From Table I,

and

$$
\sqrt{2} A_{0}+A_{+}-A_{-}=0.22 \pm 0.09
$$$$
\sqrt{2} B_{0}+B_{+}-B_{-}=2.7 \pm 1.0 \text {. }
$$

If we neglect the $\Delta I=5 / 2$ amplitudes and assume all amplitudes to be real we can solve for possible $\Delta I=3 / 2$ amplitudes. The result is

$$
\frac{A_{3}}{A_{-}}=-0.061 \pm 0.024
$$

and

$$
\frac{B_{3}}{B_{+}}=-0.074 \pm 0.027 \text {. }
$$

Thus for hyperon decay, present experimental data limit $\Delta I=3 / 2$ amplitudes to less than about $5 \%$.

3. The Lee-Sugawara Relation

From Table I the Lee-Sugawara relation, $7,8 \sqrt{3} \Sigma_{0}^{+}+\Lambda_{-}^{0}-2 \equiv \Xi^{-}$ $=0$, is satisfied to $-0.07 \pm 0.11$ for the $A$ amplitudes, and to $3.0 \pm 1.9$ for the $B$ amplitudes.

\section{References}

1. J. P. Berge, in Proceedings of the 13th International Conference on High-Energy Physics, Berkeley, (1966) (University of California Press, Berkeley, 1967), p. 46.

2. N. P. Samios, International Conference on Weak Interactions, Argonne, (1965), p. 189.

3. J. D. Jackson, private communication (1973).

4. See O. E. Overseth and S. Pakvasa, Phys. Rev. 184, 1663 (1969). The expression for $\Gamma_{0} / \Gamma_{-}$for $\Lambda$ decay should read

$$
\begin{aligned}
& \frac{\Gamma_{0}}{\Gamma_{2}} \approx \frac{1}{2}\{1+3 \sqrt{2} \\
& \left.\times\left[\frac{S_{11} S_{33} \cos \left(\delta_{1}-\delta_{3}\right)+P_{11} P_{33} \cos \left(\delta_{11}-\delta_{31}\right)}{S_{11}^{2}+P_{11}^{2}}\right]\right\} .
\end{aligned}
$$

5. See A. A. Belavin and I. M. Narodetsky, Yadern. Fiz. $\underline{8}, 978$ (1968) [Soviet J. Nucl. Phys. 8,568 (1969)].

6. G. W. Intemann, private communication (1973).

7. See B. W. Lee, Phys. Rev. Lett. 12, 83 (1964).

8. See H. Sugawara, Prog. Theor. Phys. 31, 213 (1964).

\section{Appendix III}

\section{A. SU(3) CLASSIFICATION OF BARYON RESONANCES}

A. Barbaro-Galtieri

Lawrence Berkeley Laboratory

It is established that a symmetry higher than $\mathrm{SU}(3)$ is necessary to classify the known baryon resonances. However, many higher-symmetry schemes have been proposed, and even for $S U(6)$ various versions exist (for a review see Dalitz ${ }^{1}$ ). Since it is not clear which one of these schemes best fits the data, we do not review them here, but we report once again fits of baryon states into SU(3) multiplets.

For the reader's convenience, we collect here the relevant formulae.

Exact SU(3) symmetry predicts that all the members of a multiplet should have the same mass and the same couplings for decays into other multiplets. It has been found, however, that the members of the octet of stable baryons lie within $20 \%$ of their mean mass; therefore a symmetry-breaking interaction has been introduced by Gell-Mann and Okubo independently. ${ }^{2}$ In addition, for the isospin-0 vector mesons ( $\omega$ and $\phi)$, an additional symmetry-breaking interaction has been introduced

\begin{tabular}{|c|c|c|c|}
\hline Decuplet & $\Delta-\Sigma=\Sigma-\Xi=\Xi-\Omega$ & GMO & (1) \\
\hline Octet & $2(N+\equiv)=3 \Lambda+\Sigma$ & GMO & (2) \\
\hline $\begin{array}{l}\text { Octet- } \\
\text { Singlet }\end{array}$ & $\sin ^{2} \theta=\frac{\Lambda-M_{8}}{\Lambda-\Lambda^{\prime}}$ & $\begin{array}{l}\text { Mixing } \\
\text { angle }\end{array}$ & (3) \\
\hline & $M_{8}=\frac{2(N+\Xi)-\Sigma}{3}$ & GMO & (4) \\
\hline
\end{tabular}
by Sakurai ${ }^{3}$ to take care of octet-singlet mixing. The relevant formulae for masses and decay rates are given below.

Broken SU(3) gives:

Here GMO stands for the Gell-Mann-Okubo formula; the particle symbol indicates its mass. The formulae would be the same if squared masses were used. For the nonet case, $\Lambda$ is the "mostly-octet" particle, $\Lambda^{\prime}$ is the "mostly-singlet" particle.

\section{Decay Rates}

In terms of a relativistically invariant matrix element $T$, the decay rate for two-body decay of a resonance of mass $M_{R}$ is

$$
\Gamma \propto \frac{|T|^{2} R_{2}}{M_{R}},
$$

where $R_{2}=k / M_{R}$ is the two-body phase space factor. Since the numerator is an invariant, and since $\Gamma$ must transform as $1 / E$, we introduce the denominator $M_{R} \cdot{ }^{5}$ 
For meson decays (see below) the rates are calculated according to Eq. (5); for baryon resonance decays into $1 / 2^{+}$ baryons and $0^{-}$mesons, one next takes into account the fact that spin sums in $|T|^{2}$ introduce another factore $M_{R}$, cancelling the $1 / M_{R}$. We are then left with

$$
\begin{aligned}
\Gamma & =\frac{|T|^{2} k}{M_{R}} M_{N}, \text { for baryons } \\
& =\frac{|T|^{2} k}{M_{R}^{2}} M_{N}^{2}, \text { for mesons. }
\end{aligned}
$$

The powers of the nucleon mass $M_{N}$ or $M_{N}^{2}$ have been introduced so that we can treat $|\mathrm{T}|$ as dimensionless.

$|T|^{2}$ contains centrifugal barrier factors, which we call $B_{\ell}$. We then have

$$
\begin{aligned}
& \left.\begin{array}{l}
\text { Decuplet } \\
\text { Singlet }
\end{array}\right\} \Gamma=(\mathrm{cg})^{2} \mathrm{~B}_{\ell}(\mathrm{k}) \frac{\mathrm{M}_{\mathrm{N}}}{\mathrm{M}_{\mathrm{R}}} \mathrm{k} \\
& \text { octet } \quad \Gamma=\left(c_{D} g_{D}+c_{F} g_{F}\right)^{2} B_{l}(k) \frac{M_{N}}{M_{R}} k \\
& \begin{array}{l}
\text { Octet - } \\
\text { Singlet }
\end{array} \quad \Lambda=\mathrm{G}_{8} \cos \theta+\mathrm{G}_{1} \sin \theta \\
& \text { mixing }\left\{\begin{array}{l}
\text { Singlet } \\
\Lambda^{\prime}=-G_{8} \sin \theta+G_{1} \cos \theta
\end{array}\right. \\
& \text { with } \quad G_{8}=c_{D} g_{D}+c_{F} g_{F} \\
& \mathrm{G}_{1}=\mathrm{c}_{1} \mathrm{~g}_{1} \text {. }
\end{aligned}
$$

Here $c_{i}$ are the $S U(3)$ coefficients with the sign convention adopted in this article [see note in the Table of SU(3) Iscoscalar Factors and Fig. 2 in the text]. $M_{N}$ is the nucleon mass, $M_{R}$ is the resonance mass for which $\Gamma$ is calculated, $k$ is the center-of-mass momentum for the channel being considered, and $g_{i}$ are the relevant couplings. For the case of singlet-octet mixing, formula ( 8 ) has to be used in conjunction with (6) and (7). $G_{8}$ and $G_{1}$ represent the couplings for the multiplet, and $\Lambda$ and $\Lambda^{\prime}$ represent the couplings for the physical states.

The relation between $g_{D}, g_{F}$. and the parameter $\alpha$ is

$$
a=\left[\begin{array}{ll}
1+\frac{\sqrt{5}}{3} & \frac{g_{F}}{g_{D}}
\end{array}\right]^{-1} \text {. }
$$

Exact SU(3) predicts that the couplings $g_{i}$ for all the members of a multiplet are the same; however, since the symmetry is broken for the masses, it is probably broken for the widths. In the case of the $3 / 2^{+}$decuplet, for broken SU(3) sum rules have been derived by Becchi, ${ }^{6}$ Gupta, ${ }^{7}$ and Konuma ${ }^{8}$ independently. The form derived by Gupta relates the $g_{i}$ for the members of the decuplet by the relation

$$
2(\Delta+\equiv)=3 \Sigma^{*}(\Lambda \pi)+\Sigma^{*}(\Sigma \pi),
$$

where $\Sigma^{*}(\Lambda \pi)$ is the coupling for the $\Sigma(1385) \rightarrow \Lambda \pi$ decay and $\Sigma^{*}(\Sigma \pi)$ is the coupling for the decay $\Sigma(1385) \rightarrow \Sigma \pi$.

As mentioned in the text (Sec. IV B) the determination of the relative signs of resonant amplitudes can be useful in making an $\mathrm{SU}(3)$ assignment of resonances. In fact the resonant amplitude $T \propto \sqrt{x_{e} x_{i}} \propto G_{e} G_{i}$, where the subscript $e$ refers to the elastic channel and the $G_{e}, G_{i}$ are the couplings of Eqs. (6) through (9). Assuming that all $g_{i}$ are positive, the sign of the $G_{i}$ are dependent upon the sign of the Clebsh-Gordon coefficients $c_{i}$. Once a sign convention is adopted (we use the Levi-Setti ${ }^{9}$ convention, see Fig. 2 in the text) and the signs for a $\Sigma$ state $(I=1)$ and a $\Lambda$ state $(I=0)$ of known $S U(3)$ assignment have been chosen for reference, the signs of all the other amplitudes can be useful in determining multiplet assignments. For exact SU(3) all the decays of members of a decuplet have the same sign. For octets the relative sign depends upon the value of $g_{D} / g_{F}$ and the mixing angle, as seen from Eqs. (7) through (9).

\section{Fits to the Data}

Fits of baryon decay rates within $\mathrm{SU}(3)$ can be found in, among others, papers by Tripp, ${ }^{10,11}$ Levi-Setti, ${ }^{9}$ Samios, ${ }^{12}$ and Plane. ${ }^{13}$ The most recent fits were made by Barbaro-Galtieri ${ }^{14}$ and Samios. ${ }^{15}$ A fit of the decay rates within $S U(6)_{w}$ can be found in Litchfield et al. ${ }^{16}$ Analysis of the baryon mass spectrum using the quark shell model has been done by Jones et al. ${ }^{17}$ An analysis of baryon couplings in a quark model with chromodynamics has been done recently by Koniuk and Isgur. ${ }^{18}$

For our SU(3) analysis in fitting the data a choice for $\mathrm{B}_{\ell}$ has to be made. Plane ${ }^{13}$ tried two forms for $B_{\ell}$ :

(a) The form $B_{\ell}=(k r)^{2 l_{1}} D_{\ell}(k r), r$ being the radius of interaction and $D_{\ell}$ the polynomials in $k r$ given by Blatt and Weisskopf. ${ }^{19}$ Usually $r$ is taken to be 1 fermi. ${ }^{10}$

(b) The form $B_{\ell}=k^{2 \ell}$.

However, for final results form (b) was chosen. A discussion of the differences among these two forms has been given by Barbaro-Galtieri. ${ }^{20}$. As shown in Ref. 20, not only the values of the couplings, $g_{i}$, depend upon the form used for $B_{\ell}$, but also the value obtained for the mixing angle. For the $3 / 2^{-}$singlet, $\Lambda(1520)$, and the isospin-0 member of the octet, $\Lambda(1690)$, the mixing angles obtained in the two cases were

$$
\theta_{a}=\left(-16.1_{-1.3}^{+1.4}\right)^{\circ}, \theta_{b}=\left(-27.5_{-3.4}^{+3.6}\right)^{\circ} \text {. }
$$

in disagreement by a few standard deviations. However, if a 
radius of interaction of $r=0.15$ fermi was used for form (a), the two values of $\theta$ agreed. This value of $r$ does not fit resonance shapes when used in the Breit-Wigner resonant form.

Samios ${ }^{15}$ used form (b) for $B_{t}$.

Table $I$ is a summary of the fits made by us (update of Barbaro-Galtieri ${ }^{14}$ ) using the barrier factor form (a) and exact $\mathrm{SU}(3)$. The values of the masses, widths, and amplitudes used in the fits are taken from this edition's Tables and Listings.

\section{$1 / 2^{-}$Nonet (Baryon-Eta Resonances)}

For this nonet Eq. (7) was multiplied by the factor.

$$
\left[\frac{M_{R}-M_{B}}{M_{R}-M_{B}}\right]^{2} \text {, }
$$

where $M_{B}$ is the decay baryon and $\bar{M}_{R}-\bar{M}_{B}=564 \mathrm{MeV}$ is the difference of the mean $1 / 2^{-}$and $1 / 2^{+}$baryon octet masses. This kinematic factor comes from PCAC arguments (i.e., the assumption that the axial vector current remains an octet in the presence of symmetry breaking) and it was advocated by Graham. ${ }^{21}$ For the $1 / 2^{-}$nonet it was used in this form first by Gell-Mann. 22

\section{$3 / 2^{+}$Decuplet}

The agreement among the coupling constants obtained for the four rates in this decuplet is very bad. The fit made using form (a) for $B_{l}$ has $\chi^{2}=58$ for 3 degrees of freedom; the one made with form (b) for $B_{\ell}$ has $x^{2} / D F=13 / 3$. The broken $S U(3)$ relation (11), however, is very well satisified.

\section{B. SU(3) CLASSIFICATION OF MESON RESONANCES}

All of the discussion above applies, except that for bosons the GMO formula is usually applied to the square of the masses, as opposed to the first power for fermions. Thus for example, Eq. (2) becomes

$$
4 \hat{\mathrm{K}}=3 \hat{\eta}+\hat{\pi} .
$$

The symbol $\hat{K}$ was introduced by Glashow and Socolow ${ }^{4}$ for the square of the $K$ mass, etc.

Because of the difference between Eqs. $\left(5^{\prime}\right)$ and ( $\left.5^{\prime \prime}\right)$, there is also an extra factor of $\left(M_{N} / M_{R}\right)$ in Eqs. (6) and (7). The three established nonets $\left(0^{-}, 1^{-}, 2^{+}\right)$and their mixing angles are listed at the bottom of the Meson Table.

Table I. SU( 3 ) baryon multiplets with two or more known members. Values of $\theta$ and $a$ [defined by Eqs. (8) and (10)] are the result of fits made to all the

\begin{tabular}{|c|c|c|c|c|c|c|c|}
\hline${ }^{P}$ & & \multicolumn{3}{|c|}{ Octet members ${ }^{a}$} & Singlet & \multicolumn{2}{|c|}{$\theta(\operatorname{deg})^{b} \quad a$} \\
\hline $1 / 2^{-}$ & $N(1535)$ & $\Lambda(1670)$ & $\Sigma(1750)$ & $\left\{\begin{array}{l}{[\equiv(1850)]} \\
{[\equiv(1737)]}\end{array}\right\}$ & $\Lambda(1405)$ & $\left\{\begin{array}{r}-2 \pm 6 \\
-32 \pm 6\end{array}\right.$ & $\left.\begin{array}{l}0.94 \pm .14 \\
0.38 \pm .08\end{array}\right\}$ \\
\hline $3 / 2^{-}$ & $N(1520)$ & $\Lambda(1690)$ & $\Sigma(1670)$ & {$[\equiv(1819)]$} & $\Lambda(1520)$ & $-21 \pm 3$ & $0.32 \pm .04$ \\
\hline $5 / 2^{-}$ & $N(1670)$ & $\Lambda(1830)$ & $\Sigma(1765)$ & & & & $1.21 \pm .04$ \\
\hline \multirow[t]{2}{*}{$5 / 2^{+}$} & $N(1688)$ & $\Lambda(1815)$ & $\Sigma(1915)$ & {$[\equiv(2087)]$} & $\Lambda(2110)$ & $24 \pm 4$ & $0.72 \pm .02$ \\
\hline & & \multicolumn{3}{|c|}{ Decuplet members ${ }^{d}$} & $\mathrm{~g}_{10}$ & & \\
\hline $3 / 2^{+}$ & $\Delta(1232)$ & $\Sigma(1385)$ & $\Xi(1530)$ & $\Omega^{-}$ & $1.0-1.5$ & $2 / D F=58 /$ & \\
\hline $7 / 2^{+}$ & $\Delta(1950)$ & $\Sigma(2030)$ & & & & & \\
\hline
\end{tabular}
measured two-body decay rates of each mutiplet.

a Masses in parentheses are the nominal masses used in the Baryon Table. The Imembers have masses as calculated by using formulae (1) and (2) with the mixing angle $\theta$ derived from the decay widths.

bee text for a discussion of the $1 / 2^{-}$mixing angle.

bee text for a discussion of the $1 / 2$ mixing angle. of both $N(1535) \rightarrow \mathrm{N} \eta$ and $\Lambda(1670) \rightarrow \Lambda \eta$. The second values use a minus sign for the second amplitude. Both fits, however, have a bad $x^{2}$, mostly due to the two baryon $-\eta$ amplitudes.

dCoupling constants updated from Ref. 14, using new $\equiv(1530)$ data. 


\section{References}

1. R. H. Dalitz, in Fundamentals of Quark Models (Proceedings of the $17^{\text {th }}$ Scottish Universities Summer School in Physics, St. Andrews, August 1976), edited by I. M. Barbour and A. T. Davies, pg. 151.

2. M. Gell-Mann, Phys. Rev. 125, 1067 (1962); S. Okubo, Prog. Theor. Phys. (Kyoto) 27, 949 (1962).

3. J. J. Sakurai, Phys. Rev. Letters 9, 472 (1962).

4. The formula has been calculated from analogy with the formula for mixing of meson states, first put in this form by S. L. Glashow and R. H. Socolow, Phys. Rev. Letters 15, 329 (1966).

5. See. R. P. Feynman, Theory of Fundamental Processes, W. A. Benjamin, Inc., New York, 1962.

6. C. Becchi, E. Eberle, and G. Morpurgo, Phys Rev. 136B, 808 (1964).

7. V. Gupta and V. Singh, Phys. Rev. 135B, 1442 (1964).

8. M. Konuma and Y. Tomozawa, Phys. Lett. 10, 347 (1964).

9. R. Levi-Setti, in Proceedings of the Lund International Conference on Elementary Particles, Lund, 1969.

10. R. D. Tripp, in Proceedings of the 14th International Conference on High Energy Physics, Vienna, 1968, p. 173.

11. R. D. Tripp, in Proceedings of the 3rd Hawaiian Topical Conference on Particle Physics; UCRL-19361 (1969).

12. N. P. Samios, in Proceedings of the 15th International Conference oi High Energy Physics, Kiev, 1970, p. 187.

13. D. E. Plane et al., Nuclear Physics B22, 93 (1970). Also J. Meyer and D. E. Plane, Nuclear Physics B25, 428 (1971).

14. A. Barbaro-Galtieri, LBL-1366 and in Proceedings of the 16th International Conference on High Energy Physics, National Accelerator Laboratory, Vol. 1, page 159 (1972).

15. N. P. Samios, M. Goldberg, and B. T. Meadows in Hadrons and SU(3): A Critical Review, BNL Report BNL-17851 (1973)

16. P. J. Litchfield, R. J. Cashmore, and A. J. G. Hey in Proceedings of the Topical Conference on Baryon Resonances (Oxford, 1976), edited by R. T. Ross and D. H. Saxon, pg. 477.

17. M. Jones, R. H. Dalitz, and R. R. Horgan, Nucl. Phys. B129, 45 (1977).

18. R. Koniuk and N. Isgur, "Baryon Decays in a Quark Model with Chromodynamics", University of Toronto preprint, November 1979.

19. J. M. Blatt and V. F. Weisskopf, Theoretical Nuclear Physics, Wiley, New York, 1952.

20. A. Barbaro-Galtieri, in Properties of Fundamental Interactions, Erice, July 8-26, 1971, edited by A. Zichichi, Editrice Compositori, page 533 (1973).

21. R. Graham, S. Pakvasa, and K. Raman, Phys. Rev. 163, 1774 (1967).

22. M. Gell-Mann, R. Oakes, and B. Renner, Phys. Rev. 175,2195 (1968).

\section{Appendix IV}

\section{GROWTH OF INFORMATION}

From time to time we have presented figures demonstrating the amount of experimental work which has gone into spectroscopy, and the amount of new information available as a result. The 1980 versions of these figures are shown as Figs. 1 and 2 .

Figure 1 is a simple count of the number of meson resonances listed in the Tables, categorized as those "understood" - - i.e., all quantum numbers are believed known -- and those simply "listed". The rapid recent increase in both of these categories occurred because of the discovery of the $\mathrm{J} / \psi$ and related particles.

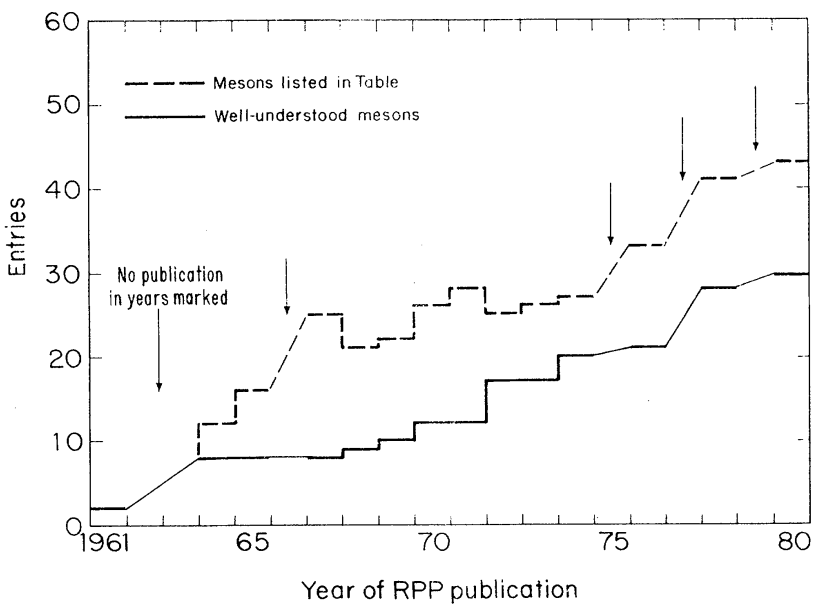

Fig. 1. Number of meson resonances listed in the Tables (dashed line) and those for which all quantum numbers are known (solid line), as a function of year of publication of the Review of Particle Properties.

In Figure 2 we present similar information for the baryon resonances, but concentrate here on the "growth of understanding". That is, the number of known baryons (we include for this figure only those with known $\mathrm{J}^{\mathrm{P}}$ ) has grown only very slowly vith time (dashed line); the real progress has been in the measurement of the properties of those baryons. Therefore we show as the solid line a count of the number of baryonic properties - - mass, width, and branching ratios. Most of these results are from partial-wave analyses. 


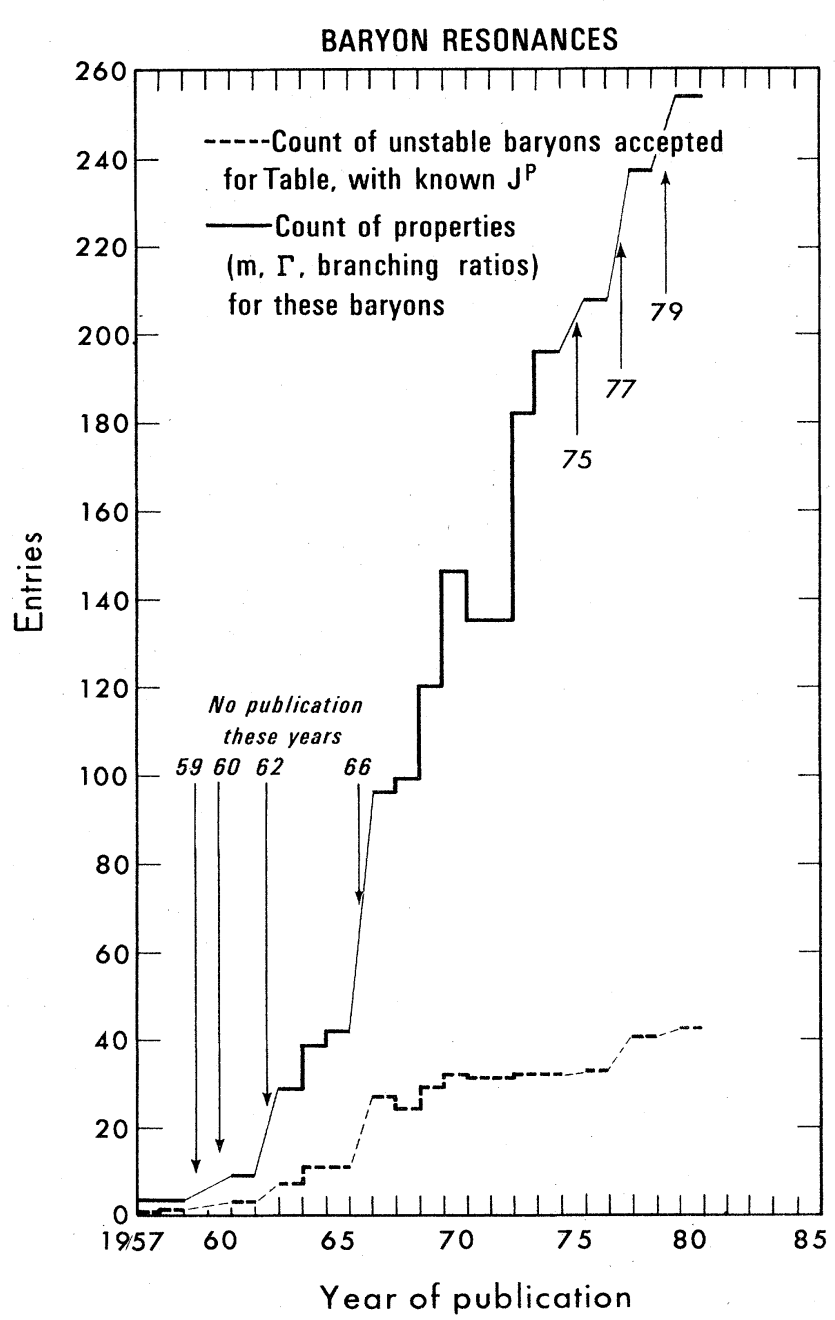

Fig. 2. Total amount of information (mass + width + branching ratios) on baryon resonances listed in the Tables, restricted to those with well-established ${ }^{P}$ (solid line). Dashed line shows numbers of such resonances listed. Abscissa shows year of publication of Review of Particle Properties.

A history of the values of some of the constants in the Review of Particle Properties is presented in Figs. 3-7. It may be said that one can estimate the age of a high energy physicist by asking him or her the mass of the $\Lambda$. If the answer is 1115.44 $\mathrm{MeV}$, he probably was deep into his graduate training in 1965.

A history of this sort has more than whimsical value. We may use it as a guide to develop a "feel" for the reliability of current values. In Fig. 3 we show how the generally accepted values for the speed of light and a couple of other constants have changed with time. The "generally accepted value" is usually an average over several experiments, performed by a compiler (in Fig. 3, the compiler is other than the Particle Data Group in all cases, although we do quote the compiled results). The abscissa on all these figures is the date of publication of the value shown. Clearly there is a general progression toward better understanding - - at least as measured by the size of the error bars. However, the size of the error bars does not tell the full story, as we can see by the frequency with which the "best" value has changed by more than one standard deviation. Changes in these values can come from several sources: a new experimental measurement, re-evaluation of an old measurement (which can come about if a previously unrecognized source of bias is discovered and corrected, or if a new value for one of the input constants, e.g. the electric charge, is available), or a change in the averaging procedure.

In Fig. 4 we show the history of some masses (including the $\Lambda$, for radioactive $\Lambda$ dating of your colleagues), based on averages which we ourselves performed. These are adapted from those originally presented by Rosenfeld ${ }^{1}$ in 1975. The publication date refers to the publication of the Review of Particle Properties.

In Fig. 5 we show the best estimates for the lifetimes of some of the particles stable against strong decay. These and subsequent figures have been compiled since publication of the Rosenfeld article. ${ }^{1}$ In Fig. 6 we show the widths of some of the resonances, and in Fig. 7, the values of some of the branching fractions. All values are taken from the Tables. Before 1964, very few branching fractions were listed in the Tables. In all cases, a representative sample is chosen, usually from those with a lot of activity (a limited number of special requests for a more complete set of such figures may be honored, for those seriously interested in the history of the "best" values of physical constants). In each figure, the heavy inner error bar represents the statistical error computed in the averaging procedure, and the thin outer error bars, when present, indicate the increase in the error due to the "scale factor". The scale factor is described in the int,roductory text, Sec. VII. It represents an attempt to quantify the increase in the uncertainty which is present in the case of experiments which disagree by more than a certain amount. In the case where the error represents an "educated guess," rather than a calculation, the inner error bar is absent.

On the whole, the number of times the values have changed by more than one standard deviation over the years is remarkably few. Even those branching fractions which involve rare decays and which are therefore presumably difficult to measure (Fig. 7) are, for the most part, within one or two standard deviations in 1978 of their value in any year since 1960. This is in spite of the vast amount of new experimental input, and indicates the general reliability of the results.

of course, the data points for a given quantity are hardly independent of each other, but those differing by several years frequently have quite different experimental input. The relative lack of change is a comment both on the experiments and on the averaging procedures. We, of course, are responsible only for the averages (except Fig. 3). These averages entail considerable exercise of judgment: there are conflicting experiments, experiments with impossibly small errors, "preliminary" results, and so forth. Statistical procedures will tell us that two experiments do not agree; they do not give a clue as to which (if either) is a good representation of the truth. 

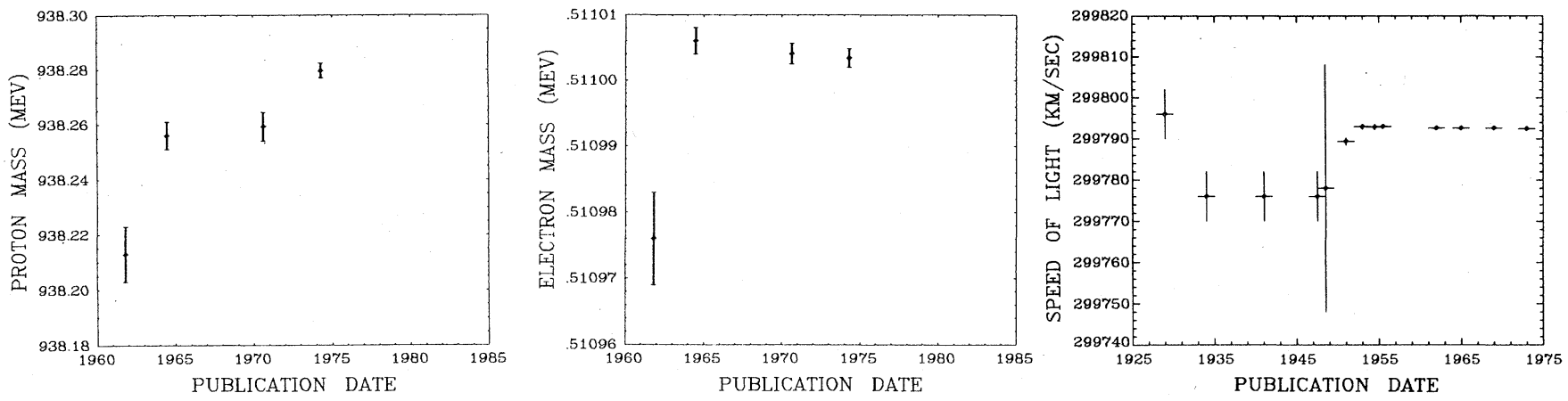

Fig. 3. The "generally accepted values" of the proton mass, the electron mass, and the speed of light, as a function of the publication date of the compilation used (not done by the Particle Data Group). Data for the speed of light plot courtesy of E. R. Cohen, Rockwell International Science Center. See the Stable Particle Data Card Listings for references on proton and electron masses.
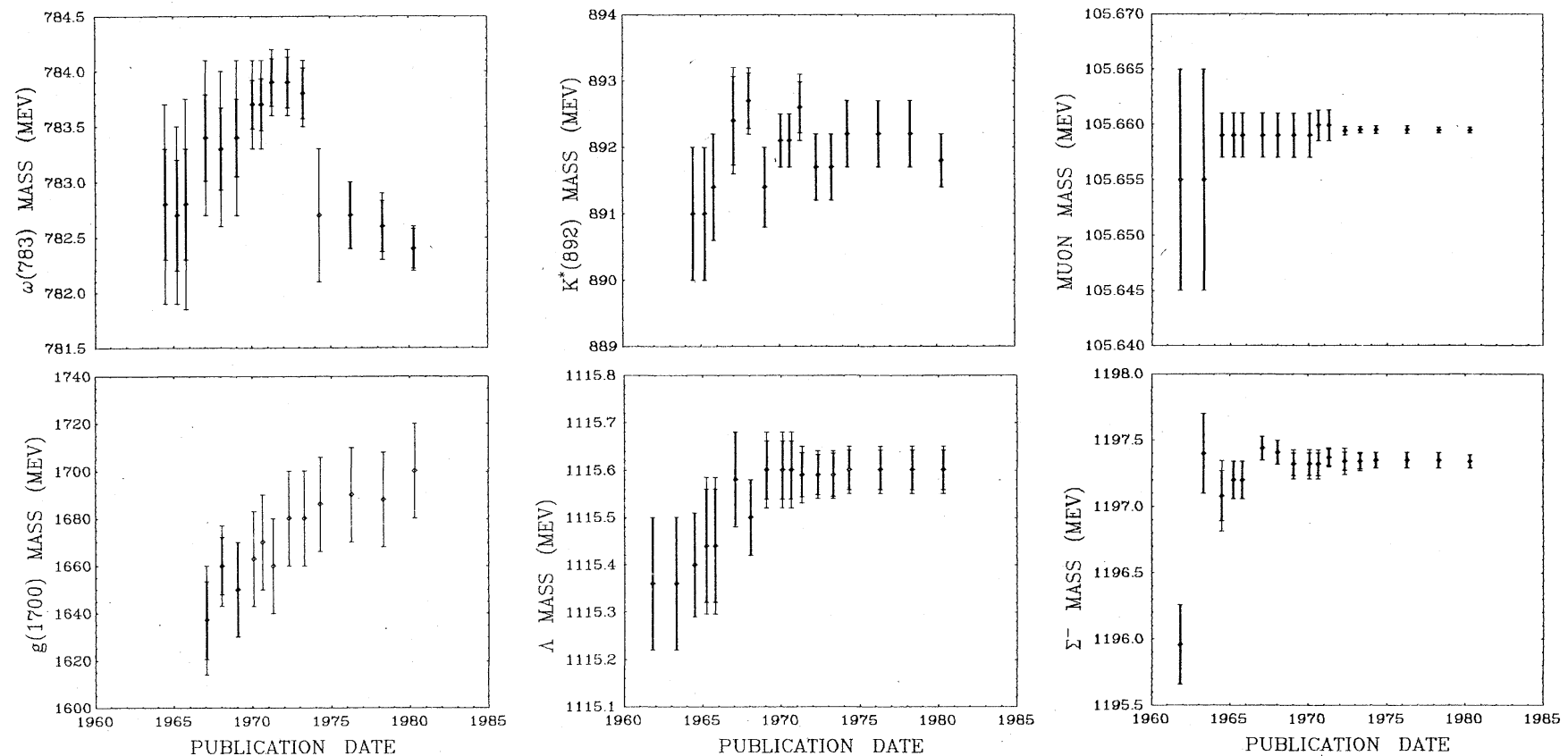

Fig. 4. Particle Data Group averages of the masses of various particles, as a function of date of publication of Review of Particle Properties (Adapted, with permission, from Annual Review of Nuclear Science, Volume 25. Copyright 1975 by Annual Reviews, Inc. All rights reserved). Full error bar indicates quoted error; thick-lined portion indicates quoted error with "scale factor" removed (see Sec. VII of introductory text).
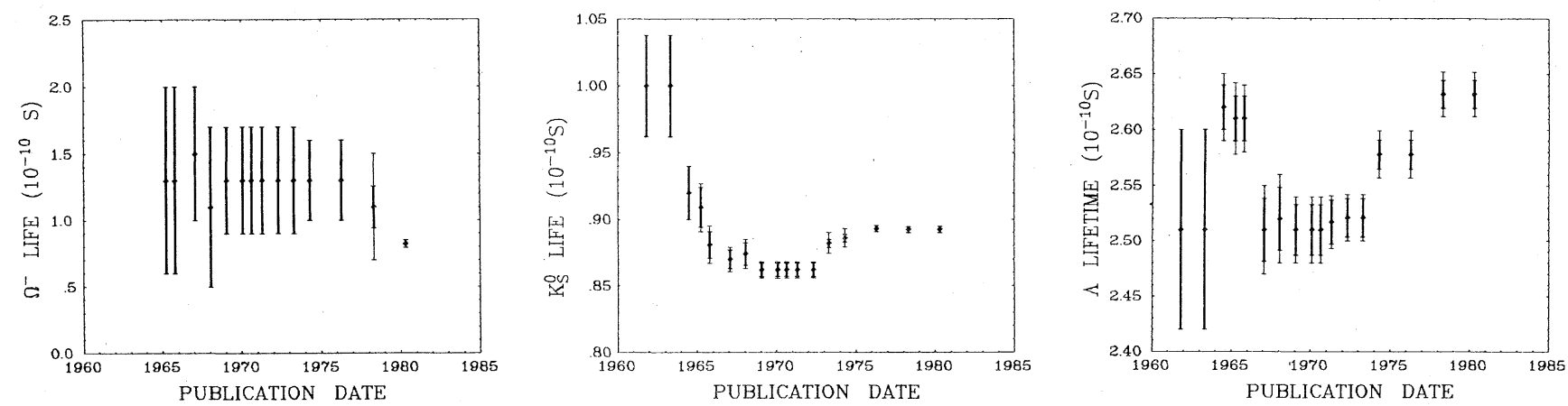

Fig. 5. Particle Data Group averages of the lifetimes of various particles, as a function of publication date of RPP. 

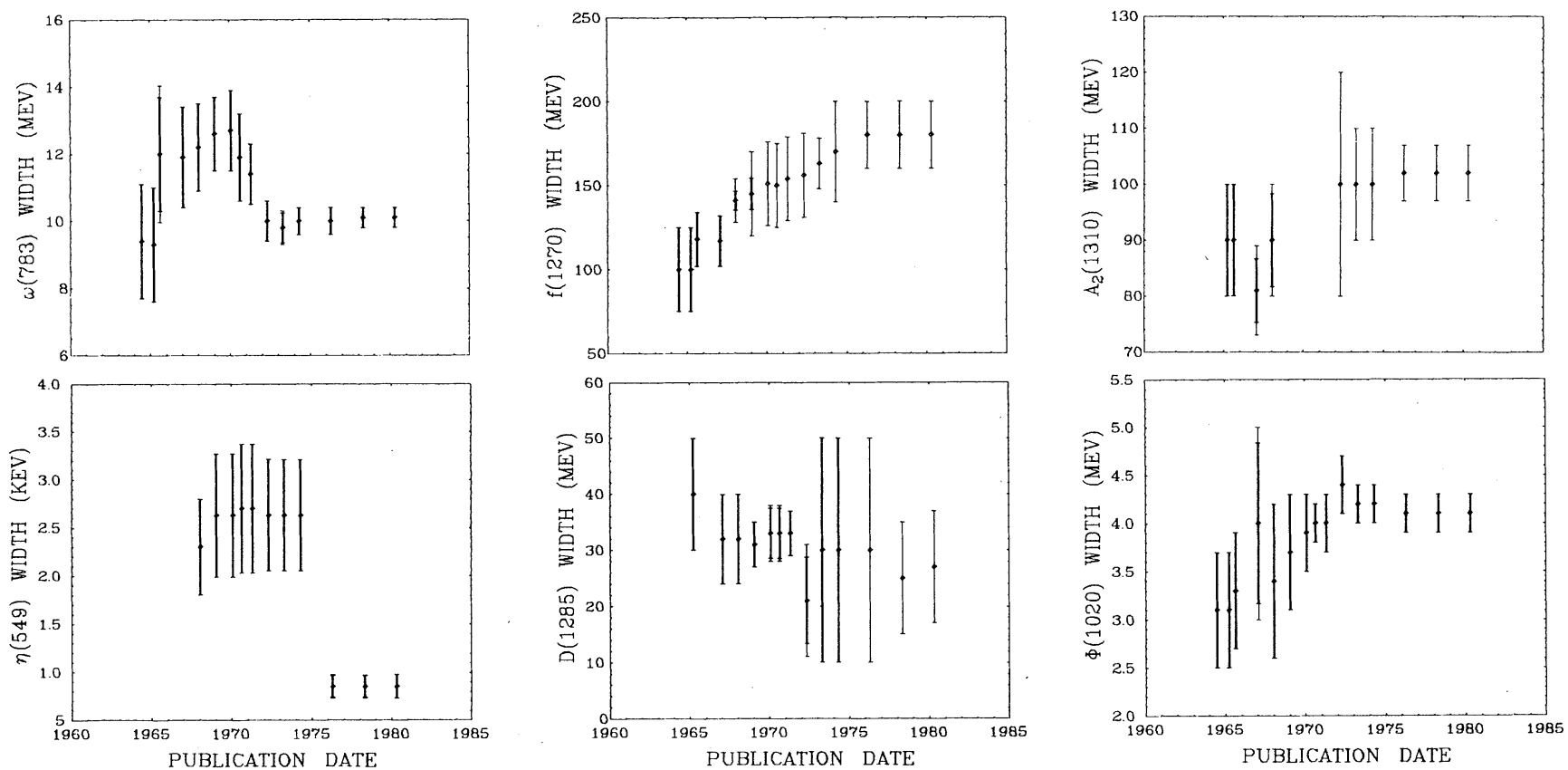

Fig. 6. Particle Data Group averages of the widths of various resonances, as a function of date of publication of RPP. The gap in the $A_{2}$ data indicates the years when the $A_{2}$ was thought to be split.
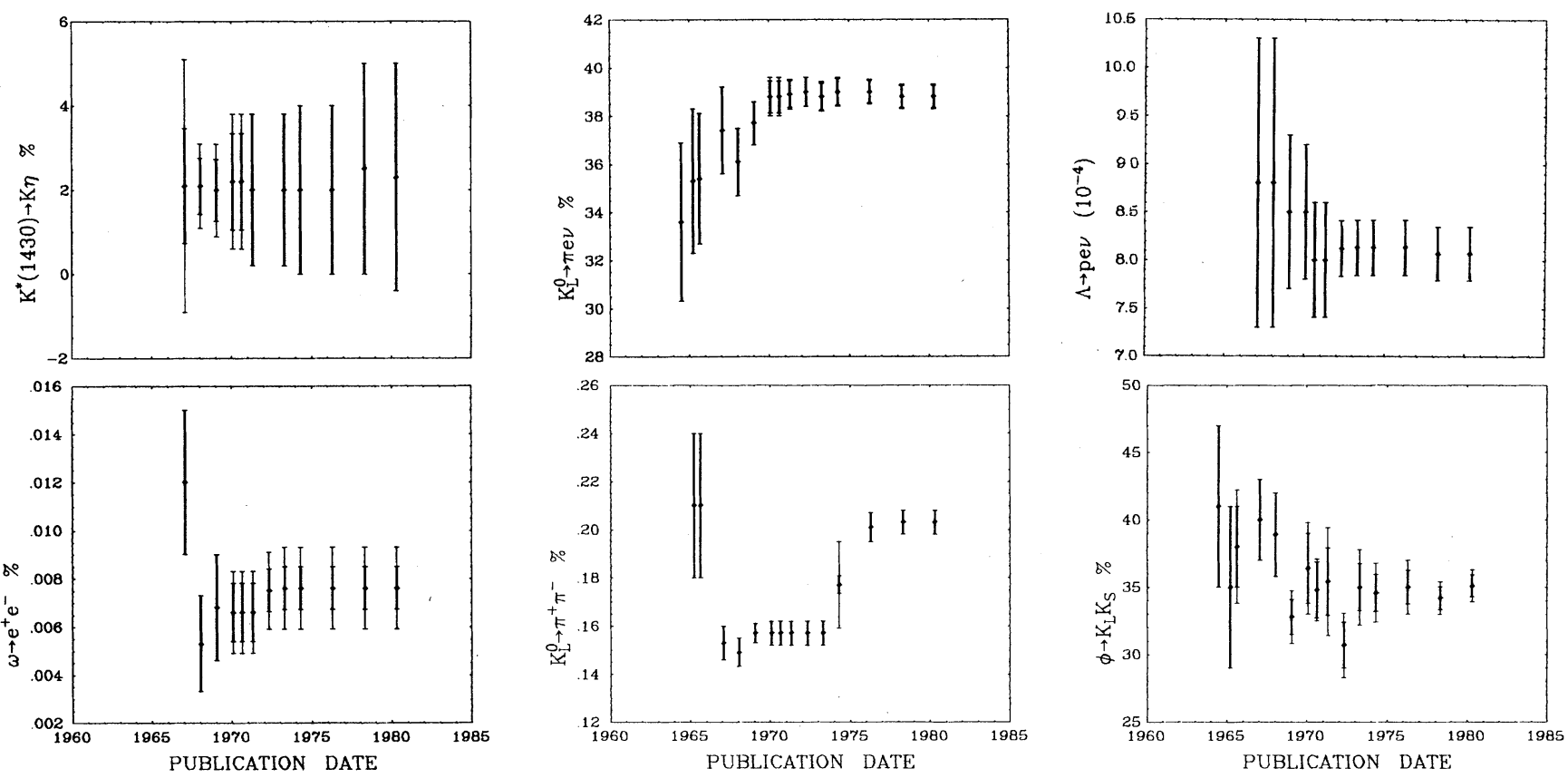

Fig. 7. Particle Data Group averages of various branching fractions, as a function of date of publication of RPP. 
Major decisions, and their motivations, are usually discussed on a case-by-case basis in the Data Card Listings; general comments may be found in Sec. II of the text and in Rosenfeld ${ }^{1}$. Note that, occasionally, the error bars increase from one publication to the next. This is usually the result of decision making by the compiler, e.g., to cease using a particular result, or because of new results in poor agreement with the old results.

We show these figures not only to demonstrate that there is not much change in these averages in the usual case, but also to show that there exist cases with relatively large changes. There is a psychological danger in preparing tables of "right" answers.
The old joke about the experimenter who fights the systematics until he or she gets the "right" answer (read "agrees with previous experiments"), and then publishes, contains a germ of truth (presumably, those who compile and average experimental results are also not immune to this disease). A result can disagree with the average of all previous experiments by five standard deviations, and still be right! Hence, perhaps it is of value to show that large changes can (and do) sometimes occur.

Reference

1. A. H. Rosenfeld, Ann. Rev. Nucl. Sci. 25,555 (1975). 